\title{
Review of particle properties
}

\section{Particle Data Group}

C.G. Wohl, R. N. Cahn, A. Rittenberg, T. G. Trippe, and G. P. Yost

Lawrence Berkeley Laboratory, ${ }^{\dagger}$ University of California, Berkeley, California 94720, USA

F.C. Porter

Department of Physics, California Institute of Technology, Pasadena, California 91125, USA

J. J. Hernandez and L. Montanet

CERN, European Laboratory for Particle Physics, CH-1211 Geneva 23, Switzerland

R. E. Hendrick

Department of Radiology, University of Colorado Medical Center, Denver, Colorado 80262, USA

R. L. Crawford

Department of Natural Philosophy, University of Glasgow, G12 8QQ Glasgow, Scotland

\section{Roos and N. A. Törnqvist}

Department of High Energy Physics, University of Helsinki, SF-00170 Helsinki 17, Finland,

and CERN, European Laboratory for Particle Physics, CH-1211 Geneva 23, Switzerland

G. Höhler

University of Karlsruhe, D-75 Karlsruhe, Federal Republic of Germany

M. Aguilar-Benitez

Junta de Energia Nuclear, Madrid 3, Spain,

and CERN, European Laboratory for Particle Physics, CH-1211 Geneva 23, Switzerland

\section{T. Shimada}

Meiji University, Kanda-Surugadai 1-1, Chiyoda-ku, Tokyo 101, Japan

M. J. Losty

Division of Physics, National Research Council, Ottawa, Ontario K1A OR6, Canada

G. P. Gopal

Rutherford Appleton Laboratory, Chilton, Didcot, Oxon. OX11 OQX, England

Ch. Walck

Department of Physics, University of Stockholm, S-11346 Stockholm, Sweden

R. E. Shrock

Department of Physics, State University of New York, Stony Brook, New York 11794, USA

\section{R. Frosch}

Swiss Institute for Nuclear Research, SIN, CH-5234 Villigen, Switzerland

\section{D. Roper and W. P. Trower}

Department of Physics, Virginia Polytechnic Institute and State University, Blacksburg, Virginia 24061, USA

B. Armstrong (Technical Associate)

Lawrence Berkeley Laboratory, ${ }^{\dagger}$ University of California, Berkeley, California 94720, USA

This review of the properties of leptons, mesons, and baryons is an updating of the Review of Particle Properties, Particle Data Group [Phys. Lett. 111B (1982)]. Data are evaluated, listed, averaged, and summarized in tables. Numerous tables, figures, and formulae of interest to particle physicists are also included. A data booklet is available.

${ }^{\dagger}$ The Berkeley Particle Data Group is supported by the Director, Office of Energy Research, Office of High Energy and Nuclear Physics, Division of High Energy Physics of the U.S. Department of Energy under Contract No. DE-AC03-76SF00098, and by the U.S. National Science Foundation under Agreement No. PHY83-18358. 


\section{CONTENTS}

\section{Introduction}

I. Overview

II. Authors and consultants

III. Reorganization

IV. Nomenclature

V. Procedures

A. Selection and treatment of data

B. Criteria for new states

C. Statistical procedures

1. Unconstrained averaging

2. Constrained fits

D. Discussion

Acknowledgments

References (for above sections)

Tables of Particle Properties

Stable particles

Mesons

Addendum

Baryons

Miscellaneous Tables, Figures, and Formulae

Physical constants (rev.)

Clebsch-Gordan coefficients, spherical harmonics, and $\mathrm{d}$ functions

SU(3) isoscalar factors

SU(n) multiplets and Young diagrams (new)

Tests of conservation laws (new)

Kinematics, decays, and scattering (new)

C.M. energy and momentum vs. beam momentum

Standard model of electroweak interactions (new)

Cabibbo and Kobayashi-Maskawa mixing (new)

model for deep inelastic scattering (new) S43

Nonrelativistic quark model (new) S44

Probability and statistics (rev.) S46

Particle detectors, absorbers, and ranges (rev.) $\quad$ S48

Electromagnetic relations (rev.) $\quad$ S58

Radioactivity and radiation protection (rev.) S59

Periodic table of the elements $\quad$ S60

Plots of cross sections and related quantities (rev.) S61

\section{Data Card Listings}

Illustrative key

S73

Stable particles

S76

Gauge bosons $\quad$ S76

Leptons $\quad \mathbf{S 7 7}$

Mesons $\quad$ S91

Baryons $\quad \mathbf{S 1 2 0}$

Searches $\quad$ S136

Mesons

$S=0$

S144

S144

$\mathbf{S}= \pm 1$

S193

Charmed mesons $\quad$ S203

Bottom (beauty) meson $\quad$ S204

Baryons

$\mathbf{S}=0$

S205

S205

S243

$\begin{array}{ll}S=-1 & S 245 \\ S=-2 & S 282\end{array}$

$S=-3 \quad S 288$

Charmed baryons $\quad \mathbf{S 2 8 8}$

Bottom (beauty) baryon $\quad \mathbf{S 2 8 8}$

Dibaryons $\quad \mathbf{S 2 8 8}$

\section{Appendices}

I. Status of the standard model of electroweak interactions (new)

II. The perturbative QCD coupling constant (new) S294

III. Kobayashi-Maskawa mixing matrix (new) S296

Recent Particle Physics Compilations

Accessing and Using Particle Physics Databases

Index

\section{OVERVIEW}

This review is an updating through December 1983 of the Review of Particle Properties [Particle Data Group (1982)], a compilation of experimental results on the properties of particles studied in elementary particle physics. These properties include masses, widths or lifetimes, branching ratios, and other experimentally determined properties. Where feasible, we provide a suggested "best" value of each parameter based on our own judgment, using the best available data. A discussion of some of the procedures that we apply, and a brief review of the historical performance of averages of measurements, may be found below (Section V Part D).

The results of this compilation are presented in two sections, the "Tables of Particle Properties" and the "Data Card Listings." The Tables give our estimates of the properties of those states whose existence we consider well established. Our opinion of whether or not a particle's existence is well established can change as new data become available. We attempt to be conservative, so particles whose existence awaits confirmation are not included, even if they may be theoretically well understood. Reported states which have been omitted from the Tables are indicated there by a short arrow $(\rightarrow)$.

The Listings contain a complete record of our compiled data on all states whose existence is either well established or unconfirmed, but do not include reported states of historical interest only. All data used for the numerical estimates in the Tables are listed here, with references and our comments, if any. Those measurements considered recent enough or important enough to mention, but which for some reason were not used in the averaging, appear in parentheses.

The Listings also contain short reviews, which we call "mini-reviews," about subjects of particular interest or data which have particular problems. These are usually written by the same experts who have assisted with the data compilation itself. 
In the past, we have attempted to use the Listings as an archive of all reported data on particles of interest. This is no longer possible because the growth of information would require a 5 to $10 \%$ per year expansion in this Review. Therefore we refer interested readers to previous editions [for example, Particle Data Group (1982)] for references to data considered obsolete.

We categorize the particles into types, intended to correspond roughly to the different types of data and problems encountered:

STABLE PARTICLES - All particles stable under the strong interaction. This includes the truly stable particles as well as those which decay weakly or electromagnetically, including the $\eta, D(1865), F, \Lambda_{c}, W$, $Z^{0}$, and so on.

MESONS - All meson resonances, including the $\psi, \chi$, and $r$ families.

BARYONS - All baryon resonances, including the resonant $\mathrm{N}$ and $\Delta$ families, dibaryon candidates, and so on.

This classification scheme is used to organize the Tables and the Listings.

We include a section of "Miscellaneous Tables, Figures, and Formulae." These are designed as a quick reference for the practicing elementary particle physicist. They normally presuppose some understanding of the subject matter, and do not attempt to serve as a textbook. We welcome all suggestions and comments regarding topics for inclusion or deletion, any errors or confusing passages, etc.

A pocket-sized Particle Properties Data Booklet is available. This contains the complete Tables of Particle Properties and the Miscellaneous Section, but not the Listings. For North and South America, Australia, and the Far East, write to Technical Information Department, Lawrence Berkeley Laboratory, Berkeley, CA 94720, USA. For all other areas, write to CERN Scientific Information Service, CH-1211 Geneva 23, Switzerland.

This year we are beginning a multiyear effort aimed at modernization and reorganization. The first fruits are visible immediately in the form of improved readability. We also include, for the first time, an Index at the back. The Miscellaneous Section has been almost completely reorganized. Some new material has been added, and some of the old material is now in different sections. A detailed description of the changes is given in Section III below.

\section{AUTHORS AND CONSULTANTS} lows:

The primary responsibilities of the authors are as fol-

(1) Stable particles: R. Frosch, T. Shimada, R.E. Shrock T.G. Trippe, W.P. Trower, and C.G. Wohl.

(2) Meson resonances: M. Aguilar-Benitez, J.J. Hernandez, M.J. Losty, L. Montanet, F.C. Porter, M. Roos, N.A. Törnqvist, and $\mathrm{Ch}$. Walck

(3) Baryon resonances: R.L. Crawford, G.P. Gopal, R.E. Hendrick, G. Höhler, L.D. Roper, and C.G. Wohl.

(4) General, including Introduction: All authors.

Of increasing importance to the production of this Review is a world-wide network of consultants, experts in particular topics. We wish to mention the following people with thanks:
- R.A. Arndt (Virginia Polytechnic Institute and State University)

- S. Aronson (BNL)

- W.B. Atwood (SLAC)

- C. Baltay (Columbia University)

- A. Barbaro-Galtieri (LBL)

- B. Barish (California Institute of Technology)

- A.V. Barnes (LBL)

- M.J. Berger (U.S. National Bureau of Standards)

- D. Besset (Stanford University)

- C. Bricman (CERN)

- W. Carithers (LBL)

- J. Carr (LBL)

- M.S. Chanowitz (LBL)

- J.M. Dorfan (SLAC)

- J. Engler (DESY)

- G. Feldman (SLAC)

- V. Flaminio (University of Pisa)

- F. Foster (University of Lancaster)

- M.K. Gaillard (LBL)

- G. Gidal (LBL)

- F.J. Gilman (SLAC)

- G. Goldhaber (LBL)

- M. Goldhaber (BNL)

- R. Hagstrom (ANL)

- G. Hall (Imperial College, London)

- I. Hinchliffe (LBL)

- J.H. Hubbell (U.S. National Bureau of Standards)

- J.D. Jackson (LBL)

- D.A. Jensen (University of Massachusetts at Amherst)

- J. Learned (University of Hawaii)

- G.M. Lewis (University of Glasgow)

- M. Matsuda (University of Hiroshima)

- T. Mizutani (Virginia Polytechnic Institute and State University)

- W.G. Moorhead (CERN)

- D.R.O. Morrison (CERN)

- B. Nefkens (University of California at Los Angeles)

- P. Némethy (LBL)

- O.E. Overseth (University of Michigan)

- S.I. Parker (University of Hawaii)

- N. Rivoire (CERN)

- D.N. Schramm (University of Chicago)

- M. Shaevitz (Nevis Laboratory)

- M. Suzuki (LBL)

- B.N. Taylor (U.S. National Bureau of Standards)

- J.A. Thompson (University of Pittsburgh)

- G.H. Trilling (LBL)

- R.D. Tripp (LBL)

- Y.S. Tsai (SLAC)

- L. Wolfenstein (Carnegie-Mellon University)

- G.B. Yodh (University of Maryland)

In addition, the Berkeley Particle Data Group has benefited from the advice of the PDG Advisory Committee, which meets annually to discuss matters of importance to the group, including the structure and content of this Review. The members of the 1983 committee are G. Feldman (SLAC) (chair), C.M. Lederer (University of California, Berkeley), J. Rosner (University of Chicago), R. Thun (University of Michigan), and L. Wolfenstein (CarnegieMellon University). In addition, G. Gidal (LBL) served in 1982.

The usefulness of this compilation depends in large part on the interaction between the users and the authors and 
consultants. We appreciate comments, criticisms, and suggestions for improvements of all stages of data retrieval, evaluation, and presentation.

\section{REORGANIZATION}

This year we have reorganized the Introduction and Miscellaneous Sections, added an Index (at the back), changed the Appendices to reflect modern interest, and improved the readability of most of the Review. This is the first stage of our modernization of the Review of Particle Properties system. The input of our readership into this process is welcome and encouraged. Many of the planned future changes will be internal, e.g., in the data-handling programs, but we do plan some additional improvements in the appearance and layout of the Review.

The changes in the Introduction Section have been to move the portions with physics discussion to other places. The discussion on the quantum numbers of mesons is now found (condensed) in the new Nonrelativistic Quark Model discussion in the Miscellaneous Section. The discussion of partial-wave amplitudes is partly in the Kinematics, Decays, and Scattering portion of the Miscellaneous Section and partly in some of the baryon mini-reviews, along with the discussion of sign conventions for resonance couplings. The conventions and parameters for weak and electromagnetic decays are now in various mini-reviews in the Stable Particle Section, preceding the appropriate particles.

The changes to the Miscellaneous Section and the Appendices are as follows. Sections which have been substantially revised or which are new appear in italics. Physical Constants:

General reorganization and updating. Most of the numerical constants have been removed to improve readability.

Clebsch-Gordan Coefficients, Spherical Harmonics, and

d Functions:

No change.

SU(3) Isoscalar Factors: No change.

SU(n) Multiplicities: Removed; see new SU(n) Multiplets and Young Diagrams Section.

SU(n) Multiplets and Young Diagrams: New.

Properties of Quarks: Replaced by Nonrelativistic Quark Model Section.

Tests of Conservation Laws: New.

Weak Interactions of Quarks and Leptons; Relativistic Kinematics; Lorentz Invariant Phase Space Formulae: Incorporated into the following five new sections:

Kinematics, Decays, and Scattering

Standard Model of Electroweak Interactions

Cabibbo and Kobayashi-Maskawa Mixing

Quark Parton Model for Deep Inelastic Scattering

Nonrelativistic Quark Model

C.M. Energy and Momentum vs. Beam Momentum: No change.

Probability and Statistics: Minor revisions.

Particle Detectors, Absorbers, and Ranges:

Revised. Silicon detectors added. Mean Range in
$\mathrm{Pb}, \mathrm{Cu}, \mathrm{Al}, \mathrm{C}$ figure revised. Photon Mass Attenuation figures slightly revised. Atomic and Nuclear Properties of Materials revised.

Electromagnetic Relations:

Revised to include motion of charged particles in magnetic and electric fields, conversion of units.

Radioactivity and Radiation Protection:

Revised to include SI units, lethal dose, other changes.

Periodic Table: No change.

Plots of Cross Sections and Related Quantities: Revised. New $\nu \mathrm{N}$ figure. Structure Functions expanded. New $\mathrm{e}^{+} \mathrm{e}^{-}$figures. $\mathrm{K}_{\mathrm{L}}^{0}$ Regeneration figure deleted. $\pi^{+} n\left(=\pi^{-} p\right)$ deleted.

Appendices:

Old Appendices $[\Delta I=1 / 2$ Rule, SU(3) Classification of Baryon Resonances, Growth of Information] deleted. Portions of the Growth of Information Appendix moved to Introduction, Section V Part D.

Appendix I: Status of the Standard Model of Electroweak Interactions: New.

Appendix II: The Perturbative QCD Coupling Constant: New.

Appendix III: Kobayashi-Maskawa Mixing Matrix: New.

\section{NOMENCLATURE}

Our particle name conventions are summarized in Table I.

Stable particles tend to have a well-accepted colloquial name, e.g., e, $\tau, \eta, \pi, \mathrm{K}, \mathrm{D}, \mathrm{B}, \Lambda, \Sigma$, etc., and we use that name. If a meson resonance has such a well-accepted name, we use that. If not, or if there is conflict in the literature, we follow the naming conventions of Table I. For baryon resonances, the naming conventions of Table I are regularly followed in the literature. All of the resonances incorporate the mass value in the name, e.g., $\rho(770), \omega(783), N(1440)$, $\Lambda(1520), \Sigma(1385)$, etc. This allows one to distinguish, e.g., the charmed and bottomed mesons, D and B (stable under strong decay), from the meson resonances $D(1285)$ and $\mathrm{B}(1235)$. It also distinguishes all of the $\mathrm{N}, \Delta, \Lambda$, etc., baryon resonances from one another.

The meson resonance naming convention of Table $I$ incorporates some information as to the spin-parity $\mathrm{J}^{\mathrm{P}}$ into the name. For a few such states believed to have identical quantum numbers, the colloquial names in the literature may incorporate primes into the name, e.g., $\rho(770)$, $\rho^{\prime}(1600), f(1270), f^{\prime}(1525)$, etc. However, we discourage this practice except for isosinglets within one $\mathrm{SU}(3)$ nonet. Thus $\eta^{\prime}(958)$ and $f^{\prime}(1525)$ keep their primes, whereas $\rho^{\prime}(1600)$ becomes $\rho(1600)$ in analogy with the radial excitations of the $\psi(3100)$ and the $\Upsilon(9460)$. We have taken the liberty of changing some colloquial meson names in accordance with Table I, having been urged for years to standardize the jungle of letters. The old names will still appear next to the new ones, to facilitate this conversion. We have restricted ourselves to relatively minor changes in this edition, but are considering more radical possibilities for the future. For example, it may be argued that, since the $D(1285)$ and the 
$E(1420)$ have the same quantum numbers, they should get the same letter in spite of historical considerations. For a second example, there are several possible states whose interpretation is unclear [e.g., $\iota(1440), \theta(1690), \xi(2220)$, $g_{S}(1240)$, and $\left.g_{T}(2240)\right]$. To the extent that some of these may be ordinary $q \bar{q}$ mesons, their exotic names should presumably be changed to more conventional ones. To the extent that some may not be ordinary mesons, it is less clear what naming convention, if any, should be adopted. We hope the reader will understand the rationale for the

Table I. Particle name conventions. These names are used in the absence of a different generally accepted name in the literature.

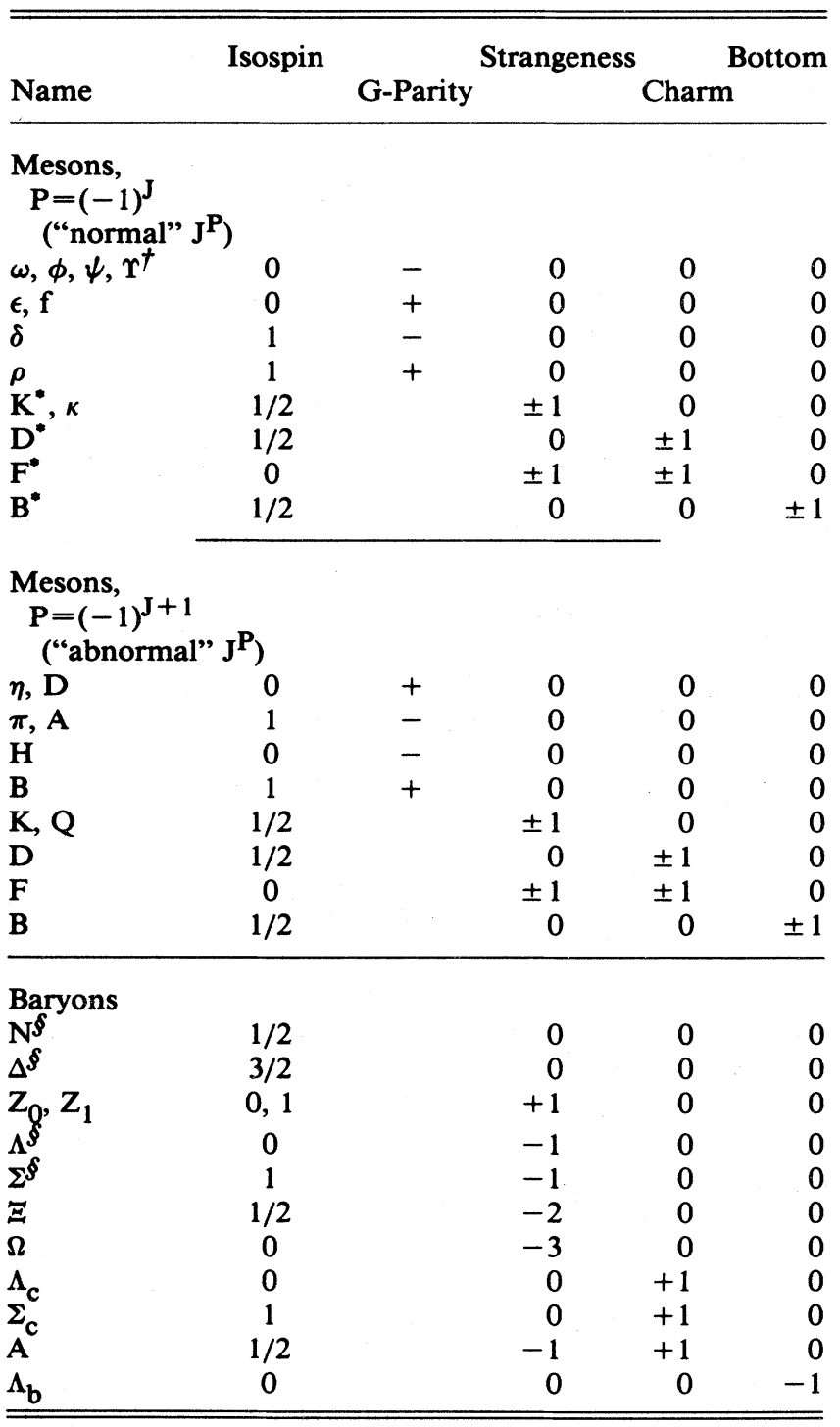

$\dagger^{\dagger}$ We use the symbol $\omega$ for $\mathrm{I}^{\mathrm{G}}=0^{-}$mesons which are

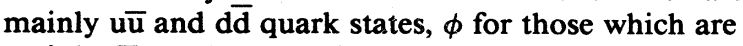
mainly ss quark states, $\psi$ for mainly $\overline{c \bar{c}}$ states, and $\Upsilon$ for mainly b $\bar{b}$ states.

$\S$ We occasionally use the symbol $\mathrm{N}^{*}$ to refer to the $\mathrm{N}$ and $\Delta$ resonances together, and $\mathrm{Y}^{*}$ to refer to the $\Lambda$ and $\Sigma$ resonances together. changes we have made and, in particular, not take any name change as an offense.

For baryon resonances, the strangeness, charm, "bottomness," and isospin are incorporated into the name. The $J^{P}$ is listed separately along with the spectroscopic notation for the partial-wave amplitude in which the particle is produced or decays. Our convention for spectroscopic notation is as follows:

$$
\mathrm{L}_{2 \mathrm{I} \cdot 2 \mathrm{~J}}
$$

for $\mathrm{N}, \Delta$, and $\Xi$ resonances, and

$$
\mathbf{L}_{\mathbf{I} \cdot 2 \mathrm{~J}}
$$

for $\Lambda$ and $\Sigma$ resonances. Here the orbital angular momentum $L$ is represented by $S, P, D, F, \cdots$ as $L=0,1,2,3$, $\cdots$. For example, the $J^{P}=7 / 2^{-} \Lambda(2100)$ is $G_{07}$ and the $J^{P}=5 / 2^{+} \Delta(1905)$ is $F_{35}$. Where more than one resonance with the same quantum numbers exists, we add a prime to the lowest mass state, two primes to the second lowest, etc. Thus, for example, the $J^{P}=5 / 2^{+}$states $\Lambda(1820)$ and $\Lambda(2100)$ are listed as $F_{05}^{\prime}$ and $F_{05}^{\prime \prime}$, respectively.

Some discussion about quantum numbers is found under the Nonrelativistic Quark Model in the Miscellaneous Section.

\section{PROCEDURES}

\section{A. Selection and treatment of data}

The Listings contain a complete record of all relevant data known to us from the journals listed in the Illustrative Key at the start of the Listings. As a general rule, we do not include results from preprints or conference reports. It is our experience that preprinted results may change before publication. In some cases, such results may be cited but not used in computing the estimates given in the Tables. There are a few exceptions to this exclusion, which we decide on a case-by-case basis after consultation with the experimenters.

As mentioned earlier, we no longer attempt to maintain an archival record of data of historical importance only. Thus, in this edition, results from many early papers on the baryon resonances have been omitted.

If data are included in the Listings but not used in the final average given in the Tables, they are set off by parentheses. We give explanatory comments in some such cases. If no comment is given, the reason the data were excluded is one or more of the following:

- No error was given.

- The data were contained in a preprint or conference report.

- The result involves some assumptions we do not wish to incorporate.

- The measurement has poor signal-to-noise ratio, low statistical significance, or is otherwise of much poorer quality than other data which are available.

- The measurement is clearly inconsistent with other results which appear to be highly reliable (see discussion in Section V Part D below).

- The measurement is not independent of other results, e.g., it is from one of several partial-wave analyses, all of which use the same data, rendering averaging meaningless. 
In some cases, none of the measurements pass all these criteria and no statistically meaningful average is quoted. For example, the masses of many of the baryon resonances, obtained from partial-wave analyses, are quoted as a range thought to probably include the true value rather than as an average with error. This is discussed in more detail in some of the Listings mini-reviews in the Baryon Section.

Our treatment of upper limits is normally to quote in the Tables the strongest limit available from a single experiment. We do not average or combine upper limits except in a very few cases where they may be re-expressed as measured numbers with Gaussian errors.

Our treatment of quantum number assignments is to indicate in the Tables those which are either well established or probable. For the Meson Table, we underline those which we consider well established; the others are inferred from whatever experimental evidence is available. In the Stable Particles Section, nearly all quantum numbers are well established and we do not underline; those which are not well established are indicated by a footnote.

As is customary, we assume that antiparticles are the result of operating with CPT on particles, so both share the same spins, masses, and mean lives. There is a new entry in the Miscellaneous Section, Tests of Conservation Laws, which contains tests of CPT and other conservation laws.

\section{B. Criteria for new states}

An experimentalist who sees indications of a new state will of course want to know what has been seen in that region in the past. Hence, we include in the Listings all reported states which have not been, in our opinion, disproved by better (e.g., more reliable) data.

For the Tables we are much more conservative in our judgment. We include only those reported states which we feel have a large chance of survival. An arrow $(\rightarrow)$ at the left of the Tables of Particle Properties indicates that a questionable candidate has been omitted from the Table in that mass region, but that it can be found in the corresponding part of the Data Card Listings. One's betting odds for survival are of course subjective; therefore no precise criteria can be defined. For more detailed discussions, see the mini-reviews in the Listings. In what follows we shall attempt to specify some guidelines.

(a) When energy-independent partial-wave analyses are available (mostly for $\pi \mathrm{N}$ resonances), approximate BreitWigner behavior of the amplitude appears to us to be the most satisfactory test for a resonance. We can check that the Argand plot follows roughly a left-hand circle, and that the "speed" of the amplitude also shows a maximum near the resonance energy; further, there should be data well above the resonance, showing that the speed again decreases. Indeed proper behavior of the partial-wave amplitude could accredit a resonance even if its elasticity is too small to make a noticeable peak in the cross section.

Of course even if Argand plots are available, it may still be a matter of opinion as to what behavior constitutes a resonance. Such an example is the $Z_{0}(1780)$ state seen in $\mathrm{KN}$ total cross-section experiments and in partial-wave analyses. The partial-wave analyses of Giacomelli (1974) and Martin (1975) find preferred solutions which exhibit a resonance-like loop in the $P_{01}$ wave near $1740 \mathrm{MeV}$. However, Giacomelli et al. and Martin point out that, despite the resonant-like appearance of the loop, the evidence for resonant energy dependence is inconclusive. Thus we omit the $Z_{0}(1780)$ from the Baryon Table. A similar quandary has existed for some time concerning the $Z_{1}(1900)$, and it too has been omitted from the Tables.

(b) When there are insufficient data to perform energyindependent analyses, one often resorts to energy-dependent partial-wave analyses. In this case Breit-Wigner behavior is an input. We therefore require that resonance solutions be found by several different analyses, preferably in different channels $(\overline{\mathrm{K}} \mathrm{N} \rightarrow \overline{\mathrm{K}} \mathrm{N}, \pi \Sigma$, etc.), before putting the claim in the Tables.

(c) Stable particles, most meson resonances, $\Xi$ resonances, and high-mass $N^{*}$ and $Y^{*}$ resonances fall into a category for which no partial-wave analyses exist. In general, we accept such states if they are experimentally reliable, of high statistical significance, or observed in several different production processes.

(d) Partial-wave analyses of three-body final states $(\pi \mathrm{N}$ $\rightarrow \pi \pi \mathrm{N})$ are also available. While these analyses are based on the isobar model $(\pi N \rightarrow \rho N, \pi \Delta$, etc.) and are subject to theoretical objections of varying importance, they provide increasingly reliable information on inelastic decay modes of otherwise-established resonances.

Thus, we enter into the Tables of Particle Properties only states for which there is experimentally convincing evidence, and we expect that nearly all of them will survive.

\section{Statistical Procedures}

We divide this discussion on obtaining averages and errors into two sections:

1. The unconstrained case, or "simple averaging;" and

2. The constrained case.

In what follows, the term "error" means one standard deviation $(1 \sigma)$; that is, for central value $\bar{x}$ and error $\delta \bar{x}$, the range $\bar{x} \pm \delta \bar{x}$ constitutes a $68.3 \%$ confidence interval.

\section{Unconstrained averaging}

We use a standard Gaussian procedure with a "scale factor" applied to the errors as our method of averaging the data. The Student's t-distribution, the basis of an earlier experiment of ours in data averaging, would give more conservative (and perhaps more realistic) errors at the twostandard-deviation $(2 \sigma)$ and higher level, but we do not choose to quote such errors. It is worth bearing in mind, however, that a $2 \sigma$ error might more realistically be somewhat larger than twice a $1 \sigma$ error, owing to the nonGaussian character of some sets of real measurements. This is a persistent problem in data averaging arising from the existence of mildly discrepant measurements.

We begin by assuming that measurements of a given quantity obey a Gaussian distribution, and thus we calculate a weighted average and error

$$
\begin{aligned}
& \bar{x} \pm \delta \bar{x}=\left(\sum_{i} w_{i} x_{i} / \sum_{i} w_{i}\right) \pm\left(\sum_{i} w_{i}\right)^{-1 / 2}, \\
& w_{i}=\left[1 /\left(\delta x_{i}\right)^{2}\right]
\end{aligned}
$$

where $x_{i}$ and $\delta x_{i}$ are the value and error, respectively, reported by the $i$ th experiment, and the sums run over $N$ experiments. We also calculate $\chi^{2}$ and compare it with its expectation value of $N-1$. 
If $\chi^{2} /(N-1)$ is less than or equal to 1 , and there are no known problems with the data, we accept the above results.

If $\chi^{2} /(N-1)$ is very large, or if there is prior knowledge of extremely large inconsistencies among experiments, we may choose not to average the data at all. Alternatively, we may quote the calculated average, but then give an educated guess as to the error; such a guess is generally a quite conservative estimate designed to take into account known problems with the data.

Finally, if $\chi^{2} /(N-1)$ is greater than 1 , but not greatly so, we still average the data, but then also do the following:

(a) We plot an ideogram to display the pattern of the data. Sometimes only one or two data points lie apart from the main body; other times the data split into two or more roughly equal-sized groups. The reader may use this information in deciding upon an alternative average, but caution is urged, as "outlying" data points are sometimes the "correct" ones. An example of such an ideogram is given in Fig. 1 below. Each experiment appearing in the plot is represented by a Gaussian with central value $x_{i}$, error $\delta x_{i}$, and area proportional to $1 / \delta x_{i}$. The choice of area is somewhat arbitrary; it assumes that an experimenter will work to reduce the systematic errors until they are slightly smaller (but seldom much smaller) than the statistical errors. Thus, as a physicist collects more events, he or she will use them both to reduce the statistical errors and to study the biases. Our confidence that a significant systematic error has not been made in a given experiment, as compared with other contradictory experiments, then tends to go up as $1 / \delta x_{i}$.

But why not assign a weight $1 /\left(\delta x_{i}\right)^{2}$, as is done when computing a weighted average? We feel that this assignment is equivalent to assuming that large systematic errors are as infrequent as large statistical fluctuations, and that this assumption is unrealistic.

We emphasize the difference between least-squares averaging (where the weighting factor is the inverse square of the error) and the ideograms prepared for visual display. The former arithmetic is of course best if one has unbiased

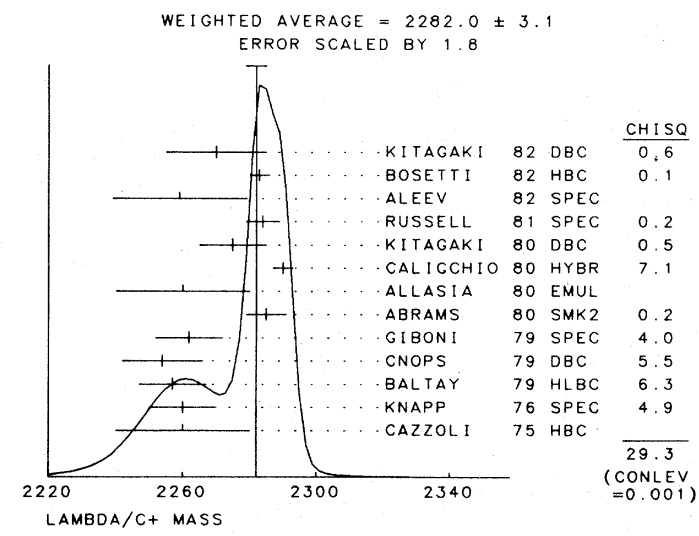

Fig. 1. Ideogram of measurements of the $\Lambda_{c}^{+}$mass. The vertical line indicates the position of the weighted average, while the horizontal bar atop the line gives the error in the average after scaling by the SCALE factor. Only those experiments indicated by + error flags were precise enough to be accepted in the calculation of the SCALE factor; the column on the far right gives the $\chi^{2}$ contribution of each of these experiments. The less precise experiments were included in the calculation of the weighted average, but not SCALE; they have $\perp$ error flags. data whose errors are well understood. In particular, the error analysis assumes that the true error on each datum is sampled from a Gaussian whose width is correctly reported. Then we obtain a narrow Gaussian distribution centered at the weighted mean for the answer. The ideogram (often multipeaked and certainly not Gaussian) is based on the opposite hypothesis that some of the input is systematically in error. The idea behind least-squares averaging is that experiments 1, 2, 3, etc., are all valid (so we should multiply their probabilities). Our ideograms are based on the assumption that 1 or 2 or 3 , etc., is valid, "hedged" with $1 / \delta x_{i}$ betting odds; we then add their probabilities. Both approaches cannot simultaneously be right; we allow the reader to choose. However, we quote the least-squares result in the Tables. This is the most precise value if the data satisfy the appropriate assumptions. A glance at the ideogram will show that the difference between the two approaches is usually not severe.

(b) The second way in which we try to take account of $\chi^{2} /(N-1)$ being greater than 1 is to scale up our quoted error $\delta \bar{x}$ in Eq. (1) by a factor

$$
\operatorname{SCALE}=\left[\chi^{2} /(N-1)\right]^{1 / 2}
$$

Our reasoning is as follows. Since we do not know which of the experiments are wrong, we assume that all experimentalists underestimated their errors by the same scale factor (2). If we scale up all input errors by this factor, $\chi^{2}$ becomes $N-1$, and of course the output error scales up by the same factor.

If we are to combine experiments with widely varying errors, we modify this procedure slightly. This is because it is the more precise experiments that most influence not only the average value $\bar{x}$, but also the error $\delta \bar{x}$. Now, on the average, the low-precision experiments each contribute about unity to both the numerator and the denominator of SCALE, hence the $\chi^{2}$ contribution of the sensitive experiments is diluted, i.e., reduced. Therefore, we evaluate SCALE by using only experiments for which the errors are not much greater than those of the more precise experiments, i.e., only those experiments with errors less than $\delta_{0}$, where the ceiling $\delta_{0}$ is (arbitrarily) chosen to be

$$
\delta_{0}=3 N^{1 / 2} \delta \bar{x} \text {. }
$$

Here $\delta \bar{x}$ is the unscaled error of the mean of all the experiments. Note that if each experiment had the same error $\delta x_{i}$, then $\delta \bar{x}$ would be $\delta x_{i} / N^{1 / 2}$, so each individual experiment would be well under the ceiling on SCALE.

This scaling approach has the property that if there are two values with comparable errors separated by much more than their stated errors (with or without a number of other experiments of lower accuracy), the error on the mean value $\delta \bar{x}$ is increased so that it is approximately half the interval between the two discrepant values.

We wish to emphasize the fact that our scaling procedures for errors in no way affect central values. In addition, if one wishes to recover the unscaled error $\delta \bar{x}$, one need only divide the given error by the SCALE factor for that error.

\section{Constrained fits}

Except for trivial cases, all branching ratios and rate measurements are analyzed by making a simultaneous 
least-squares fit to all the data and extracting the partial decay fractions $P_{i}$, the width $\Gamma$, the partial widths $\Gamma_{i}$, and the associated error matrix.

Assume, for a simple example, that a state has only three partial decay fractions, $P_{1}, P_{2}$, and $P_{3}\left(\Sigma P_{i}=1\right)$, which have been measured in four different ratios, $R_{1}, \cdots, R_{4}$, where, e.g., $R_{1}=P_{1} / P_{2}, R_{2}=P_{1} / P_{3}$, etc. $\ddagger$ Further assume that each ratio $r$ has been measured by $N_{r}$ experiments (we designate each experiment with a subscript $x$, e.g., $R_{1 x}$ ). We then find the best values of $P_{1}, P_{2}$, and $P_{3}$ by minimizing $\chi^{2}$ :

$$
\chi^{2}=\sum_{r=1}^{4}\left[\sum_{x=1}^{N_{r}}\left(\frac{R_{r x}-R_{r}\left(P_{1}, P_{2}, P_{3}\right)}{\delta R_{r x}}\right)^{2}\right] .
$$

In addition to the fitted values $\bar{P}_{j}$, we calculate an error matrix $\left\langle\delta \bar{P}_{i} \delta \bar{P}_{j}\right\rangle$. We tabulate the diagonal elements of $\delta \bar{P}_{i}$ $=\left\langle\delta \bar{P}_{i} \delta \bar{P}_{i}\right\rangle^{1 / 2}$ (except that some errors are scaled as discussed below). In the Listings we give the complete error matrix; we also calculate the fitted value of each ratio, for comparison with the input data, and list it below the relevant input, along with a simple unconstrained average of the same input.

Two further comments on the example above:

(1) There was no connection between measurements of the width and the branching ratios. But often we also have information on partial widths $\Gamma_{i}$ as well as total width $\Gamma$. In this case we must introduce $\Gamma$ as a parameter into the fit, along with the relations $\Gamma_{i}=\Gamma P_{i}, \Sigma \Gamma_{i}=\Gamma$. When appropriate, we tabulate the $\Gamma_{i}$ along with the $P_{i}$, and give error matrices in the Listings.

(2) We do not allow for correlations between input data. We do try to pick those ratios and widths which are as independent and as close to the original data as possible.

For asymmetric errors, we use a continuous function of $\delta(P)^{+}$and $\delta(P)^{-}$in the fitting. When no errors are reported, we merely list the data for inspection.

Inconsistent constrained data. According to Eq. (3), the double sum for $\chi^{2}$ is first summed over experiments $x=1$ to $N_{r}$, leaving a single sum over ratios

$$
\chi^{2}=\sum_{r} x_{r}^{2}
$$

We test for SCALE factors after the fit. Knowing the fitted $\chi_{r}^{2}$ and its expectation value $\left\langle\chi_{r}^{2}\right\rangle$, we form SCALE factors (just as before), i.e.,

$$
(\mathrm{SCALE})_{r}^{2}=\chi_{r}^{2} /\left\langle\chi_{r}^{2}\right\rangle,
$$

and if any (SCALE) $r$ is greater than 1 , all $N_{r}$ of the measurements of that particular ratio are equally penalized by having their errors increased by (SCALE) $r$. We then recycle the full fit, yielding new values $\delta \bar{P}_{i}^{\prime}$ for the errors in the partial decay modes, as well as new central values $\bar{P}_{i}^{\prime}$.

Because of the constraint $\left(\Sigma P_{i}=1\right)$, some of the new

$\ddagger$ We can handle any $R$ of the form $R=\sum \alpha_{i} P_{i} / \sum \beta_{i} P_{i}$, where $\alpha_{i}$ and $\beta_{i}$ are constants, usually 1 or 0 . The forms $R=P_{i} \cdot P_{j}$ and $R=\left(P_{i} \cdot P_{j}\right)^{1 / 2}$ are also allowed.
SCALE factors may still be greater than 1. If this is so, the whole procedure (i.e., increasing errors by the new SCALE factors and recycling through the fit) is repeated until the process converges.

At the end, we have final estimated errors $\delta \bar{P}_{i}^{\prime}$ for the $\bar{P}_{i}^{\prime}$. If SCALE factors have been used, they normally will have caused a shift in the central fitted values $\bar{P}_{i}^{\prime}$, as well as having given larger errors $\delta \bar{P}_{i}^{\prime}$. Often we find that the shift $\left|\bar{P}_{i}-\bar{P}_{i}^{\prime}\right|$ due to the SCALE factors is the same size as (or greater than) the $\delta \bar{P}_{i}^{\prime}$. We have decided to incorporate this shift into our errors as a reflection of the uncertainty due to the introduction of the SCALE factor; we tabulate an error

$$
\left(\delta \bar{P}_{i}\right)_{\mathrm{tab}}=\left[\left(\delta \bar{P}_{i}^{\prime}\right)^{2}+\left(\bar{P}_{i}-\bar{P}_{i}^{\prime}\right)^{2}\right]^{1 / 2},
$$

where $\bar{P}_{i}$ is the fitted value of the $i$ th partial decay mode before scaling, $\bar{P}_{i}^{\prime}$ is its value after all scaling, and $\delta \bar{P}_{i}^{\prime}$ is the error in $\bar{P}_{i}^{\prime}$. The SCALE factors we finally list in such cases are defined by

$$
(\mathrm{SCALE})_{i}=\left(\delta \bar{P}_{i}\right)_{\mathrm{tab}} / \delta \bar{P}_{i}
$$

However, in line with our policy of not letting SCALE affect the central values, we quote the values of $\bar{P}_{i}$ obtained from the original (unscaled) fit [which are always less than or equal to one standard deviation from $\bar{P}_{i}^{\prime}$, by construction of $\left.\left(\delta \bar{P}_{i}\right)_{\text {tab }}\right]$.

\section{Discussion}

The entire question of averaging data containing discrepant values is nicely discussed by Taylor (1982). He considers a number of algorithms which attempt to incorporate data which are not completely consistent into a meaningful average. Problems occur because it is very difficult to develop a procedure which handles simultaneously in a reasonable way two basic types of situations: (a) data which seem to lie apart from the main body of the data are incorrect (contain unreported errors); and (b) the opposite (the main body of the data is systematically wrong). Unfortunately, as Taylor shows, case (b) is not infrequent. His conclusion is that the choice of procedure is less significant than the initial choice of data to include or exclude.

We place a great emphasis on the choice of data to include or exclude. Unfortunately, the volume of data precludes spending as much time on the problem as we would like. We address this problem by soliciting the help of as many outside experts (consultants) as possible. In the final analysis, however, it is often impossible to determine which (if either) of two discrepant measurements is correct. Our SCALE factor technique is an attempt to address this ignorance by increasing the error above that suggested by least-squares analysis. In effect, we are saying that present experiments do not allow a precise determination of this constant because of unresolvable discrepancies, and one must await further measurements. The reader is warned of this situation by the size of the SCALE factor; he or she is then able to go back to the literature (via the Listings) and redo the average as desired.

Our situation with regard to discrepant data is easier to handle than most of the cases Taylor considers, such as estimates of the fundamental constants like $\hbar$, etc. Most of the errors in his case are dominated by systematic effects. In 
particle properties data, statistical effects are often at least as large as systematic effects, and statistical errors are usually easier to estimate. A notable exception occurs in partial-wave analyses, where different techniques applied to the same data yield different results. In this case, as stated earlier, we often do not attempt an average, but just quote a range of values.

A brief history of Particle Data Group averages is given in Rosenfeld (1975). Updated versions of some of Rosenfeld's figures are shown in Fig. 2. The least-squares error is shown by the thick portion of the error bars; the full error bar exhibits the SCALE factor extension.

Some cases of rather wild fluctuation are shown; this usually represents the introduction of significant new data or the discarding of some older data. Older data are sometimes discarded in favor of more modern data if it is felt that the newer data had fewer systematic errors, had more checks on their systematic errors, made some corrections unknown at the time of the older experiments, or some such reason. Near the time at which a large jump takes place, the SCALE factor sometimes becomes large, reflecting the uncertainty introduced by the new existence of partly inconsistent data.

By and large, a full scan of our history plots shows a rather dull progression toward greater precision at a central value completely consistent with the first data point shown. These plots are available on request from the Berkeley Particle Data Group.

We conclude that the reliability of the combination of experimental data and Particle Data Group averaging procedures is usually good, but it is important to realize that fluctuations outside of the quoted errors can and do occur, perhaps with more frequency than expected for truly Gaussian errors.

\section{ACKNOWLEDGMENTS}

The Particle Data Group wishes to acknowledge with appreciation the efforts made over many years by Robert Kelly. Kelly was in charge of the Baryon Section for a number of years and served a term as Berkeley group leader. His contributions will be missed, and we wish him well in his new endeavors.

We thank all those who have assisted in the many phases of preparing this Review. In particular, we acknowledge the usefulness of feedback from the physics community, especially those who have made suggestions or pointed out errors.

The European members of the Particle Data Group wish to acknowledge the generous support of CERN, in particular Division EP and Dr. A. Günther and his services.

\section{REFERENCES}

G. Giacomelli, et al., Nucl. Phys. B71, 138 (1974).

B.R. Martin, Nucl. Phys. B94, 413 (1975).

Particle Data Group: M. Roos, F.C. Porter, M. AguilarBenitez, L. Montanet, Ch. Walck, R.L. Crawford, R.L. Kelly, A. Rittenberg, T.G. Trippe, C.G. Wohl, G.P. Yost, T. Shimada, M.J. Losty, G.P. Gopal, R.E. Hendrick, R.E. Shrock, R. Frosch, L.D. Roper, and B. Armstrong, Phys. Lett. 111B (1982).

A.H. Rosenfeld, Ann. Rev. Nucl. Sci. 25, 555 (1975).

B.N. Taylor, "Numerical Comparisons of Several Algorithms for Treating Inconsistent Data in a Least-Squares Adjustment of the Fundamental Constants," U.S. National Bureau of Standards NBSIR 81-2426 (1982).
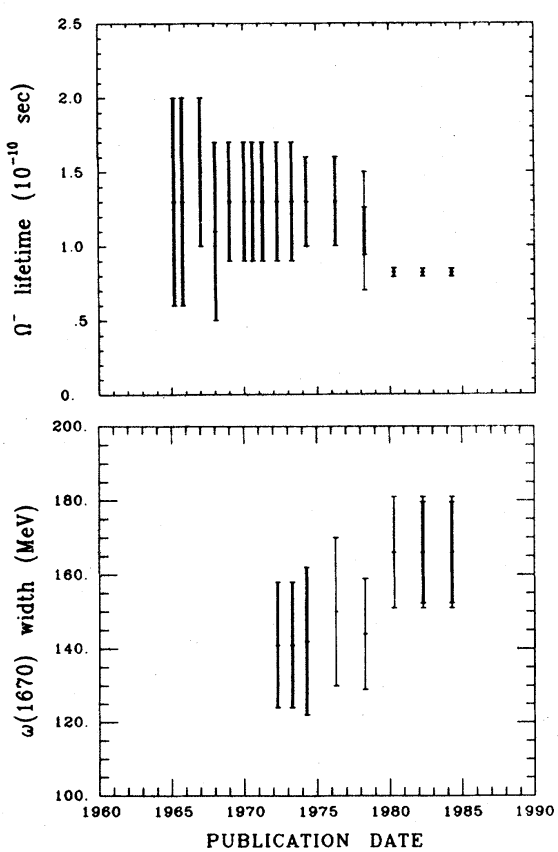
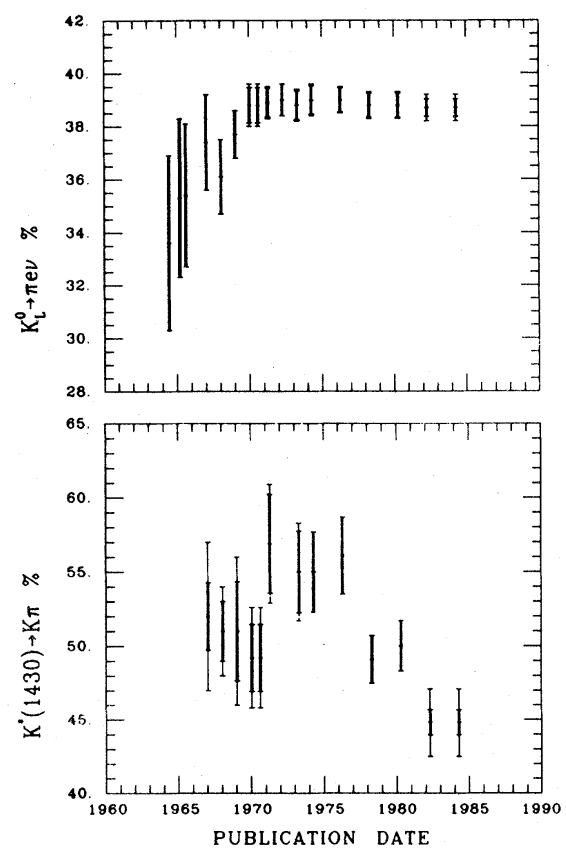
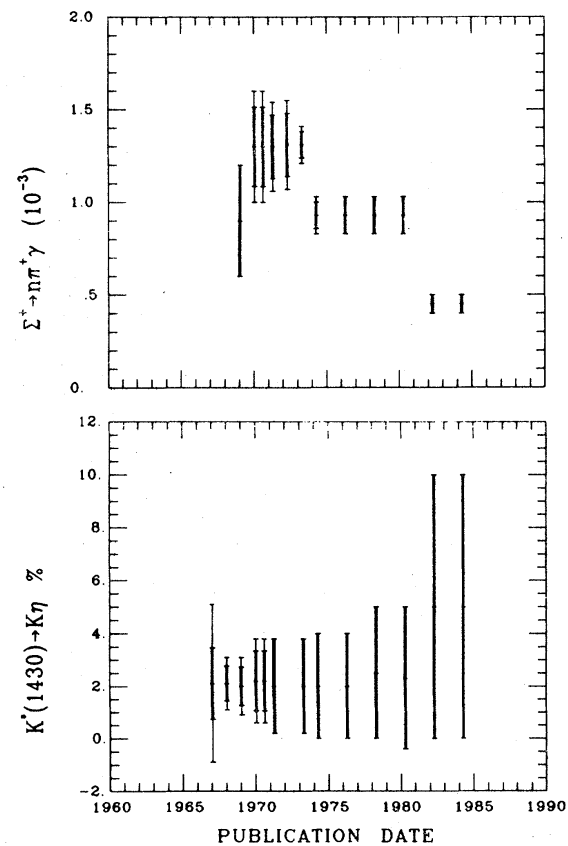

Fig. 2. Historical perspective of a few quantities tabulated in the Review of Particle Properties; abcissa specifies date of publication of the Review. Data measured by a variety of different techniques are included. The general reliability of the averages is good; very few are presently more than two standard deviations from their first tabulated values. Full error bar indicates quoted error; thick-lined portion indicates quoted error with "scale factor" removed (see Section V Part C above). 


\title{
TABLES OF PARTICLE PROPERTIES
}

\author{
April 1984
}

M. Aguilar-Benitez, R.N. Cahn, R.L. Crawford, R. Frosch, G.P. Gopal, R.E. Hendrick,

J.J. Hernandez, G. Höhler, M.J. Losty, L. Montanet, F.C. Porter, A. Rittenberg,

M. Roos, L.D. Roper, T. Shimada, R.E. Shrock, N.A. Törnqvist, T.G. Trippe,

W.P. Trower, Ch. Walck, C.G. Wohl, G.P. Yost, and B. Armstrong (Technical Associate)

(Closing date for data: Jan. 1, 1984)

\section{Stable Particle Table}

For additional parameters, see Addendum to this table.

Quantities in italics are new or have changed by more than one (old) standard deviation since April 1982.

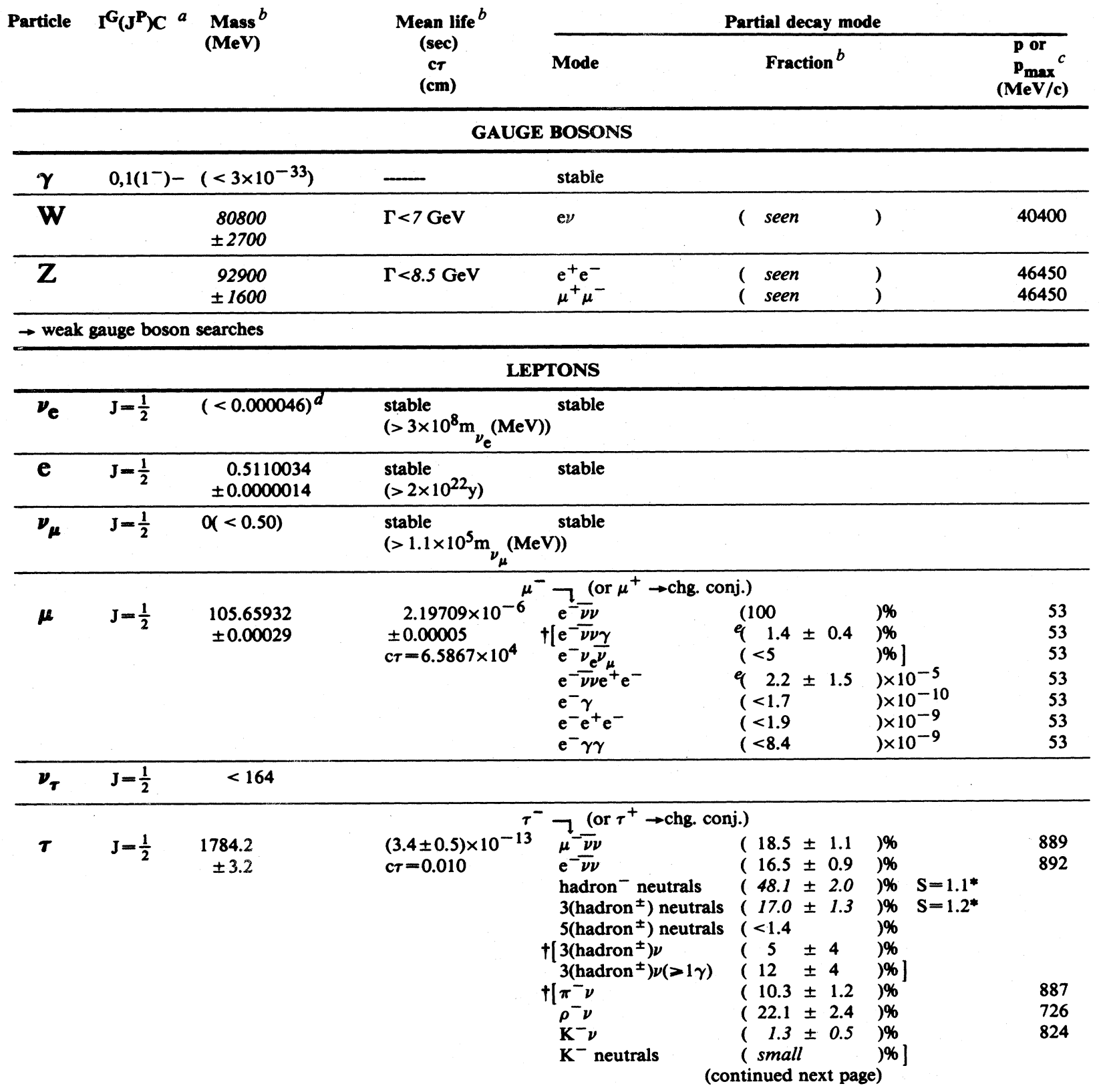




\section{Stable Particle Table (cont'd)}

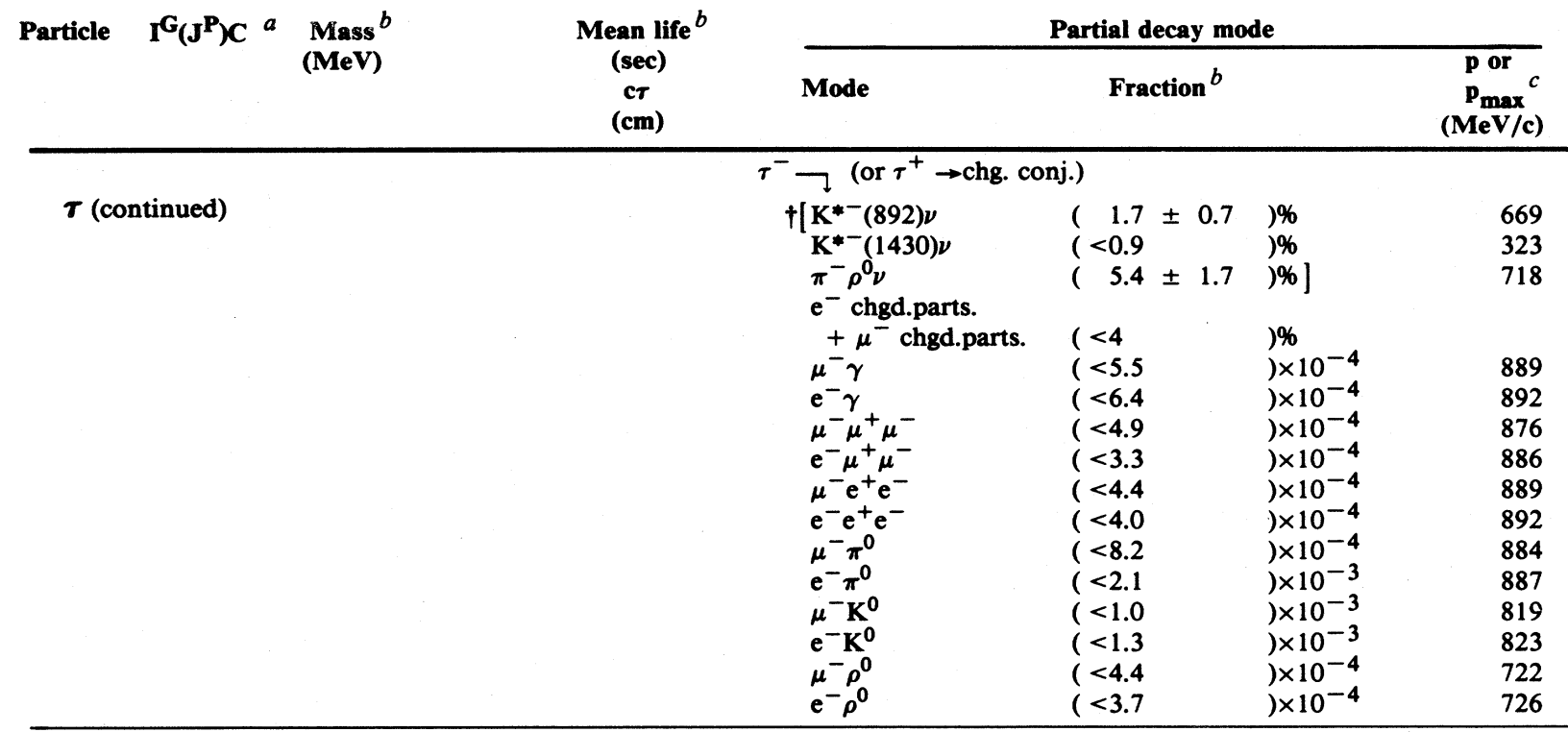

$\rightarrow$ searches for massive neutrinos and lepton mixing $\rightarrow \nu$ bounds from astrophysics and cosmology

$\rightarrow$ heavy lepton searches

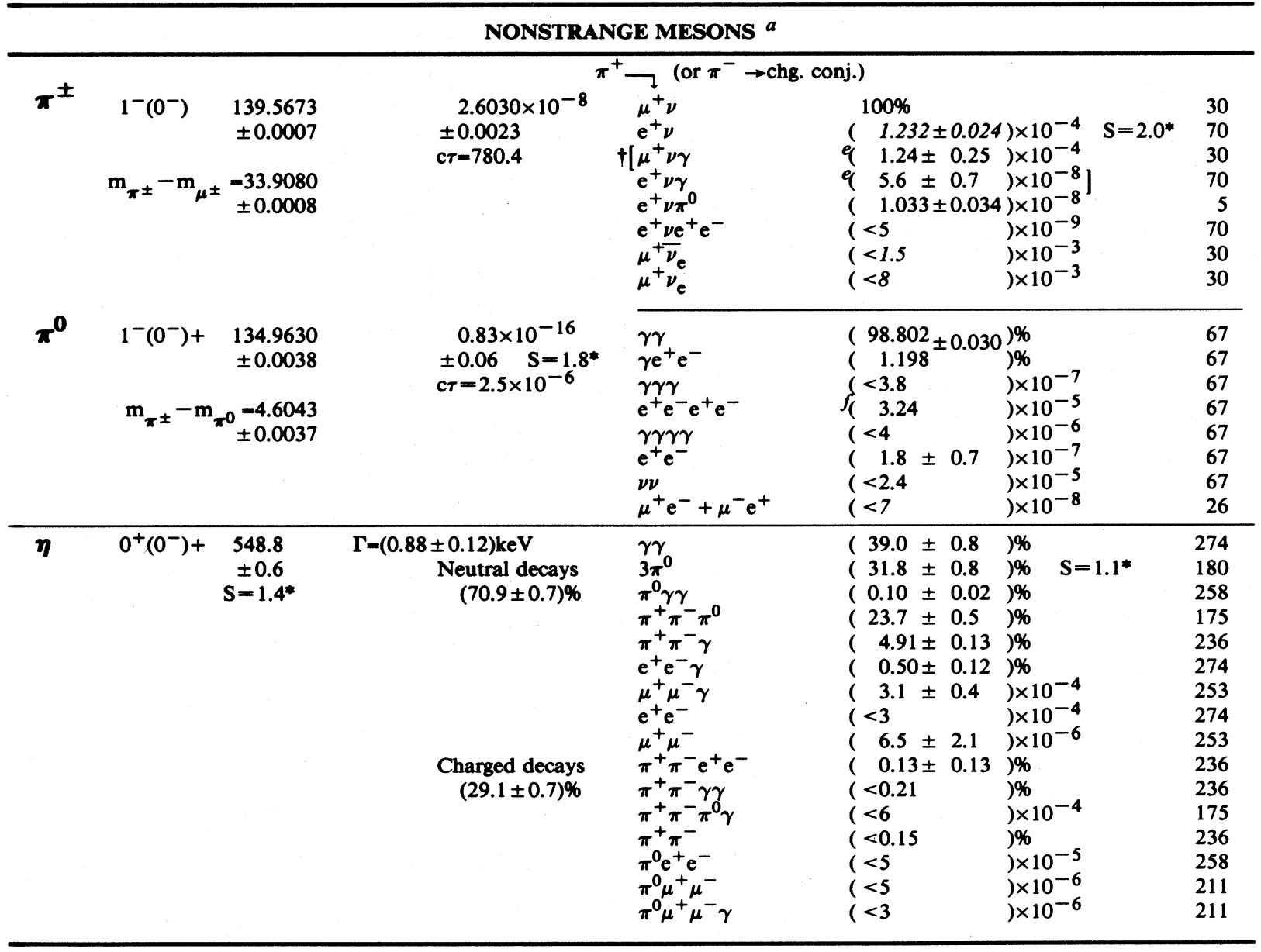


Stable Particle Table (cont'd)

\begin{tabular}{|c|c|c|c|}
\hline Particle & $\mathbf{I}_{\left(\mathbf{J}^{\mathbf{P}}\right)^{a}}$ & $\begin{array}{l}\text { Mass }^{b} \\
(\mathrm{MeV})\end{array}$ & $\begin{array}{c}\text { Mean life } \\
\text { (sec) } \\
\text { ct } \\
(\mathrm{cm})\end{array}$ \\
\hline
\end{tabular}

\begin{tabular}{ccc}
\multicolumn{3}{c}{ Partial decay mode } \\
\hline Mode & Fraction $^{b}$ & $\begin{array}{r}\text { p or } c \\
\mathbf{p}_{\max } \\
(\mathrm{MeV} / \mathrm{c})\end{array}$ \\
\hline
\end{tabular}

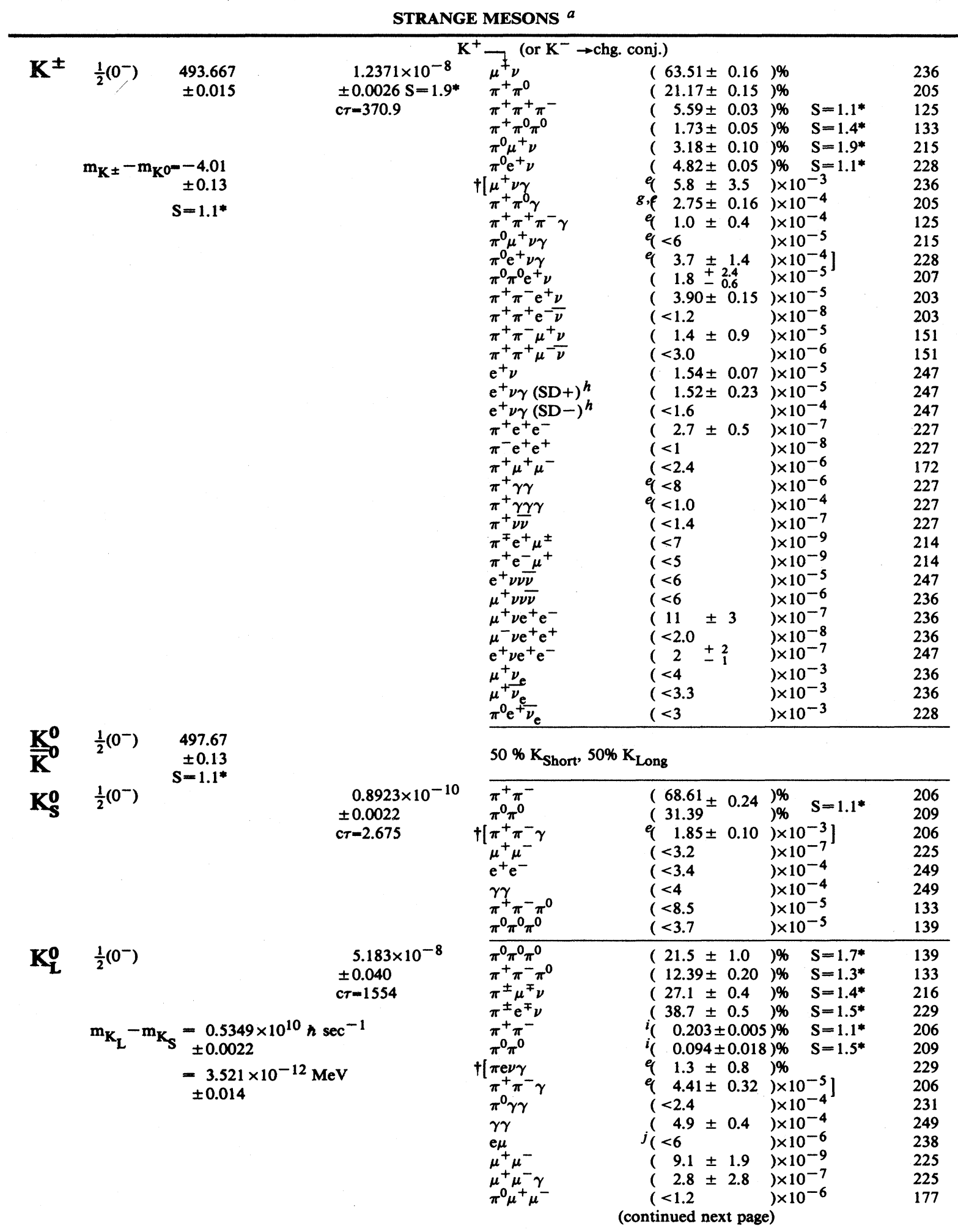


Particle Data Group: Review of particle properties

\section{Stable Particle Table (cont'd)}

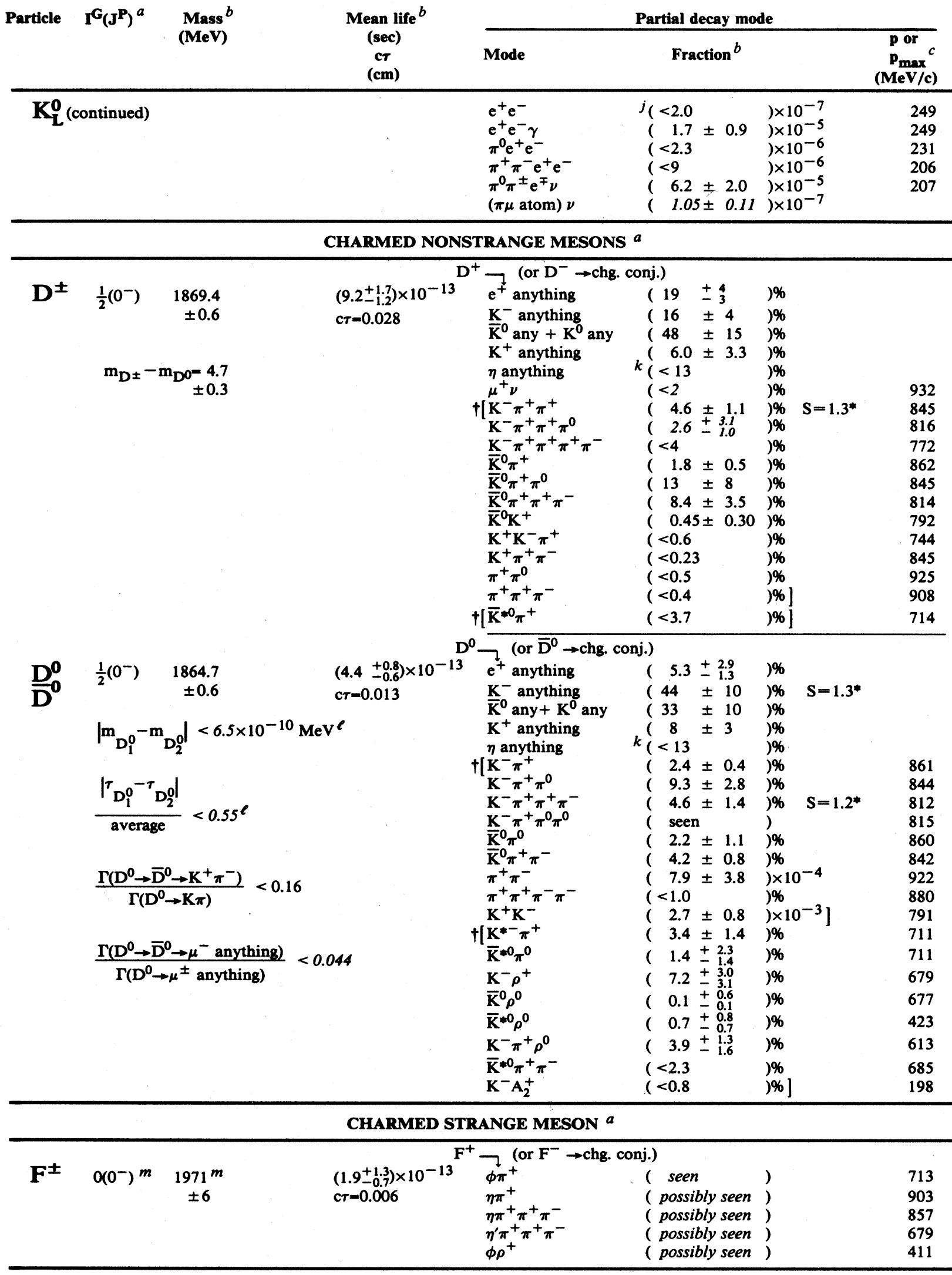


Stable Particle Table (cont'd)

\begin{tabular}{|c|c|c|c|c|c|c|}
\hline \multirow[t]{2}{*}{ Particle } & \multirow{2}{*}{$\underset{(\mathrm{MeV})}{\operatorname{Mass} b}$} & \multirow{2}{*}{$\begin{array}{c}\text { Mean life }{ }^{b} \\
(\mathrm{sec}) \\
\text { cr } \\
(\mathrm{cm})\end{array}$} & \multicolumn{4}{|c|}{ Partial decay mode } \\
\hline & & & Mode & Fraction $^{b}$ & & $\begin{array}{c}\text { p or } \\
\mathbf{p}_{\max } c \\
(\mathrm{MeV} / \mathrm{c})\end{array}$ \\
\hline \multicolumn{7}{|c|}{ BOTTOM MESONS ${ }^{a}$} \\
\hline \multicolumn{7}{|c|}{$\mathrm{B}^{+} \rightarrow$ (or $\mathrm{B}^{-} \rightarrow$ chg. conj.) } \\
\hline $\mathbf{B}^{ \pm} \quad \frac{1}{2}\left(0^{-}\right)^{n}$ & $\begin{array}{r}5270.8 \\
\pm 3.0\end{array}$ & & $\begin{array}{l}\overline{\mathrm{D}}^{0} \pi^{+} \\
\mathrm{D}^{*-} \pi^{+} \pi^{+}\end{array}$ & $\begin{array}{l}4.2 \pm 4.2 \\
(4.8 \pm 3.0\end{array}$ & $\%$ & $\begin{array}{l}2303 \\
2243\end{array}$ \\
\hline$\frac{\mathbf{B}_{0}^{0}}{\mathbf{B}^{0}} \quad \frac{1}{2}\left(0^{-}\right)^{n}$ & $\begin{array}{r}5274.2 \\
\pm 2.8\end{array}$ & & $\begin{array}{l}\text { (or } \overline{\mathrm{B}}^{0} \rightarrow \text { chg. } \\
\overline{\mathrm{D}}^{0} \pi^{+} \pi^{-} \\
\mathrm{D}^{*-} \pi^{+}\end{array}$ & 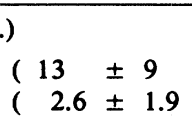 & $\begin{array}{l}\% \% \\
\%\end{array}$ & $\begin{array}{l}2298 \\
2253\end{array}$ \\
\hline $\begin{array}{l}\mathbf{B}^{ \pm}, \mathbf{B}^{0}, \overline{\mathbf{B}}^{\mathbf{0}} \\
\text { (not separated) }^{p}\end{array}$ & & $\begin{array}{l}(14 \pm 4) \times 10^{-13} \\
\mathrm{c} \tau=0.042\end{array}$ & $\begin{array}{l}\mathrm{e}^{ \pm} \nu \text { hadrons } \\
\mu^{ \pm} \nu \text { hadrons } \\
\mathrm{D}^{0} \text { anything } \\
\mathrm{K} \text { anything } \\
\mathrm{p} \text { anything } \\
\Lambda \text { anything } \\
\mathrm{e}^{+} \mathrm{e}^{-} \text {anything } \\
\mu^{+} \mu^{-} \text {anything }\end{array}$ & $\begin{array}{l}(13.0 \pm 1.3 \\
(12.4 \pm 3.5 \\
(80 \pm 28 \\
(\text { seen } \\
(>3.6 \\
(>2.2 \\
(<0.8 \\
(<0.7\end{array}$ & $\begin{array}{l}\% \% \\
\% \% \\
\% \% \\
\% \% \\
\% \% \\
\%\end{array}$ & \\
\hline
\end{tabular}

\section{NONSTRANGE BARYONS $a$}

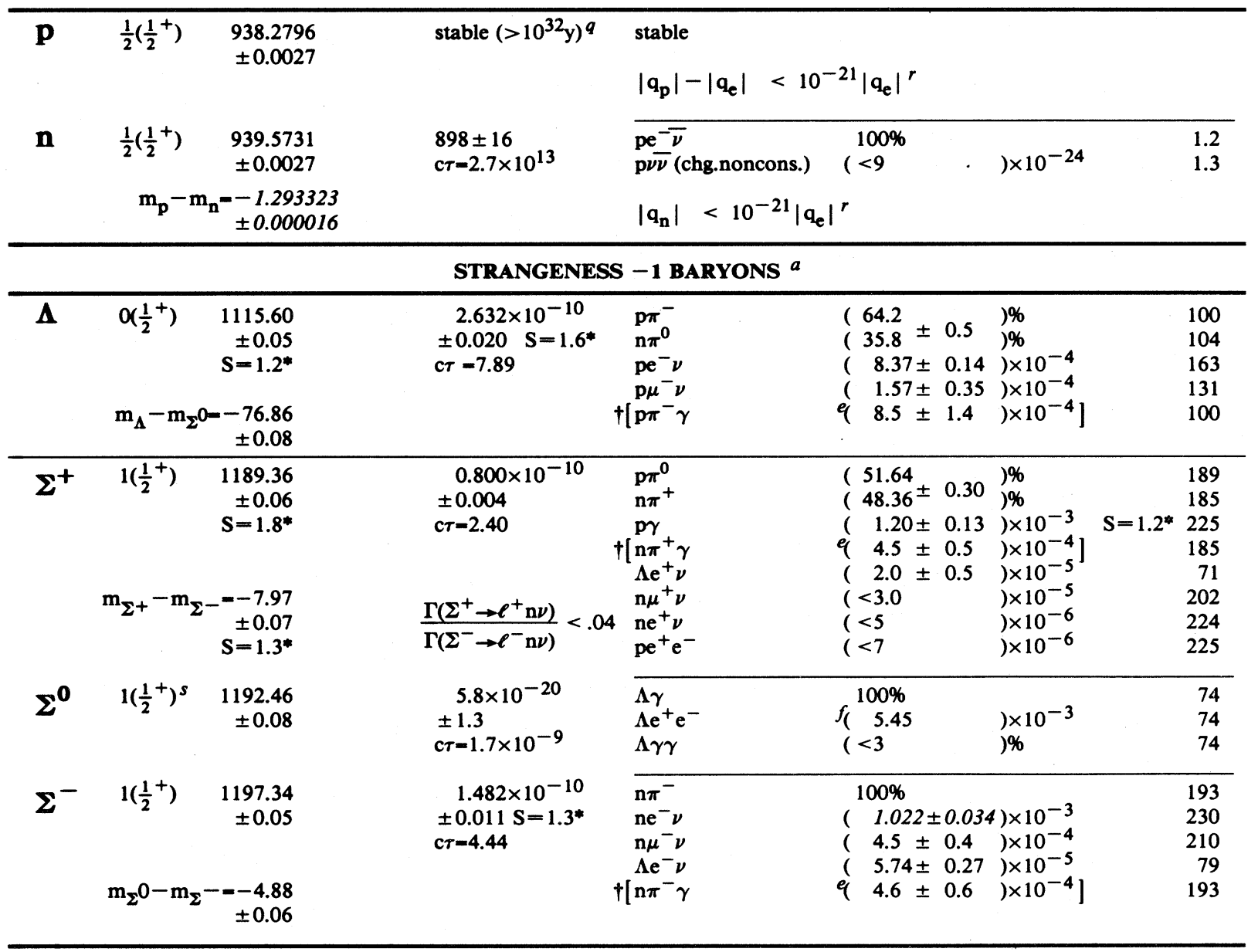


Stable Particle Table (cont'd)

\begin{tabular}{|c|c|c|c|c|c|c|}
\hline \multirow[t]{2}{*}{ Particle } & \multirow[t]{2}{*}{$\mathbf{I}_{\left(\mathbf{J}^{\mathbf{P}}\right)^{a}}^{a}$} & \multirow{2}{*}{$\begin{array}{c}\text { Mean life }{ }^{b} \\
\text { (sec) } \\
\text { cr } \\
(\mathrm{cm})\end{array}$} & \multicolumn{4}{|c|}{ Partial decay mode } \\
\hline & & & Mode & Fraction $^{b}$ & & $\begin{array}{c}\mathbf{p}_{\text {or }} \\
\mathbf{p}_{\max } \\
(\mathrm{MeV} / \mathrm{c})\end{array}$ \\
\hline \multicolumn{7}{|c|}{ STRANGENESS - 2 BARYONS ${ }^{a}$} \\
\hline$\Xi^{0}$ & 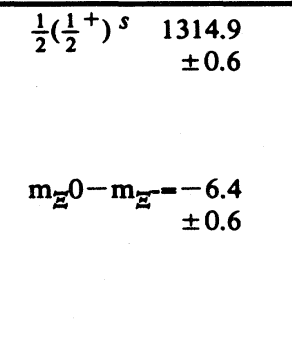 & $\begin{array}{l}2.90 \times 10^{-10} \\
\pm 0.10 \\
c \tau=8.69\end{array}$ & $\begin{array}{l}\Lambda \pi^{0} \\
\Lambda \gamma \\
\Sigma^{0} \gamma \\
\mathrm{p} \pi^{-} \\
\mathrm{pe}^{-} \nu \\
\Sigma^{+} \mathrm{e}^{-} \nu \\
\Sigma^{-} \mathrm{e}^{+} \nu \\
\Sigma^{+} \mu^{-} \nu \\
\Sigma^{-} \mu^{+} \nu \\
\mathrm{p} \mu^{-} \nu\end{array}$ & 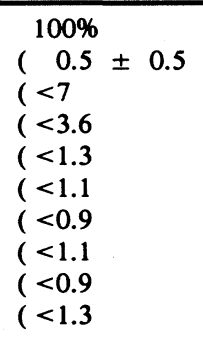 & $\begin{array}{l}\% \% \\
) \% \\
) \times 10^{-5} \\
) \times 10^{-3} \\
) \times 10^{-3} \\
) \times 10^{-3} \\
) \times 10^{-3} \\
) \times 10^{-3} \\
) \times 10^{-3}\end{array}$ & $\begin{array}{r}135 \\
184 \\
117 \\
299 \\
323 \\
120 \\
112 \\
65 \\
49 \\
309\end{array}$ \\
\hline$\underline{\Xi}$ & 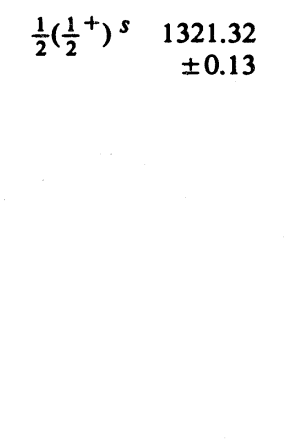 & $\begin{array}{l}1.641 \times 10^{-10} \\
\pm 0.016 \\
\mathrm{c} \tau=4.92\end{array}$ & $\begin{array}{l}\Lambda \pi^{-} \\
\Lambda \mathrm{e}^{-} \nu \\
\Sigma^{0} \mathrm{e}^{-} \nu \\
\Lambda \mu^{-} \nu \\
\Sigma^{0} \mu^{-} \nu \\
\mathrm{n} \pi^{-} \\
\mathrm{ne}^{-} \nu \\
\mathrm{n} \mu^{-} \nu \\
\Sigma^{-} \gamma \\
\mathrm{p} \pi^{-} \pi^{-} \\
\mathrm{p} \pi^{-} \mathrm{e}^{-} \nu \\
\mathrm{p} \pi^{-} \mu^{-} \nu \\
\Xi^{0} \mathrm{e}^{-} \nu\end{array}$ & 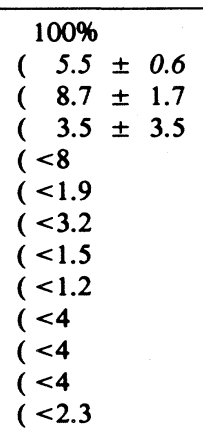 & $\begin{array}{l}) \times 10^{-4} \\
) \times 10^{-5} \\
) \times 10^{-4} \\
) \times 10^{-4} \\
) \times 10^{-5} \\
) \times 10^{-3} \\
) \% \\
) \times 10^{-3} \\
) \times 10^{-4} \\
) \times 10^{-4} \\
) \times 10^{-4} \\
) \times 10^{-3}\end{array}$ & $\begin{array}{r}139 \\
S=2.0^{*} \quad 190 \\
123 \\
163 \\
70 \\
303 \\
327 \\
313 \\
118 \\
223 \\
304 \\
250 \\
6\end{array}$ \\
\hline \multicolumn{7}{|c|}{ STRANGENESS -3 BARYON $a$} \\
\hline $\mathbf{\Omega}^{-}$ & $\begin{array}{rr}\left(\frac{3}{2}^{+}\right)^{s} & 1672.45 \\
& \pm 0.32\end{array}$ & $\begin{array}{l}0.819 \times 10^{-10} \\
\pm 0.027 \\
c \tau=2.46\end{array}$ & $\begin{array}{l}\Lambda \mathrm{K}^{-} \\
\Xi^{0} \pi^{-} \\
\Xi^{-} \pi^{0} \\
\Xi^{0} \mathrm{e}^{-} \nu \\
\Xi^{0}(1530) \pi^{-} \\
\Lambda \pi^{-} \\
\Xi^{-} \gamma\end{array}$ & $\begin{array}{l}(68.6 \pm 1.3 \\
(23.4 \pm 1.3 \\
(8.0 \pm 0.8 \\
(\sim 1 \\
(\sim 2 \\
(<1.3 \\
(<3.1\end{array}$ & $\begin{array}{l}\% \% \\
\% \% \\
\% \% \\
\% \times 10^{-3} \\
) \times 10^{-3} \\
) \times 10^{-3}\end{array}$ & $\begin{array}{l}211 \\
294 \\
290 \\
319 \\
\\
449 \\
314\end{array}$ \\
\hline \multicolumn{7}{|c|}{ NONSTRANGE CHARMED BARYON $a$} \\
\hline $\mathbf{\Lambda}_{\mathbf{c}}^{+}$ & $\begin{array}{cc}0\left(\frac{1}{2}^{+}\right)^{s} & 2282.0 \\
& \pm 3.1 \\
& S=1.8^{*}\end{array}$ & $\begin{array}{l}\left(2.3_{-0.6}^{+1.9} \times 10^{-13}\right. \\
\text { c } \tau=0.007\end{array}$ & $\begin{array}{c}\mathrm{pK}^{-} \pi^{+} \\
\mathrm{p} \overline{\mathrm{K}}^{0} \\
\mathrm{p} \overline{\mathrm{K}}^{0} \pi^{+} \pi^{-} \\
\Lambda \text { anything } \\
+\left[\Lambda \pi^{+}\right. \\
\Lambda \pi^{+} \pi^{+} \pi^{-} \\
\Sigma^{0} \pi^{+} \\
+\left[\mathrm{pK}^{* 0}\right. \\
\Delta^{++} \mathrm{K}^{-} \\
\mathrm{pK}^{*-} \pi^{+} \\
\mathrm{e}^{+} \text {anything } \\
+\left[\mathrm{pe}^{+} \text {anything }\right. \\
\Lambda \mathrm{e}^{+} \text {anything }\end{array}$ & 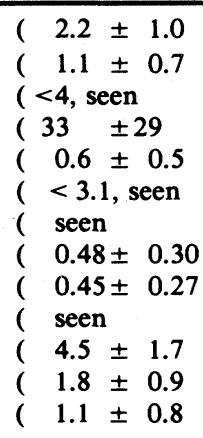 & 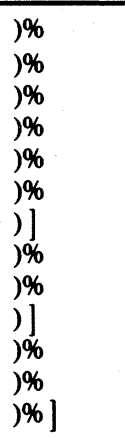 & $\begin{array}{l}820 \\
870 \\
751 \\
\\
861 \\
804 \\
822 \\
681 \\
706 \\
575\end{array}$ \\
\hline $\begin{array}{l}\rightarrow \mathbf{A}^{+} \\
\rightarrow \Lambda_{\mathbf{b}}^{\mathbf{0}} \\
\rightarrow \text { top } \mathbf{h} \\
\rightarrow \text { free q } \\
\rightarrow \text { magn } \\
\rightarrow \text { axion } \\
\rightarrow \text { other }\end{array}$ & $\begin{array}{l}\text { adron searches } \\
\text { uark searches } \\
\text { etic monopole searches } \\
\text { searches } \\
\text { stable particle searches }\end{array}$ & & & & & \\
\hline
\end{tabular}




\section{ADDENDUM TO \\ Stable Particle Table}

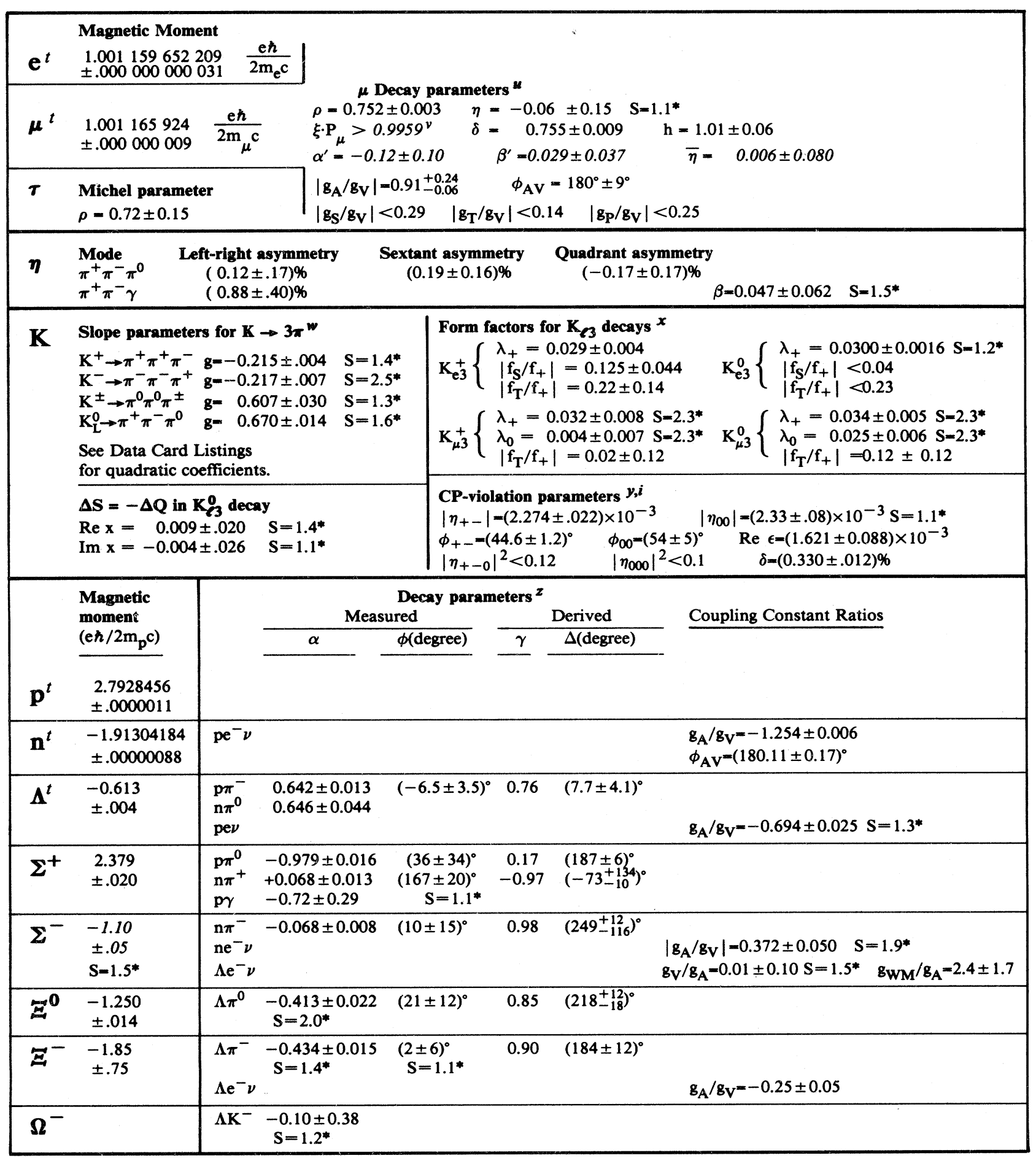




\section{Stable Particle Table (cont'd)}

$\rightarrow$ Indicates an entry in the Stable Particle Data Card Listings not entered in the Stable Particle Table.

* $\mathrm{S}$ - Scale factor $=\sqrt{\chi^{2} /(\mathrm{N}-1)}$, where $\mathrm{N} \approx$ number of experiments. $\mathrm{S}$ should be $\approx 1$. If $\mathrm{S}>1$, we have enlarged the error of the mean, $\delta \bar{x}$; i.e., $\delta \bar{x} \rightarrow S \delta \bar{x}$. This convention is still inadequate, since if $S \gg 1$ the experiments are probably inconsistent, and therefore the real uncertainty is probably even greater than $S \delta x$. See the Introduction, and ideograms in Stable Particle Data Card Listings.

$\dagger$ Square brackets indicate subreactions of some previous unbracketed decay mode(s). Reactions in one set of brackets may overlap with reactions in another set of brackets. A radiative mode such as $\pi \rightarrow \mu \nu \gamma$ is a subreaction of its parent mode $\pi \rightarrow \mu \nu$.

a. The strangeness $S$, charm $C$, and bottomness (beauty) $B$ of the hadrons which appear in the Table are as follows:

\begin{tabular}{|c|c|c|c|c|c|c|c|c|c|c|}
\hline Mesons & $S$ & $C$ & $B$ & Mesons & $S$ & $C$ & $B$ & Baryons & $S$ & $C$ \\
\hline$\pi, \eta$ & 0 & 0 & 0 & $\mathrm{~F}^{+}$ & +1 & +1 & 0 & $\mathrm{p}, \mathrm{n}$ & 0 & 0 \\
\hline $\mathbf{K}^{+}, \mathbf{K}^{0}$ & +1 & 0 & 0 & $\mathbf{F}^{-}$ & -1 & -1 & 0 & $\Lambda, \Sigma$ & -1 & 0 \\
\hline $\mathbf{K}^{-}, \overline{\mathbf{K}}^{0}$ & -1 & 0 & 0 & $\mathrm{~B}^{+}, \mathrm{B}^{0}$ & 0 & 0 & +1 & $\Xi$ & -2 & 0 \\
\hline $\mathrm{D}^{+}, \underline{\mathrm{D}}^{0}$ & 0 & +1 & 0 & $\mathbf{B}^{-}, \overline{\mathbf{B}}^{0}$ & 0 & 0 & -1 & $\mathbf{\Omega}^{-}$ & -3 & 0 \\
\hline$D^{-}, \overline{\mathbf{D}}^{0}$ & 0 & -1 & 0 & & & & & $\Lambda_{c}^{+}$ & 0 & +1 \\
\hline
\end{tabular}

b. Quoted upper limits correspond to a $90 \%$ confidence level. Masses, mean lives, and partial rates evaluated assuming equality for particles and antiparticles. See Conservation Laws Section for further details.

c. In decays with more than two bodies, $p_{\max }$ is the maximum momentum that any particle can have.

d. 99\% confidence level. See footnote in Stable Particle Data Card Listings.

e. See Stable Particle Data Card Listings for energy limits used in this measurement.

f. Theoretical value; see also Stable Particle Data Card Listings.

g. The direct emission branching fraction is $(1.56 \pm .35) \times 10^{-5}$.

h. Structure-dependent part with positive $(\mathrm{SD}+)$ and negative $(\mathrm{SD}-)$ photon helicity.

$i$. The $\mathrm{K}_{\mathrm{S}}^{0} \rightarrow \pi \pi$ and $\mathrm{K}_{\mathrm{L}}^{0} \rightarrow \pi \pi$ branching fractions are from our branching fraction and rate fits and do not include results of $\mathrm{K}_{\mathrm{L}}^{0}-\mathrm{K}_{\mathrm{S}}^{0}$ interference experiments. The $\pi \pi$ rate results are combined with the interference results to obtain the $\left|\eta_{+-}\right|$ and $\left|\eta_{00}\right|$ values given in the addendum.

j. The stronger limit $<2 \times 10^{-9}$ of Clark et al., Phys. Rev. Lett. 26, 1667 (1971) is not listed because of possible (but unknown) systematic errors. See Stable Particle Data Card Listings.

$k$. This is a weighted average of $\mathrm{D}^{ \pm}(44 \%)$ and $\mathrm{D}^{0}(56 \%)$ branching fractions.

$\ell$. $\mathrm{D}_{1}^{0}-\mathrm{D}_{2}^{0}$ limits inferred from limit on $\mathrm{D}^{0} \rightarrow \overline{\mathrm{D}}^{0} \rightarrow \mu^{-}$anything.

$m$. F mass determined from $\phi \pi$ mode. See note on conflicting F meson results in Stable Particle Data Card Listings. Quantum numbers shown are favored but not yet established.

n. Quantum numbers not measured. Values shown are quark model predictions.

p. Except for the neutral-current decay modes $\left(\ell^{+} \ell^{-}\right.$anything), only data from $\Upsilon(105752$ decays are used. Behrends et al. [Phys. Rev. Lett. 50, 881 (1983)] estimate the $\Upsilon(10575) \rightarrow B^{+} B^{-}$and $\Upsilon(10575) \rightarrow B^{0} B^{0}$ branching fractions to be $60 \pm 2$ and $40 \pm 2 \%$.

q. Partial mean life for $\mathrm{p} \rightarrow \mathrm{e}^{+} \pi^{0}$ mode. For antiprotons the best mean life limit, inferred from observation of cosmic ray $\overline{\mathrm{p}} \mathrm{s}$, is $\tau_{\overline{\mathrm{p}}}>10^{7}$ yrs, the cosmic ray storage time.

r. Limit from neutrality-of-matter experiments. Assumes $\left|q_{n}\right|=\left|q_{p}\right|-\left|q_{e}\right|$.

s. $\mathbf{P}$ for $\Xi, \mathrm{J}^{\mathrm{P}}$ for $\Omega^{-}$and $\Sigma^{0}$, and $\mathrm{J}$ for $\Lambda_{c}^{+}$not yet measured. Values shown are quark model predictions.

t. For limits on electric dipole moment, see Conservation Laws Section. Forbidden by $P$ and $T$ invariance.

u. $\left|\mathrm{g}_{\mathrm{A}} / \mathrm{g}_{\mathrm{V}}\right|$ defined by $\mathrm{g}_{\mathrm{A}}^{2}=\left|\mathrm{C}_{\mathrm{A}}\right|^{2}+\left|\mathrm{C}_{\mathrm{A}}^{\prime}\right|^{2}, \mathrm{~g}_{\mathrm{V}}^{2}=\left|\mathrm{C}_{\mathrm{V}}\right|^{2}+\left|\mathrm{C}_{\mathrm{V}}^{\prime}\right|^{2}$, and $\Sigma\left[\overline{\mathrm{e}} \Gamma_{\mathrm{i}} \mu\right]\left[\bar{\nu} \Gamma_{\mathrm{i}}\left(\mathrm{C}_{\mathrm{i}}+\mathrm{C}_{\mathrm{i}}^{\prime} \gamma_{5}\right) \nu\right] ; \phi$ defined by $\cos \phi=-\operatorname{Re}\left(C_{A}^{*} C_{V}^{\prime}+C_{A}^{\prime} C_{V}^{*}\right) / g_{A} g_{V}$. For more details, see Data Card Listings. v. Value assumes $\rho=\delta$. $\mathrm{P}_{\mu}$ is muon longitudinal polarization from $\pi$ decay. In standard $\mathrm{V}$-A theory, $\mathrm{P}_{\mu}=1$ and $\rho=\delta=$
$3 / 4$.

w. The definition of the slope parameter of the Dalizz plot is as follows [see also note in Data Card Listings]:

$$
|M|^{2}=1+g\left(\frac{s_{3}-s_{0}}{m_{\pi^{+}}^{2}}\right)
$$

$x$. For definitions of form factors $f_{+}, f_{S}$, and $f_{T}$, and linear $t$ dependences $\lambda_{+}$and $\lambda_{0}$ of $f_{+}(t)$ and $f_{0}(t)$, see note in $K^{+}$section of Data Card Listings.

y. The definition for the CP violation parameters is as follows [see also note in Data Card Listings]:

$$
\begin{aligned}
& \eta_{+-}=\left|\eta_{+-}\right| \mathrm{e}^{\mathrm{i} \phi_{+-}}=\frac{\mathrm{A}\left(\mathrm{K}_{\mathrm{L}}^{0} \rightarrow \pi^{+} \pi^{-}\right)}{\mathrm{A}\left(\mathrm{K}_{\mathrm{S}}^{0} \rightarrow \pi^{+} \pi^{-}\right)} \quad \eta_{00}=\left|\eta_{\infty 0}\right| \mathrm{e}^{\mathrm{i} \phi_{00}}=\frac{\mathrm{A}\left(\mathrm{K}_{\mathrm{L}}^{0} \rightarrow \pi^{0} \pi^{0}\right)}{\mathrm{A}\left(\mathrm{K}_{\mathrm{S}}^{0} \rightarrow \pi^{0} \pi^{0}\right)} \\
& \delta=\frac{\Gamma\left(\mathrm{K}_{\mathrm{L} \rightarrow \ell^{+}}^{0}\right)-\Gamma\left(\mathrm{K}_{\mathrm{L}}^{0} \rightarrow \ell^{-}\right)}{\Gamma\left(\mathrm{K}_{\mathrm{L}}^{0} \rightarrow \ell^{+}\right)+\Gamma\left(\mathrm{K}_{\mathrm{L}}^{0} \rightarrow \ell^{-}\right)}, \quad\left|\eta_{+-0}\right|^{2}=\frac{\Gamma\left(\mathrm{K}_{\mathrm{S}}^{0} \rightarrow \pi^{+} \pi^{-} \pi^{0}\right)^{C P} \text { viol. }}{\Gamma\left(\mathrm{K}_{\mathrm{L}}^{0} \rightarrow \pi^{+} \pi^{-} \pi^{0}\right)}, \quad\left|\eta_{000}\right|^{2}=\frac{\Gamma\left(\mathrm{K}_{\mathrm{S}}^{0} \rightarrow \pi^{0} \pi^{0} \pi^{0}\right)^{C P} \text { viol. }}{\Gamma\left(\mathrm{K}_{\mathrm{L}}^{0} \rightarrow \pi^{0} \pi^{0} \pi^{0}\right)} .
\end{aligned}
$$

z. The definition of these quantities is as follows [for more details and sign convention, see note in Data Card Listings]:

$$
\begin{array}{lll}
\alpha=\frac{2|\mathrm{~s}||\mathrm{p}| \cos \Delta}{|\mathrm{s}|^{2}+|\mathrm{p}|^{2}} & \beta=\sqrt{1-\alpha^{2}} \sin \phi & \mathrm{g}_{\mathrm{A}}, \mathrm{g}_{\mathrm{V}}, \mathrm{g}_{\mathrm{WM}} \text { defined by }\left\langle\mathrm{B}_{\mathrm{f}}\left|\gamma_{\lambda}\left(\mathrm{g}_{\mathrm{V}}-\mathrm{g}_{\mathrm{A}} \gamma_{5}\right)+\left(\mathrm{g}_{\mathrm{WM}} / \mathrm{m}_{\mathrm{B}_{\mathrm{i}}}\right) \sigma^{\lambda \nu} \mathrm{q}_{\nu}\right| \mathrm{B}_{\mathrm{i}}\right\rangle \\
\beta=\frac{-2|\mathrm{~s}||\mathrm{p}| \sin \Delta}{|\mathrm{s}|^{2}+|\mathrm{p}|^{2}} & \gamma=\sqrt{1-\alpha^{2}} \cos \phi & \phi_{\mathrm{AV}} \text { defined by } \mathrm{g}_{\mathrm{A}} / \mathrm{g}_{\mathrm{V}}=\left|\mathrm{g}_{\mathrm{A}} / \mathrm{g}_{\mathrm{V}}\right| \mathrm{e}^{\mathrm{i} \phi} \mathrm{AV}
\end{array}
$$




\section{Meson Table}

April 1984

In addition to the entries in the Meson Table, the Meson Data Card Listings contain al substantial claims for meson resonances. See Contents of Meson Data Card Listings at end of this Table.

Quantities in italics are new or have changed by more than one (old) standard deviation since April 1982.

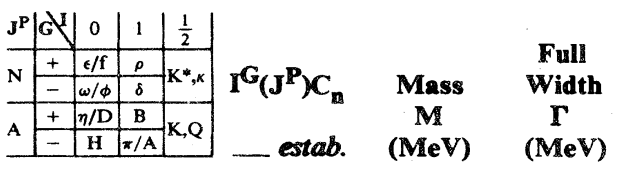

Partial decay mode

$\begin{array}{lcc}\text { Mode } & \text { Fraction(\%) } & \begin{array}{c}\text { p or } \\ \mathrm{P}_{\max } b \\ (\mathrm{MeV} / \mathrm{c})\end{array}\end{array}$

NONSTRANGE MESONS

\begin{tabular}{|c|c|c|c|c|c|c|c|}
\hline $\begin{array}{l}\overline{\pi^{ \pm}} \\
\pi^{0}\end{array}$ & $1-\left(0^{-}\right)+$ & $\begin{array}{l}139.57 \\
134.96\end{array}$ & $\begin{array}{l}0.0 \\
7.95 \mathrm{eV} \\
\pm 0.55 \mathrm{eV}\end{array}$ & \multicolumn{3}{|c|}{ See Stable Particle Table } & \\
\hline$\eta$ & $0^{+}\left(0^{-}\right) \pm$ & $\begin{array}{r}548.8 \\
\pm 0.6\end{array}$ & $\begin{array}{r}0.83 \mathrm{keV} \\
\pm 0.12 \mathrm{keV} \\
\end{array}$ & $\begin{array}{l}\text { Neutral } \\
\text { Charged }\end{array}$ & $\begin{array}{l}70.9 \\
29.1\end{array}$ & \multicolumn{2}{|c|}{$\begin{array}{l}\text { See Stable } \\
\text { Particle Table }\end{array}$} \\
\hline$\overline{\rho(770)}$ & $1^{+}\left(1^{-}\right)-$ & $\begin{array}{c}769^{\ddagger} \\
\pm 3^{\S}\end{array}$ & $\begin{array}{c}154^{\ddagger} \\
\pm 5^{\S}\end{array}$ & $\begin{array}{l}\pi \pi \\
\pi \gamma \\
\mu^{+} \mu^{-} \\
\mathrm{e}^{+} \mathrm{e}^{-} \\
\eta \gamma\end{array}$ & \multicolumn{2}{|c|}{$\begin{array}{c}\approx 100 \\
0.046 \pm 0.005 \\
0.0067 \pm 0.0012^{d} \\
0.0046 \pm 0.0002^{d} \\
\text { seen }^{+}\end{array}$} & $\begin{array}{l}358 \\
372 \\
370 \\
384 \\
189\end{array}$ \\
\hline \multicolumn{4}{|c|}{$M$ and $\Gamma$ from neutral mode. } & \multicolumn{4}{|c|}{ For upper limits, see footnote $e$} \\
\hline$\omega(783)$ & $0^{-}\left(1^{-}\right)=$ & $\begin{array}{c}782.6 \\
\pm 0.2 \\
S=1.1 *\end{array}$ & $\begin{array}{r}9.9 \\
+0.3\end{array}$ & $\begin{array}{l}\pi^{+} \pi^{-} \pi^{0} \\
\pi^{0} \gamma \\
\pi^{+} \pi^{-} \\
\pi^{0} \mu^{+} \mu^{-} \\
\mathrm{e}^{+} \mathrm{e}^{-} \\
\eta \gamma \\
\text { For uppe }\end{array}$ & $\begin{aligned} & 89.9 \pm 0.5 \\
& 8.7 \pm 0.5 \\
& 1.4 \pm 0.2 \\
& 0.010 \pm 0.002 \\
& 0.0067 \pm q .000 \\
& \text { seen } \\
& \text { otnote } f\end{aligned}$ & $4 S=1.2^{*}$ & $\begin{array}{l}327 \\
380 \\
366 \\
349 \\
391 \\
199\end{array}$ \\
\hline$\overline{\eta^{\prime}(958)}$ & $0^{+}\left(0^{-}\right)+^{f}$ & $\begin{array}{r}957.57 \\
\pm 0.25\end{array}$ & $\begin{array}{r}0.29 \\
\pm 0.05\end{array}$ & $\begin{array}{l}\eta \pi \pi \\
\rho^{0} \gamma \\
\omega \gamma \\
\gamma \gamma \\
\mu^{+} \mu^{-} \gamma \\
\text { For uppe }\end{array}$ & $\begin{array}{l}\quad 65.3 \pm 1.6 \\
30.0 \pm 1.6 \\
2.8 \pm 0.5 \\
1.9 \pm 0.2 \\
0.009 \pm 0.002 \\
\text { otnote } g\end{array}$ & & $\begin{array}{l}231 \\
170 \\
159 \\
479 \\
467\end{array}$ \\
\hline $\begin{array}{l}S(975) \\
\text { or } S^{*} \\
\text { See note }\end{array}$ & $\begin{array}{l}0^{+}\left(0^{+}\right) \pm \\
\pi \pi \text { and } \mathrm{K} \overline{\mathrm{K}}\end{array}$ & $\begin{array}{l}975^{c} \\
\pm 4 \\
\text { S=1.4 } \\
\text { S wave. }\end{array}$ & $\begin{array}{l}33^{c} \\
\pm 6\end{array}$ & $\begin{array}{l}\pi \pi \\
K \bar{K}\end{array}$ & $\begin{array}{l}78 \pm 3 \\
22 \pm 3\end{array}$ & & 467 \\
\hline$\delta(980)^{\ddagger}$ & $1-\left(0^{+}\right)+$ & $\begin{array}{l}983^{h} \\
\pm 2\end{array}$ & $\begin{array}{c}54^{h} \\
\pm 7\end{array}$ & $\begin{array}{l}\eta \pi \\
\mathbf{K} \overline{\mathbf{K}}\end{array}$ & $\begin{array}{l}\text { seen } \\
\text { seen }\end{array}$ & & 320 \\
\hline$\phi(1020)$ & $0^{-}\left(1^{-}\right)=$ & $\begin{array}{c}1019.5 \\
\pm 0.1 \\
S=1.2\end{array}$ & $\begin{array}{r}4.22 \\
\pm 0.13\end{array}$ & $\begin{array}{l}\mathrm{K}^{+} \mathrm{K}^{-} \\
\mathbf{K}_{\mathbf{L}} \mathbf{K}_{\mathbf{S}} \\
\pi^{+} \pi^{0} \pi^{0} \\
\eta \gamma \\
\pi^{0} \gamma \\
\mathrm{e}^{+} \mathrm{e}^{-} \\
\mu^{+} \mu^{-} \\
\pi^{+} \pi^{-} \\
\text {For uppe }\end{array}$ & $\begin{array}{c}49.3 \pm 1.0 \\
34.7 \pm 1.0 \\
14.8 \pm 0.7 \\
1.2 \pm 0.2 \\
0.14 \pm 0.05 \\
0.031 \pm 0.001 \\
0.025 \pm 0.003 \\
0.02 \pm 0.01 \\
\text { otnote } i\end{array}$ & $\begin{array}{l}S=1.3^{*} \\
S=1.3^{*} \\
S=1.2^{*} \\
S=1.4^{*}\end{array}$ & $\begin{array}{l}127 \\
110 \\
462 \\
362 \\
501 \\
510 \\
499 \\
490\end{array}$ \\
\hline $\begin{array}{l}H(1190) \\
\text { Seen in o }\end{array}$ & $\begin{array}{l}0^{-}\left(1^{+}\right)- \\
\text {experiment }\end{array}$ & $\begin{array}{c}1190 \\
\pm 60 \\
\text { only. }\end{array}$ & $\begin{array}{r}320 \\
\pm 50\end{array}$ & $\rho \pi$ & seen & & 327 \\
\hline $\mathrm{B}(1235)$ & $1^{+}\left(1^{+}\right)=$ & $\begin{array}{c}1234 \\
\pm 10^{\S}\end{array}$ & $\begin{array}{c}150 \\
\pm 10^{\S}\end{array}$ & $\begin{array}{l}\omega \pi \\
{[\mathrm{D} / \mathrm{S} \text { am }} \\
\text { For uppe }\end{array}$ & $\begin{array}{l}\text { only mode see } \\
0.29 \pm 0.05] \\
\text { otnote } j\end{array}$ & & 350 \\
\hline
\end{tabular}


Meson Table (cont'd)

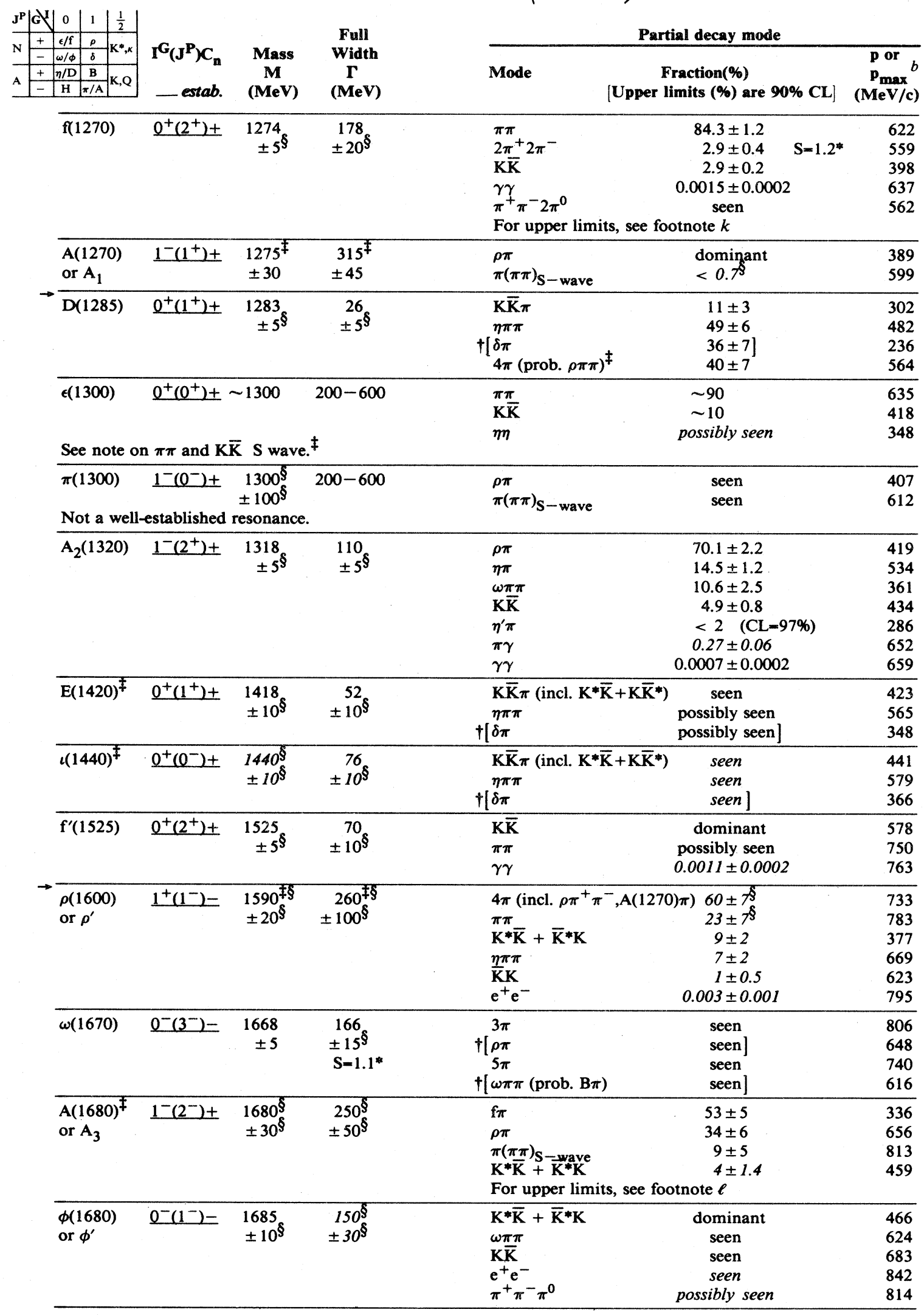




\section{Meson Table (cont'd)}

\begin{tabular}{|c|c|c|c|c|c|c|c|c|}
\hline & \begin{tabular}{l|l|l|l|l|l|l|l|l|l|} 
& 0 & 1 & $\frac{1}{2}$ \\
+ & $\epsilon / \mathrm{f}$ & 0
\end{tabular} & & & & & Partial & 1 decay mode & \\
\hline & 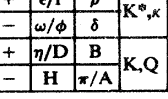 & 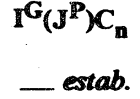 & $\begin{array}{c}\text { Mass } \\
\text { M } \\
(\mathbf{M e V})\end{array}$ & $\begin{array}{l}\text { Width } \\
\Gamma \\
(\mathbf{M e V})\end{array}$ & Mode & $\begin{array}{r}\text { Frac } \\
\text { [Upper limi }\end{array}$ & $\begin{array}{l}\text { action(\%) } \\
\text { nits (\%) are } 90 \% \mathrm{CL} \text { ) }\end{array}$ & $\begin{array}{c}\mathrm{p} \text { or } \\
\mathrm{p}_{\max } \\
(\mathrm{MeV} / \mathrm{c}\end{array}$ \\
\hline & $g(1690)$ & $1^{+}\left(3^{-}\right)=$ & $\begin{array}{l}1691 \\
\pm 5^{\S}\end{array}$ & $\begin{array}{r}200^{\S} \\
\pm 20^{\S}\end{array}$ & $\begin{array}{l}2 \pi \\
4 \pi \text { (in } \\
\mathrm{K} \overline{\mathbf{K}} \pi \\
\mathrm{K} \overline{\mathbf{K}}\end{array}$ & $\begin{array}{l}\rho \rho, \mathbf{A}_{2} \frac{\pi, \omega \pi)}{\mathrm{K}+\mathbf{K}^{*}} \\
\left.\bar{K}^{*}\right)\end{array}$ & $\begin{array}{r}23.8 \pm 1.3 \\
70.9 \pm 1.9 \\
3.8 \pm 1.2 \\
1.5 \pm 0.3\end{array}$ & $\begin{array}{l}834 \\
787 \\
625 \\
684\end{array}$ \\
\hline
\end{tabular}

$\mathrm{J}^{\mathrm{P}}, \mathrm{M}$, and $\Gamma$ from the $2 \pi$ and $\mathrm{K} \overline{\mathrm{K}}$ modes.

\begin{tabular}{|c|c|c|c|c|c|c|}
\hline$\theta(1690)$ & $0^{+}\left(2^{+}\right)+$ & $\begin{array}{r}1690 \\
\pm 30 \\
\end{array}$ & $\begin{array}{r}180 \\
\pm 50\end{array}$ & $\eta \overline{\mathbf{K}}$ & $\begin{array}{l}\text { seen } \\
\text { seen }\end{array}$ & $\begin{array}{l}643 \\
683\end{array}$ \\
\hline$\phi(1850)$ & $0^{-}\left(3^{-}\right)-$ & $\begin{array}{r}1853 \\
\pm 10\end{array}$ & $\begin{array}{r}96 \\
\pm 32\end{array}$ & $\begin{array}{l}\mathbf{K} \overline{\mathbf{K}} \\
\mathbf{K}^{*} \overline{\mathbf{K}}+\overline{\mathbf{K}}^{*} \mathbf{K}\end{array}$ & $\begin{array}{l}\text { seen } \\
\text { seen }\end{array}$ & $\begin{array}{l}784 \\
601\end{array}$ \\
\hline$h(2030)$ & $0^{+}\left(4^{+}\right)+$ & $\begin{array}{r}2027 \\
\pm 12\end{array}$ & $\begin{array}{r}220 \\
\pm 30\end{array}$ & $\begin{array}{l}\pi \pi \\
\mathrm{K} \overline{\mathrm{K}}\end{array}$ & $\begin{array}{c}17 \pm 2 \\
0.7 \pm 0.4\end{array}$ & $\begin{array}{r}1004 \\
883\end{array}$ \\
\hline$\eta_{c}(2980)$ & $0^{+}\left(0^{-}\right) \pm$ & $\begin{array}{r}2981 \\
\pm 6\end{array}$ & $<20$ & $\begin{array}{l}\eta \pi^{+} \pi^{-} \\
2\left(\pi^{+} \pi^{-}\right) \\
\mathrm{K}^{+} \mathrm{K}^{-} \pi^{+} \pi^{-} \\
\mathrm{p} \overline{\mathrm{p}}\end{array}$ & $\begin{array}{l}\text { seen } \\
\text { seen } \\
\text { seen } \\
\text { seen }\end{array}$ & $\begin{array}{l}1426 \\
1458 \\
1343 \\
1158 \\
\end{array}$ \\
\hline$J / \psi(3100)$ & $0^{-}\left(1^{-}\right)=$ & $\begin{array}{r}3096.9 \\
\pm 0.1\end{array}$ & $\begin{array}{r}0.063 \\
\pm 0.009\end{array}$ & $\begin{array}{l}\mathrm{e}^{+} \mathrm{e}^{-} \\
\mu^{+} \mu^{-} \\
\text {hadrons + radiative }\end{array}$ & $\begin{array}{l}7.4 \pm 1.2 \\
7.4 \pm 1.2 \\
85 \pm 2\end{array}$ & $\begin{array}{l}1548 \\
1545\end{array}$ \\
\hline
\end{tabular}

Decay modes into stable hadrons

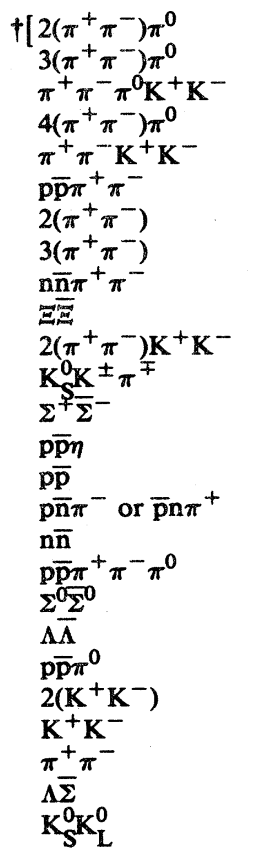

1496

1433

1368

1345

1407

1107

1517

1466

1106

818

1320

1440

988

948

1232

1174

1231

1033

988

1074

1176

1131

1468

1542

1032

1466

Decay modes into hadronic resonances

$+[\rho \pi+1.22 \pm 0.12 \quad 1449$

$\omega 2 \pi^{+} 2 \pi^{-} \quad 0.85+0.34$

$\rho \mathrm{A}_{2} \quad 0.84 \pm 0.45 \quad 1126$

$\omega \pi \pi \quad 0.68 \pm 0.19 \quad 1435$

$\mathrm{K}^{* 0}(892) \overline{\mathrm{K}} * 0(1430)+$ c.c. $0.67 \pm 0.26 \quad 1009$

$\mathrm{K}^{ \pm \mathrm{K}^{*}{ }^{\mp}(892)} \quad 0.34 \pm 0.05 \quad 1373$

$\begin{array}{lll}\mathrm{B}^{ \pm}(1235) \pi^{\mp} & 0.29 \pm 0.07 & 1298\end{array}$

$\mathrm{K}^{0} \overline{\mathrm{K}}^{* 0}(892)+$ c.c. $\quad 0.27 \pm 0.06 \quad 1370$

wf $\quad 0.23 \pm 0.08 \quad S=1.2 * \quad 1143$

$\begin{array}{lll}\phi \pi^{+} \pi^{-} & 0.21 \pm 0.09 & 1365\end{array}$

$\eta^{\prime} \mathbf{p} \overline{\mathrm{p}} \quad 0.18 \pm 0.06 \quad 596$

$\phi \mathrm{K} \overline{\mathrm{K}} \quad 0.18 \pm 0.08 \quad 1176$

$\omega \mathrm{p} \overline{\mathrm{p}} \quad 0.16 \pm 0.03 \quad 768$

$\omega \mathrm{K} \overline{\mathrm{K}} \quad 0.16 \pm 0.10 \quad 1265$

$\phi \eta \quad 0.10 \pm 0.06 \quad 1320$

$\phi \mathrm{f}^{\prime}(1525) \quad 0.037 \pm 0.013 \quad 871$

$\phi \mathrm{S}(975) \quad 0.026 \pm 0.006 \quad 1184$

$\pi^{ \pm} \mathrm{A}_{2}^{\mp}<0.43 \quad 1263$

$\mathrm{K}^{* 0}\left(\mathrm{I}_{430)} \overline{\mathrm{K}}^{* 0}(1430)<0.29 \quad 606\right.$

$\mathrm{K}^{0} \mathrm{~K}^{* 0}(1430)+$ c.c. $\quad<0.2 \quad 1158$

$\mathrm{K}^{ \pm \mathrm{K}^{*}}{ }^{\mathrm{F}}(1430) \quad<0.2$

$\phi 2 \pi^{+} 2 \pi^{-}<0.15 \quad 1318$

$\phi \eta^{\prime}<0.13<1192$

$\mathrm{K}^{* 0}(892) \overline{\mathrm{K}}^{* 0}(892) \quad<0.05 \quad 1261$

$\phi f \quad<0.037 \quad 1037$

$\left.\omega \mathrm{f}^{\prime}(1525)<0.016\right] \quad 1003$

Radiative decay modes

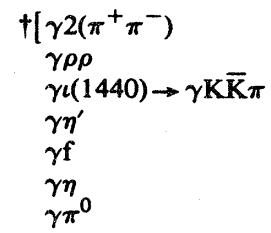
$0.49 \pm 0.17$ seen
$0.42 \pm 0.12^{n}$
$0.36 \pm 0.05$
$0.15 \pm 0.04$
$0.086 \pm 0.009$
$0.007 \pm 0.005$

1517

1344

1214

1400

1286

1500

1546

Radiative decay modes (cont'd)

\begin{tabular}{llr}
$\gamma \eta_{\mathrm{c}}(2980)$ & seen & 114 \\
$\gamma \theta(1690)$ & seen & 1087 \\
$\gamma \eta \pi \pi$ & seen & 1487 \\
$\gamma \mathrm{D}(1285)$ & $<0.6$ & 1283 \\
$2 \gamma$ & $<0.05$ & 1548 \\
$\gamma \mathrm{f}^{\prime}(1525)$ & $<0.03$ & 1173 \\
$\gamma \mathrm{p} \overline{\mathrm{p}}$ & $<0.01$ & 1232 \\
$3 \gamma$ & $<0.006]$ & 1548 \\
\hline
\end{tabular}




\section{Meson Table (cont'd)}

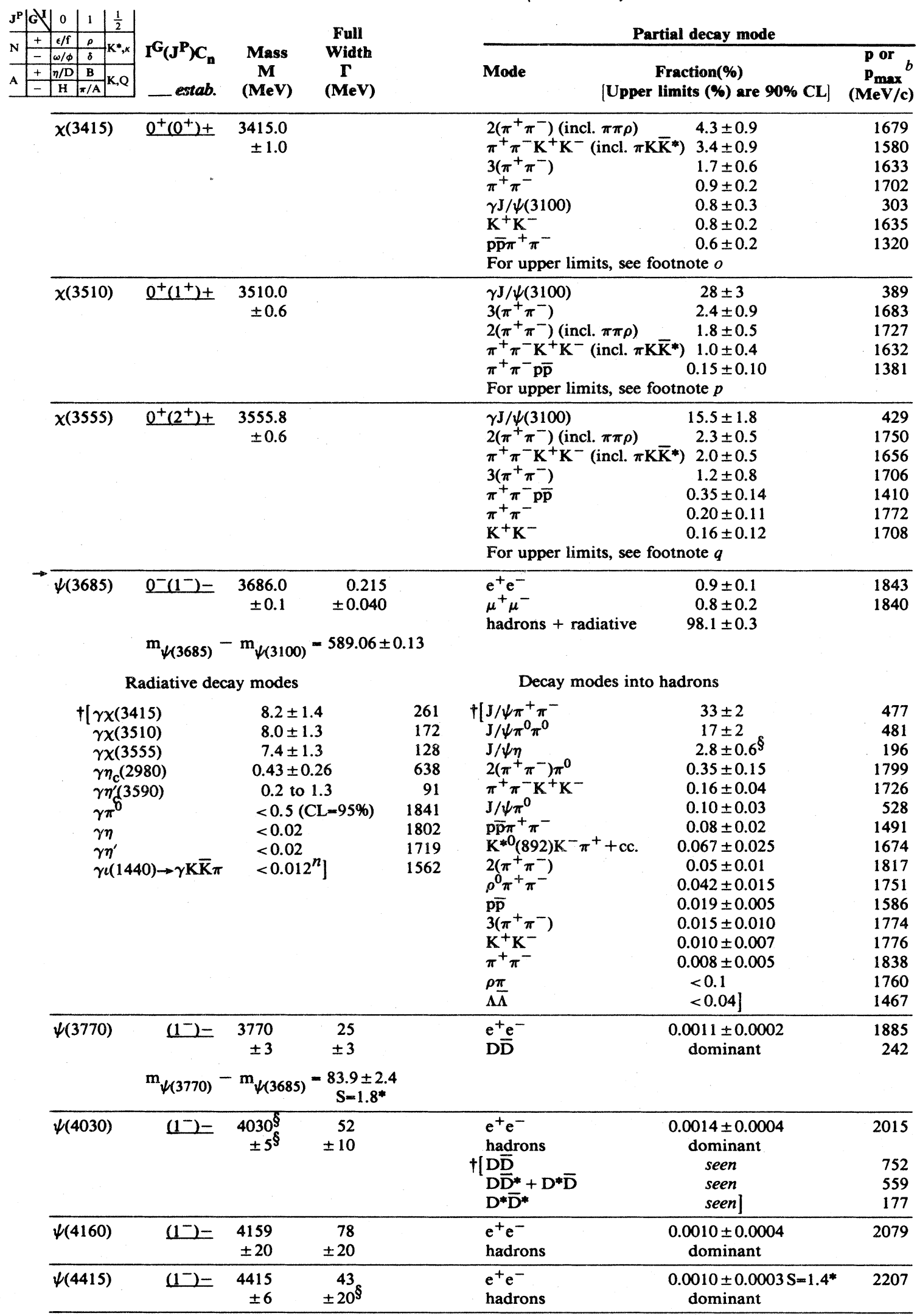




\section{Meson Table (cont'd)}

\begin{tabular}{|c|c|c|c|c|c|c|c|}
\hline & 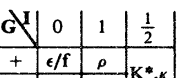 & & & Full & & Partial decay mode & \\
\hline & 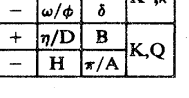 & $\begin{array}{l}\mathbb{I}^{0}\left(\mathrm{~J}^{2}\right) C_{\mathbf{n}} \\
\text { - estab. }\end{array}$ & $\begin{array}{c}\text { Mass } \\
\text { M } \\
(\mathbf{M e V})\end{array}$ & $\begin{array}{c}\text { Width } \\
\mathbb{T} \\
(\mathrm{MeV})\end{array}$ & Mode & $\begin{array}{l}\text { Fraction(\%) } \\
\text { [Upper limits (\%) are } 90 \% \mathrm{CL}]\end{array}$ & $\begin{array}{c}p_{\text {or }} \\
p_{\max } b \\
(\mathrm{MeV} / \mathrm{c})\end{array}$ \\
\hline & $\begin{array}{l}\Upsilon(9460) \\
\text { or } \Upsilon(1 S)\end{array}$ & $(1-)=$ & $\begin{array}{c}9460.0 \\
\pm 0.3 \\
S=1.6^{*}\end{array}$ & $\begin{array}{r}0.0443 \\
\pm 0.0066\end{array}$ & $\begin{array}{l}\mu^{+} \mu^{-} \\
\mathrm{e}^{+} \mathrm{e}^{-} \\
\tau^{+} \tau^{-}\end{array}$ & $\begin{array}{l}2.9 \pm 0.5 \\
2.5 \pm 0.5 \\
3.4 \pm 0.8\end{array}$ & $\begin{array}{l}4729 \\
4730 \\
4381 \\
\end{array}$ \\
\hline & $\begin{array}{l}x_{b}(9875) \\
\text { or } x_{b}\left(1{ }^{3} P_{0}\right) \\
\end{array}$ & $r^{r}()+$ & $\begin{array}{r}9872.9 \\
\pm 5.8 \\
\end{array}$ & & $\gamma \Upsilon(9460)$ & seen & 404 \\
\hline & $\begin{array}{l}\chi_{b}(9895) \\
\text { or } x_{b}\left(1{ }^{3} P_{1}\right) \\
\end{array}$ & $r^{(1)+}$ & $\begin{array}{r}9894.5 \\
\pm 3.5 \\
\end{array}$ & & $\gamma \Upsilon(9460)$ & $43 \pm 11$ & 425 \\
\hline & $\begin{array}{l}\chi_{b}(9915) \\
\text { or } \chi_{b}\left(1^{3} P_{2}\right) \\
\end{array}$ & $r^{r}(1)+$ & $\begin{array}{r}9914.6 \\
\pm 2.4 \\
\end{array}$ & & $\gamma \mathrm{r}(9460)$ & $20.0 \pm 4.4$ & 444 \\
\hline & $\begin{array}{l}\mathrm{r}(10025) \\
\text { or } \mathrm{Y}(2 \mathrm{~S})\end{array}$ & $\begin{array}{c}\left(1^{-}\right)- \\
m_{r(10025)}\end{array}$ & $\begin{array}{c}10023.4 \\
\pm 0.3 \\
-\mathrm{m}_{\Upsilon(9460)}\end{array}$ & $\begin{aligned} & 0.0296 \\
\pm & 0.0047 \\
- & 563.3 \pm 0.4\end{aligned}$ & $\begin{array}{l}\mu^{+} \mu^{-} \\
\mathrm{e}^{+} \mathrm{e}^{-} \\
\Upsilon(9460) \pi \pi \\
\gamma \chi_{\mathrm{b}}(9875) \\
\gamma \chi_{\mathrm{b}}(9895) \\
\gamma \chi_{\mathrm{b}}(9915)\end{array}$ & $\begin{array}{r}1.9 \pm 1.8 \\
1.6 \pm 0.3 \\
19.5 \pm 1.7 \\
3.5 \pm 1.4 \\
5.9 \pm 1.4 \\
6.1 \pm 1.4\end{array}$ & $\begin{array}{r}5011 \\
5012 \\
476 \\
149 \\
128 \\
108\end{array}$ \\
\hline & $\begin{array}{l}\chi_{b}(10255) \\
\text { or } \chi_{b}\left(2^{3} P_{1}\right)\end{array}$ & )$^{r(1)+}$ & $\begin{array}{r}10253.7 \\
\pm 3.4 \\
\end{array}$ & & $\begin{array}{l}\gamma r(9460) \\
\gamma r(10025)\end{array}$ & $\begin{array}{l}\text { seen } \\
\text { seen }\end{array}$ & $\begin{array}{l}763 \\
228 \\
\end{array}$ \\
\hline & $\begin{array}{l}\chi_{b}(10270) \\
\text { or } \chi_{b}\left(2^{3} P_{2}\right) \\
\end{array}$ & $r^{r}()+$ & $\begin{array}{r}10271.0 \\
\pm 2.4 \\
\end{array}$ & & $\begin{array}{l}\gamma r(9460) \\
\gamma r(10025)\end{array}$ & $\begin{array}{l}\text { seen } \\
\text { seen }\end{array}$ & $\begin{array}{l}779 \\
245 \\
\end{array}$ \\
\hline & $\begin{array}{l}\mathrm{r}(10355) \\
\text { or } \mathrm{r(3S)}\end{array}$ & $\begin{array}{c}\left(1^{-}\right)- \\
m_{r(10355)}\end{array}$ & $\begin{array}{l}10355.5 \\
\pm 0.5 \\
-\mathrm{m}_{\mathrm{\Upsilon}(9460)}\end{array}$ & $\begin{array}{c}0.0177 \\
\pm 0.0051 \\
-895.5 \pm .6\end{array}$ & $\begin{array}{l}\mathrm{e}^{+} \mathrm{e}^{-} \\
\mu^{+} \mu^{-} \\
\Upsilon(9460) \pi^{+} \pi^{-} \\
\Upsilon(10025) \pi^{+} \pi^{-} \\
\gamma \chi_{\mathrm{b}}(10235) \\
\gamma \chi_{\mathrm{b}}(10255) \\
\gamma \chi_{\mathrm{b}}(10270)\end{array}$ & $\begin{array}{c}2.0 \pm 0.7 \\
3.3 \pm 2.0 \\
5.1 \pm 1.1 \\
3 \pm 3 \\
7.6 \pm 3.5 \\
15.6 \pm 4.2 \\
12.7 \pm 4.1\end{array}$ & $\begin{array}{r}5178 \\
5177 \\
814 \\
177 \\
122 \\
101 \\
84\end{array}$ \\
\hline & $\begin{array}{l}r(10575) \\
\text { or } \Upsilon(4 S)\end{array}$ & $\left(1^{-}\right)-$ & $\begin{array}{r}10573 \\
\pm 4\end{array}$ & $\begin{array}{r}14 \\
\pm 5\end{array}$ & $\mathrm{e}^{+} \mathrm{e}^{-}$ & $0.0017 \pm 0.0007$ & $\overline{5286}$ \\
\hline & & $\mathrm{m}_{\mathrm{r}(10575)}$ & $-m_{r(946}$ & $=1113 \pm 4$ & & & \\
\hline
\end{tabular}

STRANGE MESONS

\begin{tabular}{lllllcr}
\hline $\mathrm{K}^{+}$ & $1 / 2\left(0^{-}\right)$ & 493.67 & & & See Stable Particle Table \\
$\mathrm{K}^{0}$ & & 497.67 & & & \\
\hline $\mathrm{K}^{*}(892)$ & $1 / 2\left(1^{-}\right)$ & 892.1 & 51.3 & $\mathrm{~K} \pi$ & 100 & 288 \\
& & \pm 0.4 & \pm 1.0 & $\mathrm{~K} \gamma$ & $0.10 \pm 0.01$ & 309 \\
& & $\mathrm{~S}=1.4^{*}$ & $\mathrm{~S}=1.1^{*}$ & $\mathrm{~K} \pi \pi$ & $<0.05$ & 216
\end{tabular}

$M$ and $\Gamma$ from charged mode; $\mathrm{m}^{0}-\mathrm{m}^{ \pm}=6.7 \pm 1.2 \mathrm{MeV}$.

\begin{tabular}{|c|c|c|c|c|c|c|}
\hline $\begin{array}{l}\mathrm{Q}(1280) \\
\text { or } \mathrm{Q}_{1}\end{array}$ & $1 / 2\left(1^{+}\right)$ & $\begin{array}{l}1270^{\S} \\
\pm 10^{\S}\end{array}$ & $\begin{array}{r}90^{\S} \\
\pm 20^{\S}\end{array}$ & $\begin{array}{l}\mathrm{K} \rho \\
\kappa(1350) \pi \\
\mathrm{K}^{*}(892) \pi \\
\mathrm{K} \omega \\
\mathrm{K} \epsilon \\
\end{array}$ & $\begin{array}{r}42 \pm 6 \\
28 \pm 4 \\
16 \pm 5 \\
11 \pm 2 \\
3 \pm 2 \\
\end{array}$ & $\begin{array}{r}45 \\
298\end{array}$ \\
\hline $\begin{array}{l}\alpha(1350) \\
\text { See note o }\end{array}$ & $\frac{1 / 2\left(0^{+}\right)}{K \pi S \text { wa }}$ & $e^{1350}$ & $\sim 250$ & $\mathrm{~K} \pi$ & seen & 574 \\
\hline $\begin{array}{l}Q(1400) \\
\text { or } Q_{2}\end{array}$ & $1 / 2\left(1^{+}\right)$ & $\begin{array}{l}1406 \\
\pm 10\end{array}$ & $\begin{array}{r}184 \\
\pm 9\end{array}$ & $\begin{array}{l}\mathrm{K}^{*}(892) \pi \\
\mathrm{K} \rho \\
\mathrm{K} \epsilon \\
\mathrm{K} \omega\end{array}$ & $\begin{array}{r}94 \pm 6 \\
3 \pm 3 \\
2 \pm 2 \\
1 \pm 1\end{array}$ & $\begin{array}{l}403 \\
299 \\
285\end{array}$ \\
\hline
\end{tabular}




\section{Meson Table (cont'd)}

\begin{tabular}{|c|c|c|c|c|c|c|c|c|c|}
\hline \multirow{3}{*}{\multicolumn{2}{|c|}{\begin{tabular}{l|l}
$\mathbf{J}^{\mathbf{P}}$ & $\mathbf{G}$ \\
$\mathbf{N}$ & + \\
\cline { 2 - 2 } & - \\
\cline { 2 - 2 } & +
\end{tabular}}} & \begin{tabular}{|l|l|l|l|}
$Y$ & 0 & 1 & $\frac{1}{2}$ \\
\end{tabular} & \multirow{3}{*}{$\begin{array}{l}\mathbf{I}_{\left(\mathbf{J}^{\mathbf{P}}\right)} \\
- \text { estab. }\end{array}$} & \multirow{3}{*}{\multicolumn{2}{|c|}{$\begin{array}{c}\text { Full } \\
\text { Width } \\
\Gamma \\
(\mathrm{MeV})\end{array}$}} & \multicolumn{4}{|c|}{ Partial decay mode } \\
\hline & & \begin{tabular}{|l|l|l|}
$\omega / \phi$ & $\delta$ & $K^{*}, \kappa$ \\
\end{tabular} & & & & & & & \\
\hline & \begin{tabular}{|l|}
+ \\
- \\
\end{tabular} & \begin{tabular}{|l|l|l|}
$\eta / \mathrm{D}$ & $\mathrm{B}$ & $\mathrm{K}, \mathrm{Q}$ \\
$\mathrm{H}$ & $\pi / \mathrm{A}$ & \\
\end{tabular} & & & & Mode & $\begin{array}{c}\text { Fraction(\%) } \\
\text { [Upper limits (\%) are } 9\end{array}$ & $0 \% \mathrm{CL}]$ & $\begin{array}{c}\mathbf{p}_{\max } \\
(\mathrm{MeV} / \mathrm{c})\end{array}$ \\
\hline & & $\mathrm{K}^{*}(1430)$ & $1 / 2\left(2^{+}\right)$ & $\begin{array}{r}1425^{\S} \\
\pm 5^{\S}\end{array}$ & $\begin{array}{r}100^{\S} \\
\pm 10^{\S}\end{array}$ & $\begin{array}{l}\mathrm{K} \pi \\
\mathrm{K}^{*}(892) \pi \\
\mathrm{K}^{*}(892) \pi \pi \\
\mathrm{K}_{\rho} \\
\mathrm{K} \omega \\
\mathrm{K} \boldsymbol{\gamma} \\
\mathrm{K}_{\gamma}\end{array}$ & $\begin{array}{c}44.8 \pm 2.3 \\
24.5 \pm 2.0 \\
13.0 \pm 2.6 \\
8.8 \pm 1.0 \\
4.2 \pm 1.5 \\
5 \pm 5^{\S} \\
0.24 \pm 0.05\end{array}$ & $\begin{array}{l}S=2.7^{*} \\
S=1.1^{*} \\
S=1.1^{*} \\
S=1.2^{*}\end{array}$ & $\begin{array}{l}618 \\
417 \\
366 \\
324 \\
310 \\
485 \\
627\end{array}$ \\
\hline & $\rightarrow$ & See note on & $\begin{array}{l}1 / 2\left(2^{-}\right) \\
L(1770)^{\ddagger}\end{array}$ & $\sim 1770^{\S}$ & $\sim 200^{\S}$ & $\begin{array}{l}\mathrm{K}^{*}(1430) \pi \\
\mathrm{K}^{*}(892) \pi \\
\mathrm{Kf} \\
\mathrm{K} \phi\end{array}$ & $\begin{array}{c}\text { dominant } \\
\text { seen } \\
\text { seen } \\
\text { seen }\end{array}$ & & $\begin{array}{l}286 \\
651 \\
816\end{array}$ \\
\hline & & $\begin{array}{l}K^{*}(1780)^{\frac{1}{\ddagger}} \\
\text { or } K^{*} \\
\text { See note on }\end{array}$ & $K^{*}(1780)$ & $\begin{array}{c}1780 \\
\pm 10^{\S}\end{array}$ & $\begin{array}{c}160 \\
\pm 20^{\S}\end{array}$ & $\begin{aligned} & \mathbf{K} \pi \pi \\
&+ \mathbf{K} \rho \\
&+ \mathbf{K}^{*}(892) \pi \\
& \mathbf{K} \pi\end{aligned}$ & $\begin{array}{c}\text { large } \\
\text { large } \\
\text { large } \\
17 \pm 5^{\$}\end{array}$ & & $\begin{array}{l}796 \\
620 \\
657 \\
815\end{array}$ \\
\hline & $\rightarrow$ & Not a well- & established & $\begin{array}{c}2060^{\S} \\
\pm 30^{\S} \\
\end{array}$ & $\begin{array}{r}210^{\S} \\
\pm 40^{\S}\end{array}$ & $\begin{array}{l}\mathrm{K} \pi \\
\mathrm{K}^{*}(892) \pi \pi \\
\rho \mathrm{K} \pi \\
\omega \mathrm{K} \pi \\
\mathrm{K}^{*}(892) \pi \pi \pi\end{array}$ & $\begin{array}{l}7 \pm 1 \\
\text { seen } \\
\text { seen } \\
\text { seen } \\
\text { seen }\end{array}$ & & $\begin{array}{l}966 \\
809 \\
751 \\
744 \\
775\end{array}$ \\
\hline & $\rightarrow$ & & & & CHARI & NSTRANGE MI & SONS & & \\
\hline & & $\begin{array}{l}\mathrm{D}^{+} \\
\mathrm{D}^{0}\end{array}$ & $1 / 2\left(0^{-}\right)$ & $\begin{array}{l}1869.4 \\
1864.7\end{array}$ & & See $S$ & able Particle Table & & \\
\hline & & $\mathrm{D}^{*+}(2010)$ & $\begin{array}{l}1 / 2\left(1^{-}\right) \\
\mathrm{m}_{\mathrm{D}^{*+}}\end{array}$ & $\begin{array}{c}2010.1 \\
\pm 0.7 \\
m_{D^{0}}=145 .\end{array}$ & $\begin{array}{c}<2.0 \\
4 \pm 0.2 \mathrm{Me}\end{array}$ & $\begin{array}{l}\mathrm{D}^{0} \pi^{+} \\
\mathrm{D}^{+} \pi^{0} \\
\mathrm{D}^{+} \gamma\end{array}$ & $\begin{array}{c}64 \pm 11 \\
28 \pm 9 \\
8 \pm 7\end{array}$ & & $\begin{array}{r}39 \\
38 \\
136\end{array}$ \\
\hline & & $D^{* 0}(2010)$ & $1 / 2\left(1^{-}\right)$ & $\begin{array}{r}2007.2 \\
\pm 2.1\end{array}$ & $<5$ & $\begin{array}{l}D^{0} \pi^{0} \\
D^{0} \gamma\end{array}$ & $\begin{array}{l}55 \pm 15 \\
45 \pm 15\end{array}$ & & $\begin{array}{r}44 \\
137\end{array}$ \\
\hline & & & & & CHA & STRANGE MES & & & \\
\hline & & $\mathrm{F}^{+}$ & $O\left(0^{-}\right)$ & 1971 & & See & able Particle Table & & \\
\hline & & & & & BOTT & NSTRANGE MI & SON & & \\
\hline & & $\begin{array}{l}\mathrm{B}^{+} \\
\mathrm{B}^{0}\end{array}$ & $1 / 2\left(0^{-}\right)$ & $\begin{array}{l}5271 \\
5274\end{array}$ & & See $s$ & able Particle Table & & \\
\hline
\end{tabular}

$\rightarrow$ Indicates an entry in the Meson Data Card Listings not entered in the Meson Table. We do not regard these as established resonances. All the entries in the Listings can be found in the Table of Contents of the Meson Data Card Listings immediately following these footnotes.

¥ See Meson Data Card Listings.

* Quoted error includes scale factor $S=\sqrt{\chi^{2} /(N-1)}$. See footnote to Stable Particle Table.

$\dagger$ Square brackets indicate a subreaction of the previous (unbracketed) decay mode(s).

$\S$ This is only an educated guess; the error given is larger than the error on the average of the published values. (See the Meson Data Card Listings for the latter.)

a. $\Gamma M$ is approximately the half-width of the resonance when plotted against $\mathbf{M}^{2}$.

$b$. For decay modes into $\geqslant 3$ particles, $p_{\max }$ is the maximum momentum that any of the particles in the final state can have. The momenta have been calculated by using the averaged central mass values, without taking into account the widths of the resonances.

c. From pole position $(\mathrm{M}-\mathrm{i} \Gamma / 2)$. 


\section{Meson Table (cont'd)}

d. The $\mathrm{e}^{+} \mathrm{e}^{-}$branching fraction is from $\mathrm{e}^{+} \mathrm{e}^{-} \rightarrow \pi^{+} \pi^{-}$experiments only. The $\omega \rho$ interference is then due to $\omega \rho$ mixing only, and is expected to be small. See note in the Meson Data Card Listings. The $\mu^{+} \mu^{-}$branching fraction is compiled from 3 experiments, each possibly with substantial $\omega \rho$ interference. The error reflects this uncertainty; see notes in the Meson Data Card Listings. If $\mathrm{e} \mu$ universality holds, $\Gamma\left(\rho^{0} \rightarrow \mu^{+} \mu^{-}\right)=\Gamma\left(\rho^{0} \rightarrow \mathrm{e}^{+} \mathrm{e}^{-}\right) \times 0.99785$.

e. Empirical limits on fractions for other decay modes of $\rho(770)$ are $\pi^{ \pm} \eta<0.8 \%(\mathrm{CL}=84 \%), \pi^{+} \pi^{+} \pi^{-} \pi^{-}<0.15 \%, \pi^{ \pm} \pi^{+} \pi^{-} \pi^{0}$ $<0.2 \%(\mathrm{CL}-84 \%)$.

f. Empirical limits on fractions for other decay modes of $\omega(783)$ are $\pi^{+} \pi^{-} \gamma<5 \%, \pi^{0} \pi^{0} \gamma<1 \%, \eta+$ neutral(s) $<1.5 \%, \mu^{+} \mu^{-}$ $<0.02 \%$.

g. Empirical limits on fractions for other decay modes of $\eta^{\prime}(958)$ are $\pi^{+} \pi^{-}<2 \%(\mathrm{CL}=84 \%), \pi^{+} \pi^{-} \pi^{0}<5 \%(\mathrm{CL}=84 \%)$, $\pi^{+} \pi^{+} \pi^{-} \pi^{-}<1 \%(C L=95 \%), \pi^{+} \pi^{+} \pi^{-} \pi^{-} \pi^{0}<1 \%(C L=84 \%), 6 \pi<1 \%, \pi^{+} \pi^{-} \mathrm{e}^{+} \mathrm{e}^{-}<0.6 \%, \pi^{0} \mathrm{e}^{+} \mathrm{e}^{-}<1.3 \%(\mathrm{CL}=84 \%)$, $\eta \mathrm{e}^{+} \mathrm{e}^{-}<1.1 \%, \pi^{0} \rho^{0}<4 \%, \eta \mu^{+} \mu^{-}<1.5 \times 10^{-5}, \pi^{0} \mu^{+} \mu^{-}<6 \times 10^{-5}$.

$h$. The mass and width are from the $\eta \pi$ mode only. If the $\mathrm{K} \overline{\mathbf{K}}$ channel is strongly coupled, the width may be larger.

i. Empirical limits on fractions for other decay modes of $\phi(1020)$ are $\pi^{+} \pi^{-} \gamma<0.7 \%, \omega \gamma<5 \%(C L=84 \%), \rho \gamma<2 \%(C L=84 \%)$, $2 \pi^{+} 2 \pi^{-} \pi^{0}<1 \%(\mathrm{CL}=95 \%), 2 \pi^{+} 2 \pi^{-}<0.1 \%$.

j. Empirical limits on fractions for other decay modes of $\mathrm{B}(1235)$ are $\pi \pi<15 \%, \mathrm{~K} \overline{\mathrm{K}}<2 \%(\mathrm{CL}=84 \%), 4 \pi<50 \%(\mathrm{CL}=84 \%), \phi \pi$ $<1.5 \%(\mathrm{CL}=84 \%), \eta \pi<25 \%,(\overline{\mathrm{KKK}})^{ \pm} \pi^{0}<8 \%, \mathrm{~K}_{\mathrm{S}} \mathrm{K}_{\mathrm{S}} \pi^{ \pm}<2 \%, \mathrm{~K}_{\mathrm{S}} \mathrm{K}_{\mathrm{L}} \pi^{ \pm}<6 \%$.

$k$. Empirical limits (CL-95\%) on fractions for other decay modes of $\mathrm{f}(1270)$ are $\eta \pi \pi<1 \%, \mathrm{~K}^{0} \mathrm{~K}^{-} \pi^{+}+$c.c. $<0.4 \%, \eta \eta<2 \%$.

$\ell$. Empirical limits on fractions for other decay modes of $\mathrm{A}(1680)$ are $\eta \pi<10 \%, 5 \pi<10 \%$.

$m$. Includes $\overline{\mathrm{p}} \overline{\mathrm{p}} \boldsymbol{\pi}^{+} \pi^{-} \gamma$ and excludes $\overline{\mathrm{p}} \boldsymbol{\eta}, \mathrm{p} \overline{\mathrm{p}} \omega, \mathrm{p} \overline{\mathrm{p}} \eta^{\prime}$.

$n$. See $E(1420)$ mini-review.

o. Empirical limits on fractions for other decay modes of $\chi(3415)$ are $2 \gamma<0.17 \%, p \bar{p}<0.11 \%$.

p. Empirical limits on fractions for other decay modes of $\chi(3510)$ are $\left(\pi^{+} \pi^{-}\right.$and $\left.\mathrm{K}^{+} \mathrm{K}^{-}\right)<0.2 \%, \gamma \gamma<0.16 \%, \mathrm{p} \overline{\mathrm{p}}<0.13 \%$.

q. Empirical limits on fractions for other decay modes of $\chi(3555)$ are $2 \gamma<0.06 \%, \mathrm{p} \overline{\mathrm{p}}<0.10 \%, \mathrm{~J} / 4 \pi^{+} \pi^{-} \pi^{0}<1.5 \%$.

$r$. Spectroscopic labeling for these states is theoretical, pending experimental information.

Contents of Meson Data Card Listings

\begin{tabular}{|c|c|c|c|c|c|c|c|c|c|c|}
\hline \multicolumn{9}{|c|}{ Non-strange $(\mathrm{S}=0 ; \mathrm{C}, \mathrm{B}=0)$} & \multicolumn{2}{|c|}{ Strange $(|\mathbf{S}|=1 ; \mathbf{C}, \mathbf{B}=0)$} \\
\hline \multicolumn{2}{|r|}{ entry } & ${ }^{\mathbf{I}}\left(\mathrm{J}^{\mathbf{P}}\right) \mathrm{C}_{\mathbf{n}}$ & \multicolumn{2}{|c|}{ entry } & $\mathrm{I}^{\mathrm{G}}\left(\mathrm{J}^{\mathrm{P}}\right) \mathrm{C}_{\mathrm{n}}$ & \multicolumn{2}{|c|}{ entry } & \multirow{2}{*}{$\frac{I^{G_{(}}\left(J^{P}\right) C_{n}}{\left(1^{-}\right)}$} & entry & I $\left(\mathrm{J}^{\mathrm{P}}\right)$ \\
\hline & $\pi$ & $1^{-}\left(0^{-}\right)+$ & $\omega$ & (1670) & $0^{-}\left(3^{-}\right)-$ & $\rightarrow \mathrm{e}^{+} \mathrm{e}^{-}$ & $(1100-2200)$ & & $\mathbf{K}$ & $1 / 2\left(0^{-}\right)$ \\
\hline & $\boldsymbol{\eta}$ & $0^{+}\left(0^{-}\right)+$ & $\mathbf{A}$ & $(1680)$ & $1^{-}\left(2^{-}\right)+$ & $\rightarrow \bar{N} N$ & $(1200-3600)$ & & $K^{*} \quad(892)$ & $1 / 2\left(1^{-}\right)$ \\
\hline & (770) & $1^{+}\left(1^{-}\right)-$ & $\phi$ & (1680) & $0^{-}\left(1^{-}\right)-$ & $\rightarrow \mathrm{X}$ & $(1900-3600)$ & & $Q \quad(1280)$ & $1 / 2\left(1^{+}\right)$ \\
\hline & $\omega \quad(783)$ & $0^{-}\left(1^{-}\right)-$ & g & $(1690)$ & $1^{+}\left(3^{-}\right)-$ & $\eta_{c}$ & (2980) & $0^{+}$ & $\times \quad(1350)$ & $1 / 2\left(0^{+}\right)$ \\
\hline & $\eta^{\prime} \quad(958)$ & $0^{+}\left(0^{-}\right)+$ & $\theta$ & (1690) & $0^{+}\left({ }^{+}\right)+$ & $\mathrm{J} / \psi$ & $(3100)$ & $0^{-}\left(1^{-}\right)-$ & $Q \quad(1400)$ & $1 / 2\left(1^{+}\right)$ \\
\hline & S (975) & $0^{+}\left(0^{+}\right)+$ & $\rightarrow \eta$ & $(1700)$ & + & $x$ & (3415) & $0^{+}\left(0^{+}\right)+$ & $\rightarrow \mathrm{K} \quad(1400)$ & $1 / 2\left(0^{-}\right)$ \\
\hline & $\delta \quad(980)$ & $1^{-}\left(0^{+}\right)+$ & $\rightarrow \mathrm{S}$ & (1730) & $0^{+}\left(0^{+}\right)+$ & $x$ & (3510) & $0^{+}\left(1^{+}\right)+$ & $K^{*}(1430)$ & $1 / 2\left(2^{+}\right)$ \\
\hline & $\phi \quad(1020)$ & $0^{-}\left(1^{-}\right)-$ & $\rightarrow \pi$ & (1770) & $1^{-}\left(0^{-}\right)+$ & $x$ & (3555) & $0^{+}\left(2^{+}\right)+$ & $\rightarrow \mathrm{L} \quad(1580)$ & $1 / 2\left(2^{-}\right)$ \\
\hline & H (1190) & $0^{-}\left(1^{+}\right)-$ & $\rightarrow \mathrm{f}$ & (1810) & $0^{+}\left(2^{+}\right)+$ & $\rightarrow \eta_{\mathrm{c}}$ & (3590) & + & $\rightarrow \mathrm{K}^{*}(1650)$ & $1 / 2\left(1^{-}\right)$ \\
\hline & B (1235) & $1^{+}\left(1^{+}\right)-$ & & $(\mathbf{1 8 5 0 )}$ & 0 & $\psi$ & (3685) & $0^{-}\left(1^{-}\right)-$ & L (1770) & $1 / 2\left(2^{-}\right)$ \\
\hline$\rightarrow$ & gs $(1240)$ & $0^{+}\left(0^{+}\right)+$ & $\rightarrow \mathrm{S}$ & (1935) & & $\psi$ & (3770) & $\left(1^{-}\right)-$ & $K^{*}(1780)$ & $1 / 2\left(3^{-}\right)$ \\
\hline 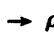 & $\rho \quad(1250)$ & $1^{+}\left(1^{-}\right)-$ & h & (2030) & $0^{+}\left(4^{+}\right)+$ & $\psi$ & (4030) & $\left(1^{-}\right)-$ & $\rightarrow \mathrm{K} \quad(1830)$ & $1 / 2\left(0^{-}\right)$ \\
\hline & f $(1270)$ & $0^{+}\left(2^{+}\right)+$ & $\rightarrow \delta$ & (2040) & $1^{-}\left(4^{+}\right)+$ & $\psi$ & $(4160)$ & $\left(1^{-}\right)-$ & $\mathrm{K}^{*}(2060)$ & $1 / 2\left(4^{+}\right)$ \\
\hline & A (1270) & $1^{-}\left(1^{+}\right)+$ & $\rightarrow \mathbf{A}$ & (2050) & $1^{-}\left(3^{+}\right)+$ & $\psi$ & (4415) & $\left(1^{-}\right)-$ & $\rightarrow \mathrm{K} \quad(2250)$ & $1 / 2\left(2^{-}\right)$ \\
\hline & $\eta \quad(1275)$ & $0^{+}\left(0^{-}\right)+$ & $\rightarrow \mathbf{A}$ & $(2100)$ & $1^{-}\left(2^{-}\right)+$ & $r$ & (9460) & $\left(1^{-}\right)-$ & $\rightarrow \mathrm{K} \quad(2320)$ & $1 / 2\left(3^{+}\right)$ \\
\hline & D (1285) & $0^{+}\left(1^{+}\right)+$ & $\rightarrow \rho$ & $(2150)$ & $1^{+}\left(1^{-}\right)-$ & $x_{b}$ & (9875) & ()$+$ & $\rightarrow \mathrm{K} \quad(2500)$ & $1 / 2\left(4^{-}\right)$ \\
\hline & $\epsilon \quad(1300)$ & $0^{+}\left(0^{+}\right)+$ & $\rightarrow \epsilon$ & (2150) & $0^{+}\left(2^{+}\right)+$ & $x_{b}$ & (9895) & ()$+$ & Charmed $(\mid C$ & $1=1$ \\
\hline & $\pi \quad(1300)$ & $1^{-}\left(0^{-}\right)+$ & $\rightarrow \xi$ & (2220) & $0\left(^{+}\right)$ & $x_{b}$ & (9915) & ()$+$ & D & $1 / 2\left(0^{-}\right)$ \\
\hline & $A_{2}(1320)$ & $1^{-}\left(2^{+}\right)+$ & $\rightarrow \mathbf{8}_{\mathrm{T}}$ & (2240) & $0^{+}\left(2^{+}\right)+$ & $r$ & (10025) & $\left(1^{-}\right)-$ & $D^{*}(2010)$ & $1 / 2\left(1^{-}\right)$ \\
\hline E & E (1420) & $0^{+}\left(1^{+}\right)+$ & $\rightarrow \rho$ & (2250) & $1^{+}\left(3^{-}\right)-$ & & (10235) & & $\mathbf{F}$ & $0\left(0^{-}\right)$ \\
\hline & ८ (1440) & $0^{+}\left(0^{-}\right)+$ & $\rightarrow \epsilon$ & $(2300)$ & $0^{+}\left(4^{+}\right)+$ & & (10200) & $(2)$ & $\rightarrow F^{*}(2140)$ & \\
\hline & $f^{\prime} \quad(1525)$ & $0^{+}\left(2^{+}\right)+$ & $\rightarrow \rho$ & $(2350)$ & $1^{+}\left(5^{-}\right)-$ & & & & Bottom (Beal & aty) $(|B|=1)$ \\
\hline I & D (1530) & $0^{+}\left(1^{+}\right)+$ & $\rightarrow \delta$ & (2450) & $1^{-}\left(6^{+}\right)$ & $x_{b}$ & (1027) & $(5)+$ & B & \\
\hline & $\rho \quad(1600)$ & $1^{+}\left(1^{-}\right)-$ & $\rightarrow r$ & $(2510)$ & $0^{+}\left(6^{+}\right)$ & $\begin{array}{l}\Upsilon \\
\Upsilon\end{array}$ & $\begin{array}{l}(10355) \\
(10575)\end{array}$ & $\begin{array}{l}\left(1^{-}\right)- \\
\left(1^{-}\right)-\end{array}$ & $\rightarrow$ Exotics & \\
\hline
\end{tabular}


Baryon Table

April 1984

The following short list gives the name, the nominal mass, the quantum numbers (where known), and the status of each of the Baryon States in the Data Card Listings. States with 3- or 4-star status are included in the Baryon Table below; the others are omitted because the evidence for the existence of the effect and/or for its interpretation as a resonance is open to question.

\begin{tabular}{|c|c|c|c|c|c|c|c|c|c|}
\hline N(939) P11 & $* * * *$ & $\Delta(1232)$ P33 & $* * *$ & $\mathrm{ZO}(1780) \mathrm{P} 01$ & * & $\Sigma(1193) P 11$ & 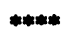 & $\Xi(1318)$ P11 & $* * *$ \\
\hline$N(1440) P 11$ & $m+m * *$ & $\Delta(1550)$ P31 & $*$ & ZO(1865) D03 & 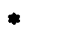 & $\Sigma(1385) \quad P 13$ & $* * * *$ & $\Xi(1530) \mathrm{P} 13$ & $* * * * *$ \\
\hline$N(1520) \mathrm{D} 13$ & $* * * *$ & $\Delta(1600)$ P33 & $*$ & Z1(1900) P13 & $*$ & $\Sigma(1480)$ & $*$ & $\Xi(1630)$ & $*$ \\
\hline$N(1535) S 11$ & $* * * *$ & $\Delta(1620) \mathrm{S} 31$ & $* * *$ & $\mathrm{Z1}(2150)$ & * & $\Sigma(1560)$ & ** & $\Xi(1680)$ & $*$ \\
\hline$N(1540) P 13$ & $*$ & $\Delta(1700)$ D33 & $* * *$ & $\mathrm{Z1}(2500)$ & 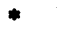 & $\Sigma(1580)$ D13 & $* *$ & $\Xi(1820) \quad 13$ & $* * *$ \\
\hline$N(1650) S 11$ & $* * * *$ & $\Delta(1900) \mathrm{S} 31$ & $* * *$ & & & $\Sigma(1620) S 11$ & $*$ & $\Xi(1940)$ & $* *$ \\
\hline$N(1675)$ D15 & $m * * *$ & $\Delta(1905)$ F35 & $* * * *$ & $\Lambda(1116) \mathrm{P} 01$ & $* * * * *$ & $\Sigma(1660) P 11$ & $* * *$ & $\Xi(2030) \quad 1$ & $* * *$ \\
\hline$N(1680)$ F15 & $* * * *$ & $\Delta(1910)$ P31 & $* * *$ & $\Lambda(1405)$ S01 & $* * * * *$ & $\Sigma(1670)$ D13 & $* * * * *$ & $\Xi(2120)$ & $*$ \\
\hline$N(1700) D 13$ & $* *$ & $\Delta(1920)$ P33 & $* * *$ & $\Lambda(1520) \mathrm{D03}$ & $* * * *$ & $\Sigma(1690)$ & $* *$ & $\Xi(2250)$ & $* *$ \\
\hline$N(1710)$ P11 & $* * *$ & $\Delta(1930)$ D35 & $* * *$ & $\Lambda(1600)$ P01 & $* * *$ & $\Sigma(1750) S 11$ & $* * * *$ & $\Xi(2370) \quad 1$ & $* *$ \\
\hline$N(1720)$ P13 & $* * * *$ & $\Delta(1940)$ D33 & * & $\Lambda(1670)$ S01 & $* * * * *$ & $\Sigma(1770) P 11$ & $*$ & $\Xi(2500)$ & $*$ \\
\hline$N(1990)$ F17 & $*$ & $\Delta(1950)$ F37 & $* * * *$ & $\Lambda(1690) \mathrm{D} 03$ & thin* & $\Sigma(1775)$ D15 & $* * * *$ & & \\
\hline$N(2000)$ F15 & $* *$ & $\Delta(2150) \mathrm{S31}$ & $*$ & $\Lambda(1800)$ S01 & $* * *$ & $\Sigma(1840)$ P13 & $*$ & $\Omega(1672) P 03$ & $* * *$ \\
\hline$N(2080)$ D13 & $*$ & $\Delta(2200) G 37$ & $*$ & $\Lambda(1800) \mathrm{P} 01$ & $+*$ & $\Sigma(1880) P 11$ & ** & & $* \neq * *$ \\
\hline$N(2090) S 11$ & $*$ & $\Delta(2300)$ H39 & $* *$ & $\Lambda(1820)$ F05 & $+* * * *$ & $\Sigma(1915) F 15$ & $* * * *$ & 6) & \\
\hline$N(2100) P 11$ & $*$ & $\Delta(2350)$ D35 & * & $\Lambda(1830)$ D05 & $* * * *$ & $\Sigma(1940)$ D13 & $* * *$ & $\Sigma_{c}(2450)$ & $* *$ \\
\hline$N(2190) \mathrm{G} 17$ & 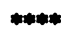 & $\Delta(2390)$ F37 & $*$ & $\Lambda(1890)$ P03 & $* * * *$ & $\Sigma(2000) S 11$ & $*$ & & \\
\hline$N(2200)$ D15 & $* *$ & $\Delta(2400)$ G39 & $* *$ & $\Lambda(2000)$ & $*$ & $\Sigma(2030) \mathrm{F} 17$ & $* * * *$ & $A(2460)$ & * \\
\hline$N(2220) \mathrm{H} 19$ & 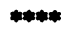 & $\Delta(2420)$ H311 & $* * *$ & $\Lambda(2020)$ F07 & $*$ & $\Sigma(2070) F 15$ & $*$ & & * \\
\hline$N(2250) G 19$ & $+* * *$ & $\Delta(2750) 1313$ & $*$ & $\Lambda(2100)$ G07 & $* * * *$ & $\Sigma(2080)$ P13 & $* *$ & $\Lambda_{b}(5000)$ & 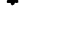 \\
\hline$N(2600) 1111$ & $*$ & $\Delta(2950)$ K315 & $*$ & $\Lambda(2110)$ F05 & $* * *$ & $\Sigma(2100) \mathrm{G} 17$ & * & Dibaryons & \\
\hline$N(2700) \mathrm{K} 113$ & $*$ & $\Delta(\sim 3000)$ & & $\Lambda(2325) \mathrm{D} 03$ & $*$ & $\Sigma(2250)$ & $* * *$ & $\mathrm{NN}(2170) 1 \mathrm{D} 2$ & $* *$ \\
\hline \multirow[t]{4}{*}{$N(\sim 3000)$} & & & & $\Lambda(2350)$ & $* * *$ & $\Sigma(2455)$ & $* *$ & $\mathrm{NN}(2250) 3 \mathrm{~F} 3$ & $* *$ \\
\hline & & & & $\Lambda(2585)$ & $* *$ & $\Sigma(2620)$ & $*$ & NN(?) & $*$ \\
\hline & & & & & & $\Sigma(3000)$ & $*$ & $\Lambda N(2130) 3 S 1$ & ** \\
\hline & & & & & & $\Sigma(3170)$ & $*$ & $\Xi N(?)$ & * \\
\hline
\end{tabular}

\begin{tabular}{|c|c|c|c|c|c|c|c|}
\hline$*$ & $\begin{array}{l}\text { Good, clear, and u } \\
\text { Good, but in need } \\
\text { Not established; ne } \\
\text { Evidence weak; co }\end{array}$ & $\begin{array}{l}\text { Inmistakable. } \\
\text { of clarification or } \\
\text { eeds confirmation. } \\
\text { uld disappear. }\end{array}$ & not absol & ely certain. & & & \\
\hline \multirow[b]{2}{*}{ Particle $^{a}$} & \multirow[b]{2}{*}{$\mathbf{I}\left(\mathbf{J}^{\mathbf{P}}\right) \mathbf{L}_{\mathbf{2 I} \cdot \mathbf{2 J}}^{b}$} & \multirow[b]{2}{*}{$\begin{array}{l}P_{\text {beam }}^{c}(\mathrm{GeV} / \mathrm{c}) \\
\sigma=4 \pi X^{2}(\mathrm{mb})\end{array}$} & \multirow{2}{*}{$\underset{\substack{\text { Mass } \\
(\mathrm{MeV})}}{d}$} & \multirow{2}{*}{$\begin{array}{l}\text { Full }^{e} \\
\text { width } \mathrm{T} \\
(\mathrm{MeV})\end{array}$} & \multicolumn{3}{|c|}{ Partial decay modes } \\
\hline & & & & & Mode $^{f}$ & $\begin{array}{l}\text { Fraction } 8 \\
\text { (\%) }\end{array}$ & $\frac{\mathrm{p}^{h}}{(\mathrm{MeV} / \mathrm{c})}$ \\
\hline \multicolumn{8}{|c|}{$S=0 \quad I=1 / 2$ NUCLEON RESONANCES (N) } \\
\hline $\begin{array}{l}\mathbf{p} \\
\mathbf{n}\end{array}$ & $1 / 2\left(1 / 2^{+}\right)$ & & $\begin{array}{l}938.3 \\
939.6\end{array}$ & & \multicolumn{3}{|c|}{ See Stable Particle Table } \\
\hline$N(1440)$ & $1 / 2\left(1 / 2^{+}\right) \mathrm{P}_{11}^{\prime}$ & $\begin{array}{l}p=0.61 \\
\sigma=31.0\end{array}$ & $\begin{array}{r}1400 \text { to } \\
1480\end{array}$ & $\begin{array}{r}120 \text { to } \\
350 \\
(200)\end{array}$ & $\begin{array}{l}\mathbf{N} \pi \\
N \eta \\
N \pi \pi \\
{\left[\begin{array}{l}\Delta \pi \\
N \rho \\
N \epsilon\end{array}\right.}\end{array}$ & $\left.\begin{array}{c}50-70 \\
8-18 \\
\sim 30 \\
12-28 \\
\sim 7 \\
\sim 5\end{array}\right]^{*}$ & $\begin{array}{r}397 \\
+ \\
342 \\
143 \\
+ \\
+\end{array}$ \\
\hline $\mathrm{N}(1520)$ & $1 / 2\left(3 / 2^{-}\right) D_{13}^{\prime}$ & $\begin{array}{l}p=0.74 \\
\sigma=23.5\end{array}$ & $\begin{array}{r}1510 \text { to } \\
1530\end{array}$ & $\begin{array}{r}100 \text { to } \\
140 \\
(125)\end{array}$ & $\begin{array}{l}\mathrm{N} \pi \\
\mathrm{N} \eta \\
\mathrm{N} \pi \boldsymbol{\pi} \\
\Delta \pi \\
\mathrm{N} \rho \\
\mathrm{N} \epsilon\end{array}$ & $\left.\begin{array}{c}50-60 \\
\sim 0.1 \\
35-50 \\
15-25 \\
15-25 \\
<5\end{array}\right]^{*}$ & $\begin{array}{r}456 \\
149 \\
410 \\
228 \\
+ \\
+\end{array}$ \\
\hline
\end{tabular}




\section{Baryon Table (cont'd)}

\begin{tabular}{|c|c|c|c|c|c|c|c|}
\hline \multirow[b]{2}{*}{ Particle $^{a}$} & \multirow[b]{2}{*}{$\mathbf{I}\left(\mathbf{J}^{\mathbf{P}}\right) \mathbf{L}_{\mathbf{2 I} \cdot \mathbf{2 J}}^{\boldsymbol{b}}$} & \multirow[b]{2}{*}{$\begin{array}{l}P_{\text {beam }}^{c}(\mathrm{GeV} / \mathrm{c}) \\
\sigma=4 \pi X^{2}(\mathrm{mb})\end{array}$} & \multirow{2}{*}{$\begin{array}{c}\text { Mass }^{d} \\
\text { M } \\
(\mathrm{MeV})\end{array}$} & \multirow{2}{*}{$\begin{array}{c}\text { Full }^{e} \\
\text { width } \Gamma \\
(\mathrm{MeV})\end{array}$} & \multicolumn{3}{|c|}{ Partial decay modes } \\
\hline & & & & & Mode $^{f}$ & $\begin{array}{l}\text { Fraction }^{g} \\
(\%)\end{array}$ & $\begin{array}{c}\mathbf{p}^{h} \\
(\mathrm{MeV} / \mathrm{c})\end{array}$ \\
\hline $\mathrm{N}(1535)$ & $1 / 2\left(1 / 2^{-}\right) S_{11}^{\prime}$ & $\begin{array}{l}p=0.76 \\
\sigma=22.5\end{array}$ & $\begin{array}{r}1520 \text { to } \\
1560\end{array}$ & $\begin{array}{r}100 \text { to } \\
250 \\
(150)\end{array}$ & $\begin{array}{c}\mathrm{N} \pi \\
\mathrm{N} \eta \\
\mathrm{N} \pi \boldsymbol{\pi} \\
{\left[\begin{array}{l}\Delta \pi \\
\mathrm{N} \rho \\
\mathrm{N} \epsilon\end{array}\right.}\end{array}$ & $\left.\begin{array}{r}35-50 \\
\sim 35 \\
\sim 5 \\
\sim 1 \\
\sim 3 \\
\sim 2\end{array}\right]^{*}$ & $\begin{array}{r}467 \\
182 \\
422 \\
+\quad 242 \\
+ \\
+\end{array}$ \\
\hline $\mathrm{N}(1650)$ & $1 / 2\left(1 / 2^{-}\right) S_{11}^{\prime \prime}$ & $\begin{array}{l}p=0.96 \\
\sigma=16.4\end{array}$ & $\begin{array}{r}1620 \text { to } \\
1680\end{array}$ & $\begin{array}{r}100 \text { to } \\
200 \\
(150)\end{array}$ & 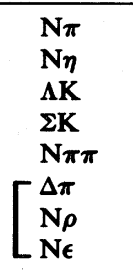 & $\left.\begin{array}{c}55-65 \\
\sim 1.5 \\
\sim 8 \\
3-10 \\
\sim 30 \\
4-15 \\
\sim 20 \\
<5\end{array}\right]^{*}$ & $\begin{array}{r}547 \\
346 \\
161 \\
+ \\
511 \\
+\quad 344 \\
\dagger \\
\dagger\end{array}$ \\
\hline$N(1675)$ & $1 / 2\left(5 / 2^{-}\right) D_{15}^{\prime}$ & $\begin{array}{l}p=1.01 \\
\sigma=15.4\end{array}$ & $\begin{array}{r}1660 \text { to } \\
1690\end{array}$ & $\begin{array}{r}120 \text { to } \\
180 \\
(155)\end{array}$ & $\begin{array}{c}\mathbf{N} \boldsymbol{\pi} \\
\mathbf{N} \boldsymbol{\eta} \\
\mathbf{\Lambda} \mathbf{K} \\
\mathbf{N} \boldsymbol{\pi} \boldsymbol{\pi} \\
{\left[\begin{array}{l}\Delta \boldsymbol{\pi} \\
\mathbf{N} \rho\end{array}\right.}\end{array}$ & $\left.\begin{array}{r}30-40 \\
\sim 1 \\
\sim 0.1 \\
55-70 \\
50-65 \\
\sim 5\end{array}\right]^{*}$ & $\begin{array}{r}563 \\
374 \\
209 \\
529 \\
364 \\
+\end{array}$ \\
\hline$N(1680)$ & $1 / 2\left(5 / 2^{+}\right) F_{15}^{\prime}$ & $\begin{array}{l}p=1.01 \\
\sigma=15.2\end{array}$ & $\begin{array}{r}1670 \text { to } \\
1690\end{array}$ & $\begin{array}{r}110 \text { to } \\
140 \\
(125)\end{array}$ & $\begin{array}{c}\mathbf{N} \pi \\
\mathbf{N} \eta \\
\Lambda \mathbf{K} \\
\mathbf{N} \pi \boldsymbol{\pi} \\
{\left[\begin{array}{l}\Delta \pi \\
\mathbf{N} \rho \\
\mathbf{N} \epsilon\end{array}\right.}\end{array}$ & $\left.\begin{array}{r}55-65 \\
<1 \\
\text { not seen } \\
\sim 40 \\
\sim 12 \\
\sim 10 \\
\sim 20\end{array}\right]^{*}$ & $\begin{array}{r}567 \\
379 \\
218 \\
532 \\
+369 \\
\dagger \\
+\end{array}$ \\
\hline$N(1700)$ & $1 / 2\left(3 / 2^{-}\right) D_{13}^{\prime \prime}$ & $\begin{array}{l}p=1.05 \\
\sigma=14.5\end{array}$ & $\begin{array}{r}1670 \text { to } \\
1730\end{array}$ & $\begin{array}{r}70 \text { to } \\
120 \\
(100)\end{array}$ & $\begin{array}{c}\mathbf{N} \pi \\
\mathbf{N} \eta \\
\Delta \mathrm{K} \\
\mathbf{N} \pi \boldsymbol{\pi} \\
{\left[\begin{array}{l}\Delta \pi \\
\mathbf{N} \rho \\
\mathrm{N} \epsilon\end{array}\right.}\end{array}$ & $\left.\begin{array}{r}8-12 \\
\sim 4 \\
\sim 0.2 \\
\sim 85 \\
15-40 \\
\sim 5 \\
<40\end{array}\right]^{*}$ & $\begin{array}{r}580 \\
400 \\
250 \\
547 \\
385 \\
\dagger \\
\dagger\end{array}$ \\
\hline $\mathrm{N}(1710)$ & $1 / 2\left(1 / 2^{+}\right) \mathrm{P}_{11}^{\prime \prime}$ & $\begin{array}{l}p=1.07 \\
\sigma=14.2\end{array}$ & $\begin{array}{r}1680 \text { to } \\
1740\end{array}$ & $\begin{array}{r}90 \text { to } \\
130 \\
(110)\end{array}$ & 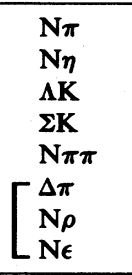 & $\left.\begin{array}{r}10-20 \\
\sim 25 \\
\sim 15 \\
2-10 \\
>50 \\
10-25 \\
25-65 \\
15-40\end{array}\right]^{*}$ & $\begin{array}{r}587 \\
410 \\
264 \\
138 \\
554 \\
\quad 393 \\
48 \\
+\end{array}$ \\
\hline$N(1720)$ & $1 / 2\left(3 / 2^{+}\right) P_{13}^{\prime \prime}$ & $\begin{array}{l}p=1.09 \\
\sigma=13.9\end{array}$ & $\begin{array}{r}1690 \text { to } \\
1800\end{array}$ & $\begin{array}{r}125 \text { to } \\
250 \\
(200)\end{array}$ & $\begin{array}{c}\mathbf{N} \pi \\
\mathbf{N} \eta \\
\Delta \mathrm{K} \\
\Sigma \mathrm{K} \\
\mathbf{N} \pi \boldsymbol{\pi} \\
{\left[\begin{array}{l}\Delta \pi \\
\mathrm{N} \rho \\
\mathrm{N} \epsilon\end{array}\right.} \\
\end{array}$ & $\left.\begin{array}{r}10-20 \\
\sim 3.5 \\
\sim 5 \\
2-5 \\
\sim 70 \\
\sim 20 \\
45-70 \\
\sim 20\end{array}\right]^{*}$ & $\begin{array}{r}594 \\
420 \\
278 \\
162 \\
561 \\
401 \\
104 \\
\dagger \\
\end{array}$ \\
\hline $\mathrm{N}(2190)$ & $1 / 2\left(7 / 2^{-}\right) G_{17}$ & $\begin{array}{l}p=2.07 \\
\sigma=6.21\end{array}$ & $\begin{array}{r}2120 \text { to } \\
2230\end{array}$ & $\begin{array}{r}200 \text { to } \\
500 \\
(350)\end{array}$ & $\begin{array}{l}\mathbf{N} \boldsymbol{\pi} \\
\mathbf{N} \boldsymbol{\eta} \\
\mathbf{\Lambda}\end{array}$ & $\begin{array}{r}\sim 14 \\
\sim 3 \\
\sim 0.3\end{array}$ & $\begin{array}{l}888 \\
790 \\
712\end{array}$ \\
\hline
\end{tabular}




\section{Baryon Table (cont'd)}

\begin{tabular}{|c|c|c|c|c|c|c|c|}
\hline \multirow[b]{2}{*}{ Particle $^{a}$} & \multirow[b]{2}{*}{$\mathbf{I}\left(\mathbf{J}^{\mathbf{P}}\right) \mathbf{L}_{\mathbf{2} \mathbf{I} \cdot \mathbf{2 J}}^{\boldsymbol{b}}$} & \multirow[b]{2}{*}{$\begin{array}{l}P_{\text {beam }}^{c}(\mathrm{GeV} / \mathrm{c}) \\
\sigma=4 \pi \chi^{2}(\mathrm{mb})\end{array}$} & \multirow{2}{*}{$\begin{array}{c}\text { Mass }^{d} \\
\text { M } \\
(\mathrm{MeV})\end{array}$} & \multirow{2}{*}{$\begin{array}{l}\text { Full }^{e} \\
\text { width } \Gamma \\
(\mathrm{MeV})\end{array}$} & \multicolumn{3}{|c|}{ Partial decay modes } \\
\hline & & & & & Mode $^{f}$ & $\begin{array}{c}\text { Fraction }{ }^{g} \\
(\%)\end{array}$ & $\frac{p^{h}}{(M e V / c)}$ \\
\hline $\mathrm{N}(2220)$ & $1 / 2\left(9 / 2^{+}\right) \mathrm{H}_{19}$ & $\begin{array}{l}p=2.14 \\
\sigma=5.97\end{array}$ & $\begin{array}{r}2150 \text { to } \\
2300\end{array}$ & $\begin{array}{r}300 \text { to } \\
500 \\
(400) \\
\end{array}$ & $\begin{array}{l}\mathbf{N} \boldsymbol{\pi} \\
\mathbf{N} \boldsymbol{\eta} \\
\mathbf{\Lambda K}\end{array}$ & $\begin{array}{l}\sim 18 \\
\sim 0.5 \\
\sim 0.2 \\
\end{array}$ & $\begin{array}{l}905 \\
811 \\
732 \\
\end{array}$ \\
\hline$N(2250)$ & $1 / 2\left(9 / 2^{-}\right) G_{19}^{\prime}$ & $\begin{array}{l}p=2.21 \\
\sigma=5.74\end{array}$ & $\begin{array}{r}2130 \text { to } \\
2270\end{array}$ & $\begin{array}{r}200 \text { to } \\
500 \\
(300) \\
\end{array}$ & $\begin{array}{l}\mathbf{N} \boldsymbol{\pi} \\
\mathbf{N} \boldsymbol{\eta} \\
\mathbf{\Lambda K}\end{array}$ & $\begin{array}{r}\sim 10 \\
\sim 2 \\
\sim 0.3\end{array}$ & $\begin{array}{l}923 \\
831 \\
754 \\
\end{array}$ \\
\hline $\mathbf{N}(2600)$ & $1 / 2\left(11 / 2^{-}\right) I_{111}$ & $\begin{array}{l}p=3.12 \\
\sigma=3.86\end{array}$ & $\begin{array}{r}2580 \text { to } \\
2700\end{array}$ & $\begin{array}{l}>300 \\
(400)\end{array}$ & $\mathbf{N} \pi$ & $\sim 5$ & 1126 \\
\hline \multicolumn{8}{|c|}{$S=0 \quad I=3 / 2$ DELTA RESONANCES ( $\Delta)$} \\
\hline$\Delta(1232)$ & $3 / 2\left(3 / 2^{+}\right) \mathrm{P}_{33}^{\prime}$ & $\begin{array}{l}p=0.30 \\
\sigma=94.8\end{array}$ & $\begin{array}{r}1230 \text { to } \\
1234\end{array}$ & $\begin{array}{r}110 \text { to } \\
120 \\
(115) \\
\end{array}$ & $\begin{array}{l}\mathbf{N}_{\boldsymbol{\pi}} \\
\mathbf{N}_{\boldsymbol{\gamma}}\end{array}$ & $\begin{array}{r}99.4 \\
0.6\end{array}$ & $\begin{array}{l}227 \\
259\end{array}$ \\
\hline$\Delta(1620)$ & $3 / 2\left(1 / 2^{-}\right) \mathrm{S}_{31}^{\prime}$ & $\begin{array}{l}p=0.91 \\
\sigma=17.7\end{array}$ & $\begin{array}{r}1600 \text { to } \\
1650\end{array}$ & $\begin{array}{r}120 \text { to } \\
160 \\
(140)\end{array}$ & $\begin{array}{c}\mathbf{N} \pi \\
\mathbf{N} \pi \boldsymbol{\pi} \\
-\Delta \pi \\
-\mathbf{N} \rho \\
\end{array}$ & $\left.\begin{array}{r}25-35 \\
\sim 70 \\
35-50 \\
<40\end{array}\right]$ & $\begin{array}{r}526 \\
488 \\
318 \\
+ \\
\end{array}$ \\
\hline$\Delta(1700)$ & $3 / 2\left(3 / 2^{-}\right) D_{33}^{\prime}$ & $\begin{array}{l}p=1.05 \\
\sigma=14.5\end{array}$ & $\begin{array}{r}1630 \text { to } \\
1740\end{array}$ & $\begin{array}{r}190 \text { to } \\
300 \\
(250)\end{array}$ & $\begin{array}{l}\mathbf{N} \pi \\
\mathbf{N} \pi \boldsymbol{\pi} \\
-\Delta \pi \\
\mathbf{N} \rho \\
\end{array}$ & $\left.\begin{array}{r}10-20 \\
\sim 85 \\
<50 \\
\sim 40\end{array}\right]$ & $\begin{array}{r}580 \\
547 \\
385 \\
+ \\
\end{array}$ \\
\hline$\Delta(1900)$ & $3 / 2\left(1 / 2^{-}\right) S_{31}^{\prime \prime}$ & $\begin{array}{l}p=1.44 \\
\sigma=9.71\end{array}$ & $\begin{array}{r}1850 \text { to } \\
2000\end{array}$ & $\begin{array}{r}130 \text { to } \\
300 \\
(150)\end{array}$ & $\begin{array}{l}\mathbf{N} \boldsymbol{\pi} \\
\mathbf{2 K}\end{array}$ & $\begin{array}{r}6-12 \\
\sim 10\end{array}$ & $\begin{array}{l}710 \\
410\end{array}$ \\
\hline$\Delta(1905)$ & $3 / 2\left(5 / 2^{+}\right) F_{35}$ & $\begin{array}{l}p=1.45 \\
\sigma=9.62\end{array}$ & $\begin{array}{r}1890 \text { to } \\
1920\end{array}$ & $\begin{array}{r}250 \text { to } \\
400 \\
(300)\end{array}$ & $\begin{array}{l}\mathbf{N} \pi \\
\Sigma K \\
\mathbf{N} \pi \boldsymbol{\pi} \\
-\Delta \pi \\
-\mathbf{N} \rho \\
\end{array}$ & $\left.\begin{array}{r}8-15 \\
<3 \\
\sim 80 \\
10-30 \\
\sim 60\end{array}\right]$ & $\begin{array}{l}713 \\
415 \\
687 \\
542 \\
421 \\
\end{array}$ \\
\hline$\Delta(1910)$ & $3 / 2\left(1 / 2^{+}\right) \mathrm{P}_{31}^{\prime \prime}$ & $\begin{array}{l}p=1.46 \\
\sigma=9.54\end{array}$ & $\begin{array}{r}1850 \text { to } \\
1950\end{array}$ & $\begin{array}{r}200 \text { to } \\
330 \\
(220)\end{array}$ & $\begin{array}{l}\mathbf{N} \pi \\
\Sigma \mathrm{K} \\
\mathrm{N} \pi \boldsymbol{\pi} \\
\Delta \pi \\
-\mathrm{N} \rho \\
\end{array}$ & $\left.\begin{array}{c}20-25 \\
2-20 \\
>40 \\
\text { small } \\
<40\end{array}\right]$ & $\begin{array}{l}716 \\
421 \\
691 \\
545 \\
426 \\
\end{array}$ \\
\hline$\Delta(1920)$ & $3 / 2\left(3 / 2^{+}\right) P_{33}^{\prime \prime \prime}$ & $\begin{array}{l}p=1.48 \\
\sigma=9.38\end{array}$ & $\begin{array}{r}1860 \text { to } \\
2160\end{array}$ & $\begin{array}{r}190 \text { to } \\
300 \\
(250)\end{array}$ & $\begin{array}{l}\mathbf{N} \pi \\
\mathbf{\Sigma K}\end{array}$ & $\begin{array}{r}14-20 \\
\sim 5\end{array}$ & $\begin{array}{l}722 \\
431\end{array}$ \\
\hline$\Delta(1930)$ & $3 / 2\left(5 / 2^{-}\right) \mathrm{D}_{35}^{\prime}$ & $\begin{array}{l}p=1.50 \\
\sigma=9.21\end{array}$ & $\begin{array}{r}1890 \text { to } \\
1960\end{array}$ & $\begin{array}{r}150 \text { to } \\
350 \\
(250)\end{array}$ & $\begin{array}{l}\mathbf{N} \boldsymbol{\pi} \\
\mathbf{\Sigma K}\end{array}$ & $\begin{array}{l}4-14 \\
<10\end{array}$ & $\begin{array}{l}729 \\
441\end{array}$ \\
\hline$\Delta(1950)$ & $3 / 2\left(7 / 2^{+}\right) F_{37}^{\prime}$ & $\begin{array}{l}p=1.54 \\
\sigma=8.91\end{array}$ & $\begin{array}{r}1910 \text { to } \\
1960\end{array}$ & $\begin{array}{r}200 \text { to } \\
340 \\
(240)\end{array}$ & $\begin{array}{l}\mathbf{N} \pi \\
\Sigma K \\
N_{\pi} \pi \\
-\Delta \pi \\
-N_{\rho} \\
\end{array}$ & $\begin{array}{c}35-45 \\
<1 \\
\sim 60 \\
\sim 40 \\
\sim 20\end{array}$ & $\begin{array}{l}741 \\
460 \\
716 \\
574 \\
469 \\
\end{array}$ \\
\hline$\Delta(2420)$ & $3 / 2\left(11 / 2^{+}\right) \mathrm{H}_{311}$ & $\begin{array}{l}p=2.64 \\
\sigma=4.68\end{array}$ & $\begin{array}{r}2380 \text { to } \\
2450\end{array}$ & $\begin{array}{r}300 \text { to } \\
500 \\
(300)\end{array}$ & $\mathbf{N} \pi$ & $5-15$ & 1023 \\
\hline
\end{tabular}


Baryon Table (cont'd)

\begin{tabular}{|c|c|c|c|c|c|c|c|}
\hline \multirow[b]{2}{*}{ Particle $^{a}$} & \multirow[b]{2}{*}{$\mathbf{I}\left(\mathbf{J}^{\mathbf{P}}\right) \mathbf{L}_{\mathbf{I} \cdot \mathbf{2} \mathbf{J}}^{b}$} & \multirow[b]{2}{*}{$\begin{array}{l}P_{\text {beam }}^{c}(\mathrm{GeV} / \mathrm{c}) \\
\sigma=4 \pi \boldsymbol{\lambda}^{2}(\mathrm{mb})\end{array}$} & \multirow{2}{*}{$\begin{array}{l}\underset{\mathbf{M a s s}}{ }{ }^{d} \\
(\mathbf{M e V})\end{array}$} & \multirow{2}{*}{$\begin{array}{l}\text { Full }{ }^{e} \\
\text { width } \Gamma \\
\text { (MeV) }\end{array}$} & \multicolumn{3}{|c|}{ Partial decay modes } \\
\hline & & & & & Mode & $\begin{array}{l}\text { Fraction }^{8} \\
\quad(\%)\end{array}$ & $\begin{array}{c}\mathbf{p}^{h} \\
(\mathbf{M e V} / \mathrm{c})\end{array}$ \\
\hline \multicolumn{8}{|c|}{$S=-1$ I=0 LAMBDA RESONANCES $(\Lambda)$} \\
\hline$\Lambda$ & $0\left(1 / 2^{+}\right)$ & & 1115.6 & & See Sta & ble Particle & Table \\
\hline$\Lambda(1405)$ & $\alpha\left(1 / 2^{-}\right) S_{01}^{\prime}$ & $\begin{array}{l}\text { Below } \\
\mathbf{K}^{-} \mathbf{p} \\
\text { threshold }\end{array}$ & $\begin{array}{l}1405 \\
\quad \pm 5^{i}\end{array}$ & $40 \pm 10^{i}$ & $\Sigma \pi$ & 100 & 152 \\
\hline$\Lambda(1520)$ & $\alpha\left(3 / 2^{-}\right) D_{03}^{\prime}$ & $\begin{array}{l}p=0.395 \\
\sigma=82.3\end{array}$ & $\begin{array}{l}1519.5 \\
\pm 1.0^{i}\end{array}$ & $15.6 \pm 1.0^{i}$ & $\begin{array}{l}N \overline{\mathrm{K}} \\
\Sigma \pi \\
\Lambda \pi \pi \\
\Sigma \pi \pi \\
\Lambda \gamma \\
\end{array}$ & $\begin{array}{l}45 \pm 1 \\
42 \pm 1 \\
10 \pm 1 \\
0.9 \pm 0.1 \\
0.8 \pm 0.2 \\
\end{array}$ & $\begin{array}{l}244 \\
267 \\
252 \\
152 \\
351 \\
\end{array}$ \\
\hline$\Lambda(1600)$ & $O\left(1 / 2^{+}\right) P_{01}^{\prime}$ & $\begin{array}{l}\mathrm{p}=0.58 \\
\sigma=41.6\end{array}$ & $\begin{array}{r}1560 \text { to } \\
1700\end{array}$ & $\begin{array}{r}50 \text { to } \\
250 \\
(150)\end{array}$ & $\begin{array}{l}\mathbf{N \overline { K }} \\
\Sigma \pi\end{array}$ & $\begin{array}{l}15-30 \\
10-60\end{array}$ & $\begin{array}{l}343 \\
336\end{array}$ \\
\hline$\Lambda(1670)$ & $O\left(1 / 2^{-}\right) S_{01}^{\prime \prime}$ & $\begin{array}{l}\mathrm{p}=0.74 \\
\sigma=28.5\end{array}$ & $\begin{array}{r}1660 \text { to } \\
1680\end{array}$ & $\begin{array}{r}25 \text { to } \\
50 \\
(35) \\
\end{array}$ & $\begin{array}{l}\mathrm{N} \overline{\mathrm{K}} \\
\Sigma \boldsymbol{\pi} \\
\Lambda \eta \\
\end{array}$ & $\begin{array}{l}15-25 \\
20-60 \\
15-35 \\
\end{array}$ & $\begin{array}{r}414 \\
393 \\
64 \\
\end{array}$ \\
\hline$\Lambda(1690)$ & $O\left(3 / 2^{-}\right) D_{03}^{\prime \prime}$ & $\begin{array}{l}p=0.78 \\
\sigma=26.1\end{array}$ & $\begin{array}{r}1685 \text { to } \\
1695\end{array}$ & $\begin{array}{r}50 \text { to } \\
70 \\
(60)\end{array}$ & $\begin{array}{l}N \overline{\mathrm{K}} \\
\Sigma \pi \\
\Lambda \pi \pi \\
\Sigma \pi \pi\end{array}$ & $\begin{array}{r}20-30 \\
20-40 \\
\sim 25 \\
\sim 20\end{array}$ & $\begin{array}{l}433 \\
409 \\
415 \\
350\end{array}$ \\
\hline$\Lambda(1800)$ & $\alpha\left(1 / 2^{-}\right) S_{01}^{\prime \prime \prime}$ & $\begin{array}{l}\mathrm{p}=1.01 \\
\sigma=17.5\end{array}$ & $\begin{array}{r}1720 \text { to } \\
1850\end{array}$ & $\begin{array}{r}200 \text { to } \\
400 \\
(300)\end{array}$ & $\begin{array}{c}\mathrm{N} \overline{\mathrm{K}} \\
\Sigma \pi \\
\Sigma(1385) \pi \\
\mathrm{NK}^{*}(\mathbf{8 9 2}) \\
\end{array}$ & $\begin{array}{r}25-40 \\
\text { seen } \\
\text { seen } \\
\text { seen } \\
\end{array}$ & $\begin{array}{r}528 \\
493 \\
345 \\
\dagger \\
\end{array}$ \\
\hline$\Lambda(1800)$ & $O\left(1 / 2^{+}\right) P_{01}^{\prime \prime}$ & $\begin{array}{l}\mathrm{p}=1.01 \\
\sigma=17.5\end{array}$ & $\begin{array}{r}1750 \text { to } \\
1850\end{array}$ & $\begin{array}{l}50 \text { to } \\
250 \\
(150)\end{array}$ & $\begin{array}{c}\mathrm{N} \overline{\mathrm{K}} \\
\Sigma \pi \\
\Sigma(1385) \pi \\
\mathrm{N}^{*}(892)\end{array}$ & $\begin{array}{r}20-50 \\
10-40 \\
\text { seen } \\
30-60\end{array}$ & $\begin{array}{r}528 \\
493 \\
345 \\
+\end{array}$ \\
\hline$\Lambda(1820)$ & $0\left(5 / 2^{+}\right) F_{05}^{\prime}$ & $\begin{array}{l}\mathrm{p}=1.06 \\
\sigma=16.5\end{array}$ & $\begin{array}{r}1815 \text { to } \\
1825\end{array}$ & $\begin{array}{r}70 \text { to } \\
90 \\
(80)\end{array}$ & $\begin{array}{c}\mathbf{N \overline { K }} \\
\Sigma \pi \\
\Sigma(1385) \pi\end{array}$ & $\begin{array}{r}55-65 \\
8-14 \\
5-10\end{array}$ & $\begin{array}{l}545 \\
508 \\
362\end{array}$ \\
\hline$\Lambda(1830)$ & $O\left(5 / 2^{-}\right) D_{05}$ & $\begin{array}{l}\mathrm{p}=1.08 \\
\sigma=16.0\end{array}$ & $\begin{array}{r}1810 \text { to } \\
1830\end{array}$ & $\begin{array}{l}60 \text { to } \\
110 \\
(95)\end{array}$ & $\begin{array}{c}\mathbf{N \overline { K }} \\
\Sigma \pi \\
\Sigma(1385) \pi\end{array}$ & $\begin{array}{r}3-10 \\
35-75 \\
>15\end{array}$ & $\begin{array}{l}553 \\
515 \\
371\end{array}$ \\
\hline$\Lambda(1890)$ & $O\left(3 / 2^{+}\right) P_{03}^{\prime}$ & $\begin{array}{l}p=1.21 \\
\sigma=13.6\end{array}$ & $\begin{array}{r}1850 \text { to } \\
1910\end{array}$ & $\begin{array}{l}60 \text { to } \\
200 \\
(100)\end{array}$ & $\begin{array}{c}\mathrm{N} \overline{\mathrm{K}} \\
\Sigma \pi \\
\Sigma(1385) \pi \\
\mathrm{NK}^{*}(892)\end{array}$ & $\begin{array}{r}20-35 \\
3-10 \\
\text { seen } \\
\text { seen }\end{array}$ & $\begin{array}{l}599 \\
559 \\
420 \\
233\end{array}$ \\
\hline$\Lambda(2100)$ & $\alpha\left(7 / 2^{-}\right) G_{07}$ & $\begin{array}{l}\mathrm{p}=1.68 \\
\sigma=8.68\end{array}$ & $\begin{array}{r}2090 \text { to } \\
2110\end{array}$ & $\begin{array}{r}100 \text { to } \\
250 \\
(200)\end{array}$ & $\begin{array}{c}\mathbf{N} \overline{\mathbf{K}} \\
\Sigma \pi \\
\Lambda \eta \\
\Xi \mathrm{K} \\
\Lambda \omega \\
\mathrm{NK}^{*}(892) \\
\end{array}$ & $\begin{array}{r}25-35 \\
\sim 5 \\
<3 \\
<3 \\
<8 \\
10-20 \\
\end{array}$ & $\begin{array}{l}751 \\
704 \\
617 \\
483 \\
443 \\
514 \\
\end{array}$ \\
\hline$\Lambda(2110)$ & $O\left(5 / 2^{+}\right) F_{05}^{\prime \prime}$ & $\begin{array}{l}p=1.70 \\
\sigma=8.53\end{array}$ & $\begin{array}{r}2090 \text { to } \\
2140\end{array}$ & $\begin{array}{r}150 \text { to } \\
250 \\
(200)\end{array}$ & $\begin{array}{c}N \bar{K} \\
\Sigma \pi \\
\Lambda \omega \\
\Sigma(1385) \pi \\
N^{*} \bar{K}^{*}(892)\end{array}$ & $\begin{array}{r}5-25 \\
10-40 \\
\text { seen } \\
\text { seen } \\
10-60\end{array}$ & $\begin{array}{l}757 \\
711 \\
455 \\
589 \\
524\end{array}$ \\
\hline$\Lambda(2350)$ & $0\left(9 / 2^{+}\right)$ & $\begin{array}{l}\mathrm{p}=2.29 \\
\sigma=5.85\end{array}$ & $\begin{array}{r}2340 \text { to } \\
2370\end{array}$ & $\begin{array}{r}100 \text { to } \\
250 \\
(150)\end{array}$ & $\begin{array}{l}\mathrm{N} \overline{\mathrm{K}} \\
\Sigma \pi\end{array}$ & $\begin{array}{l}12 \\
\sim 10\end{array}$ & $\begin{array}{l}915 \\
867\end{array}$ \\
\hline
\end{tabular}


Baryon Table (cont'd)

\begin{tabular}{|c|c|c|c|c|c|c|c|}
\hline \multirow[b]{2}{*}{ Particle $^{a}$} & \multirow[b]{2}{*}{$\mathbf{I}\left(\mathbf{J}^{\mathbf{P}}\right) \mathbf{L}_{\mathbf{I} \cdot \mathbf{2 J}}^{b}$} & \multirow[b]{2}{*}{$\begin{array}{l}P_{\text {beam }}^{c}(\mathrm{GeV} / \mathrm{c}) \\
\sigma=4 \pi X^{2}(\mathrm{mb})\end{array}$} & \multirow{2}{*}{$\underset{\text { Mass }}{\operatorname{MeV}}{ }^{d}$} & \multirow{2}{*}{$\begin{array}{l}\text { Full }{ }^{e} \\
\text { width } \Gamma \\
\text { (MeV) }\end{array}$} & \multicolumn{3}{|c|}{ Partial decay modes } \\
\hline & & & & & Mode & $\begin{array}{c}\text { Fraction } 8 \\
\text { (\%) }\end{array}$ & $\bar{g} \begin{array}{c}\mathrm{p}^{h} \\
(\mathrm{MeV} / \mathrm{c})\end{array}$ \\
\hline \multicolumn{8}{|c|}{$S=-1$ Im SIGMA RESONANCES ( $\Sigma$ ) } \\
\hline $\bar{\Sigma}$ & $1\left(1 / 2^{+}\right)$ & ( & $\begin{array}{l}+) 1189.4 \\
(0) 1192.5 \\
-) 1197.3\end{array}$ & & See Sta & ole Particle & e Table \\
\hline$\Sigma(1385)$ & $1\left(3 / 2^{+}\right) P_{13}^{\prime}$ & $\begin{array}{l}\begin{array}{l}\text { Below } \\
\mathbf{K}^{-} \mathbf{p} \\
\text { threshold }\end{array} \\
\\
(0) 138 \\
(-) 138\end{array}$ & $\begin{array}{c}32.3 \pm 0.4 \\
S=1.6^{j} \\
32.0 \pm 2.5 \\
S=1.6^{j} \\
37.4 \pm 0.6 \\
S=2.2^{j}\end{array}$ & $\begin{array}{c}35 \pm 1 \\
S=1.0^{j} \\
\sim 35 \\
\\
40 \pm 2 \\
S=1.9^{j}\end{array}$ & $\begin{array}{l}\Delta \pi \\
\Sigma \pi\end{array}$ & $\begin{array}{l}88 \pm 2 \\
12 \pm 2\end{array}$ & $\begin{array}{l}208 \\
127\end{array}$ \\
\hline$\Sigma(1660)$ & $1\left(1 / 2^{+}\right) \mathrm{P}_{11}^{\prime}$ & $\begin{array}{l}p=0.72 \\
\sigma=29.9\end{array}$ & $\begin{array}{r}1630 \text { to } \\
1690\end{array}$ & $\begin{array}{r}40 \text { to } \\
200 \\
(100)\end{array}$ & $\begin{array}{l}\mathbf{N K} \\
\Lambda \pi \\
\Sigma \pi\end{array}$ & $\begin{array}{r}10-30 \\
\text { seen } \\
\text { seen }\end{array}$ & $\begin{array}{l}405 \\
439 \\
385 \\
\end{array}$ \\
\hline$\Sigma(1670)$ & $1\left(3 / 2^{-}\right) D_{13}^{\prime \prime}$ & $\begin{array}{l}p=0.74 \\
\sigma=28.5\end{array}$ & $\begin{array}{r}1665 \text { to } \\
1685\end{array}$ & $\begin{array}{r}40 \text { to } \\
80 \\
(60)\end{array}$ & $\begin{array}{l}\mathbf{N} \overline{\mathbf{K}} \\
\Lambda \pi \\
\Sigma \pi\end{array}$ & $\begin{array}{r}7-13 \\
5-15 \\
30-60\end{array}$ & $\begin{array}{l}414 \\
447 \\
393\end{array}$ \\
\hline$\Sigma(1750)$ & $1\left(1 / 2^{-}\right) S_{11}^{\prime \prime}$ & $\begin{array}{l}p=0.91 \\
\sigma=20.7\end{array}$ & $\begin{array}{r}1730 \text { to } \\
1800\end{array}$ & $\begin{array}{r}60 \text { to } \\
160 \\
(90)\end{array}$ & $\begin{array}{l}\mathbf{N K} \\
\Lambda \pi \\
\Sigma \pi \\
\Sigma \eta\end{array}$ & $\begin{array}{r}10-40 \\
\text { seen } \\
<8 \\
15-55\end{array}$ & $\begin{array}{r}486 \\
507 \\
455 \\
81\end{array}$ \\
\hline$\Sigma(1775)$ & $1\left(5 / 2^{-}\right) D_{15}$ & $\begin{array}{l}p=0.96 \\
\sigma=19.0\end{array}$ & $\begin{array}{r}1770 \text { to } \\
1780\end{array}$ & $\begin{array}{r}105 \text { to } \\
135 \\
(120)\end{array}$ & $\begin{array}{c}\mathbf{N \overline { K }} \\
\Lambda \pi \\
\Sigma \pi \\
\Sigma(1385) \pi \\
\Lambda(1520) \pi\end{array}$ & $\begin{array}{r}37-43 \\
14-20 \\
2-5 \\
8-12 \\
17-23\end{array}$ & $\begin{array}{l}508 \\
525 \\
474 \\
324 \\
198\end{array}$ \\
\hline$\Sigma(1915)$ & $1\left(5 / 2^{+}\right) F_{15}^{\prime}$ & $\begin{array}{l}p=1.26 \\
\sigma=12.8\end{array}$ & $\begin{array}{r}1900 \text { to } \\
1935\end{array}$ & $\begin{array}{r}80 \text { to } \\
160 \\
(120)\end{array}$ & $\begin{array}{c}\mathrm{N} \overline{\mathrm{K}} \\
\Lambda \pi \\
\Sigma \boldsymbol{\pi} \\
\Sigma(1385) \pi\end{array}$ & $\begin{array}{l}5-15 \\
\text { seen } \\
\text { seen } \\
<5\end{array}$ & $\begin{array}{l}618 \\
622 \\
577 \\
440 \\
\end{array}$ \\
\hline$\Sigma(1940)$ & $1\left(3 / 2^{-}\right) D_{13}^{\prime \prime \prime}$ & $\begin{array}{l}p=1.32 \\
\sigma=12.1\end{array}$ & $\begin{array}{r}1900 \text { to } \\
1950\end{array}$ & $\begin{array}{r}150 \text { to } \\
300 \\
(220)\end{array}$ & $\begin{array}{c}\mathbf{N K} \\
\Lambda \pi \\
\Sigma \bar{K} \\
\Sigma(1385) \pi \\
\Lambda(1520) \pi \\
\Delta(1232) \bar{K} \\
N^{*} \bar{K}^{*(892)}\end{array}$ & $\begin{array}{l}<20 \\
\text { seen } \\
\text { seen } \\
\text { seen } \\
\text { seen } \\
\text { seen } \\
\text { seen }\end{array}$ & $\begin{array}{l}637 \\
639 \\
594 \\
460 \\
354 \\
410 \\
320\end{array}$ \\
\hline$\Sigma(2030)$ & $1\left(7 / 2^{+}\right) F_{17}$ & $\begin{array}{l}p=1.52 \\
\sigma=9.93\end{array}$ & $\begin{array}{r}2025 \text { to } \\
2040\end{array}$ & $\begin{array}{r}150 \text { to } \\
200 \\
(180)\end{array}$ & $\begin{array}{c}\mathbf{N \overline { K }} \\
\Lambda \pi \\
\Sigma \pi \\
\Xi K \\
\Sigma(1385) \pi \\
\Lambda(1520) \pi \\
\Delta(1232) \bar{K} \\
N^{*} \bar{K}^{*}(892)\end{array}$ & $\begin{array}{r}17-23 \\
17-23 \\
5-10 \\
<2 \\
5-15 \\
10-20 \\
10-20 \\
<5\end{array}$ & $\begin{array}{l}702 \\
700 \\
657 \\
412 \\
529 \\
430 \\
498 \\
438\end{array}$ \\
\hline $2(2250)$ & $1(?)$ & $\begin{array}{l}p=2.04 \\
\sigma=6.76\end{array}$ & $\begin{array}{r}2210 \text { to } \\
2280\end{array}$ & $\begin{array}{r}60 \text { to } \\
150 \\
(100)\end{array}$ & $\begin{array}{l}\mathbf{N K} \\
\Lambda \pi \\
\Sigma \pi\end{array}$ & $\begin{array}{l}<10 \\
\text { seen } \\
\text { seen }\end{array}$ & $\begin{array}{l}851 \\
842 \\
803\end{array}$ \\
\hline
\end{tabular}




\section{Baryon Table (cont'd)}

\begin{tabular}{|c|c|c|c|c|c|c|}
\hline \multirow[b]{2}{*}{ Particle $^{a}$} & \multirow[b]{2}{*}{$\mathbf{I}\left(\mathbf{J}^{\mathbf{P}}\right) \mathbf{L}_{\mathbf{2 I} \mathbf{I} \cdot \mathbf{2 J}}^{b}$} & \multirow{2}{*}{$\begin{array}{c}\text { Mass }^{d} \\
\mathbf{M} \\
(\mathbf{M e V})\end{array}$} & \multirow{2}{*}{$\begin{array}{c}\text { Full }{ }^{e} \\
\text { width } \Gamma \\
(\mathrm{MeV})\end{array}$} & \multicolumn{3}{|c|}{ Partial decay modes } \\
\hline & & & & Mode & $\begin{array}{l}\text { Fraction } \\
\text { (\%) }\end{array}$ & $\underset{(\mathrm{MeV} / \mathrm{c})}{\mathbf{p}^{h}}$ \\
\hline \multicolumn{7}{|c|}{$S=-2 \quad I=1 / 2$ CASCADE RESONANCES (Z) } \\
\hline$\Xi$ & $1 / 2\left(1 / 2^{+}\right)$ & $\begin{array}{l}(0) 1314.9 \\
(-) 1321.3 \\
\end{array}$ & & \multicolumn{3}{|c|}{ See Stable Particle Table } \\
\hline$\Xi(1530)$ & $1 / 2\left(3 / 2^{+}\right) \mathrm{P}_{13}$ & $\begin{array}{r}(0) 1531.8 \pm 0.3 \\
\mathrm{~S}=1.3^{j} \\
(-) 1535.0 \pm 0.6\end{array}$ & $\begin{array}{r}9.1 \pm 0.5 \\
10.1 \pm 1.9\end{array}$ & $\Xi \pi$ & 100 & 148 \\
\hline$\Xi(1820)$ & $1 / 2(3 / 2)$ & $\begin{array}{r}1823 \\
\pm 6^{i}\end{array}$ & $20_{-10}^{+15^{i}}$ & $\begin{array}{c}\Lambda \overline{\mathbf{K}} \\
\Sigma \overline{\mathbf{K}} \\
\Xi \pi \\
\Xi(1530) \pi\end{array}$ & $\begin{array}{c}\sim 45 \\
\sim 10 \\
\text { small } \\
\sim 45\end{array}$ & $\begin{array}{l}396 \\
306 \\
413 \\
231\end{array}$ \\
\hline$\Xi(2030)$ & $1 / 2(?)$ & $\stackrel{2024}{ \pm 6^{i}}$ & $16_{-5}^{+15^{i}}$ & $\begin{array}{c}\Lambda \overline{\mathbf{K}} \\
\Sigma \overline{\mathbf{K}} \\
\Xi \pi \\
\Xi(1530) \pi\end{array}$ & $\begin{array}{c}\sim 20 \\
\sim 80 \\
\sim 8 m a l l \\
\text { small }\end{array}$ & $\begin{array}{l}587 \\
524 \\
573 \\
418 \\
\end{array}$ \\
\hline \multicolumn{7}{|c|}{ OTHER BARYONS } \\
\hline$\overline{\Omega^{-}}$ & $0\left(3 / 2^{+}\right)$ & 1672.4 & & See $S t$ & ble Particl & le Table \\
\hline$\Lambda_{c}^{+}$ & $0\left(1 / 2^{+}\right)$ & 2282 & & See $\mathbf{S t}$ & ble Particl & le Table \\
\hline
\end{tabular}

$\rightarrow$ Each arrow in the left-hand margin indicates there is an entry in the Data Card Listings for a baryon that is not well enough established (status less than 3 stars) to be included here. There is a short list of all the baryons in the Listings, whatever their status, at the front of this Table.

$\dagger$. This mode is energetically forbidden when the nominal mass of the decaying resonance (and of any resonance in the final state) is used, but is in fact allowed due to the nonzero widths of the resonance(s).

* The modes in brackets are subreactions of the $N \pi \pi$ mode.

a. The nominal mass here (in $\mathrm{MeV}$ ) is used for identification. See column $\mathbf{4}$ for the actual mass.

$b$. When there is more than one baryon with the same quantum numbers, one prime is attached to the spectroscopic symbol for the first of them (e.g., $S_{11}^{\prime}$ ), two primes to the second, etc.

c. The quantities here are calculated using the nominal mass of column 1 .

$d$. Usually a conservatively large range of masses rather than a statistical average of the various determinations of the mass is given. In these cases, the mass determinations are nearly entirely from various phase-shift analyses of more or less the same data. It is thus not appropriate to treat the determinations as independent measurements or to average them together. The masses, widths, and branching fractions in this Table are Breit-Wigner parameters. The Data Card Listings also include pole parameters where they are available.

e. Usually a conservatively large range of widths rather than a statistical average of the various determinations of the width is given (see note $d$ for the reason). The nominal value in parentheses is then simply a best guess.

f. For information on the $\mathrm{N} \gamma$ decay modes, see the Note on $\mathrm{N}$ and $\Delta$ Resonances in the Listings.

g. Most of the inelastic branching fractions come from partial-wave analyses, and these determine $\sqrt{\mathrm{xx}^{\prime}}$, where $\mathrm{x}$ and $x^{\prime}$ are the elastic and inelastic branching fractions, not $x^{\prime}$ directly. Thus any uncertainty (and it is often considerable) in $x$ carries over into $x^{\prime}$. When $x^{\prime}$ so determined is really poorly known, we here simply note that the mode is seen. The values of $\sqrt{\mathbf{x x}^{\prime}}$ are given in the Data Card Listings.

$h$. For a 2-body decay mode, this is the momentum of the decay products in the rest frame of the decaying particle. For a mode with more than two decay products, this is the maximum momentum any of the products can have in this frame. The nominal mass of column 1 is used, as is the nominal mass of any resonance in the final state.

$i$. The error given here is only an educated guess. It is larger than the error on the weighted average of the published values (the error on this average is given in the Listings).

$j$. The error given here has been scaled up by the "S factor" (see the * footnote to the Stable Particle Table for how $S$ is defined) because the various measurements disagree more seriously than one would expect from statistics. 
PHYSICAL CONSTANTS*

\begin{tabular}{|c|c|c|c|}
\hline Quantity & Symbol, equation & Value & Uncert. (ppm) \\
\hline speed of light & c & $2.99792458(1.2) \times 10^{10} \mathrm{~cm} \mathrm{~s}^{-1} \quad\left(\right.$ see note $\left.{ }^{* *}\right)$ & 0.004 \\
\hline Planck constant & $\mathbf{h}$ & $6.626176(36) \times 10^{-27} \mathrm{erg} \mathrm{s}$ & 5.4 \\
\hline \multirow[t]{2}{*}{ Planck constant, reduced } & $h=h / 2 \pi$ & $1.0545887(57) \times 10^{-27} \mathrm{erg} \mathrm{s}$ & 5.4 \\
\hline & & $=6.582173(17) \times 10^{-22} \mathrm{MeV} \mathrm{s}$ & 2.6 \\
\hline \multirow[t]{2}{*}{ electron charge magnitude } & e & $4.803242(14) \times 10^{-10} \mathrm{esu}$ & 2.9 \\
\hline & & $=1.6021892(46) \times 10^{-19}$ coulomb & 2.9 \\
\hline conversion constant & hc & $197.32858(51) \mathrm{MeV} \mathrm{fm}$ & 2.6 \\
\hline conversion constant & $(n c)^{2}$ & $0.3893857(20) \mathrm{GeV}^{2}$ mbarn & 5.2 \\
\hline \multirow{3}{*}{$\begin{array}{l}\text { electron mass } \\
\text { proton mass }\end{array}$} & $\mathrm{m}_{\mathrm{e}}$ & $0.5110034(14) \mathrm{MeV} / \mathrm{c}^{2}=9.109534(47) \times 10^{-28} \mathrm{~g}$ & $2.8,5.1$ \\
\hline & $\mathrm{m}_{\mathrm{p}}$ & $938.2796(27) \mathrm{MeV} / \mathrm{c}^{2}=1.6726485(86) \times 10^{-24} \mathrm{~g}$ & $2.8,5.1$ \\
\hline & & $=1.007276470(11) \mathrm{amu}=1836.15152(70) \mathrm{m}_{\mathrm{e}}$ & $0.011,0.38$ \\
\hline deuteron mass & & $1875.6280(53) \mathrm{MeV} / \mathrm{c}^{2}$ & 2.8 \\
\hline atomic mass unit (amu) & $\left(\right.$ mass $C^{12}$ atom $) / 12=(1 \mathrm{~g}) / \mathrm{N}_{\mathrm{A}}$ & $931.5016(26) \mathrm{MeV} / \mathrm{c}^{2}=1.6605655(86) \times 10^{-24} \mathrm{~g}$ & $2.8,5.1$ \\
\hline \multirow{3}{*}{$\begin{array}{l}\text { fine structure constant } \\
\text { classical electron radius } \\
\text { electron Compton wavelength }\end{array}$} & \multirow{6}{*}{$\begin{array}{l}\alpha=\mathrm{e}^{2} / \hbar \mathrm{c} \\
\mathrm{r}_{\mathrm{e}}=\mathrm{e}^{2} / \mathrm{m}_{\mathrm{e}} \mathrm{c}^{2} \\
\lambda_{\mathrm{e}}=\hbar / \mathrm{m}_{\mathrm{e}} \mathrm{c}=\mathrm{r}_{\mathrm{e}} \alpha^{-1} \\
\mathrm{a}_{\infty}=h^{2} / \mathrm{cm}_{\mathrm{e}} \mathrm{e}^{2}=\mathrm{r}_{\mathrm{e}} \alpha^{-2} \\
\mathrm{hcR_{ \infty }}=\mathrm{m}_{\mathrm{e}} \mathrm{e}^{4} / 2 h^{2}=\mathrm{m}_{\mathrm{e}} \mathrm{c}^{2} \alpha^{2} / 2 \\
\sigma_{\mathrm{T}}=8 \pi \mathrm{r}_{\mathrm{e}}^{2} / 3\end{array}$} & $1 / 137.03604(11)$ & 0.82 \\
\hline & & $2.8179380(70) \mathrm{fm}$ & 2.5 \\
\hline & & $3.8615905(64) \times 10^{-11} \mathrm{~cm}$ & 1.6 \\
\hline Bohr radius $\left(m_{\text {nucleus }}=\infty\right)$ & & $0.52917706(44) \times 10^{-8} \mathrm{~cm}$ & 0.82 \\
\hline Rydberg energy & & $13.605804(36) \mathrm{eV}$ & 2.6 \\
\hline Thomson cross section & & $0.6652448(33)$ barn & 4.9 \\
\hline \multirow{4}{*}{$\begin{array}{l}\text { Bohr magneton } \\
\text { nuclear magneton } \\
\text { electron cyclotron frequency/field } \\
\text { proton cyclotron frequency/field }\end{array}$} & \multirow{4}{*}{$\begin{array}{l}\mu_{\mathrm{B}}=\mathrm{eh} / 2 \mathrm{~m}_{\mathrm{e}} \mathrm{c} \\
\mu_{\mathrm{N}}=\mathrm{eh} / 2 \mathrm{~m}_{\mathrm{p}} \mathrm{c} \\
\omega_{\text {cycl }}^{\mathrm{e}} / \mathrm{B}=\mathrm{e} / \mathrm{m}_{\mathrm{e}} \mathrm{c} \\
\omega_{\text {cycl }}^{\mathrm{p}} / \mathrm{B}=\mathrm{e} / \mathrm{m}_{\mathrm{p}} \mathrm{c}\end{array}$} & $5.7883785(95) \times 10^{-15} \mathrm{MeV}_{\text {gauss }}{ }^{-1}$ & 1.6 \\
\hline & & $3.1524515(53) \times 10^{-18} \mathrm{MeV}_{\text {gauss }^{-1}}$ & 1.7 \\
\hline & & $1.7588047(49) \times 10^{7} \mathrm{rad} \mathrm{s}^{-1}$ gauss $^{-1}$ & 2.8 \\
\hline & & $9.578756(28) \times 10^{3} \mathrm{rad} \mathrm{s}^{-1}$ gauss $^{-1}$ & 2.8 \\
\hline \multirow{3}{*}{$\begin{array}{l}\text { gravitational constant } \\
\text { grav. acceleration, sea level, } 45^{\circ} \text { lat. } \\
\text { Fermi coupling constant }\end{array}$} & \multirow{3}{*}{$\begin{array}{l}\mathrm{G}_{\mathrm{N}} \\
\mathrm{g} \\
\mathrm{G}_{\mathrm{F}} /(\hbar \mathrm{hc})^{3} \\
\end{array}$} & $6.6720(41) \times 10^{-8} \mathrm{~cm}^{3} \mathrm{~g}^{-1} \mathrm{~s}^{-2}$ & 615 \\
\hline & & $980.62 \mathrm{~cm} \mathrm{~s}^{-2}$ & - \\
\hline & & $1.16637(2) \times 10^{-5} \mathrm{GeV}^{-2}$ & 17 \\
\hline \multirow{3}{*}{$\begin{array}{l}\text { Avogadro number } \\
\text { Boltzmann constant }\end{array}$} & \multirow{3}{*}{$\mathbf{N}_{\mathbf{A}}$} & $6.022045(31) \times 10^{23} \mathrm{~mol}^{-1}$ & 5.1 \\
\hline & & $1.380662(44) \times 10^{-16} \mathrm{erg} \mathrm{K}^{-1}$ & 32 \\
\hline & & $=8.61735(28) \times 10^{-5} \mathrm{eV} \mathrm{K}^{-1}$ & 32 \\
\hline molar volume, ideal gas at STP & $\mathrm{N}_{\mathrm{A}} \mathrm{k}(273.15 \mathrm{~K}) /(1$ atmosphere $)$ & $22413.83(70) \mathrm{cm}^{3} \mathrm{~mol}^{-1}$ & 31 \\
\hline Stefan-Boltzmann constant & $\sigma=\pi^{2} k^{4} / 60 h^{3} c^{2}$ & $5.67032(71) \times 10^{-5} \mathrm{erg} \mathrm{s}^{-1} \mathrm{~cm}^{-2} \mathrm{~K}^{-4}$ & 125 \\
\hline
\end{tabular}

$$
\pi=3.141592653589793238
$$

e $=2.718281828459045235$

$\gamma=0.577215664901532861$

\begin{tabular}{|c|c|c|c|}
\hline $\begin{aligned} 1 \text { in } & =2.54 \mathrm{~cm} \\
1 \AA & =10^{-8} \mathrm{~cm} \\
1 \mathrm{fm} & =10^{-13} \mathrm{~cm} \\
1 \mathrm{barn} & =10^{-24} \mathrm{~cm}^{2}\end{aligned}$ & $\begin{aligned} 1 \text { newton } & =10^{5} \text { dyne } \\
1 \text { joule } & =10^{7} \mathrm{erg} \\
1 \mathrm{eV} & =1.6021892 \times 10^{-12} \mathrm{erg} \\
1 \mathrm{eV} / \mathrm{c}^{2} & =1.782676 \times 10^{-33} \mathrm{~g}\end{aligned}$ & $\begin{aligned} 1 \text { coulomb } & =2.99792458 \times 10^{9} \mathrm{esu} \\
1 \text { tesla } & =10^{4} \text { gauss } \\
1 \mathrm{~atm} . & =1.01325 \times 10^{6} \mathrm{dyne} / \mathrm{cm}^{2} \\
0^{\circ} \mathrm{C} & =273.15 \mathrm{~K}\end{aligned}$ & $\begin{aligned} 1 \text { tropical year } & \simeq 3.15569 \times 10^{7} \mathrm{~s} \\
1 \text { light year } & =9.460528 \times 10^{17} \mathrm{~cm} \\
1 \text { parsec } & =3.261633 \text { light year } \\
1 \text { astro. unit } & =1.495979 \times 10^{13} \mathrm{~cm}\end{aligned}$ \\
\hline
\end{tabular}

* Revised 1984 by Barry N. Taylor, based mainly on the "1973 Least-Squares Adjustment of the Fundamental Constants," by E.R. Cohen and B.N. Taylor, J. Phys. Chem. Ref. Data 2, 663 (1973). The figures in parentheses give the 1-standard-deviation uncertainties in the last digits of the main numbers; the uncertainties in parts per million (ppm) are given in the last column. The uncertainties of the output values of a least-squares adjustment are in general correlated, and the laws of error propagation must be used in calculating additional quantities.

The set of constants resulting from the 1973 adjustment of Cohen and Taylor has been recommended for international use by CODATA (Committee on Data for Science and Technology), and is the most up-to-date, generally accepted set currently available. Since the publication of the 1973 adjustment, new experiments have yielded better values for some of the constants: $\mathrm{N}_{\mathrm{A}}=6.0220978(63) \times 10^{23}$ $\mathrm{mol}^{-1}(1.04 \mathrm{ppm}) ; \alpha^{-1}=137.035963(15)(0.11 \mathrm{ppm}) ;$ and $\mathrm{m}_{\mathrm{p}} / \mathrm{m}_{\mathrm{e}}=1836.152470(79)(0.043 \mathrm{ppm})$. However, since a change in the measured value of one constant usually leads to changes in the adjusted values of others, one must be cautious in using together the values from the 1973 adjustment and the results of more recent experiments.

A new adjustment of the fundamental constants is planned for completion in 1984.

** In October 1983, the Conference Génerale des Poids et Mesures adopted a new definition of the meter. The meter is the length of the path traveled by light in vacuum during a time interval of $1 / 299792458 \mathrm{~s}$. Thus the speed of light is defined to be $299792458 \mathrm{~m} / \mathrm{s}$. For a discussion of this change, see B.W. Petley, Nature 303, 373 (1983). 


\section{CLEBSCH-GORDAN COEFFICIENTS, SPHERICAL HARMONICS, AND D FUNCTIONS}

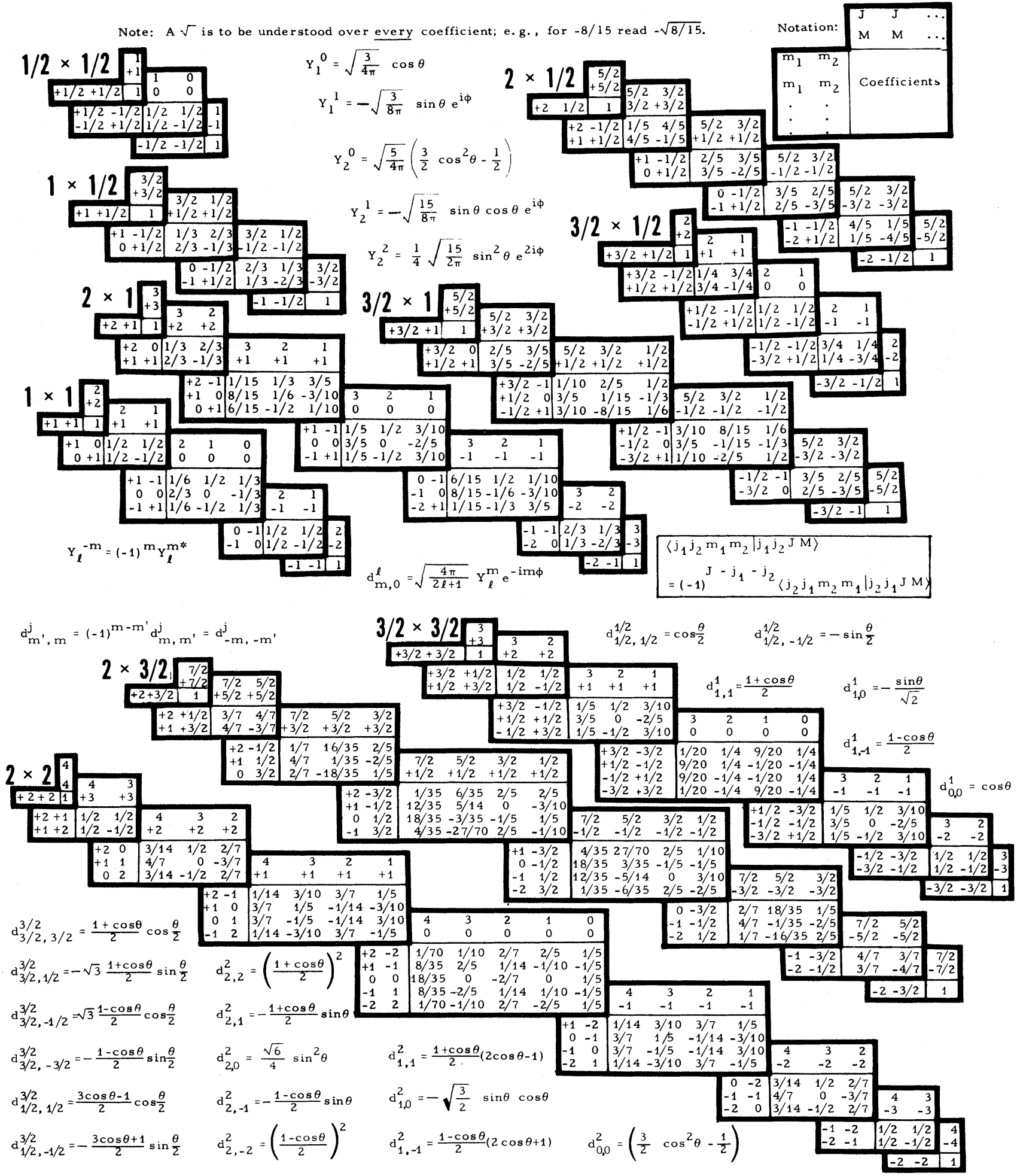

Sign convention is that of Wigner (Group Theory, Academic Press, New York, 1959), also used by Condon and Shortley (The Theory of Atomic Spectra, Cambridge Univ. Press, New York, 1953), Rose (Elementary Theory of Angular Momentum, Wiley, New York, 1957), and Cohen (Tables of the Clebsch-Gordan Coefficients, North American Rockwell Science Center, Thousand Oaks, Calif., 1974). The signs and numbers in the current tables have been calculated by computer programs written independently by Cohen and at LBL. (Table extended April 1974.) 


\section{SU(3) ISOSCALAR FACTORS}

The most commonly used isoscalar factors, corresponding to the singlet, octet, and decuplet content of $8 \otimes 8$ and $10 \otimes 8$, are displayed at the right. The notation uses particle names to identify the coefficients, so that the pattern of relative couplings can be seen at a glance. We illustrate the use of the coefficients by example; see J.J de Swart, Rev. Mod Phys. 35, 916 (1963) for detailed explanation and phase conventions.

$A \sqrt{ }$ is understood over every integer in the matrices; the exponent $1 / 2$ is a reminder of this. For example, in de Swart's notation the $\Xi \rightarrow \Omega \mathrm{K}$ element of our $10 \rightarrow 10 \otimes 8$ matrix reads

$$
\left(\begin{array}{cc|c}
10 & 8 & 10 \\
0-2 & 1 / 2 & 1 / 2-1
\end{array}\right)=\frac{-\sqrt{6}}{\sqrt{24}} .
$$

Intramultiplet relative decay strengths can be read directly from our matrices. Thus, the partial widths for $\Delta \rightarrow(N \pi)_{I=3 / 2}$ and $\Omega^{*}$ $\rightarrow(\Xi \bar{K})_{I=0}$ are in the ratio

$$
\frac{\Gamma\left(\Omega^{*} \rightarrow(\Xi \bar{K})_{I=0}\right)}{\Gamma\left(\Delta \rightarrow(N \pi)_{I=3 / 2}\right)}=\frac{12}{6} \times(\text { phase space factors }) .
$$

Supplying isospin Clebsch-Gordan coefficients, one obtains, e.g.,

$$
\frac{\Gamma\left(\Omega^{*-} \rightarrow \Xi^{0} K^{-}\right)}{\Gamma\left(\Delta^{+} \rightarrow p \pi^{0}\right)}=\frac{1 / 2}{2 / 3} \times \frac{12}{6} \times \text { p.s.f. }=\frac{3}{2} \times \text { p.s.f. }
$$

Partial widths for $8 \rightarrow 8 \otimes 8$ involve a linear superposition of $8_{1}$ (symmetric) and $8_{2}$ (antisymmetric) couplings. For example,

$$
\Gamma\left(\Xi^{*} \rightarrow \Xi \pi\right) \sim\left(-\sqrt{\frac{9}{20}} g_{1}+\sqrt{\frac{3}{12}} g_{2}\right)^{2} .
$$

The relation between $g_{1}, g_{2}$ (with de Swart's normalization) and the standard D,F couplings appearing in the interaction Lagrangian,

$$
\mathscr{L}=-\sqrt{2} \mathrm{D} \operatorname{Tr}\left([\overline{\mathrm{B}}, \mathrm{B}]_{+} \mathrm{M}\right)+\sqrt{2} \mathrm{~F} \operatorname{Tr}([\overline{\mathrm{B}}, \mathrm{B}]-\mathrm{M}),
$$

is

$$
D=\frac{\sqrt{30}}{40} g_{1}, F=\frac{\sqrt{6}}{24} g_{2} .
$$

Thus,

$$
\Gamma\left(\Xi^{*} \rightarrow \Xi \pi\right) \sim(1-2 \alpha)^{2}
$$

where $\alpha \equiv D /(D+F)$.

$$
1 \rightarrow 8 \otimes 8
$$

$$
(\Lambda)_{1} \rightarrow\left(\begin{array}{lllll}
\mathrm{N} \bar{K} & \Sigma \pi & \Lambda \eta & \Xi \mathrm{K}
\end{array}\right)_{8 \otimes 8}=\frac{1}{\sqrt{8}}\left(\begin{array}{llll}
2 & 3 & -1 & -2
\end{array}\right)^{\frac{1}{2}}
$$

$8_{1} \rightarrow \mathbf{8} \otimes 8$

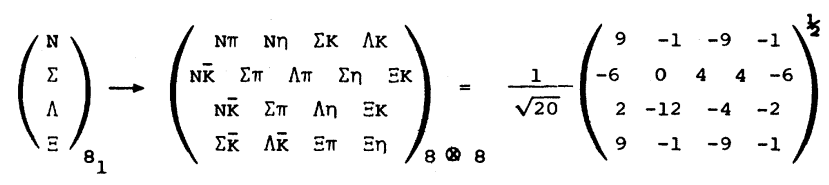

$8_{2} \rightarrow 8 \otimes 8$

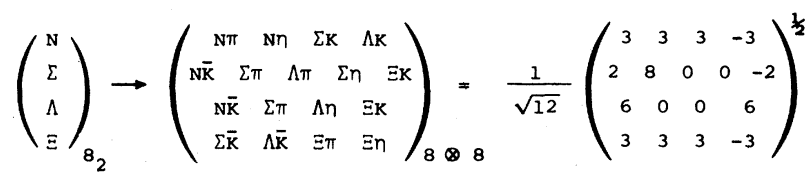

$10 \rightarrow 8 \otimes 8$

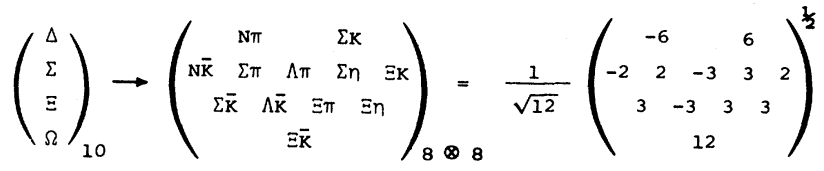

\section{$8 \rightarrow 10 \otimes 8$}

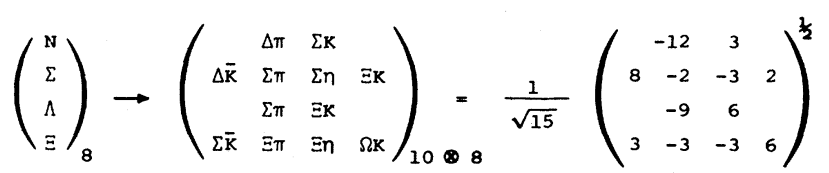

$10 \rightarrow 10 \otimes 8$

$$
\left(\begin{array}{l}
\Delta \\
\Sigma \\
\Xi \\
\Omega
\end{array}\right)_{10} \rightarrow\left(\begin{array}{cccc}
\Delta \bar{K} & \Delta \pi & \Sigma \mathrm{K} \\
\Sigma \overline{\mathrm{K}} & \Xi \pi & \Sigma \eta & \Xi \mathrm{K} \\
& \Xi \overline{\mathrm{K}} & \Omega \mathrm{K} & \mathrm{K}
\end{array}\right)_{10 \otimes 8}=\frac{1}{\sqrt{24}}\left(\begin{array}{rrrr}
15 & 3 & -6 \\
8 & 8 & 0 & -8 \\
12 & 3 & -3 & -6 \\
12 & -12
\end{array}\right)^{\frac{1}{2}}
$$




\section{SU(N) MULTIPLETS AND YOUNG DIAGRAMS}

This note tells how SU(n) particle multiplets are identified or labeled, how to find the number of particles in a multiplet from its label, how to draw the Young diagram for a multiplet, and how to use Young diagrams to determine the overall multiplet structure of a composite system, such as a 3-quark or a meson-baryon system.

(1) Multiplet labels - An SU(n) multiplet is uniquely identified by a string of $(n-1)$ nonnegative integers: $(\alpha, \beta, \gamma, \cdots)$. Any such set of integers specifies a multiplet. For an SU(2) multiplet such as an isospin multiplet, the single integer $\alpha$ is the number of steps from one end of the multiplet to the other (i.e., it is one fewer than the number of particles in the multiplet). In SU(3), the two integers $\alpha$ and $\beta$ are the numbers of steps across the top and bottom levels of the multiplet diagram. Thus the labels for the SU(3) octet and decuplet
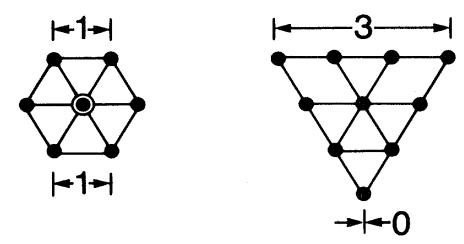

are $(1,1)$ and $(3,0)$. For larger $n$, the interpretation of the integers in terms of the geometry of the multiplets, which exist in an (n-1)dimensional space, is not so readily apparent.

The label for the $\mathrm{SU}(\mathrm{n})$ singlet is $(0,0, \ldots, 0)$. In a flavor $\mathrm{SU}(\mathrm{n})$, the $n$ quarks together form a $(1,0, \ldots, 0)$ multiplet, and the $n$ antiquarks belong to a $(0, \ldots, 0,1)$ multiplet. These two multiplets are conjugate to one another, which means their labels are related by $(\alpha, \beta, \ldots) \leftrightarrow(\ldots, \beta, \alpha)$.

(2) Number of particles - The number of particles in a multiplet, $\mathbf{N}=\mathrm{N}(\alpha, \beta, \ldots)$, is given as follows (note the pattern of the equations). In $\mathrm{SU}(2), \mathrm{N}=\mathrm{N}(\alpha)$ is

$$
\mathbf{N}=\frac{(\alpha+1)}{1}
$$

In $\mathrm{SU}(3), \mathrm{N}=\mathrm{N}(\alpha, \beta)$ is

$$
\mathrm{N}=\frac{(\alpha+1)}{1} \cdot \frac{(\beta+1)}{1} \cdot \frac{(\alpha+\beta+2)}{2} \text {. }
$$

In $\mathrm{SU}(4), \mathrm{N}=\mathrm{N}(\alpha, \beta, \gamma)$ is

$N=\frac{(\alpha+1)}{1} \cdot \frac{(\beta+1)}{1} \cdot \frac{(\gamma+1)}{1} \cdot \frac{(\alpha+\beta+2)}{2} \cdot \frac{(\beta+\gamma+2)}{2} \cdot \frac{(\alpha+\beta+\gamma+3)}{3}$.

Note that there is no factor with $(\alpha+\gamma+2)$ : only a consecutive sequence of the label integers appears in any factor. One more example should make the pattern clear for any SU(n). In SU(5), N $=\mathrm{N}(\alpha, \beta, \gamma, \delta)$ is

$$
\begin{aligned}
N= & \frac{(\alpha+1)}{1} \cdot \frac{(\beta+1)}{1} \cdot \frac{(\gamma+1)}{1} \cdot \frac{(\delta+1)}{1} \cdot \frac{(\alpha+\beta+2)}{2} \cdot \frac{(\beta+\gamma+2)}{2} \times \\
& \frac{(\gamma+\delta+2)}{2} \cdot \frac{(\alpha+\beta+\gamma+3)}{3} \cdot \frac{(\beta+\gamma+\delta+3)}{3} \cdot \frac{(\alpha+\beta+\gamma+\delta+4)}{4} .
\end{aligned}
$$

Multiplets that are conjugate to one another obviously have the same number of particles, but so can other multiplets. For example, the SU(4) multiplets $(3,0,0)$ and $(1,1,0)$ each have 20 particles.

(3) Young diagrams - A Young diagram consists of an array of boxes (or some other symbol) arranged in one or more left-justified rows, with each row being at least as long as the row beneath. The correspondence between a diagram and a multiplet label is: The top row juts out $\alpha$ boxes to the right past the end of the second row, the second row juts out $\beta$ boxes to the right past the end of the third row, etc. A diagram in $S U(n)$ has at most $n$ rows. There can be any number of "completed" columns of $n$ boxes buttressing the left of a diagram; these don't affect the label. Thus in SU(3) the diagrams

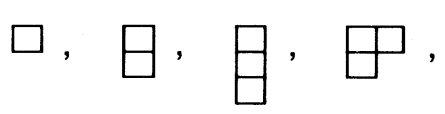

represent the multiplets $(1,0),(0,1),(0,0),(1,1)$, and $(3,0)$. In any $\mathrm{SU}(\mathrm{n})$, the quark multiplet is represented by a single box, the antiquark multiplet by a column of $(n-1)$ boxes, and a singlet by a completed column of $\mathbf{n}$ boxes.

(4) Coupling multiplets together - The following recipe tells how to find the multiplets that occur in coupling two multiplets together. To couple together more than two multiplets, first couple two, then couple the third with each of the multiplets obtained from the first two, etc.

First a definition: A sequence of the letters $a, b, c, \ldots$ is admissible if at any point in the sequence at least as many $a$ 's have been reached as $b$ 's, at least as many $b$ 's have been reached as $c$ 's, etc. Thus $a b c d$ and $a a b c b$ are admissible sequences and $a b b$ and $a c b$ are not. Now the recipe:

(a) Draw the Young diagrams for the two multiplets, but in one of the diagrams replace the boxes in the first row with $a$ 's, the boxes in the second row with $b$ 's, etc. The unlettered diagram forms the upper left-hand corner of all the enlarged diagrams constructed below.

(b) Add the $a$ 's from the lettered diagram to the unlettered diagram to form all possible legitimate Young diagrams that have no more than one $a$ per column. (All the $a$ 's appear in each new diagram.)

(c) Use the $b$ 's to further enlarge the diagrams already obtained, subject to the same rules. Throw away any diagram in which the sequence of letters formed by reading right to left in the first row, then the second row, etc., is not admissible.

(d) Proceed as in (c) with the $c$ 's, etc.

Thus, for example, the calculation to find the multiplets that can occur in a system made up of two SU(3) octets (one might be the $\pi$-meson octet, the other the N-baryon octet) is as follows:

$\otimes{ }_{b}^{a}=$

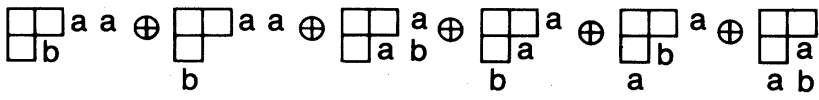

where only the diagrams with admissible sequences and with fewer than four rows (since $n=3$ ) have been kept. In terms of multiplet labels, the above may be written

$$
(1,1) \otimes(1,1)=(2,2) \oplus(3,0) \oplus(0,3) \oplus(1,1) \oplus(1,1) \oplus(0,0),
$$

or in terms of numbers of particles,

\section{$8 \otimes 8=27 \oplus 10 \oplus \overline{10} \oplus 8 \oplus 8 \oplus 1$.}

The product of the numbers of the left is equal to the sum on the right. (See the section on the Nonrelativistic Quark Model for results for 3-quark systems.) 
TESTS OF CONSERVATION LAWS*

\section{INTRODUCTION}

In response to the current interest in tests of conservation laws, we have made a list of experimental limits on all weak and electromagnetic decays, mass differences, and moments, whose observation would violate conservation laws. The list is in two parts, "Number Conservation Laws," i.e., lepton, baryon, hadronic flavor, and charge conservation, and "Discrete Space Time Symmetries," i.e. C, P, T, CP, and CPT. The references for these data can be found in the Stable Particle Section of the Data Card Listings in this Review. A discussion of these tests follows.

\section{CONSERVATION OF LEPTON NUMBERS}

Present experimental evidence and the standard electroweak theory are consistent with the absolute conservation of three separate lepton numbers: electron number $L_{e}$, muon number $L_{\mu}$, and $\tau$-number $L_{\tau}$. Searches for violations are of the following types:

a) $\Delta L=2$ for one type of lepton. The best limit comes from the search for neutrinoless double beta decay $(\mathrm{Z}, \mathrm{A}) \rightarrow$ $(\mathrm{Z}+2, \mathrm{~A})+\mathrm{e}^{-}+\mathrm{e}^{-}$. The best laboratory limit is $\mathrm{t}_{1 / 2}>2 \times 10^{22} \mathrm{yr}$ for ${ }^{76} \mathrm{Ge}$ [E. Bellotti et al., Phys. Lett. 121B, 72 (1983)].

b) Conversion of one lepton type to another. For purely leptonic processes, the best limit is on $\mu \rightarrow \mathrm{e} \gamma$. For semileptonic processes, the best limit comes from the coherent conversion process in a muonic atom $\mu^{-}+(Z, A) \rightarrow e^{-}+(Z, A)$. Of special interest is the case in which the hadronic flavor also changes, as in $K_{L} \rightarrow \mu^{ \pm} e^{\mp}$. Limits on the conversion of $\tau$ into e or $\mu$ are found in $\tau$ decay and are much less stringent that those for $\mu \rightarrow$ e conversion.

c) Conversion of one type of lepton into another type of antilepton. The case most studied is $\mu^{-}+(\mathrm{Z}, \mathrm{A}) \rightarrow \mathrm{e}^{+}+(\mathrm{Z}-2, \mathrm{~A})$.

d) Relation to neutrino mass. If neutrinos have masses then it is expected even in the standard electroweak theory that separate lepton numbers are not conserved. With small neutrino masses this would be observed first in neutrino oscillations which have been the subject of extensive experimental searches. If the $\Delta L=2$ type of violation occurs, it is expected that neutrinos will have a nonzero mass of the Majorana type.

\section{CONSERVATION OF HADRONIC FLAVORS}

The conversion of quarks of a given charge, $(d, s, b)$ or $(u, c, t)$, into one another is forbidden in strong and electromagnetic interactions by the conservation of hadron flavors: $S$ (strangeness), $C$ (charm), $B$ (bottomness), and $T$ (topness). The weak interactions violate these conservation laws as a result of the Cabibbo or Kobayashi-Maskawa mixing (see Appendix III in the complete Review of Particle Properties). The way in which these conservation laws are violated is tested as follows:

a) $\Delta S=\Delta Q$ rule. In the semileptonic decay of strange particles, the strangeness change equals the change in charge of the hadrons. Tests come from limits on decay rates such as $\mathbf{\Sigma}^{+} \rightarrow$ $\mathrm{ne}^{+} \nu$ and from a detailed analysis of $\mathrm{K}_{\mathrm{L}} \rightarrow \pi \mathrm{e} \nu$, which yields the parameter $\mathrm{x}$. A corresponding rule for charm decays is $\Delta C=\Delta Q$.

b) Change of flavor by 2 units. In the standard model this occurs only in second-order weak interactions. The one example for which this has been measured is the $\Delta S=2 \mathrm{~K}^{0}-\overline{\mathrm{K}}^{0}$ mixing, which is directly measured by $m\left(K_{S}\right)-m\left(K_{L}\right)$. A limit on the $\Delta C$ $=2 D^{0}-\bar{D}^{0}$ mixing provides a limit on $\left|m\left(D_{1}^{0}\right)-m\left(D_{2}^{0}\right)\right|$.

c) Flavor-changing neutral-currents. In the standard model the neutral-current interactions do not change flavor. The low rate of $\mathbf{K}_{\mathbf{L}} \rightarrow \mu^{+} \mu^{-}$puts limits on such interactions; the nonzero value for this rate is attributed to a combination of the weak and electromagnetic interactions. The best test should come from a limit on $\mathrm{K}^{+}$ $\rightarrow \pi^{+} \overline{\nu \nu}$, which occurs in the standard model only as a secondorder weak process with a branching fraction of $10^{-10}$ to $10^{-11}$. Limits for charm-changing or bottom-changing neutral currents are much less stringent.

\section{CPT INVARIANCE}

General principles of relativistic field theory require invariance under the combined transformation CPT. The simplest tests of CPT invariance are the equality of the masses and lifetimes of a particle and its antiparticle. The best test comes from a limit on the mass difference between $\mathrm{K}^{0}$ and $\overline{\mathrm{K}}^{0}$. Any such mass difference contributes to the CP-violating parameter $\epsilon$. In fact $\epsilon$ can be explained by a CPT-conserving but CP-violating mixing of $\mathrm{K}^{0}$ and $\overline{\mathbf{K}}^{0}$, which yields a prediction that $\phi_{+-} \approx 44^{\circ}$, while a $\mathrm{K}^{0}-\overline{\mathbf{K}}^{0}$ mass difference would yield $\phi_{\alpha_{-}} \approx 44^{\circ}+90^{\circ}$. It is thus possible to deduce that $\left|m\left(K^{0}\right)-m\left(K^{0}\right)\right|<10^{-4}\left|m\left(K_{S}\right)-m\left(K_{L}\right)\right|<$ $3 \times 10^{-10} \mathrm{eV}$. Also, an upper limit on $\left|\mathrm{m}\left(\mathrm{D}^{0}\right)-\mathrm{m}\left(\overline{\mathrm{D}}^{0}\right)\right|$ can be derived from the bound $\left|m\left(D_{1}^{0}\right)-m\left(D_{2}^{0}\right)\right|<0.65 \times 10^{-9} \mathrm{MeV}$ (inferred from bound on $D^{0} \rightarrow \bar{D}^{0} \rightarrow \mu^{-}$anything), given an input value of, or bound on, the CP-violation parameter $\epsilon$ for $D^{0}-\bar{D}^{0}$ mixing.

\section{CP AND T INVARIANCE}

Given CPT invariance, $\mathrm{CP}$ violation and $\mathrm{T}$ violation are equivalent. So far the only evidence for $\mathrm{CP}$ or $\mathrm{T}$ violation comes from the measurements of $\eta_{+-}, \eta_{00}$, and the semileptonic decay charge asymmetry for $K_{L}$. Other searches for CP or $T$ violation should be divided into (a) those that involve weak interactions or parity violation, and (b) those that involve processes allowed by the strong or electromagnetic interactions. In class (a) the most sensitive is probably the search for an electric dipole moment of the neutron, which requires both $\mathrm{P}$ and $\mathrm{T}$ violation to be nonzero. Class (b) searches involve looking for $C$ or $T$ violation in strong or electromagnetic processes. Examples are the search for $C$ violation in $\eta$ decay, believed to be an electromagnetic process, and the search for $\mathrm{T}$ violation in a number of nuclear and electromagnetic reactions.

\footnotetext{
Prepared April 1984 by R.E. Shrock, T.G. Trippe, and L. Wolfenstein.
} 
TESTS OF CONSERVATION LAWS (Cont'd)

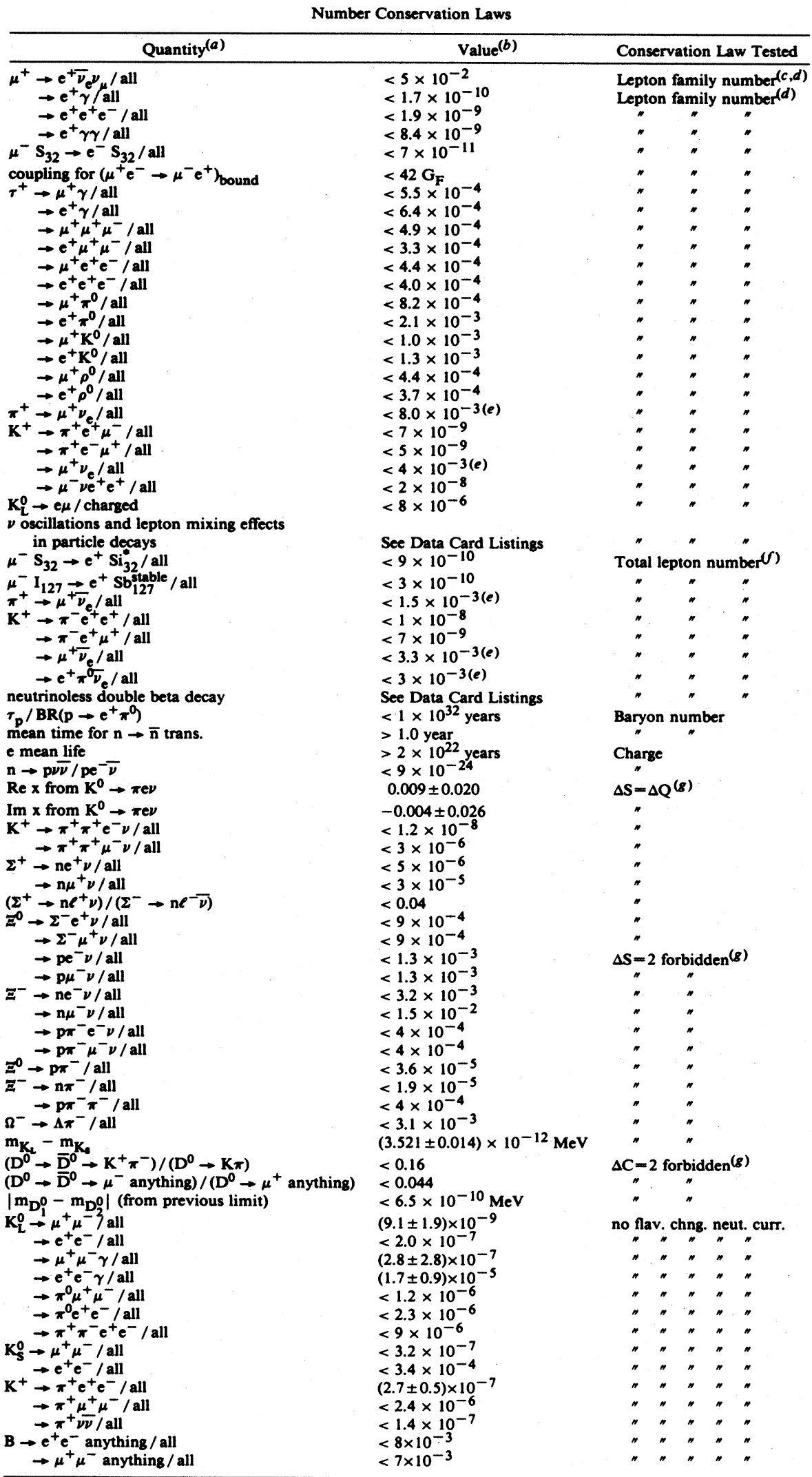


TESTS OF CONSERVATION LAWS (Cont'd)

Discrete Space Time Symmetries

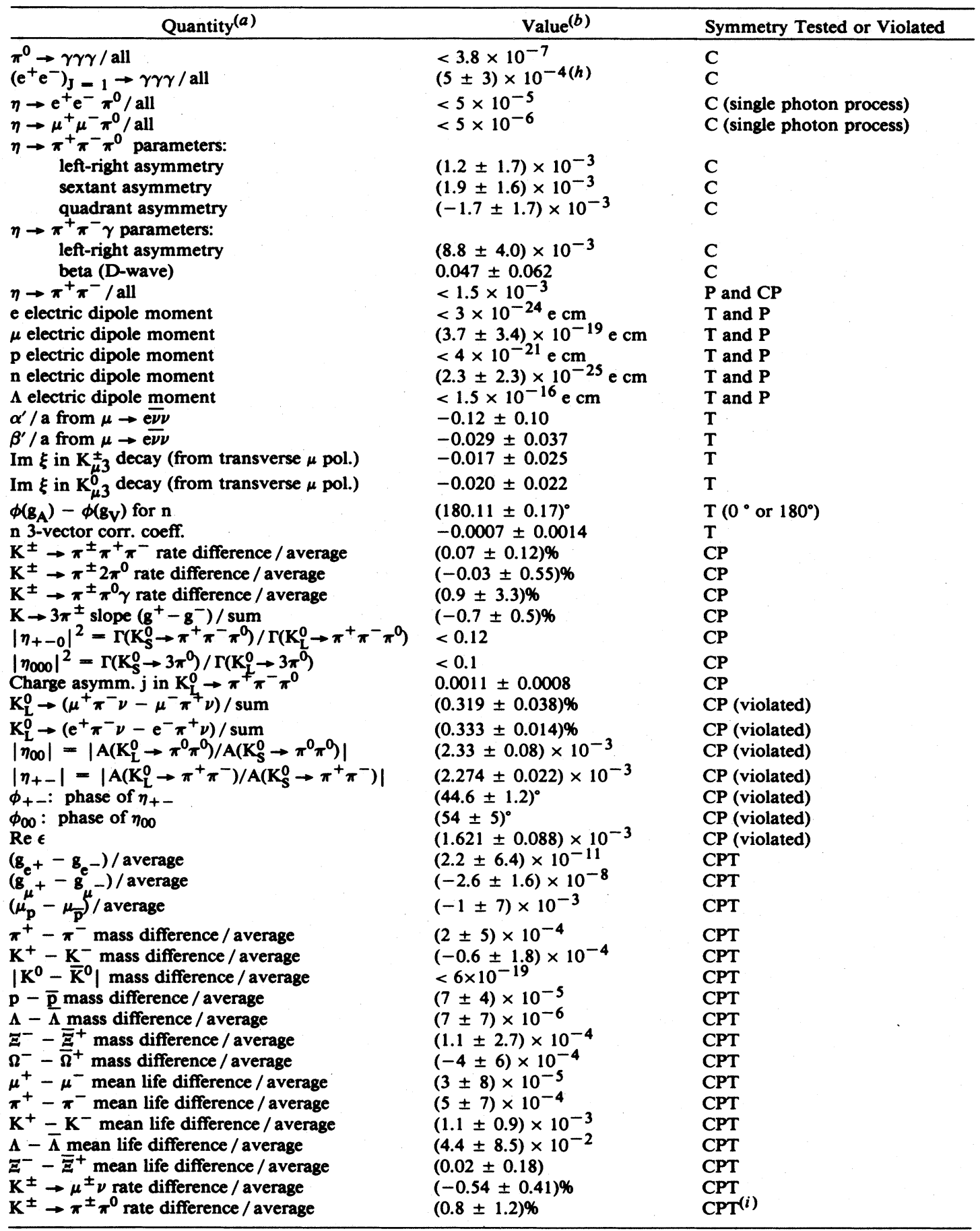

a. Branching fractions are described by a shorthand notation, e.g., " $\mu^{+} \rightarrow \mathrm{e}^{+} \gamma /$ all" means $\Gamma\left(\mu^{+} \rightarrow \mathrm{e}^{+} \gamma\right) / \Gamma\left(\mu^{+} \rightarrow\right.$ all).

$b$. Limits are given at $90 \%$ confidence level while errors are given as \pm 1 standard deviation.

c. Test of additive vs. multiplicative lepton family number conservation.

$d$. Lepton family number conservation means separate conservation of e-number, $\mu$-number, and $\tau$-number.

$e$. These limits are derived from the analysis of neutrino oscillation experiments.

$f$. Violation of total lepton number conservation also implies violation of lepton family number conservation.

g. Can be violated in second-order weak interactions.

h. Orthopositronium data are from Liu and Roberts, Phys. Rev. Lett. 16, 67 (1966).

i. Neglecting photon channels. See, e.g., A. Pais and S.B. Treiman, Phys. Rev. D12, 2744 (1975). 


\section{KINEMATICS, DECAYS, AND SCATTERING}

\section{A. LORENTZ TRANSFORMATIONS}

The energy $E$ and three-momentum $\vec{p}$ of a particle form a fourvector $p=(E, \vec{p})$. Viewed from a second frame with velocity $\vec{v}=\beta c \hat{z}$ relative to the original frame, the components of $p$ are $\left(E^{\prime}\right.$, $\overrightarrow{\mathbf{p}}$ ), where

$$
\begin{aligned}
& \mathrm{E}^{\prime}=\gamma \mathrm{E}-\beta \gamma \mathrm{p}_{\mathrm{z}}, \\
& \mathrm{p}_{\mathrm{z}}^{\prime}=\gamma \mathrm{p}_{\mathrm{z}}-\beta \gamma \mathrm{E}, \\
& \mathrm{p}_{\mathrm{x}}^{\prime}=\mathrm{p}_{\mathrm{x}} ; \mathrm{p}_{\mathrm{y}}^{\prime}=\mathrm{p}_{\mathbf{y}},
\end{aligned}
$$

and where $\gamma=\left(1-\beta^{2}\right)^{-1 / 2}$. It follows that the scalar product of two momenta, $\mathrm{p}_{1} \cdot \mathrm{p}_{2}=\mathrm{E}_{1} \mathrm{E}_{2}-\overrightarrow{\mathrm{p}}_{1} \cdot \overrightarrow{\mathrm{p}}_{2}$, is invariant, that is, frame independent.

If $\overrightarrow{\mathrm{p}}$ makes an angle $\theta$ with the $\mathrm{z}$-axis, then $\overrightarrow{\mathrm{p}}^{\prime}$ makes an angle $\theta^{\prime}$ with the $\mathrm{z}$-axis,
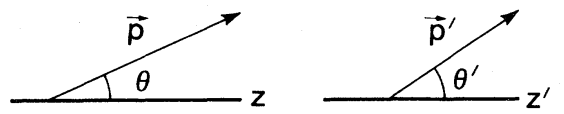

where

$$
\tan \theta^{\prime}=\frac{|\vec{p}| \sin \theta}{\gamma|\vec{p}| \cos \theta-\beta \gamma E} .
$$

In particular, if the unprimed frame is the center of mass and the primed frame is the lab, and if the velocity of the center of mass in the lab frame is $\beta^{*} \hat{z}$, we use $\beta=-\beta^{*}$ above to find (denoting $\left.\mathrm{p}_{\mathrm{cm}}=\left|\overrightarrow{\mathrm{p}}_{\mathrm{cm}}\right|\right)$

$$
\tan \theta_{\mathrm{lab}}=\frac{\mathrm{p}_{\mathrm{cm}} \sin \theta_{\mathrm{cm}}}{\gamma^{*} \mathrm{p}_{\mathrm{cm}} \cos \theta_{\mathrm{cm}}+\beta^{*} \gamma^{*} \mathrm{E}_{\mathrm{cm}}} .
$$

If $\beta^{*}>\mathrm{p}_{\mathrm{cm}} / \mathrm{E}_{\mathrm{cm}}$, the particle is necessarily moving forward in the lab and

$$
\left(\tan \theta_{\mathrm{lab}}\right)_{\max }=\frac{\mathrm{p}_{\mathrm{cm}}}{\gamma^{*} \mathrm{E}_{\mathrm{cm}}} \frac{1}{\sqrt{\beta^{* 2}-\mathrm{p}_{\mathrm{cm}}^{2} / \mathrm{E}_{\mathrm{cm}}^{2}}} .
$$

We denote $\mathrm{p}_{\perp}=\mathrm{p}_{\perp}{ }^{\prime}=|\overrightarrow{\mathrm{p}}| \sin \theta_{\mathrm{cm}}$. Then given a fixed $\mathrm{p}_{\mathrm{cm}}$ and $\mathrm{E}_{\mathrm{cm}}$, as, for example, in a two-to-two scattering process, as $\theta_{\mathrm{cm}}$ varies from 0 to $2 \pi$ the lab momentum describes an ellipse:

$$
\frac{\left(\mathrm{p}_{\mathrm{z}}^{\prime}-\beta^{*} \gamma^{*} \mathrm{E}_{\mathrm{cm}}\right)^{2}}{\gamma^{* 2} \mathrm{p}_{\mathrm{cm}}^{2}}+\frac{\mathrm{p}_{\perp}^{\prime 2}}{\mathrm{p}_{\mathrm{cm}}^{2}}=1
$$

\section{B. DECAYS}

\section{B.1.a Two-body kinematics:}

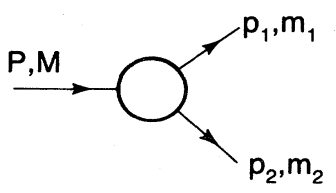

In the rest frame of the decaying particle,

$$
\begin{aligned}
& E_{1}=\frac{M^{2}+m_{1}^{2}-m_{2}^{2}}{2 M}, \\
& \left|\vec{p}_{1}\right|=\left[\frac{\left[M^{2}-\left(m_{1}+m_{2}\right)^{2}\right]\left[M^{2}-\left(m_{1}-m_{2}\right)^{2}\right]}{4 M^{2}}\right]^{1 / 2} .
\end{aligned}
$$

B.1.b Two-body partial decay rate: If $\mathscr{M}$ is the Lorentz invariant matrix element (see Section $D$ below), the partial decay rate in the rest frame of the decaying particle is

$$
\mathrm{d} \Gamma=\frac{1}{32 \pi^{2}}|\mathscr{M}|^{2} \frac{\left|\overrightarrow{\mathrm{p}}_{1}\right| \mathrm{d} \Omega}{\mathrm{M}^{2}},
$$

where $d \Omega$ is the differential solid angle in the rest frame of the decaying particle.

B.2.a Three-body kinematics:

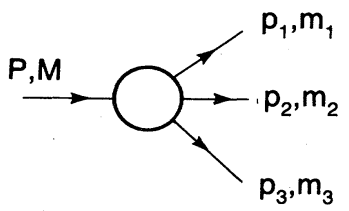

We denote

$$
p_{12}=p_{1}+p_{2}, m_{12}^{2}=p_{12}^{2}, \text { etc. }
$$

Then

$$
m_{12}^{2}+m_{23}^{2}+m_{13}^{2}=M^{2}+m_{1}^{2}+m_{2}^{2}+m_{3}^{2} .
$$

The invariant mass of the pair 1-2 is related to the energy of particle 3 in the rest frame of $M$,

$$
m_{12}^{2}=\left(P-p_{3}\right)^{2}=M^{2}+m_{3}^{2}-2 M E_{3} .
$$

B.2.b Dalitz plot: If the orientation of the decaying particle is ignored, there are two kinematic variables, which may be chosen to be $m_{12}^{2}$ and $m_{13}^{2}$. For fixed $m_{12}^{2}$, the range of $m_{13}^{2}$ is determined by letting $\vec{p}_{1}$ be parallel or antiparallel to $\vec{p}_{3}$. In the rest frame of $\left(p_{1}+p_{2}\right)$, the energy of particle 3 is $E_{3}^{*}=\left(M^{2}-m_{12}^{2}-m_{3}^{2}\right) /\left(2 m_{12}\right)$, and that of particle 1 is $E_{1}^{*}=\left(m_{12}^{2}+m_{1}^{2}-m_{2}^{2}\right) /\left(2 m_{12}\right)$. Thus for $a$ given $\mathrm{m}_{12}^{2}$,

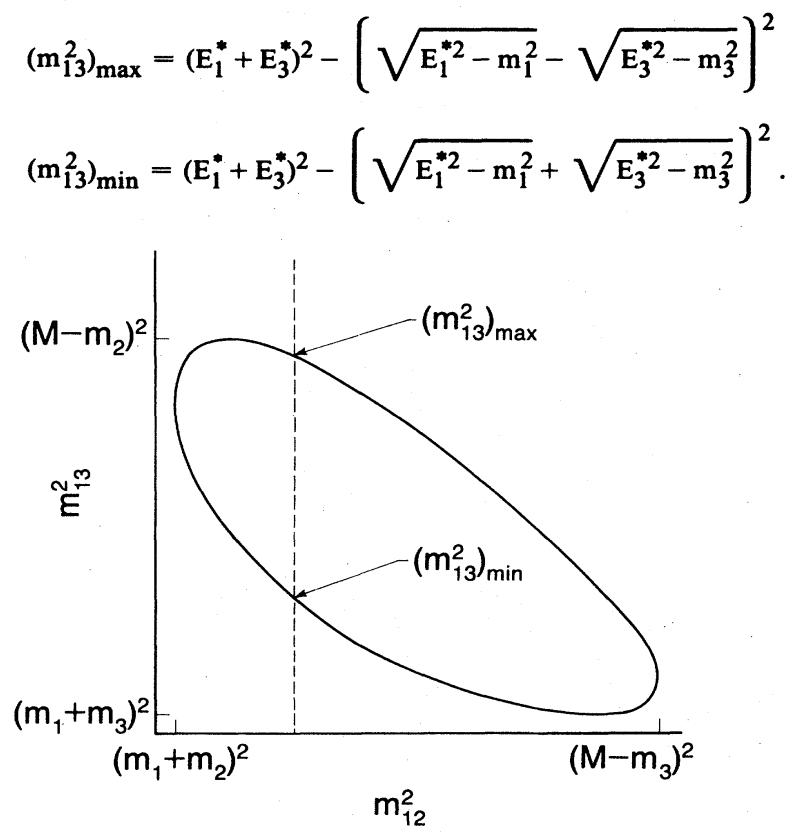

The scatter plot in $\mathrm{m}_{12}^{2}$ and $\mathrm{m}_{13}^{2}$ is called a Dalitz plot. Phase space density is uniform across the plot. See below. 


\section{KINEMATICS, DECAYS, AND SCATTERING (Cont'd)}

B.2.c Three-body phase space: Fixing the energies $E_{1}$ and $E_{2}$ of two of the final state particles in the $M$ rest frame determines the relative orientation of the three outgoing particles. Their momenta may then be regarded as a rigid body whose orientation with respect to the initial particle is specified by the Euler angles $\alpha, \beta$, and $\gamma$. The partial decay rate in the $M$ rest frame is

$$
\mathrm{d} \Gamma=\frac{(2 \pi)^{-5}}{16 \mathrm{M}}|\mathscr{\mu}|^{2} \mathrm{dE}_{1} \mathrm{dE}_{2} \mathrm{~d} \alpha \mathrm{d} \cos \beta \mathrm{d} \gamma \text {. }
$$

If the angles are integrated out, we have the Dalitz plot form,

$$
\mathrm{d} \Gamma=\frac{(2 \pi)^{-3}}{8 \mathrm{M}}|\mathscr{M}|^{2} \mathrm{dE}_{1} \mathrm{dE}_{2}=\frac{(2 \pi)^{-3}}{32 \mathrm{M}^{3}}|\mathscr{M}|^{2} \mathrm{dm}_{12}^{2} \mathrm{dm}_{23}^{2} \text {. }
$$

An alternative expression is

$$
\mathrm{d} \Gamma=\frac{(2 \pi)^{-5}}{16 \mathrm{M}^{2}}|\mathscr{M}|^{2}\left|\overrightarrow{\mathrm{p}}_{1}^{*}\right|\left|\overrightarrow{\mathrm{p}}_{3}\right| \mathrm{dm_{12 }} \mathrm{d} \Omega_{1}^{*} \mathrm{~d} \Omega_{3},
$$

where

$$
\left|\vec{p}_{1}^{*}\right|=\left[\frac{\left[m_{12}^{2}-\left(m_{1}+m_{2}\right)^{2}\right]\left[m_{12}^{2}-\left(m_{1}-m_{2}\right)^{2}\right]}{4 m_{12}^{2}}\right]^{1 / 2}
$$

is the momentum of particle 1 in the rest frame of $m_{12}$,

$$
\left|\vec{p}_{3}\right|=\left[\frac{\left[M^{2}-\left(m_{12}+m_{3}\right)^{2}\right]\left[M^{2}-\left(m_{12}-m_{3}\right)^{2}\right]}{4 M^{2}}\right]^{1 / 2}
$$

is the momentum of particle 3 in the $M$ rest frame, $d \Omega_{1}^{*}$ is the solid angle element for particle 1 in the 1-2 rest frame, and $d \Omega_{3}$ is the solid angle element for particle 3 in the $M$ rest frame.

B.3 n-body phase space:

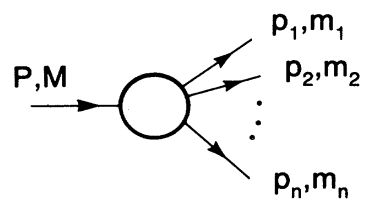

The partial decay rate in the $M$ rest frame is

$$
\mathrm{d} \Gamma=\frac{(2 \pi)^{4}}{2 M}|M|^{2} \mathrm{~d} \Phi_{\mathrm{n}}\left(P ; p_{1}, \cdots, p_{n}\right),
$$

where

$$
d \Phi_{n}\left(P ; p_{1}, \cdots, p_{n}\right)=\delta^{4}\left(P-\sum_{i=1}^{n} p_{i}\right) \prod_{i=1}^{n} \frac{d^{3} p_{i}}{(2 \pi)^{3} 2 E_{i}} .
$$

In particular,

$$
\mathrm{d} \Phi_{2}\left(P ; p_{1}, p_{2}\right)=(2 \pi)^{-6} \frac{\left|\vec{p}_{1}^{*}\right|}{4 M} d \Omega_{1}^{*},
$$

where $\left|\overrightarrow{\mathbf{p}}_{1}^{*}\right|$ is the momentum of particle 1 in the $M$ rest frame and $d \Omega_{1}^{*}$ is the solid angle element in the same frame.

Phase space for $n$ particles can be related to that for $n-1$ by treating particles 1 and 2 as a single system of momentum $p_{12}=$ $p_{1}+p_{2}$ and mass squared $m_{12}^{2}=p_{12}^{2}$. Thus

$$
\begin{gathered}
d \Phi_{n}\left(P ; p_{1}, p_{2}, \cdots, p_{n}\right)=d \Phi_{n-1}\left(P ; p_{12}, p_{3}, \cdots, p_{n}\right) \\
\quad \times d \Phi_{2}\left(p_{12} ; p_{1}, p_{2}\right)(2 \pi)^{3} d_{12}^{2} .
\end{gathered}
$$

\section{SCATTERING}

Throughout Section C, we set $\hbar=1, c=1$. Use $\hbar c=197.3$ $\mathrm{MeV}$ fermi, and $(h \mathrm{c})^{2}=0.3894 \mathrm{GeV}^{2} \mathrm{mb}$ for conversions.

C.1 Partial waves: The amplitude in the center of mass for elastic scattering of spinless particles may be written in a partial wave expansion

$$
\mathrm{f}(\mathrm{k}, \theta)=\frac{1}{\mathrm{k}} \sum_{\ell}(2 \ell+1) \mathrm{a}_{\ell} \mathrm{P}_{\ell}(\cos \theta),
$$

where $\mathrm{k}$ is the c.m. momentum, $\theta$ is the c.m. scattering angle, $a_{\ell}=$ $\left(\eta_{\ell} \mathrm{e}^{2 \mathrm{i} \delta_{\ell}}-1\right) / 2 \mathrm{i}, 0 \leqslant \eta_{\ell} \leqslant 1$, and $\delta_{\ell}$ is the phase shift of the $\ell^{\text {th }}$ partial wave. For purely elastic scattering, $\eta_{\ell}=1$. The differential cross section is

$$
\frac{d \sigma}{d \Omega}=|f(k, \theta)|^{2} .
$$

The optical theorem is

$$
\sigma_{\text {tot }}=\frac{4 \pi}{k} \operatorname{Im~} f(k, 0),
$$

and the cross section in the $\ell^{\text {th }}$ partial wave is

$$
\sigma_{\ell}=\frac{4 \pi}{k^{2}}(2 \ell+1)\left|a_{\ell}\right|^{2} \leqslant \frac{4 \pi(2 \ell+1)}{k^{2}}
$$

The partial-wave amplitude $a_{\ell}$ can be displayed in an Argand plot.

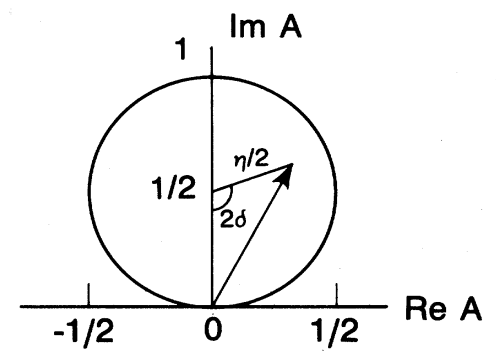

The usual Lorentz invariant matrix element $\mathscr{M}$ (see Section D below) for the elastic process is related to $f(k, \theta)$ by

$$
\mathscr{M}=-8 \pi \sqrt{\mathrm{s}} \mathrm{f}(\mathrm{k}, \theta) \text {, }
$$

so

$$
\sigma_{\text {tot }}=-\frac{1}{2 k \sqrt{s}} \operatorname{Im} \mathscr{K}(\mathrm{t}=0),
$$

where $s$ and $t$ are the center-of-mass energy squared and momentum transfer squared, respectively (see Section C.3.a).

C.2 Resonances: The Breit-Wigner form for $\mathrm{a}_{\ell}$ with a resonance at c.m. energy $E_{R}$, elastic width $\Gamma_{\text {el }}$, and total width $\Gamma_{\text {tot }}$ is

$$
a_{\ell}=\frac{\frac{1}{2} \Gamma_{e l}}{E_{R}-E-\frac{i}{2} \Gamma_{\text {tot }}},
$$

where $\mathrm{E}$ is the c.m. energy. This gives a circle in the Argand plot with center $\mathrm{ix}_{\mathrm{el}} / 2$ and radius $\mathrm{x}_{\mathrm{el}} / 2$, where $\mathrm{x}_{\mathrm{el}}=\Gamma_{\mathrm{el}} / \Gamma_{\text {tot }}$. The quantity $x_{e l}$ is called the elasticity. The amplitude has a pole at $E=$ $\mathrm{E}_{\mathbf{R}}-\mathrm{i} \Gamma_{\text {tot }} / 2$. 


\section{KINEMATICS, DECAYS, AND SCATTERING (Cont'd)}

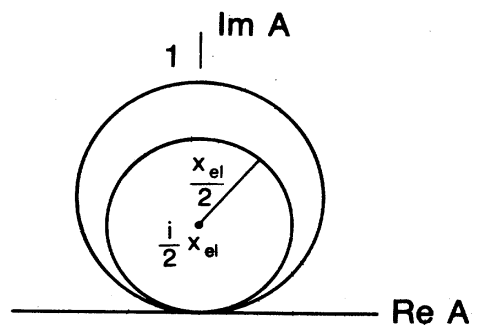

The Breit-Wigner cross section for a spin-J resonance produced in the collision of particles of spin $S_{1}$ and $S_{2}$ is

$$
\sigma_{\mathrm{BW}}(\mathrm{E})=\frac{(2 \mathrm{~J}+1)}{\left(2 \mathrm{~S}_{1}+1\right)\left(2 \mathrm{~S}_{2}+1\right)} \frac{\pi}{\mathrm{k}^{2}} \frac{\mathrm{B}_{\text {in }} \mathrm{B}_{\text {out }} \Gamma_{\text {tot }}^{2}}{\left(E-E_{\mathrm{R}}\right)^{2}+\Gamma_{\text {tot }}^{2} / 4},
$$

where $k$ is the c.m. momentum, $E$ is the c.m. energy, and $B_{\text {in }}$ and $B_{\text {out }}$ are the branching fractions of the resonance into the entrance and exit channels. The $2 S+1$ factors are the multiplicities of the incident spin states, so they are replaced by 2 for photons, etc.

C.3.a Two-body scattering kinematics:

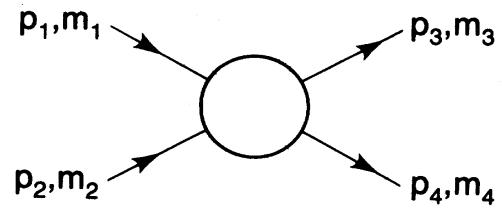

In the center of mass,

$$
\begin{aligned}
E_{1 \mathrm{~cm}} & =\frac{s+m_{1}^{2}-m_{2}^{2}}{2 \sqrt{s}}, \\
p_{1 \mathrm{~cm}} & =\left[\frac{\left[s-\left(m_{1}+m_{2}\right)^{2}\right]\left[s-\left(m_{1}-m_{2}\right)^{2}\right]}{4 s}\right]^{1 / 2} \\
& =\frac{p_{1 l a b} m_{2}}{\sqrt{s}},
\end{aligned}
$$

where $\sqrt{s}$ is the total c.m. energy. The Lorentz invariant Mandelstam variables are

$$
\begin{aligned}
s & =\left(p_{1}+p_{2}\right)^{2}=\left(p_{3}+p_{4}\right)^{2} \\
& =m_{1}^{2}+2 E_{1} E_{2}-2 \vec{p}_{1} \cdot \vec{p}_{2}+m_{2}^{2}, \\
t & =\left(p_{1}-p_{3}\right)^{2}=\left(p_{2}-p_{4}\right)^{2} \\
& =m_{1}^{2}-2 E_{1} E_{3}+2 \vec{p}_{1} \cdot \vec{p}_{3}+m_{3}^{2}, \\
u & =\left(p_{1}-p_{4}\right)^{2}=\left(p_{2}-p_{3}\right)^{2} \\
& =m_{1}^{2}-2 E_{1} E_{4}+2 \vec{p}_{1} \cdot \vec{p}_{4}+m_{4}^{2},
\end{aligned}
$$

and they satisfy

$$
s+t+u=m_{1}^{2}+m_{2}^{2}+m_{3}^{2}+m_{4}^{2} .
$$

If $\theta_{\mathrm{cm}}$ is the c.m. scattering angle between particles 1 and 3 , then (denoting $\mathbf{p}_{1 \mathrm{~cm}}=\left|\overrightarrow{\mathbf{p}}_{1 \mathrm{~cm}}\right|, \mathbf{p}_{3 \mathrm{~cm}}=\left|\overrightarrow{\mathbf{p}}_{3 \mathrm{~cm}}\right|$ )

$$
t=\left(E_{1 \mathrm{~cm}}-E_{3 \mathrm{~cm}}\right)^{2}-\left(p_{1 \mathrm{~cm}}-p_{3 \mathrm{~cm}}\right)^{2}-4 p_{1 \mathrm{~cm}} p_{3 \mathrm{~cm}} \sin ^{2}\left(\theta_{\mathrm{cm}} / 2\right) \text {. }
$$

For $\theta_{\mathrm{cm}}=0,-t$ is a minimum.
C.3.b Two-body differential cross sections: In the center of mass or lab,

$$
\frac{\mathrm{d} \sigma}{\mathrm{dt}}=\frac{1}{64 \pi \mathrm{s}} \frac{1}{\mathrm{p}_{1 \mathrm{~cm}}^{2}}|\mathscr{M}|^{2} .
$$

In the center of mass,

$$
\frac{\mathrm{d} \sigma}{\mathrm{d} \Omega_{\mathrm{cm}}}=\frac{\mathrm{p}_{1 \mathrm{~cm}} \mathrm{p}_{3 \mathrm{~cm}}}{\pi} \frac{\mathrm{d} \sigma}{\mathrm{dt}}
$$

\section{C.4 n-body differential cross sections:}

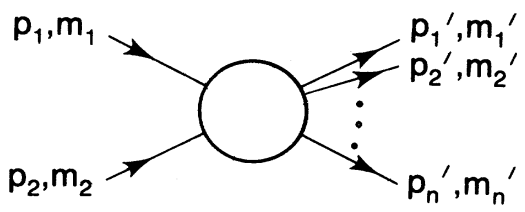

In the c.m. or lab

$$
\mathrm{d} \sigma=\frac{(2 \pi)^{4}|\mathscr{M}|^{2}}{4 \sqrt{\left(\mathrm{p}_{1} \cdot \mathrm{p}_{2}\right)^{2}-\mathrm{m}_{1}^{2} \mathrm{~m}_{2}^{2}}} \mathrm{~d} \Phi_{\mathrm{n}}\left(\mathrm{p}_{1}+\mathrm{p}_{2} ; \mathrm{p}_{1}^{\prime}, \mathrm{p}_{2}^{\prime}, \cdots, \mathrm{p}_{\mathrm{n}}^{\prime}\right),
$$

where $n$-body phase space, $d \Phi_{n}$, is described in Section B.3 above. Note that $\sqrt{\left(p_{1} \cdot p_{2}\right)^{2}-m_{1}^{2} m_{2}^{2}}=p_{1 l a b} m_{2}=p_{1 c m} \sqrt{s}$.

C.5.a Leptoproduction kinematics:

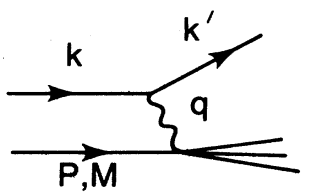

$q=k-k^{\prime}$ is the four-momentum transferred to the target.

Invariant quantities:

$\nu=\frac{\mathrm{g} \cdot \mathrm{P}}{\mathrm{M}}=\mathrm{E}-\mathrm{E}^{\prime}$ is the lepton's energy loss in the lab (in earlier literature sometimes $\nu=q \cdot P$ ). Here, $E$ and $E^{\prime}$ are the initial and final lepton energies in the lab.

$Q^{2}=-q^{2}=2\left(E E^{\prime}-\vec{k} \cdot \vec{k}^{\prime}\right)-m_{\ell}^{2}-m_{\ell^{\prime}}^{2}$ where $m_{\ell}\left(m_{\ell^{\prime}}\right)$ is the initial (final) lepton mass. If $\mathrm{EE}^{\prime} \sin ^{2}(\theta / 2) \gg \mathrm{m}_{\ell}^{2}$, $\mathrm{m}_{\ell^{\prime}}^{2}$, then

$\simeq 4 \mathrm{EE}^{\prime} \sin ^{2}(\theta / 2)$, where $\theta$ is the lepton's scattering angle in the lab.

$\mathrm{x}=\frac{\mathrm{Q}^{2}}{2 \mathrm{M} \nu}$ In the parton model, $\mathrm{x}$ is the fraction of the target nucleon's momentum carried by the struck quark. See section on Quark Parton Model.

$y=\frac{q \cdot P}{k \cdot P}=\frac{\nu}{E}$ is the fraction of the lepton's energy lost in the lab.

$W^{2}=(P+q)^{2}=M^{2}+2 M \nu-Q^{2}$ is the mass squared of the system recoiling against the lepton.

C.5.b Leptoproduction cross sections:

$$
\frac{d^{2} \sigma}{d x d y}=2 M \nu E \frac{d^{2} \sigma}{d \nu d Q^{2}}=\frac{2 \pi M \nu}{E^{\prime}} \frac{d^{2} \sigma}{d \Omega_{1 a b} d E^{\prime}}=2 x M E \frac{d^{2} \sigma}{d x d Q^{2}} .
$$




\section{KINEMATICS, DECAYS, AND SCATTERING (Cont'd)}

\section{C.5.b.i Electroproduction structure functions:}

$$
\begin{aligned}
\frac{d^{2} \sigma}{d x d y}=\frac{8 \pi \alpha^{2} M E}{Q^{4}}\left[\frac{1+(1-y)^{2}}{2} 2 x F_{1}^{e m}\right. \\
\left.+(1-y)\left(F_{2}^{e m}-2 x F_{1}^{e m}\right)-\frac{M}{2 E} x y F_{2}^{e m}\right] .
\end{aligned}
$$

$F_{1}^{\mathrm{em}}\left(x, Q^{2}\right)$ and $F_{2}^{\mathrm{em}}\left(x, Q^{2}\right)$ are the (unpolarized) structure functions, which are, in the naive parton model, independent of $Q^{2}$.

C.5.b.ii Neutrino production structure functions:

$$
\begin{aligned}
& \frac{d^{2} \sigma^{y}}{d x d y}=\frac{G_{F}^{2} M E}{\pi}\left[\left(1-y-\frac{M}{2 E} x y\right) F_{2}^{\nu}+\frac{y^{2}}{2} 2 x F_{1}^{\nu}\right. \\
& \left.+\left(y-\frac{y^{2}}{2}\right) \mathrm{xF}_{3}^{\nu}\right] \\
& \frac{d^{2} \sigma^{\bar{\nu}}}{d x d y}=\frac{G_{F}^{2} M E}{\pi}\left[\left(1-y-\frac{M}{2 E} x y\right) F_{2}^{\bar{\nu}}+\frac{y^{2}}{2} 2 x F_{1}^{\bar{\nu}}\right. \\
& \left.-\left(y-\frac{y^{2}}{2}\right) \mathrm{xF}_{3}^{\bar{\nu}}\right] .
\end{aligned}
$$

The structure functions $F_{i}^{,, \bar{\nu}}$ are related to quark distributions in the parton model (see section on Quark Parton Model). There are separate $F_{i}$ 's for neutral- and charged-current processes.

C.6.a $e^{+} e^{-}$annihilation: For pointlike spin-1/2 fermions in the c.m., the differential cross section for $e^{+} e^{-} \rightarrow \bar{f} \bar{f}$ via single photon annihilation is

$$
\frac{\mathrm{d} \sigma}{\mathrm{d} \Omega}=\frac{\alpha^{2}}{4 \mathrm{~s}} \beta\left[1+\cos ^{2} \theta+\left(1-\beta^{2}\right) \sin ^{2} \theta\right] \mathrm{e}_{\mathrm{Q}}^{2},
$$

where $\beta$ is the velocity of the final state fermion in the center of mass, and where $e_{Q}$ is the charge of the fermion in units of the proton charge. For $\beta \rightarrow 1$,

$$
\sigma=\frac{4 \pi \alpha^{2}}{3 \mathrm{~s}} \mathrm{e}_{\mathrm{Q}}^{2}=\frac{86.8 \mathrm{e}_{\mathrm{Q}}^{2} \mathrm{nb}}{\mathrm{s}\left(\mathrm{GeV}^{2}\right)} .
$$

C.6.b $\mathrm{e}^{+} \mathrm{e}^{-}$two-photon process: In the equivalent photon approximation, the cross section for $\mathrm{e}^{+} \mathrm{e}^{-} \rightarrow \mathrm{e}^{+} \mathrm{e}^{-} \mathrm{X}$ is related to the cross section for $\gamma \gamma \rightarrow \mathrm{X}$ by

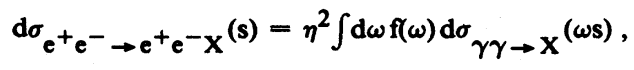

where

$$
\eta \simeq \frac{\alpha}{2 \pi} \ln \left[\frac{s}{4 m_{e}^{2}}\right]
$$

and

$$
f(\omega)=\frac{1}{\omega}\left[(2+\omega)^{2} \ln \frac{1}{\omega}-2(1-\omega)(3+\omega)\right] .
$$

For the production of a resonance of mass $m_{R}$ and spin $J$,

$$
\left.\sigma\left(\mathrm{e}^{+} \mathrm{e}^{-} \rightarrow \mathrm{e}^{+} \mathrm{e}^{-} \mathrm{R}\right)=\eta^{2(2 \mathrm{~J}+1) 8 \pi^{2} \Gamma(\mathrm{R} \rightarrow \gamma \gamma)} \frac{\mathrm{m}_{\mathrm{R}}^{2}}{\mathrm{~m}}\right) .
$$

C.7 Inclusive hadronic reactions: A particle's momentum can be parametrized by selecting a particular direction for the $\mathrm{z}$-axis and writing

$$
\left(E=m_{\perp} \cosh y, p_{z}=m_{\perp} \sinh y, p_{x}, p_{y}\right),
$$

where

$$
\begin{aligned}
& m_{\perp}^{2}=m^{2}+p_{x}^{2}+p_{y}^{2} \\
& y=\frac{1}{2} \ell n\left(\frac{E+p_{z}}{E-p_{z}}\right)=\ell n\left(\frac{E+p_{z}}{m_{\perp}}\right)=\tanh ^{-1}\left(\frac{p_{z}}{E}\right) .
\end{aligned}
$$

The variable $\mathrm{y}$ is called the rapidity. $\mathrm{A}$ boost in the $\mathrm{z}$-direction then modifies y by y $\rightarrow y+\Delta$, where $\gamma=\cosh \Delta, \beta=\tanh \Delta$. Thus the shape of the distribution $\mathrm{dN} / \mathrm{dy}$ is invariant under such a boost, and

$$
E \frac{d^{3} \sigma}{d^{3} p}=\frac{d^{3} \sigma}{d y d^{2} p_{\perp}} .
$$

Feynman's $\mathrm{x}$ variable is defined to be

$$
\mathrm{x}=\left(\frac{\mathrm{p}_{\mathrm{z}}}{\mathrm{p}_{\mathrm{z} \max }}\right)_{\mathrm{cm}} \approx \frac{2 \mathrm{p}_{\mathrm{zcm}}}{\sqrt{\mathrm{s}}} \approx \frac{2 \mathrm{~m}_{\perp} \sinh \mathrm{y}_{\mathrm{cm}}}{\sqrt{\mathrm{s}}} .
$$

For $y_{c m}$ not small $\left(e^{-2 y_{c m}} \ll 1\right)$

$$
\mathrm{x} \approx \frac{\mathrm{m}_{\perp}}{\sqrt{\mathrm{s}}} \mathrm{e}^{\mathrm{y}_{\mathrm{cm}}}
$$

and

$$
\left(\mathrm{y}_{\mathrm{cm}}\right)_{\max }=\ell \mathrm{n} \frac{\sqrt{\mathrm{s}}}{\mathrm{m}} .
$$

\section{LORENTZ INVARIANT AMPLITUDES}

The quantity $-i \mathscr{K}$ is determined in perturbation theory by the Feynman rules. Our convention above is consistent with the Appendices of Bjorken and Drell except that fermion spinors are normalized so that $\bar{u} u=2 \mathrm{~m}$, etc. In particular, the S-matrix for two-body scattering is

$$
\left\langle p_{1}^{\prime} p_{2}^{\prime}|S| p_{1} p_{2}\right\rangle=I-i(2 \pi)^{4} \delta^{4}\left(p_{1}+p_{2}-p_{1}^{\prime}-p_{2}^{\prime}\right)
$$

$$
\times \frac{\mathscr{M}\left(\mathrm{p}_{1}, \mathrm{p}_{2} ; \mathrm{p}_{1}^{\prime}, \mathrm{p}_{2}^{\prime}\right)}{\left(2 \mathrm{E}_{1}\right)^{1 / 2}\left(2 \mathrm{E}_{2}\right)^{1 / 2}\left(2 \mathrm{E}_{1}^{\prime}\right)^{1 / 2}\left(2 \mathrm{E}_{2}^{\prime}\right)^{1 / 2}}
$$

where the states are normalized so

$$
\left\langle\mathbf{p}^{\prime} \mid \mathbf{p}\right\rangle=(2 \pi)^{3} \delta^{3}\left(\overrightarrow{\mathrm{p}}-\overrightarrow{\mathrm{p}}^{\prime}\right)
$$




\section{C.M. ENERGY AND MOMENTUM VS. BEAM MOMENTUM (for scattering on a proton target)}

$$
E_{c m}{ }^{d E_{c m}}=m_{p}{ }^{d T} T_{\text {beam }}=m_{p} v_{\text {beam }}{ }^{d P_{\text {beam }}} \approx m_{p} d P_{\text {beam }}
$$

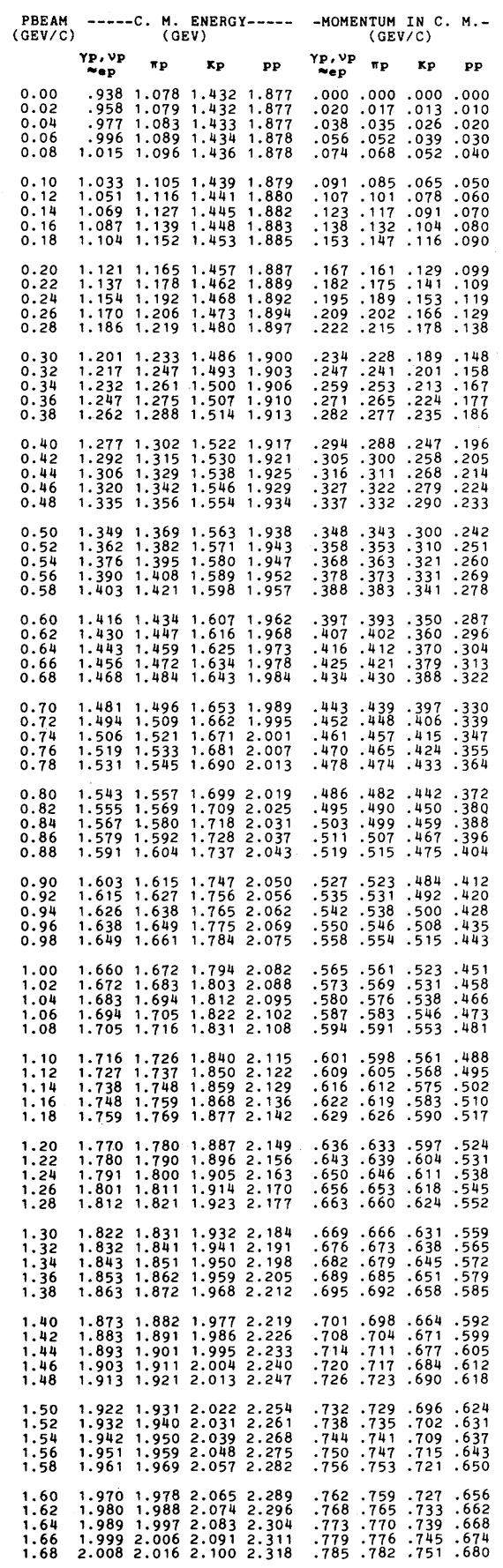

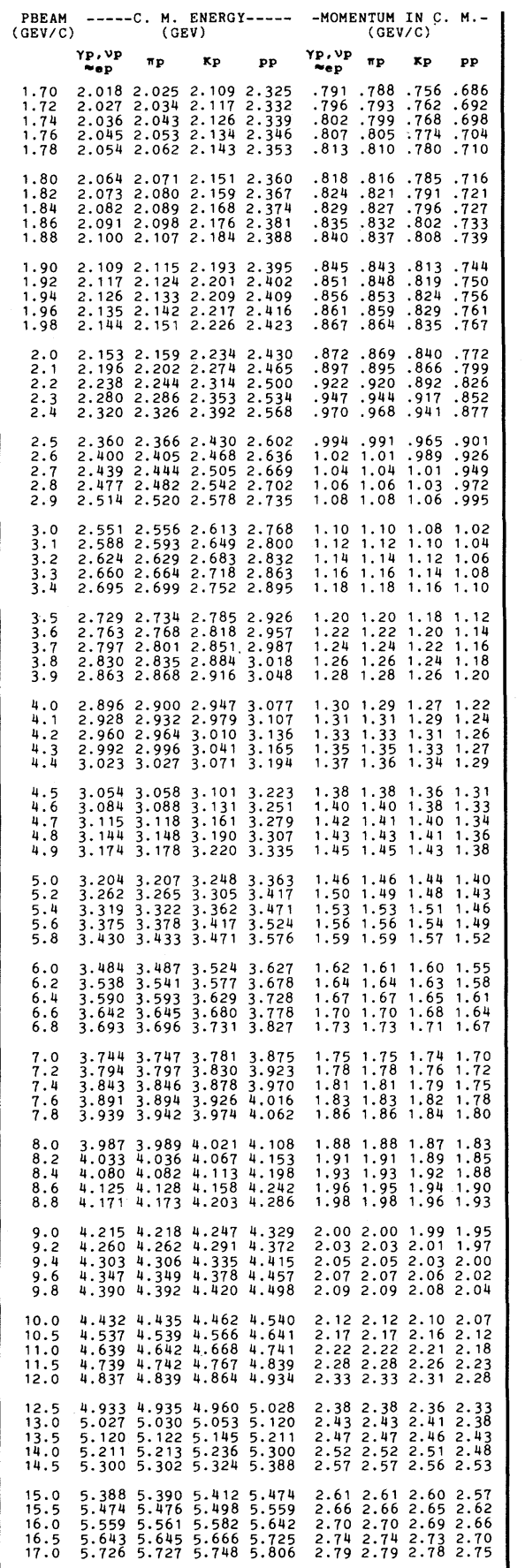

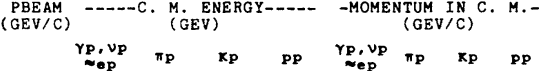

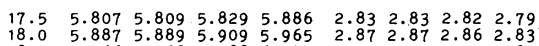

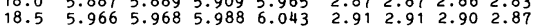

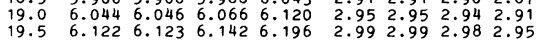

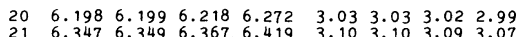

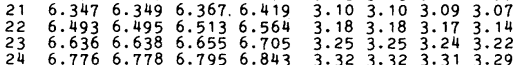

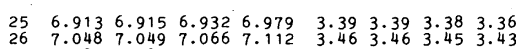

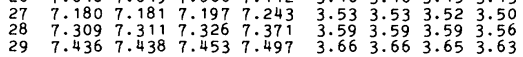

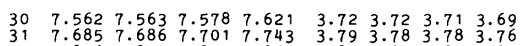

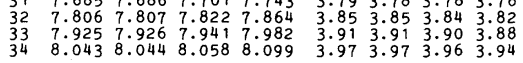

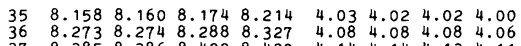

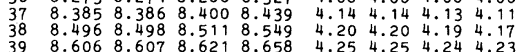
$\begin{array}{lllllllll}40 & 8.715 & 8.716 & 8.729 & 8.766 & 4.31 & 4.31 & 4.30 & 4.28 \\ 41 & 8.822 & 8.823 & 8.836 & 8.872 & 4.36 & 4 & 36 & 4 \\ 42 & 8.35 & 4.34\end{array}$ $428.927 \quad 8.928 \quad 8.9418 .978$ 4. 4144.414 .414 .39

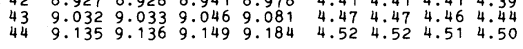

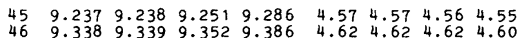

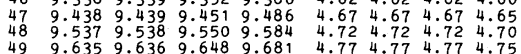
$\begin{array}{lllllllll}50 & 9.732 & 9.733 & 9.745 & 9.778 & 4.82 & 4.82 & 4.81 & 4.80 \\ 52 & 9.923 & 9.924 & 9.935 & 9.968 & 4.92 & 4.92 & 4.91 & 4.89\end{array}$

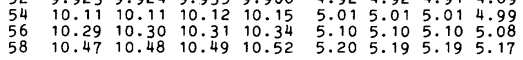
$\begin{array}{lllllllll}60 & 10.65 & 10.65 & 10.66 & 10.69 & 5.28 & 5.28 & 5.28 & 5.26\end{array}$ $\begin{array}{lllllllll}62 & 10.83 & 10.83 & 10.84 & 10.87 & 5.37 & 5.37 & 5.37 & 5.35 \\ 64 & 11.00 & 11.00 & 11.01 & 11.04 & 5.46 & 5.46 & 5.45 & 5.44 \\ 66 & 11.17 & 11.17 & 11.18 & 11.21 & 5.54 & 5.54 & 5.54 & 5.53 \\ 68 & 11.34 & 11.34 & 11.35 & 11.37 & 5.53 & 5.53 & 5.62 & 5.61\end{array}$

$\begin{array}{lllllllll}70 & 11.50 & 11.50 & 11.51 & 11.54 & 5.71 & 5.71 & 5.71 & 5.69 \\ 72 & 11.66 & 11.66 & 11.67 & 11.70 & 5.79 & 5.79 & 5.79 & 5.77\end{array}$

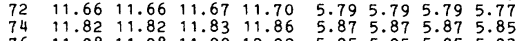

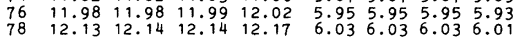
$\begin{array}{lllllllll}80 & 12.29 & 12.29 & 12.30 & 12.32 & 6.11 & 6.11 & 6.10 & 6.09 \\ 82 & 12.44 & 12.44 & 12.45 & 12.48 & 6.18 & 6.18 & 6.18 & 6.17 \\ 84 & 12.45 & 12.49 & 12.40 & 12.48 & 6 & 6\end{array}$

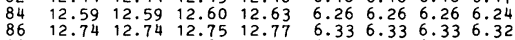

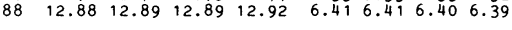

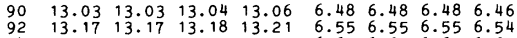

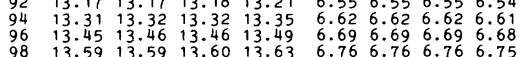

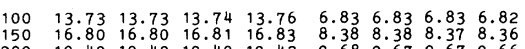

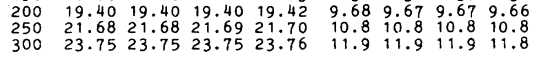

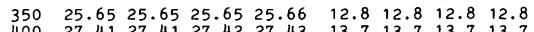

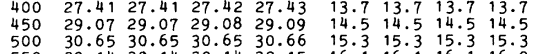
$550 \quad 32.1432 .1432 .1432 .15$ 16.1 16.816 .116 .0

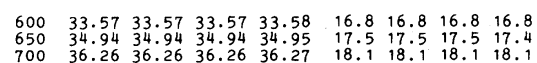

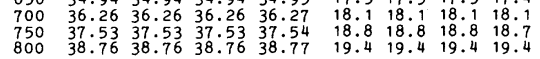
$\begin{array}{lllllllll}850 & 39.95 & 39.95 & 39.95 & 39.96 & 20.0 & 20.0 & 20.0 & 20.0 \\ 900 & 41.11 & 44.11 & 41.11 & 41.12 & 20.5 & 20.5 & 20.5 & 200.5 \\ 950 & 42.23 & 42.23 & 42.24 & 42.24 & 21.1 & 21.1 & 21.1 & 21.1\end{array}$

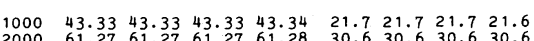

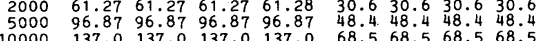

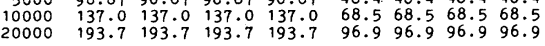
50000
100000

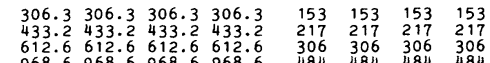




\section{STANDARD MODEL OF ELECTROWEAK INTERACTIONS}

The couplings of the photon, $\mathrm{W}^{ \pm}$, and $\mathrm{Z}$ to fundamental fermions are

$$
\begin{gathered}
\bar{\psi} \gamma^{\mu}\left[\mathrm{eQA}_{\mu}+\frac{\mathrm{e}}{\sqrt{2} \sin \theta_{\mathrm{W}}}\left(\mathrm{T}^{+} \mathrm{W}_{\mu}^{+}+\mathrm{T}^{-} \mathrm{W}_{\mu}^{-}\right)\right. \\
\left.+\frac{\mathrm{e}}{\sin \theta_{\mathrm{W}} \cos \theta_{\mathrm{W}}}\left(\mathrm{T}_{3}-\sin ^{2} \theta_{\mathrm{w}} \mathrm{Q}\right) \mathrm{Z}_{\mu}\right] \psi,
\end{gathered}
$$

where

$$
\psi \quad=\left(\begin{array}{c}
\mathrm{u} \\
\mathrm{d}^{\prime}
\end{array}\right),\left(\begin{array}{c}
\mathrm{c} \\
\mathrm{s}^{\prime}
\end{array}\right),\left(\begin{array}{c}
\mathrm{t} \\
\mathrm{b}^{\prime}
\end{array}\right),\left(\begin{array}{c}
\nu_{\mathrm{e}} \\
\mathrm{e}^{-}
\end{array}\right),\left(\begin{array}{c}
\nu_{\mu} \\
\mu^{-}
\end{array}\right),\left(\begin{array}{l}
\nu_{\tau} \\
\tau^{-}
\end{array}\right) ;
$$

for mixing effects defining $d^{\prime}, s^{\prime}$, and $b^{\prime}$ see the section on Cabibbo and Kobayashi-Maskawa Mixing;

$\mathrm{T}^{+} \quad-\frac{1}{2}\left(1-\gamma_{5}\right) \times$ weak isospin raising operator $\left(\mathrm{T}^{ \pm}\right.$act on left-handed fermions); $T_{3}=\frac{1}{2}\left(1-\gamma_{5}\right) \times$ third component of weak isospin, (i.e.,

$-1 / 2$ for $\mathrm{e}^{-}, \mu^{-}, \tau^{-}, \mathrm{d}, \mathrm{s}, \mathrm{b}$ );

$\mathrm{Q}$ - electric charge operator, in units of proton charge;

$\theta_{\mathrm{W}}=$ weak mixing angle;

A - electromagnetic vector potential.

Thus, for example, the We $\nu$ coupling is

$$
\left(\frac{\mathrm{e}}{\sqrt{2} \sin \theta_{\mathrm{W}}}\right)\left[\mathrm{w}_{\mu}^{-\overline{\mathrm{e}}} \gamma^{\mu} \frac{1}{2}\left(1-\gamma_{5}\right) \nu+\mathrm{w}_{\mu}^{+} \bar{\nu} \gamma^{\mu} \frac{1}{2}\left(1-\gamma_{5}\right) \mathrm{e}\right]
$$

and the $\mathrm{Zu} \bar{u}$ coupling is

$$
\left(\frac{\mathrm{e}}{\sin \theta_{\mathrm{W}} \cos \theta_{\mathrm{W}}}\right) \mathrm{z}_{\mu} \overline{\mathrm{u}} \gamma^{\mu}\left[\frac{1}{4}\left(1-\gamma_{5}\right)-\frac{2}{3} \sin ^{2} \theta_{\mathrm{W}}\right] \mathrm{u} \text {. }
$$

The physical neutral fields $A$ and $Z$ are mixtures of $W_{3}$, the partner of $\mathrm{W}^{ \pm}$, and another field $\mathrm{B}$ :

$$
A=W_{3} \sin \theta_{W}+B \cos \theta_{W}, Z=W_{3} \cos \theta_{W}-B \sin \theta_{W} .
$$

The $S U(2) \times U(1)$ gauge couplings $g$ and $g^{\prime}$ appear as

$$
\mathbf{g W}_{\mu} \cdot \mathbf{T}+\mathbf{g}^{\prime} \mathbf{B}_{\mu} \frac{\mathbf{Y}}{2},
$$

where electric charge $Q, T_{3}$, and $Y / 2$ are connected by $Q=T_{3}+Y / 2$. The couplings and mixing angle are related by $\tan \theta_{W}=g^{\prime} / g, \sin \theta_{W}=e / g$.

In lowest order

$$
\begin{aligned}
& \mathbf{M}_{\mathrm{W}}^{2}=\frac{\pi \alpha}{\sqrt{2} \sin ^{2} \theta_{\mathrm{W}} \mathrm{G}_{\mathrm{F}}} \simeq\left(\frac{37.3 \mathrm{GeV}}{\sin \theta_{\mathrm{W}}}\right)^{2}, \\
& \mathbf{M}_{\mathrm{Z}}^{2}=\mathbf{M}_{\mathrm{W}}^{2} / \cos ^{2} \theta_{\mathrm{W}} .
\end{aligned}
$$

See Appendix I of this Review (found only in complete version, not in booklet) for more details.

Branching fractions of the $\mathrm{W}^{ \pm}$and $\mathrm{Z}$ are predicted to be roughly

$$
\begin{array}{llll}
\mathrm{BR}\left(\mathrm{W}^{+} \rightarrow \mathrm{e}^{+} \nu_{\mathrm{e}}\right)=0.08, & & \mathrm{BR}\left(\mathrm{W}^{+} \rightarrow \mathrm{ud}\right)=0.24, \\
\operatorname{BR}\left(\mathrm{Z} \rightarrow \nu_{\mathrm{e}} \bar{\nu}_{\mathrm{e}}\right) & =0.06, & & \mathrm{BR}\left(\mathrm{Z} \rightarrow \mathrm{e}^{+} \mathrm{e}^{-}\right)=0.03, \\
\operatorname{BR}(\mathrm{Z} \rightarrow \mathrm{u} \overline{\mathrm{u}}) & =0.10, & & \mathrm{BR}(\mathrm{Z} \rightarrow \mathrm{d \overline {d }})=0.13, \text { etc. }
\end{array}
$$

and similarly for the other generations, assuming there is no suppression for phase space even for the $t$ quark. The total widths are expected to be (with $\sin ^{2} \theta_{\mathrm{W}} \simeq 0.21$ ): $\Gamma(\mathrm{W}) \simeq 2.8 \mathrm{GeV}$ and $\Gamma(\mathrm{Z}) \cong 2.8 \mathrm{GeV}$.

\section{CABIBBO AND KOBAYASHI-MASKAWA MIXING}

The quark mass eigenstates are not the weak eigenstates. The unitary matrix connecting them is known as the KobayashiMaskawa matrix. It generalizes to three generations the Cabibbo mixing which includes only the first two generations. The K-M matrix can be parametrized by three angles $\theta_{1}, \theta_{2}$, and $\theta_{3}$ and a phase $\mathrm{e}^{\mathrm{i} \delta}$, as described in Appendix II (found in the full Review of Particle Properties, not in the data booklet). Independent of such a parametrization we can write

$$
\left(\begin{array}{l}
d^{\prime} \\
s^{\prime} \\
b^{\prime}
\end{array}\right)=\left(\begin{array}{lll}
v_{u d} & v_{u s} & v_{u b} \\
v_{c d} & v_{c s} & v_{c b} \\
v_{t d} & v_{t s} & v_{t b}
\end{array}\right)\left(\begin{array}{l}
d \\
s \\
b
\end{array}\right)
$$

The primed quarks are the weak eigenstates, while the unprimed ones are the mass eigenstates. The analysis in Appendix II leads to an estimate of the $\mathrm{K}-\mathrm{M}$ matrix:

$$
\left(\begin{array}{lll|ll}
0.9705 & \text { to } 0.9770 & 0.21 \text { to } 0.24 & 0 . & \text { to } 0.014 \\
0.21 & \text { to } 0.24 & 0.971 \text { to } 0.973 & 0.036 \text { to } 0.070 \\
0 . & \text { to } 0.024 & 0.036 \text { to } 0.069 & 0.997 \text { to } 0.999
\end{array}\right) .
$$

\section{QUARK PARTON MODEL FOR DEEP INELASTIC SCATTERING}

In the naive parton model, the number of quarks, $q(x) d x$, of type $q$ carrying a fraction between $x$ and $x+d x$ of the proton's momentum (in a frame in which it is large) is independent of the $\mathrm{Q}^{2}$ of the scattering. (In more complete $\mathrm{QCD}$ models there is a logarithmic dependence on $Q^{2}$.) Thus deep inelastic leptoproduction probes $u(x), d(x), \bar{u}(x)$, etc. In particular, the structure functions for scattering from a proton (see section on Kinematics, Decays, and Scattering) are determined by these:

$$
\begin{aligned}
F_{2}^{\nu C C} & =2 x[d(x)+s(x)+\bar{u}(x)+\bar{c}(x)] \\
x F_{3}^{\nu C C} & =2 x[d(x)+s(x)-\bar{u}(x)-\bar{c}(x)] \\
F_{2}^{\bar{\nu} C C} & =2 x[u(x)+c(x)+\bar{d}(x)+\bar{s}(x)] \\
x_{3}^{\bar{\nu}} \bar{l} C C & =2 x[u(x)+c(x)-\bar{d}(x)-\bar{s}(x)] \\
F_{2}^{e m} & =x\left[\frac{4}{9}(u(x)+\bar{u}(x))+\frac{1}{9}(d(x)+\bar{d}(x))+\cdots\right] \\
F_{2}^{\nu N C} & =2 \rho^{2} x\left\{\left[\frac{1}{4}-\frac{2}{3} \sin ^{2} \theta_{W}+\frac{8}{9} \sin ^{4} \theta_{W}\right][u(x)+\bar{u}(x)]\right. \\
& \left.+\left[\frac{1}{4}-\frac{1}{3} \sin ^{2} \theta_{W}+\frac{2}{9} \sin ^{4} \theta_{W}\right][d(x)+\bar{d}(x)]\right\} \\
x_{3}^{\nu N C} & =2 \rho^{2} x\left\{\left[\frac{1}{4}-\frac{2}{3} \sin ^{2} \theta_{W}\right][u(x)-\bar{u}(x)]\right. \\
& \left.+\left[\frac{1}{4}-\frac{1}{3} \sin ^{2} \theta_{W}\right][d(x)-\bar{d}(x)]\right\} \\
F_{2}= & 2 x F_{1} \text { (in all cases. This is the Callan-Gross relation, } \\
\text { and ignores parton transverse momentum.) } & \\
F_{i}^{\nu N C} & =F_{i}^{\nu N C} .
\end{aligned}
$$

Here $\rho=M_{W}^{2} /\left(M_{Z}^{2} \cos ^{2} \theta_{W}\right)$. See section on the Standard Model of Electroweak Interactions and Appendix I of this Review (found only in complete version, not in booklet). 


\section{NONRELATIVISTIC QUARK MODEL}

\section{A. OUANTUM NUMBERS}

Each quark has spin $1 / 2$. The additive quantum numbers (other than baryon number $=1 / 3$ ) of the known (and presumed) quarks are shown in the table.

Quark type (flavor)

\begin{tabular}{|c|c|c|c|c|c|c|}
\hline Quantum number & d & $\mathbf{u}$ & s & c & $\bar{b}$ & $t$ \\
\hline - electric charge & $-\frac{1}{3}$ & $+\frac{2}{3}$ & $-\frac{1}{3}$ & $+\frac{2}{3}$ & $-\frac{1}{3}$ & $+\frac{2}{3}$ \\
\hline $\mathscr{I}_{z}-\mathrm{z}$-component of isospin & $-\frac{1}{2}$ & $+\frac{1}{2}$ & 0 & 0 & 0 & 0 \\
\hline $\mathscr{S}$ - strangeness & 0 & 0 & -1 & 0 & 0 & 0 \\
\hline $\mathscr{B}$ - charm & 0 & 0 & 0 & +1 & 0 & 0 \\
\hline $\mathscr{B}$ - bottomness & 0 & 0 & 0 & 0 & -1 & 0 \\
\hline $\mathscr{T}$ - topness & 0 & 0 & 0 & 0 & 0 & +1 \\
\hline
\end{tabular}

With these conventions the strangeness $\mathscr{S}_{\text {of }}$ the $\mathrm{K}^{+}$is +1 and the bottomness $\mathscr{B}$ of the $\mathrm{B}^{+}$is +1 .

The G-parity operator is defined to be $\mathrm{G}=\mathrm{Ce}^{-\mathrm{i} \pi \mathrm{I}_{\mathrm{y}}}$, where $\mathrm{C}$ is the charge conjugation operator. The mesons with $\mathscr{S}=\mathscr{B}=\mathscr{B}=\mathscr{T}$ $=0$ are eigenstates of $G$. If a meson is also an eigenstate of the charge conjugation operator with charge conjugation $C$, then $\mathbf{G}=$ $C(-1)^{I}$, where $I$ is its isospin; all the other particles in the same isomultiplet have the same value of $G: G\left(\pi^{ \pm}\right)=G\left(\pi^{0}\right)=-1$, $G\left(\rho^{ \pm}\right)=G\left(\rho^{9}\right)=+1$, etc.

\section{B. MESONS}

Nearly all known mesons can be understood as bound states of a quark $q$ and an antiquark $\bar{q}^{\prime}$ (the flavors of $q$ and $q^{\prime}$ may be different). If the orbital angular momentum of the $q \bar{q}^{\prime}$ state is $L$, then the parity $P=(-1)^{L+1}$. A state $q \bar{q}$ of a quark and its own antiquark is also an eigenstate of charge conjugation with $\mathrm{C}=$ $(-1)^{L+S}$, where the spin $S=0$ or 1 . The $L=0$ states are the pseudoscalars, $\mathrm{J}^{\mathrm{P}}=0^{-}$, and the vectors, $\mathrm{J}^{\mathrm{P}}=\mathrm{1}^{-}$. See table below.
States in the "normal" spin-parity series, $P=(-1)^{\mathrm{J}}$, must, according to the above, have $S=1$ and hence $C P=+1$. Thus mesons with normal spin-parity and $\mathrm{CP}=-1$ are forbidden in the $\mathrm{q} \overline{\mathrm{q}}^{\prime}$ quark model. The $\mathrm{J}^{\mathrm{PC}}=0^{--}$state is forbidden as well. Mesons with such $\mathrm{J}^{\mathrm{PC}}$ could exist, but would lie outside the $q \overline{\mathrm{q}}^{\prime}$ model.

States with the same $J^{P}$ and additive quantum numbers can mix (if they are eigenstates of charge conjugation, they must also have the same value of $\mathrm{C}$ ). Thus the physical $\mathrm{J}^{\mathrm{P}}=1^{+}$, strangeness $\mathscr{S}=$ 1 states, $Q(1280)$ and $Q(1400)$, are mixtures of $Q_{A}$ and $Q_{B}$. The $\psi(3770)$ is a mixture of ${ }^{3} S_{1}$ and ${ }^{3} D_{1}$. The $\eta$ and $\eta^{\prime}$ are mixtures of the SU(3) octet and singlet states.

For the pseudoscalar mesons, the Gell-Mann-Okubo formula is

$$
\mathrm{m}_{\eta}^{2}=\frac{1}{3}\left(4 \mathrm{~m}_{\mathrm{K}}^{2}-\mathrm{m}_{\pi}^{2}\right),
$$

assuming no octet-singlet mixing. However, the octet $\eta_{8}$ and singlet $\eta_{1}$ mix because of SU(3) breaking. The physical states $\eta$ and $\eta^{\prime}$ are given by

$$
\begin{gathered}
\eta=\eta_{8} \cos \theta_{\mathrm{P}}-\eta_{1} \sin \theta_{\mathrm{P}} \\
\eta^{\prime}=\eta_{8} \sin \theta_{\mathrm{P}}+\eta_{1} \cos \theta_{\mathrm{P}} .
\end{gathered}
$$

These combinations diagonalize the mass-squared matrix

$$
\mathbf{M}^{2}=\left(\begin{array}{ll}
\mathbf{M}_{11}^{2} & \mathbf{M}_{18}^{2} \\
\mathbf{M}_{18}^{2} & \mathbf{M}_{88}^{2}
\end{array}\right),
$$

where $M_{88}^{2}=\frac{1}{3}\left(4 m_{K}^{2}-m_{\pi}^{2}\right)$. It follows that

$$
\tan ^{2} \theta_{\mathrm{P}}=\frac{M_{88}^{2}-m_{\eta}^{2}}{m_{\eta^{\prime}}^{2}-M_{88}^{2}}
$$

The sign of $\theta_{\mathrm{P}}$ is meaningful in the quark model. If

\begin{tabular}{|c|c|c|c|c|c|c|c|c|c|}
\hline${ }^{2 S+1} L_{J}$ & $\mathrm{~J}^{\mathrm{PC}}$ & $\begin{array}{c}\mathrm{u} \overline{\mathrm{u}}, \mathrm{d} \overline{\mathrm{d}}, \overline{\mathrm{ss}} \\
\mathrm{I}=0\end{array}$ & $\begin{array}{l}\mathrm{u} \overline{\mathrm{d}}, \mathrm{u} \overline{\mathrm{u}}, \mathrm{d} \overline{\mathrm{d}} \\
\quad \mathrm{I}=\mathbf{1}\end{array}$ & $\begin{array}{c}\overline{s u}, \overline{s d} \\
I=1 / 2\end{array}$ & $\begin{array}{l}c \bar{u}, c \bar{d} \\
I=1 / 2\end{array}$ & $\begin{array}{c}\overline{\mathbf{C S}} \\
I=0\end{array}$ & $\begin{array}{c}\overline{c \bar{c}} \\
I=0\end{array}$ & $\begin{array}{l}\overline{\mathrm{b} u}, \overline{\mathrm{b} d} \\
\mathrm{I}=1 / 2\end{array}$ & $\begin{array}{c}\mathrm{b} \overline{\mathrm{b}} \\
\mathrm{I}=0\end{array}$ \\
\hline${ }^{1} \mathbf{S}_{0}$ & $0^{-+}$ & $\eta, \eta^{\prime}$ & $\pi$ & $\mathbf{K}$ & D & $\mathbf{F}$ & $\eta_{c}$ & B & \\
\hline${ }^{3} S_{1}$ & $1^{--}$ & $\phi, \omega$ & $\rho$ & $K^{*}(892)$ & $D^{*}(2010)$ & & $\mathrm{J} / \psi$ & & $r$ \\
\hline${ }^{1} \mathbf{P}_{1}$ & $1^{+-}$ & $\mathbf{H}$ & $\mathrm{B}(1235)$ & $\mathrm{Q}_{\mathbf{B}}$ & & & & & \\
\hline${ }^{3} \mathrm{P}_{0}$ & $0^{++}$ & $S(975), \epsilon$ & $\delta$ & $\kappa$ & & & $x(3415)$ & & $\chi_{b}(9875)$ \\
\hline${ }^{3} \mathbf{P}_{1}$ & $1^{++}$ & $D(1285), E$ & $A(1270)$ & $\mathrm{Q}_{\mathbf{A}}$ & & & $x(3510)$ & & $\chi_{b}(9895)$ \\
\hline${ }^{3} \mathrm{P}_{2}$ & $2^{++}$ & $f^{\prime}, f$ & $A_{2}$ & $K^{*}(1430)$ & & & $x(3555)$ & & $x_{b}(9915)$ \\
\hline${ }^{1} \mathrm{D}_{2}$ & $2^{-+}$ & & $A(1680)$ & & & & & & \\
\hline${ }^{3} \mathrm{D}_{1}$ & $1^{--}$ & & & & & & $\psi(3770)$ & & \\
\hline${ }^{3} \mathrm{D}_{2}$ & $2^{--}$ & & & L(1770) & & & & & \\
\hline${ }^{3} D_{3}$ & $3^{--}$ & $\omega(1670)$ & g & $K^{*}(1780)$ & & & & & \\
\hline
\end{tabular}

$$
\begin{aligned}
& \eta_{1}=(u \bar{u}+d \bar{d}+s \bar{s}) / \sqrt{3} \\
& \eta_{8}=(u \bar{u}+d \bar{d}-2 s \bar{s}) / \sqrt{6},
\end{aligned}
$$

then the matrix element $M_{18}^{2}$, which is due mostly to the strange

Standard quark model assignments for some of the known mesons. Some assignments, especially for $0^{++}$, are controversial. Note that only the states in the $u \bar{u}, d \bar{d}, \overline{s s}, c \bar{c}$, and $b \bar{b}$ columns and the neutral states in the $I=1$ column are eigenstates of charge conjugation $C$. 


\section{NONRELATIVISTIC QUARK MODEL (Cont'd)}

quark mass, is negative. From the relation

$$
\tan \theta_{\mathbf{P}}=\frac{\mathbf{M}_{88}^{2}-\mathbf{m}_{\eta}^{2}}{\mathbf{M}_{18}^{2}},
$$

we find $\theta_{\mathrm{p}}<0$.

For the vector mesons we replace $\pi \rightarrow \rho, \mathrm{K} \rightarrow \mathrm{K}^{*}, \eta \rightarrow \phi$, and $\eta^{\prime} \rightarrow \omega$, so

$$
\begin{aligned}
& \phi=\omega_{8} \cos \theta_{\mathrm{V}}-\omega_{1} \sin \theta_{\mathrm{V}} \\
& \omega=\omega_{8} \sin \theta_{\mathrm{V}}+\omega_{1} \cos \theta_{\mathrm{V}} .
\end{aligned}
$$

For "ideal mixing," $\phi=s \bar{s}, \tan \theta_{\mathrm{V}}=1 / \sqrt{2}$, so $\theta_{\mathrm{V}} \approx 35.3^{\circ}$. Experimentally, $\theta_{\mathrm{V}}$ is near $35^{\circ}$, the sign being determined by a formula analogous to that for $\tan \theta_{\mathrm{p}}$. Following this procedure we find the mixing angles below. There are uncertainties of a few degrees arising from electromagnetic mass splittings and uncertainties in resonance masses.

Singlet-octet mixing for the pseudoscalar, vector, and tensor mesons. The sign conventions are as above. The value of $\theta_{\text {quad }}$ is obtained from the equations above, and $\theta_{\text {lin }}$ is obtained by replacing $\mathrm{m}^{2} \rightarrow \mathrm{m}$ throughout. Of the two isosinglets, the mostly octet one is listed first.

\begin{tabular}{llrr}
\hline $\mathrm{J}^{\mathrm{PC}}$ & Nonet Members & $\theta_{\text {quad }}$ & $\theta_{\text {lin }}$ \\
\hline $0^{-+}$ & $\pi, \mathrm{K}, \eta, \eta^{\prime}$ & $-10^{\circ}$ & $-23^{\circ}$ \\
$1^{--}$ & $\rho, \mathrm{K}^{*}(892), \phi, \omega$ & $39^{\circ}$ & $36^{\circ}$ \\
$2^{++}$ & $\mathrm{A}_{2}, \mathrm{~K}^{*}(1430), \mathrm{f}^{\prime}, \mathrm{f}$ & $28^{\circ}$ & $26^{\circ}$ \\
$3^{--}$ & $\mathrm{g}(1690), \mathrm{K}^{*}(1780), \phi(1850), \omega(1670)$ & $29^{\circ}$ & $28^{\circ}$ \\
\hline
\end{tabular}

\section{BARYONS}

All the established baryons are apparently 3-quark (qqq) states, and each such state is an SU(3) color singlet, a completely antisymmetric state of the three possible colors. Since the quarks are fermions, the state function for any baryon must be antisymmetric under interchange of any two of its quarks. Thus the state is symmetric under interchange of the quantum labels other than color:

$$
\left.|\mathbf{q q q}\rangle_{\mathbf{A}}=\mid \text { color }_{\mathbf{A}} \times \mid \text { space, spin, flavor }\right\rangle_{\mathbf{S}} \text {, }
$$

where the subscripts $\mathbf{S}$ and $\mathbf{A}$ indicate symmetry or antisymmetry under interchange of any two of the quarks. Note the contrast with the state function for the three nucleons in ${ }^{3} \mathrm{H}$ or ${ }^{3} \mathrm{He}$ :

$$
\left.|\mathbf{N N N}\rangle_{\mathbf{A}}=\mid \text { space, } \text { spin, isospin }\right\rangle_{\mathbf{A}} \text {. }
$$
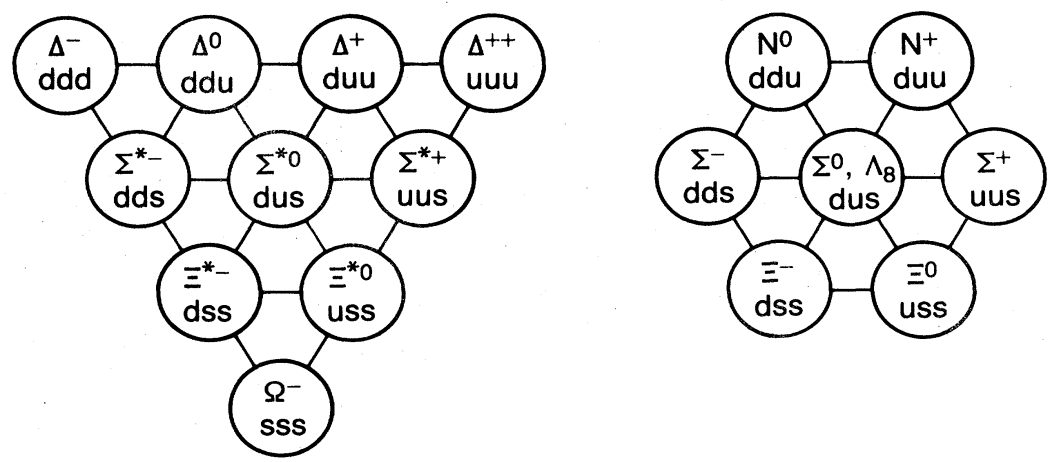

This difference has major implications for internal structure, magnetic moments, etc. (For a nice discussion, see Ref. 1.)

Few of the baryons containing $\mathrm{c}$ or heavier quarks have yet been discovered, so we restrict further attention to baryons made up of just $\mathrm{d}$, $\mathrm{u}$, and $\mathrm{s}$ quarks. The three flavors imply a flavor SU(3), which requires that baryons made of these quarks belong to the multiplets on the right side of

$$
3 \otimes 3 \otimes 3=10_{S} \oplus 8_{M} \oplus 8_{M} \oplus 1_{A}
$$

(see the section on SU(n) Multiplets and Young Diagrams). Here the subscripts indicate symmetric, mixed-symmetric, or antisymmetric states under interchange of any two quarks. The figure shows particle assignments in these multiplets. States $\Lambda_{8}$ and $\Lambda_{1}$ that have the same spin and parity can mix; an example is the mainly octet $D_{03} \Lambda(1690)$ and mainly singlet $D_{03} \Lambda(1520)$. The formalism is the same as for $\eta-\eta^{\prime}$ or $\phi-\omega$ mixing (see above), except that for baryons the mass $M$ instead of $M^{2}$ is used. The section SU(3) Isoscalar Factors shows how relative decay rates in, say, $10 \rightarrow 8 \otimes 8$ decays may be calculated. A summary of results of fits to the observed baryon masses and decay rates for the best-known SU(3) multiplets is given in Appendix II of our 1982 edition. ${ }^{2}$

Flavor and spin may be combined in a flavor-spin SU(6) in which the six basic states are $d \uparrow, d \downarrow, \cdots, s \downarrow(\uparrow, \downarrow=$ spin up, down). Then the baryons belong to the multiplets on the right side of

$$
6 \otimes 6 \otimes 6=56_{S} \oplus 70_{M} \oplus 70_{M} \oplus 20_{A} \text {. }
$$

These SU(6) multiplets decompose into flavor SU(3) multiplets as follows:

$$
\begin{aligned}
& 56={ }^{4} 10 \oplus{ }^{2} 8 \\
& 70={ }^{2} 10 \oplus{ }^{4} 8 \oplus{ }^{2} 8 \oplus^{2} 1 \\
& 20={ }^{2} 8 \oplus{ }^{4} 1,
\end{aligned}
$$

where the superscript $(2 S+1)$ gives the net spin $S$ of the quarks for each particle in the SU(3) multiplet. The $\mathrm{J}^{\mathrm{P}}=1 / 2^{+}$octet containing the nucleon and the $\mathrm{J}^{\mathrm{P}}=3 / 2^{+}$decuplet containing the $\Delta(1232)$ together make up the "ground-state" 56-plet in which the orbital angular momenta between the quarks are zero (so that the spatial part of the state function is trivially symmetric). The $\mathbf{7 0}$ and 20 require some excitation of the spatial part of the state function in order to make the overall state function symmetric.

The quark model for baryons is extensively reviewed in Ref. 3.

1. F.E. Close, in Quarks and Nuclear Forces (Springer-Verlag, 1982), p. 56.

2. Particle Data Group, Phys. Lett. 111B (1982).

3. A.J.G. Hey and R.L. Kelly, Phys. Reports 96, 71 (1983). 


\section{PROBABILITY AND STATISTICS}

\section{A. PROBABIUTY DISTRIBUTIONS AND CONFIDENCE LEVELS}

We give here properties of the three probability distributions most commonly used in high energy physics: normal (or Gaussian), chi-squared $\left(\chi^{2}\right)$, and Poisson. We warn the reader that there is no universal convention for the term "confidence level"; thus, explicit definitions that correspond to common usage are given for each distribution. It is explained below how confidence levels for all three distributions may be extracted from the following figure.

CONFIDENCE LEVEL VS. $x^{2}$ FOR $n_{D}$ DEGREES OF FREEDOM

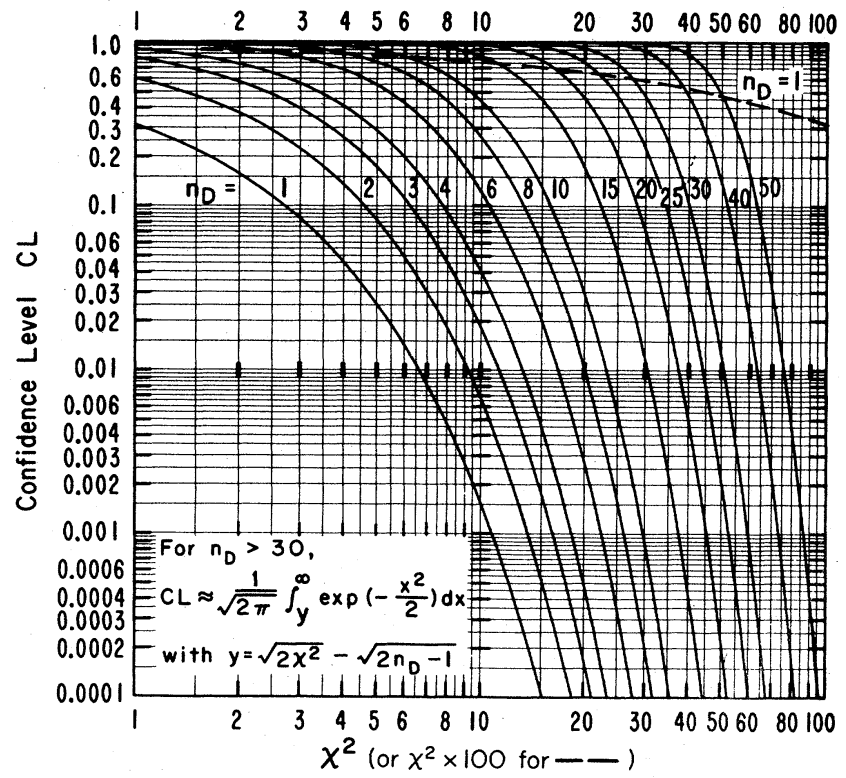

\section{A.1 Normal distribution}

The normal distribution with mean $\bar{x}$ and standard deviation $\sigma$ (variance $\sigma^{2}$ ) is:

$$
P(x) d x=\frac{1}{\sigma \sqrt{2 \pi}} e^{-(x-\bar{x})^{2} / 2 \sigma^{2}} d x .
$$

The confidence level associated with an observed deviation $\delta$ from the mean is the probability that $|x-\bar{x}|>\delta$, i.e.,

$$
C L=2 \int_{\bar{x}+\delta}^{\infty} d x P(x),
$$

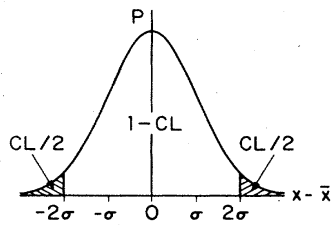

since the distribution is symmetric about $\overline{\mathrm{x}}$. The small figure in Eq (2) is drawn with $\delta-2 \sigma$. CL is given by the ordinate of the $n_{D}=1$ curve in the large figure at $\chi^{2}=(\delta / \sigma)^{2}$. The confidence level for $\delta=1 \sigma$ is $31.7 \% ; 2 \sigma, 4.6 \% ; 3 \sigma, 0.3 \%$. The odds against exceeding $\delta,(1-C L) / C L$, for $\delta=1 \sigma$ are $2.15: 1 ; 2 \sigma, 21: 1 ; 3 \sigma, 370: 1$; $4 \sigma, 16,000: 1 ; 5 \sigma, 1,700,000: 1$. Relations between $\sigma$ and other measures of the width: probable error $(C L=0.5)=0.67 \sigma$; mean absolute deviation $=0.80 \sigma$; RMS deviation $=\sigma$; half width at half $\max -$ imum $=1.18 \sigma$.
A.2 $\boldsymbol{\chi}^{2}$ distribution

The $\chi^{2}$ distribution for $\mathrm{n}_{\mathrm{D}}$ degrees of freedom is:

$$
P_{n_{D}}\left(\chi^{2}\right) d \chi^{2}=\frac{1}{2^{h} \Gamma(h)}\left(\chi^{2}\right)^{h-1} e^{-\chi^{2} / 2} d \chi^{2} \quad\left(\chi^{2} \geq 0\right),
$$

where $h$ (for "half") $=n_{D} / 2$. The mean and variance are $n_{D}$ and $2 \mathrm{n}_{\mathrm{D}}$ respectively. In evaluating Eq. (3) one may use Stirling's approximation: $\Gamma(\mathrm{h}) \simeq 2.507 \mathrm{e}^{-\mathrm{h}} \mathrm{h}^{(\mathrm{h}-1 / 2)}(1+0.0833 / \mathrm{h})$, which is accurate to $\pm 0.1 \%$ for all $h \geq 1 / 2$. The confidence level associated with a given value of $n_{D}$ and an observed value of $\chi_{0}^{2}$ is the probability of the $\chi^{2}$ exceeding the observed value, i.e.,

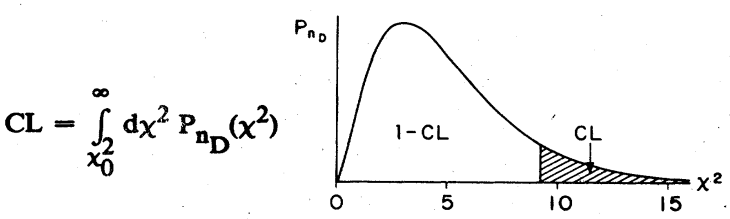

The small figure in Eq. (4) is drawn with $n_{D}=5$ and $C L=10 \%$. $C L$ is plotted as a function of $\chi^{2}$ for several values of $n_{D}$ in the large figure. For large $n_{D}, \chi^{2}$ becomes normally distributed about $\mathbf{n}_{\mathrm{D}}$. Thus,

$$
y_{1}=\left(\chi^{2}-n_{D}\right) / \sqrt{2 n_{D}}
$$

becomes normally distributed with unit standard deviation and mean zero. A better approximation is that $\chi$, not $\chi^{2}$, becomes normally distributed; specifically

$$
y_{2}=\sqrt{2 x^{2}}-\sqrt{2 n_{D}-1}
$$

approaches normality with unit standard deviation and mean zero. For small CL's in particular, $y_{2}$ is much more accurate than $y_{1}$. Thus, for $\mathrm{n}_{D}=50$ and $\chi^{2}-80$, the true $C L=0.45 \%$, but $y_{1}$ is 3.0 corresponding to a CL of $0.13 \%$, while $y_{2}$ is 2.7 corresponding to a CL of $0.35 \%$

\section{A.3 Poisson distribution}

The Poisson distribution with mean $\overline{\mathbf{n}}$ is:

$$
\mathbf{P}_{\overline{\mathbf{n}}}(\mathbf{n})=\frac{\mathrm{e}^{-\overline{\mathbf{n}}} \overline{\mathbf{n}^{\mathbf{n}}}}{\mathbf{n} !}(\mathbf{n}=0,1,2, \cdots) \text {. }
$$

The variance is equal to the mean. Confidence levels for Poisson distributions are usually defined in terms of quantities called "upper limits" as follows: The confidence level associated with a given upper limit $\mathbf{N}$ and an observed value $n_{0}$ of $n$ is the probability that $n>n_{0}$ if $\bar{n}=N$, i.e.,

$$
\begin{aligned}
C L & =\sum_{n=n_{0}+1}^{\infty} P_{N}(n) \\
& =1-\sum_{n=0}^{n_{0}} P_{N}(n)
\end{aligned}
$$

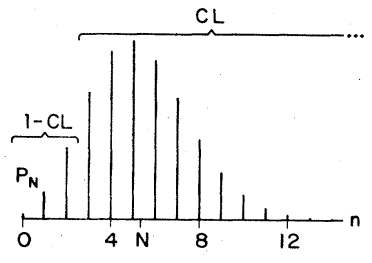

The small figure in Eq. (8) is drawn with $\mathrm{n}_{0}=2$ and $\mathrm{CL}=90 \%$. $A$ useful relation between Poisson and $\chi^{2}$ confidence levels allows one to look up this quantity on the large figure. Specifically, the quantity $1-C L$ is given by the ordinate of the $n_{D}=2\left(n_{0}+1\right)$ curve at $\chi^{2}=2 N$. Thus, $90 \%$ confidence level upper limits for $n_{0}=0,1$, and 2 are given by half the $\chi^{2}$ value corresponding to an ordinate of 0.1 on the $n_{D}=2,4$, and 6 curves, respectively; the values are $N$ $-2.3,3.9$, and 5.3 .

Tables of confidence levels for all three of these distributions, the relation between Poisson and $\chi^{2}$ confidence levels, and numerous other useful tables and relations may be found in Ref. 1 . 


\section{PROBABILITY AND STATISTICS (Cont'd)}

\section{B. STATISTICS}

Suppose one is presented with $N$ independent data, $y_{n} \pm \sigma_{n}$, and it is desired to make some inference about the "true" value of the quantity represented by these data. For this purpose we interpret each datum $y_{n}$ as a single sample point drawn randomly (and independently of the other data) from a distribution having true mean $\bar{y}_{n}$ (which we wish to estimate) and variance $\sigma_{n}^{2}$. We do not require that they be normally distributed. (Identification of the true $\sigma_{n}$ with the $\sigma_{n}$ datum is often an approximation which may become seriously inaccurate when $\sigma_{n}$ is an appreciable fraction of $y_{n}$.) Some methods of estimation commonly used in high energy physics are given below; see Ref. 2 for numerous applications. Section B.1 deals with the case in which all $\bar{y}_{n}$ are the same, e.g., several different measurements of the same quantity; Sec. B.2 deals with the case in which $\bar{y}_{n}=\bar{y}\left(x_{n}\right)$, where $x_{n}$ represents some set of independent variables, e.g., cross-section measurements at various values of energy and angle, $x_{n}=\left\{E_{n}, \theta_{n}\right\}$.

B.1 Single mean and variance estimates

(1) If the $y_{n}$ represent a set of values all supposedly drawn from a single distribution with mean $\bar{y}$ and variance $\sigma^{2}$ (i.e., the $\sigma_{n}$ are all the same, but their common value is unknown), then

$$
\begin{aligned}
& \hat{y}=\frac{1}{N} \sum_{n=1}^{N} y_{n} \text { and } \\
& \hat{\sigma}^{2}=\frac{1}{N-1} \sum_{n=1}^{N}\left(y_{n}-\hat{y}\right)^{2}=\frac{N}{N-1}\left[\left\langle\left(y^{2}\right)\right\rangle-(\hat{y})^{2}\right]
\end{aligned}
$$

are unbiased estimates of $\bar{y}$ and $\sigma^{2}$; the angular brackets denote an average over the data. The variance of $\hat{y}$ is $\sigma^{2} / N$. If the parent distribution is normal and $N$ is large, the variance of $\hat{\sigma}^{2}$ is $2 \sigma^{4} / N$.

(2) If the $y_{n}$ are independent estimates of the same $\bar{y}$, and the $\sigma_{n}$ are known, then the weighted average

$$
\hat{y}=\frac{1}{w} \sum_{n} w_{n} y_{n}
$$

where $w_{n}=1 / \sigma_{n}^{2}$ and $w-\Sigma w_{n}$, is an appropriate unbiased estimate of $\frac{\mathrm{n}}{\mathrm{y}}$. This choice of weighting factors in Eq. (11) minimizes the variance of the estimate; the variance is $1 / w$.

\section{B.2 Linear least-squares fit}

We wish to determine the best fit of independent unbiased data $y_{n} \pm \sigma_{n}$, measured at points $x_{n}$, to the form $y(x)=\Sigma a_{i} f_{i}(x)$, where the $f_{i}$ are known, linearly independent functions (e.g., Legendre polynomials) one-to-one over the allowed range of $x$. The estimates for the linear coefficients $a_{i}$ which minimize the sum of the squared deviations are

$$
\hat{a}_{i}=\sum_{j, n} v_{i j} f_{j}\left(x_{n}\right) y_{n} / \sigma_{n}^{2} .
$$

Here $\mathrm{V}$ is the covariance matrix of the fitted parameters

$$
v_{i j}=\left(\overline{\left.a_{i}-\bar{a}_{i}\right)\left(\hat{a}_{j}-\bar{a}_{j}\right)},\right.
$$

where the overbar denotes the unknown true value; $\mathrm{V}$ is estimated by

$$
\left(V^{-1}\right)_{i j}=\sum_{n} f_{i}\left(x_{n}\right) f_{j}\left(x_{n}\right) / \sigma_{n}^{2}
$$

The estimated variance of an interpolated or extrapolated value of $y$ at point $x, \hat{y}=\Sigma \hat{a}_{i} f_{i}(x)$, is:

$$
\left.(\hat{y}-\bar{y})^{2}\right|_{e s t}=\sum_{i j} v_{i j} f_{i}(x) f_{j}(x) .
$$

For the case of a straight line fit, $\mathrm{y}(\mathrm{x})=\mathrm{a}+\mathrm{bx}$, one obtains the following estimates of $a$ and $b$,

$$
\begin{aligned}
& \hat{a}=\left(S_{y} S_{x x}-S_{x} S_{x y}\right) / D, \\
& \hat{b}=\left(S_{1} S_{x y}-S_{x} S_{y}\right) / D,
\end{aligned}
$$

where

$$
S_{1}, S_{x}, S_{y}, S_{x x}, S_{x y}=\Sigma\left(1, x_{n}, y_{n}, x_{n}^{2}, x_{n} y_{n}\right) / \sigma_{n}^{2},
$$

respectively, and

$$
D=S_{1} S_{x x}-S_{x}^{2} .
$$

The covariance matrix of the fitted parameters is:

$$
\left(\begin{array}{ll}
\mathrm{v}_{\mathrm{aa}} & \mathrm{v}_{\mathrm{ab}} \\
\mathrm{V}_{\mathrm{ab}} & \mathbf{v}_{\mathrm{bb}}
\end{array}\right)=\frac{1}{\mathrm{D}}\left(\begin{array}{cc}
\mathrm{S}_{\mathrm{xx}} & -\mathrm{S}_{\mathrm{x}} \\
-\mathrm{S}_{\mathrm{x}} & \mathrm{S}_{1}
\end{array}\right) \text {. }
$$

The estimated variance of an interpolated or extrapolated value of $y$ at point $x$ is:

$$
(\hat{y}-\bar{y})^{2} l_{\text {est }}=\frac{1}{s_{1}}+\frac{s_{1}}{D}\left(x-\frac{s_{x}}{s_{1}}\right)^{2} .
$$

A least-squares fit gives estimates for the $a_{i}$ [Eq.(12)] with the smallest variance, under the conditions that the expansion of $y$ in terms of $\mathrm{a}_{\mathrm{i}} \mathrm{f}_{\mathrm{i}}$ is the correct model and that the $\mathrm{y}_{\mathrm{n}}$ are independent, unbiased measurements whose variances $\sigma_{n}^{2}$ are known.

\section{ERROR PROPAGATION}

Suppose one wishes to calculate the value and error of a function of some other quantities with errors, e.g., in a Monte Carlo program. Let $\{y\}$ be a set of random variables with means $\{y\}$ and covariance matrix $\mathrm{V}$. Then the mean and variance of a function of these variables are approximately (to second order in $\{y-\bar{y}\}$ ):

$$
\begin{aligned}
& \bar{f} \simeq f(\{y\})+\frac{1}{2} \sum_{m n} v_{m n}\left(\frac{\partial^{2} f}{\partial y_{m} \partial y_{n}}\right)_{\{y\}-\{y\}} \\
& \overline{(f-\bar{f})^{2}} \simeq \sum_{m n} v_{m n}\left(\frac{\partial f}{\partial y_{m}}\right)_{\{y\}-\{y\}}\left(\frac{\partial f}{\partial y_{n}}\right)_{\{y\}-\{y\}} .
\end{aligned}
$$

E.g., the mean and variance of a function of a single variable with mean $\bar{y}$ and variance $\sigma^{2}$ are

$$
\begin{aligned}
& \bar{f} \simeq f(\bar{y})+\frac{1}{2} \sigma^{2} f^{\prime \prime}(y), \\
& \overline{(f-\bar{f})^{2}} \simeq \sigma^{2} f^{\prime}(\bar{y})^{2} .
\end{aligned}
$$

Note that these equations will usually be applied by substituting measured quantities, $\{$ y $\}$ say, for the true means, $\{$ y $\}$. If, as is often the case, $\tilde{y}_{n}-\bar{y}_{n}$ is of order $\sqrt{V_{n n}}$, then the second-order terms in Eqs. (20) and (22) may be small compared with the first-order errors introduced by the substitution.

1. M. Abramowitz and I. Stegun, eds., Handbook of Mathematical Functions (Dover, New York, 1972).

2. W.T. Eadie, D. Drijard, F.E. James, M. Roos, and B. Sadoulet, Statistical Methods in Experimental Physics (North Holland, Amsterdam and London, 1971); S.L. Meyer, Data Analysis for Scientists and Engineers (John Wiley and Sons, Inc., New York, 1975); A.G. Frodesen, O. Skjeggestad, and H. Tфfte, Probability and Statistics in Particle Physics (Universitetsforlaget, Oslo, Norway, 1979). 


\section{PARTICle DeTECTORS, ABSORBERS, AND RANGES*}

\section{A. DETECTOR PARAMETERS}

In this section we give various parameters for common detectors. The quoted numbers are usually based on some typical apparatus, and obviously should be regarded as rough approximations, valid only for preliminary design when applied to other cases. A more detailed introduction to detectors can be found in "A Consumer's Guide to Particle Detectors," by D.J. Miller, Rutherford Lab Report RL-76-072, July 1976.

A.1 Scintillators: The photon yield in the frequency range of practical photomultiplier tubes is $\approx 1 \gamma$ per $100 \mathrm{eV}$ of charged particle ionization energy loss in plastic scintillator ${ }^{1}$ and $\simeq 1 \gamma / 25 \mathrm{eV}$ in NaI. 1,2

A.2 Cerenkov: ${ }^{3}$ The half-angle $\theta_{c}$ of the Cerenkov cone aperture in terms of the velocity $\beta$ and the index of refraction $n$ is:

$$
\theta_{c}=\arccos \left(\frac{1}{\beta n}\right) \simeq\left[2\left(1-\frac{1}{\beta n}\right)\right]^{1 / 2} .
$$

The threshold velocity is: $\beta_{\mathrm{t}}=1 / \mathrm{n} ; \gamma_{\mathrm{t}}=1 / \sqrt{1-\beta_{\mathrm{t}}^{2}}$. Therefore, $\beta_{t} \gamma_{t}=1 / \sqrt{2 \delta+\delta^{2}}$, where $\delta=n-1$. Values of $\delta$ for various commonly used gases are given as a function of pressure and wavelength in Ref. 4; for values at atmospheric pressure, see the Table of Atomic and Nuclear Properties, following.

The number of photons $\mathbf{N}$ per $\mathrm{cm}$ of path length is given by:

$\mathrm{N}=\frac{\alpha}{\mathrm{c}} \int\left(1-\frac{1}{\beta^{2} \mathrm{n}^{2}}\right) 2 \pi \mathrm{d} \nu=\frac{\alpha}{\mathrm{c}} \beta_{\mathrm{t}}^{2} \int\left(\frac{1}{\beta_{\mathrm{t}}^{2} \gamma_{\mathrm{t}}^{2}}-\frac{1}{\beta^{2} \gamma^{2}}\right) 2 \pi \mathrm{d} \nu$

$\simeq 500 \sin ^{2} \theta_{\mathrm{c}} / \mathrm{cm}$ (visible spectrum) .

A.3 Photon collection: In addition to the photon yield, one should take into account the light collection efficiency ( $\leqslant 10 \%$ for typical 1 -cm-thick scintillator), the attenuation length $(\simeq 1$ to $4 \mathrm{~m}$ for typical scintillators ${ }^{5}$ ), and the quantum efficiency of the photomultiplier cathode $(\$ 25 \%)$.

\section{A.4 Typical detector characteristics:}

\begin{tabular}{lccc} 
Detector Type & Accuracy $(\mathrm{rms})$ & $\begin{array}{c}\text { Resolution } \\
\text { Time }\end{array}$ & $\begin{array}{c}\text { Dead } \\
\text { Time }\end{array}$ \\
\hline Bubble chamber & $\simeq \pm 10$ to $\simeq \pm 150 \mu$ & $\simeq 1 \mathrm{~ms}$ & $\simeq 1 / 20 \mathrm{~s}$ \\
Streamer chamber & $\pm 300 \mu$ & $\cong 2 \mu \mathrm{s}$ & $\simeq 100 \mathrm{~ms}$ \\
Proportional chamber & $\geq \pm 300 \mu, c$ & $\cong 50 \mathrm{~ns}$ & $\cong 200 \mathrm{~ns}$ \\
Drift chamber & \pm 50 to $300 \mu$ & $\simeq 2 \mathrm{~ns}$ & $\simeq 100 \mathrm{~ns}$ \\
Scintillator & - & $\simeq 150 \mathrm{ps}$ & $\simeq 10 \mathrm{~ns}$ \\
Emulsion & $\pm 1 \mu$ & $\bar{e}$ & $\bar{e}$ \\
Silicon strip & $\pm 5 \mu$ & $e$
\end{tabular}

$a^{a}$ Multiple pulsing time.

$b 300 \mu$ is for $1 \mathrm{~mm}$ pitch.

${ }^{c}$ Delay line cathode readout can give $\pm 150 \mu$

parallel to anode wire.

$d$ For two chambers.

$e^{e}$ Limited at present by noise and readout time of attached electronics.

A.5 Shower detectors: We give below typical energy resolutions (FWHM) for an incident electron in the $1 \mathrm{GeV}$ range; $\mathrm{E}$ is in $\mathrm{GeV}$. For a fixed number of radiation lengths, FWHM in the last three detectors would be expected to be proportional to $\sqrt{t}$ for $t(-$ plate thickness) $>0.2$ radiation lengths. ${ }^{6}$ For all detectors, operational resolution may be up to $50 \%$ worse due to dead areas, nonnormally incident tracks, and other effects.
NaI (20 rad. lengths): ${ }^{7} \frac{2 \%}{E^{1 / 4}}$

Lead glass (14 rad. lengths): $8 \frac{10-12 \%}{\sqrt{E}}$

Lead-liquid argon (15.75 rad. lengths): 6

(42 cells: $1.1 \mathrm{~mm}$ lead, $2 \mathrm{~mm}$ liquid argon, $\frac{\sqrt{E}}{\sqrt{6}}$ $2.3 \mathrm{~mm}$ lead-G10, $2 \mathrm{~mm}$ liquid argon)

Lead-scintillator sandwich (12.5 rad. lengths): $9 \frac{17 \%}{\sqrt{E}}$

( 66 cells: $1 \mathrm{~mm}$ lead, $5 \mathrm{~mm}$ scintillator) $\sqrt{\mathrm{E}}$

Proportional wire shower chamber (17 rad. lengths): $10 \frac{40 \%}{\sqrt{E}}$

( 36 cells: $0.474 \mathrm{rad}$. length type-metal $+\mathrm{Al}$,

$9.5 \mathrm{~mm} \mathrm{80 \%} \mathrm{Ar} \mathrm{-} 20 \% \mathrm{CH}_{4}$ gas)

A.6 dE/dx resolution in Argon: Particle identification (relativistic, $Q=1$ incident particles) by $\mathrm{dE} / \mathrm{dx}$ is dependent on the width of the distribution:

Multiple-sample Ar gas counters (no lead): ${ }^{11}$

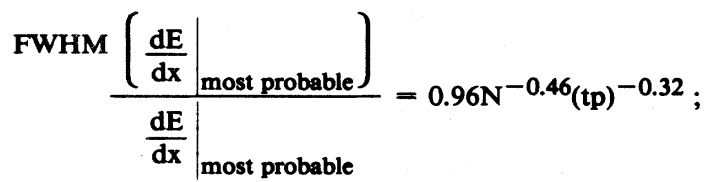

$\mathbf{N}=$ no. samples, $\mathrm{t}$ - thickness per sample $(\mathrm{cm}), \mathbf{p}=$ pressure (atm.); most commonly used chamber gases (except Xe) give approximately the same resolution.

A.7. Proportional chamber wire instability: The limit on the voltage $\mathrm{V}$ for a wire tension $\mathrm{T}$, due to mechanical effects when the electrostatic repulsion of adjacent wires exceeds the restoring force of wire tension, is given by (MSKA) ${ }^{12}$

$$
\mathrm{V}<\frac{\mathrm{S}}{\ell \mathrm{C}} \sqrt{4 \pi \epsilon_{0} \mathrm{~T}},
$$

where $s, \ell$, and $C$ are the wire spacing, length, and capacitance per unit length. An approximation to $C$ for chamber half-gap $t$ and wire diameter $d(g o o d$ for $s \leqslant t)$ gives $^{13}$

$$
\mathrm{v} \leqslant 59 \mathrm{~T}^{1 / 2}\left[\frac{\mathrm{t}}{\ell}+\frac{\mathrm{s}}{\pi \ell} \ell \mathrm{n}\left(\frac{\mathrm{s}}{\pi \mathrm{d}}\right)\right],
$$

where $\mathrm{V}$ is in $\mathrm{kV}$, and $\mathrm{T}$ is in grams-weight equivalent.

A.8 Proportional and drift chamber potentials: The potential distributions and fields in a proportional or drift chamber can usually be calculated with good accuracy from the exact formula for the potential around an array of parallel line charges $q(\mathrm{coul} / \mathrm{m})$ along $z$ and located at $y=0, x=0, \pm s, \pm 2 s, \ldots$,

$V(x, y)=-\frac{q}{4 \pi \epsilon_{0}} \ln \left\{4\left[\sin ^{2}\left(\frac{\pi x}{s}\right)+\sinh ^{2}\left(\frac{\pi y}{s}\right)\right]\right\}$.

Errors from the presence of cathodes, mechanical defects, TPC-type edge effects, etc., are usually small and are beyond the scope of this review.

A.9 Silicon strip detectors: These are silicon diodes operated with a reverse bias voltage $V$ (typically $30-300$ volts) sufficient to deplete the sensitive volume of most mobile charge carriers (electrons and holes). The active (depletion layer) thickness $t$ is given in a simple model by (MKSA) 


\section{PARTICLE DETECTORS, ABSORBERS, AND RANGES (Cont'd)}

$$
t=\sqrt{\frac{2 \epsilon V}{n e}}=\sqrt{2 \rho \mu \epsilon V},
$$

where

$$
\begin{aligned}
\mathrm{n} & =\text { number of impurity centers } / \mathrm{m}^{3} \\
\mathrm{e} & =\text { electron charge } \\
\epsilon & =\text { dielectric constant } \simeq 105 \mathrm{pF} / \mathrm{m} \simeq 11.9 \epsilon_{0} \\
\rho & =\text { resistivity } \simeq 10-200 \Omega-\mathrm{m} \\
\mu & =\text { majority charge carrier mobility } \\
& \simeq 0.13-0.15 \mathrm{~m}^{2} / \mathrm{volt-sec} \text { (electrons) } \\
& \simeq 0.045-0.06 \mathrm{~m}^{2} / \text { volt-sec (holes) }
\end{aligned}
$$

A minimum-ionizing particle has a Landau energy-loss distribution with average energy loss $39 \mathrm{keV} / 100 \mu \mathrm{m}$ and full width at halfmaximum of $0.071 \mathrm{t} / \beta^{2} \mathrm{keV}$, where $t$ is the detector thickness in microns and $\beta=v_{\text {ind }} / c$. The width is usually increased further by electronic noise $(\sigma \sim 1-10 \mathrm{keV})$ and for thin layers by a Gaussian contribution due to atomic effects $[\sigma \sim(0.3-0.4) \sqrt{\mathrm{t}} \mathrm{keV}]$. The average energy required to produce an electron-hole pair is $3.62 \mathrm{eV}$, from which one can estimate total charge of either sign released. Silicon detectors can tolerate integrated charged-particle fluxes of up to $\sim 10^{14}-10^{18} / \mathrm{m}^{2}$ and still operate as efficient detectors.

\section{B. COSMIC RAY FLUXES}

The fluxes of particles of different types depend on the latitude, their energy, and the conditions of measurement. Some typical sea-level values ${ }^{14}$ for charged particles are given below:

Iv flux per unit solid angle about vertical direction crossing unit horizontal area

$J_{1}$ perpendicular component of total flux crossing unit horizontal area from above

$\mathrm{J}_{2}$ total flux crossing unit horizontal area Total Hard Soft Intensity Compone Component

$\begin{array}{ll}\mathrm{I}_{\mathrm{v}} & 1.1 \times 10^{2} \\ \mathrm{~J}_{1} & 1.8 \times 10^{2}\end{array}$ $0.8 \times 10^{2}$ $1.3 \times 10^{2}$ $\overline{0.3 \times 10^{2}} \mathrm{~m}^{-2} \mathrm{sec}^{-1}$ sterad $^{-1}$

$\mathrm{J}_{2} \quad 2.4 \times 10^{2}$ $1.7 \times 10^{2}$ $\begin{array}{ll}0.3 \times 10^{2} & \mathrm{~m}^{-2} \mathrm{sec}^{-1} \\ 0.5 \times 10^{2} & \mathrm{~m}^{-2} \mathrm{sec}^{-1}\end{array}$ $\begin{array}{ll}0.5 \times 10^{2} & \mathrm{~m}^{-2} \mathrm{sec}^{-1} \\ 0.7 \times 10^{2} & \mathrm{~m}^{-2} \mathrm{sec}^{-1}\end{array}$

Very approximately, about $75 \%$ of all particles at sea level are penetrating, and are muons. The sea-level vertical flux ratio for protons to muons (both charges together) is about $3 \frac{1}{2} \%$ at $1 \mathrm{GeV} / \mathrm{c}$, decreasing to about $1 / 2 \%$ at $10 \mathrm{GeV} / \mathrm{c}$.

The muon flux at sea level has a mean energy of $2 \mathrm{GeV}$ and a differential spectrum falling as $E^{-2}$, steepening smoothly to $E^{-3.6}$ above a few TeV. The angular distribution is $\cos ^{2} \theta$, changing to $\sec \theta$ at energies above a $\mathrm{TeV}$, where $\theta$ is the zenith angle at production. The +- charge ratio is $1.25-1.30$. The mean energy of muons originating in the atmosphere is roughly $300 \mathrm{GeV}$ at slant depths $z$ a few hundred meters. Beyond slant depths of $\sim 10 \mathrm{~km}$ water-equivalent, the muons are due primarily to in-the-earth neutrino interactions (roughly $1 / 8$ interaction ton $^{-1}$ year $^{-1}$ for $E_{y}>$ $300 \mathrm{MeV}, \sim$ constant throughout the earth). ${ }^{15}$ Muons from this source arrive with a mean energy of $20 \mathrm{GeV}$, and have a flux of $2 \times 10^{-9} \mathrm{~m}^{-2} \sec ^{-1}$ sterad $^{-1}$ in the vertical direction and about twice that in the horizontal, ${ }^{16}$ down at least as far as the deepest mines.

\section{PASSAGE OF PARTICleS THROUGH MATTER}

C.1 Energy loss rates for heavy charged projectiles: A heavy projectile (much more massive than an electron) of charge $Z_{\text {inc }} e$, incident at speed $\beta c(\beta \gg 1 / 137)$ through a slowing medium, dissipates energy principally via interactions with the electrons of the medium. The mean rate of such energy loss per unit path length $x$, called the stopping power, is given by the Bethe-Bloch equation: ${ }^{17}$

$$
\begin{aligned}
& \left(\frac{\mathrm{dE}}{\mathrm{dx}}\right)_{\text {inc }}=\frac{\mathrm{D} \mathrm{z}_{\mathrm{med}} \rho_{\mathrm{med}}}{\mathrm{A}_{\mathrm{med}}}\left(\frac{\mathrm{Z}_{\mathrm{inc}}}{\beta}\right)^{2} \\
& \times\left[\ln \left(\frac{2 \mathrm{~m}_{\mathrm{e}} \gamma^{2} \beta^{2} \mathrm{c}^{2}}{I}\right)-\beta^{2}-\frac{\delta}{2}-\frac{\mathrm{C}}{\mathrm{Z}_{\mathrm{med}}}\right]\{1+\nu\},
\end{aligned}
$$

where $D=4 \pi N_{A} r_{e}^{2} m_{e} c^{2}=0.3070 \mathrm{MeV} \mathrm{cm} / g$ (see Physical Constants Table). Mean range and energy loss figures appear at the end of this section.

Here, $Z_{\text {med }}$ and $A_{\text {med }}$ are the charge and mass numbers of the medium and $\rho_{\text {med }}$ is the mass density of the medium; $I, \delta, C$, and $\nu$ are phenomenological functions. Frequently, the values of $\delta, C$, and $\nu$ are negligibly small; the parameter $I$ characterizes the binding of the electrons of the medium. As a rule of thumb, we may estimate $I$ for an idealized medium as $I \cong 16\left(\mathrm{Z}_{\text {med }}\right)^{0.9} \mathrm{eV}$ when $Z_{\text {med }}>1$. For realistic media the value of $I$ will vary at the $10 \%$ level from this estimate. Variations of this order occur due to atomic effects such as completion of a shell, also due to chemical binding, and even due to the phase of the substance. Hydrogen, perhaps the most sensitive, has $I$ of about $15 \mathrm{eV}$ in the atomic mode, rising to about $19.2 \mathrm{eV}$ as $\mathrm{H}_{2}$ gas and to $21.8 \mathrm{eV}$ as $\mathrm{H}_{2}$ liquid. ${ }^{18}$ For many substances, the transition from gas to solid is accompanied by a $20-30 \%$ increase in $1 .{ }^{18}$ We may approximately treat media which are chemical mixtures or compounds by computing

$$
\frac{d E}{d x} \simeq \sum_{n=1}^{N}\left(\frac{d E}{d x}\right)_{n},
$$

with (dE/dx) appropriate to the $\mathrm{n}^{\text {th }}$ chemical constituent (using $\rho_{\text {med }}^{(n)}$ as the partial density in the formula for $\mathrm{dE} / \mathrm{dx}$ ). ${ }^{19}$ For many chemical compounds, small corrections to this additivity rule may be found in Ref. 18.

The function $\delta$ represents the density effect upon the energy loss rate; it is non-negligible only for highly relativistic projectiles in denser media. ${ }^{20}$ For ultra-relativistic projectiles, $\delta$ approaches $2 \ell \mathrm{n} \gamma+$ constant, where the value of the constant depends upon the density of the medium as well as its chemical composition.

The function $\mathrm{C}$ represents shell corrections to the energy loss rate. ${ }^{17}$ These effects are non-negligible only for projectiles with speeds not much faster than the speeds of the fastest electrons bound in the medium.

The function $\nu$ represents corrections due to higher order electrodynamics. ${ }^{21}$ These effects become important when $\left|Z_{\text {inc }} / \beta\right|$ is comparable to 137. For relativistic unit-charge projectiles, $|\nu|$ is of the order of $1 \%$; positively charged projectiles lose energy more rapidly than do their charge conjugates. 21,22

For nonrelativistic projectiles, our formulae above are inapplicable. At the very slowest speeds, total energy loss rates are believed to be proportional to $\beta$, rising through a peak at projectile speeds comparable to atomic speeds ( $\beta$ on the order of $\alpha$ ), after having passed through a smaller peak (due to elastic Coulomb collisions with the nuclei of the slowing medium ${ }^{23}$ ) at intermediate speeds. For example, for protons in $\mathrm{Si}, \mathrm{dE} / \mathrm{dx}=61.23 \beta \mathrm{GeV} /\left(\mathrm{gm} \mathrm{cm}^{-2}\right.$ ) for $\beta<0.005$; the peak occurs at $\beta=0.0126$ where $\mathrm{dE} / \mathrm{dx}=522$ $\mathrm{MeV} /\left(\mathrm{gm} \mathrm{cm}^{-2}\right)$. In some cases, energy loss rates depend significantly upon the relation of the projectile trajectory to the crystalline structure of the slowing medium. ${ }^{24}$

For relativistic projectiles, $(\mathrm{dE} / \mathrm{dx})_{\text {inc }}$ falls rapidly with increasing $\beta$ until reaching a minimum around $\beta=0.96$ (almost independent of medium), followed by a slow rise. Because of the density 


\section{PARTICLE DETECTORS, ABSORBERS, AND RANGES (Cont'd)}

effect, the quantity in square brackets approaches $\ell \mathrm{n} \gamma+$ constant for large $\gamma$.

The quantity $(\mathrm{dE} / \mathrm{dx})_{\text {inc }} \delta \mathrm{x}$ is the mean total energy loss via interactions with electrons of the medium in a layer of thickness $\delta x$. For any finite $\delta x$, Poisson fluctuations can cause the actual energy loss to deviate from the mean. For thin layers, the distribution is broad and skewed, being peaked below $(\mathrm{dE} / \mathrm{dx}) \delta \mathrm{x}$, and having a long tail toward large energy losses. ${ }^{25}$ Only for a very thick layer $\left[(\mathrm{dE} / \mathrm{dx}) \delta \mathrm{x} \gg 2 \mathrm{me}_{\mathrm{e}} \beta^{2} \gamma^{2} \mathrm{c}^{2}\right]$ will the distribution of energy losses become nearly Gaussian. The large fluctuations of the total energy loss rate from the mean are due to a small number of collisions involving large energy transfers. The fluctuations are greatly reduced for the so-called restricted energy loss rate, described in Section C.4.

C.2 Ionization yields: Physicists frequently relate total energy loss to the number of ion pairs produced near the projectile's track. This relation becomes complicated for relativistic projectiles due to the wandering of energetic knock-on electrons whose ranges exceed the dimensions of the fiducial volume. For a qualitative appraisal of the nonlocality of energy deposition by such modestly energetic knock-on electrons in various media, see Ref. 26. Furthermore, the mean local energy dissipation per local ion pair produced, $W$, while essentially constant for relativistic projectiles, increases at slow projectile speeds. ${ }^{27}$ The numerical value of $W$ for gases can be surprisingly sensitive to trace amounts of various contaminants. ${ }^{27}$ Of course, in addition to the preceding effects, practical ionization yields may be greatly influenced by subsequent recombinations and other factors. ${ }^{28}$

C.3 Energetic knock-on electrons: For a relativistic point-charge projectile, the production of high energy (kinetic energy $\mathrm{T} \gg I$ ) electrons is given by: 29

$$
\frac{d^{2} N}{d T d x}=\frac{1}{2} D\left(\frac{Z_{\text {med }}}{A_{\text {med }}}\right)\left(\frac{Z_{\text {inc }}}{\beta}\right)^{2} \rho_{\text {med }} \frac{1}{T^{2}} F,
$$

for $I \ll \mathrm{T}<\mathrm{T}_{\max }$, where

$$
T_{\max }=\frac{2 m_{e} \beta^{2} \gamma^{2} c^{2}}{1+2 \gamma \frac{m_{e}}{M_{i n c}}+\left(\frac{m_{e}}{M_{i n c}}\right)^{2}},
$$

$M_{\text {inc }}$ is the mass of the incident projectile, and all other quantities except $F$ are as in Sec. C.1. $F\left(\simeq 1\right.$ for $\left.T \ll T_{\max }\right)$ is a factor dependent upon the spin of the projectile.

For spin-0 projectiles,

$$
F=1-\beta^{2} \frac{T}{T_{\max }}
$$

for spin-1/2 projectiles,

$$
F=1-\beta^{2} \frac{T}{T_{\max }}+\frac{1}{2}\left(\frac{T}{T_{i n c}+M_{i n c} c^{2}}\right)^{2},
$$

where $T_{\text {inc }}$ is the kinetic energy of the projectile; for electrons incident,

$$
\mathrm{F}=\beta^{2} \mathrm{~T}^{2}\left[\frac{\mathrm{T}_{\text {inc }}}{\mathrm{T}\left(\mathrm{T}_{\text {inc }}-\mathrm{T}\right)}-\frac{1}{\mathrm{~T}_{\text {inc }}}\right]^{2} ;
$$

and for positrons incident,

$$
\mathrm{F}=\beta^{2}\left[1-\frac{\mathrm{T}}{\mathrm{T}_{\mathrm{inc}}}+\left(\frac{\mathrm{T}}{\mathrm{T}_{\mathrm{inc}}}\right)^{2}\right]^{2} .
$$

For incident electrons, the indistinguishability of projectile and target means that the range of $T$ is only up to $T_{i n c} / 2$. For additional formulas see Ref. 30. Our formula is inaccurate for $\mathrm{T}$ close to $I$; for $2 I \leqslant T \leqslant 10 I$, the $1 / T^{2}$ dependence above becomes $\cong T^{-} \eta$ with $3 \leqslant \eta \leqslant 5.31$

C.4 Rates of restricted energy loss for relativistic charged projectiles: The variability of energy loss for heavy projectiles is due primarily to the variability in the production of energetic knock-on electrons. Bremsstrahlung and pair-production processes make this variability even greater for electrons than for heavy particles as projectiles (see, e.g., the figure "Fractional Energy Loss for Electrons and Positrons in Lead," following). If an instrument, such as a bubble chamber, is capable of isolating these high-energy-loss interactions, then it is appropriate to consider the rate of energy loss excluding them, i.e., a restricted energy loss rate. The mean energy loss rate via all collisions which have energy transfer $T$ such that $\mathrm{T} \ll \mathrm{E}_{\max } \ll \mathrm{T}_{\max }$ is: $^{17}$

$$
\begin{aligned}
& \left(\frac{\mathrm{dE}}{\mathrm{dx}}\right)_{<\mathrm{E}_{\max }}=\frac{1}{2} \mathrm{D} \frac{\mathrm{Z}_{\text {med }} \rho_{\text {med }}}{A_{\text {med }}}\left(\frac{\mathrm{Z}_{\text {inc }}}{\beta}\right)^{2} \\
& \times\left[\ell \mathrm{n}\left(\frac{\mathrm{E}_{\max } \mathrm{T}_{\max }}{I^{2}}\right)-\beta^{2}-\delta-\frac{2 \mathrm{C}}{\mathrm{Z}_{\text {med }}}\right] .
\end{aligned}
$$

Notice the overall factor of $1 / 2$. See Sec. C. 1 above for definitions of the quantities in this equation.

The density effect causes the restricted energy loss rate to approach a constant, the Fermi plateau value, for the fastest projectiles.

C.5 Multiple scattering through small angles: As a charged particle traverses a medium it is deflected by many small-angle elastic scatterings. The bulk of this deflection is due to elastic Coulomb scattering from the nuclei within the medium, hence the usual identification as multiple Coulomb scattering (note, however, that strong interactions do contribute to the total multiple scattering for hadronic projectiles). For both Coulomb and strong interactions, the Central Limit Theorem provides little useful guidance in establishing the precise nature of the distribution of the total deflections resulting from multiple scattering. The true distribution is roughly Gaussian only for small deflection angles, while it shows much greater probability for large-angle scatterings ( $\gtrsim$ a few $\theta_{0}$, see below, depending on absorber) than the Gaussian would suggest. These tails on the distribution (a few per cent of peak height in the region where the Gaussian part becomes negligible) are more pronounced for hadrons than for muons as projectiles. The large-angle behavior of these distributions is best estimated by computing the exact distribution for the vectorial sum of the largest deflections based upon the true elastic scattering cross section of the projectile against the medium, ${ }^{32}$ or, when applicable, by interpolation from tabular data. ${ }^{33}$ An easier alternative which may suffice for noncritical applications would be to use a Gaussian approximation with the following width: ${ }^{34}$

$\theta_{0}=\frac{14.1 \mathrm{MeV} / \mathrm{c}}{\mathrm{p} \beta} \mathrm{Z}_{\mathrm{inc}} \sqrt{\mathrm{L} / \mathrm{L}_{\mathrm{R}}}\left[1+\frac{1}{9} \log _{10}\left(\mathrm{~L} / \mathrm{L}_{\mathrm{R}}\right)\right]$ (radians),

where $p, \beta$, and $\mathrm{Z}_{\text {inc }}$ are the momentum (in $\mathrm{MeV} / \mathrm{c}$ ), velocity, and charge number of the incident particle, and $L / L_{R}$ is the thickness, in radiation lengths, of the scattering medium. $\mathbf{L}_{R}$ for certain 


\section{PARTICLE DETECTORS, ABSORBERS, AND RANGES (Cont'd)}

materials is given in the Table of Atomic and Nuclear Properties of Materials, following. See also Sec. C.7 below. The angle, $\theta_{0}$, is a fit to Moliere 32 theory, accurate to about $5 \%$ for $10^{-3}<L / L_{R}<$ 10 except for very light elements or low velocity where the error is about 10 to $20 \%$. In this Gaussian approximation, $\theta_{0}$ has the meaning

$$
\theta_{0}=\theta_{\text {plane }}^{\mathrm{rms}}=\frac{1}{\sqrt{2}} \theta_{\mathrm{space}}^{\mathrm{rms}} .
$$

The nonprojected (space) and projected (plane) angular distributions are given approximately ${ }^{22}$ by the Gaussian forms:

$$
\begin{aligned}
& \frac{1}{2 \pi \theta_{0}^{2}} \exp \left(-\frac{\theta_{\text {space }}^{2}}{2 \theta_{0}^{2}}\right) \mathrm{d} \Omega \\
& \frac{1}{\sqrt{2 \pi} \theta_{0}} \exp \left(-\frac{\theta_{\text {plane }}^{2}}{2 \theta_{0}^{2}}\right) \mathrm{d} \theta_{\text {plane }}
\end{aligned}
$$

where $\theta$ is the deflection angle.

Other quantities are sometimes used to describe the amount of multiple Coulomb scattering: the auxiliary quantities $\psi_{\text {plane }}$ $y_{\text {plane, }}$ and $s_{\text {plane }}$ (see the figure) obey:

$$
\begin{array}{ll}
\psi_{\text {plane }}^{\text {rms }} & =\frac{1}{\sqrt{3}} \theta_{\text {plane }}^{\text {rms }}=\frac{1}{\sqrt{3}} \theta_{0}, \\
\text { and } \quad & y_{\text {plane }}^{\text {rms }}=\frac{1}{\sqrt{3}} L \theta_{\text {plane }}^{\text {rms }}=\frac{1}{\sqrt{3}} L \theta_{0}, \\
s_{\text {plane }}^{\text {rms }} & =\frac{1}{4 \sqrt{3}} \mathrm{~L} \theta_{\text {plane }}^{\text {rms }}=\frac{1}{4 \sqrt{3}} L \theta_{0} .
\end{array}
$$

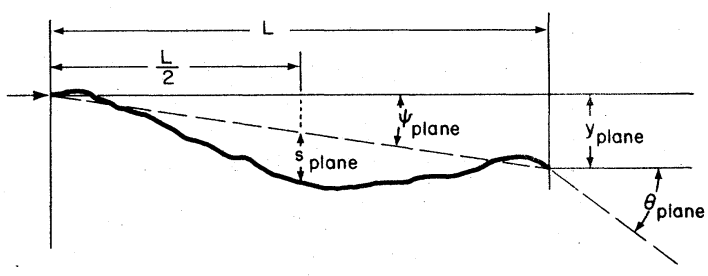

All the quantitative estimates in this section apply only in the limit of small $\theta_{\text {plane }}^{\text {rms }}$ and in the absence of large-angle scatters.

C.6 Longitudinal distribution of electromagnetic showers: A photon of energy $E>0.1 \mathrm{GeV}$ converting in a semi-infinite medium produces an electromagnetic cascade whose intensity initially increases with depth and then falls off. The average number of $e^{ \pm}$ with kinetic energy above $1.5 \mathrm{MeV}$, crossing a plane at a depth of $\mathrm{L}$ radiation lengths from the beginning of the medium, in a material of atomic number $\mathrm{Z}$, calculated using the Monte Carlo program EGS, ${ }^{35}$ can be fit by the empirical formula ${ }^{36}$

$$
\mathbf{N}=\mathbf{N}_{0} \mathbf{L}^{\mathbf{a}} \mathbf{e}^{-\mathbf{b L}},
$$

where $N_{0}=5.51 \mathrm{E}(\mathrm{GeV}) \sqrt{\mathrm{Z}} \mathrm{b}^{\mathrm{a}+1} / \mathrm{\Gamma}(\mathrm{a}+1)$ and $\mathrm{b}=0.634$

$-0.0021 Z$. For $Z>26, a=2.0-Z / 340+(0.664-Z / 340) \ell n$ E. For $Z=13, a=1.77-0.52 \ell n E$. The maximum intensity, $N_{\max }$, occurs at the depth $L=a / b$. The maximum error of the fit occurs in the vicinity of this depth and is less than $0.15 \mathrm{~N}_{\max }$. The integral of the tail, $\int_{1.5 \mathrm{a} / \mathrm{b}}^{\infty} \mathrm{N} \mathrm{dL}$ is fit to better than $2.5 \%$. The total longitudinally projected $\mathrm{e}^{ \pm}$path length, $\int_{0}^{\infty} \mathrm{N} d L=5.51 \mathrm{E} \sqrt{\mathrm{Z}}$, is less than the total $\mathrm{e}^{ \pm}$path length due primarily to multiple Coulomb scattering.

C.7 Radiation length: For the passage of electromagnetically interacting particles through a medium it is convenient to measure thickness in terms of radiation length. ${ }^{37}$ For most electromagnetic processes (Bremsstrahlung, Coulomb scattering, showering, pair production, etc.), over large energy intervals, some or all of the dependence upon the medium is contained in the radiation length.

The radiation length may be defined as the distance $L_{R}$ over which a high energy electron ( $~(1 \mathrm{GeV}$ for most materials) loses all but a fraction 1/e of its energy to Bremsstrahlung, on average. For a homogeneous monoatomic medium, $Z \geqslant 5$,

$$
\begin{gathered}
\frac{1}{\mathrm{~L}_{\mathrm{R}}}=\frac{4 \alpha \mathrm{r}_{\mathrm{e}}^{2} \mathrm{~N}_{\mathrm{A}} \mathrm{Z}^{2}}{\mathrm{~A}}\left\{\ln \left(\frac{184.15}{\mathrm{Z}^{1 / 3}}\right)+\frac{1}{\mathrm{Z}} \ln \left(\frac{1194}{\mathrm{Z}^{2 / 3}}\right)-1.202 \alpha^{2} \mathrm{Z}^{2}\right. \\
\left.+1.0369 \alpha^{4} \mathrm{Z}^{4}-\frac{1.008 \alpha^{6} \mathrm{Z}^{6}}{1+\alpha^{2} \mathrm{Z}^{2}}\right\}=\frac{\mathrm{Z}^{2}\{\}}{716.405 \mathrm{~A}},
\end{gathered}
$$

where $\alpha, r_{e}$, and $N_{A}$ are found in the Physical Constants Table, and $Z$ and $A$ are the atomic number and weight of the medium. For $Z$ $<5$, a more complex numerical calculation is required. Radiation lengths for many substances are tabulated in the Table of Atomic and Nuclear Properties of Materials, following. For media which are chemical mixtures or compounds,

$$
\frac{1}{L_{R}} \simeq \sum_{i} \frac{f_{i}}{L_{R}^{i}}
$$

where $f_{i}$ is the fraction by mass of atoms of type $i$, radiation length $L_{R}^{i}$. Chemical binding can lower $L_{R}$ from this, typically by a few per cent.

For electrons of energy below about one $\mathrm{GeV}$, the average fractional energy loss per unit length decreases as the energy decreases (see Fractional Energy Loss for Electrons and Positrons in Lead figure, following). With distances measured in units of $L_{R}$, dependence of the Bremsstrahlung fractional energy loss upon $Z$ of the medium in the low energy region $(z 10 \mathrm{MeV})$ is of order a few percent or less.

For photons of infinite energy, the total pair-production cross section is

$$
\sigma=\frac{7}{9}\left(\mathrm{~A} / \mathrm{L}_{\mathrm{R}} \mathrm{N}_{\mathrm{A}}\right)
$$

This is accurate to within a few per cent down to $\sim 1 \mathrm{GeV}$ for most materials. For energies below about $1 \mathrm{GeV}$, the cross section varies in a manner which may be determined from the Photon Mass Attenuation figures, following. See also Contributions to Photon Cross Section in Carbon and Lead figure, following.

C.8 Electron practical range: The electron "practical range" - a common measure of straight-line penetration distance - is shorter than the total path length because of multiple Coulomb scattering, which becomes increasingly important as the electron slows down. E.g., for a fast electron the rms projected angle due to multiple Coulomb scattering reaches 1 radian by the time the electron has slowed to $0.4 \mathrm{MeV}$ in hydrogen, $1.5 \mathrm{MeV}$ in carbon, $9 \mathrm{MeV}$ in copper, and $24 \mathrm{MeV}$ in lead. Electrons which have energy less than 


\section{PARTICLE DETECTORS, ABSORBERS, AND RANGES (Cont'd)}

$0.2 \mathrm{MeV}$ in $\mathrm{Ar}, 1.5 \mathrm{MeV}$ in $\mathrm{Cu}, 3.5 \mathrm{MeV}$ in $\mathrm{Sn}$, and $5 \mathrm{MeV}$ in $\mathrm{Pb}$ are likely to deposit $10 \%$ of their energy behind their starting plane. The practical range, $R_{p}$, is defined as that absorber thickness obtained by extrapolating to zero the linearly decreasing part of the curve of penetration probability vs. absorber thickness. Data for $\mathrm{Al}$ in the $\mathrm{T}$ range up to about $10 \mathrm{MeV}$ are available, and fit (to $\sim \pm 10 \%) R_{p}=A T[1-B /(1+C T)] \mathrm{mg} \mathrm{cm}^{-2}$, a form suggested in Ref. 38, with $\mathrm{A}=0.55 \mathrm{mg} \mathrm{cm}^{-2} \mathrm{keV}^{-1}, \mathrm{~B}=0.9841$, and $\mathrm{C}=0.0030$ $\mathrm{keV}^{-1}$. At this penetration depth, $90-95 \%$ of the incident electrons have stopped. Data for other elements are sketchy, but suggest that higher-Z ( $\$ 50)$ elements have $1 \leqslant R_{p} / R_{p}(A l) \leqslant 1.4$ below $\sim 10 \mathrm{keV}$, and $0.6 \leqslant \mathrm{R}_{\mathrm{p}} / \mathrm{R}_{\mathrm{p}}(\mathrm{Al}) \leqslant 1 \mathrm{above} \sim 100 \mathrm{keV}$. The "critical energy" (above which the energy loss due to bremsstrahlung exceeds that due to ionization, and showering becomes important) is $400 \mathrm{MeV}$ for hydrogen, $100 \mathrm{MeV}$ for carbon, $25 \mathrm{MeV}$ for copper, and $10 \mathrm{MeV}$ for lead. The mean positron range may differ from the mean electron range by several percent. See Refs. 39 and 40. Electron energy deposition and penetration probability vs. range are discussed in Refs. 26, 41, and 42.

C.9 Atomic and nuclear properties of matter: See table following.

C.10 Range and energy loss for heavy projectiles in lead, copper, aluminum, and carbon: See figure following.

C.11 Range and energy loss for heavy projectiles in liquid hydrogen: See figure following.

C.12 Photon mass attenuation coefificients, energy deposition: See figures following.

C.13 Fractional energy loss for electrons and positrons in lead: See figure following.

C.14 Contributions to photon cross section in lead and carbon: See figures following.

* Revised April 1984 by Sherwood Parker, Ray Hagstrom, and Geoff Hall.

1. Methods of Experimental Physics, L.C.L. Yuan and C.-S. Wu, editors, Academic Press, 1961, Vol. 5A, p.127.

2. R.K. Swank, Ann. Rev. Nucl. Sci. 4, 137 (1954), and G.T. Wright, Proc. Phys. Soc. B68, 929 (1955).

3. Methods of Experimental Physics, L.C.L. Yuan and C.-S. Wu, editors, Academic Press, 1961, Vol. SA, p.163.

4. E.R. Hayes, R.A. Schluter, and A. Tamosaitis, "Index and Dispersion of Some Cerenkov Counter Gases," ANL-6916 (1964).

5. Nuclear Enterprises Catalogue.

6. D. Hitlin et al., Nucl. Instr. and Meth. 137, 225 (1976). See also W.J. Willis and V. Radeka, Nucl. Instr. and Meth. 120, 221 (1974), for a more detailed discussion.

7. E.B. Hughes et al., IEEE Transactions on Nuclear Science NS19, No. 3, 126 (1972)

8. M. Holder et al., Phys. Letters 40B, 141 (1972), and J.S. Beale et al., "A Lead-Glass Cerenkov Detector for Electrons and Photons," CERN Writeup, Intl. Conf. on Instrumentation in H.E.P., Frascati (1973).

9. W. Hofmann et al., DESY 81/045 (July 1981). See also S.L. Stone et al., Nucl. Instr. and Meth. 151, 387 (1978).

10. R.L. Anderson et al., "Tests of Proportional Wire Shower Counter and Hadron Calorimeter Modules," SLAC-PUB-2039 (1977).

11. W.W.M. Allison and J.H. Cobb, "Relativistic Charged Particle Identification by Energy-Loss," Ann. Rev. Nucl. Part. Sci. 30, 253 (1980).

12. T. Trippe, CERN NP Internal Report 69-18 (1969).

13. S. Parker and R. Jones, LBL-797 (1972), and P. Morse and H. Feshbach, Methods of Theoretical Physics, McGraw-Hill, New York, 1953, p.1236.
14. B. Rossi, Rev. Mod. Phys. 20,537 (1948). See also C. Grupen, "News from Cosmic Rays at High Energies," Siegen University preprint SI-84-01; flux ratio for protons at sea-level from G. Brook and A.W. Wolfendale, Proc. of the Phys. Soc. of London, Vol. 83 (1964), p. 843.

15. J.G. Learned, F. Reines, and A. Soni, Phys. Rev. Lett. 43, 907 (1979).

16. M.F. Crouch et al., Phys. Rev. D18, 2239 (1978).

17. U. Fano, Ann. Rev. Nucl. Sci. 13, 1 (1963).

18. M.J. Berger and S.M. Seltzer, "Mean Excitation Energies for Use in Bethe's Stopping-Power Formula", p. 57-74, Proceedings of Hawaii Conference on Charge States and Dynamic Screening of Swift Ions (1982).

19. H.A. Bethe and J. Ashkin, Experimental Nuclear Physics, Vol. 1, E. Segrè, editor, John Wiley, New York, 1959.

20. A. Crispin and G.N. Fowler, Rev. Mod. Phys. 42, 290 (1970); R.M. Sternheimer, S.M. Seltzer, and M.J. Berger, "The Density Effect for the Ionizatin Loss of Charged Particles in Various Substances," BNL preprint 31435, to be published in Atomic Data \& Nucl. Data Tables.

21. For $Z^{3}$ calculations with $Z=1$, see J.D. Jackson and R.L. McCarthy, Phys. Rev. B6, 4131 (1972).

22. For an approximate treatment of high-Z projectiles, see P.B. Eby and S.H. Morgan, Phys. Rev. A5, 2536 (1972).

23. See, for instance, G. Sidenius, Det Kong. Danske Viden. Selskab Mat.-Fysk. Med. 39, No. 4 (1974)

24. See, for instance, S. Datz, "Atomic Collisions in Solids" in "Structure and Collisions of Ions and Atoms," Springer Verlag, Berlin, 1978, p. 309.

25. See, for instance, K.A. Ispirian, A.T. Margarian, and A.M. Zverev, Nucl. Instr. and Meth. 117, 125 (1974).

26. L.V. Spencer "Energy Dissipation by Fast Electrons," Nat'1 Bureau of Standards Monograph No. 1 (1959).

27. "Average Energy Required to Produce an Ion Pair," ICRU Report No. 31 (1979).

28. N. Hadley et al., "List of Poisoning Times for Materials," Lawrence Berkeley Lab Report TPC-LBL-79-8 (1981).

29. B. Rossi, High Energy Particles, Prentice-Hall, Inc., Englewood Cliffs, NJ, 1952.

30. For unit-charge projectiles, see E.A. Uehling, Ann. Rev. Nucl. Sci. 4, 315 (1954). For highly charged projectiles, see J.A. Doggett and L.V. Spencer, Phys. Rev. 103, 1597 (1956). A Lorentz transformation is needed to convert these center-of-mass data to knock-on energy spectra.

31. N.F. Mott and H.S.W. Massey, The Theory of Atomic Collisions, Oxford Press, London, 1965.

32. For a thorough discussion of simple formulae for single scatters and methods of compounding these into multiple-scattering formulae, see W.T. Scott, Rev. Mod. Phys. 35, 231 (1963). For detailed summaries of formulae for computing single scatters, see J.W. Motz, H. Olsen, and H.W. Koch, Rev. Mod. Phys. 36, 881 (1964).

33. E.V. Hungerford and B.W. Mayes, Atomic Data and Nuclear Data Tables 15, 477 (1975).

34. V.L. Highland, Nucl. Inst. and Meth. 129, 497 (1975) and important modification Nucl. Inst. and Meth. 161, 171 (1979).

35. R. Ford and W. Nelson, SLAC-210 (1978).

36. A similar form has been used by E. Longo and I. Sestili, Nucl. Inst. and Meth. 128, 283 (1975), and J. Sass and M. Spiro, CERN p̄p Tech. Note 78-32 (1978).

37. Y.S. Tsai, Rev. Mod. Phys. 46, 815 (1974).

38. K.-H. Weber, Nucl. Instr. and Meth. 25, 261 (1964).

39. M.J. Berger and S.M. Seltzer, NASA SP-3012 (1964) and SP3036 (1966).

40. P. Trower, UCRL-2426, Vol. III, Rev. (1966).

41. S.M. Seltzer, "Transmission of Electrons through Foils," NBSIR 74, 457 (1974).

42. M.J. Berger and S.M. Seltzer, "Stopping Powers and Ranges of Electrons and Positrons" ( $2^{\text {nd }}$ Ed.), U.S. National Bureau of Standards Report NBSIR 82-2550-A (1982). 


\section{PARTICLE DETECTORS, ABSORBERS, AND RANGES (Cont'd)}

\section{Atomic and Nuclear Properties of Materials*}

\begin{tabular}{|c|c|c|c|c|c|c|c|c|c|c|c|c|}
\hline Material & $\mathbf{z}$ & $\mathbf{A}$ & $\begin{array}{l}\text { Nuclear }^{a} \\
\text { total } \\
\text { cross } \\
\text { section } \\
\sigma_{\mathrm{T}}[\text { barn] }\end{array}$ & $\begin{array}{c}\text { Nuclear } b \\
\text { inelastic } \\
\text { cross } \\
\text { section } \\
\sigma_{I}[\text { barn] }\end{array}$ & $\begin{array}{c}\begin{array}{c}\text { Nuclear } \\
\text { collision } \\
\text { length } \\
\lambda_{\mathbf{T}} \\
{\left[\mathrm{g} / \mathrm{cm}^{2}\right]}\end{array} \\
43.3\end{array}$ & $\begin{array}{c}\begin{array}{c}\text { Nuclear } \\
\text { interaction } \\
\text { length } \\
\lambda_{\mathrm{I}} \\
{\left[\mathrm{g} / \mathrm{cm}^{2}\right]}\end{array} \\
50.8\end{array}$ & \multicolumn{2}{|c|}{ 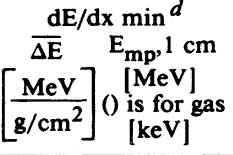 } & \multicolumn{2}{|c|}{$\begin{array}{l}\text { Radiation length }^{e} \\
\mathrm{~L}_{\mathrm{rad}} \\
{\left[\mathrm{g} / \mathrm{cm}^{2}\right] \text { [ }[\mathrm{cm}]} \\
\quad(\text { is for gas }\end{array}$} & $\begin{array}{l}\text { Density } f \\
{\left[\mathrm{~g} / \mathrm{cm}^{3}\right]} \\
() \text { is for gas } \\
{[\mathrm{g} / \ell]}\end{array}$ & $\begin{array}{l}\text { Refractive } \\
\text { index } n f \\
() \text { is }(n-1) \times 10^{6} \\
\quad \text { for gas }\end{array}$ \\
\hline $\begin{array}{l}\mathrm{H}_{2} \\
\mathrm{D}_{2} \\
\mathrm{He} \\
\mathrm{Li} \\
\mathrm{Be}\end{array}$ & $\begin{array}{l}1 \\
1 \\
2 \\
3 \\
4\end{array}$ & $\begin{array}{l}1.01 \\
2.01 \\
4.00 \\
6.94 \\
9.01\end{array}$ & $\begin{array}{l}0.0387 \\
0.073 \\
0.133 \\
0.211 \\
0.268 \\
\end{array}$ & $\begin{array}{l}0.033 \\
0.061 \\
0.102 \\
0.157 \\
0.199 \\
\end{array}$ & $\begin{array}{l}43.3 \\
45.7 \\
49.9 \\
54.6 \\
55.8 \\
\end{array}$ & $\begin{array}{l}50.8 \\
53.7 \\
65.1 \\
73.4 \\
75.2 \\
\end{array}$ & $\begin{array}{l}4.12 \\
2.07 \\
1.94 \\
1.58 \\
1.61 \\
\end{array}$ & $\begin{array}{l}(0.19) \\
(0.17) \\
(0.16) \\
0.70 \\
2.61 \\
\end{array}$ & $\begin{array}{c}61.28 \\
122.6 \\
94.32 \\
82.76 \\
65.19 \\
\end{array}$ & $\begin{array}{c}865 \\
757 \\
755 \\
155 \\
35.3 \\
\end{array}$ & $\begin{array}{c}0.0708(0.090) \\
0.162(0.177) \\
0.125(0.178) \\
0.534 \\
1.848 \\
\end{array}$ & $\begin{array}{c}1.112(140) \\
1.128 \\
1.024(35) \\
= \\
-\end{array}$ \\
\hline $\begin{array}{l}\mathrm{C} \\
\mathrm{N}_{2} \\
\mathrm{O}_{2} \\
\mathrm{Ne} \\
\mathrm{Al} \\
\mathrm{Si} \\
\mathrm{Ar}\end{array}$ & $\begin{array}{r}6 \\
7 \\
8 \\
10 \\
13 \\
14 \\
18 \\
\end{array}$ & $\begin{array}{l}12.01 \\
14.01 \\
16.00 \\
20.18 \\
26.98 \\
28.09 \\
39.95 \\
\end{array}$ & $\begin{array}{l}0.331 \\
0.379 \\
0.420 \\
0.507 \\
0.634 \\
0.660 \\
0.868 \\
\end{array}$ & $\begin{array}{l}0.231 \\
0.265 \\
0.292 \\
0.347 \\
0.421 \\
0.440 \\
0.566 \\
\end{array}$ & $\begin{array}{l}60.2 \\
61.4 \\
63.2 \\
66.1 \\
70.6 \\
70.6 \\
76.4 \\
\end{array}$ & $\begin{array}{r}86.3 \\
87.8 \\
91.0 \\
96.6 \\
106.4 \\
106.0 \\
117.2 \\
\end{array}$ & $\begin{array}{l}1.78 \\
1.82 \\
1.82 \\
1.73 \\
1.62 \\
1.66 \\
1.51 \\
\end{array}$ & $\begin{array}{c}3.57 \\
(0.93) \\
(1.31) \\
(0.75) \\
3.81 \\
3.36 \\
(1.30) \\
\end{array}$ & $\begin{array}{l}42.70 \\
37.99 \\
34.24 \\
28.94 \\
24.01 \\
21.82 \\
19.55 \\
\end{array}$ & $\begin{array}{c}18.8 \\
44.5 \\
28.7 \\
24.0 \\
8.9 \\
9.36 \\
14.0 \\
\end{array}$ & $\begin{array}{c}2.265^{g} \\
0.808(1.25) \\
1.14(1.43) \\
1.207(0.90) \\
2.70 \\
2.33 \\
1.40(1.78) \\
\end{array}$ & $\begin{array}{c}1.205(300) \\
1.22(266) \\
1.092(67) \\
= \\
\overline{-} \\
1.233(283) \\
\end{array}$ \\
\hline $\begin{array}{l}\mathrm{Fe} \\
\mathrm{Cu} \\
\mathrm{Sn} \\
\mathrm{Xe} \\
\mathrm{W} \\
\mathrm{Pb} \\
\mathrm{U} \\
\end{array}$ & $\begin{array}{l}26 \\
29 \\
50 \\
54 \\
74 \\
82 \\
92 \\
\end{array}$ & $\begin{array}{r}55.85 \\
63.54 \\
118.69 \\
131.30 \\
183.85 \\
207.19 \\
238.03 \\
\end{array}$ & $\begin{array}{l}1.120 \\
1.232 \\
1.967 \\
2.120 \\
2.767 \\
2.960 \\
3.378 \\
\end{array}$ & $\begin{array}{l}0.703 \\
0.782 \\
1.21 \\
1.29 \\
1.65 \\
1.77 \\
1.98 \\
\end{array}$ & $\begin{array}{r}82.8 \\
85.6 \\
100.2 \\
102.8 \\
110.3 \\
116.2 \\
117.0 \\
\end{array}$ & $\begin{array}{l}131.9 \\
134.9 \\
163 \\
169 \\
185 \\
194 \\
199 \\
\end{array}$ & $\begin{array}{l}1.48 \\
1.44 \\
1.26 \\
1.24 \\
1.16 \\
1.13 \\
1.09 \\
\end{array}$ & $\begin{array}{l}10.7 \\
11.85 \\
8.3 \\
(3.57) \\
21.1 \\
11.7 \\
19.3 \\
\end{array}$ & $\begin{array}{r}13.84 \\
12.86 \\
8.82 \\
8.48 \\
6.76 \\
6.37 \\
6.00 \\
\end{array}$ & $\begin{array}{r}1.76 \\
1.43 \\
1.21 \\
2.77 \\
0.35 \\
0.56 \\
\approx 0.32 \\
\end{array}$ & $\begin{array}{c}7.87 \\
8.96 \\
7.31 \\
3.057(5.89) \\
19.3 \\
11.35 \\
\approx 18.95 \\
\end{array}$ & $\begin{array}{l}\overline{-} \\
\bar{z} \\
\overline{-} \\
\overline{-}\end{array}$ \\
\hline \multicolumn{5}{|c|}{$\begin{array}{l}\text { Air, } 20^{\circ} \mathrm{C}, 1 \text { atm. (STP in paren.) } \\
\mathrm{H}_{2} \mathrm{O} \\
\text { Shielding concrete } \\
\mathrm{SiO}_{2} \text { (quartz) }\end{array}$} & $\begin{array}{l}62.0 \\
60.1 \\
67.4 \\
67.0\end{array}$ & $\begin{array}{l}90.0 \\
84.9 \\
99.9 \\
99.2 \\
\end{array}$ & $\begin{array}{l}1.82 \\
2.03 \\
1.70 \\
1.72 \\
\end{array}$ & $\begin{array}{l}(1.12) \\
1.72 \\
3.68 \\
3.28 \\
\end{array}$ & $\begin{array}{l}36.66 \\
36.08 \\
26.7 \\
27.05 \\
\end{array}$ & $\begin{array}{c}3420) \\
36.1 \\
10.7 \\
12.3 \\
\end{array}$ & $\begin{array}{c}.001205(1.29) \\
1.00 \\
2.5 \\
2.2 \\
\end{array}$ & $\begin{array}{c}1.000273(293) \\
1.33 \\
-\overline{1.458} \\
\end{array}$ \\
\hline \multicolumn{5}{|c|}{$\begin{array}{l}\left.\mathrm{H}_{2} \text { (bubble chamber } 26^{\circ} \mathrm{K}\right) \\
\left.\mathrm{D}_{2} \text { (bubble chamber } 31^{\circ} \mathrm{K}\right) \\
\mathrm{H} \text {-Ne mixture }(50 \text { mole percent) } \\
\end{array}$} & $\begin{array}{l}43.3 \\
45.7 \\
65.0\end{array}$ & $\begin{array}{l}50.8 \\
53.7 \\
94.5\end{array}$ & $\begin{array}{l}4.12 \\
2.07 \\
1.84 \\
\end{array}$ & $\begin{array}{l}0.20 \\
0.22 \\
0.59 \\
\end{array}$ & $\begin{array}{c}61.28 \\
122.6 \\
29.70 \\
\end{array}$ & $\begin{array}{r}\approx 1000 \\
\approx 900 \\
73.0\end{array}$ & $\begin{array}{c}\approx 0.063^{i} \\
\approx 0.140^{i} \\
0.407\end{array}$ & $\begin{array}{l}1.100 \\
1.110 \\
1.092 \\
\end{array}$ \\
\hline \multicolumn{5}{|c|}{ 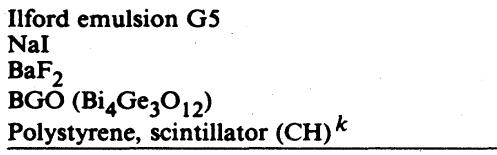 } & $\begin{array}{l}82.0 \\
94.8 \\
92.1 \\
97.4 \\
58.4 \\
\end{array}$ & $\begin{array}{c}134 \\
152 \\
146 \\
156 \\
82.0 \\
\end{array}$ & $\begin{array}{l}1.44 \\
1.32 \\
1.35 \\
1.27 \\
1.95\end{array}$ & $\begin{array}{l}4.79 \\
4.13 \\
3.78 \\
8.07 \\
1.72 \\
\end{array}$ & $\begin{array}{c}11.0 \\
9.49 \\
9.91 \\
7.98 \\
43.8 \\
\end{array}$ & $\begin{array}{r}2.89 \\
2.59 \\
2.05 \\
1.12 \\
42.4 \\
\end{array}$ & $\begin{array}{l}3.815 \\
3.67 \\
4.83 \\
7.1 \\
1.032 \\
\end{array}$ & $\begin{array}{l}1 . \overline{775} \\
1.56 \\
2.15 \\
1.581 \\
\end{array}$ \\
\hline \multicolumn{5}{|c|}{$\begin{array}{l}\text { Lucite, Plexiglas }\left(\mathrm{C}_{5} \mathrm{H}_{8} \mathrm{O}_{2}\right) \\
\text { Polyethylene }\left(\mathrm{CH}_{2}\right) \\
\text { Mylar }\left(\mathrm{C}_{5} \mathrm{H}_{4} \mathrm{O}_{2}\right) \\
\text { Borosilicate glass (Pyrex) } \\
\end{array}$} & $\begin{array}{l}59.2 \\
56.9 \\
60.2 \\
66.2 \\
\end{array}$ & $\begin{array}{l}83.6 \\
78.8 \\
85.7 \\
97.6 \\
\end{array}$ & $\begin{array}{l}1.95 \\
2.09 \\
1.86 \\
1.72 \\
\end{array}$ & $\begin{array}{l}1.98 \\
1.68 \\
2.24 \\
3.32 \\
\end{array}$ & $\begin{array}{l}40.55 \\
44.8 \\
39.95 \\
28.3 \\
\end{array}$ & 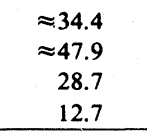 & $\begin{array}{l}1.16-1.20 \\
0.92-0.95 \\
1.39 \\
2.23 \\
\end{array}$ & $\begin{array}{c}=1.49 \\
- \\
1.474 \\
\end{array}$ \\
\hline \multicolumn{5}{|c|}{$\begin{array}{l}\mathrm{CO}_{2} \\
\text { Methane } \mathrm{CH}_{4} \\
\text { Isobutane } \mathrm{C}_{4} \mathrm{H}_{1}\end{array}$} & $\begin{array}{l}62.4 \\
54.7 \\
56.3\end{array}$ & $\begin{array}{l}90.5 \\
74.0 \\
77.4\end{array}$ & $\begin{array}{l}1.82 \\
2.41 \\
2.22\end{array}$ & $\begin{array}{l}(1.92) \\
(0.91) \\
(3.43)\end{array}$ & $\begin{array}{l}36.2 \\
46.5 \\
45.2\end{array}$ & $\begin{array}{l}(18310) \\
(64850) \\
(16930)\end{array}$ & $\begin{array}{c}(1.977) \\
0.423(0.717) \\
(2.67)\end{array}$ & $\begin{array}{r}(410) \\
(444) \\
(1270)\end{array}$ \\
\hline \multirow{2}{*}{\multicolumn{5}{|c|}{ Freon $12\left(\mathrm{CCl}_{2} \mathrm{~F}_{2}\right)$ gas, $26^{\circ} \mathrm{C}, 1$ atm. ${ }^{m}$}} & 70.6 & 106 & 1.62 & 4.49 & 23.7 & 4810 & 4.93 & 1.001080 \\
\hline & & & & & $\begin{array}{l}65.5 \\
62.6 \\
\end{array}$ & $\begin{array}{l}95.7 \\
90.2 \\
\end{array}$ & $\begin{array}{l}1.83 \\
1.87 \\
\end{array}$ & $\begin{array}{l}0.28 \\
2.7 \\
\end{array}$ & $\begin{array}{l}29.85 \\
33.0 \\
\end{array}$ & $\begin{array}{c}\approx 150 \\
\quad 19.4\end{array}$ & $\begin{array}{c}0.1-0.3 \\
1.7 \\
\end{array}$ & $\begin{array}{c}1.0+0.25 \rho \\
- \\
\end{array}$ \\
\hline
\end{tabular}

- Table revised April 1984 by Joachim Engler. For details, see Report KfK 3386B, Kernforschungzentrum, D 7500 Karlsruhe, P.O. Box 3640, FRG.

a. $\sigma_{\text {total }}$ at $80-240 \mathrm{GeV}$ for neutrons ( $\approx \sigma$ for protons) from Murthy et al., Nucl. Phys. B92, 269 (1975).

b. $\sigma_{\text {inelastic }}=\sigma_{\text {total }}-\sigma_{\text {elastic }}-\sigma_{\text {quasielastic }}$; for neutrons at $60-375 \mathrm{GeV}$ from Roberts et al., Nucl. Phys., B159, 56 (1979). For protons and other particles, see Carroll et al., Phys. Lett. $80 \mathrm{~B}, 319$ (1979); note that $\sigma_{\mathrm{I}}(\mathrm{p}) \approx \sigma_{\mathrm{I}}(\mathrm{n})$.

c. Mean free path between collisions $\left(\lambda_{T}\right)$ or inelastic interactions $\left(\lambda_{I}\right)$, calculated from $\lambda=A /(N \times \sigma)$.

d. For minimum-ionizing protons and pions. $\overline{\Delta \mathrm{E}}$ is energy loss per $\mathrm{g} / \mathrm{cm}^{2}$ from Barkas and Berger, Tables of Energy Losses and Ranges of Heavy Charged Particles, NASA-SP-3013 (1964). For electrons and positrons see: M.J. Berger and S.M. Seltzer, Stopping Powers and Ranges of Electrons and Positrons ( $2^{\text {nd }}$ Ed.), U.S. National Bureau of Standards report NBSIR 82-2550-A (1982). $\mathrm{E}_{\mathrm{mp}}$ is the most probable deposited energy in one cm, in $\mathrm{MeV}$ for solids and liquids, in $\mathrm{keV}$ for gases. $\mathrm{E}_{\mathrm{mp}}$ varies with depth in a nonproportional manner. (See Sec. C.1 preceding.) Parentheses refer to gaseous form at STP $\left(0^{\circ} \mathrm{C}, 1 \mathrm{~atm}.\right)$.

e. From Y.S. Tsai, Rev. Mod. Phys. 46, 815 (1974). Corrections for molecular binding applied for $\mathrm{H}_{2}$, and $\mathrm{D}_{2}$. Parentheses refer to gaseous form at STP $\left(0^{\circ} \mathrm{C}, 1 \mathrm{~atm}.\right)$

f. Values for solids, or the liquid phase at boiling point, except as noted. Values in parentheses for gaseous phase at STP $\left(0^{\circ} \mathrm{C}\right.$, 1 atm.). Refractive index given for sodium $D$ line.

g. For pure graphite; industrial graphite density may vary $2.1-2.3 \mathrm{~g} / \mathrm{cm}^{3}$

h. Standard shielding blocks, typical composition $\mathrm{O}_{2} 52 \%, \mathrm{Si} 32.5 \%, \mathrm{Ca} 6 \%, \mathrm{Na} 1.5 \%$, Fe $2 \%$, Al $4 \%$, plus reinforcing iron bars. The attenuation length, $\ell=115 \pm 5 \mathrm{~g} / \mathrm{cm}^{2}$, is also valid for earth (typical $\rho=2.15$ ), from CERN-LRL-RHEL Shielding exp., UCRL-17841 (1968).

$i$. Density may vary about $\pm 3 \%$, depending on operating conditions.

$j$. Values for typical working conditions with $\mathrm{H}_{2}$ target: 50 mole percent, $29^{\circ} \mathrm{K}, 7 \mathrm{~atm}$.

$k$. Typical scintillator; e.g., PILOT $B$ and $\mathrm{NE} 102 \mathrm{~A}$ have an atomic ratio $\mathrm{H} / \mathrm{C}=1.10$.

$\ell$. Main components: $80 \% \mathrm{SiO}_{2}+12 \% \mathrm{~B}_{2} \mathrm{O}_{3}+5 \% \mathrm{Na}_{2} \mathrm{O}$.

$m$. Used in Cerenkov counters. Values at $26^{\circ} \mathrm{C}$ and 1 atm. Indices of refraction from E.R. Hayes, R.A. Schluter, and A. Tamosaitis, ANL-6916 (1964).

n. $\mathrm{n}\left(\mathrm{SiO}_{2}\right)+2 \mathrm{n}\left(\mathrm{H}_{2} \mathrm{O}\right)$ used in Cerenkov counters, $\rho=$ density in $\mathrm{g} / \mathrm{cm}^{3}$. From M. Cantin et al., Nucl. Instr. Meth. 118, 177 (1974).

o. G10-plate, typical $60 \% \mathrm{SiO}_{2}$ and $40 \%$ Epoxy. 
PARTICLE DETECTORS, ABSORBERS, AND RANGES (Cont'd)

Mean Range and Energy Loss in Lead, Copper, Aluminum, and Carbon

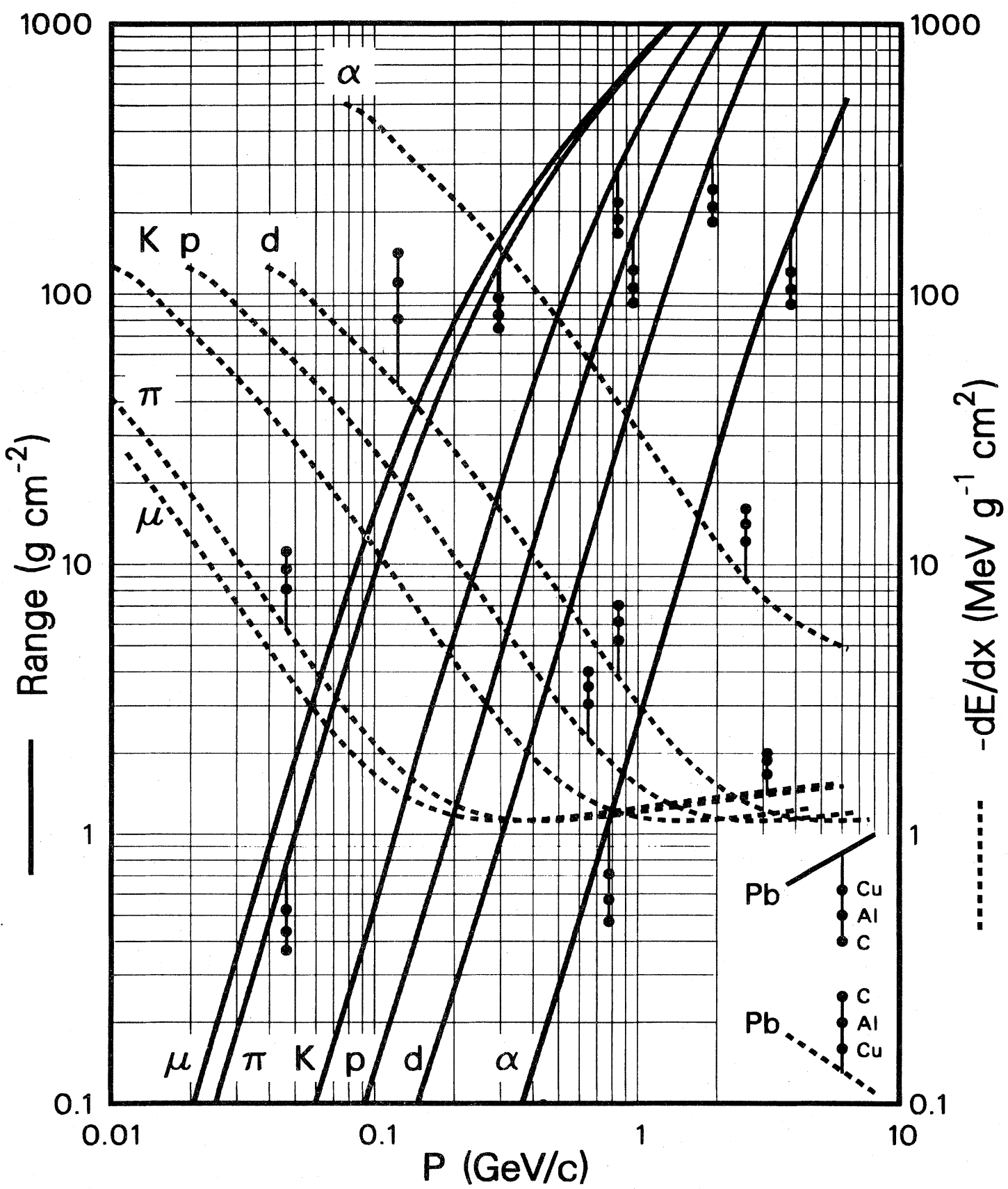

Mean range and energy loss due to ionization for the indicated particles in $\mathrm{Pb}$, with scaling to $\mathrm{Cu}, \mathrm{Al}$, and $\mathrm{C}$ indicated, using Bethe- $\mathrm{Bloch}$ equation (Section C.1 above) with corrections. Calculated by M.J. Berger, using ionization potentials and density effect corrections as discussed in M.J. Berger and S.M. Seltzer, "Stopping Powers and Ranges of Electrons and Positrons," (2 ${ }^{\text {nd }}$ ed.), U.S. National Bureau of Standards Report NBSIR 82-2550-A (1982). The average ionization potentials (I) assumed were: $\mathrm{Pb}(823 \mathrm{eV}), \mathrm{Cu}(322 \mathrm{eV}), \mathrm{Al}(166 \mathrm{eV})$, and C $(78.0 \mathrm{eV})$. Figure indicates total path length; observed range may be smaller (by $\sim 1 \%-2 \%$ in heavy elements) due to multiple scattering, primarily from small energy-loss collisions with nuclei. The functional forms have not been experimentally verified to better than roughly $\pm 1 \%$. For higher energies refer to discussion by Cobb ["A Study of Some Electromagnetic Interactions of High Velocity Particles with Matter," University of Oxford Report HEP/T/55 (1973)] and by Turner ["Penetration of Charged Particles in Matter: A Symposium," National Academy of Sciences, Washington D.C. (1970), p. 48]. Scaling to other beam particles is, to a good approximation, described by the expression on the next page. 
PARTICLE DETECTORS, ABSORBERS, AND RANGES (Cont'd)

\section{Mean Range and Energy Loss in Liquid Hydrogen}

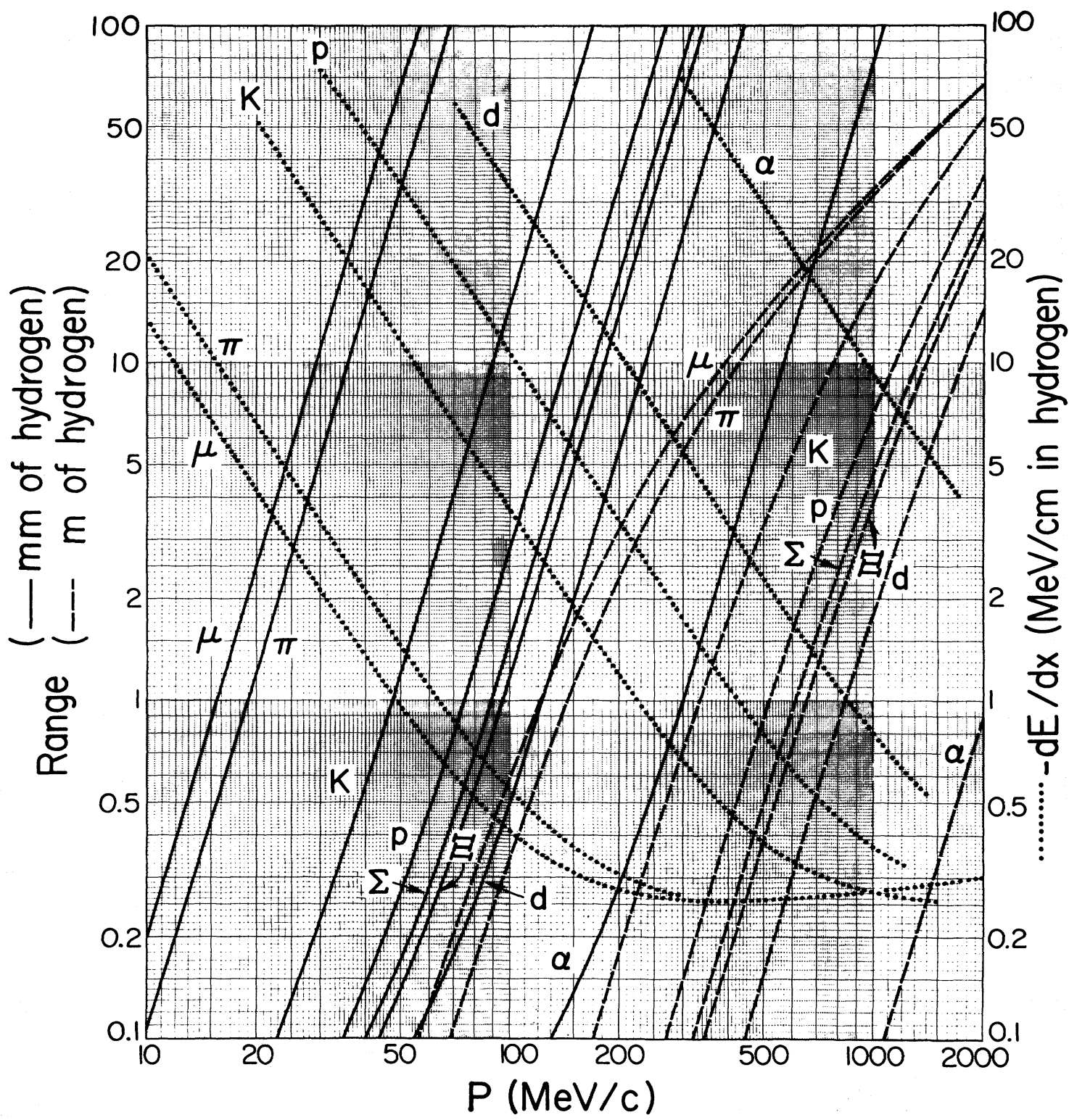

Range and energy loss in liquid hydrogen bubble chamber, based on Bethe-Bloch equation (Section C.1 above), using an average ionization potential for $\mathrm{H}_{2}$ of $\mathrm{I}=20.0 \mathrm{eV}$, which is an approximate average of the experimental result of Garbincius and Hyman [Phys. Rev. A2, 1834 (1970)] and the theoretical result of Ford and Browne [Phys. Rev. A7, 418 (1973)]. Bubble chamber conditions are chosen to be those of Garbincius and Hyman: parahydrogen of density $=0.0625 \mathrm{~g} / \mathrm{cm}^{3}$ (note: range $\propto 1 /$ density), with vapor-pressure $60.8 \mathrm{lb} / \mathrm{in}^{2}$ (absolute) and temperature $26.2^{\circ} \mathrm{K}$. The functional dependence of the Bethe-Bloch equation is not experimentally verified to better than about $\pm 1 \%$ over large momentum ranges. It should be noted that the number of bubbles per $\mathrm{cm}$ of a track in a bubble chamber is nearly proportional to $1 / \beta^{2}$, not $\mathrm{dE} / \mathrm{dx}$. For the linear portions of the range curves, $R \propto \mathrm{p}^{3.6}$. Scaling law for particles of other mass or charge (except electrons): for a given medium, the range $R_{b}$ of any beam particle with mass $\mathbf{M}_{b}$, charge $z_{b}$, and momentum $p_{b}$ is given in terms of the range $R_{a}$ of any other particle with mass $M_{a}$, charge $z_{a}$, and momentum $p_{a}=p_{b} M_{a} / M_{b}$ (i.e., having the same velocity) by the expression:

$$
\mathbf{R}_{b}\left(M_{b}, \mathbf{z}_{b}, p_{b}\right)=\left[\frac{M_{b} / M_{a}}{z_{b}^{2} / z_{a}^{2}}\right] R_{a}\left(M_{a}, z_{a}, p_{a}=p_{b} M_{a} / M_{b}\right) .
$$


PARTICle DETECTORS, ABSORBERS, AND RANGES (Cont'd)

Photon Mass Attenuation Coefficients, Energy Deposition

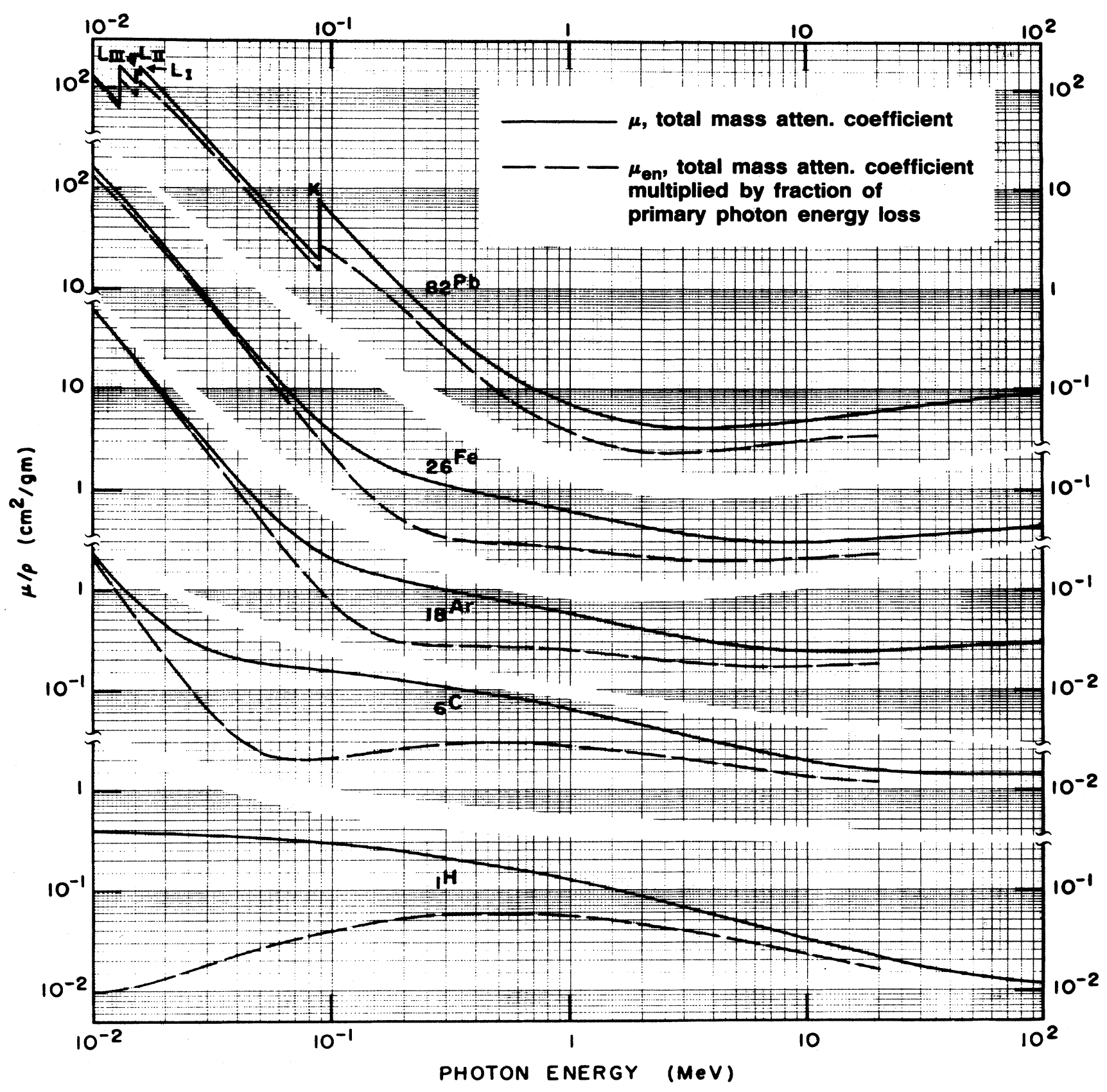

The photon mass attenuation coefficient $\mu$ for various absorbers as a function of photon energy (solid curves). For a homogeneous medium of density $\rho$, the intensity $I$ remaining after traversal of thickness $t$ is given by $I=I_{0} \exp (-\mu t)$. The accuracy is a few percent. Interpolation to other $\mathrm{Z}$ should be done in the cross section $\sigma=(\mu / \rho) \mathrm{A} / \mathrm{N}_{\mathrm{A}} \mathrm{cm}^{2} /$ atom, where $\mathrm{A}$ is the atomic weight of the absorber material and $\mathrm{N}_{\mathrm{A}}$ is Avogadro's number. For a chemical compound or mixture, use $(\mu / \rho)_{e f f} \cong \Sigma w_{i}(\mu / \rho)_{i}$, accurate to a few percent, where $w_{i}$ is the proportion by weight of the $\mathrm{i}^{\text {th }}$ constituent. See next page for high energy range. The dashed curves give the mass energy-absorption coefficient $\mu_{\text {en }}$, which is $\mu$ multiplied by the fraction of photon energy deposited in a small volume (assumed large enough to contain the ranges of most secondary electrons) about the interaction. This fraction is smaller than 1.0 because such processes as Compton scattering and electron bremsstrahlung imply radiation of some of the energy away from the immediate area. The absorption coefficient is an approximation to the energy available for chemical, biological, and other effects associated with exposure to ionizing radiation. At high energies, the range of secondary electrons tends to become comparable to the photon mean free path, and $\mu_{\text {en }}$ is not a useful approximation. From Hubbell, Gimm, and Фverbф, J. Phys. Chem. Ref. Data 9, 1023 (1980). See also J.H. Hubbell, Int. J. of Applied Rad. and Isotopes 33, 1269 (1982). Figures courtesy J.H. Hubbell. 


\section{PARTICLE DETECTORS, ABSORBERS, AND RANGES (Cont'd)}

\section{Photon Mass Attenuation Coefficients (High Energy)}

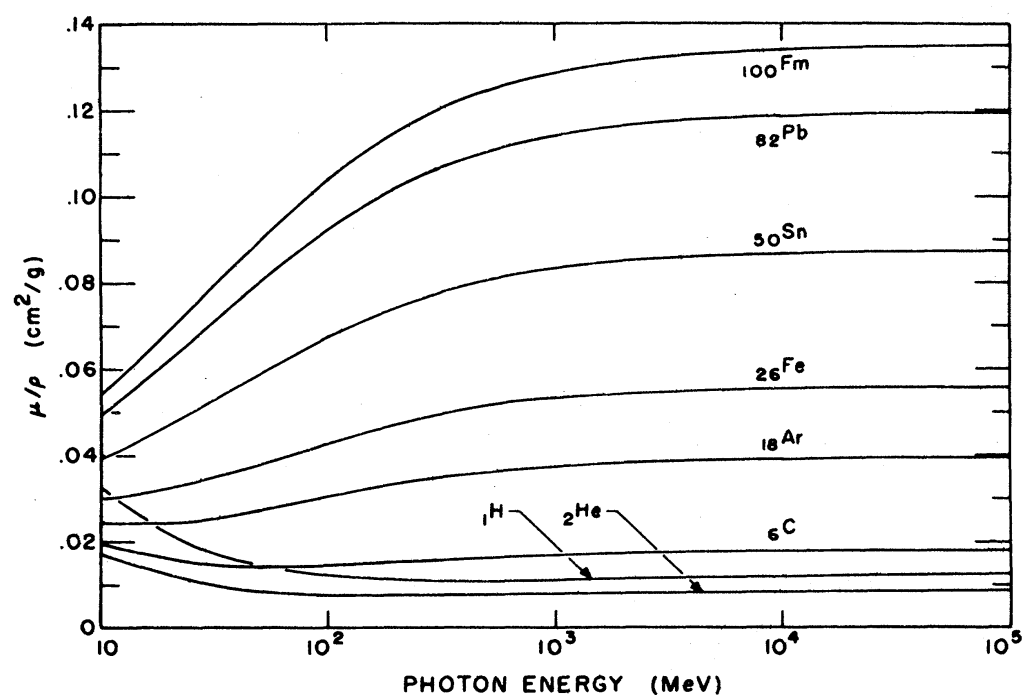

The photon mass attenuation coefficient, high energy range (note that ordinate is linear scale). See caption on previous page for details.
Fractional Energy Loss for Electrons and Positrons in Lead

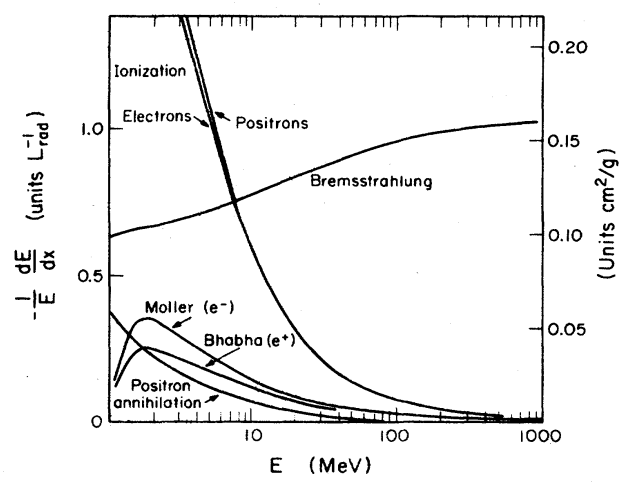

Fractional energy loss per radiation length in lead as a function of electron or positron energy. Electron (positron) scattering is considered as ionization when the energy loss per collision is below $0.255 \mathrm{MeV}$, and as Moller (Bhabha) scattering when it is above. Adapted from Fig. 3.2 from Messel and Crawford, Electron-Photon Shower Distribution Function Tables for Lead, Copper, and Air Absorbers, Pergamon Press, 1970. Messel and Crawford use $\mathrm{L}_{\mathrm{r}}(\mathrm{Pb})=5.82 \mathrm{~g} / \mathrm{cm}^{2}$, but we have modified the figures to reflect the value given in the Table of Atomic and Nuclear Properties of Materials (following), namely $L_{r}(\mathrm{~Pb})=6.4 \mathrm{~g} / \mathrm{cm}^{2}$. The development of electron-photon cascades is approximately independent of absorber when the results are expressed in terms of inverse radiation lengths (i.e., scale on left of plot).

\section{Contributions to Photon Cross Section in Carbon and Lead}
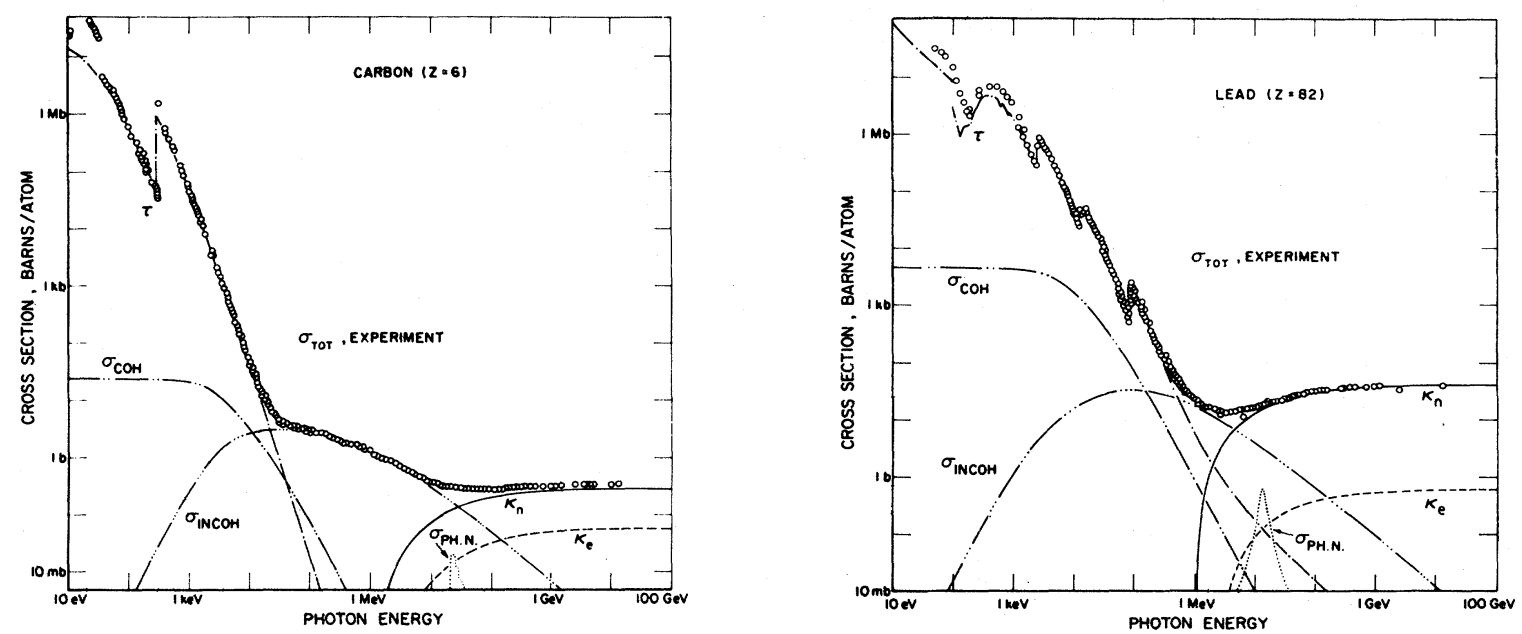

Photon total cross sections as a function of energy in carbon and lead, showing the contributions of different processes.

$$
\begin{aligned}
& \tau \text { - Atomic photo-effect (electron ejection, photon absorption) } \\
& \sigma_{\mathrm{COH}}-\text { Coherent scattering (Rayleigh scattering - atom neither ionized nor excited) } \\
& \sigma_{\text {INCOH }}=\text { Incoherent scattering (Compton scattering off an electron) } \\
& \kappa_{n} \text { - Pair production, nuclear field } \\
& \kappa_{\mathrm{e}} \quad-\text { Pair production, electron field } \\
& \sigma_{\text {PH.N. }} \text { - Photonuclear absorption (nuclear absorption, usually followed by emission of } \\
& \text { a neutron or other particle) }
\end{aligned}
$$

From Hubbell, Gimm, and $\emptyset$ verb $\phi$ J. Phys. Chem. Ref. Data 9, 1023 (1980). Figures courtesy J.H. Hubbell. 


\section{ELECTROMAGNETIC RELATIONS}

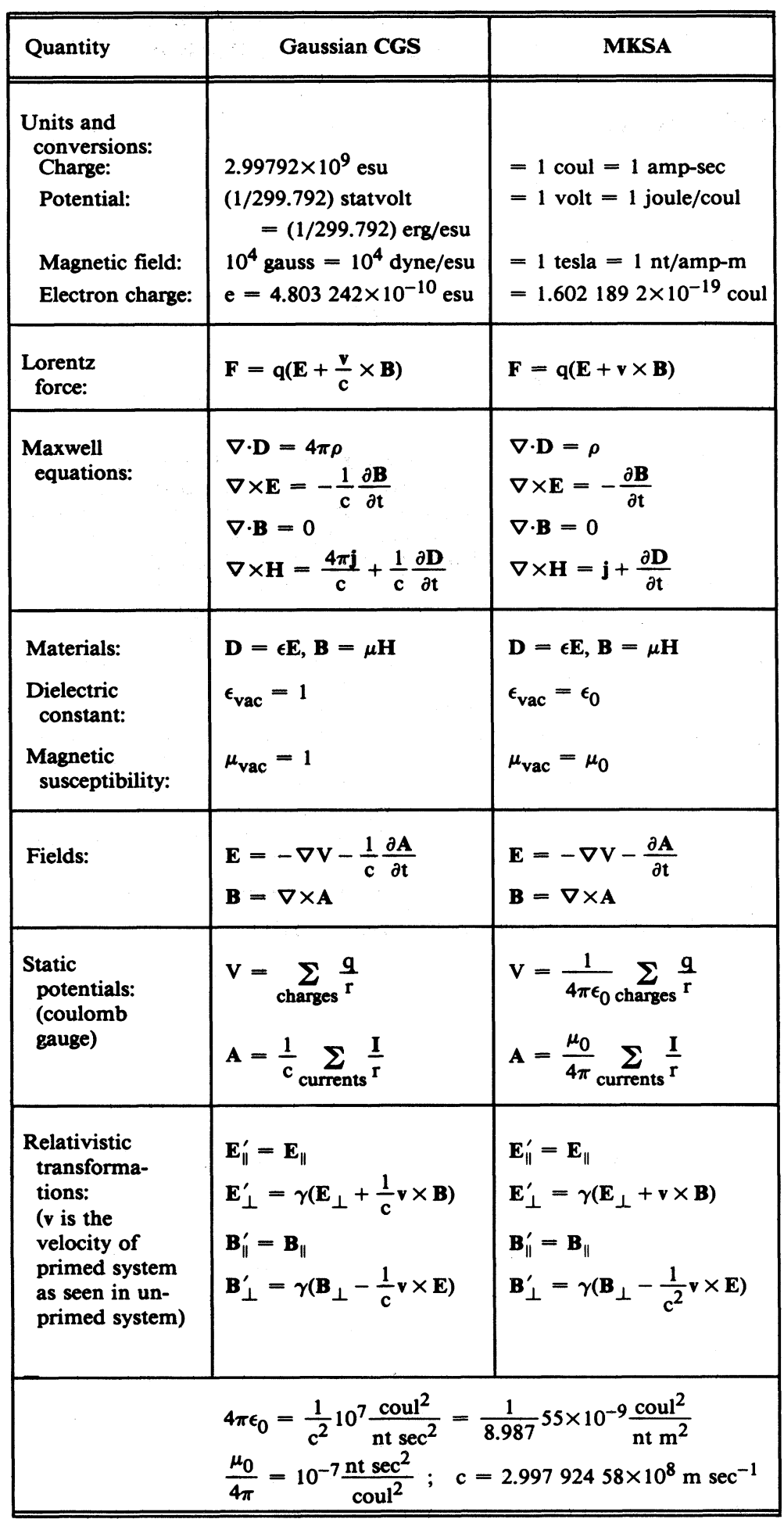

Impedances (MKSA)

$\rho=$ resistivity in $10^{-8} \Omega \mathrm{m}$ :

$$
\begin{aligned}
& \sim 1.7 \text { for } \mathrm{Cu} \\
& \sim 2.4 \text { for } \mathrm{Au} \\
& \sim 2.8 \text { for } \mathrm{Al} \\
& \text { (Al alloys may have } \\
& \text { double this value.) }
\end{aligned}
$$

$$
\begin{aligned}
& \sim 5.5 \text { for } W \\
& \sim 73 \text { for SS } 304 \\
& \sim 100 \text { for Nichrome }
\end{aligned}
$$

For alternating currents, instantaneous current $I$, voltage $V$, angular frequency $\omega$ :

$$
\mathbf{V}=\mathbf{V}_{0} \mathrm{e}^{\mathrm{i} \omega t}=\mathbf{Z I}
$$

Impedance of self-inductance $L: Z=i \omega L$.

Impedance of capacitance $C: Z=1 / i \omega C$.

Impedance of free space: $Z=\sqrt{\mu_{0} / \epsilon_{0}}=376.7 \Omega$.

Impedance per unit length of a flat conductor of width $w$ (high frequency, $\nu$ ):

$$
\begin{aligned}
& Z=\frac{(1+\mathrm{i}) \rho}{w \delta}, \text { where } \delta=\text { effective skin depth } \\
& \delta=\sqrt{\frac{\rho}{\pi \nu \mu}} \cong \frac{6.6 \mathrm{~cm}}{\sqrt{\nu\left(\sec ^{-1}\right)}} \text { for } \mathrm{Cu} .
\end{aligned}
$$

Capacitance $\hat{\mathbf{C}}$ and inductance $\hat{\mathbf{L}}$ per unit length (MKSA)

Flat rectangular plates of width $w$, separated by $d \ll w$ :

$$
\begin{aligned}
& \hat{\mathrm{C}}=\epsilon \frac{\mathrm{w}}{\mathrm{d}} ; \hat{\mathrm{L}}=\mu \frac{\mathrm{d}}{\mathrm{w}} ; \\
& \frac{\epsilon}{\epsilon_{0}}=2 \text { to } 6 \text { for plastics; } 4 \text { to } 8 \text { for porcelain, glasses. }
\end{aligned}
$$

Coaxial cable of inner radius $r_{1}$, outer radius $r_{2}$ :

$$
\hat{\mathrm{C}}=\frac{2 \pi \epsilon}{\ell \mathrm{n}\left(\mathrm{r}_{2} / \mathrm{r}_{1}\right)} ; \hat{\mathrm{L}}=\frac{\mu}{2 \pi} \ln \left(\mathrm{r}_{2} / \mathrm{r}_{1}\right) .
$$

Transmission lines (no loss):

$$
\begin{aligned}
& \text { Impedance: } Z=\sqrt{\hat{\mathrm{L}} / \hat{\mathrm{C}}} . \\
& \text { Velocity: } v=1 / \sqrt{\hat{\mathrm{L}} \hat{\mathrm{C}}}=1 / \sqrt{\mu \epsilon} .
\end{aligned}
$$

Motion of charged particles in a uniform, static, magnetic field

The path of motion of a charged particle of momentum $p$ is a helix of constant radius $R$ and constant pitch angle $\lambda$, with the axis of the helix along $B$ :

$$
\mathrm{p}(\mathrm{GeV} / \mathrm{c}) \cos \lambda=0.29979 \mathrm{q} \mathrm{B}(\text { tesla }) \mathrm{R}(\mathrm{m}),
$$

where the charge $q$ is in units of the electronic charge. The angular velocity about the axis of the helix is

$$
\omega(\mathrm{rad} \mathrm{sec}-1)=8.98755 \times 10^{7} \mathrm{q} \mathrm{B}(\text { tesla }) / \mathrm{E}(\mathrm{GeV}),
$$

where $E$ is the energy of the particle. 


\section{ELECTROMAGNETIC RELATIONS (Cont'd)}

\section{Synchrotron radiation (CGS)}

For a relativistic particle of charge e, velocity $\beta, \gamma$, energy $E$, traveling in a circular orbit of radius $\mathbf{R}$ :

Energy loss/revolution (MeV) $=\frac{4 \pi}{3} \frac{\mathrm{e}^{2}}{\mathrm{R}} \beta^{3} \gamma^{4}$

$$
\simeq 0.0885[\mathrm{E}(\mathrm{GeV})]^{4} / \mathrm{R}(\mathrm{m}) \text { for } \mathrm{e}^{ \pm} \text {if } \beta \cong 1
$$

Energy spectrum: The energy radiated into the photon energy interval $d(h \omega)$ is

$$
\mathrm{dI}=\alpha \gamma \mathrm{F}\left(\omega / \omega_{\mathrm{c}}\right) \mathrm{d}(\hbar \omega),
$$

where $\alpha=\mathrm{e}^{2} /(h \mathrm{c})$ is the fine-structure constant, $F(y)=2 \sqrt{3} y \int_{2 y}^{\infty} d x K_{5 / 3}(x)$, with $K_{5 / 3}(x)$ a modified spherical Bessel function of the third kind, and $\omega_{c}=3 \gamma^{3} \mathrm{c} / \mathrm{R}$ is a critical frequency;

$$
h \omega_{\mathrm{c}}(\mathrm{keV}) \simeq 4.44[\mathrm{E}(\mathrm{GeV})]^{3} / \mathrm{R}(\mathrm{m}) \text { for } \mathrm{e}^{ \pm} \text {if } \beta \simeq 1
$$

In the limit $\gamma \gg 1$,

$$
\begin{aligned}
& \text { for } \omega \ll \omega_{c} \text { : } \\
& \frac{\mathrm{dI}}{\mathrm{d}(h \omega)} \cong 3.3 \alpha\left(\frac{\omega \mathrm{R}}{\mathrm{c}}\right)^{1 / 3} \text {; } \\
& \text { for } \frac{\omega}{\omega_{c}}=(0.01,0.1,0.2,1.0,2.0) \text { : } \\
& \frac{d I}{d(h \omega)} \simeq(1.0,1.6,1.6,0.5,0.08) \alpha \gamma, \text { respectively; }
\end{aligned}
$$

for $\omega \gtrsim 2 \omega_{\mathrm{c}}$ :

$$
\frac{\mathrm{dI}}{\mathrm{d}(h \omega)} \cong \sqrt{3 \pi} \alpha \gamma\left(\frac{\omega}{\omega_{\mathrm{c}}}\right)^{1 / 2} \mathrm{e}^{-2 \omega / \omega_{\mathrm{c}}} .
$$

The radiation is confined to angles $\leqslant 1 / \gamma$ relative to the instantaneous direction of motion.

See J.D. Jackson, Classical Electrodynamics, 2nd edition (John Wiley \& Sons, New York, 1975) for more formulae and details. (Prepared April 1974; revised April 1984.)

\section{RADIOACTIVITY AND RADIATION PROTECTION}

The International Commission on Radiation Units and Measurements (ICRU) recommends the use of SI units. Therefore we list SI units first, followed by cgs (or other common) units in parentheses, where they differ.

Unit of activity = becquerel (curie):

$1 \mathrm{~Bq}=1$ disintegration $/ \mathrm{sec}\left[=1 /\left(3.7 \times 10^{10}\right) \mathrm{Ci}\right]$.

Unit of exposure, the quantity of $X$ - or $\gamma$-radiation at a point in space integrated over time, in terms of charge of either sign produced by showering electrons in a small volume of air about the point:

$=1 \mathrm{coul} / \mathrm{kg}$ of air (roentgen; $1 \mathrm{R}=2.58 \times 10^{-4} \mathrm{coul} / \mathrm{kg}$

$=1 \mathrm{esu} / \mathrm{cm}^{3}=87.8 \mathrm{erg}$ released energy per $\mathrm{g}$ of air); implicit

in the definition is the assumption that the small test volume is embedded in a sufficiently large uniformly irradiated volume that the number of secondary electrons entering the volume equals the number leaving.

Unit of absorbed dose = gray (rad):

$1 \mathrm{~Gy}=1$ joule $/ \mathrm{kg}\left(=10^{4} \mathrm{erg} / \mathrm{g}=10^{2} \mathrm{rad}\right)$

$=6.24 \times 10^{12} \mathrm{MeV} / \mathrm{kg}$ deposited energy.

Unit of dose equivalent (for biological damage) $=$ sievert $\left[=10^{2}\right.$ rem (roentgen equivalent for man)]:

Dose equivalent in $\mathbf{S v}=$ grays $\times \mathrm{Q}$, where $\mathrm{Q}$ (quality factor) expresses long-term risk (primarily cancer and leukemia) from low-level chronic exposure; it depends upon the type of radiation and other factors. For $\gamma$ rays and $\beta$ particles, $Q \simeq 1$; for protons, $Q \simeq 1$ at $\sim 10 \mathrm{MeV}$, rising gradually to $\simeq 2$ at $\sim 1$ $\mathrm{GeV}$; for thermal neutrons, $Q \simeq 3$; for fast neutrons, $Q$ ranges up to 10; and for $\alpha$ particles and heavy ions (assuming internal deposition - skin and clothing are usually sufficient protection against external sources), $Q \simeq 20$.

Natural annual background, all sources: Most world areas, wholebody dose equivalent rate $\simeq(0.4-4) \mathrm{mSv}(40-400$ millirems) .
Can range up to $50 \mathrm{mSv}$ ( 5 rems) in certain areas. U.S. average $\simeq 0.8 \mathrm{mSv}$. The lungs receive an additional $\cong 0.1 \mathrm{mSv}(\simeq 10$ mrem) from inhaled natural radioactivity, mostly radon and radon daughters (good to $\cong$ factor of 2 in open areas; can range an order of magnitude higher in buildings and up to $1000 \times$ in poorly ventilated mines).

Cosmic ray background in counters (Earth's surface): $\sim 10^{4} / \mathrm{min} / \mathrm{m}^{2} / \mathrm{ster}$. For more accurate estimates and more details, see Sec. B of Particle Detectors, Absorbers, and Ranges.

Fluxes (per $\mathrm{m}^{2}$ ) to deposit one $\mathrm{Gy}$ in one $\mathrm{kg}$ of matter, assuming uniform irradiation:

$\simeq$ (charged particles) $6.24 \times 10^{12} /(\mathrm{dE} / \mathrm{dx})$, where $\mathrm{dE} / \mathrm{dx}(\mathrm{MeV}$ $\mathrm{m}^{2} / \mathrm{kg}$ ), the energy loss per unit length, may be obtained (after conversion of units) from the Mean Range and Energy Loss figures.

$\cong 3.5 \times 10^{13}$ minimum-ionizing singly charged particles in carbon. $\cong$ (photons) $6.24 \times 10^{12} /\left[\mathrm{E}(\mathrm{MeV})\left(\mu_{\mathrm{en}} / \rho\right)\left(\mathrm{m}^{2} / \mathrm{kg}\right)\right]$, for photons of energy $E$, mass energy absorption coefficient $\mu_{\mathrm{en}}$, and density $\rho$ (see Photon Mass Attenuation Coefficients, Energy Deposition figure), for samples of thickness enough to contain the secondary electrons but $\ll 1 / \mu$

$\simeq 2 \times 10^{15}$ photons of $1 \mathrm{MeV}$ energy on carbon. (Quoted fluxes good to about a factor of 2 for all materials.)

U.S. maximum permissible occupational dose for the whole body: $50 \mathrm{mSv} /$ year (5 rem/year).

Lethal dose: Whole-body dose from penetrating ionizing radiation resulting in $50 \%$ mortality in $\mathbf{3 0}$ days (assuming no medical treatment), 2.5-3.0 Gy (250-300 rads) as measured internally on body longitudinal center line; surface dose varies due to variable body attenuation and may be a strong function of energy.

For a recent review, see E. Pochin, Nuclear Radiation: Risks and Benefits (Clarendon Press, Oxford, 1983). 
PERIODIC TABLE OF THE ELEMENTS

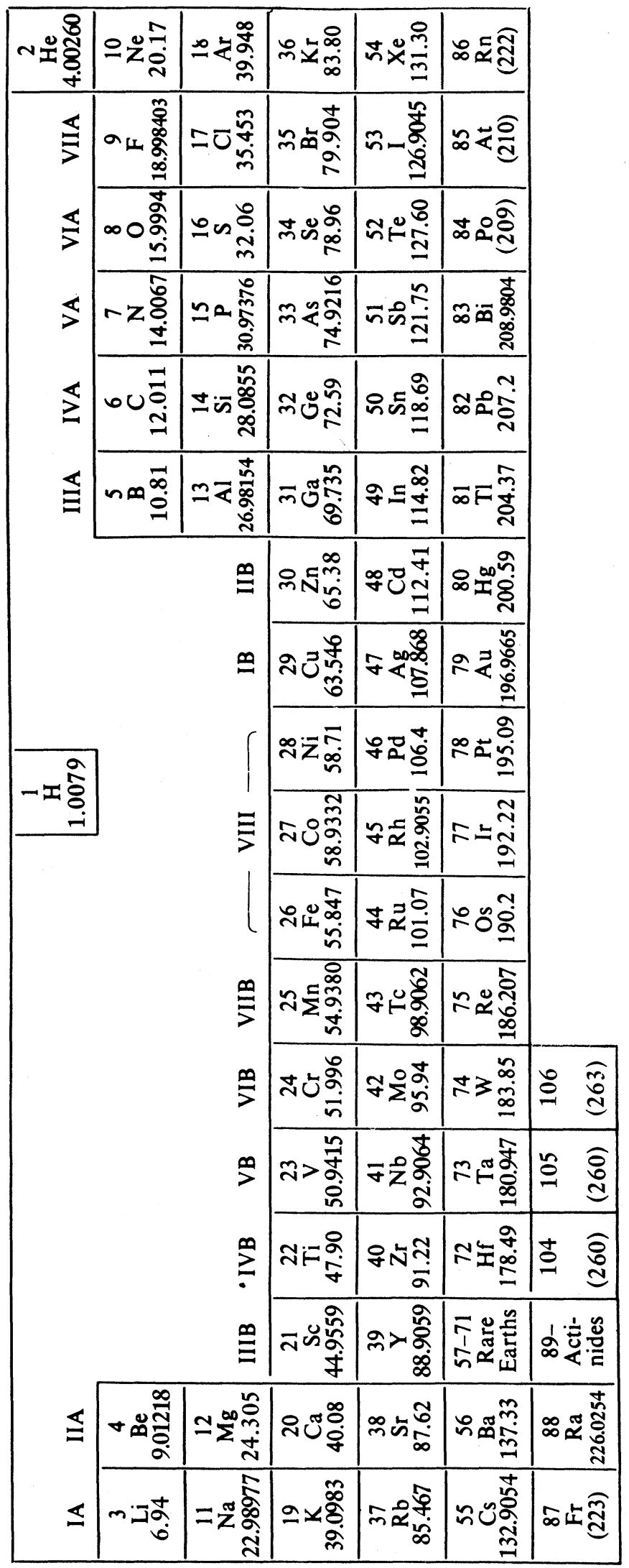

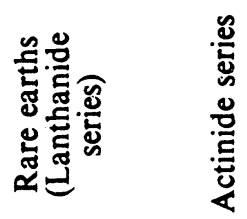

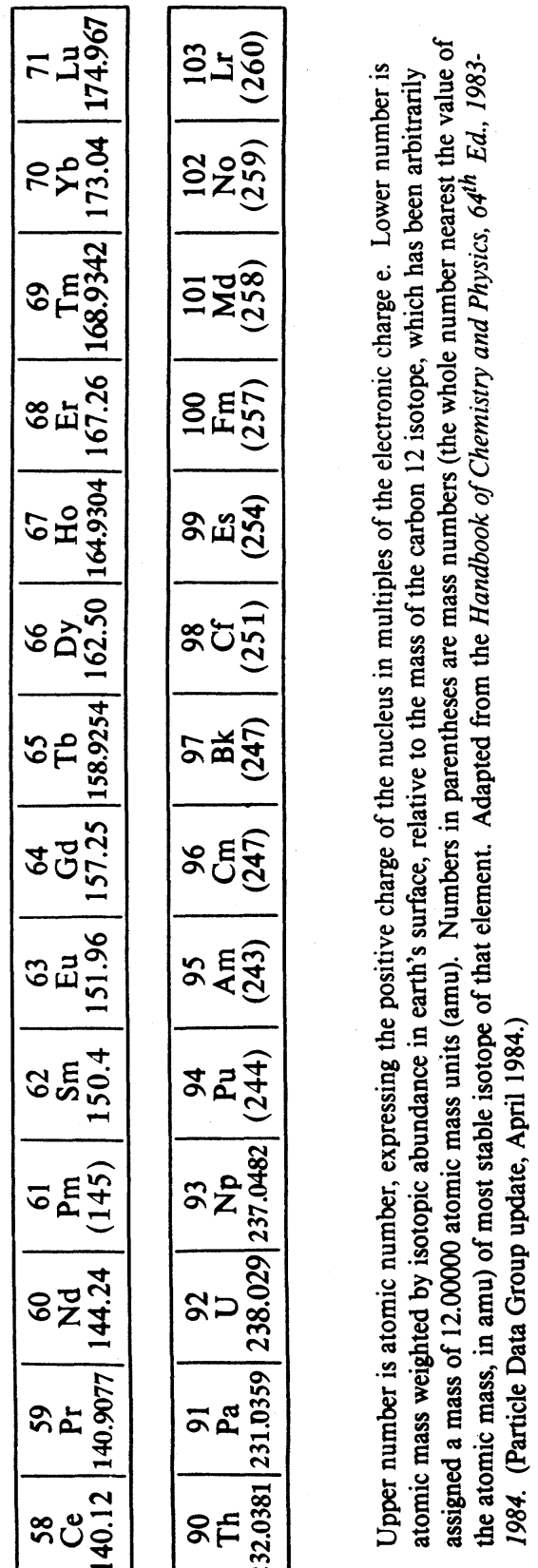




\section{PLOTS OF CROSS SECTIONS AND RELATED QUANTITIES}

NOTE: THE FIgURES IN THIS SECTION ARE INTENDED TO SHOW THE "BEST" OR "MOST REPRESENTATIVE" DATA IN THE OPINION OF THE COMPILER. THEY ARE NOT NECESSARILY COMPLETE COMPILATIONS OF ALL THE WORLD'S RELIABLE DATA.

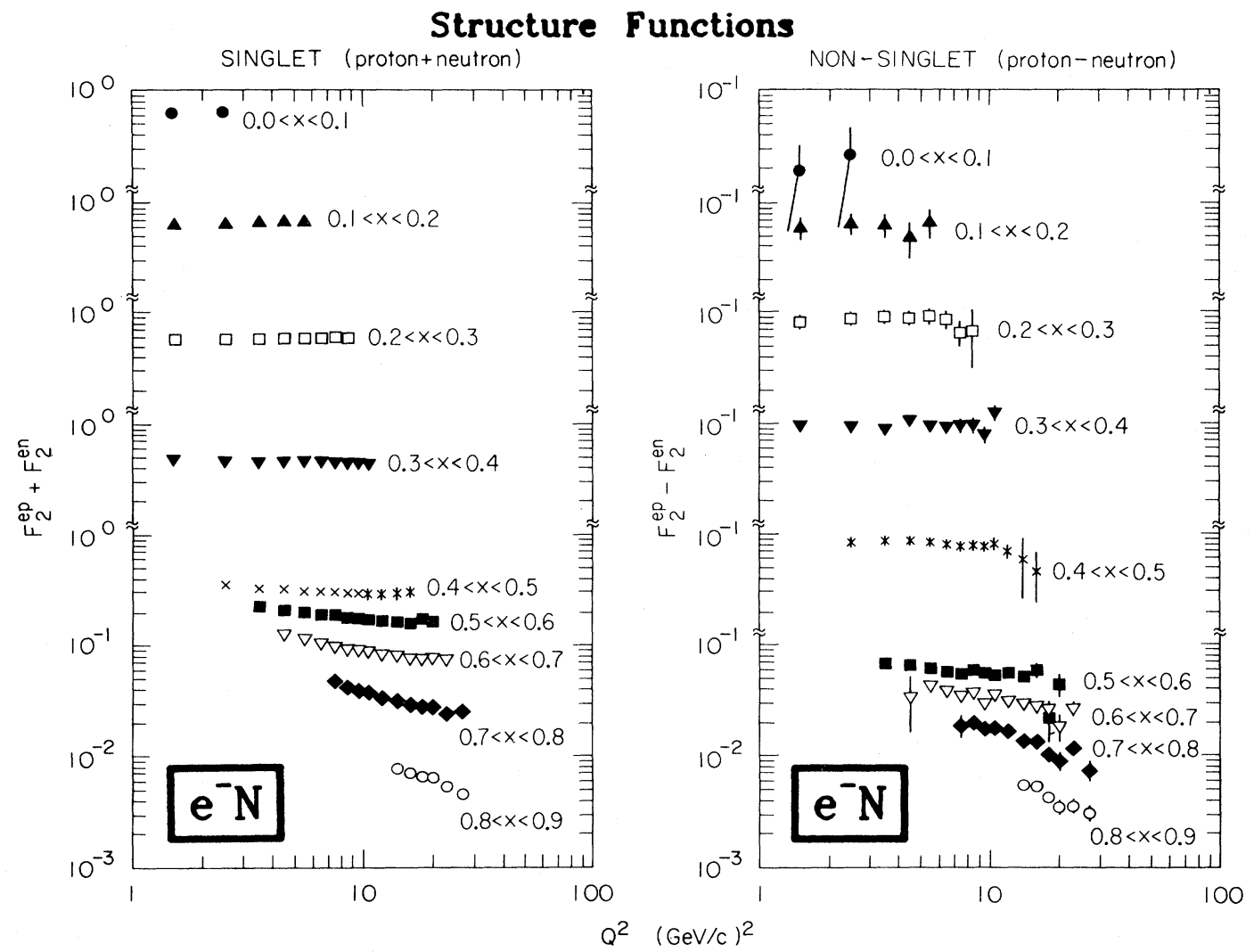

$F_{2}$ structure functions derived from inelastic electron-nucleon data taken at SLAC ${ }^{1-4}$ with recoil mass $>.2 \mathrm{GeV}$ and four-momentum transfer squared $Q^{2}>1(\mathrm{GeV} / \mathrm{c})^{2}$ are shown. For definitions of $F_{2}, x$, and $Q^{2}$, see the Kinematics, Decays, and Scattering Section. $\mathbf{R}=\sigma_{\mathrm{L}} / \sigma_{\mathrm{T}}=0.21^{3}$ was assumed. Systematic errors are comparable in size to the data point symbols. Corrections for nucleon motion in deuterium have been made. These corrections are small except for $\mathbf{x}>0.7$. No error was included to account for uncertainties in this correction. References: 1) A. Bodek et al., Phys. Rev. D20, 1471 (1979); 2) W.B. Atwood et al., SLAC Report No. 185 (1975); 3) M.D. Mestayer, SLAC Report No. 214 (1978); 4) S. Stein et al., Phys. Rev. D12, 1884 (1975). Courtesy W.B. Atwood, SLAC.

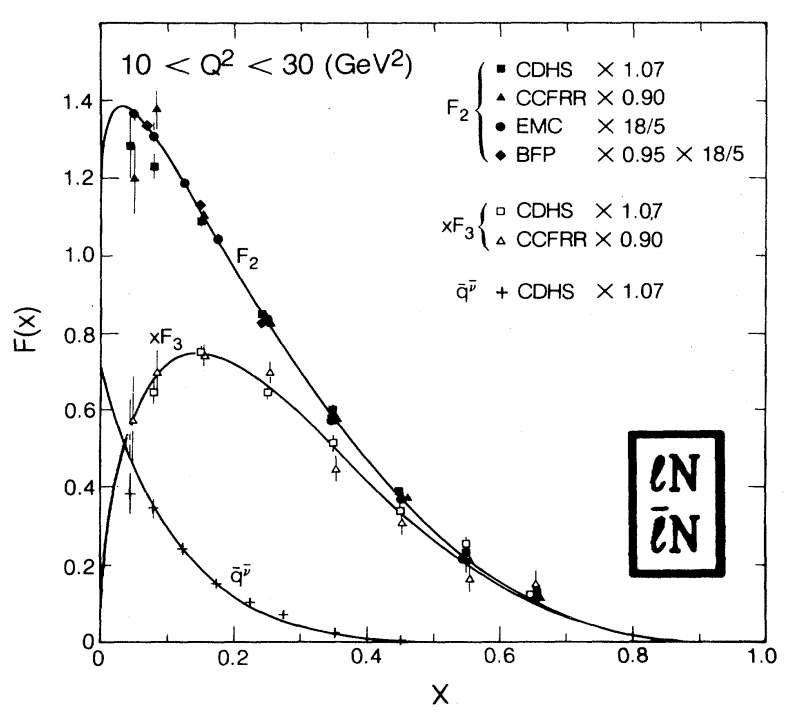

Structure functions $F_{2}, x F_{3}$, and $\overline{q^{\nu}}$, measured in different experiments, for fixed $Q^{2}$ versus $x$, plotted assuming $R=\sigma_{L} / \sigma_{T}=0$. The electromagnetic structure function $\mathrm{F}_{2}^{\mu \mathrm{N}}$ measured by $\mathrm{EMC}$ and BFP is compared with the charged-current structure function $\mathrm{F}_{2}^{\nu \mathrm{N}}$ using the 18/5 factor from the average charge squared of the quarks. No correction has been applied for the difference between the strange and charm sea quarks so the interpretation is $F_{2}=$ $x\left[q+\bar{q}-\frac{3}{5}(s+\bar{s}-c-\bar{c})\right]$. (In this $Q^{2}$ range, $F_{2}^{\nu N}$ is depleted by a similar amount due to charm threshold effects in the transition $s \rightarrow c$.) The antiquark distribution measured from antineutrino scattering is $\overline{\mathrm{q}}^{\bar{v}}=x(\bar{u}+\bar{d}+2 s)$. The solid lines have the forms: $F_{2}=3.9 x^{0.55}(1-x)^{3.2}+1.1(1-x)^{8}, x_{3}=3.6 x^{0.55}(1-x)^{3.2}$, $\frac{\bar{q}^{\nu}}{\mathrm{q}^{2}}=0.7(1-x)^{8}$. Relative normalization factors have been fitted to optimize agreement between the different data sets, and absolute changes have been arbitrarily chosen as indicated. References: CDHS - H. Abramowicz et al., Zeit. Phys. C17, 283 (1983); CCFRR - F. Sciulli, private communication; EMC - J.J. Aubert et al., Phys. Lett. 105B, 322 (1981); and A. Edwards, private communication; BFP - A.R. Clark et al., Phys. Rev. Lett. 51, 1826 (1983); and P. Meyers, Ph.D. Thesis, LBL-17108 (1983), Univ. of Calif., Berkeley. Courtesy J. Carr, LBL. 


\section{PLOTS OF CROSS SECTIONS AND RELATED QUANTITIES (Cont'd)}

\section{Structure Functions}
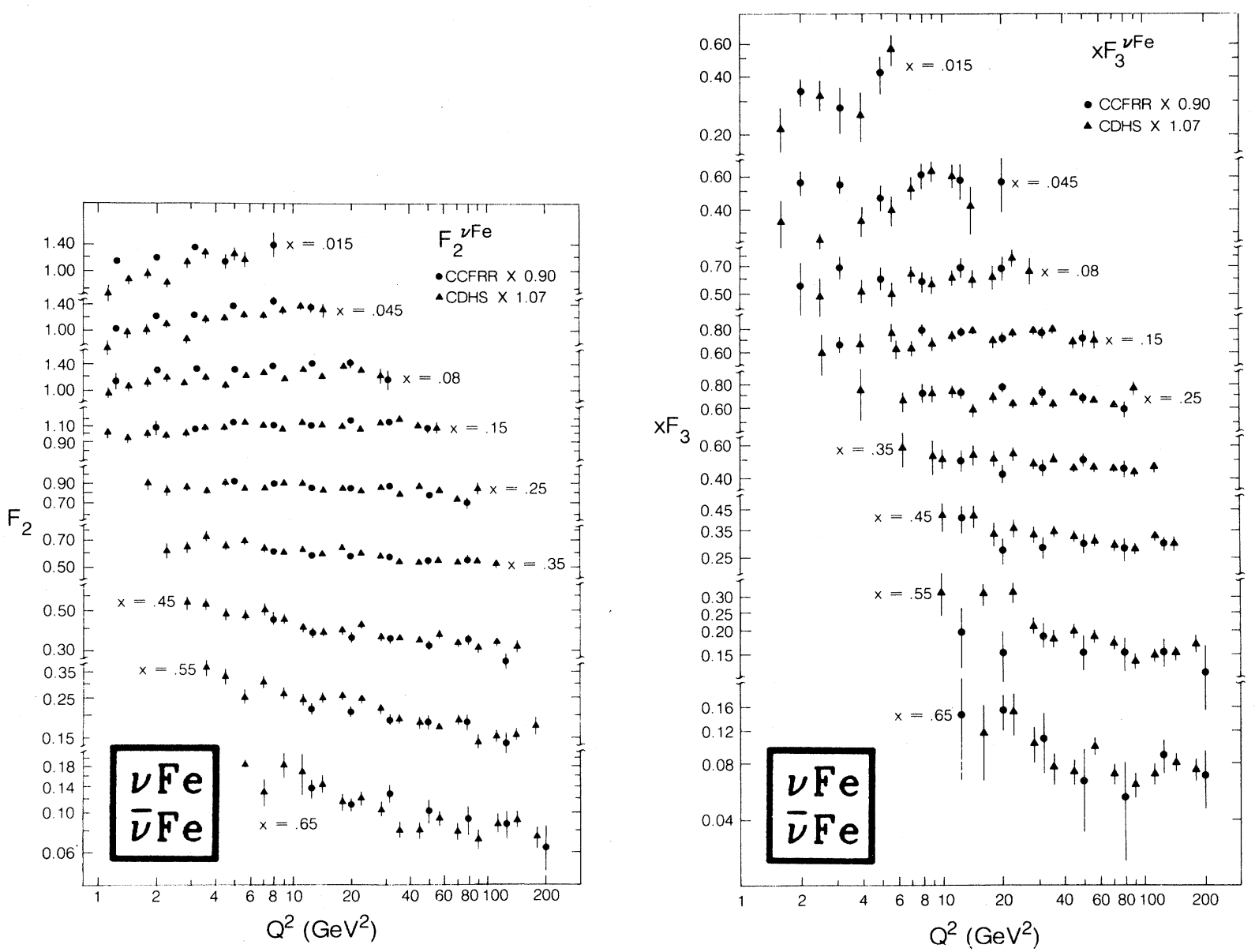

Structure functions $\mathrm{F}_{2}$ and $\mathrm{xF}_{3}$ for nucleons, measured in charged-current neutrino and antineutrino scattering from iron targets with $20<$ $\mathrm{E}_{\nu}<300 \mathrm{GeV}$, versus $\mathrm{Q}^{2}$ for fixed bins of $\mathrm{x}$, plotted assuming $\mathrm{R}=\sigma_{\mathrm{L}} / \sigma_{\mathrm{T}}=0.1$. A relative normalization factor has been fitted to optimize the agreement between the different data sets, and absolute changes to the published data have been arbitrarily chosen as indicated. The point-to-point systematic errors for both experiments are generally smaller or of the same order as the statistical errors. In addition, CDHS quote an overall scale error of $\pm 6 \%$ for $F_{2}$ and $\pm 8 \%$ for $\mathrm{xF}_{3}$, while for the CCFRR data the scale error is estimated to be $\pm 4 \%$. References: CDHS - H. Abramowicz et al., Zeit. Phys. C17, 283 (1983); CCFRR - F. Sciulli, private communication. Courtesy J. Carr, LBL.

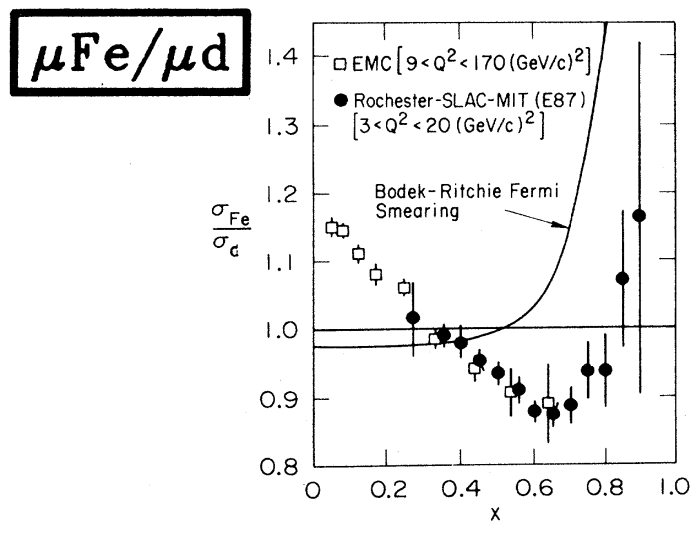

The "EMC" effect: the ratio of the differential cross section per nucleon, $\sigma_{\mathrm{Fe}} / \sigma_{\mathrm{d}}$, measured in electromagnetic deep inelastic scattering on iron and deuterium targets. (For equal values of $\mathbf{R}=$ $\sigma_{\mathrm{L}} / \sigma_{\mathrm{T}}$ on each target, $\left.\sigma_{\mathrm{Fe}} / \sigma_{\mathrm{d}}=\mathrm{F}_{2} \mathrm{Fe} / \mathrm{F}_{2}^{\mathrm{d}}\right)$. The curve indicates the contribution to the ratio from Fermi motion in the nucleus [Phys. Rev. D23, 1070 (1981) and D24, 1400 (1981)]. The errors plotted are statistical only. The systematic errors are estimated as \pm 0.011 for the Rochester-SLAC-MIT data, and \pm 0.015 at $x=0.35$ and \pm 0.06 at $x=0.05$ and $x=0.65$ for the EMC data. References: Rochester-SLAC-MIT (electrons) - Phys. Rev. Lett. 50, 1431 (1983); and EMC (muons) - Phys. Lett. 123B, 275 (1983). Courtesy D. Coward, SLAC. 


\section{PLOTS OF CROSS SECTIONS AND RELATED QUANTITIES (Cont'd)}

\section{Structure Functions}

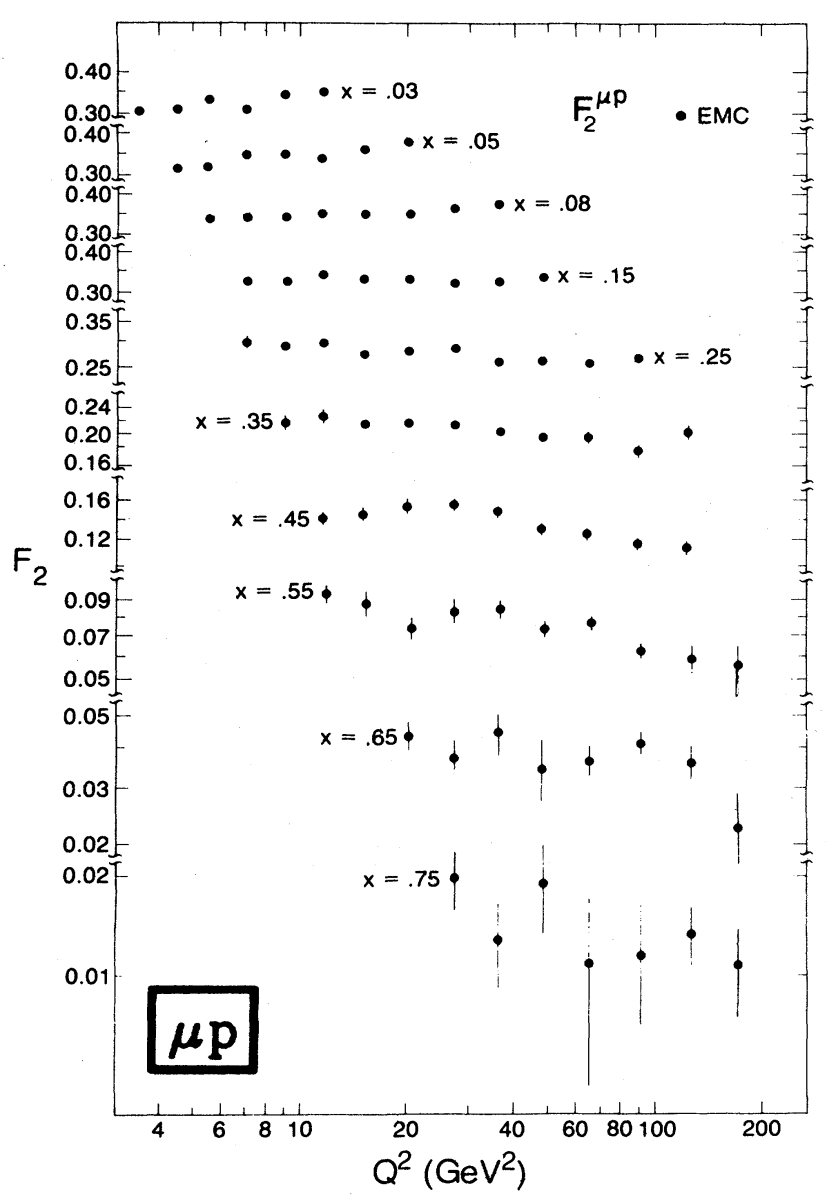

Structure function FP, measured in electromagnetic muon scattering from a hydrogen target with beam energies $120,200,240,280$ $\mathrm{GeV}$, versus $\mathbf{Q}^{2}$ for fixed bins of $x$, plotted assuming $R=\sigma_{\mathrm{L}} / \sigma_{\mathrm{T}}=$ 0. References: J.J. Aubert et al., Phys. Lett. 105B, 315 (1981); and A. Edwards, private communication. Courtesy J. Carr, LBL.

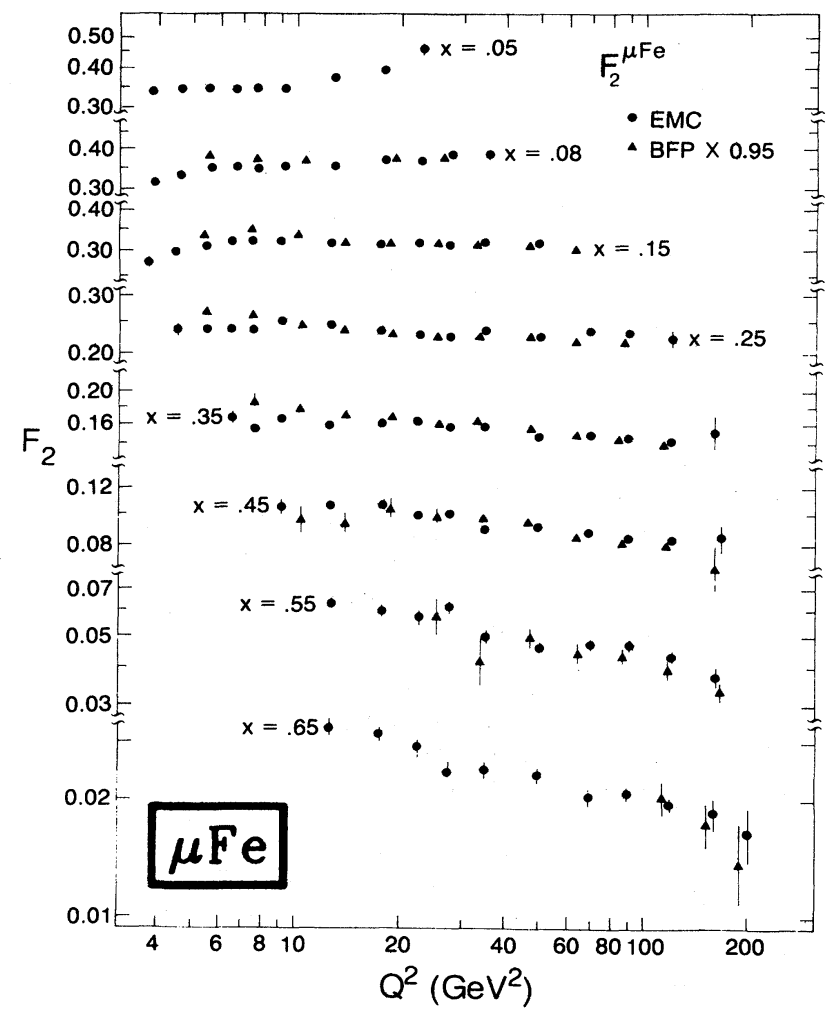

Structure function $F_{2}$ per nucleon, measured in electromagnetic muon scattering from iron targets with beam energies 120,200 , 250, $280 \mathrm{GeV}$ (EMC) and 93, $215 \mathrm{GeV}$ (BFP), versus $Q^{2}$ for fixed bins of $x$, plotted assuming $R=\sigma_{I} / \sigma_{T}=0$. A relative normalization factor has been fitted to optimize agreement between the different data sets and has been arbitrarily applied to one data set as indicated. References: EMC - J.J. Aubert et al., Phys. Lett. 105B, 322 (1981); and A. Edwards, private communication; BFP - A.R. Clark et al., Phys. Rev. Lett. 51, 1826 (1983); and P. Meyers, Ph.D. Thesis, LBL-17108 (1983), Univ. of Calif., Berkeley. Courtesy J. Carr, LBL. 


\section{PLOTS OF CROSS SECTIONS AND RELATED QUANTITIES (Cont'd)}

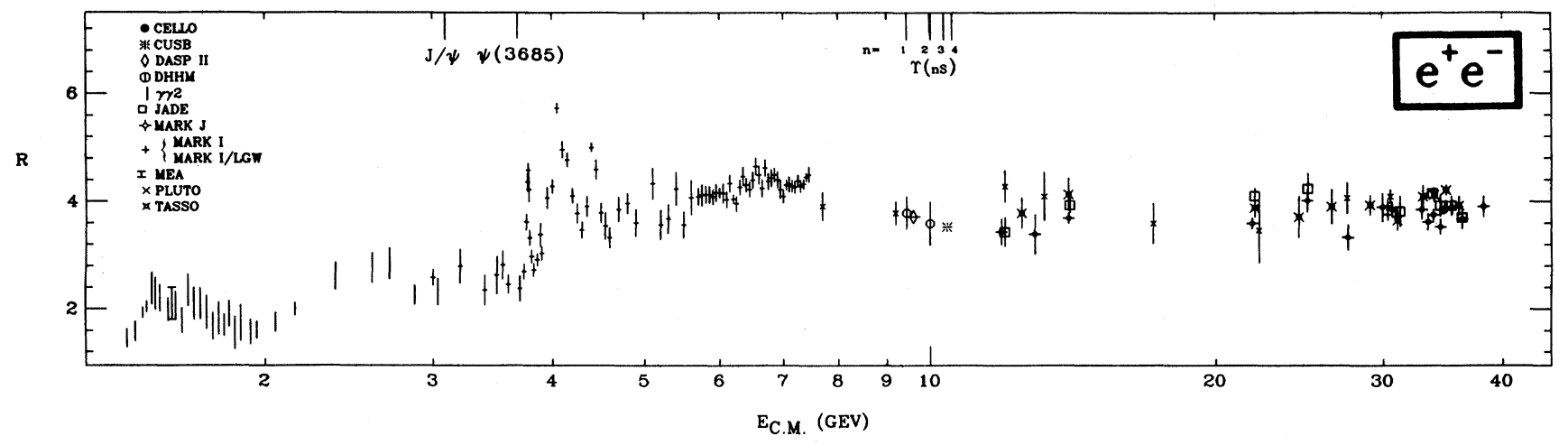

Measurements of $\mathbf{R} \equiv \sigma\left(\mathrm{e}^{+} \mathrm{e}^{-} \rightarrow\right.$ hadrons $) / \sigma\left(\mathrm{e}^{+} \mathrm{e}^{-} \rightarrow \mu^{+} \mu^{-}\right)$, where the annihilation proceeds via one photon. The denominator is a calculated quantity; see the section on Kinematics, Decays, and Scattering. Radiative corrections and, where important, corrections for two-photon processes and $\tau$ production have been made. Note that the ADONE data $(\gamma \gamma 2$ and MEA) is for $\geqslant 3$ hadrons. The points in the $\psi(3770)$ region are from the MARK I - Lead Glass Wall experiment. The DASP [R. Brandelik et al., Phys. Lett. 76B, 361 (1978)] and PLUTO (see references below) measurements have been omitted in the charm threshold region for clarity. Also for clarity, some points have been combined or shifted slightly $(<4 \%)$ in $\mathrm{E}_{\mathrm{c} . \mathrm{m}}$. and some points with low statistical significance have been omitted. Systematic normalization errors are not included; they range from $\sim 5-20 \%$, depending on experiment. Note the suppressed zero. The horizontal extent of the plot symbols has no significance. The positions of the $J / \psi(3100), \psi(3685)$, and the four known $\Upsilon$ vector-meson resonances are indicated at the top of the figure. References: CELLO - H.-J. Behrend et al., Phys. Lett. B, to be published (preprint DESY 81-029); CUSB - E. Rice et al., Phys. Rev. Lett. 48, 906 (1982); DASP II - Phys. Lett. 116B, 383 (1982); DHHM - P. Bock et al. (DESY-

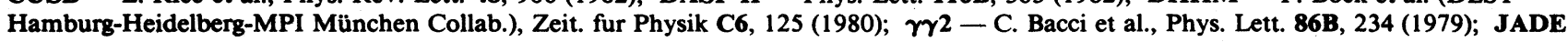
- W. Bartel et al., Phys. Lett. 129B, 145 (1983); MARK J - B. Adeva et al., Phys. Rev. Lett. 50, 799 (1983); and H. Newman, private communication; MARK I - J.L. Siegrist et al., Phys. Rev. D26, 969 (1982); MARK I + Lead Glass Wall - P.A. Rapidis et al., Phys. Rev. Lett. 39, 526 (1977); and P.A. Rapidis, thesis, SLAC-Report-220 (1979); MEA - B. Esposito et al., Lett. Nuovo Cimento 19, 21 (1977); PLUTO - A. Bäcker, thesis Gesamthochschule Siegen, DESY F33-77/03 (1977); C. Gerke, thesis, Hamburg Univ. (1979); Ch. Berger et al., Phys. Lett. 81B, 410 (1979); and W. Lackas, thesis, RWTH Aachen, DESY PLUTO-81/11 (1981); TASSO - R. Brandelik et al., Phys. Lett. 113B, 499 (1982).

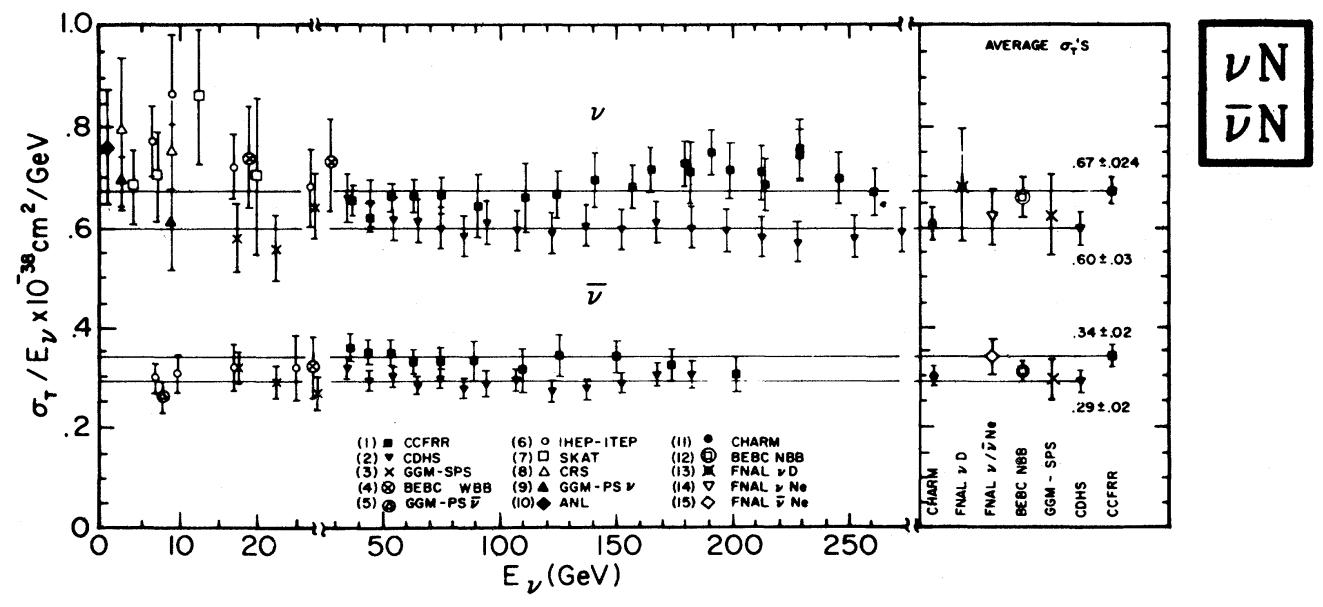

$\sigma_{\mathrm{T}} / \mathrm{E}_{\nu}$ for the muon neutrino and antineutrino charged-current total cross section as a function of neutrino energy. The error bars include both statistical and systematic errors. The straight lines are averages for the CCFRR and CDHS measurements. Note the change in the energy scale between 20 and $50 \mathrm{GeV}$. The data points on the right give averages for other high energy measurements. References: (1) R. Blair et al., Phys. Rev. Lett. 51, 343 (1983), and J.R. Lee, Ph.D. Thesis, Caltech (1981), "Measurements of $\nu$ N Charged Current Cross Sections from $E_{\nu}=25 \mathrm{GeV}$ to $\mathrm{E}_{\nu}=260 \mathrm{GeV}$;" (2) H. Abramowicz et al., Zeit. fur Physik C17, 283 (1983); (3) J. Morfin et al., Phys. Lett. 104B, 235 (1981); (4) D.C. Colley et al., Zeit. fur Physik C2, 187 (1979); (5) O. Erriquez et al., Phys. Lett. 80B, 309 (1979); (6) A.S. Vovenko et al., Sov. J. Nucl. Phys. 30, 527 (1979); (7) D.S. Baranov et al., Phys. Lett. 81B, 255 (1979); (8) C. Baltay et al., Phys. Rev. Lett. 44, 916 (1980); (9) S. Ciampolillo et al., Phys. Lett. 84B, 281 (1979); (10) S.J. Barish et al., Phys. Rev. D19, 2521 (1979); (11) M. Jonker et al., Phys. Lett. 99B, 265 (1981), $\mathrm{E}_{\nu}=20-200 \mathrm{GeV}$; (12) P. Bosetti et al., Phys. Lett. 110B, 167 (1982), $\mathrm{E}_{\nu}=20-200 \mathrm{GeV}$; (13) T. Kitagaki et al., Phys. Rev. Lett. 49, 98 (1982), $\mathrm{E}_{\nu}=10-200 \mathrm{GeV}$; (14) N.J. Baker et al., Phys. Rev. Lett. 51, 735 (1983), E $\boldsymbol{\nu}_{\nu} 10-240 \mathrm{GeV}$; (15) G.N. Taylor et al., Phys. Rev. Lett. 51, 739 (1983), $E_{\nu}=5-250 \mathrm{GeV}$. Courtesy M.H. Shaevitz, Columbia University (Nevis Laboratory). 


\section{PLOTS OF CROSS SECTIONS AND RELATED QUANTITIES (Cont'd)}

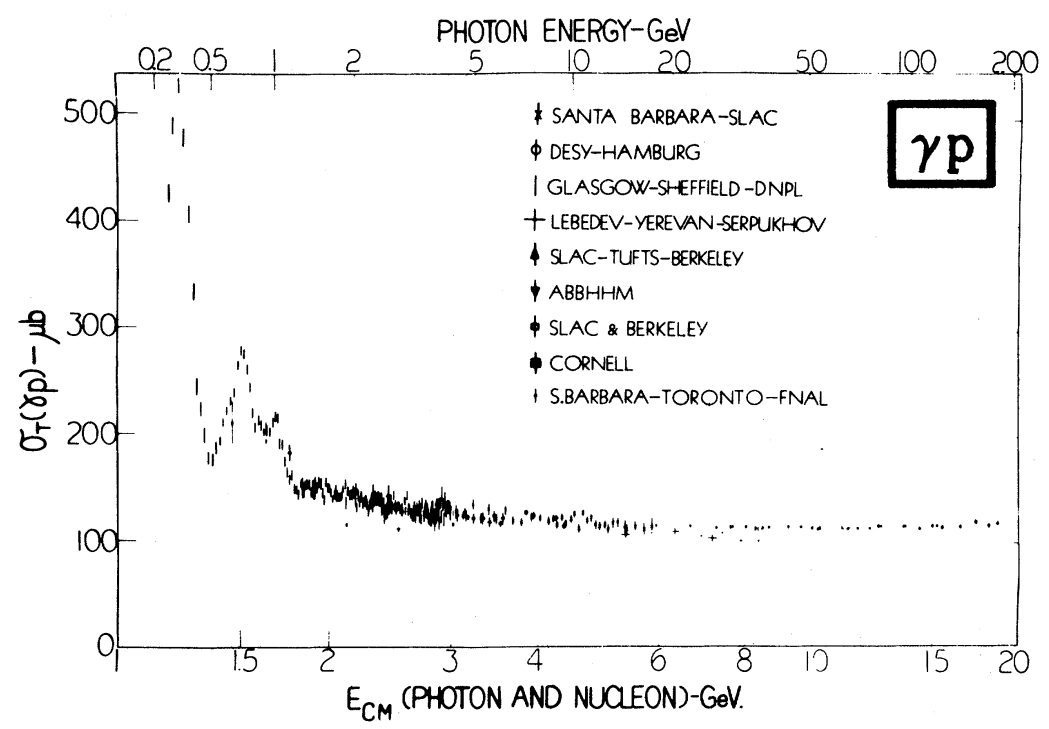

$\gamma$ p total cross section versus photon energy (top scale) and photon-plus-nucleon total center-of-mass energy (lower scale). References: SANTA BARBARA-SLAC - D.O. Caldwell et al., Phys. Rev. D7, 1362 (1973); DESY-HAMBURG - H. Meyer et al., Phys. Lett. 33B, 189 (1970); GLASGOW-SHEFFIELD-DNPL - T.A. Armstrong et al., Phys. Rev. D5, 1640 (1972); LEBEDEV-YEREVAN-

SERPUKHOV - A.S. Belousov et al., Preprint 19, Moscow, (1970); A. S. Belousov et al., Sov. Phys. Doklady 19, 123 (1974); and A. S. Belousov et al., Sov. J. Nucl. Phys. 21(3), 289 (1975); SLAC-BERKELEY-TUFTS - J. Ballam et al., Phys. Rev. D5, 545 (1972);

ABBHHM - H.G. Hilpert et al., Phys. Lett. 27B, 474 (1968); SLAC and BERKELEY - J. Ballam et al., Phys. Rev. Lett. 21, 1544 (1968), and H.H. Bingham et al., Phys. Rev. D8, 1277 (1973); CORNELL - S. Michalowski et al., Phys. Rev. Lett. 39, 737 (1977); SANTA BARBARA-TORONTO-FNAL - D.O. Caldwell et al., Phys. Rev. Lett. 40, 1222 (1978). See, also, the ep data of E.D. Bloom et al., SLAC-PUB-653 (1969). Courtesy Gething M. Lewis, Glasgow.

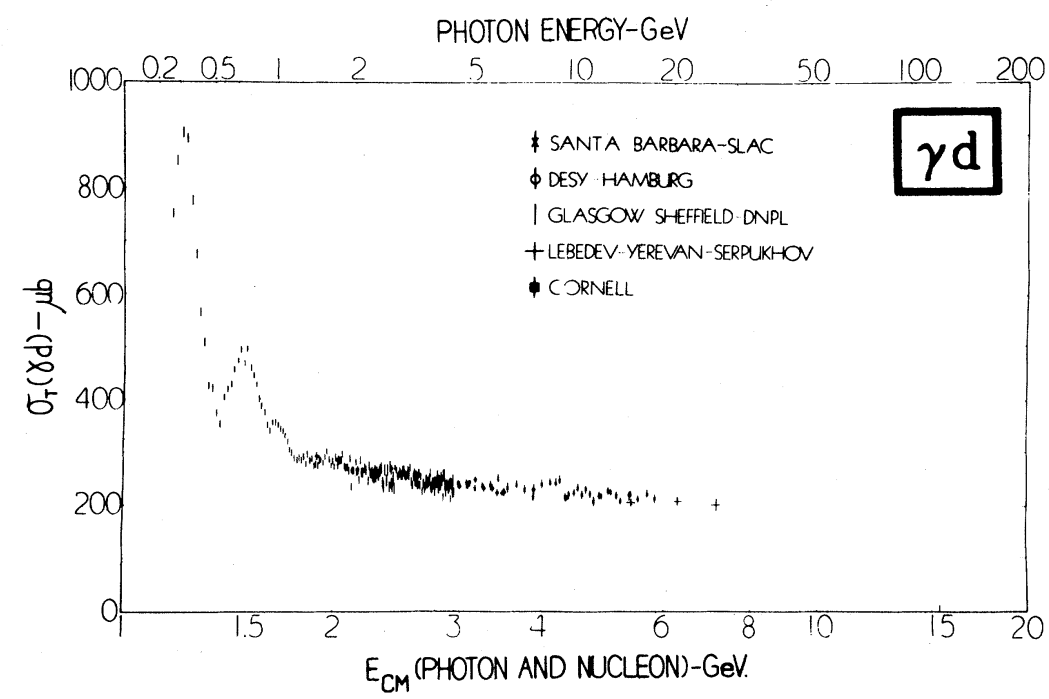

$\gamma \mathrm{d}$ total cross section versus photon energy (top scale) and photon-plus-single-nucleon total center-of-mass energy (lower scale). References: SANTA BARBARA-SLAC - D.O. Caldwell et al., Phys. Rev. D7, 1362 (1973); DESY-HAMBURG - H. Meyer et al., Phys. Lett. 33B, 189 (1970); GLASGOW-SHEFFIELD-DNPL - T.A. Armstrong et al., Nucl. Phys. B41, 445 (1972); LEBEDEV-YEREVANSERPUKHOV - A.S. Belousov et al., Sov. J. Nucl. Phys. 21(3), 289 (1975); CORNELL - S. Michalowski et al., Phys. Rev. Lett. 39, 737 (1977). Courtesy Gething M. Lewis, Glasgow. 
PLOTS OF CROSS SECTIONS AND RELATED QUANTITIES (Cont'd)
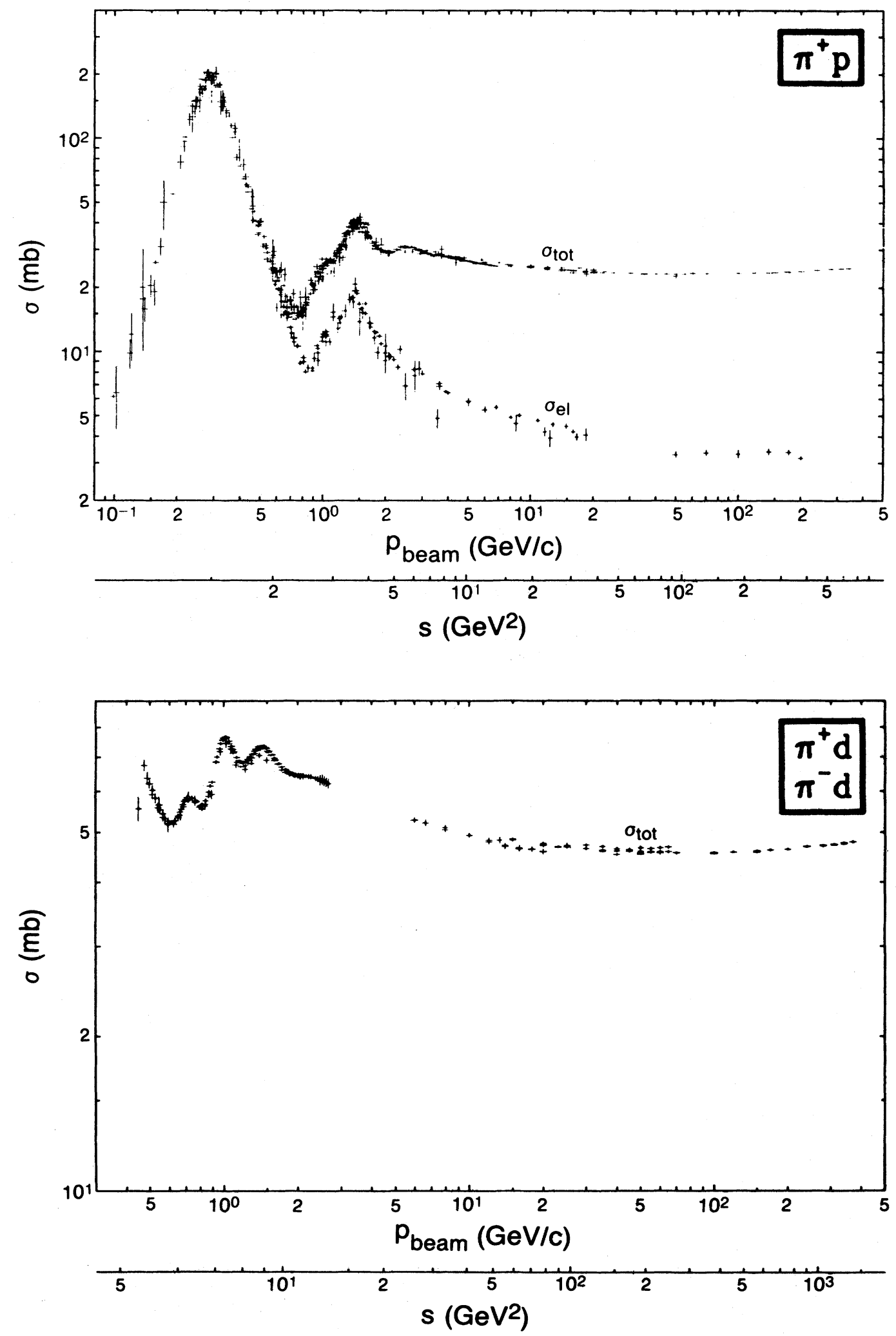

Hadronic total and elastic cross sections vs. laboratory beam momentum $p_{\text {beam }}$ and center-of-mass enersy squared s. Figures courtesy V. Flaminio, W.G. Moorhead, D.R.O. Morrison, and N. Rivoire, CERN. 


\section{PLOTS OF CROSS SECTIONS AND RELATED QUANTITIES (Cont'd)}
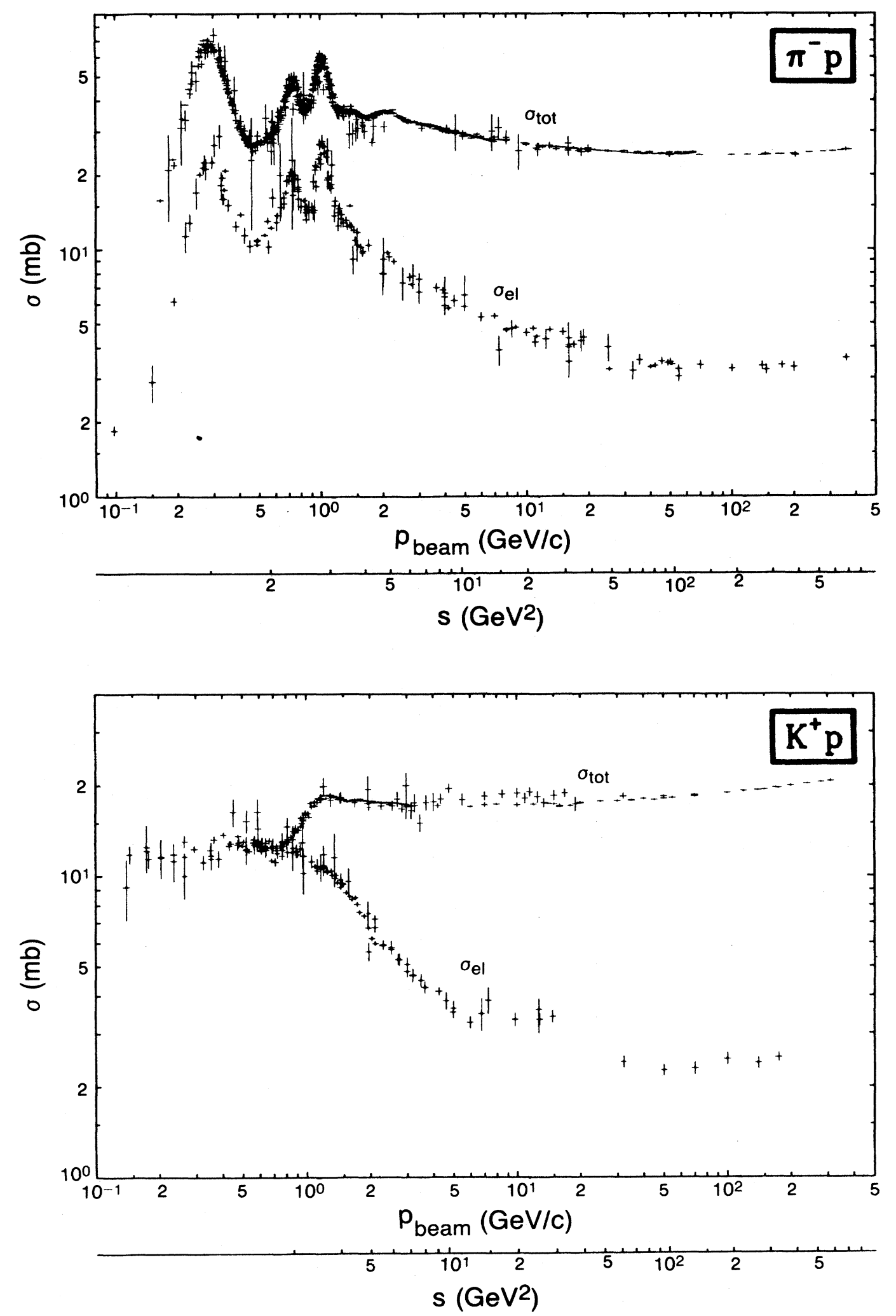

Hadronic total and elastic cross sections vs. laboratory beam momentum pbeam and center-of-mass energy squared s. Figures courtesy V. Flaminio, W.G. Moorhead, D.R.O. Morrison, and N. Rivoire, CERN. 


\section{PLOTS OF CROSS SECTIONS AND RELATED QUANTITIES (Cont'd)}
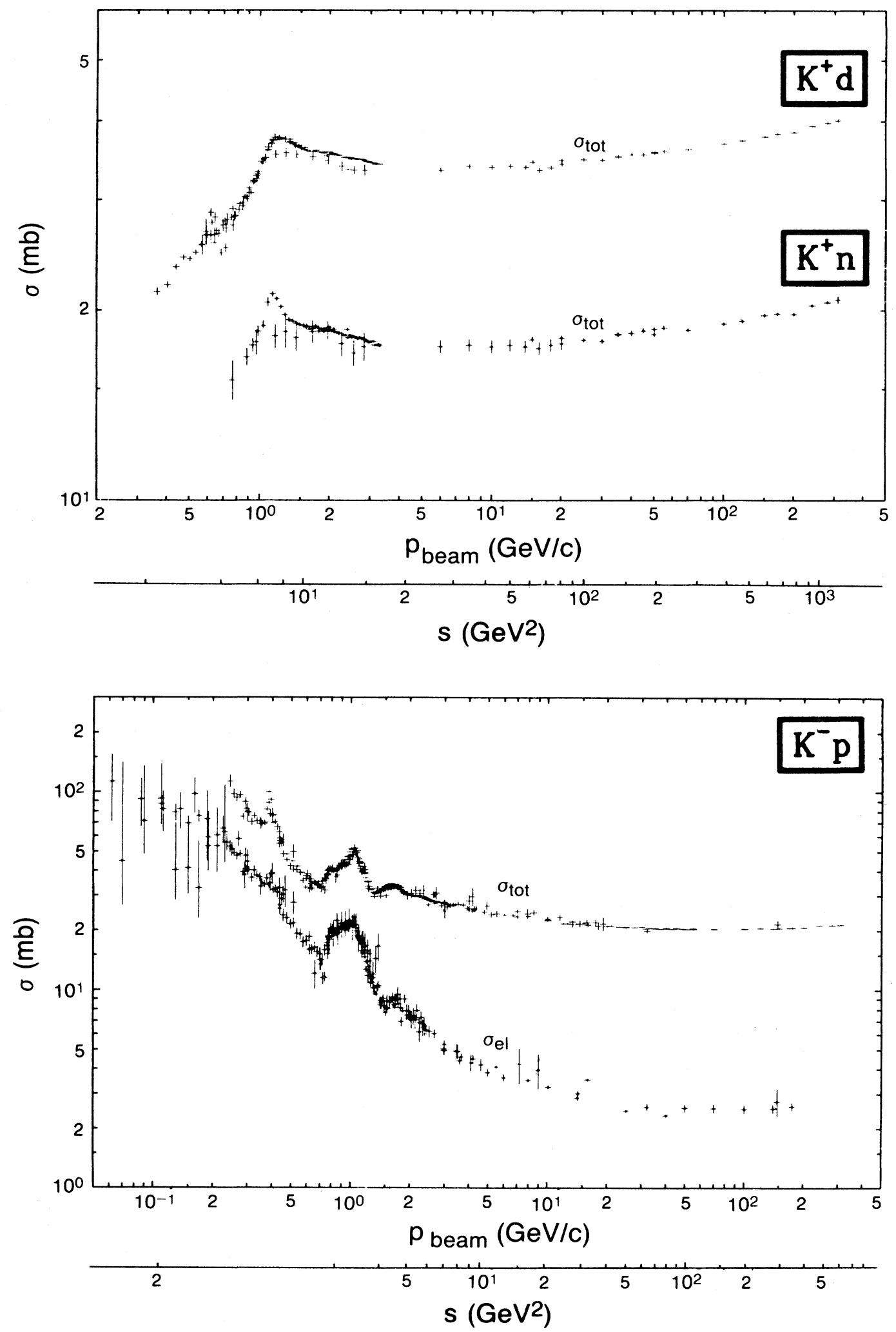

Hadronic total and elastic cross sections vs. laboratory beam momentum $p_{\text {beam }}$ and center-of-mass energy squared s. Figures courtesy V. Flaminio, W.G. Moorhead, D.R.O. Morrison, and N. Rivoire, CERN. 
PLOTS OF CROSS SECTIONS AND RELATED QUANTITIES (Cont'd)
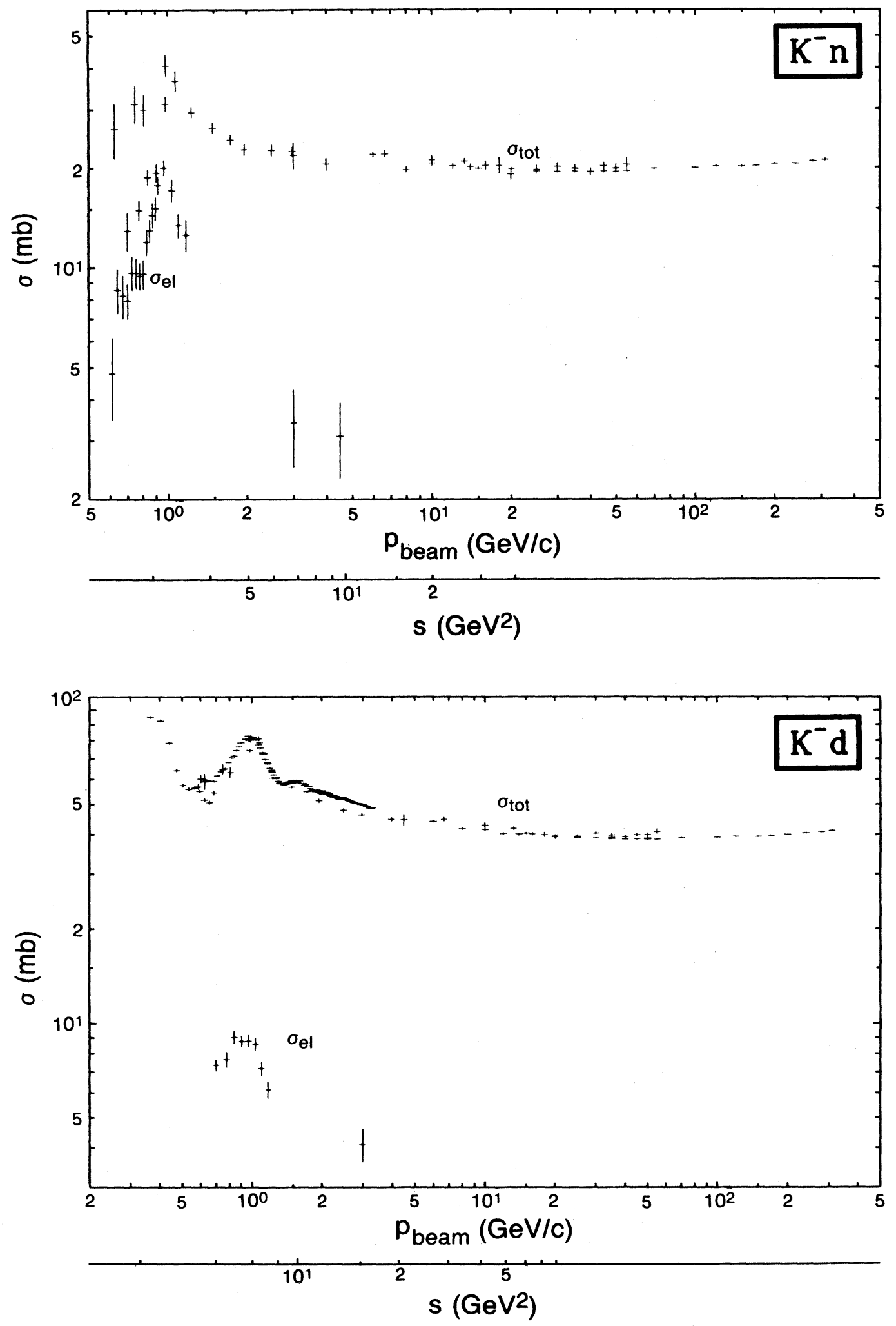

Hadronic total and elastic cross sections vs. laboratory beam momentum $p_{\text {beam }}$ and center-of-mass energy squared s. Figures courtesy V. Flaminio, W.G. Moorhead, D.R.O. Morrison, and N. Rivoire, CERN. 
PLOTS OF CROSS SECTIONS AND RELATED QUANTITIES (Cont'd)
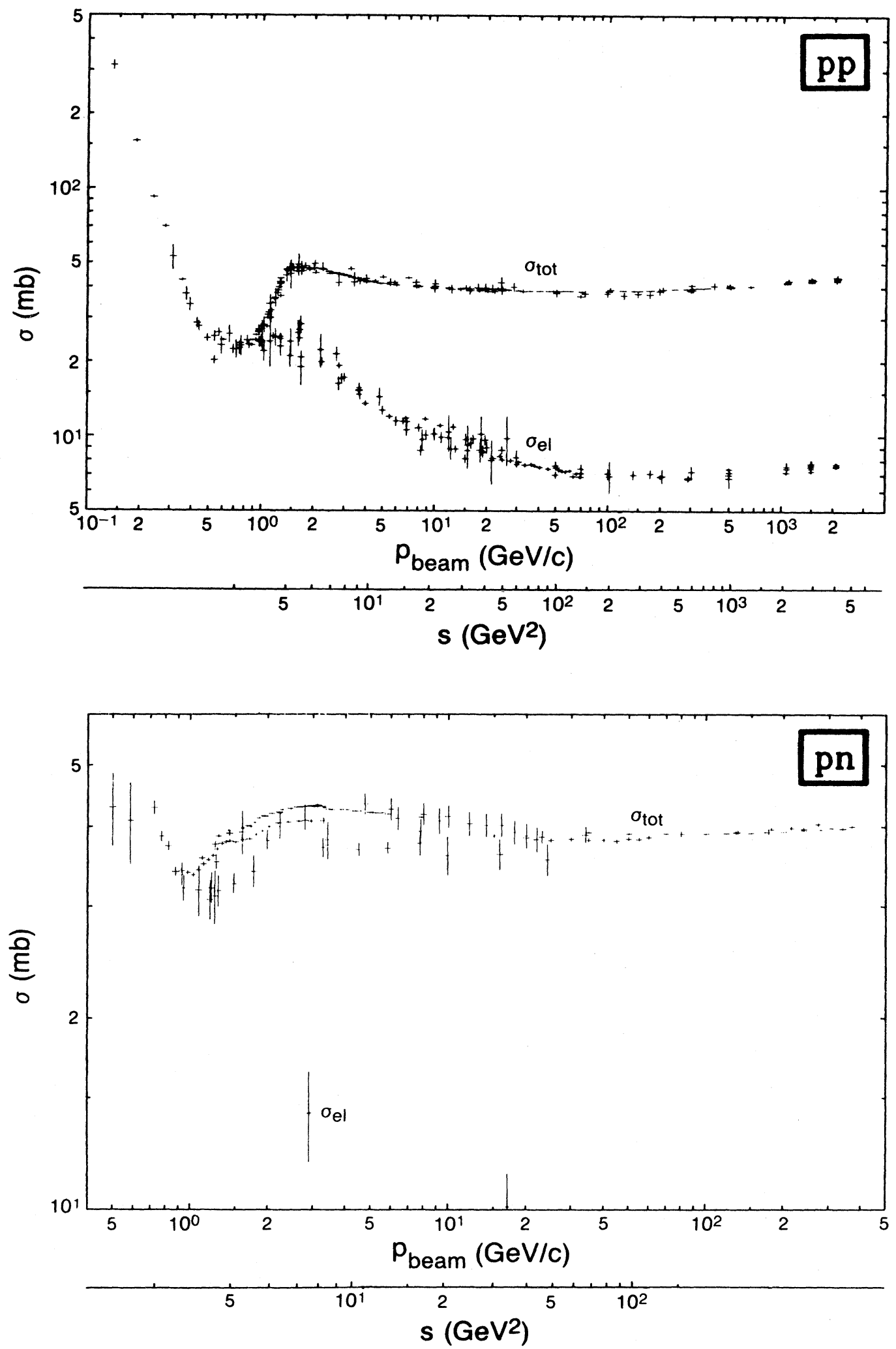

Hadronic total and elastic cross sections vs. laboratory beam momentum $p_{\text {beam }}$ and center-of-mass energy squared s. Figures courtesy V. Flaminio, W.G. Moorhead, D.R.O. Morrison, and N. Rivoire, CERN. 


\section{PLOTS OF CROSS SECTIONS AND RELATED QUANTITIES (Cont'd)}
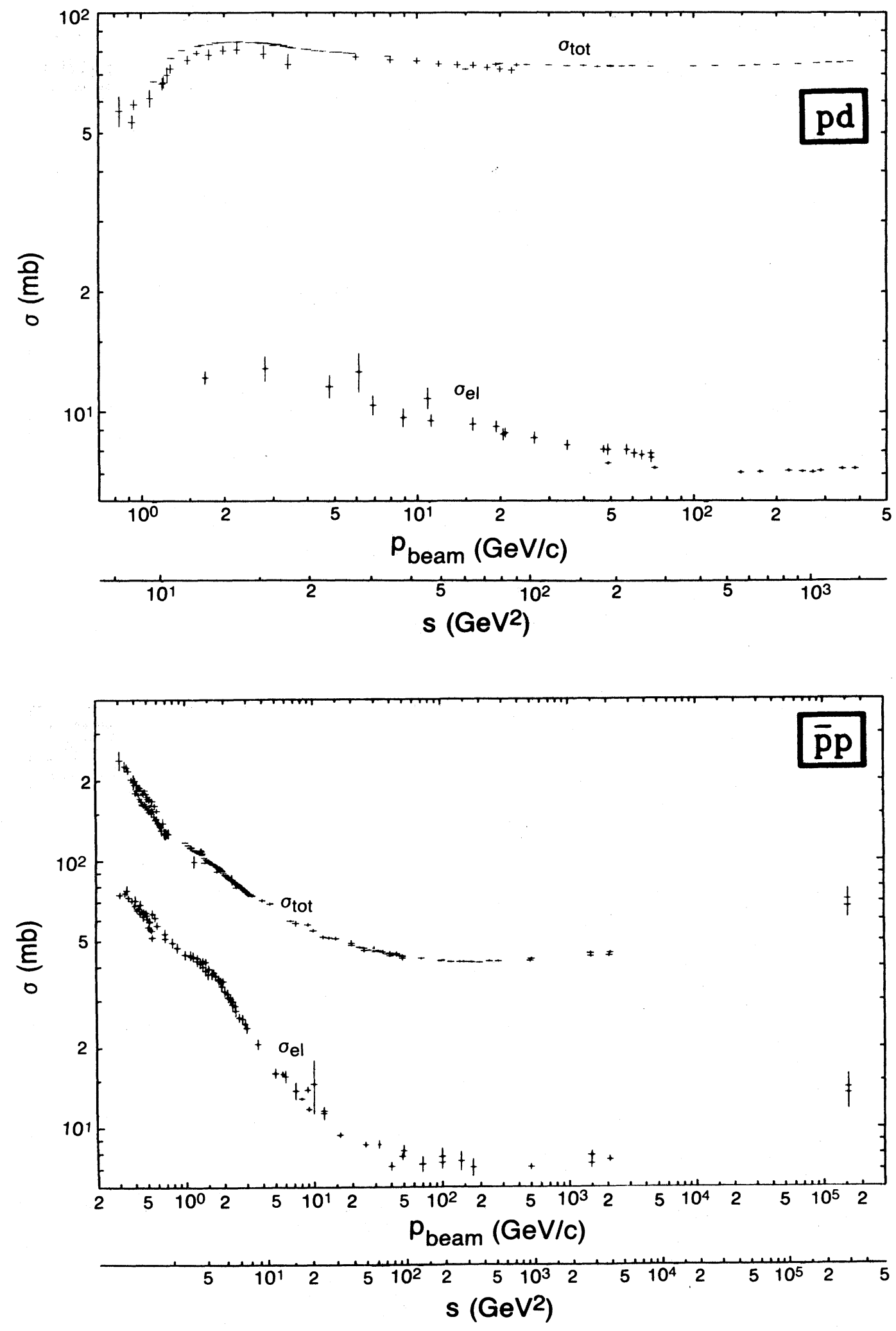

Hadronic total and elastic cross sections vs. laboratory beam momentum $p_{\text {beam }}$ and center-of-mass energy squared s. Figures courtesy V. Flaminio, W.G. Moorhead, D.R.O. Morrison, and N. Rivoire, CERN. 
PLOTS OF CROSS SECTIONS AND RELATED QUANTITIES (Cont'd)
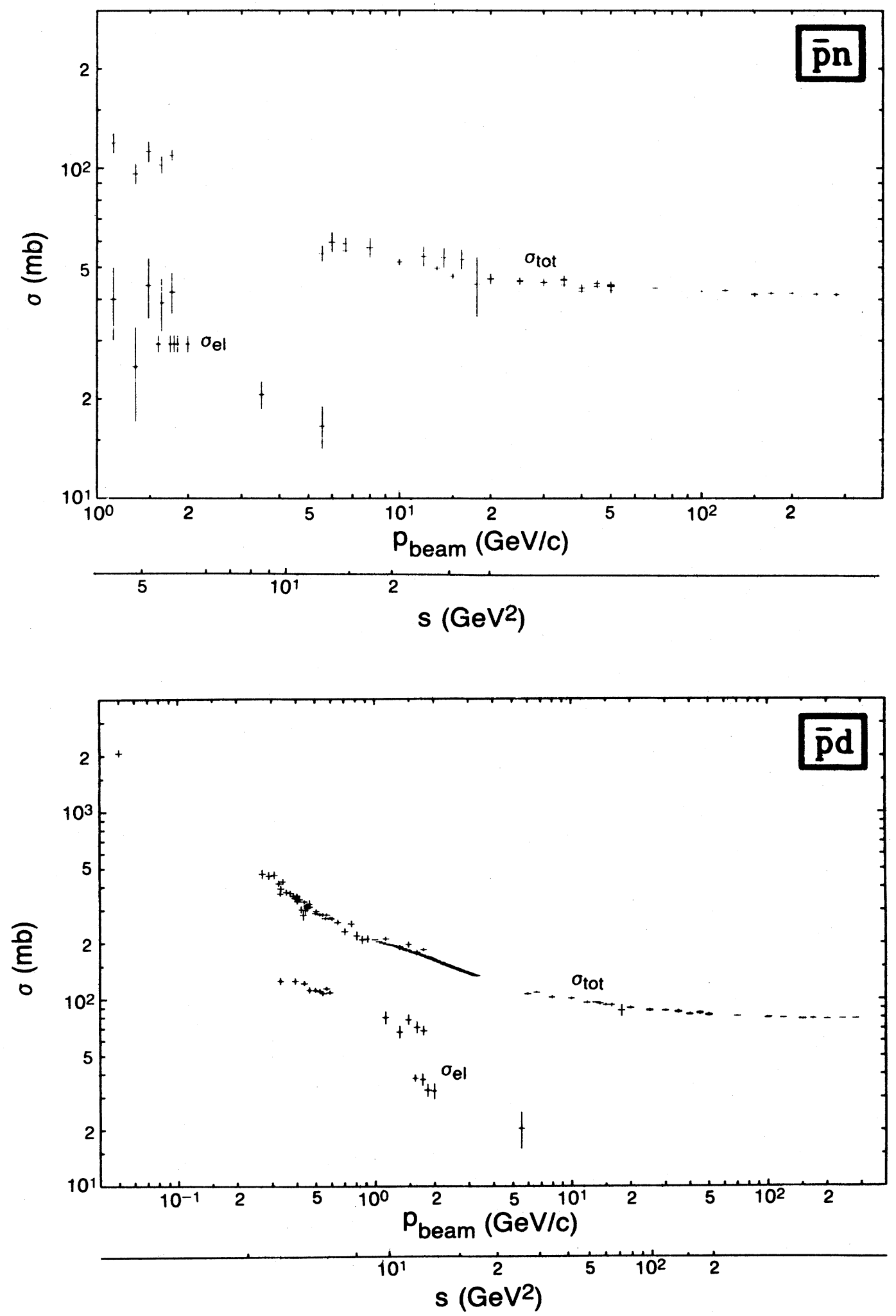

Hadronic total and elastic cross sections vs. laboratory beam momentum $P_{\text {peam }}$ and center-of-mass enersy squared s. Figures courtesy V. Flaminio, W.G. Moorhead, D.R.O. Morrison, and N. Rivoire, CERN. 


\section{DATA CARD LISTINGS}

\section{Illustrative Key}

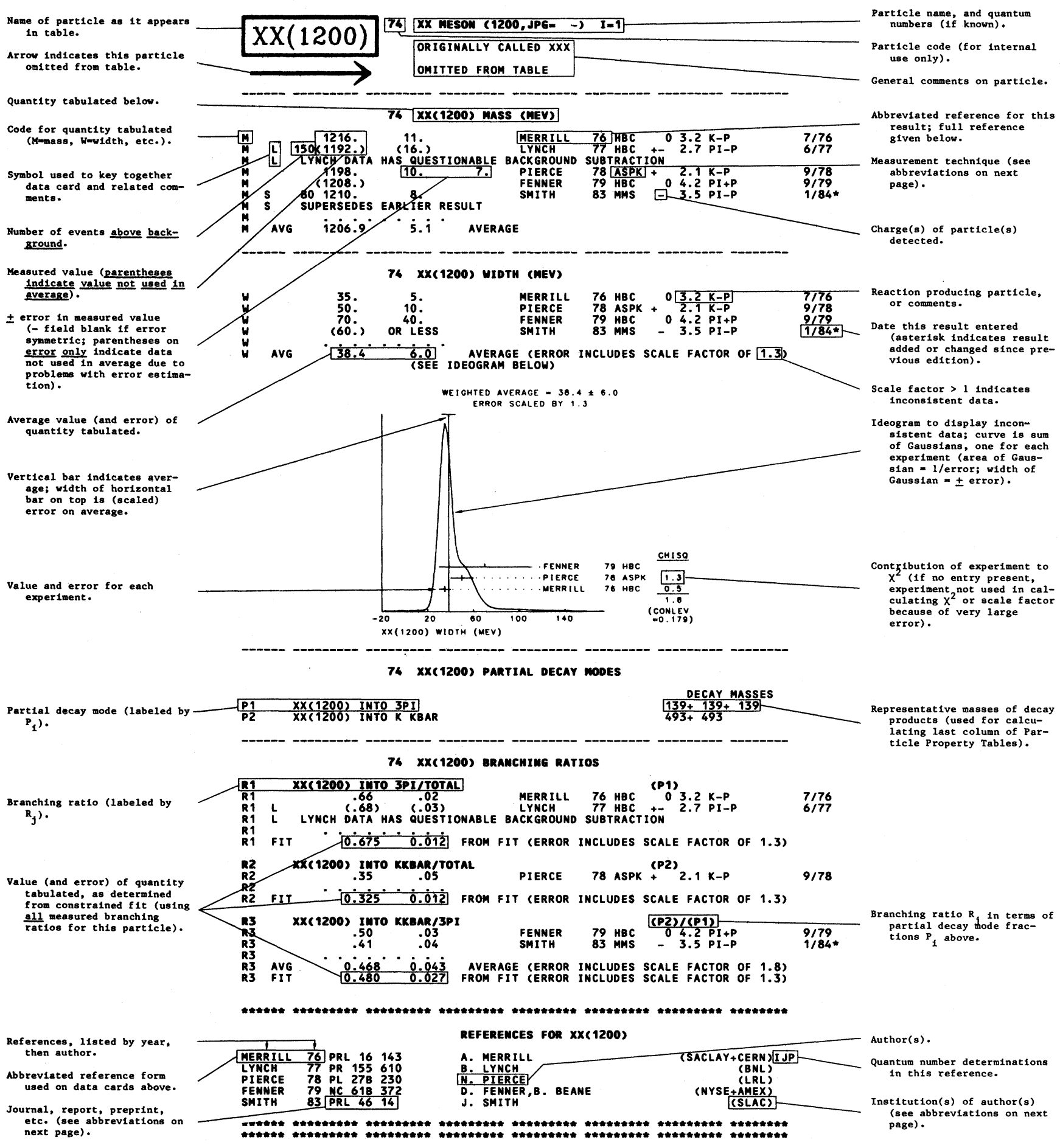




\section{Illustrative Key (cont'd)}

\section{Abbreviations}

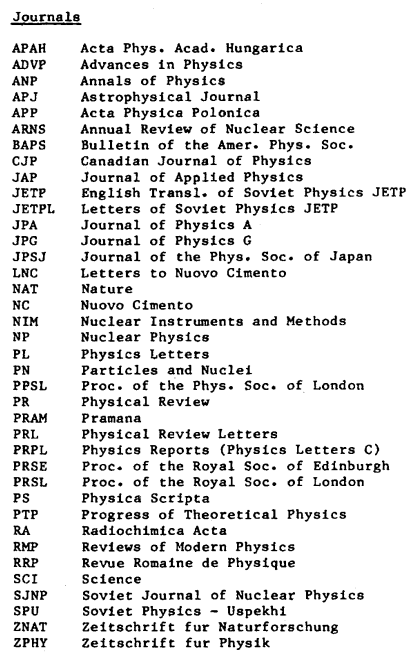

Conferences

Conferences are referred to by the location in which
they were held (e.g., DUBNA, BOULDER, LUND, etc.).

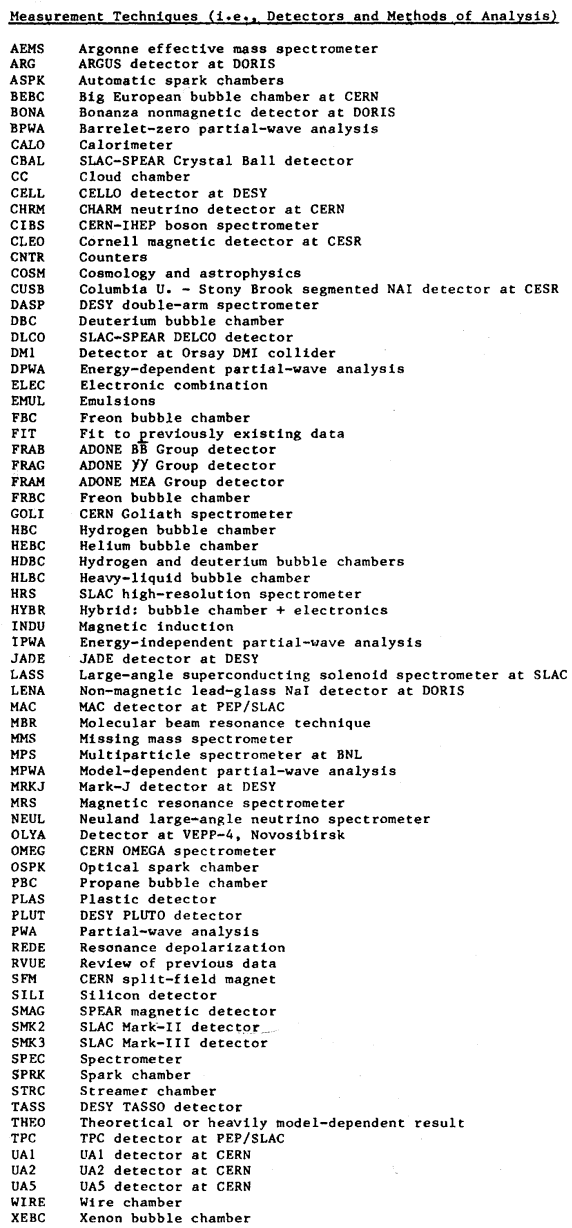

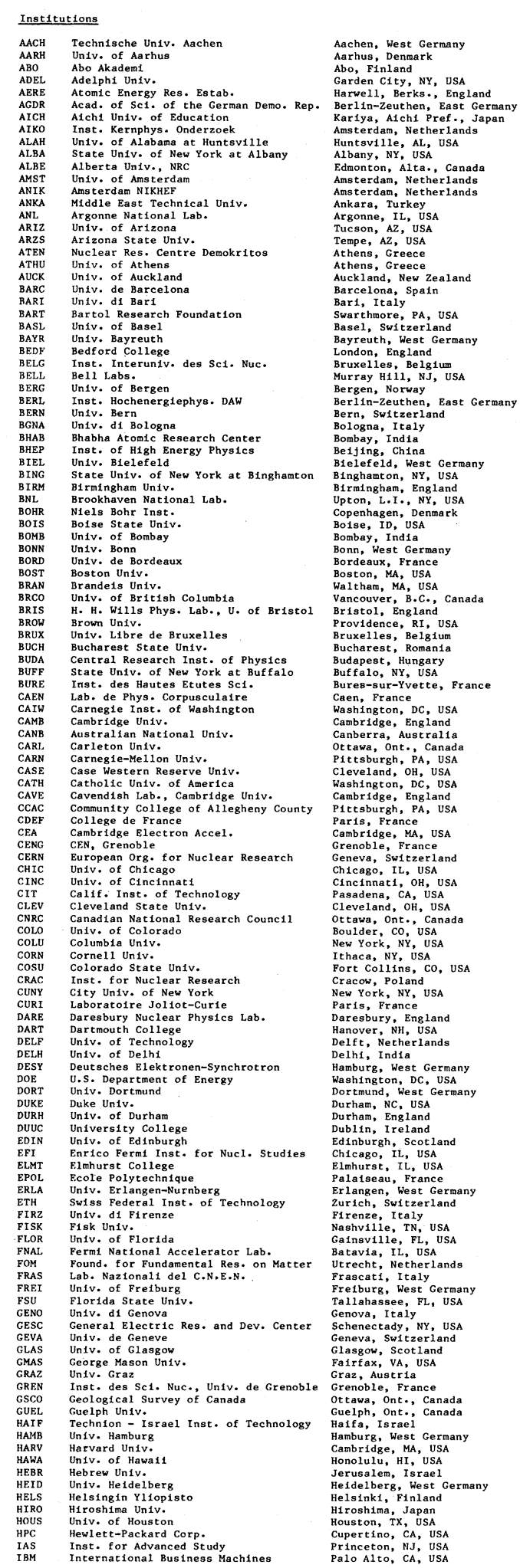




\section{Illustrative Key (cont'd)}

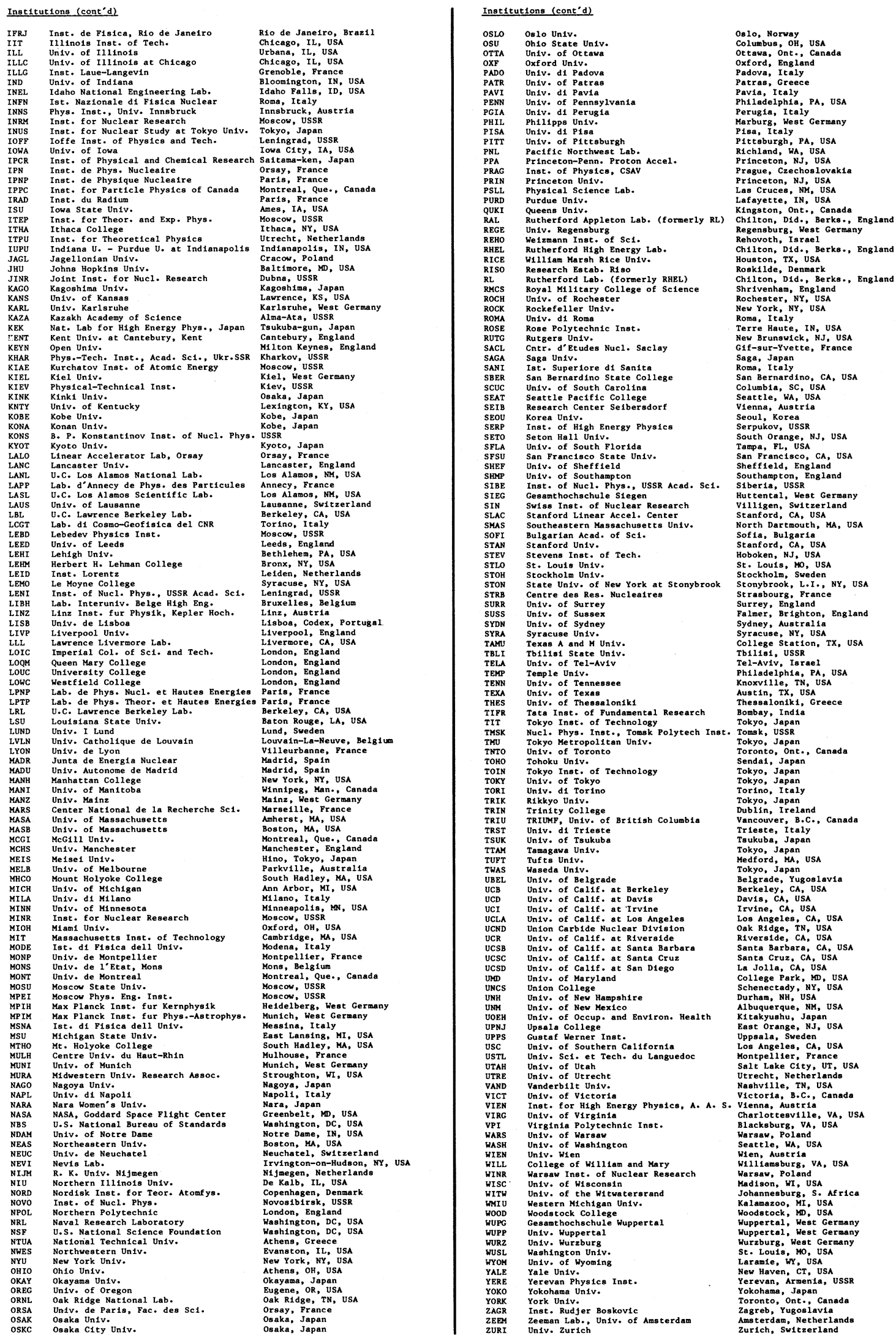




\section{Stable Particles}

\section{$\gamma$, W, $Z$, WEAK GAUGE BOSON SEARCHES}

\section{Data Card Listings}

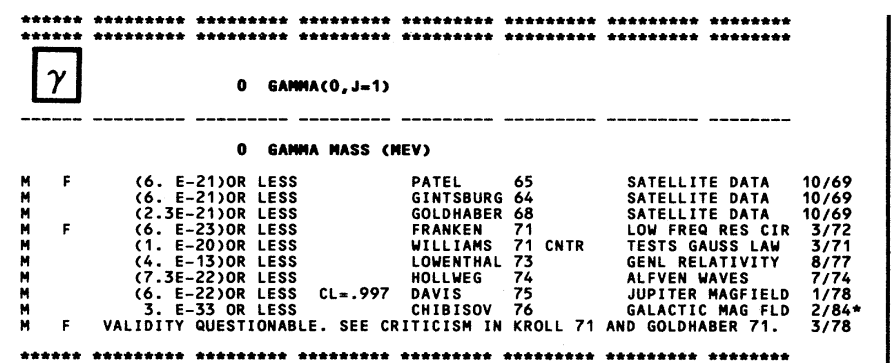

REFEREMCES FOR GAMMA

GINTSBUR 64 SOV. ASTR.AJ7 536 M. A. GINTSBURG
PATEL 65 PL 14 (ACAD SCI,USSR)
(DURHAM)

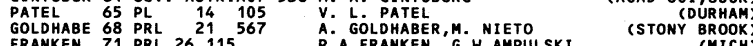

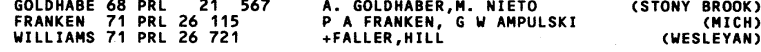

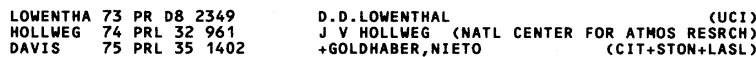

DAVIS 75 PRL 351402
CHIBISOV 76 SPU 19624
G.GOLDHABER,NIETO

PAPERS NOT REFERRED TO IN DATA CARDS

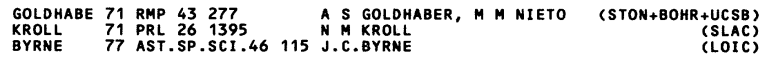

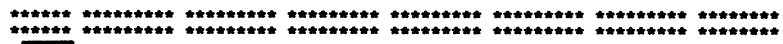

W 43 W(80000, JP = ,

MEASUREMENTS OF CHARGED WeAK GAUGE BOSON PARAMETERS
ARE LISTED HERE. SEE ALSO WEAK GAUGE BOSON SEARCHES SECTION BELOW.

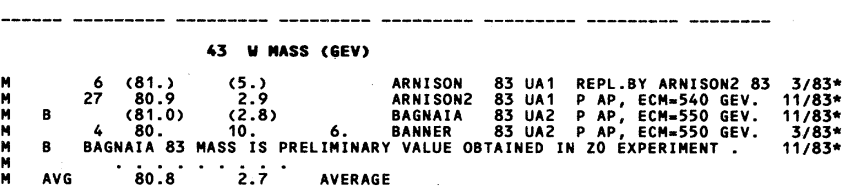

AV $\quad 80.8 \cdot 2.7^{\circ}$ aVERAGE

43 W WIOTH (GEV)

W 27 7. OR LESS CL=.90 ARNISON2 83 UA1 P AP, ECM=540 GEV. 11/83* 43 w PARTial decaY modes

10/83*

P1 W+- INTO E+- NEU

$511++0$ DECAY MASSES

43 W BRAMCHING RATIOS

10/83*

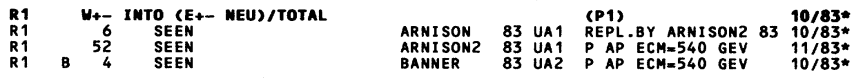

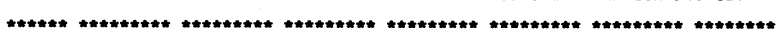

REFEREMCES FOR *

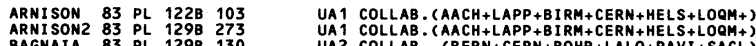

$\begin{array}{llll}\text { BAGNAIA } 83 & \text { PL } 1298 & 130 \\ \text { BANNER } & 83 & \text { PL. } 1228 & 476\end{array}$

UA2 COLLAB. (BERN+CERN+BOHR+LALO+PAVI+SACL)

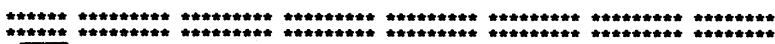

Z

$44 \mathrm{Z}(95000, \mathrm{JP}=$,

MEASUREMENTS OF MEUTRAL WEAK GaUGe BOSON PARAMETERS
ARE LISTED HERE. SEE AL SO WEAK GAUGE BOSON SEARCHES SECTION BELOH.

442 MASS (GEV)

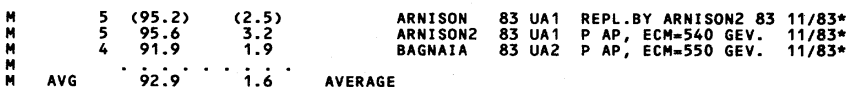

442 WIDTH (GEV)

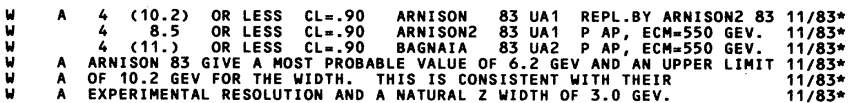

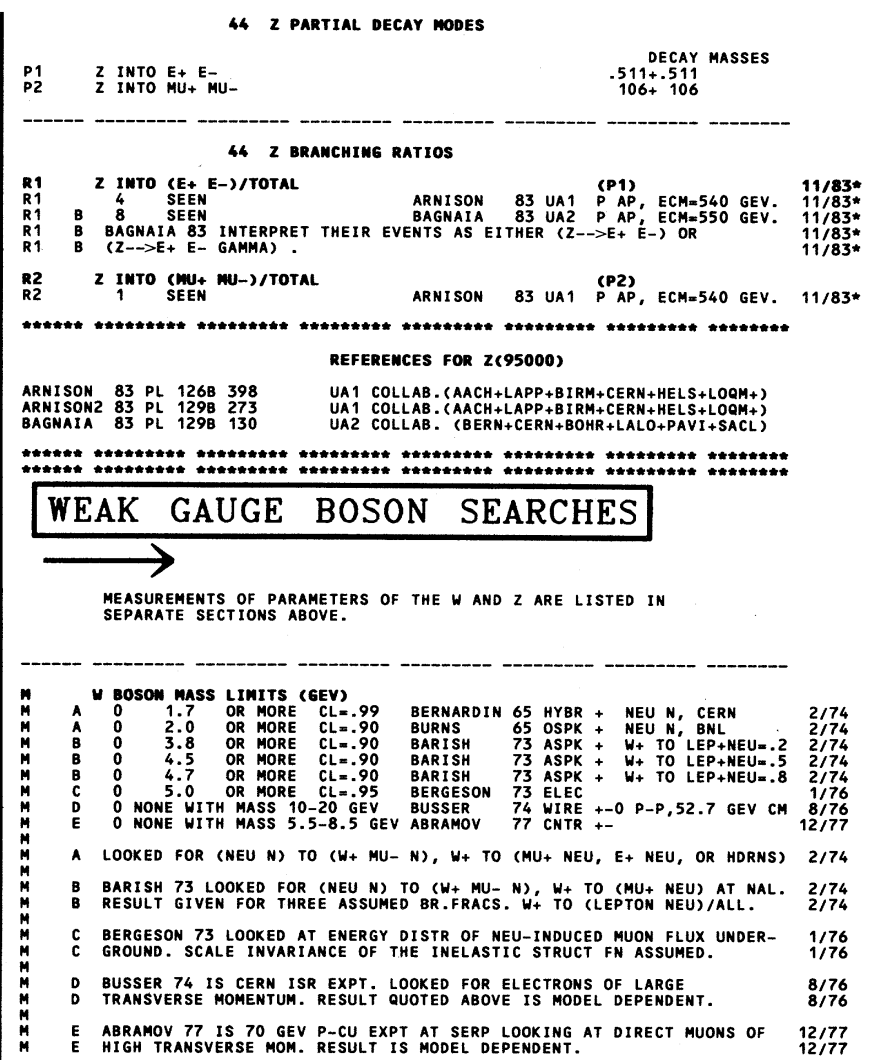

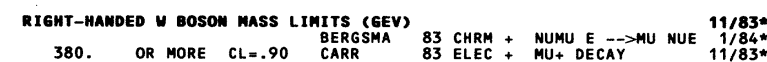

$M R$
$M R$ A BERGSMA 83 SET LIMIT M(H2)/M(W1) > 1.9 AT CL $=.90$.

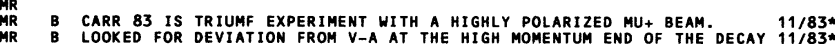

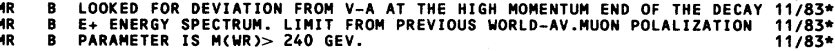

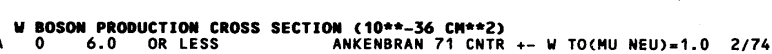

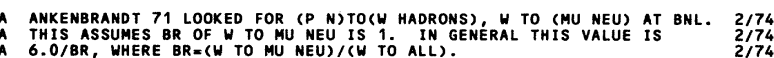

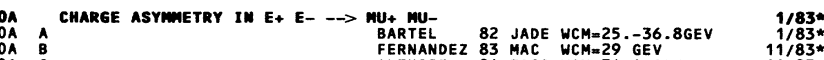

$\begin{array}{llll}20 A & B & & \\ 20 A & C & A L T H O F F & 84 \\ 20 A & \text { TASS WCM=34.4 GEV } & 11 / 83 \\ 20 A & A & 11 / 83\end{array}$

$\begin{array}{llll}20 A & \text { BARTEL } 82 \text { OBTAINED A=-0.118+-.038 AFTER RAD. COR. AND FOR } & 1 / 83 * \\ 20 A & \text { COS TTHETA, WITHIN }(+-0.8) \text {. CONSISTENT WITH STANDARD MODEL } & 1 / 83 * \\ 20 A & A \\ \text { PREDICTION A=-0.078). } & 1 / 83 *\end{array}$

20A B FERNANDEZ 83 GOT A=-.076+.018-.003.ST.MODEL(INCL.RC) GIVES A=-.060. 11/83*

ZOA C ALTHOFF 84 OBTAINED A=-.098+-.023+-.005, SIN(THETA/W)**2=.27+-.07. 11/83*

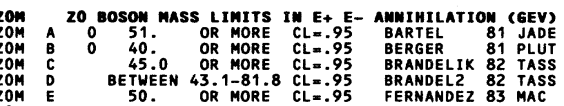

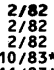

ABOVE LIMITS ARE IN STANDARD SU(2)XU(1) MODEL. FOR BOUND-PLOTS IN $2 / 82$
EXTENDED MODELS WITH TWO OR MORE ZO'S, SEE INDIVIDUAL PAPERS.
$2 / 82$

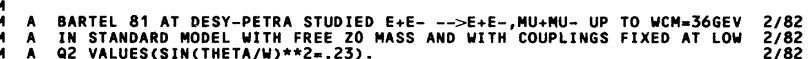

B BERGER 81 AT DESY-PETRA ANALYZED E+E-- - >E+E-, MU+MU-, TAU+TAU- DATA $2 / 82$

B AT WCM-27.5-31.6 GEV IN STANDARD MODEL. CL $=95$ CONTOUR IN GV*\#2 AND $2 / 82$
B GA*\#2 PLANE IS INCONSISTENT W. NU E RESULT IF MZ BELOW ABOVE LIMIT. 2/82

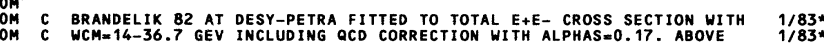




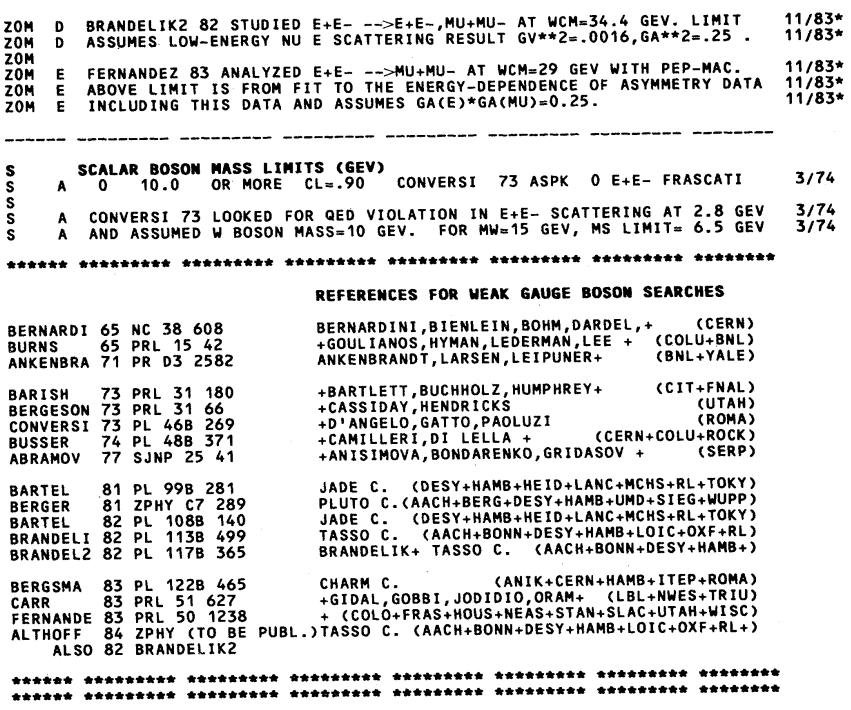

\section{NOTE ON NEUTRINOS}

(by R.E. Shrock, State Univ. of New York, Stony Brook)

With the 1982 edition of this Review, the section on neutrino properties was expanded and reorganized. As before, there are listings which deal specifically with $\nu_{\mathrm{e}}$, $\nu_{\mu}$, and $\nu_{\tau}$. In addition, in the category of searches near the end of the Stable Particle Listings, we include sections which deal with correlated bounds on neutrino masses and lepton mixing but which do not pertain to any one weak eigenstate individually. Furthermore, we include constraints from cosmological and astrophysical data. (Since this Review is a compendium of data traditionally derived more or less directly from particle and nuclear physics, we have been somewhat less comprehensive in our coverage of astrophysical data.)

In contrast to the other particles in this Review, the neutrinos $\nu_{\mathrm{e}}, \nu_{\mu}$, and $\nu_{\tau}$ are defined as weak eigenstates (that is, states which couple weakly with unit strength to e, $\nu$, and $\tau$ ) and are not, in general, states of definite mass. In the conventional case, where all neutrinos were assumed to be massless and hence degenerate, it was possible to define the weak eigenstates to be simultaneously mass eigenstates. However, in the general case of massive (nondegenerate) neutrinos, the weak eigenstates have no well-defined masses, but instead are linear combinations of mass eigenstates. Let us denote the charged leptons as the set $\left\{\ell_{\mathbf{a}}\right\}, \mathrm{a}=1, \cdots, \mathrm{n}$, where $\mathrm{n} \geqslant 3$ is the number of generations, with $\ell_{1} \equiv \mathrm{e}, \ell_{2} \equiv$ $\mu, \ell_{3} \equiv \tau$. In the standard $\mathrm{SU}(2)_{\mathrm{L}} \times \mathrm{U}(1)$ electroweak theory ${ }^{1}$ the mixing of the left-handed components of the mass eigenstates $\left(\nu_{\mathrm{j}}\right)_{\mathrm{L}}$ to form the weak gauge-group eigenstates $\left(\nu_{\ell_{\mathrm{a}}}\right)_{\mathrm{L}}$ is specified by the transformation

$$
\left(\nu_{\ell_{\mathrm{a}}}\right)_{\mathrm{L}}=\sum_{\mathrm{j}=1}^{\mathrm{n}} \mathrm{U}_{\mathrm{aj}}\left(\nu_{\mathrm{j}}\right)_{\mathrm{L}},
$$

where $U^{\dagger}=U^{-1}$. (In the case of Dirac neutrinos there are right-handed components of the $\nu_{j}$, but they are singlets under the gauge group; in the case of Majorana neutrinos in the standard theory there are no righthanded components.) The ordering of the mass eigenbasis is defined such that $U$ is as nearly diagonal as possible, i.e., $\left|U_{j j}\right|$ (no sum on $\left.\mathbf{j}\right) \geqslant\left|U_{j k}\right|, k \neq j$. This does not imply that $m\left(\nu_{j}\right)>m\left(\nu_{k}\right)$ if $j>k$, although this ordering might be regarded as natural in view of the similar one that obtains in the quark sector. The virtue of this convention is that a mass limit on " $\mathrm{m}\left(\nu_{\ell_{\mathrm{a}}}\right)$ " can be used as a definite limit on $\nu_{j}, j=a$, the dominantly coupled mass eigenstate in $\nu_{\ell_{\mathrm{a}}}$.

Thus, in this general case of $\mathbf{n}$ massive (Dirac or Majorana) neutrinos, decays such as $\mathrm{H}^{3} \rightarrow$ $\mathrm{He}^{3}+\mathrm{e}^{-}+\bar{\nu}_{\mathrm{e}}$ and $\pi^{+} \rightarrow \mu^{+}+\nu_{\mu}$, which have been used to set the best bounds on the respective neutrino masses, really consist of incoherent sums of the separate decay modes $\mathrm{H}^{3} \rightarrow \mathrm{He}^{3}+\mathrm{e}^{-}+\bar{\nu}_{\mathrm{j}}$ and $\pi^{+} \rightarrow \mu^{+}+\nu_{\mathrm{k}}$, where the $\nu_{\mathbf{j}}, \nu_{\mathbf{k}}$ are mass eigenstates, and the indices $\mathrm{j}$ and $k$ range over the subset $\{1, \cdots, n\}$ allowed by phase space in these two respective decays. ${ }^{2}$ The coupling strengths for the $\mathrm{j}^{\text {th }}$ modes are given for the two decays by the factors $\left|U_{1 j}\right|^{2}$ and $\left|U_{2 j}\right|^{2}$, respectively. There are, in addition, certain kinematic factors depending on the $m\left(\nu_{j}\right)$ which enter in determining the branching ratio for the $\mathrm{j}^{\text {th }}$ decay mode. Assuming that the offdiagonal elements of the lepton mixing matrix $U$ are small relative to the diagonal elements, the dominantly ccupled decays are the ones with coupling strength $\left|\mathrm{U}_{\mathrm{aj}}\right|^{2}, \mathrm{a}=\mathrm{j}$, i.e., $\mathrm{H}^{3} \rightarrow \mathrm{He}^{3}+\mathrm{e}^{-}+\bar{\nu}_{1}$ and $\pi^{+} \rightarrow$

It follows that the old neutrino mass limits quoted in the literature for " $\mathrm{m}\left(\nu_{\mathrm{e}}\right)$ ", " $\mathrm{m}\left(\nu_{\mu}\right)$ " and " $\mathrm{m}\left(\nu_{\tau}\right)$ " are meaningful only insofar as they are reinterpreted as limits on the corresponding mass eigenstates. Specifically, a bound such as the Bergkvist limit, 3 " $\mathrm{m}\left(\nu_{\mathrm{e}}\right)$ " $<60 \mathrm{eV}$ $(90 \% \mathrm{CL})$, really constitutes a weighted limit on each of the mass eigenstates $\nu_{\mathrm{j}}$ in the weak eigenstate $\nu_{\mathrm{e}}$ which are kinematically allowed to occur in tritium decay and which are coupled with strength $\left|U_{1 j}\right|^{2}$ sufficiently 


\section{Stable Particles}

\section{Neutrinos}

large to make a significant contribution to the observed spectrum. It is thus certainly a limit on $\nu_{1}$. If leptonic mixing is hierarchical as quark mixing is known to be (at least for the first three generations), i.e., $\left|U_{j j}\right|^{2} \gg$ $\left|\mathrm{U}_{\mathrm{jk}}\right|^{2}, \mathrm{j} \neq \mathrm{k}$, then $\nu_{1}$ is the only mass eigenstate significantly constrained by a bound on " $\mathrm{m}\left(\nu_{\mathrm{e}}\right)$." Furthermore, a neutrino mass limit cannot be stated in isolation; it always contains some implicit dependence on the relevant lepton mixing angles. Fortunately, this dependence is relatively unimportant for the dominantly coupled decay modes, i.e., $\overline{\mathrm{\nu}}_{1}, \mu \bar{\nu}_{2}$, and $\tau \bar{\nu}_{3}$. Since these modes were the ones responsible for the mass limits given previously, the latter can be reinterpreted without significant complication as proper limits on $m\left(\nu_{j}\right), j=1$, 2 , and 3 , respectively.

In addition to mass and lifetime limits, we have added data on neutrino magnetic dipole moments. These are of interest because a massless, purely chiral (empirically, left-handed) Dirac neutrino cannot have a magnetic (or electric) dipole moment. The same is true for a Majorana neutrino, whether massless or massive, because of its defining property of being self-conjugate.

If one considers the possibility of nonzero masses for neutrinos, for consistency one must also consider the leptonic mixing which would in general occur concomitantly. Accordingly we have devoted one category in the searches section to correlated bounds on neutrino masses and lepton mixing angles. These can be divided into two types. First, there are those due to decays involving neutrinos in the final state, which must be recognized to have the general multimode structure pointed out above. In the two most sensitive cases suggested as tests for neutrino masses and mixing, ${ }^{2}$ one obtains a limit on $\mathrm{m}\left(\nu_{\mathrm{j}}\right)$ and $\left|\mathrm{U}_{\mathrm{aj}}\right|^{2}$ individually for each $\mathrm{j}$. Second, there are those due to processes involving the propagation and subsequent interaction of neutrinos. The latter are often called neutrino "oscillation" 3 limits, although this term is correct only if the differences in neutrino masses are sufficiently small relative to their momenta that the propagation is effectively coherent in a quantum mechanical sense; otherwise, the individual $\nu_{\mathrm{j}}$ from a given decay such as $\pi_{\mu 2}$ or $\mathrm{k}_{\mu 2}$ propagate in a measurably incoherent manner and there is no "oscillation." Experimentalists usually present their results in terms of a simplifying model in which mixing is assumed to occur only between two neutrino species. Then the transformation equation becomes

\section{Data Card Listings}

Let the distance between the source of the neutrinos and their point of interaction be labeled as $x$, and their energy as E. Assume furthermore that the $m\left(\nu_{j}\right)$ are such that the coherence assumption is valid. Then, the probability of an initial $\nu_{\ell_{\mathrm{a}}}$ being equal to $\nu_{\ell_{\mathrm{b}}}$ at time $\mathrm{t}$, or equivalently (given the above assumption) at distance $\mathrm{x}=\mathrm{t}$, is

$$
\left|\left\langle\nu_{\ell_{\mathrm{b}}}(0) \mid \nu_{\ell_{\mathrm{a}}}(\mathrm{t})\right\rangle\right|^{2}=\sin ^{2} 2 \theta \sin ^{2}\left(\frac{\Delta \mathrm{m}^{2} \mathrm{x}}{4 \mathrm{E}}\right),
$$

where

$$
\Delta \mathrm{m}^{2}=\mathrm{m}\left(\nu_{\mathrm{i}}\right)^{2}-\mathrm{m}\left(\nu_{\mathrm{j}}\right)^{2} .
$$

Thus, neutrino oscillation experiments cannot measure individual neutrino masses, but only differences of masses squared, and indeed these are generally weighted in a more complicated way by mixing-matrix coefficients than in the two-species model. Experimental results are presented as allowed regions on a plot, the axes of which are $\left|\Delta \mathrm{m}^{2}\right|$ and $\sin ^{2} 2 \theta$. These are often summarized in terms of the asymptotic limits $\left|\Delta \mathrm{m}^{2}\right|_{\max }$ for $\sin ^{2} 2 \theta=1$, and $\sin ^{2} 2 \theta$ for "large" $\left|\Delta \mathrm{m}^{2}\right|$, i.e., sufficiently large $\left|\Delta \mathrm{m}^{2}\right|$ that the detector averages over many cycles of oscillation (or there ceases to be any coherence). We refer the reader to the original papers for the two-dimensional plots; for the purpose of these Listings, we shall give only the asymptotic limits.

An important question has to do with whether neutrinos are Dirac or Majorana (self-conjugate) particles. In the former case neutrinoless double beta decay, $(\mathrm{Z}, \mathrm{A})$ $\rightarrow(Z+2, A)+e^{-}+e^{-}$, is forbidden from occurring. ${ }^{4}$ In the Majorana case it may occur if (a) neutrinos are massive and/or (b) there are right-handed leptonic currents. In the light-neutrino case an upper limit on neutrinoless double beta decay yields a correlated upper bound on the quantity

$$
\overline{\mathbf{m}} \equiv\left|\sum_{\mathrm{j}=1}^{\mathrm{n}} \mathrm{U}_{1 \mathrm{j}}^{2} \mathrm{~m}\left(\nu_{\mathrm{j}}\right)\right|
$$

and $\eta$, the fractional admixture of right-handed leptonic current.

The correlated limits given in the section on Massive Neutrinos and Lepton Mixing are in digital form. For 
recent compendia of limits in convenient graphical form, see Refs. 5 and 6, and Figs. 1 and 2 (pp. 332-333) of Ref. 7.

Further explanatory notes are included in the Listings.

\section{References}

1. S. Weinberg, Phys. Rev. Lett. 19, 1264 (1967); A. Salam, in Elementary Particle Theory: Relativistic Groups and Analyticity, ed. N. Svartholm (Alqvist and Wiksell, Stockholm, 1968), p. 367. See also S. Glashow, Nucl Phys. 22, 579 (1961); S. Glashow; J. Iliopoulos, and L. Maiani, Phys. Rev. D2, 1285 (1970); and, for the $n=3$ case, M. Kobayashi and T. Maskawa, Prog. Theor. Phys. 49, 652 (1973).

2. R.E. Shrock, Phys. Lett. 96B, 159 (1980); Phys. Rev. D24, 1232 (1981); Phys. Rev. D24, 1275 (1981); and Phys. Lett. 112B, 382 (1982).

3. Z. Maki, M. Nakagawa, and S. Sakata, Prog. Theor. Phys. 28, 870 (1962); B. Pontecorvo, Sov. Phys. JETP 6, 429 (1957), and 7, 172 (1958); Zh. Ek. Theor. Fiz. 53, 1717 (1967); Sov. Phys. JETP 26, 984 (1968); V. Gribov and B. Pontecorvo, Phys. Lett. 28B, 493 (1969).

4. For recent studies of neutrinoless double beta decay, see H. Primakoff and S.P. Rosen, Ann. Rev. Nucl. Sci. 31, 145 (1981); S.P. Rosen, Proceedings of 1981 International Conference on Neutrino Physics and Astrophysics (Maui, Hawaii), eds. R.J. Sens et al., v.2, p. 76; W.C. Haxton, G.L. Stephenson, Jr., and D. Strottman, Phys. Rev. Lett. 47, 153 (1981); and M. Doi, T. Kotani, H. Nishiura, K. Okuda, and E. Takasugi, Phys. Lett. 103B, 219 (1981), and Prog. Theor. Phys. 66, 1739 and 1765 (1981).

5. M.H. Shaevitz, in Proceedings of the 1983 International Symposium on Lepton and Photon Interactions at High Energies, Cornell, eds. D.G. Cassel and D.L. Kreinich (Cornell University, 1983), p. 132.

6. F. Boehm and P. Vogel, Ann. Rev. Nucl. Part. Sci. 34 (1984).

7. R.E. Shrock, in Proceedings of the Third LAMPF II Workshop, eds. J.C. Allred et al. (Los Alamos National Laboratory, 1983), p. 316.
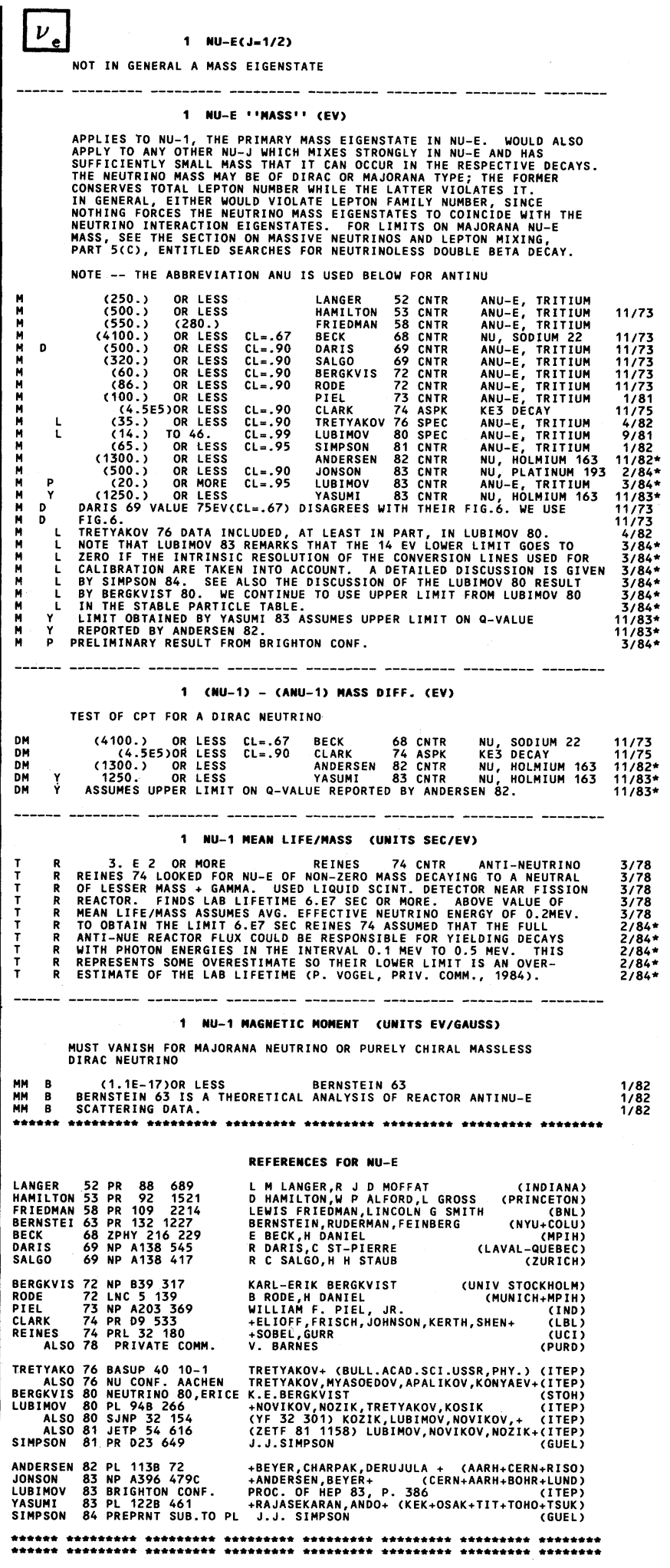


\section{Stable Particles}

e, $\nu_{\mu}$

\section{Data Card Listings}

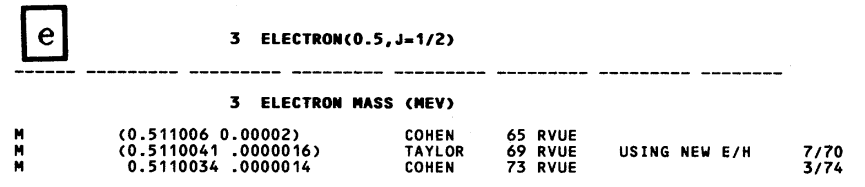

3 ELECTRON MEAM LIFE / BRAMCHIMG FRACTION (UMITS YRS) TEST OF CHARGE CONSERVATION

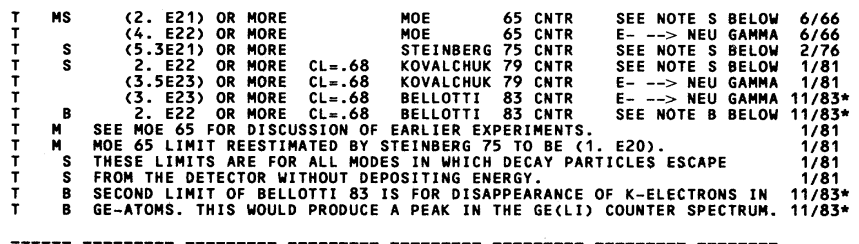

3 ELECTRON MAGMETIC MOMEMT(E/2ME)

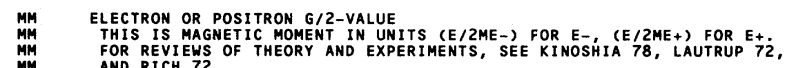
AND RICH 72.

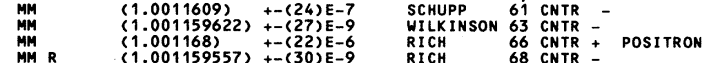

$\begin{array}{lll}\text { MMM R } & (1.001159557)+-(30) E-9 \\ \text { MMM } & (1.0011596389)+-(31) E-10 & R \\ \text { MMY } & (1.00115964)+-(7) E-9\end{array}$

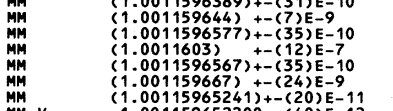

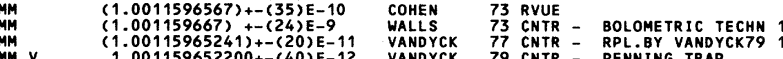

MM
MMM

MM R RICH 68 IS REEVALUATION OF WILKINSON 63 . PRT. COMM.

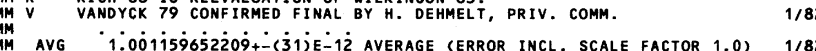

MM AVERAGE ASSUMING EQUAL G/2-VALUES FOR E + AND E- BY CPT.

MMR POSITRON TO ELECTRON G-FACTOR RATIO MINUS ONE, (G+/G-)-1

MMR
MMR
MMR
TIST OF CPT

\begin{tabular}{|c|c|c|c|c|c|c|}
\hline \multirow[b]{2}{*}{$\begin{array}{l}\text { EDM } \\
\text { EDM } \\
\text { EDM } \\
\text { EDM }\end{array}$} & \multicolumn{6}{|c|}{$\begin{array}{l}3 \text { ELECTRON ELECTRIC DIPOLE MOMENT(UNITS 10**-23 E-CH) } \\
\text { FORBIDDEN BY BOTH TINVARIANCE AND P INVARIANCE }\end{array}$} \\
\hline & $\begin{array}{l}0.3 \\
(0.07) \\
(0.19) \\
(8.1)\end{array}$ & $\begin{array}{cc}\text { OR LESS } & C L=.90 \\
(0.22) & C L=.90 \\
(0.34) & C L=.90 \\
(11.6) & \end{array}$ & $\begin{array}{l}\text { WEISSKOPF } \\
\text { PLAYER } \\
\text { SANDARS } \\
\text { VASILEV }\end{array}$ & $\begin{array}{l}68 \text { MRS } \\
70 \text { MRS } \\
75 \text { MRS } \\
78\end{array}$ & $\begin{array}{l}\text { CESIUM } \\
\text { XENON } \\
\text { THALLIUM }\end{array}$ & $\begin{array}{r}12 / 79 \\
4 / 82 \\
4 / 82 \\
12 / 79\end{array}$ \\
\hline
\end{tabular}

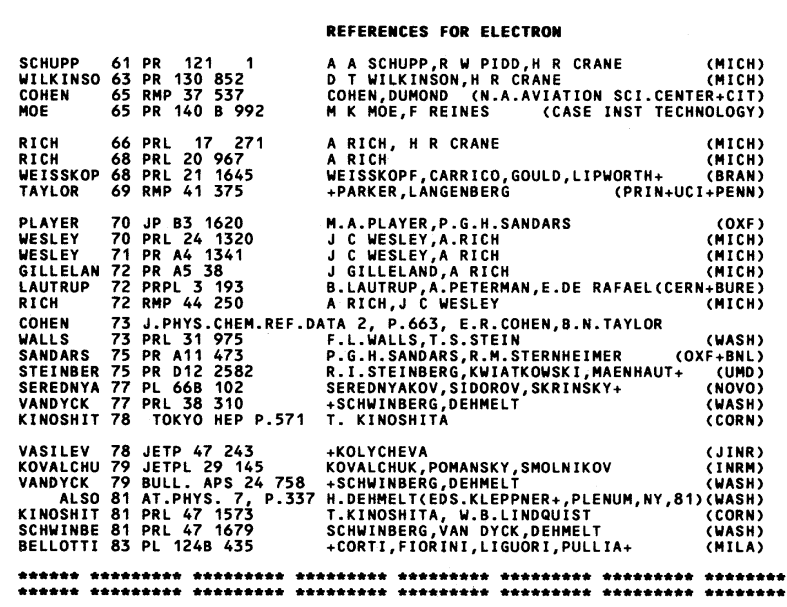

$\nu_{u}{ }_{2}$ MU-MU(J-1/2)
NOT IN GENERAL A MASS EIGENSTATE. SEE NOTE ON NEUTRINOS
IN THE ELECTRON NEUTRINO SECTION ABOVE.

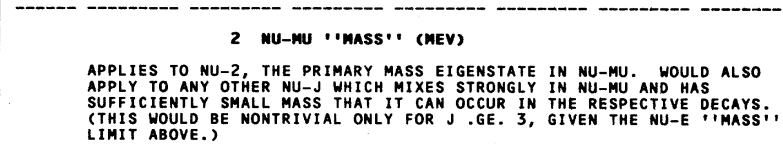

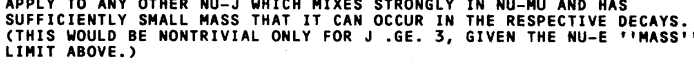

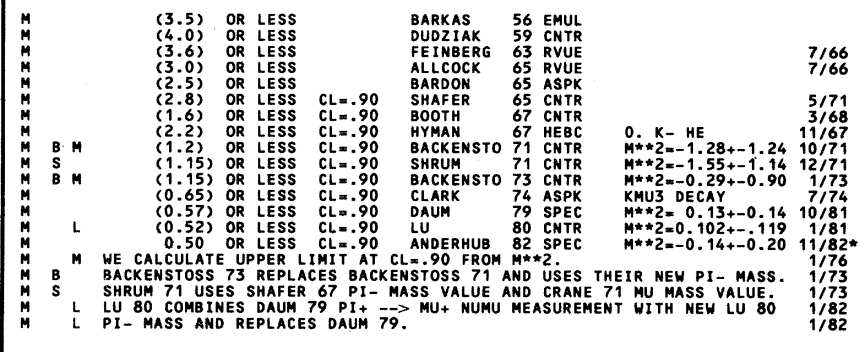

2 (MU-2) - (AMU-2) MASS DIFF. (MEV)

TEST OF CPT FOR A DIRAC NEUTRINO

OM (0.45) OR LESS CL=.90 CLARK 74 ASPK KMU3 DECAY $11 / 75$

2 MU-2 MEAN LIFE/MASS (UNITS SEC/EV)

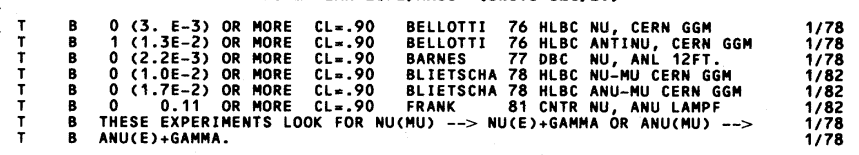

- - - - - - - - - - -

2 C - - (MU-2 VELOCITY) - ABS(CV-C)/C) (UMITS $10 * *-4)$

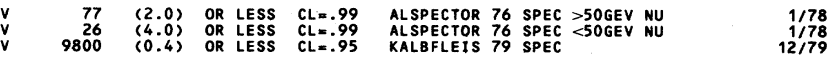

2 MU-2 MAGMETIC MOMENT (UMITS EV/GAUSS)

MUST VANISH FOR MAJORANA NEUTRINO OR PURELY CHIRAL MASSLESS

MM $K$ MM $K$ KIM ${ }^{(4} 74$ E-17) OR A THEORETICAL ANALYSIS OF ANTINU-MU REACTION DATA.

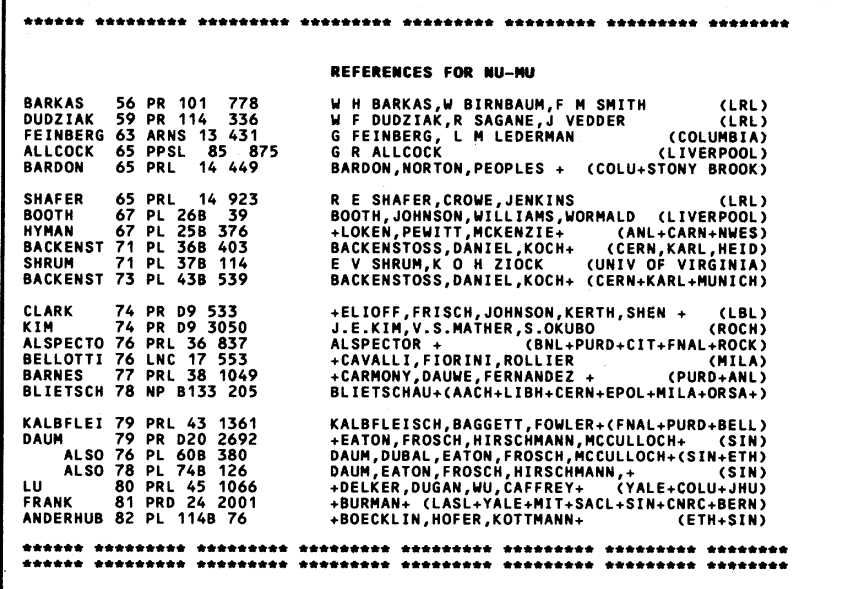




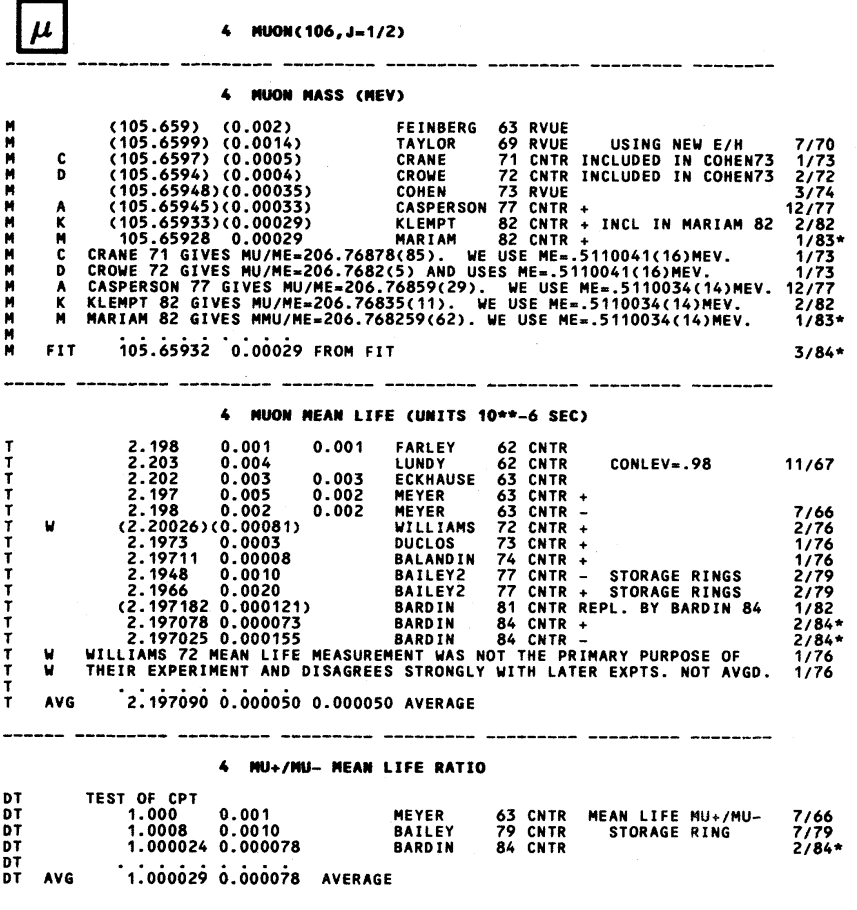

4 MUOW aMOMALOUS MAGW. MOMENT (10**-6*E/(2*MU MASS))

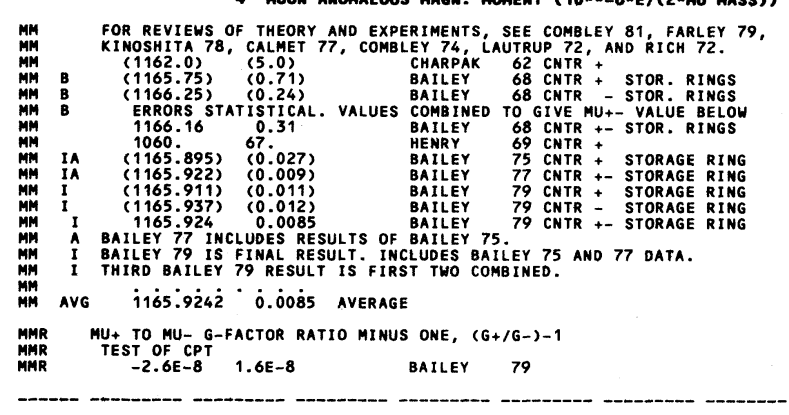

4 MUOW ELECTRIC DIPOLE MOMENT (UNITS 10**-19 E-CW) FORBIDDEN BY BOTH T INVARIANCE AND P INVARIANCE

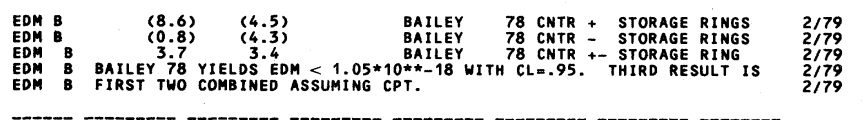

\section{MUON TO PROTON MAGNETIC MOMEMT RATIO}

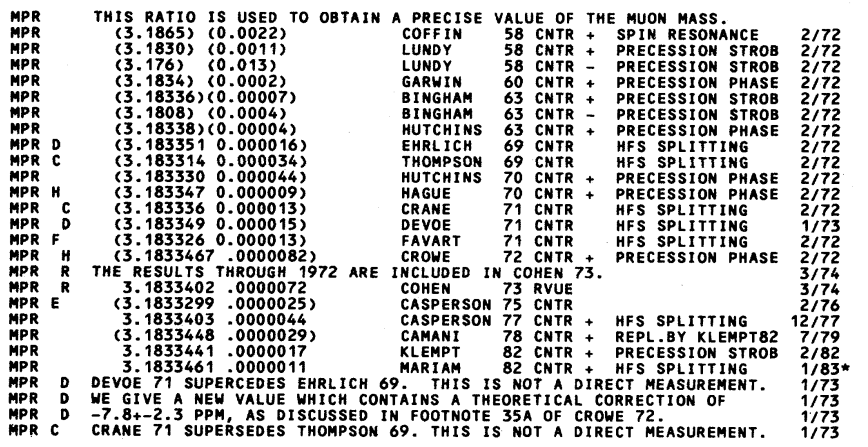

$2 / 84 *$
MPR H CROWE 72 SUPERSEDES HAGUE 70.

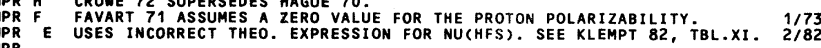
MPR AVG 3.1833452 :00000 io AVERAGE (ERROR INCLUDES SCALE FACTOR OF 1.1)

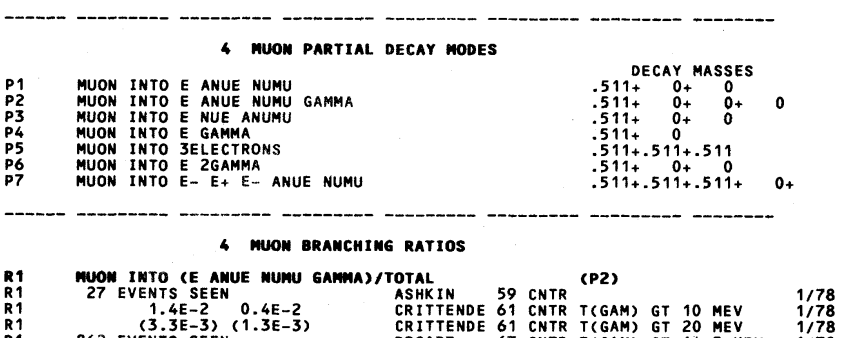

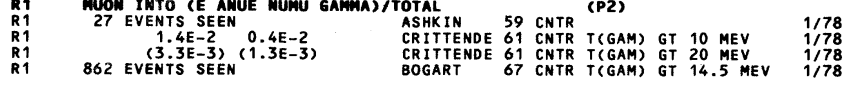

MUOW. INTO (EF AMUE MUMU), TOTAL
FORB IDDEN BY ADDITIVE CONSERVATION LAW FOR LEPTON FAMILY NUMBER.

MULTIPLICATIVE LAH PREDICTS THIS BRANCHING RATIO TO BE $1 / 2$.

A RECENT REVIEU SEE NEMETHY 81.
$(0.25)$ OR LESS CL .90

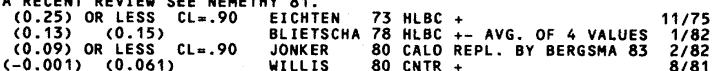

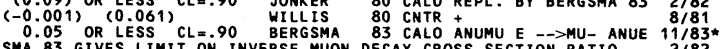

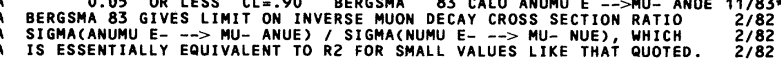

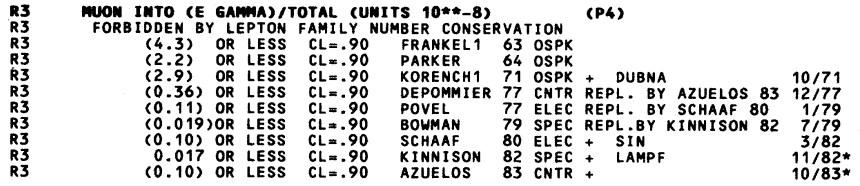

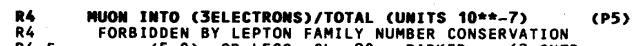

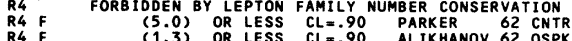

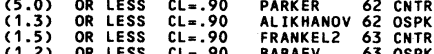

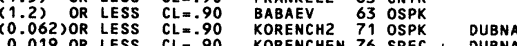

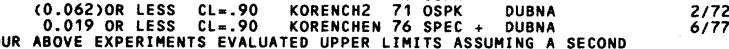

ORDER VAA NEUTRIN LOOP DIAGRAM. LIMITS NOT SIGNIFICANTLY CHANGED

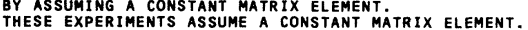

$10 / 77$

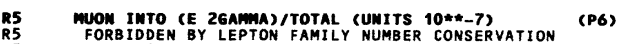

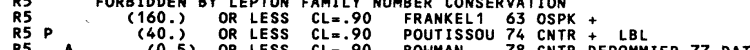

(0.5) OR LESS CL=.90 BOWMAN 78 CNTR DEPOMMIER 77 DATA

POUTIISSOU 74 LIMIT APPLIES TO SUM OF ALL NEUTRINOLESS MU+ DECAYS. $10,183$.

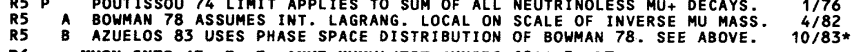

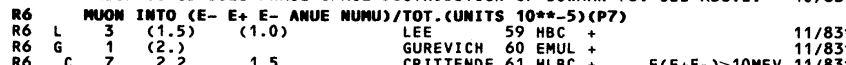

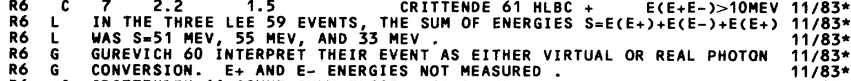

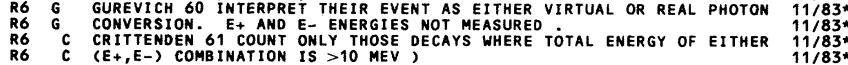

旅最

4 LIMIT ON MUON --> ELECTron CONVERSIOM

FORBIDDEN BY LEPTON FAMILY NUMBER CONSERVATION

RE (MUU- SULFUR32 $\rightarrow$ RAT- SULFUR32)/(CUU-SULFUR32 $\rightarrow$ RE MUMU PHOSPHORUS32*)

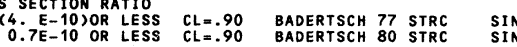

$1 / 82$

6 LIMIT ON MUON $\rightarrow \rightarrow$ POSITRON Conversion

FORBIDDEN BY TOTAL LEPTON NUMBER CONSERVATION

RP1 (MU- SULFUR32 $\rightarrow$ Et SILICOM32*) (MUU-SULF32 $\rightarrow$ MUMU PHOSPHORUS32*)

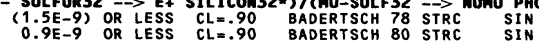

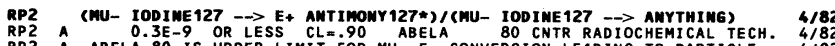

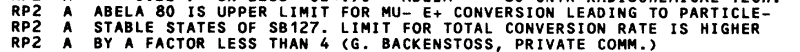

- Linit on (NU+, E-) BMo. State conversion to (MU-,E+)

FORB IDDEN BY LEPTON FAMILY NUMBER CONSERVATION

RG $=$ GC/GF

WHERE GF = 1.16637 G 5 GEV**(-2) IS THE FERMI CONSTANT AND

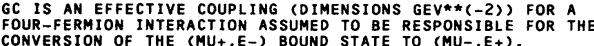

42 OR LESS CL=.95 MARSHALL 82 CNTR

$12 / 83$ 


\section{Stable Particles}

Data Card Listings

$\mu$

\section{NOTE ON MUON DECAY PARAMETERS}

The $\mu$ decay parameters describe the momentum spectrum ( $\rho$ and $\eta$ ), the asymmetry ( $\xi$ and $\delta$ ), and the helicity (h) of the electron in the process $\mu^{-} \rightarrow \mathrm{e}^{-}+\nu_{\mu}$ $+\bar{\nu}_{\mathrm{e}}$. Assuming a local and lepton-conserving interaction, the matrix element in the charge-retention form may be written as

$$
\sum_{i}\left[\overline{\mathrm{e}} \Gamma_{\mathrm{i}} \mu\right]\left[\bar{\nu}_{\mu} \Gamma_{\mathrm{i}}\left(\mathrm{C}_{\mathrm{i}}+\mathrm{C}_{\mathrm{i}}^{\prime} \gamma_{5}\right) \nu_{\mathrm{e}}\right]
$$

where the summation is taken over $i=S, V, T, A, P$. Using the definitions and sign conventions of Sachs and Sirlin ${ }^{1}$ and Scheck $^{2}$ for the Lorentz-covariant operators, we have for the momentum parameters:

$$
\begin{aligned}
& \rho=\left(3 \mathrm{~g}_{\mathrm{A}}^{2}+3 \mathrm{~g}_{\mathrm{V}}^{2}+6 \mathrm{~g}_{\mathrm{T}}^{2}\right) / \mathrm{D}, \\
& \eta=\left(\mathrm{g}_{\mathrm{S}}^{2}-\mathrm{g}_{\mathrm{P}}^{2}+2 \mathrm{~g}_{\mathrm{A}}^{2}-2 \mathrm{~g}_{\mathrm{V}}^{2}\right) / \mathrm{D} ;
\end{aligned}
$$

for the asymmetry parameters:

$$
\begin{aligned}
& \xi=\frac{-6 g_{\mathrm{S}} g_{\mathrm{P}} \cos \phi_{\mathrm{SP}}-8 \mathrm{~g}_{\mathrm{A}} \mathrm{g}_{\mathrm{V}} \cos \phi_{\mathrm{AV}}+14 \mathrm{~g}_{\mathrm{T}}^{2} \cos \phi_{\mathrm{TT}}}{\mathrm{D}} \\
& \delta=\left(-6 \mathrm{~g}_{\mathrm{A}} \mathrm{g}_{\mathrm{V}} \cos \phi_{\mathrm{AV}}+6 \mathrm{~g}_{\mathrm{T}}^{2} \cos \phi_{\mathrm{TT}}\right) / \mathrm{D} \xi
\end{aligned}
$$

and for the parameter describing the helicity of the electron:

$$
h=\frac{2 g_{S} g_{P} \cos \phi_{S P}+8 g_{A} g_{V} \cos \phi_{A V}+6 g_{T}^{2} \cos \phi_{T T}}{D} .
$$

Here

$$
\begin{aligned}
& \mathrm{D}=\mathrm{g}_{\mathrm{S}}^{2}+\mathrm{g}_{\mathrm{P}}^{2}+4 \mathrm{~g}_{\mathrm{V}}^{2}+6 \mathrm{~g}_{\mathrm{T}}^{2}+4 \mathrm{~g}_{\mathrm{A}}^{2}, \\
& \mathrm{~g}_{\mathrm{i}}^{2}=\left|\mathrm{C}_{\mathrm{i}}\right|^{2}+\left|\mathrm{C}_{\mathrm{i}}^{\prime}\right|^{2},
\end{aligned}
$$

and

$$
\cos \phi_{i j}=\operatorname{Re}\left(C_{i}^{*} C_{j}^{\prime}+C_{i}^{\prime} C_{j}^{*}\right) / g_{i} g_{j} \cdot
$$

The quantities $\mathrm{g}_{\mathrm{i}}$ are defined to be real non-negative numbers, and the $\phi_{i j}$ are phase angles between the i-type and j-type interactions. Under the assumption of twocomponent neutrinos, $C_{V}^{\prime}=-C_{V}$ and $C_{A}^{\prime}=-C_{A}$, the $S, P$, and $T$ terms vanish, and $\phi_{A V}$ is the phase angle between $\mathrm{C}_{\mathrm{A}}$ and $\mathrm{C}_{V}$ in the complex plane.
By using the above equations and the experimental determinations of $\rho, \eta, \xi, \delta$, and h, limits can be placed on $\left|g_{S} / g_{V}\right|,\left|g_{A} / g_{V}\right|,\left|g_{T} / g_{V}\right|,\left|g_{P} / g_{V}\right|$, and $\phi_{A V}$. The results, given in the Data Card Listings, assume neither two-component neutrinos nor time-reversal invariance. If, however, two-component neutrinos are assumed, then $\sin \phi_{\mathrm{AV}}$ is the amplitude of time-reversal violation. Note that most experiments study only the upper end of the spectrum where $\rho$ and $\eta$ are highly correlated, so they can only report $\rho$ for $\eta=0$ and $\eta$ for $\rho=3 / 4$. The values for $\rho$ and $\eta$ we use here were obtained by combining measurements of both upper and lower ends of the spectrum and turn out to be nearly uncorrelated.

Note also that the radiative corrections are unambiguous only when $g_{S}=g_{T}=g_{P}=0$. The same limits on $\mathrm{g}_{\mathrm{A}} / \mathrm{g}_{\mathrm{V}}$ and $\phi_{\mathrm{AV}}$ are obtained, however, as when $\mathrm{g}_{\mathrm{S}}, \mathrm{g}_{\mathrm{T}}$, and $g_{\mathbf{P}}$ are left free.

\section{References}

1. A.M. Sachs and A. Sirlin, in Muon Physics II, eds. C.S. Wu and V. Hughes (Academic Press, New York, 1975), p. 49.

2. F. Scheck, Phys. Rep. 44, 187 (1978).

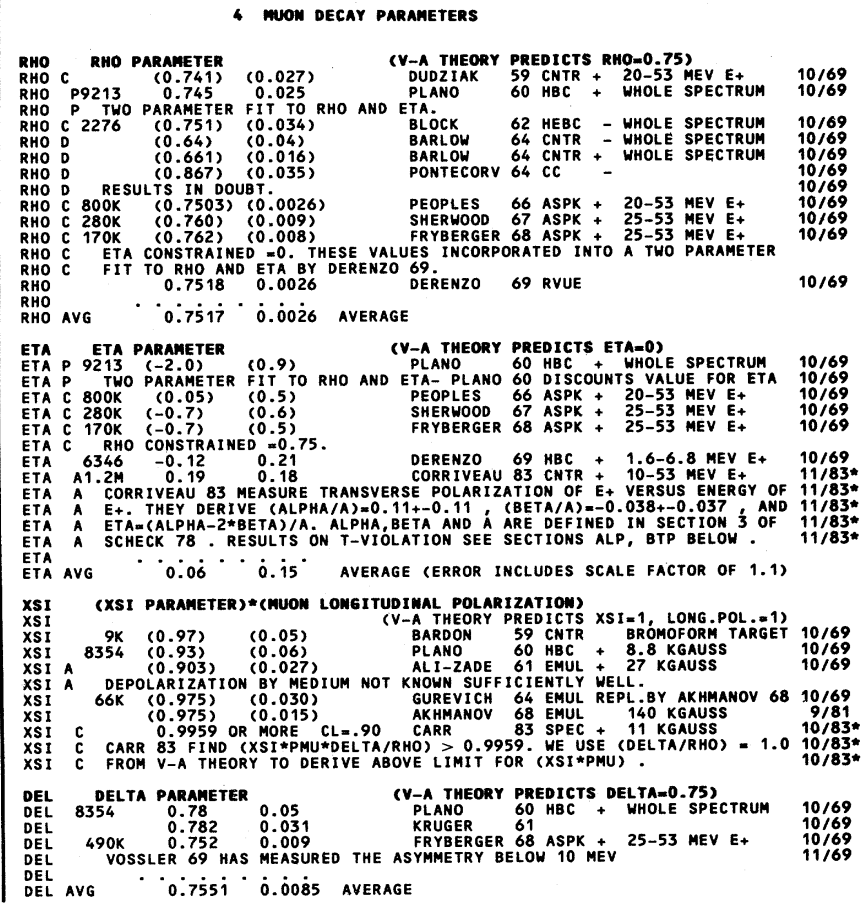


For notation, see key at front of Listings.

\section{Stable Particles}

$\mu, \nu_{\tau}$
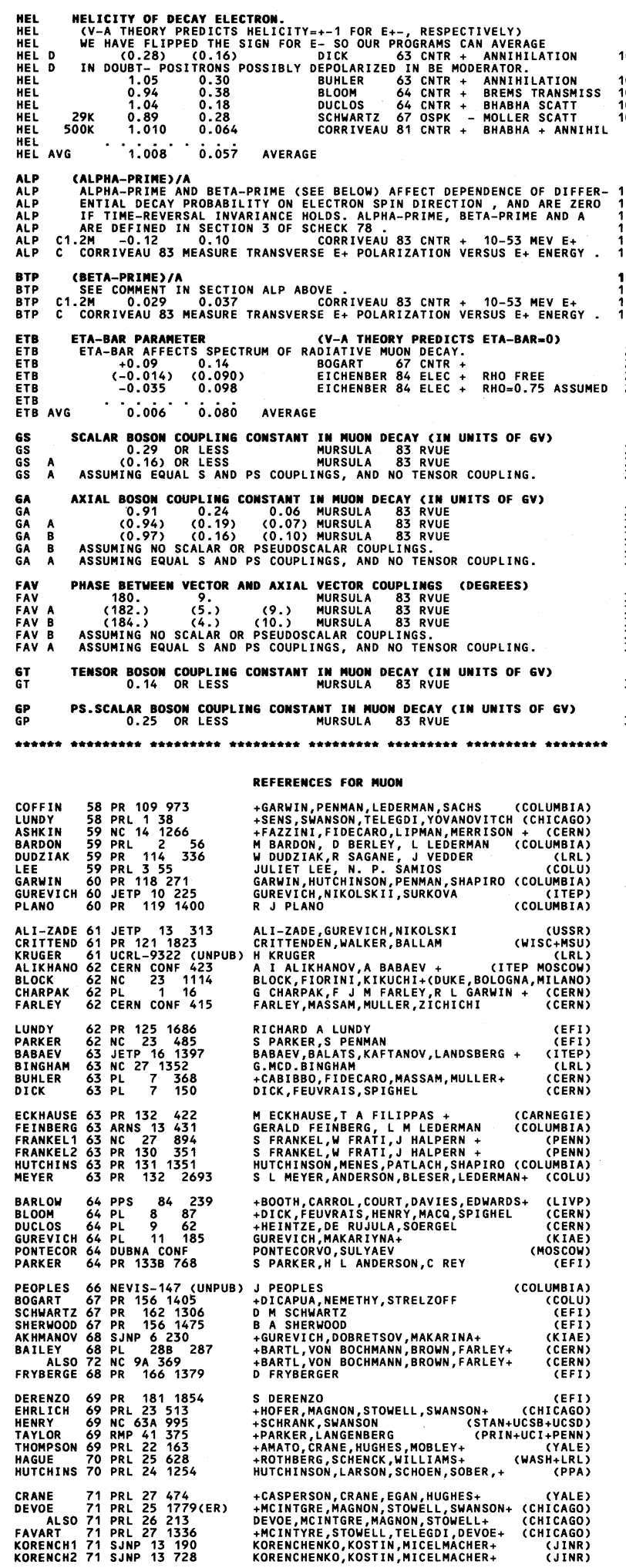

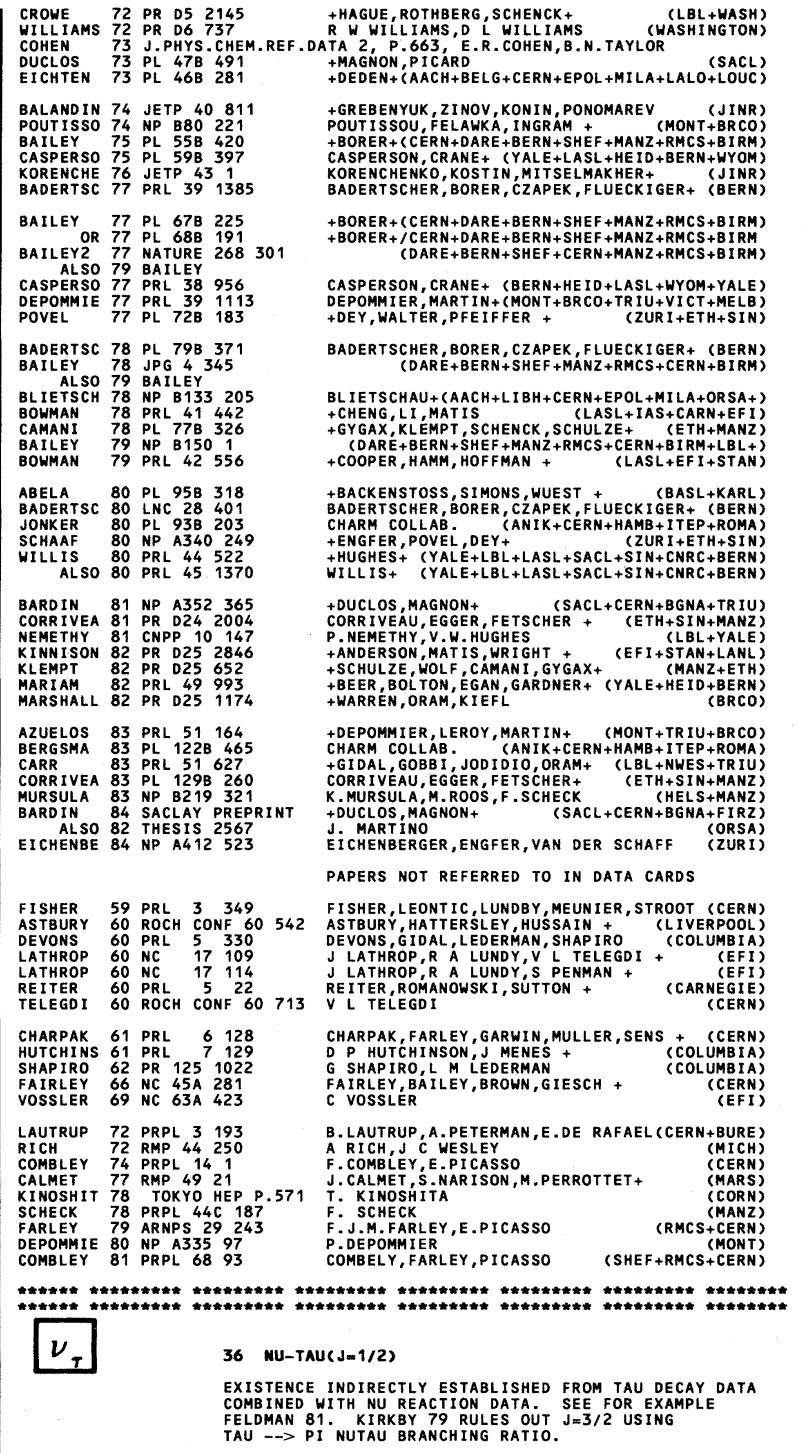

NOT IN GENERAL A MASS EIGENSTATE. SEE NOTE ON NEUTRINOS
IN THE ELECTRON NEUTRINO SECTION ABOVE.

$$
36 \text { MU-TAU " 'MASS" (MEV) }
$$

APPLIES TO NU-3, THE PRIMARY MASS EIGENSTATE IN NU-TAU. WOULD ALSO

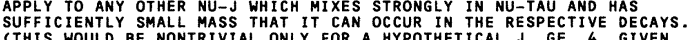
TTHS WOUL BE NONTRIVIAL ONLY FOR A HPOTHETICAL J . GE. 4, GIVEN
THE NU-E AND NU-MU , MASS. 'LIMITS ABOVE.)
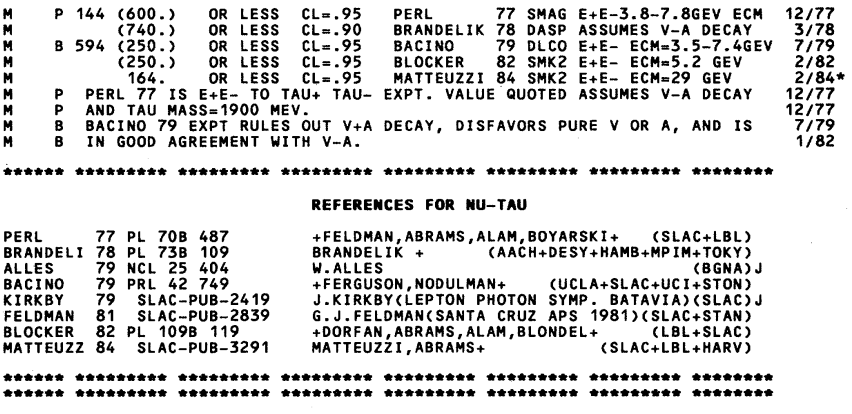


\section{Stable Particles}

$\tau^{ \pm}$

$\tau^{ \pm}$

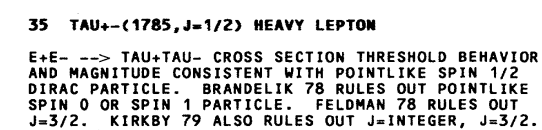

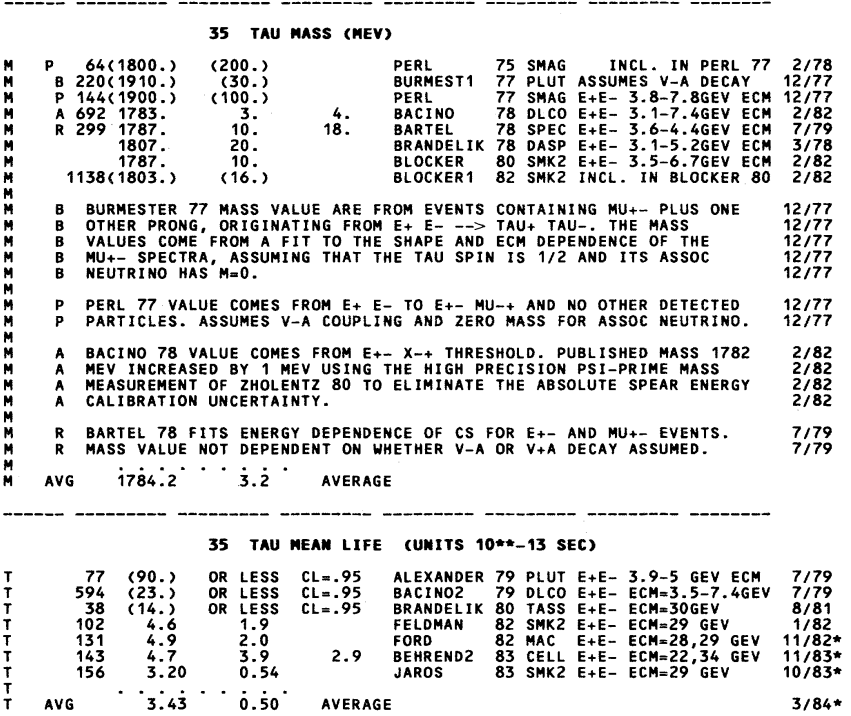

35 TAU PARTIAL decaY moDes

\begin{tabular}{|c|c|c|}
\hline 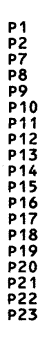 & 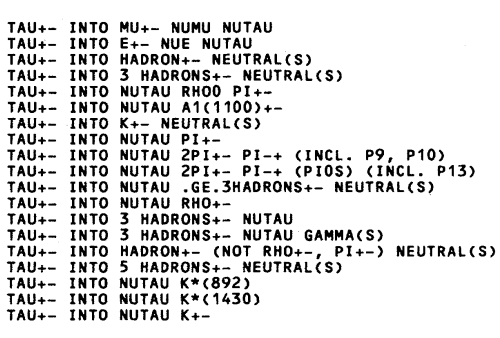 & 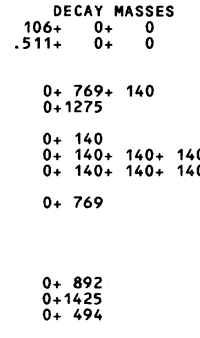 \\
\hline 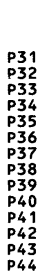 & 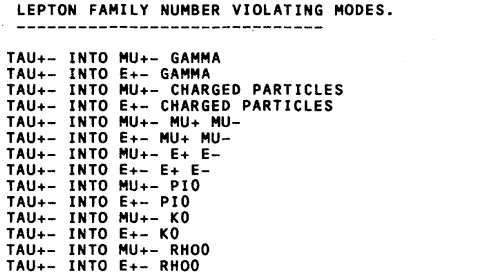 & 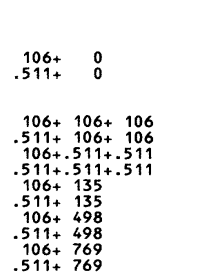 \\
\hline
\end{tabular}

\section{FITTED PARTIAL DECAY MODE BRANCHING FRACTIONS}

The matrix below is derived from the error matrix for the fitted partial decay mode branching fractions, $P_{i}$, as follows: The diagonal elements are $P_{i} \pm \delta P_{i}$, where

${ }^{\delta} \mathrm{P}_{\mathrm{i}}=\sqrt{\left\langle\delta \mathrm{P}_{\mathrm{i}} \delta \mathrm{P}_{\mathrm{i}}\right\rangle}$, while the off-diagonal elements are the normalized correlation coefficients $\left\langle\delta \mathrm{P}_{i} \delta \mathrm{P}_{j}\right\rangle /\left(\delta \mathrm{P}_{i}, \delta \mathrm{P}_{\mathrm{j}}\right)$. For the definitions of the individual $\mathrm{P}_{i}$, see the listings above; only those $P_{i}$ appearing in the matrix are assumed in the fit to be nonzero and $\begin{array}{llllll}\text { are thus constrained to add to } 1 . & \text { P12 } & \text { P16 } & \text { P17 } & \text { P18 } & \text { P19 }\end{array}$

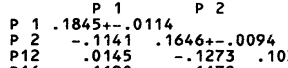

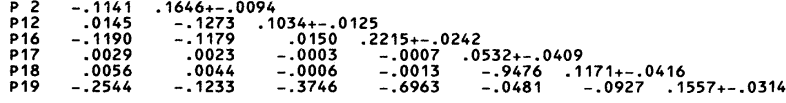

\section{Data Card Listings}

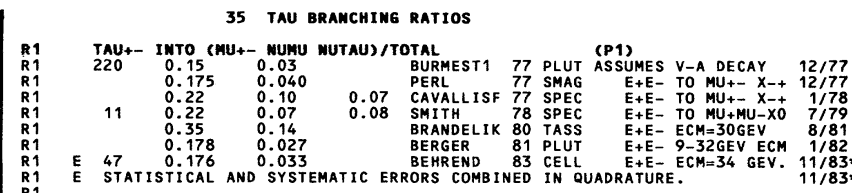

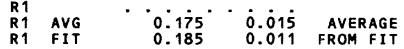

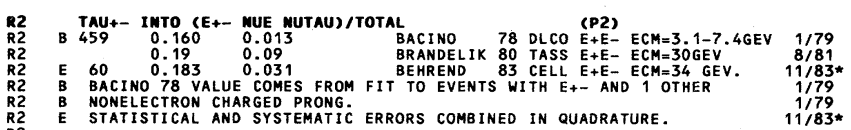
$\begin{array}{lllll}\text { R2 } & \text { AVG } & 0.164 & 0.012 & \text { AVERAGE } \\ \text { R2 } & \text { FIT } & 0.1640 & 0.0094\end{array}$

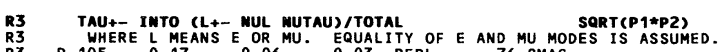

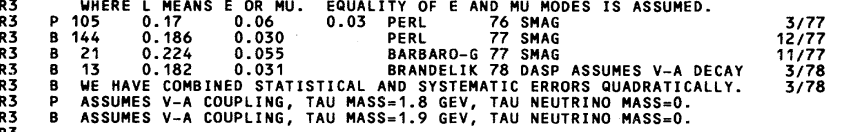

$\begin{array}{lllll}\text { R3 } & \text { AVG } & 0.186 & 0.018 & \text { AVERAGE } \\ \text { R3 } & \text { FIT } & 0.1743 & 0.0069 & \text { FROM FIT }\end{array}$

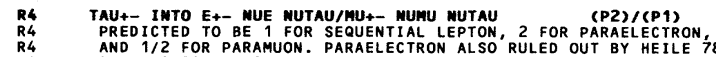

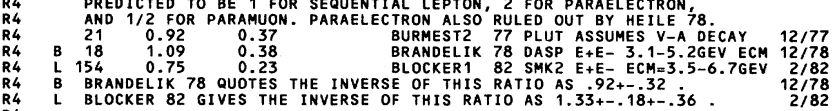

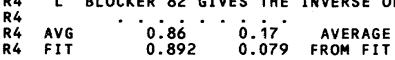

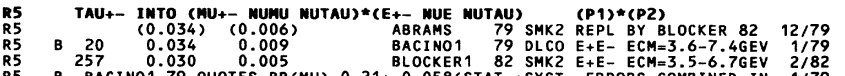

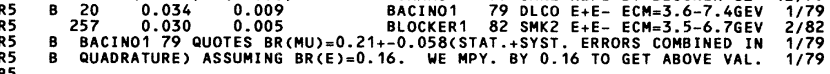
$\begin{array}{llll}R & \\ R 5 & \text { AVG } & 0.0309 \\ \text { R5 FIT } & 0.0304 & 0.0044 & \text { AVERAGE } \\ \text { RIT } & 0.0024 \text { FROM FIT }\end{array}$

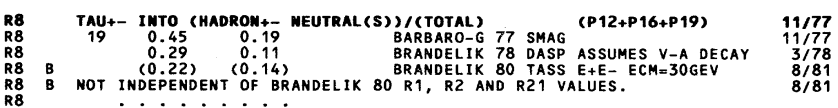

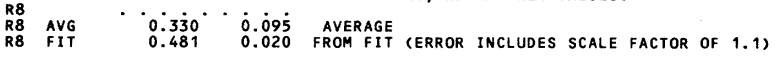

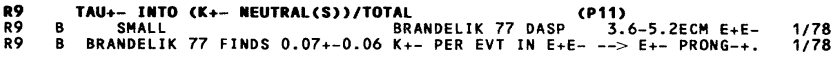

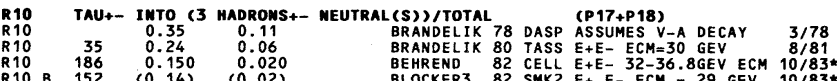

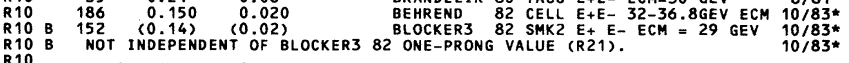

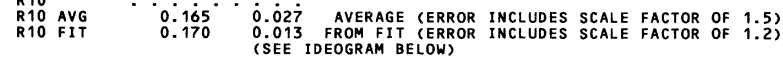

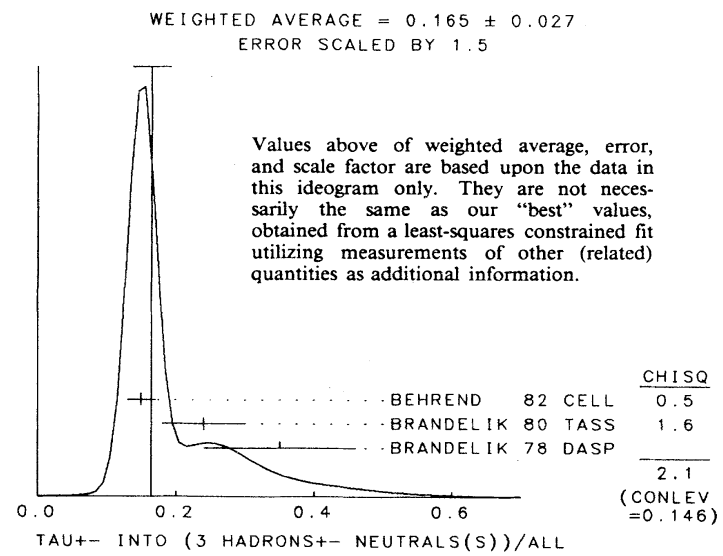




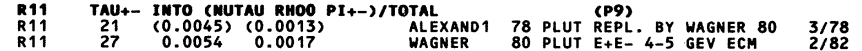

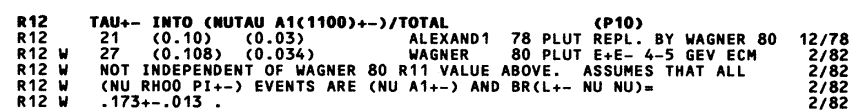

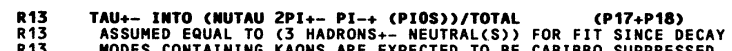

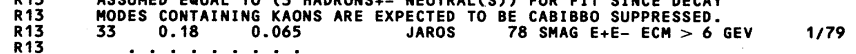

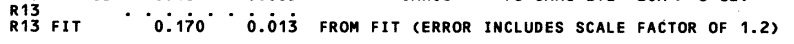

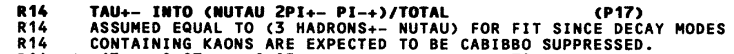

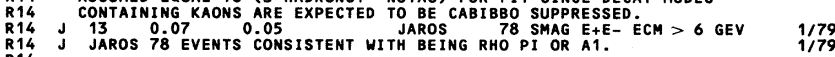

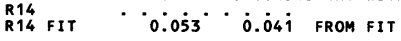

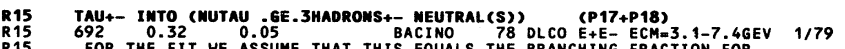

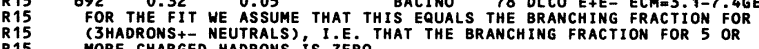
R15 FIT $0.170^{\circ} \cdot 0.013$ FROM FIT (ERROR INCLUDES SCALE FACTOR OF 1.2)

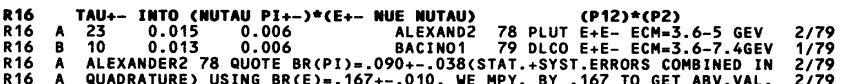

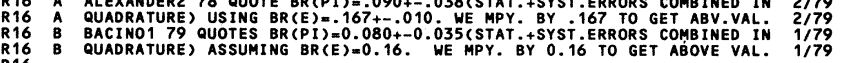
$\begin{array}{llll}\text { R16 } & \text { RVG } \\ \text { R16 FIT } & 0.0140^{\circ} & 0.0042 & \text { AVERAGE } \\ \text { R.0170 } & 0.0022 \\ \text { FROM FIT }\end{array}$

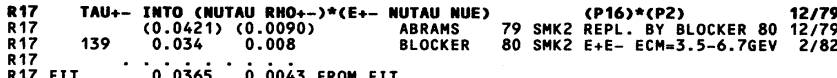

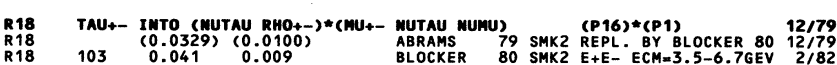

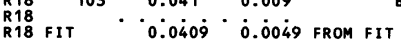

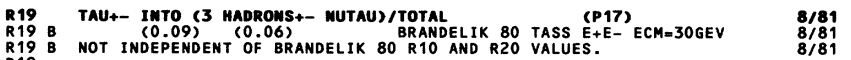
R19B NOT INDEPENDENT OF BRANDELIK 80
R19
R19 FIT O.053 $0.04 \mathrm{~T}$ FROM FIT

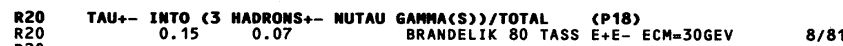

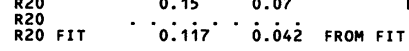

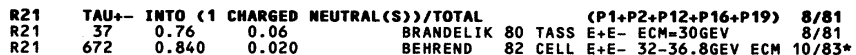

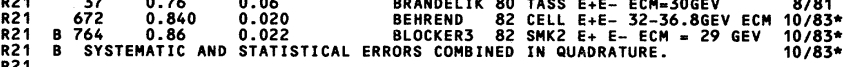

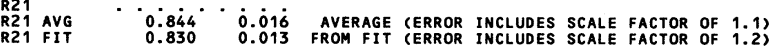

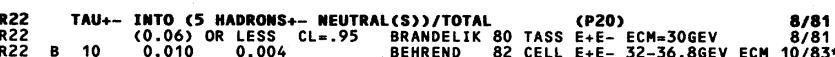

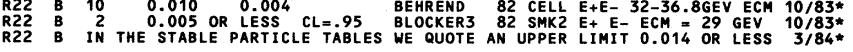
R23 TAU+- INTO (MUTAU K*(892)+-)/TOTAL
R23
0.011
0.007

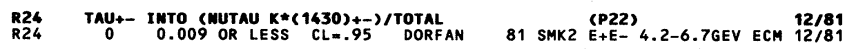

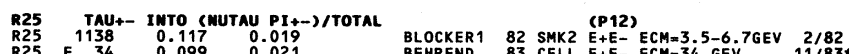

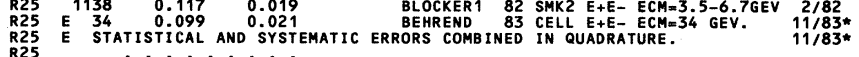

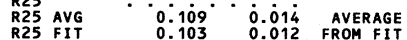

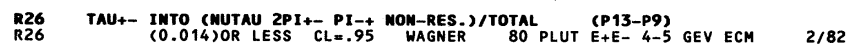

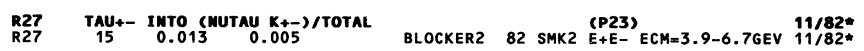

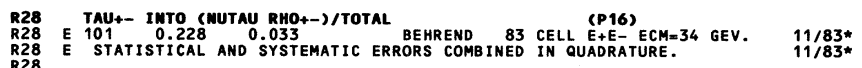
R28 FIT $0.221 \cdots 0.024$ FROM FIT

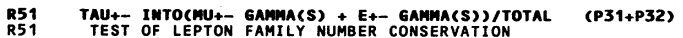

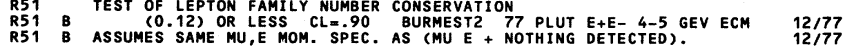

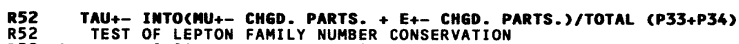

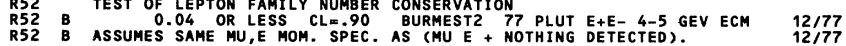

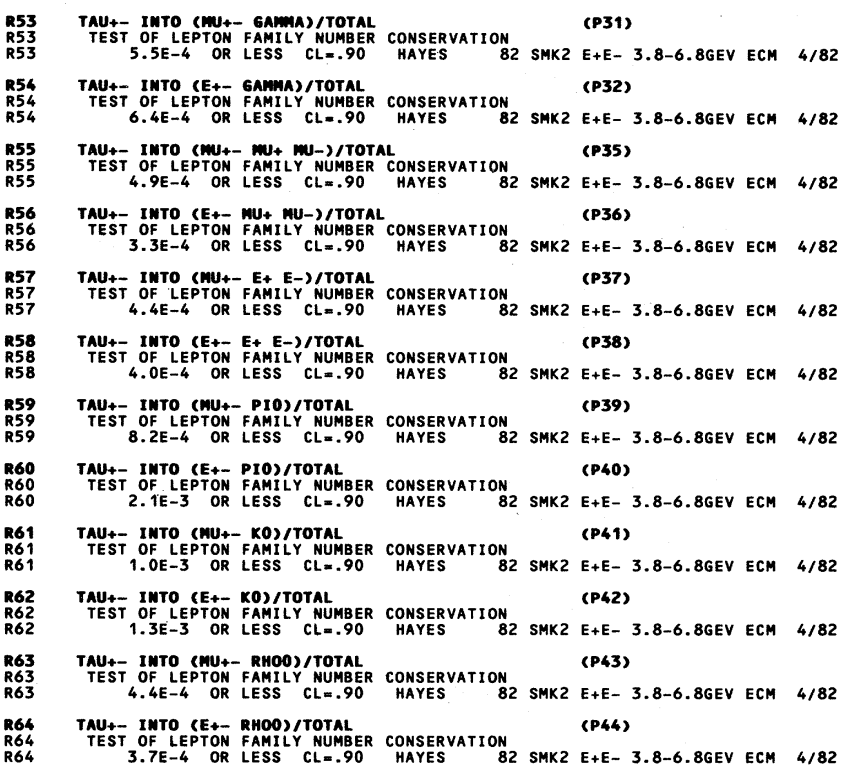
35 TAU decay parameters

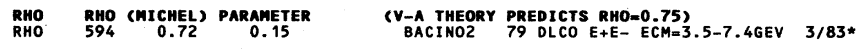

\begin{tabular}{|c|c|c|}
\hline RL & 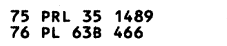 & $\begin{array}{l}\text { S, BOYARSK I, BREIDENBACH } \\
\text { AN, ABRAMS, ALAM, BOYARSKI }+{ }^{+} \begin{array}{l}(L B L+S L A C) \\
(S L A C+L B L)\end{array}\end{array}$ \\
\hline $\begin{array}{l}\text { RBARO- } \\
\text { ANDELI } \\
\text { ANMEST1 } \\
\text { RMEST } \\
\text { VALLIS } \\
\text { RL IS }\end{array}$ & 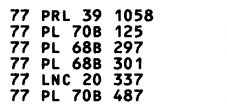 & 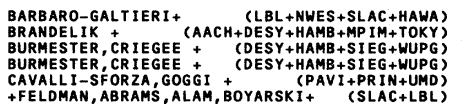 \\
\hline $\begin{array}{l}\text { ILEXAND1 } \\
\text { ILEXAND2 } \\
\text { ACINO } \\
\text { ALSO } \\
\text { ALSO } \\
\text { AARTEL }\end{array}$ & 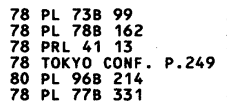 & 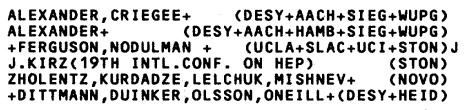 \\
\hline $\begin{array}{l}\text { RANDELI } \\
\text { IILE } \\
\text { ROS } \\
\text { IITH }\end{array}$ & $\begin{array}{l}78 \mathrm{PL} \\
78 \\
78 \\
78 \mathrm{PRL} \\
78 \mathrm{PR}\end{array}$ & 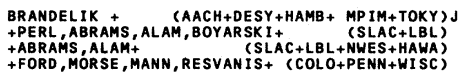 \\
\hline $\begin{array}{l}\text { BRAMS } \\
\text { LEXANDE } \\
\text { LLES } \\
\text { LCINO1 } \\
\text { ACINO2 }\end{array}$ & 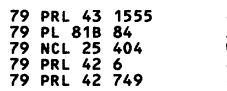 & 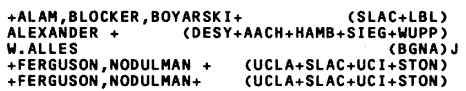 \\
\hline $\begin{array}{l}\text { BLOCKER } \\
\text { BRANDELI } \\
\text { WAGNER } \\
\text { ZHOLENTZ } \\
\text { BELSO } \\
\text { BERGER } \\
\text { DORFAN }\end{array}$ & 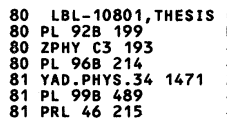 & 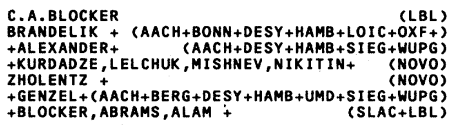 \\
\hline $\begin{array}{l}\text { BEHREND } \\
\text { BLOCKER1 } \\
\text { BLOCKER2 } \\
\text { BLOCKER3 } \\
\text { FELDMAN } \\
\text { FORDAN } \\
\text { HAYES }\end{array}$ & 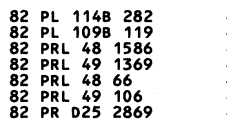 & 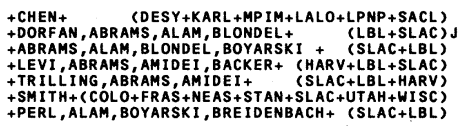 \\
\hline $\begin{array}{l}\text { BEHREND } \\
\text { BEHREND2 } \\
\text { JAROS }\end{array}$ & 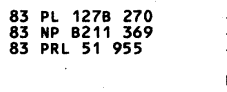 & 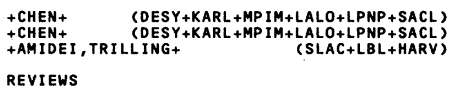 \\
\hline E & $\begin{array}{l}\text { G SYMP. } \\
\text { CONF.BOSTON }\end{array}$ & $\begin{array}{l}\text { ALSO ISSUED AS SLAC-PUB-2022, M.PERL (SLAC) } \\
\text { ALSO ISSUED AS DESY } 77-35, G \text {,FLUGGE (DESY) }\end{array}$ \\
\hline $\begin{array}{l}\text { ZIMOV } \\
\text { ELDMAN } \\
\text { ERL }\end{array}$ & $\begin{array}{l}78 \text { SPU } \\
78 \text { TOKY } \\
78 \text { SLAC }\end{array}$ & $\begin{array}{l}\text { RT KHOZE } \\
\text { MAN (19TH INTL. CONF. ON HEP) } \\
\text { (KARLSRUHE SUMMER INST.1978) }\end{array}$ \\
\hline $\begin{array}{l}\text { KIRKBY } \\
\text { PERL }\end{array}$ & $\begin{array}{l}79 \text { ZPHY C1 } 121 \\
79 \text { SLAC-PUB-2419 } \\
80 \text { ARNPS } 30299\end{array}$ & $\begin{array}{l}\text { G. FLUGGE } \\
\text { J.KIRKBY(LEPTON PHOTON SYMP. BATAVIA) (SLAY), } \\
\text { M.L.PERL } \\
\text { (SLAC) }\end{array}$ \\
\hline
\end{tabular}




\section{Stable Particles}

Data Card Listings

SEARCHES FOR MASSIVE $\nu^{\prime}$ ' \& LEPTON MIXING

\section{SEARCHES FOR MASSIVE NEUTRINOS AND LEPTON MIXING}

SEE THE NOTE ON MEUTRINOS BY R.E. SHROCK IN THE ELECTRON NEUTRINO SEARCHES FOR INDIRECT EFFECTS OF NEUTRINO MASSES AND LEPTON MIXING
ARE LISTED HERE. DIRECT SEARCHES FOR MASSES OF DOMINANTLY COUPLED NEUTRINOS ARE LISTED IN THE APPROPRIATE SECTION ON NU-MU, NU-E, OR NER BOUNDS ON MIXING MATRIX COEFFI IENTS U(A., J) VERSUS
UPEUTRINO MASS. THESE RESULTS ARE DIVIDED INTO THREE SECTIONS (A) BOUNOS FROM PART ICLE AND NUCLEAR DECAYS
(B) BOUNDS PROM (C) BOUNDS FROM NEUTRINO REACTIONS

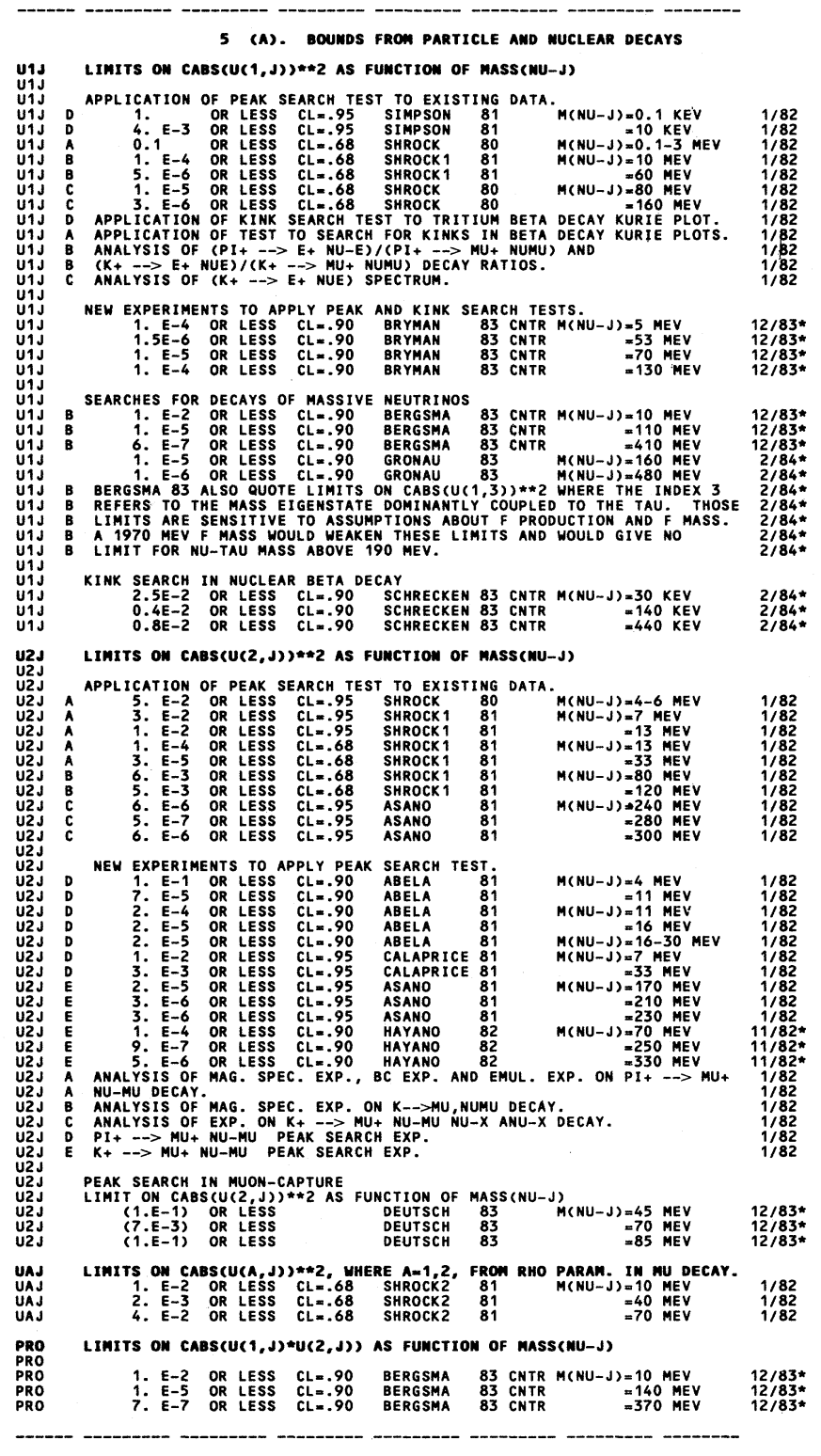

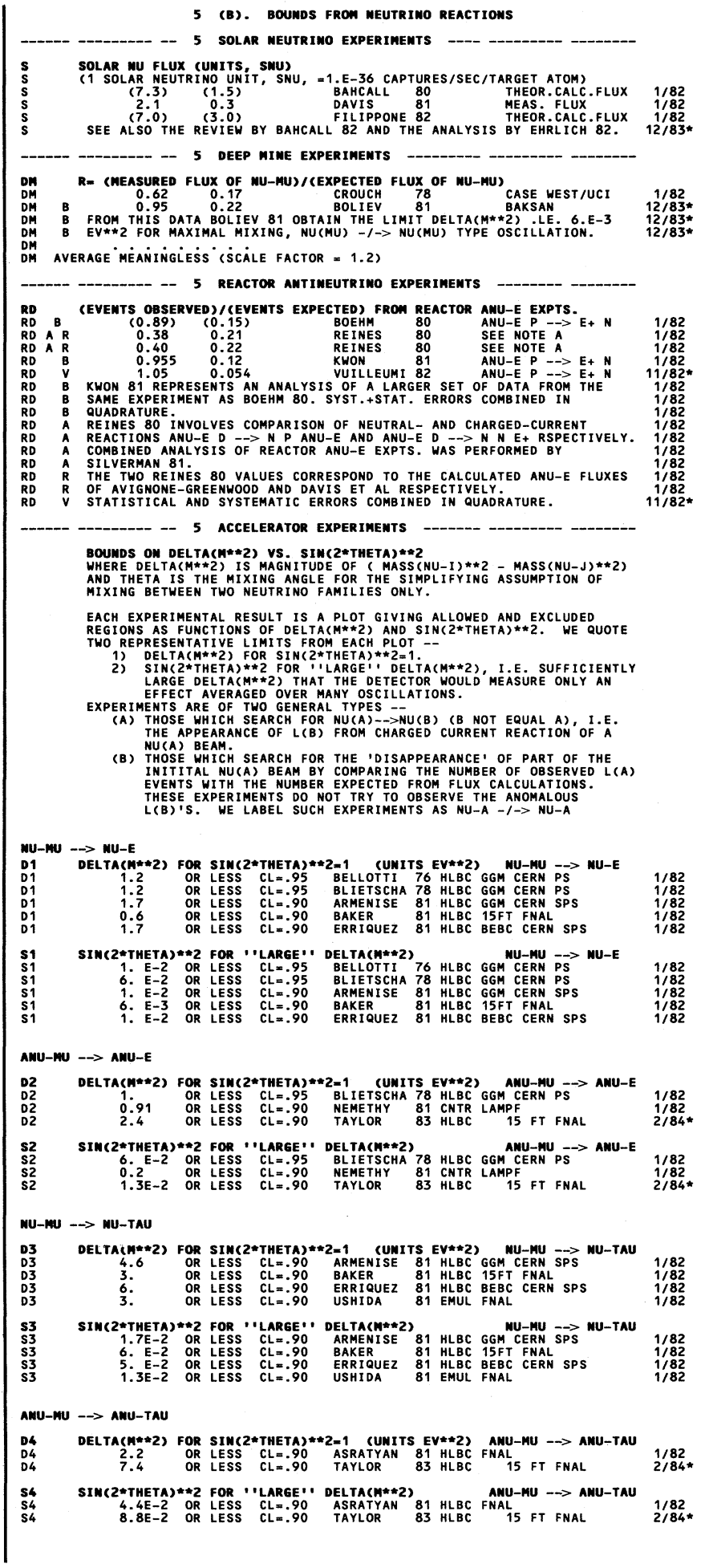



MASSIVE $\nu$ 'S \& LEPTON MIXING, $\nu$ BOUNDS FROM ASTROPHYSICS \& COSMOLOGY

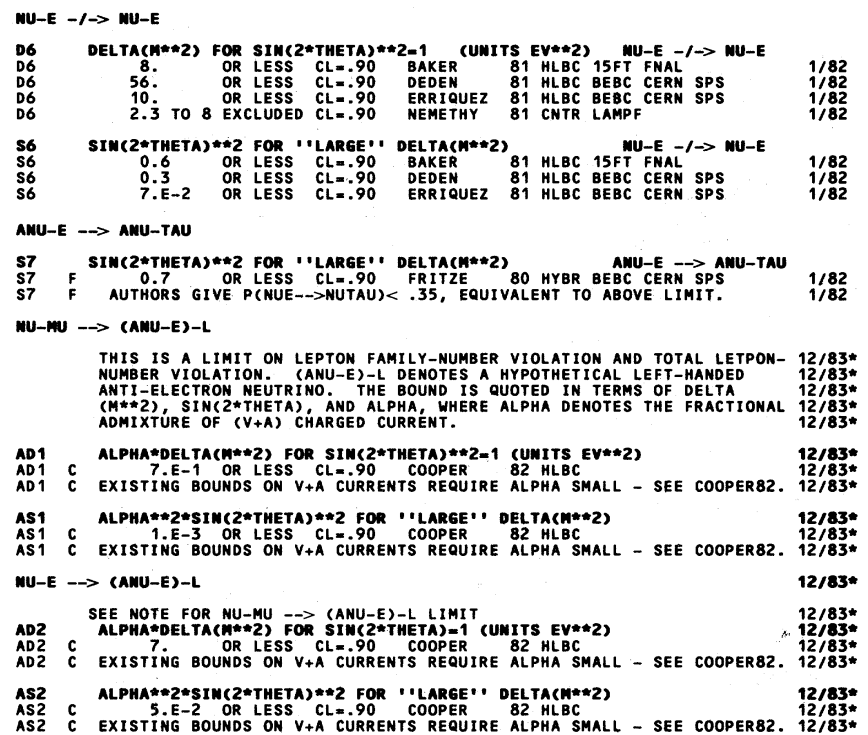

5 (C). SEARCHES For MEUTRIMOLESS DOUBLE BETA DECAY

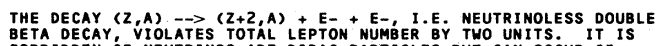

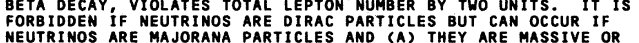

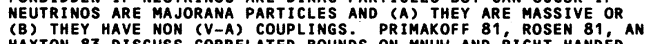

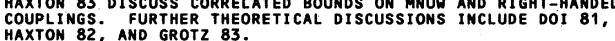

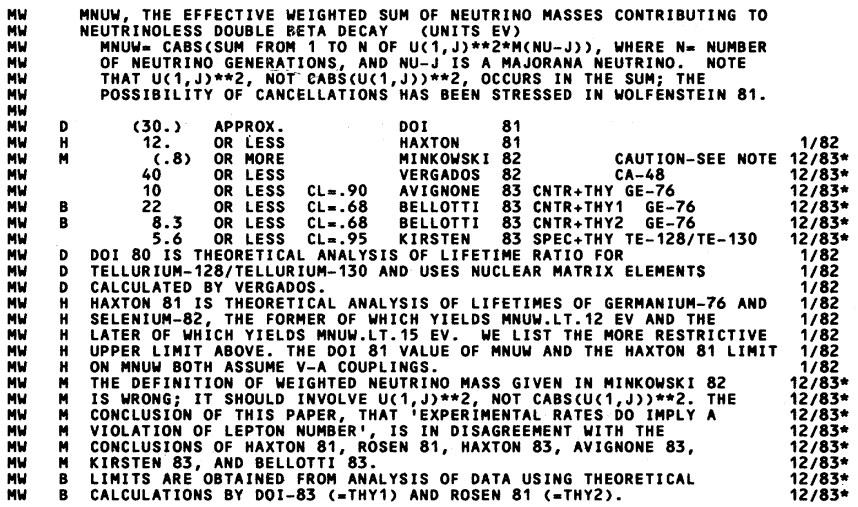

ETA LIMIS ON LEPTON-NUMBER VIOLATING (V+A) CURRENT ADMIXTURE

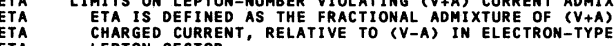

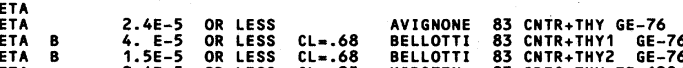

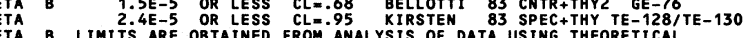
ETA B LIMITS ARE OBTA INED FROM ANALYSIS OF DATA USTNG THEOTE
ETA B CALCULATIONS BY DOI-83 (-THY1) AND ROSEN 81 ( $=$ THY2).

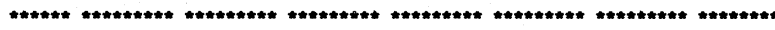

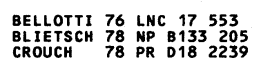

REFEREMCES FOR COR. BOUMDS ON MU MASS, Mixime.

BAHCALL 80 PRL 45945

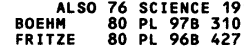

+CAVALLI, FIORINI, ROLLIER

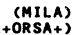
BLIESCHAU+(AACH+LIIH+CERM+EPOL+MILA+ORSA)
+LANDECKER, LATHROP, REINES+ (CASE+UCI I HITW)

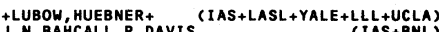

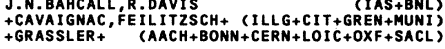

F.REINES, H.H. SOBEL, E. PASIERB

F. RENEES C. C. COHA

F. A.MEZRR CK, F.REINES
F.REINES,H.S.GURR, H.W. SOBEL
R.E.SHROCK

(UCI)
(LASL)
(CASE)
(USI)

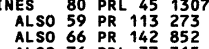

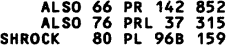

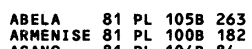

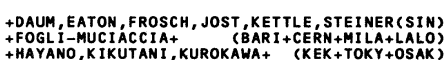

\section{NOTE ON $\nu$ MASS LIMITS}

These limits apply to $\mathrm{m}_{\text {tot }}$ given by

$$
\mathrm{m}_{\text {tot }}=\sum_{j=1, n}\left(\frac{\mathrm{g}_{\nu_{j}}}{2}\right) \mathrm{m}_{\nu_{j}},
$$

where $n$ is the number of neutrino species and $g_{\nu_{j}}$ is the number of independent components in the neutrino field: $g_{\nu_{j}}=4$ for Dirac neutrinos; $g_{\nu_{j}}=2$ for chiral Majorana neutrinos.

6 MU Mass (EV)

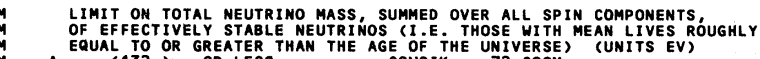

$\hat{A}$

\begin{tabular}{c}
132 \\
$(100)$ \\
Cows $(100$ \\
\hline
\end{tabular}

A COWSIK T2 AND LEE 77 HAVE BEEN OLENERALIZ 81 COSM TO APPLY TO M(TOT) AS

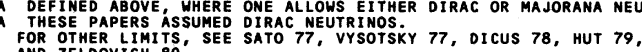

$9 / 81$
$1 / 82$
$4 / 82$
$4 / 82$
$4 / 82$
$2 / 82$
$2 / 82$ 


\section{Stable Particles $\nu$ BOUNDS FROM ASTROPHYSICS \& COSMOLOGY, HEAVY LEPTON SEARCHES}

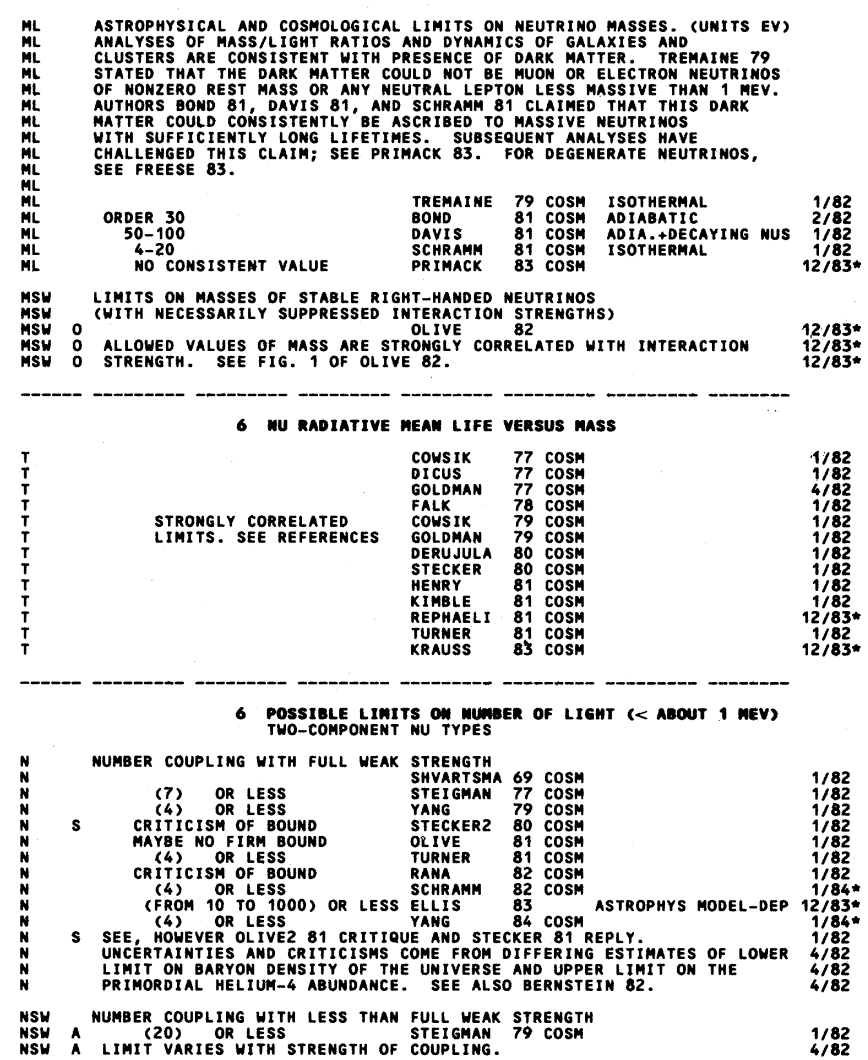

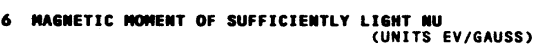

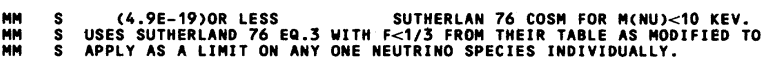

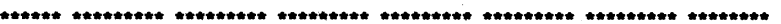

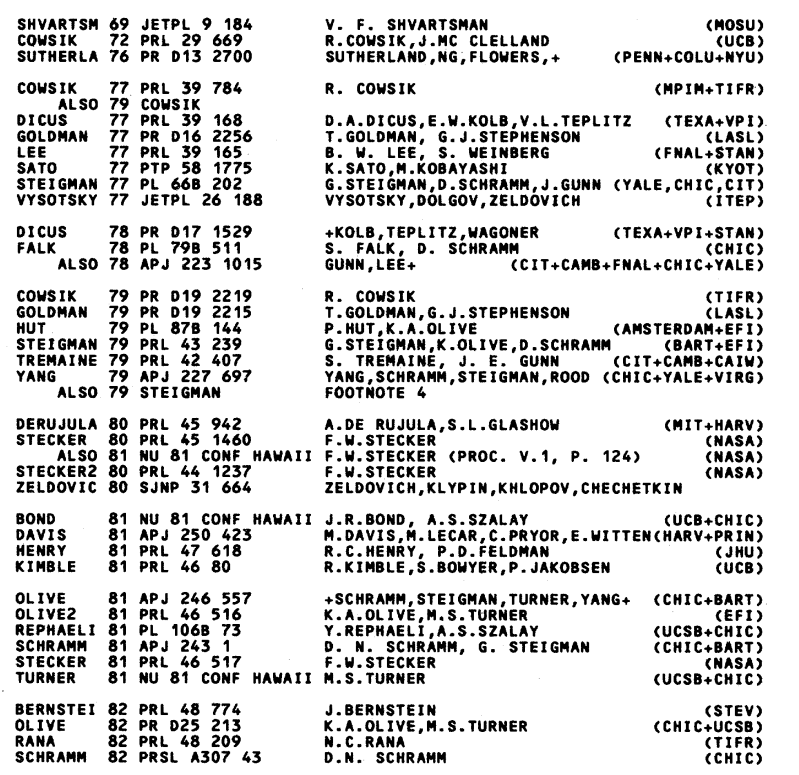

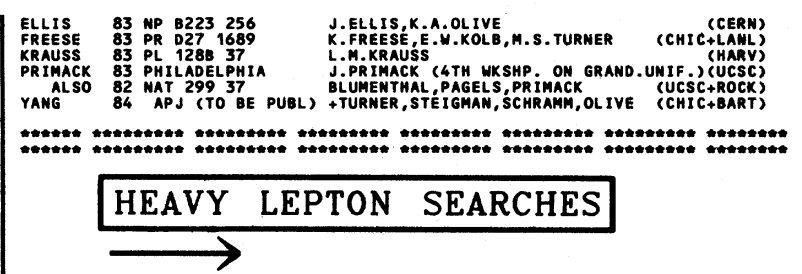

Data on the $\tau^{ \pm}$are listed in a separate section above, following the e and $\mu$ listings.

The following section contains information on searches for heavy leptons of other types and searches for the $\tau^{ \pm}$in collisions other than $\mathrm{e}^{+} \mathrm{e}^{-}$.

Several types of heavy leptons (that is, non-stronglyinteracting fermions other than $e$ and $\mu$ ) have been proposed. In the Data Card Listings we distinguish four types. ${ }^{1,2}$ Each has a corresponding antiparticle with opposite charge and lepton number. For convenience we omit writing the antiparticles in the following descriptions. The four types are:

Sequential leptons $\left(L^{-}, \nu_{L}\right)$. Such a pair is assumed to have its own separately strictly conserved lepton number $n_{L}=+1$. This means that the radiative decays

$$
\left.\begin{array}{l}
\mathrm{L}^{-} \rightarrow \mathrm{e}^{-} \boldsymbol{\gamma} \\
\mathrm{L}^{-} \rightarrow \mu^{-} \gamma
\end{array}\right\} \text { are forbidden, }
$$

while the weak decays (assuming $\mathrm{m}_{\mathrm{L}^{-}}$- sufficiently large)

$$
\left.\begin{array}{l}
\mathrm{L}^{-} \rightarrow \nu_{\mathrm{L}} \mathrm{e}^{-} \bar{\nu}_{\mathrm{e}} \\
\mathrm{L}^{-} \rightarrow \nu_{\mathrm{L}} \mu^{-} \bar{\nu}_{\mu} \\
\mathrm{L}^{-} \rightarrow \nu_{\mathrm{L}} \text { hadrons }
\end{array}\right\} \text { are allowed }
$$

There could be an increasing mass sequence of such pairs. It is frequently assumed that the neutrinos are massless.

Decay rates are assumed calculable from conventional weak interaction theory. For an $\mathrm{L}^{-}$mass between 1 and $3 \mathrm{GeV}$, the branching fraction to each of the two leptonic modes above should be roughly 10 to $20 \%$. For an $\mathrm{L}^{-}$mass above $1 \mathrm{GeV}$, the mean life should be $\leqslant 10^{-12}$ seconds.

Paraleptons $\left(E^{+}, E^{9}\right)$ and $\left(M^{+}, M^{9}\right)$. These pairs have the same lepton numbers as the opposite-charge ordinary leptons, i.e., $\mathrm{e}^{-}$and $\mu^{-}$, respectively. Radiative decays are again forbidden and decays similar to those allowed for $\mathrm{L}^{-}$are allowed here, e.g.,

$$
\mathrm{M}^{+} \rightarrow \nu_{\mu} \mathrm{e}^{+} \nu_{\mathrm{e}}
$$


For notation, see key at front of Listings.

$$
\mathbf{M}^{+} \rightarrow \nu_{\mu} \mu^{+} \nu_{\mu} .
$$

However, the lightest member is not stable as is the case for sequential leptons, so that bizarre decay schemes such as (assuming $\mathrm{m}_{\mathrm{E}^{0}}<\mathrm{m}_{\mathrm{E}^{+}}$)

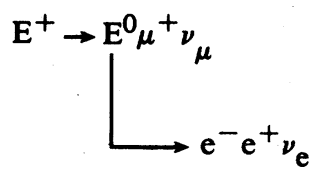

are allowed.

Heavy leptons of this type (and/or a neutral intermediate boson $\mathrm{Z}^{0}$ ) are desired in unified gauge theories of weak and electromagnetic interactions to cancel unphysical high energy behavior in such processes as $\mathrm{e}^{+} \mathrm{e}^{-} \rightarrow \mathrm{w}^{+} \mathrm{w}^{-} .^{3}$

Ortholeptons $\left(\mathrm{F}^{-}\right.$and $\left.\mathrm{N}^{-}\right)$. These have the same lepton numbers as $\mathrm{e}^{-}$and $\mu^{-}$, respectively. They may or may not have associated neutral leptons. Radiative decays are allowed in addition to weak modes similar to those of sequential leptons. The radiative mode can dominate or can be relatively unimportant depending on the model. ${ }^{4}$ Decays such as

$$
\mathrm{F}^{-} \rightarrow \mathrm{e}^{-}+\text {hadrons }
$$

are also allowed.

Long-lived penetrating particles. Heavy leptons could have long mean lives under certain circumstances. For example, if $m_{\nu^{\prime}}>m_{L^{-}}$, then $L^{-}$, the sequential lepton, is completely stable since its lepton number is conserved.

Experimental results. The results are summarized in the Data Card Listings below. Mass limits for sequential leptons are listed in subsection MS, while all other types are listed together in subsection $M$.

The Listings also contain cross-section upper limits reported as results of unsuccessful searches. We no longer list cross sections for anomalous $\mathrm{e} \mu$ events in $\mathrm{e}^{+} \mathrm{e}^{-}$collisions. These cross sections are consistent with coming from $\mathrm{e}^{+} \mathrm{e}^{-} \rightarrow \tau^{+} \tau^{-}$where the $\tau^{ \pm}$is assumed to be a spin-1/2 Dirac point particle with a mass about $1785 \mathrm{MeV}$.

\section{References}

1. M.L. Perl and P. Rapidis, SLAC-PUB-1496 (October 1974).

\section{Stable Particles} HEAVY LEPTON SEARCHES

2. C.H. Llewellyn Smith, Invited paper presented at the Royal Society Meeting on New Particles and New Quantum Numbers, 11 March 1976, Oxford Ref. 33/76.

3. J.D. Bjorken and C.H. Llewellyn Smith, Phys. Rev. D7, 887 (1973).

4. F. Wilczek and A. Zee, Nucl. Phys. B106, 461 (1976).

SEE PERL 81 FOR A REVIEW

PROPERTIES OF THE TAU+-(1785) HEAVY LEPTON AND ITS ASSOCIATED NEUTRINO ARE LISTED SEPARATELY ABOVE FOLLOWING THE E AND MU FOR HEAVY LEPTONS OF OTHER TYPES AND SEARCHES FOR TAU +- IN
COLLLISIONS OTHER THAN E+E-. WE LIST MASS LIMITS AND CROSS SECTION COLLISTONS OTHER THAN E+E. WE LIST MASS LIMITS AND CROSS SECTION
UPPER LIMITS REPORTED AS NEGATIVE SEARCH RESULTS. WE NO LONGER LIST CROSS SECTIONS FOR THE ESTABLISHE

25 MEAVY LEPTON MASS Limits

LIMITS APPLY ONLY TO HEAVY LEPTON TYPE GIVEN IN COMMENT AT RIGHT ON IN COMMENTS BELOW, ALL BEAMS ARE MU TYPE NEUTRINO OR ANTINEUTRINO. OR, M, M, N STAND FOR SEQUENTIAL LEPTON, PARA-ELECTRON, PARA-MUON,

SEQUENTIAL HEAVY LEPTON MASS LIMITS (GEV)

NONE 4 GEV TO $14.5 \mathrm{GEV}$ CL $=.95$
NONE BELOW $15.5 \mathrm{GEV}$ CL $=.95$

NONE BELOW 14 GEV $\mathrm{CL}=.95$

AZIMOV
BARBER
BERGER
BRANDELIK
A BOEALA

BRANDE

80 CNTR +- SEQUENTIAL (L)

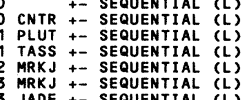

AZIMOV 80 ESTIMATED PROBABILITIES FOR M+N TYPE EVENTS IN E+ E- $->$

A L- DEDUCING SEMI-HADRONIC DEEAY MULTIPLICITIES OF L FROM E+ E-
ANMIHILATION DATA AT WCM=(2/3)*ML. OBTAINED ABOVE LIMIT COMPARING
THESE WITH E+E- DATA (BRANDELIK 80, PL 92B 199).

BARBER 80 LOOKED FOR E+ E- - $>$ L+ L-, L-->NEU(L)+X WITH MARK-J AT

C BERGER 81 IS DESY DORIS AND PETRA EXPT. LOOKING FOR E+E- --> L+L-.

D brandelik 81 is desy petra expt. Looking for E+E- --> L+L-.

E ADEVA 83 LOOKED FOR MUON OPPOSITE AGAINST A HADRON JET.

F bartel 83 Limit is from petra eie- exp With average wCM=34.2 Gev. 11/83*

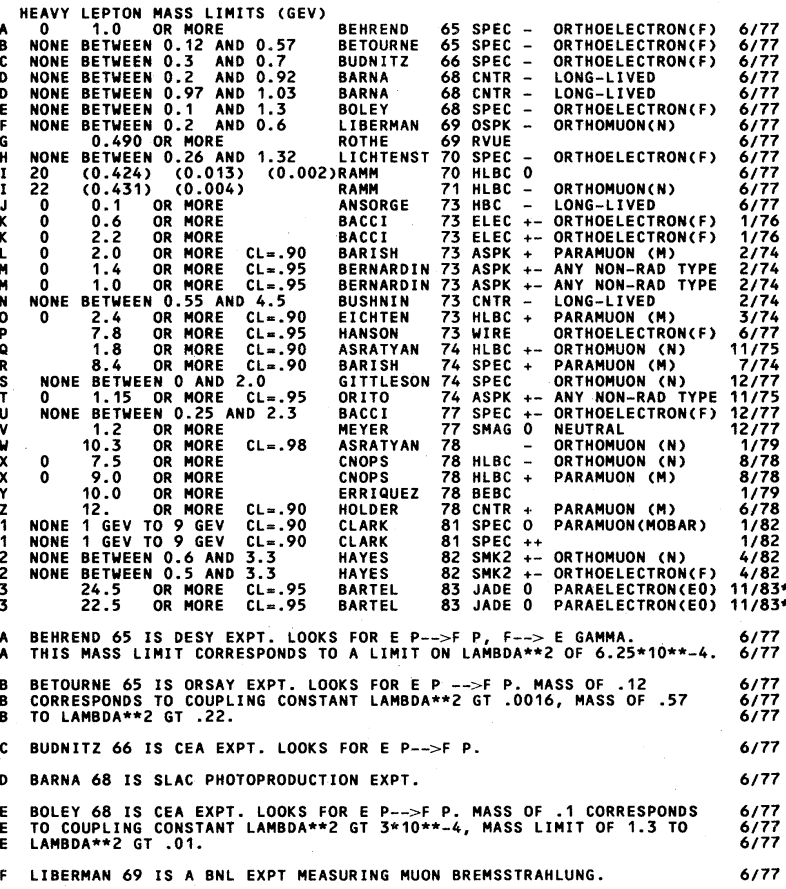




\section{Stable Particles}

\section{HEAVY LEPTON SEARCHES}

\section{Data Card Listings}

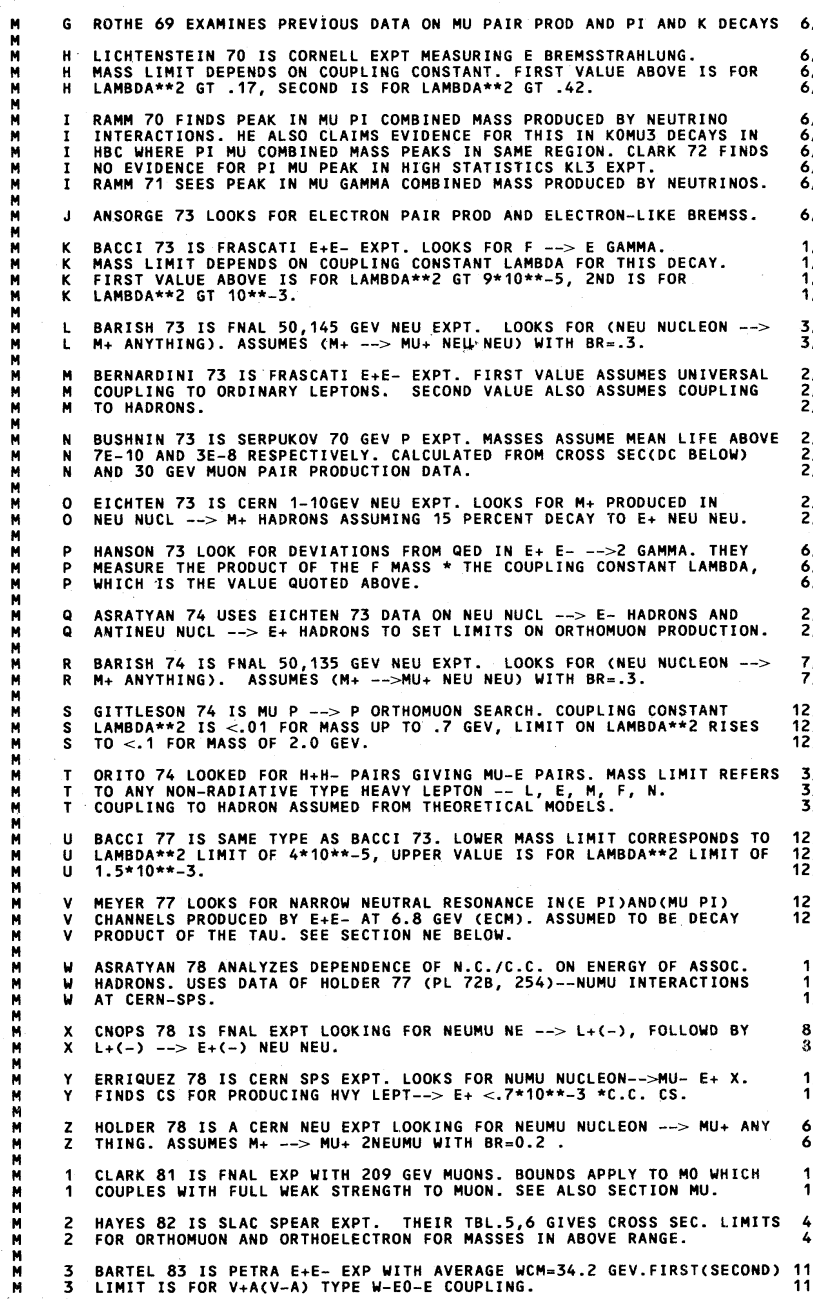

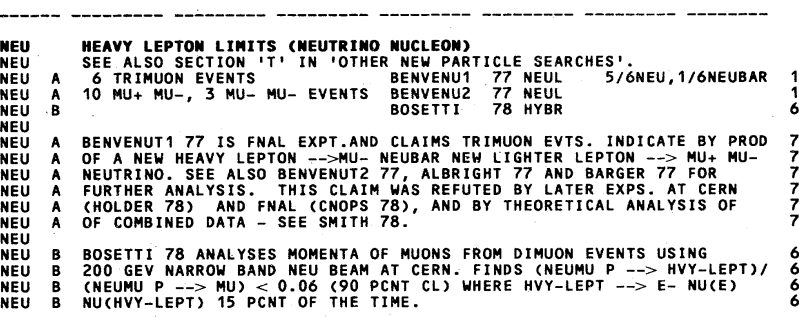

MU MEANY LEPTON PRODUCTIOM CROSS SECTION (MU MUCLEOM) (CMN*2)

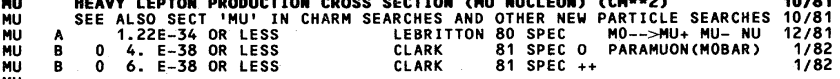
$\begin{array}{ll}\text { MUU A L LERITTON } 80 \text { IS BNL EXP HITH } 10.5 \text { GEV MUONS. TRIMUONS ARE CONSISTENT } & 12 / 81 \\ \text { MU A WITH QED TRIDENT AND DIFFRACTIVELY PRODUCED RHO DECAY. } & 12 / 81\end{array}$ MU B CLARK 81 IS FNAL EXP WITH 209 GEV MUON. LOOKED FOR MUU + N- $\rightarrow$ MOBAR X, $1 / 82$

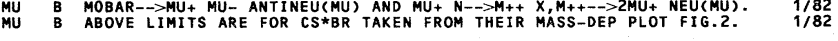

DC MEAVY LEPTOM PRODUCTION DIFF. CROSS SEC. (P MUCLEOM) (CMN*2/SR-GEY)
DC A

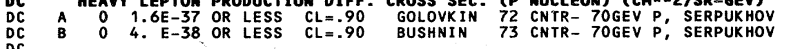
OC A MASS Range 1 to $4.5 \mathrm{GeV}$, THeTA=0, P=25 GeV/C.

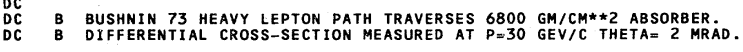

$6 / 777$ $6 / 77$ $6 / 77$
$6 / 77$
$6 / 77$ $6 / 77$
$6 / 77$ $6 / 77$ $1 / 76$
$1 / 76$
$1 / 76$
$1 / 76$ $3 / 77$ $2 / 74$
$2 / 74$
$2 / 74$ $2 / 74$
$2 / 74$ $2 / 76$
$2 / 76$ $6 / 77$
$6 / 77$
$6 / 77$ $2 / 76$
$2 / 76$ $7 / 74$ $12 / 77$
$12 / 77$
$12 / 77$ $3 / 74$
$3 / 74$
$3 / 74$ $12 / 77$
$12 / 77$
$12 / 77$ $12 / 77$ $1 / 79$
$1 / 79$
$1 / 79$ $8 / 78$
$3 / 78$ $1 / 79$
$1 / 79$ $6 / 78$
$6 / 78$ $1 / 82$
$1 / 82$ $4 / 82$
$4 / 82$ $11183 *$

年

$1 / 76$

$2 / 74$
$3 / 74$

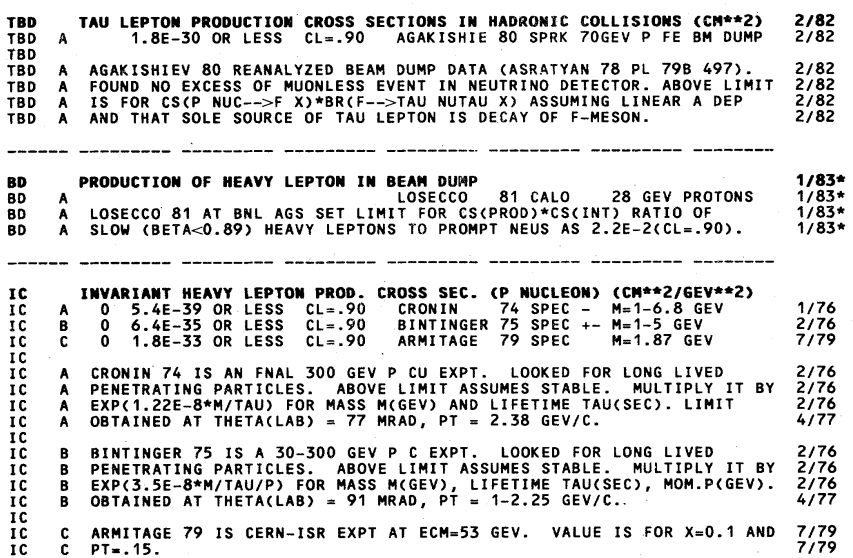

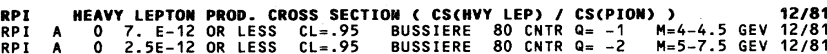

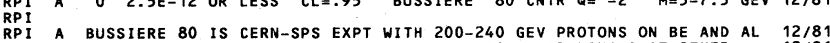

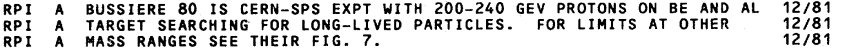

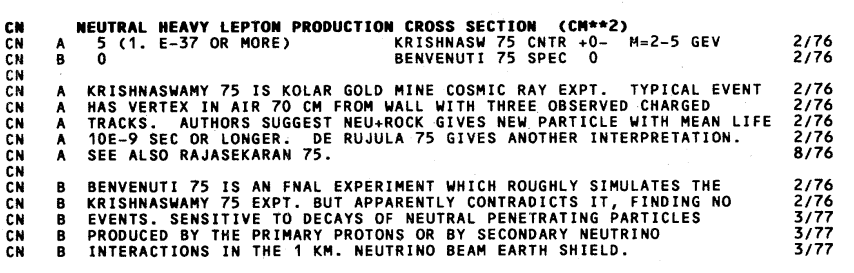

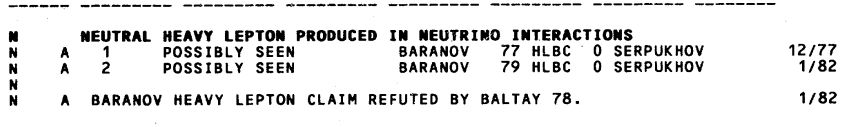

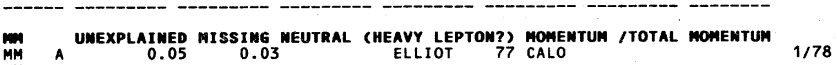

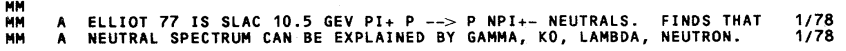

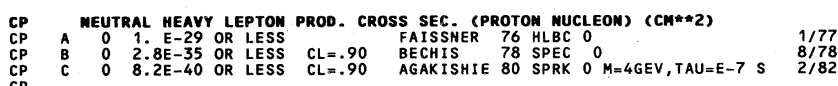

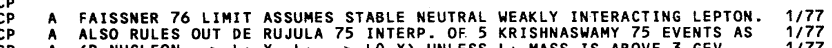
${ }_{C P}^{C P}$ A (P NUCLEON $\rightarrow-\rightarrow+x, L+-\rightarrow>$ LO $x$ ) UNLESS L+ MASS 15 ABOVE 3 GEV.

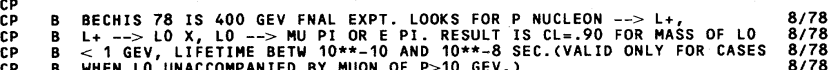
CP $C$ C AGAKISHIEV 80 REANALYZD BEAM DUMP DATA FROM 70 GEV PROTON ON IRON $2 / 82$

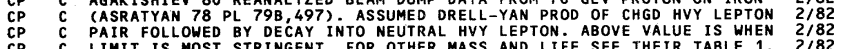

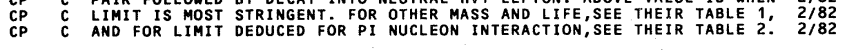

ME MEUTRAL MEAYY LEPTOM PROD. CROSS SECTIOM (E* E-) (CN**2)
NE A
N.5E-36 OR LESS

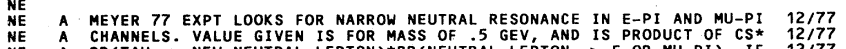

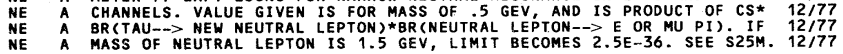

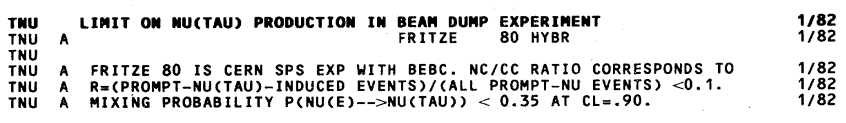

$E X C$
$E X C$
$E X C$
$E X C$
$E X C$
$E X C$
$E X C$
$E X C$

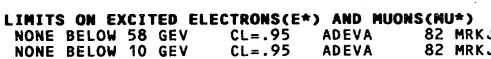

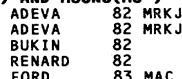

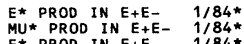

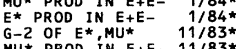




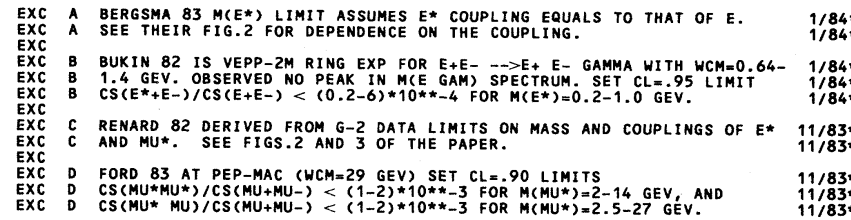

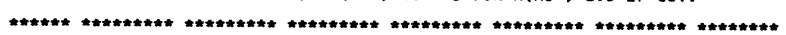

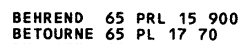

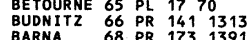

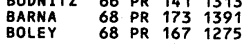
$\begin{array}{lll}\text { LIBERMAN } 69 & \text { PRL } 22 & 663 \\ \text { ROTHE } & 69 & \\ \text { NP } B 10 & 241\end{array}$ RICHTENS 70 PR D1 825

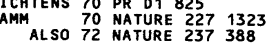

REFEREMCES FOR HEAVY LEPTON SEARCHES +BRASSE, ENGLER, GANSSAUGE+
+NGUYEN NGOC, PEREZ Y JORBA+ (DESY + KARL)
(ORSA) TDUNNING, GOOTEIN, RAMSEY, WALKER, WILSON HARV

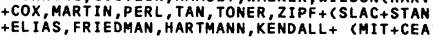
+HOFFMAN, ENGELS, IMRIE+(HARV+CASE+MCGI+SLAC)

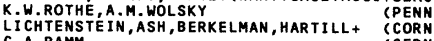
C.A.RAMM
CLARR, ELIOFF, FIELD, FRISCH, JOHNSON+ $\quad$ (CERN)

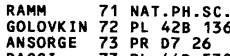

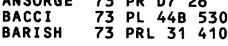
$\begin{array}{rlll}\text { BERNARDI } 73 & \text { NC } 17 A & 173 \\ \text { ALSO } 70 \text { LNC } 41156\end{array}$ USHAIN 73 NP 858476 EICHTEN 73 PL 468281
HANSON 73 NCL 7587

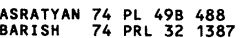

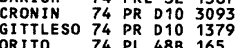
BENVENUT 75 PRL 351486

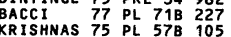

ALSO 75 PRL 35 628
ALSO 75 PRAMANA 578

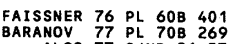
ALSO 77 SJNP 2657

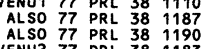
ELLIOT 77 PR 0151851 MEYER
ASRATAN 77 PL PL 708 B 237
BALTAY 78 TOKY CONF.
BECHT $\begin{array}{llll}\text { BECHIS } & 78 \text { PRL } 40602 \\ \text { BOSETTI } & 78 \text { PL } 7383802\end{array}$

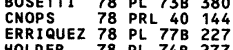
$\begin{array}{llll}\text { HOLOER } & 78 & \text { PL } 748 & 277 \\ \text { SMITH } & 78 & \text { NU } 78 \text { CONF }\end{array}$ ARMITAGE 79 NP 815087
BARANOV 79 PL 8118261
ALSO 79 SJNP 29622 AGAKISHI 80 SJNP 32345

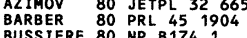

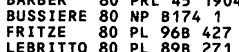
$\begin{array}{llll}\text { BERGER } & 81 & \text { PL } 998 & 489 \\ \text { BRANELI } & 81 & \text { PL } & 998 \\ \text { BRAB } & 163\end{array}$

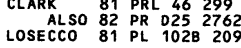

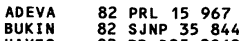

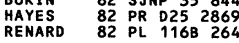

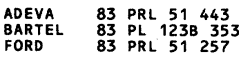

C. A. RAMM
GRACHEV, KHODYREV, KUBAROVSKY,

(CERN)
(SERP)

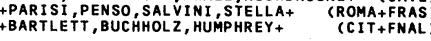
BERNARDINI, BOLLINI, BRUNINI + (CERN+ BGNA+FRAS)

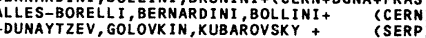

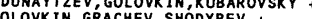

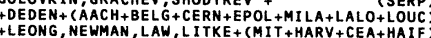
+GERSHTEIN, KAFTANOV, KUBANTZEV, LAPIN+ (SERP)

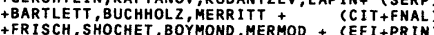

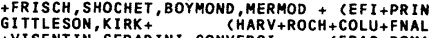
BENVENUTI, CLINE, FORD+ (HARV+PENN+WISC+FNAL) (EFI+HARV+PENN+WISC) KRISHAASHAMY, MENON+N
(BOMBAY OSAARA DE RUJULA,GEORGI, GLASHOW (HARV)
RAJASEKARAN, SARMA +HASERT+CAACH+BELG+CERN+EPOL +MILA+OXF+LOUCC)
+ VOLKOV GERSHTENT IVANILOV + BARANOV, VOLKOV, GERSHTEIN, IVANILOV (SERP)
+ (SERP

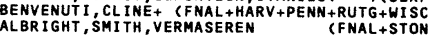

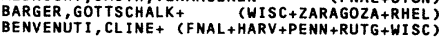
+FORTNEY, GOSHAW, LAMSA, LOOS+ (DUKE+ALBA)
+NGGYENABRAS, ALAM+ (SLAC+LBL+NHES+HAHA) ASRAYYA, KUBANTSEV
C.BALTAY (19TH INTL. CONF. ON HEP) (ITEP)
(COLU) +CHANG, DOMBECK, ELLSWORTH, GLASSER, LAU, (UMMD)

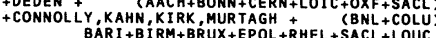

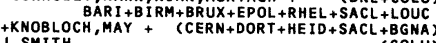
+BENZ, BOBBINK+ (CERN+DARE+FOM+MCHS +UTRECHT) (IAANIL, KONYUSHKO, KORABLEV+
BAANOV, + VOLKOV, IVANILOV, KONYUSHKO, + (SERP) AGAR ISH EV V VOVENKO, GOR VACHEV, MUKHIN (SERP)

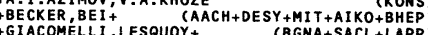

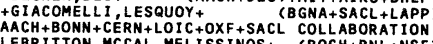
+GENZEL+(AACH+BERG+DESY+HAMB +UMD+SIEG+WUPP)

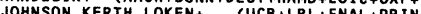

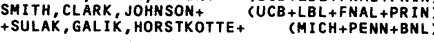

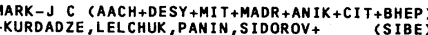
P.PRLLALAAM, BOYARSKI, BRE IDENBACH+ (SLAC+EBL)
(CERN) MARKJ C. (AACH+DESY+MIT+MADR+AIKO+BHEP+CIT)

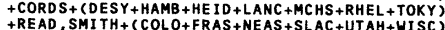
REVIENS

PERL 81 SLAC-PUB-2752 M.L.PERL, PHYS. IN COLL. CONF, V.P.I. (SLAC)

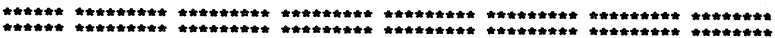
$\pi^{ \pm}$ 8 CHARGED PIOM(140,JPG=0--) I=1

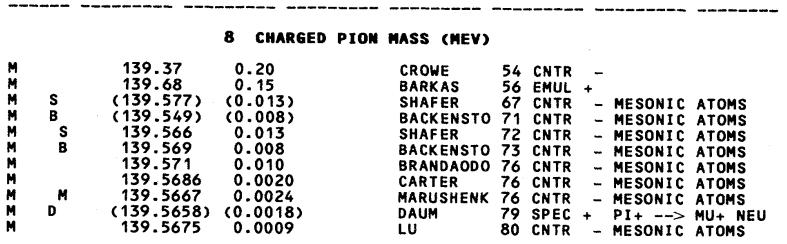
$6 / 68$
$10 / 71$
$1 / 73$
$1 / 73$
$1 / 78$
$6 / 77$
$12 / 77$
$10 / 81$
$1 / 81$

$1 / 84 *$
$1 / 84 *$
$1 / 84 *$
$1 / 84 *$
$1184 *$
$11 / 83 *$
$11 / 83 *$
$11 / 83 *$
$11 / 83 *$
$11 / 83 *$
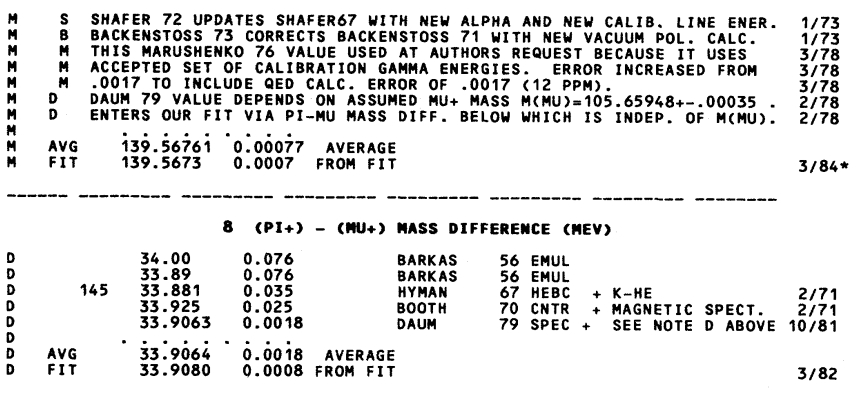

8 ((PI+) - (PI-))/AVG., MASS DIFFERENCE (PERCENT)

$\underset{D M}{M}$ AYRES 71 CNTR $3 / 71$

8 charged pion mean life (UNits 10**-9 Sec)

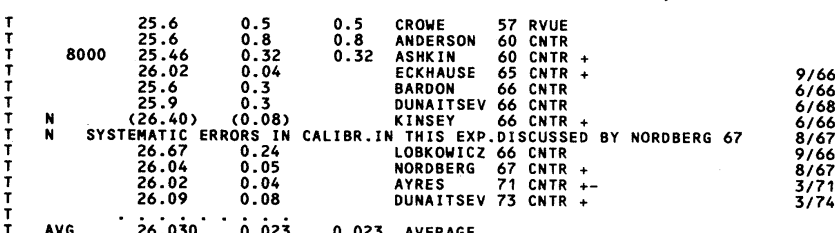

AvG $26.030^{\circ} \cdots .023 \quad 0.023$ average

8 ((PI+) - (PI-))/AVG., MEAN Life diff. (PERCENT)

Dt $N$ this Quantity is a measure of CPT Invariance IN W.t.

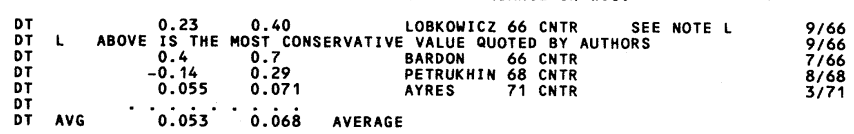

8 charged pion partial deecay modes

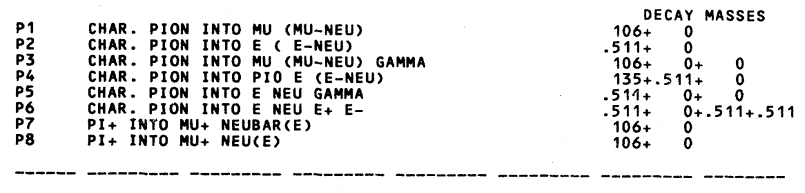

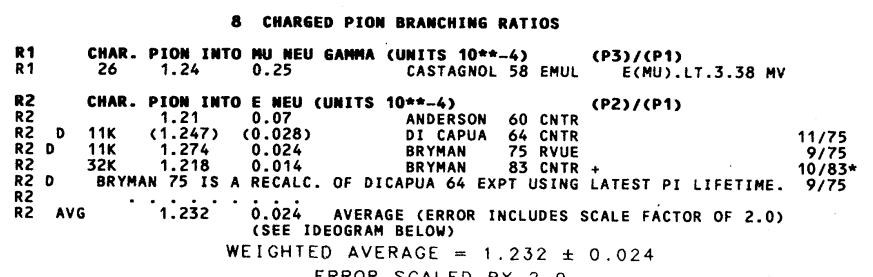

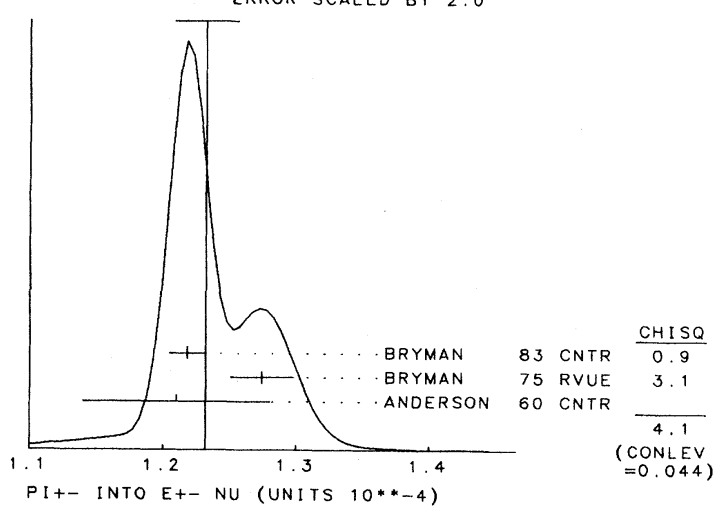


Stable Particles

$\pi^{ \pm}, \pi^{0}$

\section{Data Card Listings}

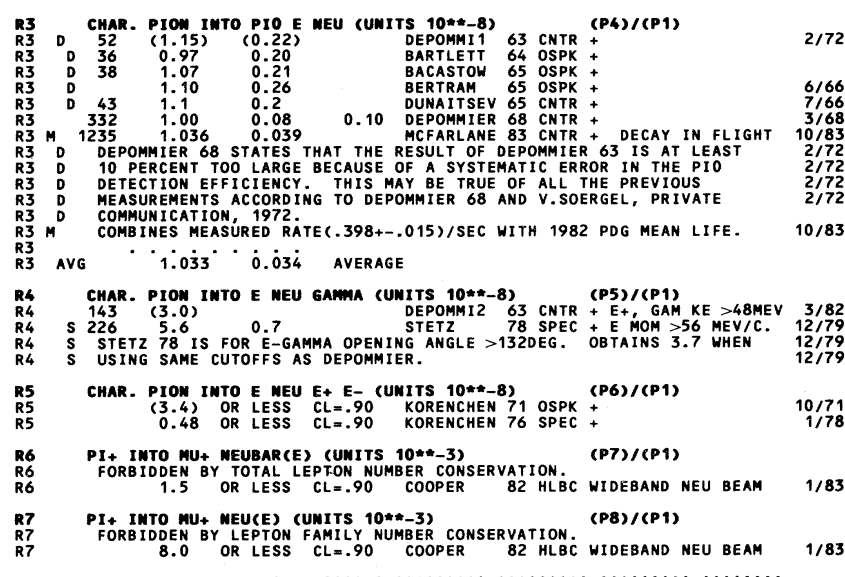

*********************************************************************

REFERENCES FOR CMARGED PION

$\begin{array}{lllll}\text { CROWE } & 54 & \text { PR } & 96 & 470 \\ \text { BARKAS } & 56 & \text { PR } & 101 & 778\end{array}$

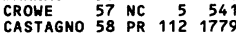

ANDERSON 60 PR 1192050

DEPOMMII 63 PL 561

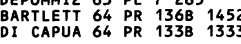

BACASTOW 65 PR 139 B 4007

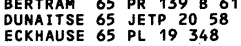

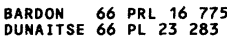

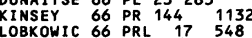

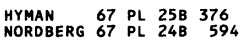

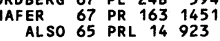

DEPOMMIE 68 NP $B 4189$

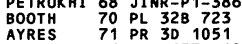

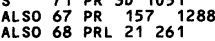

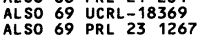

BACKENST 71 PL 368 B 403

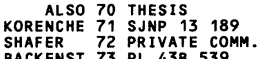

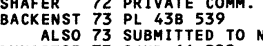

DUNATTSE 73 SJNP 16292

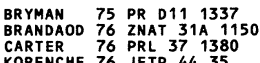

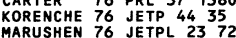

ALSO 76
ALSO 78 PRIVATE COMM.

$\begin{array}{lllll}\text { STETZ } & 78 & \text { NP } & B 138 & 285 \\ \text { DAUM } & 79 & \text { PR } & \text { D20 } 2692\end{array}$

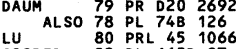

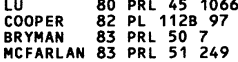

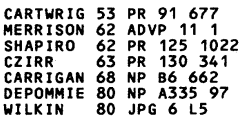

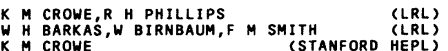

$K$ M CROWE. W M MUCHNIK (STANFORD MEPL)
(ROMA)

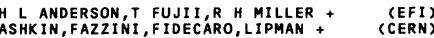

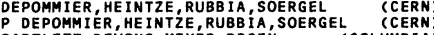

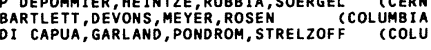

+GHESQUIERE, HIEGAND, LARSEN (LRL L SLAC)

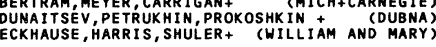

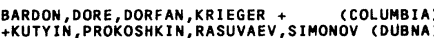

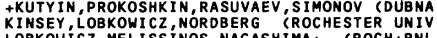

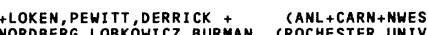
ROBRT E, SHAFER,
SHAFE, CROWE, JENKINS (LRL)
(LRL)

DEPOMMIER, DUCLOS, HEINTFE, KLET IKNEECHT +(CERN)

AYRSON, HILLIAMS, HORMALD

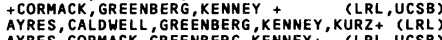

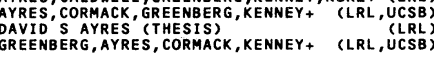

BACKENSTOSS, DANIEL, KOCH+ (CERN, KARL, HEID)
(HEIDELBERG)

C. VON DER MALSBURG
KORENCHENKO, KOST IN, MICELMACHER+ (HEIDELBERG)
(JINR)

(FNAL)
BACKENSTOSS, DANIEL, KOCH+ (CERN+KARL+MUNICH)

DUNAITSEV, PROKOSHKIN, RAZUVAEV+ + (SERP)
DuUS

PPICCIOTTO (UNIV OF VICTORIA)
BRANDAO D'OLIVERA, DANIEL, VON EGIDY+ (MUNI)

+DIXIT, SUNOARESAN, (CARL+CNRC+CHIC+CIT)

KORENCHENKO, KOSTIN, MICELMACHER+
MARUSHENKO, MEZENTSEV, PETRUNIN+

R. SHAFER

+CARROLL, ORTENDAHL, PEREZ-MENDEZ+ (LBL +UCLA)

(STI)

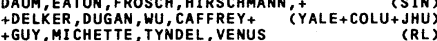

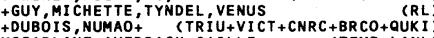

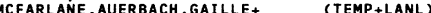

PAPERS NOT REFERRED to IN DATA CARDS

CARTHRIGHT, RICHMAN, WHI TEHEAD, HILCOX (LRL)J
(LIVERPOOL)

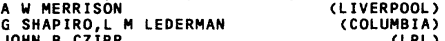

R.A.CARRIGAN JR.

P. DEPOMMIIN
C. WILKIN

(CARNT)
(LONC)P
(LOUC)

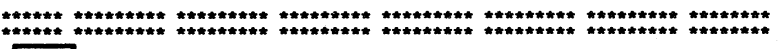

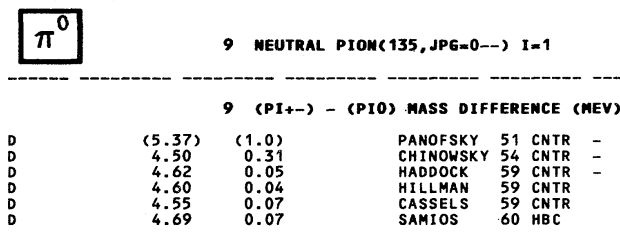

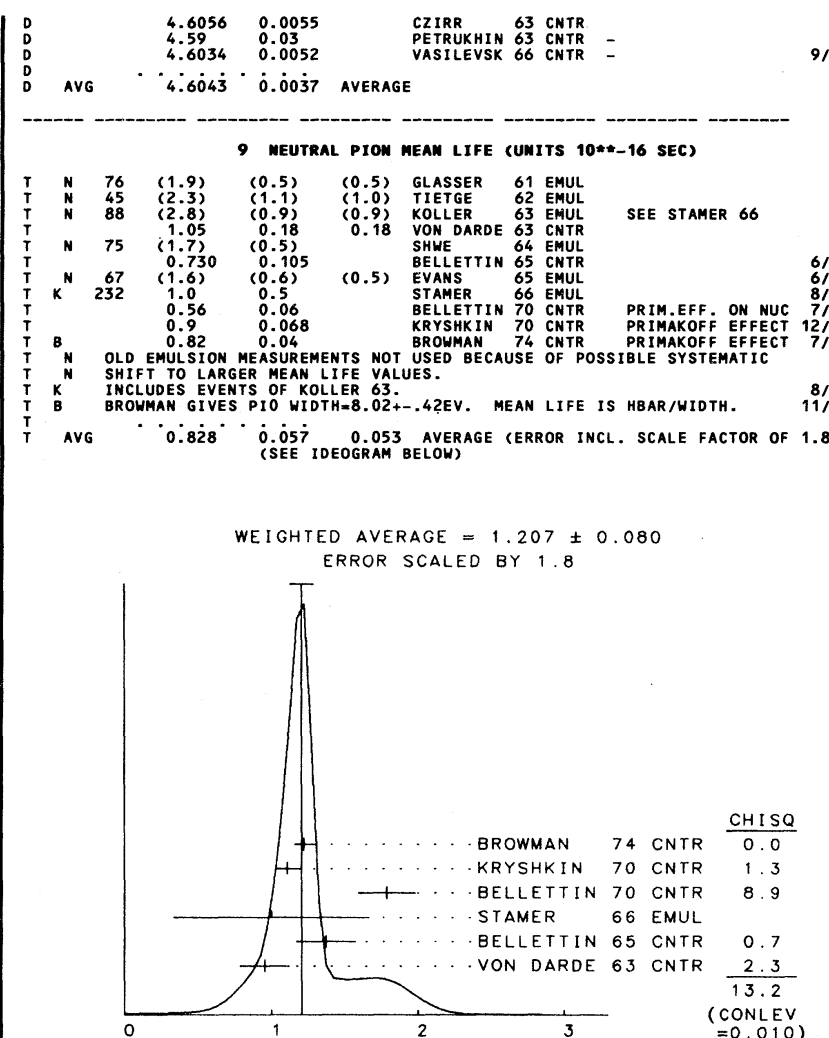

NeUtRal PI DECAY RATE(UNITS $10 * * 16$ SEC-1)

9 meutral piom partial decay modes

DECAY MASSES

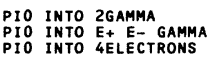

PIO INT O
PEE ECTRON INTO 3 GAMMA
PIO

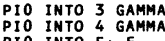

PID INTO E+ EE-

$511+.511+$
$511+.511+.511+.511$

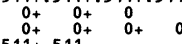
.+50
$106+.511$

9 meutral pion branching ratios

PIO IMTO (GAMTA EE E-)/(2GAMTA) (PERCEET)

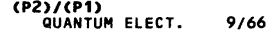

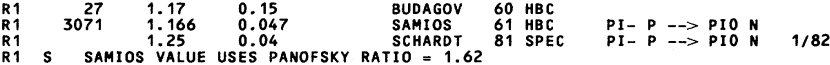

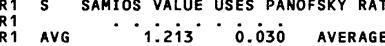

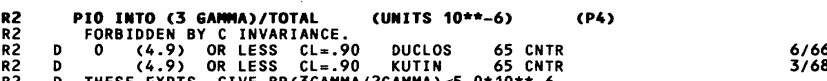

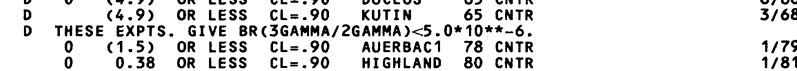

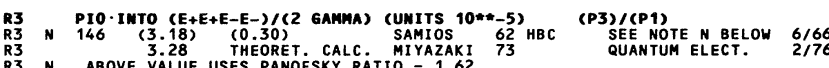

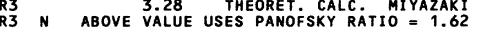

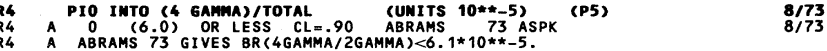

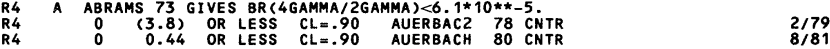

R5 DIO INTO (E+ E-)/TOTAL (UNITS 10**-6)

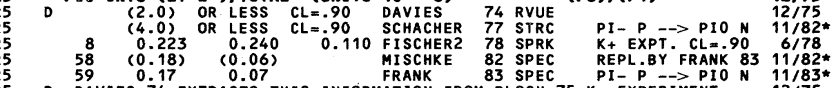

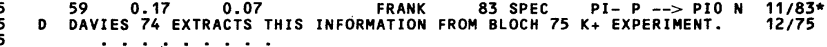
R5 AVG $0.177^{\circ} \cdots .065$ aVerage

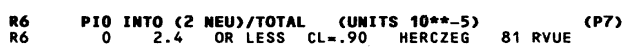

${ }_{2 / 82}^{2 / 82}$ 


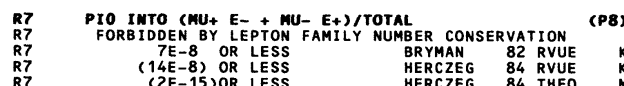

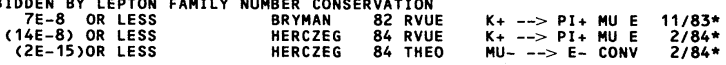

9 MEUtral PION ELECTROMAgmetic Form factor THE AMPLITUDE FOR THE PROCESS PIO $->$ E+ E- GAMMA CONTAINS A
FORM FACTOR GAMMA $X * \star 2)$ AT THE (PIO GAMMA GAMMA) VERTEX WHERE $X=M A S S$ (E+E-)/MASS (PIO). THE PARAMETER A IN THE LINEAR

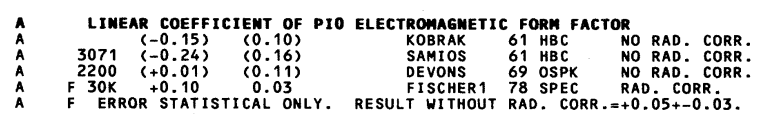

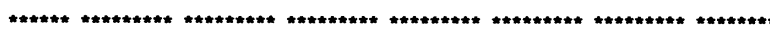

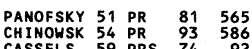
$\begin{array}{lllll}\text { CASSELS } & 59 & \text { PPS } & 74 & 92 \\ \text { HADDCK } & 59 & \text { PRL } & 3 & 478 \\ \text { HILLMAN } & 59 & \text { NC } & 14 & 887\end{array}$ BUDAGOV 60 JETP $11 \quad 755$

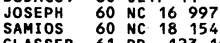

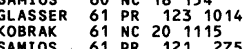

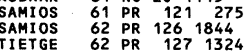
$\begin{array}{lrlrr}\text { CZIRR } & 63 & \text { PR } & 130 & 341 \\ \text { KOLLER } & 63 & \text { NC } & 27 & 1405\end{array}$ ALSO
P6 $\begin{array}{llllll}\text { SHWE } & 64 & \text { PR } & 1368 & 1839 \\ \text { BELLLETTI } & 65 & \text { NC } & 40 & \text { A } & 1139 \\ \text { DUCLOS } & 65 & \text { PL } & 19 & 253\end{array}$

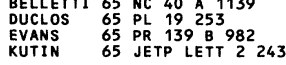
$\begin{array}{llllll}\text { STAMER } & 66 & \text { PR } & 151 & 1108 \\ \text { VASILEVS } & 66 & \text { PL } & 23 & 281\end{array}$ DEVONS 69 PR $184 \quad 1356$ $\begin{array}{llll}\text { BELLETII } 70 & \text { NC } & 664 & 243 \\ \text { KRYSHKIN } 70 & \text { JETP } 30 & 1037\end{array}$ $\begin{array}{llll}\text { ABRAMS } & 73 & \mathrm{PL} & 458 \\ \text { MIYAZAKI } & 73 & \mathrm{PR} & 08 \\ 08 & 2051\end{array}$ BROWMAN 74 PRL $33 \quad 1400$
DAVIES 74 NC $24 A 324$ SCHACHER 77 LNC 20177 $\begin{array}{llll}\text { AUERBAC2 } 78 & \text { PL } 78 B & 353 \\ \text { AISCHER1 } & 78 & \text { PL } 73 B & 359\end{array}$

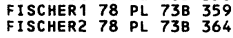

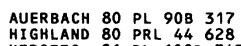
HIGHLAND 80 PRL 44628
HERCZEG 81 PL $100 \mathrm{~B} 347$

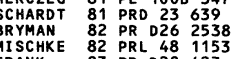

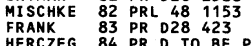
REFEREMCES FOR MEUTRAL PION $W K$ K PANOFSKY R L AAMODT, J HADLEY (COLURL)
W CHINOWSY, STTEINERGER CASSELS, JONES, MURP HY, O. NEILL (LIVERPOOL)

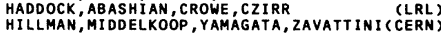
BUDAGOV, VIKTOR, DZHELEPOV, ERMOLOV + (JINR) (EFI)
D W D DSEPH
N PAMIOS
(COLUMBIA)
(NRL GLASER, SEEMAN, B STILLER R G GLASSER, N SEEMAN, B STILLER (NRL) (
(EFI)
H.KOBRAK (COLUMBIA+BNL)
(COLMBIA+BNL)
SAMIOS,PLANO, PRODELL +
$J$ TIETGE, PUESCHEL
(MAX PLANCK INST) JOHN B CZIRR
E L KOLLER, S TAYLOR, T HUETTER
(STEVENS) VI PETRUKHIN, YU D PROKOSHKIN
VON DARDEL, DEKKERS, MERMOD, VAN PUTTEN+(CERN)
(JINR) H SHWE, F M SMITH, W H BARKAS (LRL) BELLETTINI, BEMPORAD, BRACCINI +(PISA+FIRENZE)
DUCLOS, FREYTAG, HEINTZE + (CERN+HEIDELBERG)
(OXFORD)
(OXVANS DA EVANS
KUTIN, PETRUKHIN, PROKOSHKIN (OXFORD)
(JINR) STAMER, TAYLOR, KOLLER, HUETTER+
VASILEVSKY, VISHNYAKOV, DUNAITSEV + (STEVENS)
(DUBNA) VASILEVSKY, VISHNYAKOV, DUNAITSEV + ( (DUBNA)
+ NEMENTHY, NISSIM-SABAT, DI CAPUA + (COLU+ROMA)

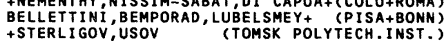
+CARROLL, KYCIA,LI, MICHAEL, MOCKETT + (BNL)

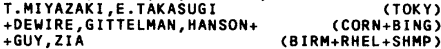
+CZAPEK, HAHN, MARTI

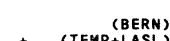
AUERBACH,' HIGHLAND, JOHNSON, + (TEMP+LASL) \begin{tabular}{l} 
+EXTERMANN, GUISAN, MERMOD, + \\
+ +EXTERMANN, GUISAN, MERMOD, MOREL + (GEVA+SACL) \\
\hline
\end{tabular} + HAIK, HIGHLAND, MCFARLANE, MACEK+ (TEMP+LASL) +AUERBACH, HAIK, MCFARLANE, MACEK + (TEMP+LASL) + FRANK, HOFFMANN, MISCHKE, MOIR + (ARZS + (LANL) D. BRYMAN
+ FRANK, HOFFMAN, MOIR, SARRACINO + (LANL+ARZS)
(TRIU)

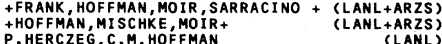

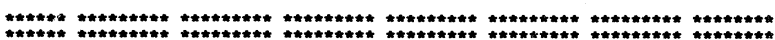
$\eta$ 14 ETA(549, JPG=0-+) I=0 14 ETA MASS (MEV)
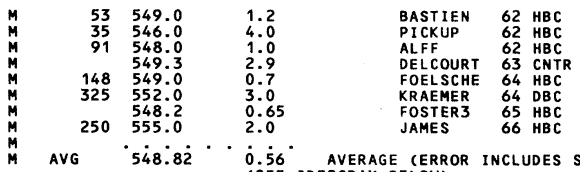

$0 . \dot{5} 6^{\circ}$ AVERAGE (ERROR INCLUDES SCALE FACTOR OF 1.4)
(SEE IDEOGRAM BELOW)

14 ETA WIDTH

W ETA WIDTH DETERMINED FROM MASS SPECTRUM (UNITS MEV)
91 (10.0) OR LESS

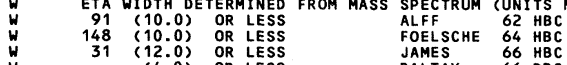

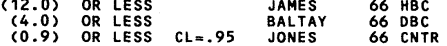

ETA WIDTH DETERMINED FROM DECAY RATE (UNITS KEV)
THIS IS THE PARTIAL DECAY RATE (WI) FOR THE MODE (ETA INTO 2GAMMA)

THIS IS THE PARTIAL DECAY RATE (W1) FOR THE MODE (ETA INTO
DIVIDED BY THE FITTED BRANCHING FRACTION (P1) FOR THAT MODE.

FIT $0.88^{\circ} \cdot{ }^{\circ}$. iz $^{\circ}$ from Fit

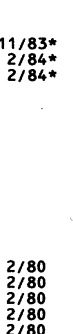

.

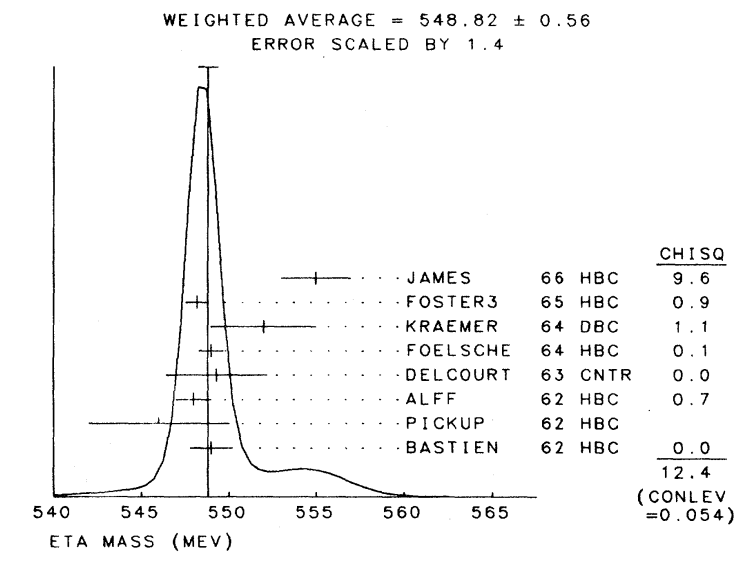

14 eta partial decay modes

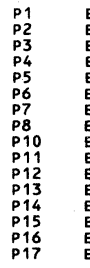

ETA INTO 2 2GAMA

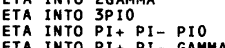

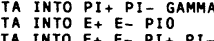

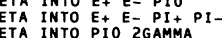

ETA INTO Et E- GAMAMA

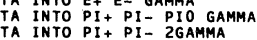

TIA INTO MUT MU-

ETA INTO MU+ MU- GAMMA

ETA INTO MU+ MU- PIO GAMMA

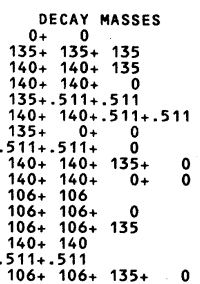

FITTED PARTLAL DECAY MODE BRANCHING FRACTIONS

The matrix below is derived from the error matrix for the fitted partial decay mode branching fractions, $P_{i}$, as follows: The diagonal elements are $P_{i} \pm \delta P_{i}$, where

$\delta P_{i}=\sqrt{\left.i \delta P_{i} \delta P_{i}\right\rangle}$, while the off-diagonal element $s$ are the normalized correlation coefficients $\left\langle\delta \mathrm{P}_{i} \delta \mathrm{P}_{j}\right\rangle /\left(\delta \mathrm{P}_{i} \cdot \delta \mathrm{P}_{j}\right)$. For the definitions of the individual $\mathrm{P}_{i}$, see the listings above; only those $P_{i}$ appearing in the matrix are assumed in the fit to be nonzero and are thus constrained to add to 1 .

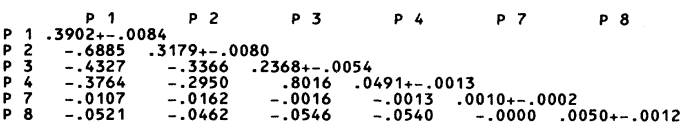

FITTED PARTIAL DECAY MODE RATES

The matrix below is the branching fraction matrix above, transformed into rate space; i.e., $G_{i} \equiv \Gamma_{i}=\Gamma_{i} \quad P_{i}$, in appropriate units. In analogy to the matrix above, the diagonal elements are $G_{i} \pm \delta G_{i}$, where $\delta G_{i}=\sqrt{\left\langle\delta G_{i} \delta G_{j}\right\rangle}$, while the off-diagonal elements are the normalized correlation coefficients $\left\langle\delta G_{i} \delta G_{j}\right\rangle /\left(\delta G_{i} \cdot \delta G_{j}\right)$. Note that, because of the error in $\Gamma_{\text {total }}$, the errors and correlations here are not directly derivable

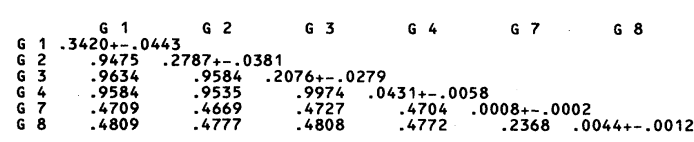

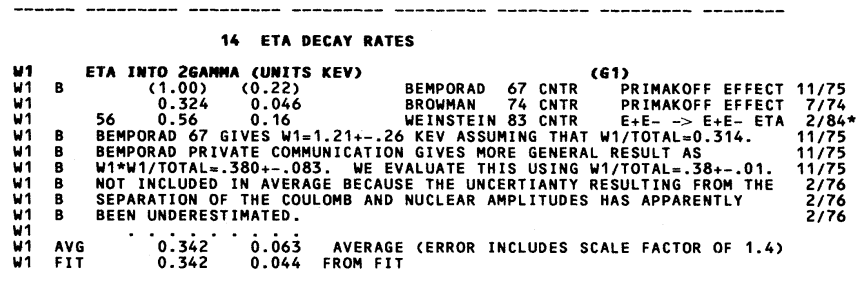




\section{Stable Particles}

$\eta$

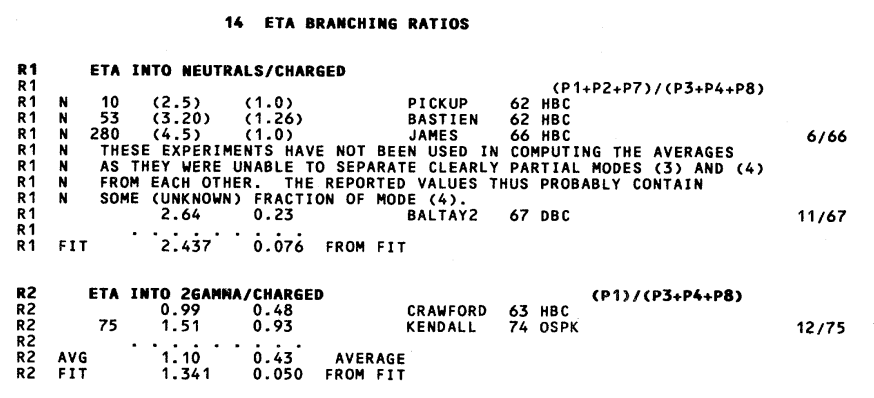

\section{NOTE ON $\eta \rightarrow \pi^{0} \gamma \gamma$}

It appears that earlier problems with the $\eta \rightarrow \pi^{0} \gamma \gamma$ branching fraction have been resolved by the excellent new measurement of BINON 82. Interested readers are referred to their paper and to the note in our 1982 edition. ${ }^{1}$

\section{Reference}

\section{Particle Data Group, Phys. Lett. 111B (1982).}
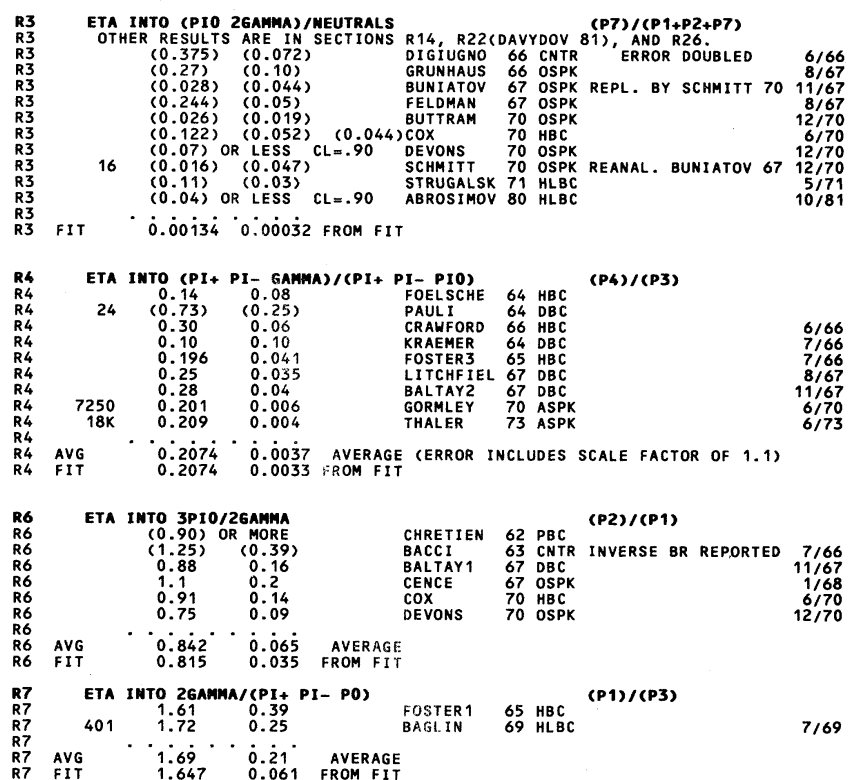

$\begin{array}{lllll}R 7 & \text { AVG } & 1.69 & 0.21 & \text { AVERAGE } \\ R 7 & \text { FIT } & 1.647 & 0.061 & \text { FROM FIT }\end{array}$

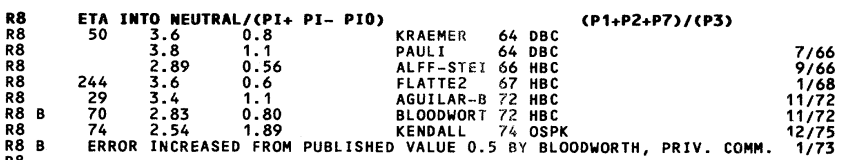

R8 B ERROR INCREASED FROM PUBLISHED

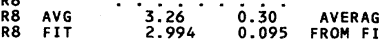

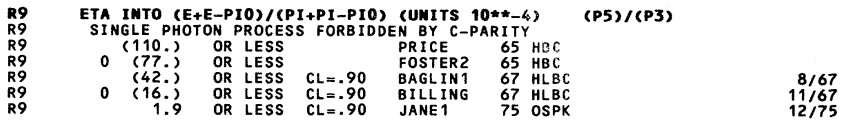

Data Card Listings

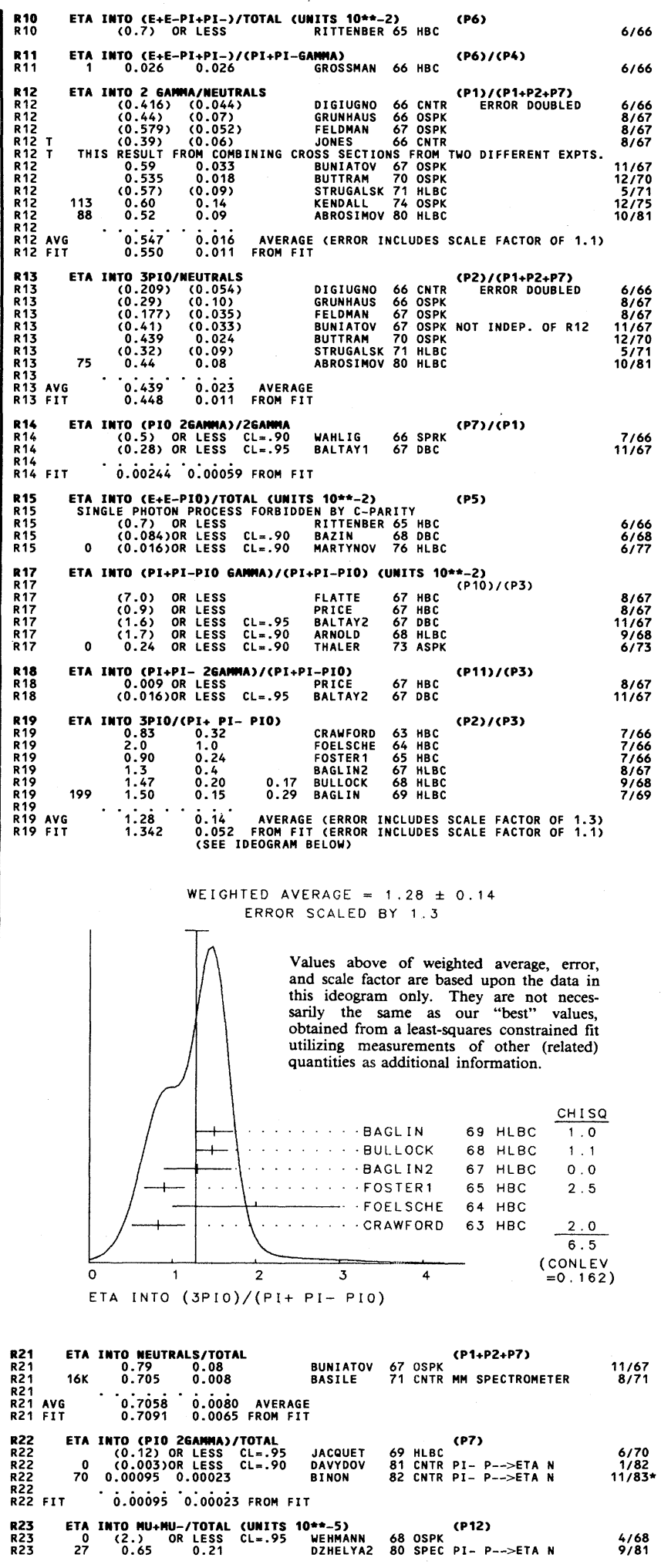




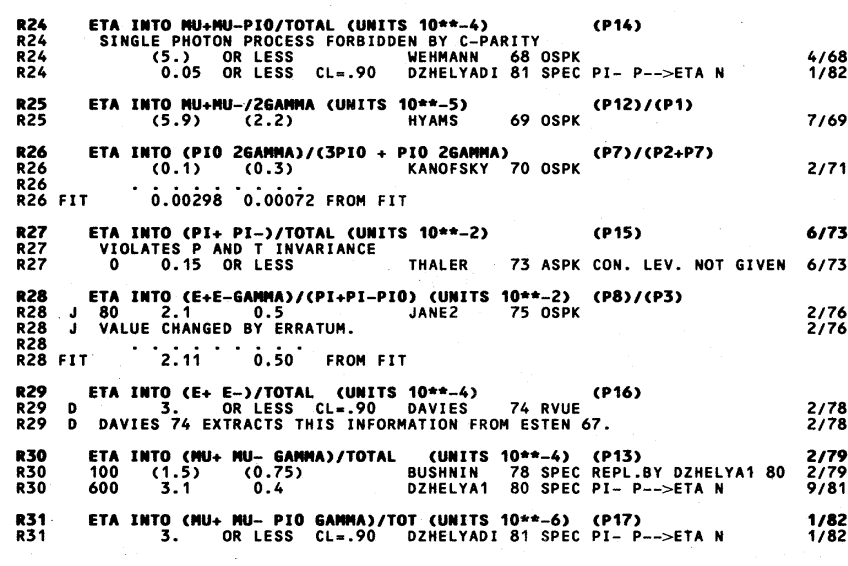

\section{NOTE ON $\boldsymbol{\eta}$ DECAY PARAMETERS}

\section{$C$ violation in $\boldsymbol{\eta}$ decays}

As a test of possible $\mathrm{C}$ violation in electromagnetic interactions, a number of experiments have looked for possible charge asymmetries in the decays $\eta \rightarrow \pi^{+} \pi^{-} \pi^{0}$ and $\eta \rightarrow \pi^{+} \pi^{-} \gamma$. We list the following parameters:

(a) The left-right asymmetry

$$
A=\left(\mathbf{N}^{+}-\mathbf{N}^{-}\right) /\left(\mathbf{N}^{+}+\mathbf{N}^{-}\right),
$$

where $\mathrm{N}^{ \pm}$means the number of events with the $\pi^{ \pm}$ energy greater than the $\pi^{\mp}$ energy in the $\eta$ rest frame.

(b) The sextant asymmetry

$$
A_{s}=\frac{N_{1}+N_{3}+N_{5}-N_{2}-N_{4}-N_{6}}{N_{1}+N_{2}+N_{3}+N_{4}+N_{5}+N_{6}}
$$

for the decay $\eta \rightarrow \pi^{+} \pi^{-} \pi^{0}$. The numbers refer to sextants of the Dalitz plot (see, for example, Layter et al. ${ }^{1}$ ). $A_{s}$ is sensitive to an $I=0 \mathrm{C}$-violating asymmetry.

(c) The quadrant asymmetry $A_{q}$, defined in a similar way as $A_{s}$, but with each sector of the Dalitz plot now containing $\pi / 2$ rather than $\pi / 3$ radians. $A_{q}$ is sensitive to an I = 2 C-violating final state.

(d) The D-wave contribution to the C-violating amplitude in the decay $\eta \rightarrow \pi^{+} \pi^{-} \gamma$. The upper limit for this contribution is measured by the parameter $\beta$, defined by

$$
\mathrm{dN} / \mathrm{d}|\cos \theta| \propto \sin ^{2} \theta\left(1+\beta \cos ^{2} \theta\right),
$$

where $\theta$ is the angle between the $\pi^{+}$and the $\gamma$ in the dipion center of mass. A term proportional to $\cos ^{2} \theta$ could also be due to P- and F-wave interference.

We list $A$ for the decay modes $\eta \rightarrow \pi^{+} \pi^{-} \pi^{0}$ and $\eta \rightarrow \pi^{+} \pi^{-} \gamma, \mathrm{A}_{\mathrm{s}}$ and $\mathrm{A}_{\mathrm{q}}$ for the decay $\eta \rightarrow \pi^{+} \pi^{-} \pi^{0}$,

and $\beta$ for the decay $\eta \rightarrow \pi^{+} \pi^{-} \gamma$ in the Data Card Listings below.

\section{Dalitz plot for $\eta \rightarrow \pi^{+} \pi^{-} \pi^{0}$}

The Dalitz plot for the decay $\eta \rightarrow \pi^{+} \pi^{-} \pi^{0}$ may be fit by the distribution

$$
|M(x, y)|^{2} \propto 1+a y+b y^{2}+c x+d x^{2}+e x y .
$$

Here,

$$
x=\sqrt{3}\left(T_{+}-T_{-}\right) / Q, \quad y=\left(3 T_{0} / Q\right)-1,
$$

$T_{+}, T_{-}$, and $T_{0}$ are the kinetic energies of the $\pi^{+}, \pi^{-}$, and $\pi^{0}$ in the $\eta$ rest system, and $\mathrm{Q}=\mathrm{m}_{\eta}-\mathrm{m}_{\pi^{+}}-$ $\mathrm{m}_{\pi^{-}}-\mathrm{m}_{\pi^{0}}$. The coefficient of the term linear in $\mathrm{x}$ is sensitive to ${ }^{\pi} \mathrm{C}$-violation due to an $\mathrm{I}=0$ or $\mathrm{I}=2$ final state. We list papers presenting determinations of the parameters $a, b, c$, and $d$ in the section DP below. However, we do not tabulate values of these parameters because the assumptions made by different authors are not compatible and do not allow comparison of the numerical values.

Dalitz plot for $\boldsymbol{\eta} \rightarrow \boldsymbol{\pi}^{0} \boldsymbol{\pi}^{\mathbf{0}} \boldsymbol{\pi}^{0}$

The Dalitz plot for the decay $\eta \rightarrow \pi^{0} \pi^{0} \pi^{0}$ may be fit to the expression

$$
|\mathbf{M}|^{2} \propto 1+2 \alpha z
$$

where

$$
z=\frac{2}{3} \sum_{i=1}^{3}\left[3\left(m_{\eta}-3 m_{\pi}\right)^{-1}\left(E_{i}-\frac{1}{3} m_{\eta}\right)\right]^{2}=\rho^{2} / \rho_{\max }^{2} .
$$

Here $E_{i}$ is the energy of the $i^{\text {th }}$ pion in the $\eta$ rest frame, and $\rho$ is the distance to the center of the Dalitz plot. We list the parameter $\alpha$ in section A0 below.

\section{Reference}

1. J.G. Layter et al., Phys. Rev. Lett. 29, 316 (1972).

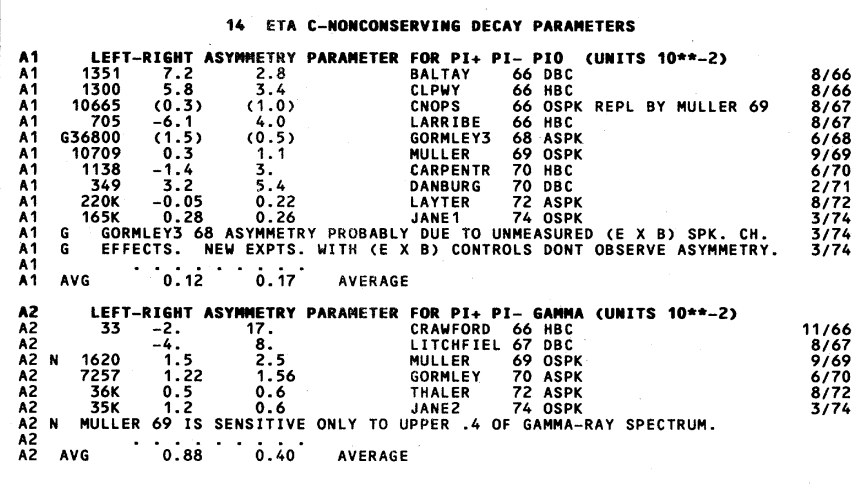


Stable Particles

$\eta, \mathrm{K}^{ \pm}$

\section{Data Card Listings}
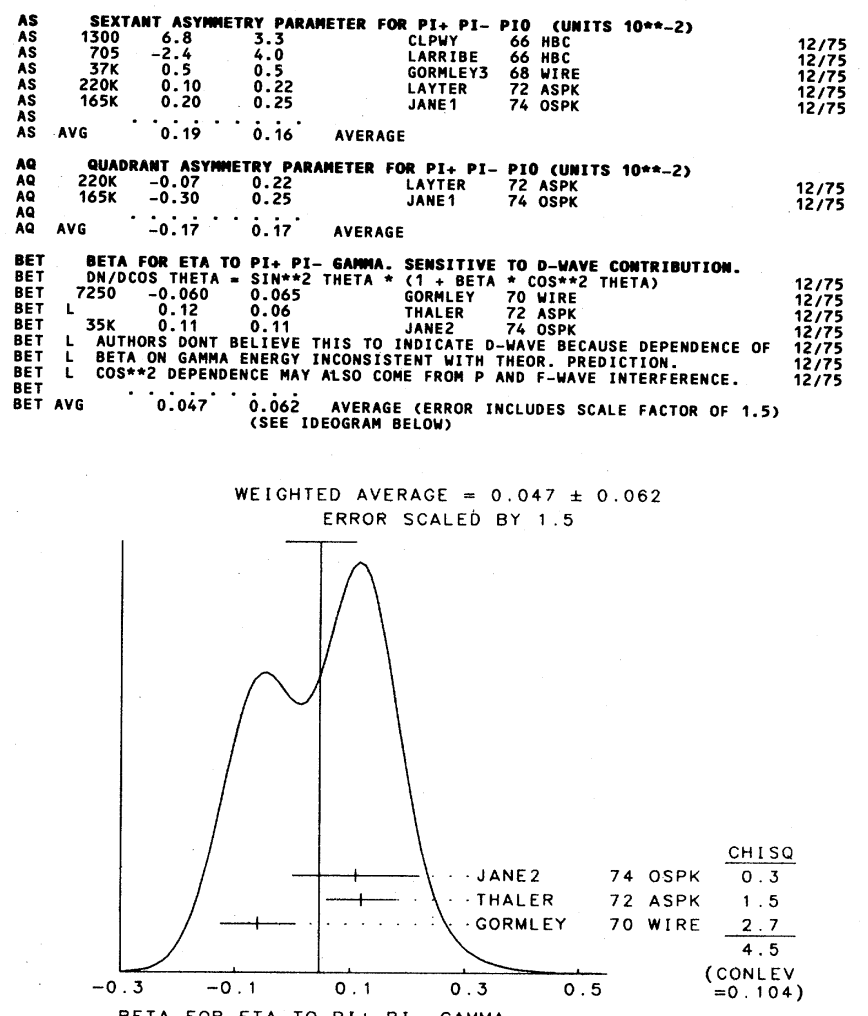

BETA FOR ETA TO PI+ PI- GAMMA

14 EMERGY DEPEMDEMCE OF ETA DALITZ PLOT

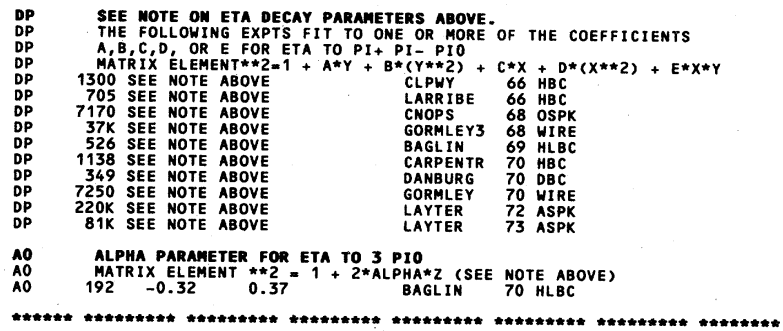

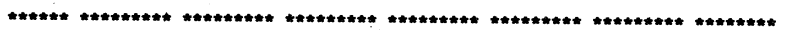

PEVSNER 61 PRL 7421

$\begin{array}{lllll}\text { ALFF } & 62 & \text { PRL } & 9 & 322 \\ \text { BASTIEN } & 62 & \text { PRL } 8 & 114\end{array}$

$\begin{array}{ll}\text { CHRETIEN } 62 & \text { PRL } 91127 \\ \text { PICKUP } 62 \text { PRL } 8329\end{array}$

$\begin{array}{lllll}\text { BACCI } & 63 & \text { PRL } & 11 & 37 \\ \text { CRAMFORD } & 63 & \text { PRL } & 10 & 546\end{array}$

$\begin{array}{lll}\text { CRALASO } 66 & \text { PRL } 16907 \\ \text { DELCOURT } 63 & \text { PL } 7215\end{array}$

$\begin{array}{llllll}\text { FOELSCHE } & 64 & \text { PR } & 134 & \text { B } & 1138 \\ \text { KRAEMER } & 64 & \text { PR } & 136 & \text { B } & 496 \\ \text { PAUL I } & 64 & \text { PL } & 13 & 351\end{array}$

PAULI 64 PL 13351

$\begin{array}{lll}\text { FOSTER } & 65 & \text { PR } \\ \text { FOSTER2 } & 65 & \text { ATHENS } \\ \text { FOSTER3 } & 65 & \text { THESIS }\end{array}$

$\begin{array}{lll}\text { FOSTER3 } & 65 & \text { THESIS } \\ \text { PRICE } & 65 & \text { PRL } 15 \\ \text { RITTENBE } & 65 & 123 \\ \text { PRL } 15 & 556\end{array}$

ALFF-STE 66 PR 1451072

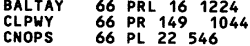

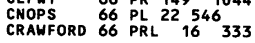

$\begin{array}{lllll}\text { DIGIUGNO } & 66 & \text { PRL } 16 & 767 \\ \text { GROSSMAN } & 66 & \text { PR } & 146 & 993\end{array}$
REFEREMCES FOR ETA

PEVSNER, KRAEMER, NUSSBAUM, RICHARDSON + (JHU)

ALFF, BERLEY, COLLEY, BRUGGER + (COLU+RUTGERS)

BASTIEN,BERGE, DAHL, FERRO-LUZZI + +RU (LRL)
CHRETIEN+ (BRAN+BROWN+HARVARD+MIT+PADOVA)
E PICKUP, ROB INSON, SALANT

BACCI, PENSO, SALVINI +
F S CRAWFORD, LLOYD, FOWLER (ROMA+FRAS)
(LRL+DUKE)

F C CRAWFORD, L LLOYD, E FOWLER (LRL + DUKE)
(LRL+DUKE)
DELCOURT, LEFRANCOIS, PEREZ Y JORBA+ (ORSAY)

H W FOELSCHE, H L KRAYBILL
KRAEMER, MADANSKY, FIELOS +
E PAULI, A MULLER

FOSTER, PETERS, MEER, LOEFFLER + (WISC+PURDUE)
FOSTER,GOOD, MEER

F.C.FOSTER , MEER (HISCONSIN)
MISCONSIN)

L.R.PRICE, F.S. CRAWFORD
RITTENBERG, KALBFLEISCH (LRL)
(LRL+BNL)

ALFF-STEINBERGER, BERLEY+ (COLUMBIA+RUTGERS)
+FRANZINI, KIM, KIRSCH+(COLUMBIA+STONY BROOK) COLUMB IA, LRL, PURDUE, WI SCONSIN, YALE CNOPS, FI INOCCHIARS, LÁSSALLE, + (CERN, ETH, SACL)
F.S. CRAWFORD, L.R.PRICE
(LRL)

DIGIUGNO, GIORGI, SILVESTRI+ (NAPL, TRST, FRAS)
R GROSSMAN, L PRICE, F CRAWFORD (COLUMBIA)
J.GRUNHAUS

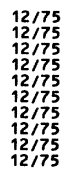

$12 / 75$

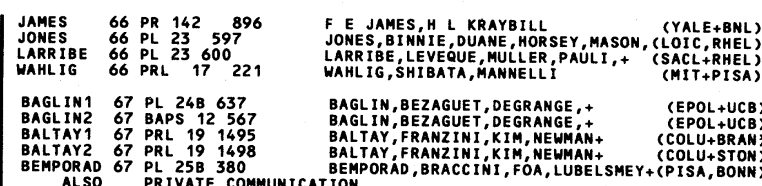

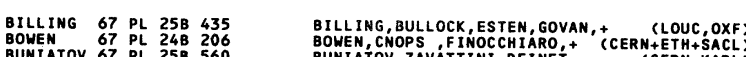
BOWEN 67 PL 248206
BUNIATOV $67 \mathrm{PL} 258560$ BOWEN, CNOPS FINOCCHIARO,+' (CERN+ETH+SACL)
(CERR +KARL)

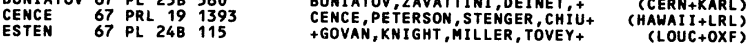

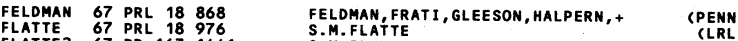

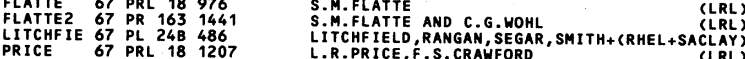

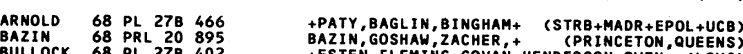
BULLOCK 68 PL 278402

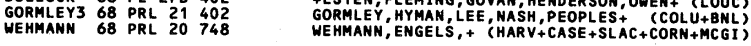
BAGLIN $69 \mathrm{PL} 298445$
ALSO 70 NP B22 66
BAGLIN, BEZAGUET, HYAMS
JACQUET 69 PL 58 NC 58 743
MULLER 69 THES IS

$\begin{array}{lll}\text { BAGLIN } 70 \text { NP } 822 & 66 & \text { +BEZAGUET, DEGRANGE, MUSSET+ (EPOL+MADR+STRB) }\end{array}$

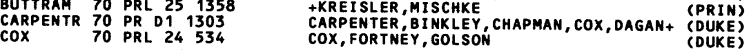
$\begin{array}{llll}\text { DANBURG } 70 & \text { PR D2 } 2564 & \text { +ABOL INS, DAHL, DAVIES, HOCH, KIRZ,+ } \\ \text { DEVONS } 70 & \text { PR DO } & \text { (LRL) } \\ \text { +GRUNHAUS, KOZLOWSKI, NEMETHY + } & \text { (COLU, SYRA) }\end{array}$

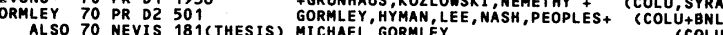

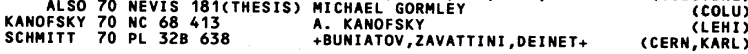
BASILE 71 NC 39796 +BOLLINI, DALPIAZ, FRABETTI+ (CERN, BGMA, STRB) AGUILAR-BENITEZ, CHUNG, EISNER, SAMIOS (BNL)

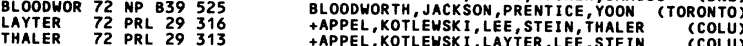

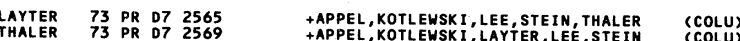

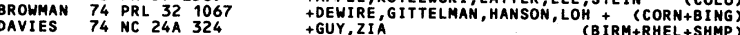
JANE1 74 PL $48 B 260$ + JONES, LIPMAN, OWEN, PENNEY+ (RHEL+LOWC+SUSS

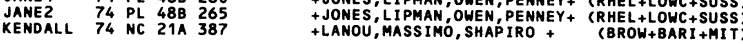

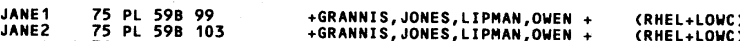

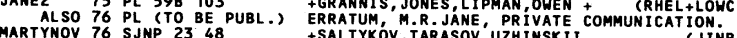

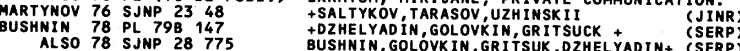

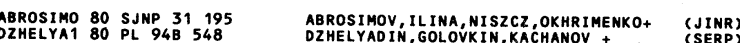

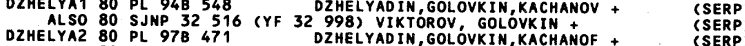

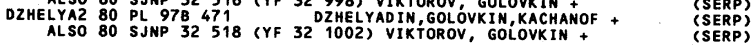

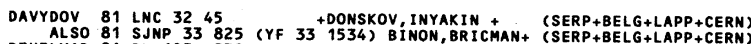
ALSO 81 SJNP 33825 (YF 33 1534) BINON, BR ICMAN+ (SERP+BELG+LAPP+CERN)
DZHELYAD 81 PL 1058239 (SERELYADIN, GOLOVKIN, KONSTANTINOV + (SEPP)

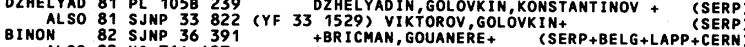

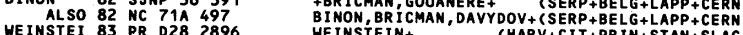
QUANTUM NUMBER DETERMinations NOT REFERRED TO IN THE DATA CARDS

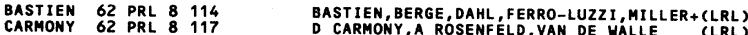

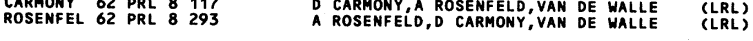

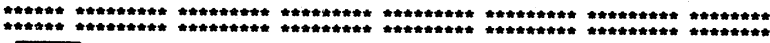

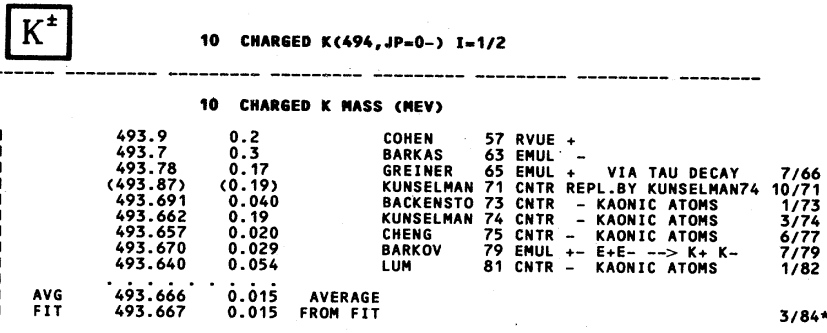
$10\left(K_{+}\right)-(K-)$ MASS DIFFEREMCE (MEV)

DM F TEST OF CPT 

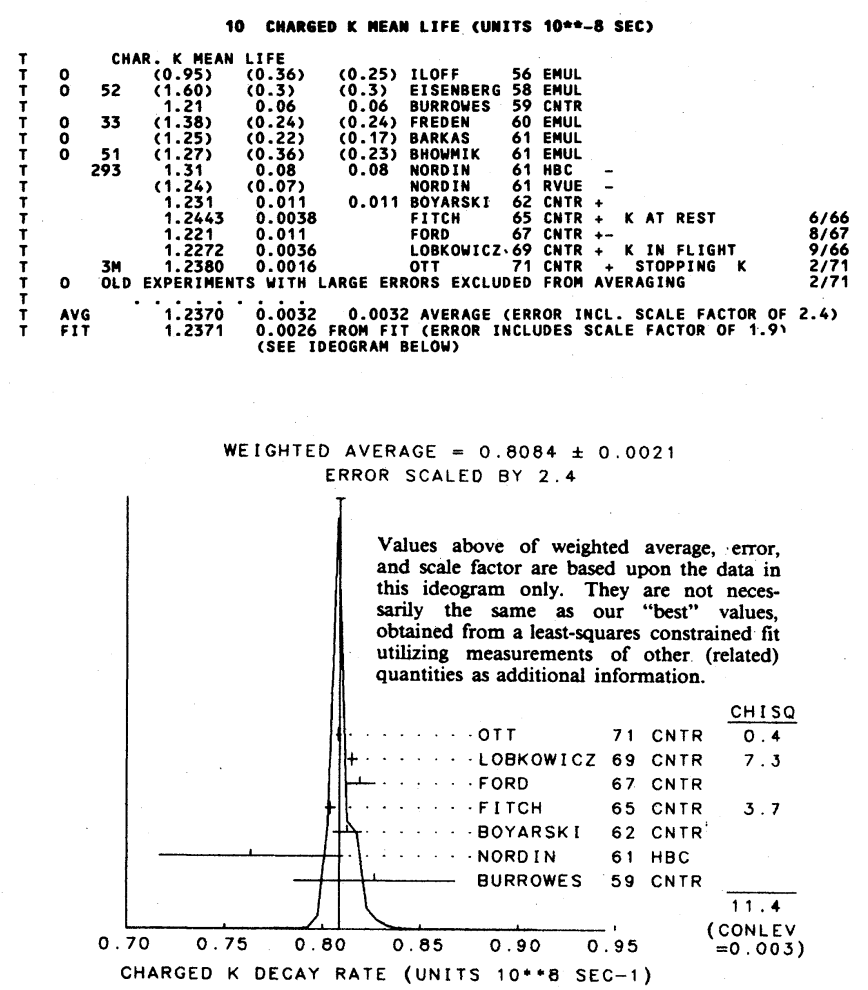

$10\left(\left(K_{+}\right)\right.$- (K-))/AVG., MEAM LIFE DifFEREMCE (PERCEWT)

ot N THIS QUANTITY IS A MEASURE OF CPT IMVARIANCE IN U.I.

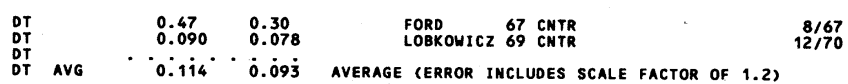

10 CMARGED K PARTIAL DECAY MODES

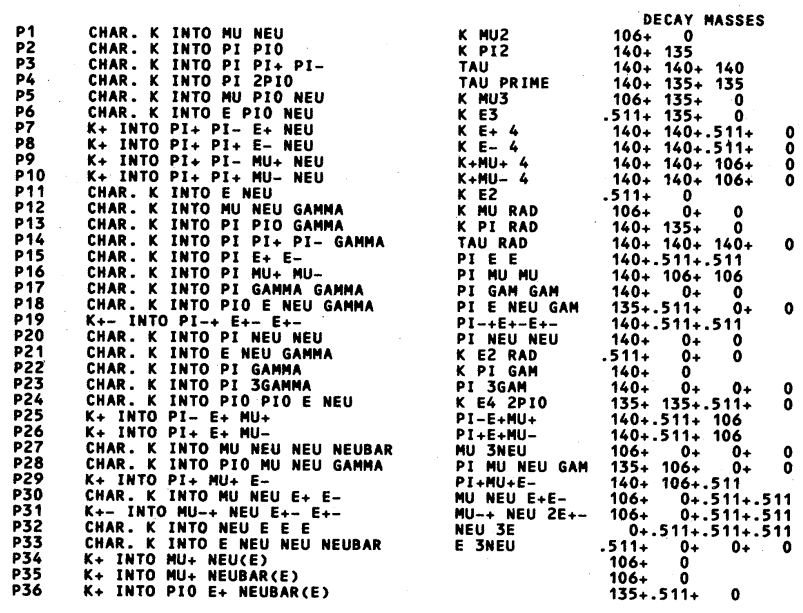

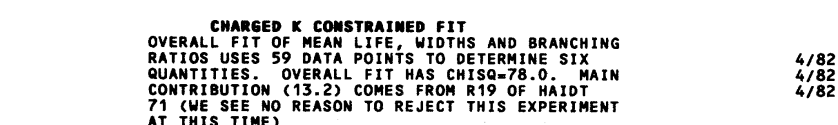

FITTED PARTLAL DECAY MODE BRANCHING FRACTIONS

The matrix below is derived from the error matrix for the fitted partial decay mode branching fractions, $P_{i}$, as follows: The diagonal elements are $P_{i} \neq 6 P_{i}$, where

$\left.\delta P_{i}=\sqrt{\left\langle\delta P_{i} \delta P_{i}\right\rangle}\right\rangle$, while the off-diagonal elements are the normalized correlation coeffi-

cients $\left\langle\delta \mathrm{P}_{\mathrm{i}} \delta \mathrm{P}_{\mathrm{j}}\right\rangle /\left\langle\delta \mathrm{P}_{\mathrm{i}} \cdot \delta \mathrm{P}_{\mathrm{j}}\right)$. For the definitions of the individual $\mathrm{P}_{\mathrm{i}}$, see the listings

above; only those $P_{i}$ appearing in the matrix are as sumed in the fit to be nonzero and

$\begin{array}{llllll}\text { P } 1.6351 .0016 & \text { P } 2 & \text { P } 3 & \text { P } 4 & \text { P } 5 & \text { P } 6\end{array}$

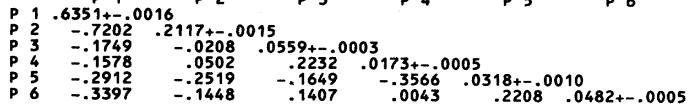

FITTED PARTLAL DECAY MODE RATES

The matrix below is the branching fraction matrix above, transformed into rate space; i. e., $G_{i} \equiv r_{i}=r_{\text {total }} p_{i}$, in appropriate units. In analogy to the matrix above, the diagonal elements are $G_{i} \neq \delta G_{i}$, where $\delta G_{i}=\sqrt{\left\langle\delta G_{i} \delta G_{j}\right\rangle}$, while the off-diagonal elements are the normalized correlation coefficients $\left\langle\delta \mathrm{G}_{i} \mathrm{G}_{\mathrm{j}}\right\rangle /\left(\delta \mathrm{G}_{\mathrm{i}} \cdot{ }_{\mathrm{G}} \mathrm{G}_{\mathrm{j}}\right)$. Note that, because of the error in $\Gamma_{\text {total }}$, the errors and correlations here are not directly derivable from those above.

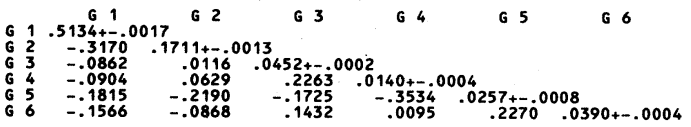

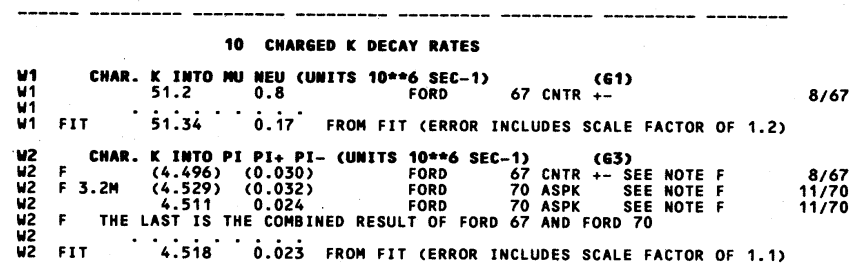

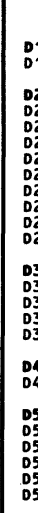

$10((K+)$ - ( $K-))$ /AVG., DECAY RATE difFEREMCE (PERCEWT)

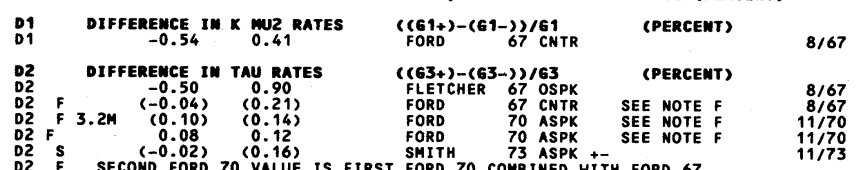

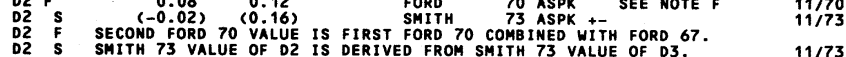

${ }_{02}^{D 2}$ avg ${ }^{\circ} 0.07^{\prime}{ }^{\prime} \dot{0}$.iz ${ }^{\circ}$ average

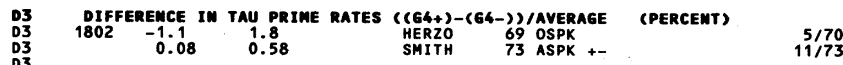

$D 3$
03 AVG $-0.03^{3} \cdots \dot{0.55^{\circ}}$ average

DQ DIFFEREWCE IN K P12 RATES
0.8
0.2

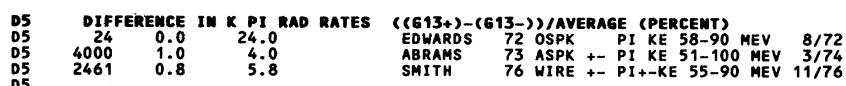
D5 avg $0.9^{\circ} \cdots 3^{3.3} \cdot$ average

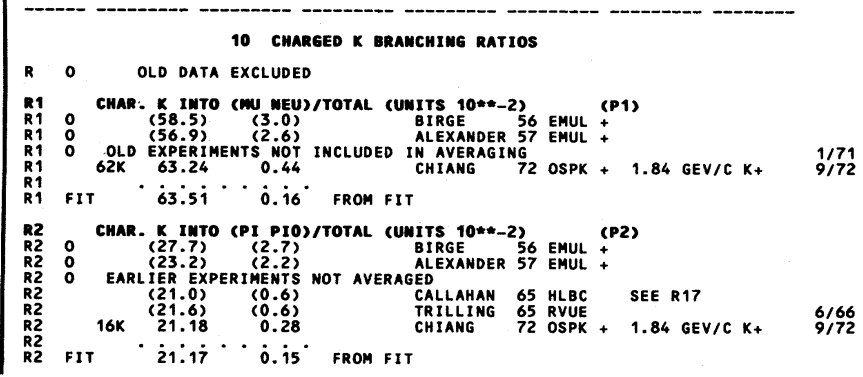




\section{Stable Particles}

$\mathrm{K}^{ \pm}$
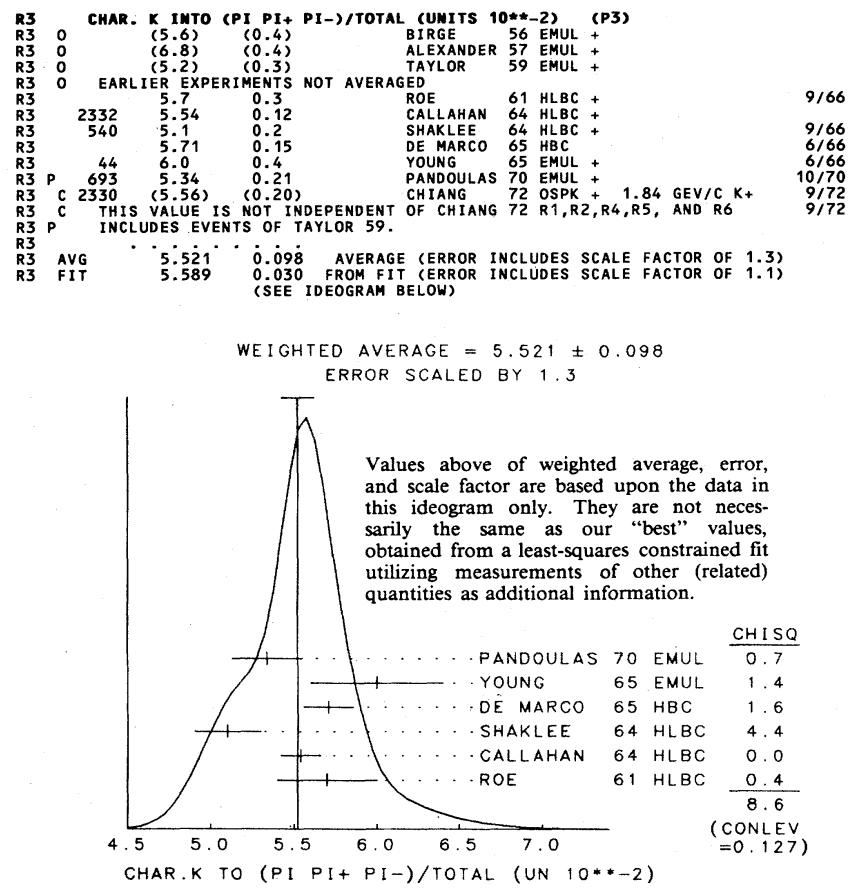

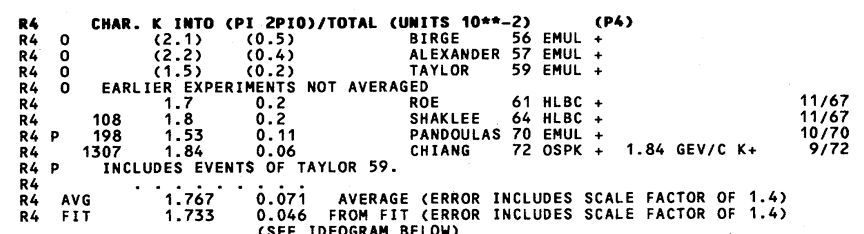

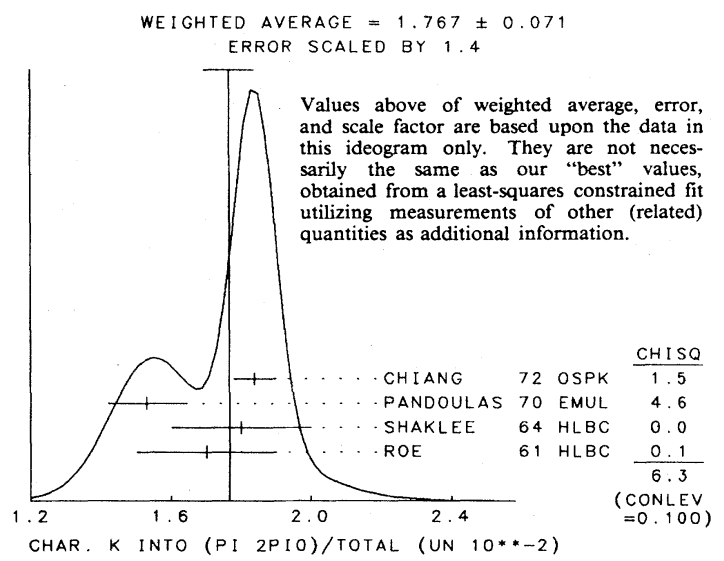

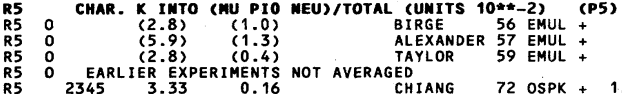

$23453.33 \quad 0.16$
25

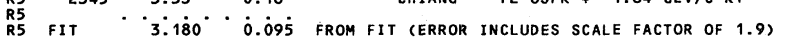

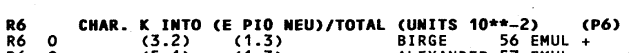

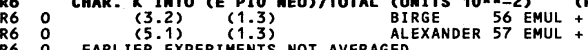

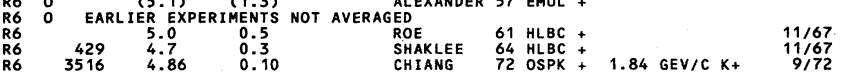

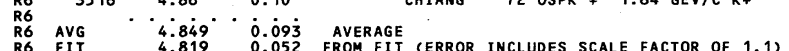

\section{Data Card Listings}

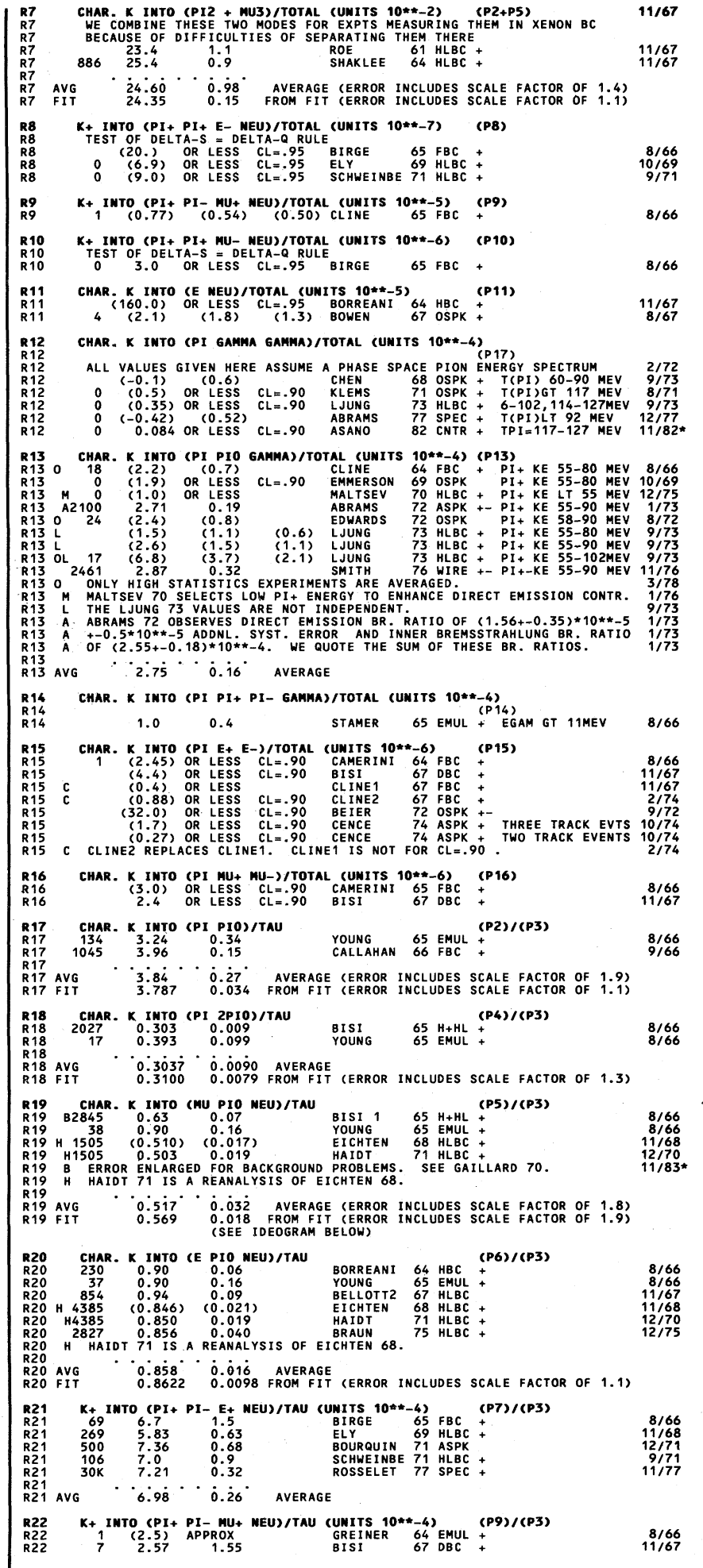




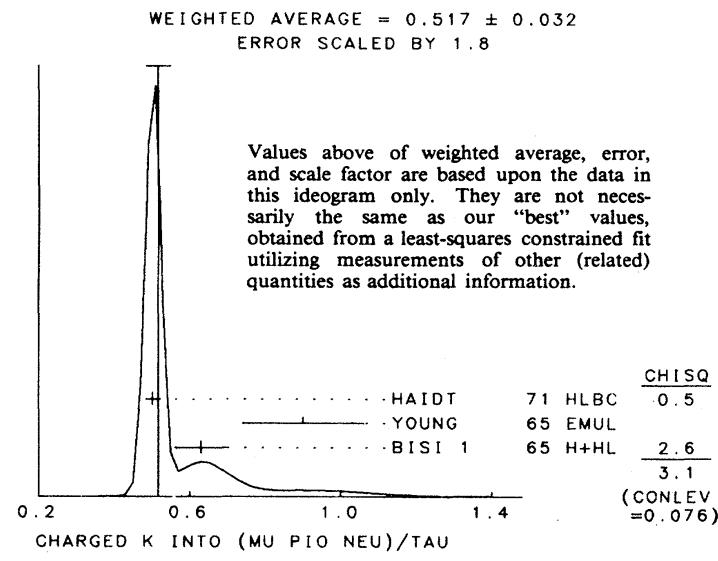

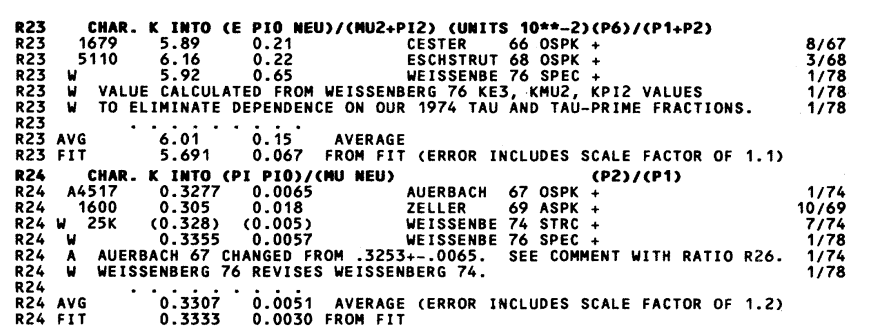
$\begin{array}{lll}\text { R24 AVG } & 0.3307^{\circ} & 0.0051 \\ \text { R24 FIT } & 0.3333 & 0.0030 \text { FRORAGE (ERROR INCLUDES SCALE FACTOR OF 1.2) } \\ \text { (SEE IDEOGRAM BELOW) }\end{array}$
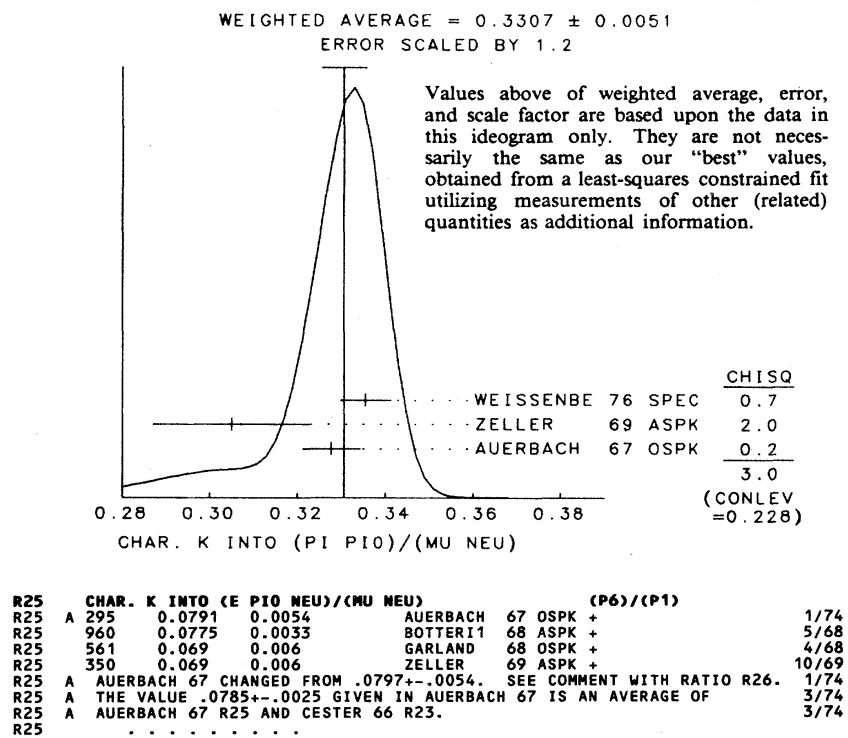

$\begin{array}{llll}\text { R25 AVG } & 0.0752 & 0.002 & \\ \text { R25 FIT } & 0.07588 & 0.00091 & \text { AVERAGE FR FIT (ERROR INCLUDES SCALE FACTOR OF 1.1) }\end{array}$

R26 CHAR. K INTO (MU P1O MEU)/(MU MEU)

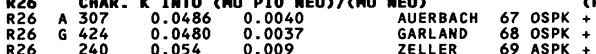

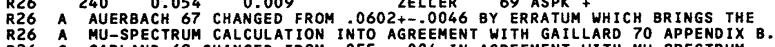
R26 A MU-SPECTRUM CALCULATION INTO AGREEMENT WITH GAILLARD 70 APPENDIX $B$.
R26 G GARLAND 68 CHAGED FROM .055+-.004 IN AGREEMENT WITH MU-SPECTRUM

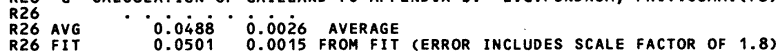

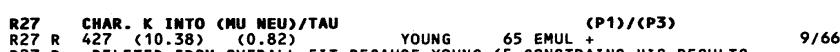

$R 27 R$ D DELETED FROM OVERALL FIT BECAUSE YOUNG 65 CONSTRAINS HIS RESULTS
$R 27 R$ TO ADD UP TO 1 . ONLY YOUNG MEASURED MU2 DIRECTLY.

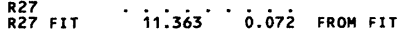

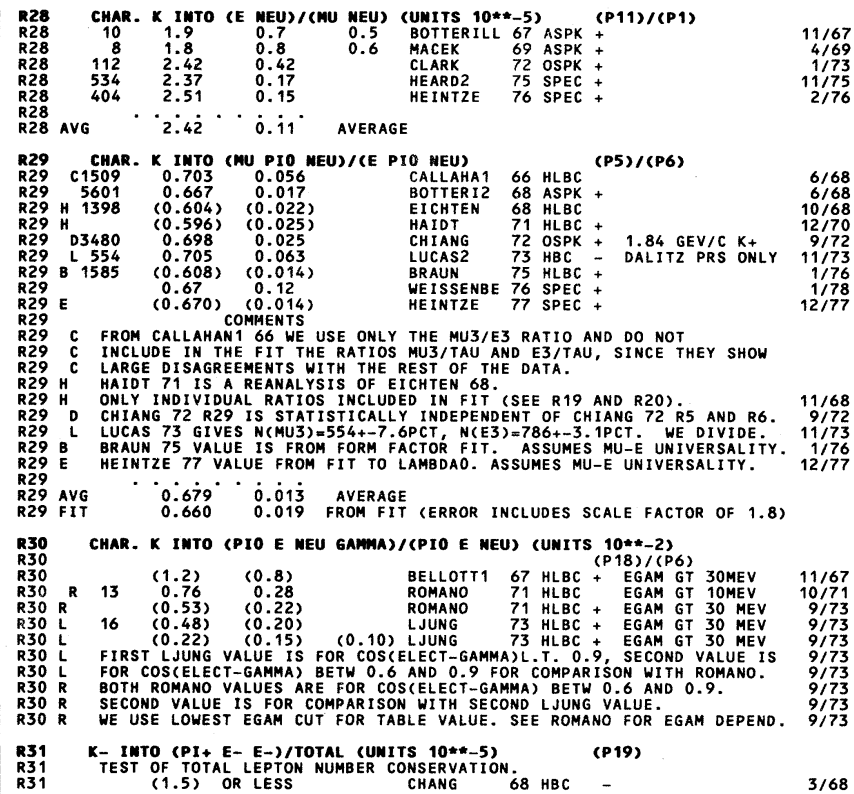

R32 CHAR. K IMTO (PI MEU MEU)/TOTAL (UNITS 10**-6) (P20)

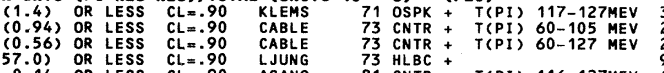

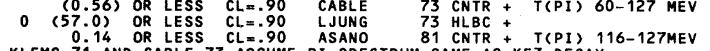
KLEMS 71 AND CABLE 73 ASSUME PI SPECTRUM SAME AS KE 3 DECAY. INT.
SECOND CABLE LIMIT COMBIES CABLE AND KLEMS DATA FOR VECTOR INT.

R33 CHAR. K IMTO (E MEU GAMMA)/TOTAL CUNITS 10**-5) (P21)
R33 M M
R33 (7).1) OR LESS R34 CHAR. K InTO (PI GAMMA) PTOTAL (UNITS 10**-6) (P22)
R34 VIOLATES ANGULAR MOMENTUM CONSERVATION. NOT LISTED IN TABLES.

R34
R34

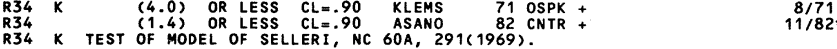
R36 CHAR K IMTO (PI 3GAMMA) PTOTAL (UNITS 10**-4) (P23)
R36 VALUES GIVEN HERE ASSUME A PHASE SPACE PION ENERGY SPECTRUM.

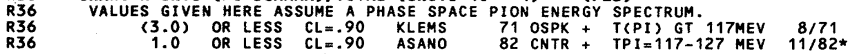

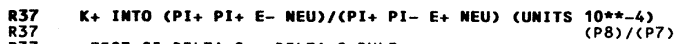

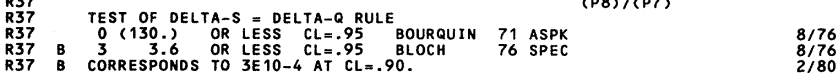

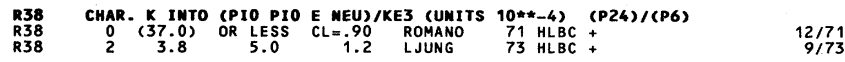

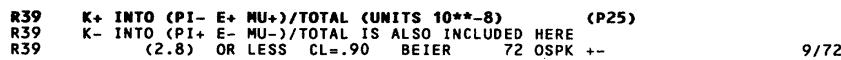

$\begin{array}{lll}\text { R40 } & K+\text { INTO (PI+ E+ MU-)/TOTAL (UNITS 10**-8) } \\ \text { R40 } & K-\text { INTO (PI- E- MU+)/TOTAL IS ALSO INCLUED HERE } \\ \text { R40 } & \text { (P26) }\end{array}$

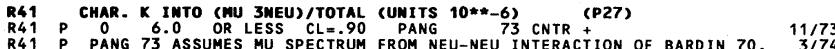

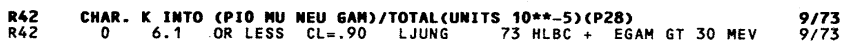

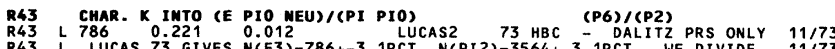

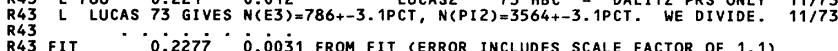

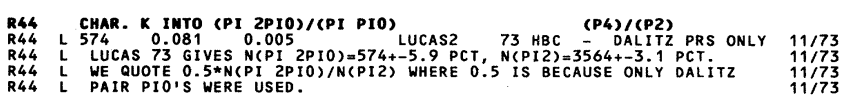

R44 FIT $0.0819 \cdot 0.0022$ FROM FIT (ERROR INCLUDES SCALE FACTOR OF 1.3)

R45 CHAR. $K_{5}$ INTOC(NU MEU GAMMA)/TOTAL CUMITS
R45
12

R46 CHAR. K INTO (PI E+ E-)/(PI+ PI- E MEU) (UNITS 10**-3) (P15)/(PP)
R46 TEST FOR DELTA-S $=1$ WEAK NEUTRAL CURRENT. ALLOWED BY COMBINED

R46 TEST FOR DELTA-S = 1 WEAK NEUTRAL CURRENT. ALLOH
R46
R46 IIRST ORDER WEAK AND E.M. INTERACTIONS.

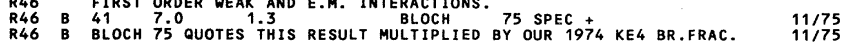

RG7 CHAR. K INTO (E MEU GAM)/(E MEU)
R47 STRUCTURE DEPENDENT PART WITH + GAMMA HELICITY. (P21)/(P11)

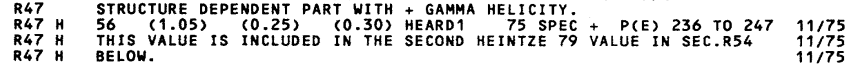




\section{Stable Particles}

$\mathrm{K}^{ \pm}$

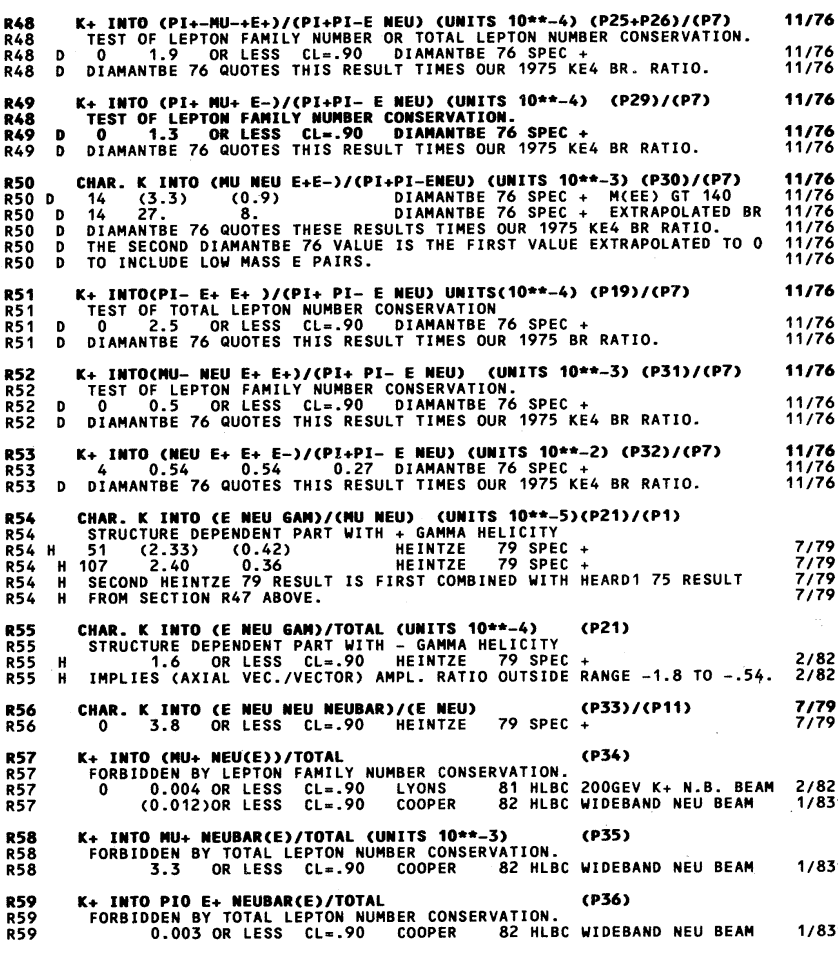

NOTE ON DALITZ PLOT PARAMETERS FOR $\mathrm{K} \rightarrow 3 \pi$ DECAYS

The Dalitz plot distribution for $\mathrm{K}^{ \pm} \rightarrow \pi^{ \pm} \pi^{ \pm} \pi^{\mp}$, $\mathrm{K}^{ \pm} \rightarrow \pi^{0} \pi^{0} \pi^{ \pm}$, and $\mathrm{K}_{\mathrm{L}}^{0} \rightarrow \pi^{+} \pi^{-} \pi^{0}$ can be parametrized by a series expansion such as that introduced by Weinberg. ${ }^{1}$ We use the form

$$
\begin{aligned}
& |\mathrm{M}|^{2} \propto 1+\mathrm{g} \frac{\left(\mathrm{s}_{3}-\mathrm{s}_{0}\right)}{\mathrm{m}_{\pi^{+}}^{2}}+\mathrm{h}\left(\frac{\mathrm{s}_{3}-\mathrm{s}_{0}}{\mathrm{~m}_{\pi^{+}}^{2}}\right)^{2} \\
& +\mathrm{j} \frac{\left(\mathrm{s}_{2}-\mathrm{s}_{1}\right)}{\mathrm{m}_{\pi^{+}}^{2}}+\mathrm{k}\left(\frac{\mathrm{s}_{2}-\mathrm{s}_{1}}{\mathrm{~m}_{\pi^{+}}^{2}}\right)^{2}+\cdots,
\end{aligned}
$$

where $\mathrm{m}^{2}+$ has been introduced to make the coefficients $g, \underset{h}{\mathbf{h}}, \mathrm{j}$, and $\mathrm{k}$ dimensionless, and

$$
\begin{aligned}
& s_{i}=\left(P_{K}-P_{i}\right)^{2}=\left(m_{K}-m_{i}\right)^{2}-2 m_{K} T_{i}, i=1,2,3, \\
& s_{0}=\frac{1}{3} \sum_{i} s_{i}=\frac{1}{3}\left(m_{K}^{2}+m_{1}^{2}+m_{2}^{2}+m_{3}^{2}\right) .
\end{aligned}
$$

Here the $P_{i}$ are four-vectors, $m_{i}$ and $T_{i}$ are the mass and kinetic energy of the $i^{\text {th }}$ pion, and the index 3 is used for the odd pion.

\section{Data Card Listings}

The coefficient $\mathrm{g}$ is a measure of the slope in the variable $s_{3}\left(\right.$ or $\left.T_{3}\right)$ of the Dalitz plot, while $h$ and $k$ measure the quadratic dependence on $s_{3}$ and $\left(s_{2}-s_{1}\right)$, respectively. The coefficient $j$ is related to the asymmetry of the plot and must be zero if $\mathrm{CP}$ invariance holds. Note also that if CP is good, $g, h$, and $k$ must be the same for $\mathrm{K}^{+} \rightarrow \pi^{+} \pi^{+} \pi^{-}$as for $\mathrm{K}^{-} \rightarrow \pi^{-} \pi^{-} \pi^{+}$.

Since different experiments use different forms for $|\mathbf{M}|^{2}$, in order to compare the experiments we have converted to $\mathrm{g}, \mathrm{h}, \mathrm{j}$, and $\mathrm{k}$ whatever coefficients have been measured. For details of this conversion and discussion of the data, see the April 1982 version of this note. $^{2}$

See also the review of Devlin and Dickey, ${ }^{3}$ which contains an analysis of $K \rightarrow 2 \pi$ and $K \rightarrow 3 \pi$ data in terms of transition amplitudes with appropriate energy dependence.

\section{References}

1. S. Weinberg, Phys. Rev. Lett. 4, 87 (1960).

2. Particle Data Group, Phys. Lett. 111B, 69 (1982).

3. T.J. Devlin and J.O. Dickey, Rev. Mod. Phys. 51, 237 (1979).

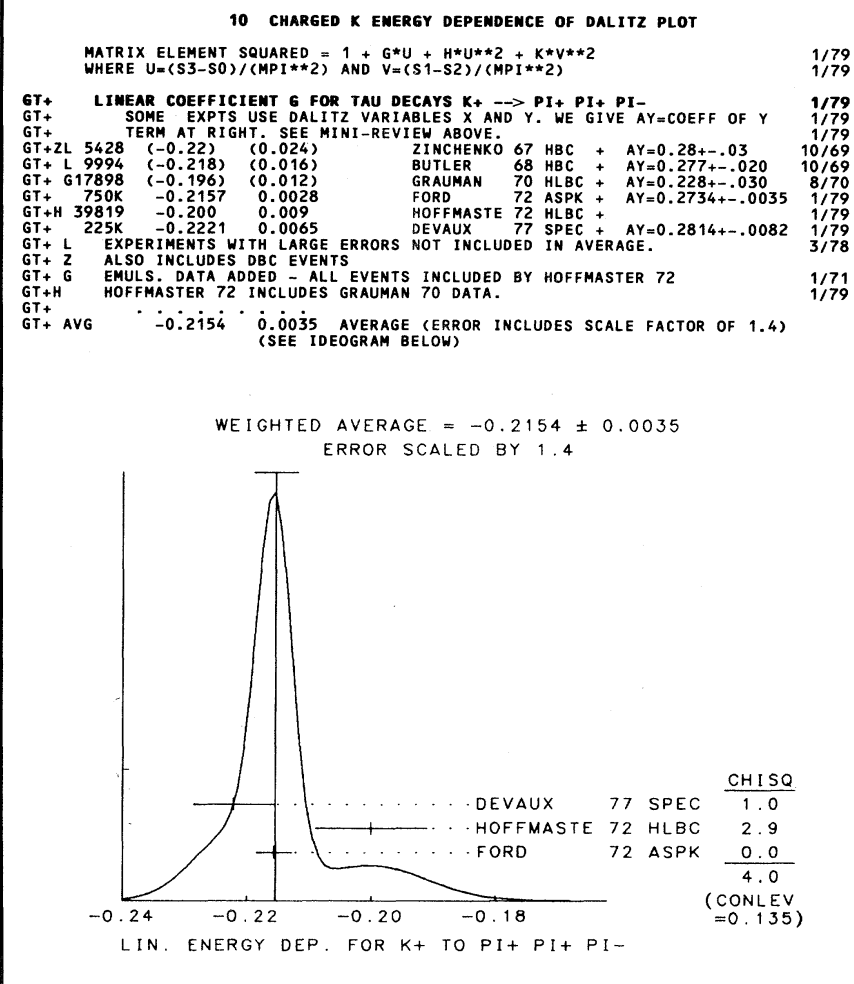




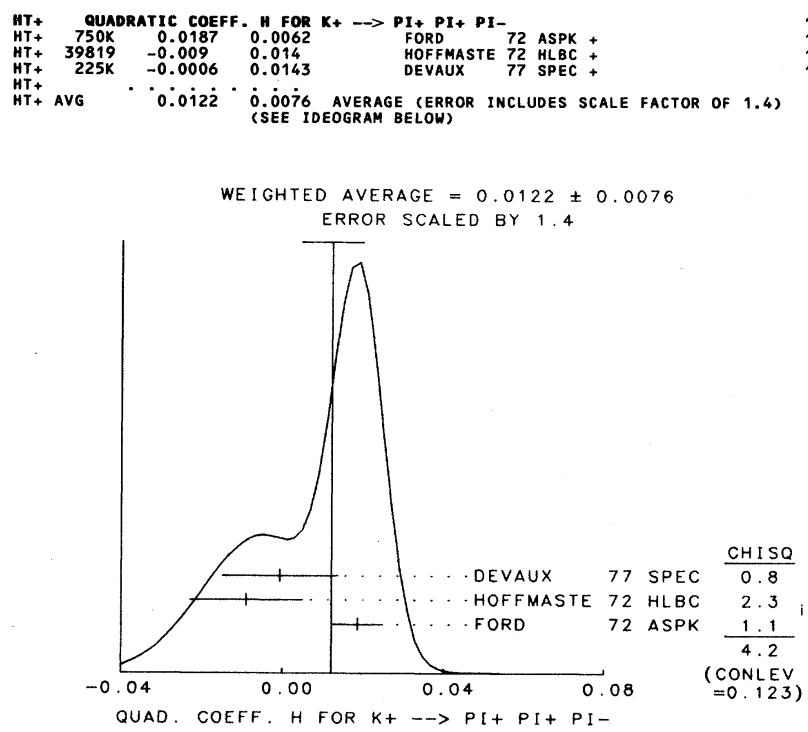

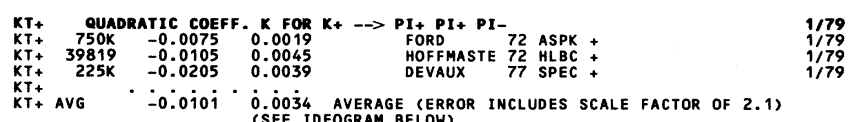

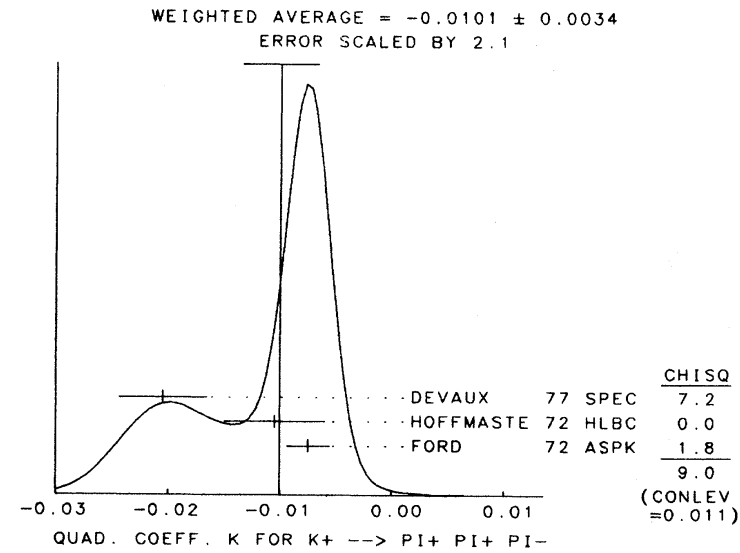

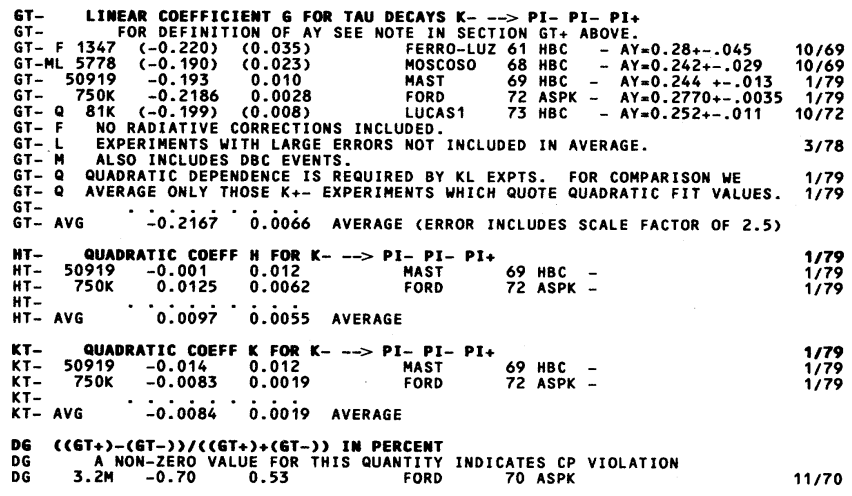
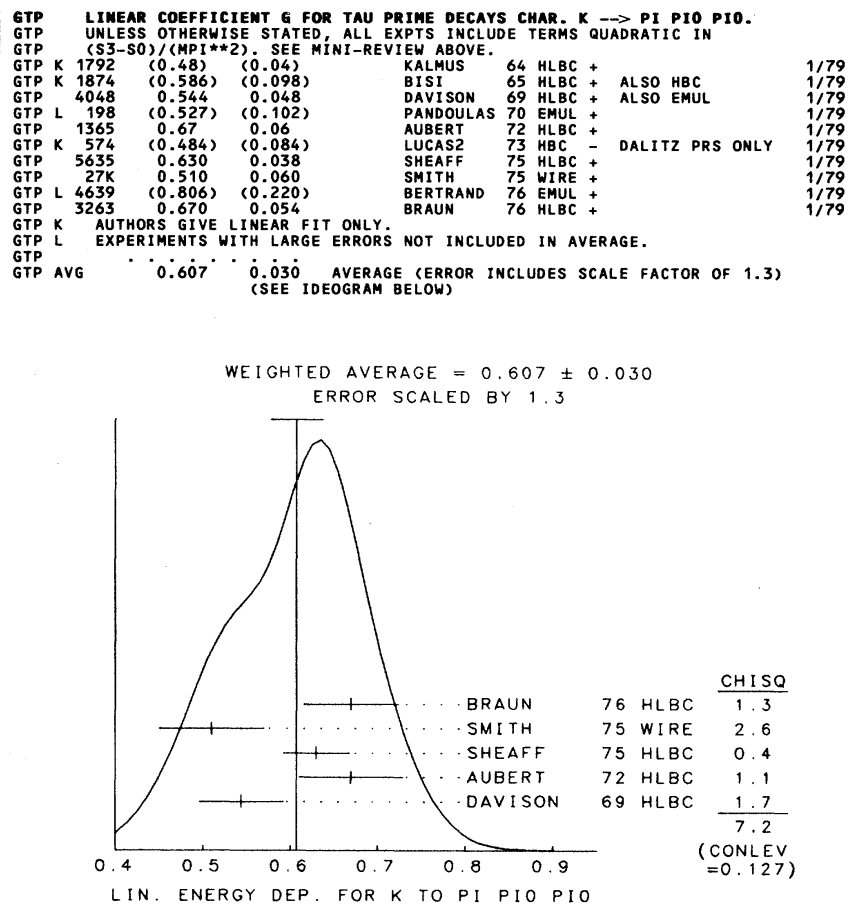

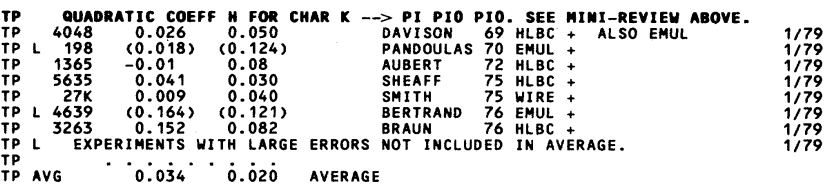

\section{NOTE ON K $K_{\ell 3}^{ \pm}$AND K $\mathrm{K}_{\ell 3}^{0}$ FORM FACTORS}

Assuming that only the vector current contributes to $\mathrm{K} \rightarrow \pi \ell \nu$ decays, we write the matrix element as

$$
\begin{aligned}
& M \propto f_{+}(t)\left[\left(P_{K}+P_{\pi}\right){ }_{\mu} \bar{\ell} \gamma_{\mu}\left(1+\gamma_{5}\right) \nu\right] \\
& +f_{-}(t)\left[m_{\ell} \bar{\ell}\left(1+\gamma_{5}\right) \nu\right],
\end{aligned}
$$

where $P_{K}$ and $P_{\pi}$ are the four-momenta of the $K$ and $\pi$ mesons, $m_{\ell}$ is the lepton mass, and $f_{+}$and $f_{-}$are dimensionless form factors which can depend only on $t$ $=\left(P_{K}-P_{\pi}\right)^{2}$, the square of the four-momentum transfer to the leptons. If time-reversal invariance holds, $f_{+}$and $f_{-}$are relatively real. $K_{\mu 3}$ experiments measure $f_{+}$and $f_{-}$, while $K_{e 3}$ experiments are sensitive only to $f_{+}$ because the small electron mass makes the $f_{-}$term negligible.

(a) $K_{\mu 3}$ experiments. Analyses of $\mathrm{K}_{\mu 3}$ data frequently assume a linear dependence of $f_{+}$and $f_{-}$on $t$, i.e., 


\section{Stable Particles}

$\mathrm{K}^{ \pm}$

$$
f_{ \pm}(t)=f_{ \pm}(0)\left[1+\lambda_{ \pm}\left(t / m_{\pi}^{2}\right)\right]
$$

Most $\mathrm{K}_{\mu 3}$ data are adequately described by Eq. (2) for $f_{+}$and a constant $f_{-}$(i.e., $\lambda_{-}=0$ ). There are two equivalent parametrizations commonly used in these analyses:

(1) $\lambda_{+}, \xi(0)$ parametrization. Analyses of $K_{\mu 3}$ data often introduce the ratio of the two form factors

$$
\xi(t)=f_{-}(t) / f_{+}(t) .
$$

The $\mathrm{K}_{\mu 3}$ decay distribution is then described by the two parameters $\lambda_{+}$and $\xi(0)$ (assuming time reversal invariance and $\lambda_{-}=0$ ). These parameters can be determined by three different methods:

Method A. By studying the Dalitz plot or the pion spectrum of $\mathrm{K}_{\mu 3}$ decay. The Dalitz plot density is (see, e.g., Chounet et $\mathrm{al}^{4}$ ):

$$
\rho\left(\mathrm{E}_{\pi}, \mathrm{E}_{\mu}\right) \propto \mathrm{f}_{+}^{2}(\mathrm{t})\left[\mathrm{A}+\mathrm{B} \xi(\mathrm{t})+\mathrm{C} \xi(\mathrm{t})^{2}\right],
$$

where

$$
\begin{aligned}
& \mathrm{A}=\mathrm{m}_{\mathrm{K}}\left(2 \mathrm{E}_{\mu} \mathrm{E}_{\nu}-\mathrm{m}_{\mathrm{K}} \mathrm{E}_{\pi}^{\prime}\right)+\mathrm{m}_{\mu}^{2}\left(\frac{1}{4} \mathrm{E}_{\pi}^{\prime}-\mathrm{E}_{\nu}\right), \\
& \mathrm{B}=\mathrm{m}_{\mu}^{2}\left(\mathrm{E}_{\nu}-\frac{1}{2} \mathrm{E}_{\pi}^{\prime}\right), \\
& \mathrm{C}=\frac{1}{4} \mathrm{~m}_{\mu}^{2} \mathrm{E}_{\pi}^{\prime}, \\
& \mathrm{E}_{\pi}^{\prime}=\mathrm{E}_{\pi}^{\max }-\mathrm{E}_{\pi}=\left(\mathrm{m}_{\mathrm{K}}^{2}+\mathrm{m}_{\pi}^{2}-\mathrm{m}_{\mu}^{2}\right) / 2 \mathrm{~m}_{\mathrm{K}}-\mathrm{E}_{\pi} .
\end{aligned}
$$

Here $\mathrm{E}_{\pi}, \mathrm{E}_{\mu}$, and $\mathrm{E}_{\nu}$ are, respectively, the pion, muon, and neutrino energies in the kaon center of mass. The density $\rho$ is fit to the data to determine the values of $\lambda_{+}, \xi(0)$, and their correlation.

Method B. By measuring the $\mathrm{K}_{\mu 3} / \mathrm{K}_{\mathrm{e} 3}$ branching ratio and comparing it with the theoretical ratio (see, e.g., Fearing et al. ${ }^{5}$ ) as given in terms of $\lambda_{+}$and $\xi(0)$, assuming $\mu$-e universality:

$$
\begin{aligned}
& \Gamma\left(\mathrm{K}_{\mu 3}^{ \pm}\right) / \Gamma\left(\mathrm{K}_{\mathrm{e} 3}^{ \pm}\right)=0.6457+1.4115 \lambda_{+}+0.1264 \xi(0) \\
& \quad+0.0192 \xi(0)^{2}+0.0080 \lambda_{+} \xi(0), \\
& \Gamma\left(\mathrm{K}_{\mu 3}^{0}\right) / \Gamma\left(\mathrm{K}_{\mathrm{e} 3}^{0}\right)=0.6452+1.3162 \lambda_{+}+0.1264 \xi(0) \\
& \quad+0.0186 \xi(0)^{2}+0.0064 \lambda_{+} \xi(0) .
\end{aligned}
$$

This cannot determine $\lambda_{+}$and $\xi(0)$ simultaneously but

\section{Data Card Listings}

simply fixes a relationship between them.

Method C. By measuring the muon polarization in $\mathrm{K}_{\mu 3}$ decay. In the rest frame of the $\mathrm{K}$, the $\mu$ is expected to be polarized in the direction $\mathbf{A}$ with $\mathbf{P}=\mathbf{A} /|\mathbf{A}|$, where $\mathbf{A}$ is given (Cabibbo and Maksymowicz ${ }^{6}$ ) by

$$
\begin{aligned}
\mathbf{A} & =\mathrm{a}_{1}(\xi) \mathbf{p}_{\mu} \\
& -\mathrm{a}_{2}(\xi)\left[\frac{\mathbf{p}_{\mu}}{\mathrm{m}_{\mu}}\left(\mathrm{m}_{\mathrm{K}}-\mathrm{E}_{\pi}+\frac{\mathbf{p}_{\pi} \cdot \mathbf{p}_{\mu}}{\left|\mathbf{p}_{\mu}\right|^{2}}\left(\mathrm{E}_{\mu}-\mathrm{m}_{\mu}\right)\right]+\mathbf{p}_{\pi}\right] \\
& +\mathrm{m}_{\mathbf{K}} \operatorname{Im} \xi(\mathrm{t})\left(\mathbf{p}_{\pi} \times \mathbf{p}_{\mu}\right) .
\end{aligned}
$$

If time-reversal invariance holds, $\xi$ is real, and thus there is no polarization perpendicular to the K-decay plane. Polarization experiments measure the weighted average of $\xi(t)$ over the $t$ range of the experiment, where the weighting accounts for the variation with $t$ of the sensitivity to $\xi(\mathrm{t})$.

(2) $\lambda_{+}, \lambda_{0}$ parametrization. Most of the more recent $\mathrm{K}_{\mu 3}$ analyses have parametrized in terms of the form factors $f_{+}$and $f_{0}$ which are associated with vector and scalar exchange, respectively, to the lepton pair. $f_{0}$ is related to $f_{+}$and $f_{-}$by

$$
f_{0}(t)=f_{+}(t)+\left[t /\left(m_{K}^{2}-m_{\pi}^{2}\right)\right] f_{-}(t) .
$$

Here $f_{0}(0)$ must equal $f_{+}(0)$ unless $f_{-}(t)$ diverges at $t=$ 0 . The earlier assumption that $f_{+}$is linear in $t$ and $f_{-}$ is constant leads to $f_{0}$ linear in $t$ :

$$
f_{0}(t)=f_{0}(0)\left[1+\lambda_{0}\left(t / m_{\pi}^{2}\right)\right]
$$

With the assumption that $f_{0}(0)=f_{+}(0)$, the two parametrizations, $\left(\lambda_{+}, \xi(0)\right)$ and $\left(\lambda_{+}, \lambda_{0}\right)$ are equivalent as long as correlation information is retained. $\left(\lambda_{+}, \lambda_{0}\right)$ correlations tend to be less strong than $\left(\lambda_{+}, \xi(0)\right)$ correlations.

The experimental results for $\xi(0)$ and its correlation with $\lambda_{+}$are listed in the $K^{ \pm}$and $K_{L}^{0}$ sections of the Stable Particle Data Card Listings in section XIA, XIB, or XIC depending on whether method A, B, or C discussed above was used. The corresponding values of $\lambda_{+}$are listed in subsection $L+M$.

Because recent experiments tend to use the $\left(\lambda_{+}, \lambda_{0}\right)$ parametrization, we include a subsection $L 0$ for $\lambda_{0}$ results. Wherever possible we have converted $\xi(0)$ results into $\lambda_{0}$ results and vice versa. 
See the 1982 version of this note ${ }^{4}$ for additional discussion of the $\mathrm{K}_{\mu 3}^{0}$ parameters, correlations, and conversion between parametrizations, and also for a comparison of the experimental results.

(b) $K_{e 3}$ experiments. Analysis of $\mathrm{K}_{\mathrm{e} 3}$ data is simpler than that of $\mathrm{K}_{\mu 3}$ because the second term of the matrix element assuming a pure vector current [Eq. (1) above] can be neglected. Here $f_{+}$is usually assumed to be linear in $t$, and the linear coefficient $\lambda_{+}$of Eq. (2) is determined.

If we remove the assumption of a pure vector current, then the matrix element for the decay, in addition to the terms in Eq. (2), would contain

$$
\begin{aligned}
+ & 2 \mathrm{~m}_{\mathrm{K}} \mathrm{f}_{\mathrm{S}} \bar{\ell}\left(1+\gamma_{5}\right) \nu \\
& +\left(2 \mathrm{f}_{\mathrm{T}} / \mathrm{m}_{\mathrm{K}}\right)\left(\mathrm{P}_{\mathrm{K}}\right)_{\lambda}\left(\mathrm{P}_{\pi}\right)_{\mu} \bar{\ell} \sigma_{\lambda \mu}\left(1+\gamma_{5}\right) \nu,
\end{aligned}
$$

where $f_{S}$ is the scalar form factor, and $f_{T}$ is the tensor form factor. In the case of the $\mathrm{K}_{\mathrm{e} 3}$ decays where the $\mathrm{f}_{-}$ term can be neglected, experiments have yielded limits on $\left|f_{S} / f_{+}\right|$and $\left|f_{T} / f_{+}\right|$.

The $K_{e 3}$ results for $\lambda_{+},\left|f_{S} / f_{+}\right|$, and $\left|f_{T} / f_{+}\right|$are listed in the subsections $\mathrm{L}+\mathrm{M}, \mathrm{FS}$, and FT, respectively, of the $\mathrm{K}^{ \pm}$and $\mathrm{K}_{\mathrm{L}}^{0}$ sections of the Stable Particle Data Card Listings.

\section{References}

1. L.M. Chounet, J.M. Gaillard, and M.K. Gaillard, Phys. Rep. 4C, 199 (1972).

2. H.W. Fearing, E. Fischbach, and J. Smith, Phys. Rev. D2, 542 (1970).

3. N. Cabibbo and A. Maksymowicz, Phys. Lett. 9, 352 (1964).

4. Particle Data Group, Phys. Lett. 111B, 73 (1982).

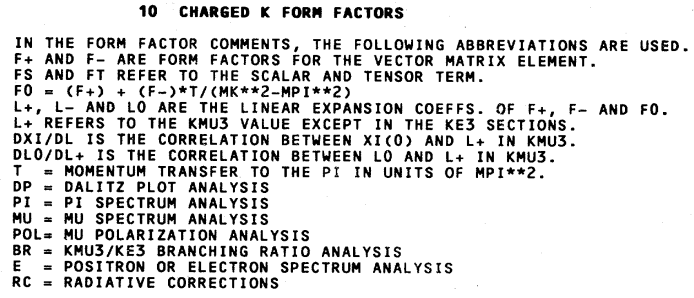

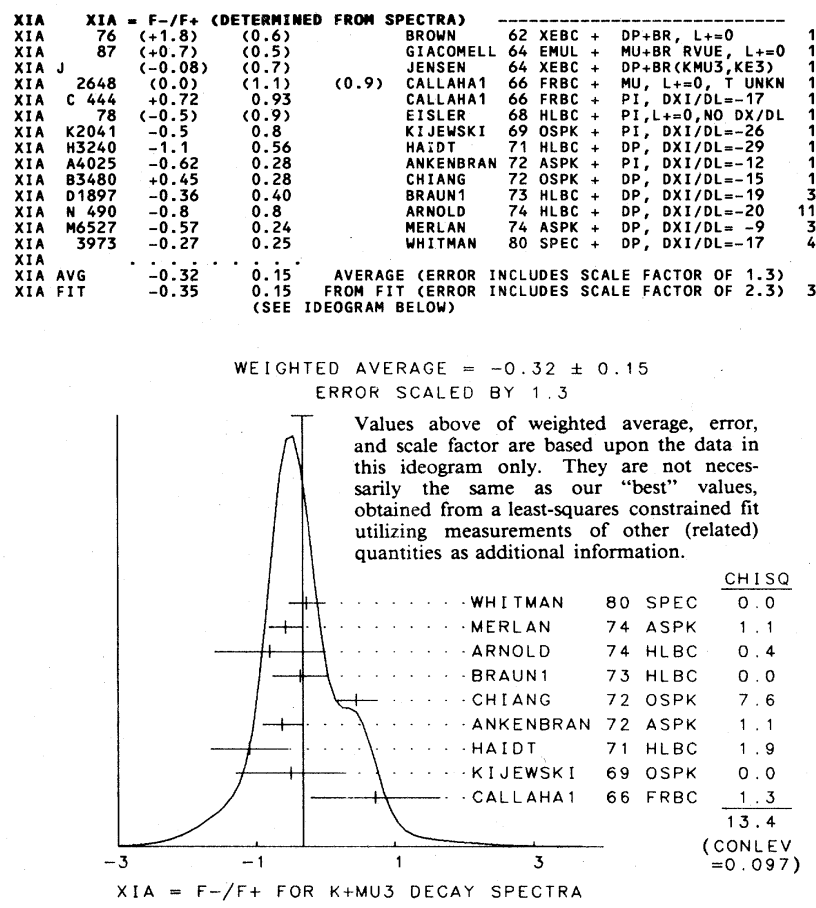

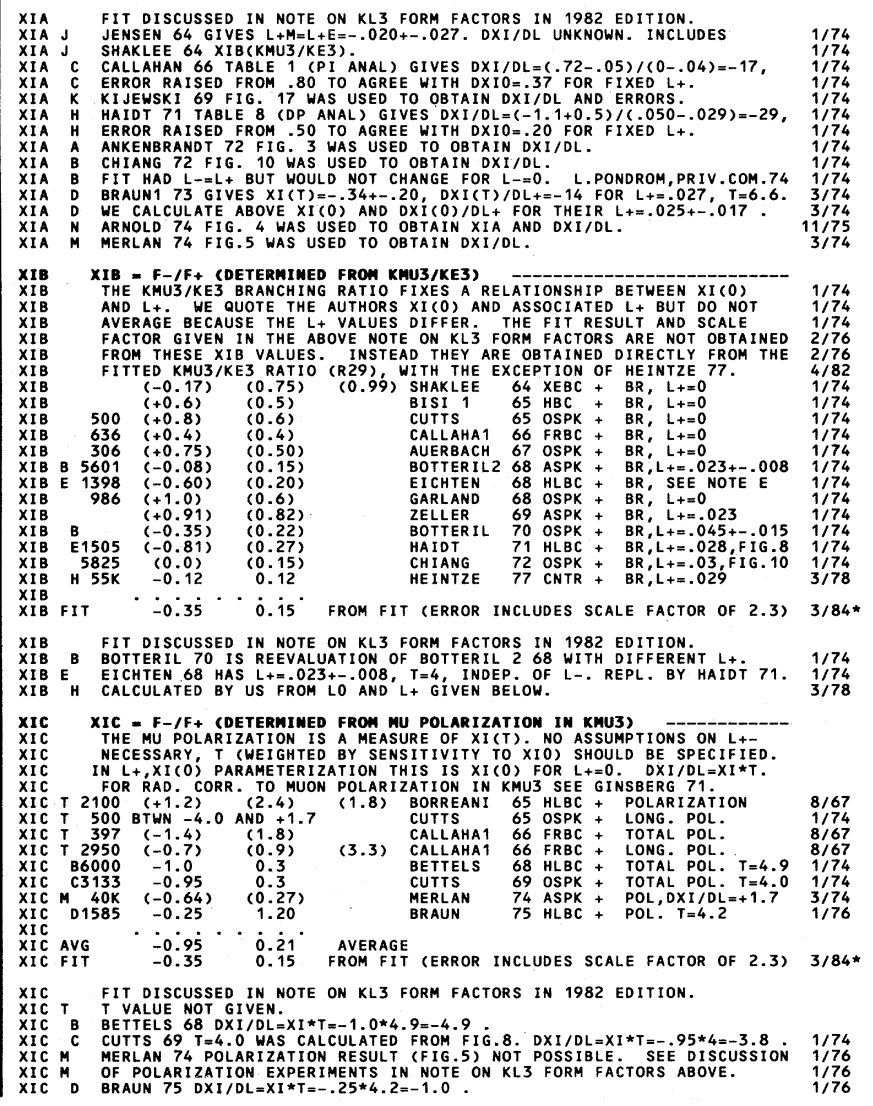




\section{Stable Particles}

\section{Data Card Listings}

$\mathrm{K}^{ \pm}$

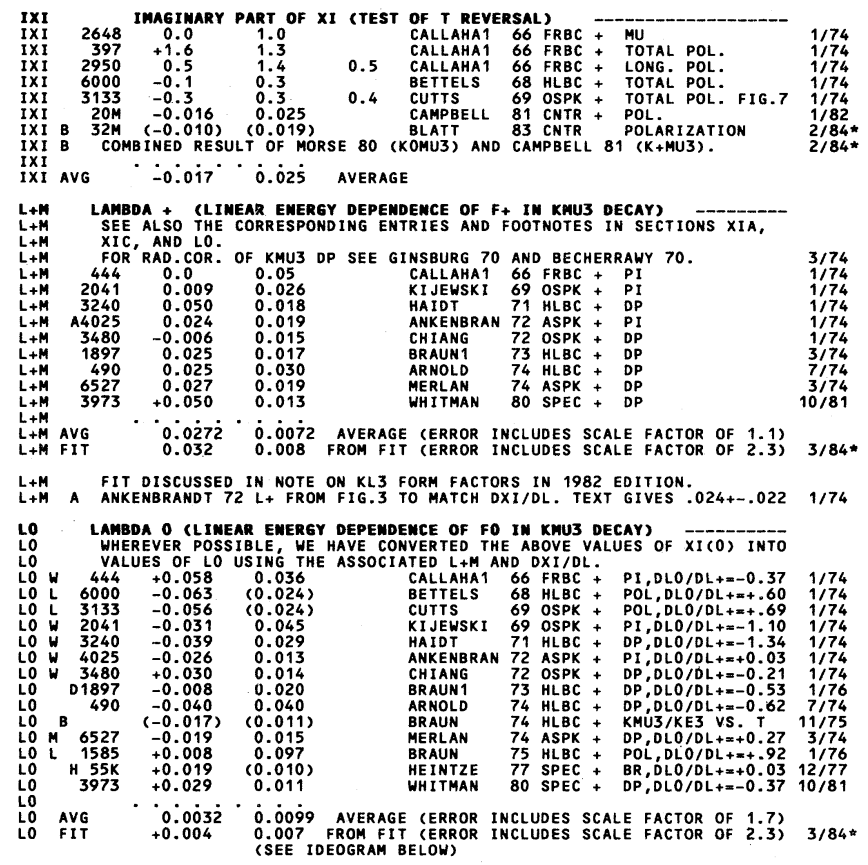

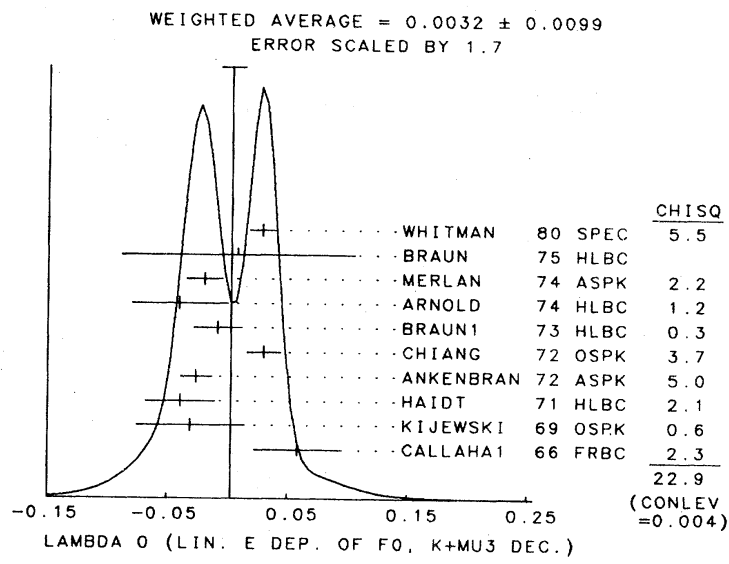

LO FIT DISCUSSED IN NOTE ON KL3 FORM FACTORS IN 1982 EDITION.

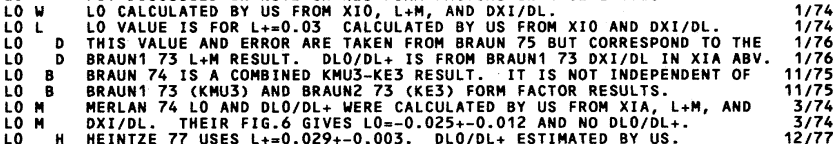

$L+E$
$L+E$
$L+E$

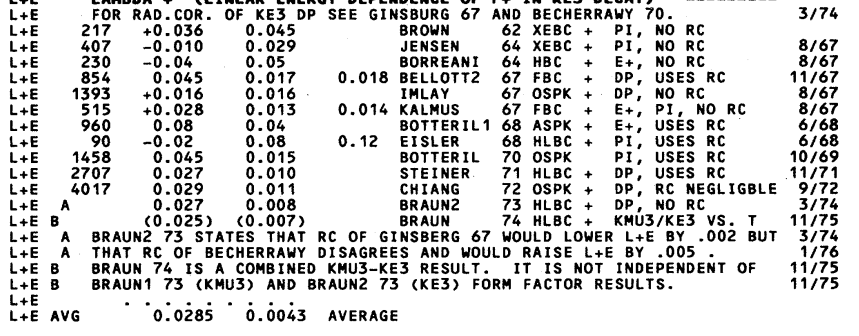

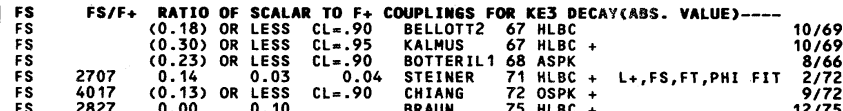

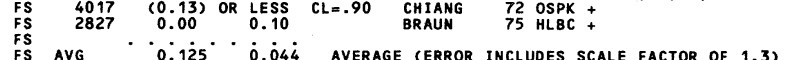

TT FT/F+ RAT10 OF TEMSOR TO F+ COUPLIMGS FOR KES DECAY (ABS. VALUE)---

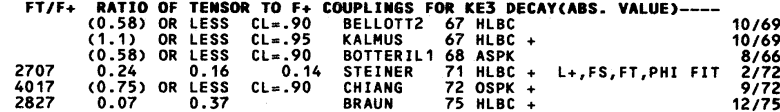

FT avg ${ }^{\text {FT }} 0.22^{\circ} \cdots . \mathrm{i}^{\circ}$ average

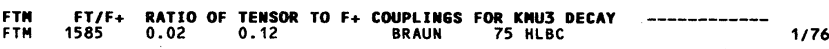

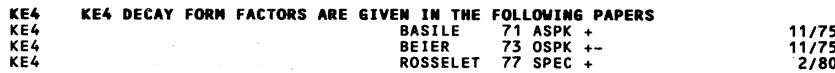

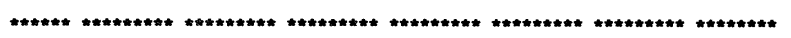

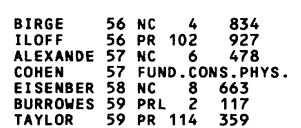

GFEREMCES FOR CHARGED

BIRGE, PERKINS, PETERSON, STORK, UHI TEHEA (LRL)

ALEXANDER, OHNSTON, OCEALALASG (DDBLIN INST)

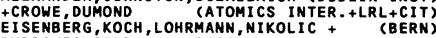

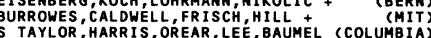

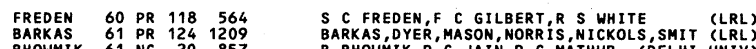

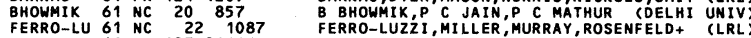

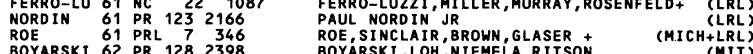

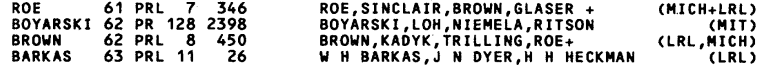

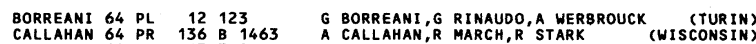

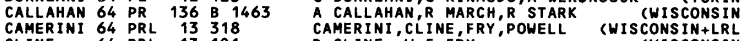

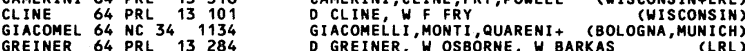

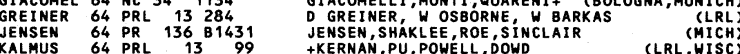

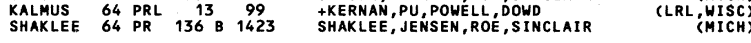

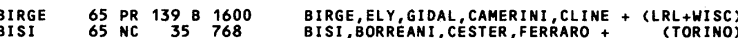

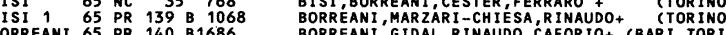

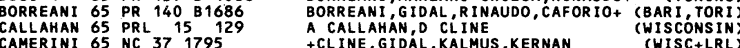

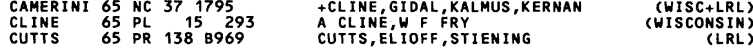

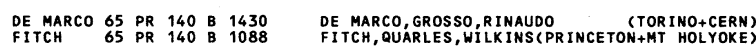

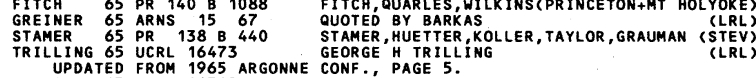

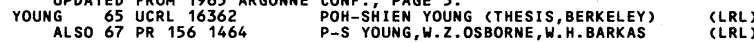

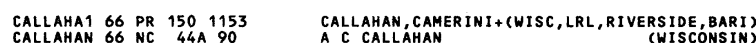

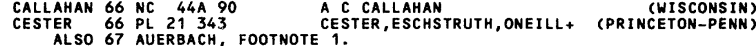

AUERBACH 67 PR 1551505

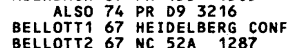

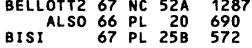

+DOBBS, MANN, MCF ARLANE, WHITE+ (PENN, PRIN)

BOTTERIL 67 PRL 19982

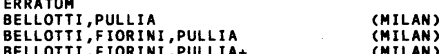

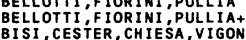

(MILAN)
(TORINO)

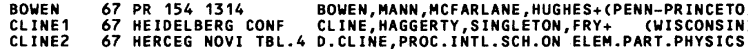

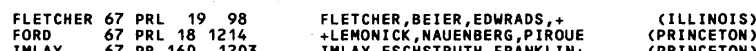

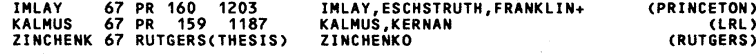

BETTELS 68 NC 56A 1106

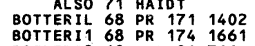

BOTTERI2 68 PRL 21766

AACHEN-BARI-BERGEN-CERN-EP-NI JMEGEN-OR SAY+

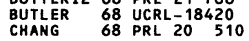

$\begin{array}{lll}\text { CHEN } & 68 & \text { PRL } 2073 \\ \text { EICHTEN } & 68 & 73 \\ \text { EISL } 278 & 586\end{array}$

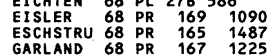

BOTTERILL, BROWN, CLEGG, CORBETT $+\quad$ (OXFORD)
BOTTERILL, BROWN, CLEGG, CORBETT $+\quad$ (OXFORDD) BOTTERILL', BROWN, CLEGG, CORBETT + (OXFORD) TBLAND, GOLOHABER, GOLDHABER, HIRATA+, (LRL)
CHANG, YOOH, EHRLITH, PLANO+(MARYLAND, RUTGERS) CHEN, CUTTS, KIJEWSKI, STIENING ${ }^{+}$(LRL, MIT)
AACHEN-BARI-CERN-EP-ORSAY-PADOVA-VALENCIA EISLER, FUNG, MARATECK, MEYER, PLANO (RUTGERS, ITSIPIS, DEVONS, ROSEN + (COLUMBA, RUTG, WIST)
(UNIV PARIS ORSAY)

CUTTS 69 PR $184 \quad 1380$

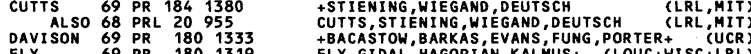

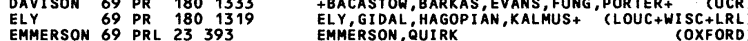

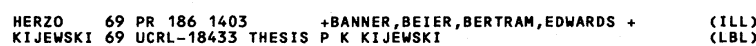

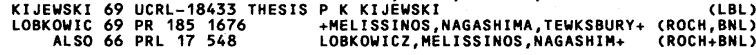




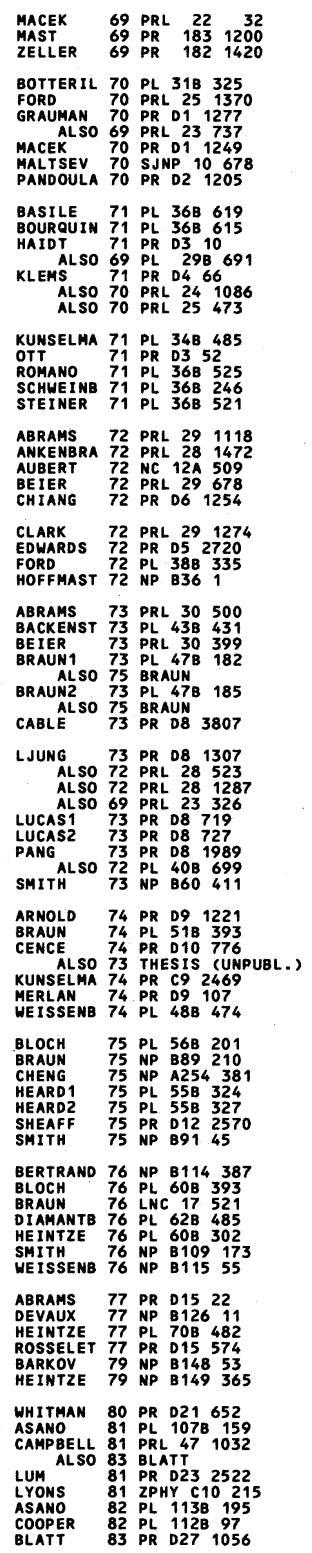

BLOCK 62 LERN CONF 371 BLOCK, LENDIMARA, MONARI (NWES+BOLOGNA)

PAPERS NOT REFERRED tO IN DATA CARDS

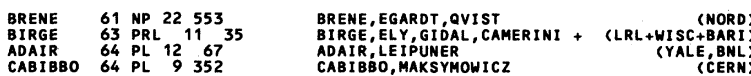

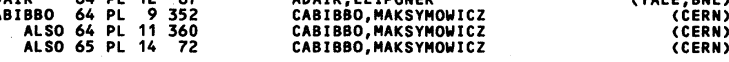

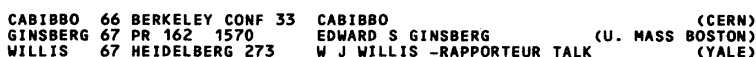

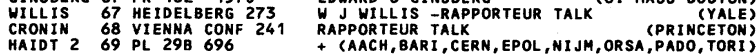

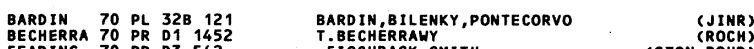

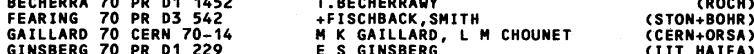

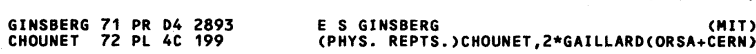

$\mathrm{K}^{0}$

11 MEUTRAL K(498,JP-0-) I=1/2

11 meUtral $K$ MASS (MEV)

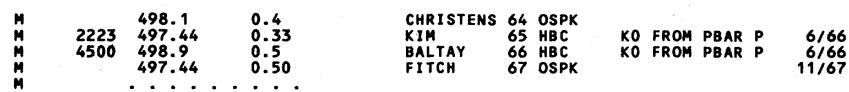

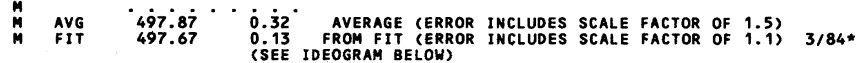

WEIGHTED AVERAGE $=497.87 \pm 0.32$

ERROR SCALED BY 1.5

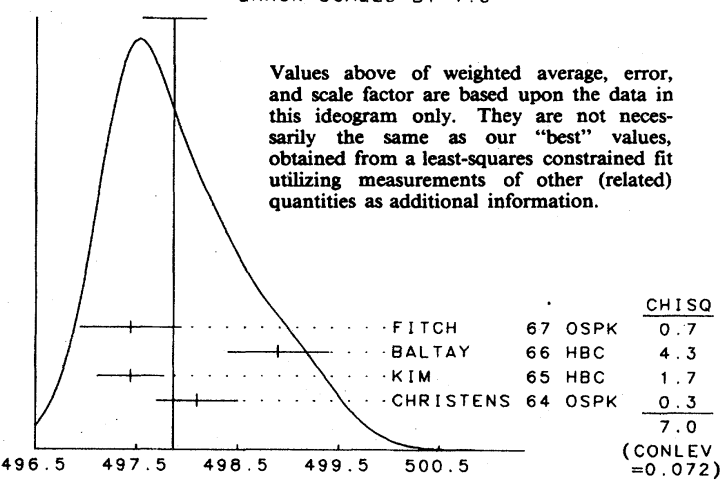

NEUTRAL K MASS (MEV)

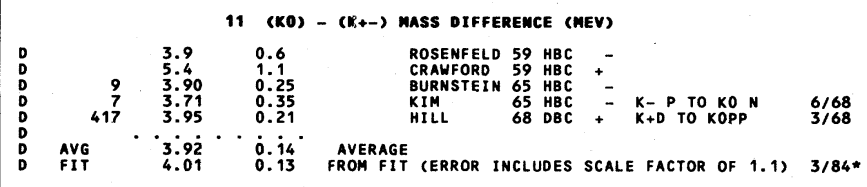

**************************************************************

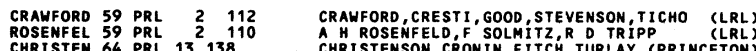

CHRISTEN 64 PRL 13138 138
BURMSTEI 65 PR 138 B 895 CHISTENSON, CRONIN,FITCH, TURLAY (PRINCETON)
(MARYLAND)

BALTAY 66 PR 142932 BALTAY, SANDWEISS, STONEHILL + (YALE+BNL)

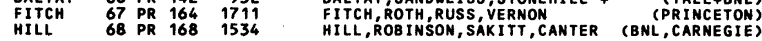

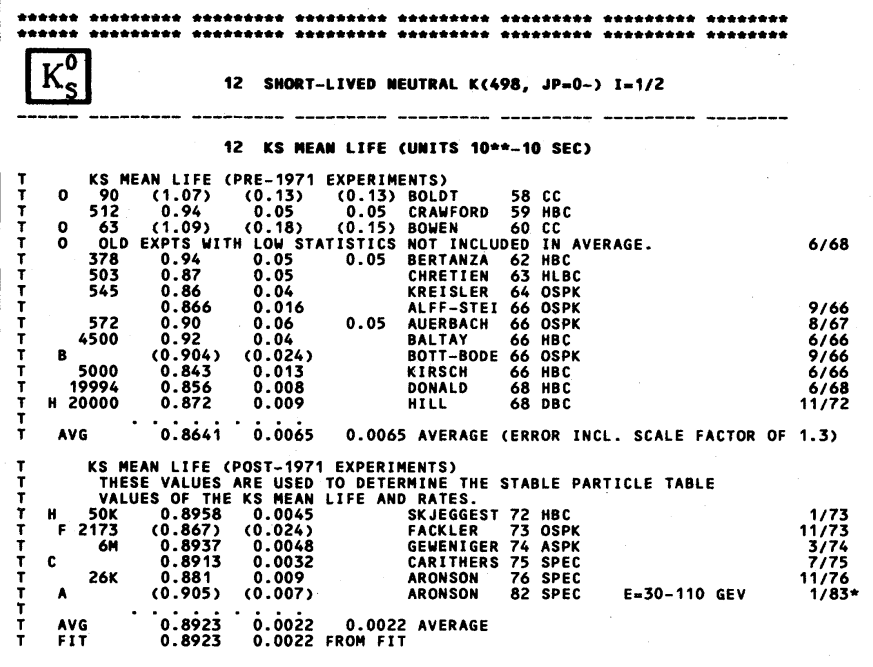




\section{Stable Particles}

Data Card Listings

$\mathrm{K}_{\mathrm{s}}^{\mathrm{o}}$
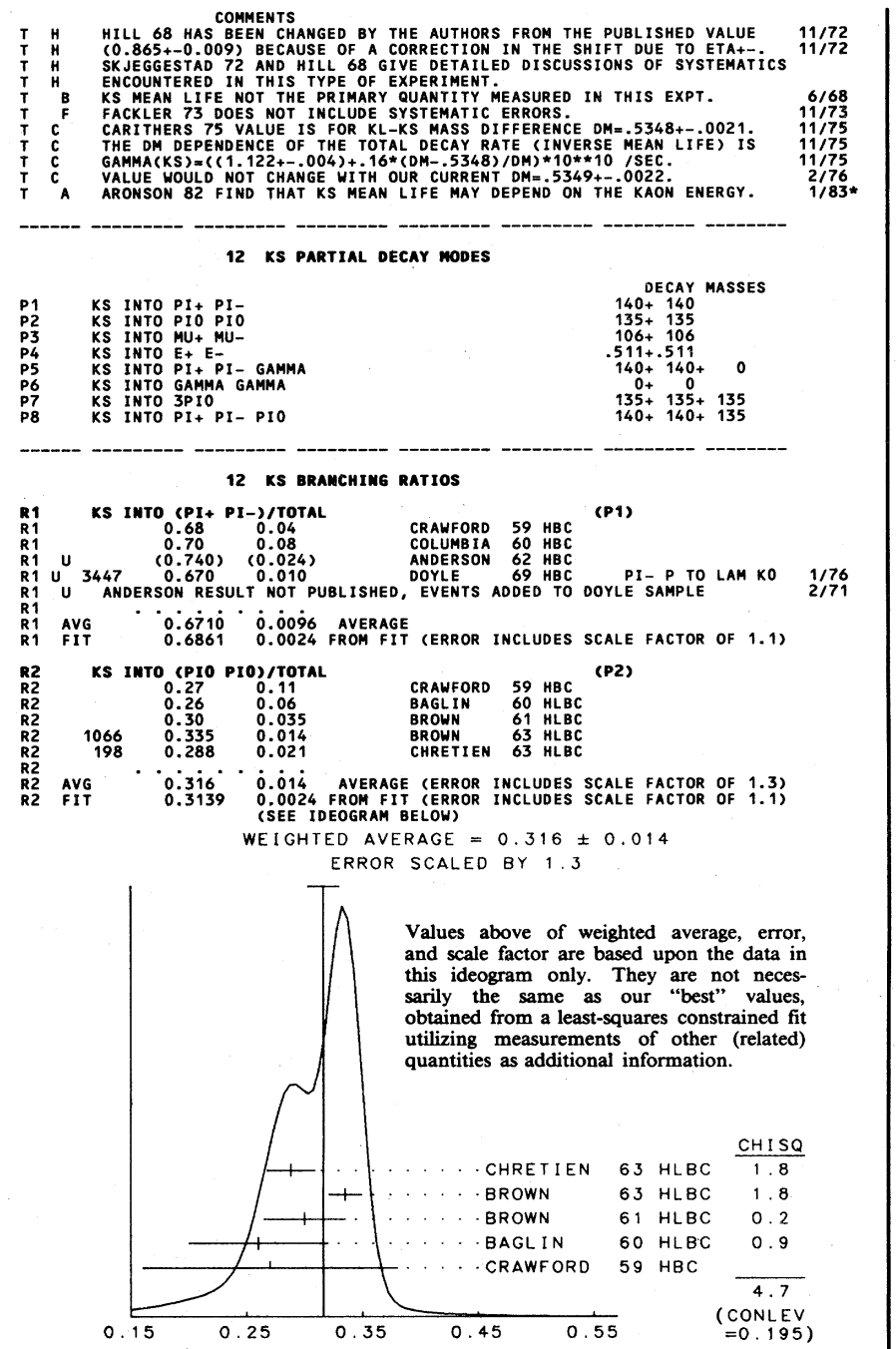

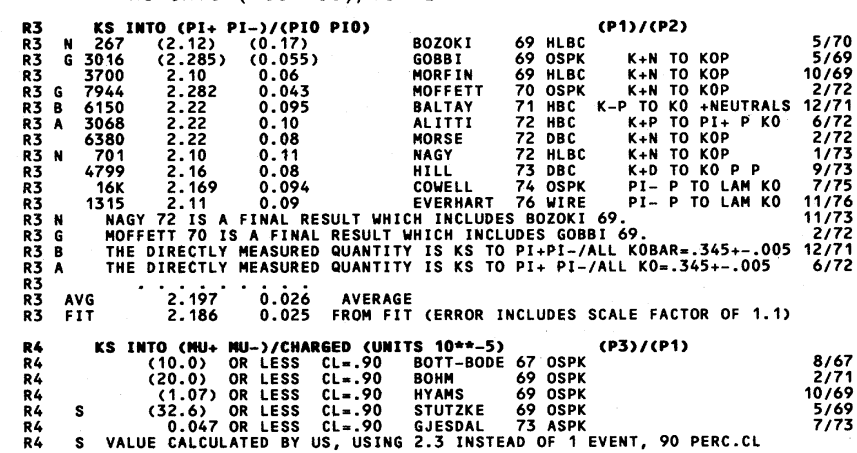

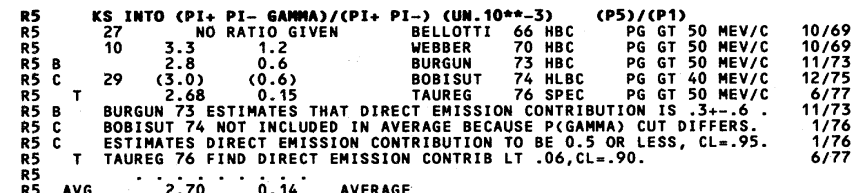

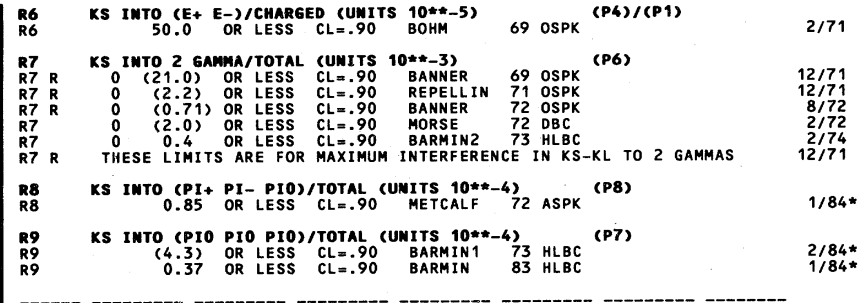

\section{NOTE ON CP VIOLATION IN $\mathrm{K}_{\mathrm{S}}^{0} \rightarrow 3 \pi$}

For $\mathrm{K}_{\mathrm{S}}^{0} \rightarrow 3 \pi$, the quantities which measure $\mathrm{CP}$ violation are the ratios of amplitudes

$$
\begin{aligned}
& \eta_{+-0}=\frac{\mathrm{A}_{\mathbf{S}}\left(\mathrm{K}_{\mathrm{S}} \rightarrow \pi^{+} \pi^{-} \pi^{0}\right)}{\mathrm{A}_{\mathrm{L}}\left(\mathrm{K}_{\left.\mathrm{L} \rightarrow \pi^{+} \pi^{-} \pi^{0}\right)},\right.} \\
& \eta_{000}=\frac{\mathrm{A}_{\mathbf{S}}\left(\mathrm{K}_{\left.\mathrm{S} \rightarrow \pi^{0} \pi^{0} \pi^{0}\right)}\right.}{\mathrm{A}_{\mathrm{L}}\left(\mathrm{K}_{\mathrm{L}} \rightarrow \pi^{0} \pi^{0} \pi^{0}\right)} .
\end{aligned}
$$

If one assumes that CPT invariance holds and that there are no transitions to $I=3$ states, then $\operatorname{Re}\left(\eta_{+-0}\right)$ and $\operatorname{Re}\left(\eta_{000}\right)$ can be neglected, and CP violation would be observed as nonzero values of $\operatorname{Im}\left(\eta_{+-0}\right)$ and $\operatorname{Im}\left(\eta_{000}\right)$. Sections ET+ and ETO list the relative rates

$$
\begin{aligned}
& \left(\operatorname{Im} \eta_{+-0}\right)^{2}=\frac{\Gamma\left(\mathrm{K}_{\mathrm{S}} \rightarrow \pi^{+} \pi^{-} \pi^{0}\right)}{\Gamma\left(\mathrm{K}_{\mathrm{L}} \rightarrow \pi^{+} \pi^{-} \pi^{0}\right)} \\
& \left(\operatorname{Im} \eta_{000}\right)^{2}=\frac{\Gamma\left(\mathrm{K}_{\mathrm{S}} \rightarrow \pi^{0} \pi^{0} \pi^{0}\right)}{\Gamma\left(\mathrm{K}_{\mathrm{L}} \rightarrow \pi^{0} \pi^{0} \pi^{0}\right)}
\end{aligned}
$$

obtained under the above assumptions.

In the above expressions the three pions are restricted to the dominant symmetric $\mathrm{I}=1$ state, a $\mathrm{CP}=-1$ state which couples to $K_{S}$ only if $C P$ is violated. The decay $\mathrm{K}_{\mathrm{S}} \rightarrow \pi^{+} \pi^{-} \pi^{0}$ also has CP-allowed amplitudes to $I=0$ and $I=2$ states of the three pions. The angular momenta in these states cannot be $S$ wave so they are strongly suppressed by centrifugal barrier effects, and, for the $I=2$ state, by the $\Delta I=1 / 2$ rule as well. For comparison with the CP-violating rate, we list in section RSQ the CP-conserving rate relative to $\mathrm{K}_{\mathrm{L}} \rightarrow \pi^{+} \pi^{-} \pi^{0}$ decay. The CP-conserving limit is large and thus determines the branching ratio limit $\Gamma\left(\mathrm{K}_{\mathrm{S}} \rightarrow \pi^{+} \pi^{-} \pi^{0}\right)$ / $\Gamma\left(\mathrm{K}_{\mathrm{S}} \rightarrow \pi^{+} \pi^{-}\right)$given in section $\mathrm{R} 8$. 


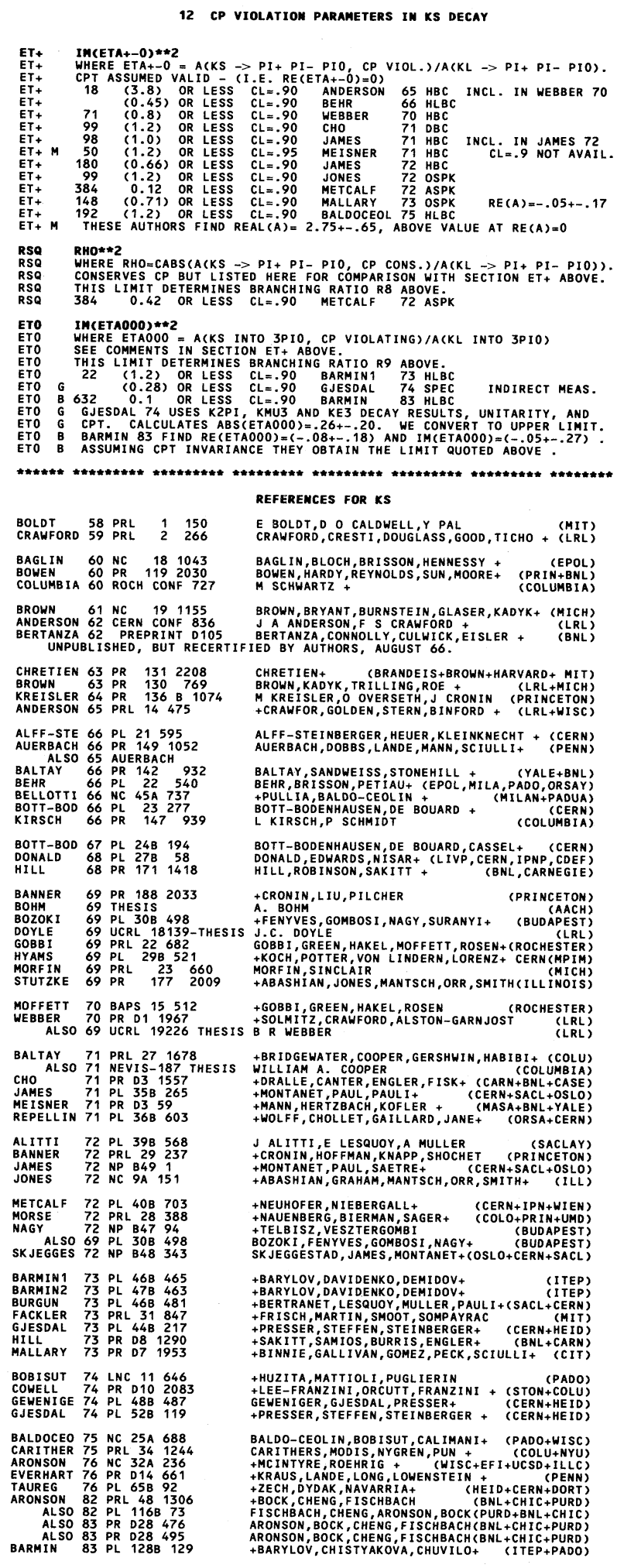

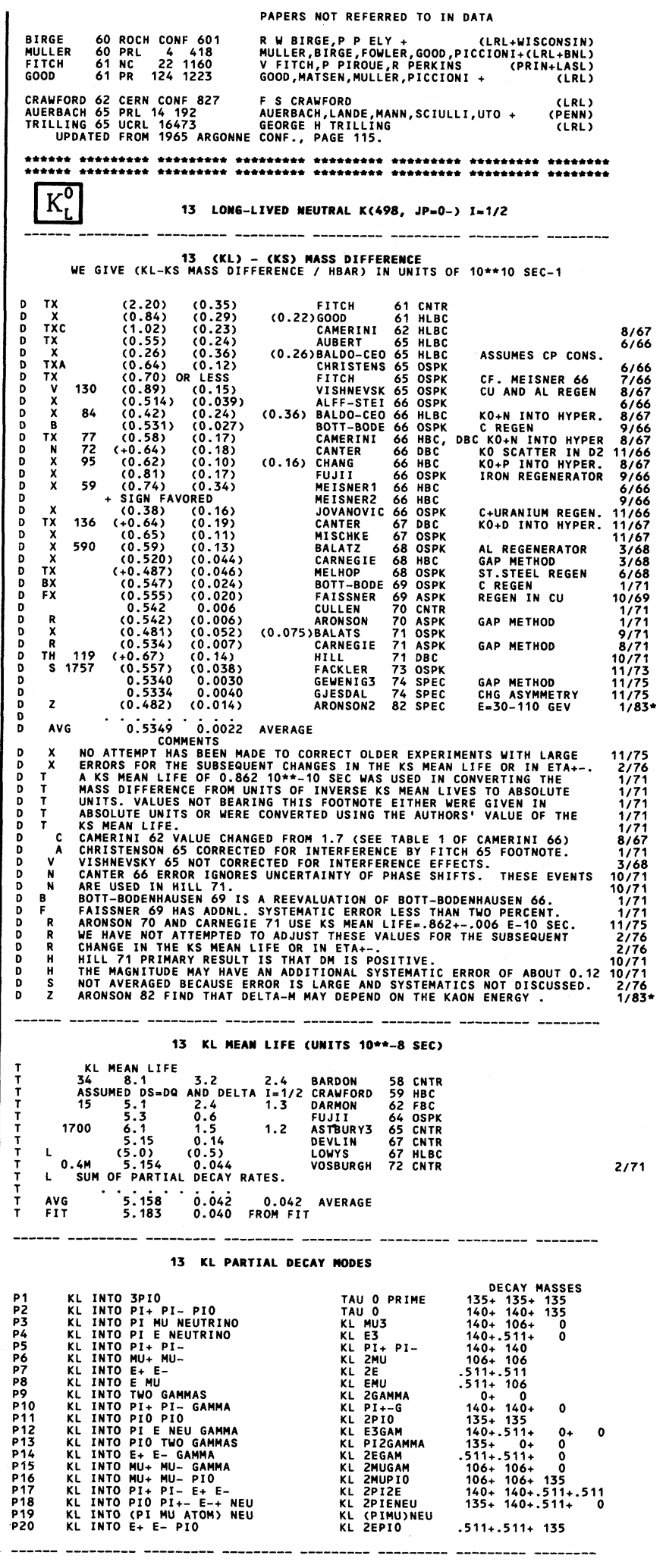


Stable Particles

$\mathrm{K}_{\mathrm{L}}^{0}$

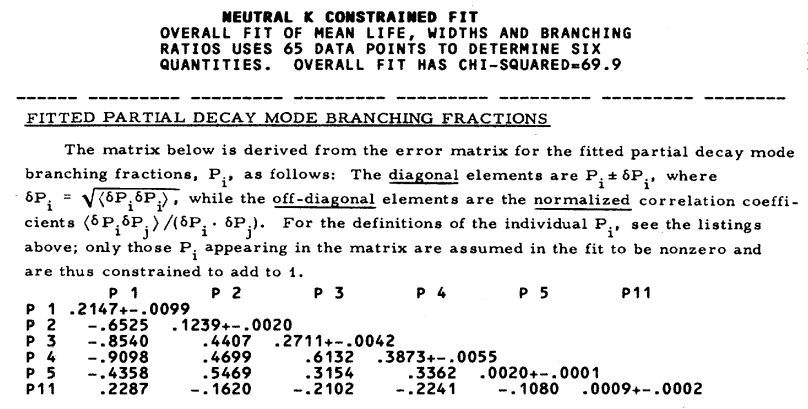

FITTED PARTIAL DECAY MODE RATES

The matrix below is the branching fraction matrix above, transformed into rate space; i.e., $G_{i}=\Gamma_{i}=\Gamma_{\text {total }} P_{i}$, in appropriate units. In analogy to the matrix above, the diagonal elements are $G_{i} \pm \delta G_{i}$, where $\delta G_{i}=\sqrt{\left\langle\delta G_{i} \delta G_{i}\right\rangle}$, while the off-diagonal elements are the normalized correlation coefficients $\left\langle\delta G_{i} \delta G_{j}\right\rangle /\left(\delta G_{i} \cdot \delta G_{j}\right)$. Note that, because of the error in $\Gamma_{\text {total }}$, the errors and correlations here are not directly derivable

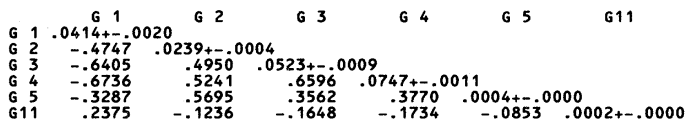

\section{KL decaY Rates}
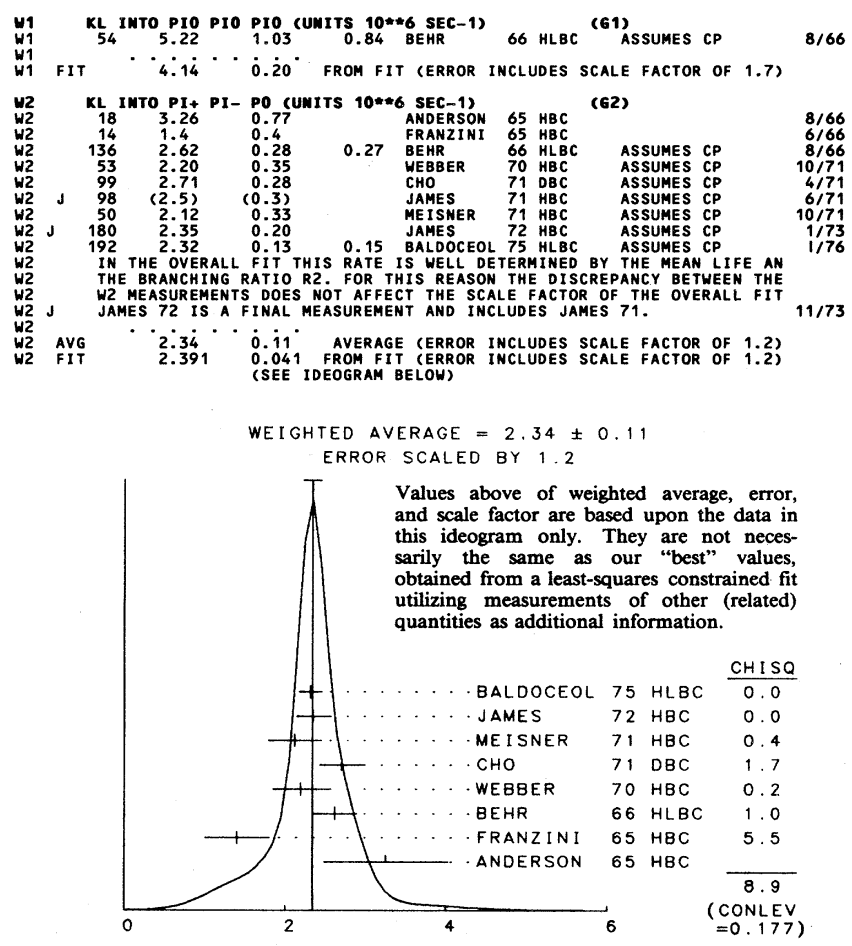

KL RATE INTO PI+ PI- PIO $(10 * * 6$ SEC-1)

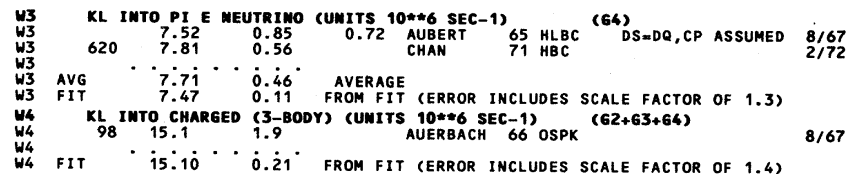

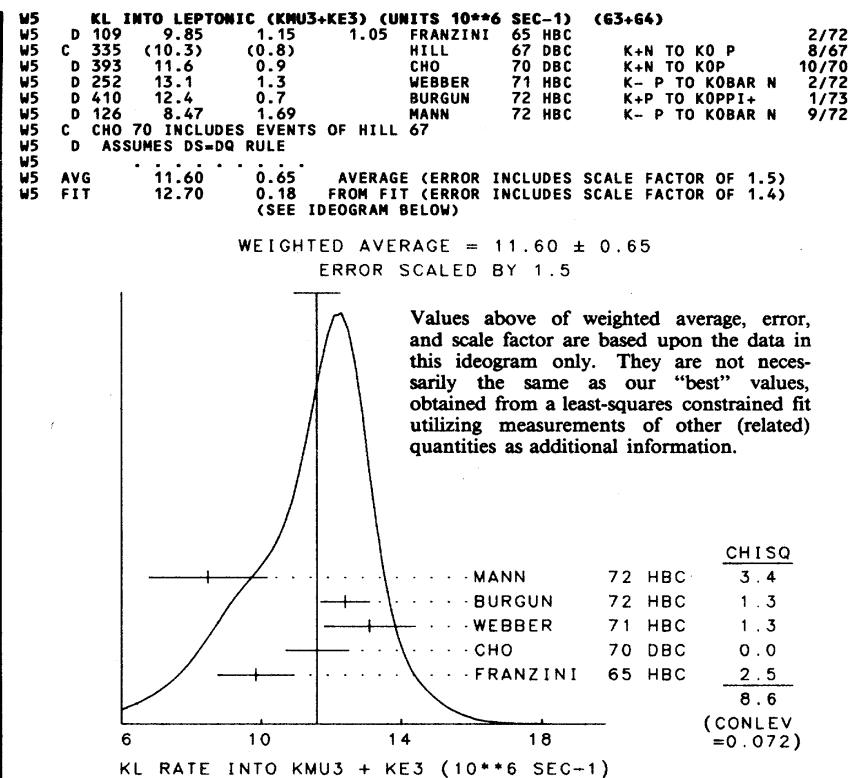

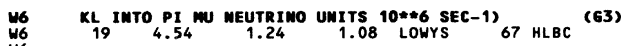

W6 FIT $-5.232^{\circ} \cdot \dot{0} .086^{\circ}$ FROM FIT (ERROR INCLUDES SCALE FACTOR OF 1.3 )

\section{KL BRAMCHIMG RATIOS}

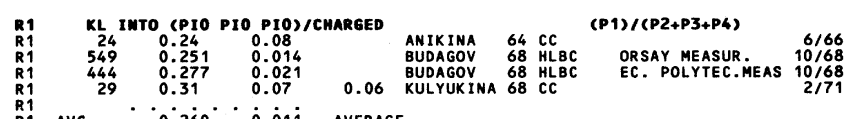

$\begin{array}{lllll}\text { R1 } & \text { AVG } & 0.260^{\circ} & 0.019 & \text { AVERAGE } \\ \text { R1 } & \text { FIT } & 0.274 & 0.016 & \text { FROM FIT (ERROR INCLUDES SCALE FACTOR OF } 1.7 \text { ) }\end{array}$

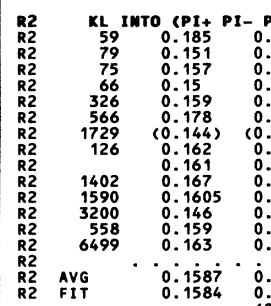

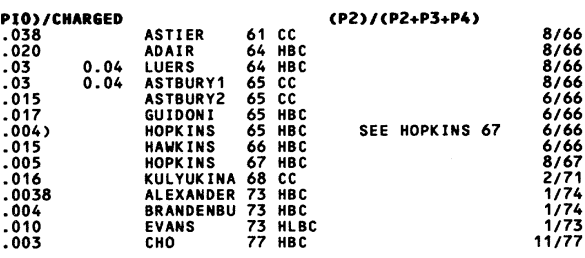

.0024 AVERAGE (ERROR INCLUDES SCALE FACTOR OF 1.3 )
.0020 FROM FIT (ERROR INCLUDES SCALE FACTOR OF 1.2 )

WEIGHTED AVERAGE $=0.1587 \pm 0.0024$ ERROR SCALED BY 1.3

Values above of weighted average, error, this ideogram only. They are not necessarily the same as our "best" values, obtained from a least-squares constrained fit utilizing measurements of other (related)

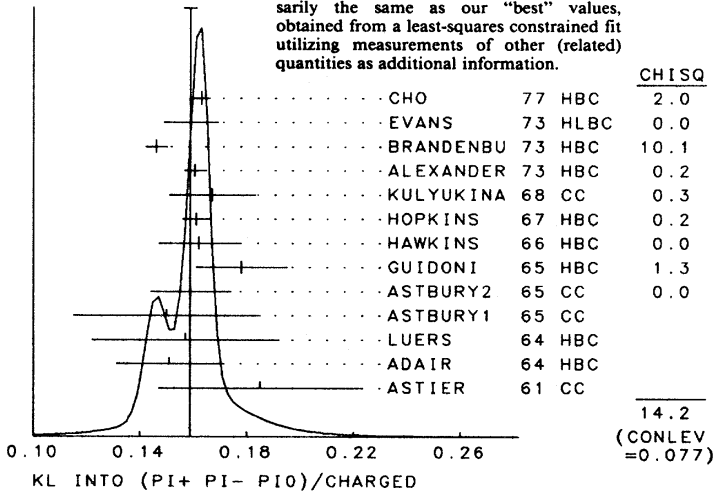

$K L$ INTO (PI+PI-PIO)/CHARGED 


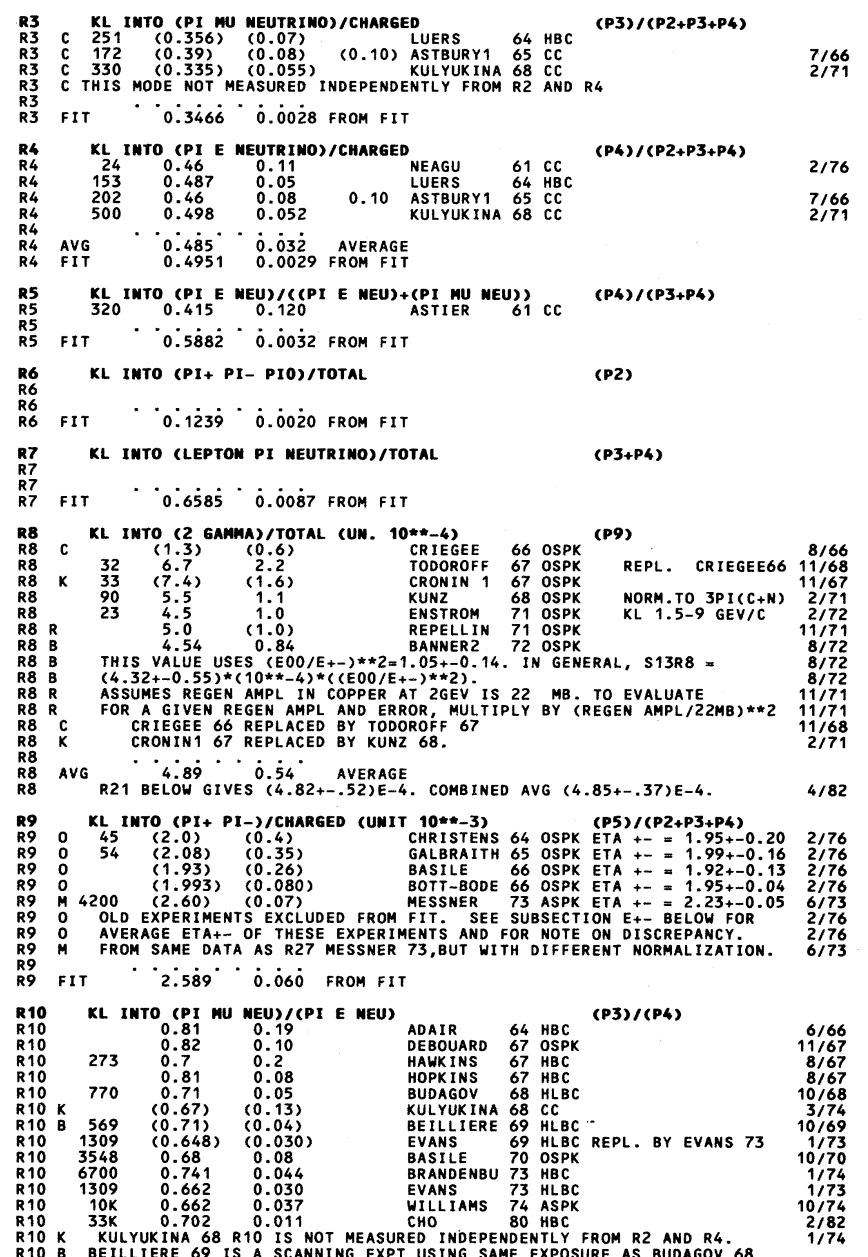

R10 K KULYUK INA 68 R10 IS NOT MEASURE INDEPENDENTLY FROM R2 AND R4.
R10 B BEILIERE 69 IS A SCANING EXPT USING SAME EXPOSURE AS BUDAGOV 68
R10

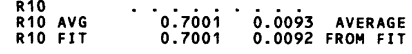

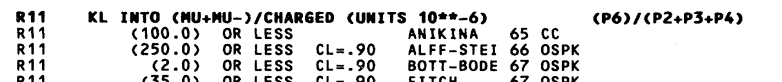

R12 KL INTO (PI+ PI- GAMMA)/TOTAL (UNITS 10**-3) 67 OSPK

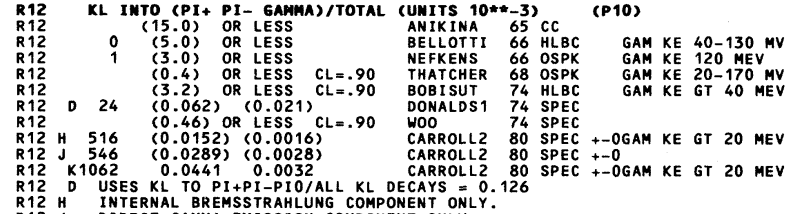

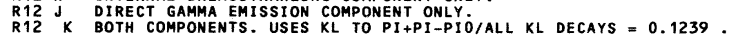

R13 KL INTO (EE E-)/CHARGED (UNITS 10**-6)
R13
$(1000.00)$

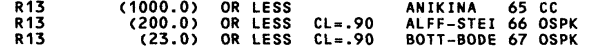

R14 KL INTO (E MU)/CHAREED (UNITS 10**-4)
R14

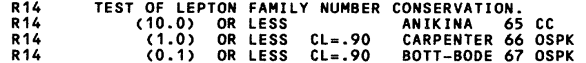

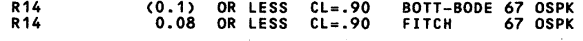

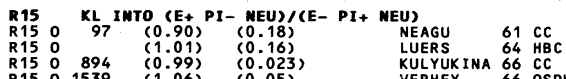

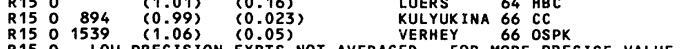

R15 : LOW PRECISION EXPTS NOT AVERAGED. FOR MORE PRECISE VALUE,
R15 O SEE S13A2 IN THE CP VIOLATION SECTION BELOW.

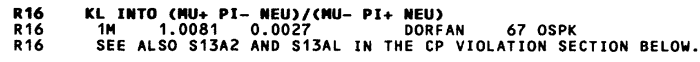

$6 / 66$
$6 / 66$
$8 / 67$

$6 / 66$
$8 / 66$
$8 / 67$
$3 / 68$

$8 / 66$
$9 / 166$
$8 / 67$

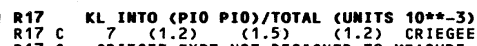

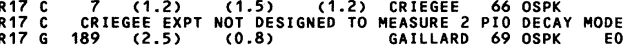

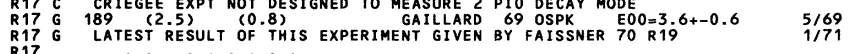

R17 FIT $0.94^{\circ}{ }^{\circ}{ }^{\circ}$. . $^{\circ}{ }^{\circ}$ FROM FIT

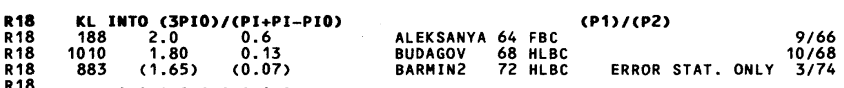

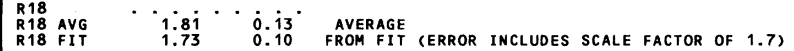

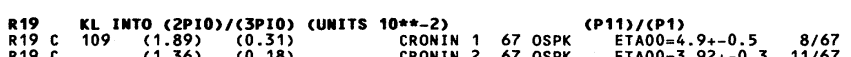

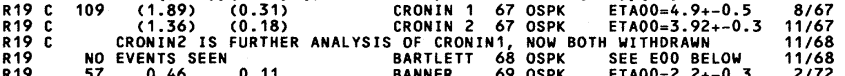

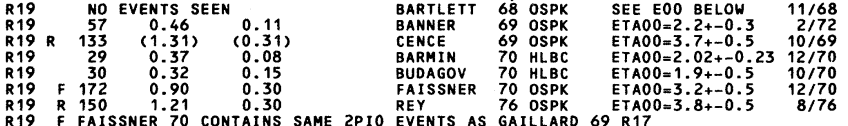

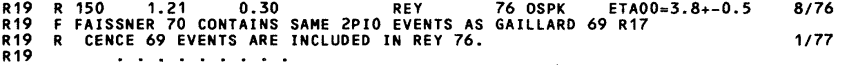

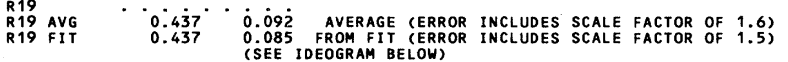

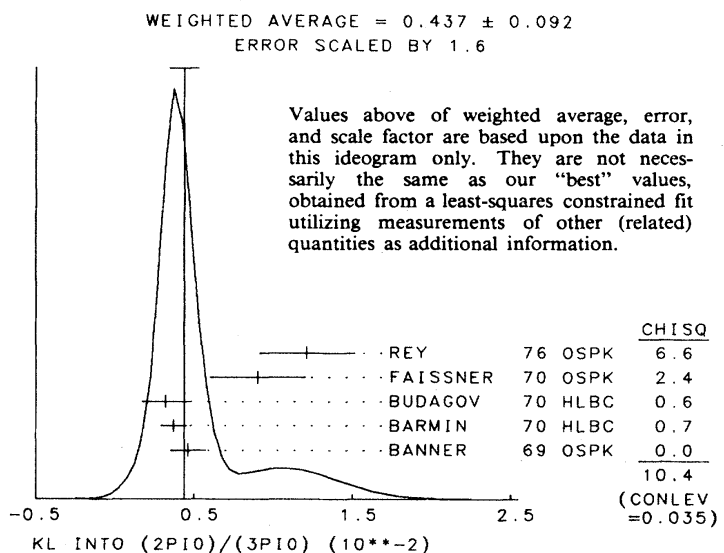

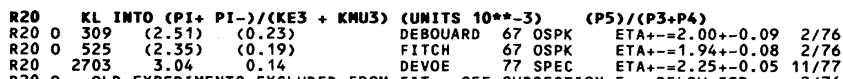

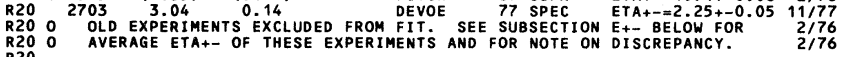

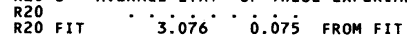

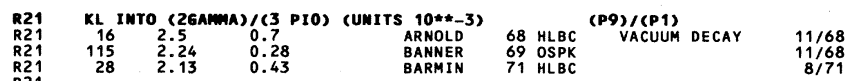
${ }_{R 21}^{R 21}$ AVG $\cdots 2.24^{\circ} \cdots \dot{0.22} 2^{\circ}$ aVerage

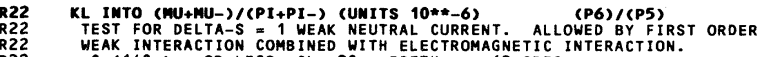

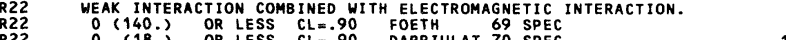

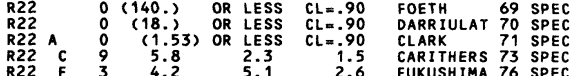

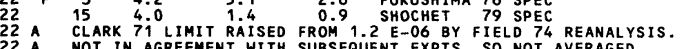

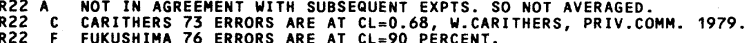

R22 AVG $\cdot 4.47^{\circ} \cdot 0.95^{\circ}$ average

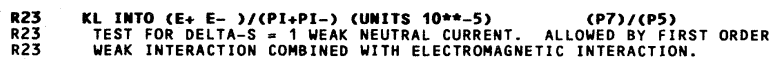

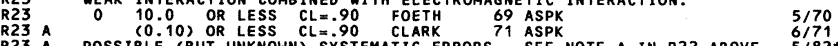

R24 KL IWTO (E MU)/(P1+PI-) (UMITS 10**-5) 71 ASPK (P8)/(P5)

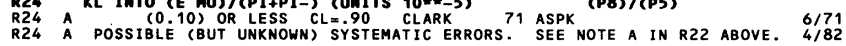

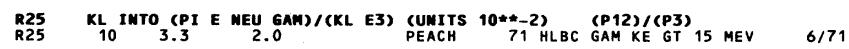

R26 KL INTO (P1O TWO GAMMAS)/(3P10) (UNIYS $10 * *-3)$
R26 (P13)/(P1)

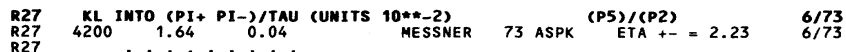




\section{Stable Particles}

$\mathrm{K}_{\mathrm{L}}^{0}$

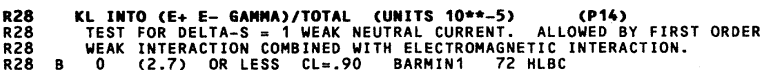

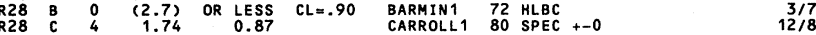

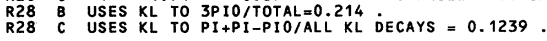

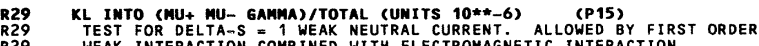

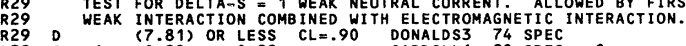

C 1 C.28 CARROLL1 80 SPEC +-0

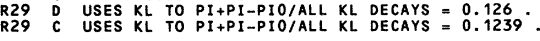

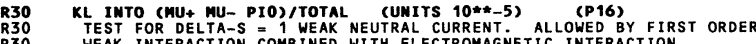
WEAK INTERACT ION COMB INED WITH ELECTROMAGNETIC INTERACTION.

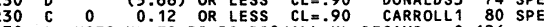

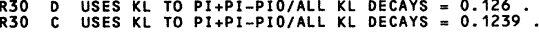

R31 KL INTO (PIIPI-E+E-) /TOTAL CUNITS 10**-6)

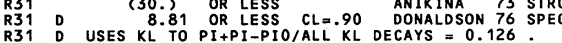

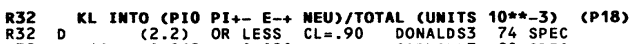

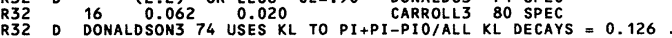

R33 KL INTO (CPI MU ATOM)MEU)/(PI MU MEU) (10*ta-7) (P19)/(P3)

$\begin{array}{llllll}\text { R33 } & 18 & \text { SEEN } \\ \text { R33 } & 155 & 3.88 & 0.41 & \text { COOMBES } & 76 \\ \text { ARONSON } & \text { WIRE } \\ \text { SPEC }\end{array}$

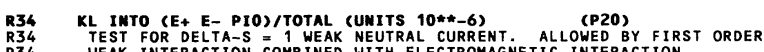

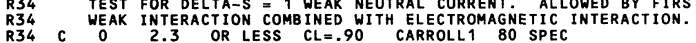
R34 C USES KL TO P1+PI-PIO/ALL KL DECAYS $=0.1239$.

13 KL EMERGY DEPEMDENCE OF DALITZ PLOT FOR DISCUSSION, SEE NOTE ON SLOPE PARAMETERS IN THE CHARGED K MATRIX ELEMENT SQUARED
WHERE $U=(S 3-S 0) /(M P I * * 2)$
AND

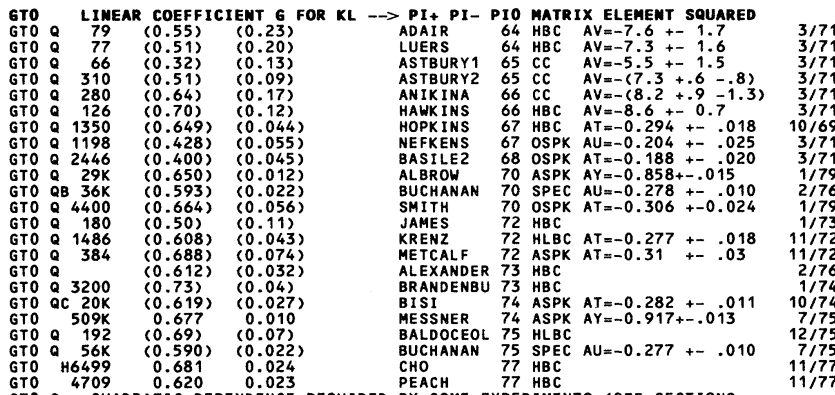

GTO O OUADRATC DEPENDENCE REQUIRED BY SOME EXPERIMENTS (SEE SECTIONS
GTO O HTO AND KTO BELOW. CORRELATIONS PREVENT US FROM AVERAGING RESULTS GTO B OF FITS NOO INCLUDING G, H, AND K TERMS S TO INCLUDE RADIATIVE COR. GTO B AND TO USE MORE RELIABLE KL MOM. SPECT OF 2 ND EXPT. SHAD SAME BEAM): 2776

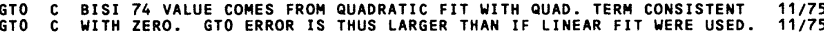
GTO AVG $0.670^{\circ} \cdots 0_{0.014}$ AVERAGE (ERROR INCLUDES SCALE FACTOR OF 1.6 )

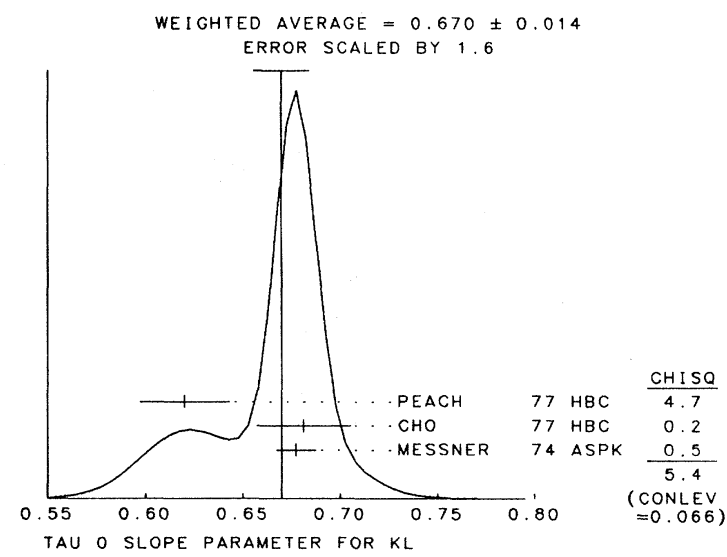

$6 / 77$
12780
61777
$12 / 80$
$12 / 75$
3378
6777
$6 / 77$
$12 / 75$
6777
9187
6177
$11 / 828$
$61 / 77$
$11 / 82$

$12 / 8$
$12 / 8$
Data Card Listings

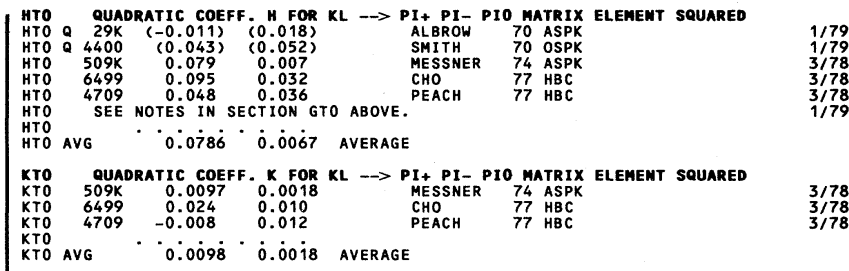

JTO LIMEAR COEFF. J FOR KL
JTO $\rightarrow$ PPI PI-PIO CP VIOLATIMG TERM
LISTED IN CP VIOLATION SECTION BELOW.

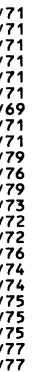

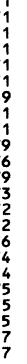

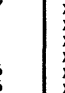

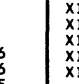

13 kL fork factors

FOR DISCUSSION, SEE NOTE ON FORM FACTORS IN THE CHARGED K
SECTION OF THE DATA CARD LISTINGS ABOVE.

IN THE FORM FACTOR COMMENTS, THE FOLLOWING ABBREVIATIONS ARE USED.
F+ AND F- ARE FORM FACOORS FOR THE VECTOR MATRE IILEMENT.

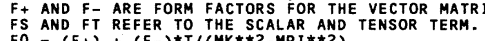

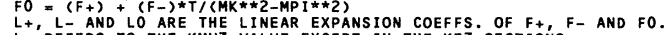

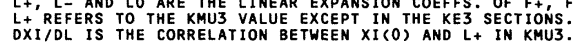

DLO/DL+ IS THE CORRELATION BETWEEN LO AND LL IN KMU3.

DP = MOMENTUM TRANS ANAY YSIS
PI $=$ PAI SPECTRUM ANALYSIS

MU = MU SPECTRUM ANALYSIS

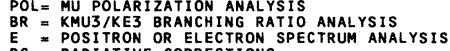

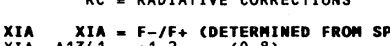

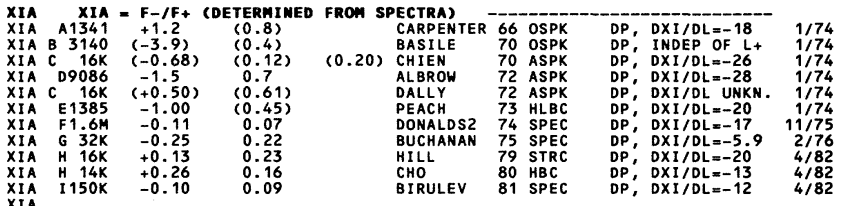

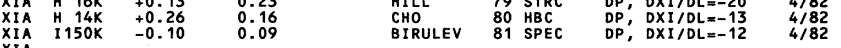

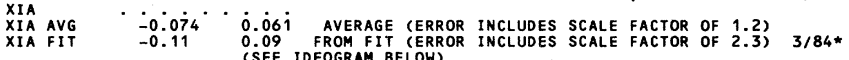

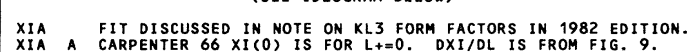

XIA B BASILE 70 IS INCOMPATIBLE WITH ALL OTHER RESULTS. AUTHORS SUGGEST 1174

XIA B TAA EFFICENCY ESTIMATES MIGHT BE RESPONSIBLE.

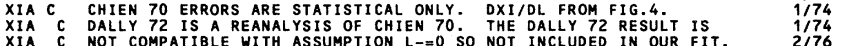

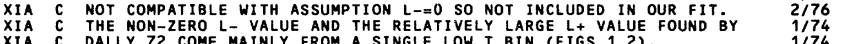

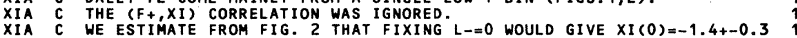

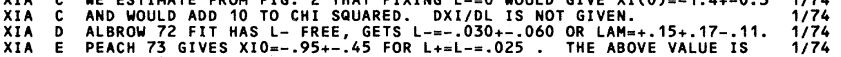

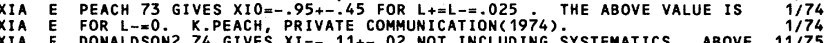

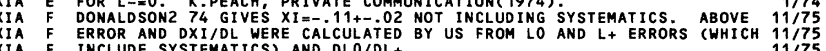

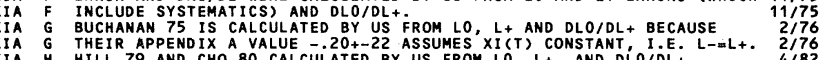

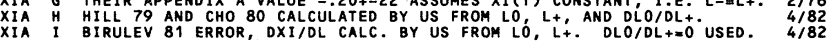

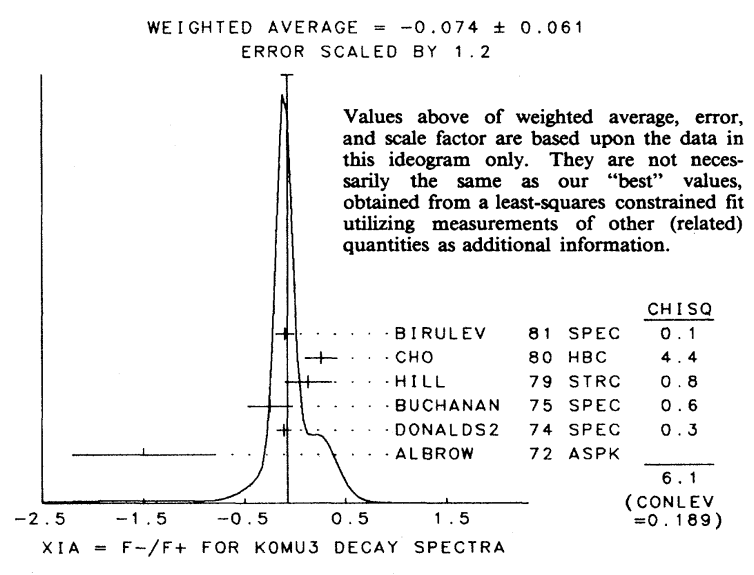




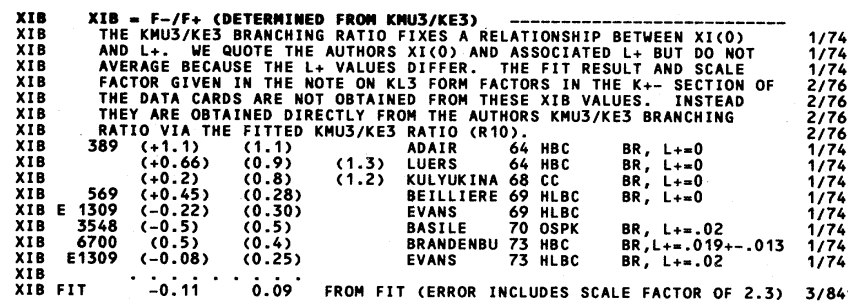

XIB
XIB E EVANS 73 REPLACES EVANS 69.

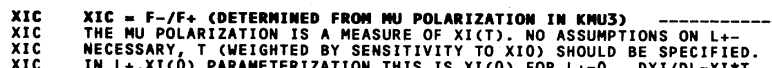
$X$ NIC
$X I C$ INESSARY, ${ }^{\top}$ (WEIGHTED BY SENSITIVITY TO XIO) SHOLD BE SPECIFIED
$X$ IC XIC FOR RAD. CORR. TO MUON POLARIZAAION IN KMU3, SEE GINSBERG T3.
XIC T $2608(-1.2) \quad(0.5)$
$X 1 C$ AUERBACH 66 OSPK POLRIZATION

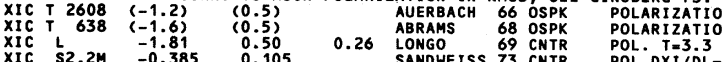
$\begin{array}{lllllllll} & & & & \\ X & & & & \\ X\end{array}$

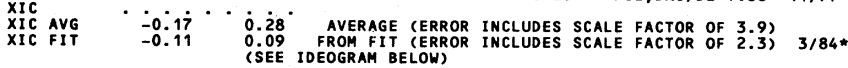

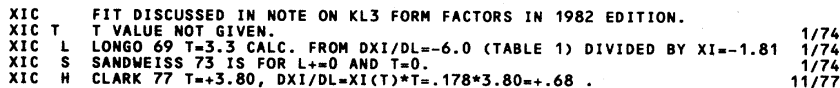
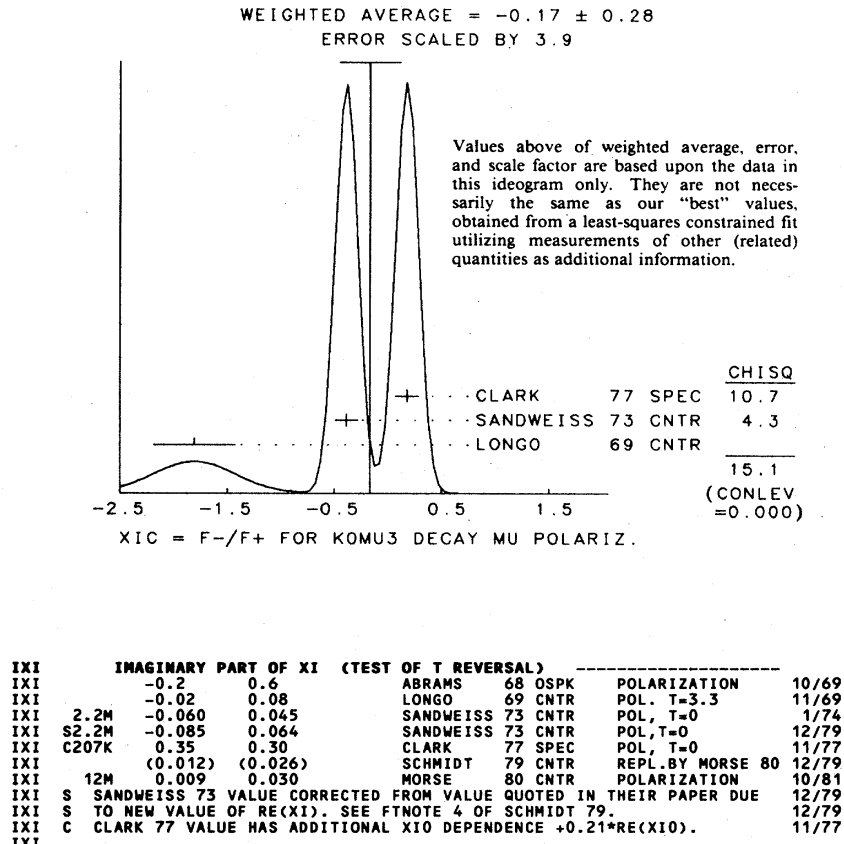

IXI AVG $-0.020^{\circ} \cdot 0.022^{\circ}$ aVERAGE

L+M LAMBDA + (LIMEAR ENERGY DEPEMDEMCE OF F+ IM KMU3 DECAY) $L+M$
$L+M E$ FOR RADO THE CORRESPOND ING ENTRIES AND NOTES IN SECTION XIA AND $1+M$ (6K 10.07$)(0.02)$

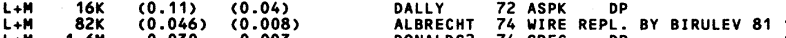
$\begin{array}{cccc} & \\ L+M & 1.6 \mathrm{M} & 0.030 & 0.003 \\ L+M & 32 \mathrm{~K} & 0.046 & 0.030 \\ L+M & 129 \mathrm{~K} & (0.0337) & (0.0033) \\ L+M & 16 \mathrm{~K} & 0.028 & 0.011\end{array}$ $\begin{array}{cccc}L+M & 16 K & 0.028 & 0.011 \\ L+M & 14 K & 0.028 & 0.010 \\ L+M & 150 K & 0.0427 & 0.0044\end{array}$

- $0.0347^{\circ} 0.0049$ AVERAGE (ERROR INCLUDES SCALE FACTOR OF 2.1 ) (SEE IDEOGRAM BELOH)

L+M FIT DISCUSSED IN NOTE ON KL3 FORM FACTORS IN 1982 EDITION.

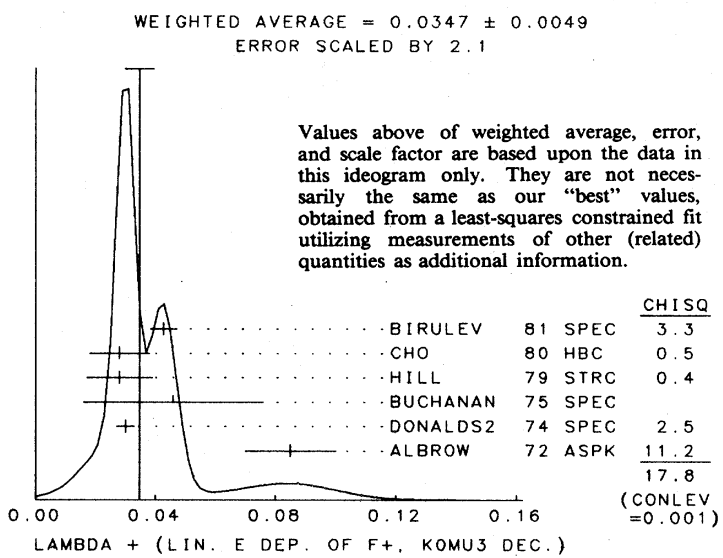

LO LAMBDA O (LIMEAR EMERGY DEPEMOEMCE OF FO IM KMUZ DECAY)

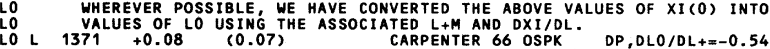

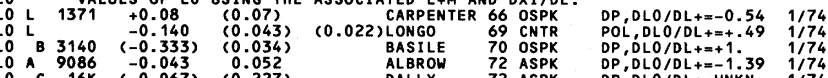

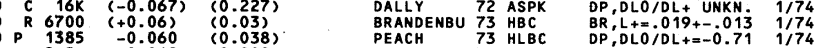

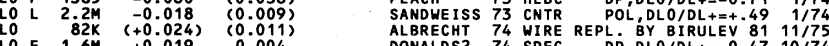

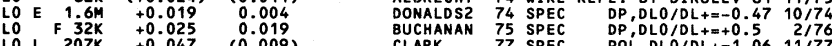

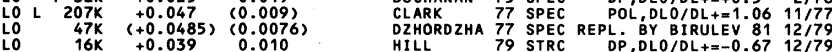

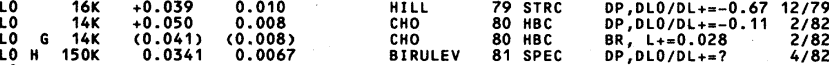
LO AVG $0.0279 \cdot 0.0057$ AVERAGE (ERROR INCLUDES SCALE FACTOR OF 1.9 ) LO FIT 0.0250 .006 FROM FIT (ERROR INCLUDES SCALE FACTOR OF 2.3 ) 3/84*

LO FIT DISCUSSED IN NOTE ON KL3 FORM FACTORS IN 1982 EDITION.

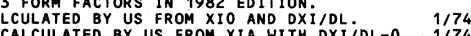
$\begin{array}{lll}10 & B \\ \text { BASILE } 70 \text { IS INCOMPATIBLE WITH ALL OTHER RESULTS. AUTHORS SUGGEST } & 1 / 74\end{array}$ LO A ALBROW 72 LO IS CALCULATED BY US FROM XIA,L+ AND DXI/DL. THEY GIVE $1 / 74$

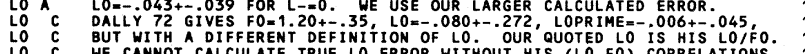
LO C SEE ALSO NOTE C IN SECTION XIA. LO PEACH 73 ASSUMES $L+=0.025$. CALCULATED BY US FROM XIO AND DXIO/DL+

LO R KMU3/KE3 RESULT FROM THIS EXPERIMENT. ${ }^{R}$ TO 18.

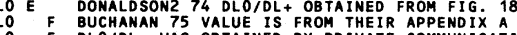
F
LO DLO/DL+ HAS OBTAINED BY PRIVATE COMMUNICATION, C. BUCHANAN, 1976. H BIRULEV 81 GIVES DLO/DL+=-1.5, GIVING AN UNREASONABLY NARROW ERROR
H ELLIPSE WHICH DOMINATES ALL OTHER RESULTS. WE USE DLO $/ 0 L+=0$.

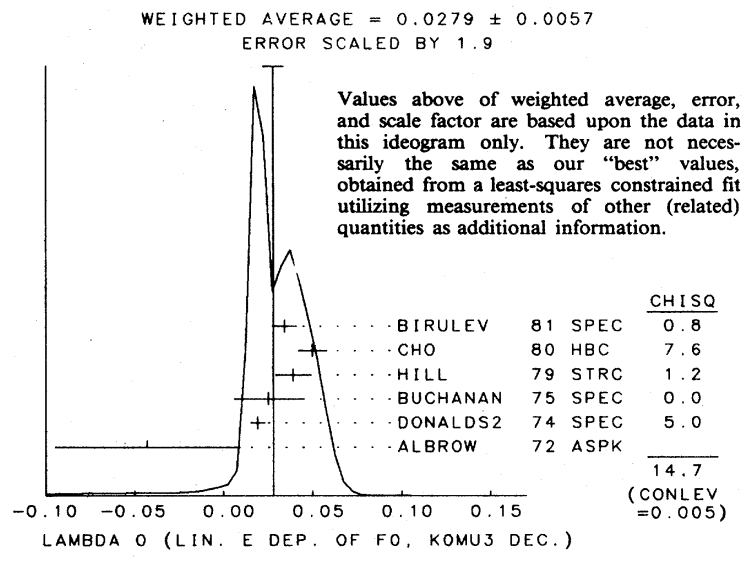




\section{Stable Particles}

$K_{L}^{0}$
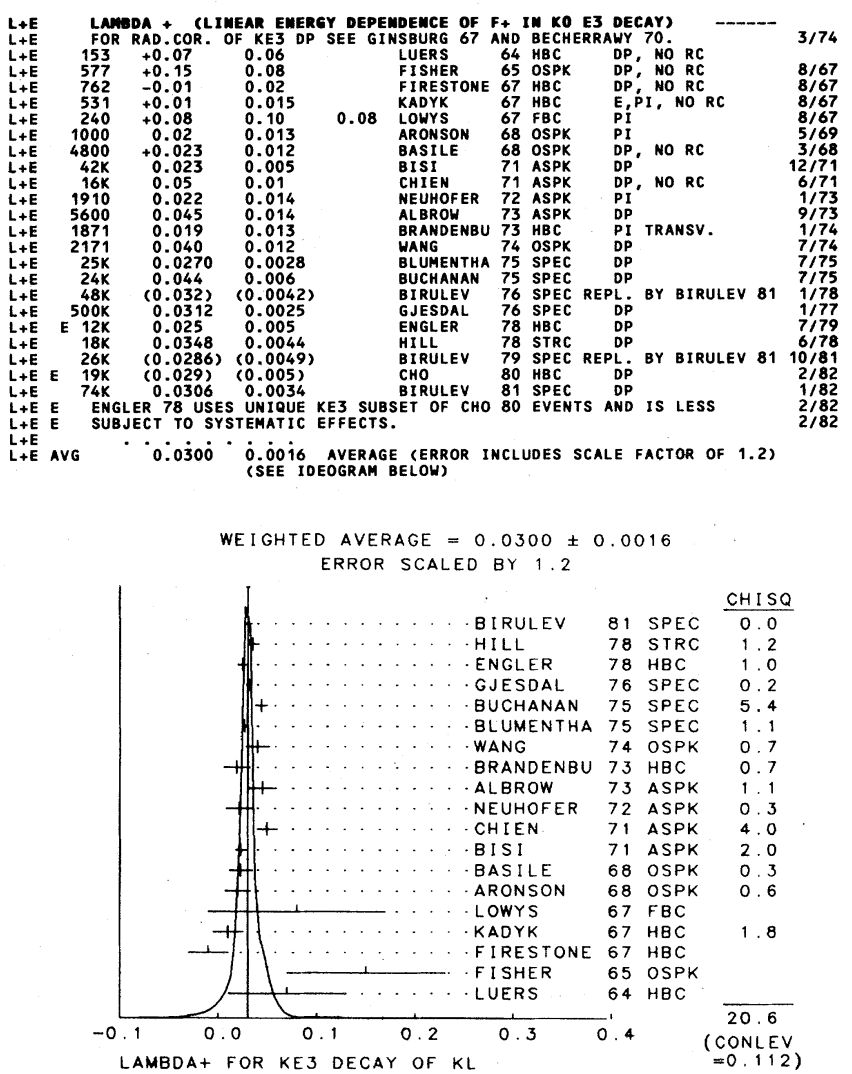

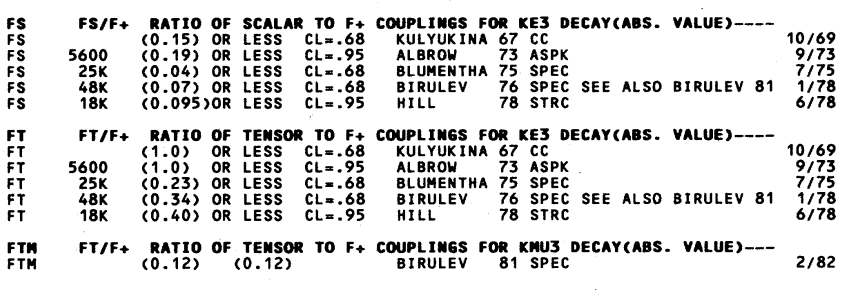

\section{NOTE ON CP VIOLATION IN $\mathbf{K}_{\mathbf{L}}^{0}$ DECAY}

We list the parameters which measure $\mathrm{CP}$ violation in $\mathrm{K}_{\mathrm{L}}^{0}$ decays and compare them with superweak model predictions.

\section{Parameters}

There are three different $\mathrm{K}_{\mathrm{L}}^{0}$ decays in which $\mathrm{CP}$ can be tested (for details, see Okun and Rubbia ${ }^{1}$, Steinberger $^{2}$, and Wolfenstein ${ }^{3}$ ).

(a) Charge asymmetry in $K_{L} \rightarrow \pi^{+} \pi^{-} \pi^{0}$ decays. As was discussed in the note on $K \rightarrow 3 \pi$ decay in the $K^{ \pm}$ section of the Data Card Listings, the Dalitz plot distribution for this decay contains a charge asymmetry term with coefficient $j$, the presence of which would indicate

\section{Data Card Listings}

$\mathrm{CP}$ violation. Experimenters have used several forms for this CP-violation term. As described in the "Note on Slope Parameters for $\mathrm{K} \rightarrow 3 \pi$ Decays" in the 1982 edition of this Review, ${ }^{4}$ we have converted all results to coefficient $j$ and have listed the results in section JT0 below. The coefficient $j$ is consistent with zero, i.e., absence of $\mathrm{CP}$ violation.

(b) Asymmetry in the $K_{L} \rightarrow \pi^{\mp} \ell^{ \pm} \nu$ decays. The quantity measured and compiled here is

$$
\delta=\frac{\Gamma\left(\mathrm{K}_{\mathrm{L}} \rightarrow \pi^{-} \ell^{+} \nu\right)-\Gamma\left(\mathrm{K}_{\mathrm{L}} \rightarrow \pi^{+} \ell^{-} \nu\right)}{\Gamma\left(\mathrm{K}_{\mathrm{L}} \rightarrow \pi^{-} \ell^{+} \nu\right)+\Gamma\left(\mathrm{K}_{\mathrm{L}} \rightarrow \pi^{+} \ell^{-} \nu\right)} .
$$

This asymmetry violates CP invariance. If CPT is good, for a pure $\mathrm{K}_{\mathrm{L}}^{0}$ beam, $\delta$ can be written as

$$
\delta=2\left[\left(1-|x|^{2}\right) /\left(|1-x|^{2}\right)\right] \operatorname{Re} \epsilon,
$$

where $\mathrm{x}$ is defined below in the "Note on the $\Delta S=\Delta \mathrm{Q}$ Rule in $\mathrm{K}^{0}$ Decay," and $\epsilon$ is the parameter of the expansion

$$
\begin{aligned}
& \left|\mathrm{K}_{\mathrm{L}}\right\rangle=[(1+\epsilon)|\mathrm{K}\rangle-(1-\epsilon)|\overline{\mathrm{K}}\rangle] /\left[2\left(1+|\epsilon|^{2}\right)\right]^{1 / 2} \\
& \left|\mathrm{~K}_{\mathrm{S}}\right\rangle=[(1+\epsilon)|\mathrm{K}\rangle+(1-\epsilon)|\overline{\mathrm{K}}\rangle] /\left[2\left(1+|\epsilon|^{2}\right)\right]^{1 / 2}
\end{aligned}
$$

We list $\delta$ separately for $\mathrm{K}_{\mathrm{L}}^{0} \rightarrow \pi \mu \nu$ and $\mathrm{K}_{\mathrm{L}}^{0} \rightarrow \pi \mathrm{e} \nu$ in sections $A 1$ and $A 2$ respectively, and list the combined values in section $A L$.

(c) $K_{L} \rightarrow 2 \pi$ decay. The relevant parameters are

$$
\begin{aligned}
\eta_{+-} & =\mathrm{A}\left(\mathrm{K}_{\mathrm{L}} \rightarrow \pi^{+} \pi^{-}\right) / \mathrm{A}\left(\mathrm{K}_{\mathrm{S}} \rightarrow \pi^{+} \pi^{-}\right) \\
& =\left|\eta_{+-}\right| \exp \left(\mathrm{i} \phi_{+-}\right) \\
\eta_{00} & =\mathrm{A}\left(\mathrm{K}_{\mathrm{L}} \rightarrow \pi^{0} \pi^{0}\right) / \mathrm{A}\left(\mathrm{K}_{\mathrm{S}} \rightarrow \pi^{0} \pi^{0}\right) \\
& =\left|\eta_{00}\right| \exp \left(\mathrm{i} \phi_{00}\right)
\end{aligned}
$$

$\epsilon$, defined in Eqs. (1) above, and

$$
\epsilon^{\prime}=\frac{1}{2} \mathrm{i} \sqrt{2} \exp \left[\mathrm{i}\left(\delta_{2}-\delta_{0}\right)\right] \operatorname{Im}\left(\mathrm{A}_{2} / \mathrm{A}_{0}\right) .
$$

Here, $A_{i}$ and $\delta_{i}$ are the amplitude and phase of $\pi \pi$ scattering at the $K$ mass, defined by

$$
\begin{aligned}
& \langle\mathrm{I}=0|\mathrm{~T}| \mathrm{K}\rangle=\exp \left(\mathrm{i} \delta_{0}\right) \mathrm{A}_{0}, \\
& \langle\mathrm{I}=2|\mathrm{~T}| \mathrm{K}\rangle=\exp \left(\mathrm{i} \delta_{2}\right) \mathrm{A}_{2} .
\end{aligned}
$$

$\mathrm{Wu}$ and Yang $^{5}$ have derived the relationships

$$
\eta_{+-}=\epsilon+\epsilon^{\prime}, \quad \eta_{00}=\epsilon-2 \epsilon^{\prime} \text {. }
$$


For notation, see key at front of Listings.

Measurements of $\left|\eta_{+-}\right|,\left|\eta_{00}\right|^{2}, \phi_{+-}$, and $\phi_{00}$ are listed in sections E+-, EOS, F+-, and F00. The FIT values given in these sections come from constrained fits which include the $\left|\eta_{00}\right| /\left|\eta_{+-}\right|$and $\phi_{00}-\phi_{+-}$ measurements from sections ER and DF, respectively.

Superweak model predictions for $\left|\eta_{00} / \eta_{+-}\right|, \phi_{+-}$, and Re $\epsilon$

The superweak model $^{6}$ predicts that ${ }^{7}$

$$
\begin{aligned}
& \left|\eta_{00} / \eta_{+-}\right|=1, \\
& \phi_{+-}=\phi_{00}=\tan ^{-1}\left(\frac{2 \Delta \mathrm{m} \tau_{S}}{\hbar}\right),
\end{aligned}
$$

and

$$
\operatorname{Re} \epsilon=\left|\eta_{+-}\right|\left[1+\left(\frac{2 \Delta \mathrm{m} \tau_{S}}{\hbar}\right)^{2}\right]^{-1 / 2} .
$$

The latter two expressions and the values of the $\mathrm{K}_{\mathrm{L}}^{0}-$ $\mathrm{K}_{\mathrm{S}}^{0}$ mass difference $\Delta \mathrm{m}=(0.5349 \pm 0.0022) \times 10^{10} h$ $\mathrm{sec}^{-1}$, the $\mathrm{K}_{S}^{0}$ mean life $\tau_{S}=(0.8923 \pm 0.0022) \times 10^{-10}$ sec, and the magnitude of the $\left(\mathrm{K}_{\mathrm{L}}^{0} \rightarrow \pi^{+} \pi^{-}\right) /\left(\mathrm{K}_{\mathrm{S}}^{0} \rightarrow\right.$ $\left.\pi^{+} \pi^{-}\right)$amplitude ratio $\left|\eta_{+-}\right|=(2.274 \pm$ $0.022) \times 10^{-3}$, all from the current edition, result in the predictions that

$$
\phi_{+-}=\phi_{00}=(43.67 \pm 0.14)^{\circ}
$$

and

$$
\operatorname{Re} \epsilon=(1.645 \pm 0.016) \times 10^{-3} .
$$

The above predictions can be compared with the experimental values

$$
\begin{aligned}
& \left|\eta_{00} / \eta_{+-}\right|=1.023 \pm 0.036, \\
& \phi_{+-}=(44.6 \pm 1.2)^{\circ}, \\
& \phi_{00}=(54.5 \pm 5.3)^{\circ}, \\
& \operatorname{Re} \epsilon=(1.621 \pm 0.088) \times 10^{-3},
\end{aligned}
$$

where $\operatorname{Re} \epsilon$ has been computed using the relation

$$
\operatorname{Re} \epsilon=\frac{\delta}{2}\left(\frac{|1-x|^{2}}{1-|x|^{2}}\right),
$$

and our current values of the charge asymmetry parameter for leptonic $K_{\mathrm{L}}^{0}$ decay $\delta=(0.330 \pm 0.012) \%$ and the $\Delta S=-\Delta Q$ amplitude $(\operatorname{Rex}, \operatorname{Imx})=(0.009 \pm 0.020$, $-0.004 \pm 0.026)$.
The superweak predictions are within one standard deviation of the data except for the measured value of $\phi_{00}$, which is two standard deviations above the prediction. This results primarily from the CHRISTENSON1 79 measurement $\phi_{00}=(55.7 \pm 5.8)^{\circ}$.

\section{References}

1. L.B. Okun and C. Rubbia, in Proceedings Heidelberg Conference on Elementary Particles, p. 301 (1967).

2. J. Steinberger, in CERN Topical Conference on Weak Interactions, CERN 69-7, p. 291 (1969).

3. L. Wolfenstein, in Theory and Phenomenology in Particle Physics, ed. A. Zichichi (Academic Press, New York, 1969) p. 218.

4. Particle Data Group, Phys. Lett. 111B, 69 (1982).

5. T.T. Wu and C.N. Yang, Phys. Rev. Lett. 13, 380 (1964).

6. L. Wolfenstein, Phys. Lett. 13, 562 (1964).

7. T.D. Lee and L. Wolfenstein, Phys. Rev. 138B, 1490 (1965).

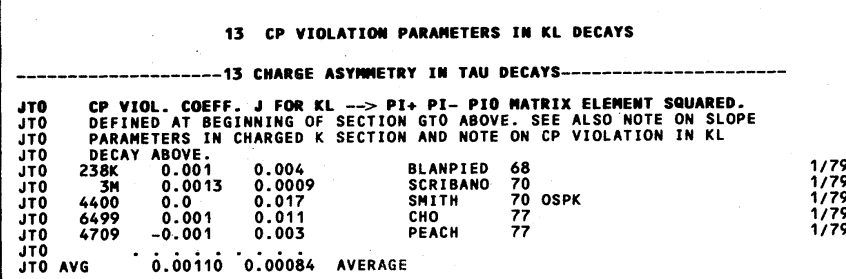

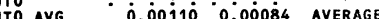

$$
\text { - } 13 \text { Charge asYmetry IN Leptomic decays (PERCENT)---_-- }
$$

SUCH ASYMMETRY VIOLATES CP. IT IS RELATED TO REAL (EPSILON).

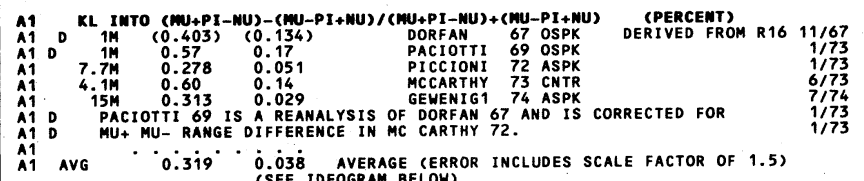

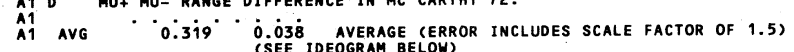

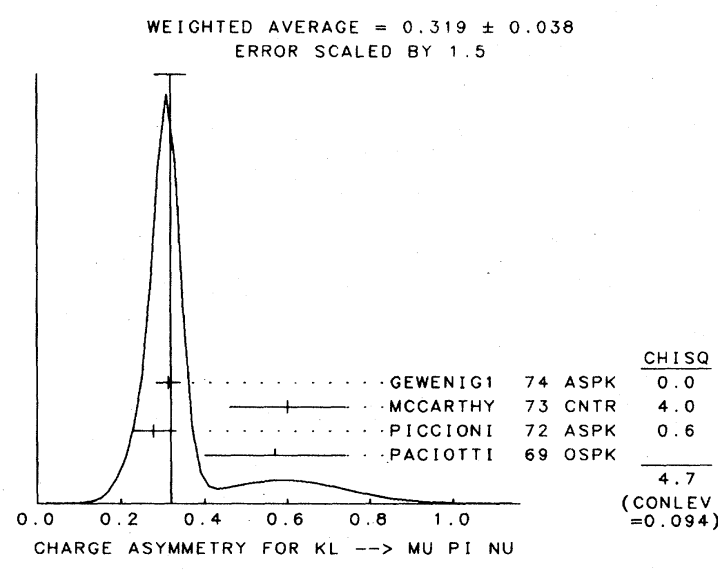


Stable Particles

$\mathrm{K}_{\mathrm{L}}^{0}$

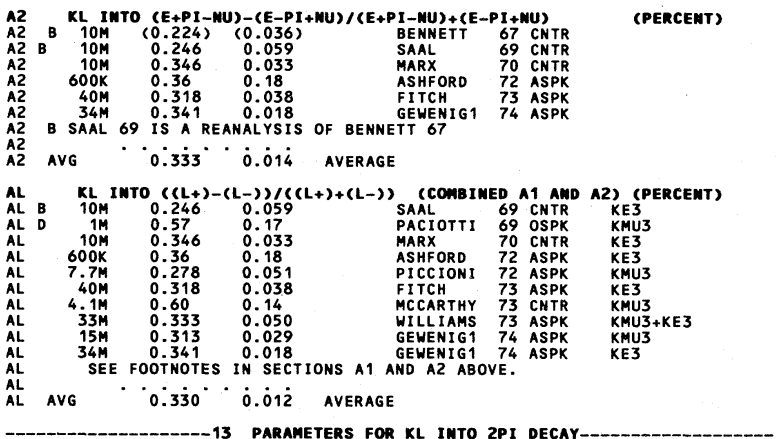

ETA+- $=A(K L$ TO PI PII ) $/ A(K S$ TO PI+PI-)
ETAOO $=A(K L$ TO PIOPIO)/A(KS TO PIOPIO)

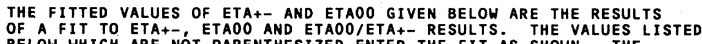
VALUES WHICH ARE PARENTHESIZZED AND BEAR THE FOOTNOTE X DD NOT ENTER WAE FIT AS SHOON. THEEE EXPERRMENTS GIVE BRANCHNG RATIDS AND ENER

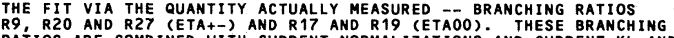

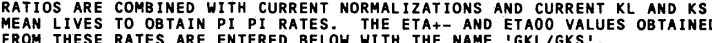
FROM THESE RATES ARE ENTERED BELOW WITH THE NAME GKL/GKS

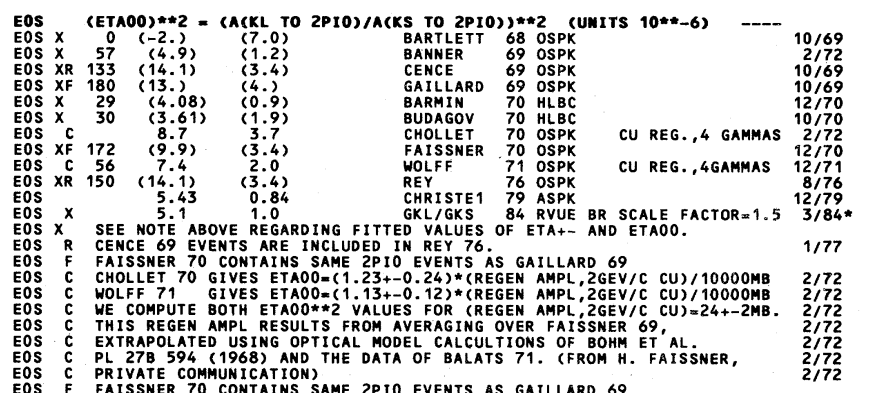

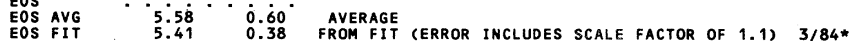

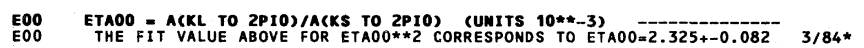

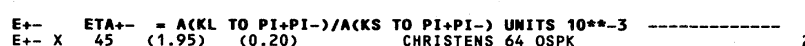

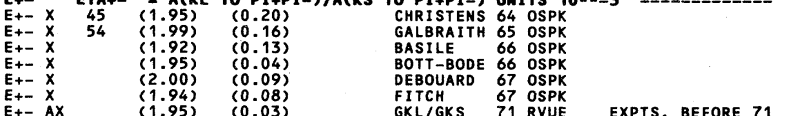

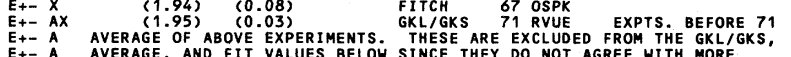
EN- A AVERGE, AND FIT VALUES BED S SINCE THEY DO NOT AGREE WITH MORE
E+- A RECENT PRECISE AND IN PRINCIPLE SUPERIOR EXPERIMENTS.

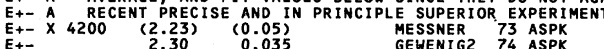

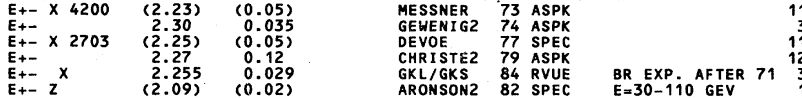

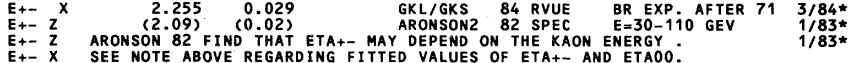

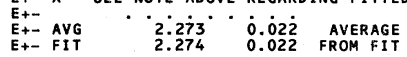

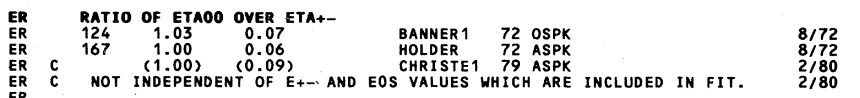

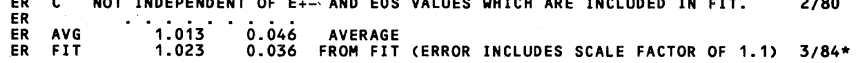

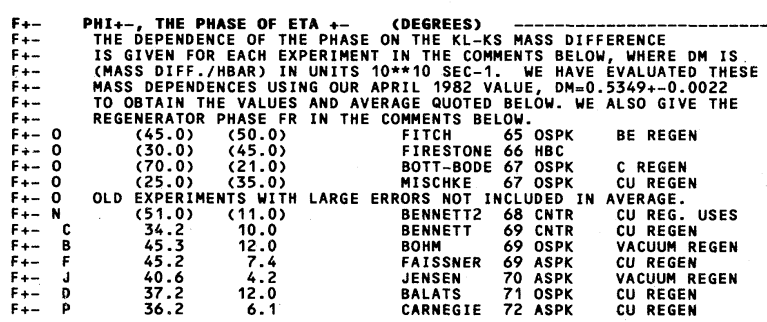

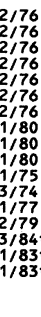
$3 / 84 *$ (n)

至

\section{Data Card Listings}

\section{NOTE ON $\Delta S=\Delta Q$ RULE IN $K^{\mathbf{0}}$ DECAYS}

The relative amount of $\Delta S \neq \Delta Q$ component present is measured by the parameter $\mathrm{x}$, defined as

$$
\mathrm{x}=\mathrm{A}\left(\overline{\mathrm{K}}^{0} \rightarrow \pi^{-} \boldsymbol{\ell}^{+} \nu\right) / \mathrm{A}\left(\mathrm{K}^{0} \rightarrow \pi^{-} \boldsymbol{\ell}^{+} \nu\right) .
$$

We list $\operatorname{Re}\{x\}$ and $\operatorname{Im}\{x\}$ for $K_{e 3}$ and $K_{\mu 3}$ combined.

$$
13 X=\text { (DS=-DQ AMPLITUDE)/(DS-+DQ AMPLITUdE) }
$$

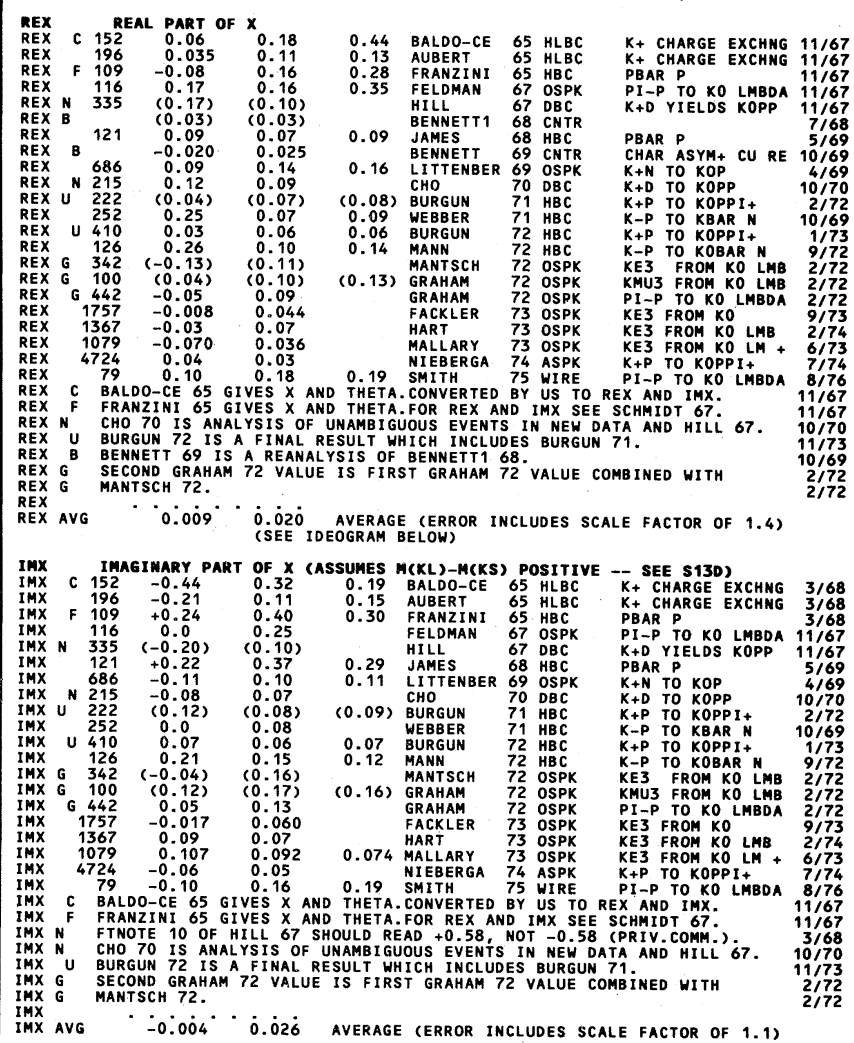




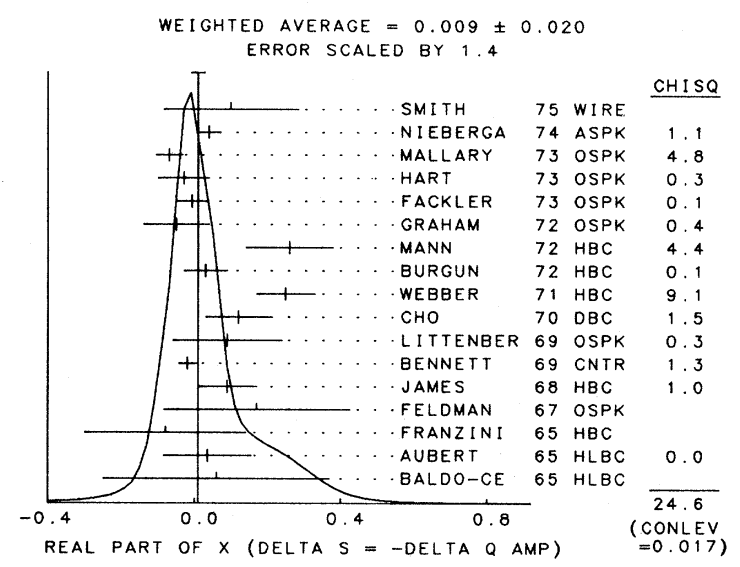

\begin{tabular}{|c|c|}
\hline & NCES FOR \\
\hline 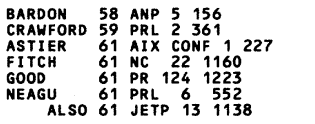 & 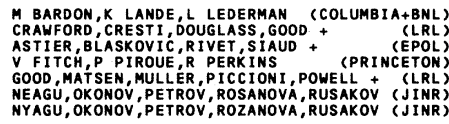 \\
\hline 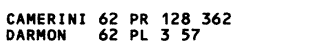 & 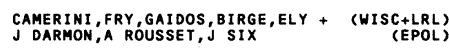 \\
\hline 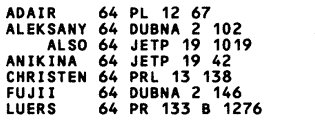 & 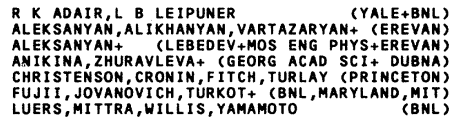 \\
\hline 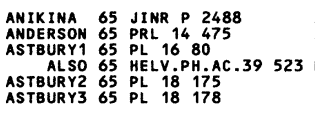 & 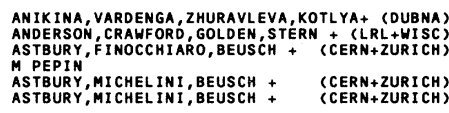 \\
\hline & AUBERT, BEHR, CANAVAN, CHOUNET+ (EPOL+ORSAY) \\
\hline 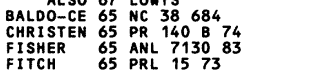 & 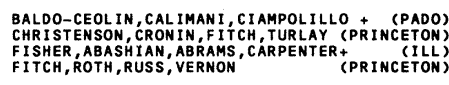 \\
\hline 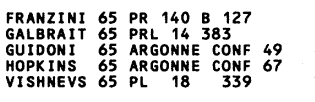 & 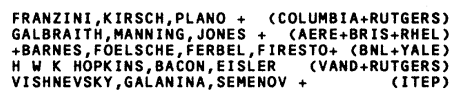 \\
\hline $\begin{array}{l}\text { FF-SSE } \\
\text { IF }\end{array}$ & 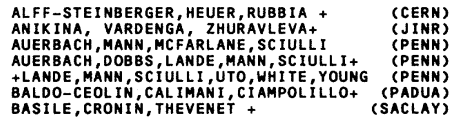 \\
\hline 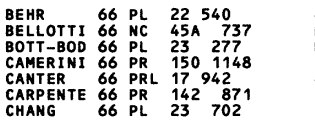 & 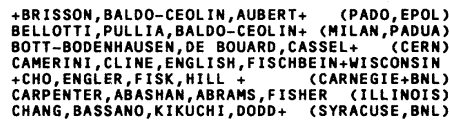 \\
\hline 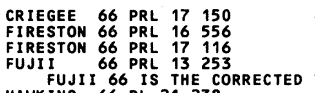 & 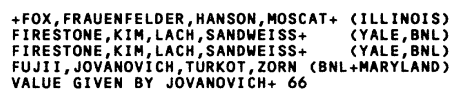 \\
\hline ALSO 67 PR $\quad 156 \quad 1444$ & $\begin{array}{l}\text { C J B HAHKINS } \\
\text { c J B HAHKINS }\end{array}$ \\
\hline 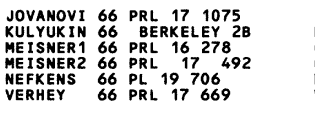 & 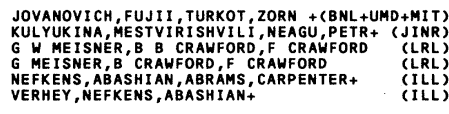 \\
\hline 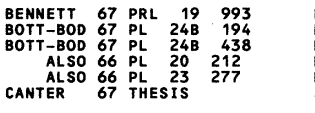 & 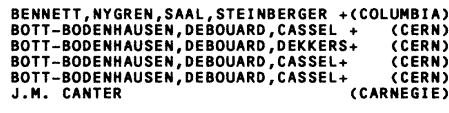 \\
\hline 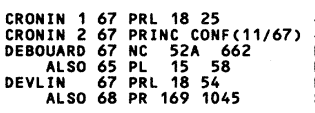 & 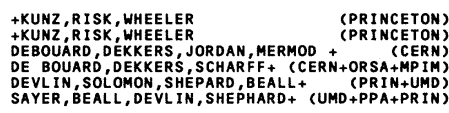 \\
\hline
\end{tabular}

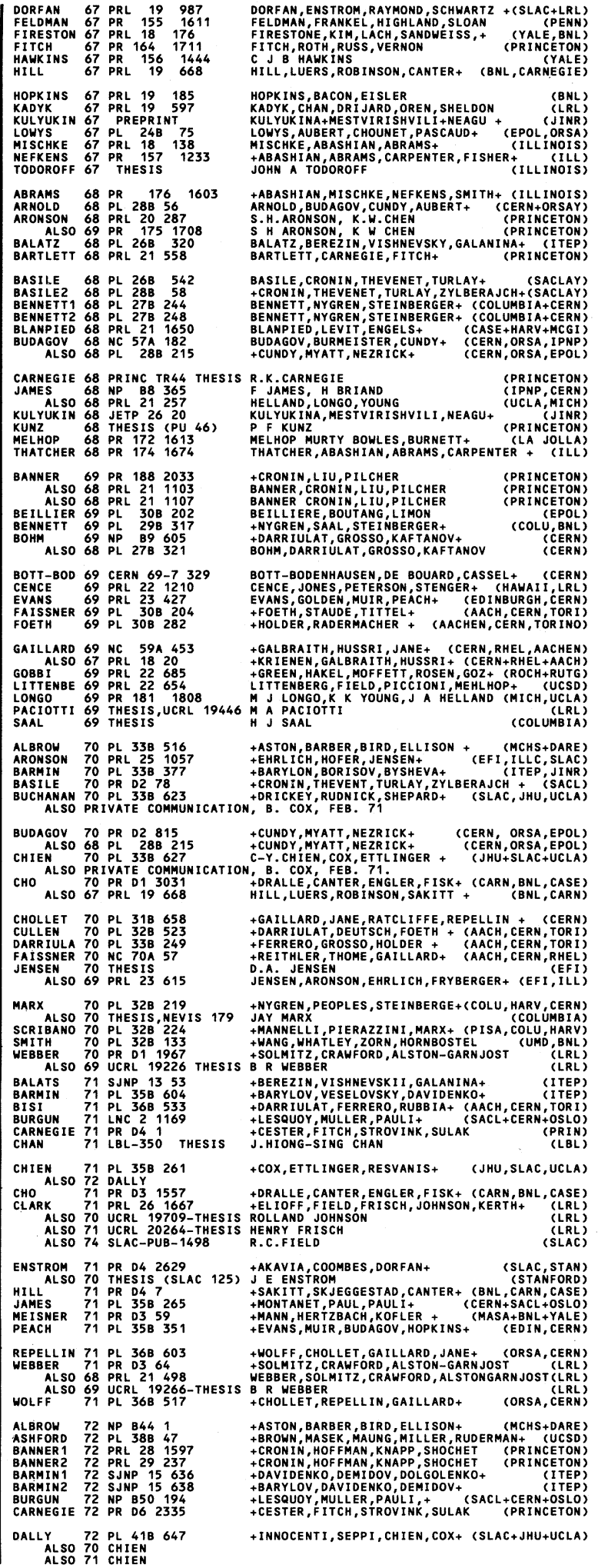




\section{Stable Particles}

$\mathrm{K}_{\mathrm{L}}^{0}, \mathrm{D}^{ \pm}$
+ABASHIAN, JONES, MANTSCH, ORR+ (ILL+NEAS)
+RADERMACHER STAUDE+
(AACH+CERN+TORI)

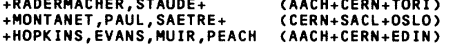
+KOFLER, MEE SNER, HERTZBACH+
+ABASHIAN, GRAHAM, JONES, ORR +
(MASA+BNL+YALE)
(ILL+NEAS) TEUHOFER, MIEBERGALL+ (CERN+ IPN+ HIEN)

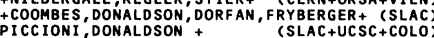

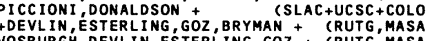
AASTON, BARBER, BIRD, ELLI ISON+ (MCHS+DARE) +BALASHOV, BANNIK, + -

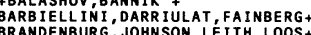

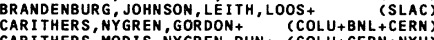

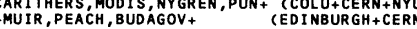
VNANS, GOLDEN, MUIR, PEACH+ (EDINBRGH+CERN)

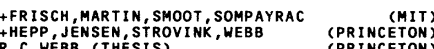
R.C. WEBB (THESTS) PBINIE, GALLIVA, GOMEZ,PECK, SCIULLI + (CIN)
SCIULLI, GALLIVAN,BINNIE, GOMEZ +
(CIT) BBREWER, BUDNITZ, ENTIS, GRAVEN, MILLER+ (LBL)
MCCARTHY, BREWER', BUONITZ, ENTIS, GRAVEN+
(LBL)

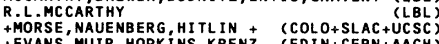
+EVANS, MUIT, HOPKI INS, KRENZ (EODN+CERN+AACH)

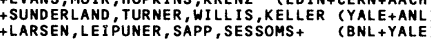
DUBNA+BERLIN+BUDAPEST+PRAGGE+SERPUKH+SOFIA
BISI, FERRERO
(TORI)

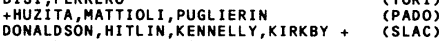

DONALDSON, FRYBERGER, HITLIN, LIUU, (SLAC+UCSC) GONALSON, RYYBERGER, HITLIN, LIU+ (SLAC+UCSC)
(SLAC)

GEWENIGER, GJESDAL, KAMAE, PRESSER+(CERN HEE ID)
VERA LUTH (THESIS- INT. REPT. 74-4)
(HEID ID GEEENGER, GJESDAL, PRESSER +

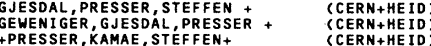
+FRANKLIN, MORSE, NAUENBERG+ (COLO+SLACCUCSC) SMITH, WHATLEY, ZORN, HORNBOSTEL (UMD + BNL)
S +LARSEN LEIPUER , NAPP, SESSOMS + (BNL+YALE)
(UCLACA) BALDO-CEOLIN, BOB ISUT, CALIMANIt (PADO+HISC)
BLUMENTHAL FRANKLL, NAGY + (PENN+CHIC+TEMP)

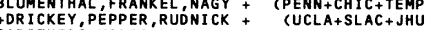

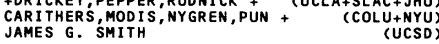
+VESTERGOMBI, VOVENKO, VOTRUBA, GENCHEV+ (JIMR)

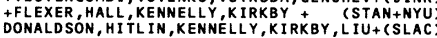

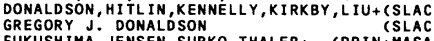

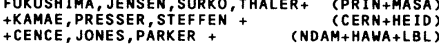
DERR ICK, LLSSAUER, MILLER, ENGLER+ (ANL+CARN)
DIST GILELD, HOLLEY, JOHNSON, KERTH, SAH, SHEN (LBL) +CRONIN, FRISCH, GROSSO-PILCHER, (EFI IANL)
DZHORDZHADE, KEKELIDZE, KR IVOKHIZHIN + (JINR) DCMMERON + (BGA + EON GLAS+PISA+RHEL)

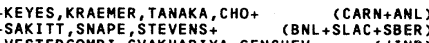

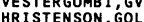
(NYU)
CHRI ITENSON, GOLOMAN, HUMMEL, ROTH+
SAKIT SNAPE STEVENS.
(BNL+SLAC+SBER +SALITT, SNAPE, STEVENST
+BATT, CAMBEL, GRANNAN+

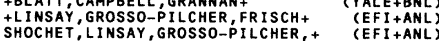
+CHIANG, KYCIA, LI, LIITENBERG, MARX+(BNL+ROCH) CHIANG, KYCIA, LI, LITTENBERG, MARX+(BNL+ROCH) (JINR) IRULEV, VESTERGOMBI, GENCHEV + (JINR)

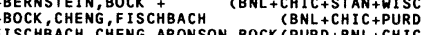
FISCHBACH, CHENG, ARONSON, BOCK (PURD $+B N L+C H I C)$
ARONSON, BOCK, CHENG, FISCHBACH BNL +CHIC+PURD ARONSON', BOCK,'CHENG', FISCHACHCWL PAPERS NOT REFERRED TO IN DATA CARDS

G ALEXANDER, S ALMEEDA, F CRAWFORD (LRL) STERN, BINF ORD LINB, ANOERSON (BNL MAARYLAND)
(WISC+LRL)

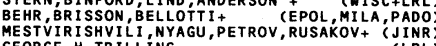
MESTVIRISHVIL, NYATI
GERGE HRILING
CONF., PAGE 115.

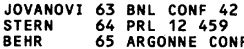
MEER 65 ARGONNE CONF 59 MESTIRI 65 JINR P 2449
TRILING 655 UCRL 16473
UPDATED FROM 1965

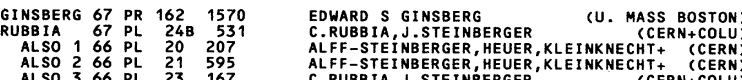

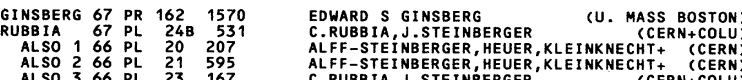

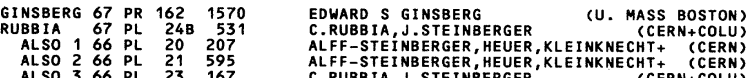

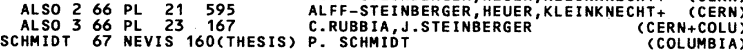
CRONIN 68 VIENAA CONF P. 281 CRONIN, RAPPORTEURS TALK
BECHERRA 70 PR D1 1452 (PRINERRAWY
(ROCH)

\section{Data Card Listings}

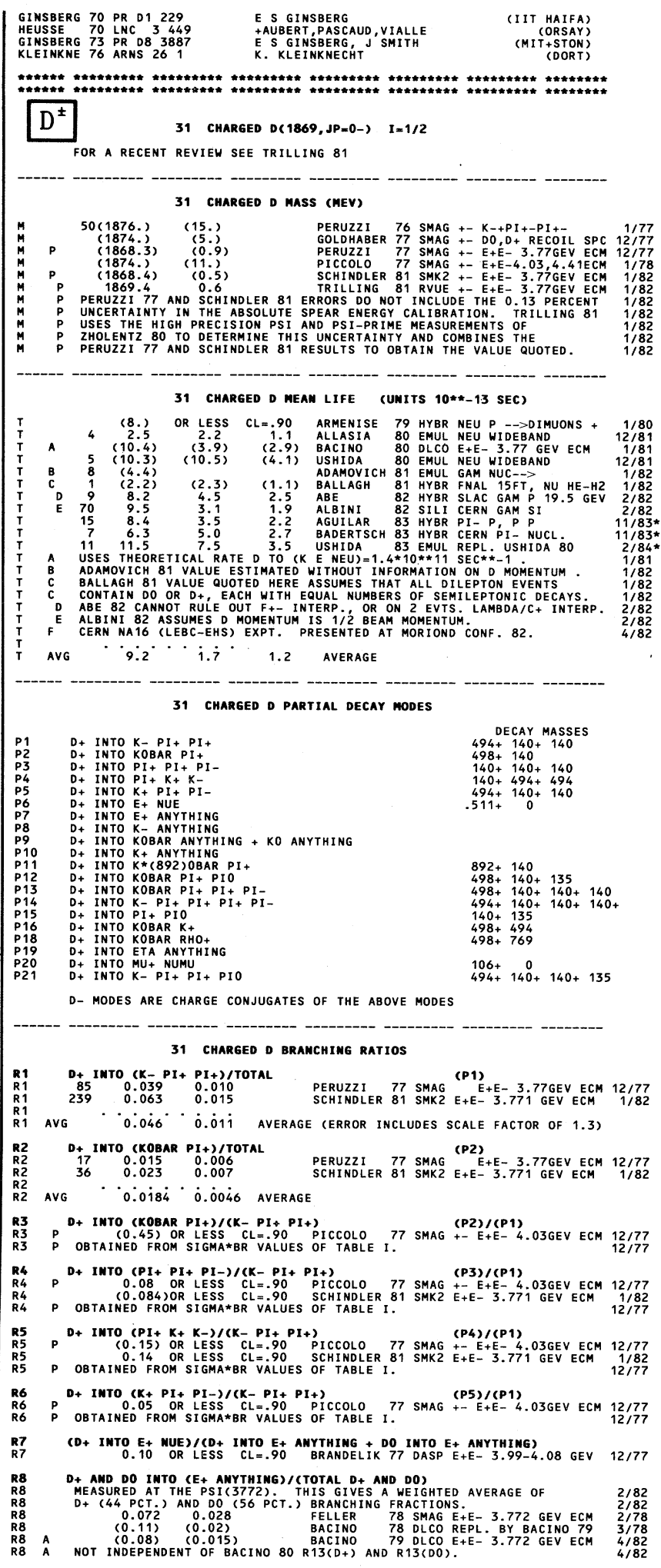




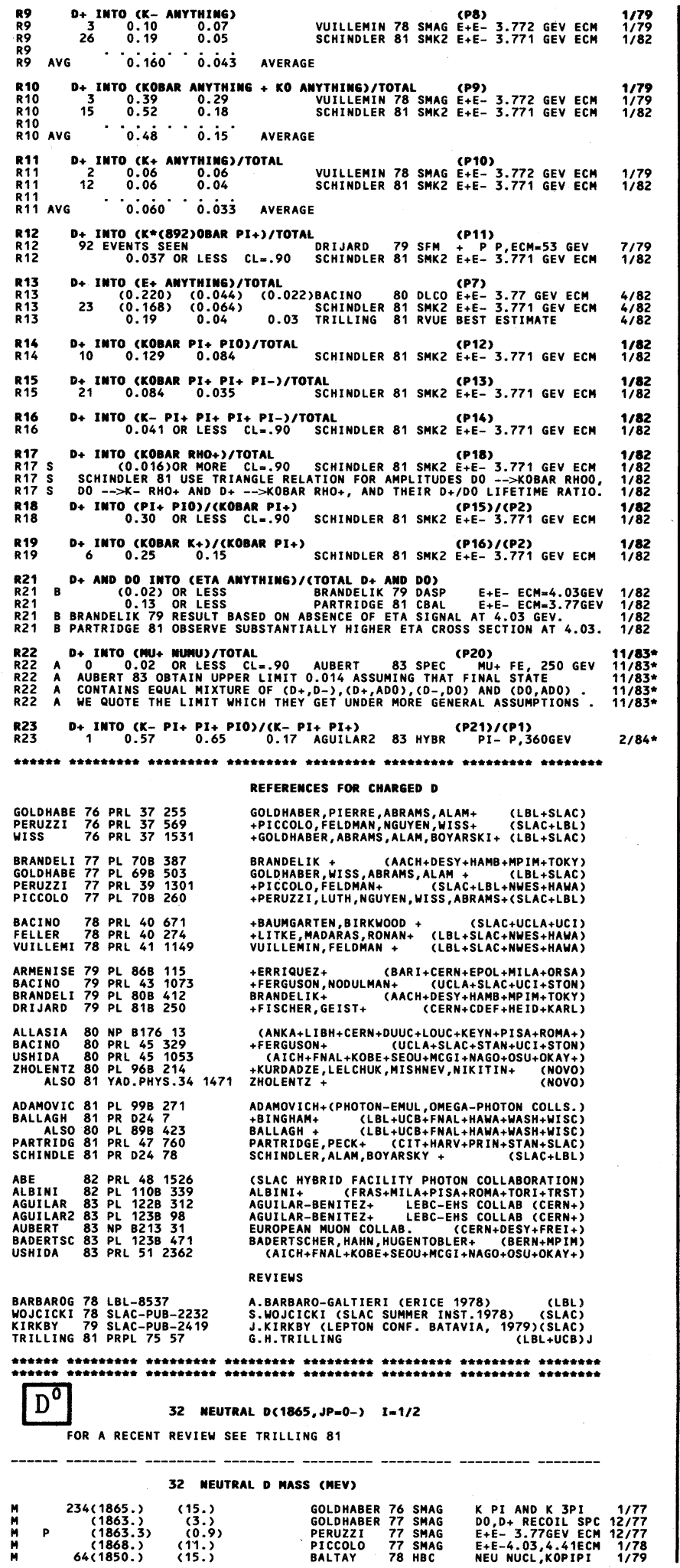

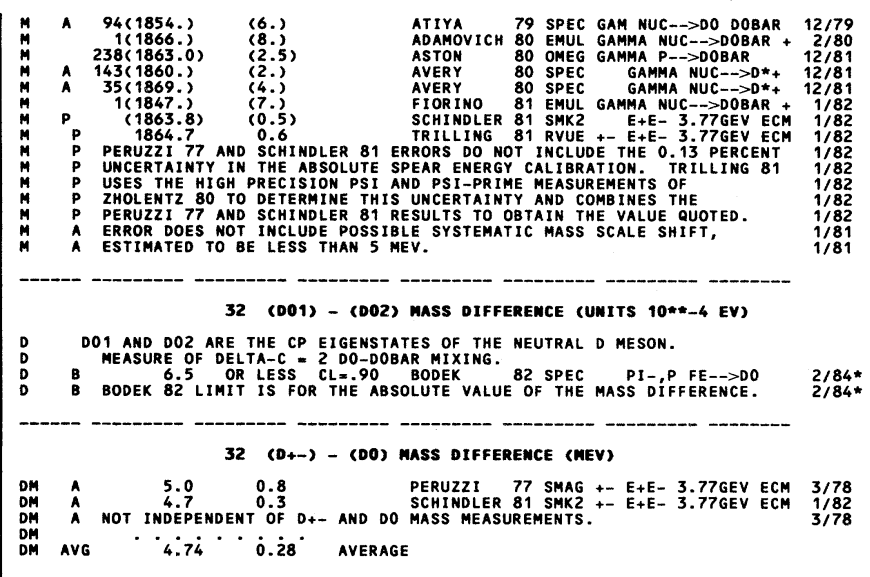

32 meUtral D MEAN LIFE (UMITS 10**-13 SEC)

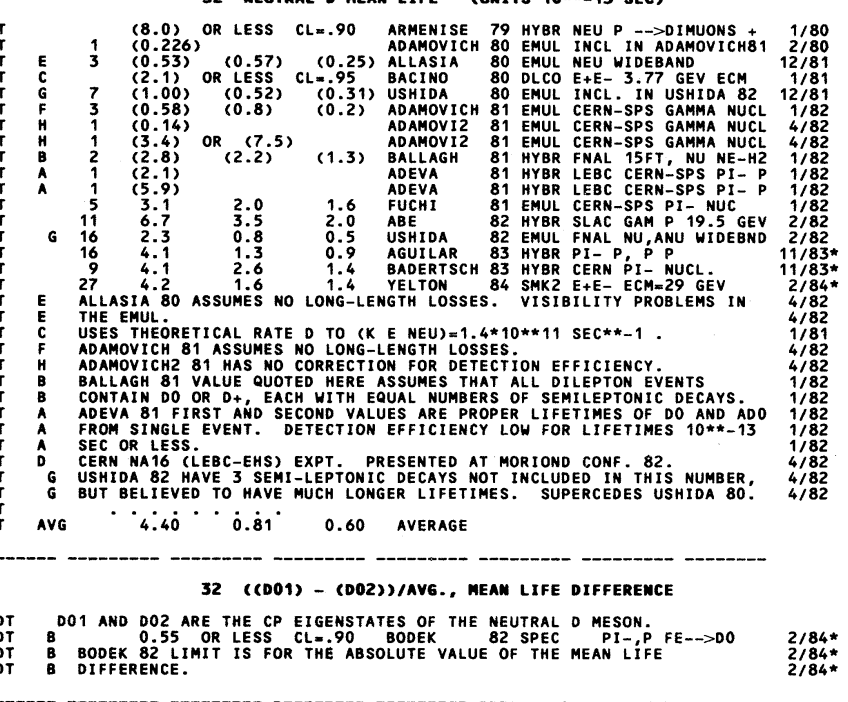

32 (CHARGed D)/(MEUTRAL D) MEAM LIFE RATIO

FROM RATIO OF D+ TO DO SEMILEPTONIC BRANCHING FRACTIONS.
NOT USED BY US TO CONSTRAIN BEST VALUES OF D LIFETIMES FOR TABLE,

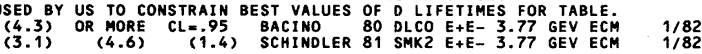

32 meutral o partial decay modes

Do INTO K- PI+

DO INTO KOBAR PI+ PI-

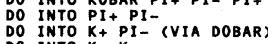

0
$D 0$
$D$

INTO E+ ANYHING

DO INTO K- ANYTHING

DO INTO KOBAR ANYTHING + KO ANYTHING

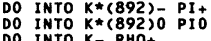

DO INTO KOBAR RHOO

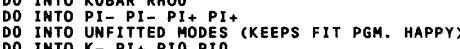

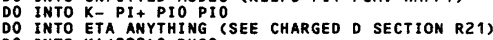

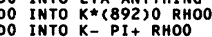

0
0
0
0

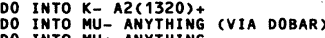

dobar modes are charge conjugates of above modes
deCAY MASSES

$494+140+140+140$

$498+140+140$
$498+140+140+140+$

$140+140$
$4994+140$

$494+494$
$494+140+135$
$498+135$

$892+140$
$892+135$

$498+769$
$140+140+140+140$

$494+140+135+135$

$892+769+769$
$494+140+769$
$892+140+140$
$494+1318$

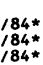

\section{2}

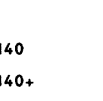


Stable Particles

$D^{0}, F^{ \pm}$

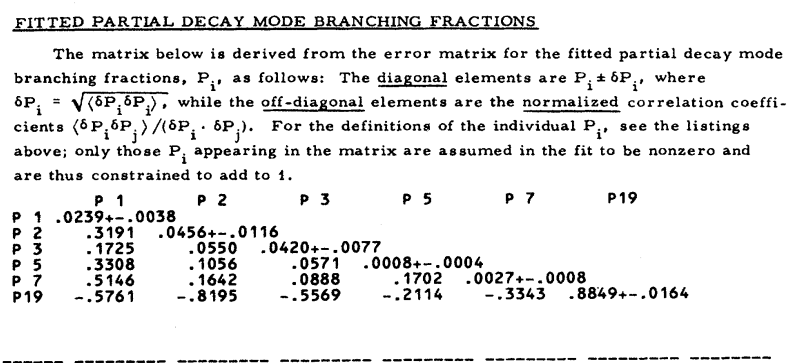

32 MEUTRAL O BRAMCHIMG RATIOS

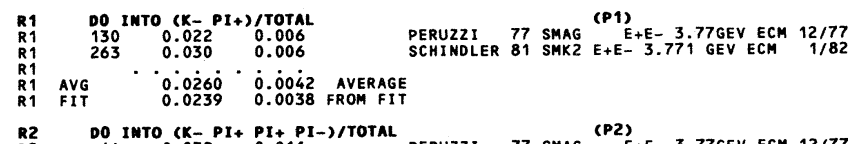

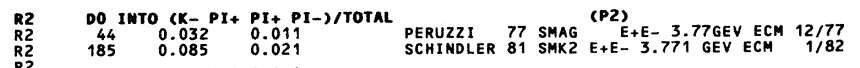

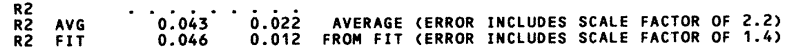

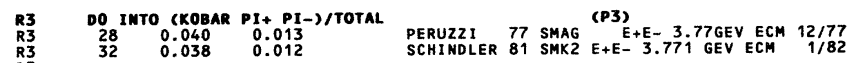

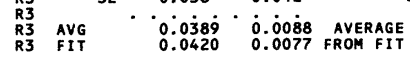

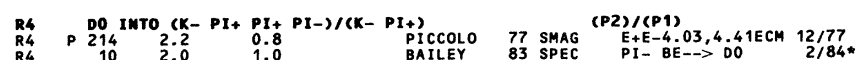

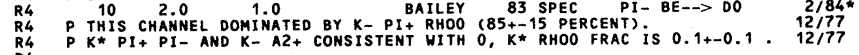

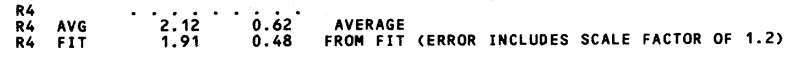

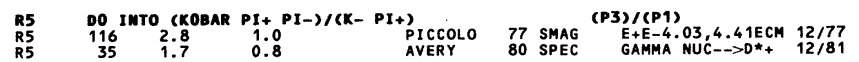

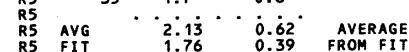

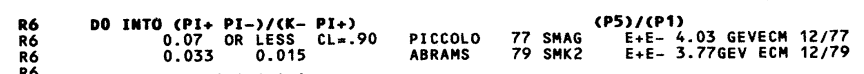

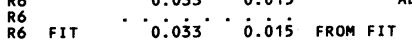

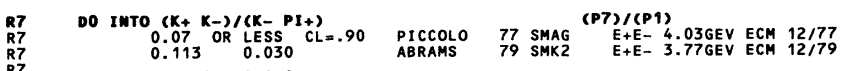

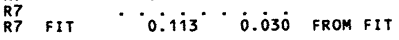

\begin{tabular}{l}
$R 8$ DO IMTO $\left(K_{+} P P_{-}\right.$, VIA DOBAR $) /(K-P 1++K+P 1-) \quad(P 6) /(P 1+P G)$ \\
\hline
\end{tabular}

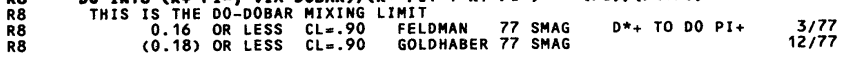

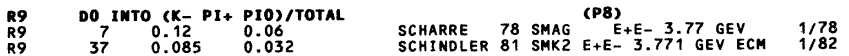

$\begin{array}{lllllll}R 9 & 37 & 0.085 & 0.032 & \text { SCHINOLER } 81 & \text { SMK2 E+E- } 3.771 \text { GEV ECM } & 1 / 82\end{array}$

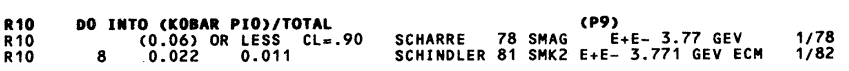

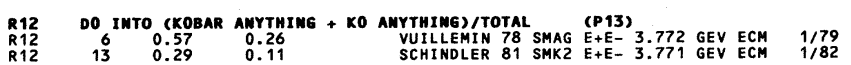

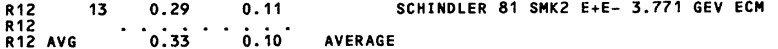

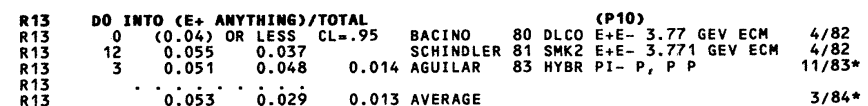

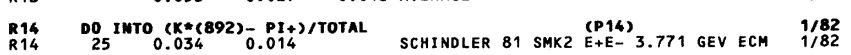

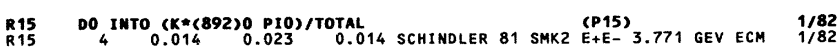

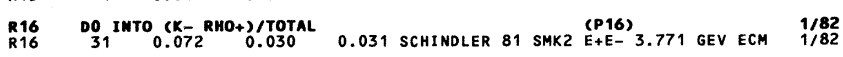

R17 DO INTO (KOBARR RHOO)/TOTAL
R17

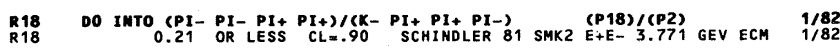

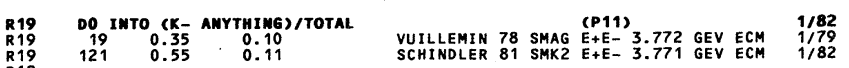

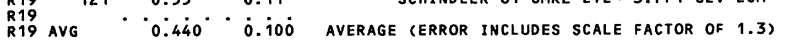

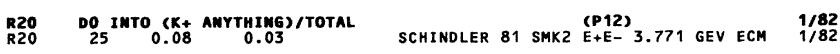

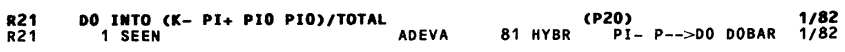

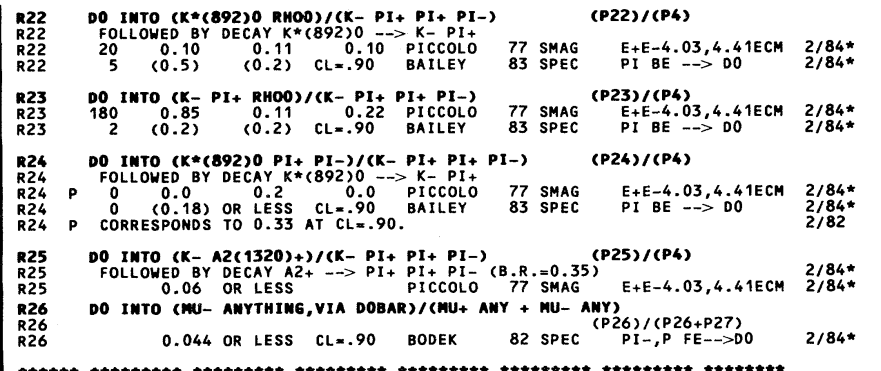

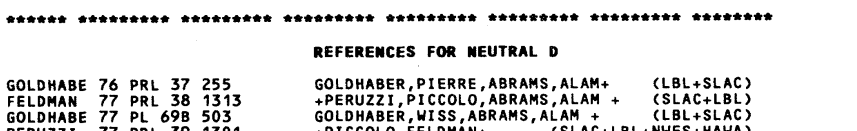

$\begin{array}{lll}\text { PERUZZZ } & 77 \text { PRL } 39 & 1301 \\ \text { PICCOLO } 77 \text { PL } 708 & 260\end{array}$

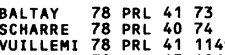

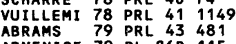

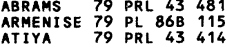

ADNMOVIC 80 PL 898427

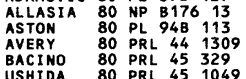

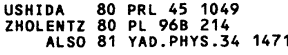

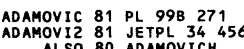

ALSO 80 ADAMOVICH

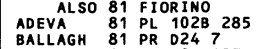

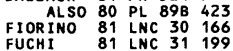

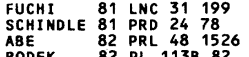

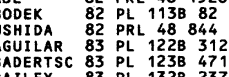

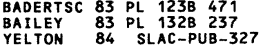
+PICCOLO, FEL DMAN
+PERUZZI, UUTH, NGUYEN, WISS, ABRAMS+ (SLACLLBLL) +CAROUMBALIS, ERENCH, HIBBS, HYL TON+(COLU+BNL)

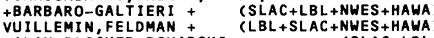

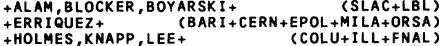
ADAMOVICH+(PHOTON-EMUL, OMEGA-PHOTON COLLS.) (ANKA+LI IBH+CERN+DUUC, +OOUC+KEFN+PISAAROMA+)

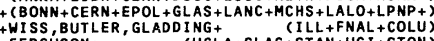
CAICH+FNAL+KOBE+SEOU + MCGI+NAGO+OSU+OKAY+)

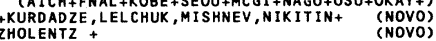
ADAMONICH+(PHOTON-EMUL, OMEGA-PHOOON COLLS.)
(PISMA JETF 34 477) (PHOT.EMUL., OMEGA-PHOT.)

QUANTUM NUMBER

+AGUILAR-BENITEZ + (LEBC-EHS COLLABORATION)

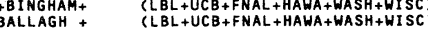

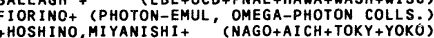
SCH NDLLR, ALAM, BOYARSKY Y TOACH (SLAC+LBD) S CAICH+FNAL+KOBE+SEOU+MCGI+NAGO+OSU+OKAY+ AGUULAR BEN
BADERSCHER, HAHN, HUGENTOBLER+

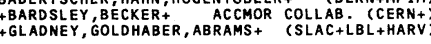

NGUYEN 77 PRL 39262 +WISS, ABRAMS, ALAM, BOYARSKI+ (LBL+SLAC)J

BARBAROG 78 LBL-8537 A.BARBARO-GALTIERI (ERICE 1978)

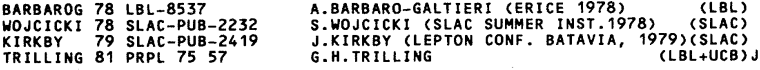

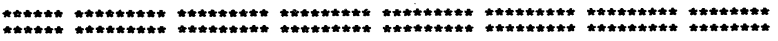

$\mathrm{F}^{ \pm}$

$34 \mathrm{~F}+-(1970, \mathrm{JP}=0-) \quad I=0$

QUANTUM NUMBERS NOT MEASURED. VALUES ARE ASSIGNED HERE ASSUMING CHARMED-STRANGE GROUND STATE F-MESON.
CHEN 83. OBSERVATIONS ARE CONSISTENT WITH J=0.

\section{NOTE ON THE F MESON}

The strongest evidence for the $F$ meson comes from the CLEO observation (CHEN 83) of a $\phi \pi^{ \pm}$signal of $104 \pm 19$ events above background with mass $(1970 \pm 5 \pm 5) \mathrm{MeV}$ and width consistent with resolution. The decay angular distributions are consistent with a spin- $0 \mathrm{~F}$ decaying to $\phi \pi$ followed by $\phi \rightarrow \mathrm{K}^{+} \mathrm{K}^{-}$. The observation of a $\phi \pi^{ \pm}$state at this mass has been confirmed by TASSO (ALTHOFF 84) and supported in a preliminary report from ARGUS (ARGUS 83).

The 1970-MeV mass is inconsistent with that found by the OMEGA Spectrometer photoproduction experiment, while the earlier DASP mass (BRANDELIK $77,79)$ is consistent with both the CLEO and OMEGA 
results. DASP (BRANDELIK 77,79) observed an $\eta \pi^{ \pm}$ signal of 6 events with negligible background at $(2030 \pm 60) \mathrm{MeV}$. OMEGA reported signals in $\eta \pi^{ \pm}$, $\eta \pi^{ \pm} \pi^{+} \pi^{-}$, and $\eta^{\prime} \pi^{ \pm} \pi^{+} \pi^{-}$at an average mass of $(2020 \pm 10 \pm 20) \mathrm{MeV}$ (ASTON 81), in $\phi \rho^{ \pm}$at (2049 \pm 15$) \mathrm{MeV}$ (ASTON2 81), and later in $\eta \pi^{ \pm}$at $(2017 \pm 13) \mathrm{MeV}$ (ATKINSON 83). The disagreement between OMEGA and CLEO masses cannot be accounted for by any known systematic errors.

Another apparent disagreement involves the $\mathrm{F} \rightarrow$ $\phi \pi^{ \pm}$branching fraction. OMEGA sets a limit $\sigma \cdot \mathrm{B}<4$ $\mathrm{nb}$ for the $\bar{F} \overline{\mathrm{F}}$ photoproduction cross section times the branching fraction to this mode (ASTON2 81), compared with $\sigma \cdot \mathrm{B}=(140 \pm 20) \mathrm{nb}$ for the sum of the four modes they reported, i.e., a branching ratio $\Gamma\left(\mathrm{F}^{ \pm} \rightarrow\right.$ $\left.\phi \pi^{ \pm}\right) / \Gamma\left(\mathrm{F}^{ \pm} \rightarrow \eta \pi^{ \pm}+\eta \pi^{ \pm} \pi^{+} \pi^{-}+\eta^{\prime} \pi^{ \pm} \pi^{+} \pi^{-}+\right.$ $\left.\phi \rho^{ \pm}\right)<3 \%$. This suggests a much smaller branching ratio to all possible modes: $\mathrm{B}(\mathrm{F} \rightarrow \phi \pi) \equiv \Gamma(\mathrm{F} \rightarrow$ $\phi \pi) / \Gamma(\mathrm{F} \rightarrow$ all $) \ll 3 \%$. In contrast CLEO (CHEN 83), using a crude estimate of the level of $\mathrm{F}^{ \pm}$production, obtains $\mathrm{B}(\mathrm{F} \rightarrow \phi \pi) \sim 4.4 \%$, while TASSO (ALTHOFF 84) finds a somewhat higher value. Theoretical estimates for this branching fraction are $2 \%-3 \% .{ }^{1}$

The hybrid-emulsion spectrometer wide-band neutrino beam experiment at Fermilab (USHIDA 83) cannot be used to establish an $F$ mass because their cuts to eliminate $D$ background effectively require their $F$ candidates to have a mass greater than $2000 \mathrm{MeV}$. In a second run this group has reduced the mass bias by improved mass resolution and by including $\phi$ decay modes. Preliminary results for this run reported at Cornell ${ }^{2}$ give a mass for $8 \mathrm{~F}^{ \pm}$candidates which is in agreement with both the CLEO and the OMEGA results.

The situation is thus by no means clear. In the Stable Particle Table we give the mass determined from $\mathrm{e}^{+} \mathrm{e}^{-}$observations of the $\phi \pi^{ \pm}$mode, $\mathrm{m}_{\mathrm{F}}=(1971 \pm 6)$ $\mathrm{MeV}$, to which we attach a cautionary footnote pointing out the conflicting results. We list decay modes of the $F$ other than $\phi \pi$ as "possibly seen" because the conflicting mass values suggest that these other modes may not be observations of the $1971-\mathrm{MeV}$ state adopted here as the F.

\section{References}

1. L. Maiani, J. Phys. (Paris), Colloq. 43, C3-631 (1982); and D. Fakirov and B. Stech, Nucl. Phys. B133, 315 (1978).

2. N.W. Reay, Proceedings 1983 International Lepton/Photon Symposium (Cornell, 1983), eds. D.G. Cassel and D.L. Kreinick, p. 244.

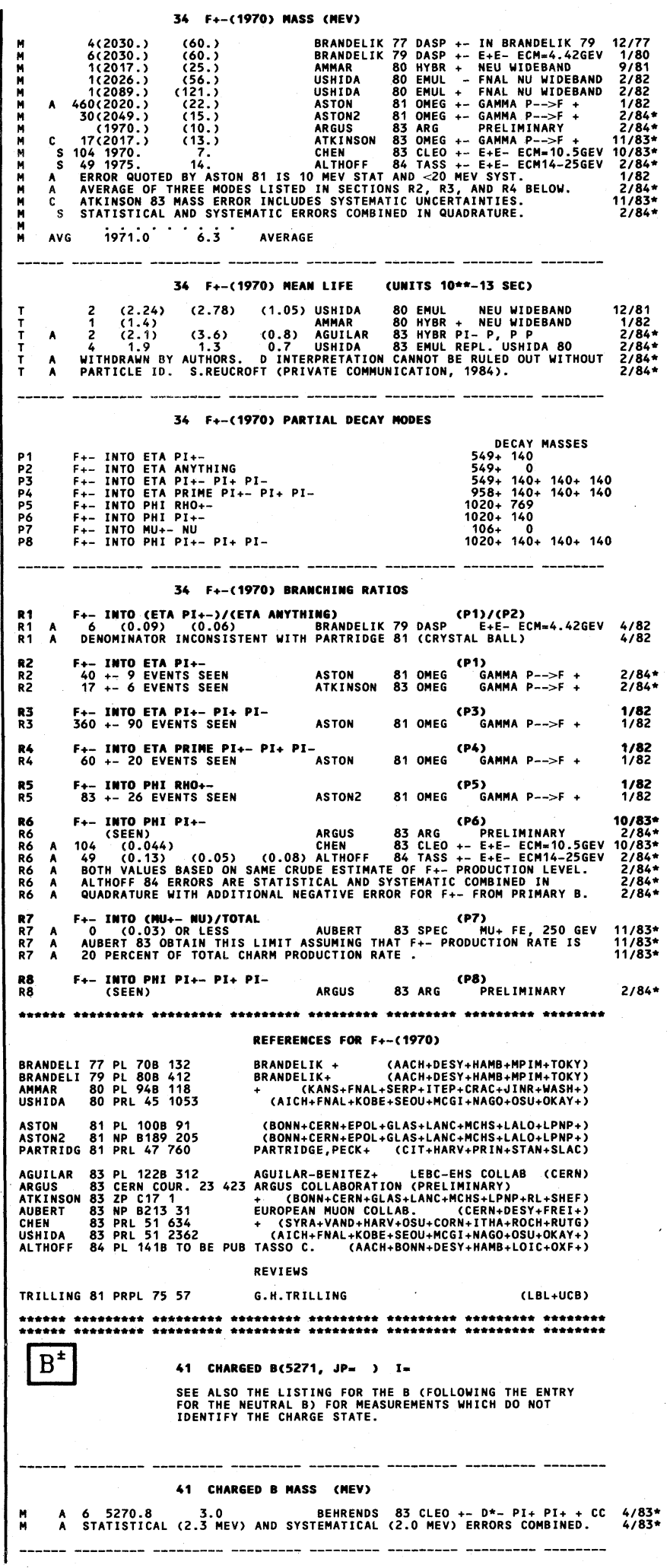




\section{Stable Particles}

$B^{ \pm}, B^{0}, B, p$

\section{Data Card Listings}

41 charged D partial decay modes

$P 1$
$P 2$

8- modes are charge conjugates of the above modes.

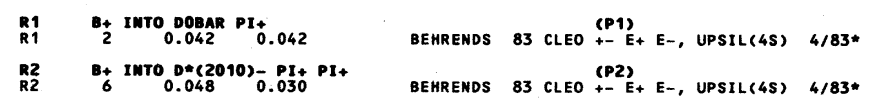

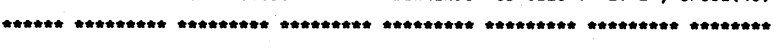
REFEREMCES FOR CHARGED B

BEHRENDS 83 PRL 50881 + (ROCH+RUTG+SYRA+VAND+CORN+ITHA+HARV+OSU) *

$\mathrm{B}^{0} \quad 42$ meUtraL B(5274, JP- , $1=$ SEE ALSO THE LISTING FOR THE B (FOLLOHING THIS ENTRY)
FOR MEASUREMENTS WHICH DO NOT IDENTIFY THE CHARGE

42 MEUTRAL B mASS (MEV)

MA A 5 A $5274.2\left(\begin{array}{l}2.8 \\ \text { MTATISTICAL }\end{array}\right.$

$42(B O)$ - (B+) MASS DIFFERENCE (MEV)

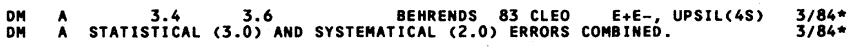

42 meutral D partial decay modes

P1 BO INTO DOBAR PI+ PI-
P2
BO INTO D*(2010) PI+

DECAY MASSES
$1865+140+140$
$2007+140+140$

BObar mOdes are charge CONJUgates of the above modes.

\section{MEUTRAL B BRAMCMIMG RATIOS}

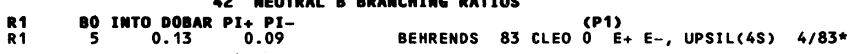

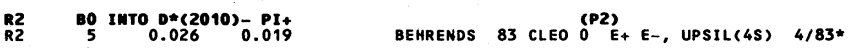

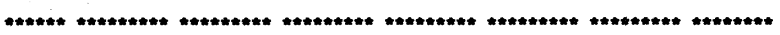
REFEREMCES FOR MEUTRAL B

BEHRENDS 83 PRL $50881+$ + (ROCH+RUTG+SYRA+VAND+CORN+1THA+HARV+OSU)

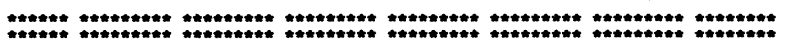
B

39 BOTTOM MESON B(5271, JP= )

THIS ENTRY LISTS MEASUREMENTS OF B MESON PARAMETERS FOR WHICH THE CHARGE STATES ARE NOT SEPARATED
MEASUREENTS IN WHICH THE CHARGE STATE IS CLEARLY IDENTIFIED ARE LISTED IN THE PRECEOING CHARGED B

39 B MASS (MEV)
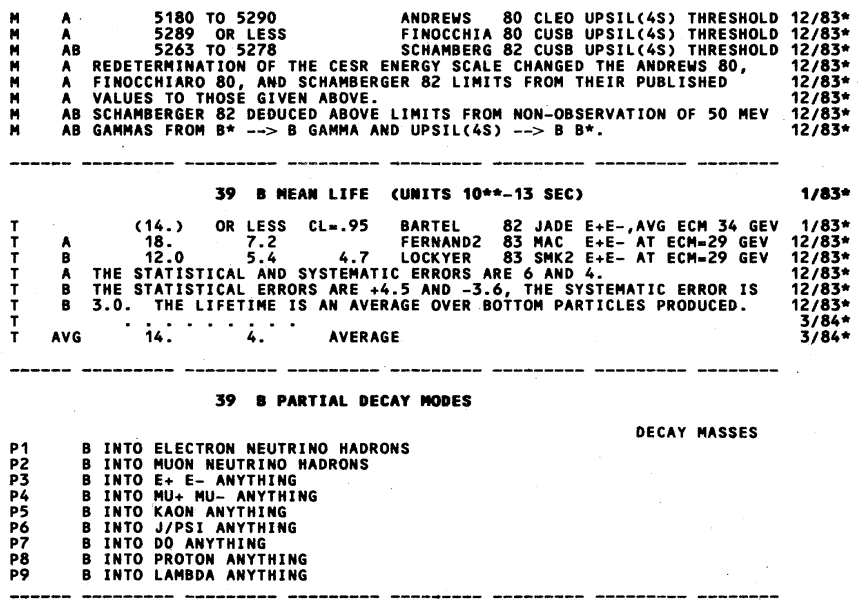

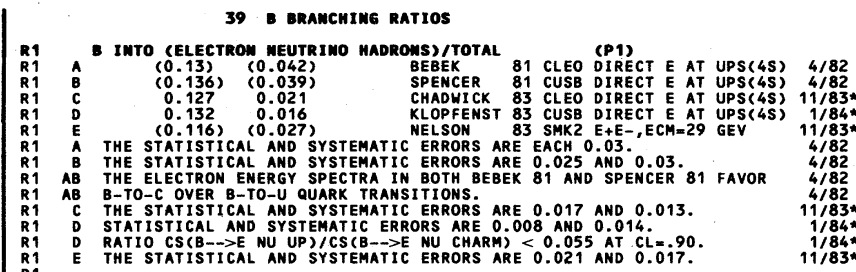

ONLY the EXPERIMENTS AT the UPSILON(4S) aRe USED IN the aVERAGE.

avg $\cdot \dot{0} 130^{\circ} \cdot \dot{0} 01 \dot{3}^{\circ}$ average

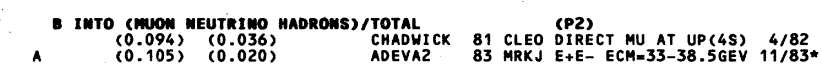

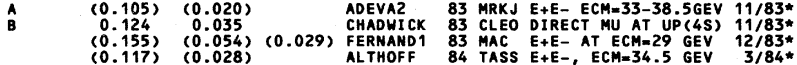

A THE STATISTICAL AND SYSTEMATIC ERRORS ARE 0.015 AND 0.013 .

THE AVERAGE OF THE THREE HIGH-ENERGY RESULTS IS 0 .113+-0.016. THE B MESON.

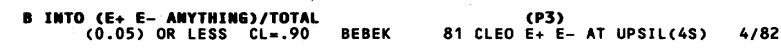

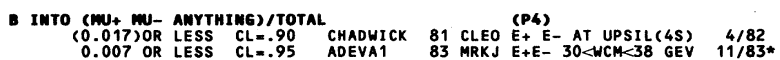

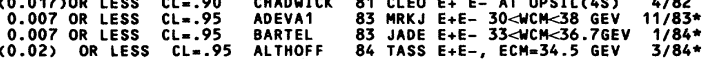

B INTO COILEPTON AMYTHIMG)
0.008 OR LESS CL=.90 MATTEUZZI 83 SMK2 E+ E- E-AT ECM=29 GEV 11/83*

c imto (KaON anYthing)/TOtal

SEEN

$\begin{array}{llll}\text { BRODY } & 82 \text { CLEO KAONS AT UPSIL( } 4 S \text { ) } & 4 / 82 \\ \text { GIANNINI } & 82 \text { CUSB KAONS AT UPSII( } 4 S \text { ) } & 2 / 84 *\end{array}$

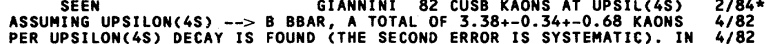

PER UPSTLON(4S) DECAY IS FOUND (THE SECOND ERROR IS SYSTEMATIC). IN $4 / 82$
THE CONTEXT OF THE STANDARD B-DECAY MODEL, THIS LEADS TO A VALUE
FOR

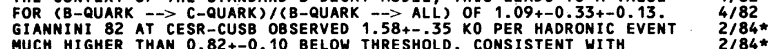

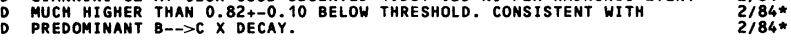

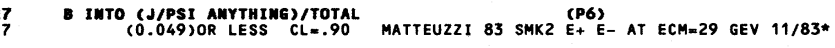

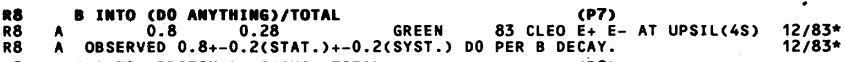

R9 A INTO (PROTOW AMYTHIMG)/TOTAL
R9 ALAM

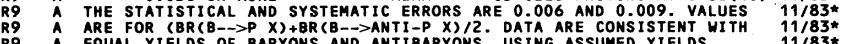

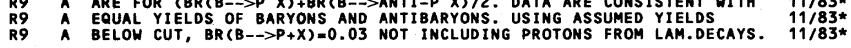

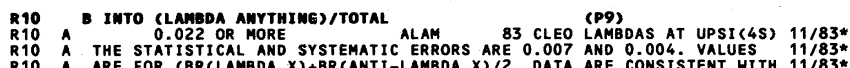

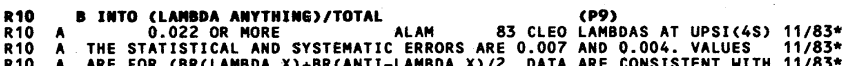

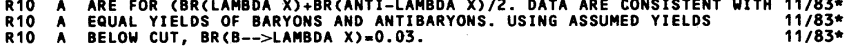

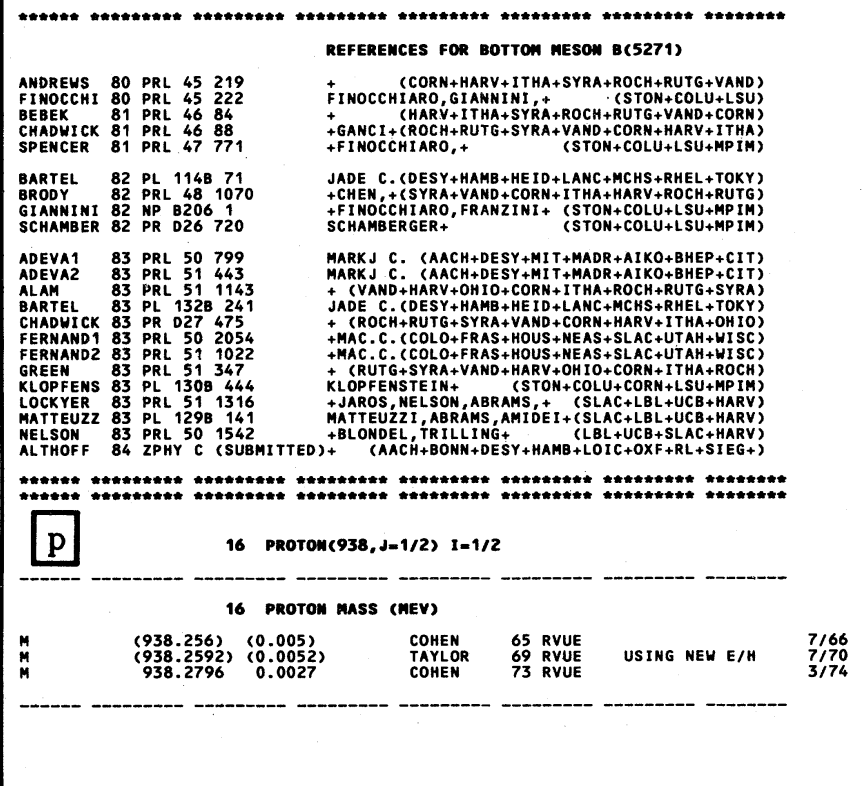




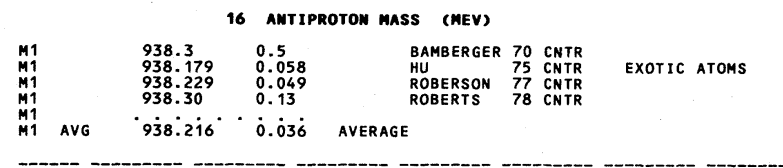

\section{NOTE ON PROTON MEAN LIFE LIMITS}

(by M. Goldhaber, Brookhaven National Laboratory, and F. Reines, University of California, Irvine)

Current ideas on the unification of the weak, electromagnetic, and strong forces suggest that baryon number might not be strictly conserved, so that the proton could decay. In the Particle Properties Tables there are nearly thirty particles listed with a mass smaller than that of the proton (if we count both particles and antiparticles and different members of multiplets separately). Ten of these particles are fermions and the remainder bosons. There are then a great many possible two-body decay modes of the proton and an even larger number of three-body, etc., decay modes which satisfy charge, energy, momentum, and angular momentum conservation. Each decay mode has to contain at least one fermion to satisfy angular momentum conservation.

The "decay signature" distributions as well as the backgrounds depend on detector characteristics (the material from which the detector is made, the method of detection, timing information, time resolution, etc.). The background, due chiefly to atmospheric neutrinos, depends also on the geomagnetic latitude and on the phase of the solar cycle with which the magnetic field of the sun is associated. The depth-dependent cosmic ray background is due to cosmic ray muons and their progeny. For each possible proton decay signature there is a finite probability of a background event with a similar signature, where the probability depends on the detector characteristics.

The Data Card Listings following this note show only published results. The lower limits quoted are partial mean life (mean life divided by branching fraction) for the proton or bound neutron decay mode listed at the right.

Since there are many new unpublished results, we also show a table of the latest (preliminary) results as reported at the ICOBAN' 84 Conference at Park City, Utah, Jan. 4-8. This table is based on the summary talk given at the conference by $W$. Allison, Oxford. The table shows the $90 \%$ confidence level lower limits on the partial mean life for decay to the mode shown. The units are $10^{30}$ years.

The number of candidate events is shown in brackets. A particular event may be listed as a candidate for several modes. The IMB collaboration considers their candidate events to be qualitatively compatible with neutrino background. An initial report on their neutrino background is given in Bionta et al., Phys. Rev. Lett. 51, 27 (1983). The Kamiokande background estimates are given after the slash (/). The Kolar-Goldfield experiment (KRISHNASWAMY 82, Data Card Listings) has a total of 6 candidates for nucleon decay. Until the above results can be reproduced consistently and above neutrino background, it is too early to draw any conclusions about proton instability.

The simplest grand unified theory, minimal SU(5), predicts $\mathrm{e}^{+} \pi^{0}$ to be the predominant proton decay mode. The IMB lower limit on the partial mean life for this mode, $2 \times 10^{32}$ years, is at least a factor of 10 higher

Lower limits on partial mean life $(\tau / \mathrm{B})$ for proton or bound neutron decay. (Adapted from W. Allison, summary talk at ICOBAN '84.)

\begin{tabular}{|c|c|c|c|}
\hline & NUSEX & IMB* & Kamiokande $^{\dagger}$ \\
\hline $\begin{array}{l}\text { Sensitivity } \\
\text { (ton-years) }\end{array}$ & 180 & 2300 & 324 \\
\hline Mode & \multicolumn{3}{|c|}{$\tau / \mathrm{B}$ limit, $\mathrm{CL}=90 \%$, units $10^{30} \mathrm{yr}$} \\
\hline $\begin{aligned} \mathrm{p} \rightarrow & \mathrm{e}^{+} \pi^{0} \\
& \mu^{+} \pi^{0} \\
& \mathrm{e}^{+} \gamma \\
& \mu^{+} \gamma\end{aligned}$ & $\begin{array}{r}>10 \\
>7\end{array}$ & $\begin{array}{l}>200 \\
>120 \\
>220 \\
>160\end{array}$ & $\begin{array}{l}>26 \\
>18\end{array}$ \\
\hline $\begin{array}{l}\mathrm{e}^{+} \eta \\
\mu^{+} \eta \\
\mathrm{e}^{+} \mathrm{K}^{0} \\
\mu^{+} \mathbf{K}^{0}\end{array}$ & $>13 \quad 1 \mathrm{ev}$ & $\begin{array}{l}>130 \\
>40 \\
>31 \\
>26\end{array} \quad[2 \mathrm{ev}]$ & $\begin{array}{rr}>18 & \\
>8 & {[1 \mathrm{ev} / 0]} \\
>18 & \\
>8 & {[1 \mathrm{ev} / 0]}\end{array}$ \\
\hline $\begin{array}{l}\mathrm{e}^{+} \omega \\
\mu^{+} \omega \\
\nu \mathbf{K}^{+} \\
\nu \pi^{+}\end{array}$ & $\begin{array}{r}>5 \\
>4 \\
\end{array}$ & $\left.\begin{array}{l}>41 \\
>51 \\
>12\end{array}\right]\left[\begin{array}{ll}1 & \mathrm{ev} \\
2 & \mathrm{ev} \\
3 \mathrm{ev}\end{array}\right]$ & $\begin{array}{ll}>6 & {[1 \mathrm{ev} / 0.2]} \\
>7 & {[3 \mathrm{ev} / 1]} \\
>3 & {[5 \mathrm{ev} / 5.5]}\end{array}$ \\
\hline $\begin{aligned} & \mathrm{n} \rightarrow \mathrm{e}^{+} \pi^{-} \\
& \mu^{+} \pi^{-} \\
& \nu \pi^{0} \\
& \nu \mathrm{K}^{0} \\
&\end{aligned}$ & $\begin{array}{r}>19 \\
>4 \\
>10 \\
>6 \\
\end{array}$ & $\underset{-8[3 \mathrm{ev}]}{-}$ & $\begin{array}{r}>9 \\
>11 \\
>15 \\
>8\end{array}$ \\
\hline
\end{tabular}

*No events observed unless listed in brackets. Background subtraction not made.

${ }^{\dagger}$ Brackets contain the number of candidates observed followed by estimated background in listed mode. 


\section{Stable Particles}

p, $\mathrm{n}$

than predicted by minimal SU(5) theory.

See also the reviews by Reines and Schultz, Surveys in High Energy Physics 1, 89 (1980); Goldhaber, Langacker, and Slansky, Science 210, 851 (1980); and Goldhaber and Sulak, Comments Nucl. Part. Phys. 10, 215 (1981).

See also the neutron-antineutron oscillations section NAN in the neutron Data Card Listings below for another test of baryon conservation.

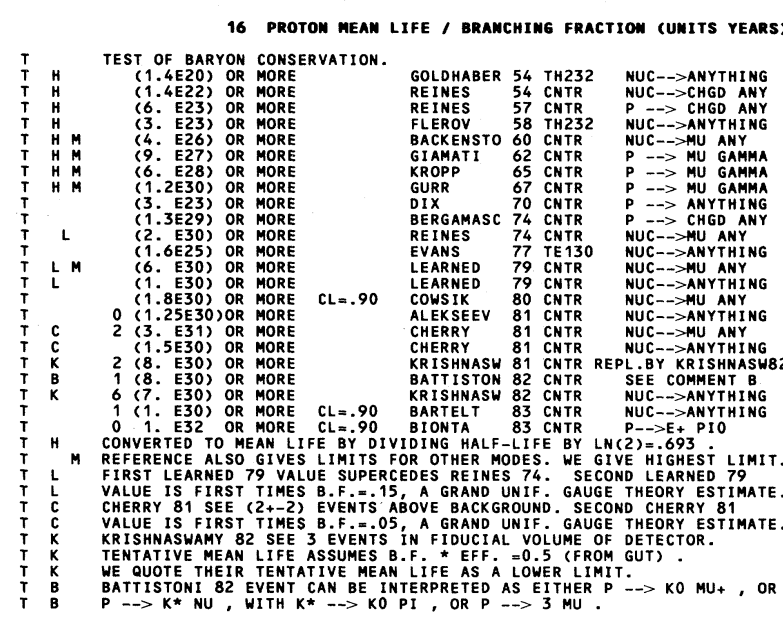

16 ANTIPROTON MEAN LIFE / BR. FRACTION (UNITS hOURS)

LIMITS SHOWN ARE PARTIAL MEAN LIFE FOR MODE SHOWN AT. RIGHT.

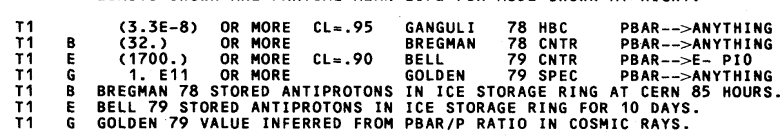

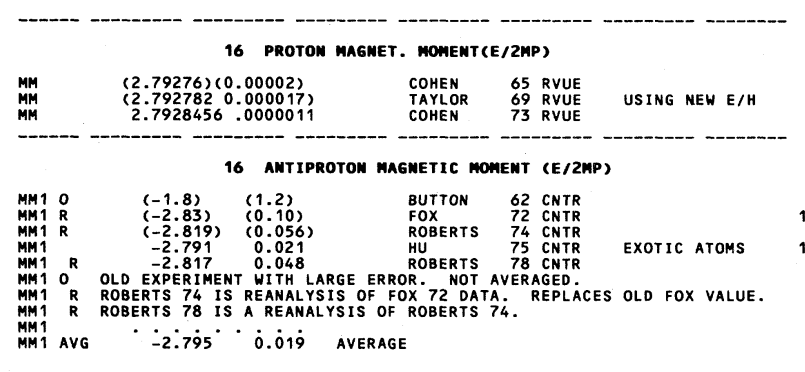

\begin{tabular}{|c|c|c|c|c|}
\hline \multirow[b]{2}{*}{$\begin{array}{l}\text { EDM } \\
\text { EDM } \\
\text { EDM }\end{array}$} & \multicolumn{3}{|c|}{$\begin{array}{l}16 \text { PROTON ELECTRIC DIPOLE } \\
\text { FORB IDDEN BY BOTH T INVARIANCE }\end{array}$} & $\begin{array}{l}\text { MOMENT (UNITS } 10 * *-23 \mathrm{E}(\mathrm{CH}) \\
\text { AND } P \text { INVARIANCE }\end{array}$ \\
\hline & $\begin{array}{l}16(700 .) \\
(55000)) \\
4000 .)\end{array}$ & $\begin{array}{l}(900 .) \\
\text { OR LESS } \\
\text { OR LESS }\end{array}$ & $\begin{array}{l}\text { HARRISON } \\
\text { KHRIPLOVI } \\
\text { RAMSEY }\end{array}$ & $\begin{array}{l}69 \\
76 \\
72 \\
82\end{array}$ \\
\hline
\end{tabular}

16 PROTON ELECTRON CHARGE DIFFEREMCE (UNITS E)

SeE also section Q in the neutron data cards listings below.

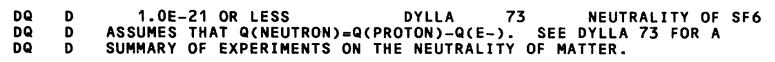

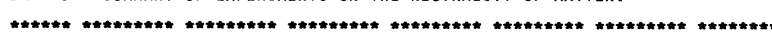

\section{Data Card Listings}

REFERENCES FOR PROTOM

GOLDHABE 54 PR 96
RENES
54

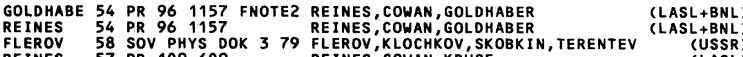

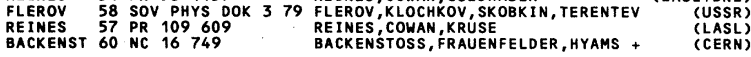

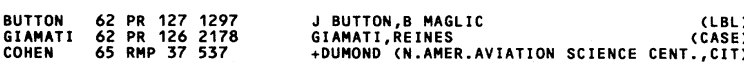

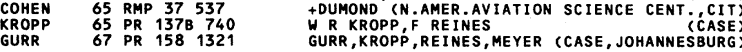
HARRISON 69 PRL $221263 \quad$ HARRISON, SANDARS, WRIGHT (CLARENDON OXFORD)
TAYLOR 69 RMP 41375
(PRIN+UCI+PENN)

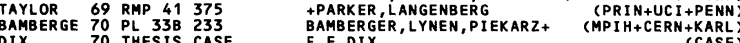

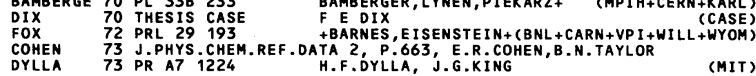
$\begin{array}{lllll}\text { BERGAMAS } 74 & \text { LNC } 11 & 636 \\ \text { REINES } 74 & \text { PRL } 32 & 493\end{array}$

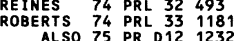

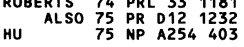
$\begin{array}{llll}\text { KHRIPLOV } 76 & \text { JETP } 44 & 25 \\ \text { EVANS } 77 & \text { SCIENCE } 1979 & 989\end{array}$ ROBERSON 77 PR C16 1945

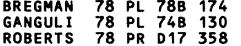
$\begin{array}{llll}\text { BELL. } & 79 & \text { PL } 86 B & 215 \\ \text { GOLDEN } & 79 & \text { PRL } 43 & 1196\end{array}$

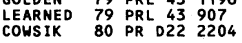

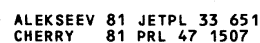
$\begin{array}{lll}\text { CHERRY } 81 \text { PRL } 47 & 1507 \\ \text { KRISHNAS } 81 \text { PL } 1068 \quad 339\end{array}$

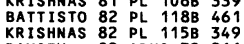

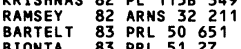
BERGAMASCO, PICCHI (MIT)
(FRAS) + + COX, ECKHAUSE+ (WILL+VPI+CARN+WYOM (UCI+CASE) ROBERTS, COX + (WILL+PPI+CARN + WYOM+CIT+BNL)
+ASANO, CHEN, CHENG, DUGAN+ (COLU+YALE) I.B.KHRIPLOVICH (NUC.PHYS.INST.,SIBERIA)

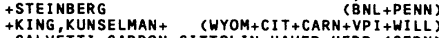
+CALVETI, CARRON, CI TOLLIN, HAUER, HERR + (CERN)
+MALHOTRA, RAGHAVAN, SUBRAMANIAN +
B. L. ROBERTS
(WILL+RHEL) +CALVETTI, CARRON, CHANEY, CITTOLIN+ (CERN) HORAN, MAUGER, BAD HWAR, LACY + (NASA+PSLL)
(UEI +REINES, SONI
R.COHSIK,V.S.NARASIMHAN (TIFI) +BAKATANOV BUTKEVICH, VOEVOOSKII + (LENI) +DEAKYNE, LANDE, LEE, STEINBERG + (PENN+BNL) X RRISHNASWAMY, MENON, MONDAL+ (TIFR+OSKC+TOKY) N. F.RAMSEY
+ COURANT, HELLER, JOYCE, MARSHAK+ (MHCO+HARV)
(MINN+ANL) QUANTUM NUMBER DETERMINATIONS NOT REFERRED TO IN THE DATA CARDS

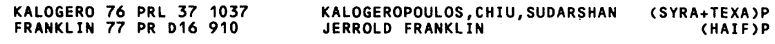

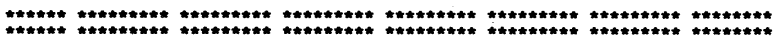

$\mathrm{n}$ 17 MEUTROM(939, J=1/2) $I=1 / 2$

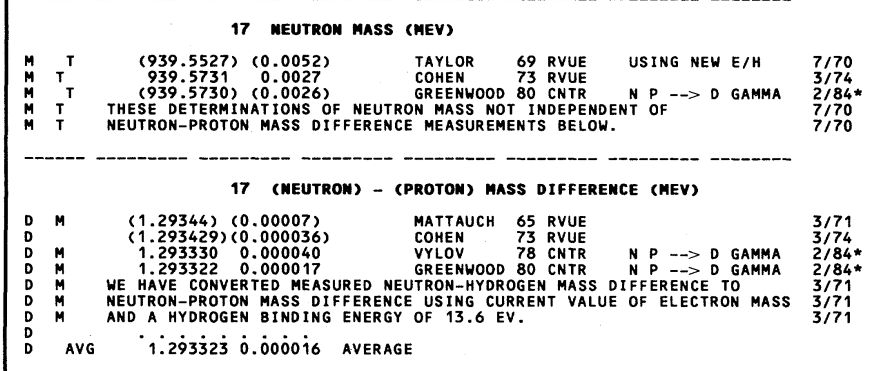
7/70 0.000016 AVERAGE

17 meutron mean Life (UNITS 10**3 SEC)

THE ORIGIN OF THE DISCREPANCIES BETWEEN MEASUREMENTS OF THE MEUTRON MEAN LIFE IS NOT KNOWN. WE AVERAGE THE HIGHEST PRECISION ERROR, AS USUAL. THE IDEOGRAM SHOWS THE INVERSE MEAN LIFE, THE
QUANTITY ACTUALIY USED IN CALCULATING OUR AVERAGE VALUE.

$\begin{array}{ll}(1.013) & (0.026) \\ (0.935) & (0.014)\end{array}$

$\begin{array}{cc}(0.935) & (0.014) \\ 0.918 & 0.014 \\ 0.881 & 0.008\end{array}$

0.93

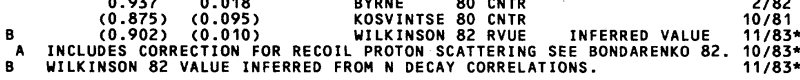

SOSNOVSKI 59 CNTR

CHRISTENS 67 CNTR BONDARENK 78 CNTR
BYRNE 80 CNTR

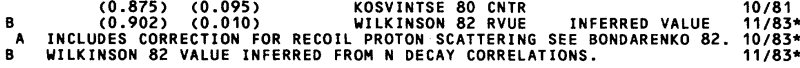
AVG $0.898^{\circ} \begin{aligned} & 0.016 \\ & \text { (SEE IDEOGRAM BeLOW) }\end{aligned}$

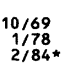

17 MEUTRON MAGMETIC MOMENT (MAGNETONS, 938.2 MEV)

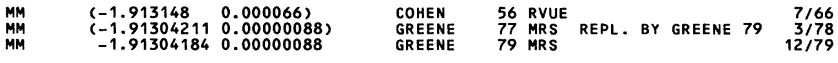




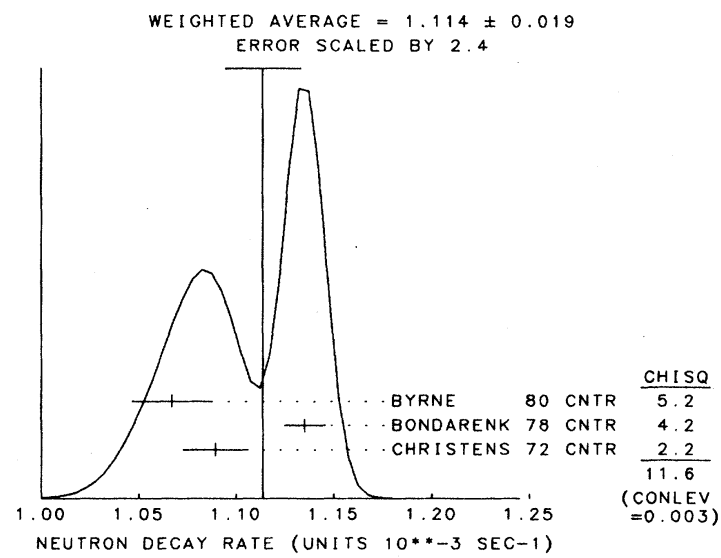

17 MEUTRON ELECTRIC DIPOLE MOMENT (UNITS 10**-23 E CM) FORBIDDEN BY BOTH T INVARIANCE AND P INVARIANCE

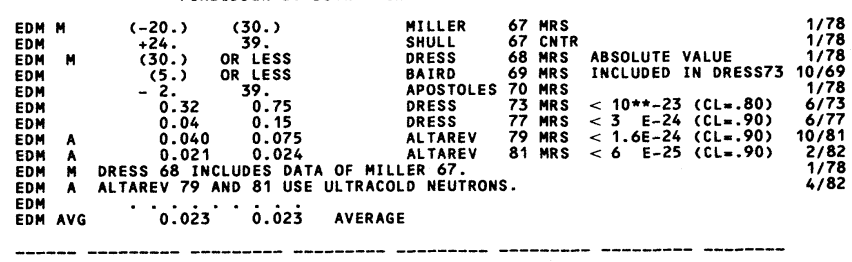

17 MEUTRON CHARGE

SEe aLSO SECTION DQ IN THE PROTON DATA CARD LISTINGS ABOVE

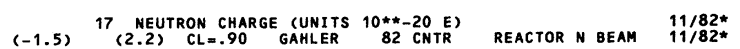

17 LIMIT ON MEUTROM-AMTIMEUTROM OSCILLATIONS

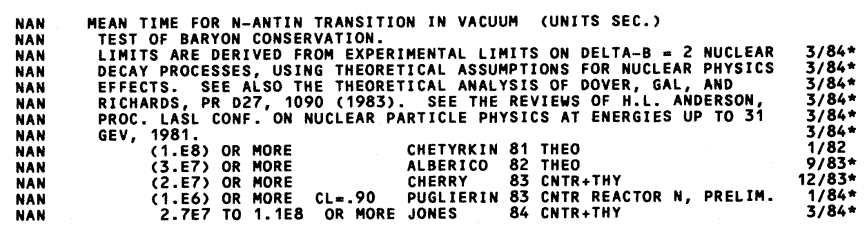

17 MEUTROM PARTial decay MODES

DECAY MASSES

P1 NEUTRON INTO PROTON E- ANTI (NUE)
P2 NEUTRON INTO PROTON NUE ANTI(NUE)

$938+.511+$
$938+$
$93+0$

17 MEUTRON BRAMCHIMG RATIOS

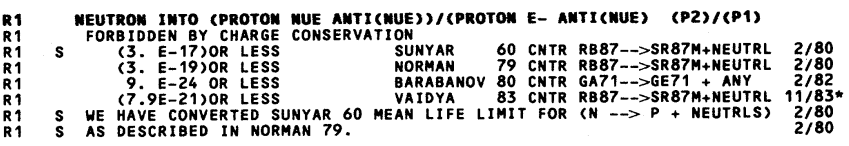

NOTE ON BARYON DECAY PARAMETERS

A/V ratio for baryon leptonic decays

Consider the decay

$\mathrm{B}_{\mathrm{i}} \rightarrow \mathrm{B}_{\mathrm{f}}+\ell+\nu$.

Assuming V, A theory, neglecting "induced" scalar, "induced" pseudoscalar, and axial weak-magnetism terms, and neglecting the $\mathrm{q}^{2}$ dependence of the form factors, the baryon part of the matrix element for these decays may be written as 1

$$
\bar{B}_{f}\left[\gamma_{\lambda}\left(g_{V}-g_{A} \gamma_{5}\right)+\left(g_{W} / m_{B_{i}}\right) \sigma^{\lambda \nu} q_{\nu}\right] B_{i}
$$

Here $B_{i}$ and $\bar{B}_{f}$ are spinors which represent initial and final baryons, $g_{A}$ and $g_{V}$ are the axial and vector coupling constants, $\mathrm{g}_{\mathbf{W}}$ is the weak magnetism coupling constant, and $\mathrm{q}_{\nu}$ is the sum of the lepton momenta. The Pauli representation is used for the $\gamma$ matrices. The ratio $g_{A} / g_{V}$ may be written as

$$
\mathbf{g}_{\mathrm{A}} / \mathbf{g}_{\mathrm{V}}=\left|\mathrm{g}_{\mathrm{A}} / \mathrm{g}_{\mathrm{V}}\right| \exp (\mathrm{i} \phi),
$$

where $\phi$ is 0 plus $n \pi$ if time reversal holds. ${ }^{2}$

Experiments on the leptonic decays of baryons other than the neutron have generally assumed $\phi$ to be either 0 or $\pi$, and have thus measured the magnitude and sign of $\mathrm{g}_{\mathrm{A}} / \mathrm{g}_{\mathrm{V}}$. In studying neutron beta decay, however, experiments have been sensitive enough to measure $\phi$ more precisely, and we include the phase angle in our Listings for this case. It is consistent with time-reversal invariance, and by using the above definition of the matrix element with the Pauli representations, the value of $g_{A} / g_{V}$ in neutron beta decay is negative.

Due to statistical limitations, the weak magnetism form factor $\mathrm{g}_{\mathrm{W}}$ is usually assumed from CVC and SU(3), so that usually only $g_{A}$ and $g_{V}$ are determined experimentally. This determination is accomplished in a variety of ways:

(a) The lepton-neutrino angular correlation provides a measure of the absolute value of $g_{A} / g_{V}$ (for relevant formulas, see, e.g., Albright ${ }^{3}$ ).

(b) The up-down asymmetry of the lepton from polarized baryon decays provides a measure of $\mathrm{g}_{\mathrm{A}} / \mathrm{g}_{\mathrm{V}}$ with its sign (for relevant formulas, see, e.g., Albright ${ }^{3}$ ).

(c) The lepton spectrum, given enough statistics, provides a measure of $\mathrm{g}_{\mathrm{A}} / \mathrm{g}_{\mathrm{V}}$ with its sign (for relevant formulas, see, e.g., Bender et al. ${ }^{4}$ ). The lepton spectrum also provides a measure of $\mathrm{g}_{\mathrm{W}} / \mathrm{g}_{\mathrm{A}}$ if the CVC-SU(3) assumption is relaxed.

(d) The polarization of the decay baryon, from polarized or unpolarized initial baryon, also provides $g_{A} / g_{V}$ with its sign (for formulas, see, e.g., Willis and Thomp$\left.\operatorname{son}^{5}\right)$.

(e) The presence of a triple correlation term proportional to 


\section{Stable Particles}

$$
\boldsymbol{\sigma}_{\mathbf{B}_{\mathrm{i}}} \cdot\left(\mathbf{p}_{\mathrm{e}} \times \mathbf{p}_{\nu}\right)
$$

where the initial baryon is polarized or

$$
\boldsymbol{\sigma}_{\mathrm{B}_{\mathrm{f}}} \cdot\left(\mathbf{p}_{\mathrm{e}} \times \mathbf{p}_{\nu}\right)
$$

where the polarization of the decay baryon is observed provides a measure of the deviation of $\phi$ from 0 or $\pi$, and is thus a test of time-reversal invariance (see, e.g., Willis and Thompson ${ }^{5}$ ).

We compile the ratio $g_{A} / g_{V}$ with its sign, for those decays for which it has been measured.

All the coupling constants and decay rates for baryon leptonic decays are related by Cabibbo's theory, ${ }^{6}$ extended to six quarks (and three mixing angles) by Kobayashi and Maskawa. ${ }^{7}$ A discussion of the Kobayashi-Maskawa mixing matrix is given in Appendix III to this edition.

\section{Asymmetry parameters in nonleptonic hyperon decays}

The transition matrix for hyperon decay may be written as

$$
\mathbf{M}=\mathbf{s}+\mathbf{p}(\boldsymbol{\sigma} \cdot \mathbf{q}),
$$

where $s$ and $p$ are the parity-changing and the parityconserving amplitudes, respectively; $\sigma$ is the Pauli spin operator, and $\mathbf{q}$ is a unit vector along the direction of the decay baryon in the hyperon rest frame.

The asymmetry parameters are defined by the relations

$$
\begin{aligned}
& \alpha=2 \operatorname{Re}\left(\mathrm{s}^{*} \mathrm{p}\right) /\left(|\mathrm{s}|^{2}+|\mathrm{p}|^{2}\right), \\
& \beta=2 \operatorname{Im}\left(\mathrm{s}^{*} \mathrm{p}\right) /\left(|\mathrm{s}|^{2}+|\mathrm{p}|^{2}\right), \\
& \gamma=\left(|\mathrm{s}|^{2}-|\mathrm{p}|^{2}\right) /\left(|\mathrm{s}|^{2}+|\mathrm{p}|^{2}\right) .
\end{aligned}
$$

With the transition matrix $M$ given by Eq. (1) above, the angular distribution of the decay baryon, in the hyperon rest system, is of the form

$$
\mathbf{I}=1+\alpha \mathbf{P}_{\mathbf{Y}^{\cdot}} \mathbf{q},
$$

where $\mathbf{P}_{\mathbf{Y}}=\langle\mathbf{Y}|\boldsymbol{\sigma}| \mathbf{Y}\rangle$ is the hyperon polarization. In the notation of Lee and Yang, ${ }^{8}$ the polarization $\mathrm{P}_{\mathrm{B}}$ of the decay baryons is

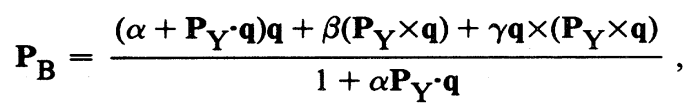

where $\mathbf{P}_{\mathbf{B}}$ is defined in that rest system of the baryon obtained by a Lorentz transformation along $q$ from the hyperon rest system in which $\mathbf{q}$ and $\mathbf{P}_{\mathbf{Y}}$ are defined.

Note that $\alpha$ is the helicity of the decay baryon for unpolarized hyperons.

The three parameters $\alpha, \beta$, and $\gamma$ satisfy the relation

$$
\alpha^{2}+\beta^{2}+\gamma^{2}=1 \text {. }
$$

It is then convenient to describe hyperon nonleptonic decays in terms of the two independent parameters $\alpha$ and the angle $\phi$ defined by

$$
\begin{aligned}
& \beta=\left(1-\alpha^{2}\right)^{1 / 2} \sin \phi, \\
& \gamma=\left(1-\alpha^{2}\right)^{1 / 2} \cos \phi,
\end{aligned}
$$

which has a more nearly Gaussian distribution of measurement error than $\beta$ or $\gamma$. Evidently

$$
\begin{array}{ll}
-\frac{1}{2} \pi \leqslant \phi \leqslant \frac{1}{2} \pi & \text { for } \gamma>0, \\
+\frac{1}{2} \pi \leqslant \phi \leqslant \frac{3}{2} \pi & \text { for } \gamma<0 .
\end{array}
$$

In discussing time-reversal invariance, the quantity of interest is $\Delta$, defined by

$$
\begin{aligned}
& \alpha=2|\mathrm{~s}||\mathrm{p}| \cos \Delta /\left(|\mathrm{s}|^{2}+|\mathrm{p}|^{2}\right), \\
& \beta=-2|\mathrm{~s}||\mathrm{p}| \sin \Delta /\left(|\mathrm{s}|^{2}+|\mathrm{p}|^{2}\right) ;
\end{aligned}
$$

that is, $\Delta$ is the phase angle of $s$ relative to $p$. Evidently

$$
\begin{array}{ll}
-\frac{1}{2} \pi \leqslant \Delta \leqslant \frac{1}{2} \pi & \text { for } \alpha>0, \\
+\frac{1}{2} \pi \leqslant \Delta \leqslant \frac{3}{2} \pi & \text { for } \alpha<0 .
\end{array}
$$

Under the assumption of time-reversal invariance, the angle $\Delta$ must satisy the relation

$$
\Delta=\delta_{\mathrm{s}}-\delta_{\mathrm{p}},
$$

modulo $\pi$, where $\delta_{\mathrm{s}}$ and $\delta_{\mathrm{p}}$ are the pion-baryon scattering phase shifts at the appropriate energy and for the appropriate isospin state. For $\Lambda$ decay, assuming the validity of the $|\Delta I|=1 / 2$ rule,

$$
\Delta=\delta_{\mathrm{s}}-\delta_{\mathrm{p}}=(7.0 \pm 1.0) \mathrm{deg} .^{9}
$$

In the Stable Particle Data Card Listings we give $\alpha$ and $\phi$ for each decay since they are the most closely related to the experiments and are essentially uncorrelated. Whenever necessary we have changed the signs of the reported values, so as to agree with our conventions. In the Stable Particle Table we give $\alpha, \phi$, and $\Delta$ with errors; and for convenience we also give the central value of $\gamma$, without an error. 


\section{References}

1. M.L. Goldberger and S.B. Treiman, Phys. Rev. 11, 354 (1958).

2. J.D. Jackson, S.B. Treiman, and H.W. Wyld Jr., Phys. Rev. 106, 517 (1957).

3. C.H. Albright, Phys. Rev. 115, 750 (1959).

4. I. Bender, V. Linke, and H.J. Rothe, Z. Physik 212, 190 (1968).

\section{7 meutrom beta decay parameters}

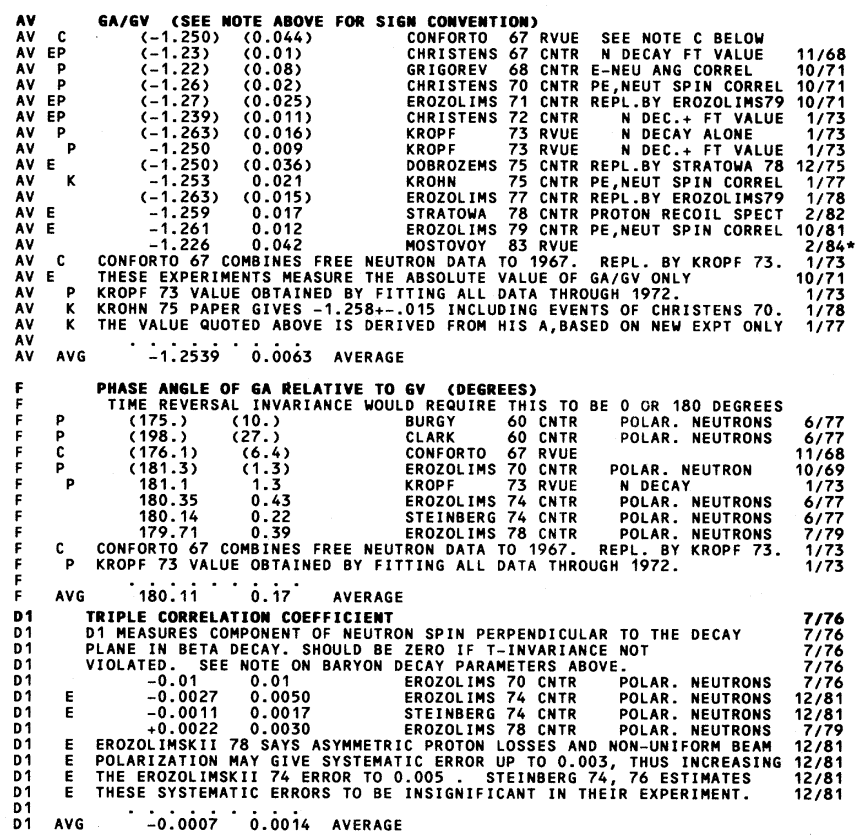

D1 AVG $-0.0007 \quad 0.0014$ AVERAge

\begin{tabular}{|c|c|}
\hline 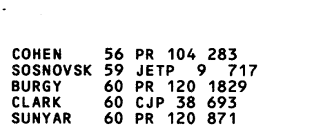 & 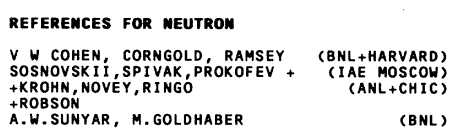 \\
\hline 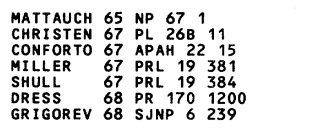 & 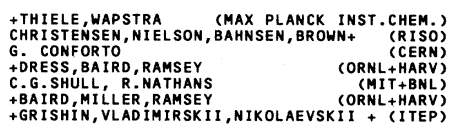 \\
\hline 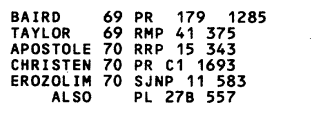 & 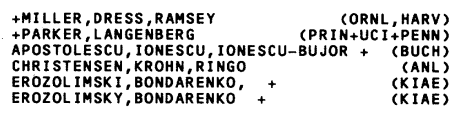 \\
\hline 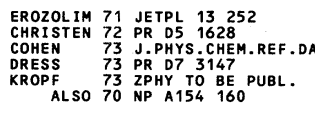 & 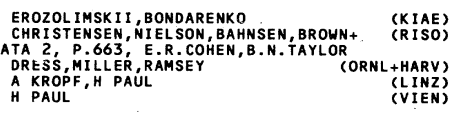 \\
\hline 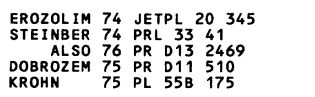 & 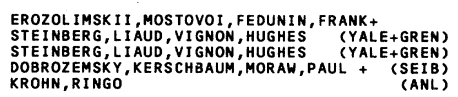 \\
\hline 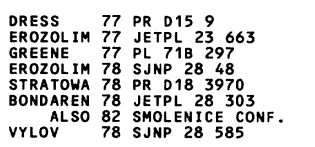 & 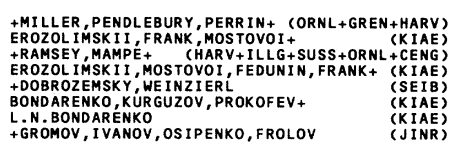 \\
\hline
\end{tabular}

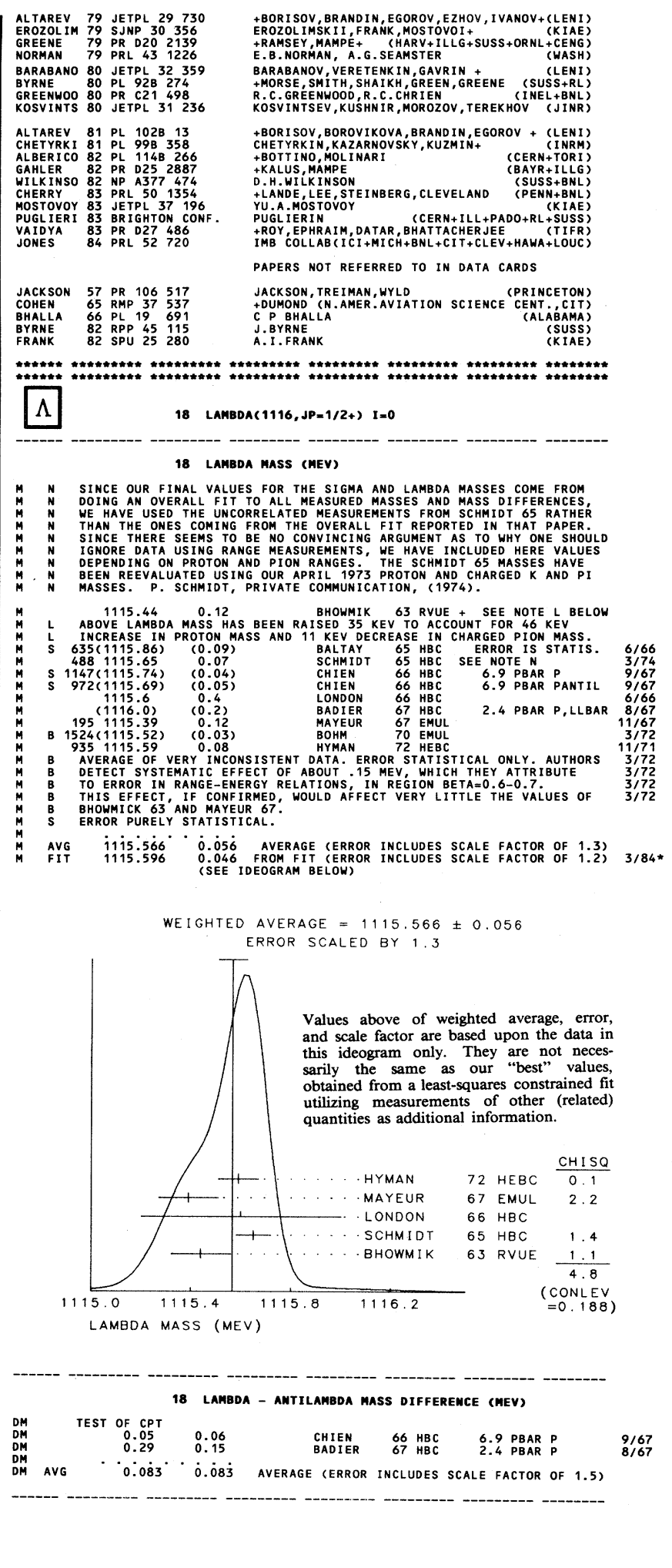




\section{Stable Particles}

\section{Data Card Listings}

$\Lambda$
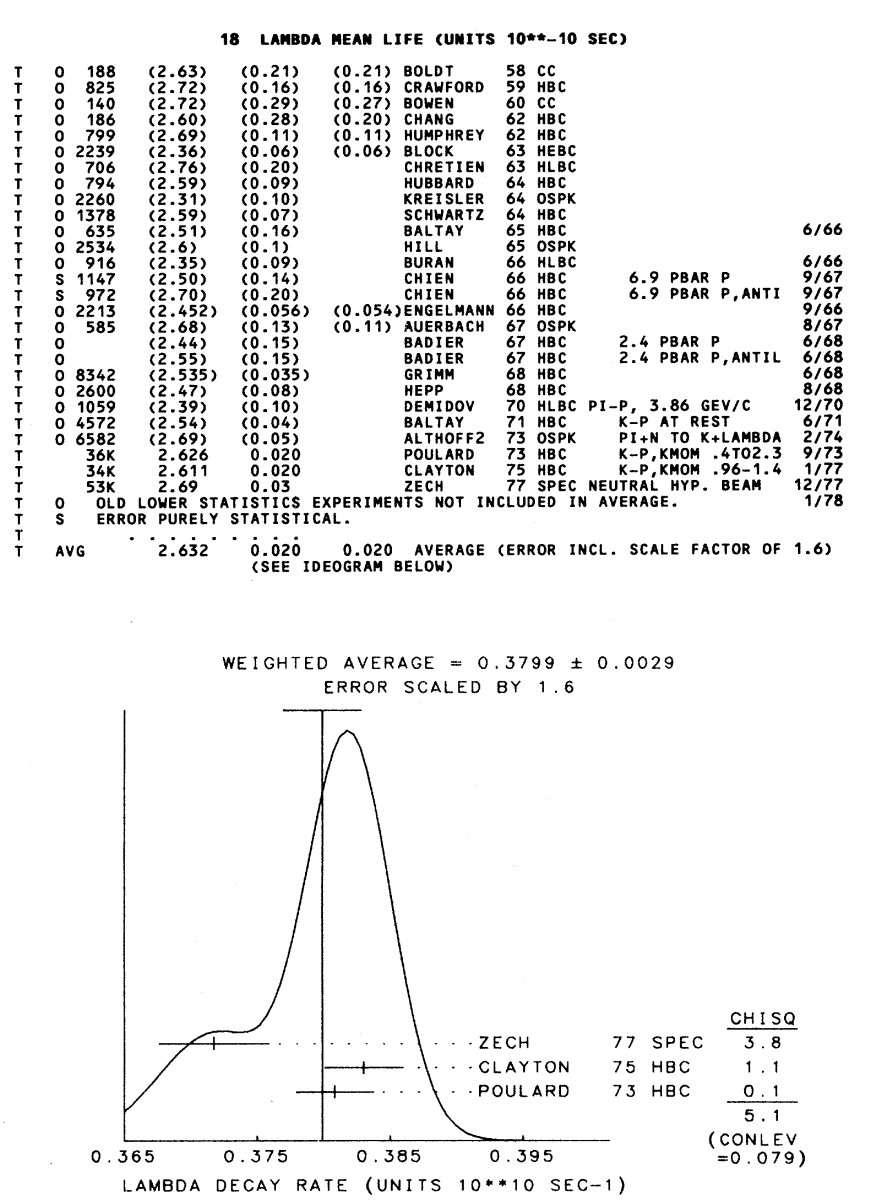

18 (LAMBda - antiLambda)/AVG., mean Life differemce
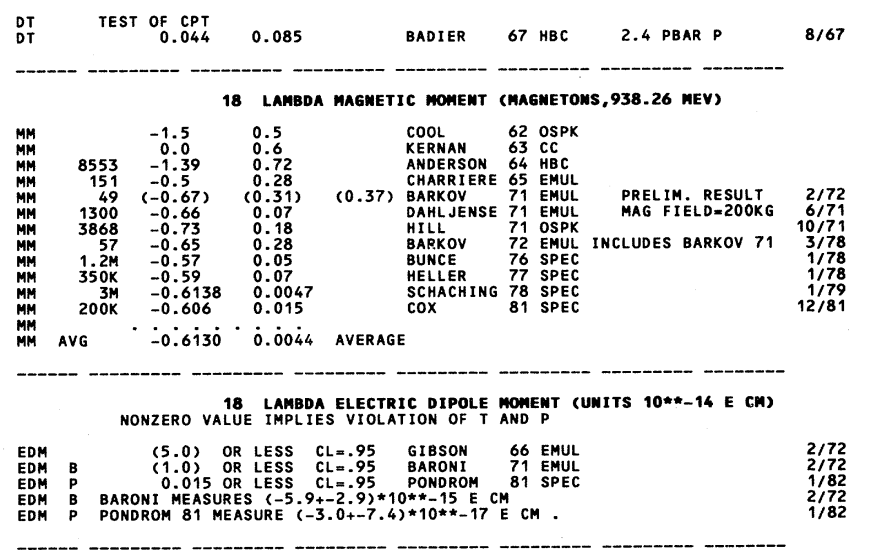

18 Lambda partial decay modes

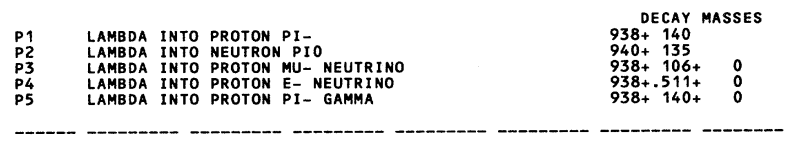

18 Lamboa BRAMChimg ratios

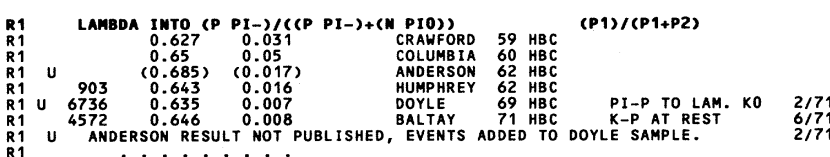

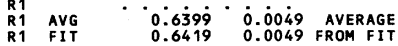

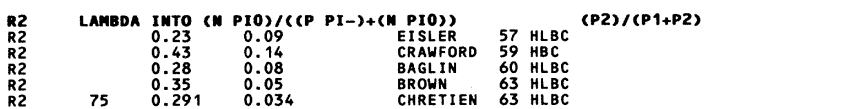

$\begin{array}{lllll}\text { R2 } & \text { AVG } & 0.300^{\circ} \cdot & 0.035 & \text { AVERAGE } \\ \text { R2 } & \text { FIT } & 0.3581 & 0.0049 \text { FROM FIT }\end{array}$

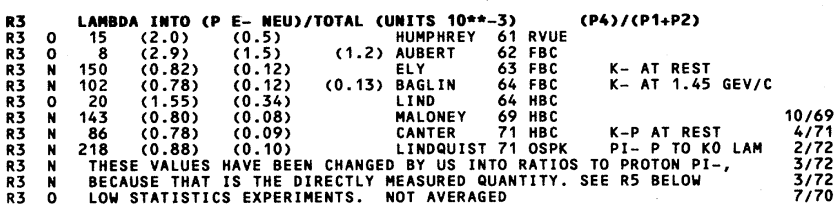

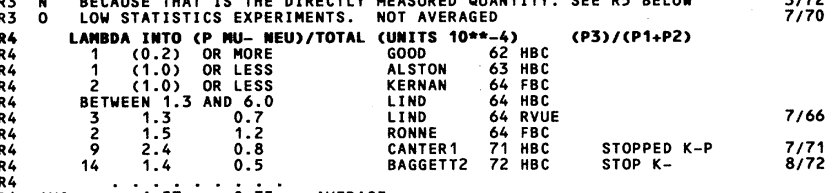

${ }^{2} 4$ avg $\cdot 1.55^{\circ} \cdot 0.35^{\circ}$ average

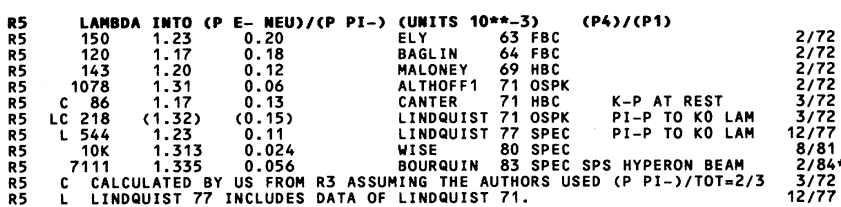

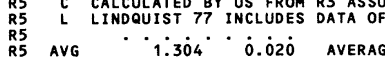

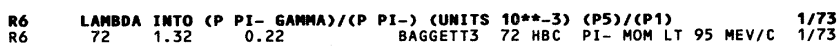

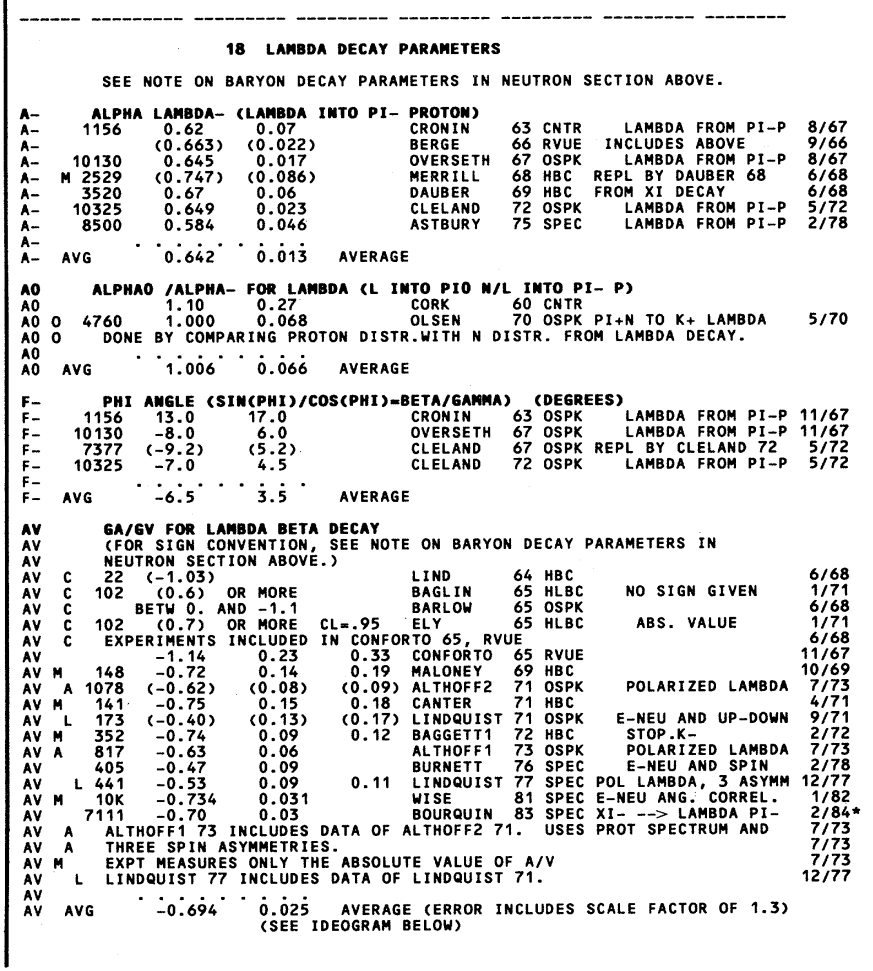



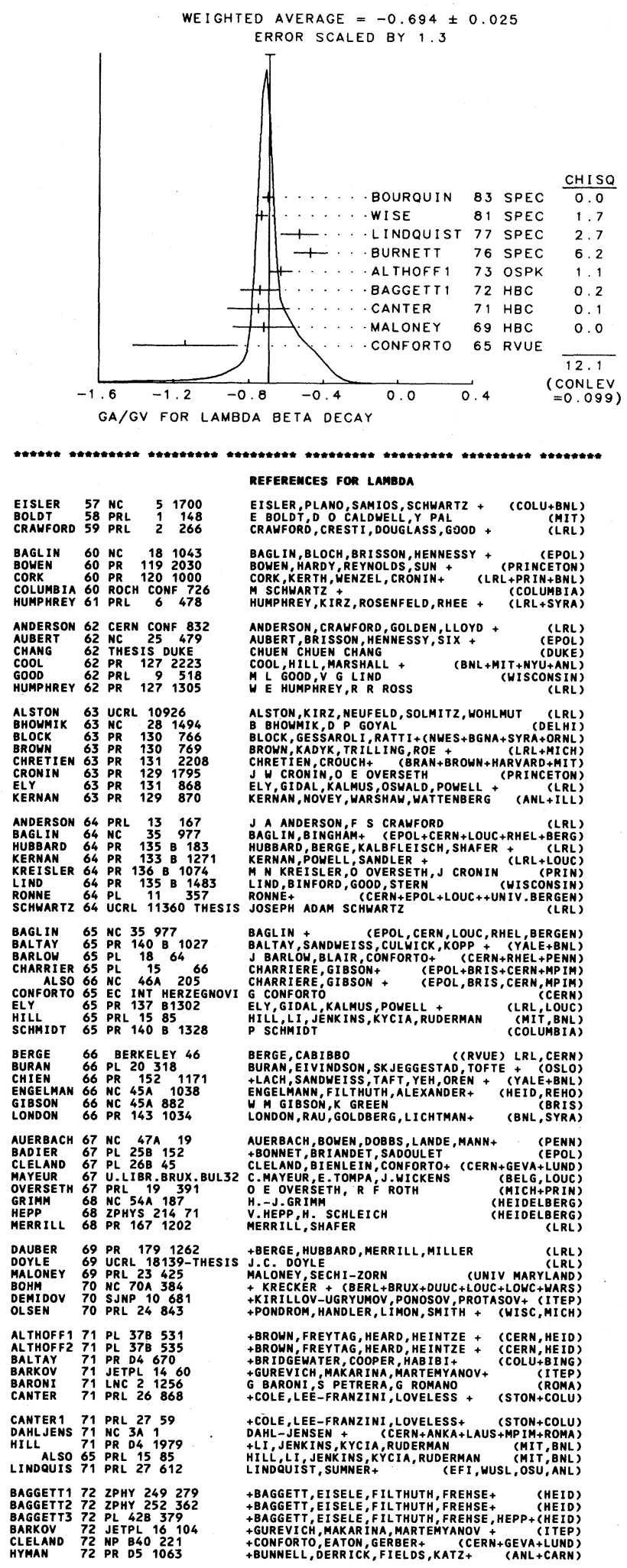

ALTHOFF1 73 PL 438237

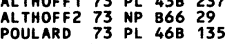

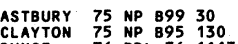

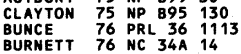

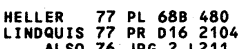

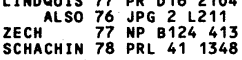

$\begin{array}{llll}\text { MISE } & 80 \text { PL } 918 & 165 \\ \text { COX } & 81 & \text { PRL } 46 & 877\end{array}$

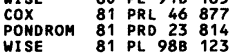

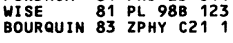

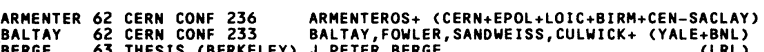

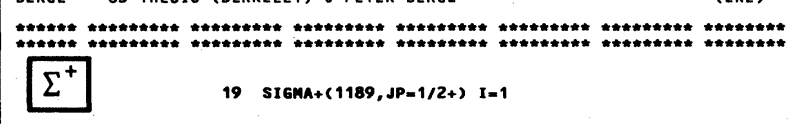

19 SIGMA+ MASS (MEV)

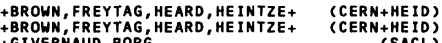
+GALLIVAN, JAFAR
+BACON, BUTTERHORTH, WATERS +
(LOIC+CERN+ETH+SACL)
(LOIC+RHEL)

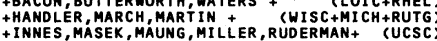
+OVERSETH, BUNCE, DVOAK + (MICH+HISC+HEID) LINDQUIST, SWALLOH, SUMAER+(EFI+HUSL+OSSUANL) SCHACHINGER, BUNCE, COX + (MICH+RUTG+WISC +JENSEN, KRE ISLER, LOMANNO, POSTER+ (MASA+BNL) HANDLER, SHEAFF, COX + (WISC+MICH+RUTG+MINM)

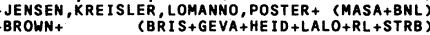
PAPERS NOT REFERRED TO IN DATA CARDS

PAPES NOT REERED TO IN OATA CARES MLTAY, FOHLER, SANOWEISS, CULUIC

4 SeE nOte preceding Lambda mass Listings

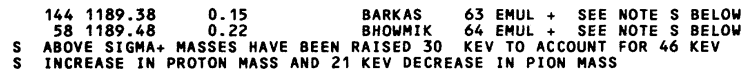

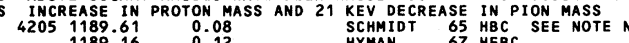
$B$
8
807189.33

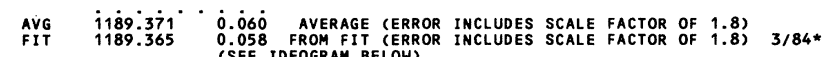

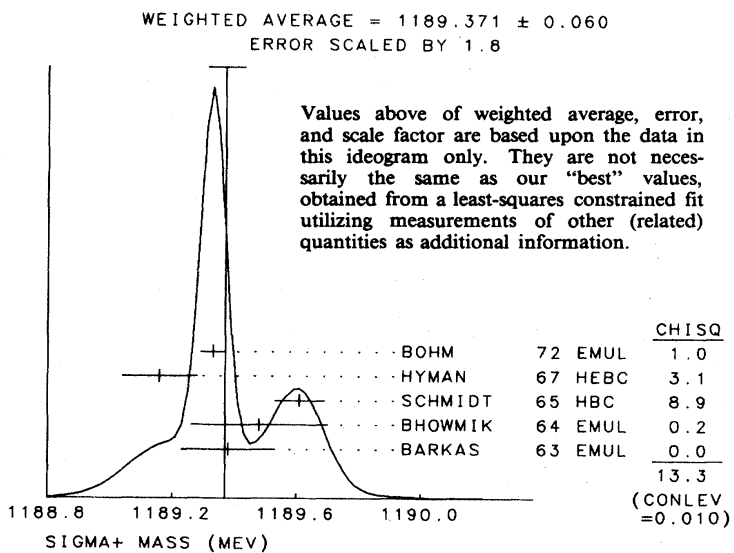

19 SIGMA+ MEAM LIFE (UNITS $10^{* * *-10 ~ S E C) ~}$

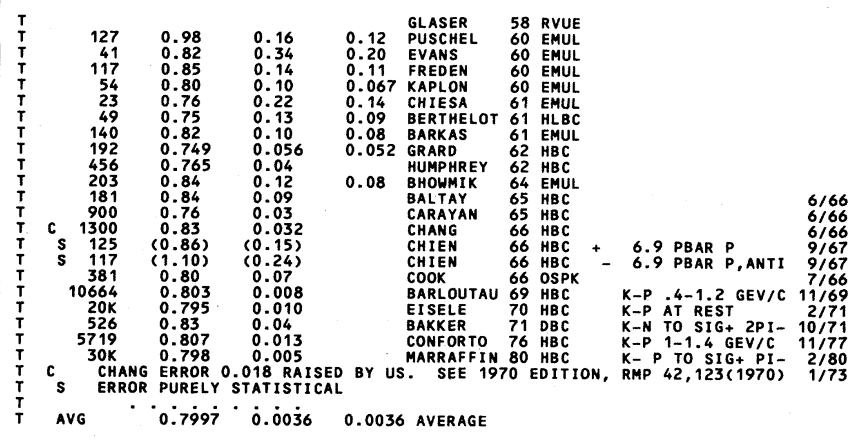




\section{Stable Particles} $\Sigma^{+}$

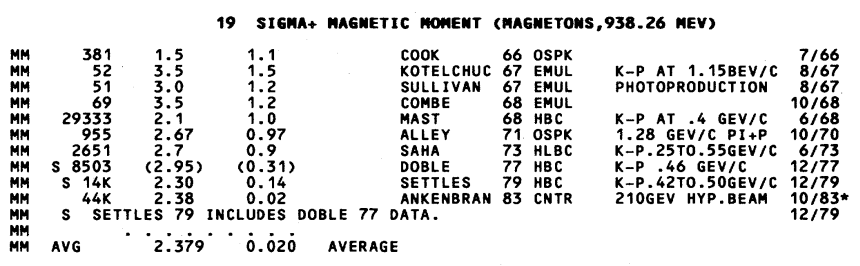

19 Sigma+ Partial decay modes

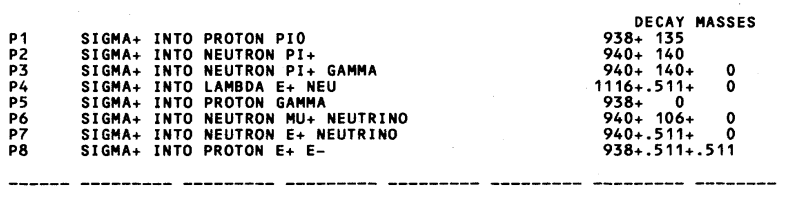

19 SIGMA+ BRAMCHIMG RATIOS

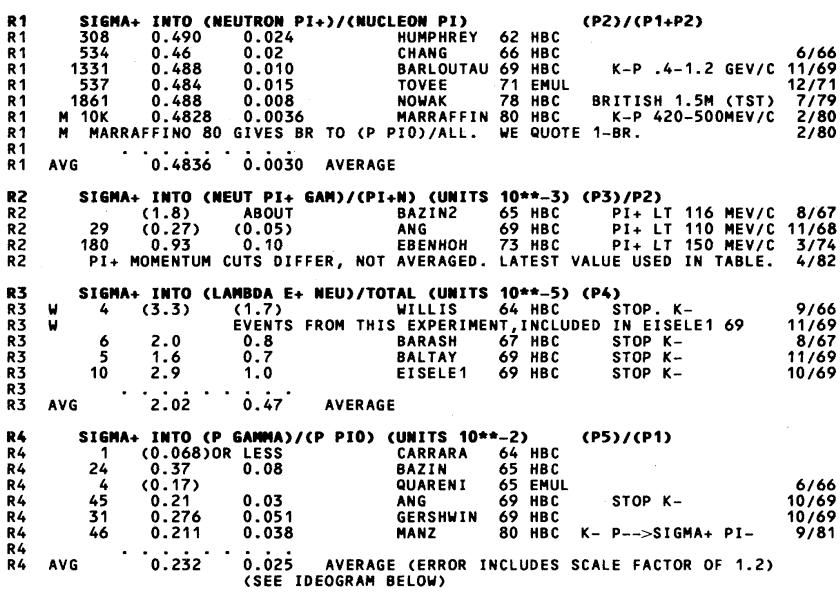
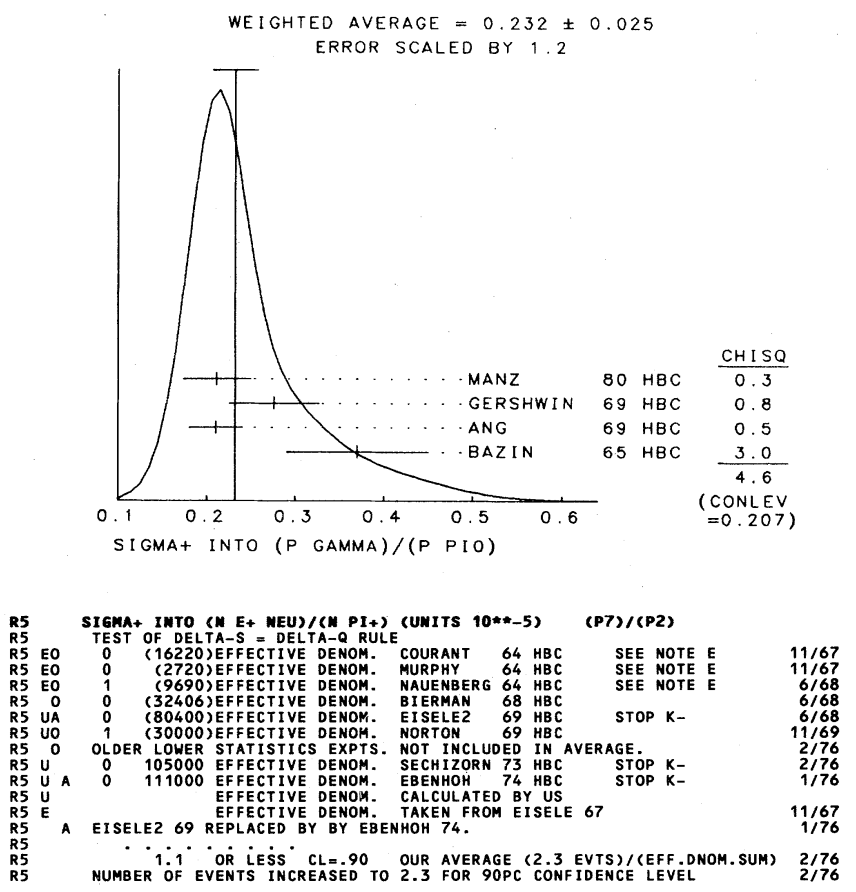

\section{Data Card Listings}

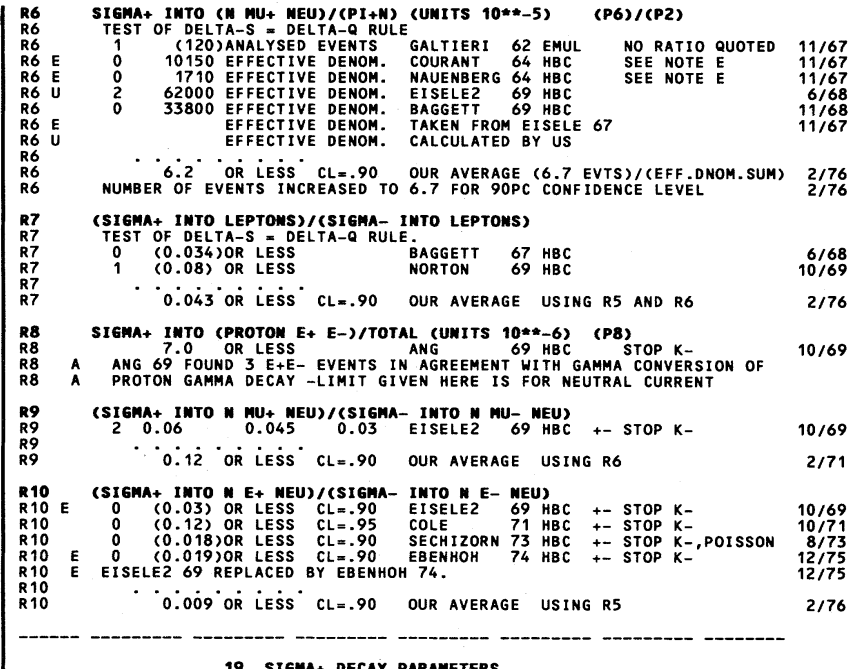

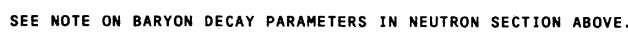

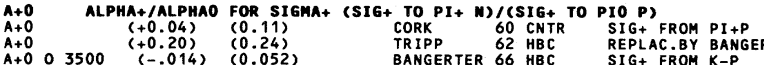

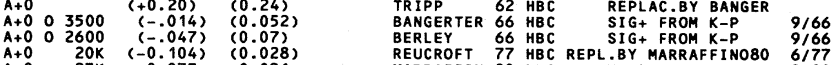

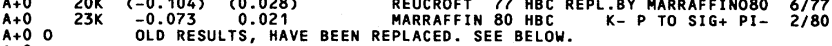

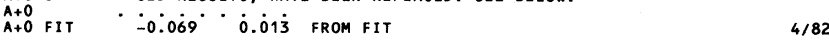

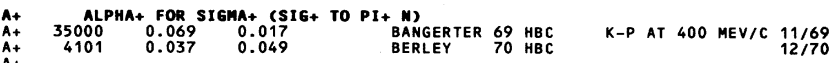

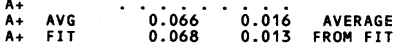

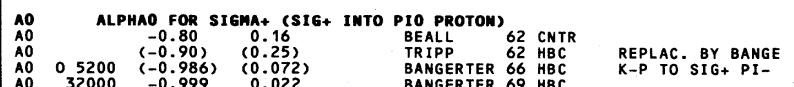

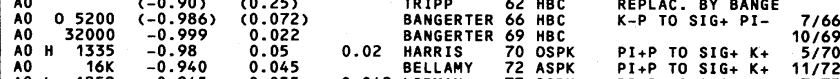

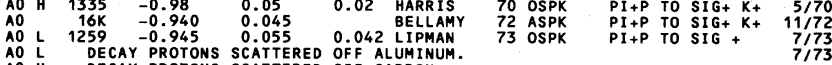
AO $L$ DECAY PROTONS SCATTERED OFF ALUMINUM.
AO H DECAY PROTONS SCATTERED OFF CARBON."

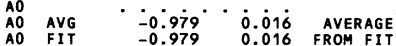

F+
$F_{+} 0 \quad 370(180$.
FHI
(30.)

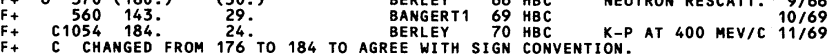

$F_{+}$
$F_{+}$

AG ALPHAG FOR SIEMA+ (SIG+ INTO PROTOM GAMMA)

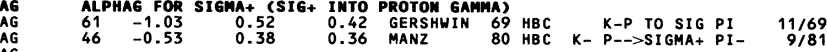

AG avg $-0.72^{\circ} \cdot \dot{0.29} 9^{\circ}$ average

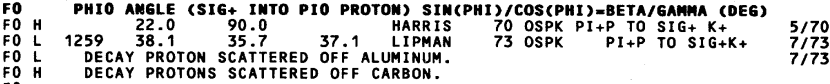

FO AVG $35.8^{\circ} \cdot 33.7^{\circ}$ AVERAGE

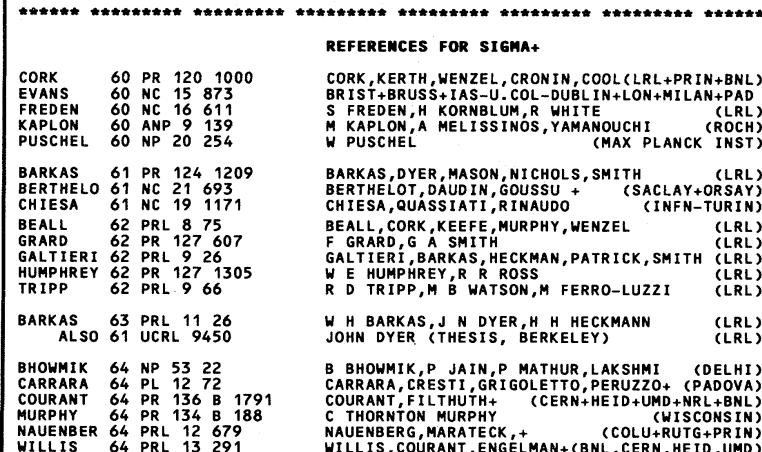




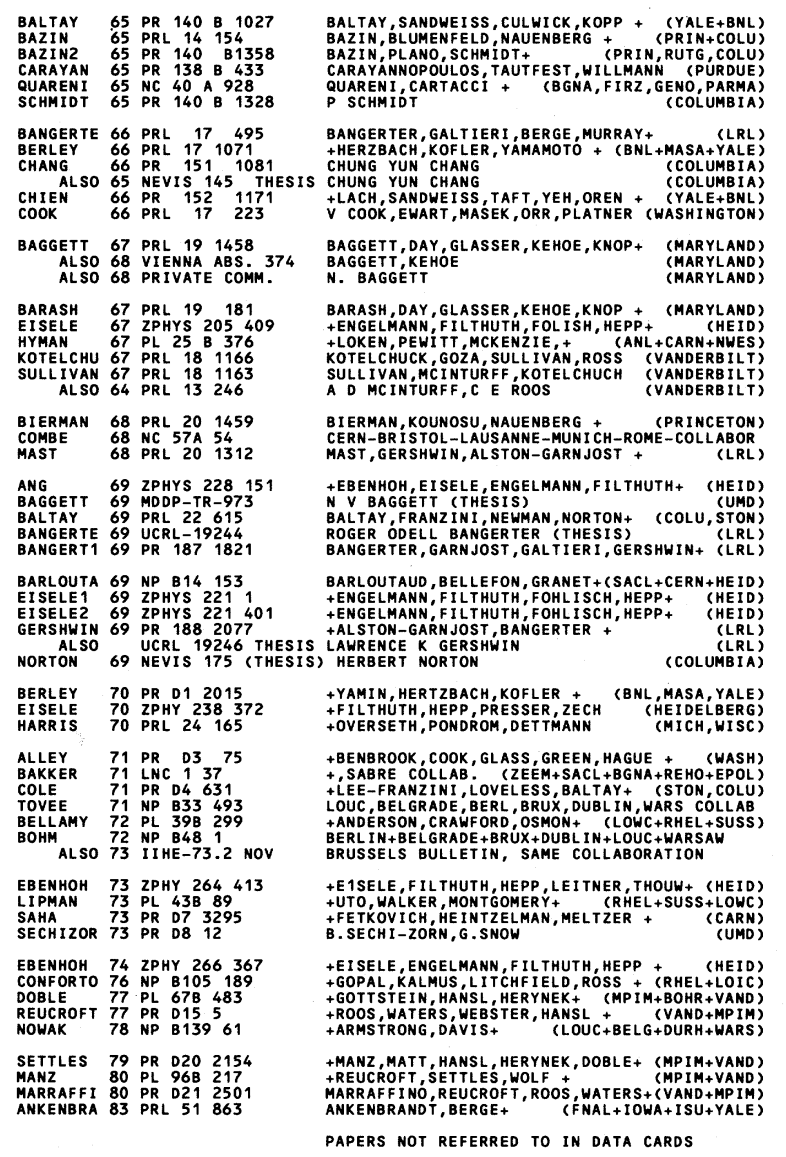

GLASER 58 CERN CONF 270 GLASER, GOOD, MORRISON (MICH+LRL) QUANTUM NUMBER DETERMINATIONS NOT REFERRED TO IN THE DATA CARDS

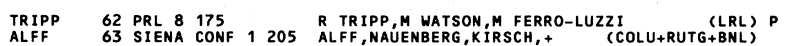

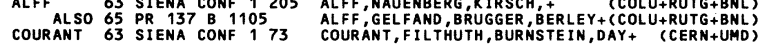

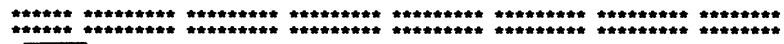

$\Sigma^{-}$

20 SIGMA-(1197, JP=1/2+) I=1

20 SIGMA- MASS (MEV)

M N SeE note preceding LAMbda mass Listings

$\begin{array}{llllllll}M & 3000 & 1197.43 & 0.08 & \text { SCHMIDT } & 65 \text { HBC } & \text { SEE NOTE N } & 3 / 74 \\ M & & 1197.24 & 0.15 & \text { DUGAN } & 75 \text { CNTR EXOEIC ATOMS } & 12 / 79\end{array}$

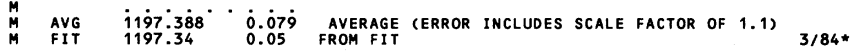

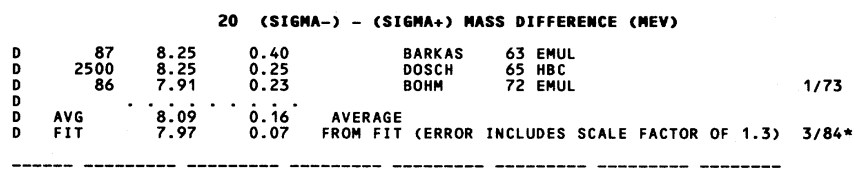

20 (SIGMA-) - (LAMBDA) MASS DifFEREMCE (MEV)

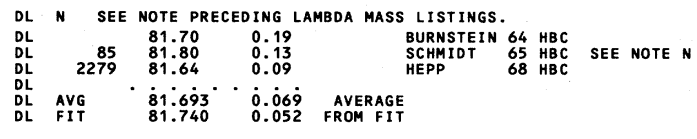

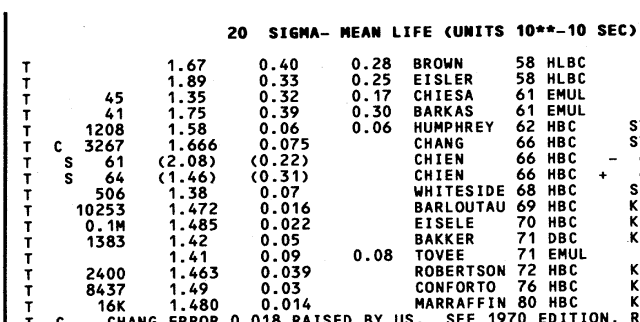

20 SIGMA- MEAN LIFE (UNITS $10 * *-10$ SEC)

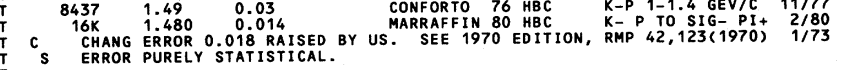

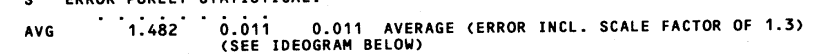

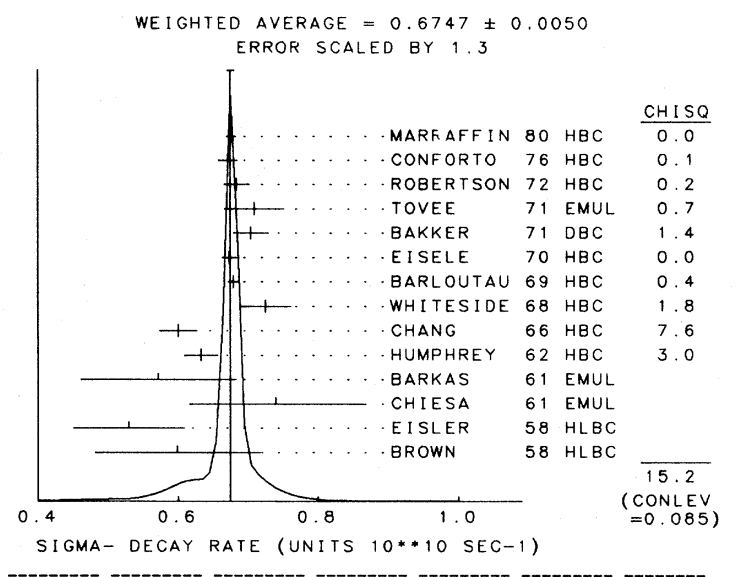

20 SIGMA- MAGMETIC MOMENT (MAgmetons, 938.26 MEV)

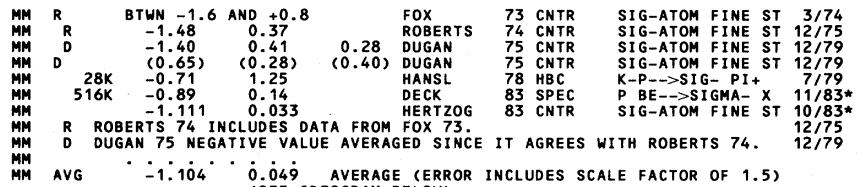

MM AVG $-1.10^{\circ} \cdot 0.04 \dot{9}^{\circ}$ AVERAGE (ERROR INCLUDES SCALE FACTOR OF 1.5)

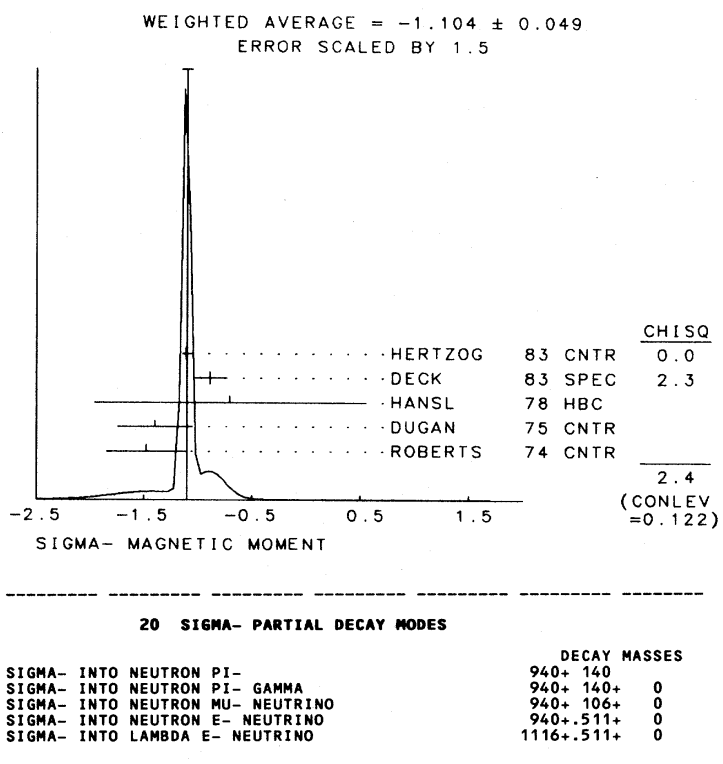




\section{Stable Particles}

$\Sigma^{-}$

\section{Data Card Listings}

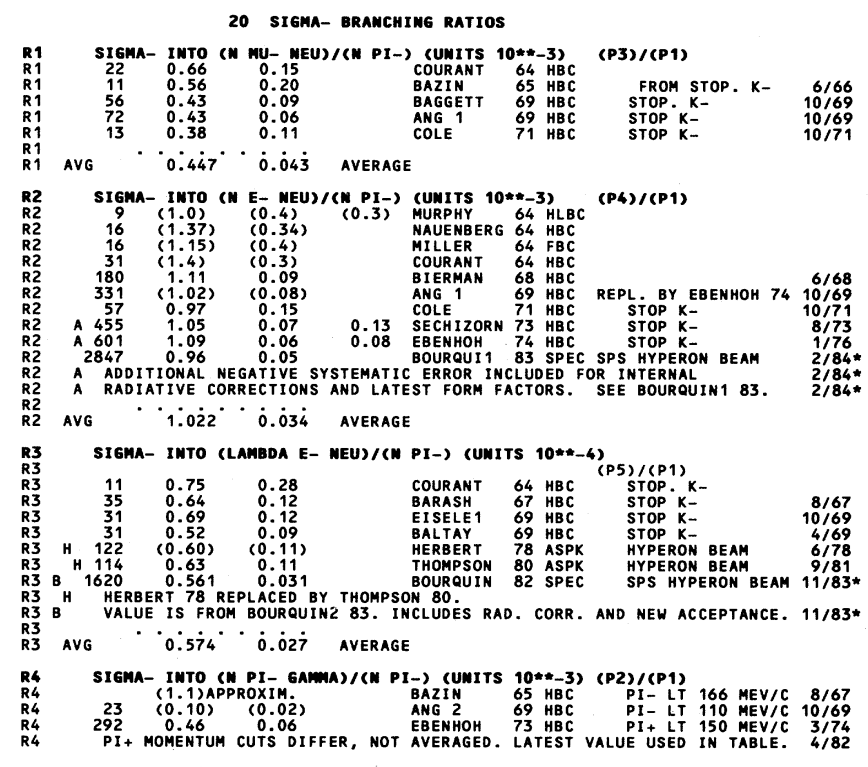

20 Sigma- Decay parameters

SEe note on baryon decay parameters in neutron section above.

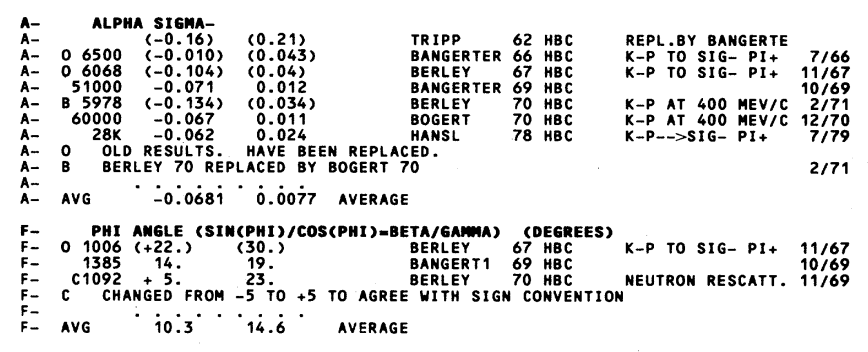

NOTE ON $\Sigma^{-} \rightarrow \Delta e^{-} \nu$

(by J.A. Thompson, University of Pittsburgh)

The decay $\Sigma^{-} \rightarrow \Lambda e^{-} \nu$ is of special interest because its form is predicted by the strong form of CVC and is not sensitive to the current octet assumptions or SU(3) structure constants which enter into Cabibbo's predictions for the other hyperon decays. For $\Delta S=0$ transitions, the weak interaction vector current is related to the electromagnetic current through a multiplicative constant, set by neutron beta decay, and an isospin rotation.

The decay $\Sigma^{0} \rightarrow \Lambda \gamma$ (the isospin-rotation analogue of $\Sigma^{-} \rightarrow \Lambda \mathrm{e}^{-} \nu$ ) is mediated predominantly through the magnetic interaction, assuming there are no inhomogeneities in the $\Sigma^{0}, \Lambda$ charge distributions. Thus we expect the $\mathbf{g}_{W M}$ term,

$$
\mathrm{g}_{\mathrm{WM}} \sim \frac{\mu_{\Sigma \Lambda}}{\sqrt{2}} \sim-\frac{\sqrt{3}}{2} \mu_{\mathrm{n}}[\text { by SU(3)] }
$$

to dominate the vector part of the weak current. The strong CVC predictions are thus: $\mathrm{g}_{\mathrm{V}} / \mathrm{g}_{\mathrm{A}}=0$ and $\mathrm{g}_{\mathrm{WM}}$ $\sim 1.6$.
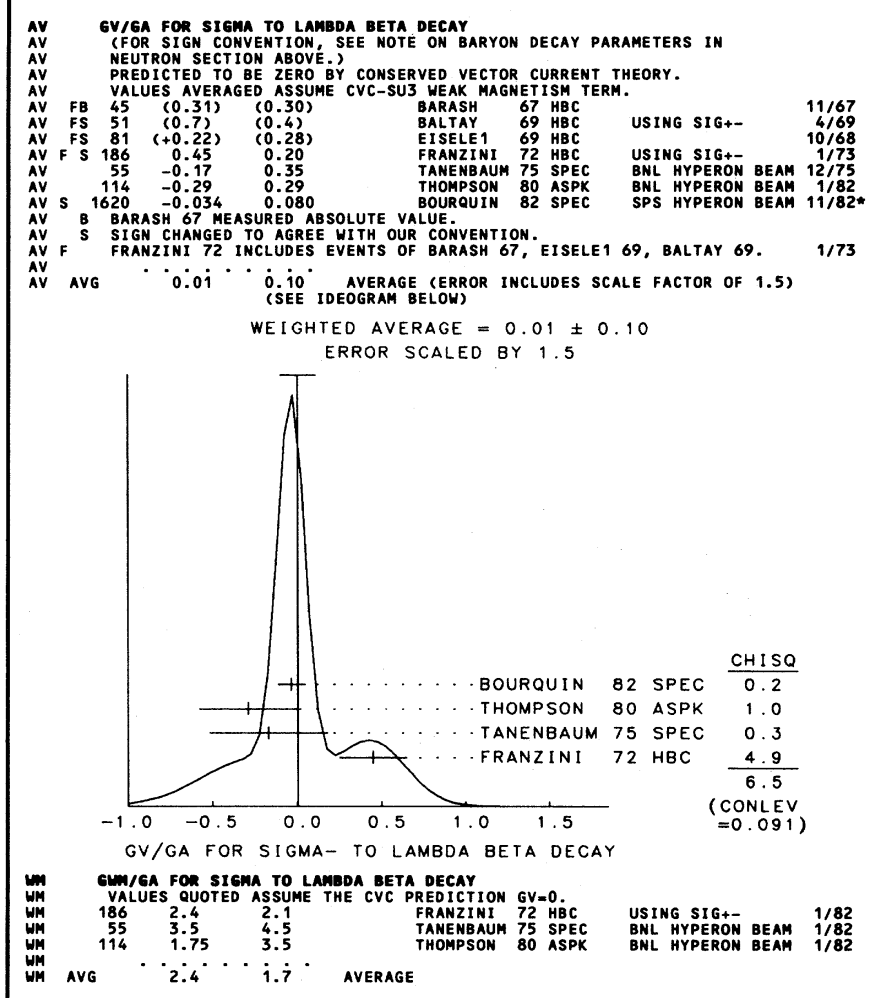

政

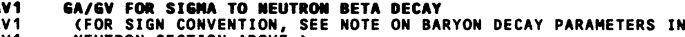
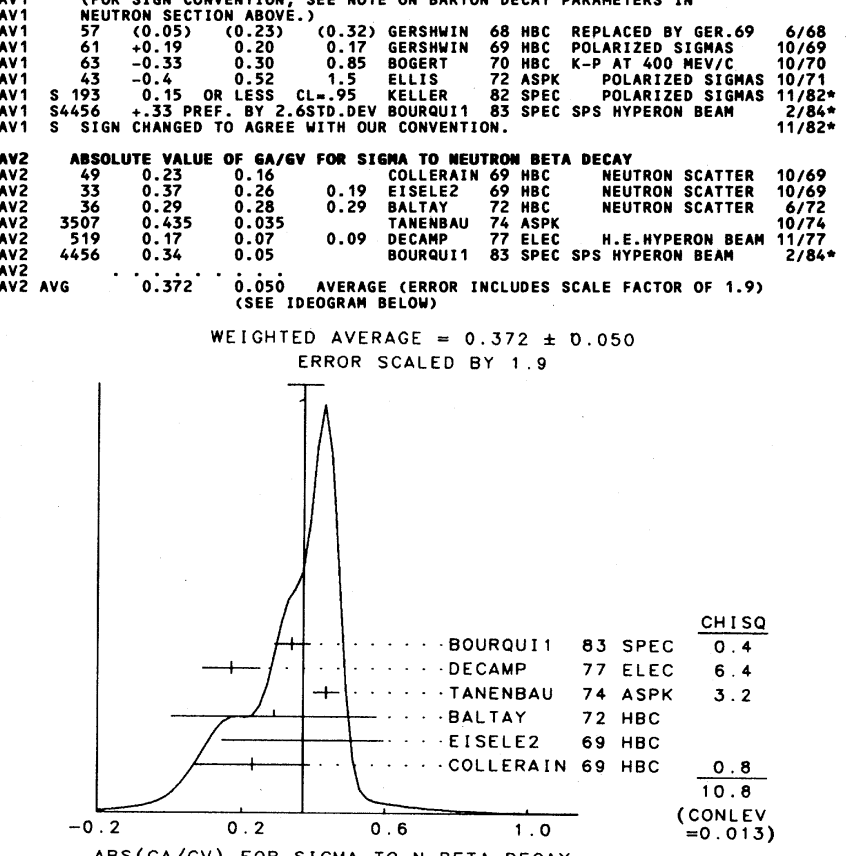

ABS (GA/GV) FOR SIGMA TO N BETA DECAY 


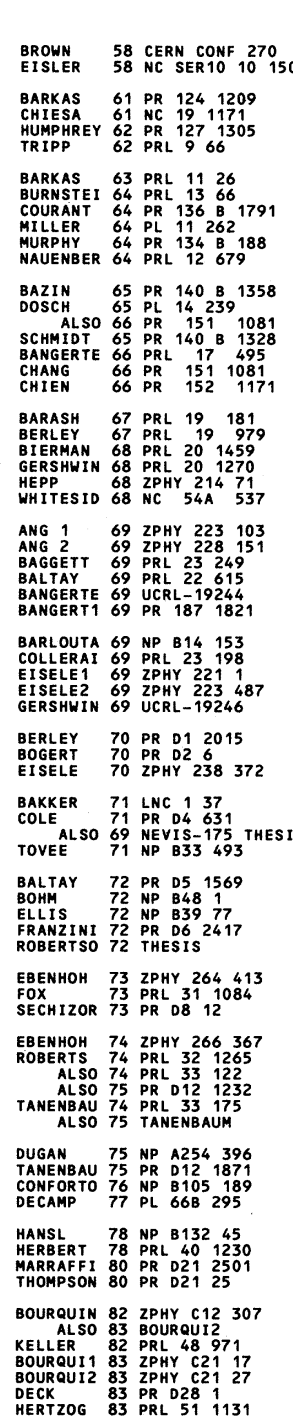

REFEREMCES FOR. SIGMA-

BRONN, GLASER, GRAVES, PERL, CRONIN ${ }^{+}$(MICM)
EISLER, BASSI, CONVERSI+ (COLU,BNL, BGNA, PISA)

BARKAS, DYER, MASON, IICKOLS, SMITH (LRL)

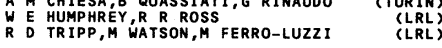

WH BARKAS, J N DYER, H H HECKMAN
BURNSTEN, DAYY KEHOE, SECHI OORN, SNOW (LRL)
(UMAD)

COURANT, F FLTHUTH, (CERN+HEID+UMD+NRL+BNL)
MILLER, STANARARD, BEZAGUET+ (LOUC, EPOL+BERG)

C THORNTON MURP HY
NAUENBERG, SCHMIDT, MARATECK+ (COLU+RUTG+PRIN)
(WISCONSIN)

BAZIN, PLANO, SCHMIDT ${ }^{+}$(PRIN+RUTG+COLU) DOSH, ENGELMANN, FILTHUTH, HEPP, KLUGEF C CHEID) CHUNG YUN CHANG
PSCHMDT (COLUMBIA) BANGERTER, GALTIERI, BERGE, MURRAY+ COLRL) CHUNG YUN, CHANG TAF, YE, OREAY+ (COLUMBIA)
+LACH, SANDWEISS, TAFT, YEH,
(YALE +BNL) BARASH, DAY, GLASSER, KEHOE, KNOP + (MARYA AND)
BERLEY, HERT ZBACH, KOFLER + (BNL, MASA, YALE) MERMN,

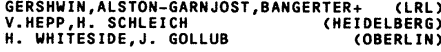
ANG, EISELE, ENGELMANN, FILTHUTH + ${ }^{+}$(HEID) BAGEETT, KEHOE, SNOW BALTAY, FRANZINI, NEEMAN, NORTON+ (COLU, STON) BANGERTER, GARNJOST, GALTIERI, GERSHWIN+ (LRL) BARLOUTAUD, BELLEFON, GRANET+ (SACL+CERH+HEID)
COLLERAINE, DAY GLLASSR KNOP + CUMIY MARYLAND)

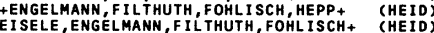
EISELE, ENGELMANN, FILTHUT, FOHLISCH+ (HEID)
LAWRENCE KENETH GERSHWIN (THESIS) (LRL)

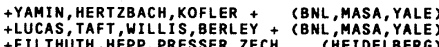
SABRE COLLAB. (ZEEM+SACL+BGMA+REHO+EPOL) TLE-FANZLINI, LOVELESS, BALTAY+ (STON, COLU) LOUC,BELGRADE, BERL, BRUX, DUBL IN, WARS COLLAB
LOEC

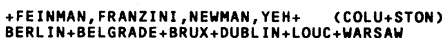
OXFFAERE+RHEL+LOOM+LYON+NWES + ITEP COLLABOR
COLUMBIA+HEIDELBERG THARYLAND STONY BROOK COLUMB A A+HE IDELBERG +MARYLAND+STONY BROOK
R.M.ROBERTSON

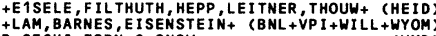
+EISELEE ENGELMANM, FILTHUTH, HEPP +
WILL+VPI+CARN+WYOH+CIT COLLABORATION ROBERTS, COX
TANENBAUM, HUNGERUELHLER+ (YALE+FNAL+BNL) FASANO, CHEN, CHENG, HU, LIDOFSKY
TANENBAUM, HUMGERBUEHLER (COLU+YALE) +GOPAL, KALMUS, LITCHFIEL, ROSS + (RHEL+LOIC)
+BADIER, BLAND, CHOLLET, GALLARD+ (LALO+EPOL) +MANZ, MATT, REUCROF, SETTLES + (MPIM+VAND)

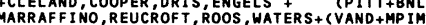

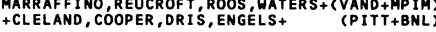
+BROWN + (BRIS+GEVA+HEID+LALO+RL+STRB) +LESNIK, ROMANOWSKI, KEIG + (OSU+CHIC+ANL)
BOURQUIN+ (BRIS+GEVA+HEID+LALO+RL+STRB) BERETVAS, DEVLIN, LUK+ (RUTG GHISC+MICH+HINN) + EEKHAUSE+
PATS ( $W I L L+B O S T+C I T+C A R N+$ HYOM) PAPERS NOT REFERRED tO IN DATA CARDS

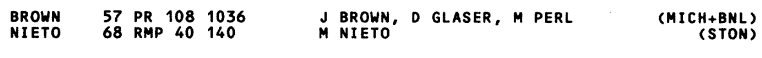

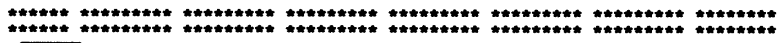

$\Sigma^{0} \quad 21$ SIGMAO(1193, JP=1/2+) I=1

JP NOT MEASURED FOR SIGMAO. ASSUMED SAME AS SIGMA+
AND SIGMA-TO ALLOH ISOTRIPLET ASSOCIATION.

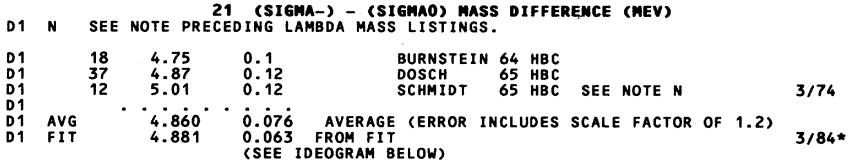

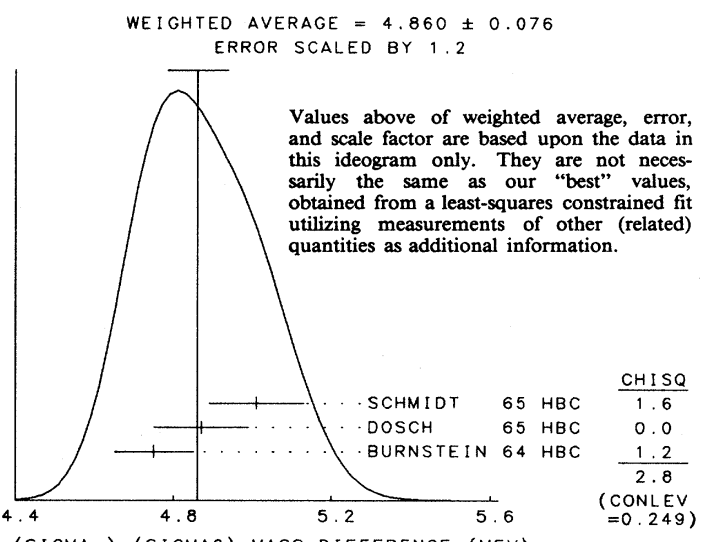

21 (SIGMAO) - (LAMBDA) MASS DIFFEREMCE (MEV)

DL N See note preceding lambda mass Listings.

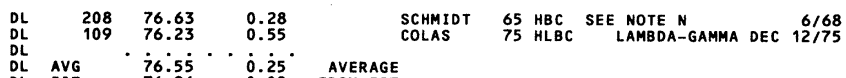
DL FIT 76.86 0.08 FROM FIT $3 / 84$ *

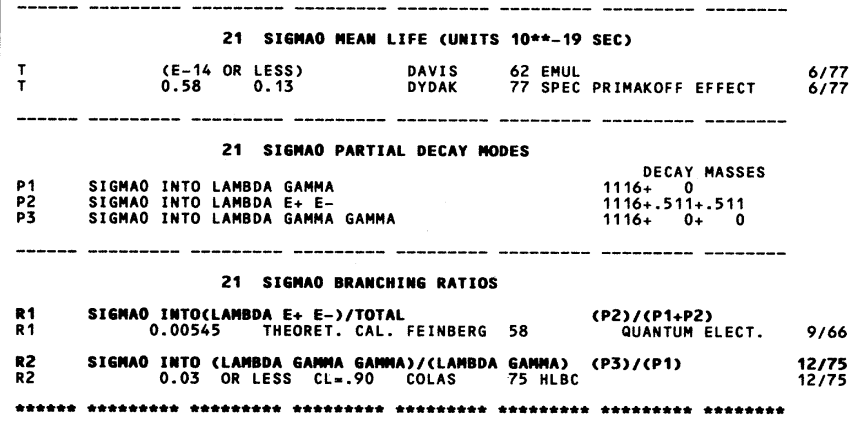

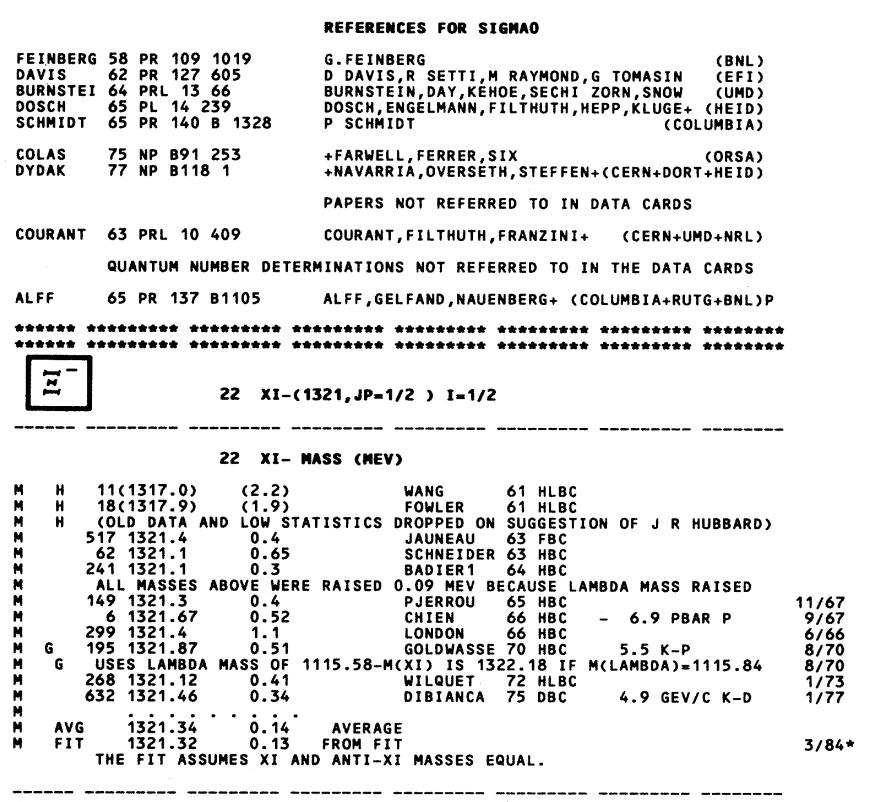




\section{Stable Particles}

Data Card Listings

$\Xi^{-}$

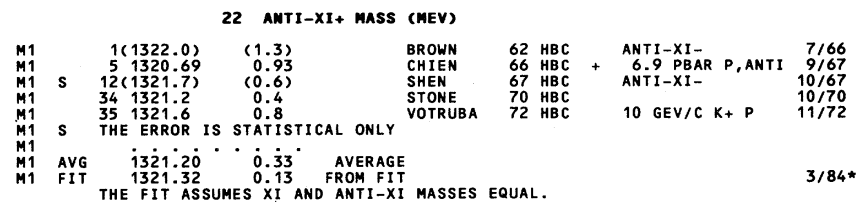

22 (XI-) - (ANTI-XI+) MASS DIFFERENCE (MEV)

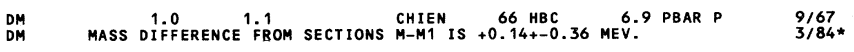

\begin{tabular}{|c|c|c|c|c|}
\hline & \multicolumn{4}{|c|}{22 XI- MAgmetic MOMENT (MAGMEtons,938.26 MEV) } \\
\hline $\begin{array}{r}\text { MM } \\
\text { MMM } \\
\text { MM }\end{array}$ & $\begin{array}{l}-0.1 \\
-2: 1 \\
-1.85\end{array}$ & $\begin{array}{l}2.1 \\
0.8 \\
0 .\end{array}$ & $\begin{array}{l}\text { BINGHAM } \\
\text { COOL }\end{array}$ & $\begin{array}{l}70 \text { OSPK }-1.8 \text { GEV/C K-P } \\
74 \text { OSPK }-1.8 \text { GEV } / C \text { K-P }\end{array}$ \\
\hline
\end{tabular}

22 XI- MEAN LIFE (UNITS 10**-10 SEC)

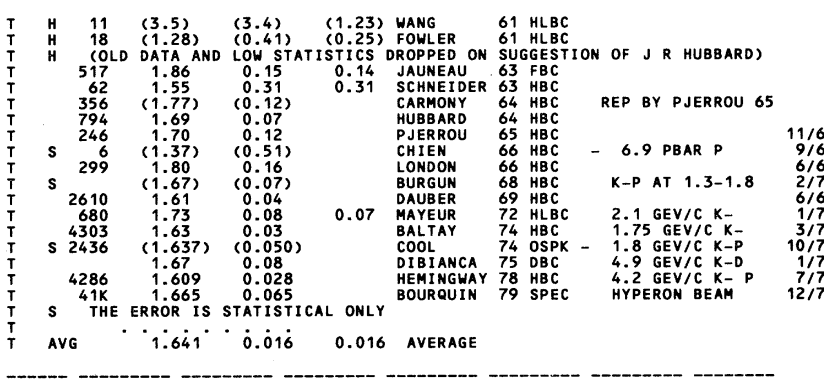

22 ANTI-XI+ MEAM LIFE (UNITS 10**-10 SEC)

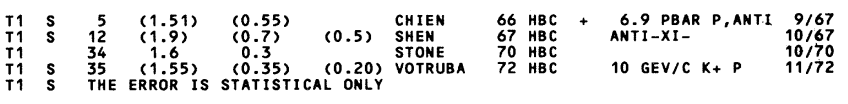

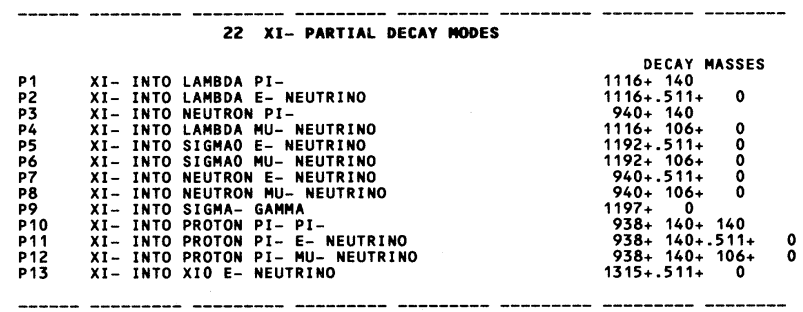

22 XI- BRAMCHING RATIOS

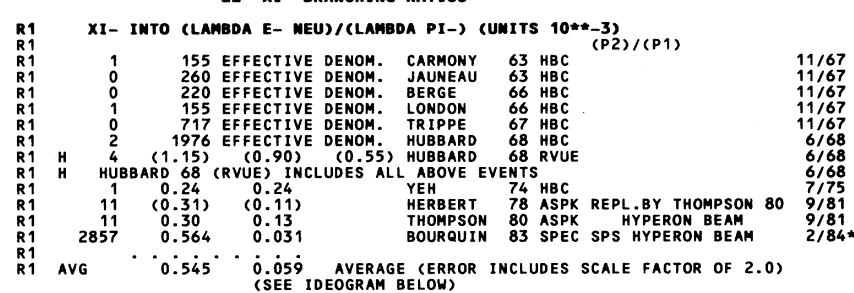

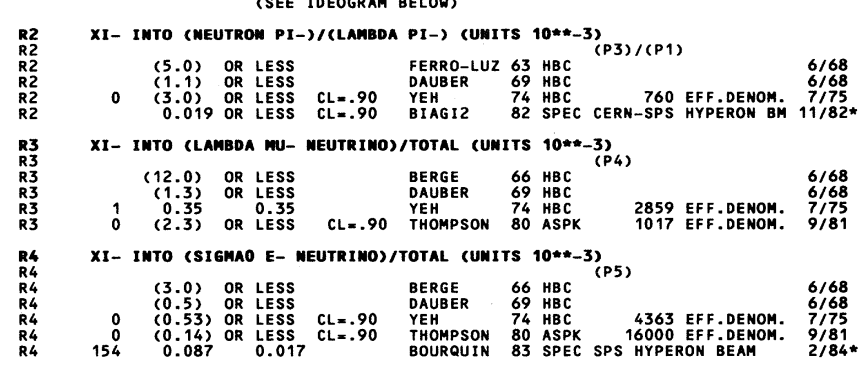

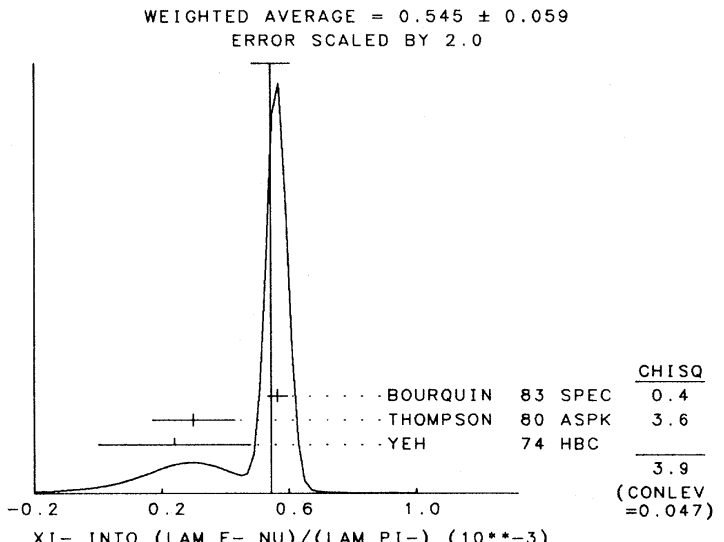

XI- INTO (LAM E- NU)/(LAM PI-) $(10 * *-3)$

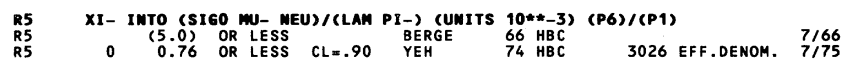
XI- INTO (M E- MEU)/(CLAMBDA PI-) (UNITS 10**-3) (P7)/(P1)

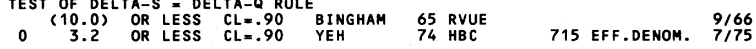
XI- ImTO (SIGMAO E- MEU + LAMBda E- MEU)/TOTAL (10**-3), (P2+P5)

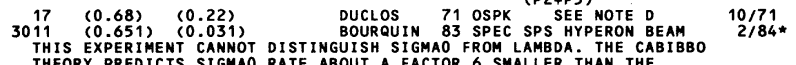
THE

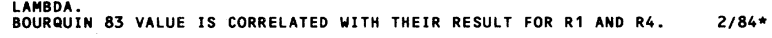

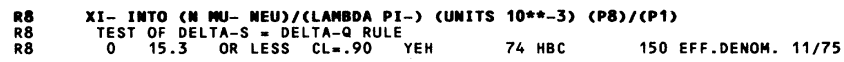
R9 XI- IWTO (SIGMA- GAMMA)/(LAM PI-) (UNITS 10**-4) (P9)/(P1)
R9

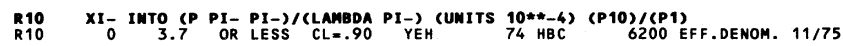
R11 XI- IWT (P PI- E- MEU)/(LAM PI-) (UMITS 10**-4) (P11)/(P1)
R11 TEST OF DELTA-S = DELTA-Q RULE

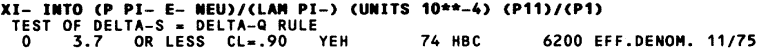

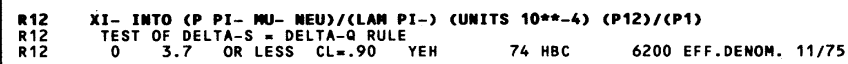

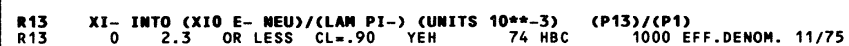
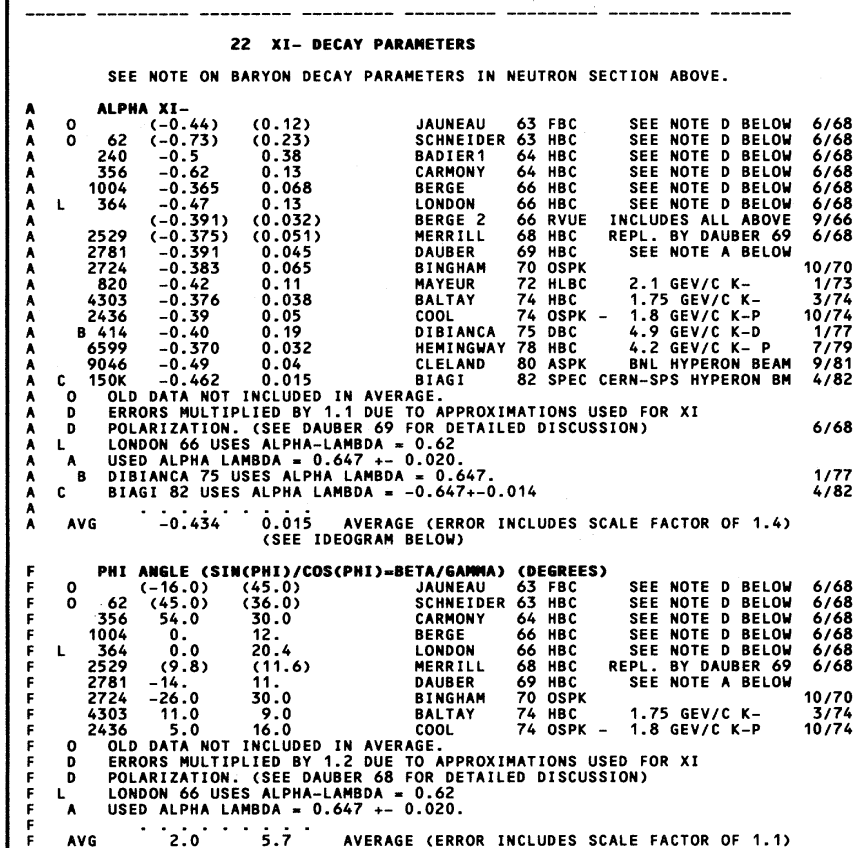


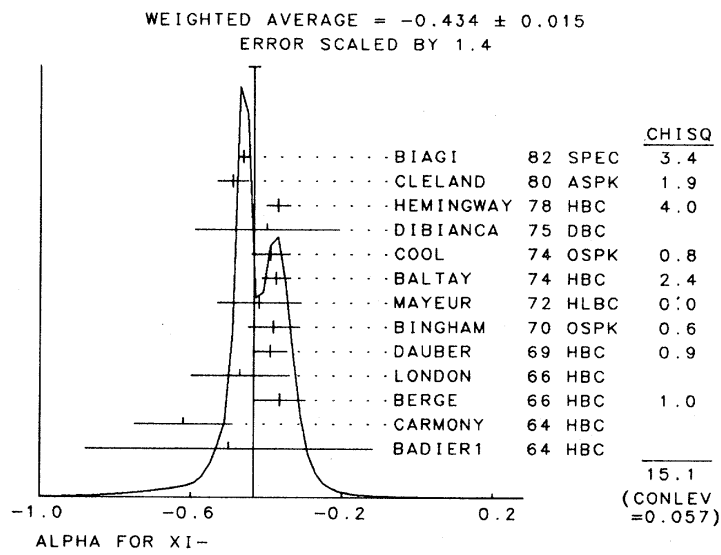

AV GAFV FOR XI- TO LAMBDA BETA DECAY
AN
AV FOR SIGN CONVENTION, SEE NOTE ON BARYON DECAY PARAMETERS IN
AV

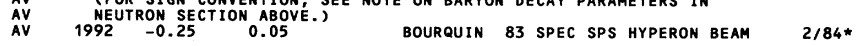

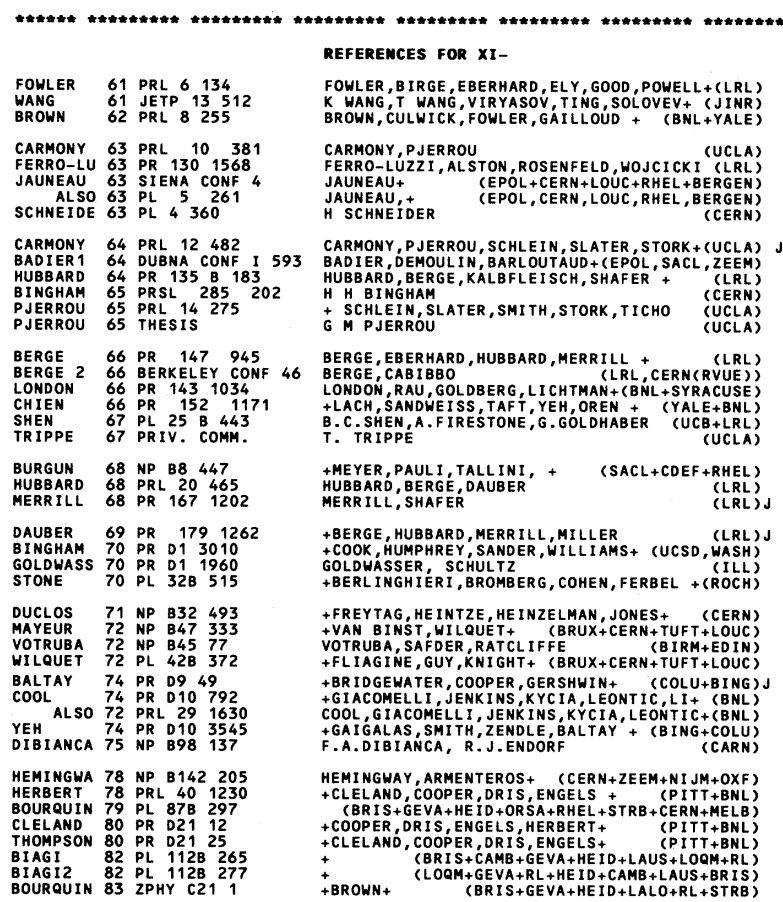

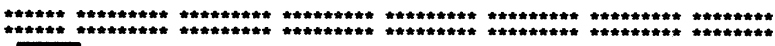
$\Xi^{0}$ $23 \times 10(1315, J P=1 / 2) I=1 / 2$

23 XIO MASS (MEV)

$\begin{array}{llll}M & 1313.4 & 1.8 & \text { PALMER } \\ M & 498 & \text { HBC } \\ \text { MILQUET } & 72 & \text { HLBC }\end{array}$

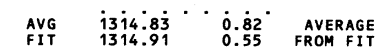

23 (XI-) - (XIO) MASS DIFFEREMCE (MEV)

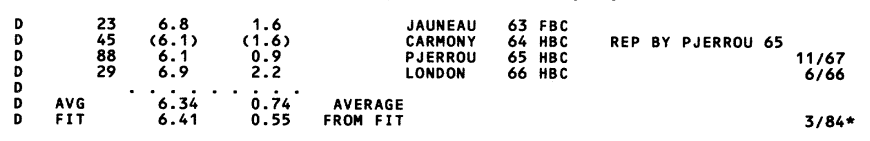

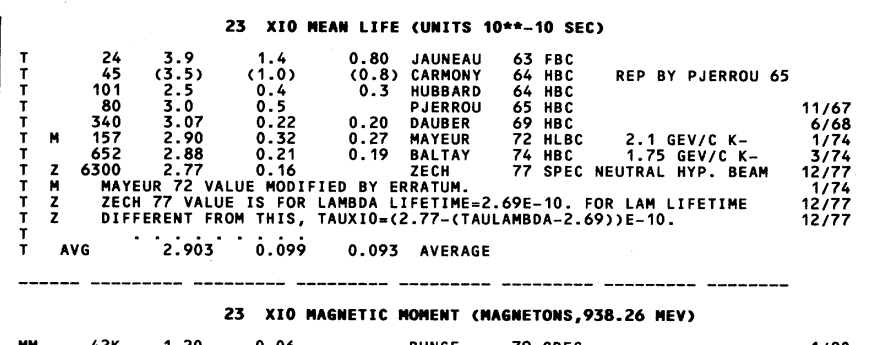

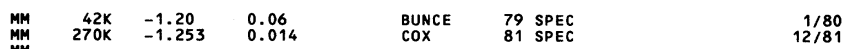

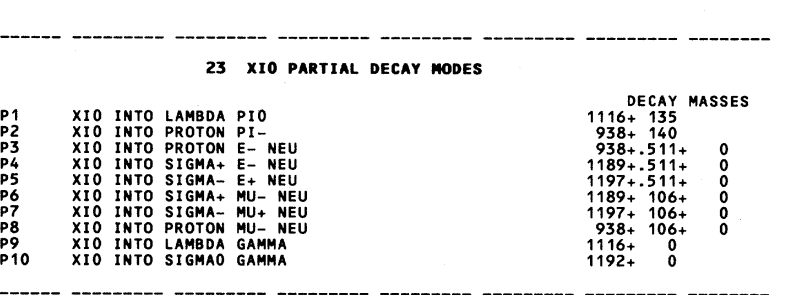

23 XIO BRAMCHIMG RATIOS

XIO INTO (PROTOM PI-)/(LAMBDA PIO) (UNITS 10**-5)

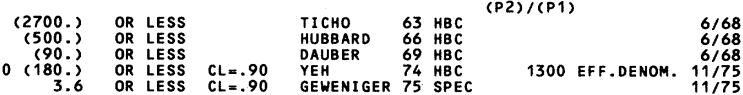
XIO INTO (PROTOM E- MEU)/(LAMBdA PIO) (UNITS 10**-3)

TEST OF DELTA-S = DELTA-Q RULE

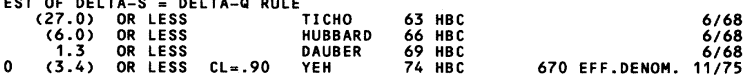
XIO INTO (SIGMA+ E- MEU)/(LAMBDA PIO) (UNITS 10**-3)

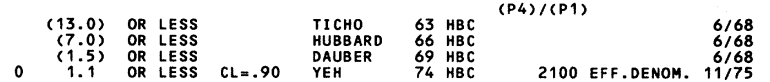
XIO IMTO (SIGMA- E+ MEU)/(LAMBDA PIO) (UNITS 10**-3)

TEST OF DELTA-S = DELTA-Q RULE

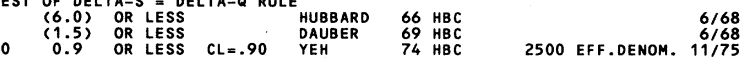
XIO INTO (SIGMA+ MU- MEU)/TOTAL (UNITS 10**-3) (P6)

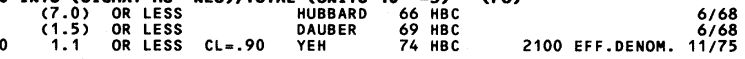
XIO INTO (SIGHA- MU+ MEU)/TOTAL (UNITS 10**-3) (P7)

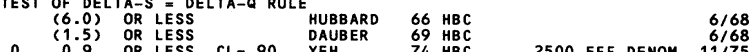
IO INTO (PROTON MU- MEU)/TOTAL (UNITS 10**-3) (P8) 2500 EFF.DENOM. 11/75 TEST OF DELTA-S = DELTA-Q RULE

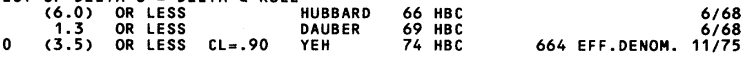

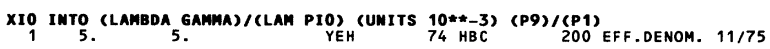
$\underset{0-1}{\text { XIO INTO (SIGMAO GAMMA)/(LAM PIO) (UMITS }} \begin{aligned} & 10 * *-2) \\ & 0.5\end{aligned}$

23 XIO DECAY PARAMETERS

SEE NOTE ON BARYon decay parameters in NEUtron SECtion above.

ALPHA XI 0

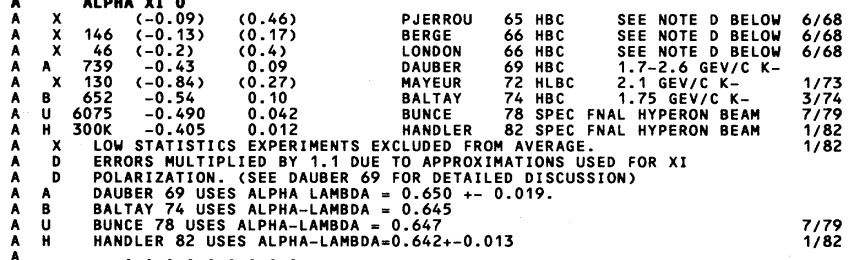

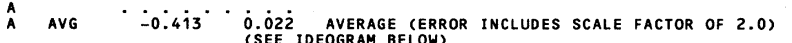




\section{Stable Particles} $\Xi^{0}, \Omega^{-}$
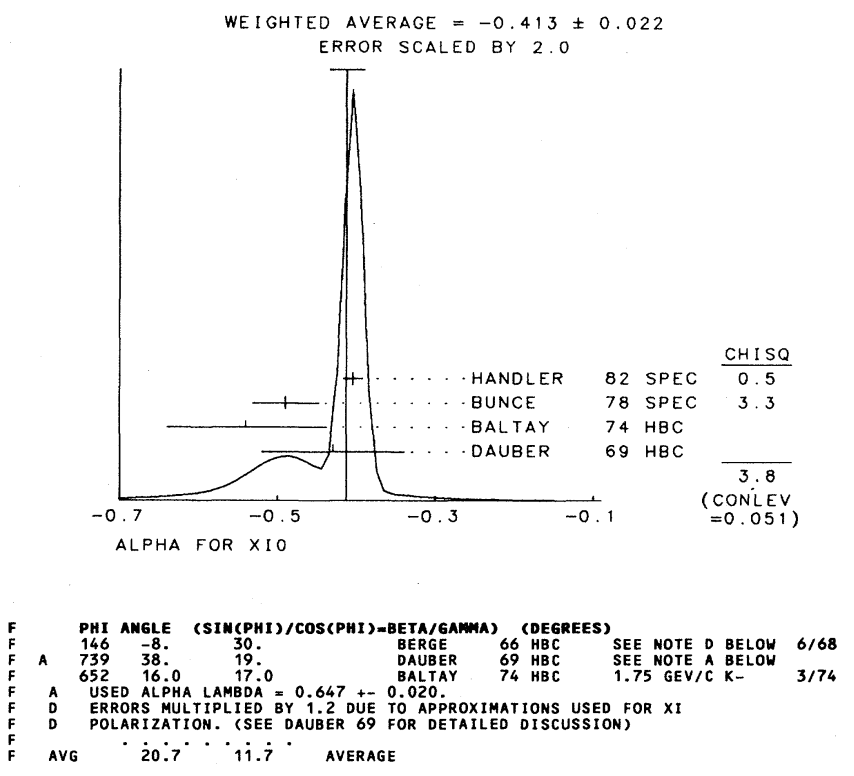

\begin{tabular}{|c|c|}
\hline $\begin{array}{l}\text { ALVAREZ } \\
\text { JAUNEAU } \\
\text { ALSO } \\
\text { TICHO }\end{array}$ & 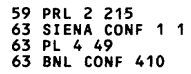 \\
\hline $\begin{array}{l}\text { CARMONY } \\
\text { HUBBARD } \\
\text { PJERROU } \\
\text { PJERROU }\end{array}$ & 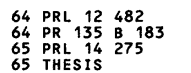 \\
\hline $\begin{array}{l}\text { BERGE } \\
\text { HUBBARD } \\
\text { LONDON }\end{array}$ & 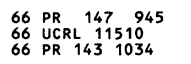 \\
\hline $\begin{array}{l}\text { PALMER } \\
\text { DAUBER } \\
\text { MAYEUR } \\
\text { ALSO } \\
\text { WILQUET }\end{array}$ & $\begin{array}{llll}68 & \mathrm{PL} & 268 & 323 \\
69 & \mathrm{PR} & 179 & 1262 \\
72 & \mathrm{NP} & 847 & 333 \\
73 & \mathrm{NP} & \mathrm{B53} & 268 \\
72 & \mathrm{PL} & 428 & 372\end{array}$ \\
\hline $\begin{array}{l}\text { BALTAY } \\
\text { YEH } \\
\text { GEWENIGE } \\
\text { ZECH }\end{array}$ & $\begin{array}{llll}74 & P R & D 9 & 49 \\
74 & P R & D 10 & 3545 \\
75 & P L & 578 & 193 \\
77 & \text { NP } & B 124 & 413\end{array}$ \\
\hline $\begin{array}{l}\text { BUNCE } \\
\text { BUNCE } \\
\text { COX } \\
\text { HANDLER }\end{array}$ & $\begin{array}{llll}78 & P R & D 18 & 633 \\
79 & P L & 868 & 386 \\
81 & P R L & 46 & 877 \\
82 & P R & 025 & 639\end{array}$ \\
\hline
\end{tabular}

REFEREMCES FOR XIO

AL VAREZ, EBERHARD, GOOD, GRAZIANO, TICHO+ (LRL) JAUNEAU+
HAROLD K TICHO (EPOL+CERN+LOUC+RHEL+BERGEN)
(UCLA) CARMONY, PJERROU, SCHLEIN, SLATER, STORK + (UCLA) + SCHLEIN, SLATER, SMI TH, STORK, TICHO (UCLA)
G M PJERROU
(ULA) BERGE, EBERHARD, HUBBARD, MERRILL ${ }^{+}$(LRL) J RICHARD HUBBARD (THESIS, BERKELEY) (LRL)
LONDON, RAU, GOLDBERG, LICHTMAN+(BNL+SYRACUSE) PALMER, RADOJICIC, RAU, RICHARDSON+ (BNL, SYRA) + VAN BINST, WILQUET+ (BRUX+CERN+TUFT+LOUC) TAN BINST, WILQUET+ (BRUX+CERN+TUFT + LOUC)
ERRATUM TO MAYEUR 72
+FLIAGINE, GUY, KNI GHT + (BRUX+CERN+TUFT + LOUC) +BR IDGEWATER, COOPER, GERSHHIN+ (COLU+BING)J +GAIGALAS, SMITH, ZENDLE, BALTAY + (BINGCOLU) GEEENIGER, GJESDAL, PRESSER + + (CERN+HEID)
+DYDAK, NAVARRIA+
(SIEG+CERN +DORT+HEID)

+HANDLER, MARCH, MARTIN+ (WISC+MICH+RUTG) +OVERSETH, COX, DWORKIN+ (BNL+MICH+RUTG+WISC) +GROBEL, PONDROM+ (WISC+MICH+MINN+RUTG)

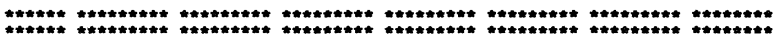

$\Omega^{-} \quad 24$ OMEGA-(1672,JP=3/2+) I=0 QUANTUM NUMBERS ASSIGNED FROM SU3

\section{OMEGA- MASS (MEV)}

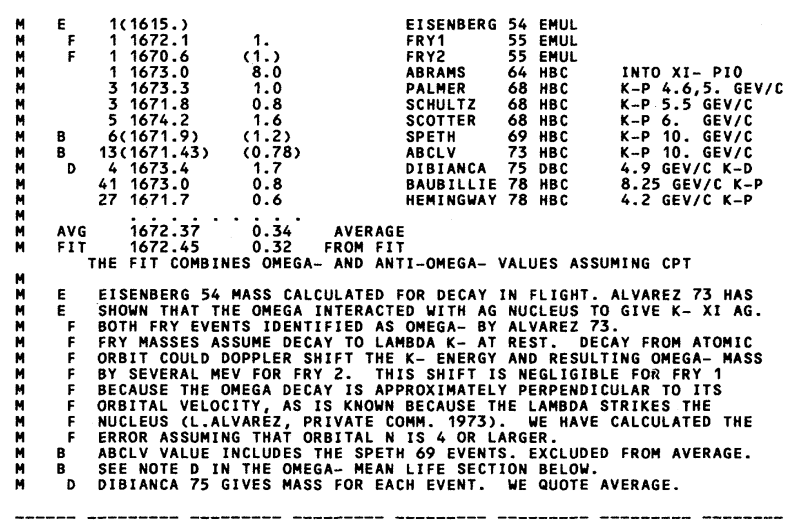

\section{Data Card Listings}

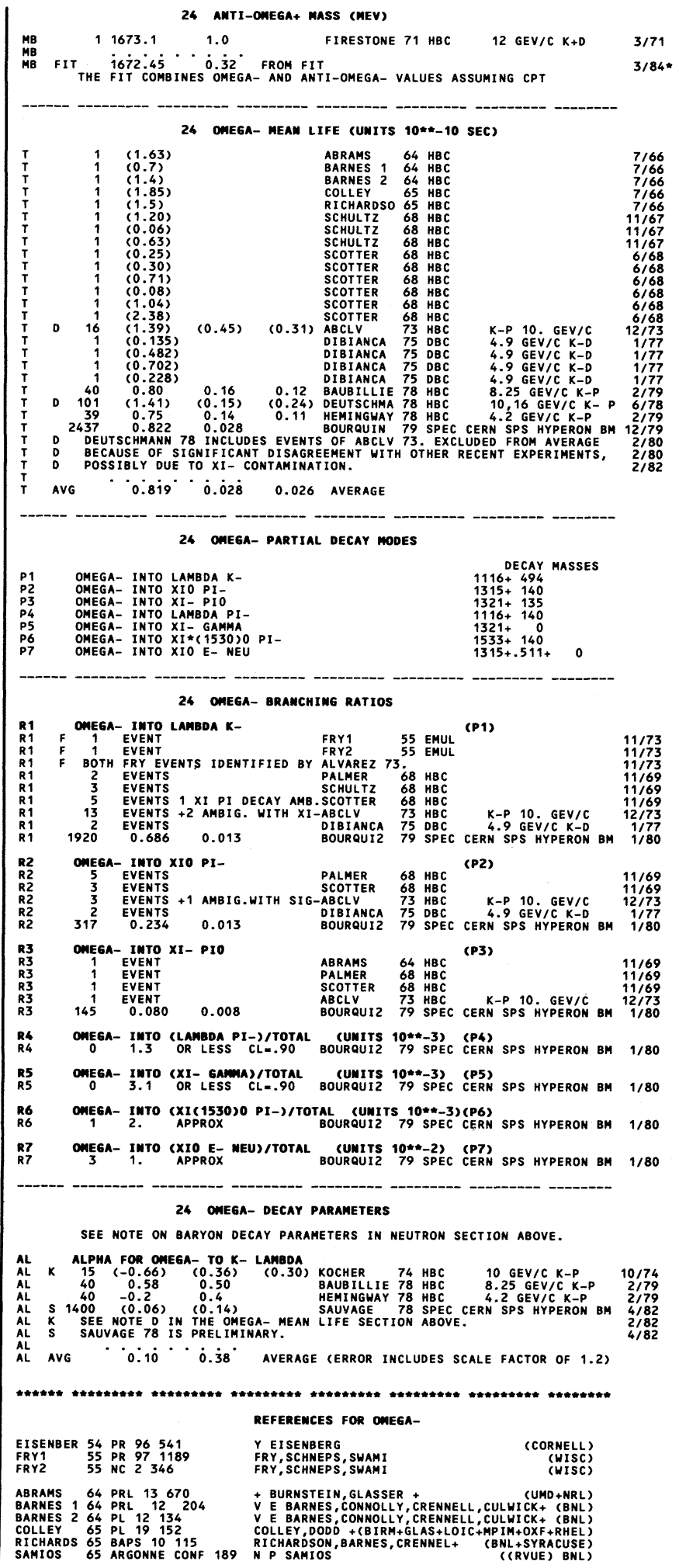




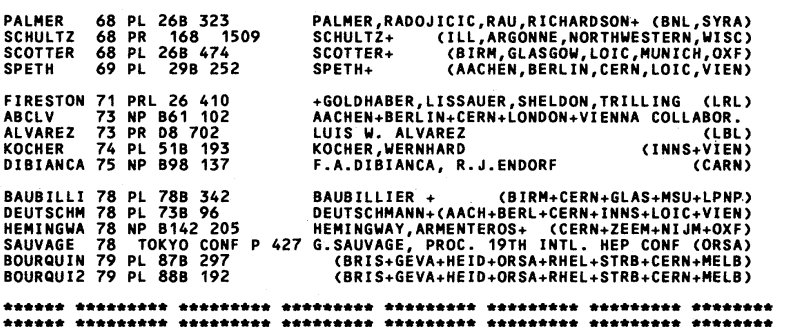

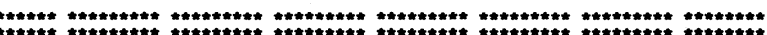

FOR THE (SIGMA/C)-(LAMBDA/C) MASS DIFFERENCE SEE THE
SIGMA/C SECTION OF THE BARYON DATA CARD LISTINGS.
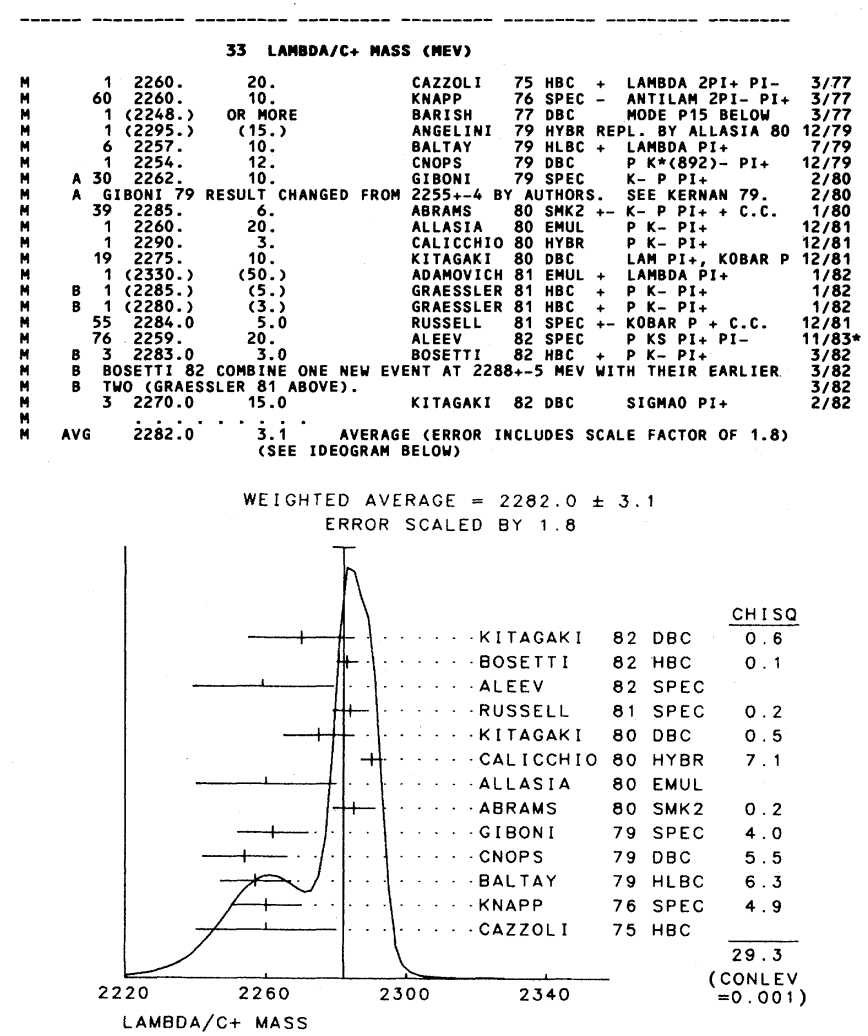

\footnotetext{
33 LAMBDA/C+ MEAM LIFE (UMITS 10**-13 SEC)

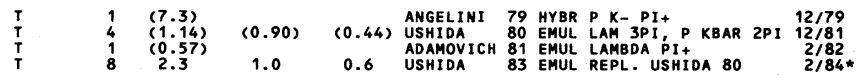

33 LAMBda/C+ PARTIAL DECAY MODES

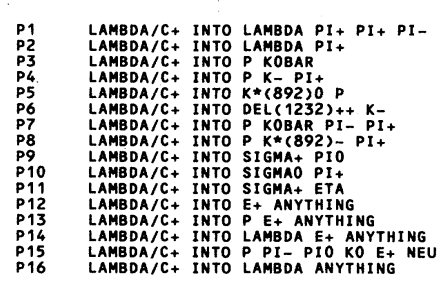

DECAY MASSES
$1116+140+140+140$

$938+498$
$938+494+140$

$938+498+140+140$

$938+892$
$1189+135$
$1192+140$

$1192+140$
$1189+549$

$938+140+135+498+$

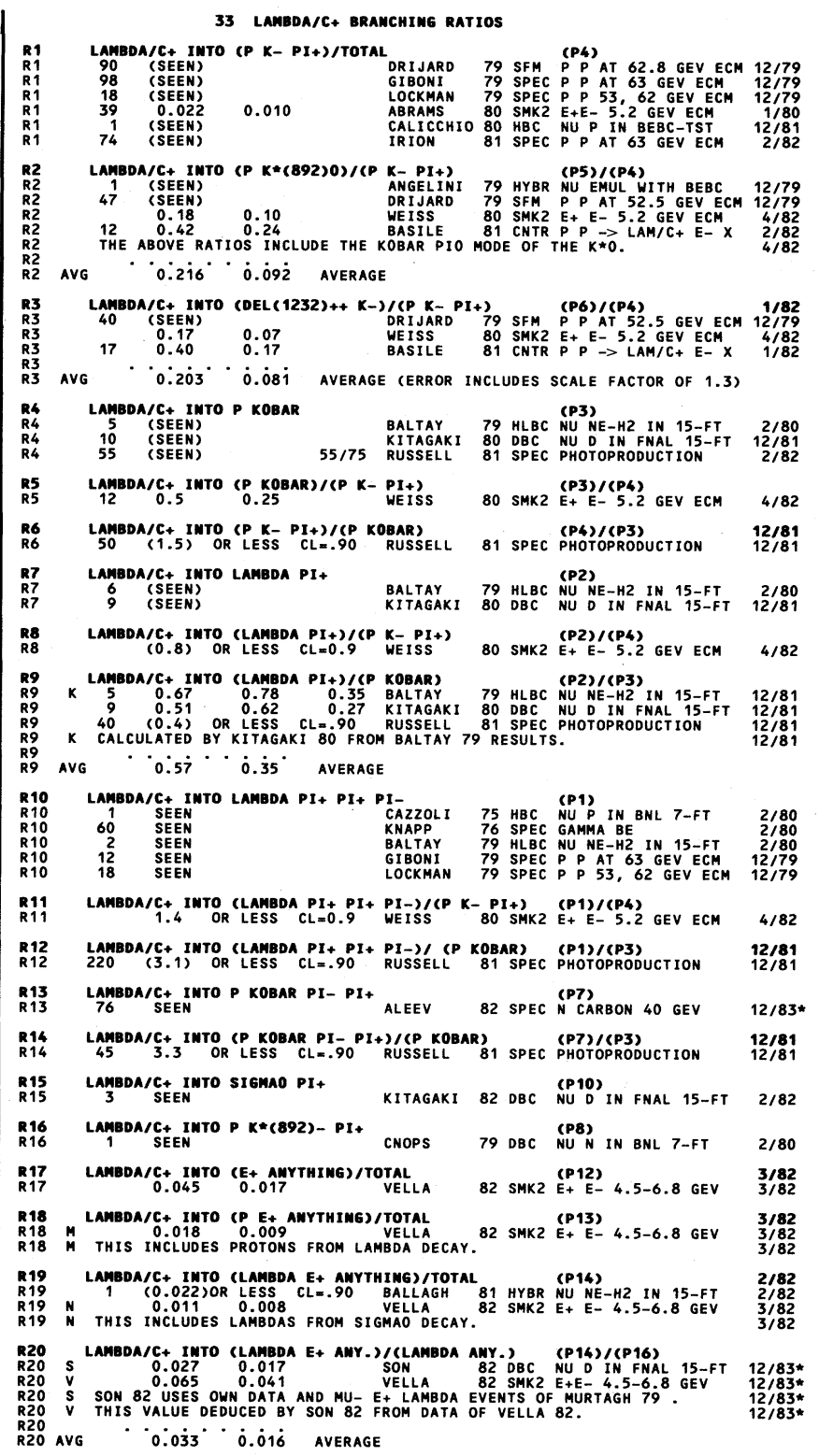

$\begin{array}{lllllll}\text { CAZZOL I } & 75 & \text { PRL } 34 & 1125 & \text { +CNOPS, CONNOLY, LOUTTIT, MURTAGH, } \\ \text { KNAPP } & 76 & \text { PRL } 37 & 882 & \text { (BNL) }\end{array}$

,

ANGELINI 79 PL $84 B 150$
BALTAY 79 PRL $421721 \quad$ (ANKA+LIBH+CERN+DUUC+LOUC+KEYN+PISA+ROMA+)
+CAROUMBALS, FRENCH, HIBBS, +
(COLU+BNL)

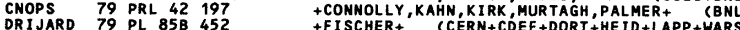

GIBONI 79 PL 85B 437 +DIBITONTO+ (AACH+CERN+HARV+ MUNI+NUES+UCR)

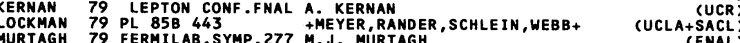

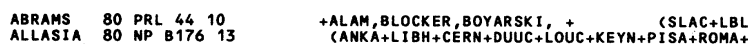

CALICCHI 80 PL $938521 \quad+$ (BARI+BIRH+BRUX +CERN+EPOL+RHEL+SACL+LOUC

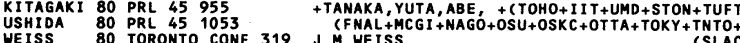

ADAMOVIC 81 PL $99 B 271$ ADAMOVICH+(PHOTON-EMUL, OMEGA-PHOTON COLLS.)

BALLAGH 81 PR D24 ?

FIORINO 81 LNC 30166 (PHOTON-EMUL COLLAB.+OMEGA-PHOTON COLLAB.)

GRAESSLE 81 PL 998159
GRAESSLER, LANSKE+ (AACH+BONN+CERN+MPIM+OXF) 
Stable Particles

Data Card Listings

$\Lambda_{\mathrm{c}}^{+}, \mathrm{A}^{+}, \Lambda_{\mathrm{b}}^{0}$, TOP HADRON SEARCHES, FREE QUARK SEARCHES
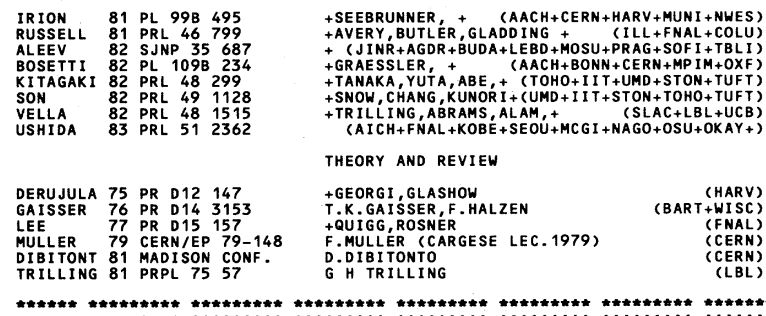

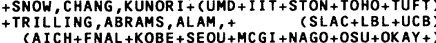
THEORY AND REVIEW

+GEORGI, GLASHOW

T.K.GAISSER, F. HALZEN
+QUIGG, ROSNER

F.MULLER (CARGESE LEC.1979) D. DIBITONTO

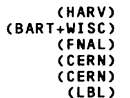

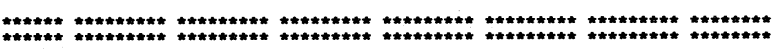

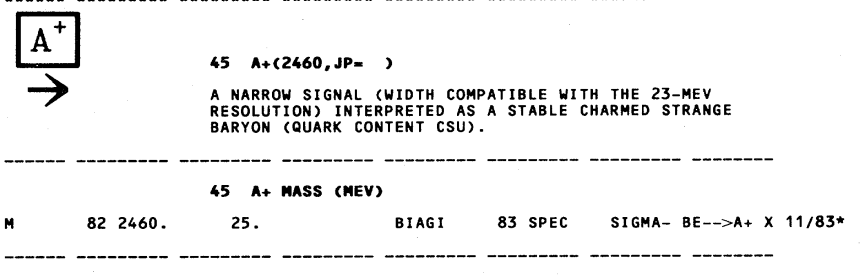

45 A+ PARtial decay modes

P1 A+ INTO LAMBDA K- PI+ PI+ DECAY MASSES
$1116+494+140+140$

45 A+ BRAMCHIMG RATIOS

R1
R1

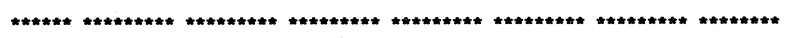
REFERENCES FOR $A+(2460)$

BIAGI 83 PL 122B $455+$ (BRIS+CERN+GEVA+HEID+LAUS+LOQM+MELB+RL)

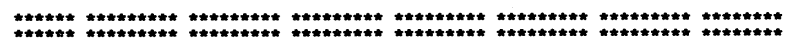

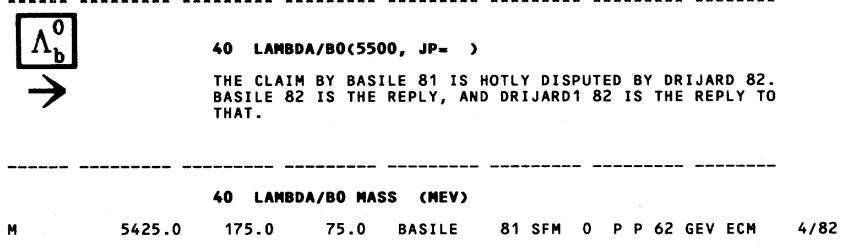

\section{Lambda/BO partial decay modes}

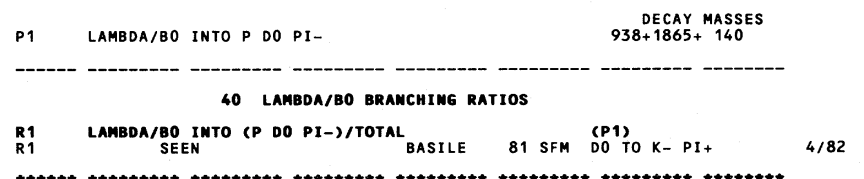

R1 LAMBDA/BO INTO (P DO PI-)/TOTAL BASILE $81 \mathrm{SFM}$ D0 TO K- PI+
R1

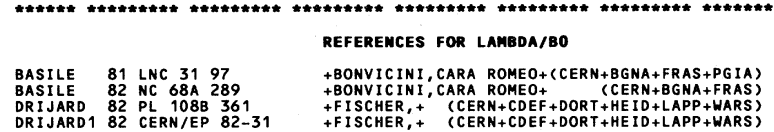

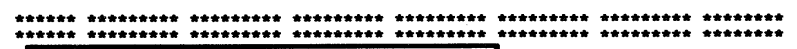
\begin{tabular}{lllll}
\hline TOP & HADRON & SEARCHES \\
\hline
\end{tabular}

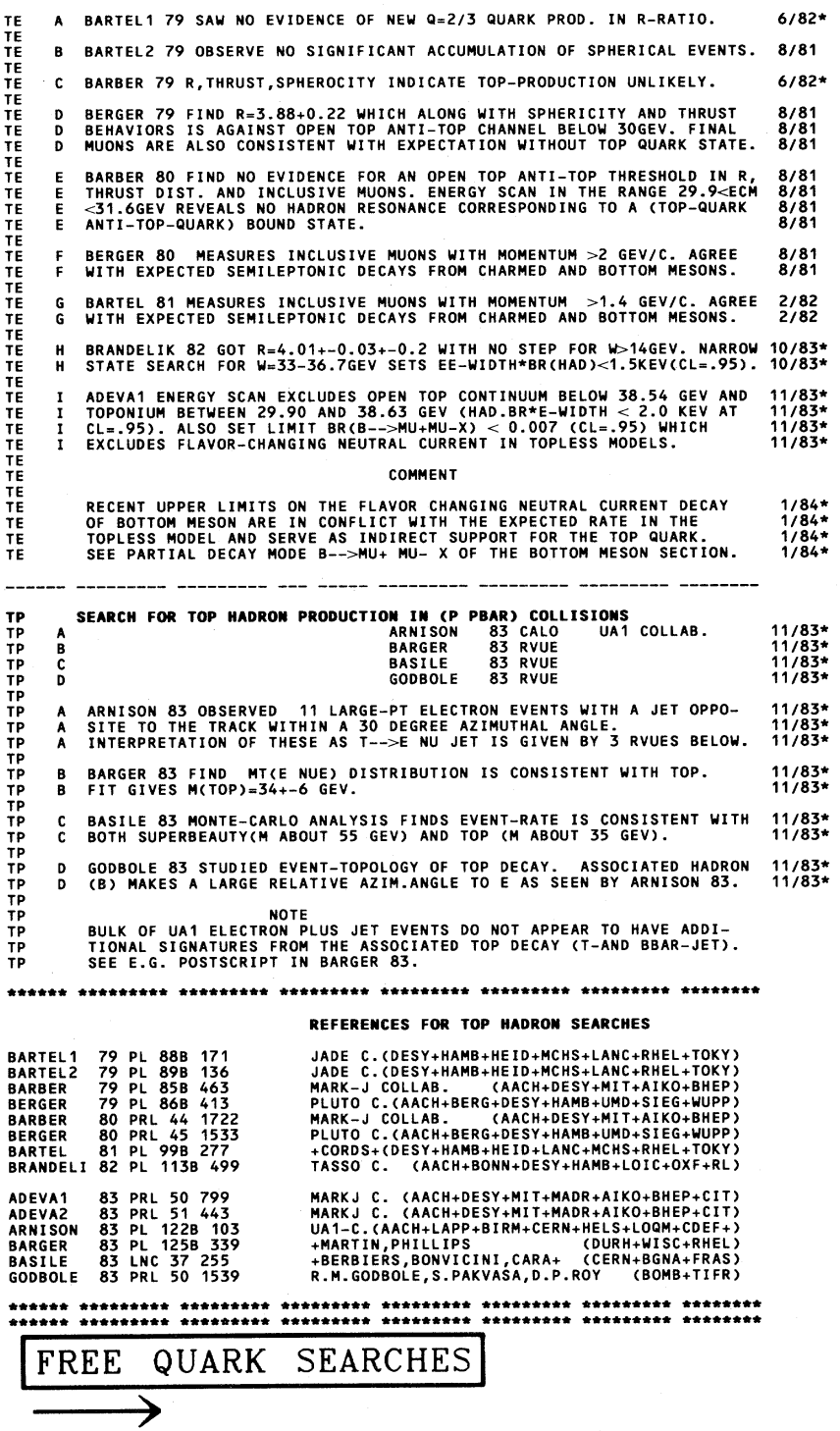

(by W.P. Trower, Virginia Polytechnic Institute and State University)

The idea that all hadrons are constructed from a set of fractionally charged constituents (quarks) is central to the quantum-chromodynamics description of particle scattering and hadron spectroscopy. Quantumchromodynamics in its usual form contains the as-yetunproven restriction that quarks must forever be confined to the mesons and baryons they make up.

Experiments support the conclusion that it is at best difficult to "unglue" quarks. Accelerator searches at increasing energies have produced no evidence for free quarks. Of the several candidate cosmic ray events, one 
still enjoys the active advocacy of its discoverer. ${ }^{1}$ The only positive searches in matter, those of LARUE, have published no new data since November 1980.

This compilation should be used as a directory to the literature since the quoted experimental limits are often only indicative.

\section{Reference}

1. C.B.A. McCusker, Aust. J. Phys. 36, 717 (1983).

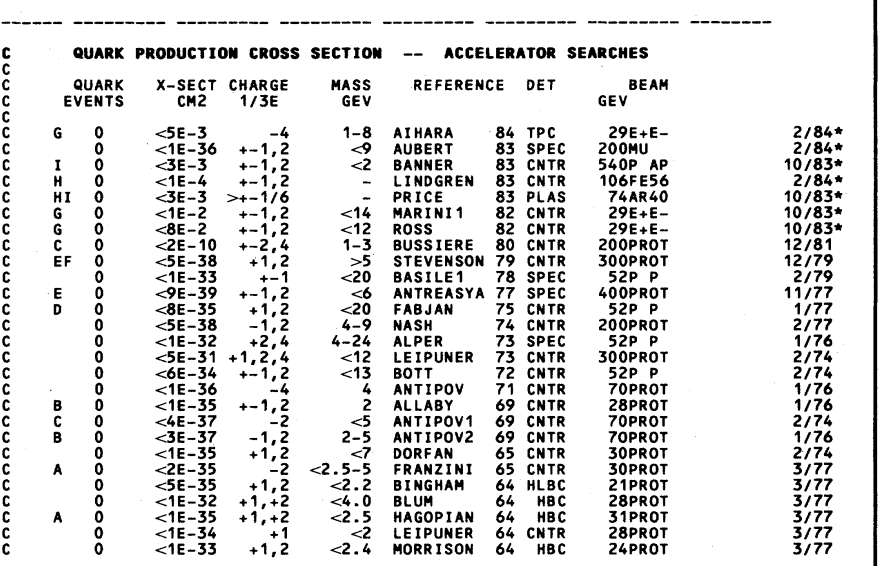

\section{I - BOUND TO NUCLEI. FOR X-SECT READ FRACTION OF FRAGMENTS. \\ G - FOR X-SECTION READ X-SECT (Q-Q X X)/X-SECT (MU-MU) \\ E - X-SECTION CM2/GEVV2.

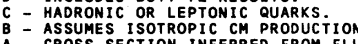

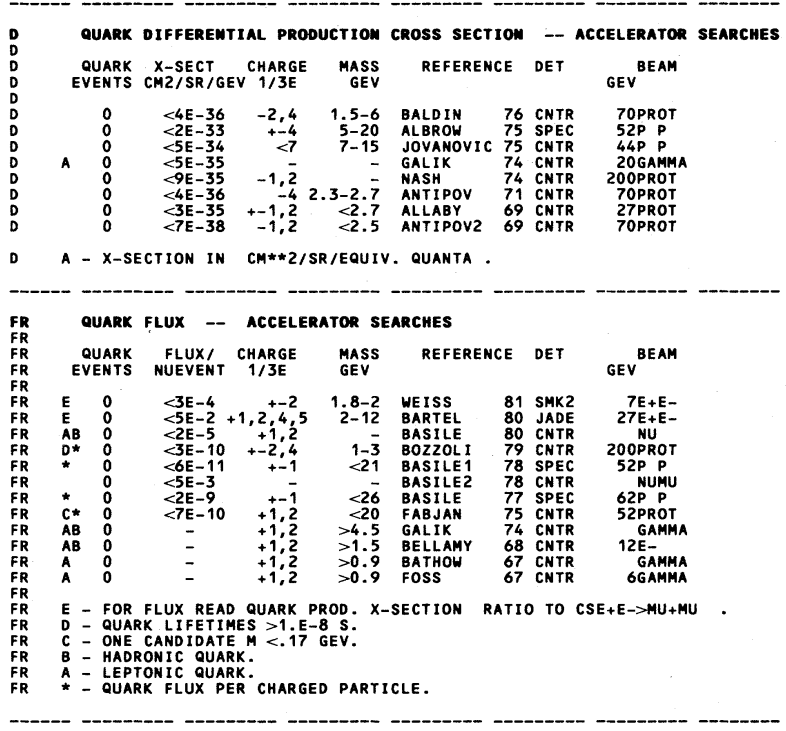

QUARK FLUX -- COSHIC RAY SEARCHES

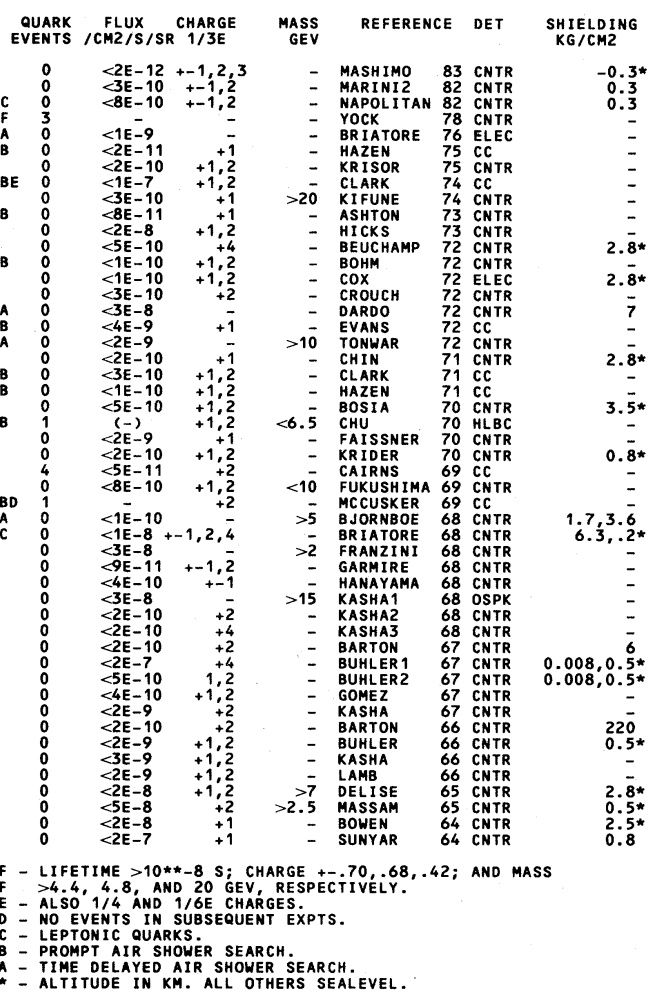

$\underset{K G / C H 2}{\text { HIELDING }}$

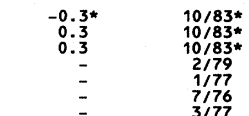

183*

A - TIME DELAYED AIR SHOWER SEARCH.
$\star$
ALTITUDE IN KM. ALL OTHERS SEALEVEL.

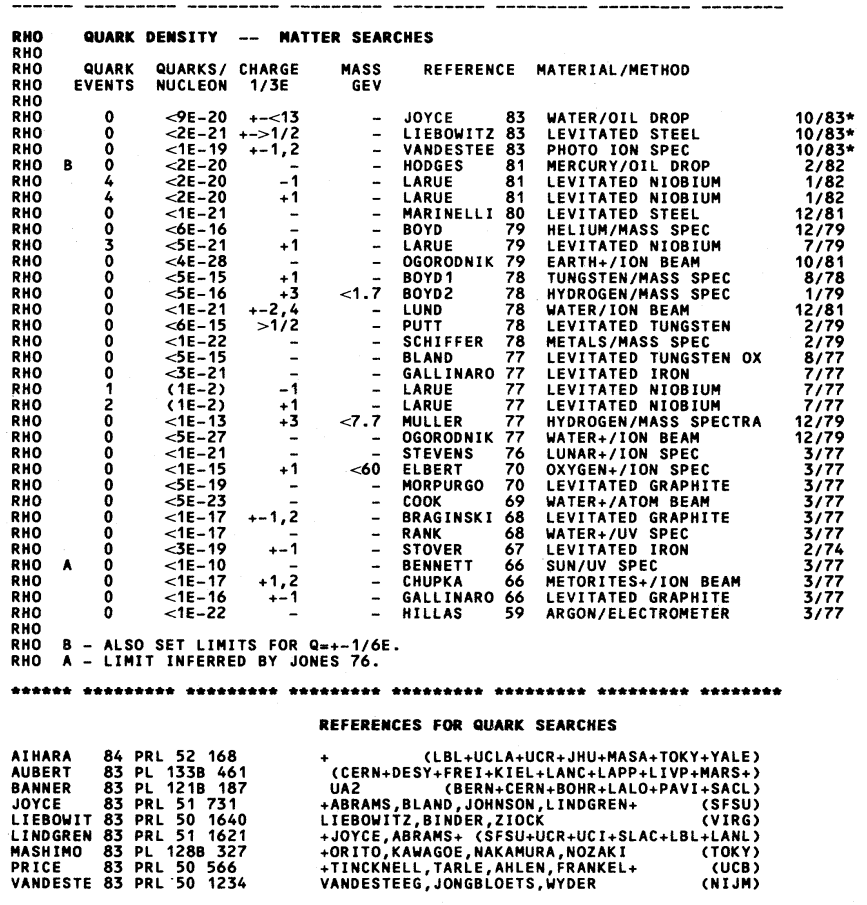


Stable Particles FREE QUARK SEARCHES, MAGNETIC MONOPOLE SEARCHES

\section{Data Card Listings}

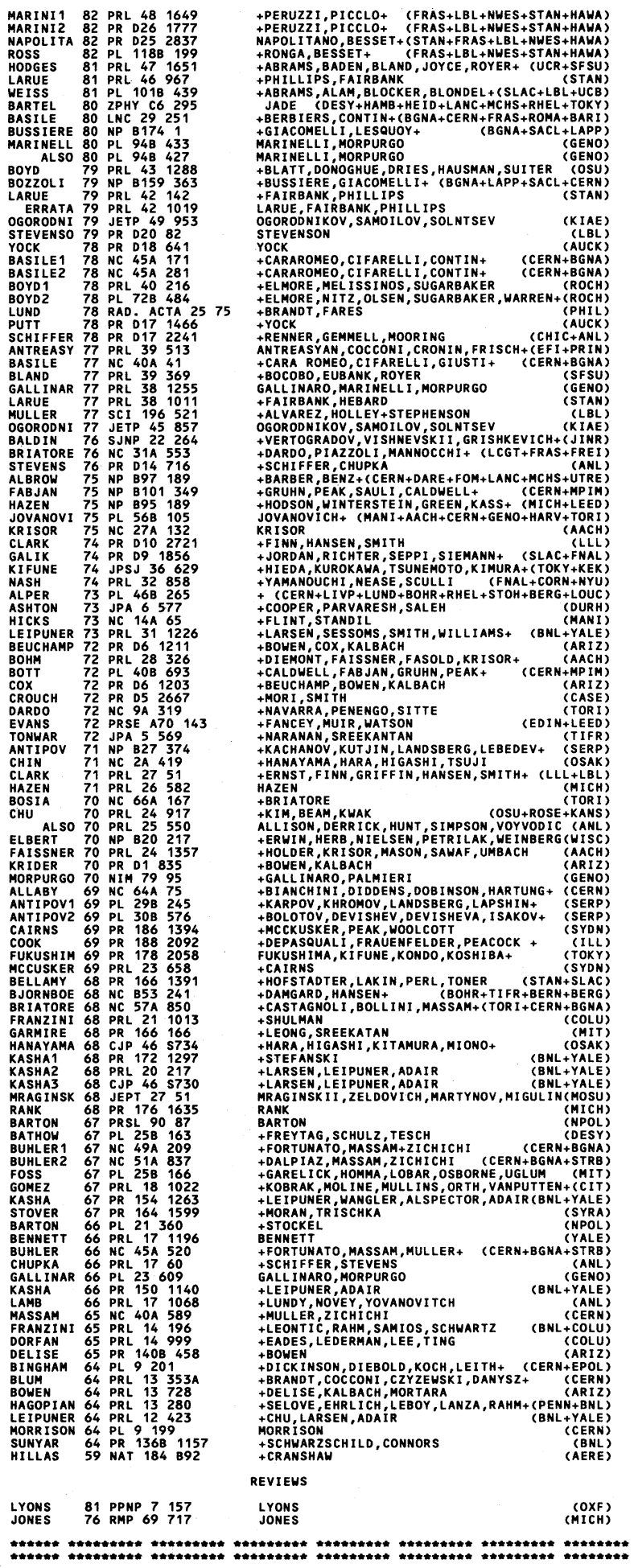

$\stackrel{\text { MAGNETIC }}{\longrightarrow}$

(by W.P. Trower, Virginia Polytechnic Institute and State University)

Although the usual formulation of Maxwell's equations suggests magnetic monopoles, no observed phenomenon requires them for its explanation. ${ }^{1}$ From the assertion that a monopole anywhere in the universe would result in electric charge quantization everywhere followed the prediction of a least magnetic charge $\mathrm{G}=$ $\mathrm{e} / 2 \alpha$, the Dirac charge. ${ }^{2}$ Observed pure multiphoton events have been attributed to virtual monopole production and annihilation. ${ }^{3}$ Monopoles have recently become indispensable to many gauge theories, which endow them with a variety of extraordinarily large masses.

Monopole detectors have predominantly used either induction or ionization. Induction experiments measure the monopole magnetic charge by detecting a change in current when a monopole passes through a loop. ${ }^{4}$

These measurements, which are independent of monopole electric charge, mass, and velocity, have produced a solitary monopole candidate event (CABRERA 82), uncorroborated by later searches (e.g., CABRERA 83).

Ionization experiments rely on a magnetic charge producing more ionization than an electrical charge with the same velocity. However, the ability to distinguish a monopole by ionization diminishes with velocity. The theory of monopole energy loss for $\beta \leqslant 10^{-3}$ is still unsettled.

Cosmic rays are the most likely source of large-mass monopoles, as accelerator energies are insufficient to produce them. Evidence for such monopoles may also be obtained from astrophysical observations.

This compilation should be used as a directory to the literature since the quoted experimental limits are often only indicative.

\section{References}

1. J.D. Jackson, CERN-77-17 (1977).

2. P.A.M. Dirac, Proc. Royal Soc. London A133, 60 (1931).

3. M.A. Ruderman and D. Zwanziger, Phys. Rev. Lett. 22, 146 (1969).

4. L.W. Alvarez, LRL Physics Note 470 (1963). 
For notation, see key at front of Listings.

Stable Particles MAGNETIC MONOPOLE SEARCHES, AXION SEARCHES

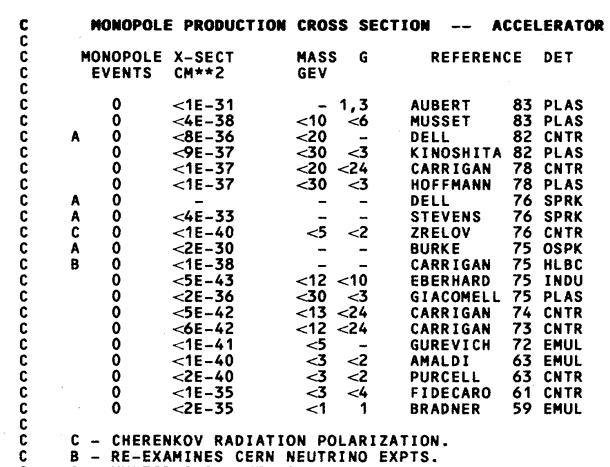

C - CHERENKOV RADIATION POLARIZATION.
B - RE-EXAMINES CERN NEUTRINO EXPTS.
A - MULTIPHOTON EVENTS.

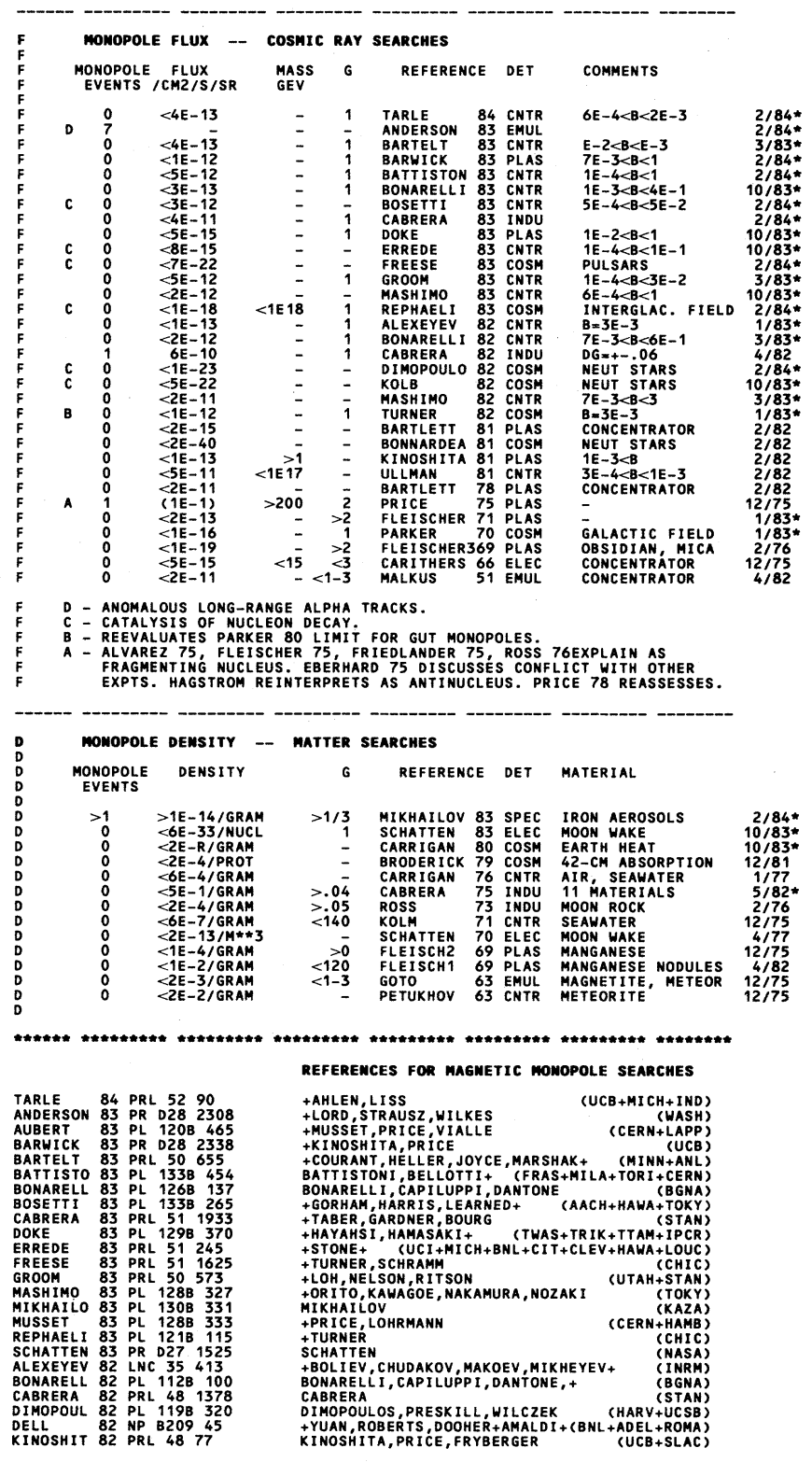

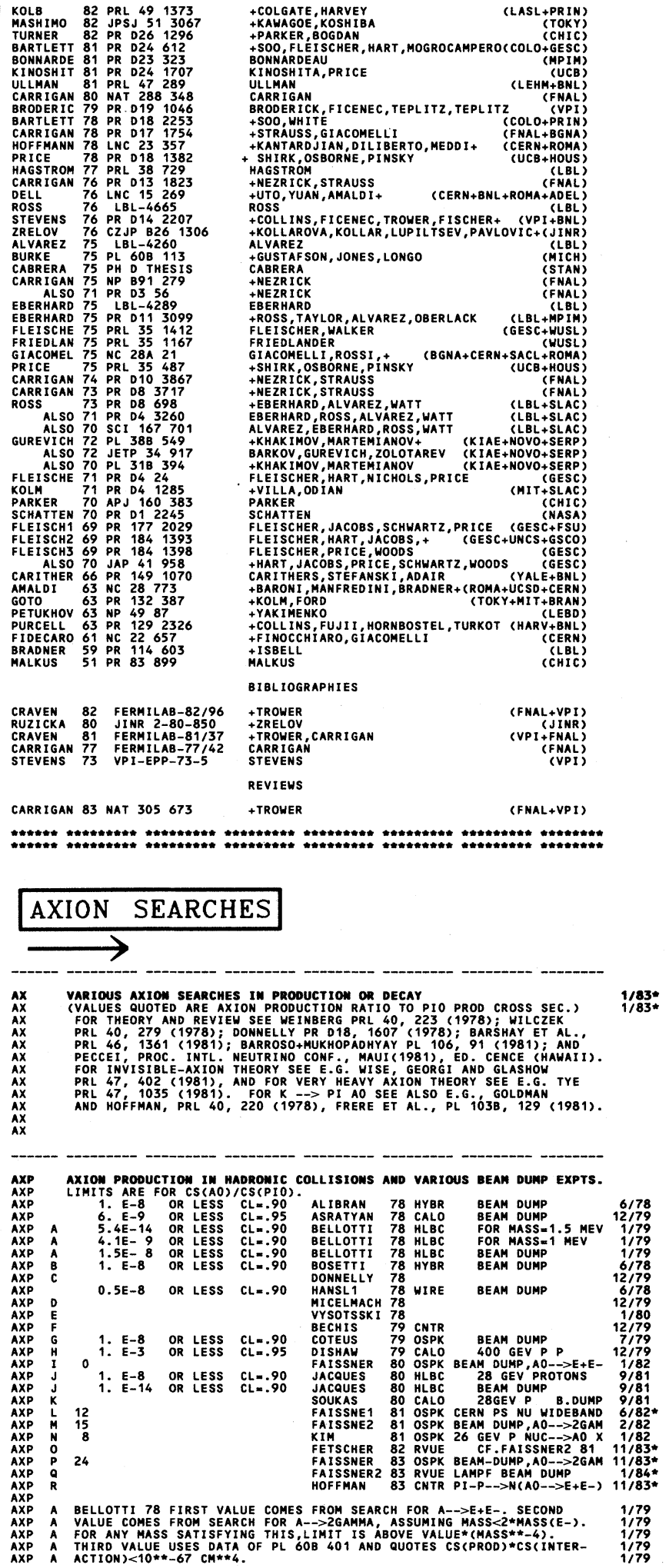


Stable Particles

Data Card Listings

\section{AXION SEARCHES, OTHER STABLE PARTICLE SEARCHES}

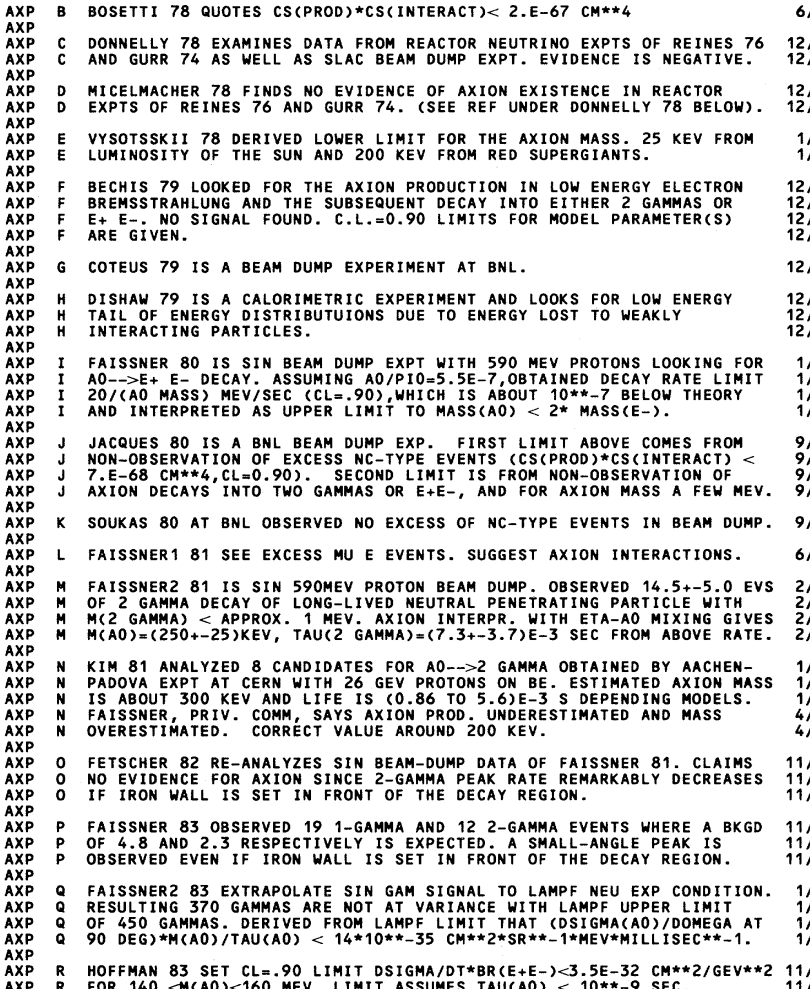

-

AXD
AXION SEARCHES IN THE DECAY OR TRAMSITION OF POSITROMIUH, QUARKOWIUm,
KAON, NUCLEON, AND RADIOACTIVE NUCLEUS. LIMITS ARE FOR BRANCHING RATIO

$A X D$
$A X D$
$A X D$
$A X D$
$A X D$
$A X D$
$A X D$
$A X D$
$A X D$
$A X D$
$A X D$
$A X D$
$A X D$
$A X D$
$A X D$
$A X D$
$A X D$
$A X D$
$A X D$
$A X D$
$A X D$
$A X D$
$A X D$
$A X D$
$A X D$
$A X D$
$A X D$
$A X D$
$A X D$
$A X D$
$A X D$
$A X D$
$A X D$
$A X D$
$A X D$
$A X D$
$A X D$
$A X D$
$A X D$
$A X D$
$A X D$
$A X D$
$A X D$
$A X D$
$A X D$
$A X D$
$A X D$
$A X D$
$A X D$
$A X D$
$A X D$
$A X D$
$A X D$
$A X D$
$A X D$
$A X D$
$A X D$
$A X D$
$A X D$
$A X D$
$A X D$
$A X D$
$A X D$
$A X D$
$A X D$
$A X D$
$A X D$
$A X D$
$A X D$
$A X D$
$A X D$
$A X D$
$A X D$
$A X D$
$A X D$
$A X D$
$A X D$
$A X D$ KAON,

0

0

1.4 E-5 OR LESS $C L=.90$

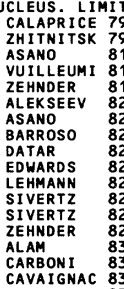

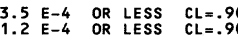

3. $E-4$ OR LESS $\mathrm{CL}=.90$

CARBONI 83 CNER UPSI (1S)-->AO GAMTA

9.1 E-4 OR LESS CL=.90 NICZYPORU 83 LENA UPSI(is)-->A0 GAMMA

CALAPRICE 79 SAW NO AXION EMISSION FROM EXCITED STATES OF CARBON.
SENSITIVE TO AXION MASS BETWEEN 1 AND 15 MEV.

ZHITNITSKII 79 ARGUE THAT A HEAVY AXION BY YANG (3 3 MC 40 MEV)
CONTRADICTS EXPERIMENTAL MUON ANOM. MAGNETIC MOMENTS.

C ASANO 81 IS KEK EXPT. SET $B R(K+-->P I+A 0)<3.8 E-8$ AT $C L=.90$.

D VUILlEUMIER 81 is at GRENOBLE REACTOR. SET LIMIT M(AO) $<280 \mathrm{KEV}$. ZEHNDER 81 LOOKED FOR BA*-->AO BA TRANSITION WITH A0-->2 GAMMA.
OBTAINED 2GAMMA COINCIDENCE RATE <2.2E-5 /SEC(CL=.95) EXCLUD ING MASS $(A O)>160$ KEV 0 (OR 200 KEV DEPENDING ON HIGGS MIX
HOWEVER, SEE BARROSO + MUKHOPADHYAY, REF. ABOVE.

ALEKSEEV 82 WITH IBR-2 PULSED REACTOR EXCLUDE STANDARD AO AT CL $x$ (DEUT* DECAY).

ASANO 82 AT KEK SET LIMITS FOR BR(K+-->PI+ AO) FOR M(AO) $<100$ MEV AS
BR $<4$. E-8 FOR TAU(AO-- $>$ N-GAMMAS) $>1$. E-9S, BR<1.4E-6 FOR TAU<1. E-9S. BARROSO 82 DERIVE IN DFS-MODEL (PL 104B, 199 ) AO MASS LIMITS FROM
STELLER-ENERGY-LOSS BOUND. ALLOWED MASS REGIONS ARE LIMIS $M(A O)<10$ EV (AXION INVISIBLE DUE TO VERY SMALL COUPLING), AND M(AO) 10 EV (AXION INVISIBLE DUE TO VERY SMAL
COMO) AROUND 200 KEV (CORESPONDING DFS-AO PARAM
COMPATIE WITH REACTOR DATA OF ZEHNDER 81). DATAR 82 LOOKED FOR AO-->2 GAMMA IN NEUTRON CAPTURE (N P-->D AO) AT
TARAPUR 500 MW REACTOR. SENSITIVE TO SUM OF I=O AND I I AMPLITUDES.
WITH ZEHNDER $81((I=0)-(I=1))$ RESULT, ASSERT NON-EX OF STANDARD AO. EDWARDS 82 LOOKED FOR J/PSI-->GAMMA+AXION DECAYS BY LOOKING FOR
EVENTS WIT A SINGLE GAMMA COF ENERGY APPROX. 1/2 THE J/PSI MASS) PLUS NOTHING ELSE IN THE DETECTOR. THE LIMIT IS INCO
THE AXION INTERPRETATION OF THE FAISSNER2 81 RESULT.

LEHMANN 82 OBTAINED A0-->2GAM RATE<6.2E-5 /SEC (CL=.95) EXCLUDING
MASS(AXION) BETWEEN 100 AND $1000 \mathrm{KEV}$.
$6 / 78$

.

$2 / 79$

$1 / 80$

$12 / 79$
$12 / 79$
$12 / 79$

$12 / 79$

$12 / 79$
$12 / 79$

$12 / 79$
$12 / 79$

$1 / 82$ $1 / 82$

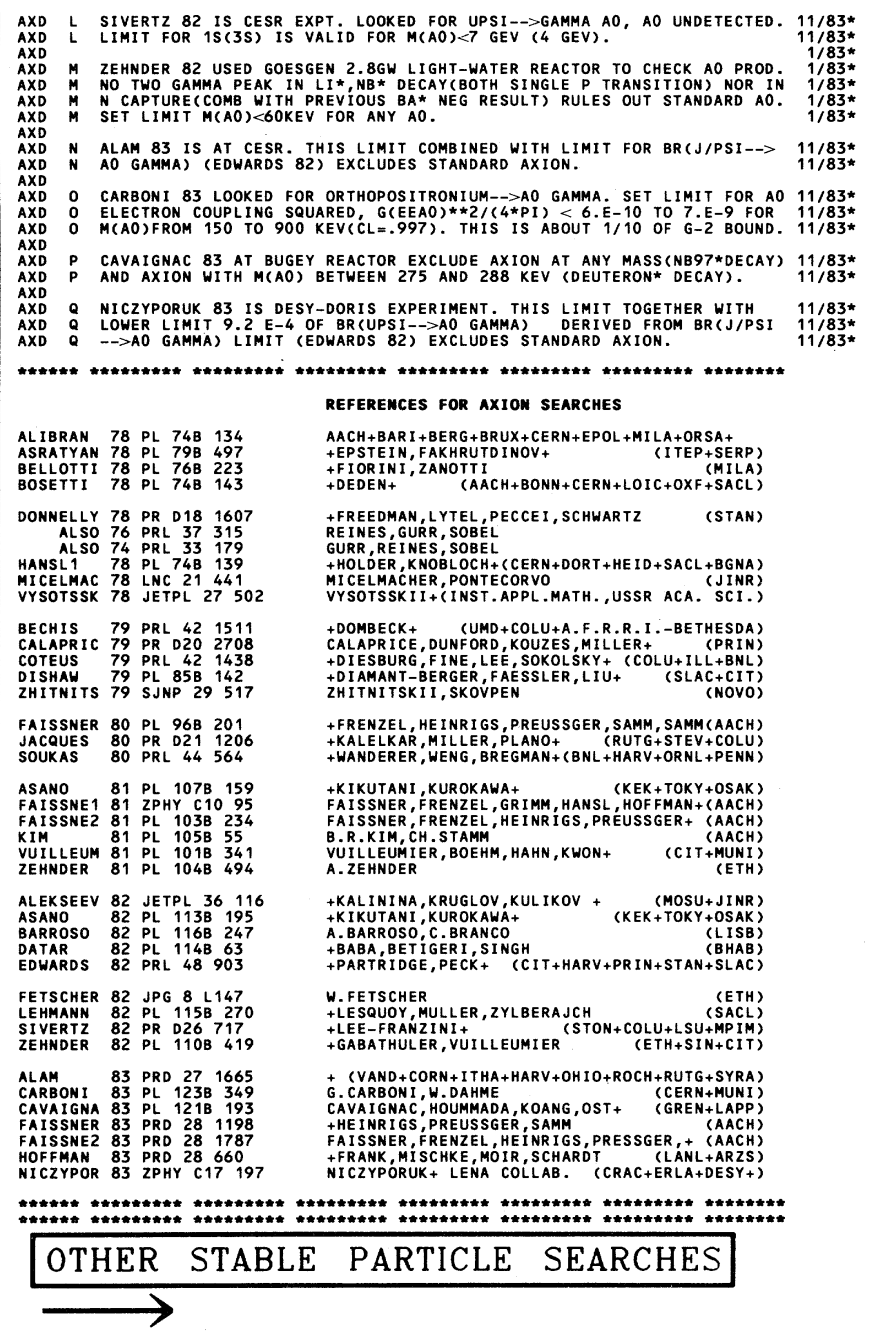

We collect here those searches which do not fit neatly into one of the above search categories. These include searches for higgs bosons, technipions, gluinos, photinos, squarks, shadrons, and sleptons (sections $\mathrm{H}$, HGC, EGT, EGP, SLP), trimuon and four-lepton production in neutrino and antineutrino reactions (T, FL), di- and trimuon production in muon interactions (MU), and heavy particle searches in accelerator experiments (EE, CH, CS, D, ICH, RPI, CA, CEN), in cosmic rays (F), and in matter (CON). Searches are also listed for light particles (C), highly-ionizing particles (ION), penetrating non-neutrino-like particles (BD), and tachyons (TCF, TCM, TCD). Note that axion searches now appear in a separate section above. 
BD
BD A PRODUCTION OF MEU PEMETRATIMG MON-MEUTRIMO LIRE STATES IN BEAM DUMP
LOSECCO

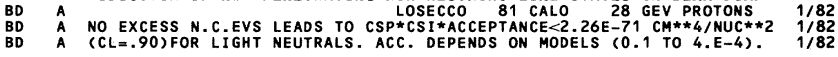

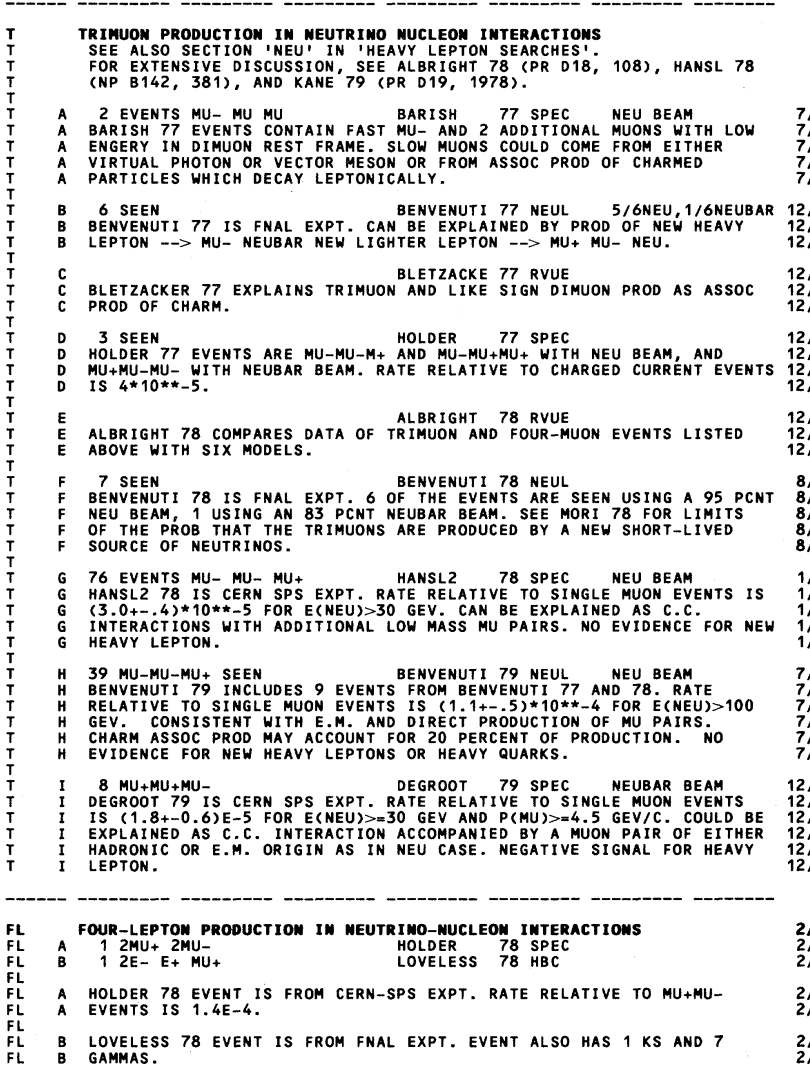

\section{MU DI- AND TRI-MUON PRODUCTION IN MU MUCLEON IMTERACTIONS
MUU SEE ALSO SECT TON PMU IN HEAVY LEPTON SEARCES ANO CHARM SEARCHES}

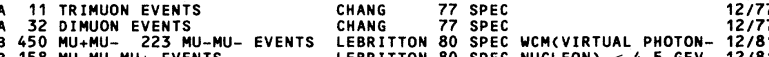
158 MU-MU-MU L ENENTS EVETS LEBG

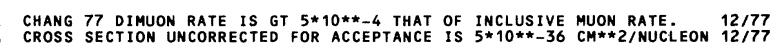

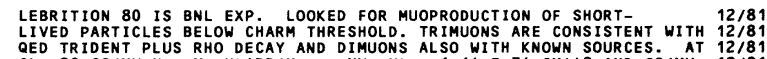

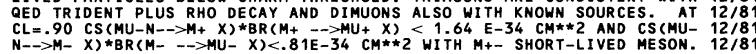

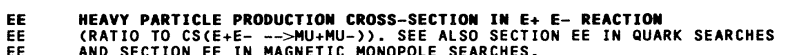

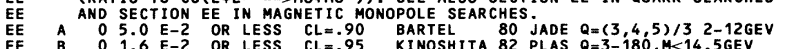
EE A BARTEL 80 IS DESY PETRA EXPT WITH WCM=27-35 GEV. ABOVE LIMIT IS FOR

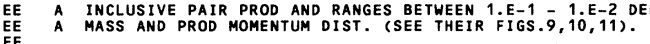
EE
EE
EE KLANOSHITA 82 IS SLAC PEP EXP AT WCMM29 GEV USING LEXAN AND CR-39
B PLASIC SHEETS SENSITIVE TO HIGHLY IONIZING PARTICLES.

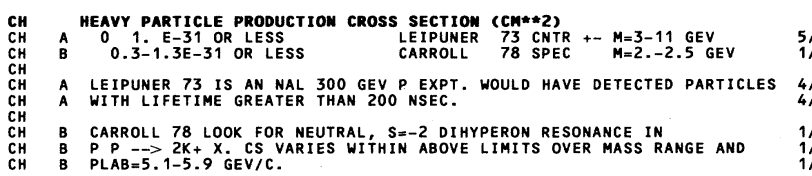

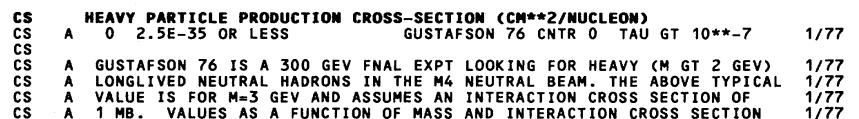
$\begin{array}{llll}\text { CS } & \text { A VALUE IS FOR MA3 GEV AND ASSUMES AN INTERACTION CROSS SECTION OF } & 1 / 77 \\ \text { CS } & \text { A MB. VALUES AS A FUNCTION OF MASS AND INTERACTION CROSS SECTION } & 1 / 77 \\ \text { CS A ARE GIVEN IN FIG. } 2 \text {. } & 1 / 77\end{array}$

A DORFAN 65 IS A $30 \mathrm{GEV} / C$ P EXPT AT BNL. UNITS ARE PER GEV MOMENTUM
A PER NUCLEUS.
$5 / 76$

ANTIPOV1 71 LIMIT INFERRED FROM FLUX RATIO. 70 GEV P EXPERIMENT. $3 / 77$

D ALPER 73 IS CERN ISR $26+26$ GEV P+P EXPT. P>.9 GEV, .2< BETA <.65. 5/76

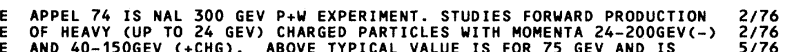

$\begin{array}{llll}F & \text { ALBROW } 75 \text { IS A CERN ISR EXPT WITH ECM=53 GEV. THETA=40 MR. SEE } & 1 / 77 \\ \text { F FIG. } 5 \text { FOR MASS RANGES UP TO } 35 \text { GEV. } & 1 / 77\end{array}$

$\begin{array}{lll}G & \text { JOVANOVICH } 75 \text { IS A CERN ISR } 26+26 \text { AND } 15+15 \text { GEV P+P EXPERIMENT. } & 2 / 76 \\ 6 \text { FIG } 4 \text { COVERS RANGES Q=1/3 TO } 2 \text { AND M=3 TO } 26 \text { GEV. } & 2 / 76 \\ G \text { VALUE IS PER GEV MOMENTUM. } & 5 / 76\end{array}$

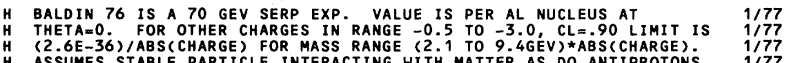

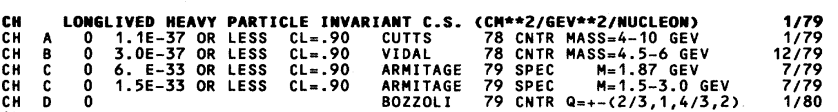

CH
CH
CH AUTS 78 IS $P$ BE EXPT AT FNAL SENSITIVE TO PARTICLES OF TAUS $\$$ ISE-8SEC $1 / 79$
A ICH
ICH
ICH
ICHDAL 78 IS FNAL 400 GEV PROTON EXPT. VALUE IS FOR X X $=0$ AND PT $=0$.

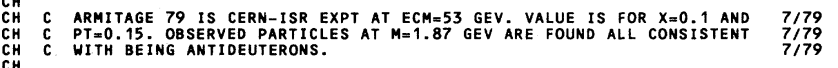
$\begin{array}{llll}\text { ICH } & \text { BOZZOLI } 79 \text { IS CERN-SPS } 200 \text { GEV P N EXPERIMENT. LOOKS FOR PARTICLE } & 1 / 80 \\ \text { ICH } & \text { D WIH TAU LARGER THAN 10**-8 SEC. SEE THEIR FIG. } 11-18 \text { FOR PRODUCTION } & 1 / 80 \\ \text { ICH } & \text { D CROSS SECTION UPPER LIMITS VS MASS. } & 1 / 80\end{array}$

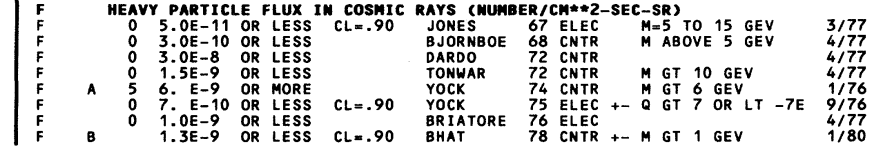




\section{Stable Particles OTHER STABLE PARTICLE SEARCHES}

Data Card Listings

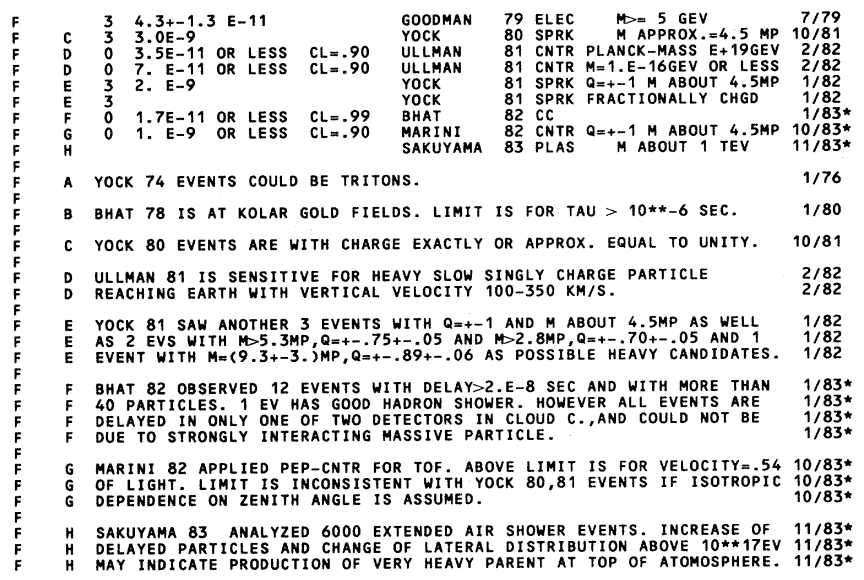

COM CONCEMTRATION OF MEAVY (CHARGE +1 ) STABLE PARTICLES IN MATTER
CON $2 . E-22$ TO $1 . E-21$ OR LESS

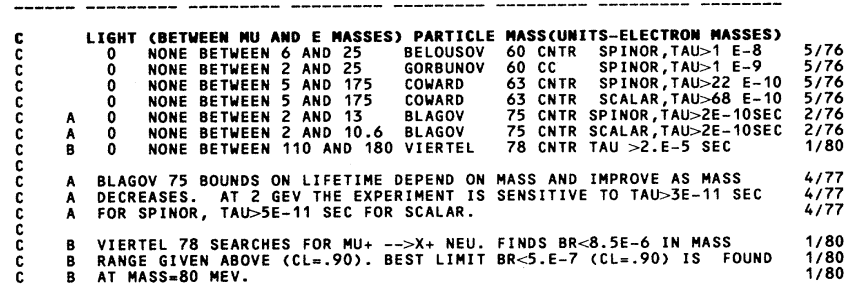

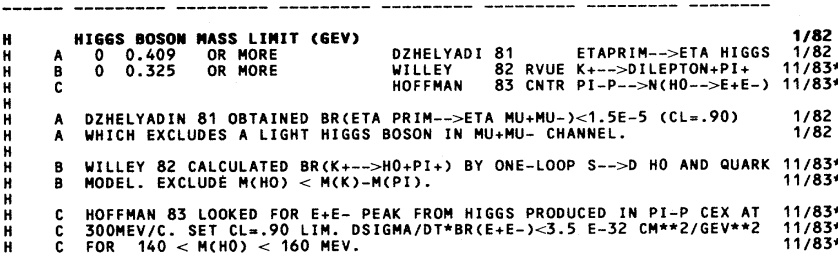

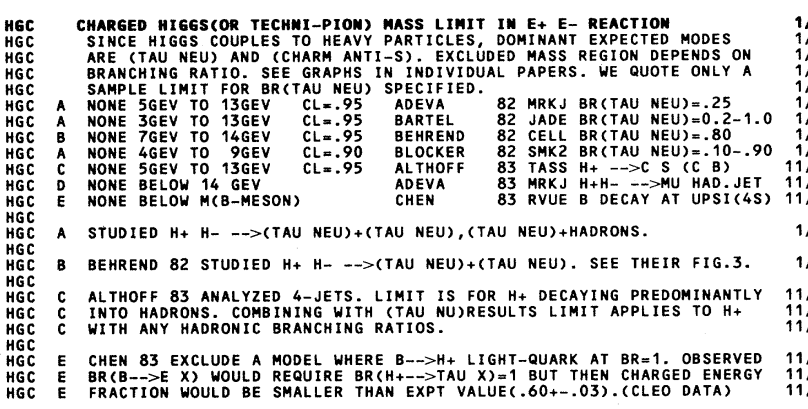

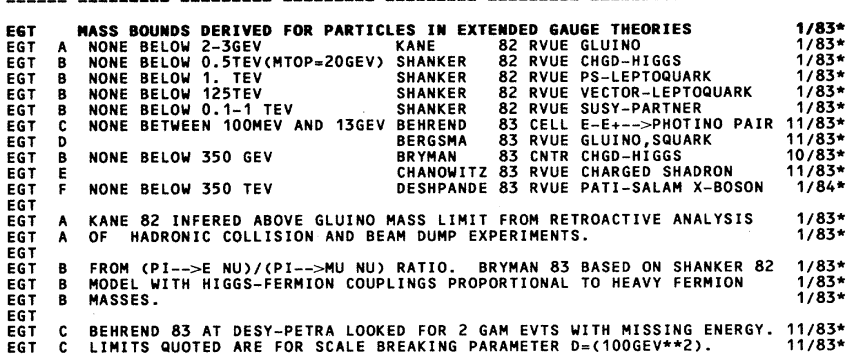

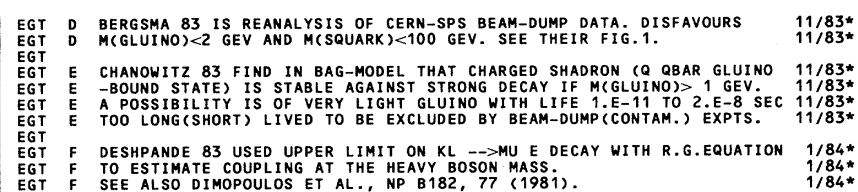

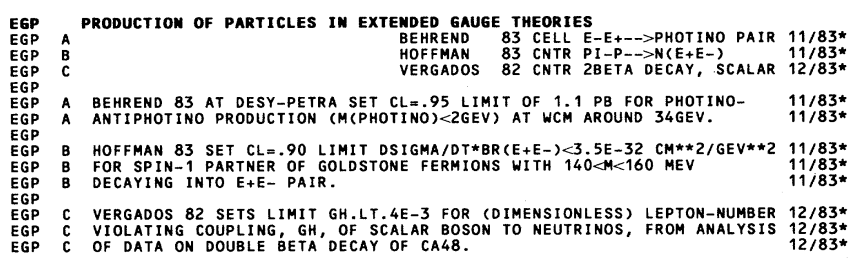

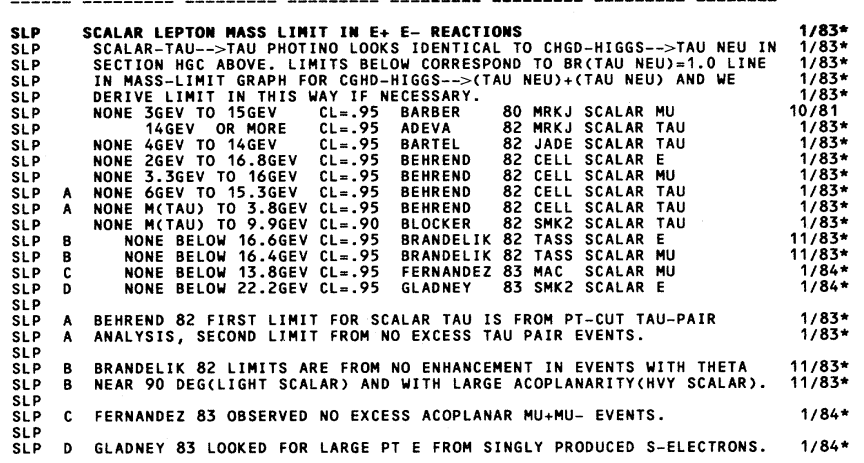

-

IOW HIGHLY TOMIZIMG PARTICLE FLUX CUMITS MUMBER/MH*2-YRP
ION
0.4 OR LESS CL..95

TCF TACHYOM FLUX IW COSHIC RAYS (MUMBER $/$ CH**2-SEC-SR)
TCF
SEE SMITH 77 FOR A REVIEW OF EARLIER COSMIC RAY AND ACCELERATOR

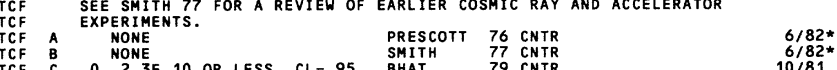

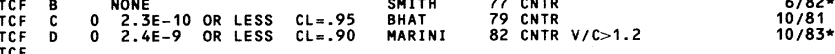

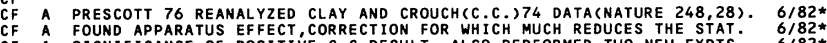

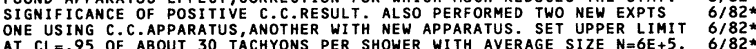

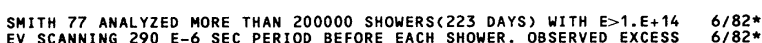

46+-40 EVS DOES NOT CONSTITUTE STATISTICALLY SI GNIFICANT EVIDENCE. 6/82*

TCF C BHAT 79 is at oOtaCaMUNo (2200M ABOVE SEA). NO SIGNAL IN 3621 HoURS. 10/81

TCF D MARINI 82 is tOF MEASUREMENT USING PEP-CNTR AT SEA LEVEL.
TCF D

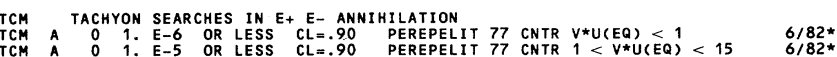

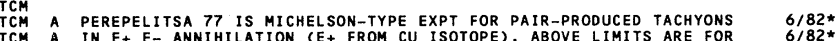

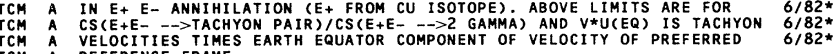

TCD SEARCHES FOR TACHYONIC DECAY (LOWER LIMIT FOR MEAN LIFE IN YEARS)

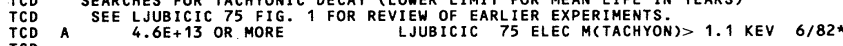

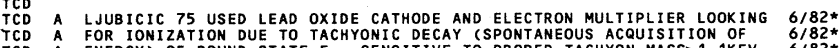

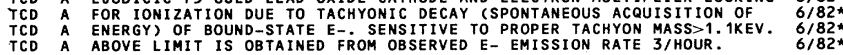

BELLOSSOV 60 JETP 111143

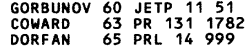

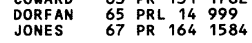

$\begin{array}{llll}\text { BJ ORNBOE } 68 & \text { NC } & 853 & 241 \\ \text { BINON } 69 \text { PL } 308 & 510\end{array}$

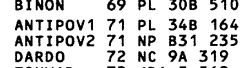

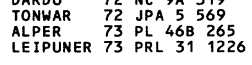

REFERENCES FOR OTHER MEU PARTICLE SEARCHES

PRSAKOV, TAMM, CERENKOV
+SPIRIDONOV CERENKOV

TGITELMAN, LYNCH, RITSON
TEA ISTAN)

(COLU)
EADES, LEDERMAN, LEE, TING
(MICH+WISC+LBL+UCLA+MINN+COSU+COLO+MURA)

+DAMGARD, HANSEN, CHATTERGEEE+
DUTEIL, KACHANOV, KHROMOV, KUTYIN+
(BOHR+BERN)
(SERP)

$\begin{array}{ll}\text { ODENISOV, DONSKOV, GORIN, KACHANOV+ } & \text { (SERP) } \\ \text { + } & \text { (SERT }\end{array}$

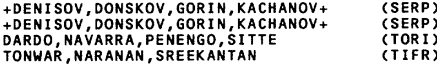

TONWAR, NARANAN, SREEKANTAN

(CERN+LIVP+LUND+BOHR+RHELSSTOH+BERG
+LARSEN, SESSOMS, SMITH, WILIIAMS + (BNL+YALE) 


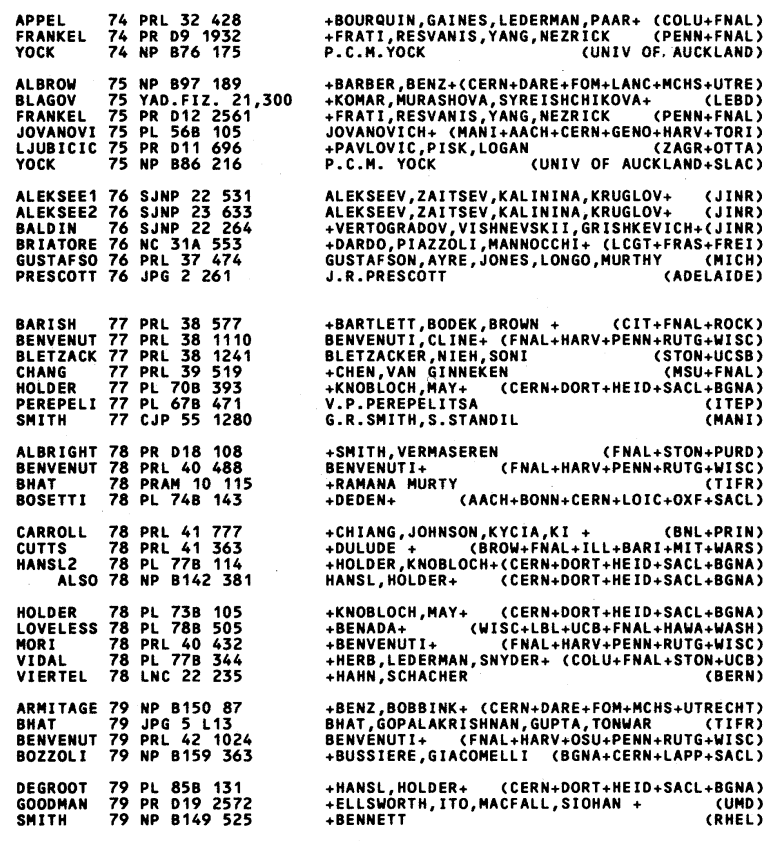

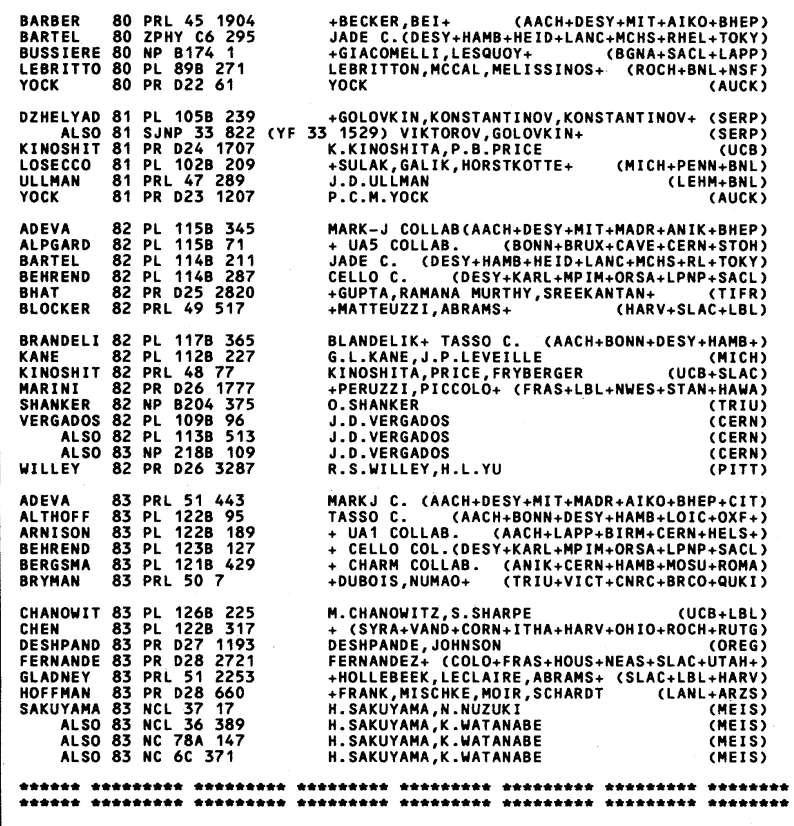




\section{Mesons}

$$
\pi^{ \pm}, \pi^{0}, \eta, \rho(770)
$$

\section{NOTE ON THE $\rho^{0}$ MASS AND WIDTH}

Because of the broadness of the $\rho$ meson, its shape is not very well described by a Breit-Wigner formula, which is a narrow-resonance approximation. Although most experimental distributions can be well described by a relativistic Breit-Wigner formula with a P-wave width and an additional shape parameter (PISUT 68), the resulting resonance parameters will clearly depend on this model. A consistent set of such determinations (PISUT 68, ESTABROOKS 74, BARTALUCCI 78, WICKLUND 78, HEYN 80) yields $\mathrm{m}_{\rho^{0}}=(769 \pm 3)$ $\mathrm{MeV}, \Gamma_{\rho^{0}}=(154 \pm 3) \mathrm{MeV}$.

Attempts have been made to determine the $\rho$ pole position in a more model-independent way (LANG 79, BOHACIK 80). It is comforting that these determinations agree with the above mass average (LANG 79, however, finds a somewhat smaller width).

A phenomenological estimate of the $\rho$ mass can be obtained with an SU(4) generalization of the GellMann-Okubo mass formula, which in the limit of ideal mixing can be written (MONTONEN 75)

$$
\rho=\frac{2\left[\psi \mathrm{K}^{*}(892)-\phi \mathrm{D}^{*}\right]+\omega\left[\mathrm{D}^{*}-\mathrm{K}^{*}(892)\right]}{2[\psi-\phi]-\left[\mathrm{D}^{*}-\mathrm{K}^{*}(892)\right]}
$$

The masses of the vector mesons on the right-hand side have all been determined to a much better precision than that of the $\rho$ and can be used to predict the mass of the $\rho=(768 \pm 2) \mathrm{MeV}$. The theoretical error due to nonideal mixing is expected to be of the order of \pm 1.5 $\mathrm{MeV}$. The result is consistent with the measured value (769 \pm 3$) \mathrm{MeV}$ given above.

\section{Data Card Listings}



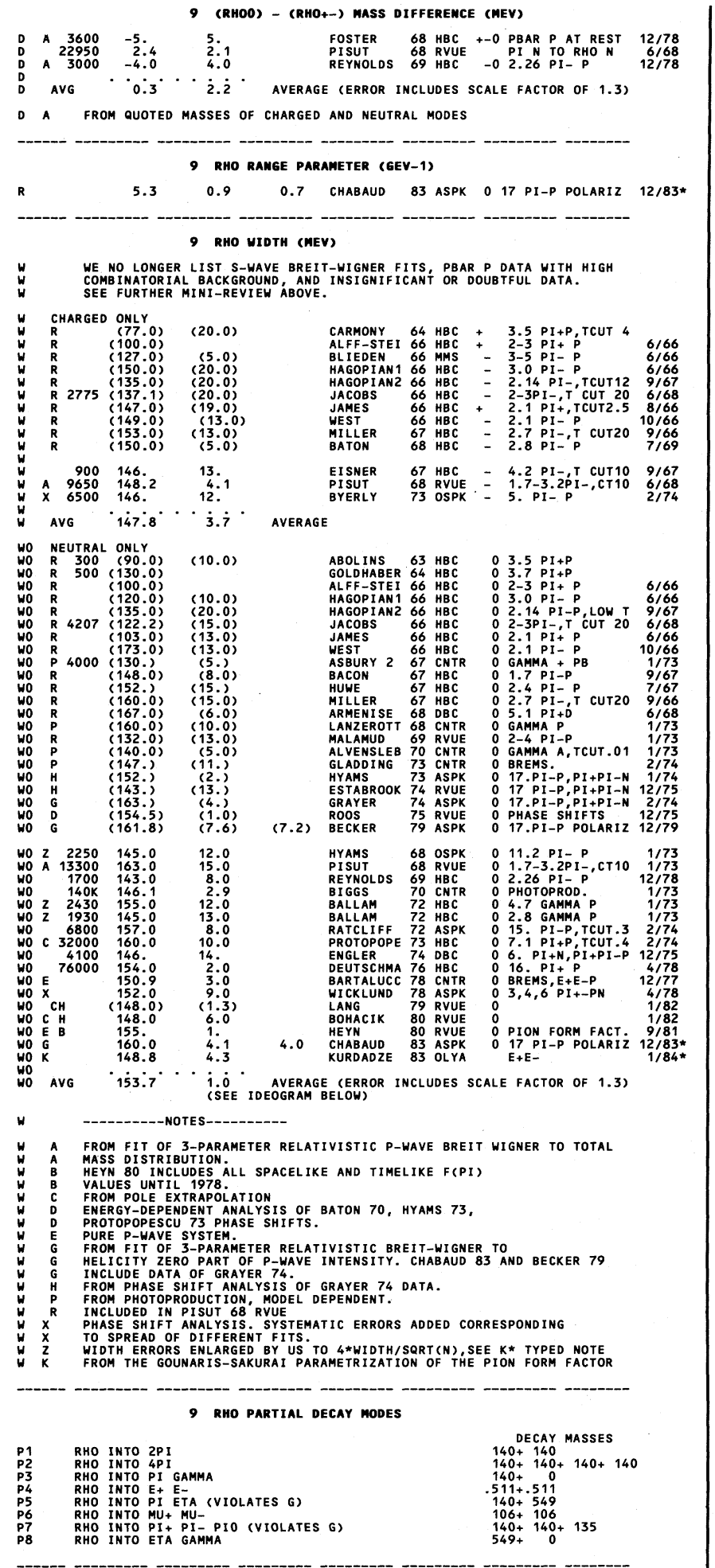

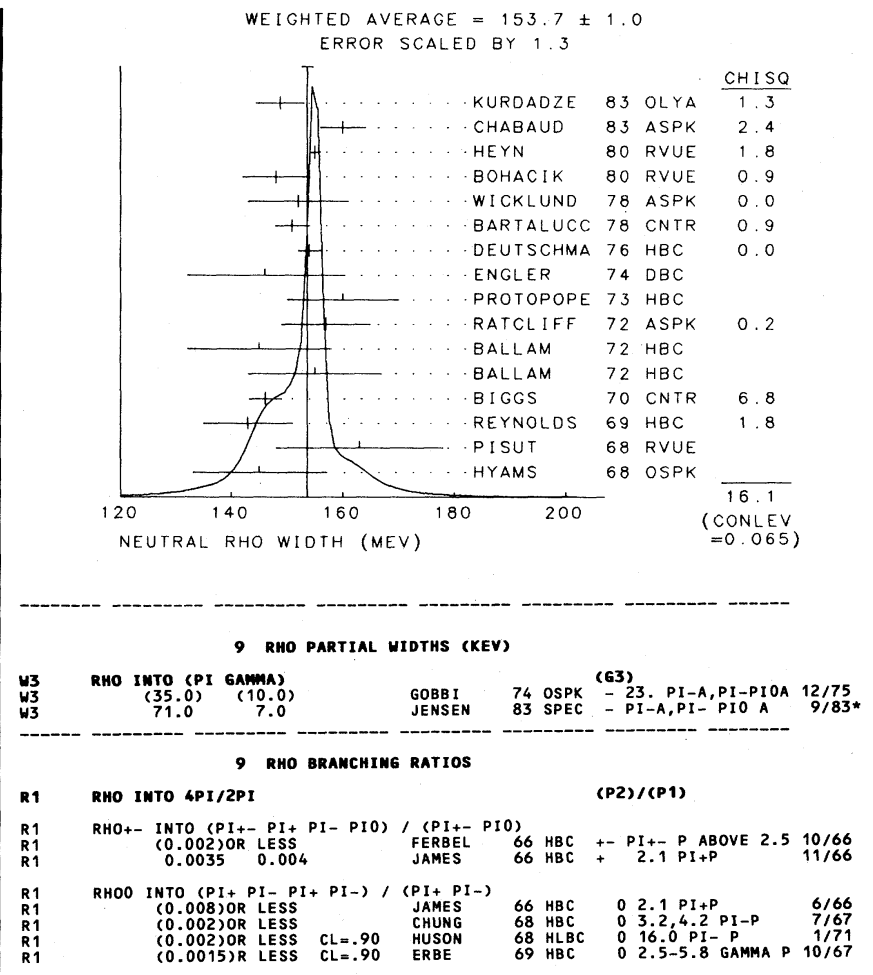

\section{NOTE ON THE $\mathrm{e}^{+} \mathrm{e}^{-}$AND $\mu^{+} \mu^{-}$DECAYS}

Extraction of a ratio for $\rho^{0} \rightarrow \mathrm{e}^{+} \mathrm{e}^{-}$is complicated by interference with $\omega$ decay. In photoproduction, $\gamma \mathrm{A}$ $\rightarrow \mathrm{e}^{+} \mathrm{e}^{-} \mathrm{A}$, there is substantial interference between the allowed $\left(\rho^{0}, \omega\right) \rightarrow \mathrm{e}^{+} \mathrm{e}^{-}$decays. The interference in the colliding-beam reaction $\mathrm{e}^{+} \mathrm{e}^{-} \rightarrow \pi^{+} \pi^{-}$is due to Gparity-violating mixing of the overlapping $\rho^{0}$ and $\omega$ resonances; it alters the results for the rate $\Gamma\left(\rho^{0} \rightarrow\right.$ $\mathrm{e}^{+} \mathrm{e}^{-}$) only by a small amount. Therefore at present we average only the values from the $\mathrm{e}^{+} \mathrm{e}^{-} \rightarrow \pi^{+} \pi^{-}$experiments.

The same comment applies to the decay $\rho^{0} \rightarrow$ $\mu^{+} \mu^{-}$.

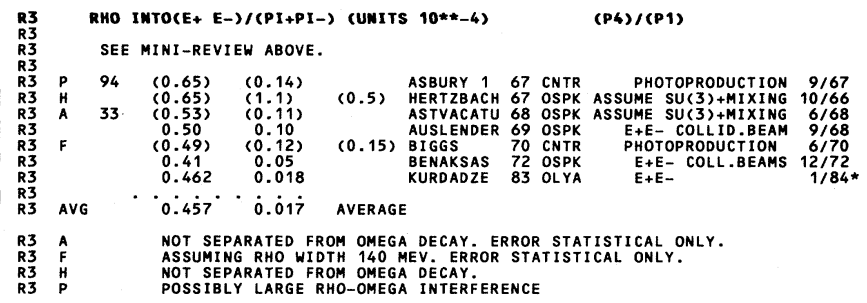


Mesons $\rho(770), \omega(783)$

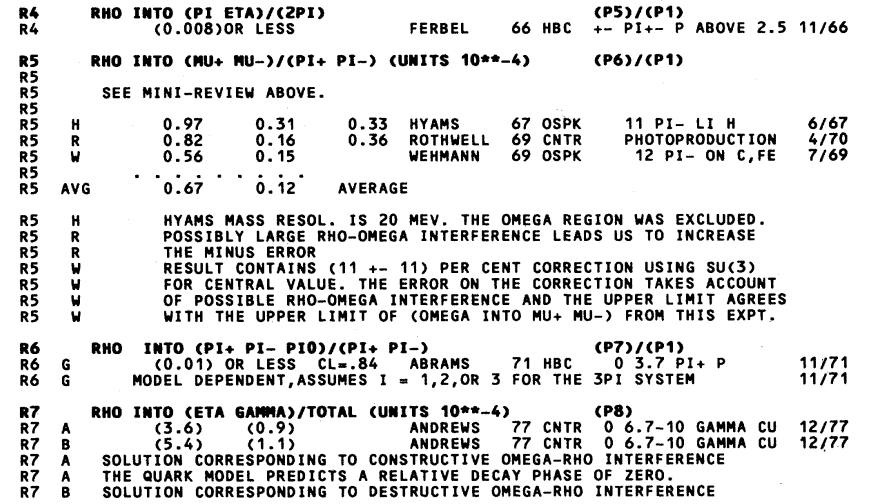

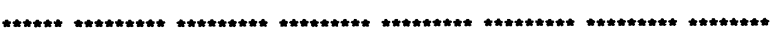

\begin{tabular}{|c|c|c|}
\hline & & \\
\hline & & $\begin{array}{l}\text { (LRL SCHMITZ } \\
\text { EST }\end{array}$ \\
\hline & 36 & $\begin{array}{l}\text { ALL (KENTUCKY) } \\
\text { UNY }+ \text { COLU+KNTY) } \\
\text { CH } \\
\text { (LRL) }\end{array}$ \\
\hline & $\begin{array}{l}1381 \\
1515 \\
062 \\
185 \\
185 \\
\text { CONF } 1239\end{array}$ & $\begin{array}{l}\text { HLHOP, NGGUYEN, YAGER (UCSD) } \\
\text { NISE+(SACL+ORSA+BARI+BGNA) } \\
\text { ERR ICK, CRESTI + (OXF+PADO) } \\
\text { N (LRL) } \\
+ \text { BOLOGNA- COLLABORATION }\end{array}$ \\
\hline & $\begin{array}{lllll}64 & \text { NC } & 31 & 729 & \\
64 & & \\
64 B N A & C O N F & 1486 \\
64 & \text { PRL } & 12 & 336\end{array}$ & 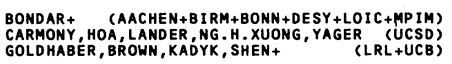 \\
\hline $\begin{array}{l}\text { YEA } \\
\text { MENISE } \\
\text { IEEDE } \\
\text { ARK } \\
\text { TAY } \\
\text { NZEROT } \\
\text { ANIS }\end{array}$ & $\begin{array}{l}1 \\
4 \\
1 \\
10 \\
21\end{array}$ & 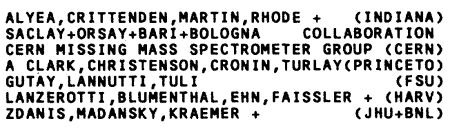 \\
\hline $\begin{array}{l}\text { ACCENSI } \\
\text { ALFF-STE } \\
\text { BALTAY } \\
\text { BLIEDEN } \\
\text { CAMBRIDG } \\
\text { CASON } \\
\text { DEUTSCHM } \\
\text { FERBEL } \\
\text { FIDECARO } \\
\text { HAGOPI }\end{array}$ & 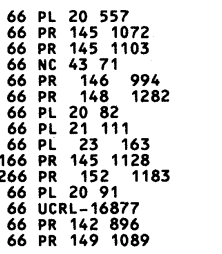 & 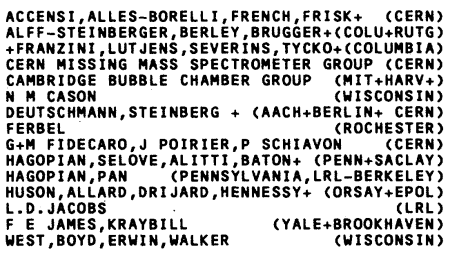 \\
\hline $\begin{array}{l}\text { ALLES-BO } \\
\text { ASBURY } \\
\text { ASBURY } \\
\text { BACON } \\
\text { BANNER } \\
\text { BARLOW } \\
\text { BATON } \\
\text { ALSO } \\
\text { CLLEAR } \\
\text { DANYSZ } \\
\text { EISNER } \\
\text { FRENCH } \\
\text { HERTZBAC } \\
\text { HUWE } \\
\text { HYAMS } \\
\text { MILLER } \\
\text { POIRIER }\end{array}$ & 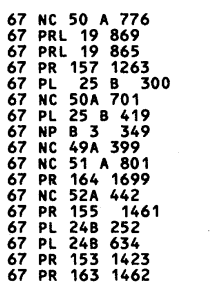 & 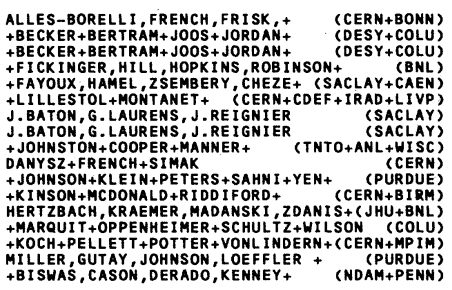 \\
\hline & 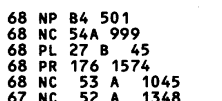 & 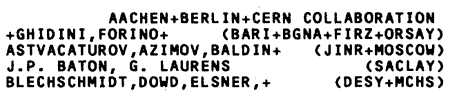 \\
\hline $\begin{array}{l}\text { FOSTER } \\
\text { HUSON } \\
\text { HYAMS } \\
\text { JONES } \\
\text { JOHNSON } \\
\text { KEY } \\
\text { LAMSA } \\
\text { LANZEROT } \\
\text { MARATECK } \\
\text { PISUT }\end{array}$ & 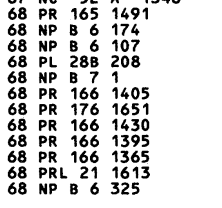 & 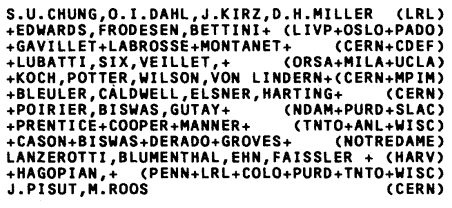 \\
\hline $\begin{array}{l}\text { GUSTI1 } \\
\text { GUSTII } \\
\text { SLENDE } \\
\text { BE } \\
\text { ISSINS } \\
\text { HALA } \\
\text { LAMUD } \\
\text { LLER } \\
\text { TTER } \\
\text { YNOLDS } \\
\text { OS } \\
\text { THWELL } \\
\text { HAREN } \\
\text { HMANN }\end{array}$ & 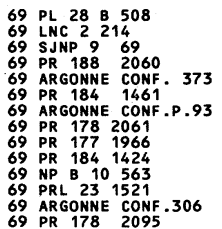 & 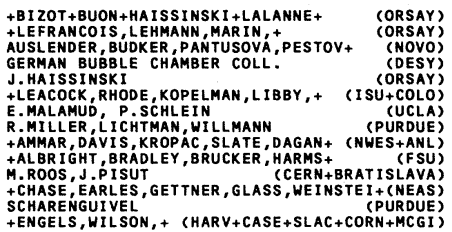 \\
\hline
\end{tabular}

Data Card Listings
ALVENSLEBEN, BECKER, BERTRAM, CHEN, COHEN(DESY)
+LAURENS, REIGNIER , (SACLAY) +BRABEN, CLIFT, GABATHULER, KITCHING+ (DARE)
+FRETTER,MFFEI T,BALAM+ (LRL+SLAC+ (UFT)
+MOTT, ALYA, (INEE, MARTIN, PRICKETT +BARNHAM, BUTLER, COYNE, GOLDHABER, HALL, +(LBL) BISWAS, CASON, GROVES, JOHNSON, + (NOTRE DAME)
+ BI SOTH + CARNEGIE, KLUGE, LEITH, LYNCH, RATCLIFF+(SLAC) BASDEVANT, FROGGATT, PETERSEN (CEACL (CERN) +COSME, JEAN-MARIE, JULLIAN, LAPLANCHE, (CORSA)
+ HEINLOTH, HOHNE, HOFMANN, RATHJE, (DESY + HAMB)

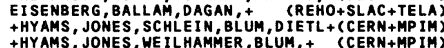
L.D.JACOBS
TAKAHES WLAMMER, BLUM, + (CERN MACLAY)
(SACLA +BULOS, CARNEGIE, KLUGE, LEITH, LYNCH, + (SLAC)
TAKAHASHI, BARI SH, +
(TOHO+PEN +NDAM+ANL) +ANTHONY, COFFIN, MEANLEY, MEYER, RICE, + (MICH) +RUSSEL, TANNENBAUM, WEISS, THOMSON R (HARV) + JONES, WEILHAMMER, BLUM, DIETL, (CERN+MPIM)
PROTOPOPESCU, GARNJOST, GALTIERI, FLATTE+(LBL) +MATTHEWS, WALKER+ (SLAC+DUKE+WISC+TNTO) +KRAEMER, TOAFF, WEISSER, DIAZ+ (CARN+CASE)
P. ESTABROOKS, A. D.MARTIN +ROSEN, SCOT ', SHAPIRO+ (NHES+ROCH+CARN)
G. GRAYER, HYAMS, BLUM, DIETL, +
(CERN+MPIM)

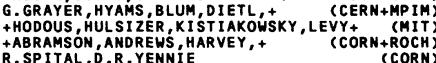
C. MONTONEN, M.ROOS, N. TORNOVIST (HELS)
(HELS) + $I$ I RK , + (AACH+BERL +BONN+CERN + CRAC+HE ID+ +HARS) +FUKUSH IMA, HARVEY, LOBKOWI CZ, MAY, + (ROCH) + CAUTIS, COHEN, CSORNA, SMITH, YEH, + (COLU+BING)
BARTALUCCI, BASINI, BERTOLUCC C I (DESY+FRAS) + RIBES, RUMPF, BERTRAND, BIZOT, CHASE, + (LALO)
+ AYYRES, DIEBOLD, GREENE, KRAMER, PAWLICKI (ANL) +BLANAR, BLUM, CERRADA+ (MPIM+CERN+ZEEM+CRAC)
C.B.LANG, A.MASS-PARARDA
(GRAZ) +CHANDLEE, BIEL, HEPPELMAN, + (ROCH+FNAL+MINN)
J.BOHACIK,H.KUHNELT
(BRATISLAVA+HIEN) J.BOHACIK, H. KUHNELT
M.F.HEYN, C.B.LANG
(BRATISLAVA+HIEN)
(GRAZ) ALEKSEEVA, KarTAMYSHEV, MAKARIN+ (KIAE)

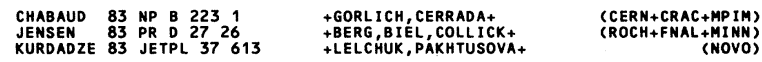

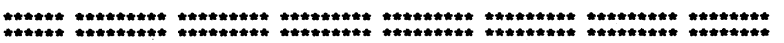
$\omega(783)$ 1 OMEGA(783,JPG=1--) I=0 1 OMEGa mass (MEV)

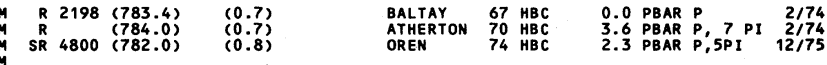

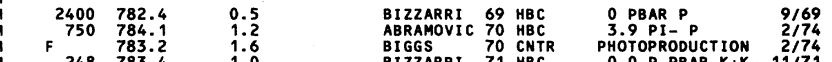

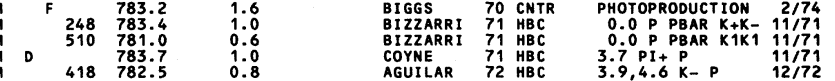

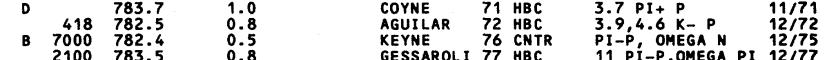
2100
535 783.5

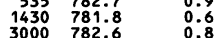

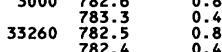

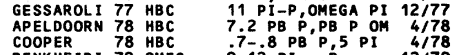

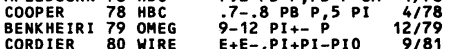

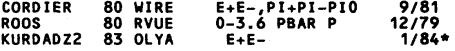
avg $7 \dot{8} 2.5 \dot{8}^{\circ} \cdot \dot{0} \cdot \dot{8}^{\circ}$ average (error includes Scale factor of 1.1 ) $M$ OBSERVED BY THRESHOLD-CROSSING TECHNIQUE. MASS RESOL. $=4.8$ MEV FWHM F
F M F ASSUMING OMEGA WIDTH 12.6 MEV.

$M$ S ERROR INCLUDES 0.5 MEV MASS SCALE ERROR

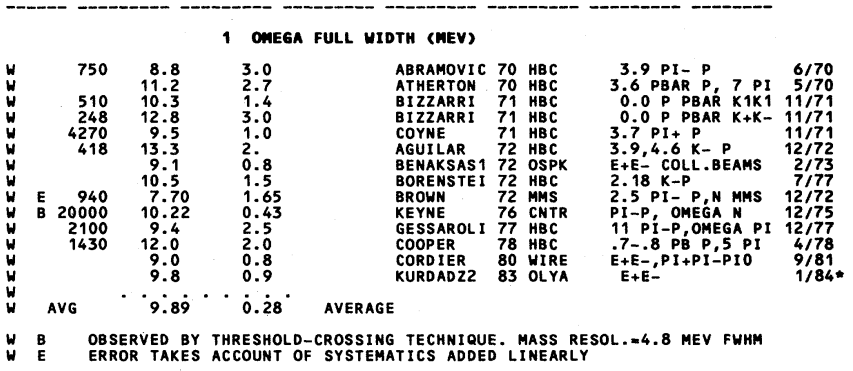




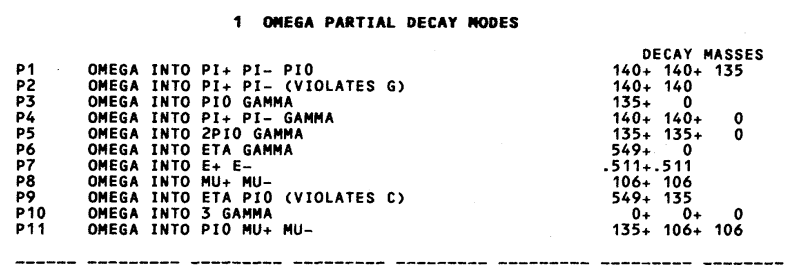

FITTED PARTIAL DECAY MODE BRANCHING FRACTIONS

The matrix below is derived from the error matrix for the fitted partial decay mode branching fractions, $P_{i}$, as follows: The diagonal elements are $P_{i} \neq \delta P_{i}$, where $\delta P_{i}=\sqrt{\left\langle\delta P_{i} \delta P_{i}\right\rangle}$, while the off-diagonal elements are the normalized correlation coefficients $\left\langle\delta P_{i} \delta P_{j}\right\rangle /\left(\delta P_{i} \cdot \delta P_{j}\right)$. For the definitions of the individual $P_{i}$, see the listings above; only those $P_{i}$ appearing in the matrix are as sumed in the fit to be nonzero and are thus constrained to add to 1 .

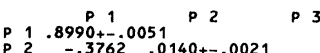

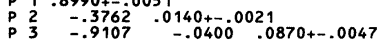

\section{1 omega bramchimg ratios}

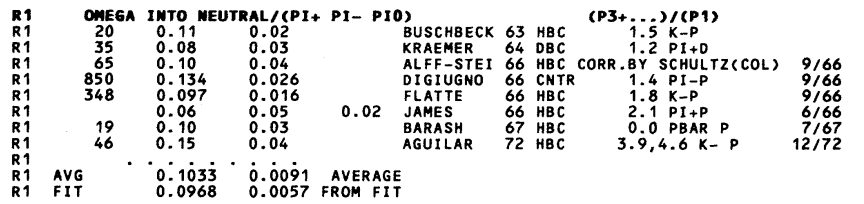

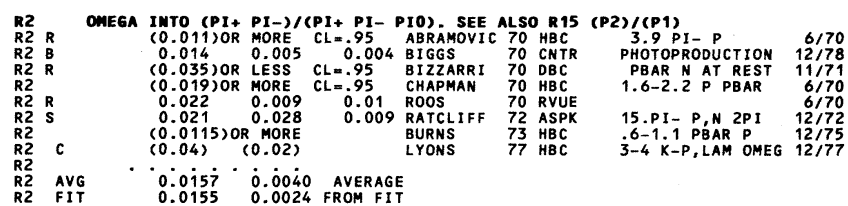

R2 B
RE B
B

R2 B
$R 2$ C TSSUMING COMPLETE RHO-OMEGA COHERENCE

$\begin{array}{ll}\text { R2 } & { }^{C} \text { ROOS } 70 \text { COMBINES ABRAMOVICH } 70 \text { AND BIZZARRI } 70 \\ \text { R2 } S & \text { SIGNIFICANT INTRFERENCE EFFECT OBSERVED. NB OF OMEGA INTO 3PI } \\ \text { R2 } S & \text { COMES FROM ANTERTRAPOLATION. }\end{array}$

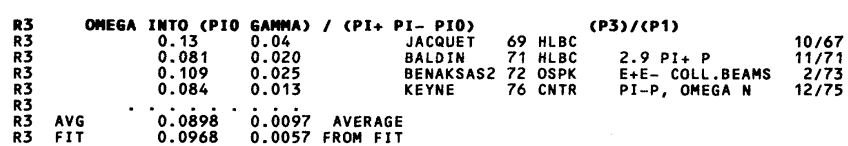

R3 FIT 0.09680 .0057 FROM FIT
R4 OMEGA INTO (PI+ PI- GAMMA)/(PI+PI-PIO)

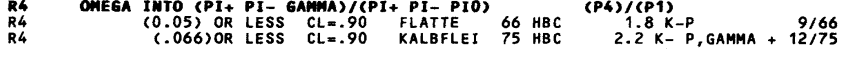

R6 OMEGA IMTO (MU+ MU-)/(PI+ PI- PIO)(UMITS 10*-3) (P8)/(P1)
R6 (1.2) OR LESS

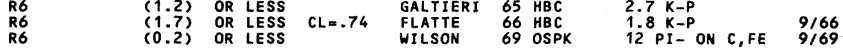

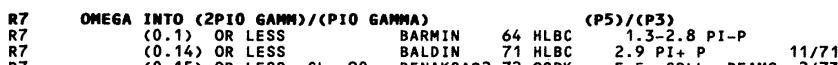

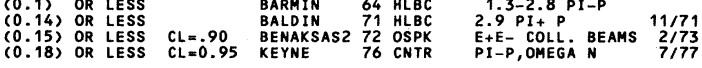
OMEGA IMTO (ETA PIO + ETA GAMMA)/(PI+ PI- PIO)
(P9+P6)/(P1)

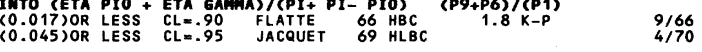

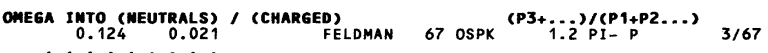
$0.0953^{\circ} \dot{0} 0.0056$ From FIT

R10 OMEGA INTO (2PIO GAMMA)/(PIPPI-PIO)
R10 (0.08) OR LESS CL. 95 JACQUET $69 \mathrm{HLBC}$ (P5)/(P1)

R11 ONEGA INTO (ETA GAMMA)/(P1O GAMMA)
R11
0.010

R12 OMEGA IHTO (PIO MU+
R12 MU-) / TOTAL (UMITS
0.096
0.023

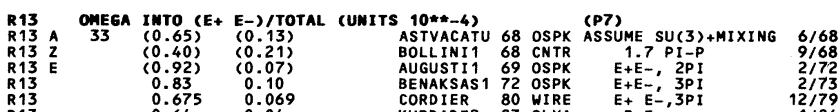

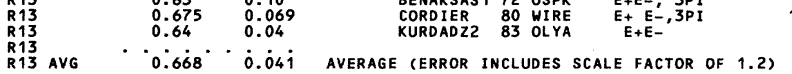

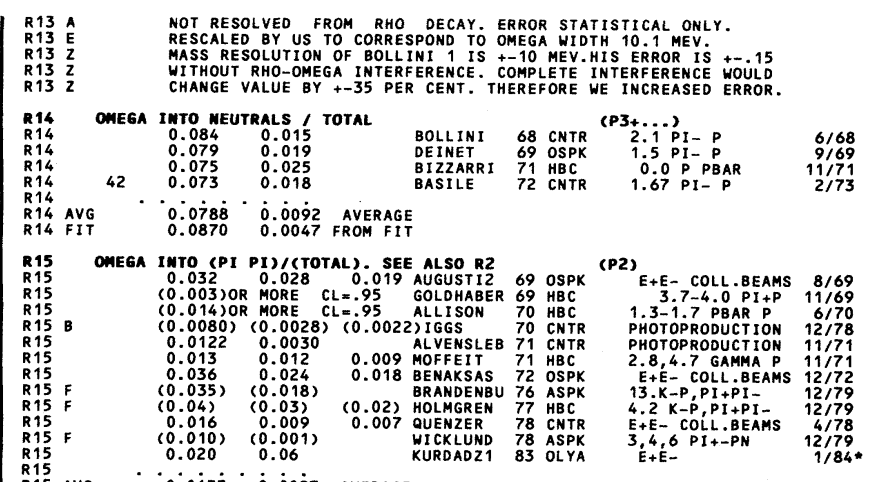

$\begin{array}{lll}\text { R15 AVG } & 0.0133 & 0.0027 \\ \text { R15 FIT } & 0.0140 & 0.0021 \\ \text { FROM FIT }\end{array}$

R15 B RE-EVALUATED UNDER R2 BY BEHREND 71 USING MORE ACCURATE OMEGA

R15 B TO RHO PHOTOPRODUCTION CROSS-SECTION RATIO.
R15 F FROM A MODEL DEPENDENT ANALYSIS ASSUMING COMPLETE COHERENCE.

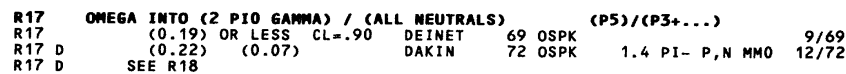

R18 OMEGA IMTO (PIO GAMmA) C (ALL MEUTRALS)
R18
(0.81) OR MORE

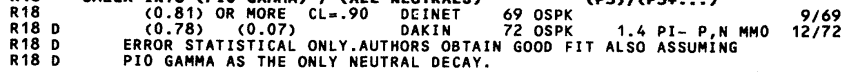

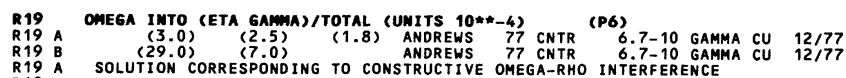

R19 B
R19 A SOLUTION CORRESPONDING TO CONSTRUEWIVE OMEGA-RHO INTERFERENCE
R19 A THE QUARK MODEL PREDICTS A RELATIVE DECAY PHASE OF ZERO

SOLUTION CORRESPONDING TO DESTRUCTIVE OMEGA-RHO INTERFERENCE

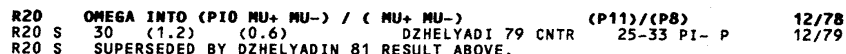

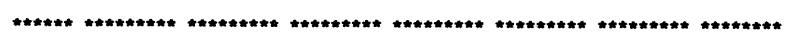

REFEREMCES FOR OMEGA

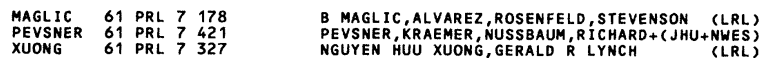

$\begin{array}{llll}\text { ALFF } & 62 & \text { PRL } 9325 & 325 \\ \text { ARMENTER } & 62 \\ \text { CERN CONF } 90 & \text { ALF, BERLEY, COLLEY, GELFAND + (COLU+RUTGERS) }\end{array}$

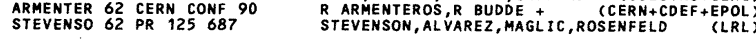

$\begin{array}{llll}\text { ARMENTER } & 63 & \text { SIENA CONF } 1296 \\ \text { BARMIN } & \text { ARMENTEROS, EDWARDS, JACOBSEN+ } & \text { (CERN+CDEF) } \\ \text { SIENA CONF } & 1207 \text { BARMIN, DOLGOLENKO, KRESTNIKOV + } & \text { (CERN (ITEP) }\end{array}$

BARM IN
BUSCHBEC 63 SIENA CONF
SIENA CONF 1106 BUSCHBECK, CAPP + KRESTNINOV+
(VIINNA+CERN+AMSTERDAM)

GELFAND 63 PRL 11436
MURRAY 63 PL 7358
GELFAND, MILLER, NUSSBAUM, RATAU, (COLU+RUTG)

$\begin{array}{llllll}\text { BARMIN } & 64 & \text { JETP } 18 & 1289 & \text { BARMIN, DOLGOLENKO, KRESTNIKOV + } \\ \text { KRAEMER } & 64 & \text { PR } 136 \text { B } & 496 \\ \text { KRAEMER, MADANSKY, MEER+ } & \text { (ITEP) }\end{array}$

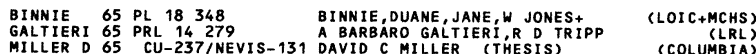

MILLER D 65 CU-237/NEVIS-131 DAVID C MILLER (THESIS)
ZDANIS 65 PRL 14 721 (COLUMBIA)
ZDANIS, MADANSKY, KRAEMER, HERTZBACH+(JHU+BNL)

$\begin{array}{lllll}\text { ALFF-STE } & 66 & \text { PR } & 145 & 1072 \\ \text { DIGIUGNO } 66 & \text { NC } & 44 A & 1272\end{array}$

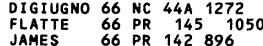

ALFF-STEINBERGER, BERLEY, BRUGGER + (COLU+RUTG)
DI GIUGNO, PERUZZI,TROISE + (NAPL+FRAS+TRST)

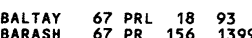

$\begin{array}{lllll}\text { BARASH } & 67 & \text { PR } & 156 & 1399 \\ \text { FEEDMAN } & 67 & \text { PR } & 159 & 1219 \\ \text { HERTZBAC } & 67 & \text { PR } & 155,1461\end{array}$

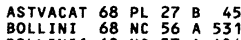

$\begin{array}{lllll}B O L L I N I 1 & 68 & \text { NC } & 57 & A \\ \text { KEY } & 404 \\ \text { PISUT } & 68 & \text { PR } 166 & 1430\end{array}$

$\begin{array}{lllll}\text { KEISUT } & 68 & \text { PR } & 166 & 1330 \\ \text { PISUT } & 68 & \text { NP B } & 325 \\ \text { WEHMANN } & 68 & \text { PRL } 20 & 748\end{array}$

AUGUST11 69 PL 28 B 513

BIZZARRI 69 NP 8 14 16

DANBURG 69 UCRL-19275

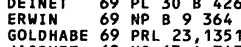

JACOUET 69 NC 63 A 743
MILLER 69 PR 1782061
STRUGALS 69 PL 29 B 532.
WILSON 69 PRIVATE COMM.
WILNAMOVI

$\begin{array}{llll}\text { ABRAMOVI } 70 & \text { NP } B & 20 & 209 \\ \text { BIZZARRI } 70 & \text { PRL } 25 & 1385\end{array}$

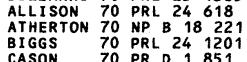

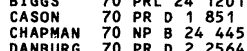

DANBURG 70
FLA

GOLDHABE 70 PHILA.CONF.P.59
HAGOPIAN 70 PRL 251050
ROOS 70 DNPL/R7 P.173
HUWE, MURRAY, BUTTON-SHAFER, SOLMITZ+ (LRL)
F E JAMES, KRAYBILL
(YALE+BROOKHAVEN)

+FRANZINI, SEVERIENS, YEH, ZANELLO (COLUMBIA) FRATI, GLEESON, HALPERN, NUSSBAUM+
HERTZBACH, KRAEMER, MADANSKI, ZDANIS+ (JHU+BNL)

ASTVACATUROV, AZIMOV, BALDIN+ (JINR+MOSCOW) BUHLER, DALPIAZ, MASSAM+ (CERN+BGMA + STRB)

J. PI SUT, M.ROOS
+ ENGELS+ (HARVARD+CASE+SLAC+CORNELL+MCGILL)
(CERN)

+BENAKSAS, BUON, GRACCO, HAISSINSKI, + (ORSAY) CORSAY)
+ FOSTER, GAVILLET, MONTANET,
+ (LRL) +WALKER, GOSHAW, WEINBERG (WISC+PR IN+VAND)
+BUTLER, COYNE, HALL, MACNAUGHTON, TRILING (LRL) NGUYEN-KHAC, HAATUFT, HALSTEINSLII (EPOL+BERG) +CHUVILO, FENYVES,
RICHARD WILSON (SEE ALSO PR (WARS +JINR + BUDA)
2095)(HARV)

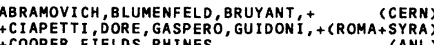
+BLAIR, CELNIKER, DOMINGO, FRENCH+ (CERN+IPN) +CLIFFT,GABATHULER, KITCHING, RAND
+ANDREWS, BISWAS, GROVES, HARRINGTON, (DARE)
(NDAM) AAVIDSON, GREEN, LYS, ROE, VANDER VELDE (MICH)

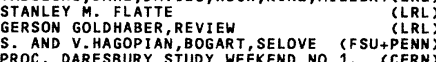




\section{Mesons} $\omega(783), \eta^{\prime}(958)$

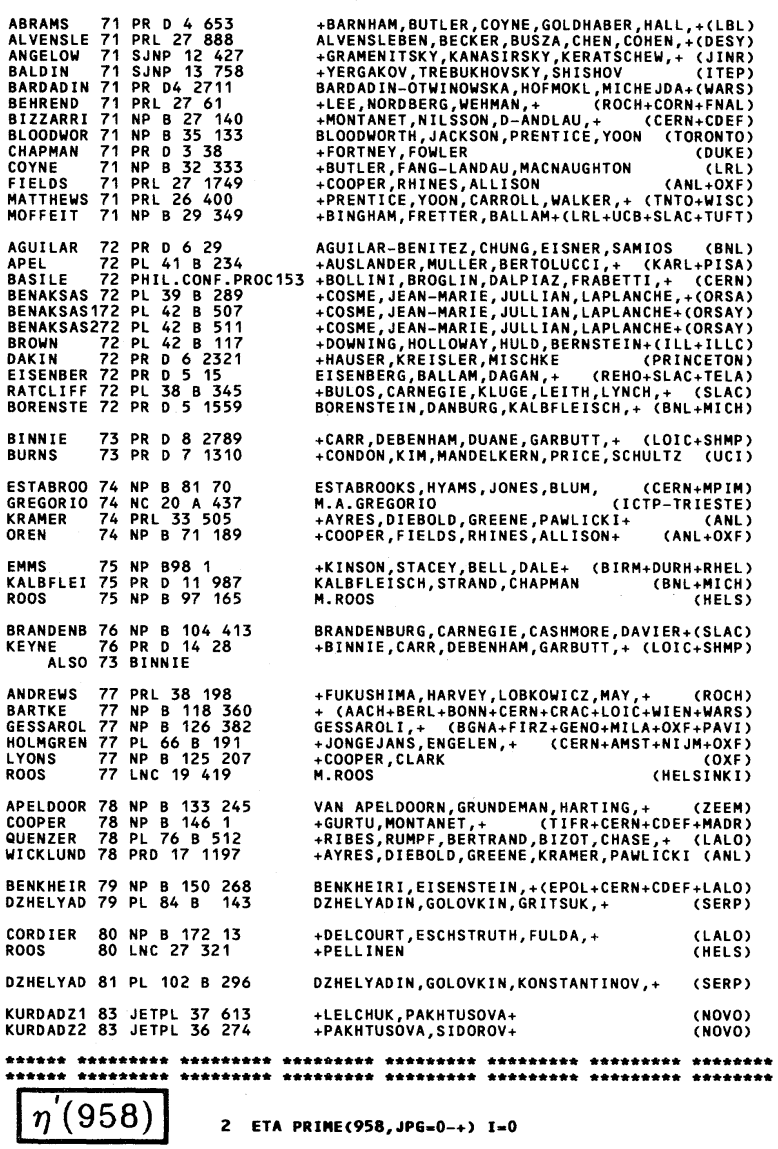

\section{NOTE ON THE JP ASSIGNMENT OF $\boldsymbol{\eta}^{\prime}$ (958)}

From the Dalitz plot analyses of the $\eta^{\prime} \rightarrow \pi \pi \eta$ and $\eta^{\prime}$ $\rightarrow \pi^{+} \pi^{-} \gamma$ decays and from the observation of an $\eta^{\prime} \rightarrow$ $\gamma \gamma$ decay mode, all assignments except $\mathrm{J}^{\mathrm{PC}}=\mathrm{0}^{-+}$and $2^{-+}$are excluded. The Dalitz plot analyses favor spin 0 , but cannot rule out spin 2. The indication of anisotropy in the decay of very forward-produced $\eta^{\prime}$ (KALBFLEISCH 73) has not been confirmed by BALTAY 74, thus again favoring spin 0 , but still not ruling out spin 2 (LEDNICKY 77).

Two analyses, however, seem to have established the spin 0 assignment of the $\eta^{\prime}$.

CERRADA 77 perform a partial-wave analysis of the $\eta \pi \pi$ system produced in the reaction $\mathrm{K}^{-} \mathrm{p} \rightarrow \eta^{\prime} \Lambda$, taking into account the $\eta^{\prime}$ and $\Lambda$ joint decay angular correlations. They conclude that $\mathrm{J}^{\mathrm{P}}$ is unambiguously $0^{-}$(see also DELAGUILA 77).

ROUSSARIE 77 analyze a large sample of events

\section{Data Card Listings}

from the reaction $\pi^{-} \mathrm{p} \rightarrow \eta^{\prime} \mathrm{n}$ at beam momenta just above threshold. They verify that the $\eta^{\prime}$ is produced in a relative S-wave state, and thus the Adair condition is satisfied by their total sample of some 1800 events. The decay angular distribution of the $\eta^{\prime}$ is consistent with isotropy, and thus ROUSSARIE 77 conclude that the spin cannot be 2 .

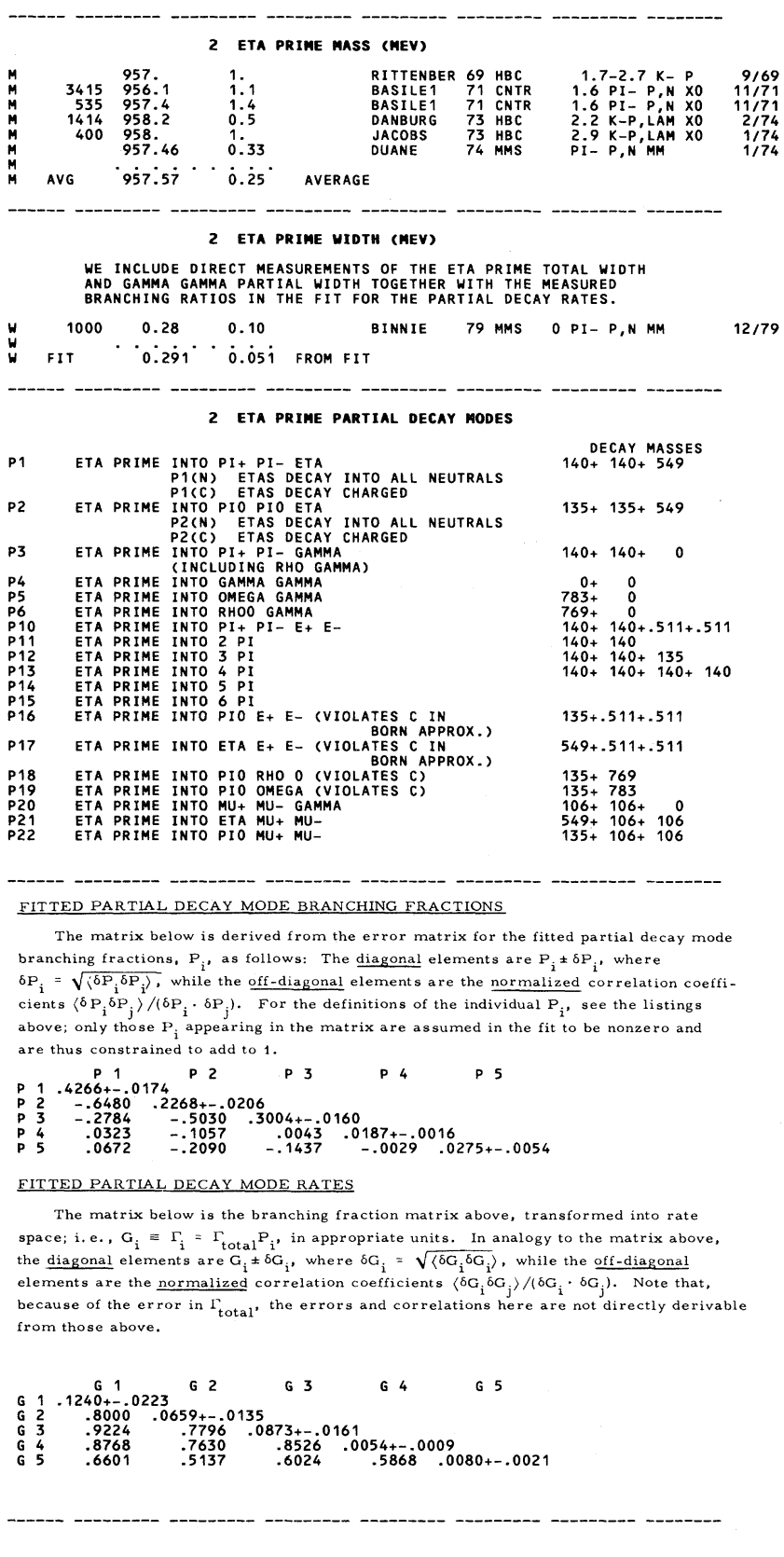




\section{NOTE ON $\boldsymbol{\eta}^{\prime}(958)$ BRANCHING FRACTIONS}

In our calculation of the branching fractions of the $\eta^{\prime}(958)$, we use the decay modes $\eta \pi \pi$ (including $\eta \pi^{0} \pi^{0}$ ), $\rho^{0} \gamma, \omega \gamma$, and $\gamma \gamma$. It is assumed that the rate $\eta \rightarrow$ neutrals is $70.9 \%$.

In the fit we do not use the constraint

$$
\mathbf{R}=\frac{\Gamma\left(\eta^{\prime} \rightarrow \eta \pi^{+} \pi^{-}\right)}{\Gamma\left(\eta^{\prime} \rightarrow \eta \pi^{0} \pi^{0}\right)}=2
$$

from isospin conservation. The result of the fit is in agreement with it: $R=1.9 \pm 0.2$.

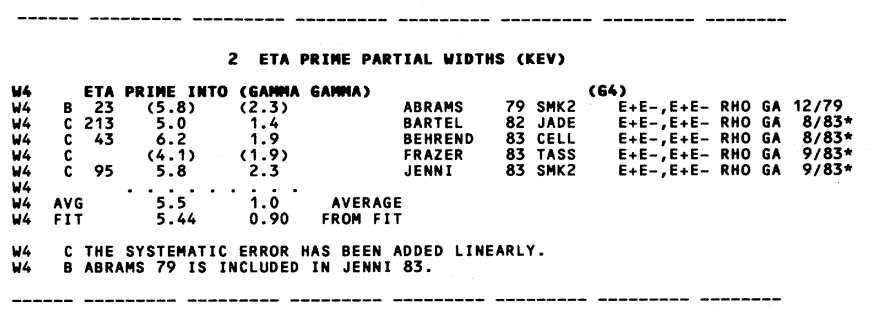

\section{ETA PRIME BRAMCHIMG RATIOS}

SEE MINI-REVIEW ABOVE.

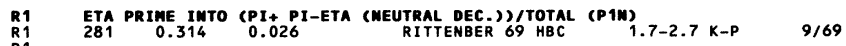

$\begin{array}{llll}R 1 & \text { RIT } & 0.3 \dot{0} 02 & \cdots \\ R 1 & 0 . \dot{0} 1 \dot{2} & \text { FROM FIT }\end{array}$

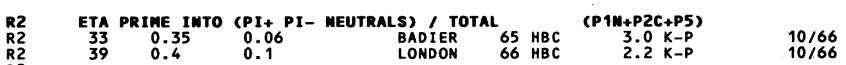

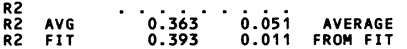

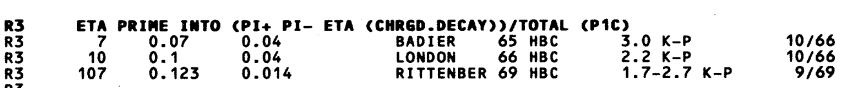

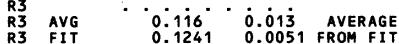

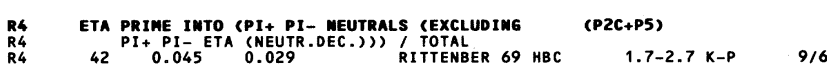

${ }_{R 4}^{R 4}$ FIT $\cdots 0.0908 \cdot 0.0069$ FROM FIT

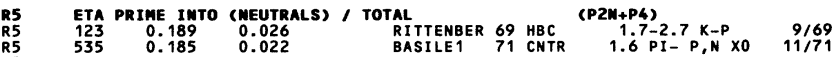

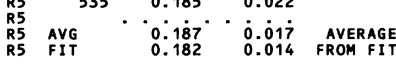

R6 ETA PRIME INTO (PI+ PI- GAMMA (IMCLUDIMG RHO GAMmA)) (P3) TOTAL

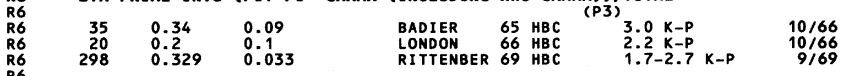

$\begin{array}{lllll}R 6 & \text { RVG } & 0.339 & 0.030 & \\ \text { R6 } & \text { AVIT } & 0.300 & 0.030 & \text { AVERAG } \\ \text { R6 } & 0.016 & \text { FROM FIT }\end{array}$

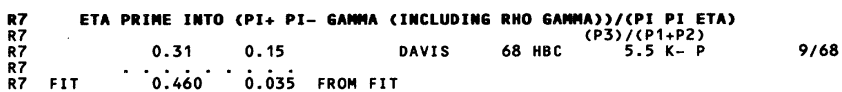

R8 ETA PRIME IHTO (PIO E+ E-)/TOTAL
R8 0.013 )

R9 ETA PRIME IMTO (ETA E+ E-)/TOTAL
R9
(0.011)OR LESS

R10 ETA PRIME INTT (P10 RHO0)/TOTAL
R10
$(0.04)$ OR LESS

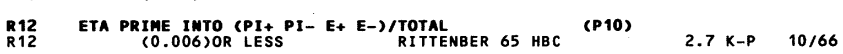

R13 ETA PRIME INTO (2 P1)/TOTAL
R13
$(0.07)$ OR LESS

R14 ETA PRIME INTO (3 P1)/TOTAL
R14 0.07 )

R15 ETA PRIME INTO (4 P1)/TOTAL
R15 0.01 ) OR LESS

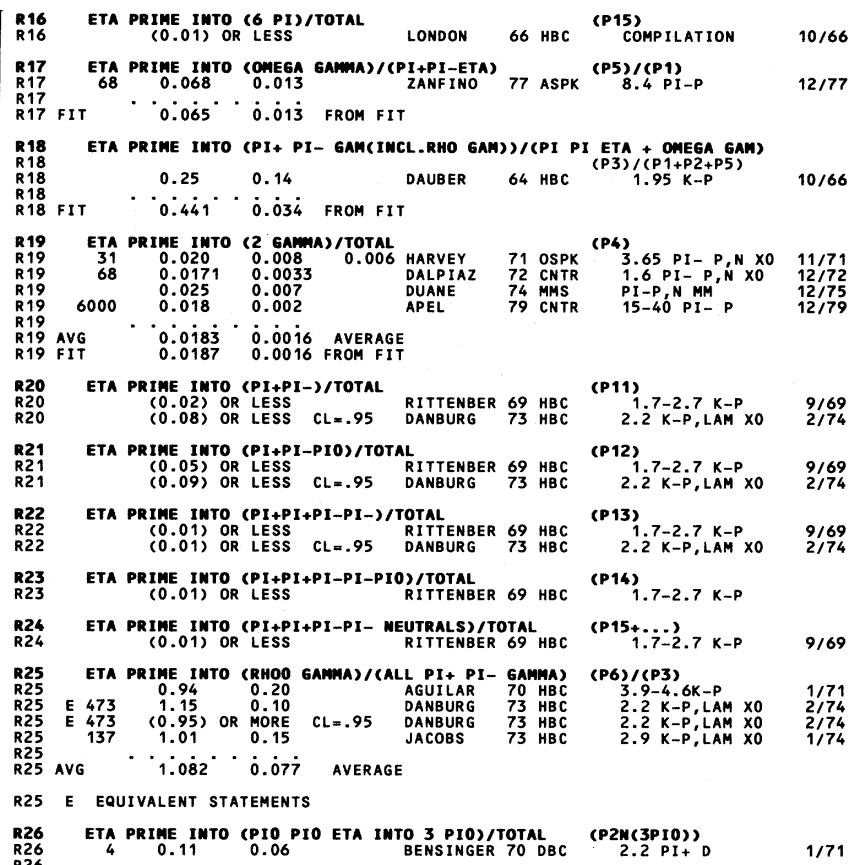

$\begin{array}{lll}\text { R26 } & 426 \\ \text { R26 FIT } & 4.11 & 0.06 \\ 0.0680 & 0.0062 \text { FROM FIT }\end{array}$

R27 ETA PRIME IMTO (PI+ PI- GAMMA)/(PI+ PI- ETA(MEUTRAL DEEC.) (P)

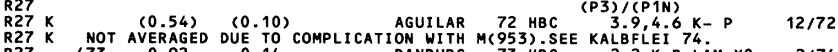

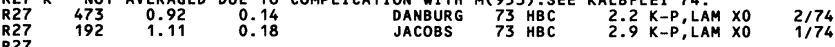

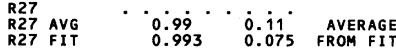

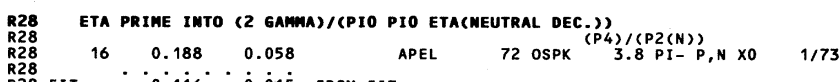

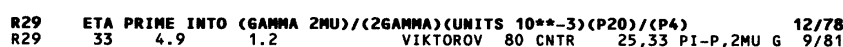

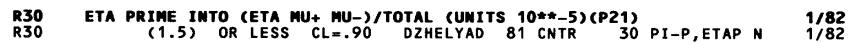

R31 ETA PRIME INTO (PIO NU+ MU-) TOTAL CUNITS
R31

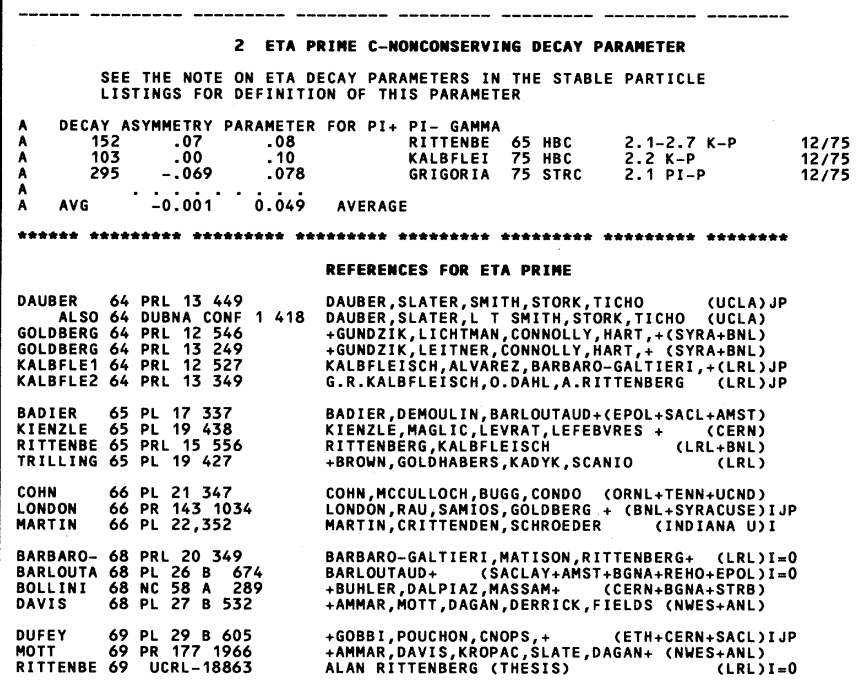


Mesons

$\eta^{\prime}(958), \mathrm{S}(975) \quad\left[\mathrm{S}^{*}\right]$

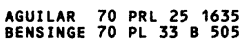

BARDADIN 71 PR D4 2711

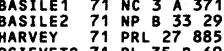

AGUILAR 72 PR ${ }^{0} 609$

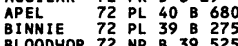

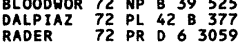

$\begin{array}{llllll}\text { DANBURG } & 73 & P R & 0 & 8 & 3744 \\ \text { JACOBS } & 73 & P R & 0 & 8 & 18 \\ \text { JACOS } & 75 & \text { PR } & 31 & 333\end{array}$

KALBFLEI 73 PRL 31333

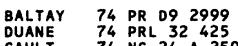

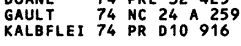

$\begin{array}{llll}\text { GRIGORIA } 75 & \text { NP } & 891 & 232 \\ \text { KALBFLE } & 75 \\ \text { PR } & 011987\end{array}$

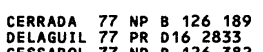

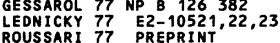

ROSSARO 77
ZANF INO 77 PUDLPEST 38 930 CONF.

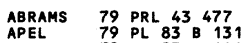

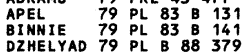

VIKTOROV 80 SJNP 32520

DZHELYAD 81 PL 105 B 239

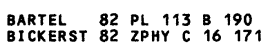

BEHREND 83 PL 125 \& 518

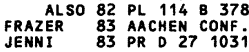

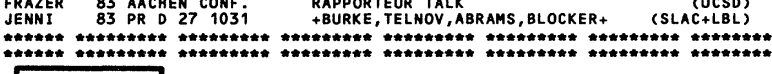

$S(975)$
or $S$

3 S(975, JPG=0++) $1=0$

FORMERLY CALLED S*
UNDER THS SENTRY WE LIST PARAMETERS OF THE POLE IN THEE
ISOSCALAR S WAVE. FOR A MINI-REVIEW SEE UNDER EPSILN.

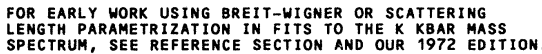
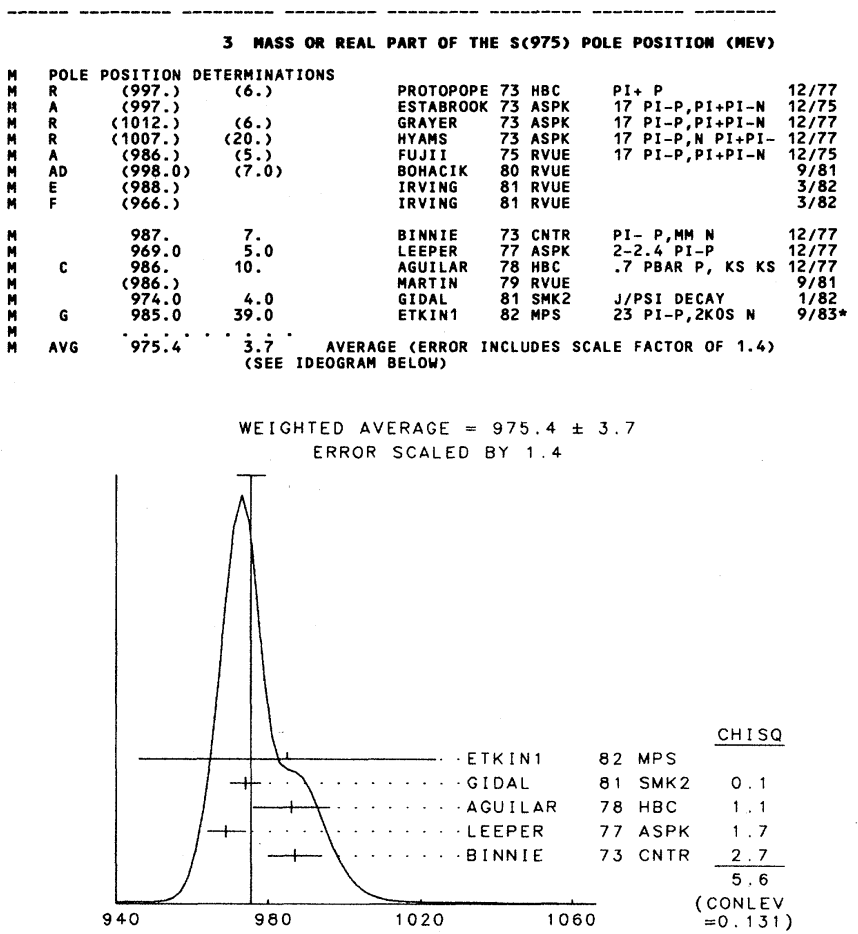

REAL PART OF $S(975)$ POLE POSITION (MEV)

\section{Data Card Listings}

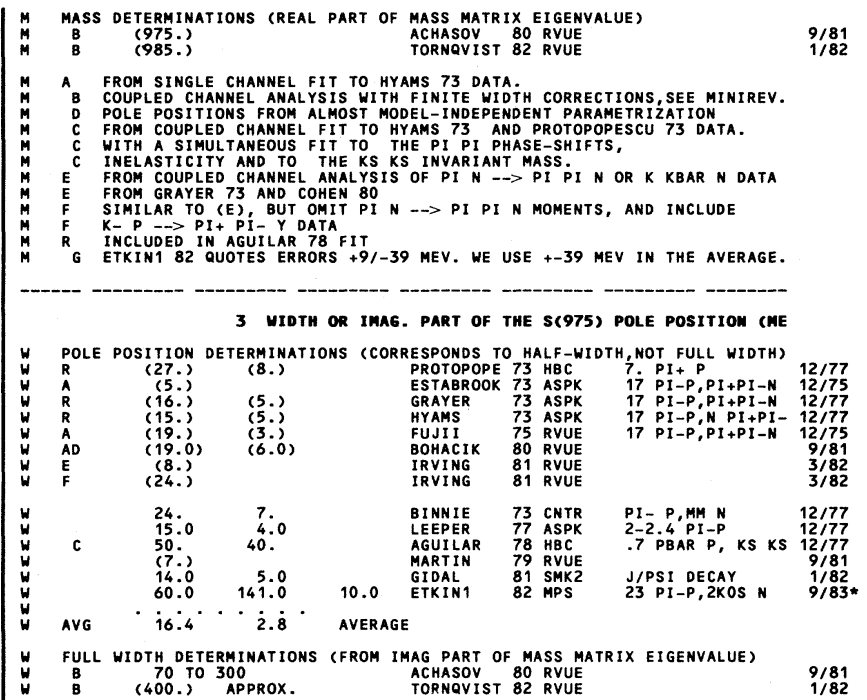

W ACR SEE NOTES UNDER REAL PART
EF SEE NOTES UNDER REAL PART

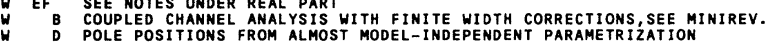

3 S(975) PARTIAL deCAY MODES

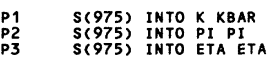

DECAY MASSES

$498+498$
$140+440$
$549+549$

3 S(975) BRAMCHIMG RATIOS

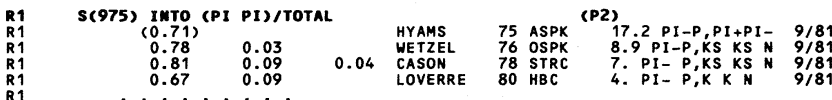
R1 avg $\cdots 0.776^{\circ} \cdot \dot{0} 0.02 \dot{\sigma}^{\circ}$ average 
For notation, see key at front of Listings.

Mesons

$S(975) \quad\left[S^{*}\right], \delta(980)$

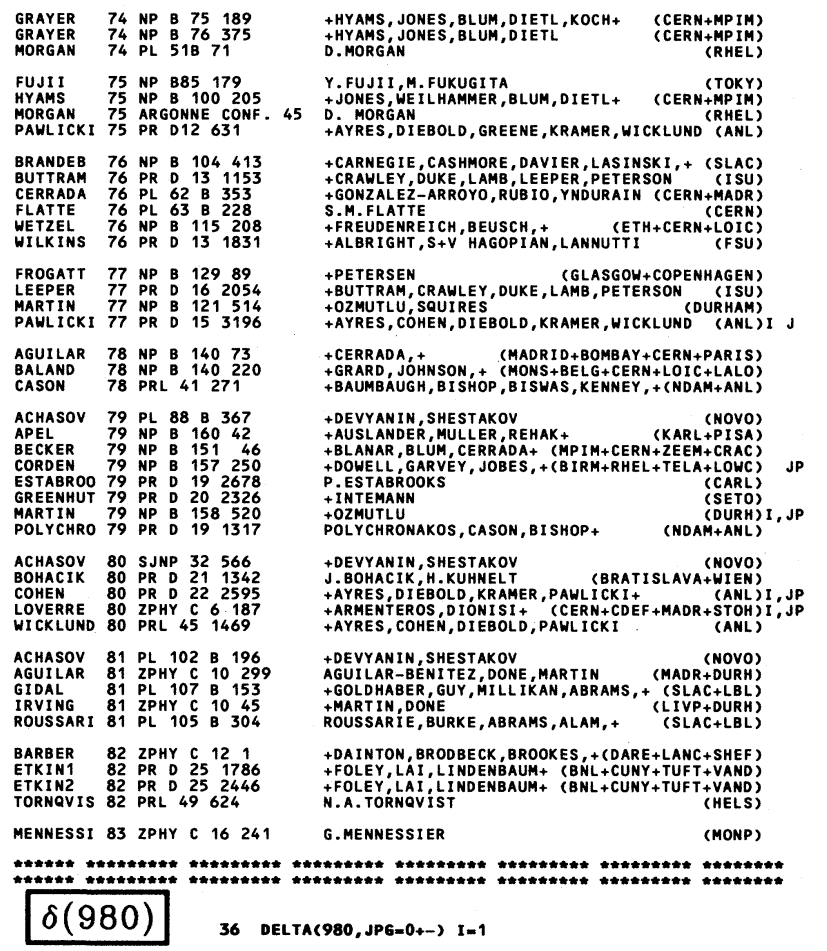

The quantum numbers of the $\delta(980)$ resonance are: $\mathrm{I}^{\mathrm{G}}=1^{-}$, deduced from its production in $\mathrm{D}^{0} \rightarrow \delta \pi$, from its $\eta \pi$ decay, and from the absence of a $\pi \pi$ decay; and $\mathrm{J}^{\mathrm{P}}=0^{+}$, deduced from the absence of a $3 \pi$ or $\rho \pi$ decay (LIPKIN 69, GRASSLER 77) and from the decay distributions of the $\eta \pi$ decay. With these quantum numbers, the $\delta(980)$ is expected to couple to the $I=1$ $\mathrm{K} \overline{\mathrm{K}}$ system, too, and to explain the nearby $\mathrm{K} \overline{\mathrm{K}}$ threshold enhancement (ASTIER 67).

The SU(3) and quark-model classification of the $\delta(980)$ has been somewhat controversial. The naive quark model would suggest that the lightest $0^{++}$states should belong to a $1 \mathrm{P}$ q $\bar{q}$ nonet. This conclusion is also supported by models with very general spin-dependent terms (SCHNITZER 82). The unconventional mass spectrum, in particular the near-degeneracy of $\delta(980)$ and $\mathbf{S}(975)$, has on the other hand led to suggestions for a 4-quark assignment of the $\delta(980)$ (JAFFE 79). A 4quark assignment would predict a very large ("superallowed") coupling to $\eta \pi$ and $\mathrm{K} \overline{\mathrm{K}}$ (ACHASOV 79,80). A comparatively large coupling is not incompatible with a narrow $\delta$ peak width (FLATTE 76 ), since the $K \bar{K}$ threshold distorts drastically the $\delta(980)$ shape.

However, a $q \bar{q}$ interpretation also requires by $\mathrm{SU}(3)$ a large $\delta \eta \pi$ coupling, and it has been shown
(TORNQVIST 82) that it is possible to understand within the unitarized quark model the unconventional features of the lightest scalar mesons. In this framework, the mass shifts, mixings, and distortions of resonance shapes induced by the nearest SU(3)-related thresholds are crucial for the light $0^{++}$states. Thus the $\delta(980)$ mass is shifted considerably by the nearby $\pi \eta$, $\mathrm{K} \overline{\mathrm{K}}$, and $\pi \eta^{\prime}$ thresholds, whereas, e.g., the $\kappa(1350)$ is shifted much less by $\mathrm{K} \pi$ and $\mathrm{K} \eta^{\prime}$, which lie relatively far from the resonance mass. Possible weaknesses of this approach have been discussed (ACHASOV 83).

A conventional $q \bar{q}$ assignment is also favored by the $\mathrm{D}(1285) / \mathrm{E}(1420) \rightarrow \rho \pi$ branching ratio, the decay $\eta^{\prime} \rightarrow$ $\delta \pi \rightarrow \eta \pi \pi$, and the tadpole contributions to e.m. mass differences and mixings (BRAMON 80,83), although some of these arguments might turn out to be inadequate (ACHASOV 83). Thus it seems plausible

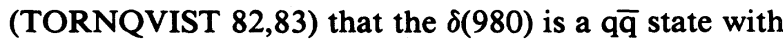
large Fock-space components of $\mathrm{q} \overline{\mathrm{q}} \mathrm{q} \overline{\mathrm{q}}$ in the form of virtual $\eta \pi, K \bar{K}$, and $\eta^{\prime} \pi$ pairs. See also the mini-reviews under $\epsilon(1300)$ and $\kappa(1350)$.

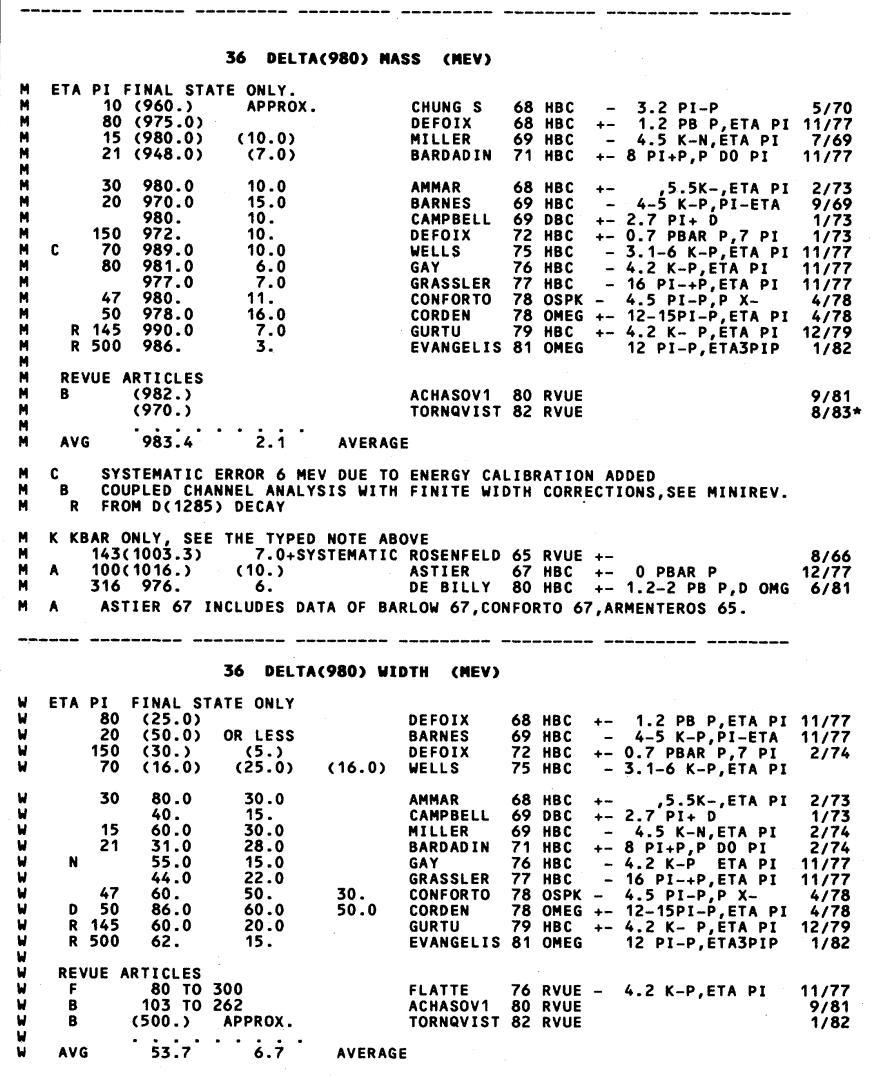


Mesons

$\delta(980), \phi(1020)$
Data Card Listings
W COUPLED CHANNEL ANALYSIS WITH FINITE WIDTH CORRECTIONS, SEE MINIREV.
W

F F USING A THO CHANNEL RESONANCE PARAMETRIZATION OF GAY 76
$W$
$W$
R FROM DRROR IN THE PAPER IS WRONGL QUOTED AT ONE POINT

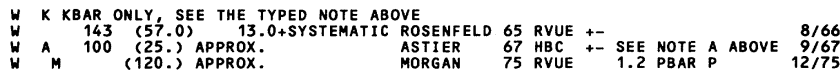

$W$
$W$ ASTIER 67 INCLUDES DATA OF BARLOW 67, CONFORTO 67 , ARMENTEROS 65.

36 deLta(980) PaRtial decay modes

$\begin{array}{llll}\text { P1 } & \text { DELTA(980) } & \text { INTO ETA PI } \\ \text { P2 } & \text { DELTA(980) } & \text { INTO RHO PI } \\ \text { P3 } & \text { DELTA(980) } & \text { INTO K KBAR }\end{array}$

$\begin{array}{lll}P 3 & \text { DEETAC980) } & \text { INTO K KBAR } \\ \text { P4 } & \text { DELTA }(980) & \text { INTO PI ETA PRIME }\end{array}$

DECAY MASSES

$549+135$
$769+135$
$498+498$
$140+958$

\section{DELTA(980) BRAMCHIMG RATIOS}

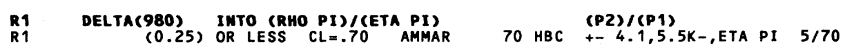

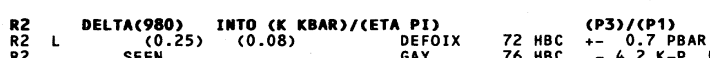

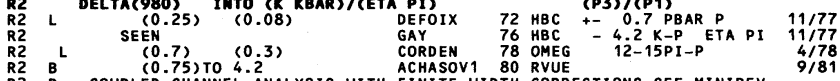
R2
R2 L COUPLED CHANNEL ANALYSIS WITH AINITE WIDTH CORRECTIONS, SEE MINIREV.
L FOM THE DECAY OF D(1285).

\begin{tabular}{|c|c|c|}
\hline & & (980) \\
\hline IRKOT & 63 SIENNA CONF 1661 & 1 + COLLINS, FUJII, KEMP+ \\
\hline $\begin{array}{l}\text { ARMENTER } \\
\text { BARASH } \\
\text { KIENZLE } \\
\text { ROSENFEL }\end{array}$ & 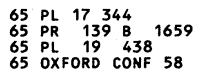 & $\begin{array}{l}\text { ARMENTEROS, EDWARDS, JACOBSEN + (CERN+CDEF) } \\
\text { +FRANZINI, KIRSCH, MILLER, STEINBERGER+ (COLU) } \\
+ \text { MALICCLEVRT, LEFEBVRES + } \\
\text { A HAROSENFELD } \\
\text { (CERN) } \\
\text { (LRL-RVUE) }\end{array}$ \\
\hline $\begin{array}{l}\text { ALLEN D } \\
\text { BALTAY } \\
\text { FOCACCI } \\
\text { OOSTENS }\end{array}$ & $\begin{array}{l}66 \mathrm{PL} 22543 \\
66 \mathrm{PR} 2142 \mathrm{~B} \\
66 \mathrm{PRL} \quad 17 \\
66 \mathrm{PL} \quad 22 \quad 708\end{array}$ & 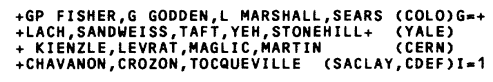 \\
\hline $\begin{array}{l}\text { ALLISON } \\
\text { ASTIER } \\
\text { ASTIER } \\
\text { BAILLON } \\
\text { BANNER } 1 \\
\text { BANNER 2 } \\
\text { BARLOW } \\
\text { CONFORTO }\end{array}$ & 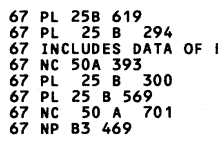 & 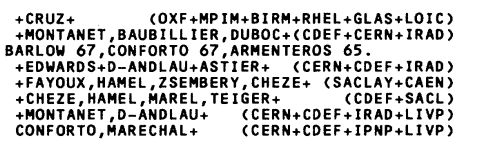 \\
\hline $\begin{array}{l}\text { AMMAR } \\
\text { CHUNG S } \\
\text { DEFOIX } \\
\text { GALTIERI } \\
\text { JUHALA } \\
\text { SABRE CO }\end{array}$ & 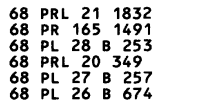 & 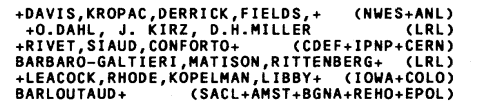 \\
\hline $\begin{array}{l}\text { BARNES } \\
\text { CAMPBELL } \\
\text { CRENAELL } \\
\text { JUHALA } \\
\text { KRUSE } \\
\text { LIPKIN } \\
\text { MILLLRR } \\
\text { ALSO } \\
\text { SCHROEDE }\end{array}$ & 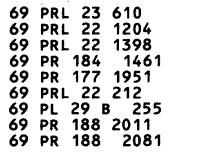 & 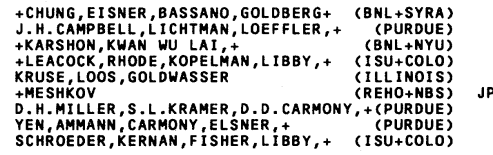 \\
\hline $\begin{array}{l}\text { ABOLINS } \\
\text { AMMAR } \\
\text { COOPER } \\
\text { YIOU }\end{array}$ & $\begin{array}{l}70 \text { PRL } 25469 \\
70 \text { PR D } 2430 \\
70 \text { NP } 823605 \\
70 \text { THESIS, A } 646\end{array}$ & 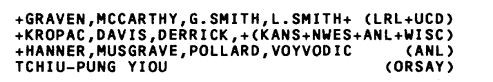 \\
\hline & & $\begin{array}{l}\text { +DIXITI (CHIC+ANL+CARL+LASL+CNRC+NAGOYA) } \\
\text { BARDADIN-OTWINOWSKA, HOFMOKL, MICHEJDA+ (WARS) }\end{array}$ \\
\hline $\begin{array}{l}\text { BINNIE } \\
\text { CHESHIRE } \\
\text { DEFOIX } \\
\text { DUBOC } \\
\text { HOLLOWAY }\end{array}$ & 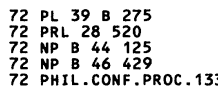 & 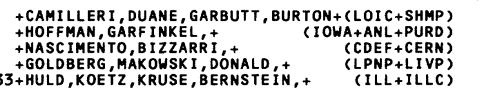 \\
\hline ATHERTON & 73 PL 43 в 249 & +FRANEK, FRENCH, GHIDINI, HILPERT, + \\
\hline $\begin{array}{l}\text { BINNIE } \\
\text { KALBFLEI } \\
\text { MORGAN }\end{array}$ & 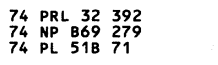 & $\begin{array}{l}\text { +CAMILLERI, CARR, DEBENHAM, + } \\
\text { KALBFLEISCH, VANDERBURG, }+ \text { (LOIC+SHMP) } \\
\text { D.MORGAN }\end{array}$ \\
\hline $\begin{array}{l}\text { BUTTRAM } \\
\text { MORGAN } \\
\text { WELLS }\end{array}$ & $\begin{array}{l}75 \text { PRL } 35970 \\
75 \text { ARGONNE CONF. } \\
75 \text { NP B } 101333 \text {. }\end{array}$ & $\begin{array}{lr}\text { + CRAWLEY, DUKE , LAMB , LEEPER , PETERSON } & \text { (ISU) } \\
\text { D. MORGAN } & \text { (RHEL) } \\
\text { +RADOJICIC , ROSCOE , LYONS } & \text { (OXF) }\end{array}$ \\
\hline $\begin{array}{l}\text { GAY } \\
\text { FLATTE }\end{array}$ & $\begin{array}{lllll}76 & \mathrm{PL} & 63 & \mathrm{~B} & 220 \\
76 & \mathrm{PL} & 63 & \mathrm{~B} & 224\end{array}$ & $\begin{array}{l}\text { +CHALOUPKA, BLOKZI JL, HEI NEN+(CERN+AMST+NI JM) } \\
\text { S.M.FLATTE } \\
\text { (CERN) }\end{array}$ \\
\hline $\begin{array}{l}\text { GRASSLER } \\
\text { IRVING } \\
\text { MARTIN } \\
\text { MAY IN }\end{array}$ & $\begin{array}{l}77 \text { NP } \\
77 \\
77\end{array}$ & $\begin{array}{l}\text { (AACH+BERL+BONN+CERN+CRAC+HEID+WARS) } \\
\text { (LIVERPOOL) } \\
\text { A.C. IRVING } \\
\text { +OZMUIUU, SQUIRES } \\
\text { +ABRAMSON, ANDREWS, BUSNELLO,+ } \\
\text { (ROCH+CORN) }\end{array}$ \\
\hline $\begin{array}{l}\text { CONFORTO } \\
\text { CORDEN } \\
\text { MARTIN }\end{array}$ & $\begin{array}{l}78 \text { LNC } 23 \quad 419 \\
78 \text { NP B } 144253 \\
78 \text { ANP } 114 \quad 1\end{array}$ & $\begin{array}{l}\text { B+G CONFORTO,KEY+(RHEL+TNTO+CHIC+FNAL+WISC) } \\
+ \text { CORBETT,ALEXANDER, (BIRM+RHEL+TELA+LOWC) } \\
\text { A.D.MARTIN, M.R.PENINGTON } \\
\text { (CERN) }\end{array}$ \\
\hline $\begin{array}{l}\text { ACHASOV } \\
\text { ESTABROO } \\
\text { GURTU } \\
\text { MARTIIN }\end{array}$ & $\begin{array}{lllll}79 & \text { PL } & 88 & \text { B } & 367 \\
79 & P R & 0 & 19 & 2678 \\
79 & \text { NP } & \text { B } & 151 & 181 \\
79 & \text { NP } & \text { B } & 158 & 520\end{array}$ & $\begin{array}{l}\text { +DEVYANIN, SHESTAKOV } \\
\text { P.ESTABROOKS (NOVO) } \\
\text { +GAVILLET, BLOKZIJL, + } \\
\text { +OZMUTLU } \\
\text { (CARL) } \\
\text { (CERN +ZEEM+NIJMOOFF) } \\
\text { (DURH) }\end{array}$ \\
\hline $\begin{array}{l}\text { ACHASOV1 } \\
\text { ACHASOV2 } \\
\text { BRAMON } \\
\text { DE BILLY }\end{array}$ & $\begin{array}{lllll}80 & \text { SJNP } & 32 & 566 \\
80 & \mathrm{PL} & 96 & \mathrm{~B} & 168 \\
80 & \mathrm{PL} & 93 & \mathrm{~B} & 65 \\
80 & \mathrm{NP} & \mathrm{B} & 176 & 176\end{array}$ & $\begin{array}{l}\text { +DEVYANIN, SHESTAKOV } \\
\text { +DEVYANIN, SHESTAKOV } \\
\text { + MAASO } \\
\text { +BRIAND, DUBOC, LEVY }\end{array}$ \\
\hline
\end{tabular}

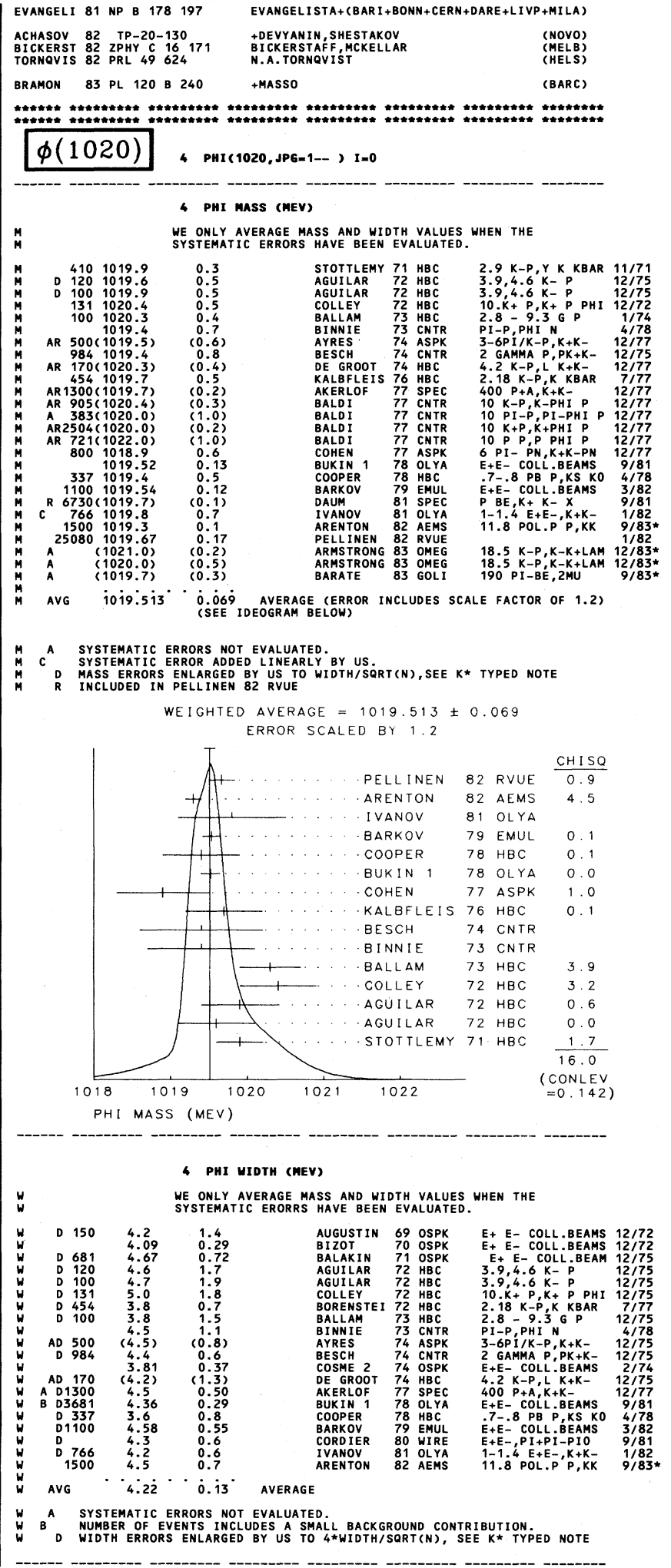




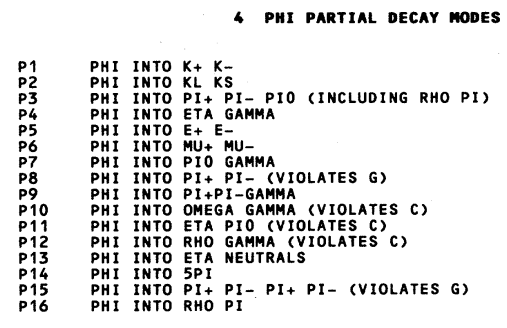

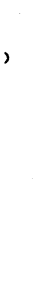

\section{FITTED PARTIAL DECAY MODE BRANCHING FRACTIONS}

The matrix below is derived from the error matrix for the fitted partial decay mode branching fractions, $P_{i}$, as follows: The diagonal elements are $P_{i} \neq \delta P_{i}$, where

$\delta P_{i}=\sqrt{\left\langle\delta P_{i} \delta P_{i}\right\rangle}$, while the off-diagonal elements are the normalized correlation coefficients $\left\langle\delta \mathrm{P}_{i} \delta \mathrm{P}_{j}\right\rangle /\left(\delta \mathrm{P}_{i}, \delta \mathrm{P}_{j}\right)$. For the definitions of the individual $\mathrm{P}_{i}$, see the listings above; only those $P_{i}$ appearing in the matrix are assumed in the fit to be nonzero and are thus constrained to add to 1 .

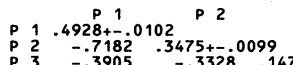

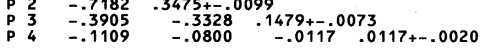

$R 1$
$R 1$
$R 1$
$R 1$
$R 1$
$R 1$
$R 1$
$R 1$
$R 1$
$R 1$
$R 1$
$R 2$
$R 2$
$R 2$
$R 2$
$R 2$
$R 2$
$R 2$
$R 2$
$R 2$
$R 2$
$R 2$

4 PHI BRAMChIMg Ratios

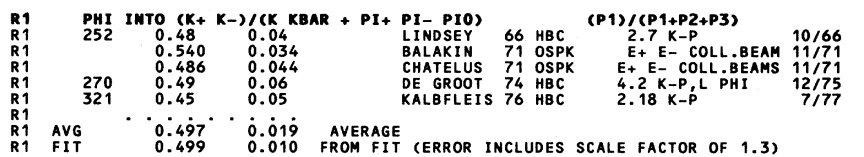

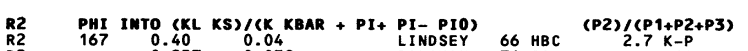

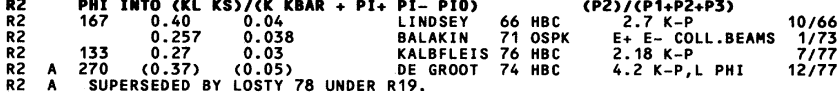

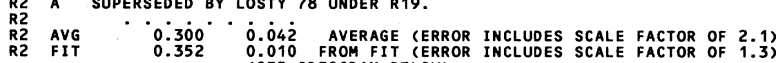

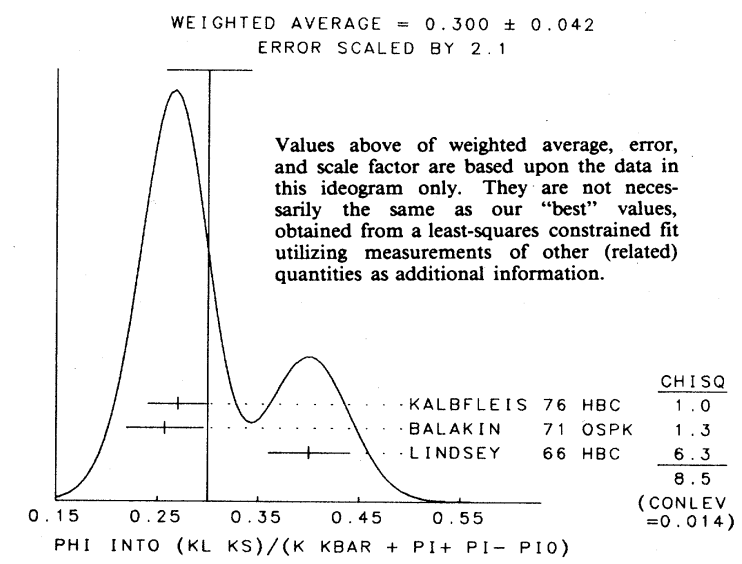

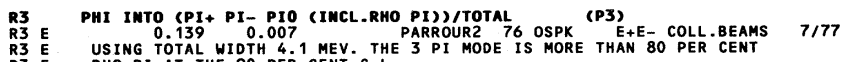
${ }_{R 3}^{R 3}$ FIT $0.1479^{\circ} 0.0073$ FROM FIT (ERROR INCLUDES SCALE FACTOR OF 1.2)

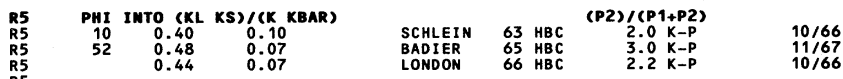
$\begin{array}{llllll}\text { R5 } & & 0.44 & 0.07 & \\ \text { R5 } & \text { AVG } & 0.448 & 0.04 & 0.044 & \text { AVERAGE } \\ \text { R5 } & \text { FIT } & 0.414 & 0.011 & \text { FROM FIT (ERROR INCLUDES SCALE FACTOR OF } 1.3 \text { ) }\end{array}$

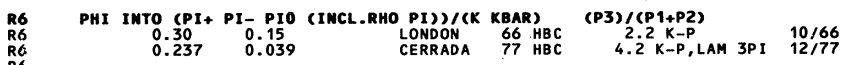
$\begin{array}{llllll}\text { R6 } & & 0.237 & 0.039 & \text { CERRADA } 77 \text { HBC } & 4.2 \mathrm{~K}-\mathrm{P}, \text { LAM 3PI } \\ \text { R6 } & \text { AVG } & 0.241 & 0.038 \\ \text { R6 } & \text { FIT } & 0.176 & 0.038 & \text { AVERAGE } & \\ \text { R6 } & 0.010 \text { FROM FIT (ERROR INCLUDES SCALE FACTOR OF 1.2) }\end{array}$

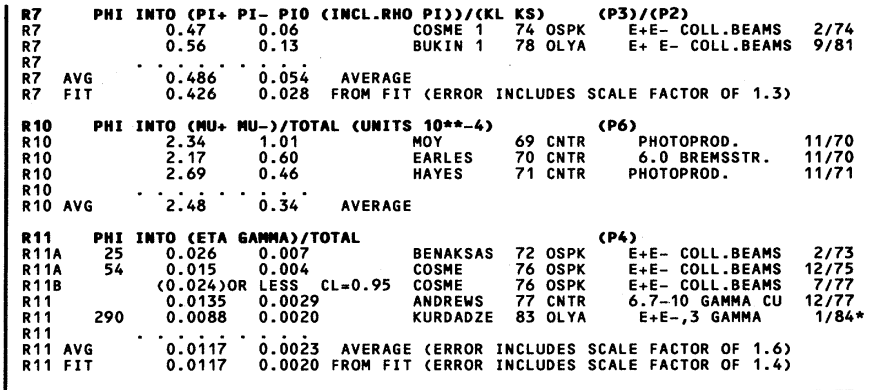

$\begin{array}{llll}\text { R11A } & \text { FROM 2 GAMMA DECAY MODE OF ETA } & 7 / 77 \\ \text { R11B } & \text { FROM PI+PI-PIO DECAY MODE OF ETA } & 7 / 77\end{array}$

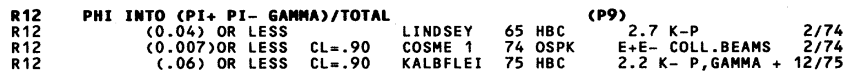

$\begin{array}{lllll}\text { R14 PHI INTO (OMEGA GAMMA) / TOTAL LINDSEY } & 66 \mathrm{HBC} & (\mathrm{P} 10) \\ \text { R14 } & 2.7 \mathrm{~K}-\mathrm{P} & 10 / 66\end{array}$

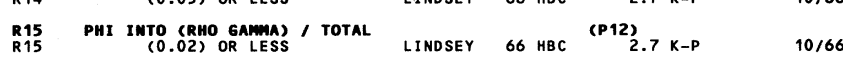

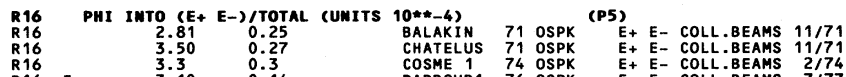

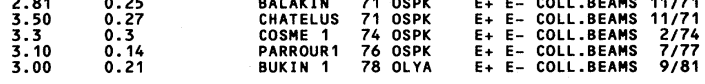

$\cdot 3.107 \cdot 0.096$ average

$\begin{array}{llll}\text { R16 } & \text { USING TOTAL WIDTH } 4.2 \text { MEV. THEY DETECT } 3 \text { PI MODE AND OBSERVE } \\ \text { R16 } & \text { E SIGNIFICANT INTERFERENGE WITH OMEGA TAIL. THIS IS ACCOUNTED FOR } \\ \text { R16 } & \text { E IN THE RESULT QUOTED ABOVE }\end{array}$

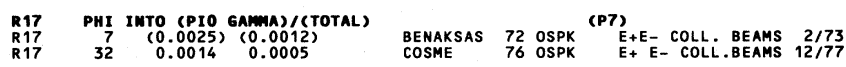

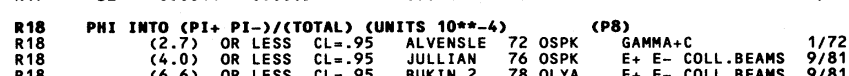

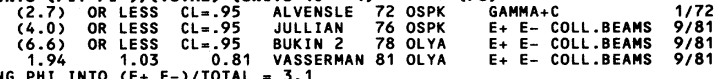
USING PHI INTO $(E+E-) /$ TOTAL $=3.1$

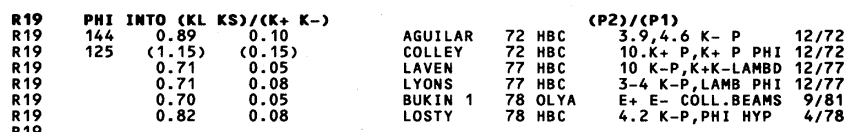

$\begin{array}{llll}\text { R19 } & & & \\ \text { R19 AVG } & 0.736^{\circ} & 0.030 & \text { AVERAGE } \\ \text { R19 FIT } & 0.705 & 0.032 & \text { FROM FIT (ERROR INCLUDES SCALE FACTOR OF 1.3) }\end{array}$

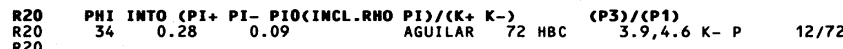

R20 FIT $0.300^{\circ} \cdot \dot{0} \dot{0} 1 \dot{8}$ FROM FIT (ERROR INCLUDES SCALE FACTOR OF 1.2)

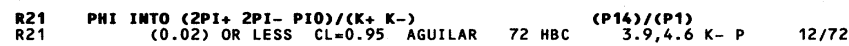

R22 PHI INTO (PI+ PI-PI+ PI-)/TOTAL (UNITS $10 * *-4)$
R22

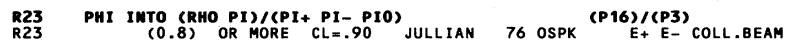

$* * * * * * * * * * * * * * * * * * * * * * * * * * * * * * * * * * * * * * * * * * * * * * * * * * * * * * * * * * * * * * * *$
REFEREMCES FOR PHI

BERTANZA 62 PRL 9180 BERTANZA, BRISSON, CONNOLLY, HART + (BNL + SYRA)

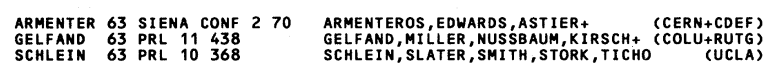

$\begin{array}{llll}\text { BADIER } & 65 \mathrm{PL} 17337 & 17 & \text { BADIER, DEMOULIN, BARLOUTAUD+ } \\ \text { BERLEY } & 65 & \text { (SACL+AMST) } \\ \text { GER } 139 \text { B } 1097 & \text { D BERLEY,N GELFAND }\end{array}$

GALTIERI 65 PRL 14279 A 279 BARBAROGALTIERI,R D TRIPP
LINDSEY 65 PRL 15221 (LRL)
(LRL)

LINDSEY 65 DATA INCLUDED IN LINDSEY 66 BELOW
MILLER D 65 CU-237 (NEVIS 131 ) DAVID C MILLER (THESIS)
(COLUMBIA)

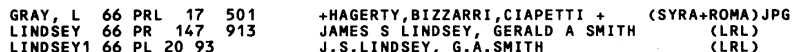

LINDSEY1 66 PL 2093
LONDON 66 PR 1431034

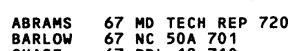

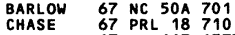

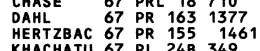

$\begin{array}{lllll}\text { ABRAMS } & 68 & \text { PR } & 175 & 1697 \\ \text { ASTVACAT } & 68 & \text { PL } & 27 \\ \text { B B } & 45\end{array}$

(LRL)
J.S. LINDSEY, G.Á. SMITH
LONDON, RAU, SAMIOS, GOLDBERG + (BNL+SYRACUSE)

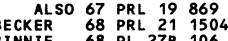

$\begin{array}{lll}\text { BINNIE } & 68 & \text { PL } 27 B \\ \text { BOLLINI } & 68 & 106 \\ \text { BC } 56 \text { A } 1171\end{array}$

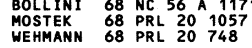

+LILLESTOL+MONTANET+ (CERN+CDEF + IRAD+LIVP)
R.C.CHASE,P.ROTHWELL, R.WEINSTEIN (CEA+NEAS)

+HARDY+HESS+KIRZ+MILLER
HERTZBACH, KRAEMER, MADANSKI, ZDANIS+ ( JHU+BNL)
(LRL) KHACHATURYAN+AZIMOV+ + BALD IN' +BELOUSOV+(DUBNA)

+GLASSER, KEHOE, SECHI-ZORN, WOL SKY (MARYLAND) ASTVACA UEOV, AZIMOV, BALDIN+ (JINR+MOSCOW) (BERTRAM, BINKLEY, JORDAN, KNASEL+ (DESY +MITI) +DUANE+FARUQI + HORSEY+
+BUHLER, (CEAPIAZ, MASSAM+ (LERN+BGNA+RTRB
+EISENHANDLER, MCCLELLAN, MISTRY + (CORNELL) +EISENHANDLER, MCCLELLAN, MISTRY+ (CORNELL)
+ +ENGELS+ (HARVARD+CASE+SLAC+CORNELL+MCGILL)
GERALD ABRAMS THESIS TMARYLAND)
+ +LILLESTOL MONTANET+ (CERN+CDEF+IRAD + IVP) 


\section{Mesons}

\section{Data Card Listings}

$\phi(1020), \mathrm{H}(1190), \mathrm{B}(1235)$

AUGUSTIN 69 PL 28 B 517 +BI 20T, BUON, OELCOURT, HAISSINSKI, (ORSAY)

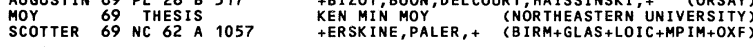

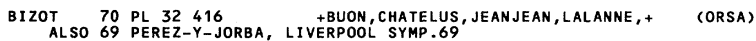

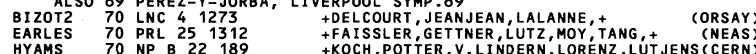
ALVENSLE 71 PRL 27441 ALVENLEEBEN, BECKER, BUSZA, CHEN, (MMIT+DESY BALAKIN 71 PL 34 B 328
CHATELUS 71 LAL 1247(THESIS) Y.CHOKER, PAKHTUSOVA, SIDOROV, SKRINSKY, +(NOVO)
(STRASBOURG)

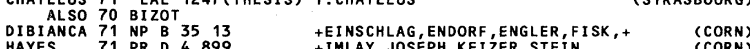

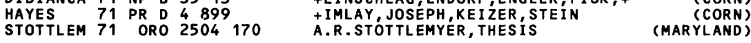

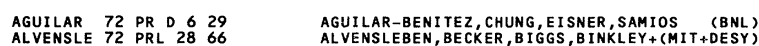

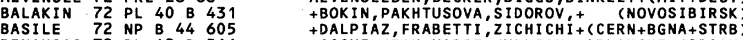
+COSM, JEA -MARIE, JULLLIAN, LAPLANCHE+ OORSAY

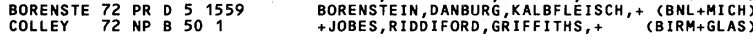

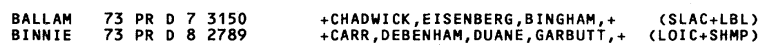

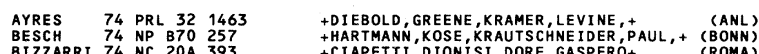
COSME $174 \mathrm{PL} 48$ B 155 +JEAN-MARIE, JULLIAN, LAPLANCHE, + (ORSAY)

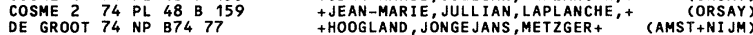
KALBFLEI 75 PR 011987 KALBFLEISCH, STRAND, CHAPMAN (BNL+MICH)

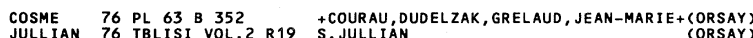

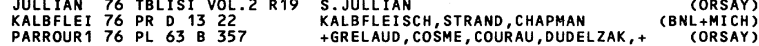
PARPOUR2 76 PL 63 B 362 +GRELAUD, 'COSME, COURAU, DUDELZAK, (ORSAY) $\begin{array}{llll}\text { AKERLOF } & 77 & \text { PRL } 39 & 861 \\ \text { ANDREWS } 77 \text { PRL } 38 & 198\end{array}$

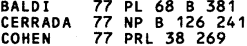

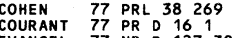

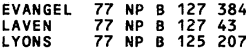

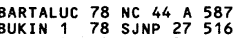

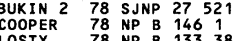
BARKOV 79 IYAF $79-93$ + ALLEY, BINTINGER, DITZLER, + (FNAL MICH+PURD) +BOHR INGER, DORSAZ, HUNGERBUHLER, (GENEVA)

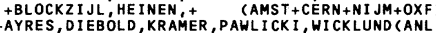

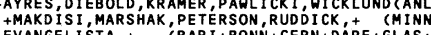
+OTTER,KLEIN +
+ COOPER, CLARK
(AACH+BERL+CERN+LOIC+WIEN)
(OXF)

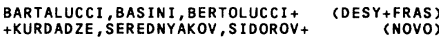

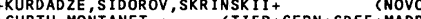

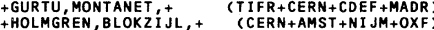
+ZOLOTOREV MAKARINA, MISHAKOVA,
+DELCOURT, ESCHSTRUTH, FULDA,
(NOVOO) $\begin{array}{lllll}\text { CORDIER } & 80 & \text { NP } & B 172 & 13 \\ \text { ROOS } & 80 & L N C & 27 & 321\end{array}$ +DELCOURT, ESCHSTRUTH, FULDA+ (ORSAY)
+PELLINEN

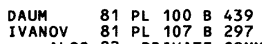

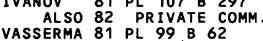
$\begin{array}{llll}\text { ARENTON } 82 & \text { PR } & 25 & 2241 \\ \text { PELLINEN } 82 \text { PS } 25599 & 59\end{array}$ +BARDSLEY+ (AMST+BRIS+CERN+CRAC+MPIM+RHEL)
+KURDADZE, LELCHUK, SIDOROV, SKR INSKY, + (NOVO) S. I. ELDELAAN
VASSERMAN, KURDADZE, SIDOROV, SKRINSKY, (NOOVO) AAYRES, OIEBOLD, MAY, SWALLOH+
A.PELLINEN, M, ROOS,
(ANL+IILL)
(HELS)

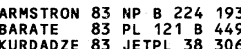
ARMSTRONG (BARI+BIRM+CERN+MILA+LPNP+PAVI)
+ BAREYRE, ASTBURY, MCEWEN (SACL+LOIC+SHMP+IND)

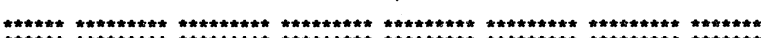

\begin{tabular}{|c|}
\hline $\mathrm{H}(1190)$ \\
30 H(1190, JPG=1+-) I=0 \\
30 H(1190) MASS (MEV)
\end{tabular}

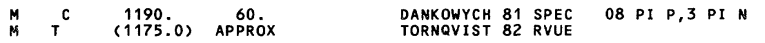
$M$
$M$ C USES THE MODEL OF BOWLER 75 FROM A UNITARIZED QUARK MODEL CALCULATION

\section{H(1190) HIDTH (MEV)}

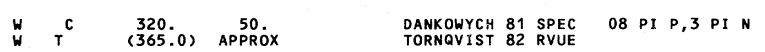
W C USES THE MODEL OF BOMLER 75
FROM A UNITAR IZED QUARK MODEL CALCULATION

30 H(1190) PARTIAL dECAY modes

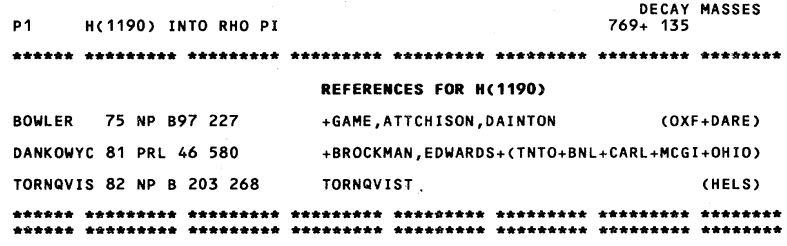

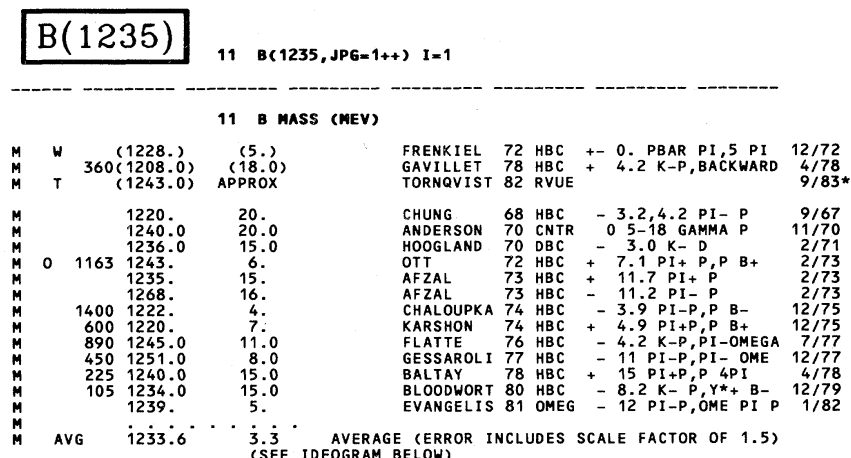

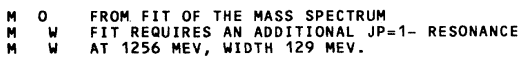

MT AT 1256 MEV
MHIDTH 129 MEV
FROM A UNITAR IZED QUARK MODEL CALCULATION

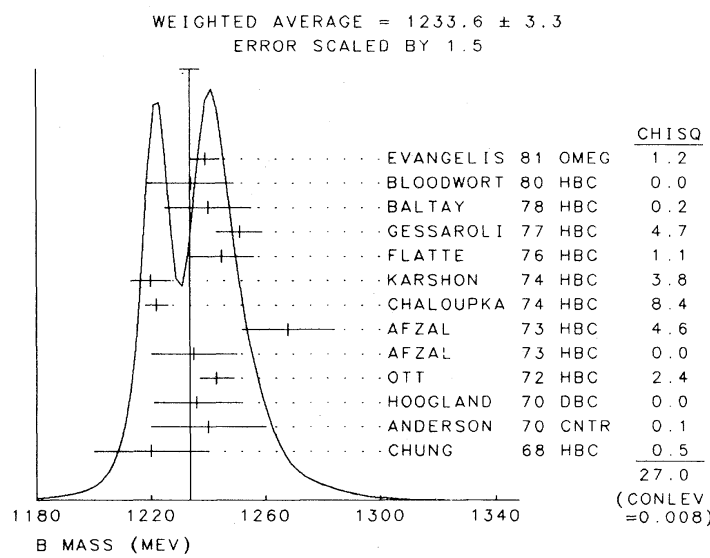

6/83*

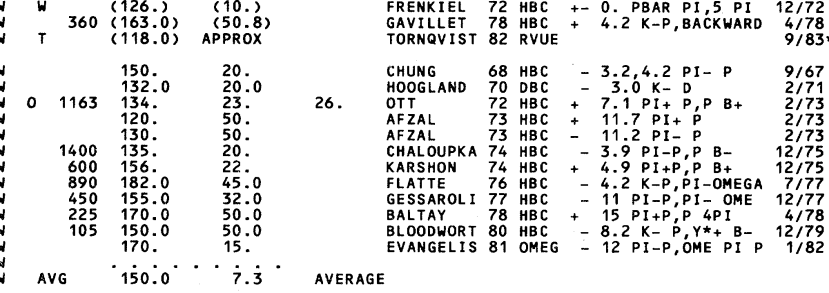

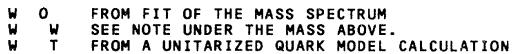

$9 / 83^{*}$ 11 B partial decay modes

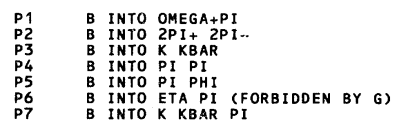
DECAY MASSES
$783+140$
$140+140+140+140$ $494+494$
$140+140$ $535+1020$ $549+140$
$494+494+140$

\section{B BRAMCHIMG RATIOS}

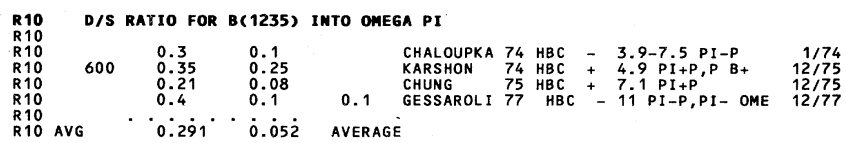


$R 1$
$R 1$
$R 1$
$R 2$
$R 22$
$R 22$
$R 2$
$R 3$
$R 3$
$R 3$
$R 3$
$R 4$
$R 4$
$R 4$
$R 4$
$R 5$
$R 5$
$R 6$
$R 6$
$R 6$
$R 7$
$R 7$
$R 8$
$R 8$
$R 8$

$R 1$
$R 1$
$R 2$
$R 2$
$R 2$
$R 2$
$R 2$
$R 3$
$R 3$
$R 3$
$R 3$
$R 4$
$R 4$
$R 4$
$R 5$
$R 5$
$R 5$
$R 6$
$R 6$
$R 7$
$R 7$
$R 7$
$R 8$
$R 8$

B IMTO (4PI)/(OMEGA PI)
(0.5) OR LESS

B INTO (K KBAR) / (OMEGA PI) $(0.02)$ OR LESS
$(0.10)$ OR LESS
$(0.08)$ OR
$(0.085$

B INTO (PI PI)/(PI OMEGA)

(PPI PI) (PI OMEGA)
(0.3) OR LES
$(0.15)$ OR LESS CL=.90

B INTO (PI PHI) ( (PI OMEGA)

(P. P P ) OR LESS OMEA)
$(0.04)$ OR LESS CL=.95

B INTO (ETA PI) (PI OMEGA)

B+- INTO (CK KBAR)+- PIO)
(0.08) OR LESS (PI OMEGA)
CL=.90 BALTAY

67 HBC +- 0.0 PBAR P

B+- INTO (KS KS PIt-) (PI OMEGA)
$(0.02)$ OR LLSS CL=.90 BALTAY

67 HBC +-0.0 PBA- $P$

B+- INTO (KS KL PI+-) C (PI OMEGA)
(0.06) OR LESS CL $=.90$ BALTAY

67 HBC +- 0.0 PBAR P

REFERENCES FOR B

$\begin{array}{ll}\text { ABOLINS } & 63 \text { PRL } 11381 \\ \text { BONDAR } & 63 \text { PL } 5209\end{array}$

$\begin{array}{ll}\text { ADERHOLZ } & 64 \text { PL } 10240 \\ \text { CARMONY } 64 \text { PRL } 12 & 254\end{array}$

GOLDHABE 65 PRL 15118

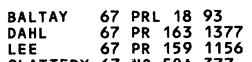

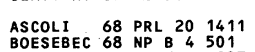

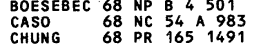

BIZZARRI 69 NP B 14169

ANDERSON 70 PR $^{0}{ }^{1} 727$
CASO

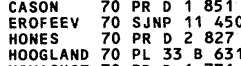

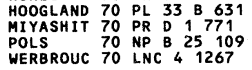

DEVONS 71 PRL 271614

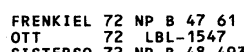

AFZAL 73 NCL 15 A 61 ARMENISE 73 L LNC 8425

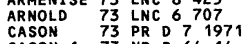

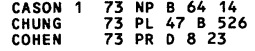

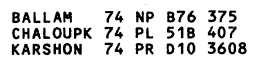

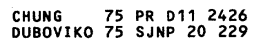

FLATTE 76 PL 64 B 225

GESSAROL 77 NP B 126382

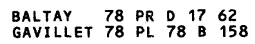

BLOODWOR 80 LNC 27555

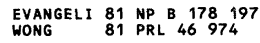

TORNAVIS 82 NP B 203268

ABOLINS, LANDER, MEHLHOP, XUONG, YAGER (UCSD)
BONDAR, DODD+ (AACHEN+BIRM+ HAMB+LOIC+MPIM)

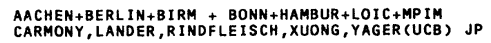

G GOLOHABER, S GOLDHABER, KADYK, SHEN (LRL)

+ SEVERIENS+YEH+ZANELLO (COLU+BNL)

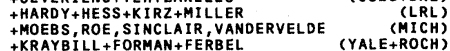

+CRAWLEY, MORTARA, SHAPIRO (ILL) JP
BOESEBECK, DEUTSCHMAN, + (AACHEN+BERLIN+CERN)

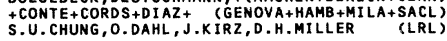

+FOster, gavillet, MONTANet, , (CERN+CDEF)

+ GUSTAVSON, JOHNSON, (SLAC+CIT+UCSB+NEAS)

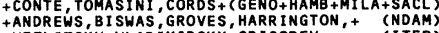

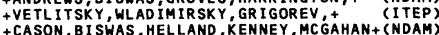

SABRE COLLABOR. CAMST SACL BGNA+REHO+EPOL)

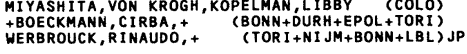

+KOZLOWSKI, HORWITZ,+ (COLU+SYRA)

+GHESQUIERE, LILLESTOL, CHUNG, + (CDEF+CERN) JP

R. L.OTT THESIS ST, HTERON, HARR ISON, HEYDA, JOHNSON, + (HARVARD)
SI STER

BASSLER,
+ FORINO, CARTACCI,
(DURH+GENO+DESY+MILA+SACL) JPARI
(BAGNA+FIRZ) JP

TEOR NO, CARTACCI,

$(B A R I+B G A+F I R Z)$
$(B A R I+B G A A+F I R Z)$

PENGEL, ESCOOBES KUURT, LLOREE, PAY,
+ (STRB

BBISWAS, KENNEY, MADDEN, SANDER, SHEPHARD (NDAM)

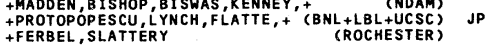

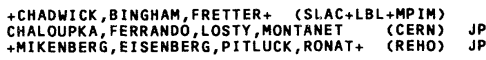
PPROTOPOPESCU, LYNCH, FLATTE, (BNL +LBL+UCSC)
M.S. JPUBOVIKOV, I.A.EROFEEV
(ITEP) + GAY, BLOKZI JL, METZGER, + (CERN+AMST+NI JM+OXF) JP GESSAROLI, + (BGNA+FIRZ+GENO+MILA+OXF+PAVI) JP

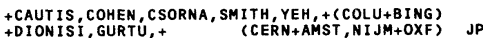

BLOOOWORTH, + (BIRM+CERN+GLAS+MSU+LPNP)

EVANGELISTA+(BARI+BONN+CERN+DARE+LIVP PMILA)
$+K E Y, F R I S K E N$, CLINE, DEBONTE+ (TNTO+YORK + PURD)

TORNQVIST

(HELS)

ATKINSON 83 CERN-EP/83-179 + (BONN+CERN+GLAS+LANC+MCHS+LPNP+RHEL+SHEF)

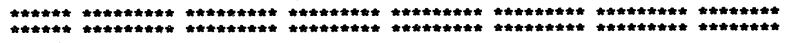

\begin{tabular}{|c|c|c|}
\hline \multicolumn{2}{|c|}{$g_{S}(1240)$} & \multirow{2}{*}{ 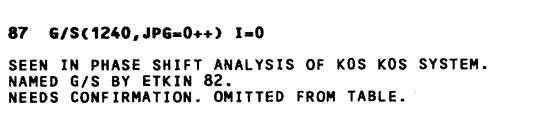 } \\
\hline & & \\
\hline & & $876 / S(1240)$ MASS (MEV) \\
\hline & 1240.0 & $82 \mathrm{MPS} \quad 023$ PI-P, $2 \mathrm{~K} 0 \mathrm{~N}$ \\
\hline & $\begin{array}{l}\text { FROM AN AA } \\
\text { ERROR ADDE }\end{array}$ & $\begin{array}{l}\text { LITUDE ANALYSIS OF THE KOS KOS SYSTEM. SYSTEMATIC } \\
\text { LINEARLY BY US. }\end{array}$ \\
\hline & & 87 G/S(1240) WIDTH (MEV) \\
\hline & 140.0 & $82 \mathrm{MPS} \quad 023 \mathrm{PI}-\mathrm{P}, 2 \mathrm{~K} 0 \mathrm{~S} \mathrm{~N}$ \\
\hline A A & $\begin{array}{l}\text { FROM AN AA } \\
\text { ERROR ADOE }\end{array}$ & $\begin{array}{l}\text { LITUDE ANALYSIS OF THE KOS KOS SYSTEM. SYSTEMATIC } \\
\text { LINEARLY BY US. }\end{array}$ \\
\hline
\end{tabular}

87 G/S(1240) PARTIAL DECAY MODES

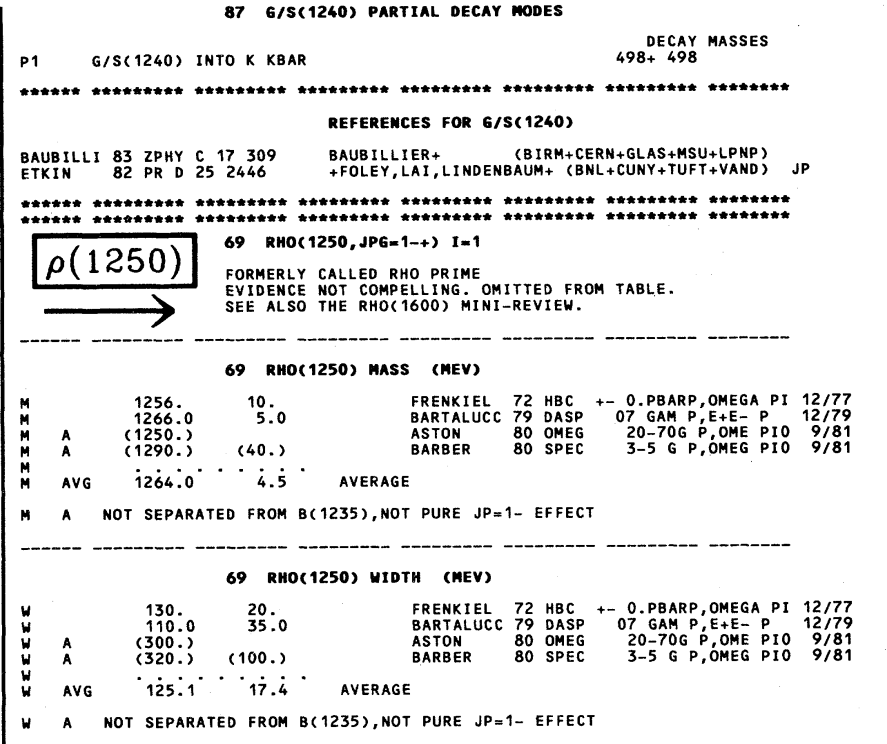

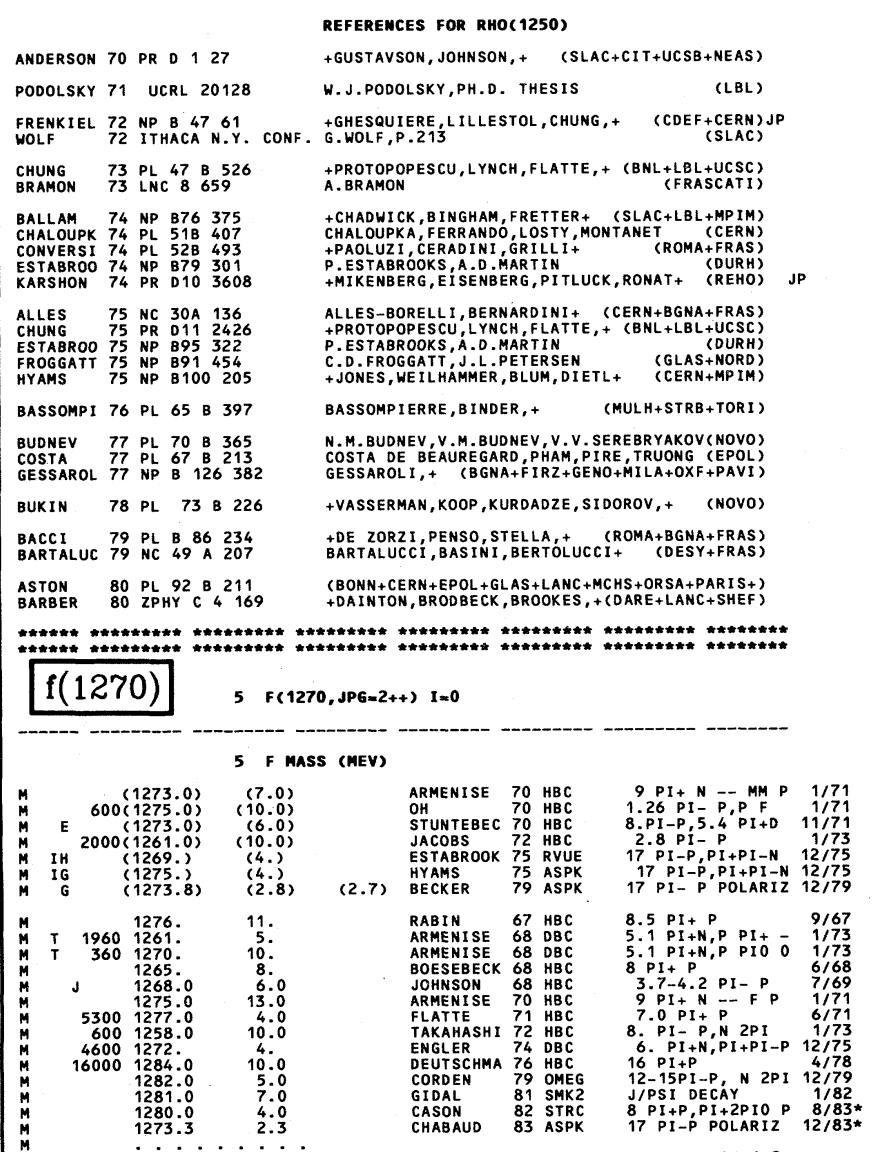

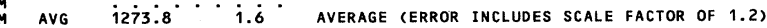

$M$
$M$ INCLUDED IN CHABAUD 83 ANALYSIS

M H USES SAME DATA AS HYAMS 75 . $M$
$M$
$M$ 


\section{Mesons}

\section{Data Card Listings}

$\mathrm{f}(1270)$

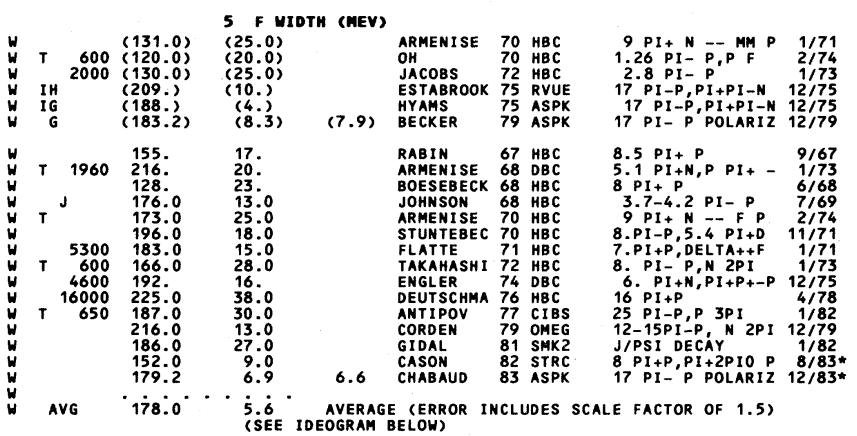

W G INCLUDED IN CHABAUD 83 ANALYSIS

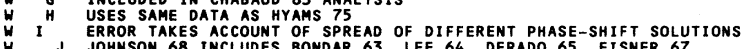

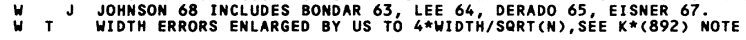

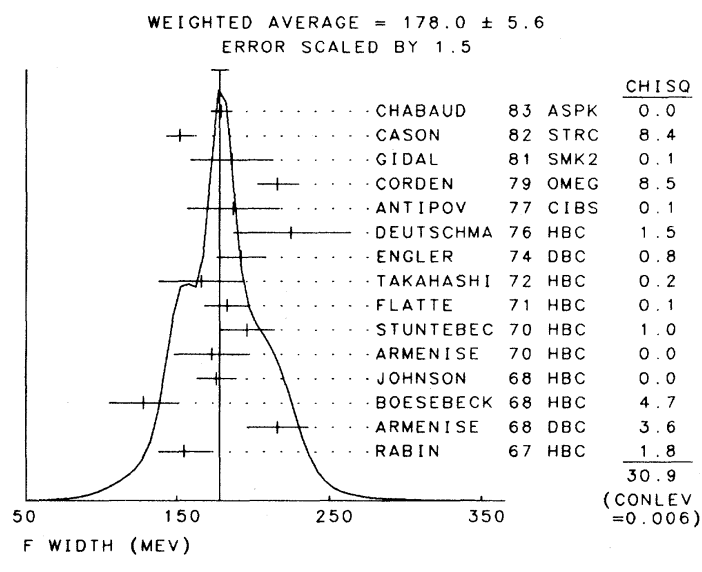

5 F PARTial decay mODEs

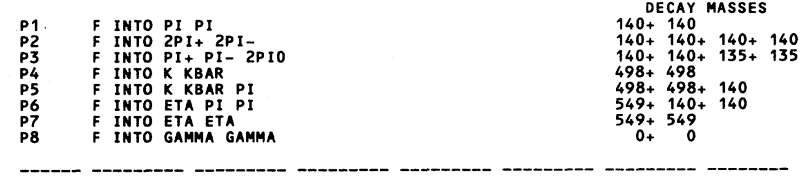

5 F PARTIAL MIDTHS

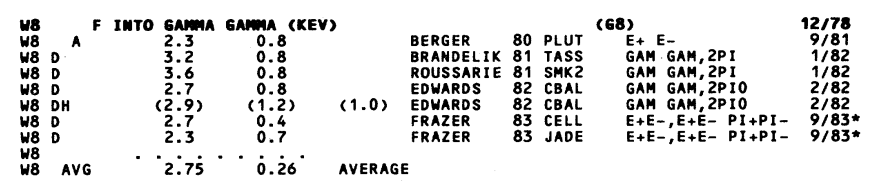

H8 A USING MASS, WIDTH AND BR(F TO 2PI) FROM PDG 1978

H8 D
WB
HY SYSEMATIC ERROR ADDED LINEARLY BY US.
IF HELICITYE ASSUMPTION IS NOT MADE

\section{F BRANCHIMG RATIOS}

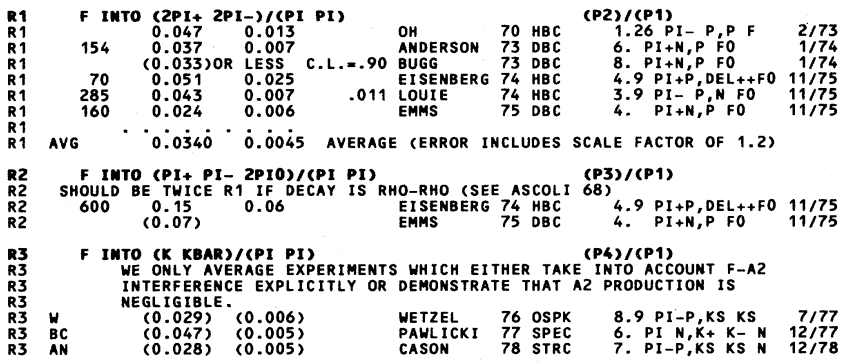

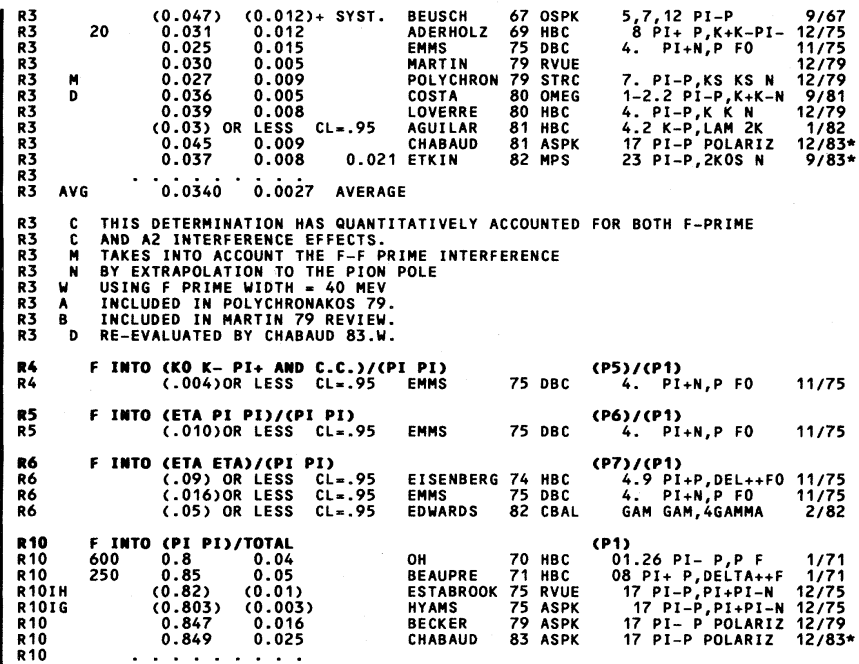

R10 AVG $0.843{ }^{\text {R }} \dot{0} \dot{0} \dot{0} 1 \dot{2}$ average

R10 G INCLUDED IN BECKER 79 ANALYSIS

R10 H" USES SAME DAA AS HYAMS 75
R10I ERROR TAKES ACCOUNT OF SPREAD OF DIFFERENT PHASE-SHIFT SOLUTIONS

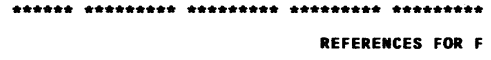

$\begin{array}{llll}\text { SELOVE } & 62 \text { PRL } 9272 \\ \text { BONDAR } & 63 & 272 \\ \text { GL } 5153\end{array}$

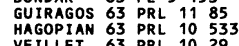

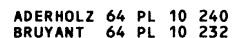

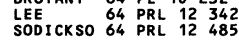

BARMIN 65 SJMP 1230

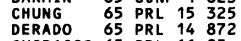

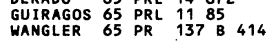

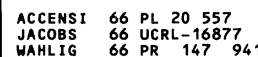

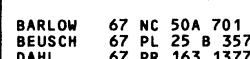

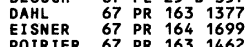

$\begin{array}{lll}\text { POIRTER } & 67 & \text { PR } 163 \\ \text { RABIN } & 67 & 1462 \\ \text { THESIS }\end{array}$

ARMENISE 68 NC 54 A 9999

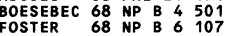

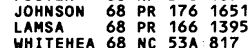

ADERHOLZ 69 NP B 11259

ARMENISE 69 LNC 2501

SELOVE, HAGOP IAN, BROOY, BAKER, LEBOY (PENN)

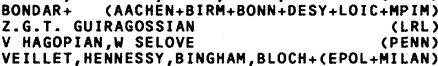

AACHEN+BERL IN+BERLIN+BONN+HAMBURG+LOIC C MPI IJ LEE, ROE, SINCLAIR, VANDERVELDE
SODICKSON, WAHLIG, MANNELLI, FRISCH+ (MICH) I

+DOLGOLENKO, ELENSKY, EROFEEV+ (ITEP MOSCOW) JP

+DOLGOLENKO+ EROOEEV KRRESTNIKOV+ (ITEP MOSC)

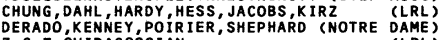

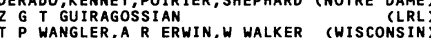

ACCENSI, ALLES-BBRELLI, FRENCH, FRISK+ (CERN)

(LRL)
TSHIBATA, JORDON, FRISCH, MANNELLI (MIT+PISA) J

+LILLESTOL +MONTANET+ (CERN+CDEF+ IRAD+LIIVP)

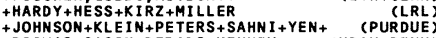

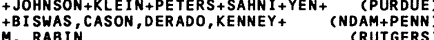

FORINO+CARTACCI+ (BARI+BGAA+EIRENZE+ORSAY

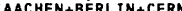

+GAYYLLET+LABROSSE MONTANET T M (CERN+CDEF)

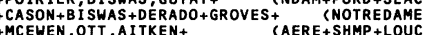

+BARTSCH, + $($ AACH+BERL+CERN+ JAGL+ HARS)

+GHIDINI FORINO, CARTACCI+ (BARI BGNA+FIRZ)

+CONTE, BENZ, ${ }^{+}$(GENO+DESY +HAMB +MILA+SACL)
+EDHARDS, BURAN, BETTINI, ${ }^{+}$(LIVP+OSLO+PADO)

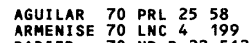

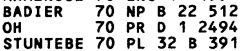

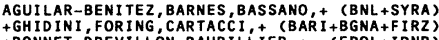

STUNTEBECK', KENNEY, OEERY', BISWAS, CASON+(NDAM)

BARDADIN 71 PR D4 2711

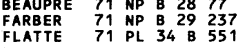

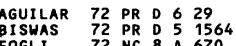

GRAYER 72 PHIL.CONF.PROC.

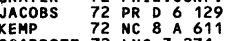

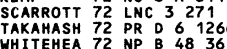

BARDANIN-OTWINOWSKA, HOFMOKL, + (HARS)

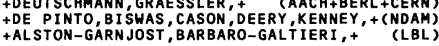

TCASON, HARRINGTON, KENAEY, SHEPHARD (NDAM)

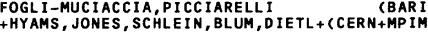

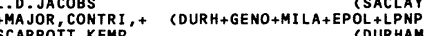

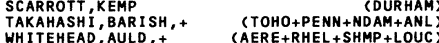

ANDERSON 73 PRL 31562

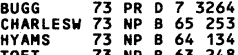

+ENGLER, KRAEMER, TOAF, DIAZ, + (CARN+CASE)

CHARLESWORTH, EMMS, BELL,+ (RHEL+BIRM+DURH

+ JONES, WEELHAMMER, BLUM, DIETL,
+ THUAN, MA JOR, RINAUDO, (NI IM B BONA+DURH+TORI)

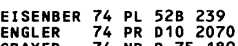

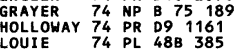

(REHO)
EISENBERG, ENGLER, HABER, KARSHON+
TKRAEMER, TOAFF, WEISSER, OIAZ+
(CARN+CASE)

G. GRAYER, HYAMS, BLUM, DIETL, ${ }^{+}$(CERN+MPIM)

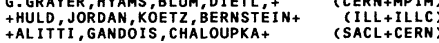




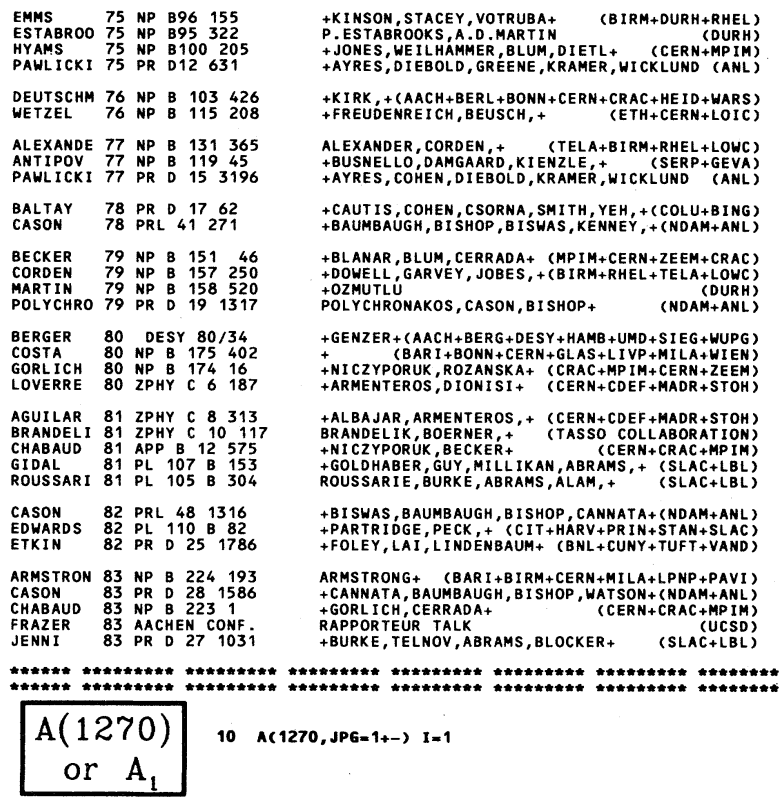

We no longer use the subscript 1 to specify this resonance.

The long-standing question concerning the resonance interpretation of the $A(1270)$ was considerably clarified at the time of our 1982 edition.

The results of the partial-wave analyses obtained in two high-statistics experiments dealing with the diffractive (DAUM 80,81) and charge-exchange (DANKOWYCH 81) production of the $3 \pi$ system in $\pi p$ interactions clearly show that the behavior of the $1^{+} \mathrm{SO}^{+}$intensity with the $3 \pi$ mass and the phase variation of the $1^{+} \mathrm{SO}^{+}(\rho \pi)$ amplitude with respect to other waves [already reported in a study of diffractive production from nuclei (PERNEGR 78)] require the presence of both Deck background and a resonance.

The resonance parameters of the $A(1270)$ are obtained by fitting the data (intensity and relative phases) to a phenomenological amplitude containing direct resonance production and a coherent Deck background which is rescattered through the resonance (BOWLER 75, BASDEVANT 77). In the context of this model-dependent analysis, the Deck background is responsible for making the peak of the $1^{+} \mathrm{SO}^{+}$intensity occur some $110 \mathrm{MeV}$ below the most-likely resonance mass.

We take the mass values for the $A(1270)$ from the above reactions (1240 $\pm 80 \mathrm{MeV}$, DANKOWYCH 81 ; $1280 \pm 30 \mathrm{MeV}$, DAUM 81). Note, however, the result reported in a study of a backwardly produced $3 \pi$ system in the reaction $\mathrm{K}^{-} \mathrm{p} \rightarrow \Sigma^{-} \pi^{+} \pi^{+} \pi^{-}(1041 \pm 13 \mathrm{MeV}$, GAVILLET 77). Based on a small statistical sample, GAVILLET 77 fitted the $3 \pi$ mass distribution with a relativistic S-wave Breit-Wigner and a background including reflections from all the competing channels.

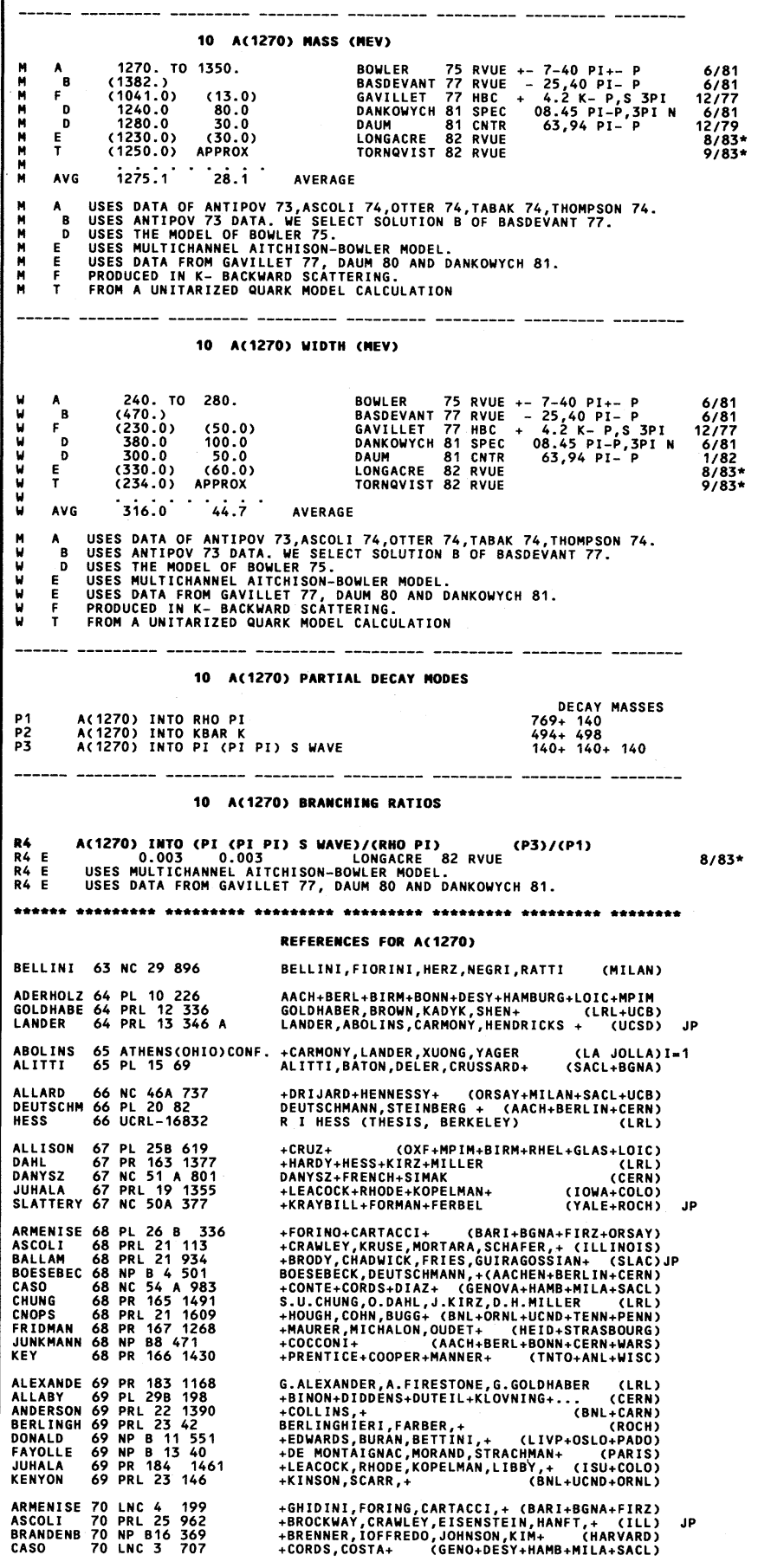




\section{Mesons}

$\mathrm{A}(1270)\left[\mathrm{A}_{1}\right], \quad \eta(1275), \mathrm{D}(1285)$
Data Card Listings

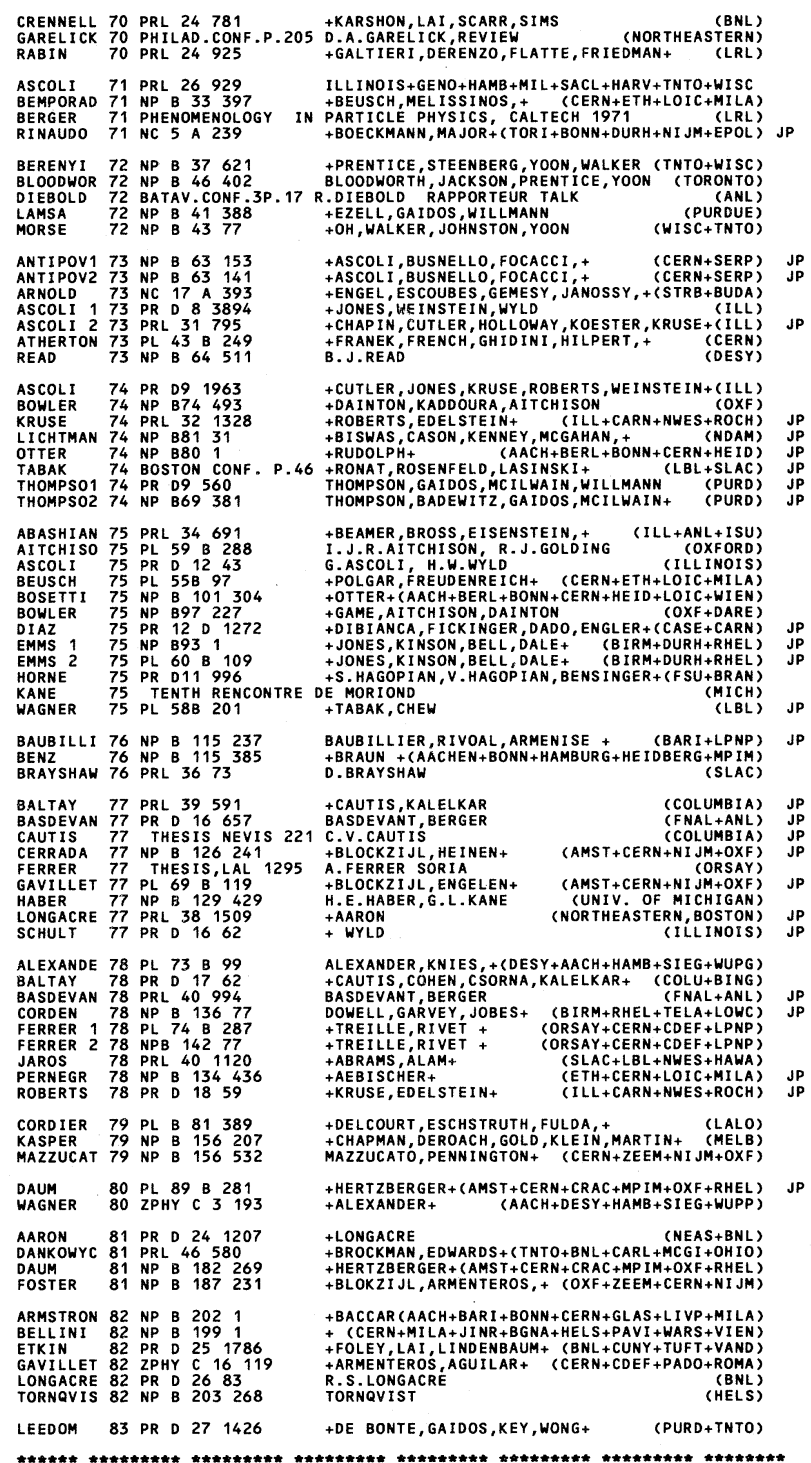

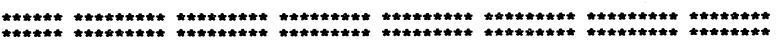

$\eta(1275) \quad 37$ ETA(1275, JPG $=0-+)$ I=0

SEEN IN PHASE SHIFTS ANALYSIS OF THE ETA PI+ PI-
SYSTEM WITH PI+ PI- IN AN S-WAVE (STANTON 79 ).

SENTEM WITH PI+ PI- IN AN S- WAE TSTANOH
HAIT CONFIRMATION. OMITED FROM TABLE.

\begin{tabular}{|c|c|c|c|c|}
\hline m & (1275.) & $\begin{array}{l}37 \text { ETAC(1275) MASS (MEV) } \\
\text { APPROX. STANTON }\end{array}$ & 79 CNTR & O 8.4PI-P,ETA 2PI \\
\hline & & 37 ETA(1275) МIDTH (MEV) & & \\
\hline w & (70.) & StANTON & 79 CNTR & O 8.4PI-P,ETA 2PI \\
\hline
\end{tabular}

$\begin{array}{ll}\text { P1 } & \text { ETAC 1275) } \\ \text { P2 } & \text { INTO DELTA PI } \\ \text { ETA(1275) INTO OETA PI+ PI- }\end{array}$

$983+140$

37 ETA(1275) Branchimg Ratios.

R1 ETAC 1275) INTO DELTA PI LARGE
R1 STANTON 79 CNTR

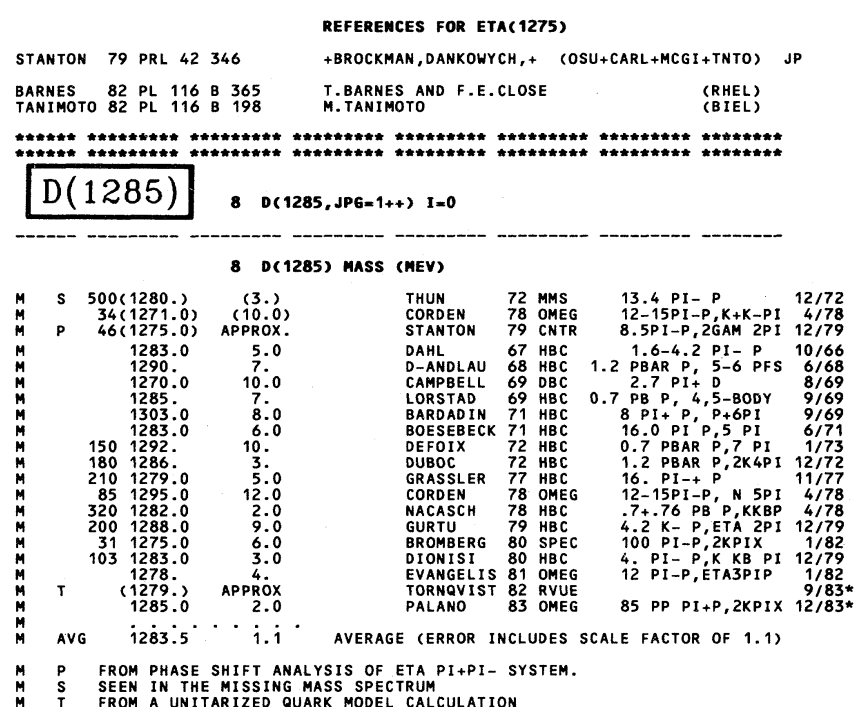

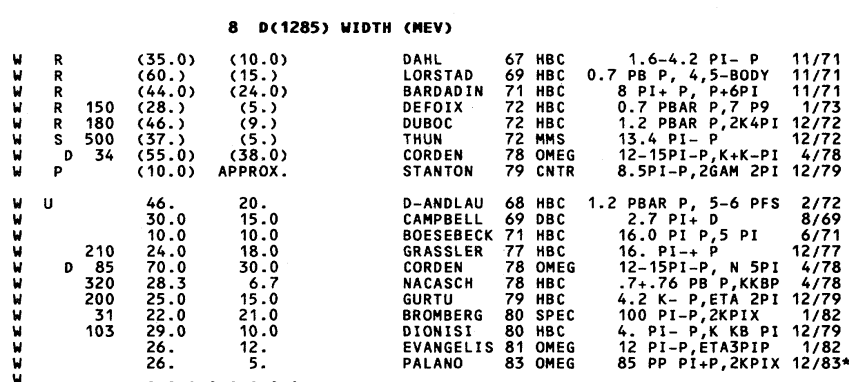

W avg $26.2^{\cdots} \cdots 3 . i^{\prime}$ average

H D HIDTH ERRORS ENLARGED BY US TO 4*HIDTH/SORT (N), SEE K*(892) NOTE

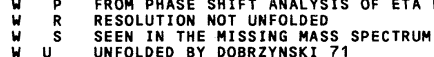

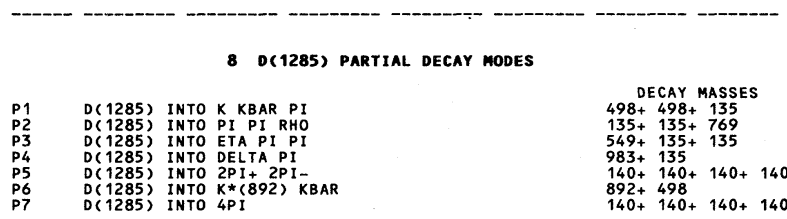

FITTED PARTIAL DECAY MODE BRANCHING FRACTIONS

The matrix below is derived from the error matrix for the fitted partial decay mode sranching fractions, $P_{i}$, as follows: The diagonal elements are $P_{i} \pm \delta P_{i}$, where

$\delta P_{i}=\sqrt{\left.i \delta P_{i} \delta P_{i}\right\rangle}$, while the off-diagonal elements are the normalized correlation coefficients $\left\langle{ }^{6} \mathrm{P}_{\mathrm{i}} \delta \mathrm{P}_{\mathrm{j}}\right\rangle /\left(\delta \mathrm{P}_{\mathrm{i}} \cdot \delta \mathrm{P}_{\mathrm{j}}\right)$. For the definitions of the individual $\mathrm{P}_{\mathrm{i}}$, see the listings

above; only those $P_{i}$ appearing in the matrix are as sumed in the fit to be nonzero and

are thus constrained to add to 1 .

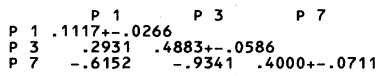

\section{D(1285) branchimg ratios}

THE D(1285) BRANCHING RATIOS FIT IS MADE WITH THE ASSUMPTION THAT
THE D(1285) INTO 4PI DECAY IS ALWAYS VIA DECAY INTO I I P PI PI PAIRS (E.G., RHO PI PI).

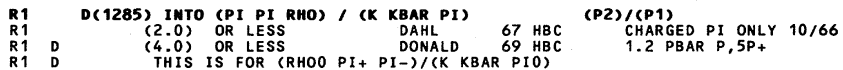




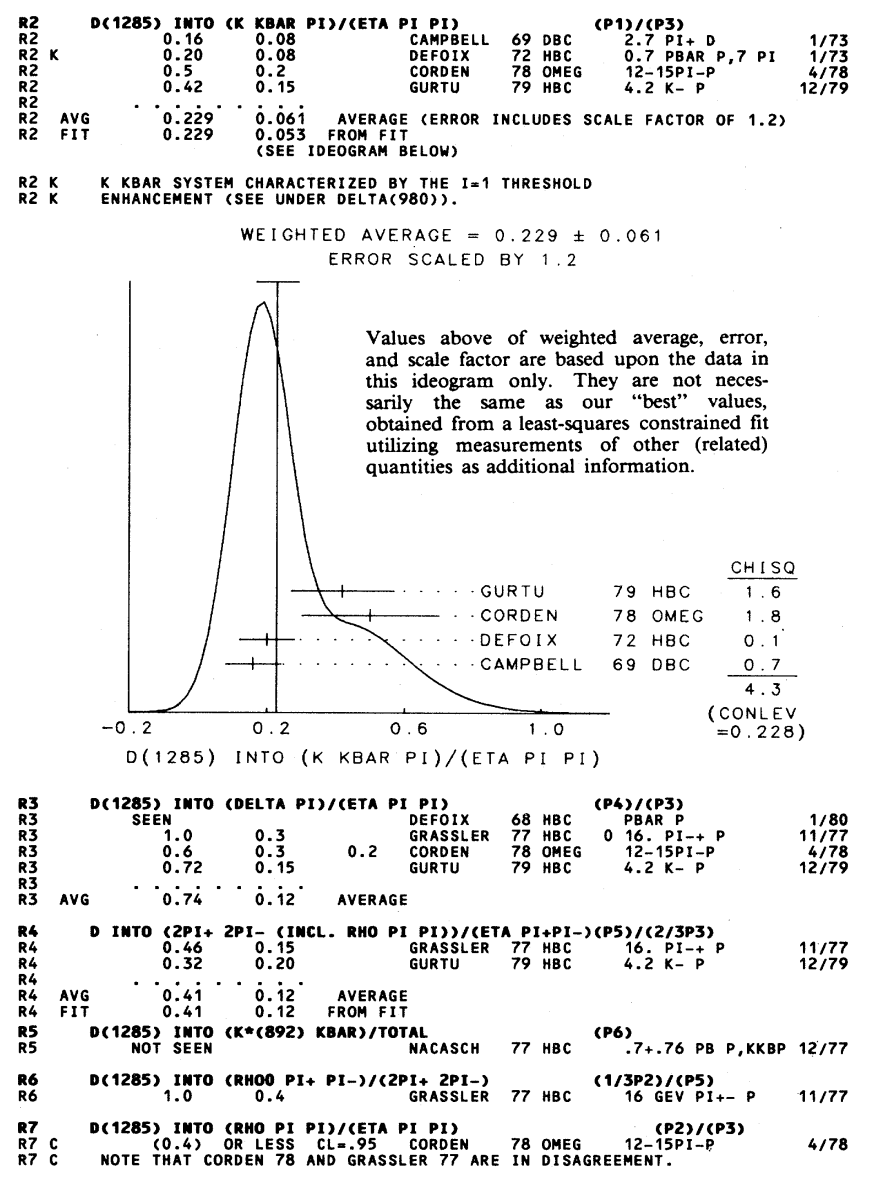

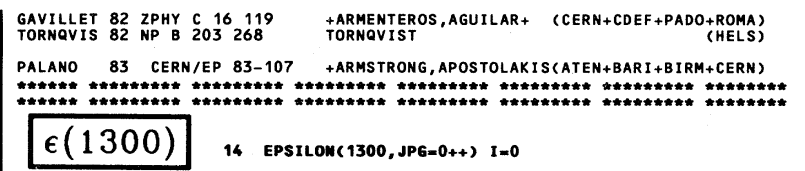

NOTE ON S-WAVE $\pi \pi$ AND KK $\bar{K}$ INTERACTIONS

In this note we discuss information on the nonstrange $\mathrm{I}_{\mathrm{J}} \mathrm{PC}=0^{+} \mathrm{O}^{++}$partial wave ( $\mathrm{S}$ wave) coupled to the $\pi \pi$ and $\mathrm{K} \overline{\mathrm{K}}$ systems.

The threshold behavior of elastic $\pi^{+} \pi^{-}$scattering involves $S, P$, and $D$ waves which can be sufficiently well described by the scattering lengths $a_{0}^{0}, a_{0}^{2}, a_{1}^{1}, a_{2}^{0}$, and $a_{2}^{2}$. The determination of these parameters has in the past met with great difficulties (see our 1978 edition). A consistent set of parameters has been proposed (see ALEKSEEVA 82 and references therein).

$\mathrm{Up}$ to the $\rho$ meson mass region, the phase shift $\delta_{0}^{0}$ is (qualitatively) uniquely determined: it rises monotonically and reaches $60^{\circ}$ to $70^{\circ}$ near $700 \mathrm{MeV}$ (SONDEREGGER 69, BATON 70, BAILLON 72, CARROL 72, FRENKIEL 72, GAIDOS 72, PROTOPOPESCU 73, HYAMS 73, OCHS 73, ENGLER 74, ESTABROOKS 74,75, GRAYER 74).

In the early phase-shift analyses two solutions for $\delta_{0}^{0}$ were found (the "up-down ambiguity") in the 700-900$\mathrm{MeV}$ region. The "up" solution corresponds to an $\epsilon$ resonance under the $\rho$ meson with mass and width similar to the $\rho$ meson, the $\epsilon(800)$. The "down" solution is characterized by an approximately energy-independent phase shift of almost $90^{\circ}$, showing no resonant behavior. This ambiguity was considered resolved in favor of the "down" solution by the observation of a very rapid decrease in the modulus of the S-wave amplitude between $900 \mathrm{MeV}$ and the $\mathrm{K} \overline{\mathrm{K}}$ threshold, followed by a sharp drop in the elasticity. $\delta_{0}^{0}$ is $\sim 90^{\circ}$ at about 900 $\mathrm{MeV}$ and reaches $\sim 180^{\circ}$ around $990 \mathrm{MeV}$ (FLATTE 72, GAIDOS 72, HYAMS 73, BINNIE 73, ENGLER 74). However, the region is complicated by the simultaneous presence of the $S(975)$ resonance and the opening of the $\mathbf{K} \overline{\mathrm{K}}$ channel, permitting almost discontinuous jumps from one solution to another.

Without polarization information, the reaction $\pi \mathrm{N}$ $\rightarrow \pi \pi \mathrm{N}$ cannot be analyzed unambiguously due to the fact that there are more helicity amplitudes than observables (see, e.g., DONOHUE 75). Thus one is obliged to make some supplementary assumptions.

An amplitude analysis (ESTABROOKS 74) of the 


\section{Mesons}

$\epsilon(1300)$

largest $\pi^{-} \mathrm{p}$ (unpolarized) $\rightarrow \pi^{+} \pi^{-} \mathrm{n}$ experiment (HYAMS 73, GRAYER 74) still finds both the "up" and "down" solutions. This analysis assumes both spin coherence (the unnatural-parity-exchange, s-channel helicity amplitudes are nucleon spin-flip, i.e., no A(1270)like exchange) and phase coherence (the S-wave amplitude and the unnatural-parity-exchange, meson helicityzero P-wave amplitude have the same phase). These assumptions may tend to bias the results (MORGAN 74, DONOHUE 75,79).

The advent of $\pi^{-} \mathrm{p}$ (polarized) $\rightarrow \pi^{+} \pi^{-} \mathrm{n}$ data (BECKER 79) has made both the spin coherence and phase coherence assumptions unnecessary. Analyzing their data in a model-independent way, BECKER2 79 also find both the "up" and the "down" solutions.

The reaction $\pi^{+} \mathrm{p} \rightarrow \pi^{+} \pi^{-} \Delta^{++}$has been analyzed in the region 660 to $860 \mathrm{MeV}$ (OWENS 76, DONOHUE 79) and in the region 600 to $920 \mathrm{MeV}$ (GELFAND 78), using all the information carried by the $\Delta^{++}$decay. The conclusion from both analyses is that the $\epsilon(800)$ of the "up" solution cannot be ruled out.

The only way to rule out a narrow $\epsilon$ under the $\rho$ meson (the "up" solution) is to study the $\pi^{0} \pi^{0}$ system. Many experiments agree that no such narrow resonance is present and that the "down" solution describes the data well (DEINET 69, SONDEREGGER 69, SHIBATA 70, BENSINGER 71, APEL 72,79, BRAUN 73, RIESTER 75, GRIVAZ 76, DAVID 77, BORREANI 79,81). The phase shifts of BISWAS 81 lie much lower than all others in the 300-700-MeV region, thus requiring a sudden phase motion in the $\rho$ region to match the "down" solution above the $\rho$.

A recent amplitude analysis of the reaction $\pi^{+} \pi^{-} \rightarrow$ $\pi^{0} \pi^{0}$ (CASON 83) has selected the so-called "downdown" solution as the only one making the $\pi^{+} \pi^{-} \rightarrow$ $\pi^{0} \pi^{0}$ and $\pi^{+} \pi^{-} \rightarrow \pi^{+} \pi^{-}$data consistent (as already noted by SKUJA 73). This solution "leads to a rather rapid phase variation at approximately $750 \mathrm{MeV}$ and a phase shift which goes through $90^{\circ}$ at about $800 \mathrm{MeV}$ " (CASON 83) (see Fig. 1).

Additional information on the $\pi \pi$ scalar states is emerging from the study of meson pair production in $\gamma \gamma$ scattering. A recent analysis of available data (MENESSIER 83) argues in favor of a very broad lowmass scalar in the 500-700-MeV region.

The region of elastic $\pi \pi$ scattering is known to extend to about $990 \mathrm{MeV}$, near the $\mathrm{K} \overline{\mathrm{K}}$ threshold (BATON 70, CARROLL 72, PROTOPOPESCU 73,
Data Card Listings

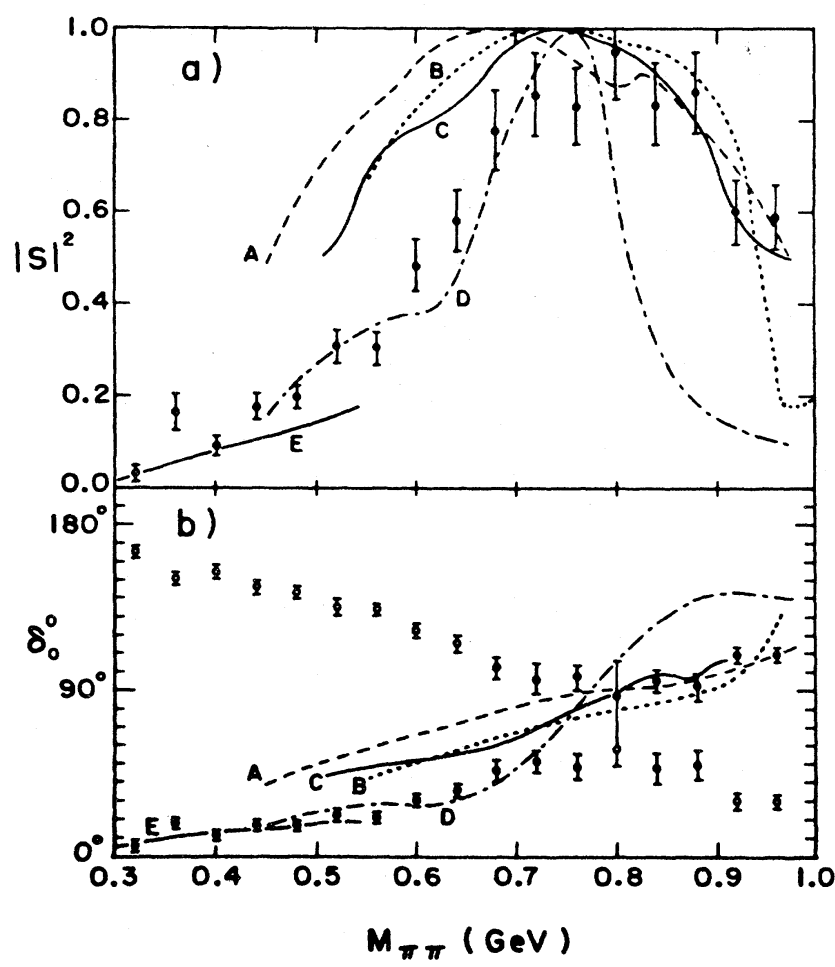

Fig. 1. (a) Extrapolated S-wave intensity as a function of the $\pi^{0} \pi^{0}$ mass. (b) $I=0, S$-wave phase shifts. From CASON 83.

HYAMS 73, OCHS 73). Beyond $1 \mathrm{GeV}$ we therefore have to consider the two channels $\pi \pi$ and $\mathrm{K} \overline{\mathrm{K}}$, and beyond $1100 \mathrm{MeV}$ the $\eta \eta$ channel also opens up. In addition, the solutions have inherent ambiguities related to the Barrelet zeros of the amplitudes. Thus HYAMS 75 find four solutions in the region 1.0 to $1.8 \mathrm{GeV}$, ESTABROOKS 74 find eight solutions, and CORDEN 79 , extending the $\pi \pi$ analysis to $2.08 \mathrm{GeV}$, find another eight solutions.

In the past many of these solutions have been ruled out by imposing continuity in various ways, as well as analyticity and unitarity (FROGGATT 75,77, COMMON 76, MARTIN 78).

One notes that a model-independent partial-wave analysis (BECKER2 79 on polarized target) agrees qualitatively with solutions $\beta$ and $\beta^{\prime}$ (of MARTIN 78).

The $\beta$ and $\beta^{\prime}$ amplitudes describe the experimental moments in each bin without any explicit smoothing; they are analytic in $\mathbf{s}$ and approximately analytic in $\cos \theta$. They take into account all waves up to $\ell=4$. The $\beta$ solution has a highly elastic $S$ wave, whereas the 
For notation, see key at front of Listings.

S wave of solution $\beta^{\prime}$ is somewhat inelastic (MARTIN 78). The unique solution of FROGGATT 77, which has explicit smoothness built in and which takes account only of $\ell \leqslant 3$ waves, is rather similar to $\beta$. However, it has problems with unitarity, apparently because of the neglected $G$ wave (MARTIN 78).

The $\mathrm{S}$ wave is clearly resonant in the data of BECKER2 79. In the 1150-1400-MeV region both the S-P and S-D phase differences show the presence of a broad resonance, and the intensity of the $S$ wave confirms this by exhibiting a peak at about $1300 \mathrm{MeV}$ with a width of about $300 \mathrm{MeV}$.

The amplitude analysis of the $\pi^{-} \mathrm{p} \rightarrow \pi^{+} \pi^{-} \mathrm{n}$ experiment of CORDEN 79 has two preferred solutions which are close to $\beta$ and $\beta^{\prime}$, giving some support for an $\epsilon(1300)$. Also the $S$ wave in the $\pi^{0} \pi^{0}$ system tends to confirm the $\epsilon(1300)$ by staying near its unitarity limit around $1200 \mathrm{MeV}$ (APEL 79).

The new results on $\pi^{+} \pi^{-} \rightarrow \pi^{0} \pi^{0}$ (CASON 83) establish that the only solutions consistent with the data are the $\beta$ and $\beta^{\prime}$, in agreement with BECKER2 79. That implies a significant $\pi \pi$ coupling of the $\rho(1600)$.

Independent evidence for the $\epsilon(1300)$ comes from studies of the $\mathrm{K} \overline{\mathrm{K}}$ systems. In the reaction $\pi^{-} \mathrm{p} \rightarrow$ $K_{S}^{0} K_{S}^{0} n$, the $S$ wave exhibits a large intensity in the 1300-MeV region (WETZEL 76, LOVERRE 80, ETKIN 82) with evidence for a bump. Moreover, the $Y_{0}^{2}$ moment shows a large negative excursion indicating $S-D$ interference (CASON 76, WETZEL 76, POLYCHRONAKOS 79, GOTTESMAN 80, LOVERRE 80, ETKIN 82). The main problem was the isospin of the bump: if OPE were the only mechanism, $\mathrm{I}=0$ would be assured. The new high-statistics data (ETKIN 82), in the restricted $t^{\prime}$ region smaller than 0.1 $\mathrm{GeV}^{2}$, strongly argues in favor of OPE dominance and assigns the observed effects to the $\mathrm{I}^{\mathrm{G}}=0^{+}$state.

An enhancement in the intensity of the $S$ wave around $1300 \mathrm{MeV}$ has also been observed in the $\mathrm{K}^{+} \mathrm{K}^{-}$ system produced in $\pi^{-} \mathrm{p}, \pi^{+} \mathrm{n}$, and $\pi^{-} \mathrm{p}$ (polarized) scattering. Again contributions from the $I=0$ and $I=1$ states may be present (PAWLICKI 77, MARTIN 79, COHEN 80, COSTA 80, GORLICH 80, WICKLUND 80).

To get from the mass-independent S-wave amplitudes and S-D relative phase to resonance parameters and $\mathrm{q} \overline{\mathrm{q}}$ composition several assumptions have to be made. Various analyses (PAWLICKI 80, ETKIN2 83) reach contradictory results concerning the number and properties of the resonance states coupled to the $\mathrm{I}^{\mathrm{G}}=$ $\mathrm{O}^{+}$system.

Recently, two fully unitarized coupled-channel analyses have been performed (ACHASOV 79,80,81, TORNQVIST 82) giving fairly consistent results.

The picture emerging (TORNQVIST 82) is that of a dominantly $\mathrm{q} \overline{\mathrm{q}}$ system with large $\mathrm{q} \overline{\mathrm{q}} \mathrm{q} \overline{\mathrm{q}}$ components in the form of virtual two-meson bound states. At the SU(3) level, two isoscalar resonances are needed, a narrow $S(975)$ superimposed on a broad $\epsilon$. The position and width of the $S(975)$ are well determined by the interference with the $\epsilon$, visible as a dip in the $\pi \pi \rightarrow \pi \pi$ cross section.

The interpretation of the $S(975)$ as a $q \bar{q} q \bar{q}$ system is another possiblity.

The mass and width of the $\epsilon$, however, are difficult to define in any simple way, its Breit-Wigner shape being completely distorted by hadronic massrenormalization effects (cusps) from the $\pi \pi, \mathrm{K} \overline{\mathrm{K}}$, and $\eta \eta$ channels (MORGAN 74, ACHASOV 79,80,81, IRVING 81, TORNQVIST 82).

For further discussion of the scalar nonet, see the mini-reviews under $\delta(980)$ and $\kappa(1350)$.

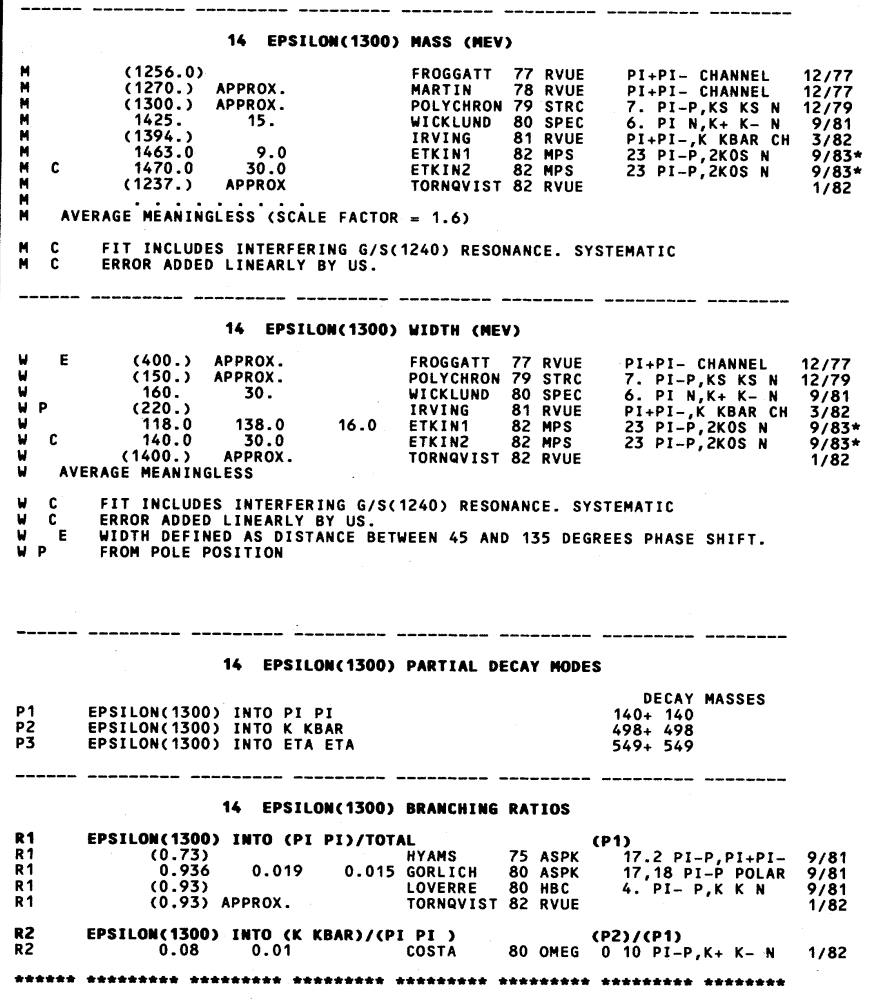




\section{Mesons}

$\epsilon(1300), \pi(1300)$

\section{Data Card Listings}

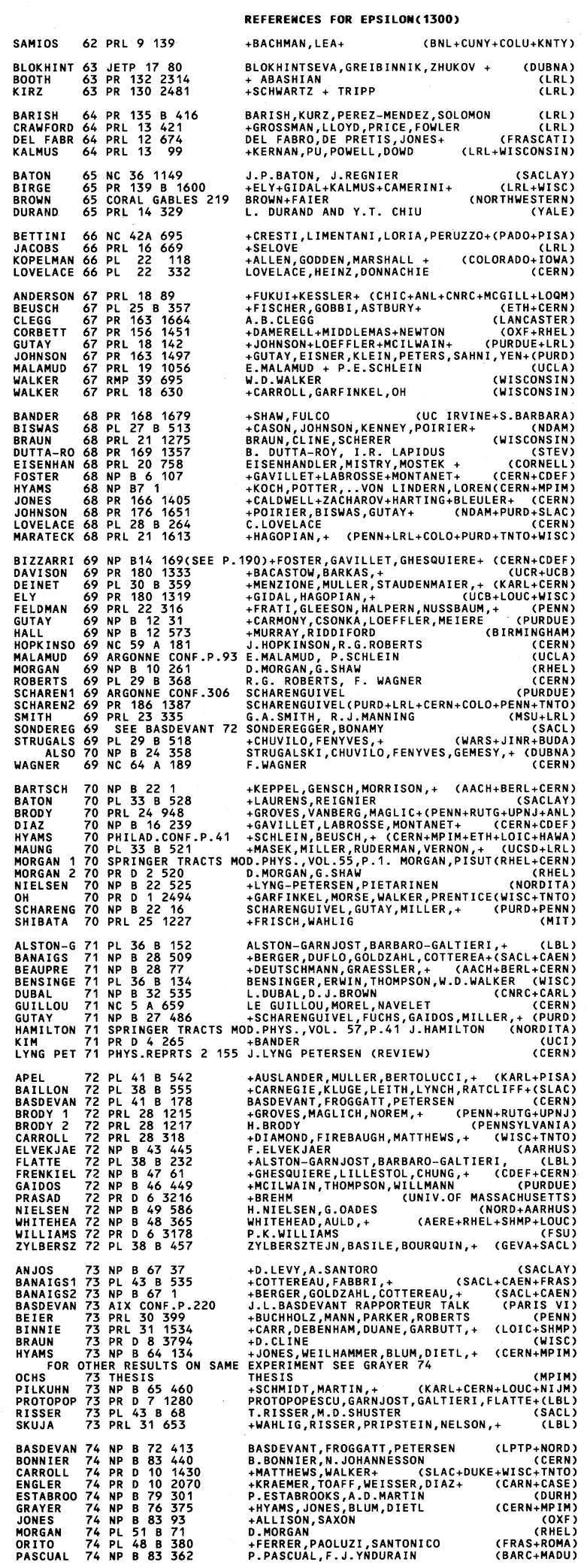

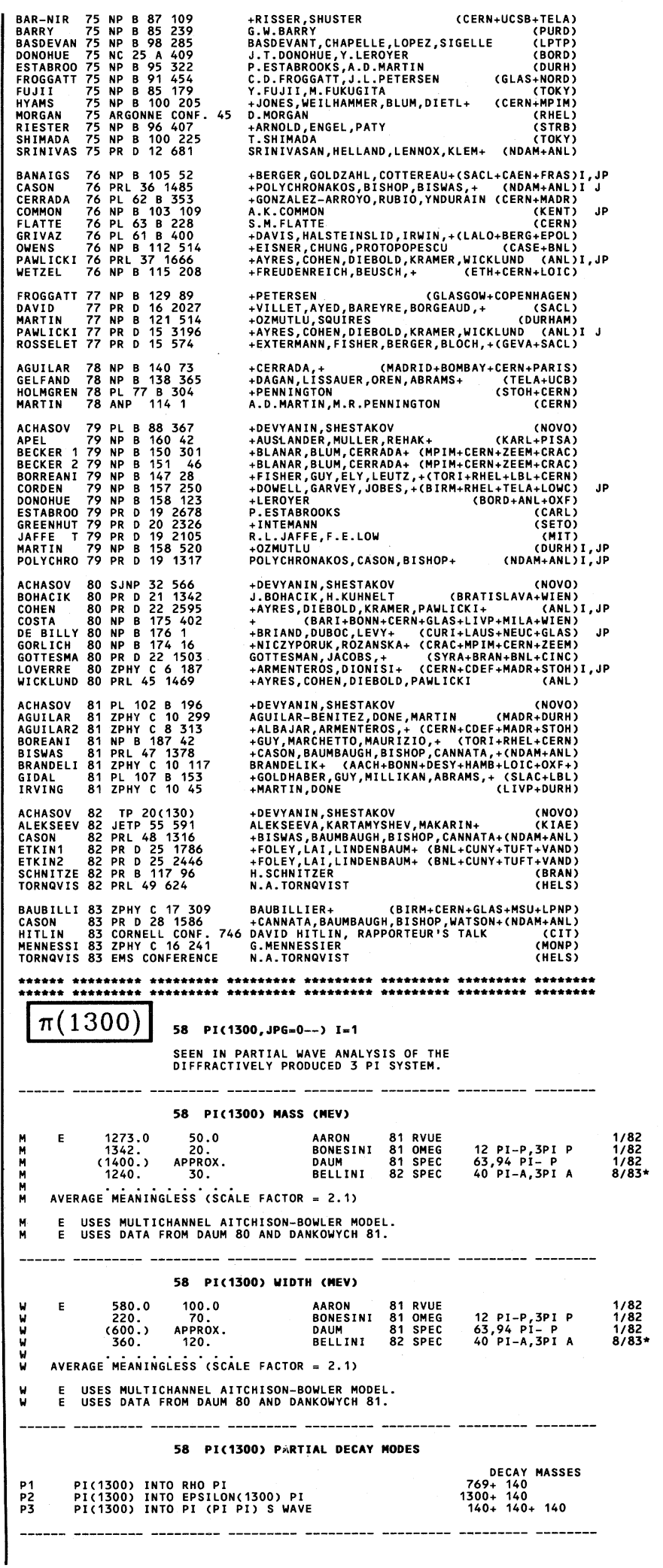


58 PI(1300) BRANCHING RATIOS

R1 PI(1300) IITTO (PI (PI PI) S WAVE/(RHO PI)
R1 E
(2.12) R1 E
R1 E USES MULTICHANNEL AITCHISON-BOWLER MODEL.
R1 E USES DATA FROM DAUM 80 AND DANKOWYCH 81.
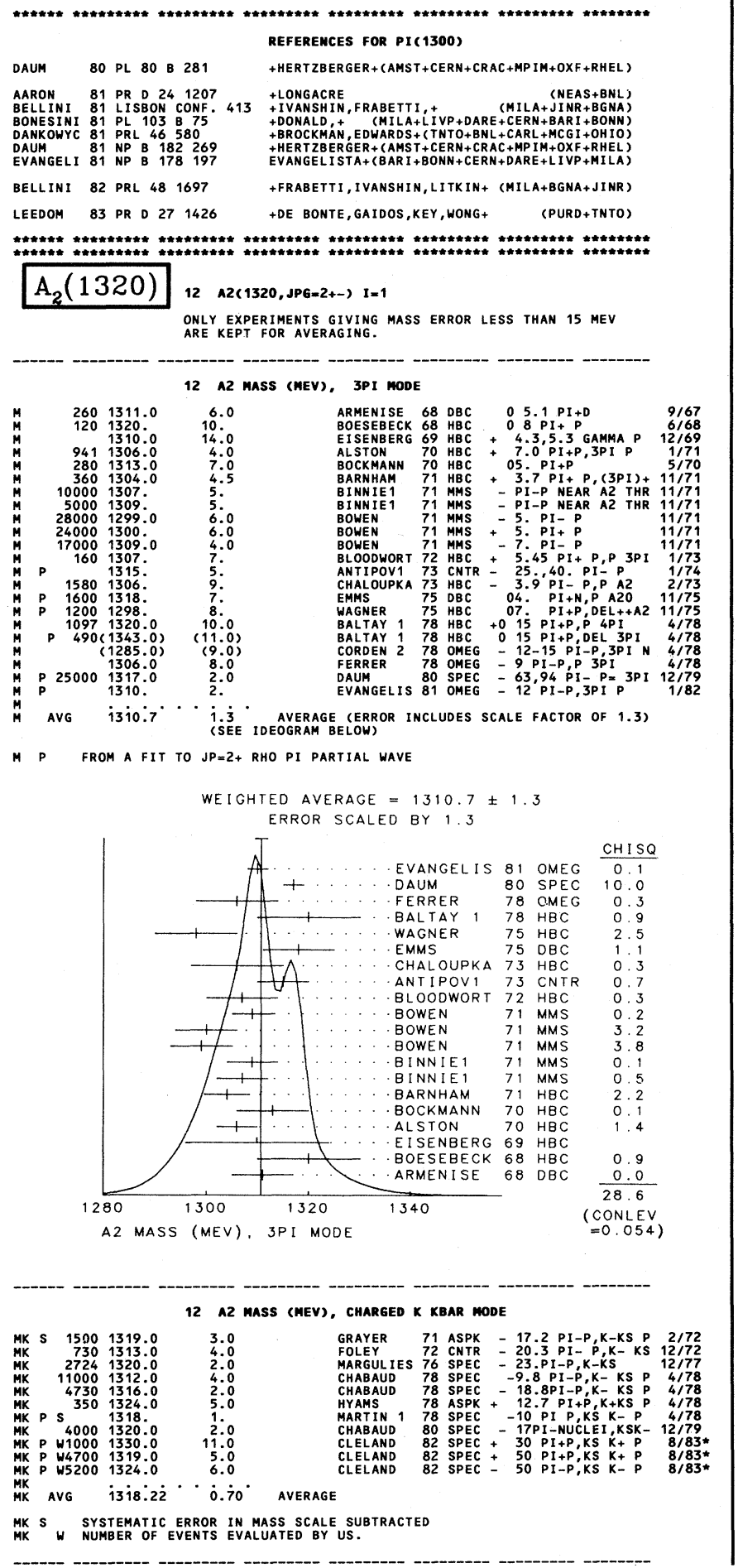

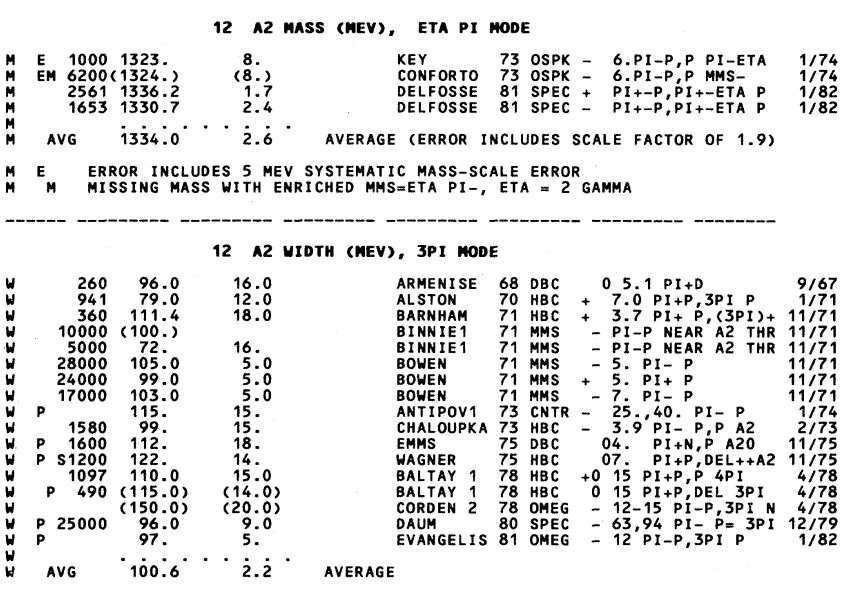

W S WIDTH ERRORS ENLARGED BY US TO 4 *WIDTH/SQRT(N), SEE K*(892) NOTE

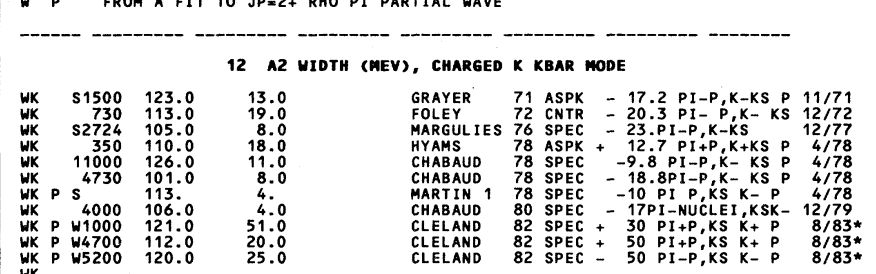
WK AVG $109.8^{\circ} \cdot \dot{2} .4^{\circ}$ aVerage

WK P P FROM A FIT TO JP=2+ PARTIAL HAVE.
WK S WIDTH ERRORS ENLARED BY US TO $4 *$ WIDTH/SORT(N), SEE K* TYPED NOTE
WK
WUMBER OF EVENTS EVALUATED BY US.

\begin{tabular}{|c|c|c|c|c|c|c|c|}
\hline \multirow{3}{*}{ W } & \multicolumn{6}{|c|}{12 A2 WIDTH (MEV), ETA PI MODE } & \multirow{3}{*}{$\begin{array}{l}1 / 74 \\
1 / 74 \\
1 / 82 \\
1 / 82\end{array}$} \\
\hline & $\begin{array}{r}1000 \\
\text { M } 6200 \\
2561 \\
1653\end{array}$ & $\begin{array}{r}108 . \\
(104 .) \\
112.2 \\
116.6\end{array}$ & $\begin{array}{l}9 . \\
9.7 \\
5.7 \\
7.7\end{array}$ & $\begin{array}{l}\text { KEY } \\
\text { CONFORTO } \\
\text { DELFOSSE } \\
\text { DELFOSSEE }\end{array}$ & $\begin{array}{l}73 \text { OSPK }- \\
73 \text { OSPK }= \\
81 \text { SPEC + } \\
81 \text { SPEC }-\end{array}$ & $\begin{array}{l}\text { 6.PI-P,P PI-ETA } \\
6 . P I-P, P \text { MMS- } \\
\text { PI+-P,PI+-ETA P } \\
\text { PI+-P,PI+-ETA P }\end{array}$ & \\
\hline & AVG & $112.6^{\circ}$ & $4 . i$ & AVERAGE & & & \\
\hline
\end{tabular}

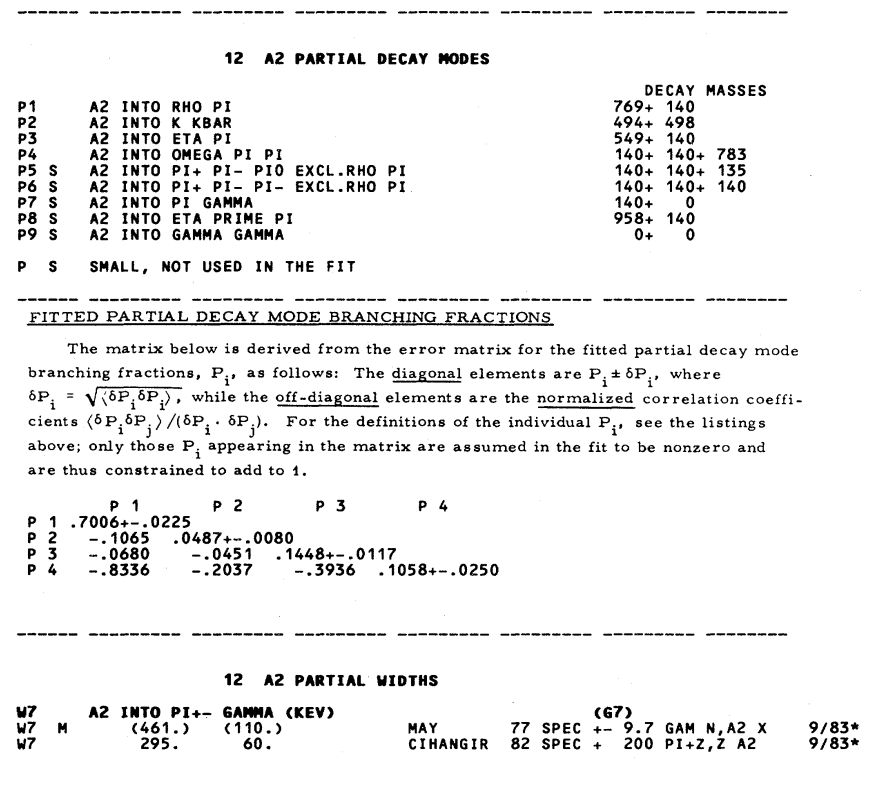




\section{Mesons}

Data Card Listings

$\mathrm{A}_{2}(1320)$

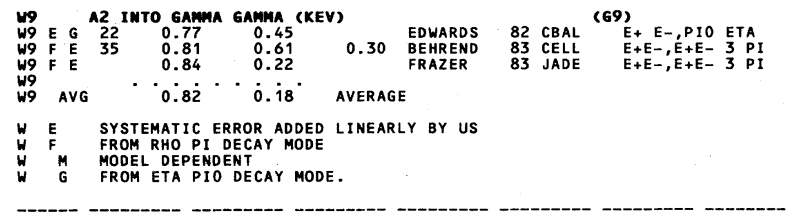

\section{A2 BRAMCHIMG RATIOS}

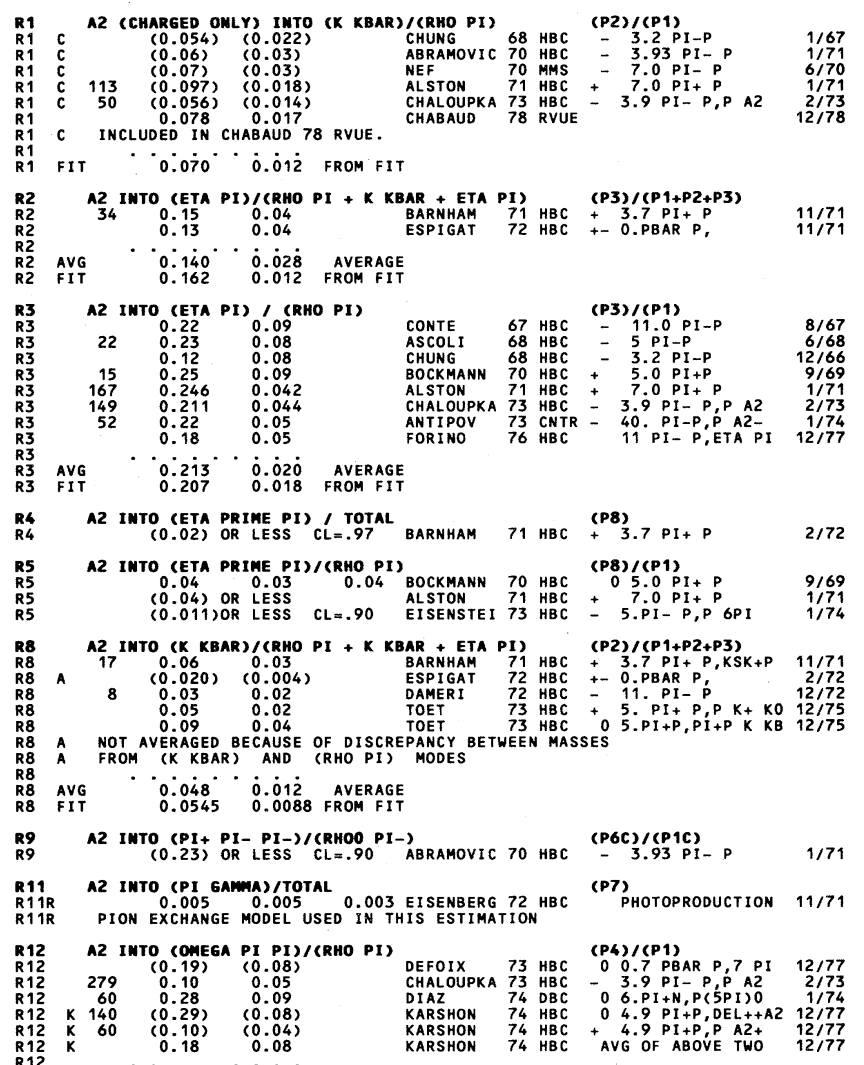

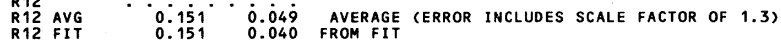

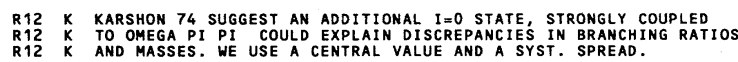

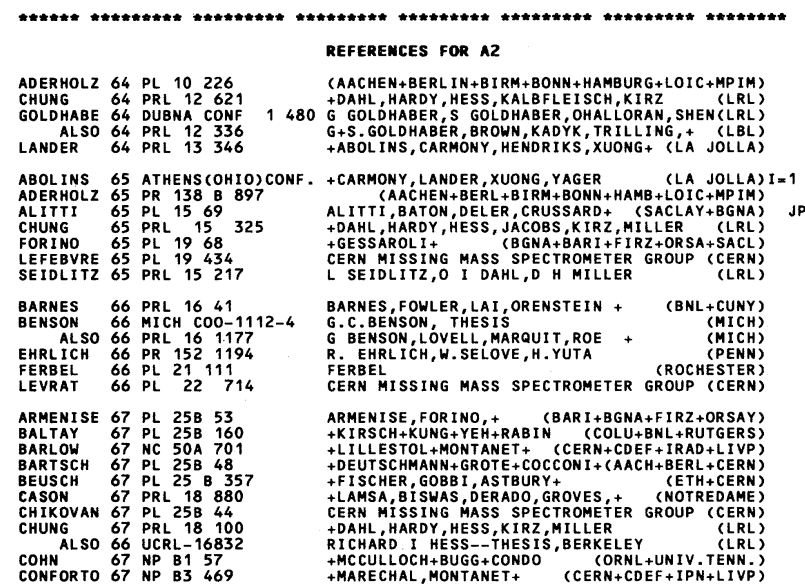

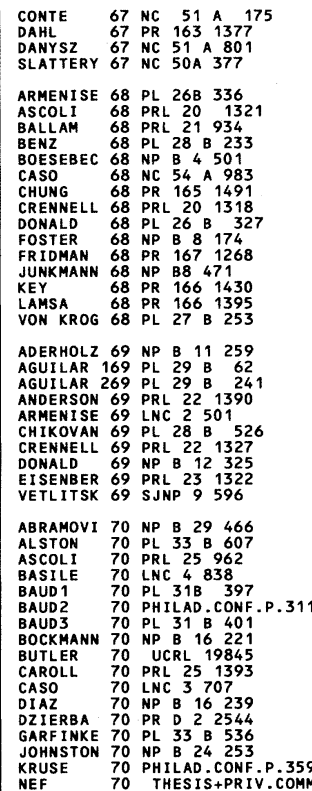

+TOMASINI, CORDS+(GENOVA+HAMB+MILANO+SACLAY)

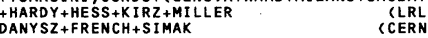
ARMENILE +FORMAN+FERBEL (YALE+ROCH)

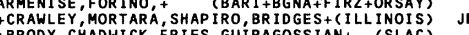

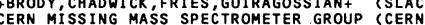

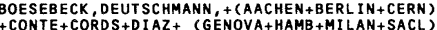

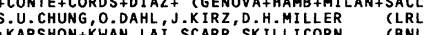
+FRODESEN+BETININIt (LIIERPOOL +OSLOPPADUA)

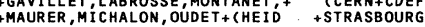

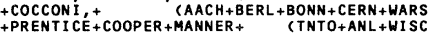
TASON+BISWAS+DERADO+GROVEST
+MIYASH ITA, KOPELMAN, MARSHALL LIBBY (NOTRDAME) +BARTSCH, ${ }^{+}$CAACH+BERL+CERN+JAGL HARS

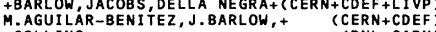
(BNL+CARN)
+ +CHLLLINS,
+GHDIN, FORINO, CARTACCI+ (BARI+BGNA+FIRZ) CERN MISS NNG MASS SPECTROMETER GROUP (CERN) JPP
TKARSHON, KWAN WU LAI,

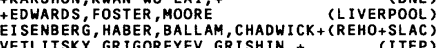
ABRAMOVICH, BLUMENFELD, BRUYANT, + (CERN) JP

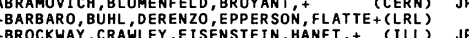

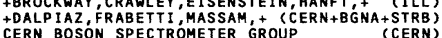
CERN BOSON SPECTROMETER GROUP
CERN BOSON SPECTROMTTER GROUP (CERN)
(CERN)

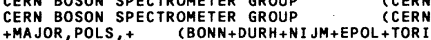

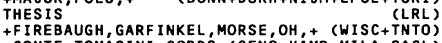

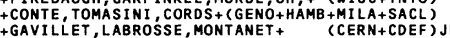
SHEPHARD, BISHAS, CASON, JOHNSON, KENNEY (NDAM)

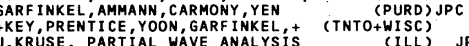

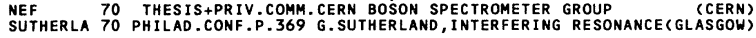

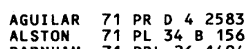

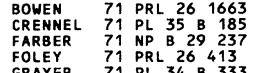

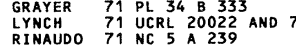
$\begin{array}{llll}\text { ANKEEBRA } 72 \text { PRL } 29 & 1688 \\ \text { BERENYI } & 72 & \text { NP B } 37 & 621\end{array}$

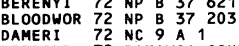

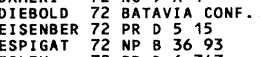

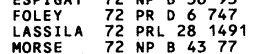

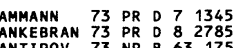

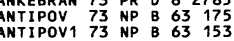
ANTIPOV2 73 NP B 63 14 14

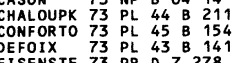

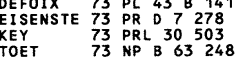
$\begin{array}{llll}\text { DIAZ } & 74 & \text { PRL } 32 & 260 \\ \text { KARSHON } & 74 & \text { PRL } 32 & 852\end{array}$

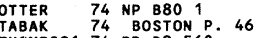

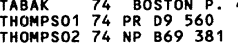

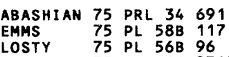

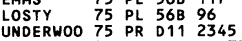

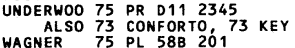

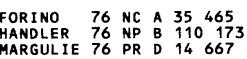
$\begin{array}{llllll}\text { CERRADA } & 77 & \text { NP } & 8 & 126 & 241 \\ \text { MAY } & 77 & \text { PR } \\ \text { MAYLICKII } & 77 & \text { PR } & 0 & 16 & 1983 \\ \text { PALI } & 3196\end{array}$

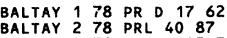
CHABAUD $78 \mathrm{NP} B 145349$

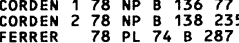

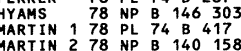

$\begin{array}{llllll}\text { CHABAUD } & 80 & \text { NP } & \text { B } & 175 & 189 \\ \text { DAUM } & 80 & \text { PL } & 89 & \text { B } & 276\end{array}$

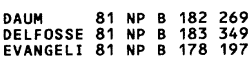

AGUILAR-BENITEZ, EISNER, KINSON (BNL)
+BARBARO BUHL DERENZO,EPPERSON, FLATTE+(LRL) + ABRAMS, BUTLER, COYNE, GOLDHABER, HALL, + (LBL)
+ SOMBKOHSKY, KONOHALOV, KRUTSCHININ, + (ITEP) +CAMILLERI, DUANE, FARUQ1, BURTON, + (LOIC+SHMP)

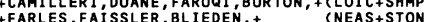

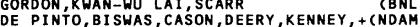

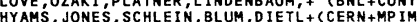

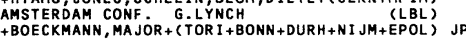
ANKENBRANDT, BRABSON, CRITTENDEN, HEINZ, + (IND) PPRENTICE, STEENBERG, YOON, WALKER (TNTO+WISC)
BLOOOHORTH, JACKSON, PRENTICE, YOON TNTOO PBORZATTA, GOUSSO
R.DIEBOLO RAPPORTEUR TALK GENO+MILA+SAAL) EISENBERG, BALLAM, DAGAN, (REHO+SLAC+TELA)
GHESOUIERE, LILLESTOL, MONTANET (CERN+COEF)

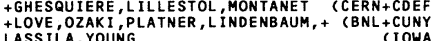

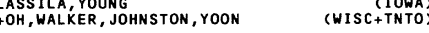
CARMONY, GARF INKEL, GUTAY, MILLER+(PURD+IUPU)

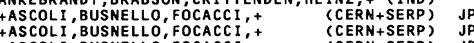

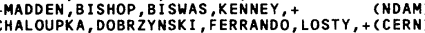

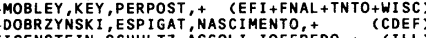
EISENSTEEN, SCHULTZ, ASCOLI, IOFFREDO, (ILL) +THUAN, MA MOR, RINAUDO, + (NNIM+BONN+DURH+TORI +DIBIANCA, FICKINGER, ANDERSON, + (CASE+CARN)
+MIKENBERG, PI TLUCK, EISENBERG, RONAT+ (REHO) TROMAT ROSENEELD LASINSKIT (LBL+SLAC) JP

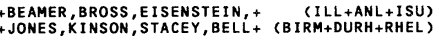

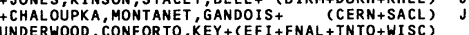

tTABAK, CHEW (LBL) JP

+GESSAROLI, BGNA+FIRZ+GENO+MILA+OXFFPAV + PLANO, BRUCKNER, KOLLER, (RUTG, STEV, SETO)
+KRAMER, FOLEY, LOVE, LINOENBAUM,
(BNL+CUNY) +BLOCKZIJL, HEINEN, + (AMST+CERN+NI JM+OXF)

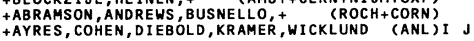
+CAUT IS, COHEN, CSORNA, SMITH, YEH, + (COLU+BING) +CAUTIS, KALELKAR MAMER BLUM, (CEER (COLU) JP

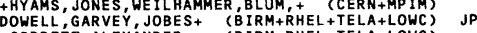

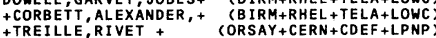
+ TOZMST +OZMUTLO+BALDI, BOHRINER, DORSAZ+ (DURH+GEVA)
+OZMUTLU, BALDI, BOHR INGER, DORSAZ+ (DURH+GEVA)

+HYAMS, PAPADOPOULOU, (CERN (MPIM+AMST)
+HERT ZBERGER+((AMST+CERN+CRAC+MP IM+OXF+RHEL) JP HERT ZBERGER+(AMST+CERN+CRAC+MPIM+OXF+RHEL)
G GUISAN MARTIN, MUHLEMANN, WEELL, +(GEVA+LAUSA)

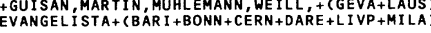

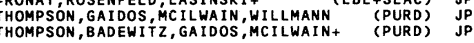




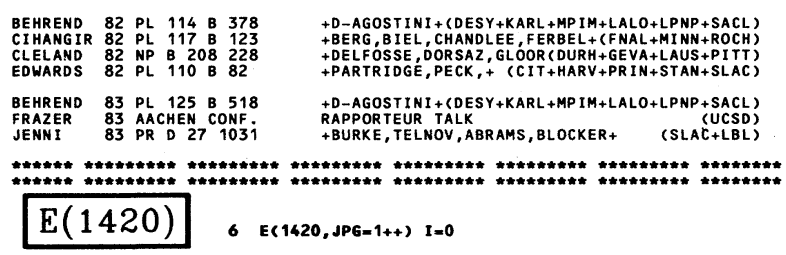

\section{NOTE ON THE E(1420) AND ८(1440)}

We are tentatively splitting the data on the $E(1420) / \iota(1440)$ into two entries according to the proposed $\mathrm{J}^{\mathrm{PC}}$ assignments.

The $\mathrm{J}^{\mathrm{PC}}=1^{++}$state (DIONISI 80), E(1420), appears to have a dominant decay mode into the $\mathrm{K}^{*}(892) \mathrm{K}$ system.

The state with $\mathrm{J}^{\mathrm{PC}}=\mathrm{O}^{-+}$is named $\iota(1440)$. Under this entry we group the results obtained in the early $\bar{p} p$ annihilation experiment at rest (BAILLON 67) and in the radiative decays of the $J / \psi$ resonance (SCHARRE $80,81$, EDWARDS 82$)$. The $\iota(1440)$ is largely coupled to the $\delta(980) \pi$ decay channel, although the lack of a signal in the $\eta \pi \pi$ system (EDWARDS 83 ) is a source of concern.

Note that the experimental situation in this mass region is still confused and that forthcoming results may drastically change present views on the subject (HITLIN 83).

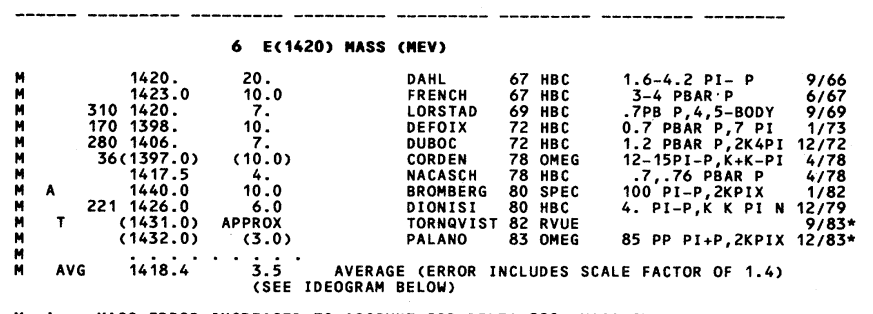

M A MASS ERROR INCREASED TO ACCOUNT FOR DELTA(980) MASS CUT UNCERTAINTIES
M $T$ FROM A UNITARIZED QUARK MODEL CALCULATION

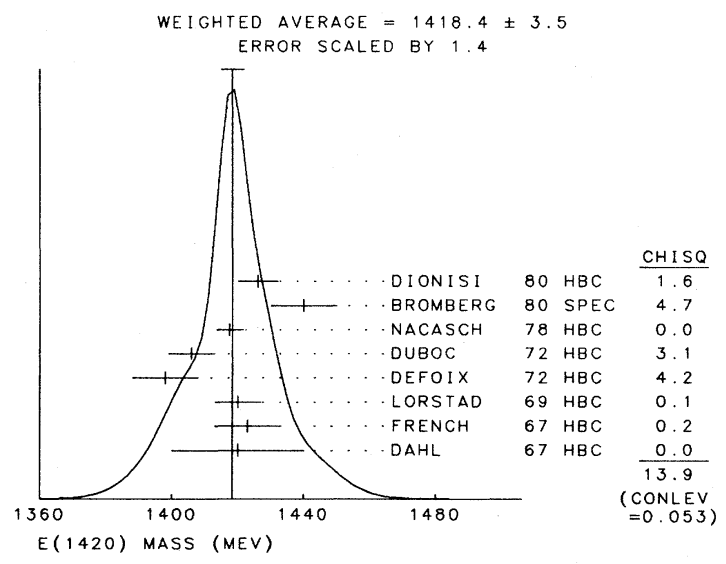

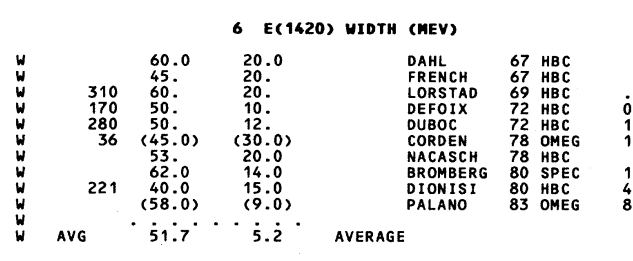

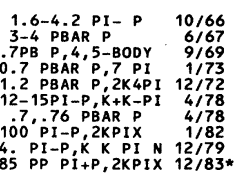

6 E(1420) Partial decay modes

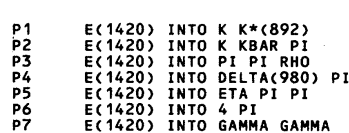

DECAY MASSES

$498+892$
$498+498+140$

$140+140+76$

$549+140+140$
$140+140+140+140$
$0+$

6 E(1420) PARTIAL WIDTHS (KEV)

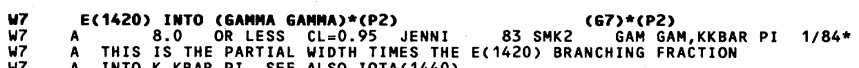

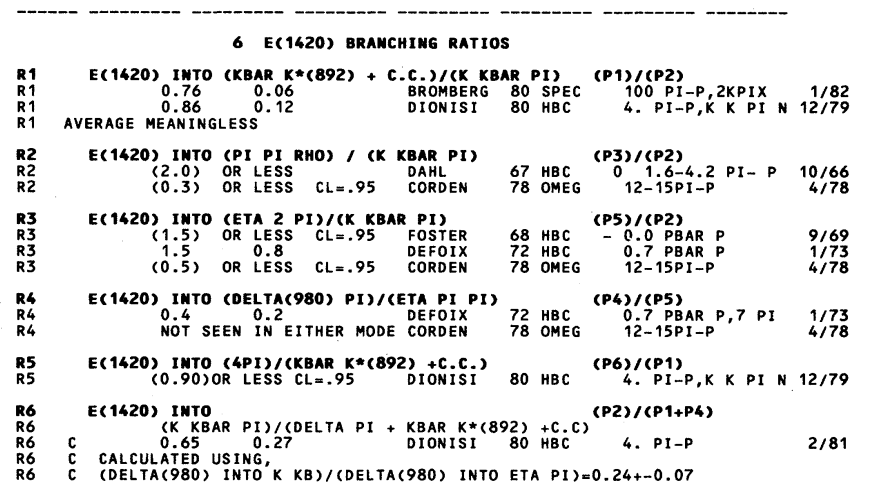

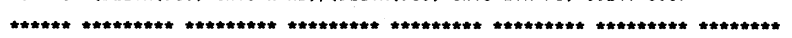

$\begin{array}{lllll}\text { BARASH } & 67 & \text { PR } & 156 & 1399 \\ \text { DAHL } & 67 & \text { PR } & 163 & 1377\end{array}$

$\begin{array}{ccccc}\text { DAHL } & 67 & \text { PR } & 163 & 1377 \\ \text { ALSO } & 65 & \text { PRL } & 14 & 1074 \\ \text { FRENCH } & 67 & \text { NC } & 52 A & 438\end{array}$

REFEREMCES FOR E(1420)

BARASH, KIRSCH, MILLER, TAN (COLUMBIA)
(LARL)I JP

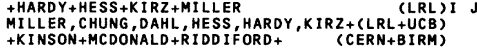

FOSTER 68 NP B 8174

$\begin{array}{llllll}\text { BETTINI } & 69 & \text { NC } & 62 & \text { A } & 1038 \\ \text { LORSTAD } & 69 & \text { NP } & \text { B } & 14 & 63\end{array}$

DEVONS 71 PRL $27 \quad 1614$

+GAVILlet, LABrosse, MONTANet,+ (CERn+CDef)

+CRESTI, LIMENTANI, BERTANZA, BIGI+ $+($ PADO+PISA)IC
B. LORSTAD, D-ANDLAU, ASTIER, $+{ }^{+}$(COEF+CERN) JP

+KOZLOWSK I, HORWITZ, + (COLU+SYRA)

+ CHURCH, LYS, MURPHY, RING, VANDER VELDE (MICH)
+ NASC IMENTO, BIZZARRI,
(CDEF+CERN) +NAS IMENTO, BIZZARR I, +
+ GOLDBERG, MAKOWSKI, DONALD ,
(CDNP+LIVP)

$\begin{array}{llllll}\text { CHAPMAN } & 72 & \text { NP } & \text { B } & 42 & 1 \\ \text { DEFOIX } & 72 & \text { NP } & \text { B } & 44 & 125 \\ \text { DUBOC } & 72 & \text { NP } & \text { B } & 46 & 429\end{array}$

VUILLEMI 75 LNC $14 \quad 165$

VUILLEMIN, + (LAUS+NEUC+LPNP+LIVP+GLAS) JP

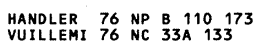

GRASSLER 77 NP B 121189

$\begin{array}{llllll}\text { CORDEN } & 78 & \text { NP } & \text { B } & 144 & 253 \\ \text { IRVING } & 78 & \text { NP } & \text { B } & 139 & 327 \\ \text { NACASCH } & 78 & \text { NP } & \text { B } & 135 & 203\end{array}$

+PLANO, BRUCKER, KOLLER+
+ (RUILLEMIN, +
(LAUS+NEUC+LPNP+ + STEV+SET + GLAS)

+ (AACHEN+BERL IN+BONN+CERN+CRACOW+HEID+WARS)

+CORBETT, ALEXANDER, + (BIRM+RHEL+TELA+LOWC)

A.C.IRVING,H.R.SEPANGI
+DEFOIX, DOBRZYNSKI, + (PARIS+MADRID+CERN)

+BROCKMAN, DANKOWYCH, + (OSU+CARL+MCGI + TNTO) JP

STANTON 79 PRL 42346

BROMBERG 80 PR D 221513

+HAGGERTY, ABRAMS, DZIERBA(CIT+FNAL+ILLC+IND)

+GAVILLET, ARMENTEROS+ (CERN+MADR+CDEF+STOH) I, JP
+ NAVELET
(SACL)

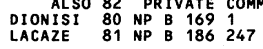

BAILLON 82 PARIS CONFERENCE

EDWARDS 82 PRL 49259

ALSO 83 PRL 50219
TORNAVIS 82 NP B 203268

BAILLON 83 CERN/EP $83-82$

(CERN)
HITLIN 83 CORNEL CARTRIDGE, PECK+ (CIT+HARV+PRIN+STAN+SLAC)

HITLN 83 CORELL CONF. 746 DAVID HITIN, RAPPORTEUR'S TALK
(JENII 83 PR D 271031 +BURKE, TELNOV, ALAM, BOYARSKI+
(SLAC+LBL)

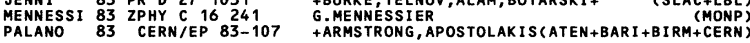

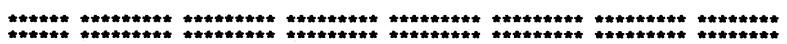


Mesons

Data Card Listings

$\iota(1440), \mathrm{f}^{\prime}(1525)$

$1(1440) \quad 27$ IOTA(1440, JPG $=0-+)$ I=0

CALLED E BY BATLLON 67.0
ANO IOTA BY SCHARRE 80.

S DER E $(1420)$

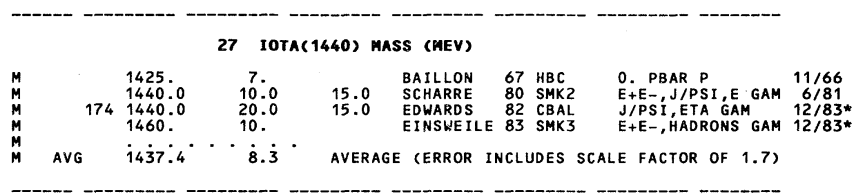

27 IOTAC(1440) WIDTH (MEV) W avg $75.9 \cdots \dot{8.2} \cdot$ average

27 IOTA(1440) DECAY MODES

\begin{tabular}{|c|c|c|}
\hline & 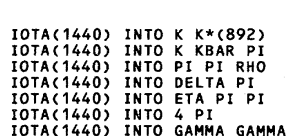 & $\begin{array}{l}\text { DECAY MASSES } \\
498+892+140 \\
498+498+140 \\
140+140+769 \\
983+140 \\
549+140+140 \\
140+140+140+140\end{array}$ \\
\hline
\end{tabular}

27 IOTA(1440) PARTIAL MIDTHS (KEV)

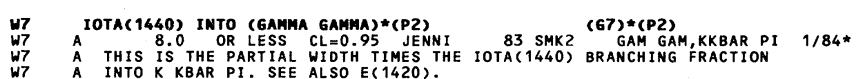

\section{IOta(1440) BRanchimg Ratios}

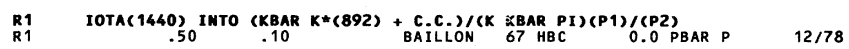

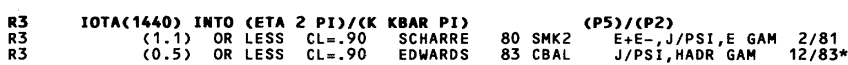

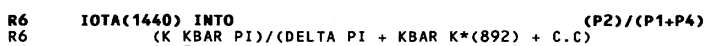

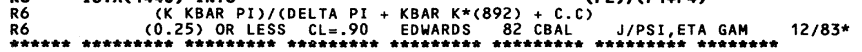

BAILLON 67 NC 50A 393 REFERENCES FOR IOTA (1440)

SCHARRE 80 PL 97 B 329 +EDWARDS+D-ANDLAU+ASTIER+ (CERN+CDEF+IRAD)

CHANOWIT 81 PRL 46981 +TR ILLING, ABRAMS, ALAM, BLOCKER+ (SLAC+LBL) CHANOWITZ

( $(\mathrm{BBL})$

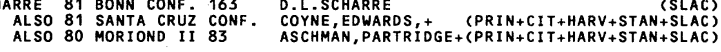

ALTHOFF 82 ZPHY C 16 13
BAILLON 82 PARIS CONFERENCE P.BORNER, BURKHARDT+ (TASSO COLLABORATION)
(CERN)

BARNES 82 PL $116 \mathrm{~B} 365$

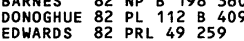

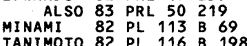

T.BARNES AND F.E.CLOSE

J.F.DONOGHUE AND H.GOMM

(ThEL+OXX)

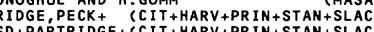

S.MINAMI
M.TANIMOTO

BAILLON 83 CERN/EP 83-82

P.BAILLON

(CIT+HARV+PRIN+STAN+SLAC)

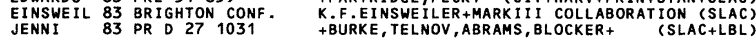

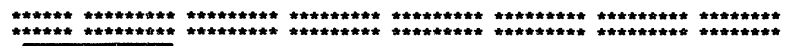

$f^{\prime}(1525)$

13 F PRIME(1525, JPG $=2++$ ) $\quad I=0$

13 F PRIME MASS (MEV)

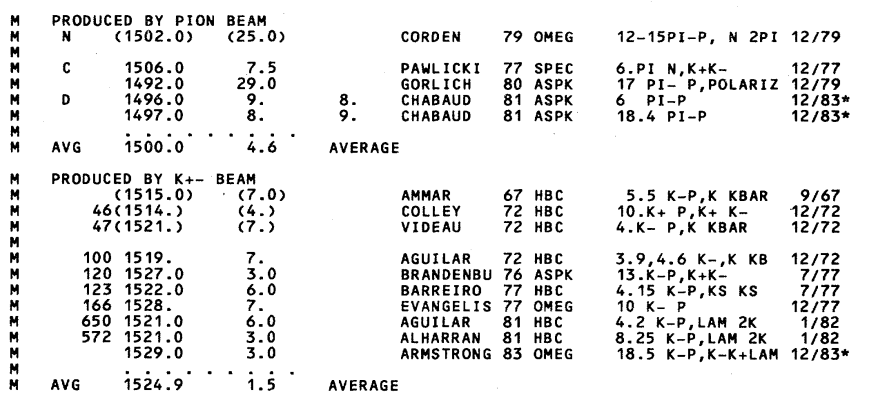

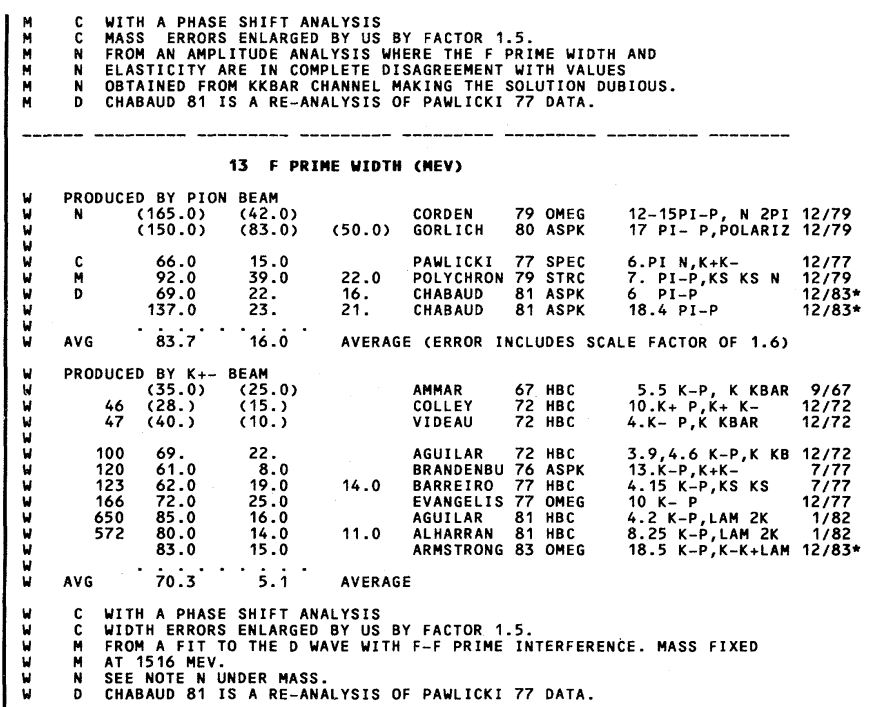

13 F PRIME PARTial decaY modes

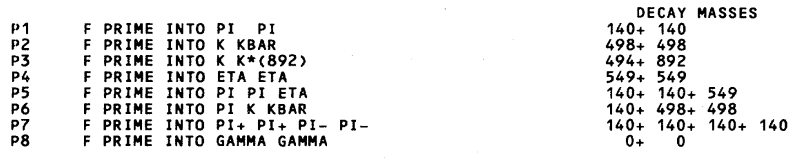

13 F PRIME PARTIAL WIDTHS 12/78

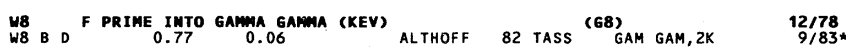

W8 B USING BRANCHING RATIO F PRIME INTO K KBAR $=1 . \quad 12 / 78$

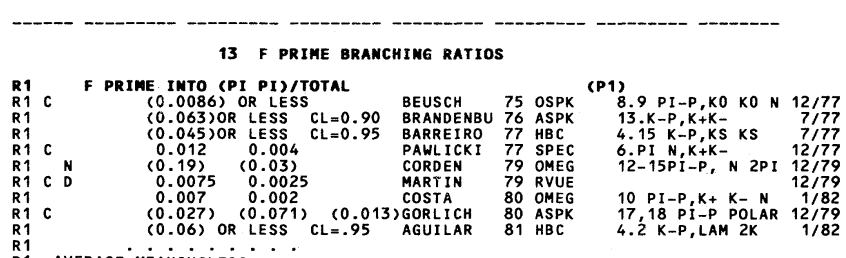

R1 average meaningless

R1 C ASSUMMNG THAT THE F PRIME IS PRODUCED BY AN OPE

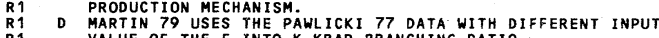

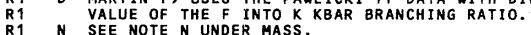

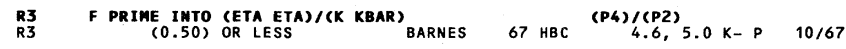

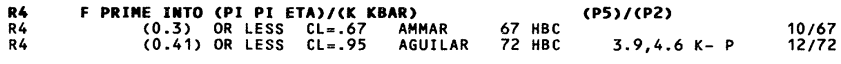

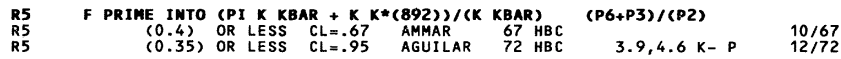

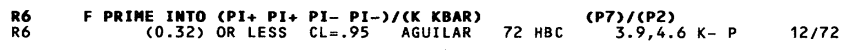

************************************************* ReFEREMCES FOR F PRIME

BARNES 65 PRL 15322

CRENNELL 66 PRL $16 \quad 1025$

$\begin{array}{lllll}\text { ABRAMS } & 67 & \text { PRL } & 18 & 620 \\ \text { AMMAR } & 67 & \text { PRL } & 19 & 1071 \\ \text { BARNES } & 67 & \text { PRL } & 19 & 964\end{array}$

ALITII 68 PRL 211705

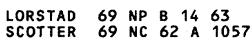

AGUILAR 72 PR $D \quad 629$

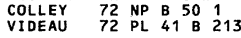

BEUSCH 75 PL 60 B 101

BRANDENB 76 NP B 104413
+CULWICK, GUIDONI, KALBFLEI SCH, GOZ+(BNL+SYRA)

+ KALBFLEISCH, LAI, SCARR, SCHUMANN + (BNL) I

+KEHOE, GLASSER, SECHI-ZORN, WOLSKY (MARYLAND) +DAVIS, HWANG, DAGAN, DERRICK + ( NAWESANL) JP
+DORNAN, GOLDBERG, LEITNER $+{ }^{+}$(BNL+SYRACUSE) ICJP +BARNES, CRENNELL, FLAMIN IO, GOLDBERG, + (BNL)

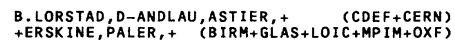
AGULLAR-BENITEZ, CHUNG, EISNER, SAMIOS (BNL)

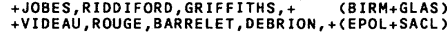

+BIRMAN, WebsDale, WETZEL (CERN+ETH) BRANDENBURG, CARNEG IE, CASHMORE, DAVI ER + (SLAC) 


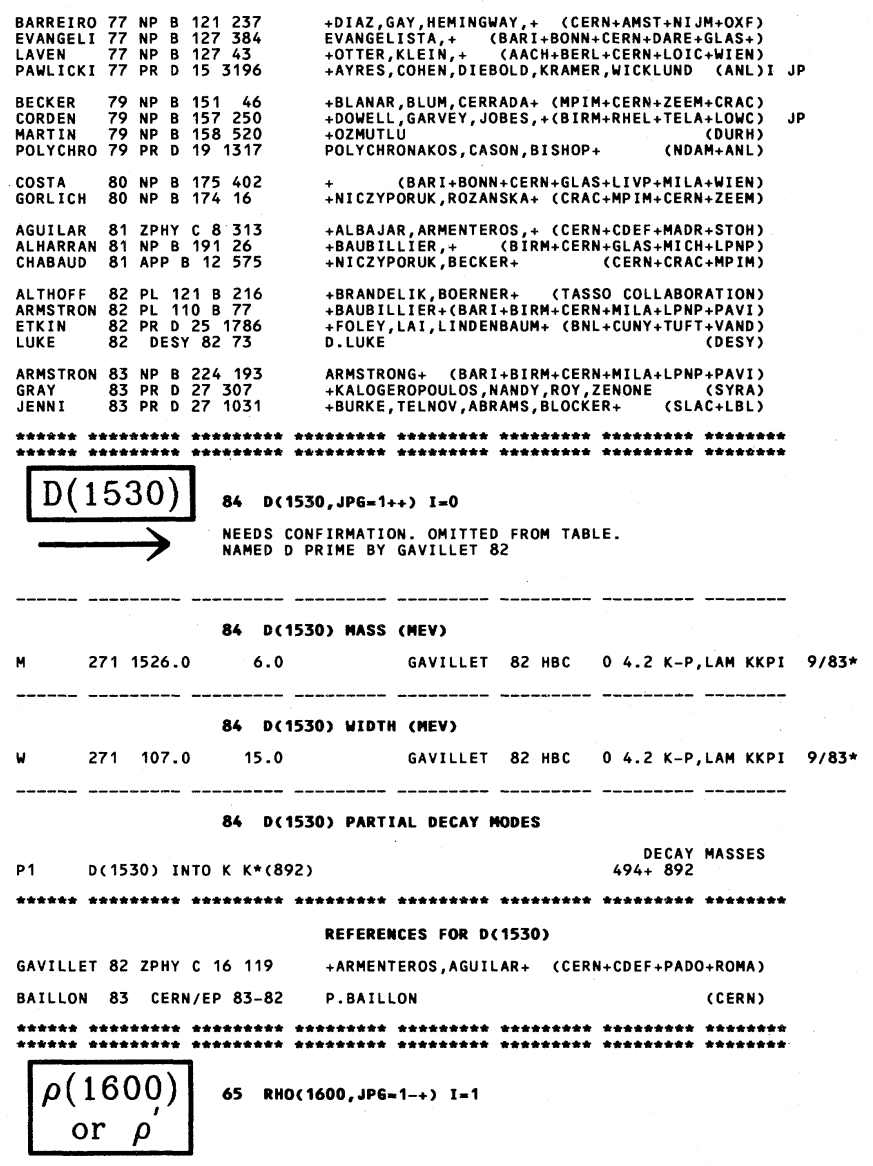

NOTE ON $\rho, \omega, \phi$ RADIAL EXCITATIONS

We no longer use primes to distinguish radial excitations: thus the mesons formerly named $\rho^{\prime}(1250)$, $\rho^{\prime}(1600)$, and $\phi^{\prime}(1680)$ have now become $\rho(1250)$, $\rho(1600)$, and $\phi(1680)$, respectively. The not-yetestablished radial excitation of the $\omega$ is temporarily called $\omega$ (rad. excit.).

The $\rho(1600)$ has been seen in the $\rho^{0} \pi^{+} \pi^{-}$final state in photoproduction (BINGHAM 72, DAVIER 73, SCHACHT 74, ALEXANDER 75, LEE 75, ATIYA 79, RICHARD 79, BARBER 80, ASTON1 81), in $\mathrm{e}^{+} \mathrm{e}^{-}$ annihilation (BARBARINO 72, CONVERSI 74, CORDIER 79, COSME 79, BACCI 80, DELCOURT 81,82, BUON 82, AUGUSTIN 83), in electroproduction (KILLIAN 80), in muoproduction (SHAMBROOM 82), and in a $\pi^{+} \mathrm{d}$ experiment (DIBIANCA 81). If the $\pi^{+} \pi^{-}$ subsystem were in an $S$ wave, as has often been assumed, one would also expect to see the $\rho(1600)$ decaying into $\rho^{0} \pi^{0} \pi^{0}$. This has, however, not been seen (ATKINSON 82). Thus the most likely decay chain is $\rho(1600) \rightarrow \mathrm{A}(1270) \pi \rightarrow \rho \pi \pi \rightarrow 4 \pi$.

For the determination of the $\rho(1600)$ parameters we turn to its relatively rare $\pi^{+} \pi^{-}$and $\mathrm{K}^{+} \mathrm{K}^{-}$decays, which do not have the problems of the above decay chain. The $\pi^{+} \pi^{-}$final state has been produced in $\pi^{-} \mathrm{p}$ interactions (HYAMS 73, BECKER 79), in photoproduction (ATIYA 79, ASTON1 80), and with weaker evidence in $\mathrm{e}^{+} \mathrm{e}^{-}$annihilation (reviewed by GENSINI 78, HEYN 80). The mass and width in these experiments are consistently $1600 \mathrm{MeV}$ and $300 \mathrm{MeV}$, respectively. Note, however, that these parameters are the results of very simplified analyses which are not adequate for such a broad resonance. An attempt to determine the $\rho(1600)$ pole position in a more model-independent way (LANG 79) from the HYAMS 73 data yields a mass at $1660 \mathrm{MeV}$.

The mass determination of BECKER 79 is very well confirmed in the $\mathrm{K}^{+} \mathrm{K}^{-}$system by CLELAND 82, and in $\mathrm{K}^{+} \mathrm{K}^{-}$and $\mathrm{K}_{\mathrm{S}} \mathrm{K}_{\mathrm{L}}$ by BUON 82 in a global fit of all the vector mesons present in this energy range (see below). The $\rho(1600)$ parameters in the Meson Table are based on these three determinations only.

The elusive $\rho(1250)$ has been reclaimed in the diffractively photoproduced $\omega \pi^{0}$ system (ASTON 80 , BARBER 80). However, the $\mathrm{J}^{\mathrm{P}}$ determinations are complicated by the simultaneously present $\mathrm{B}(1235)$ resonance. In addition, other dynamical effects obscure the interpretation of the $\rho(1250)$ as a resonance.

The radial excitations of the $\omega$ and the $\phi$ have been looked for in the channels $\mathrm{K}^{+} \mathrm{K}^{-}, \mathrm{K}_{\mathrm{S}} \mathrm{K}_{\mathrm{L}}, \mathrm{K}_{\mathrm{S}} \mathrm{K}^{\mp} \pi^{ \pm}$, $\omega \pi^{+} \pi^{-}$, and $\pi^{+} \pi^{-} \pi^{0}$, in $\mathrm{e}^{+} \mathrm{e}^{-}$annihilation and in photoproduction. We list the evidence for a state at $1680 \mathrm{MeV}$ under the name $\phi(1680)$; however, the situation is far from clear. In a global analysis of all the channels above except $\pi^{+} \pi^{-} \pi^{0}$, BUON 82 conclude that the data are well described by the tails of the $\rho, \omega$, and $\phi$, the $\rho(1600)$, a conspicuous $\phi(1680)$, and one $\omega$ (rad. excit.) hidden under the $\rho(1600)$. This view is confirmed by AUGUSTIN 83 in preliminary $\mathrm{K}^{+} \mathrm{K}^{-}$ data. The $\pi^{+} \pi^{-} \pi^{0}$ system is not yet reliably analyzed.

In photoproduction, however, no $\phi(1680)$ resonance is seen in the $\omega \pi^{+} \pi^{-}$channel, but rather a broad threshold enhancement (ASTON2 80, ATKINSON1 83), and nothing is seen in $K \bar{K} \pi$ (ATKINSON1 83). In the $\mathrm{K}^{+} \mathrm{K}^{-}$channel, the $\phi(1680)$ has been found (ASTON2 81) with the same parameters as in $\mathrm{e}^{+} \mathrm{e}^{-}$annihilation, but its interference with the $\phi$ looks quite different.

A resonance with parameters similar to the $\phi(1680)$ 


\section{Mesons} $\rho(1600)\left[\rho^{\prime}\right]$

has been found in the photoproduced $\pi^{+} \pi^{-} \pi^{0}$ system (ATKINSON3,4 83). Its interpretation is, however, complicated, as it may contain both the $\omega(\mathrm{rad}$. excit.) and the $\phi(1680)$. So far we list this evidence under the $\phi(1680)$.

$$
65 \text { RHO( } 1600) \text { MASS (MEV) }
$$

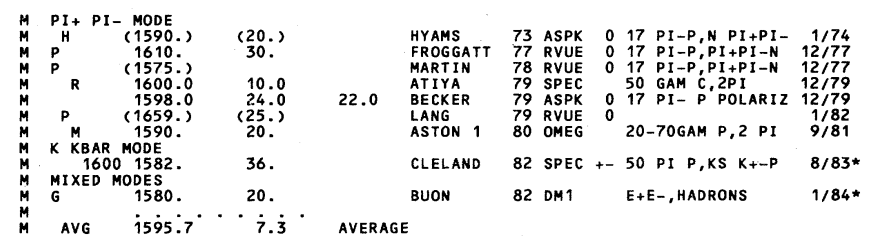

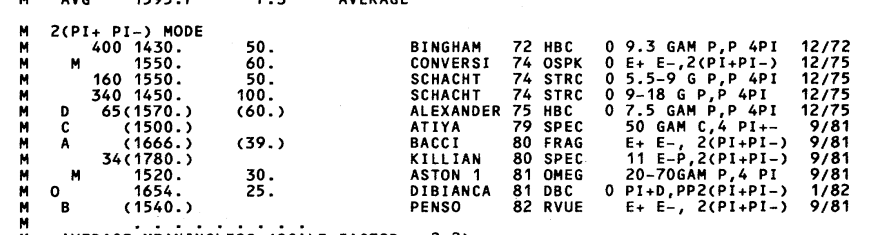

M average MEÁ̈ingiess' (SCALE Factor $=2.2$ )

$M$
$M$ A SIMPLE RELATIV. BREIT-WIGNER FIT WITH MODEL DEPENDENT WIDTH

$\begin{array}{ccc}M & B & \text { ASSUMING RHO+EPSILON(1300) DECAY MODE INTERFE } \\ M & B & \text { A } 1270)+P I \\ M & C & \text { PACKGROUND }\end{array}$

C PARAMETERS ROUGHLY ESTIMATED, NOT FROM A FIT
D SEEW MASS DISTRIBUION COMPENSATED BY ROSS-STODOLSKY FACTOR

M $M$ INCLUDED IN BECKER 79 ANALYSIS AT AITH CONSTANT WIDTH

$M$ M ONE PEAK FIT RESULT. WIS S OF HYAMS 73 DATA

$M$
$M$

$M$ M $M$ IS PRESENT DUE TO THE CHOICE OF THE BACKGROUND SHAPE. WTATIONS TO

65 RHO( 1600) UIDTH (MEV)

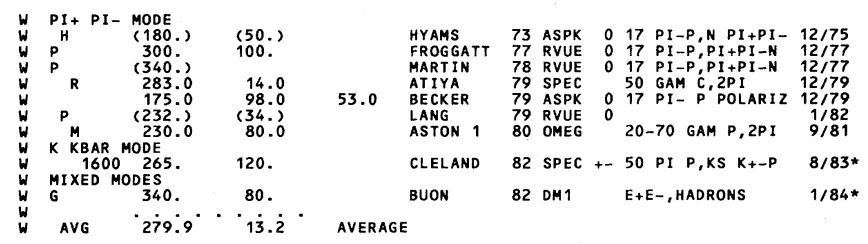

$\begin{array}{ccccc}W & 2(P I+P I-) & \text { MODE } & \\ W & 400 & 650 . & 100 . \\ W & M 60 . & 100 . \\ W & & 360 & 400 . & 120 . \\ W & E & 340 & 850 . & 200 . \\ W & D & 65 & (340 .) & (160 .)\end{array}$

$\begin{array}{rrrr}W & C & (600 .) & (160 .) \\ W & \text { A } & (700 .) & (160 .) \\ W & B & 34 & (100 .) \\ W & (230 .) & \end{array}$

W $\left.W^{M} \quad 400.\right) \quad 50$.

W average MEANingLiess (SCALE Factor $=1.4$ )

W A SIMPLE RELATIV. BREIT-WIGNER FIT WITH MODEL DEPENDENT WIOTH

$M$ M A(1270)+PI BACKGROUND

C PARAMETERS ROUGHLY ESTIMATED, NOT FROM A FIT
$W$
SKEW MASS DIITIBUTION COMPENSATED BY ROSS-STODOLSKY FACTOR
WIDTH ERRORS ENLARGED BY US TO $4 *$ HIDTH/SQRT (N), SEE K*(892) NOTE

W ${ }^{M}$ INCLUDED IN BECKER 79 ANALYSIS SIMPLE RELATIV. BREIT WIGNER FIT WITH CONSTANT WIDTH

W O ONE PEA FIT RESULT T. WS OF HYAMS 73 DATA

R AN ADDITIONAL 40 MEV UNCERTAINTY IN BOTH THE MASS AND WIDTH

$\begin{array}{lll}W & R \\ W & G & \text { IS PRESENT DUE TO THE CHOICE OF THE BACKGROUND SHAPE. } \\ W & G \text { FROM GLOBAL FIT OF RHO OMEGA, PHI AND THEIR RADIAL EXCITATIONS TO } \\ \text { CHANNELS OMEGA PI+PI-, K }+K-, K S \text { KL, KS K+- PI-+. }\end{array}$

\begin{tabular}{|c|c|c|}
\hline & & 65 RHO( 1600$)$ PARTIAL DECAY MODES \\
\hline $\begin{array}{l}\text { P1 } \\
\text { P2 } \\
\text { P3 } \\
\text { P4 } \\
\text { P5 } \\
\text { P6 } \\
\text { P7 } \\
\text { P8 } \\
\text { P9 } \\
\text { P10 } \\
\text { P11 } \\
\text { P12 }\end{array}$ & $\begin{array}{l}\text { RHO }(1600) \\
\text { RHO(1600) } \\
\text { RHO( } 1600) \\
\text { RHO(1600) } \\
\text { RHO(1600) } \\
\text { RHO(1600) } \\
\text { RHO }(1600) \\
\text { RHO(1600) } \\
\text { RHO }(1600) \\
\text { RHO(1600) } \\
\text { RHO(1600) } \\
\text { RHO(1600) }\end{array}$ & $\begin{array}{l}\text { INTO RHO PI+ PI- } \\
\text { INTO } 4 \text { PI ALL CHARGED } \\
\text { INTO RHO RHO } \\
\text { INTO PI PI } \\
\text { INTO KBAR K } \\
\text { INTO PI OMEGA } \\
\text { INTO RHOO PIO PIO } \\
\text { INTO E+ E- } \\
\text { INTO RHO PI PI-+ PIO } \\
\text { INTO KBAR K* } \\
\text { INTO PI PI ETA }+ \text { C.c. } \\
\text { INTO RHO PI PI }\end{array}$ \\
\hline
\end{tabular}

DECAY MASSES
$769+140+140$
$140+140+140+140$

$769+769$
$140+140$

$494+494$
$140+783$

$769+135+135$

$511+.511$
$769+140+135$

$494+892+549$
$140+140+54$
$769+140+140$

Data Card Listings

65 RHO(1600) PARTIAL WIDTHS (KEV)

(1)

48 RHO(1600) INTO E+ E-

W8 D (7.5) (1.5) DELCOUR2 81 DM1 E+ E-, 2(PI+PI-) 9/81

W8
W8 DODEL DEPENDENT, NOT INDEPENDENT OF DELCOUR2 81 WIDTH TIMES E+E-
BRANCHING RATIO BELOW

65 RHO(1600) BRAMCHING RATIOS

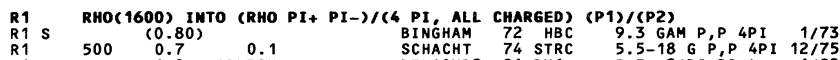

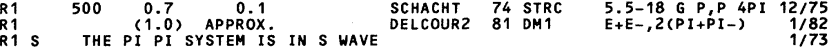

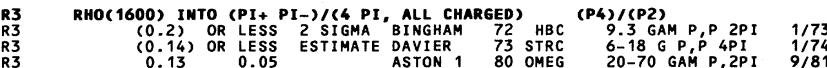

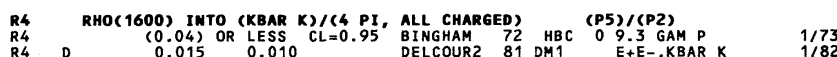

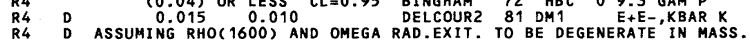

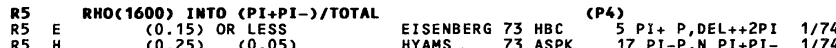

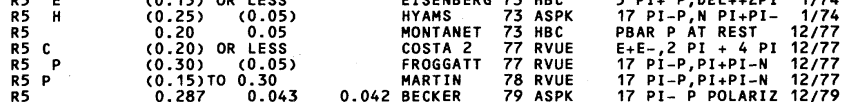

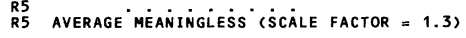

R5 C ESTIMATE USING UNITARITY,TIME REVERSAL INVARIANCE, BREIT WIGNER

R5
E

73 DATA

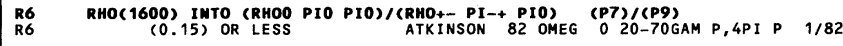

R7 RHO( 1600) IMTO (PI+ PI- + MEUTRALS)/(4PI, ALL CHARGED)

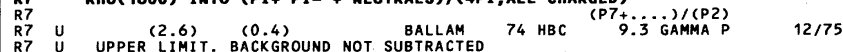

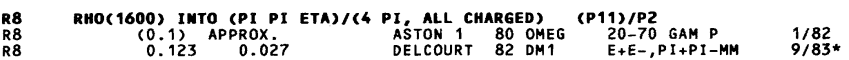

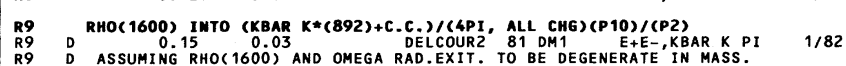

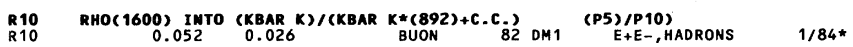

THIS COMBINATION OF A PARTIAL WIDTH WITH THE PARTIAL WIDTH INTO

$626(4 \mathrm{PI}$, ALL CHARGED)*6(E+E-)/G(TOTAL) (62)*(68)/TOTAL

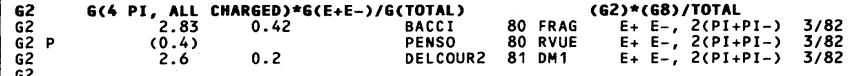

G2 AVG $\cdot 2.64 \cdot 0.4^{\circ} \cdot 0^{\circ}$ aVerage

G2 $P$ ASSUMING RHO+EPSILON DECAY MODE INTERFERES WITH A(1270)+PI
$G 2$

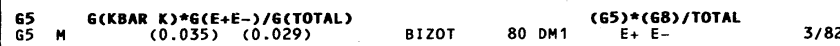

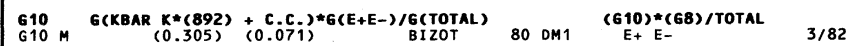

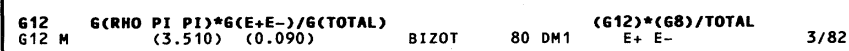

$G$ M MOdel DEPENDENT

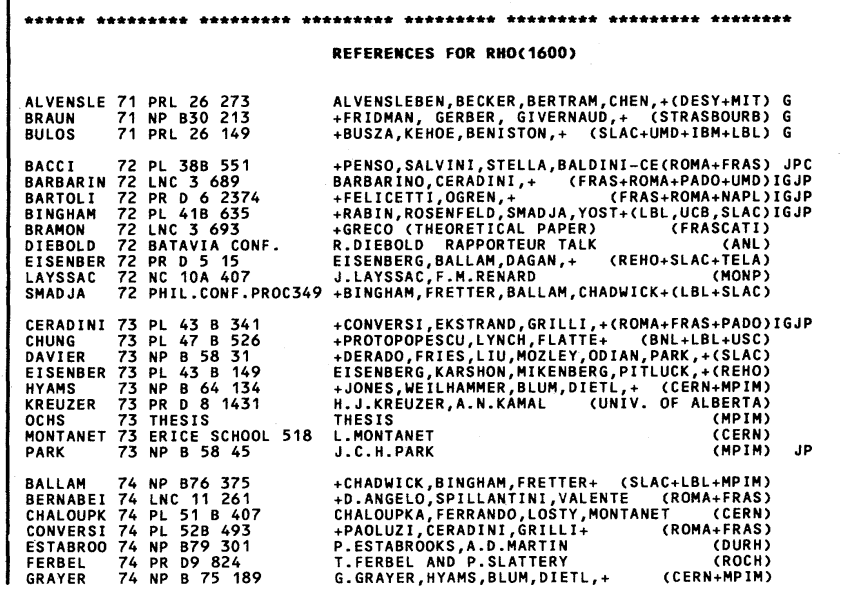




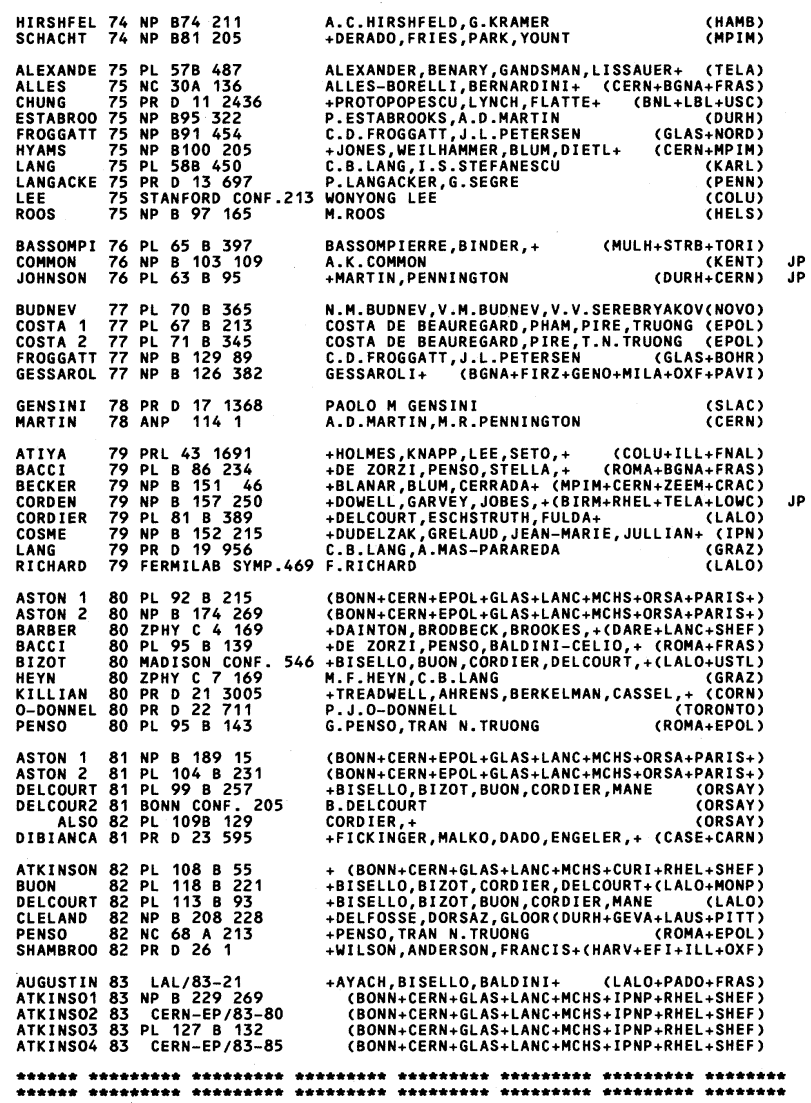

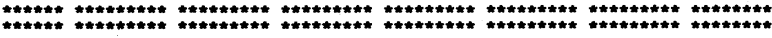

$\omega(1670)$ 45 OMEGAC 1670,JPG=3--) I=0.
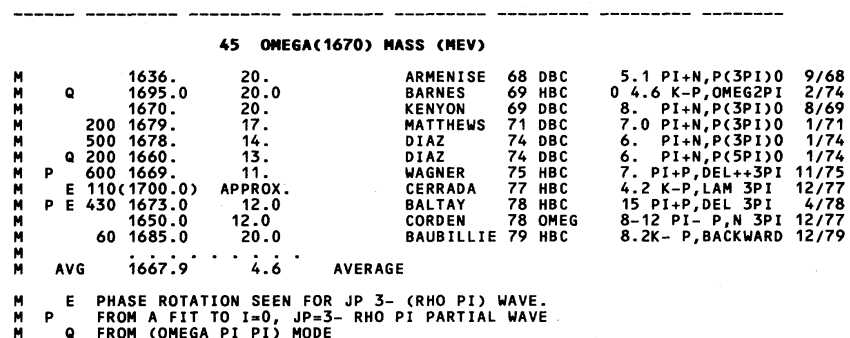

$M$
$M$

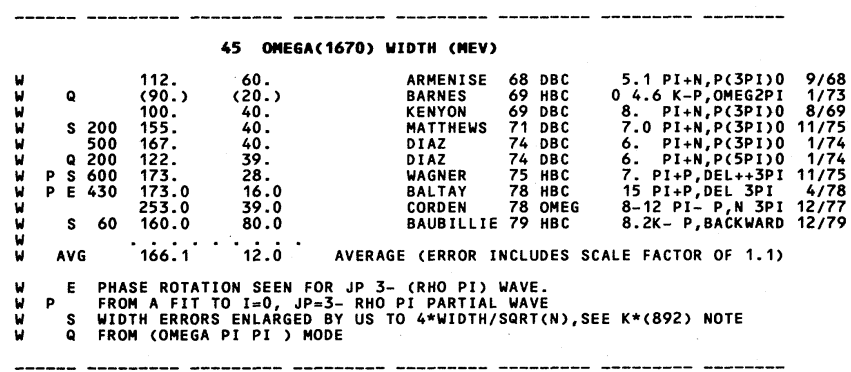

45 OMEga( 1670) PARTIAL decay modes

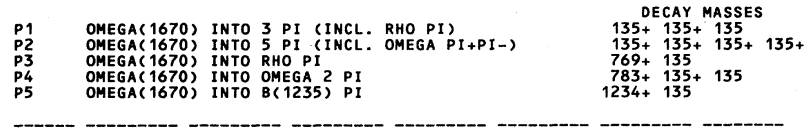

$$
\rho(1600) \quad\left[\rho^{\prime}\right], \omega(1670), \mathrm{A}(1680) \quad\left[\mathrm{A}_{3}\right]
$$

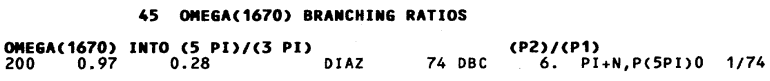

OMEGA(1670), INTO (RHO PI)/(3 PI)
$200(0.70)$ OR MORE

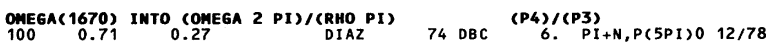

OMEGA(1670) INTO (B(1235) PI)/(RHO PI)
POSSIBLY SEEN

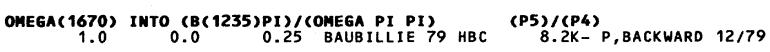

**********************************************************************

+GHIDINI, FORINO+ (BARI+BGNA +FIRZ +ORSAY)

BARNES 69 PRL $23142 \quad$ +CHUNG, EISNER, FLAMINIO,+
KENYON 69 PRL $23146 \quad$ (BNLL)
+KINSON, SCARR,

ARMENISE 70 LNC 4199 +GHIDINI, FORINO, CARTACCI, + (BARI+BGNA+FIRZ)

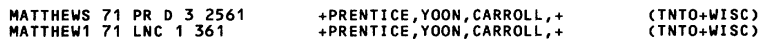

DIAZ 74 PRL 32260 +DIBIANCA, FICKINGER, ANDERSON, + (CASE+CARN)

WAGNER $75 \mathrm{PL} 58 \mathrm{~B} 201$ TTABAK, CHEW (LBL) JP

CERRADA 77 NP B 126241 +BLOCKZIJL, HEINEN, + (AMST+CERN+NIJM+OXF) JP

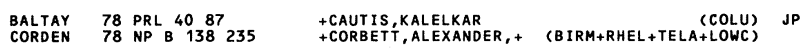

BAUBILLI 79 PL B 89131 BAUBILLIER+ (BIRM+CERN+GLAS+MSU+LPNP)

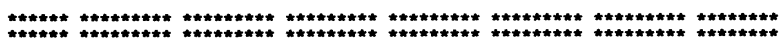

$\left.\begin{array}{c}A(1680) \\ \text { or } A_{3}\end{array}\right]$ A4 $\left.1680, J P 6=2--\right) \quad I=1$

We have dropped the subscript 3 on the $A_{3}$, and rename this meson $A(1680)$.

Evidence for the existence of the $A(1680)$ meson was previously confused due to its appearance near the $\mathrm{f} \pi$ threshold in the diffractive-like process $\pi \mathrm{N} \rightarrow \pi \pi \pi \mathrm{N}$, much like the $A(1270)$ meson. While everybody agreed that there was a $\sim 300-\mathrm{MeV}$-wide $\mathrm{f} \pi$ enhancement in the ${ }^{P^{P}} \mathbf{L M}=2^{-}$S0 partial wave at about $1650 \mathrm{MeV}$, some claimed nonresonant states (ANTIPOV1 73, ASCOLI1 73, BALTAY 77), while others saw evidence for a resonance in the phase variation with respect to other partial waves (OTTER 74, THOMPSON 74).

In the nondiffractive charge-exchange reaction $\pi^{-} p$ $\rightarrow \pi^{+} \pi^{-} \pi^{0} \Delta^{++}$(WAGNER 75, BALTAY 77 , CAUTIS 77) and in the hypercharge-exchange reaction $\mathrm{K}^{-} \mathrm{p} \rightarrow$ $\pi^{+} \pi^{-} \pi^{0} \Lambda$ at $4.2 \mathrm{GeV} / \mathrm{c}$ (CERRADA 77), there is no evidence for $A(1680)$ production.

Definitive proof for the resonant nature of the A $(1680)$ has been given by PERNEGR 78 ( $3 \pi$ system diffractively produced on nuclei) and DAUM 80,81 and EVANGELISTA 81 ( $3 \pi$ diffraction on proton target). In all these experiments, the $2^{-} \mathrm{SO}^{+}(\mathrm{f} \pi)$ partial-wave amplitude exhibits resonance-like phase variation.

In a simultaneous fit to the four $2^{-}$waves $(\epsilon \pi, \rho \pi$, twice $\mathrm{f} \pi$ ), DAUM 81 needs a heavier companion to the $A(1680)$ in addition to the Deck background. This fit probably gives the most reliable estimates of the $A(1680)$ and of its heavier companion, which we name $A(2100)$. 


\section{Mesons} $\mathrm{A}(1680)\left[\mathrm{A}_{3}\right], \phi(1680) \quad\left[\phi^{\prime}\right]$
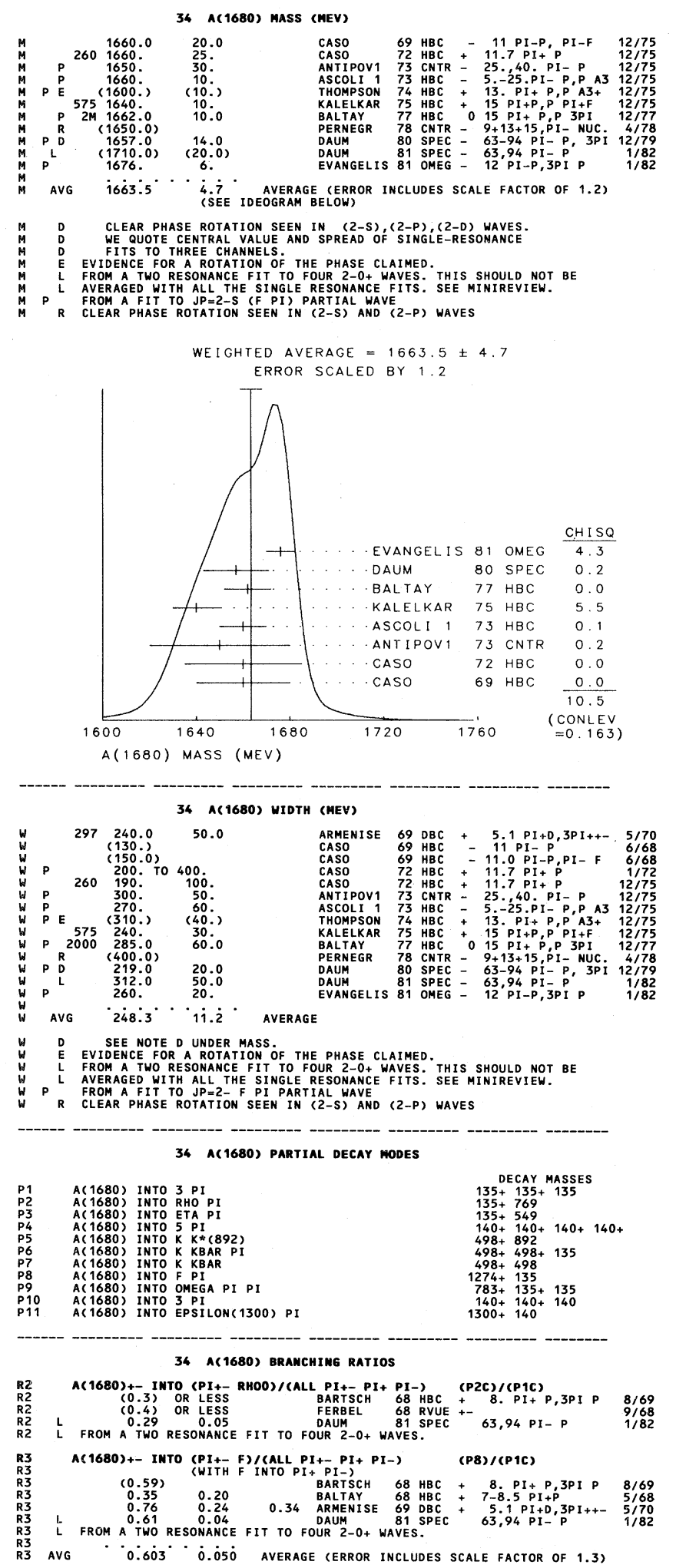

\section{Data Card Listings}

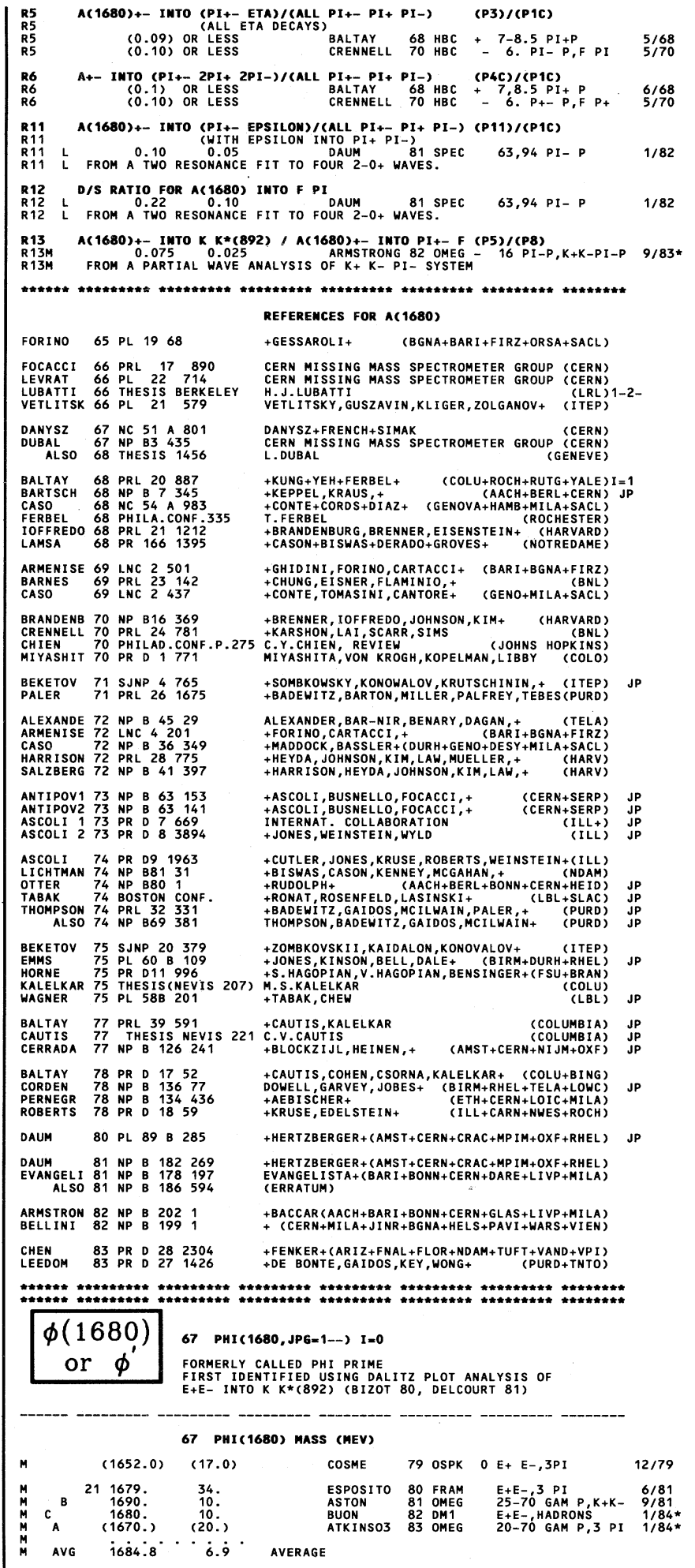




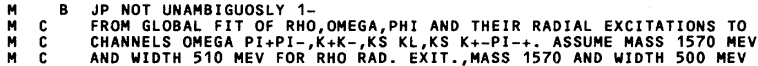

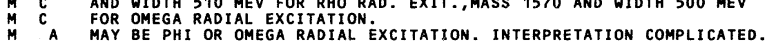

\begin{tabular}{|c|c|c|c|c|c|c|}
\hline & \multicolumn{4}{|c|}{67 PHI (1680) МIDTH (MEV) } & \multirow[b]{2}{*}{$O E+E-, 3 P I$} & \multirow[b]{2}{*}{$12 / 79$} \\
\hline & $(42.0)$ & $(17.0)$ & COSME & 79 ospk & & \\
\hline В 21 & $\begin{array}{l}99 . \\
100: \\
185:) \\
(160)\end{array}$ & $\begin{array}{c}49 . \\
40: \\
22: \\
(20)\end{array}$ & $\begin{array}{l}\text { ESPOSITO } \\
\text { ASTON } \\
\text { BUON } \\
\text { ATKINSO3 }\end{array}$ & $\begin{array}{l}80 \text { FRAM } \\
81 \text { OMEG } \\
82 \text { OM1 } \\
83 \text { OMEG }\end{array}$ & 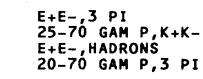 & $\begin{array}{l}6 / 81 \\
9 / 81 \\
1 / 84 * \\
1 / 84 *\end{array}$ \\
\hline
\end{tabular}

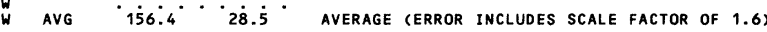

W ${ }^{B} \quad$ JP NOT UNAMBIGUOSLY 1 SE

A MAY BE PHI OR OMEGA RAdial Excitation. interpretation Complicated.

67 PHI(1680) PARTIAL DECAY MODES

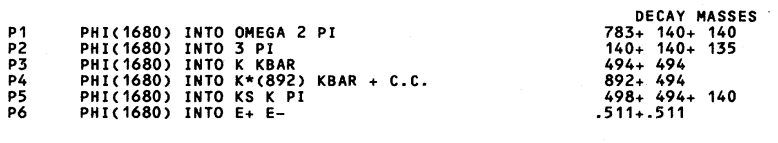

67 PHI(1680) BRANCHING RATIOS

R1 PHI (1680) INTO (OMEGA PI+PI-)/(K*(892) KBAR+C.C. (P1)/(PA)
R1
RUON

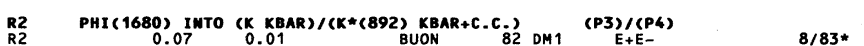

R3 PHI( 1680$)$ INTO (K*(892) KBAR)/(KS K+- PI-P)
DOMINANT
RANE

\section{PHI(1680) G(I)*G(E+E-)/G(TOTAL) (KEV) \\ THIS COMBINAT ION OF A PARTAL WIDTH WITH THE PARTIAL WIDTH INTO
EE- AND HITH THE TOAAL WIDTH IS OBATINE FOM THE INTEGRATED

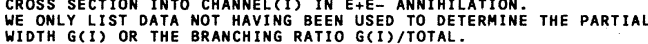 \\ G1 M1 GCOMEGA 2PPI)*G(EEE-)/G(TOTAL)
G1
$(0.017) A P P R O X$

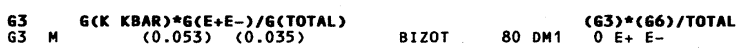

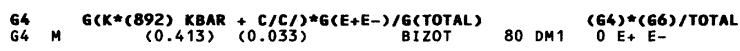 \\ $G$ M MODEL DEPENDENT}

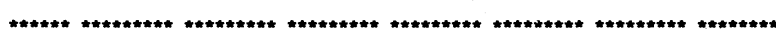

REFEREMCES FOr PHI

COSME 79 NP B 152215 +DUDELZAK, GRELAUD, JEAN-MARIE, JULL IAN+(I PNP)

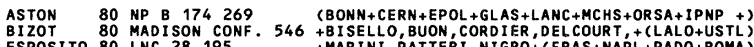

ESPOSITO 80 LNC 28195 TMARINI, PATTERI, NIGRO+(FRAS+NAPL+PADO+ROMA)

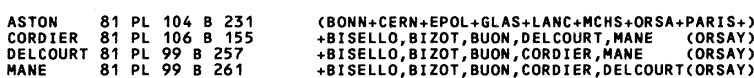

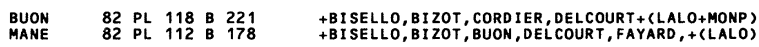

AUGUSTIN 83 LAL/83-21 AAYACH, BISELLO, BALDINI+ (LALO+PADO+FRAS)

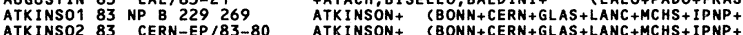

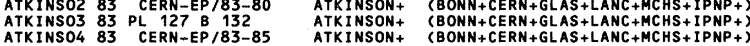

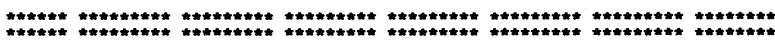

\section{$g(1690) \quad 15$ 6(1690,JPG - 3-+) I-1}

\section{MASS (MEV)}

ME ONLY INCLLDE HIGH STATISTICS EXPERTMENTS IN the AVERAGE for the M PI MODE
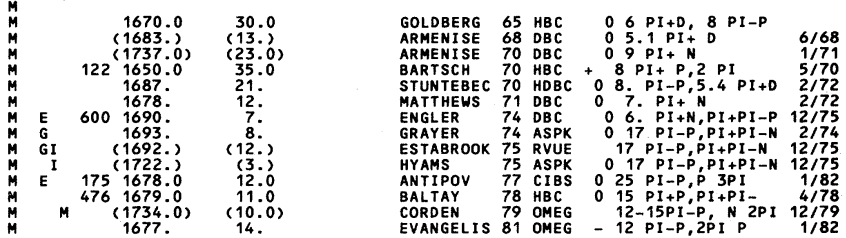
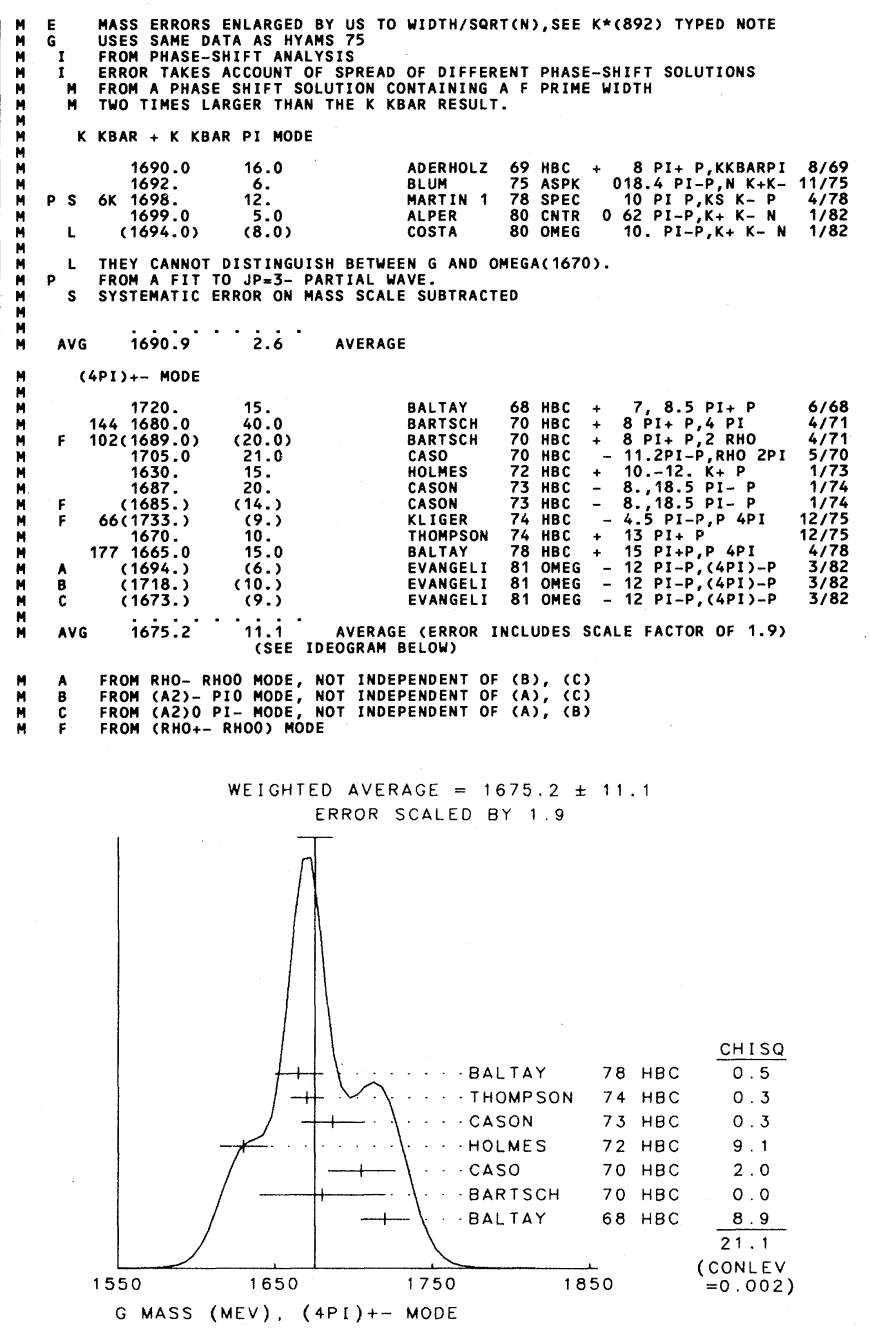

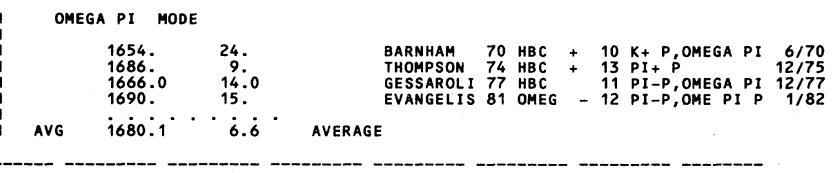

15 G MIDTH (MEV)

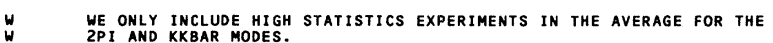
2 PI MODE

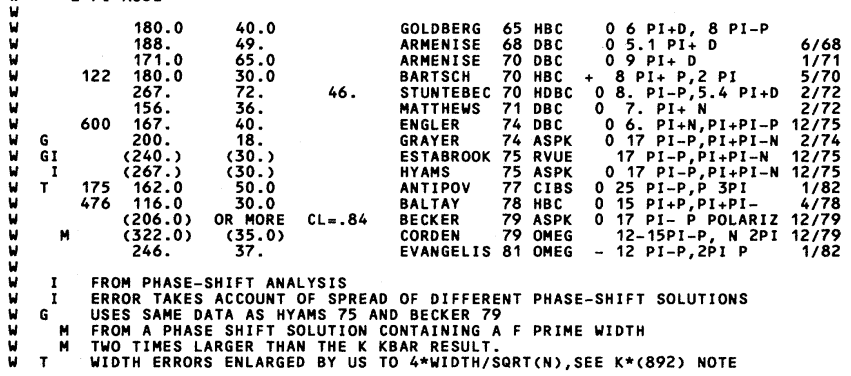


Mesons $\mathrm{g}(1690)$

\section{Data Card Listings}
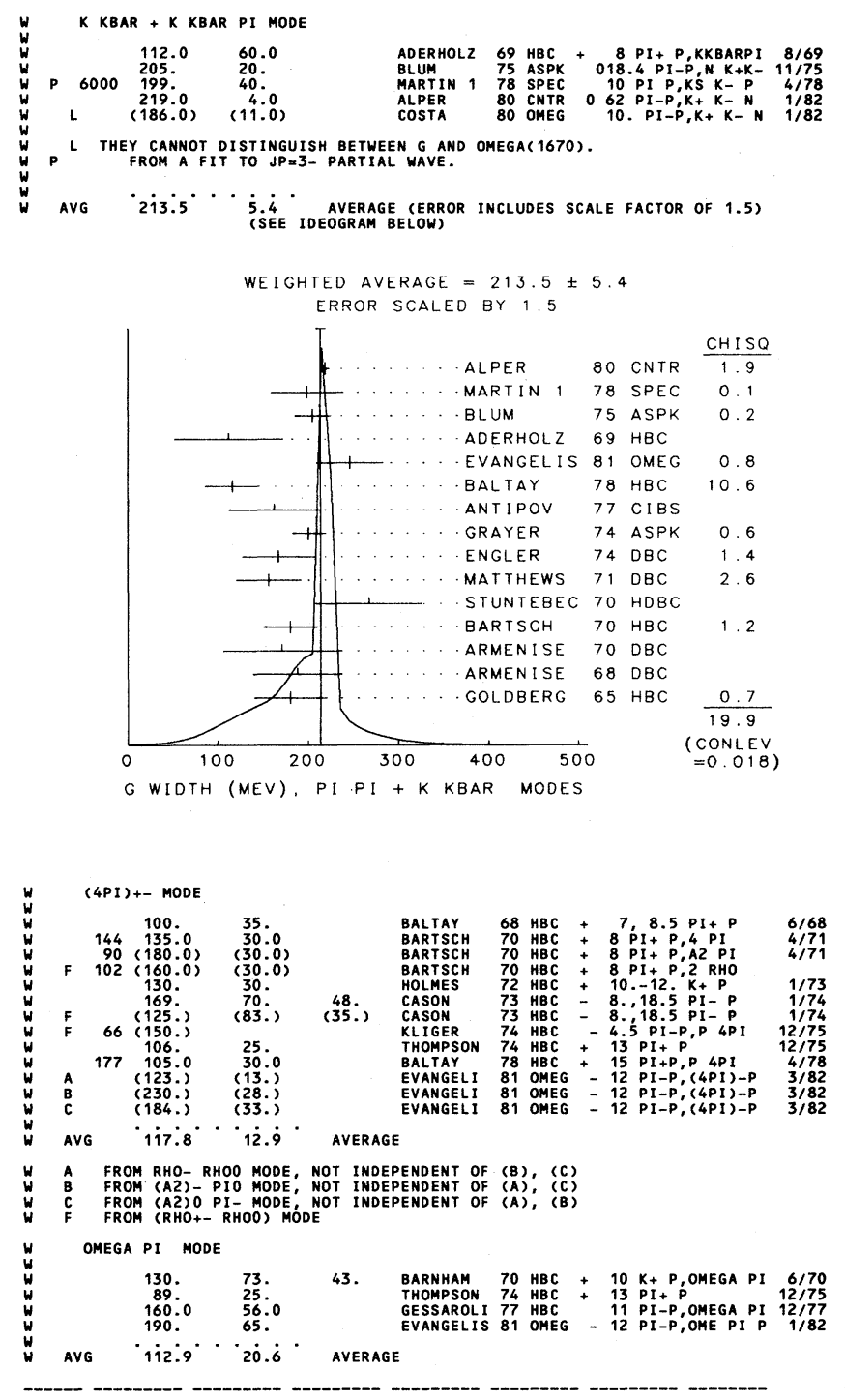

15 G Partial decay modes
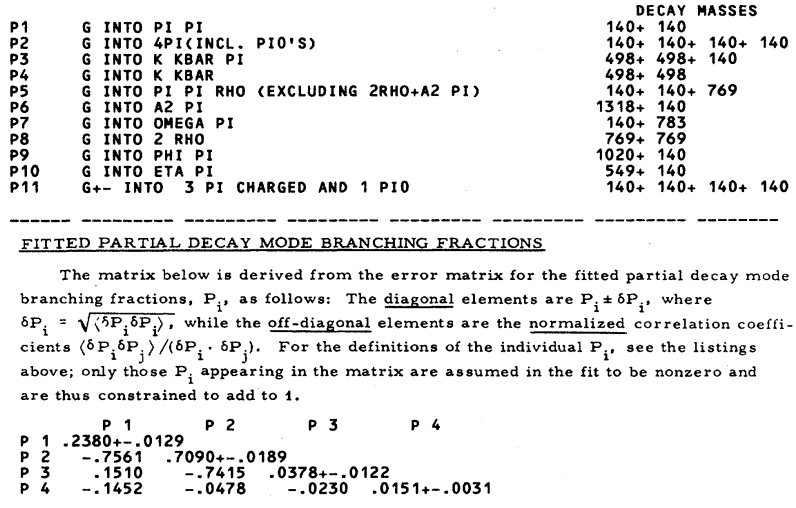

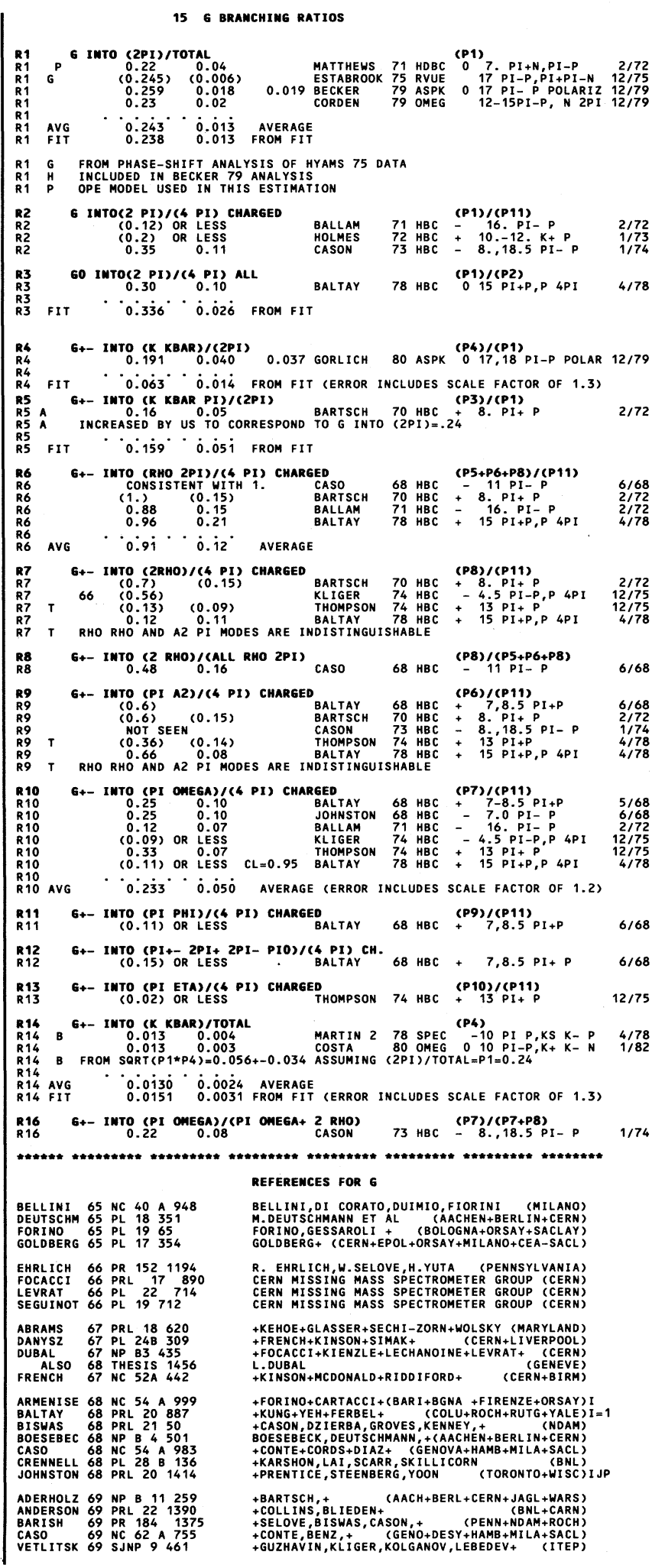




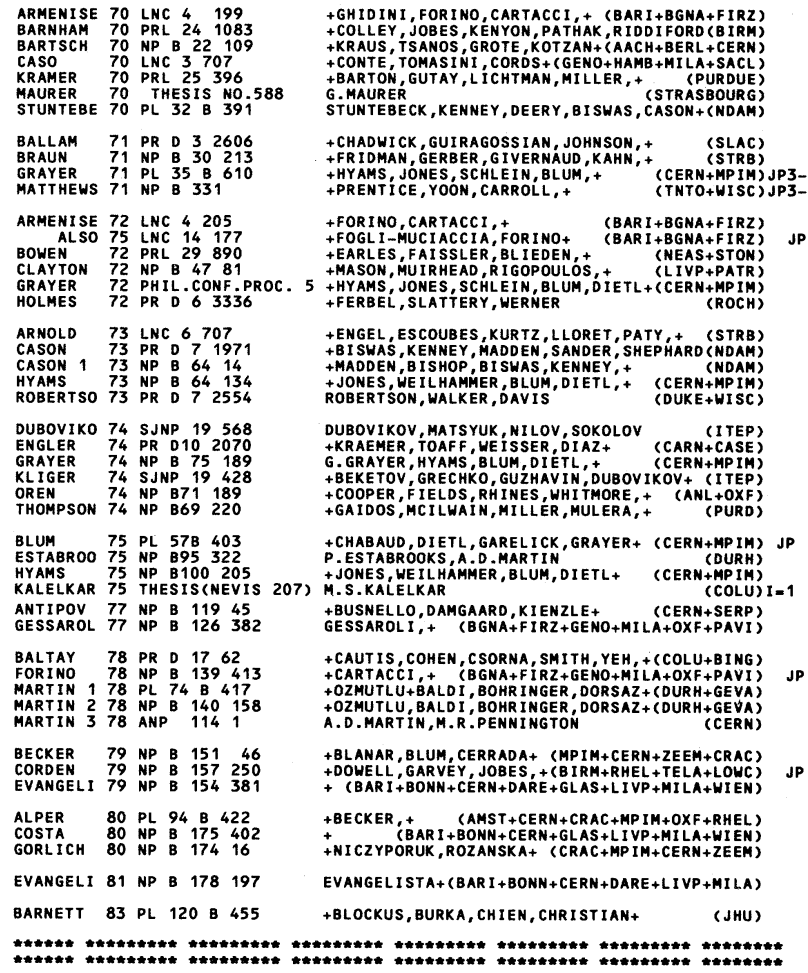

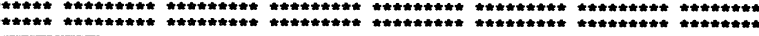

$\theta(1690)$ GS theta(1690, JPG-2++) I=0 NAMED THETA BY EDHARDS 82 TET (1690), THEREFORE C=+.

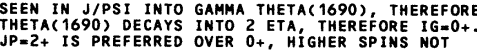

MASS AND WIDTH DSTERMINATION COMPLICATED BY OVERLAP WITH F PRIME
IN MASS SPECTRA. POSSIBLE CONNECTION OF THIS STATE WITH STRUCTURE

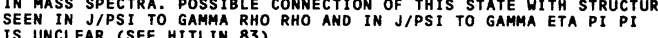

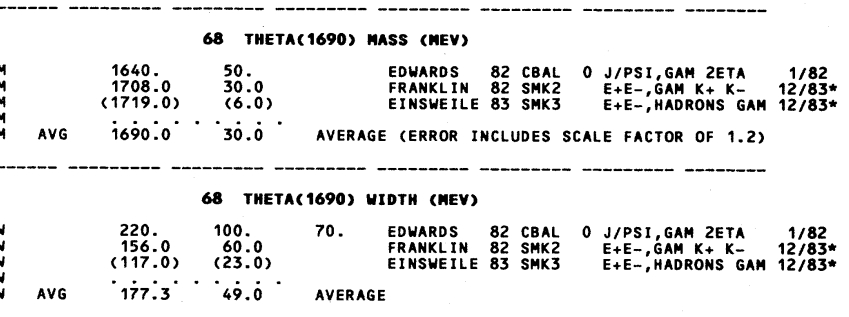

68 Theta( 1690) PARTIAL DeCAY MODES

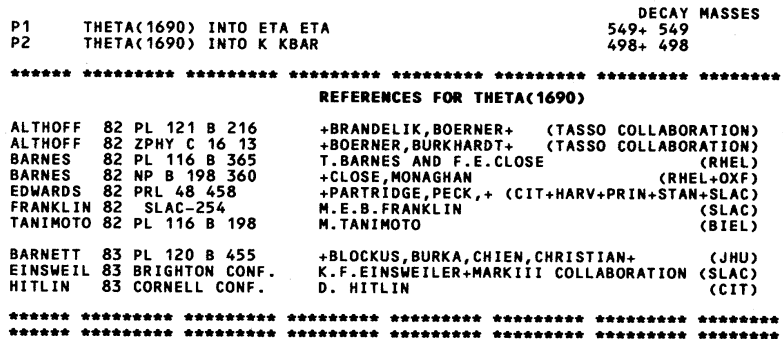

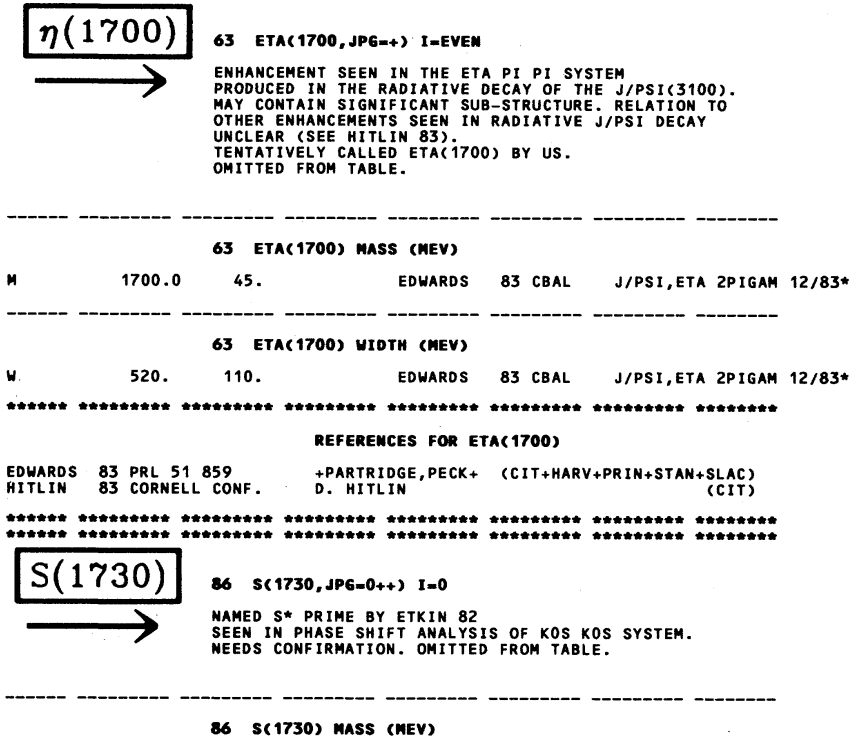

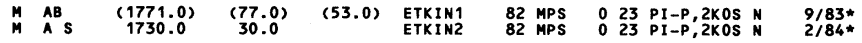

A. A FROM AN AMPLITUDE ANALYSIS OF THE KOS KOS SYSTEM.

LiNEARLY BY US.

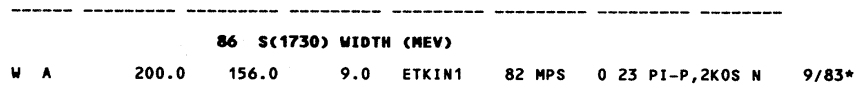

w A from an amplitude analysis of the kos kos system.

86 S(1730) PARTIAL DECAY MODES

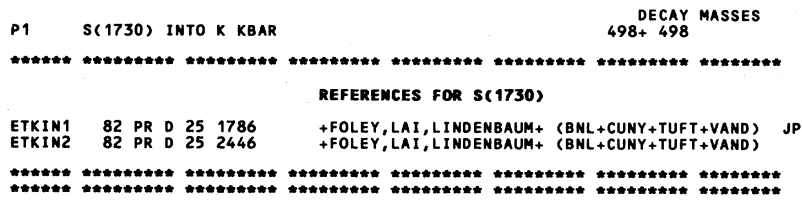

$\pi(1770)$ 75 PI(1770,JPG=0--) I=1

SEE IN PARTIAL WAVE ANALYSIS OF THE DIFFRACTIVELY

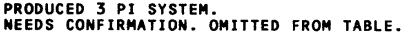

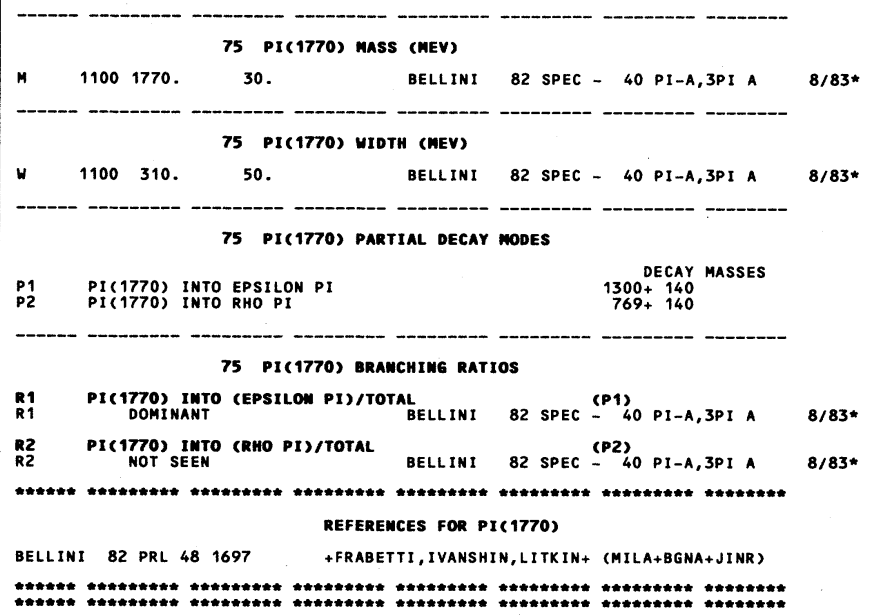




\section{Mesons}

Data Card Listings

$f(1810), \phi(1850), S(1935)$

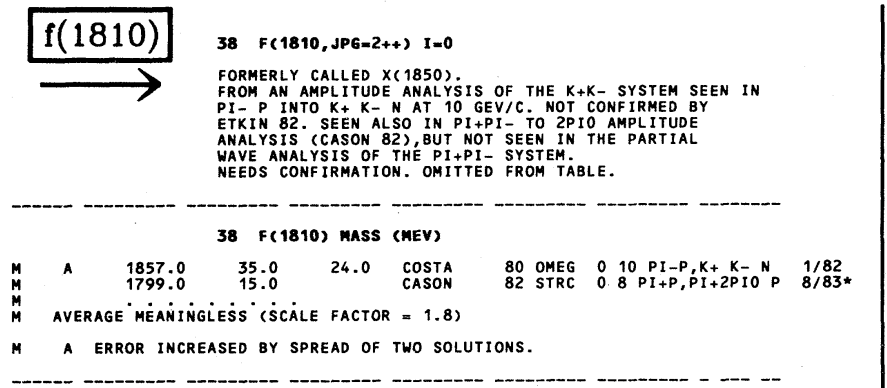

38 F(1810) WIDTH (MEV)

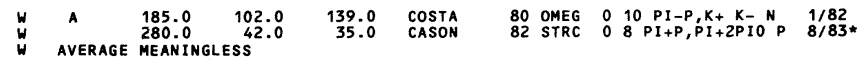

W A ERROR INCREASEd BY SPREAD OF TWO SOLUTIONS.

38 F(1810) PARTIAL DECAY MODES

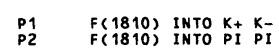

DECAY MASSES

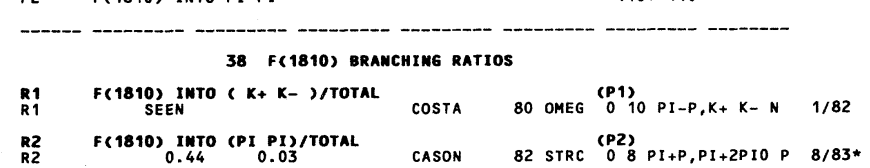

$\underset{\mathrm{S}(1935)}{\longrightarrow} 31$ s(1935,JP6= ,

A narrow enhancement called the $S(1935)$ has been observed in the antiproton-proton total cross section (CARROLL 74, CHALOUPKA 76, BRUCKNER 77, SAKAMOTO 79).

This observation has not been confirmed by other experiments (ALLEN 80, KAMAE 80, JAS-

TRZEMBSKI 81, LOWENSTEIN 81), or the effect was found to be smaller in magnitude and larger in width (HAMILTON 80).

A recent experiment (SUMIYOSHI 82) has measured with high precision the $\bar{p} p$ total cross section, by an improved transmission method, rejecting both the narrow- (CARROLL 74) and broad- (HAMILTON 80) S hypotheses.

No significant signal is observed for a narrow S(1935) in backward antiproton-proton elastic scattering (GARNJOST 79), nor in the charge-exchange cross section (GARNJOST 75, CHALOUPKA 76, HAMILTON 80).

The nonexistence of the $S$ is also confirmed by recent hadroproduction experiments (DAUM 81, BENSINGER 83, BARNETT 83).

The only observation in favor of a narrow $S$ which remains unchallenged is the one of ASTON 80 in a photoproduction experiment. The weak statistical significance of the signal does not warrant taking the S(1935) as a well-established narrow resonance.

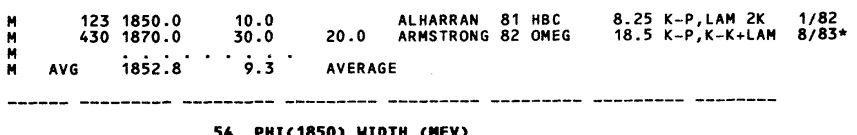

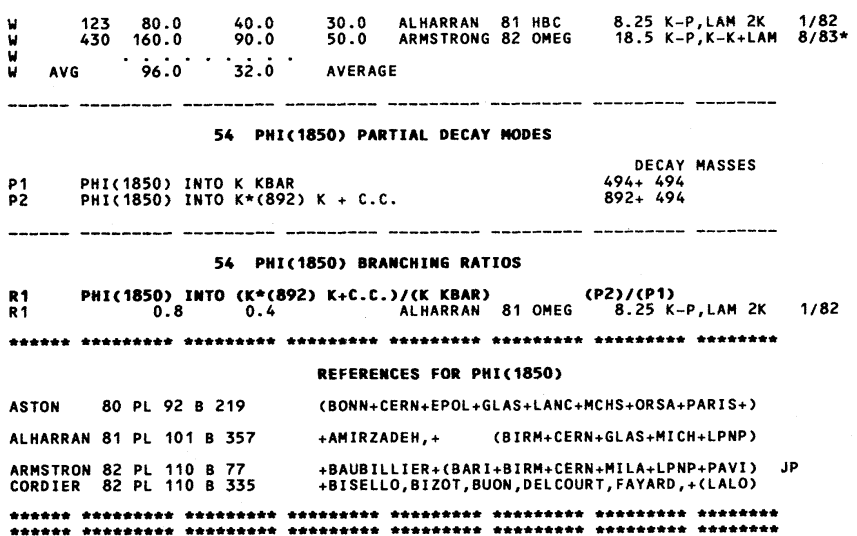

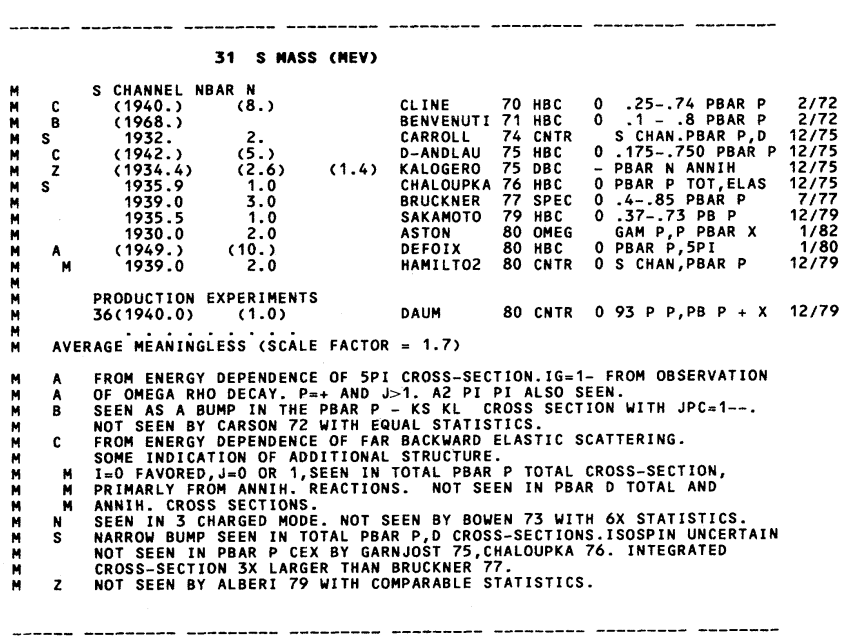




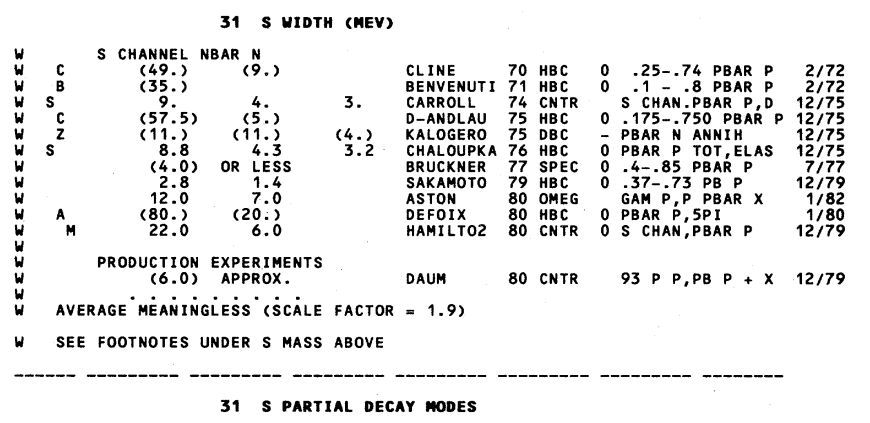

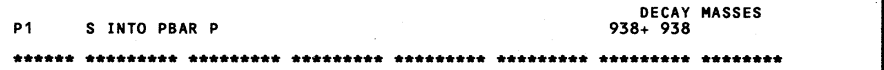

CLINE 68 PRL 211268

REFERENCES FOR S(1935)

+ENGL ISH, REEDER, TERRELL, TWITTY (WISCONSIN) D.CLINE, J.ENGLISH, D.D.REEDER (WISC)J
ASTIER RAPPORTEUR TALK

BENVENUT I, CLINE, RUTZ, REEDER, SCHERER (HISC)
STEPHEN S. PINSKY (UTAH+ARGONNE) +GUIDONI, MARZANO, CASTELLI, +
+EARLES, FAISSLER, BLIEDEN, +
(ROMA+TRST)
(NEAS+STON) +EARLES, FAISSLER, BLIEDEN, + (NEAS+STON) +EARLES, FAISSLER, BLIEDEN, + + PRICE, (NEAS+STON)
+CNDON, DONAHU, MANDELKERN, PRICE,
(CERN) +CONDON, MANDELKERN, PRICE, SCHULTZ (UCI)
+ CHIANG, KYCIA, LI, MAZUR, MICHAEL,
(BNL) +BEAMER, BROSS, EISENSTEIN, + (ILL+ANL+ISU)

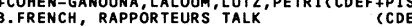
A.DONNACHIE,P.R. THOMAS (MANCHESTER) (LBL +MHCO)
(SYRA)
KALNNEG, POLLARD, ROSS, TRIPP, +
(ROCH) WATSON, GELFAND, BUT TRAM, + (ILL + +ANL+CHIC+ISU) CHALOUPKA, + (CERN+LIVP+MONS+PADO+ROMA+TRST)

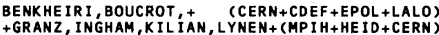
L.MONTANET
G.C.ROSSI, G.VENEZIANO
(CERN) $\begin{array}{lll}\text { MONTANET } 77 & \text { BL } 67 \\ \text { ROST } 1 & 77 & \text { PL } 70 \text { CONF. } 255\end{array}$ $\begin{array}{llllll}\text { CARTER } & 78 & \text { NP } & \text { B } & 132 & 176 \\ \text { CUTTS } & 78 & \text { PR } & \text { D } & 77 & 16 \\ \text { PENMINGT } & 78 & \text { NP } & \text { B } & 137 & 77\end{array}$ $\begin{array}{lllll}\text { ALBERI } 79 & \mathrm{PL} & 83 & \text { B } & 247 \\ \text { ALSTON-G } 79 & \text { PRL } 43 & 1901\end{array}$ CARROLL 79 PR D 191950 $\begin{array}{llll}\text { DELCOURT } 79 & \text { PL } B & 86 & 395 \\ \text { GIBBARD } 79 & \text { PRL } 42 & 1593\end{array}$ KLUYVER 79 ZPHY C 2351
RICHARD 79 BATAVIA CONF. RICHARD 79 BATAVIA CONF.
SAKAMOTO 79 NP B 158410.

$\begin{array}{ll}\text { ALLEN } & 80 \text { BRESSANONE } 175 \\ \text { ASTON } & 80 \mathrm{PL} 93 \text { B } 517\end{array}$

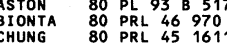

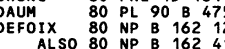

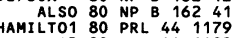
$\begin{array}{lllll}\text { HAMILTO2 } & 80 & \text { PRL } & 44 & 1182 \\ \text { KAMAE } & 80 & \text { PRL } & 44 & 1439\end{array}$ DAUM 81 PL 100 B 439

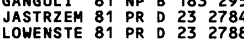
SUMIYOSH 82 PRL 49628 $\begin{array}{llllll}\text { BARNETT } & 83 & \text { PR } & 0 & 27 & 493 \\ \text { BENSINGE } & 83 & \text { PR } & D & 27 & 1417\end{array}$ A. A. CARTER
(LOQM) JP +GOOD, GRANNIS, GREEN, LEE, PITTMAN+ (STON+WISC)
(CERN) +ALVEAR, CASTELLI, POROPAT+ (TRST+CERN+IFRJ) ALSTON-GASTOST, HAMILTON+ (LBL+MTHO+BNL) AHRENS, BERKELMAN, CASSEL, DAY, HARDING + (CORN) J.C. KLUUYVER +HASHIMOTO, SAI, YAMAMOTO+ (LALO)
(TOKY)

5TH EUROPEAN SYMPOSIUM ON N-NBAR INTS. BAN+CER EDELSTEIN,
(BNL+CARN+FNAL +SMAS) + ETKIN, BEEN INGER, + (BNL+BRAN+CUNY+SMAS+ MASA) +DOBRZYNSKI ANGELINI, BIII $+{ }^{+}$(
(CDE+PISA)
ESPIGAT, DEFOIX, DOBRZYSSKI+ HAMILTON, PUN, TRIPP, LAZARUS, + (LBL+BNL+MTHO)

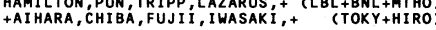
+ HERTZBERGER+ (AMST+CERN+CRAC+MPIM+OXF+RHEL)

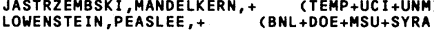
+CHIBA, FUJI I, IKEDA, TAKADA, + (TOKY+KEK + TSUK) +BLOCKUS, BURKA, CHIEN, CHRISTIAN+
BENSINGER, CHUNG (JHU)
(BRAN+BNL+CINC+FSU+SMAS)

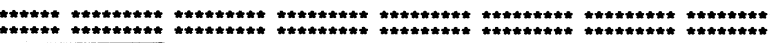
$\mathrm{h}(2030) \quad 16 \mathrm{H}(2030, \mathrm{JPG}=4++) \mathrm{I}=0$

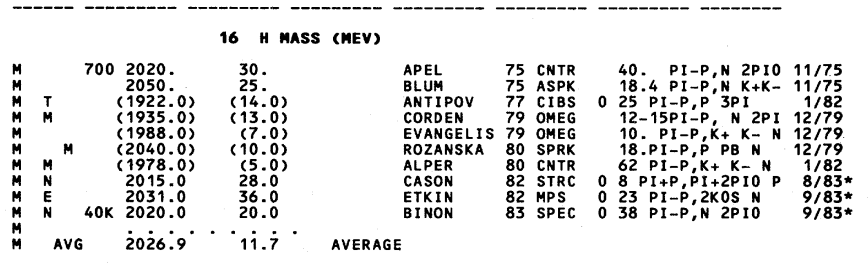

$M$ M I $=0, J P=4+$ FROM AMPLITUDE ANALYSIS ASSUMING ONE PION EXCHANGE

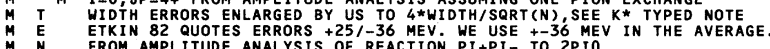

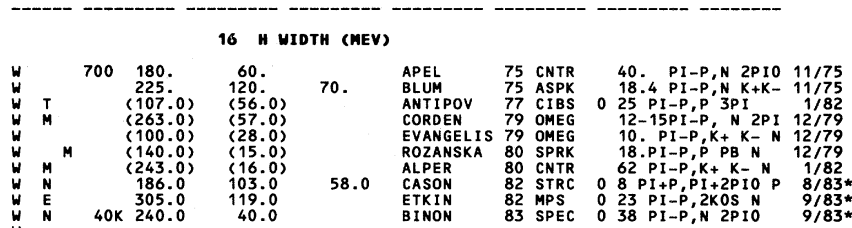
avg $2222^{\circ} \mathbf{2}^{\cdots} 2 \dot{8} . \dot{4}^{\cdot}$ average

W M I $=0, J P=4+$ FROM AMPLITUDE ANALYSIS ASSUMING ONE PION EXCHANGE W E ETKIN 82 QUOTES ERRORS +36/-119 MEV. WE USE +-119 MEV.
FROM AMPLITUDE ANALYSIS OF REACTION PI+PI- TO 2 PIO

p1 16 H PARTial decay modes

${ }_{p 1}^{p 1}$ H. INTO PI PI
HNTO K KBAR
IN

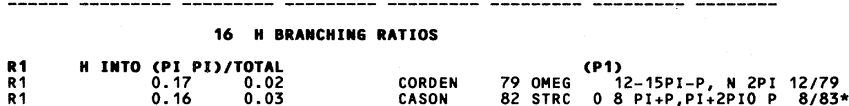
R1 AVG $0.167 \cdot 0.017$ aVerage

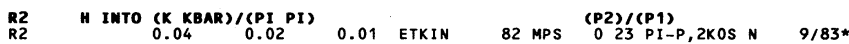
******* ********** ********** ********** ***************************************

WAGNer 74 LONDON CONF. F. HAGMER, RAPPORTEURS TALK (MPIM)

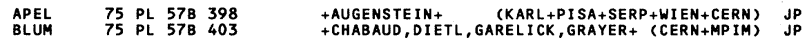
ANTIPOV 77 NP B 11945 +BUSNELLO, DAMGAARD, KIENZLE+ (CERN+SERP)
C

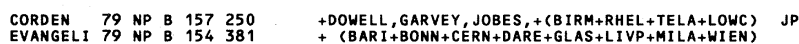
ALPER 80 PL 94 B 422 +BECKER, + (AMST+CERN+CRAC+MP IM+ OXF+RHEL)

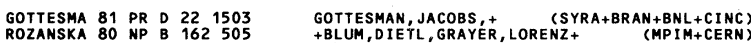

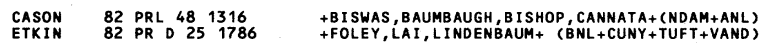

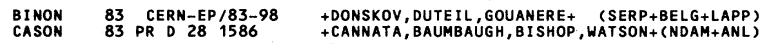

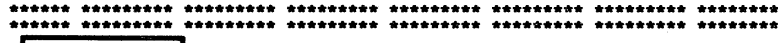

$\delta(2040) 17$ DELTA(2040,JPG=4+-) I=1

$\longrightarrow \quad \begin{aligned} & \text { SEEN IN PARTIAL WAVE ANALYSIS OF THE K KBAR AND } \\ & \text { PI PII-PIO SYSTEMS. }\end{aligned}$ PITPI-PIO SYSTEMS. OMATTE FROM TABLE.
NEES CONFIRMATION. OMITED

17 DeLtTA(2040) MASS (MEV)

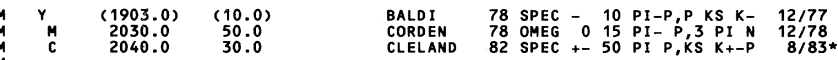
avg $2037.4^{\circ} 25.7^{\circ}$ average

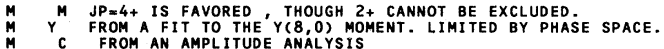

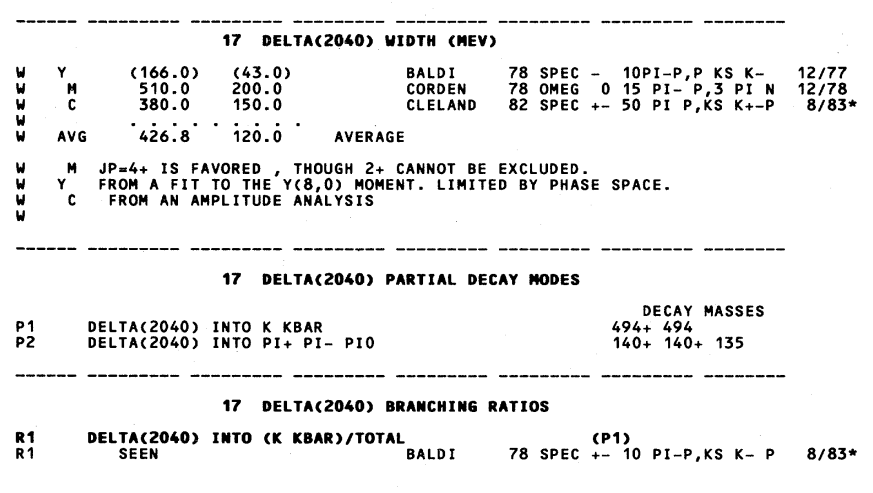


Mesons

Data Card Listings

$\delta(2040), \mathrm{A}(2050), \mathrm{A}(2100), \mathrm{T}$ and $\mathrm{U}$ Regions, $\rho(2150)$

R2 DELTA(2040) INTO (PI+ PI- PIO)/ TOTAL
CORDEN ************************************************** REFERECES FOR DELTA(2040)

$\begin{array}{llllll}\text { BALDI } & 78 & P L & 74 & 413 \\ \text { CORDEN } & 78 & \text { NP } & \text { B } & 136 & 47\end{array}$

DELFOSSE 81 NP B 183349 +BOHRINGER, DORSAZ, HUNGERBULER, + (GENEVA)
DOWELL, GARVY, JOBES + (BIRM+RHEL+TELA+LOWC) JP +DORSAZ, EXTERMANN, GLOOR, WEILL,+ (GEVA+LAUS)

CLELAND 82 NP B 208228

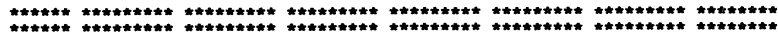

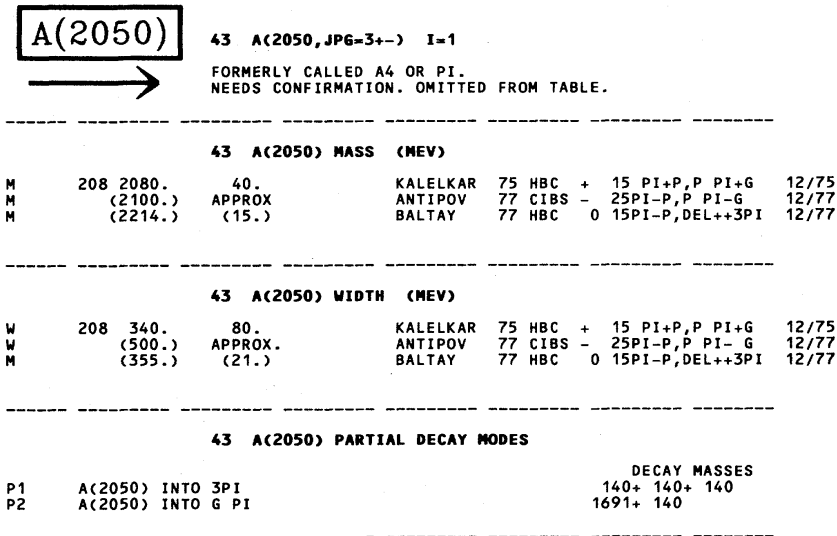

43 A(2050) BRAMCHIMG RATIOS

R1 A(2050) INTO (6 PI)/(ALL 3PI)
ROMINANT

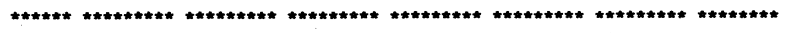

\begin{tabular}{|c|c|c|}
\hline & & REFERENCES FOR A(2050) \\
\hline HusoN & 68 PL 28 \& 208 & + +LUBATT1, BELLINI, BINGHAM, + (ORSA+MILA+LBL) \\
\hline BEMPORAD & 71 NP B 33397 & (CERN+ETH+LOIC+MILA) \\
\hline CLAYTON & 72 NP B 4781 & +MASON, MUIRHEAD, RIGOPOULOS,+ \\
\hline BASTIEN & 73 UPPSALA CONF. 73 & +DUNN, HARRIS, LUBATTI, BINGHAM,+ (SEAT+UCB) \\
\hline OREN & 74 NP B71 189 & +COOPER, F IELDS, RHINES, WHI TMORE, + (ANL+OXF) \\
\hline $\begin{array}{l}\text { DEUTSCHM } \\
\text { KALELKAR }\end{array}$ & $\begin{array}{l}75 \text { NP } 899397 \\
75 \text { THESIS (NEVIS 207) }\end{array}$ & $\begin{array}{l}\text { (ABBCCHW COLLABORATION) } \\
\text { (COLU) }\end{array}$ \\
\hline $\begin{array}{l}\text { ANTIPOV } \\
\text { BALTAY }\end{array}$ & $\begin{array}{ll}77 \\
77 \text { PRL }\end{array}$ & $\begin{array}{l}\text { +BUSNELLO, DAMGAARD, KI ENZLE+ } \\
+ \text { +CAUTIS, KALELKAR }\end{array}$ \\
\hline $\begin{array}{l}\text { MARRIS } \\
\text { CAUTIS }\end{array}$ & $\begin{array}{l}81 \text { ZPHY C } 92275 \\
77 \text { THESIS NEVIS }\end{array}$ & c.V.CAUT \\
\hline BALTAY & 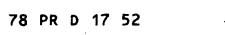 & ITIS, COHEN, CSORNA, KALELKAR + (COLU+BING) \\
\hline
\end{tabular}

:

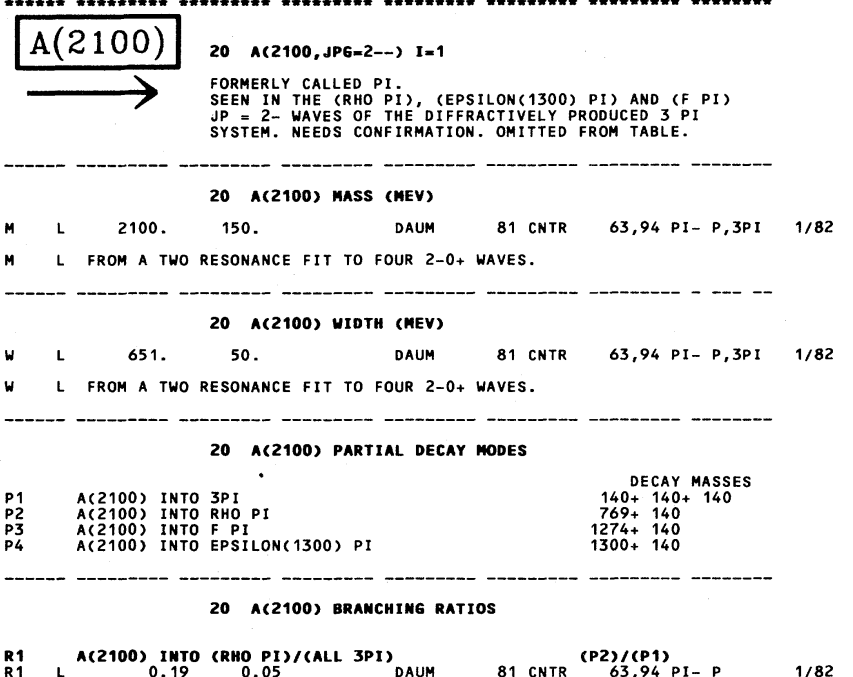

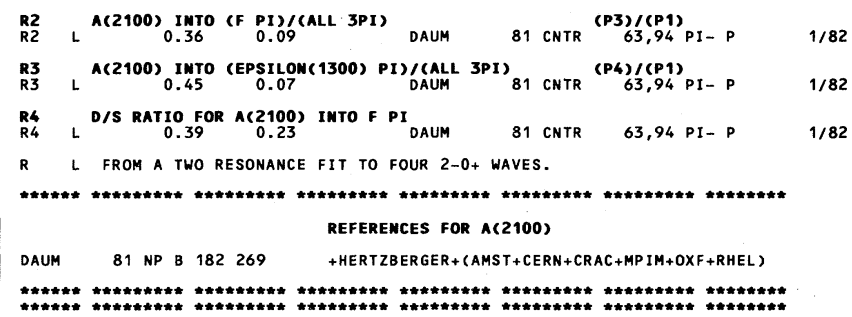

\section{NOTE ON THE T AND U REGIONS}

The observation of broad enhancements at 2190 and $2350 \mathrm{MeV}$ comes from $\overline{\mathrm{p}} \mathrm{p}$ total cross-section measurements (ABRAMS 67), $\bar{p} p$ annihilation measurements (ALSPECTOR 73), $\bar{p}$ p elastic cross-section measurements (COUPLAND 77), and $\bar{p}$ p charge-exchange cross-section measurements (CUTTS 78). The mass regions centered around $2190 \mathrm{MeV}$ and $2350 \mathrm{MeV}$ have been called $T$ and $U$ regions, respectively.

Searches for resonances in exclusive $\bar{p} p$ annihilation channels which could be coupled to the enhancements observed in the $\bar{p}$ p total cross section and in $\bar{p}$ p elastic scattering have been unsuccessful, except for the twobody annihilation channels $\pi^{+} \pi^{-}$and $\pi^{0} \pi^{0}$, where partial-wave analyses have shown that resonances are formed in the 2100-2500-MeV mass region (CARTER 77, DULUDE 78, MARTIN A 80, MARTIN B 80). We have listed the results of these analyses under the headings $\epsilon(2150)$ for the $I=0, J^{P}=2^{+}$wave; $\rho(2150)$ for the $\mathrm{I}=1, \mathrm{~J}^{\mathrm{P}}=1^{-}$wave; $\rho(2250)$ for the $\mathrm{I}=1, \mathrm{~J}^{\mathrm{P}}=3^{-}$wave; $\epsilon(2300)$ for the $I=0, J^{P}=4^{+}$wave; and $\rho(2350)$ for the $\mathrm{I}=1, \mathrm{~J}^{\mathrm{P}}=5^{-}$wave.

Various structures coupled to $\bar{p} p$ and observed in production experiments are listed under the heading $\bar{N} N(1200-3600)$.

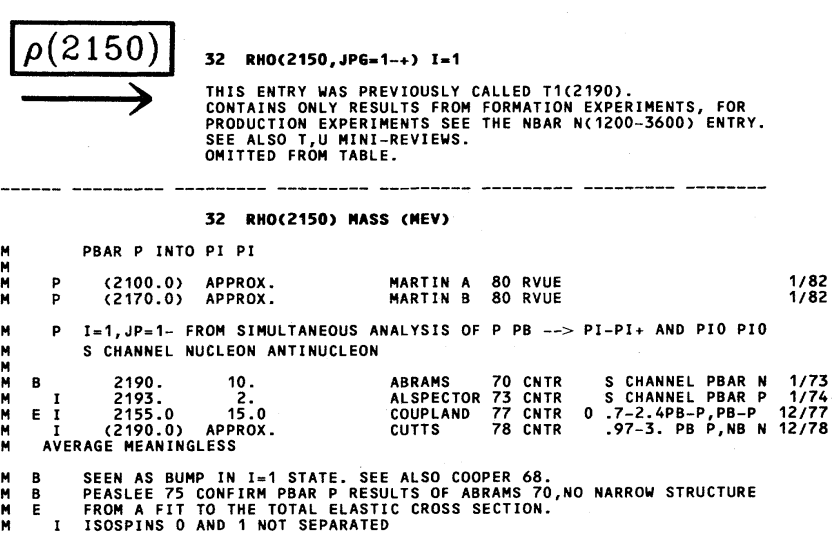




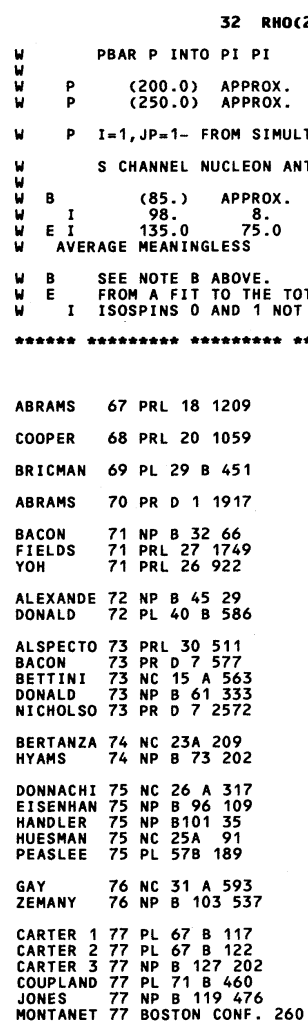

32 RHO(2150) WIOTH (MEV)

$\begin{array}{lllllll}\text { CARTER } & 1 & 78 & N P & \text { B } & 132 & 176 \\ \text { CARTER } & 278 & \text { NP } & 8 & 144 & 467 \\ \text { CUTTS } & 78 & \text { PR } & 0 & 17 & 16\end{array}$

MARTIN 79 PL 86 B 93

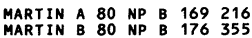

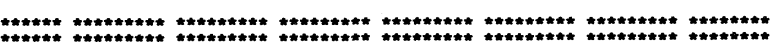

$\epsilon(2150) \quad 42$ EPSILON(2150, JPG=2++) $\quad$ I=0

THIS ENTRY HAS PREVIOUSLY CALLED TO.

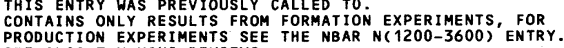

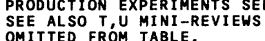

\footnotetext{
M. PBAR P INTO PI PI

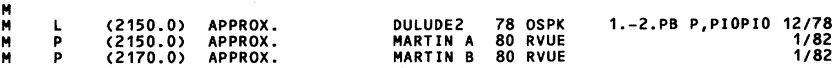

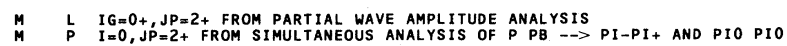
$S$ CHANNEL PBaR P OR NBAR N

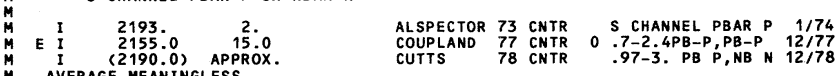

M. AVERAGE MEANINGLESS
M E I FROM A FIT TO THE TOTAL ELASTIC CROSS SECTION.
}

42 EPSILOM(2150) UIDTH (MEV)

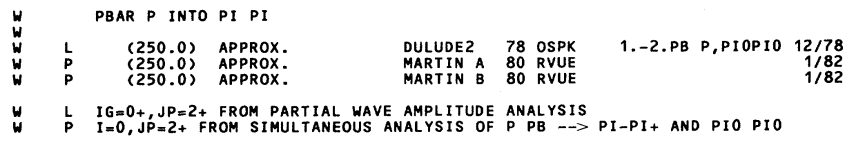

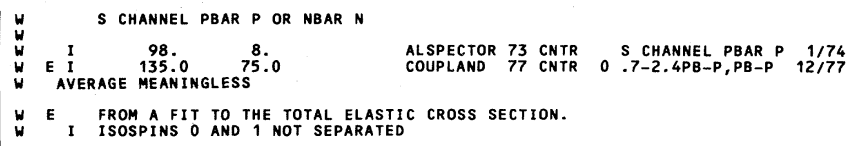

M. A SYSTEMATIC ERRoR ADDED LINEARLY BY US. 82 XI(2220) WIDTh (MEV)

W A SYSTEMATIC ERRoR ADDED LINEARLY BY BY US. 83 SMK3 E+E-, HADRONS GAM 12/83* **********************************************************************

\section{REFERENCES FOR XI(2220)}

EINSWEIL 83 BRIGHTON CONF. K. F. EINSWEILER +MARKIII COLLABORATION (SLAC)
HILIN 83 CORNELL CONF.
(CIT)

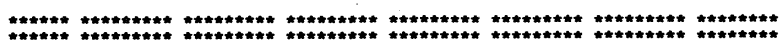
$\mathrm{g}_{\mathrm{T}}(2240) 83 \quad 6 / \mathrm{T}(2240, \mathrm{JPG}=2++)$ I=0 $\longrightarrow \begin{gathered}\text { THIS ENTRY CONTAINS VARIOUS STATES OBSERED IN } \\ \text { PARIAL WAVE ANAYSSES OF THE PHI PHI SSYTEM. } \\ \text { NEED CONFIRMATION. OMITTE FROM TABLE. }\end{gathered}$

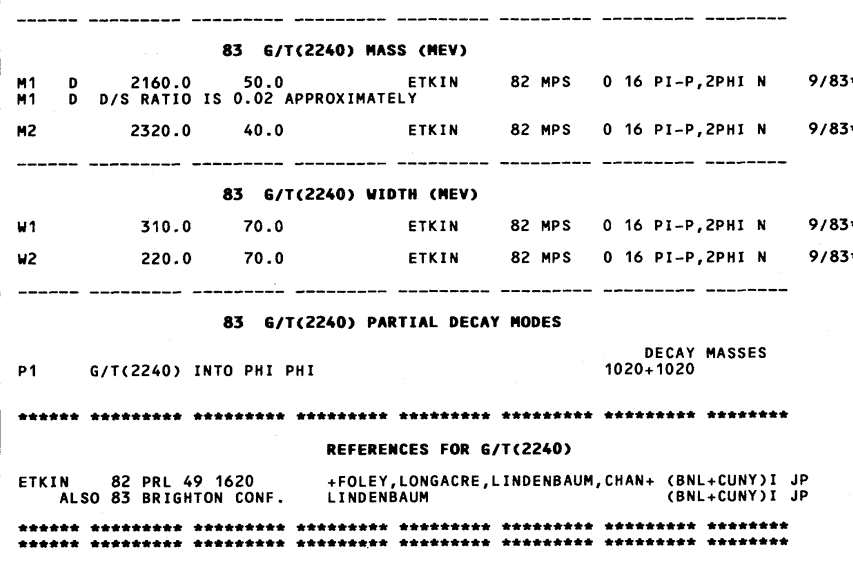




\section{Mesons}

$\rho(2250), \epsilon(2300), \rho(2350)$
4 RHO(2250,JPG=3-+) I=1

CONTAINS ONLY RESULTS FROM FORMATION EXPERIMENTS, FOR
PROOUCTION EXPERTMENTS SEE THE NBAR N $(1200-3600)$ ' ENTRY. PROUCION EXPERIMETS
SEE ALS T, M MINI-R
OMITIED FOM TABLE

44 RHO(2250) MASS (MEV)

PBAR P INTO PI PI OR K KB

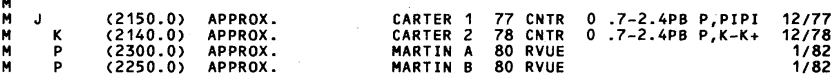

$M$ I $I=1$, P $=3$ - FROM AMPLITUDE ANALYSIS.

$K$
$M$
$M$ S CHANNEL NUCLEON ANTINUCLEON

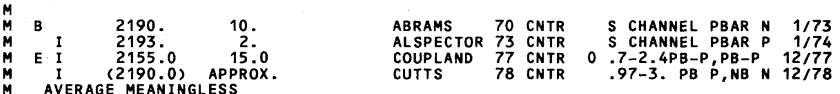

$M$
$M$
$M$ M E FROM A FIT TO THE TOTAL ELASTIC CROSS SECTION.
$M$ I ISOSPINS O AND 1 NOT SEPARATED

\section{RHO(2250) MIDTH (MEV)}

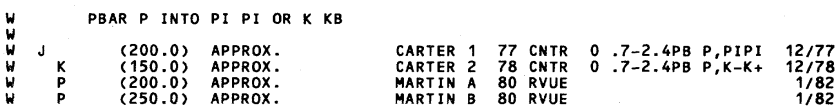

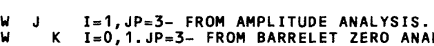

$K$
$\mathrm{P}$ $S$ CHANNEL NUCLEON ANTINUCLEON

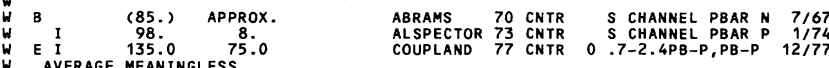

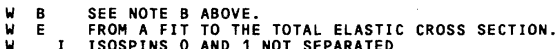

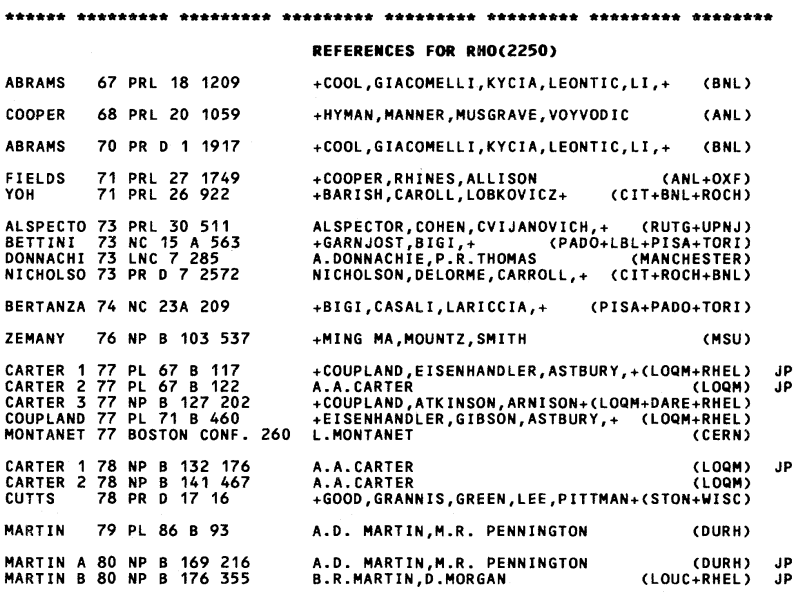

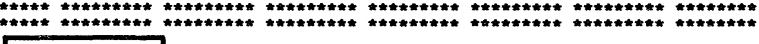

$\epsilon(2300)$ 41 EPSILOM(2300, JPG $=4++)$ I I 0

THIS ENTRY WAS PREVIOUSLY CALLED UO(2350) CONTAINS ONLY RESULTS FROM FORMATION EXPERIMENTS, FOR
PRODUCTION EXPER IMENTS SEE THE NBAR N $1200-3600)$ ENTRY SEE ALSO T, U MINI-REVIEWS.

41 EPSILON(2300) MASS (MEV)

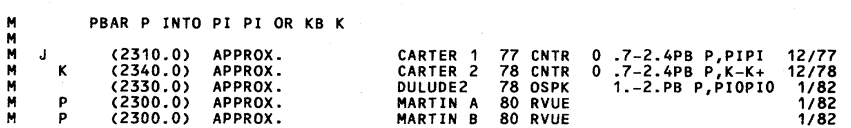

$M$ J $I=0, J P=4+$ FROM AMPLITUDE ANALYSIS.

$\stackrel{M}{M} \quad \begin{aligned} & K \\ & M\end{aligned} \quad \begin{aligned} & =00, \\ & I=0, J P=4+\end{aligned}$

\section{Data Card Listings}

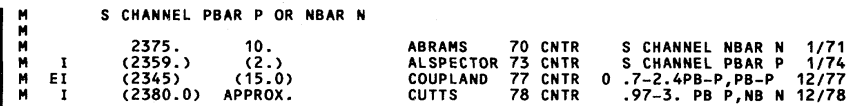

$M$
$M$

41 EPSILOM(2300) WIDTH (MEV)

PBAR P INTO PI PI OR KB $K$

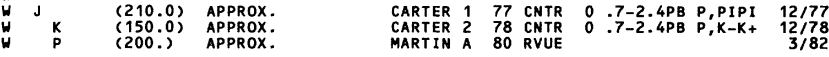

U J $\mathrm{I}=\mathrm{I}=0, \mathrm{JP}=4+$ FROM AMPLITUDE ANALYSIS.

P PBAR --> PI-PI+ AND PIO PIO

$S$ CHANNEL PBAR P OR NBAR N

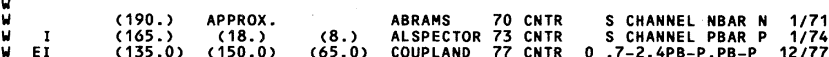
Y E
I

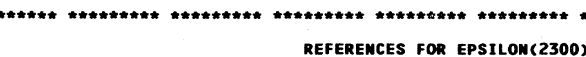

BRICMAN 69 PL 29 B 451

ABRAMS 70 PR D 11917

$\begin{array}{lllll}\text { FIELDS } & 71 & \text { PRL } & 27 & 1749 \\ \text { YOH } & 71 & \text { PRL } & 26 & 922\end{array}$

EASTMAN 72 NP B 5129

ALSPECTO 73 PRL 30511

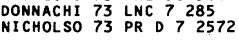

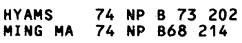

$\begin{array}{llllll}\text { DONNACHI } & 75 & \text { NC } & 26 & \text { A } & 317 \\ \text { EI SENHAN } 75 & \text { NP } & \text { B } 96 & 109\end{array}$

$\begin{array}{lllllll}\text { CARTER } & 1 & 77 & \text { PL } & 67 & \text { B } & 117 \\ \text { CARTER } & 2 & 77 & \text { PL } & 67 & \text { B } & 122\end{array}$

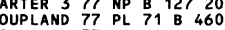

MONTANET 77 BOSTON CONF.

$\begin{array}{lllllll}\text { CARTER } & 1 & 78 & \text { NP } & \text { B } & 132 & 176 \\ \text { CARTER } & 2 & 78 & \text { NP } & \text { B } & 141 & 467\end{array}$

$\begin{array}{llllll}\text { CUTTS } & 78 & \text { PR } & \text { D } & 17 & 16 \\ \text { DULUDE } & 78 & \text { PL } & 79 & \text { B } & 329 \\ \text { DULUDE2 } & 78 & \text { PL } & 79 & \text { B } & 335\end{array}$

MARTIN 79 PL $86 \quad 8 \quad 93$

\begin{tabular}{l} 
BOWCOCK 80 LNC 2821 \\
MARTIN A 80 NP B 169216 \\
MARTIN B 80 NP B $176 \quad 355$ \\
\hline
\end{tabular}

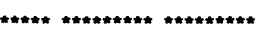

$\stackrel{\rho(2350)}{\longrightarrow}$

33 RHO(2350,JPG=5-+) $I=1$

THIS ENTRY WAS PREVIOUSLY CALLED U1(2400)

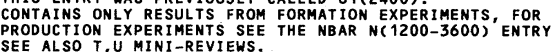
SEE ALSO T,U MINI-REVI

33 RHO(2350) MASS (MEV)

$M$
$M$
$M$

$\begin{array}{cccc}M & k & (2480.0) & \text { APPROX. } \\ M & \mathrm{P} & (2500.0) & \text { APPROX. } \\ M & (22500) & \text { APPROX. } \\ M & (2300.0) & \text { APPROX. }\end{array}$ $2250.0)$
$2300.0)$
APPROXX.

+FERRO-LUZZ1, BIZARD, + (CERN+CAEN+SACL) COOL, GIACOMELLI, KYCIA, LEONTIC,LI, + (BNL) (COOPER, RHINES, ALLISON
+ +BARISH, CAROLL, LOBKOVICZ+
+OXF) +MING MA, OH, PARKER, SMITH, SPRAFKa (MSU) ALSPECTOR, COHEN, CVIJANOVICH, + (RUTG+UPNJ) A.D DNNACHIE, P.R.THOMAS
NICHOLSON, DELORME, CAROLL, + (MANCHESTER) + JONES, WEILHAMMER, BLUM, +
+MOUNTZ, ZEMANY, SMITH A. DONNACHIE, P.R. THOMAS (MANCHESTER)
EISENHANDLER, GIBSON, + COUPLAND, EISENHANDLER, ASTBURY, + (LOQM+RHEL) JP COUPLAND ATKINSON, ARNI SON+ (LOQM+DARE+RHEL) +EISENHAND́LER, GIBSON, ASTBURY, +
L. MONTANET
(LOQMREEL)
(CERN)

A.A.CARTER
A.A.CARTER

(LOQM)
(LOQM) +GOOD, GRANNIS, GREEN, LEE, PITTMAN+(STON+WISC) $\begin{array}{lll}\text { +LANOU, MASSIMO, PEASLEE+ } & \text { (BROW+MIT+BARI) } \\ \text { (BROW +MIT+BARI) } & \text { JP }\end{array}$ A.D. MARTin,M.R. PENNINGTON (DURH) J.E.BOWCOCK, D.C. PODGSON
A.D.MARTIN,M.R. PENAINGTON
B.R.MARTIN, (BIRM)
(DURH) JPRAN
(LOUC+RHEL) JP

M J $I=1, J P=5$ - FROM AMPLITUDE ANALYSIS.

$\begin{array}{lll} & \\ \text { MARTIN A } 80 & \text { RVUE } & 1 / 82 \\ \text { MARTIN B } & 80 \text { RVUE } & 1 / 82\end{array}$

$M \quad \begin{array}{ll}I=0,1, J P=5-F R O M \\ P\end{array}$ $S$ CHANNEL NUCLEON ANTINUCLEON

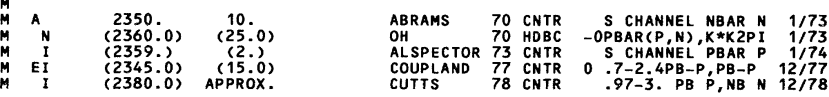

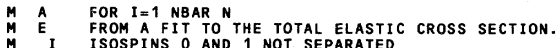

$M$
$M$
$M$

33 RHO(2350) WIDTH (MEV)

$W$
$W$
$W$

$\begin{array}{llll}W & J & (210.0) & \text { APPROX } \\ W & K & (150.0) & \text { APPROX } \\ W & P & (300.0) & \text { APPROX. } \\ W & P & (250.0) & \text { APPROX }\end{array}$

$(300.0)$ APPROX.
$(250.0)$ APPROX.

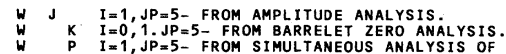

$\begin{array}{llllllll}\text { CARTER } & 1 & 77 & \text { CNTR } & 0 & -7-2.4 P B & \text { P,PIPI } & 12 / 77 \\ \text { CARTER } & 2 & 78 & \text { CNTR } & 0 & : 7-2.4 P B & \text { P }, K-K+ & 12 / 78\end{array}$ MARTIN A 80 RVUE
MARTIN B 80 RVUE 
For notation, see key at front of Listings.

Mesons $\rho(2350), \delta(2450), \mathrm{r}(2510), \mathrm{e}^{+} \mathrm{e}^{-}(1100-2200), \overline{\mathrm{N}} \mathrm{N}(1200-3600)$

W S ChanNel NUCleon ANTINUCleon

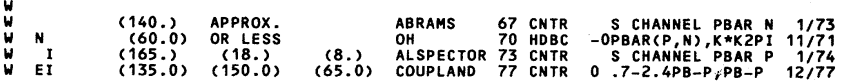

W E FROM A FIT TO THE TOTAL ELASTIC CROSS SECTION.

N N NO EVIDENCE FOR THIS BUMP SEN IN THE PBAR P DATA OF CHAPMAN 71
NAROW STATE NOT CONF IRMED BY OH 73 WITH MORE DATA.

*******************************************************************

ABRAMS 67 PRL 181209

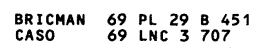

\begin{tabular}{llllll} 
ABRAMS & 70 & PR & 0 & 1 & 1917 \\
\hline OH & 70 & PRL & 24 & 1257
\end{tabular}

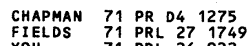

YOH 71 PRL 26922

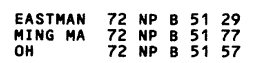

$\begin{array}{lllll}\text { ALSPECTO } & 73 & \text { PRL } & 30 & 511 \\ \text { NICHOLSO } & 73 & \text { PR } D & 7 & 2572\end{array}$

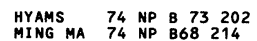

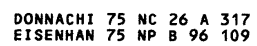

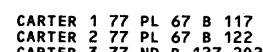

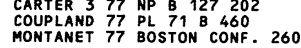

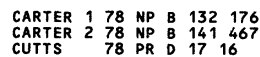

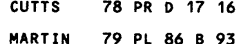

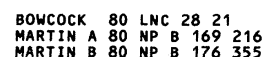

\section{REFERENCES FOR RHO(2350)}

+COOL, GIACOMELLI, KYCIA, LEONTIC, LI, + (BNL)

+ +FERRO-LUZZI, BI ZARD, + (CERN+CAEN+SACL)
+ CONTE, BENZ,

+ COOL, GIACOMELLI, KYCIA, LEONTIC, LI, + (BNL)
+ PARKER, EASTMAN, SMITH, SPRAFKA, MA
(MSU)

+GREEN, LYS, MURPHY, RING, + (MICH)

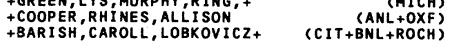

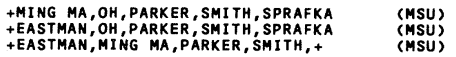

ALSPECTOR, OOHEN, CVIJANOVICH, + (RUTG (RPPNJ)
NI CHOLSON, DELORME, CARROLL, $+{ }^{+}$(CIT+ROCH + BNL)

+ JONES, WEIL HAMMER, BLUM, +
+MOUNTZ, ZEMANY, SMITH
(CERN+MPIM)

A. DDNACHIE, P.R. THOMAS (MAMCHESTER)

+ COUPLAND, EISENHANOLER, ASTBURY, + (LOQM+RHEL) JP

A.A.CARTER

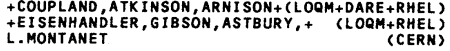

A.A. CARTER
A.A.CARTER (LOOM) JP

A.A. CARTER
+GOOD, GRANIS, GREEN, LEE, PITTMAN+(STON+WISC)

A.D. MARTIN,M.R. PENNINGTON (DURH)

J.E.BOWCOCK, D.C. HODGSON (BIRM)

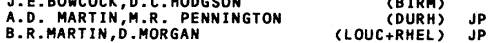

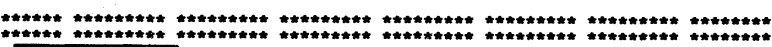

$\delta(2450)$ 24 DELTA(2450, JPG 6+6-) I=1

SEEN IN PARTIAL WAVE ANALYSIS OF THE K KBAR
SYSTEM. NEEDS CONF IRMATION. OMITTED FROM TABLE

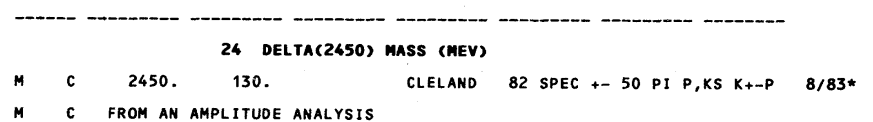

M. C from an amplitude analysis

24 DeLta(2450) Uidth (MEV)

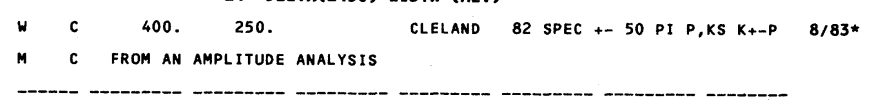

24 DELTAC2650) PARTIAL DECAY MODES

P1 DELTA(2450) INTO K KBAR DECAY MASSES
$498+498$

REFERENCES FOR DELTAC2450)

CLELAND 82 NP B 208228 +DELFOSSE, DORSAZ, GLOOR (OURH+GEVA+LAUS+PITT)

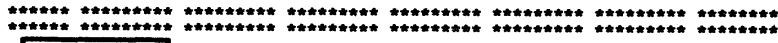

$\mathrm{r}(2510) \quad 89$ R(2510, JPG $6+6+1)$ I=0

$\longrightarrow \quad \begin{gathered}\text { SEEN IN PIO PIO } \\ \text { NeEOS CONF IRMATion. OMItTed from table. }\end{gathered}$

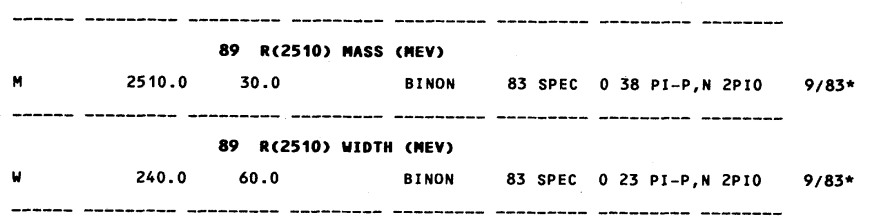

89 R(2510) PARTIAL DECAY MODES

P1 R(2510) INTO PI PI

DECAY MASSES
$135+135$
REFEREMCES FOR R(2510)

BINON 83 CERN-EP/83-98 +DONSKOV, DUTEIL, GOUANERE+ (SERP+BELG+LAPP) JP

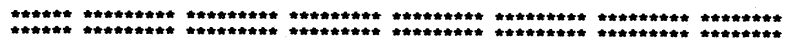

\begin{tabular}{|c|c|}
\hline$e^{+} e^{-}(1100-2200)$ & 7 E+ E-(1100-2200,JPG=1-) $I=$ \\
\hline & 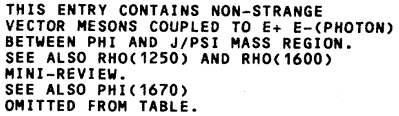 \\
\hline
\end{tabular}

\begin{tabular}{|c|c|c|c|c|c|c|c|}
\hline & & $7 \mathrm{E}+$ & $-(1100-22)$ & (00) MASSES & AND WIDTHS & (MEV) & \\
\hline M & $\begin{array}{r}(1097.0) \\
(31.0)\end{array}$ & $\begin{array}{l}(16.0) \\
(24.0)\end{array}$ & $\begin{array}{l}(19.0) \\
(20.0)\end{array}$ & $\begin{array}{l}\text { BARTALUCC } \\
\text { BARTALUCC }\end{array}$ & $\begin{array}{l}79 \text { OSPK } \\
79 \text { OSPK }\end{array}$ & $\begin{array}{l}7 \text { GAM } P, E+E-P \\
7 \text { GAM } P, E+E-P\end{array}$ & $\begin{array}{l}12 / 79 \\
12 / 79\end{array}$ \\
\hline$\stackrel{M}{W}$ & $\left(\begin{array}{c}1830.0) \\
(120.0)\end{array}\right.$ & $\begin{array}{l}\text { APPROX. } \\
\text { APPROX. }\end{array}$ & & $\begin{array}{l}\text { PETERSON } \\
\text { PETERSON }\end{array}$ & $\begin{array}{l}78 \text { SPEC } \\
78 \text { SPEC }\end{array}$ & $\begin{array}{l}\text { GAM } p, K+K-p \\
\text { GAM } P, K+K-P\end{array}$ & \\
\hline $\begin{array}{ll}M \\
W\end{array}$ & $\begin{array}{l}(2130 .) \\
(30 .)\end{array}$ & $\begin{array}{l}\text { APPROX. } \\
\text { APPROX. }\end{array}$ & & $\begin{array}{l}\text { ESPOSIT1 } \\
\text { ESPOSIT1 }\end{array}$ & $\begin{array}{l}78 \text { FRAM } \\
78 \text { FRAM }\end{array}$ & $\begin{array}{l}E+E-, K *(892)+\ldots \\
E+E-, K *(892)+\ldots\end{array}$ & $\begin{array}{l}12 / 78 \\
12 / 78\end{array}$ \\
\hline$\stackrel{M}{H} \hat{A}$ & $\begin{array}{r}(1820 .) \\
(30 .)\end{array}$ & $\begin{array}{l}\text { APPROX. } \\
\text { APPROX: }\end{array}$ & & $\begin{array}{l}\text { SPINETTI } \\
\text { SPINETTI }\end{array}$ & $\begin{array}{l}79 \text { RVUE } \\
79 \text { RVUE }\end{array}$ & $\begin{array}{lll}E+E-, 4 & P_{1+-} & 2 G A M \\
E+E-, 4 & P_{1}+- & 2 G A M\end{array}$ & $\begin{array}{l}1 / 82 \\
1 / 82\end{array}$ \\
\hline
\end{tabular}

$\hat{c}$ INTEGRATED CROSS-SECTION OF BARBIELLINI 77, BACCI 77 , ESPOSITO 77.

**********************************************************************

BACCI 75 PL 58 B 481 +BIDOLI, PENSO, STELLA, BALDINI, + (ROMA+FRAS)

BACCI 76 PL 64 B 356 +BIDOLI, PENSO, STELLA, BALDINI, (ROMA+FRAS)

BACCI $77 \mathrm{PL}$ B $68393 \quad$ +DE ZORZI, PENSO, STELLA, BALDINI, +(ROMA+FRAS)
BARBIELL 77 PL B 68397 BARBIELLINI, BARLETTA, + (FRAS+NAPL+PISA+SANI)
BARB

BARTALUC 77 NC A 39374
ESPOSITO 77 PL B 68389
PARTALUCCI BERTOLUCI, BRADASCHACDESY+FRAS)

AMBROS 1078 PL 80 B 141

ESPOSIT1 78 LNC 22305

ESPOSIT1 78 LNC 22305
ESPOSIT2 78 LNC 23.604
PETERSON 78 PR D 183955

BARTALUC 79 NC 49 A 207
DELCOURT 79 BATAVIA CONF.499

DELCOURT 79 BATAVIA CONF. 499
ESPOSITO 79 LNC 2555 S 5 SPINETII 79 BATAVIA CONF. 506

+ CERRITO, BEMPORAD, BROSCO, + (NAPL+PISA+ROMA)

BATTISTONI, CAPON, BACC1, DEZORZ1+ + (FRAS+ROMA)

ESPOSITO, FELICETTI+ (FRAS + NAPL +PADO+ROMA)
+DIXON, EHRLICH, GALIK, LARSON+ (CORN+HARV)

BARTALUCCI, BASINI, BERTOLUCCI+ (DESY+FRAS)

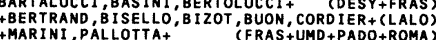

BALDINI 81 LNC 30337 +BATTISTONI, CAPON, BACCI, DEZORZI + (FRAS+ROMA)

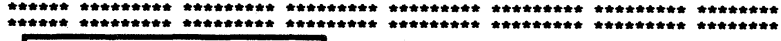

$\stackrel{\overline{\mathrm{N}} \mathrm{N}(1200-3600)}{\longrightarrow}$

51 MBAR M(1200-3600)

THIS ENTRY CONTAINS VARIOUS

THG MASS, NON-STRANGE STRUCTURES

SYSTEM AS WELL BA QUAS QUA-NUCLEAR

SEE ALSO S, T, UO DATA CARD LISTINGS AND MINIREVIEWS. EVIDENEE FOR STRUCTURES COUPLED TO THE ANTI-HYPER
NUCLEON COR C.C.) SYSTEM IS LISTED UNDER $K(2200)$.

51 mBar m(1200-3600) MASSES AMd wIOTHS (MEV)

M W 1210. 5.0 RICHTER 83 CNTR O STOPPED PBARS 9/83*

M W (1395.) PAVLOPOUL 78 CNTR STOPPED PBARS $1 / 78$

$M$ W 1638 . $3.0 \quad$ RICHTER 83 CNTR O STOPPED PBARS 9/83*

M W (1646.) PAVLOPOUL 78 CNTR STOPPED PBARS 1/78

$M$
$M G$ (1684.) PAVLOPOUL 78 CNTR STOPPED PBARS $1 / 78$

$\begin{array}{llllllll}M & \text { W } & 1694 . & 2.0 & \text { RICHTER } 83 \text { CNTR } & 0 & \text { STOPPED PBARS } & 9 / 83 * \\ \text { M W } & 1771 . & 1.0 & \text { RICHTER } & 83 \text { CNTR } & 0 \text { STOPPED PBARS } & 9 / 83\end{array}$

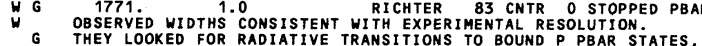

$G$ THEY LOOKED FOR RADIATIVE TRANSITIONS TO BOUND P PBAR STATES,

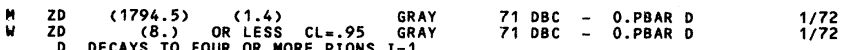

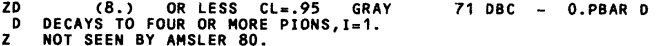

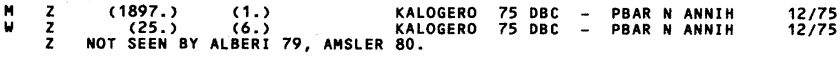

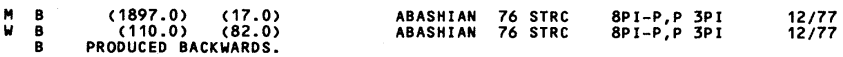

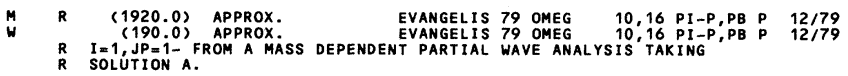

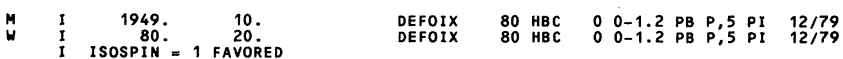


Mesons

$\overline{\mathrm{N}} \mathrm{N}(1200-3600), X(1900-3600)$
Data Card Listings

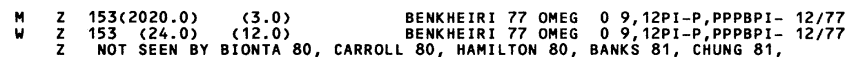

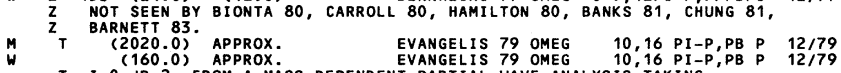
TT $I=0, \begin{aligned} & (160,0) \\ & \text { SOLUTION }=2+\text { FR }\end{aligned}$

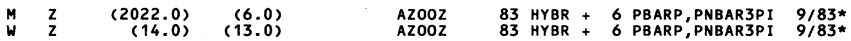

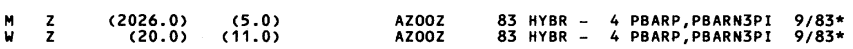

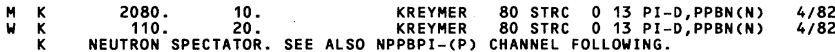

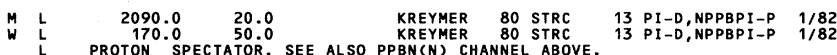

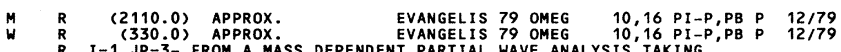
$\begin{array}{ll}R & (330,0) \\ R & I=1, J P 3-F R O H \\ R & \text { SOLUTION } A .\end{array}$

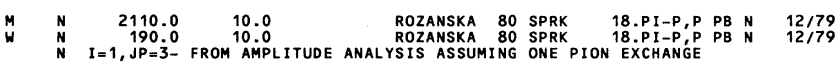

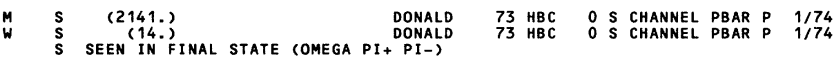

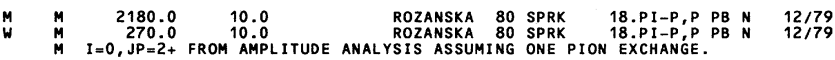

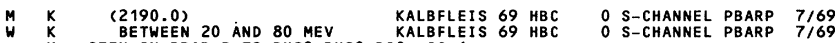
$K$
$K$
$K$

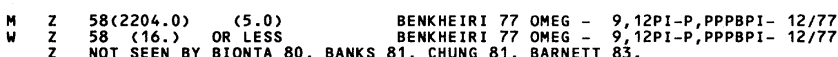

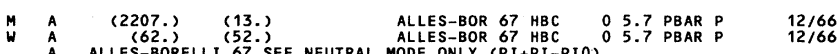

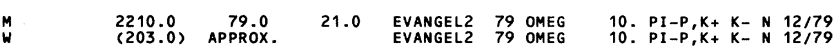

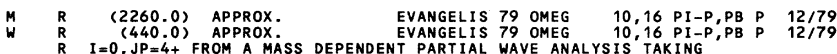

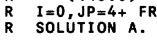

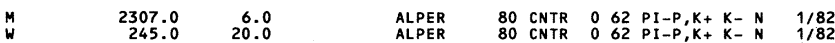

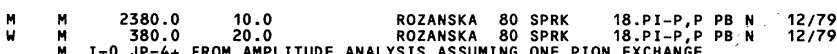

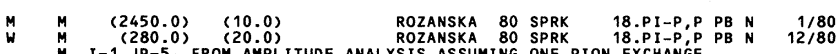

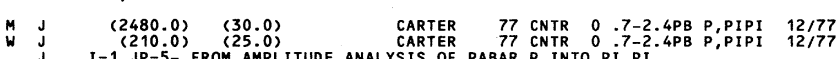

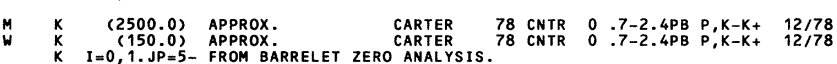

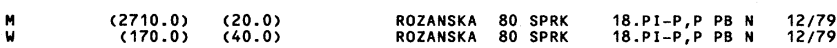

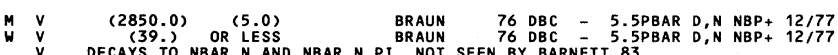

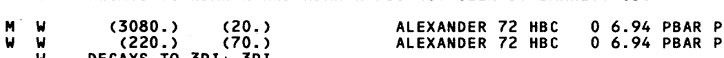

W NECAYS TO 3 PPI 1 3PI- 75 WITH 1.5 TIMES MORE DATA

$\begin{array}{cccc}M & X & (3370 .) & (10 .) \\ W & X & (150 .) & (40 .) \\ X & \text { DECAYS TO } & 4 P I+4 \mathrm{PI}-\end{array}$

$\begin{array}{llll}M & Y & (3390 .) & (20 .) \\ y & y & (220) & (100)\end{array}$

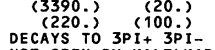

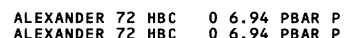

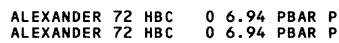

$\checkmark$ NOT SEEN BY KaLeLKar 75 WITH 1.5 TIMES MoRe DATA

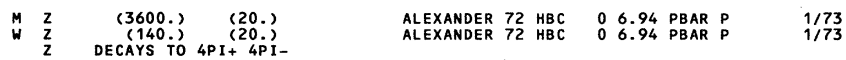

\begin{tabular}{|c|c|}
\hline & $200-3600)$ \\
\hline 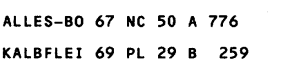 & $\begin{array}{l}\text { ALLES-BORELLI, FRENCH, FRISK, } \quad \text { (CERN +BONN) G= } \\
\text { G.KALBFLEISCH, R.STRAND, V.VANDERBURG (BNL) }\end{array}$ \\
\hline $\begin{array}{l}\text { ALEXANDE } 70 \text { PRL } 2563 \\
\text { KALBELEI } 70 \text { PHILAD.CONF.P. } 409\end{array}$ & $\begin{array}{l}\text { PBR-NIR, DAGAN, GIDAL, GRUNAAUS+ } \\
\text { G.KALBFLISCH AND D.MILLER REVUES (TELLAVIV) } \\
\text { GBNL) }\end{array}$ \\
\hline 71 PRL 261491 & +HAGERT, KALOGEROPOULOS \\
\hline 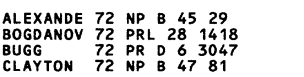 & 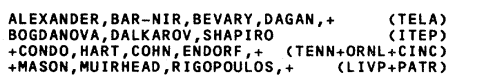 \\
\hline
\end{tabular}

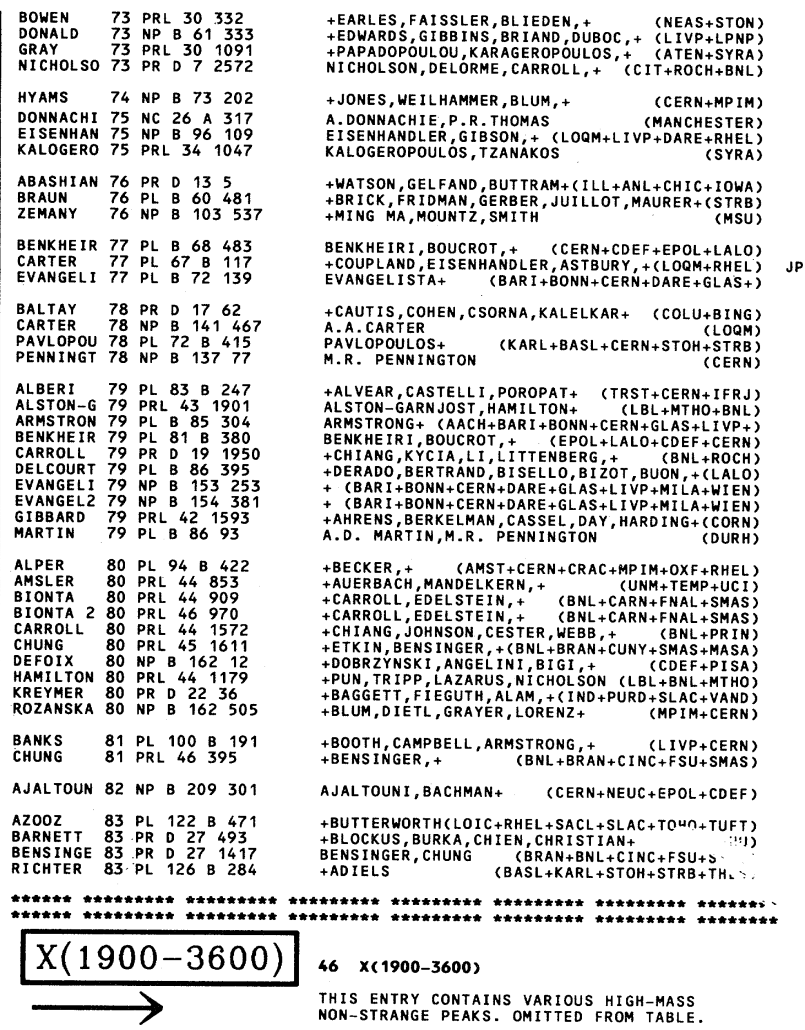

The high-mass region is covered nearly continuously by evidence for peaks of various widths and decay modes. As a satisfactory grouping into particles is not yet possible, we list all the $\mathrm{Y}=0$ bumps coupled neither to $\overline{\mathrm{N}} \mathrm{N}$ nor to $\mathrm{e}^{+} \mathrm{e}^{-}$, and having $\mathrm{M}>1900 \mathrm{MeV}$, together, ordered by increasing mass. Note that ANTIPOV $72\left(\pi^{-}\right.$p $\rightarrow$ p MM$^{-}$at 25 and $\left.40 \mathrm{GeV} / \mathrm{c}\right)$ see no narrow bumps.

$1 / 73$
$1 / 73$
$1 / 73$
$1 / 733$
$1 / 73$
$1 / 73$

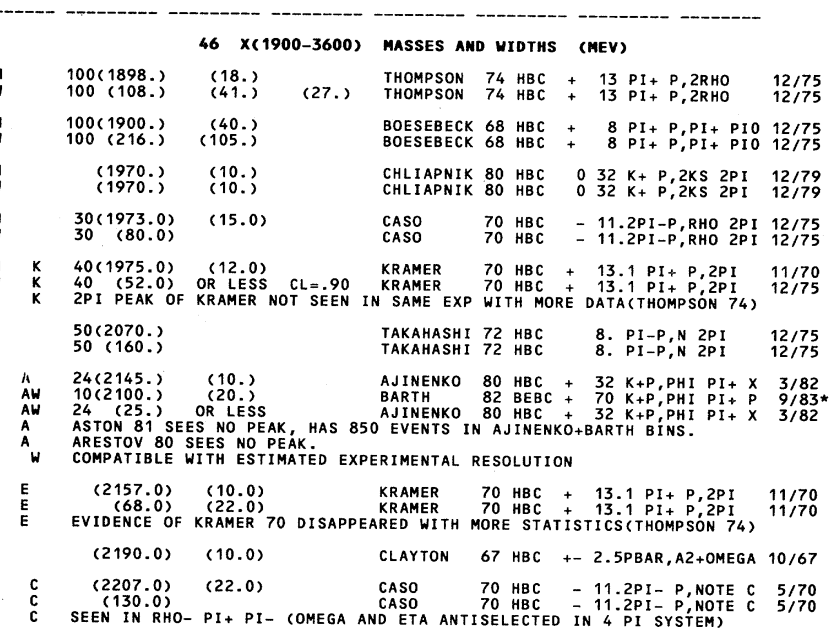


For notation, see key at front of Listings.

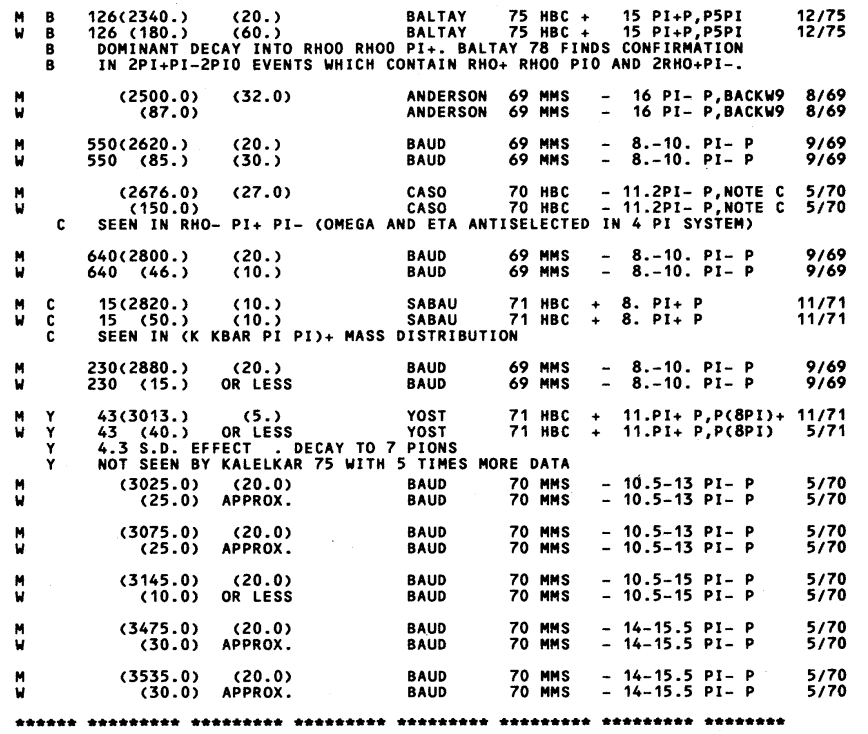

REFERENCES FOR $x(1900-3600)$

CLAYTON 67 HEIDBG.CONF.P. 57 +MASON, MUIRHEAD, FILIPPAS+ (LIVERPOOL +ATHENS) BOESEBEC 68 NP B 4501 BOESEBECK, DEUTSCHMANN, + (AACHEN+BERLIN+CERN)

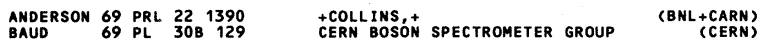
BAUD $70 \mathrm{PL} 313549$ CERN BOSON SPECTROMETER GROUP
(CERN)

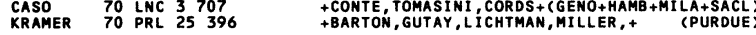

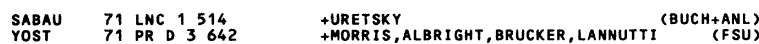
TAKAHASH 72 PR D 61266 TAKAHASHI, BARISH, + (TOHO+PENN+NDAM+ANL) THOMPSON 74 MP B69 220 +GAIDOS,MCILHAIN, MILLER, MULERA, + (PURD) BALTAY 75 PRL 35891979 +CAUTIS, COHEN, KALELKAR, PISELLO, + (COLU+BING)

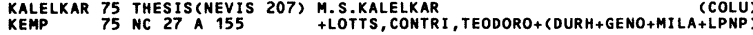
BALTAY 78 PR $D 1752 \quad$ CCAUTIS, COHEN, CSORNA, KALELKAR+ (COLU+BING)

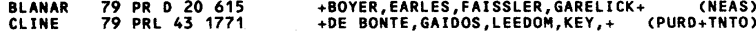
AJINENKO 80 PL 95 B $451, \quad$ CHLIAPNIKOV,
CHLIAPNI 80 ZPHY C $3285 \quad$ CHLIAPNIKOV, GERDYUKOV, (SERP+BELG+MONS+SACL)
(SERP+BRUX+MONS) ASTON 81 NP B 189205 (BONN+CERN+EPOL+GLAS+LANC+MCHS+ORSA+RHEL++) BARTH 82 PL 117 B 267 +DREVERMANN+(BEL G+CERN+GENO+MONS+NI JM+SERP) ATKINSON 83 CERN-EP/83-106 + (BONN+CERN+GLAS+LANC+MCHS+IPNP+RHEL + SHEF)

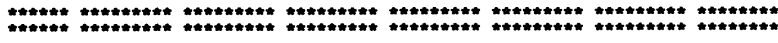

\section{NOTE ON THE CHARMONIUM SYSTEM}

We group into this system those meson states commonly believed to consist of charmed-quark-charmedantiquark pairs. Since the discovery of the $J / \psi(3100)$ (AUBERT 74, AUGUSTIN 74), this family has increased to 11 , of which we tabulate 10 as wellestablished particles. Figure 1 shows the states of charmonium below the $\psi(3685)$, interpreted by the charmonium model, as of January 1984.

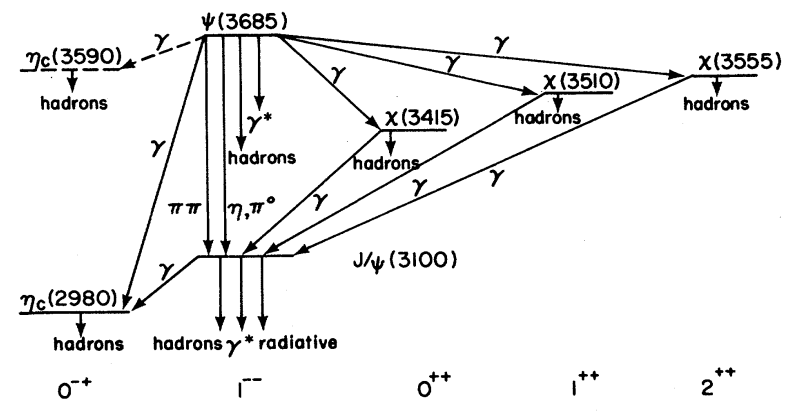

Fig. 1. The current state of knowledge of the charmonium system and transitions, as interpreted by the charmonium model. Uncertain states and transitions are indicated by dashed lines. The notation $\gamma^{*}$ refers to decay processes involving intermediate virtual photons, including decays to $\mathrm{e}^{+} \mathrm{e}^{-}$and $\mu^{+} \mu^{-}$.

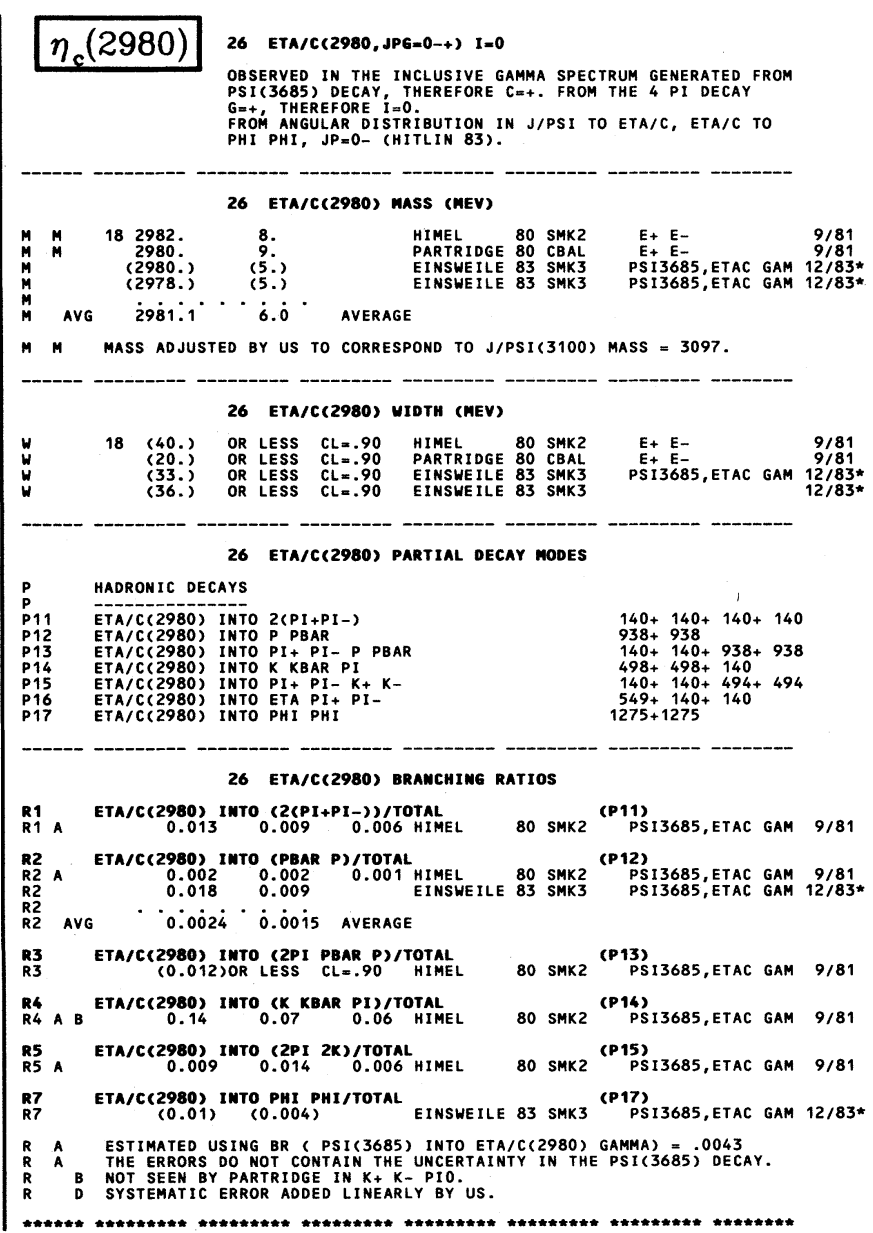


Mesons

Data Card Listings

$\eta_{\mathrm{c}}(2980), \mathrm{J} / \psi(3100)$

\begin{tabular}{|c|c|c|}
\hline & REFEREMCES FOR ETA/C(2980) \\
\hline вLоOM & 79 FERMILAB SYMP. 92 & (CIT+HARV+PRIN+SLAC+STAN) \\
\hline $\begin{array}{l}\text { HIMEL } \\
\text { PARTRID }\end{array}$ & $\begin{array}{l}80 \text { PRL } \\
80 \text { PRL }\end{array}$ & 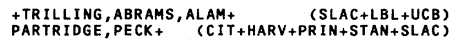 \\
\hline 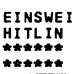 & 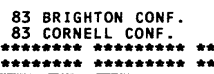 & 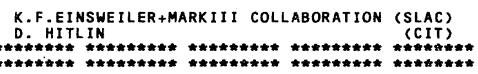 \\
\hline $\mathrm{J} /$ & $3100)$ & \\
\hline
\end{tabular}

70 J/PSI(3100) MASS (MEV)

WE USE INDEPENDENT MEASUREMENTS OF THE J/PSI 3100 )
MASS. THE PSI (3685) MASS AND THE MASS DIFFERENCE TO
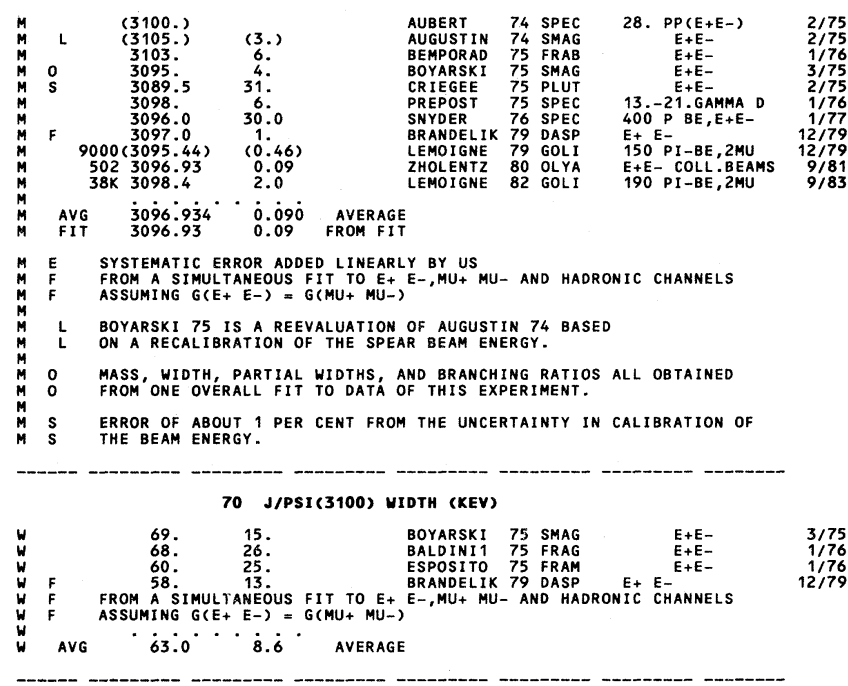

70 J/PSI(3100) PARTIAL DECAY MODES
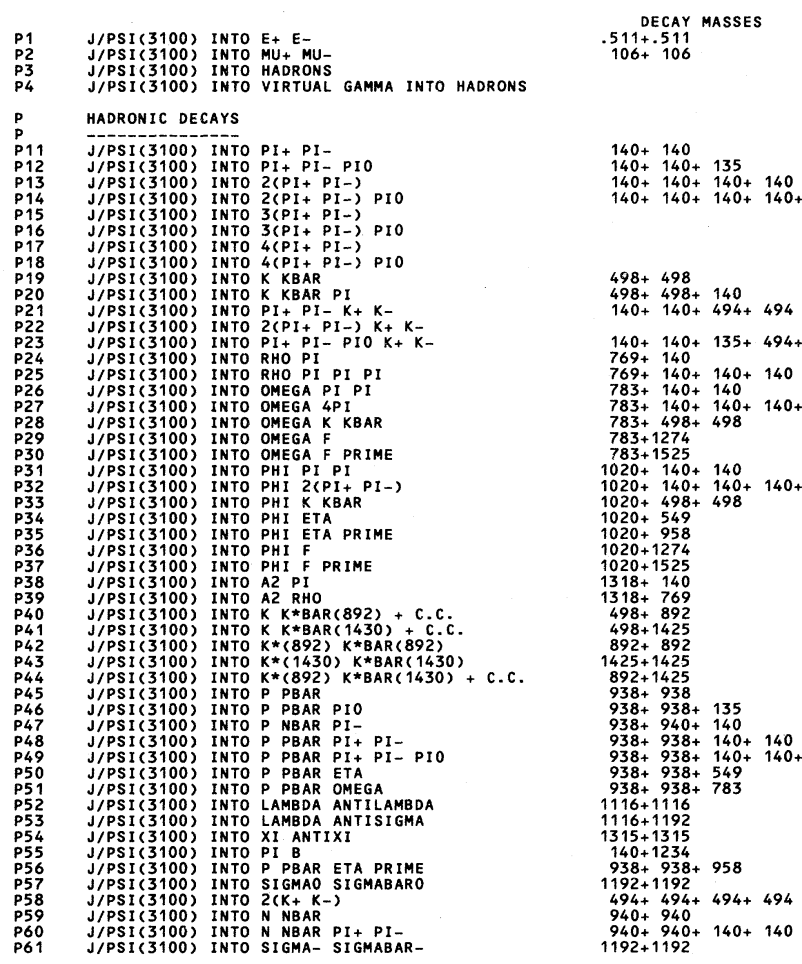

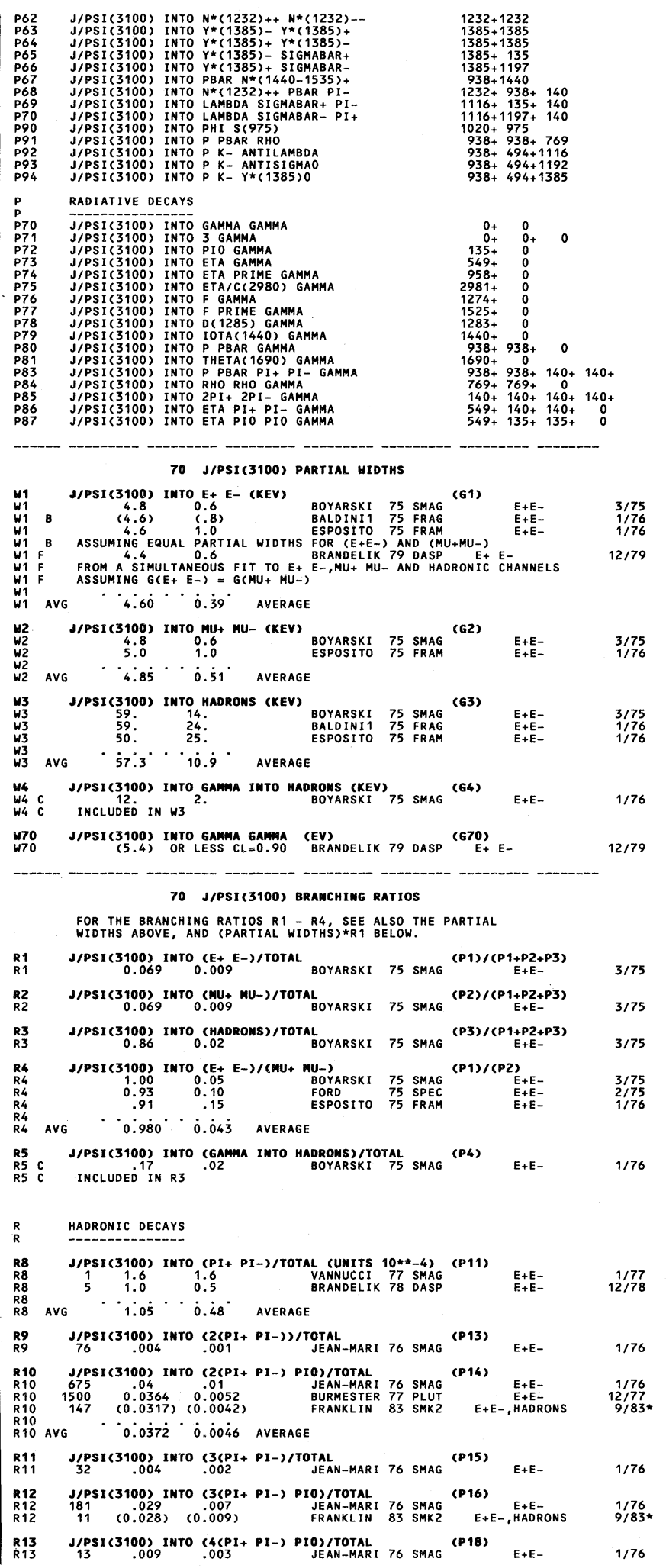




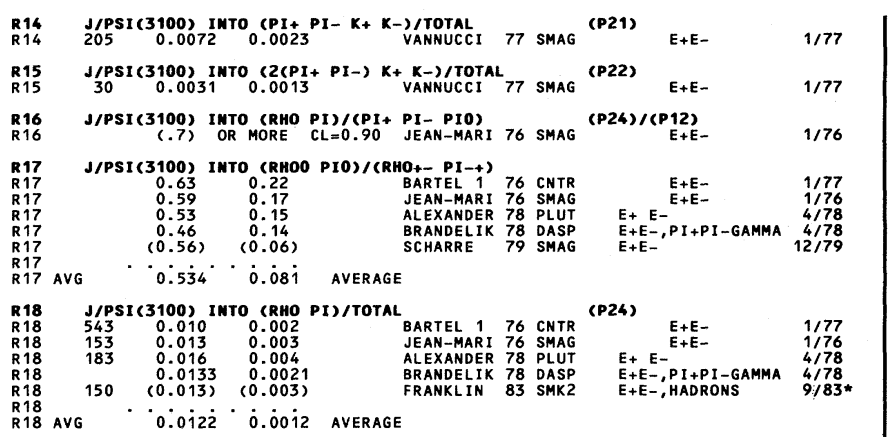

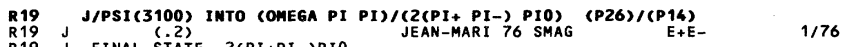

R20 J JPSI(3100) INTO (RHO PI PI PI)/(2 (PI+ PI-) PI0) (P25)/(P14)
R20
JEAN-MARI 76 SMAG

R20 J FINAL STATE $2(P I+P I-) P I 0$ JEAN-MARI 76 SMAG

R21 J/PSI(3100) INTO (PHI PI+ PI-)/TOTAL
R21
FELDMAN 73 SMAG (P31)

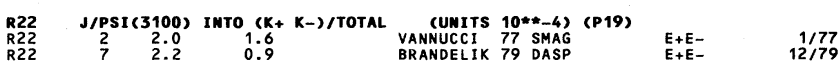

R22 AVG $2.15^{\text {R2 }} \cdot \dot{0}^{\circ} .78^{\circ}$ AVERAGE

$\begin{array}{lllll}\text { R23 J/PSI(3100) INTO (KOS KOL)/TOTAL CUMITS } & 10 * *-4) \\ \text { R23 (P19) } & \text { E+E- } & 1 / 77\end{array}$

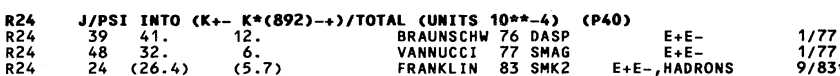

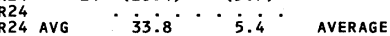

R25 J/PSI(3100) INTO (KO K*(892)0)/TOTAL
R25 UNITS $10 * *-4)$
VANNUCCI 77 SMAG

R26 J/PSI $(3100)$ INTO $(K+-K *(1430-+) /$ TOTAL
R26
$(0.0033)$ OR LESS $C L=0.90$ BRAUNSCHW 76 DASP (P41)

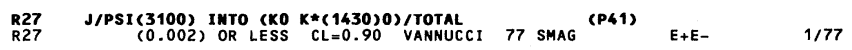

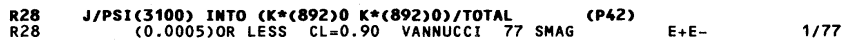

R29 J/PSI (3100) INTO $(K *(1430) 0 K *(1430) 0) /$ TOTAL
R29
$(0.0029)$ OR LESS CL $2=0.90$ VANNUCCI 77 SMAG

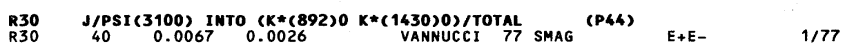

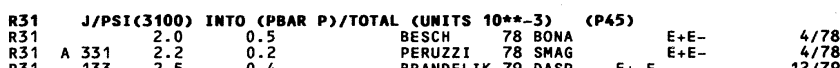

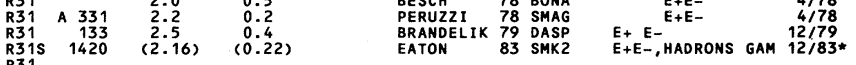

R31 AVG $2.23^{\circ} \cdot \dot{0} \cdot i^{\circ}$ average

R31S SYSTEMATIC ERROR ADDED LINEARLY BY US.
R31 A ASSUMING ANGULAR DISTRIBUTION $(1++\operatorname{COS}($ THETA $) * * 2)$

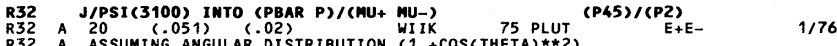

R32 A ASSUMING ANGULAR DISTRIBUTION $(1++\cos ($ THETA $) \star \star 2)$

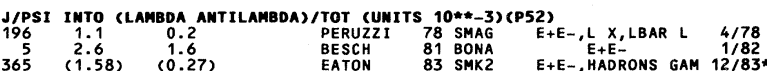

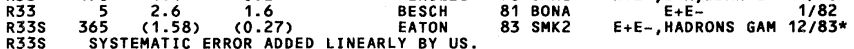

R33 AVG $\cdot 1.12^{\circ} \cdot \dot{0} 3^{\circ}$ average

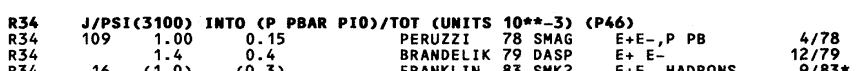

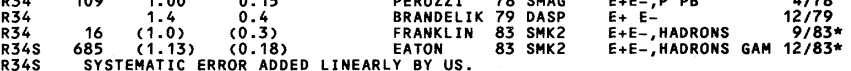

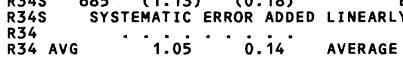

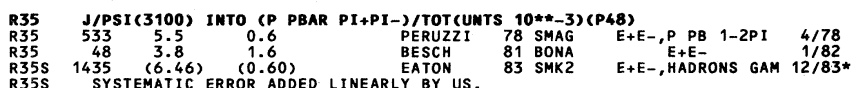

R35S SYSTEMATIC ERROR ADDED LINEARLY
R35
R35 AVG $5.29^{\circ} \cdot 0.56^{\circ}$ AVERAGE

R36 J/PSI INTO (P PBAR PI+ PI- PIO)/TOT (UNTS 10**-3) (P49)

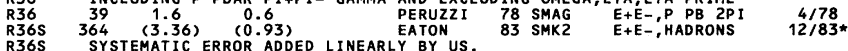

R37 J/PSI INTO (LAMBDA ANTISIGMA)/TOT CUMITS
R37
(0.15) OR LESS CL

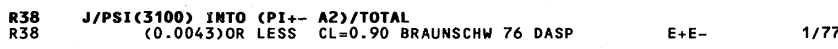

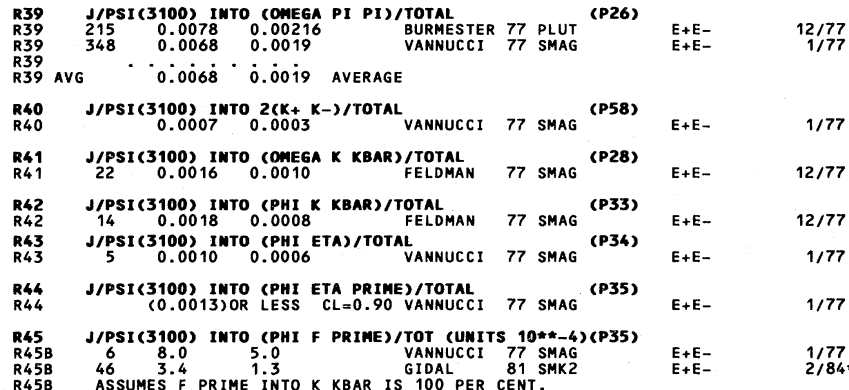

- $3.7^{\circ} \cdot i .3^{\circ}$ aVERAGE

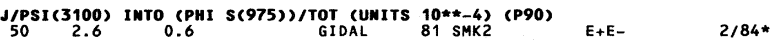

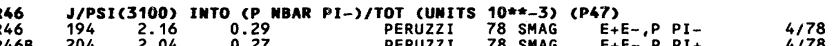

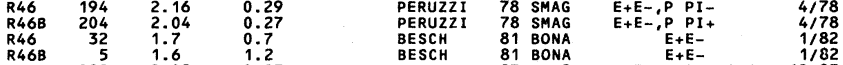

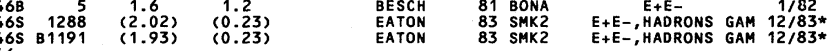

AVG $2.06^{\circ} 0.19^{\circ}$ average

R46S SYSTEMATIC ERROR ADDED LINEARLY BY US.
R46B FROM ANTI-CHANNEL (PBAR N PI+)

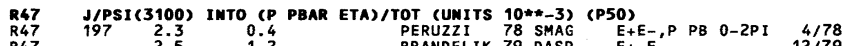

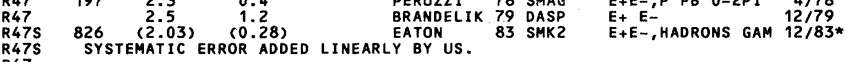
R47 AVG ' $2.32^{\circ}{ }^{\circ} \dot{0} \dot{0} 38^{\circ}$ AVERAGE

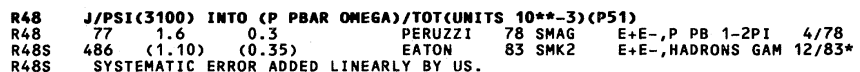

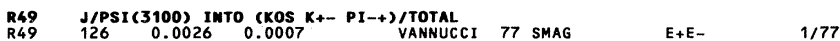

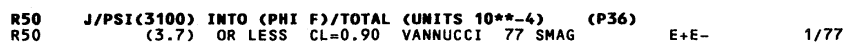

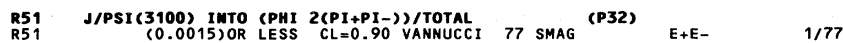

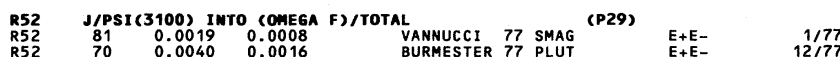
$\begin{array}{llllll}R 52 & 70 & 0.0040 & 0.0016 & \text { BURMESTER } 77 \text { PLUT } \\ \text { R52 AVG } & 0.00232 & 0.0008 & \text { E } & \text { AVERAGE (ERROR INCLUDES SCALE FACTOR OF 1.2) }\end{array}$

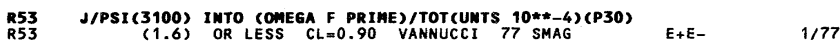

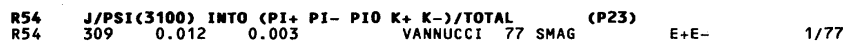

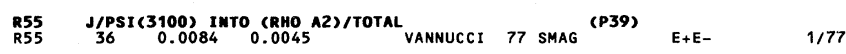

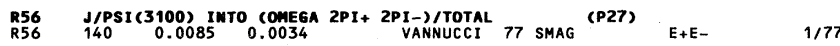

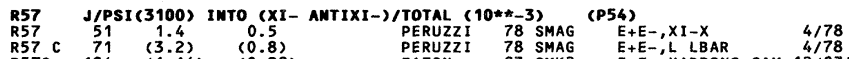
$\begin{array}{lll}\text { PERUZZ1 } & 78 \text { SMAG } & \text { E+E-, L LBAR } \\ \text { EATON } & 83 \text { SMK2 } & 4 / 78 \\ \text { E+E-, HADRONS GAM } 12 / 83^{*}\end{array}$ R57S SYSTEMATIC ERROR ADDED LINEARLY BY US.
R57C INCLUDES CHANNEL ( $X I 0$ ANTIXIO)

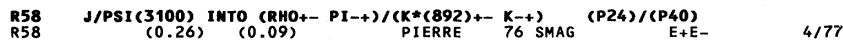

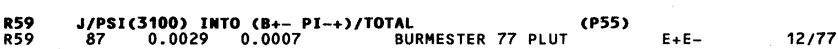
R60 J/PSI(3100) INTO (N MBAR)/TOTAL
R60 (UNITS 10**-2)
BESCH R61
R61
R61S
R61S

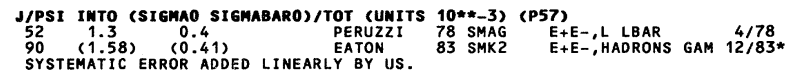

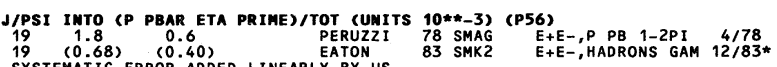
$19(0.68)(0.40)$
SYSTEMATIC ERROR ADDED LINEARLY EATON BY US.

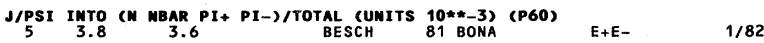

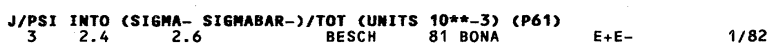

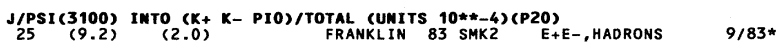

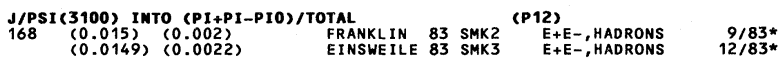

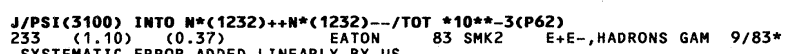




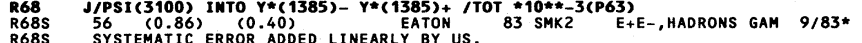

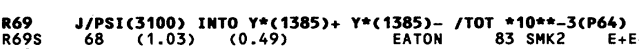

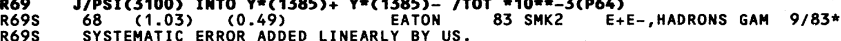

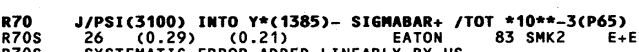

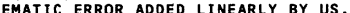

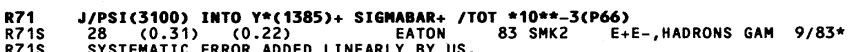

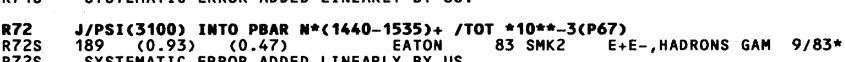

R73. JYPSI(3100) INTO NE(1232)++ PBAR PI- /TOT * 10**-3(P68)

R73S 332 (1.58) (0.63)
R73S SYSTEMATIC ERROR ADDED LINEARLY EATON US. 83 SMK2 E+E-, HADRONS GAM 9/83*

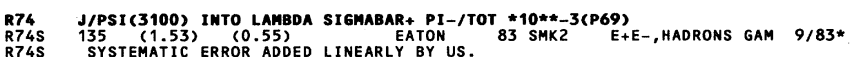

R75 J/PSI (3100) IMTO LAMBDA SIGMABAR- PI+/TOT *10**-3(P70)

R75S 118 (1.38) (0.56)
R75S SYSTEMATIC ERROR ADDED LINEARLY EATYN US. 83 SMK2 E+E-, HADRONS GAM 9/83*

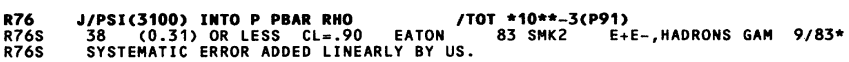

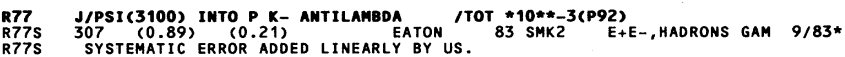

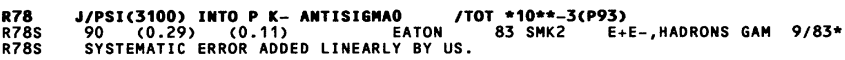

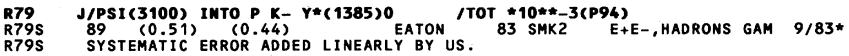

R RAdATIVE decays

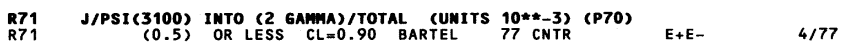

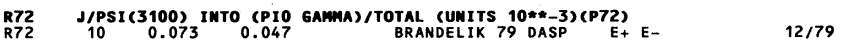

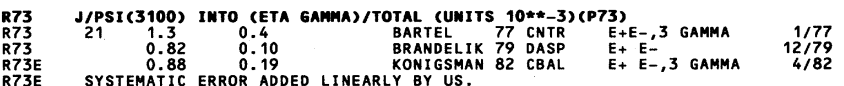

R73 AVG $0.855 \cdots 0.080$ AVERAGE

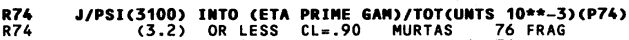

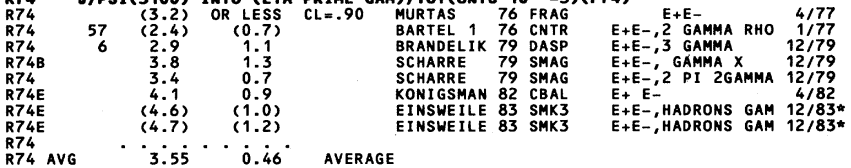

R74B
R74E SROM THE INCLUSIVE GAMMA DECAY SPECTRUM
SYSTEMATIC ERROR ADDED LINEARLY BY US.

R78 J/PSI (3100) INTO (3 GAMAA) TOOTAL CUMIS $10 \% \pm-3)$ (P71)
R78
$(0.055)$ OR LESS CL=0.90 PARTRIDGE 80 CNTR R80 J/PSI(3100) INTO (GAMMA + 2 OR MORE MEUTRALS)/TOTAL
R80
7.0

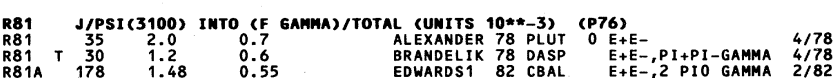

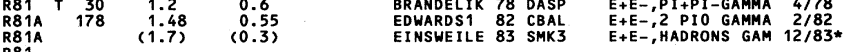
R81 AVG $\cdots 1.5 i^{\circ} \cdots \dot{0.35} 5^{\circ}$ average

R81 T' RE-STATED BY US TO TAKE ACCOUNT OF SPREAD OF E1,M2,E3 TRANSITIONS.
R81A SYSTEMATIC ERROR ADDED LINEARLY BY US

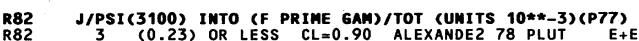

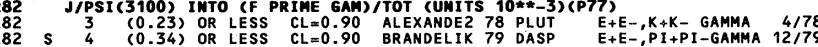

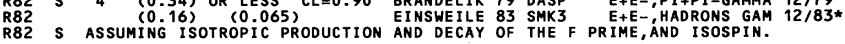

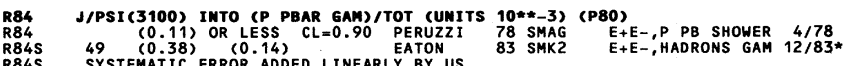

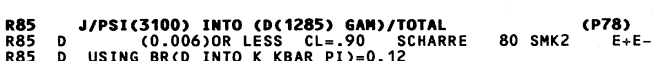

$2 / 81$

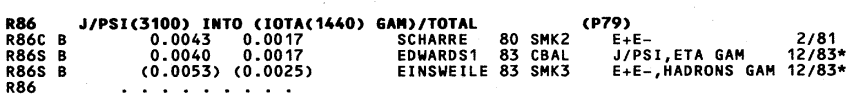

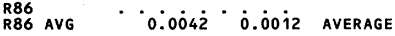

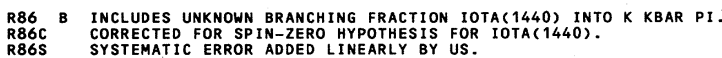

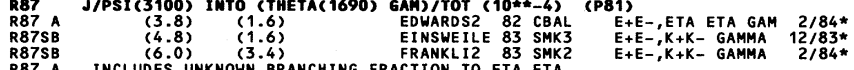 INCLUDES UNKNOON BRANCHING FRARTION TO EAT ETA.
INCLUES UNKNOWN BRANCHING FRACTION TO K+K-.}

R87 B INCLUDES UNKNOWN BRANCHING FACTION TO
R87S SYSTEMATIC ERROR ADDED LINERLIY BY US.

R88 J/PSI(3100) INTO (XI(2220) GAM)/TOT UWITS 10**-4 (P82)

R88SB
R88 R88B INCLUDE UNKNOWN BRANCHING FRACTION INT
R8BS SYSTEMATIC ERROR ADDED LINEARLY BY US.

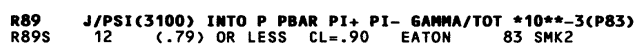

R89
R89S SYSTEMATIC ERROR ADDED LINEARLY BY US. 83 SMK2

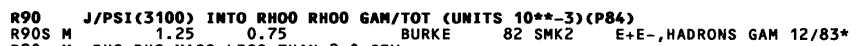
R90S M RHO RHO MASS LESS THAN 2.0 GEV BURK
R90 M9O
R90S SYSTEMATIC ERROR ADDED LINEARLY BY US.

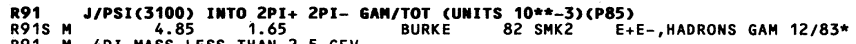
R91 M
R91 MP MASS LESS THAN 2.5 GEV
SYSTEMATIC ERROR ADDED LINEARLY BY US.

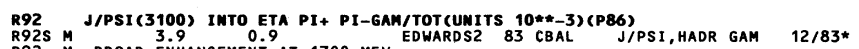

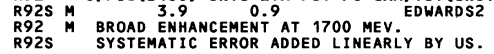

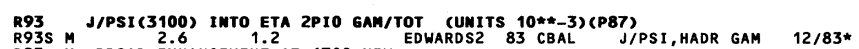

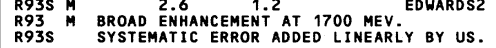

\section{J/PSI(3100) G(I)*G(E+E-)/G(TOTAL) (KEV)}

THIS COMBINATION OF A PARTIAL WIDTH WITH THE PARTIAL WIDTH
IITO EEE- AND WITH THE TOTAL WIDTH IS OBTAINED FROM THE INTEGRATED
COOSS G(E+E-)*G(E+E-)/G(TOTAL)

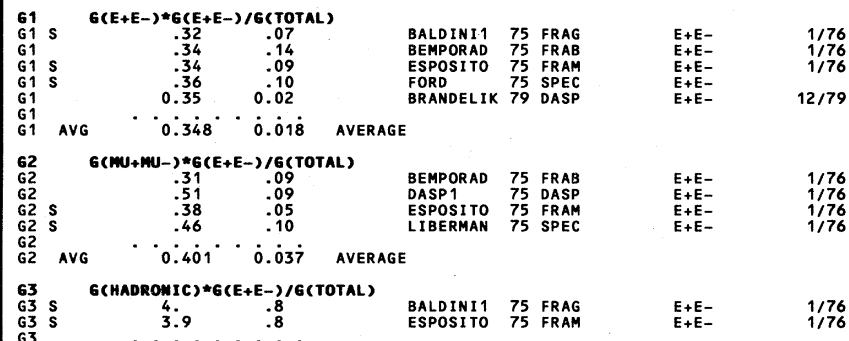

G3 AVG ${ }^{3} .95^{\circ} \cdots{ }^{\circ} \dot{0.57^{\circ}}$ average

$G$ SATA REDUNDANT WITH BRANCHING RATIOS OR PARTIAL WIDTHS ABOVE

******************************************* **************************

REFEREMCES For J/PSI(3100)

CHRISTEN 70 PRL 251523 CHRI STENSON, HI CKS, LEDERMAN+ (COLU+BNL+CERN)

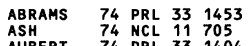
UGEUSTIN 74 PRL 331406

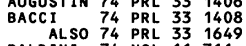

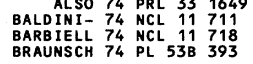

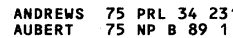

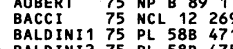

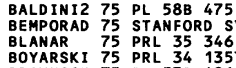
+ +BRIGGS, AUGUSTIN, BOYARSKI+
$+20 R N, B$ BRTOLI+ (LBL+SLAC)
(FRAS+UMD+NAPL+PADO+ROMA)

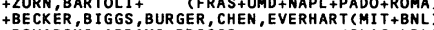
+BOYARSKI, ABRAMS, BRIGGS
+BARTOLI, BARBARINO, BARBIELLINI+ (SLACLBL)
(FRASCATI) (FRASCATI+ROMA)

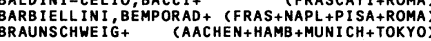
+ HARUEY, LOBKOHI CZ, MAY, NORDBERG (ROCH+CORN)

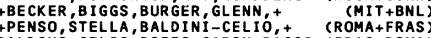
ALONI (PISA+FRASCAII)
C.BEMPORAD
BOOYER EAISSLER, GARELICK, GETTNER,
(NEAS)

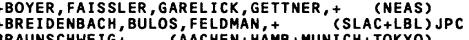

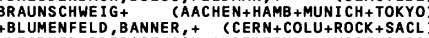

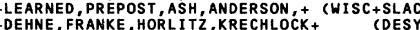
KREISLER, BOLON, HEILE+ (MASA+MIT+SLAC) RAUNSCHWEIG, KONIGS, + (AACH+DESY MP IM+ TOKY) PAUSCHU (SLAC+PENN)

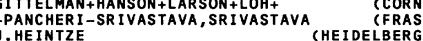

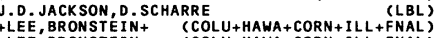

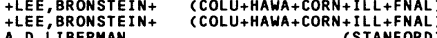

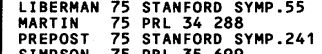

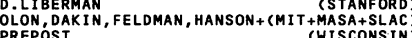

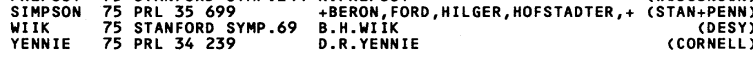

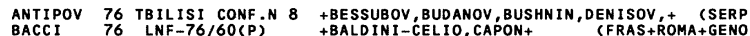

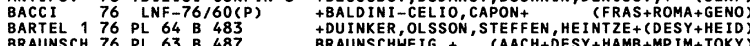

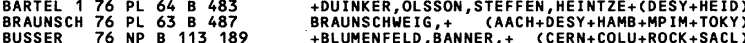

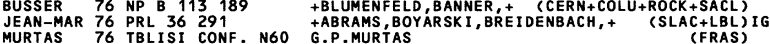

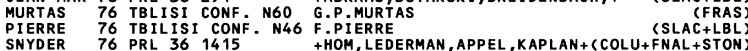




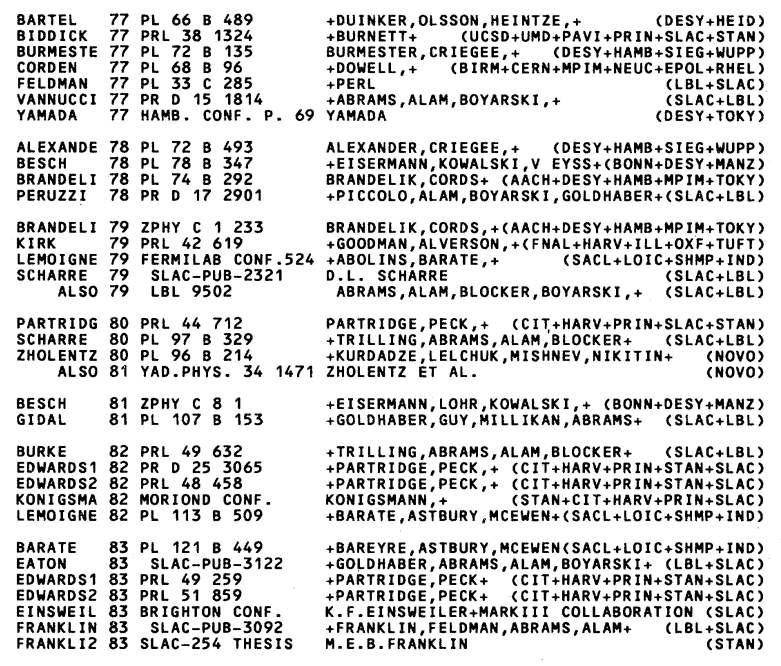

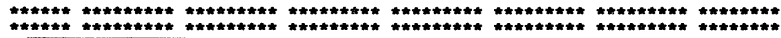

$\chi(3415) 56$ CHI(3415,JPG $=0++)$ I=0

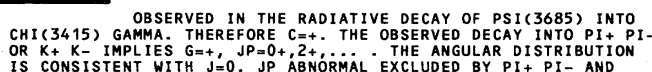

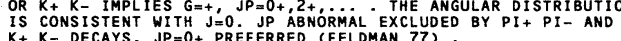

\section{CHI(3415) MASS (MEV)}

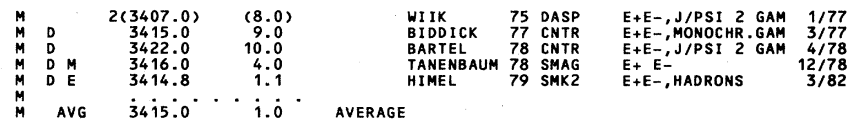

$M$
$M$

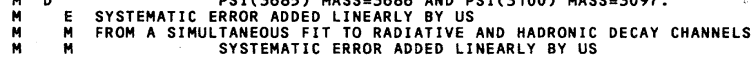

\begin{tabular}{llll}
\multicolumn{5}{c}{56 CHI(3415) PARTIAL DECAY MODES } \\
P1 \\
P1
\end{tabular}

\section{CHI (3415) BRAMCHIMG RATIOS}

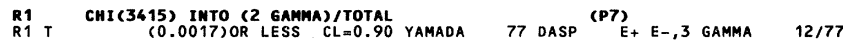

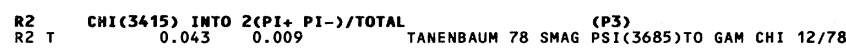

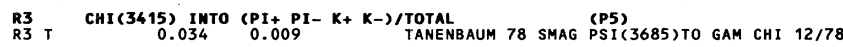

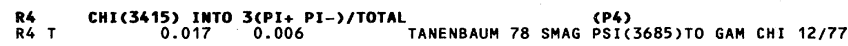

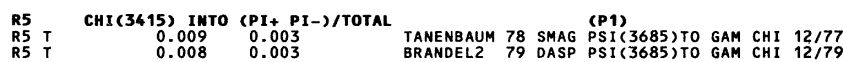
R5 AVG $0.0085 \cdot \dot{0} \cdot \dot{0} 021$ aVerage

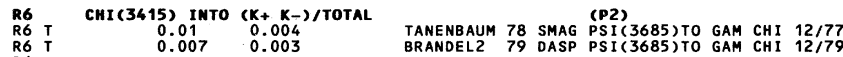
$\begin{array}{lll}R 6 & T \\ \text { R6 } \\ \text { R6 AVG }\end{array}$

R7 T CHI(3415) INTO
R7
0.006
0.002

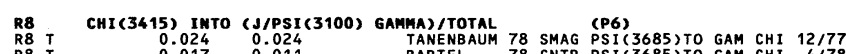

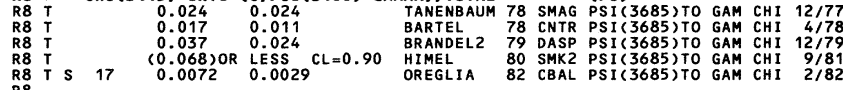
$\begin{array}{lll}R 8 & \text { T S } & 17 \\ R 8 & \text { AVG } & 0.0072 \\ \text { R8.0084 }\end{array}$
R9
R9 T CHI(3415) IIHTO (RHO0 PI+ PI-)/TOTAL
0.000

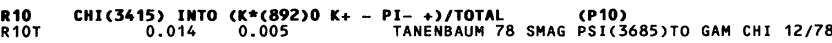

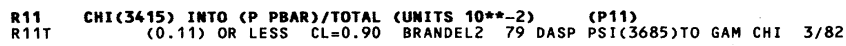
$R$
$R$ S SYSTEMATIC ERROR ADDED LINEARLY BY US. ******************************************************************************** REFEREMCES FOR CHI(3415)

FELDMAN 75 PRL 35821 J JEAN-MARIE, SADOULET, VANNUCCI, + (LBL+SLAC) TANENBA 755 PR 351323 (ERRATA) TANEABUM, HHITAKER, ABRAMS, + (LBL+SLAC)
(DESY)
WIIK 75 STANFORD SYMP. 69 B.H.WIIK

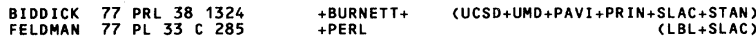
YAMADA 77 HAMB. CONF. P. 69 YAMADA
PDEST+TOKY)

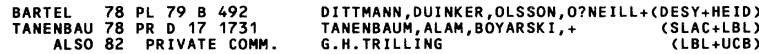

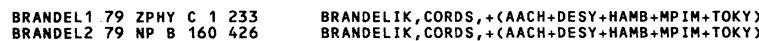

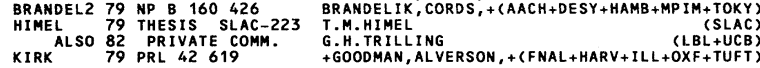
HIMEL 80 PRL 44920 +ABRAMS, ALAM, BLOCKER,+ OREGLIA 82 PR D 252259 +PARTRIDGE, BLOOM, + (SLAC+CIT+ HARV+PRIN+STAN)

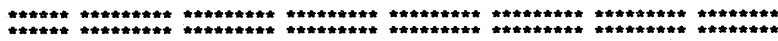

$\chi(3510) 55$ CHI(3510,JPG=1++) I=0 FORMERLY CALLED PC.

OBERVED IN THE RADIAT IVE SEQUENTIAL DECAY
OF THE PSI 3685 ) INTO CHI 3510 ) GAMMA, CHI (3510)

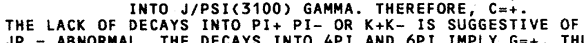

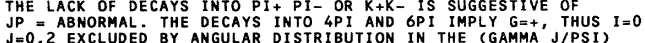
DECAY. JP $1+$ PREFERRED (FELDMAN 77, OREGLIA 82)

\section{CHI (3510) MASS (MEV)}

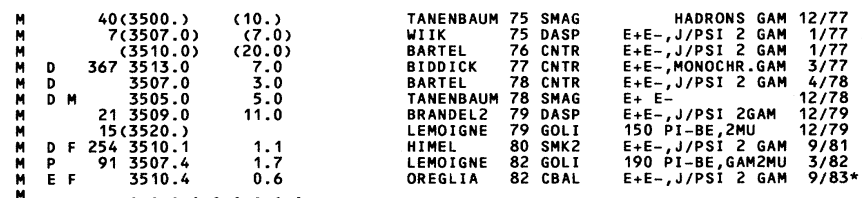

M avg $3509.95^{\circ} \cdots 0.55^{\circ}$ average (error includes Scale factor of 1.1 )

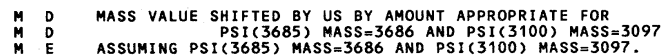

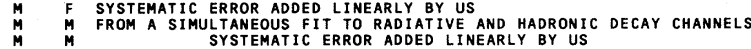

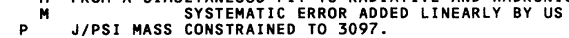

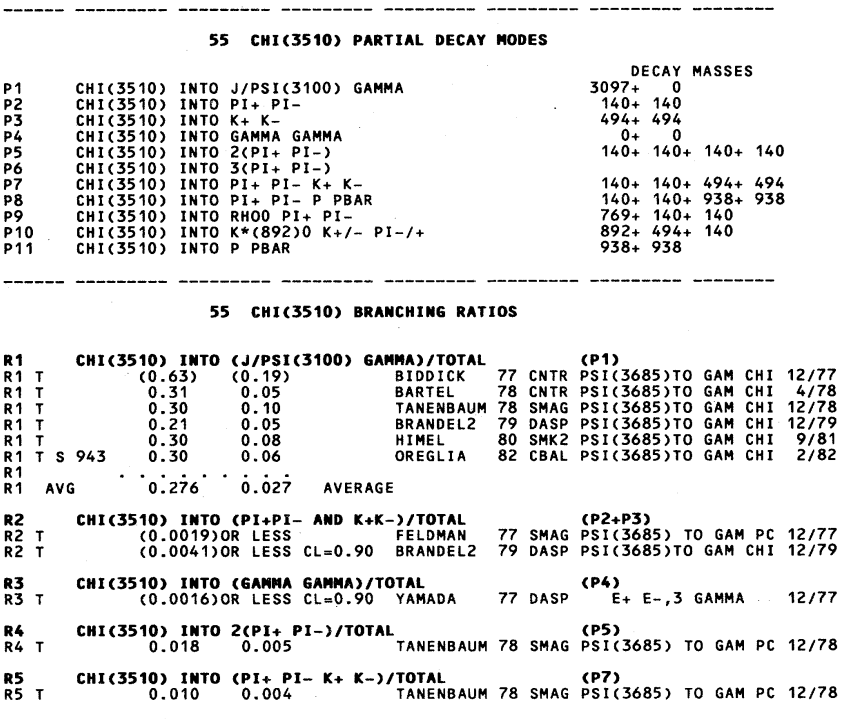




\section{Mesons}

\section{Data Card Listings}

$\chi(3510), \quad \chi(3555), \quad \eta_{\mathrm{c}}(3590)$

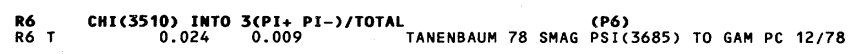

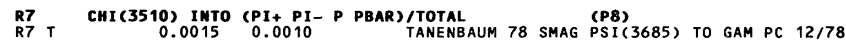

R8 CHI(3510) INTO (RHO0 PI+ PI-)/TOTAL
R8 T C.0043
R.

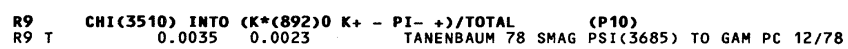

R11 CHI(3510) INTO (P PBAR)/TOTAL
R11T 0.0013$)$ OR LESS CL $=0.90$ BRANDEL2 79 DASP PSI(3685)TO GAM CHI 12/79

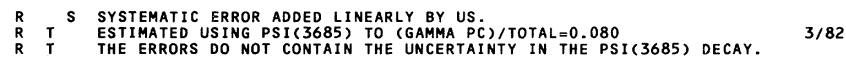

\section{******************************************************************************}

REFEREMCES FOR CHI 35 10)

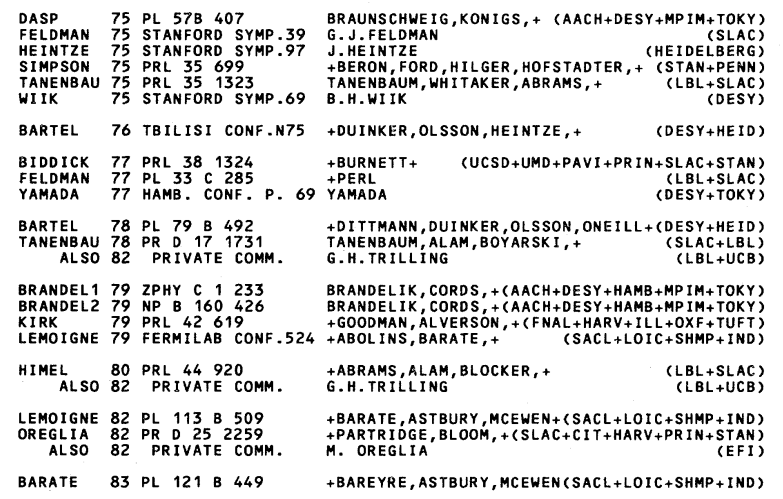

BARATE 83 PL 121 B 449 +BAREYRE, ASTBURY, MCEWEN(SACL+LOIC+SHMP+IND)
$* * * * * * * * * * * * * * * * * * * * * * * * * * * * * * * * * * * * * * * * * * * * * * * * * * * * * * * * * * * * * * * * * * * * * * * *$

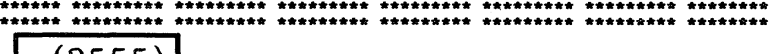

$\chi(3555) 57$ cHI(3555,JPG=2++) I=0

OBSERVE IN RADIATIVE DECAY OF PS ( 3685$)$ INTO
CHI(3555) GAMMA. THEREORE C=+. THE OBSERVED DECAY INTO 4 PI AND 6 PI IMPLY G $=+$ T THUS IN=0.
$J=0$ IS EXCLODED BY THE AGULAR DISTRIBUTION IN THE HADRONIC

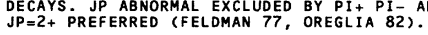

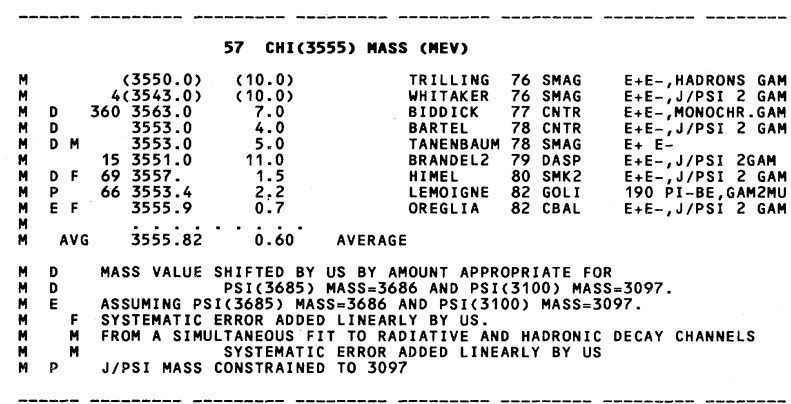

57 CHI (3555) PARTIAL DECAY MODES

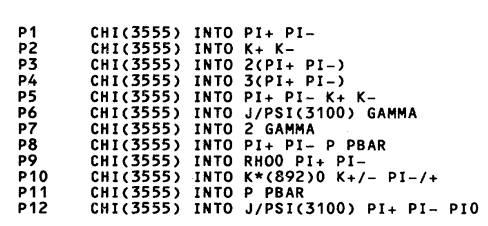

DECAY MASSES
$140+140$

$494+494$
$140+140+140+140$

$140+140+494+494$

$3097++0$

$140+140+938+938$

$769+140+140$
$892+494+140$

$3097+140+140+135$

57 CHI(3555) BRANCHIME RATIOS

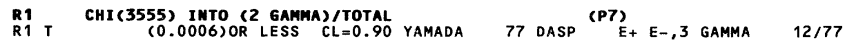

R2 CHI(3555) INTO 2(PI+ PI-)/TOTAL
R2 T
$0.023 \quad 0.005$

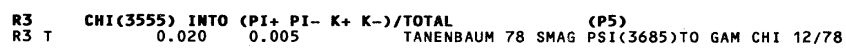

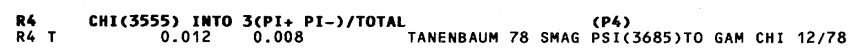

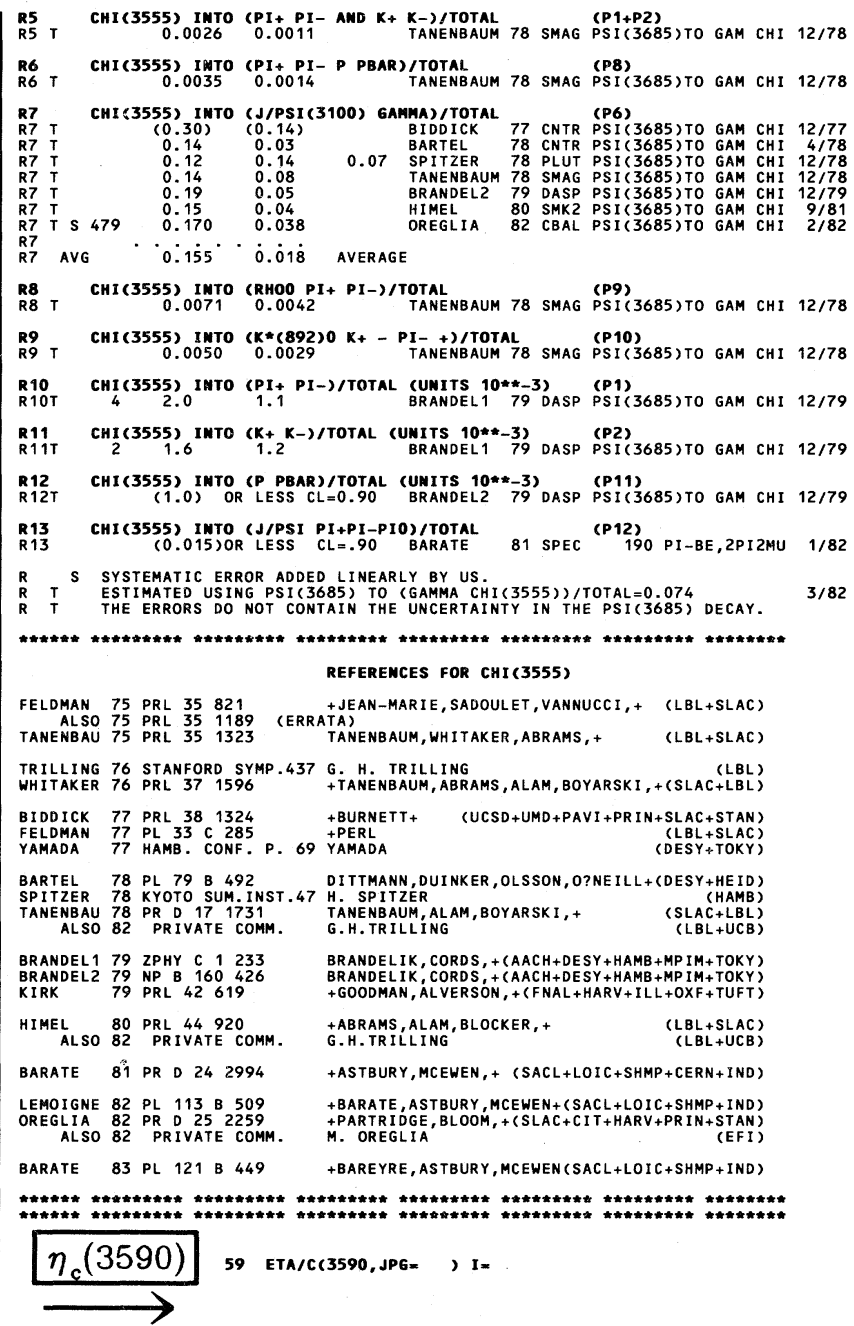

Evidence for the $\eta_{\mathrm{c}}(3590)$ is based on the observation of a monochromatic gamma line in the inclusive photon spectrum for $\psi(3685)$ decays (EDWARDS 82 ). No exclusive decay modes are known at this time. A signal had been reported for a state at similar mass in the decay $\psi(3685) \rightarrow \gamma \gamma \mathrm{J} / \psi(3100)$ (BARTEL 78), but this signal was not confirmed in an experiment with higher statistics (OREGLIA 82).

This particle needs confirmation, and thus is omitted from the Meson Table.

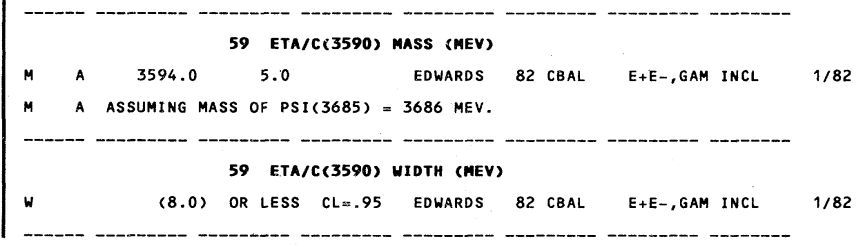




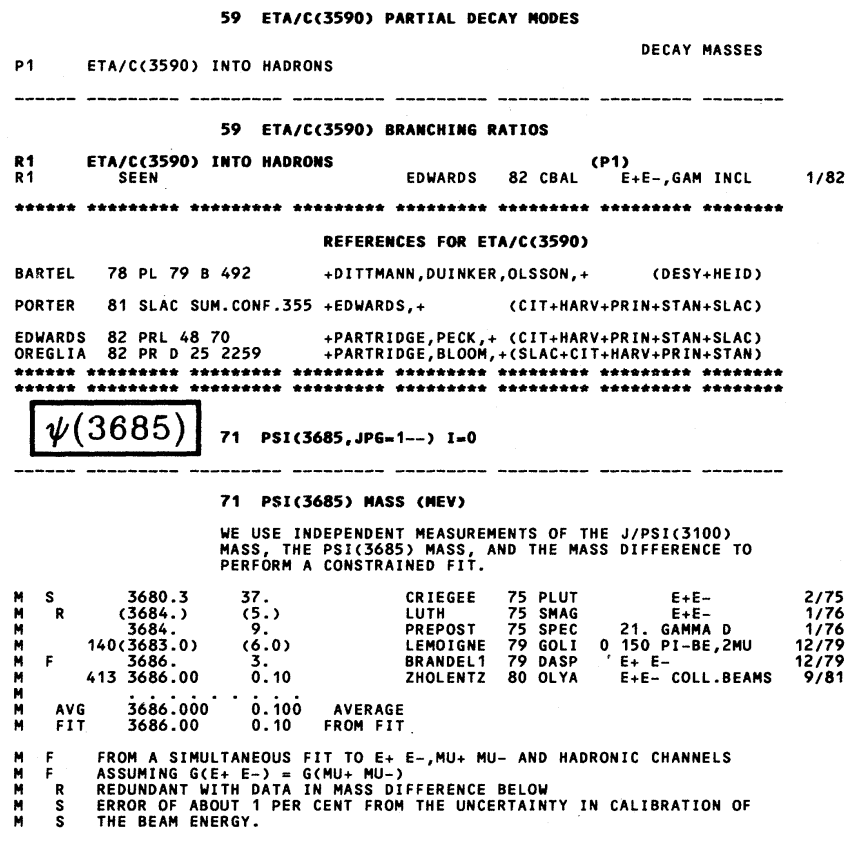

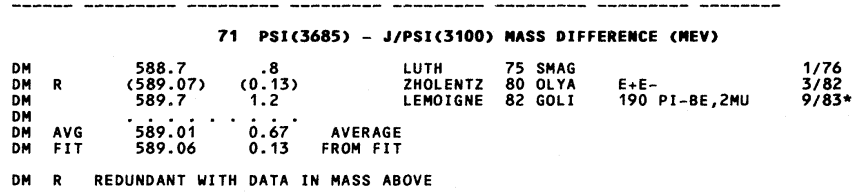

$\begin{array}{llll} & 71 \text { PSI(3685) WIDTH (KEV) } & \\ W \\ W\end{array}$

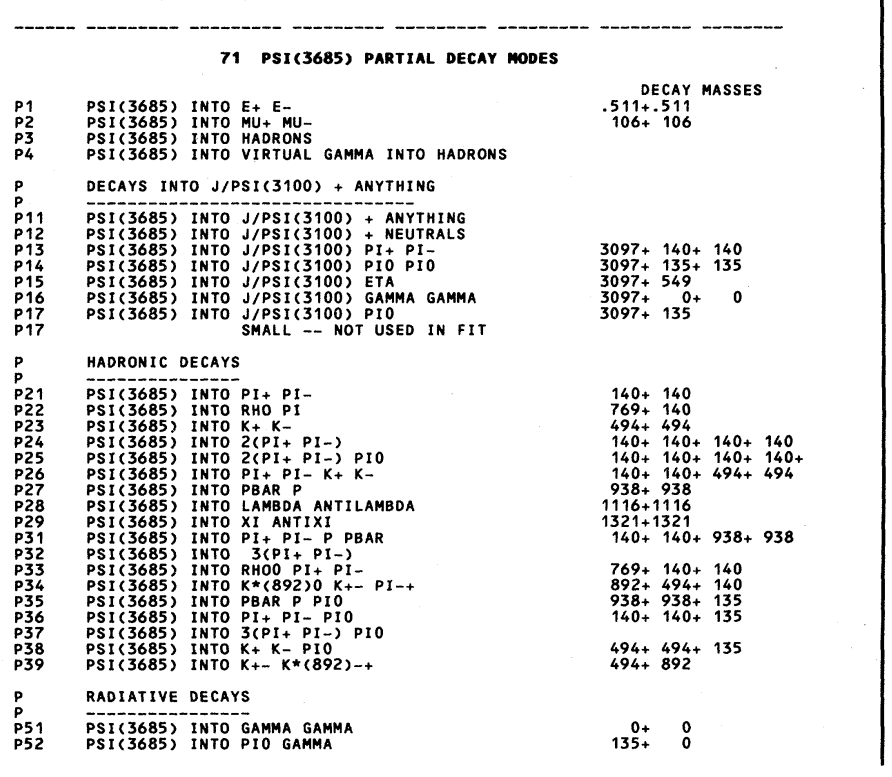

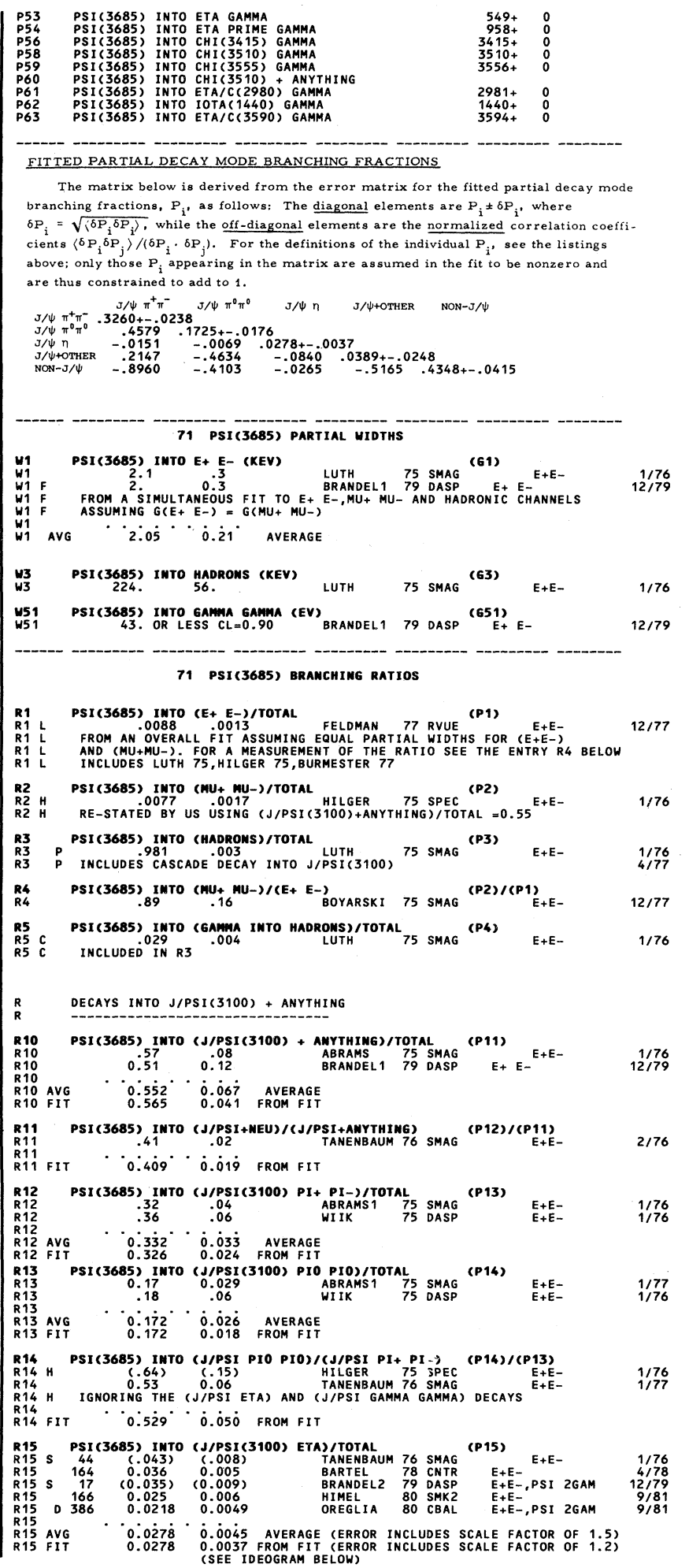


Mesons $\psi(3685)$

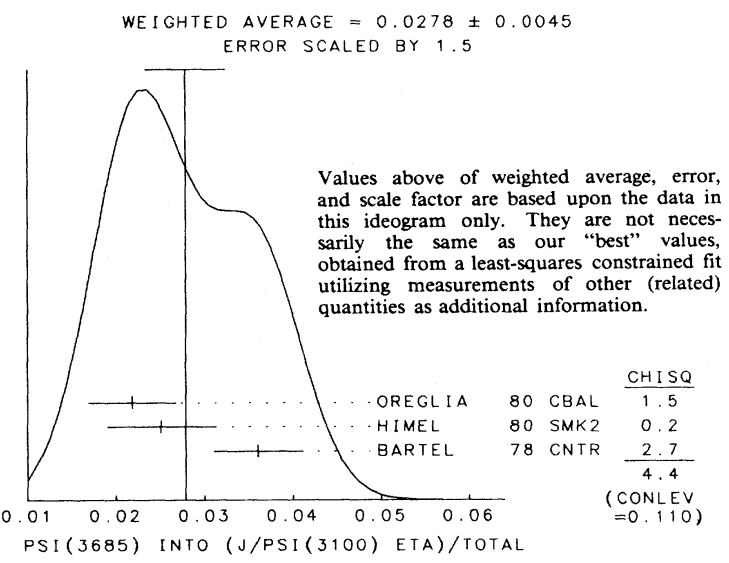

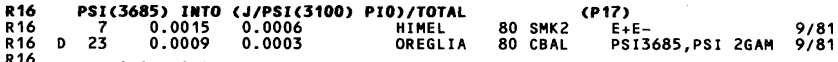
R16 AVG $0.00102 \cdot 0.00027$ AVerage

$R \quad$ HADRONIC DECAYS

R20
RR2
R20

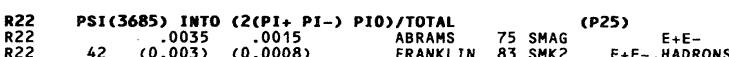

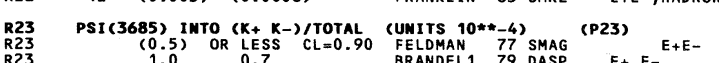

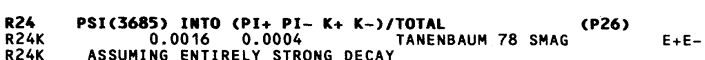

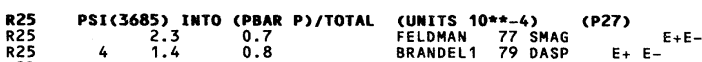

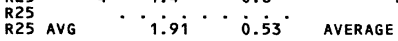

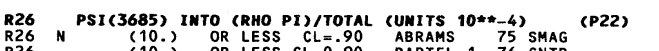

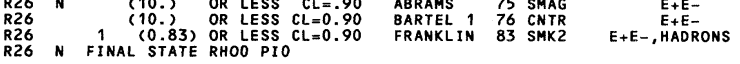

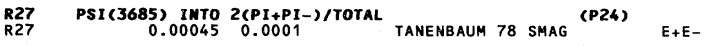

R28 PSI(3685) INTO (LAMBDA ANTILAMBDA) TTOTAL
R28
(0.0004) OR LESS
CL $=0.90$ FELOMAN 77 SMAG

R29 PSI (3685)
R29
(0.0002) (XI- ANTIXI-)/TOTAL FELDMAN 77 SMAG

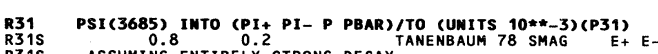

R32 PSI(3685) INTO 3 (P1+ PI-)/TOTAL CUAITS 10***-3) (P32)

R33 PSI(3685) 1NTO
R33 RHO0 PI+ PI-)/TOT (UNIS S
0.15

R34 PSI (3685) INTO(K*(892) OK+-PI-+)/TTOTCUNTS
R34
R.

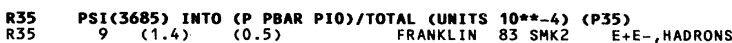

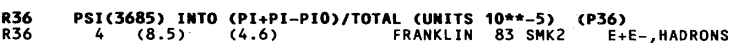

R37 PSI(3685) INTO (3) (PItPI-)PI0)/TOTAL
R37

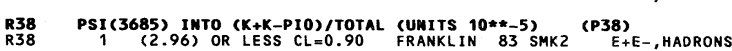

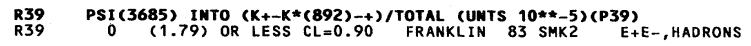

\section{$R \quad$ RADIATIVE DECAYS}

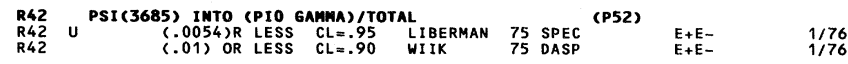

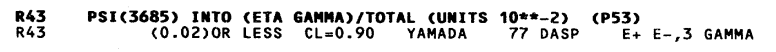

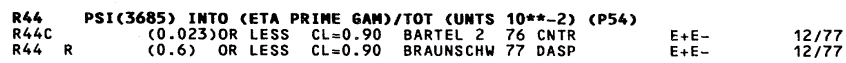

$2 / 77$

$9 / 83 *$ 12,779 $12 / 78$
$12 / 78$ 12,77
12,79

$\underset{\substack{1 \\ 1,777 \\ 9,83 *}}{1,76}$ $12 / 78$ $12 / 77$ $12 / 77$ $12 / 78$ $12 / 78$ $12 / 78$ $12 / 78$ 9/83* 9/83* 9/83* 9/83* 9/83* $12 / 77$

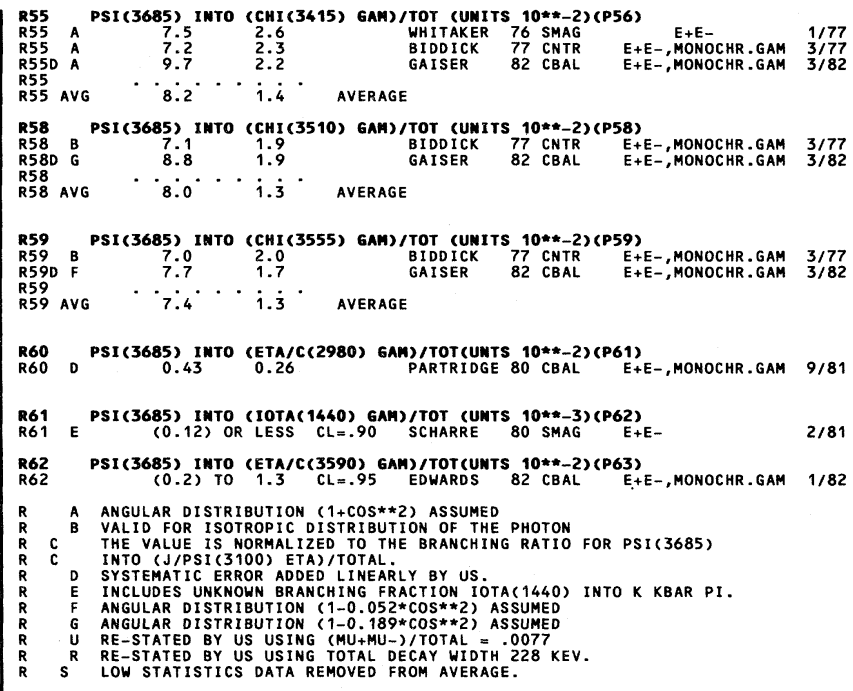
THIS COMBINATION OF A PARTIAL WIDTH WITH THE PARTIAL WIDTH
INTO E+E- AND WITH THE TOTAL WIDTH IS OBTAINED FROM THE INTEGRATED
CROSS-SECTION INTO CHANEL(I) IN THE E+E- ANNIHILATION.

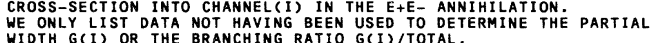

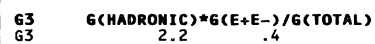

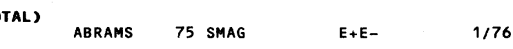


$\psi(3770)$ 53 PSI(3770,JPG=1- , I=

\section{PSI (3770) MASS (MEV)}

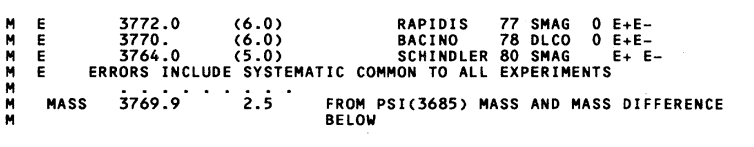

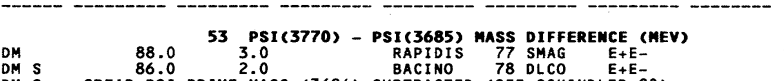

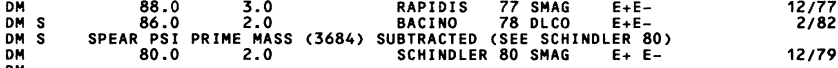

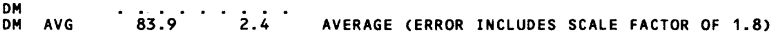

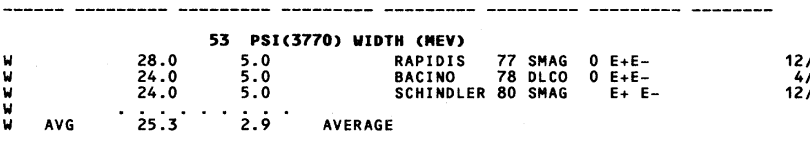

53 PSI(3770) PARTIAL DECAY MODES

$P 1$
$P 2$

\section{DECAY MASSES} $511+$
$1869+1869$

53 PSI(3770) PARTIAL MIDTHS (KEV)

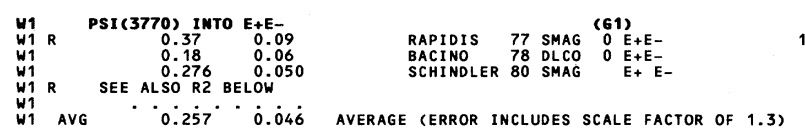

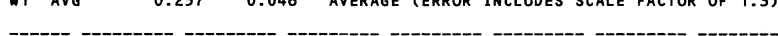

53 PSI(3770) BRAMCHIMG RATIOS

R1 PSI(3770)
ROMINANT (D DBAR)/TOTAL PERUZZI 77 SMAG

R2 PSI (3770) INTO
R2
$(\mathrm{N} 2.3$
0.2

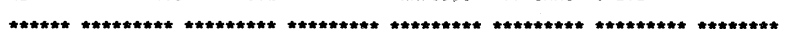

\section{REFERENCES FOR PSI (3770)}

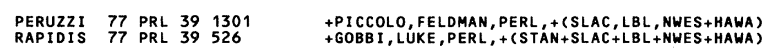

BACINO 78 PRL 40671 +BAUMGARTEN, BIRKWO00, + (SLAC+STAN+UCLA+UCI)

SCHINDLE 80 PR D 212716 SCHINDLER, SIEGRIST, ALAM, BOYARSKI+ (SLAC+LBL)

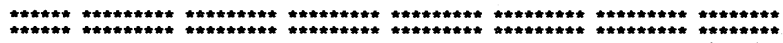

$\psi(4030) 72$ PSI(4030, JPG=1- , I-

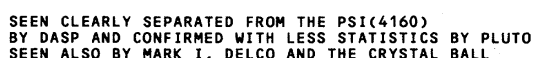

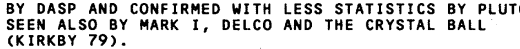

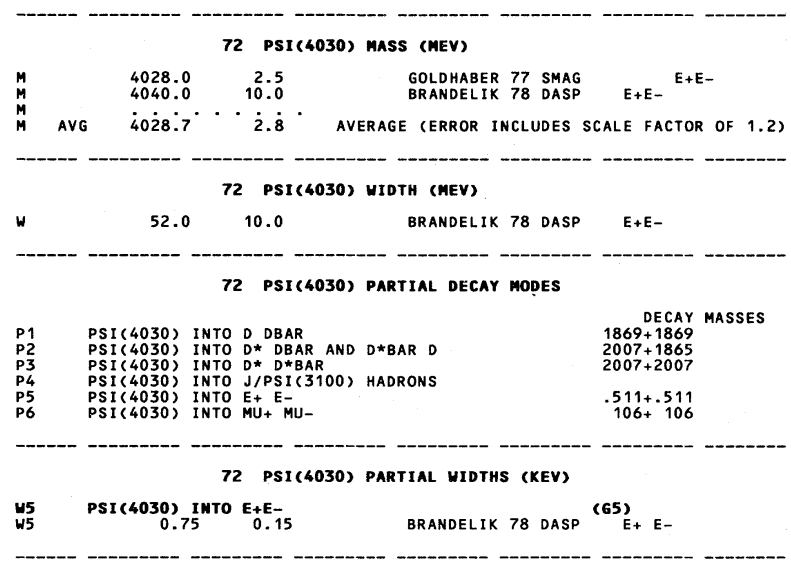

$12 / 77$
$4 / 78$
$12 / 79$

\section{PSI(4030) BRAMCHIMG RATIOS}

R1 P1 PSI (4030) IWTO
$R 1$ P DO DOBAR )
0.03

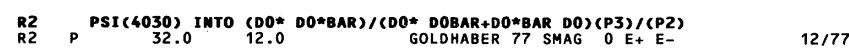

$R$ P PHASE-SPACE FACTOR (P**3) EXPLicitLY RemOVED.

R3 PSI(4030) INTO J/PSI(3100) HADROMS
R3
LOOKED FOR

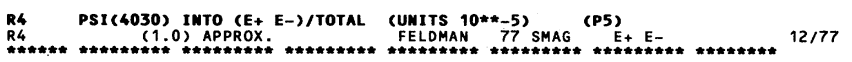

R4

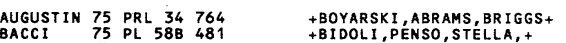

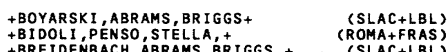

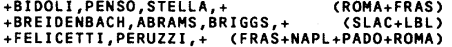

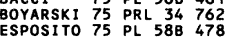
PERUZZI 76 PRL 37569

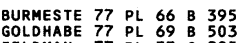
+PICCOLO, FEL DMAN, NGUYEN, WISS, + (SLAC+LBL)

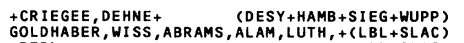
GoLHABER, WISS, ABRAMS, ALAM, LUTH, +(LBL+SLAC)
+PRL (LBL+SLAC)
+ PIERRE, ABRAMS, ALAM, BOYARSKI,
(LBL+SLAC)

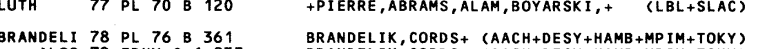
RISKY 79 FERMILAB SYMP. $107 \mathrm{~J}$. KIRKBY RAPPORTEUR
(SLAC)

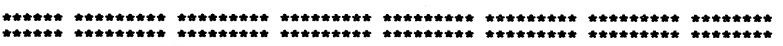
$\psi(4160) 25$ PSI (4160, JPG=1- ) I= SEEN CLEARLY SEPARATE FROM THE PSI (4030)
BY DASP AND CONF IRED WITH LESS STATISTICS BY PLUTO .
MARK I, DELCO AND THE CRYSTAL BAL SEE PROMINET

$\begin{array}{r}12 / 77 \\ 4 / 78 \\ 1 / 82 \\ \hline\end{array}$

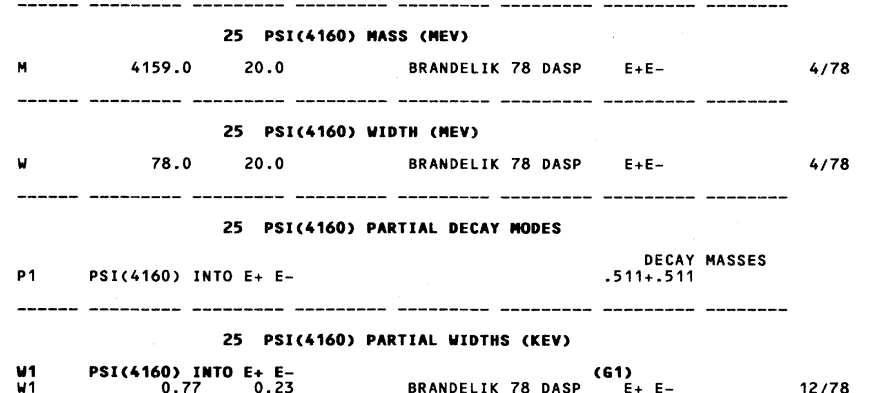

W1 PSI (6160) INTO E+ E. E-
W1

$* * * * * * * * * * * * * * * * * * * * * * * * * * * * * * * * * * * * * * * * * * * * * * * * * * * * * * * * * * * * * * * * * * * * * *$
REFEREMCES FOR PSI( $(4160)$

BURMESTE 77 PL 66 B 395 +CRIEGEE, DEHNE+ (DESY+HAMB+SIEG+WUPP) BRANDELI 78 PL 76 B 361 BRANDELIK, CORDS+ (AACH+DESY+HAMB+MPIM+TOKY) KIRKBY 79 FERMILAB SYMP.107 J. KIRKBY RAPPORTEUR
(SLAC)

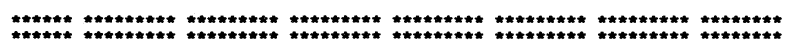
$\psi(4415) 73$ PSI (44415, JPG=1- ) I=

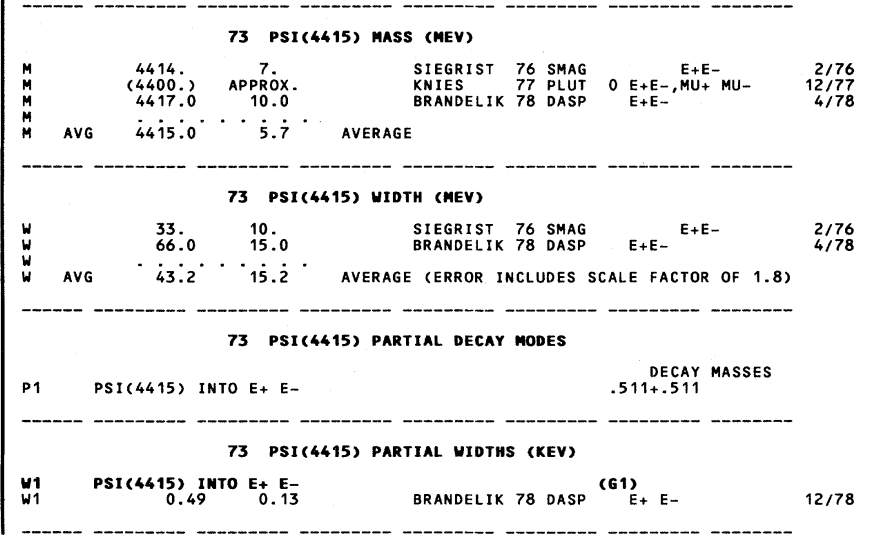


Mesons

$\psi(4415), \Upsilon(9460) \quad[\Upsilon(1 \mathrm{~S})], \quad \chi_{b}(9875) \quad\left[\chi_{b}\left(1^{3} \mathrm{P}_{0}\right)\right], \chi_{b}(9895)\left[\chi_{b}\left(1^{3} \mathrm{P}_{1}\right)\right]$

\section{Data Card Listings}

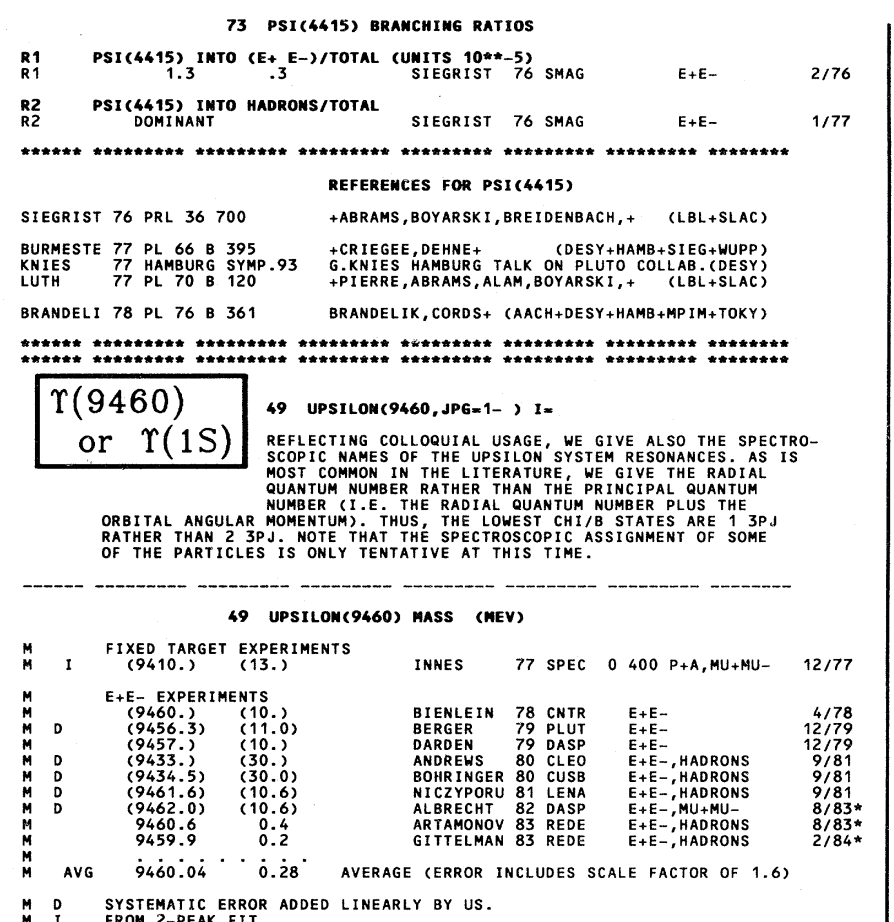

$M$ D SYSTEMATXC ERROR ADDED LINEARLY BY US.
$M$ II

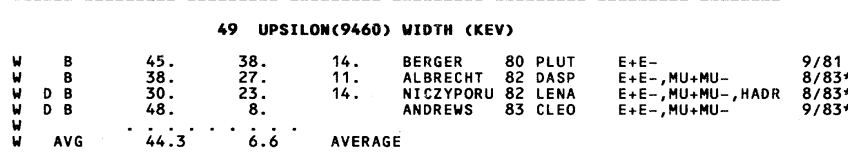

$W$
$W$ B FROM R1,R2, W2 BELLW AND ASSUMING E-MU-TAU UNIVERSALITY

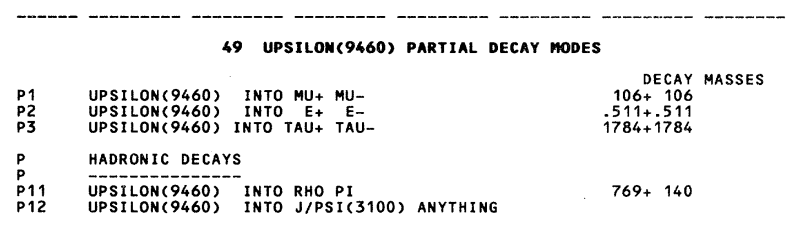

49 UPSILOM(9460) PARTIAL WIDTHS (KEV)

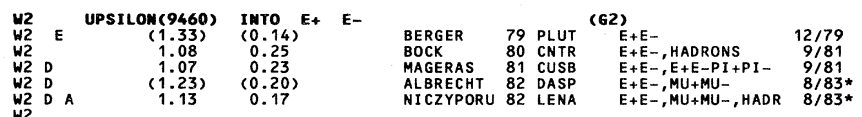

W2 avg $\cdot 1.10^{\circ} \cdot \dot{0}^{\circ} i^{\circ}$ average

W2 A ASSUMING E-MU-TAU UNIVERSALITY
W2 D
W2 ESTEMATIC ERRORS ADDED LINEARLY BY US.
ASSUMING HADRONIC PARTIAL WIDTH EQUAL TO TOTAL WIDTH

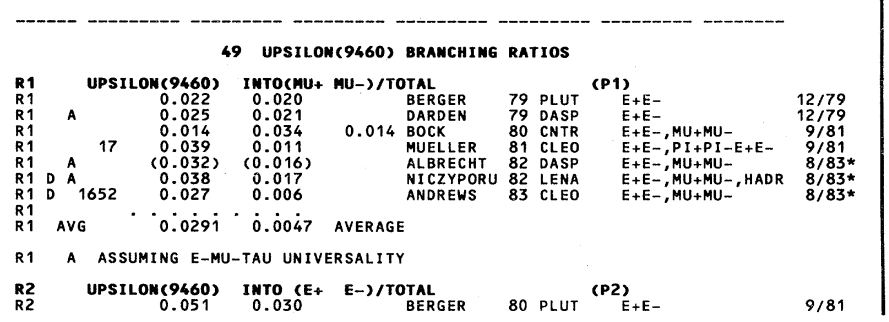

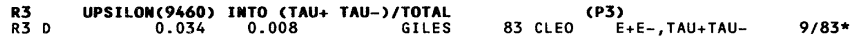
R11 UPSILOM(9460) INTO (RHO PI)/TOTAL
R11
NICZYPORU 83 LENA R12 UPSILON(9460) INTO (J/PSI(3100) AMYTHIMG)/TTOTAL (P12)
R12 (.02) OR LESS
NICZYPORU 83 LENA $R$ D SYSTEMatic ERRORS ADded Linearly by US.

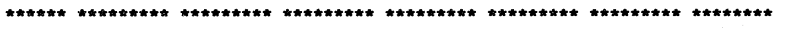

$\begin{array}{llll}\text { COBB } & 77 \text { PL } 72 \text { B } 273 \\ \text { HERB } & 77 \text { PRL } 39252\end{array}$

INNES 77 PRL 391240

$\begin{array}{llllll}\text { BERGER } & 78 & \text { PL } & 76 & \text { B } & 243 \\ \text { BIENLEIN } & 78 & \text { PL } & 78 & \text { B } & 360\end{array}$

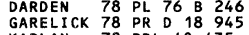

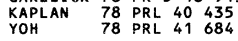

$\begin{array}{llllll}\text { ANGELIS } & 79 & \text { PL } & 87 & \text { B } & 398 \\ \text { BADIER } & 79 & \text { PL } & 86 & \text { B } & 98\end{array}$

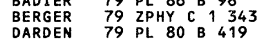

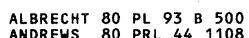
$\begin{array}{lllll}\text { ANDREWS } & 80 & \text { PRL } & 44 & 1108 \\ \text { BERGER } & 80 & \text { PL } 93 & \text { B } & 497\end{array}$ $\begin{array}{llllll}\text { BOCK } & 80 & \text { PPHY } & \text { C } & 6 & 125 \\ \text { BOHRINGE } & 80 & \text { PRL } & 44 & 1111 \\ \text { KOURKOUM } & \text { PLL } & 91 & \text { B } & 481\end{array}$ $\begin{array}{lllll}\text { MAGERAS } & 81 & \text { PRL } & 46 & 1115 \\ \text { MUELLER } & 81 & \text { PRL } & 46 & 1181\end{array}$ ALBRECHT 82 PL 116 B 383

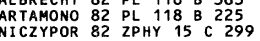
ALBRECHT 83 DESY $83-101$

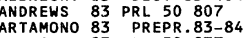
GILES 83 PRL 50877 $\begin{array}{ll}\text { GITTELMA } 83 & \text { CORNELL CONF } \\ \text { NICZYPOR } 83 \text { ZPHY } 17 \text { C } 197\end{array}$ +IWATA, FABJAN, GOLDBERG+(BNL+CERN+SYRA+YALE) + HOM, LEDERMAN, APPEL, ITO, + (COLU+FNAL+STON)
+ APPEL, BROWN, HERB, HOM, FISK + (COLU +FNAL+STON) AALEXANDER, DAUM, + (AACH+DESY+HAMB + SIEG+WUPG)
+ GLAWE BOCK, BLANAR, + (DESY+HAMB +HEID+MPIM) HOFMANN, ALBRECHT, + (DESY+DORT+HET+LUND) +APPEL, HERB , HOM, LLDERMAN,+ (STON+FNAL+COLU)
+ HERB, HOM, LEDERMAN, UENO,+ (COLU+FNAL+STON) +BESCH, BLUMENFELD, + (CERN+COLU+OXF+ROCK) +BOUCROT, BURGUN + (SACL+CERN +CDEF+EPOL+LALO) +ALEXANDER+ (AACH+DESY+HAMB +SIEG + WUPG)
+ HOFMANN, ALBRECHT, + (DESY+DORT +HEID+LUND) +CHILDERS, DARDEN+ (DESY+DORT + HEID+LUND+ ITEP) + (CORN+HARV+1THA+ $+B L A N A R, B L U M, B I E N L E I N+($ CEID+MPIM+DESY+HAMB) BOHRINGER, COSTANTINI, FINOCCHIARO (COLU+STON)
KOURKOUMEL IS + (ATHU+NTUA+BNL+CERN+SYRA+YALE)

+ BOHRINGER, FINOCCHIARO+ (COLU+STON+LSU+MPIM) + (RUTG $+S Y R A+L E M O+V A N D+C O R N+I T H A+H A R V+R O C H)$
NICZYPORUK, CHEN, VOGEL, WEGENER+(LENA COLLAB HOFMANN SHUBERT + (DESY+DORT + HEID +LUND+ITEP, BAARU, BLINOV, BONDAR, BUIN IN, GROSHEV (NOVO)
NICZYPORUK, FOLGER, BIEALEIN + (LENA COLLAB) (ARGUS COLLABORATION) + (CORN+ITHA+HARV+OSU+ROCH+RUTG+SYRA+VAND) +2 ( $H A R V+O S U+R O C H+R U T G+S Y R A+V A N D+C O R N+I T H A)$

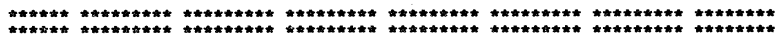
REFERENCES FOR UPSILON(9460) B. GITTELMAN
+ (CRAC+ERLA+DESY+NI JM+PITT+SACL+TELA+WURZ)
76 CHI/B(9875, JPG $=$, J J 0 PREFERRED

OBSERVED IN RADIATIVE DECAY OF THE UPSILON(10025).
INTO CHI/B GAMMA, THEREFORE C=+. $\chi_{b}(9875)$
or $\chi_{b}\left(1^{3} P_{0}\right)$

\section{CHI/B(9875) MASS (MEV) \\ M U $\quad 9872.9 \quad 5.8 \quad$ KLOPFENST 83 CUSB E+E-, GAMMAS HADR 9/83* MU FROM GAMMA ENERGY BELOW, ASSUMING UPSILON(10025) MASS $=10023.4 \mathrm{MEV}$ \\ 76 GAMMA ENERGY IM UPSILON(10025) DECAY (MEV) \\ $\begin{array}{llllll}\text { DM } & S & 149.4 & 5.7 & \text { KLOPFENST } 83 \text { CUSB E+E-,GAMMAS HADR } & 9 / 83 *\end{array}$} DM S SYSTEMATIC ERROR ADDED LINEARLY BY US.

76 CHI/B(9875) PARTIAL DECAY MODES

76 CHI/B(9875) BRANCHING RATIOS

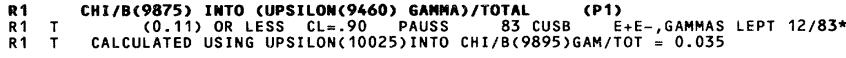

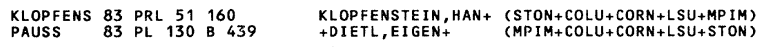

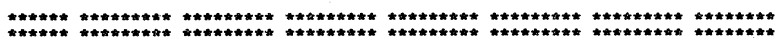

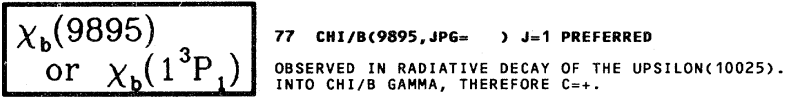

P1 CHI/B(9875) INTO UPSILON(9460) GAMMA DECAY MASSES

77 CHI/B(9895) MASS (MEV) 
For notation, see key at front of Listings.

Mesons

$$
\chi_{b}(9895)\left[\chi_{b}\left(1^{3} \mathrm{P}_{1}\right)\right], \chi_{b}(9915)\left[\chi_{b}\left(1^{3} \mathrm{P}_{2}\right)\right], \Upsilon(10025) \quad[\Upsilon(2 S)]
$$

77 GAMMA EMERGY IM UPSILOM(10025) DECAY (MEV)

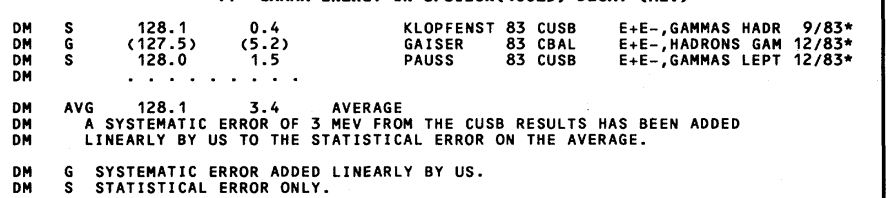

77 CHI/B(9895) PARTIAL DECAY MODES

P1 CHI/B(9895) INTO UPSILON(9460) GAMMA $9460+\underset{0}{\text { DECAY MASSES }}$

77 CHI/B(9895) BRANCHIMG RATIOS

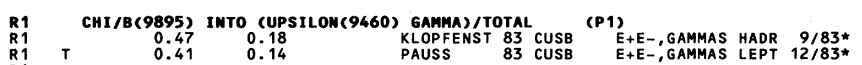

R1 AVG $\cdot 0.43^{*} \cdot \dot{0} \cdot i^{\circ}$ average

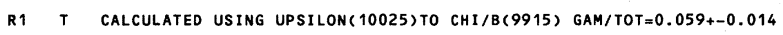

**********************************************************************

REFEREMCES FOR CHI/B(9895)

GAISER 83
KLOPFENS 83 PLAC-PUB-3232 J.

$\begin{array}{lll}\text { KLOPFENS } 83 & \text { PRL } 51160 & \text { KLOPFENSTEIN, HAN + } \\ \text { PAUSS } 83 \text { PL } 130 \text { B } 439 & \text { (STON+COLU+CORN+LSU+MPIM) } \\ \text { (MPIM+CLUT + CORN + LSU+STON) }\end{array}$

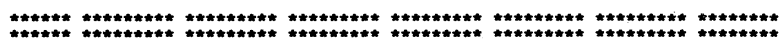

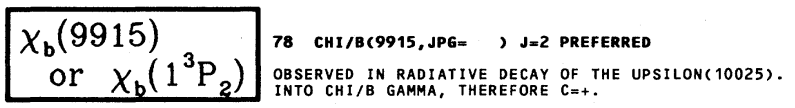

78 CHI/B (9915) MASS (MEV)

$\begin{array}{llll}M & U & 9914.6 \quad 2.4\end{array}$

M U FROM GAMMA ENERGY BELOW ASSUMING UPSILON(10025) MASS $=10023.4 \mathrm{MEV}$

78 GAMMA EMERGY IN UPSILON(10025) DECAY (MEV)

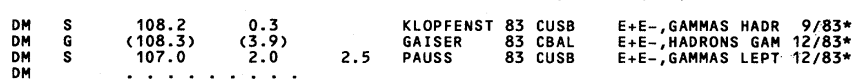

DM AVG 108.2 .2 2.3
DM AVERAGE
DM SYYSTMATIC ERROR OF 2 MEV FROM THE CUSB RESULTS HAS BEEN ADDED
LINEARLY BY US TO THE STATISTICAL ERROR ON THE AVERAGE.

$\begin{array}{lll}\text { DM } & G & \text { SYSTEMATIC ERROR ADDED LINEARLY BY US. } \\ \text { DM STATISTICAL ERROR ONLY, } & \text { SING }\end{array}$

\section{CHI/B(9915) PARTIAL DECAY MODES}

P1 CHI/B(9915) INTO UPSILON(9460) GAMMA

$9460+\quad 0$ DECAY MASSES

78 CHI/B(9915) BRANCHING RATIOS

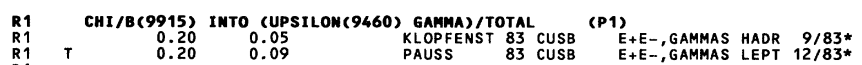

R1 AVG $\cdot 0.200^{\circ} \cdot \dot{0} \cdot \dot{0} 44_{\text {R }}$ aVerage

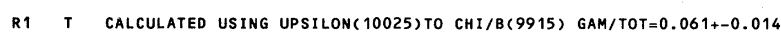

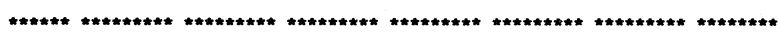

REFERENCES FOR CHI/B(9915)

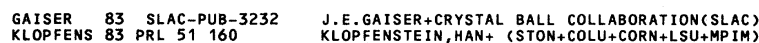

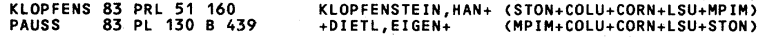

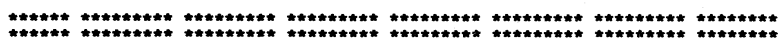
$\Upsilon(10025)$
or $\Upsilon(2 S)$ 52 UPSILONC 10025, JPG=1- , $1=$

52 UPSILON(10025) MASS (GEV)

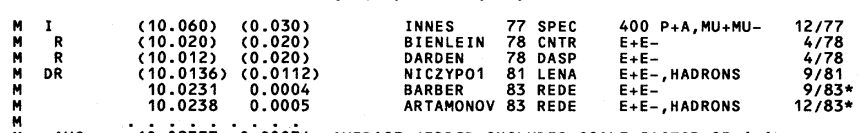

$M$ avg $10.02 \dot{3} 3 \dot{0} 0.000 \dot{3} 4$ aVerage (error includes SCALE factor of 1.1)

$\begin{array}{lll}M & \text { SYSTEMATIC ERRORS ADDEED LINEARLY BY US } \\ M & \text { I } & \text { FROM } 2 \text { PEAK FIT } \\ M & \text { R REDUDANT WITH DATA IN MASS DIFFERENCE BELOW }\end{array}$
52 UPSILON( 10025) WIDTH (KEV)

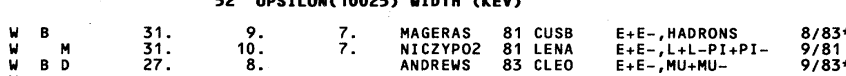
$W$ avg $29.6^{\circ} \cdot 4.7^{\circ}$ aVerage

B F FROM W2 AND R1 BELOW AND ASSUMING E-MU-TAU UNIVERSALITY

$W$
$W$ M SYSTEMATIC ERRORS ADDED LINEARLY BY US

52 UPSILON( 10025)-UPSILON(9460) MASS DIFFERENCE (MEV)

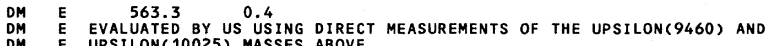
2/84*

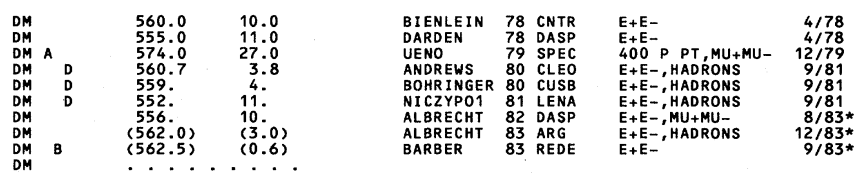

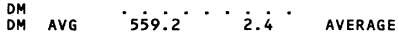

DM A FIXING THE UPSILON(9460) MASS AT 9460 MEV
DM B USING THE UPSILON $(9460)$ MASS OF ARTAMONOV 82.

DMM USING THE UPSILON ( 9460$)$ MASS OF ARTAMONOV
DM D SYSTEMATIC ERROR ADDED LINEARLY BY US.

52 UPSILON(10025) PARTIAL DECAY MODES
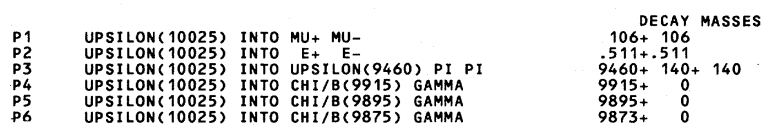

52 UPSILON(10025) PARTIAL WIDTHS (KEV)

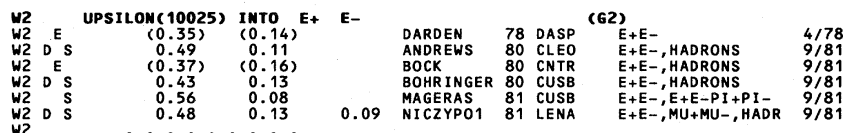
W2 AVG $0.507^{\circ} \cdot \dot{0.05} i$ aVerage

$\begin{array}{ll}\text { W2 D } & \text { SYSTEMATIC ERROR ADDED LINEARLY BY US. } \\ \text { W2 E ASSUMING HADRONIC PARTIAL WIDTH EQUAL TO TOTAL WIDTH } \\ \text { W2 }\end{array}$

52 UPSILON( 10025) BRANCHIMG RATIOS

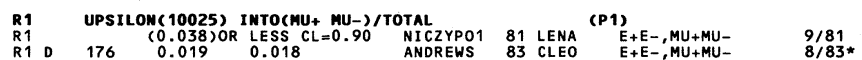

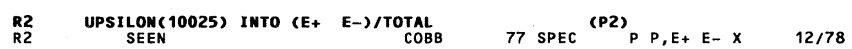

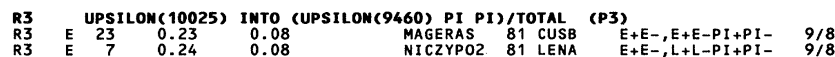

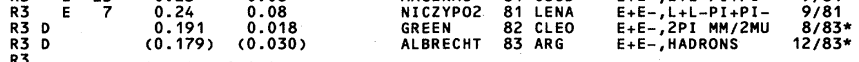

R3 AVG $0.195^{\circ} \cdot \dot{0.017}$ average

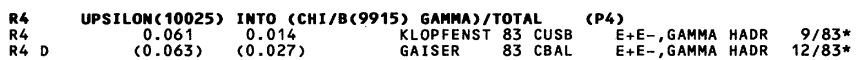

$\begin{array}{ccccccc}\text { R5 } & \text { UPSILON(10025) INTO (CH1/B(9895) GAMMA)/TOTAL } & \text { (P5) } \\ \text { R5 } & (0.059 & .014 & \text { KLOPFENST } 83 \text { CUSB } & \text { E+E-, GAMMA HADR } & 9 / 83 * \\ \text { R5 } & (0.060) & (0.027) & \text { GAISER } & 83 \text { CBAL } & \text { E+E-,GAMMA HADR } & 12 / 83 *\end{array}$

R6 UPSILON( 10025) INTO (CHI/B(9875) GAMHA)/TOTAL (P6)

$R$ DYSTEMATIC ERRORS ADDED LINEARLY BY US
$R$

盗

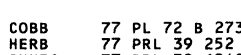

REFERENCES FOR UPSILON (10025)

INNES $\quad 77$ PRL 391240

+IWATA, FABJAN, GOL DBERG+(BNL+CERN+SYRA+YALE) + HOM, LEDERMAN, APPEE, ITO ' + (COLU+FNAL+STON)
+ +APPEL, BROWN, HERB, HOM, FISK+ (COLU+FNAL+STON)

+ HOFMANN, ALBRECHT, $+{ }^{+}$(DESY+DORT+HE ID+LUND) + APPEL, HERB, HOM, LEDERMAN, + (STON+FNAL+COLU)
+ HERB, HOM, LEDERMAN, UENO, + (COLU+FNAL+STON)

+BROWN, HERB, HOM, FISK, ITO, + (FNAL+COLU+STON)

+ (CORN+HARV+ITHA+LEMO+ROCH+RUTG+SYRA+VAND) +BOGOL JUBSKI, +
+BLANAR, BLUM, BI IENLEIN+ (HEID+MPIM+DESY (SERP) BOHINGER, COSTANTINI, FINOC CHIARO (COL+STON)
KOURKOUMEL IS+ (ATHU+NTUA+BNL+CERN+SYRA+YALE)

+ BOHR INGER, FINOCCHIARO+ (COLU+STON+LSU+MPIM) NICZYPORUK, CHEN, VOGEL, WEGENER+ (LENA COLLAB) NICZYPORUK, CHEN, FOLGER, LURZ, + (LENA COLLAB)

+ HOFMANN, SHUBERT+CDESY+DORT+HEID+LUND+ITEP) $+(R U T G+S Y R A+V A N D+C O R N+I T H A C A+H A R V+O S U+R O C H)$
$+(R U T G+S Y R A+V A N D+C O R N+I T H A C A+H A R V+O S U+R O C H)$ 
Mesons

$\chi_{b}(10235)\left[\chi_{b}\left(2^{3} \mathrm{P}_{0}\right)\right], \chi_{b}(10255)\left[\chi_{b}\left(2^{3} \mathrm{P}_{1}\right)\right], \chi_{b}(10270)\left[\chi_{b}\left(2^{3} \mathrm{P}_{2}\right)\right], \Upsilon(10355) \quad[\Upsilon(3 S)]$

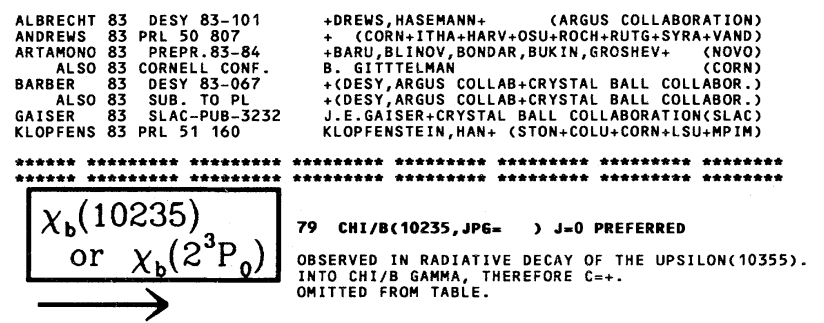

$$
79 \text { CHI/B(10235) MASS (GEV) }
$$

$\begin{array}{llll}M & U & 10.2328 & .0058\end{array}$

M U FROM GAMMA ENERGY BELOW ASSUMING UPSILON(10355) MASS $=10355.5 \mathrm{MEV}$

79 GAMMa EMERGY IN UPSILON(10355) DeCAY (MEV)

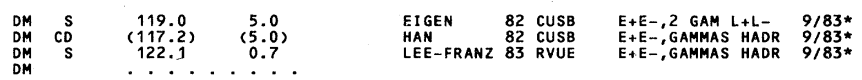

DM AVG $122.0 \quad 5.7$ AVERAGE

DM D SYSTEMATIC ERROR ADDED LINEARLY BY US.

DM
DM S STATISTICAL ERROR ONLY.

\section{CHI/B(10235) PARTIAL dECAY MODES}

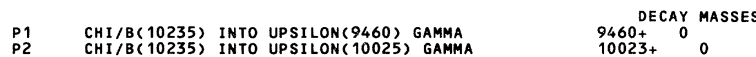

**********************************************************************

\section{REFEREMCES FOR CHI/B(10235)}

$\begin{array}{llllll}\text { EIGEN } & 82 & \text { PRL } & 49 & 1616 & \text { +BOHRINGER, HERB + (MPIM+COLU+CORN+STON+LSU) } \\ \text { HAN } & 82 & \text { PRL } & 49 & 1612 & \text { +HORSTKOTTE, IMLAY+(COLU+STON+CORN+LSU+MPIM) }\end{array}$

LEE-FRAN 83 EMS 83 CONF. 284 LEE-FRANZINI
ALSO 83 CORNELL CONF. 28 PICHAEL TUTS
(COLU)

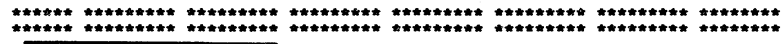
$\chi_{\mathrm{b}}(10255)$

80 CHI/B(10255,JPG= , J=1 PREFERrED

or $\chi_{b}\left(2^{3} P_{1}\right)$ observed in radiative decay of the upsilon(10355).

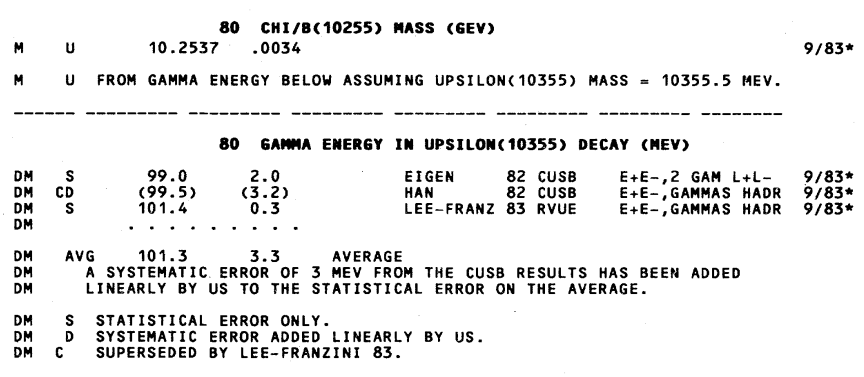

80 CHI/B(10255) PARTIAL DECAY MODES

$\begin{array}{llll}\text { P1 } & \text { CHI/B (10255) INTO UPSILON (9460) GAMMA } & \text { DECAY MASSES } \\ \text { P2 } & \text { CHI/B(10255) INTO UPSILON(10025) GAMMA } & 9460+0_{+} 0 & 10023+\end{array}$

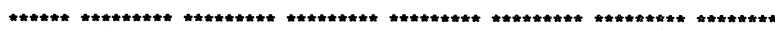

REFERENCES FOR CHI/B(10255)

$\begin{array}{lllll}\text { EIGEN } & 82 & \text { PRL } 491616 & \text { +BOHRINGER, HERB + (MPIM+COLU+CORN+STON+LSU) } \\ \text { HAN } & 82 \text { PRL } 491612 & \text { +HORSTKOT TE, IMLAY+(COLU+STON+CORN+LSU+MPIM) }\end{array}$

LEE-FRAN 83 EMS 83 CONF. 284 LEE-FRANZINI
ALSO 83 CORNELL CONF. 28 PICHAEL TUTS (COLU)
(COLU)

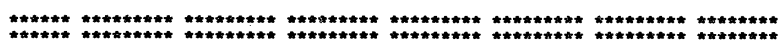

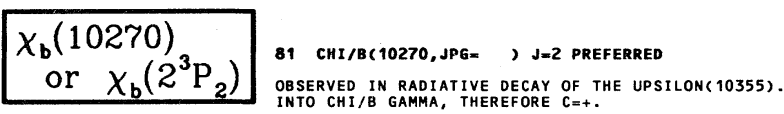

81 CHI/B(10270) MASS (GEV)

$\begin{array}{lll}\text { M U } & 10.2710 \quad .0024\end{array}$

-

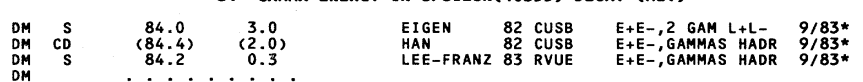

DMM AVG 84.2
DM
A SYSTEMATIC ERROR OF 2 MVERAGE FROM THE CUSB RESULTS HAS BEEN ADDED

DM STATISTICAL ERROR ONLY.
DM D SYSTEMATIC ERROR ADDED LINEARLY BY US.
DM
D SUPERSEDED BY LEE-FRANZINI 83 .

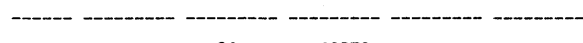

$\begin{array}{lll}\text { P1 } & \text { CHI/B(10270) INTO UPSILON(9460), GAMMA } & \text { DECAY MASSES } \\ \text { P2 } & \text { CHI/B(10270) INTO UPSILOH(10025) GAMMA } & 9460+ \\ \end{array}$

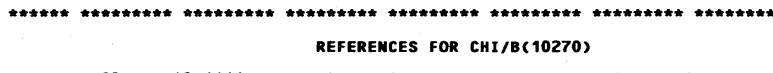

$\begin{array}{llllll}\text { EIGEN } & 82 & \text { PRL } 49 & 1616 & \text { +BOHRINGER, HERB + (MPIM+COLU+CORN+STON+LSU) } \\ \text { HAN } & 82 \text { PRL } & 49 & 1612 & \text { +HORSTKOTTE, IMLAY+ (COLU+STON+CORN+LSU+MPIM) }\end{array}$

LEE-FRAN 83 EMS 83 CONF.
ALSO 83 CORNEL CONF. 284 PEE-FRANZINI
MICHAEL TUTS
(COLU)

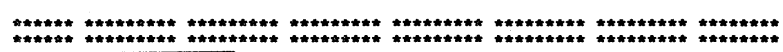

$\Upsilon(10355)$
or $\Upsilon(3 S)$ 48 upsilon(10355, JPG=1- , I=

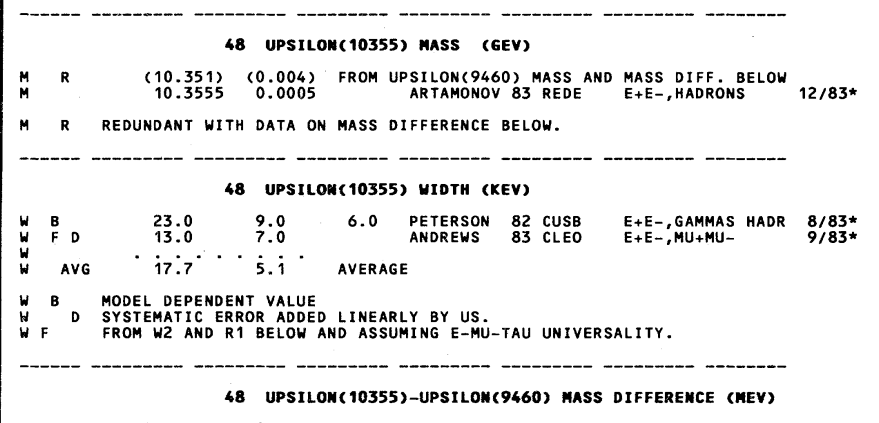

$\begin{array}{llcl}\text { DM } & \text { E } & 895.5 & 0.6 \\ \text { DM } & \text { E EVALUATED BY US USING DIRECT MEASUREMENTS OF THE UPSILON(9460) AND } & 2 / 84 * \\ \text { DM } & \text { E UPSILON(10355) MASSES ABOVE. }\end{array}$ $\begin{array}{llclllll}\text { DM A } & 950.0 & 30.0 & \text { UENO } & 79 \text { SPEC } & 400 \text { P PT,MU+MU- } & 12 / 79 \\ \text { DMM }_{\text {DM }}{ }^{D} & 891.1 & 5.7 & \text { ANDRES } & 80 \text { CLED } & \text { E+E-,HADRONS } & 9 / 81 \\ \text { DM } & 889 . & 6 . & \text { BOHRINGER } & 80 \text { CUSB } & \text { E EE-,HADRONS } & 9 / 81\end{array}$

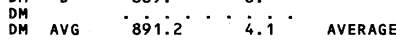

DM A FIXING THE UPSILON(9460) MASS AT 9460 MEV AND THE
DM A UPSILON(10025)-UPSILN(9460) MASS DIFFERENE AT 558 MEV.
DM D SYSTEMATIC ERROR ADDED LINEARLY BY US.

48 UPSILON(10355) PARTIAL DECAY MODES

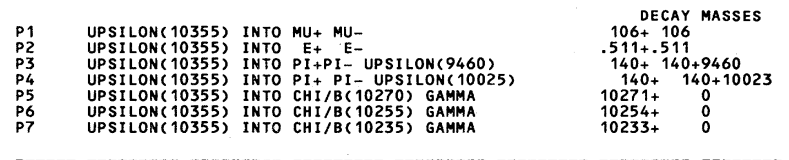

48 UPSILOM(10355) PARTIAL MIDTHS (KEV)

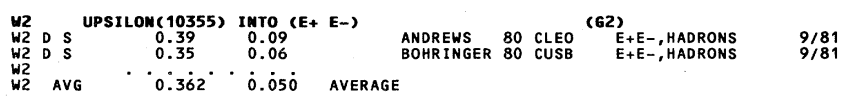

W2 ${ }^{\circ} \mathrm{S}$ SYSTEMATIC ERROR ADDED LINEARLY BY US.
W2 
For notation, see key at front of Listings.

Mesons $\Upsilon(10355)[\Upsilon(3 S)], \Upsilon(10575) \quad[\Upsilon(4 S)], K^{ \pm}, K^{0}, K^{*}(892)$

48 UPSILON(10355) BRANCHING RATIOS

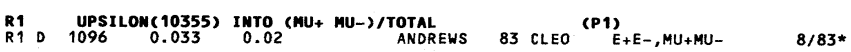
R3 UPSILON (10355) INTO (PI+PI- UPSILON(9460)) /TOTAL (P3)

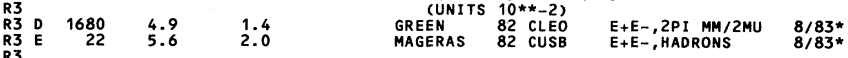
R3 AVG $\cdot 5.1^{\text {R3 }} \cdot i^{\prime} i^{\prime}$ aVerage

R4 UPSILON( 10355) INTO UPSILON (10025) PI+ P1-JTOTAL (P4)

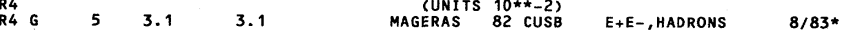

R5 UPSILON(10355) IMTO (CHI/B(10270) GAMMA)/TOTAL (P5)

R6 UPSILON(10355) IMTO (CHI/B(10255) GAMMA)/TOTAL
R6 (P6)
0.042

R7 UPSILON( 10355) INTO
R7 (CHI/B (10235) GAMMA)/TOTAL
R7.076 (P7)

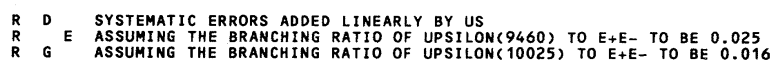

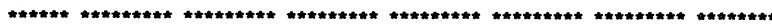

$\begin{array}{lllll}\text { COBB } & 77 & \text { PL } 72 & 273 \\ \text { HERB } & 77 & \text { PRL } 39 & 252 \\ \text { INNES } & 77 & \text { PRL } 39 & 1240\end{array}$

REFEREMCES FOR UPSILON (10355)

$\begin{array}{lllll}\text { KAPLAN } & 78 & \text { PRL } & 40 & 435 \\ \text { YOH } & 78 & \text { PRL } & 41 & 684\end{array}$

+ IWATA, FAB JAN, GOLDBERG+(BNL+CERN+SYRA+YALE)

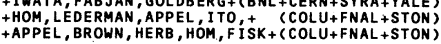

UENO 79 PRL 42486

$\begin{array}{lllll}\text { ANDREWS } & 80 & \text { PRL } & 44 & 1108 \\ \text { BOHR INGE } & 80 & \text { PRL } & 44 & 1111\end{array}$

$\begin{array}{lllll}\text { GREEN } & 82 & \text { PRL } & 49 & 617 \\ \text { HAN } & 82 & \text { PRL } & 49 & 1612\end{array}$

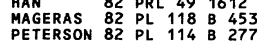

ANDREWS 83 PRL 50807

APPEL, HERB HOM, LEDERMAN, + (STON+FNAL+COLU)

+HERB, HOM, LEDERMAN, UENO,+ (COLU+FNAL+STON)

+ BROWN, HERB, HOM, FISK, ITO, + (FNAL+COLU+STON) + (CORN+HARV+ ITHA+LEMO+ROCH+RUTG+SYRA+VAND

+ (RUTG+SYRA+VAND+CORN+ITHACA+HARV+OSU+ROCH ) (HORSTKOTTE, IMLAY+ + (COLU+STON+CORN+ + SU+MPIM) +HERB, IMLAY+
+ GIANNINI, LEE-FRANZINI + (COLU+STON+LSU+MPIM) + (CORN+ITHA+HARV+OSU+ROCH+RUTG+SYRA+VAND) +BARU, BLINOV, BONDAR, BUKIN, GROSHEV+ (NOVO)
(COLU)

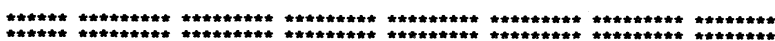
$\left.\begin{array}{c}\Upsilon(10575) \\ \text { or } \\ \Upsilon(4 S)\end{array}\right] 47$ UPSILON(10575,JPG=1- , I=

47 UPSILON(10575) MASS (GEV)

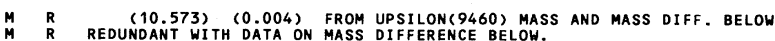

47 UPSILON (10575) UIDTH (MEV)

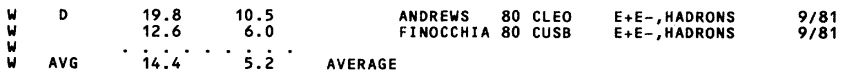

W D SYSTEMatic ERROR AdDED Lineraly By US.

47 UPSILON(10575)-UPSILOM(9460) MASS DIFFEREMCE (MEV)

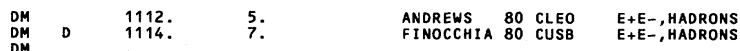
DM AVG $i 1 i 2.7^{\circ} \cdot i^{\circ} i^{\cdot}$ average

DM D SYSTEMATIC ERROR ADDED LINERALY BY US

47 UPSILON(10575) PARTIAL DECAY MODES

P1 UPSILON(10575) INTO MU+ MU-
P2 UPSILON(10575)

DECAY MASSES $106+106$
$.511+.511$

47 UPSILON(10575) PARTIAL WIDTHS (KEV)

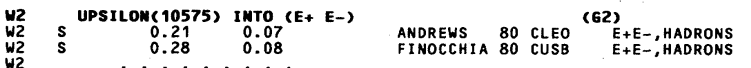

W2 AVG $\cdot 0.240^{\circ} \cdot \dot{0} .05 \dot{3}$ aVerage

W2 S USING UPSILON(9460) PARTIAL WIDTH TO E+E- $=1.10 \mathrm{KEV}$

**********************************************************************

REFEREMCES FOR UPSILON(10575)

$\begin{array}{llllll}\text { ANDREWS } & 80 & \text { PRL } & 45 & 219 \\ \text { FINOCCHI } 80 & \text { PRL } & 45 & 222 & \text { + (CORN+HARV + ITHA+LEMO+ROCH+RUTG +SYRA+VAND) }\end{array}$

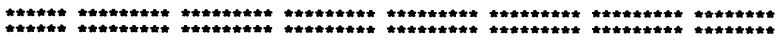

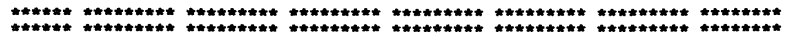

$S= \pm 1, \quad C=0, B=0$ MESON STATES

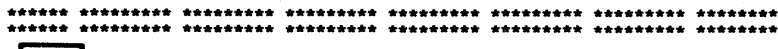

$\mathrm{K}^{ \pm} \quad 10$ CHARGED $K(494, J P=0-) \quad I=1 / 2$

SEE STABLE PARTICLE DATA CARD LISTINGS

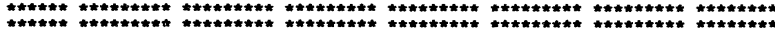

$K^{0} \quad 11$ MEUTRAL $K(498, J P=0-) \quad I=1 / 2$

SEE STABLE PARTICLE DATA CARD LISTINGS

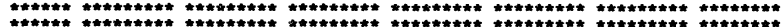

$K^{*}(892) \quad$ is $\quad K *(892, J P=1-) \quad I=1 / 2$

$18 K *(892)$ MASS (MEV)

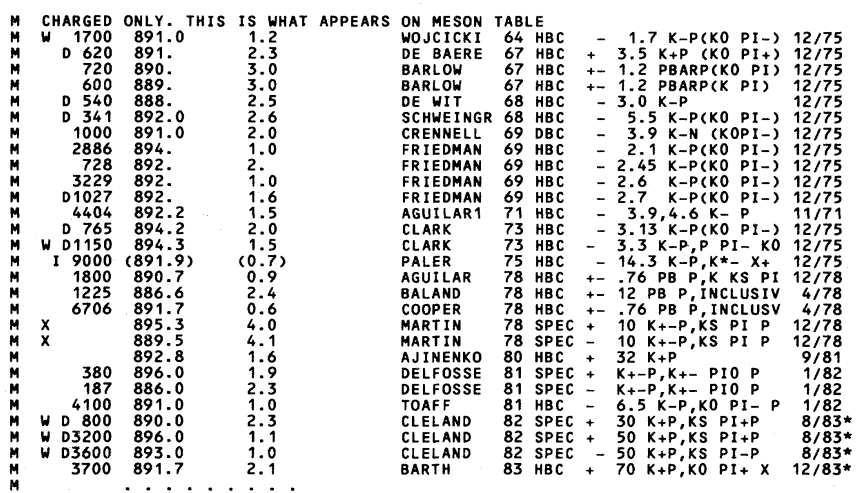

$0.37^{\circ}$ AVERAGE (ERROR INCLUDES SCALE FACTOR OF 1.4)

WEIGHTED AVERAGE $=892.08 \pm 0.37$
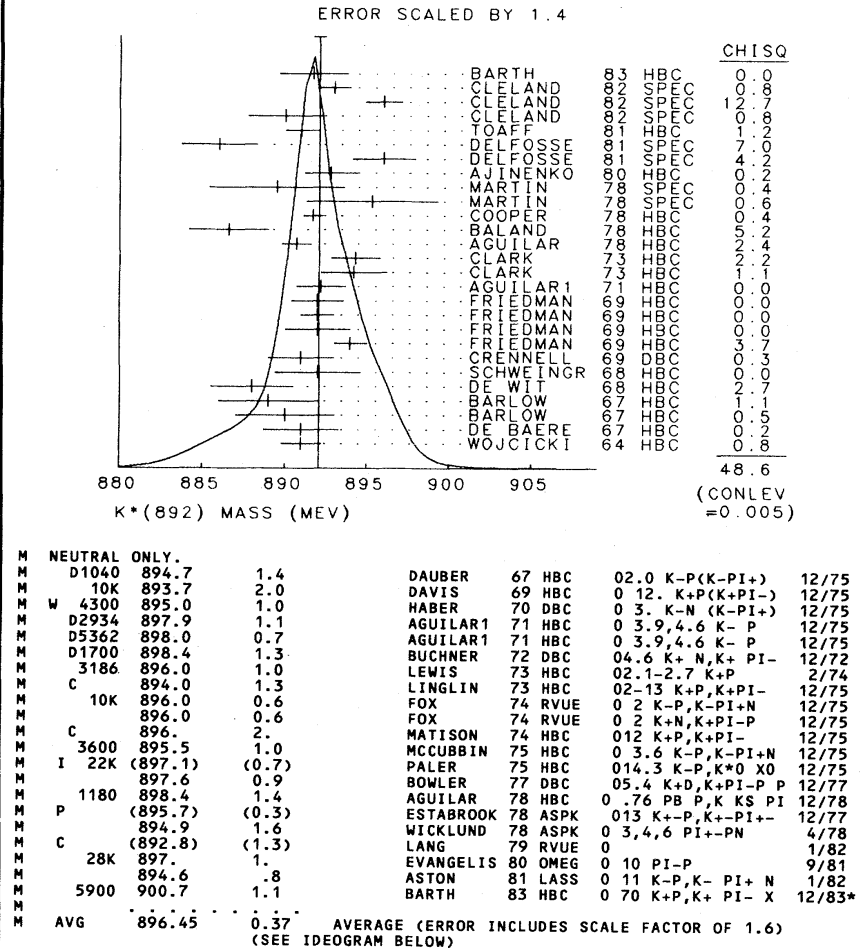


\section{Mesons}

Data Card Listings

$K^{*}(892)$

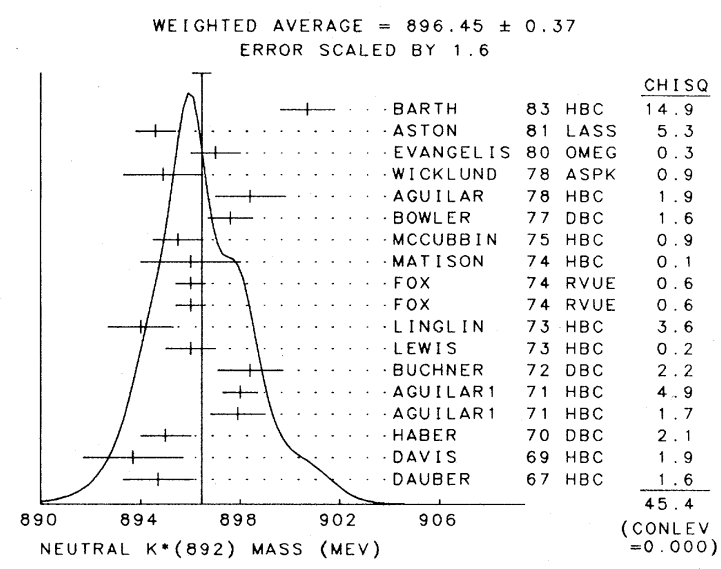

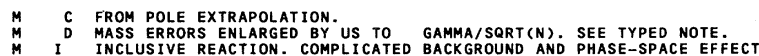
$M$
$M$
$M$

\section{NOTE ON K${ }^{\star}(892)$ MASSES AND MASS DIFFER- ENCES}

Unrealistically small errors are reported by some experiments. We use simple "realistic" tests for the minimum errors on the determination of mass and width from a sample of $\mathrm{N}$ events:

$$
\delta_{\min }(\mathrm{m})=\frac{\Gamma}{\sqrt{\mathrm{N}}}, \quad \delta_{\min }(\Gamma)=4 \frac{\Gamma}{\sqrt{\mathrm{N}}} .
$$

(For a detailed discussion, see the 1971 edition of this note.) We consistently increase unrealistic errors before averaging.
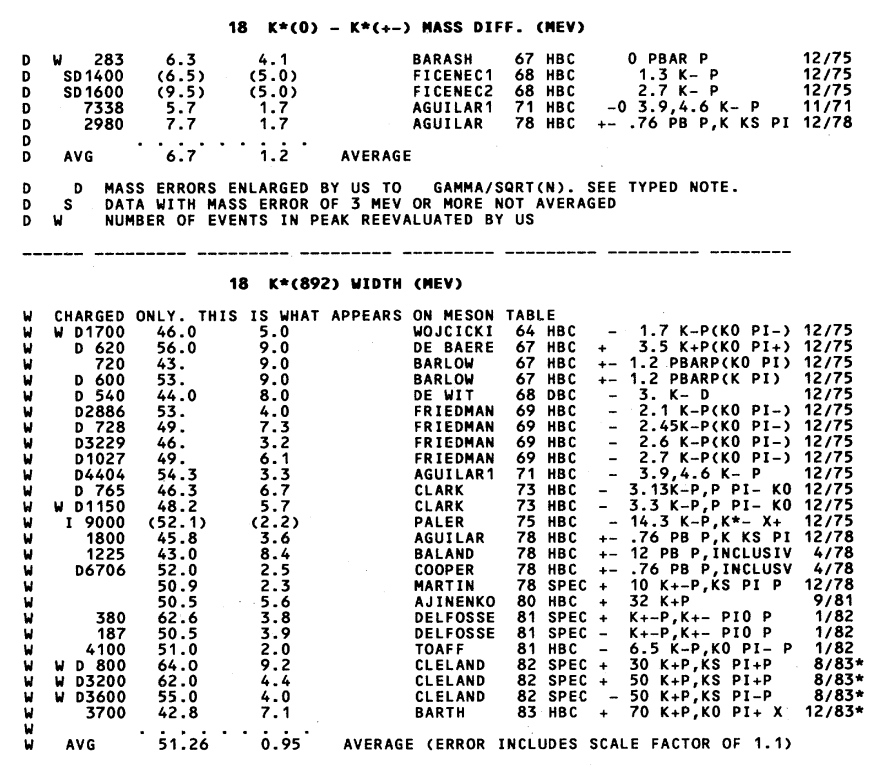

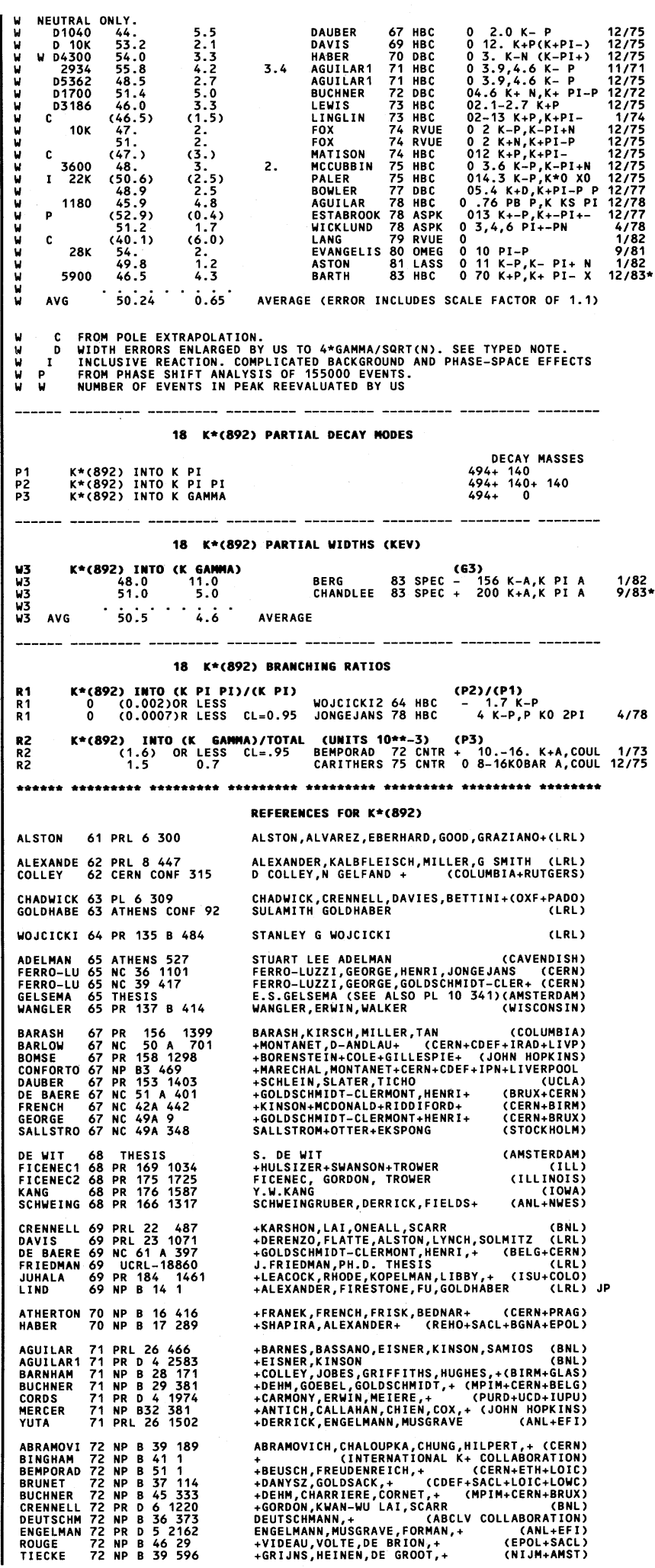




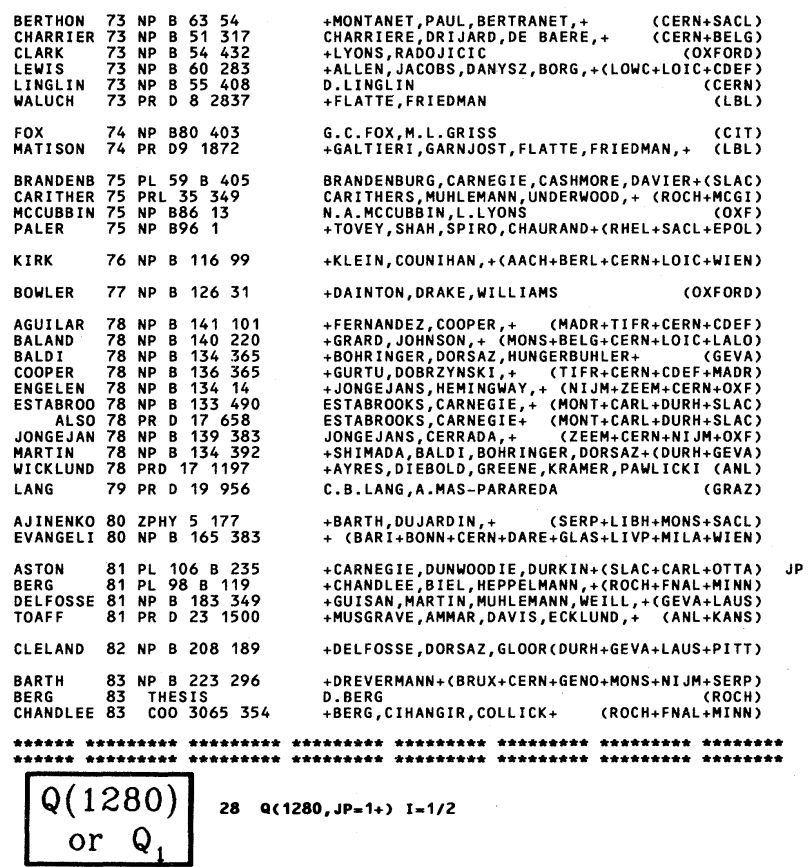

\section{NOTE ON THE Q(1280) AND Q(1400)}

We no longer use the subscripts 1 and 2 on the $Q$ states.

Since all the recent high-statistics experiments investigating the partial-wave contents of the $\mathrm{K} \pi \pi$ system diffractively produced on protons (BRANDENBURG 76, OTTER 76, VERGEEST 79, DAUM 81) give consistent evidence for the existence of two strangeness-one axial vector mesons, we have split the " $Q$ region" entry into two entries: one for the $Q(1280)$ resonance, with a mass around $1280 \mathrm{MeV}$, a width of the order of $100 \mathrm{MeV}$, and coupled mainly to the $\mathrm{K} \rho$ channel; and another one for the $Q(1400)$ resonance, with a mass around 1400 $\mathrm{MeV}$, a width of the order of $180 \mathrm{MeV}$, and coupled mainly to the $\mathrm{K}^{*}(892) \pi$ channel.

Notice that, whereas both $\mathrm{Q}(1280)$ and $\mathrm{Q}(1400)$ are produced in diffractive processes, the nondiffractive reactions (ARMENTEROS 64, CRENNELL 67,72, ASTIER 69, GAVILLET 78, ETKIN 80, RODEBACK 81 , BAUBILLIER 82 ) select preferentially the production of one of the two states.

\section{8 a(1280) mass (MEV)}

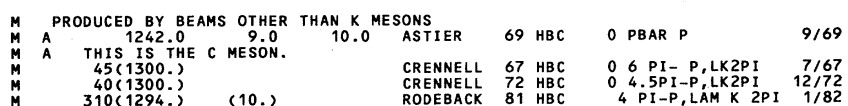
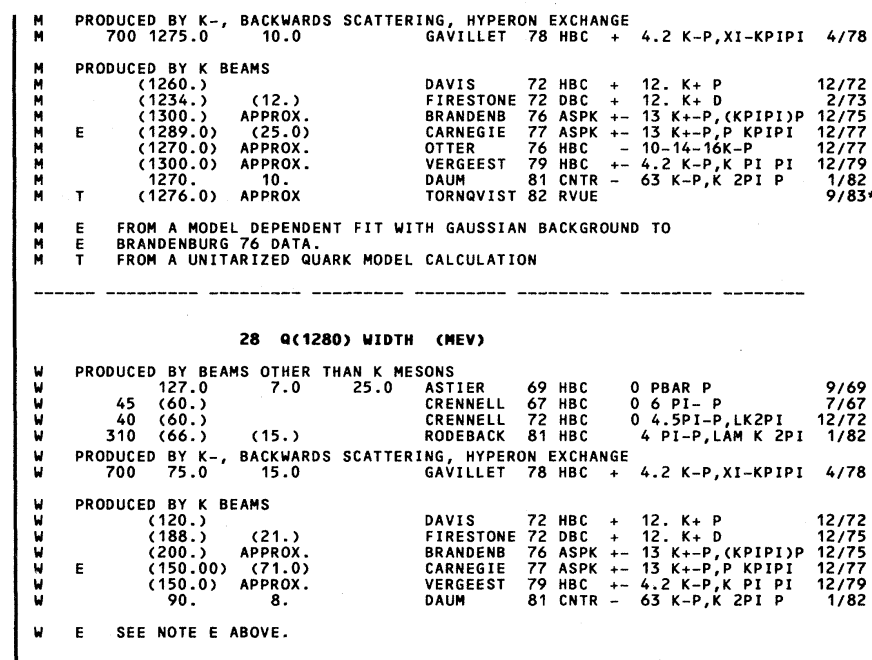

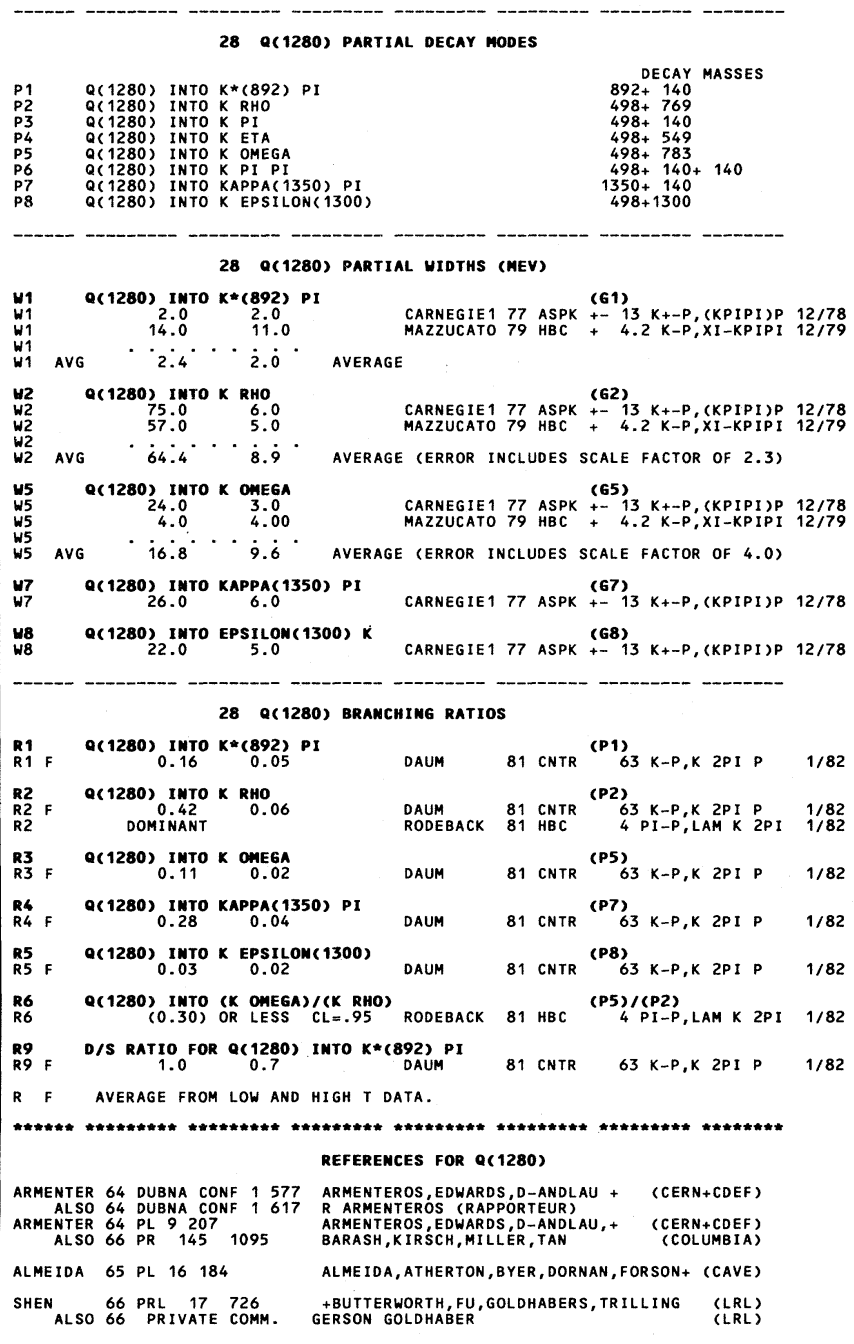




\section{Mesons}

$Q(1280)\left[Q_{1}\right], \kappa(1350)$ tion in the region of the $K^{*}(892)$ has been ruled out conclusively (MATISON 72,74, GALTIERI 73, BOWLER 77, ESTABROOKS 78).

The first inelastic two-body threshold is $\mathrm{K} \eta$, which, however, is very weakly coupled to the $\kappa(1350)$, in accordance with the SU(3) prediction for the $\kappa(1350) \mathrm{K} \eta$ coupling.

In the energy range $1350-1450 \mathrm{MeV}$, the phase shift exhibits rapid motion (BOWLER 77, ESTABROOKS 78, MARTIN 78), which has been taken as an indication of a $\kappa$ resonance at $1500 \mathrm{MeV}$ (ESTABROOKS 79). However, the phase-shift behavior can also be understood as a cusp effect due to the nearby $\mathrm{K} \eta^{\prime}$ threshold at $1455 \mathrm{MeV}$ (TORNQVIST 82). Above this energy the inelasticity due to $\mathrm{K} \eta^{\prime}$ is important. The phase shift can be fitted up to about $1500 \mathrm{MeV}$ in a unitary coupledchannel analysis with proper analytic structure with one resonance, the $\kappa(1350)$, without background

(TORNQVIST 82). This supports earlier interpretations (FIRESTONE 71,72, FRATI 72, ROUGE 72, CORDS 73, LAUSCHER 75, MORGAN 75, ENGELEN 78, ASTON 81) that this $\kappa$ mass is indeed where $\delta_{0}^{1}$ passes through $90^{\circ}$.

At still higher energies some evidence for a second scalar resonance exists (ASTON 81).

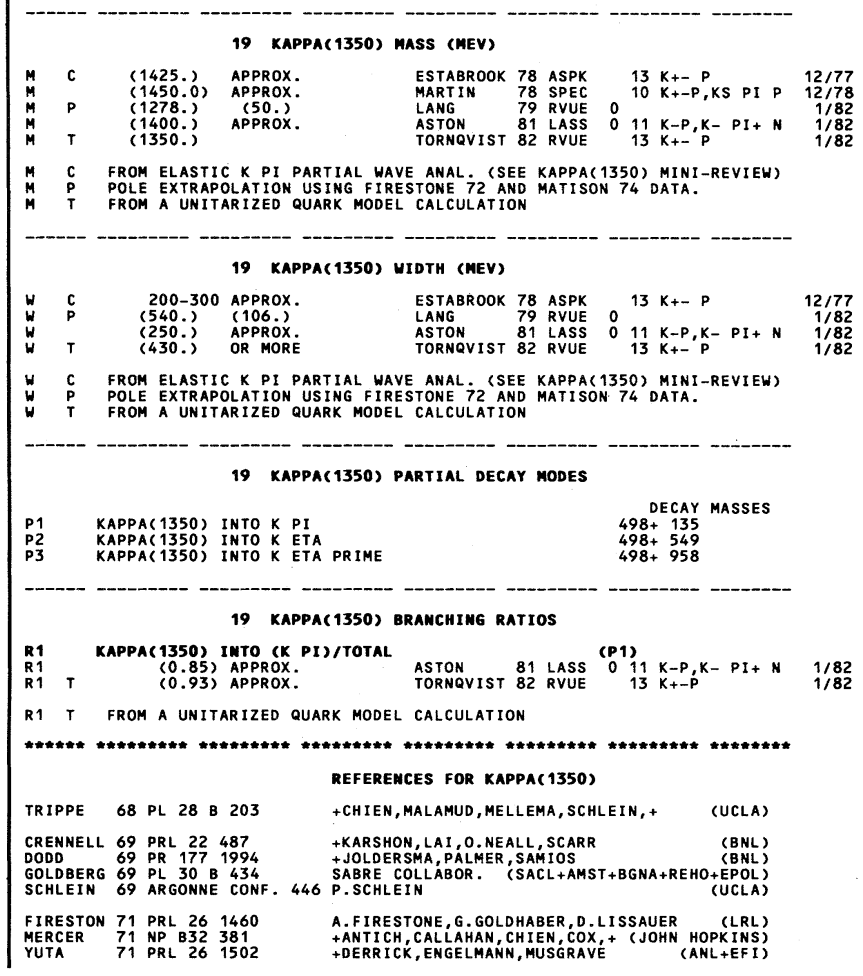




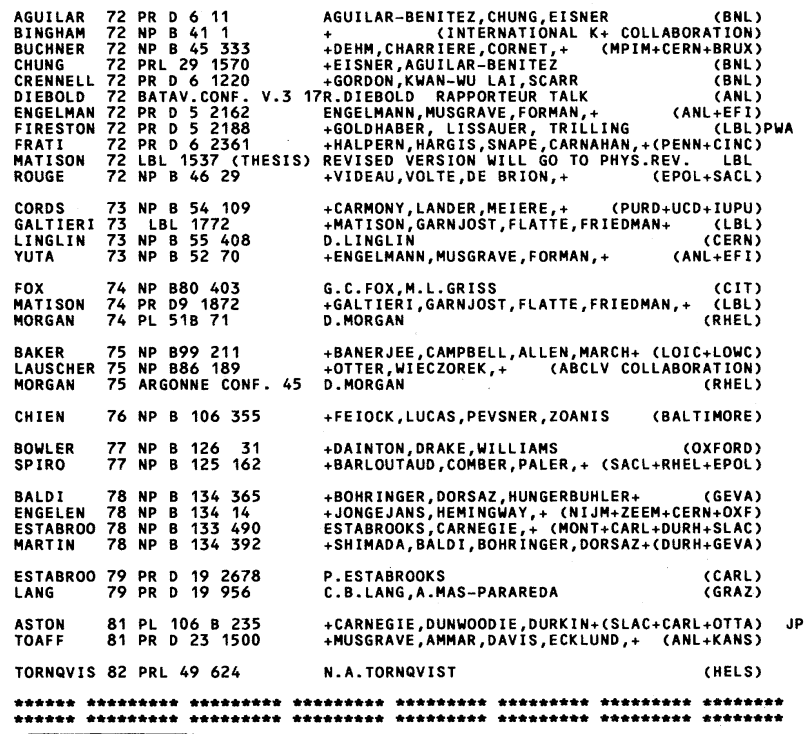

$\mathrm{Q}(1400)$ of $\mathrm{Q}(1400, \mathrm{JP}=1+) \mathrm{I}=1 / 2$

or $Q_{2}$ SEE MINI-REVIEW UNDER Q(1280)
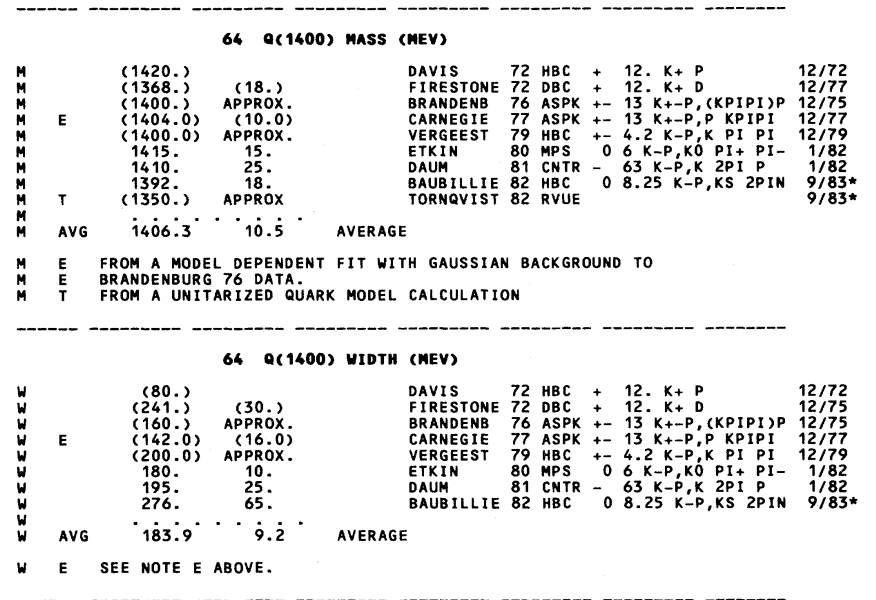

64 Q(1400) PARTIAL DECAY mODES

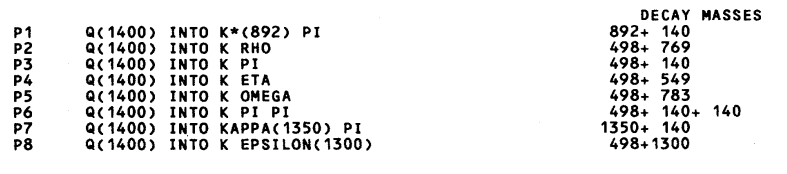

\begin{tabular}{|c|c|c|c|c|c|}
\hline & $640(1400)$ & PARTIAL WIDTHS & (MEV) & & \\
\hline $\begin{array}{l}\text { u1 } \\
\text { w1 }\end{array}$ & $\begin{array}{c}Q(1400) \text { INTO } K *(892) \\
117.0 \\
10.0\end{array}$ & CARNEGIE 1 & 177 ASPK & $\begin{array}{l}(61) \\
+-13 K+-P,(K P I P I) P\end{array}$ & $12 / 78$ \\
\hline $\begin{array}{l}\text { w2 } \\
w 2\end{array}$ & Q(1400) $\underset{2.0}{\text { INTO K RHO }}$ & CARNEGIE 1 & 77 ASPK & $\begin{array}{l}(62) \\
+-13 K+-P,(K P I P I) P\end{array}$ & $12 / 78$ \\
\hline $\begin{array}{c}45 \\
45\end{array}$ & $\underset{23.0}{Q(1400)_{23} \text { IMTO K OMEGA }}$ & CARNEGIE 1 & 177 ASPK & $\begin{array}{l}(65) \\
+-13 K+-P,(K P I P I) P\end{array}$ & $12 / 78$ \\
\hline & $64 a(1400)$ & BRAMCHING RATIOS & & & \\
\hline $\begin{array}{ll}\text { R1 } \\
\text { R1 F }\end{array}$ & $Q(1400) \underset{0.94}{\text { IWTO }} \mathrm{K*(892)} \underset{0.06}{\mathrm{PI}}$ & DAUM & 81 CNTR & ${ }_{63}^{(P 1)} K-P, K$ 2PI P & $1 / 82$ \\
\hline $\begin{array}{ll}R 2 \\
R 2\end{array}$ & $Q(1400) \underset{0.03}{\text { INTO K RHO }} \underset{0.03}{ }$ & DAUM & 81 CNTR & ${ }_{63}^{(P 2)} K-P, K 2 P 1 P$ & $1 / 82$ \\
\hline
\end{tabular}

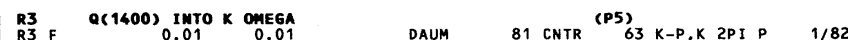

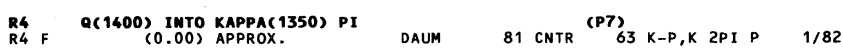

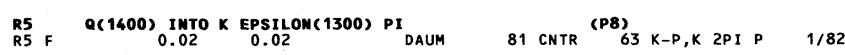

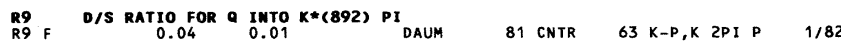
R F AVERAGE From LOW AND HIGH T DATA.

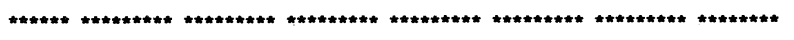
REFEREMCES FOR Q(1400)

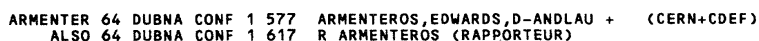

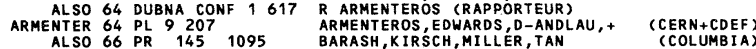
ALMEIDA 65 PL 16184 ALMEIDA, ATHERTON, BYER, DORNAN, FORSON+ (CAVE)

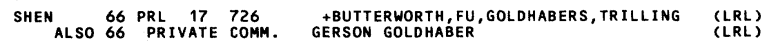

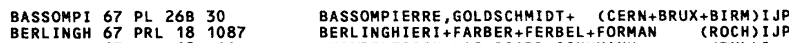

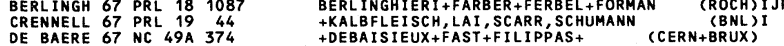

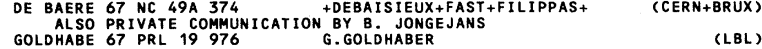

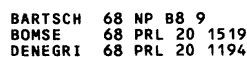
ALEXANDE 69 NP B 13503 ANDREWS 69 PRL 2273 $\begin{array}{ll}\text { BARARO } & 69 \text { PRL } 22.1207 \\ \text { BETTINI } & 69 \\ \text { NC } & 22 \text { A } 1038\end{array}$

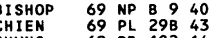
$\begin{array}{lll}\text { CHUNG } & 69 \text { PR } 1821443 \\ \text { COLLY } & 69 & 18 \\ \text { CC } & 59519\end{array}$

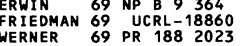

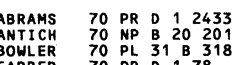
BARNHAM 71 NP $B 2549$

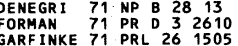
ANDERSON 72 PR $D \quad 61823$

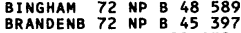

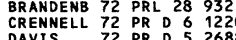

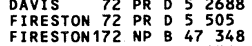

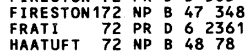
BARLOUTA 73 NP B 59374

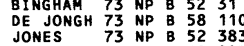

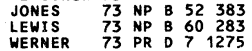

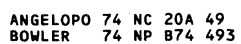

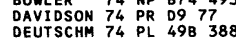
ANTIPOV $75 \mathrm{NP} B 86381$

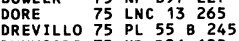

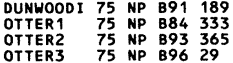

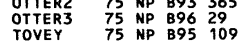

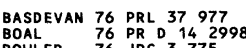

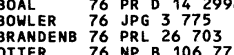

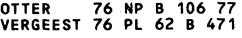
$\begin{array}{llll}\text { CARNEGIE } & 77 & \text { NP B } & 127 \\ \text { CARNEGIE } & 509 \\ \text { CAL B } & 68 & 287\end{array}$

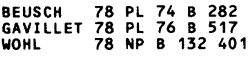

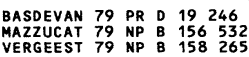
$\begin{array}{llllll}\text { BACON } & 80 & \text { NP } & \text { B } & 162 & 189 \\ \text { DIONISI } & 80 & \text { NP B } & 169 & 1\end{array}$

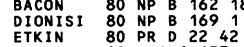

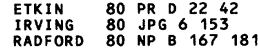
$\begin{array}{llllll}\text { DAUM } & 81 & \text { NP } & \text { B } & 187 & 1 \\ \text { OTTER } & 81 & \text { NP } & 181 & 181 \\ \text { ODOEBACK } & 81 & \text { ZPHY } & \text { C } & 9\end{array}$

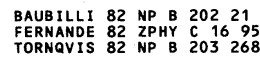

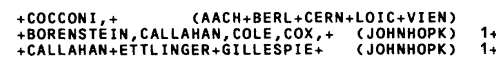
G. ALEXANDER, FIRESTONE, GOLDHABER, + (LRL)

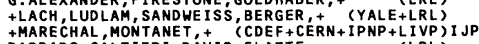

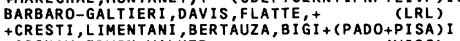
+GOSHAW, ERWIN, WALKER
+MALAMUD, MELLEMA, RUDNICK, SCHLEIN+ (WISCLA
(UCLA) (BNL)
+EISNER+BALI +LUERS
+ +EASTWOD
+AIR

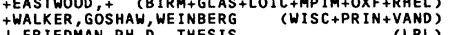
J FRIEDMAN, PH, DO THESIS
+AMMAR, DAVIS, KROPAC, YARGER, CHO, + (NMES+ARL) 1+

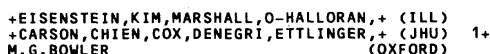
M. G. BOWLER
+FERBEL, SLATTERY, YUTA

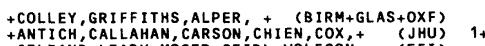

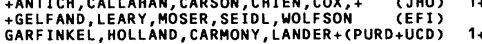
+FRANKL IN, GODDEN, KOPELMAN, L IBBY, TAN (COLO)

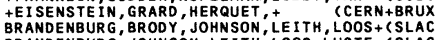
BRANENBBRRG, JOHNSON, LETTH, LOOS, LUSTE+(SLAC)

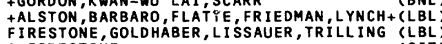

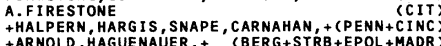

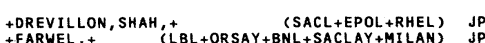

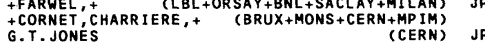

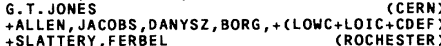

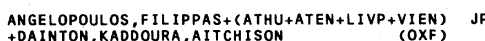

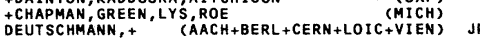
+ASCOLI, BUSNELLO, KIENZLE+ (SERP+CERN+ILL) JP +GAME, AITCHISON, DAINTON (OXF+DARE) G GUIDONI, LAAKSO MARINI, CONFORTO+ (ROMA FHEL)

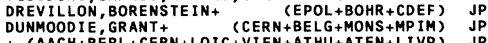

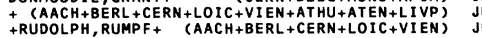

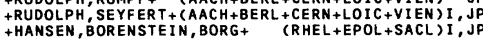
BASDEVANT, BERGER
+EDHARDS, KAMAL, TORGESON (FNAL+ANL)

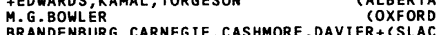

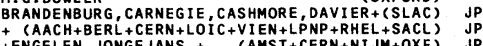

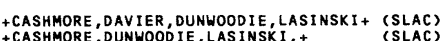

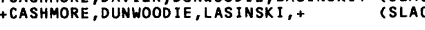

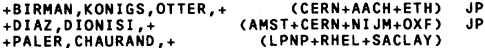
BASDEVANT, BERGER
(ANL) MAZZUCATO, PENNINGTON+ (CERN+ZEEM+NI JM+OXX)
+JONGEJANS, DIONISI, +BARREY, BUTTERWORTH, ANSORGE,
+GAVILLET, (LOIC+CAVE FOLEY, LNAENBAUM, KRAMER, +
A.C.IRVNING
(LIVP) A.C. IRVING
RADFORD, BRANDENBURG (MIT) + HERT ZBEREER+ (AMST+CERN+CRAC+MPIM+OXF+RHEL)

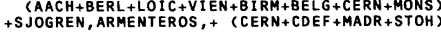
BAUBILLIER+
GERAANOER, AGUILAR+
(BIRM+CERN+GLAS+MSU+LPNP)
(MADR+CERN+CDEF + STOH

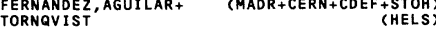


Mesons $\mathrm{K}(1400)\left[\mathrm{K}^{\prime}\right], \mathrm{K}^{*}(1430)$

\section{Data Card Listings}
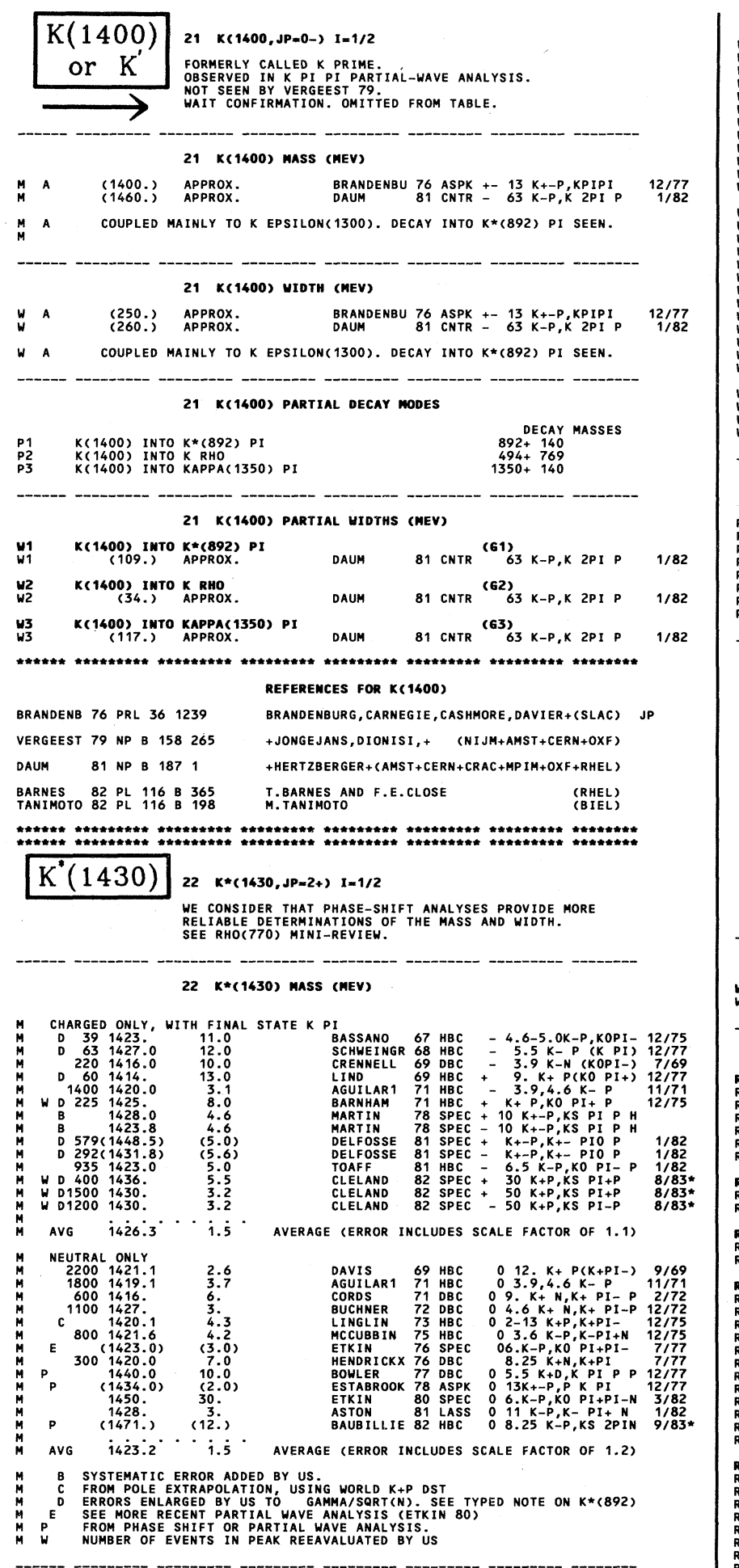

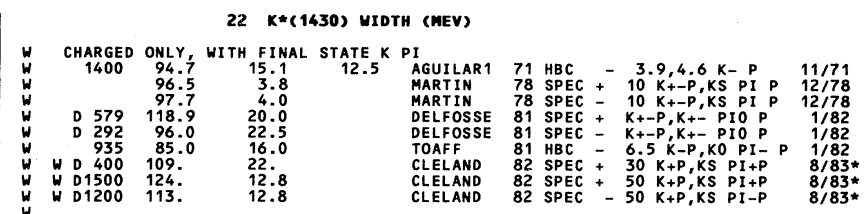

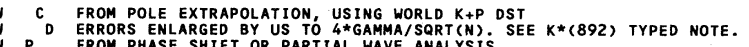

H NUMBER OF EVENTS IN PEAK REEAVALUATED BY US

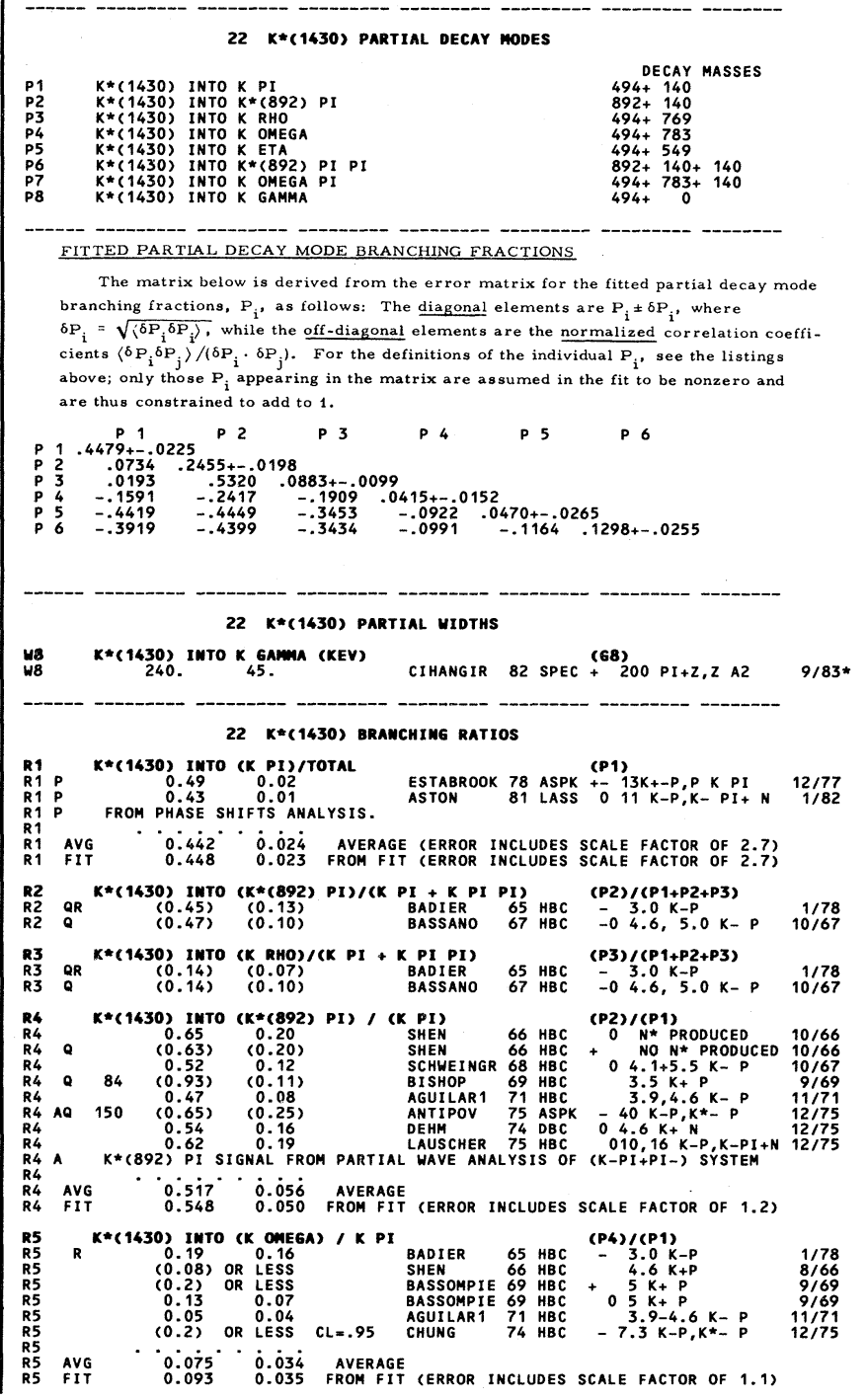



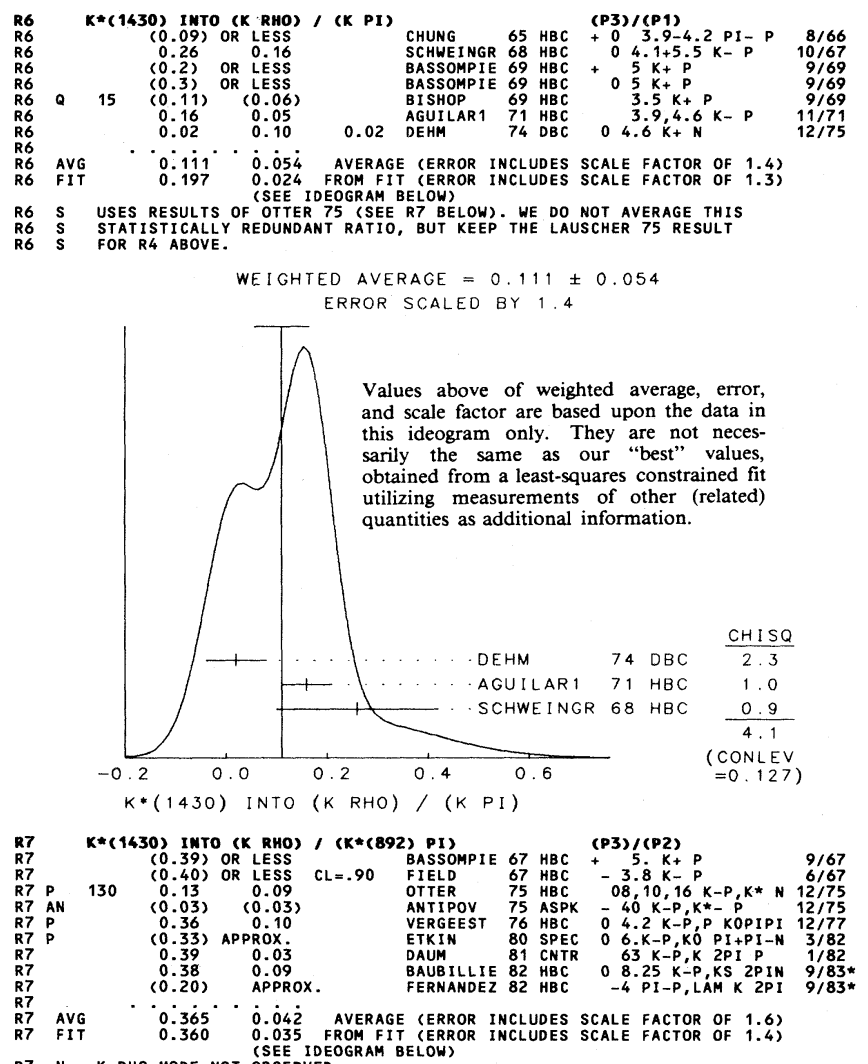

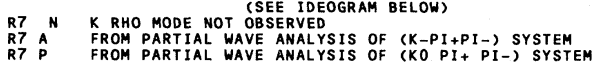

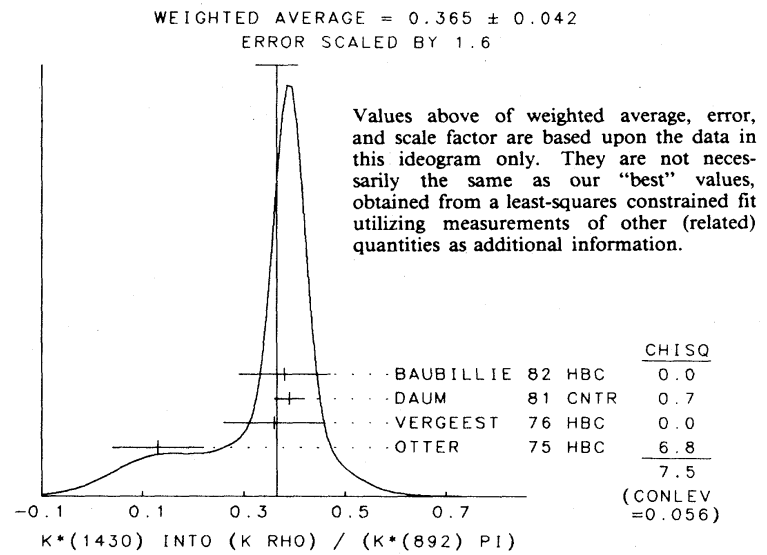

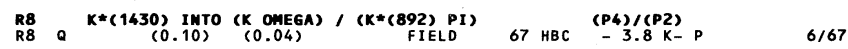

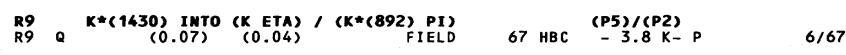

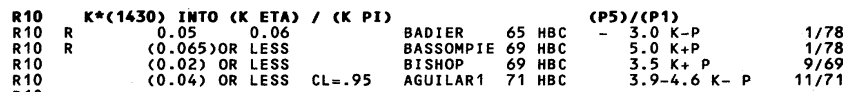
R10 FIT $0.105^{\circ} \cdot 0.062$ FROM FIT (ERROR INCLUDES SCALE FACTOR OF 1.2)

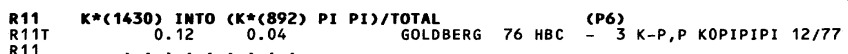
R11 FIT $\cdot 0.130^{\circ} \cdot \dot{0} 0 . \dot{0} \dot{6}$ FROM FIT (ERROR INCLUDES SCALE FACTOR OF 1.1)

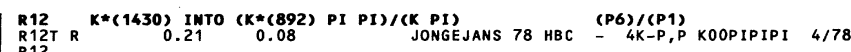
R12 FIT $\cdot 0.290^{\circ} \cdot 0.064$ FROM FIT (ERROR INCLUDES SCALE FACTOR OF 1.2)

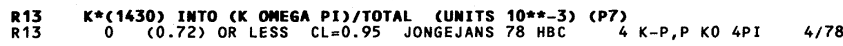
$R$ F FOLLOWING SUGGESTION BY AGUILAR 70 , WE DO NOT MAKE USE OF MEASURE-
$R$ Q MENTS WHERE THE (K PI PI) BACKGROUND SUBTRACTION IS DIFFICULT DUE Q TO THE NEARBYQ REGION.

$R$
$R$$\quad$ RESTATED BY US. ASSUMING PI PI SYSTEM HAS ISO-SPIN 1, WHICH IS SUPPORTED BY

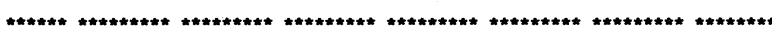

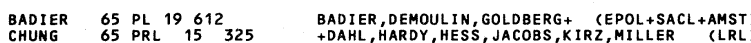

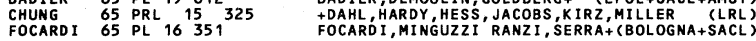

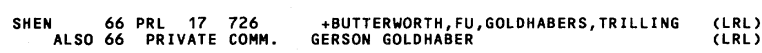
BASSANO 67 PRL 19968 $\begin{array}{lll}\text { BASSANO } 67 & \text { PRL } 19 \\ \text { BASSOMPI } & 67 & \text { PL } 26830 \\ \text { CRENNELL } 67 & \text { PL } 30\end{array}$

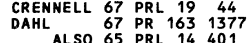
DAHL ALSO 65 PRL 14401
DE BAERE 67 NC 51 A 401
FIELD 67 PL 248 A 638
GOLDHABE 67 PRL 19.972

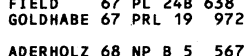

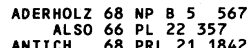
$\begin{array}{llll}\text { ANTICH } & 68 & \text { PRL } 21 & 1842 \\ \text { DUBAL } & 68 & \text { THESIS } & 1456\end{array}$ $\begin{array}{lllll}\text { KANG } & 68 & \text { PR } & 176 & 1587 \\ \text { SCHWEING } & 68 & \text { PR } & 166 & 1317 \\ \text { ALSO } & 67 & \text { THESIS }\end{array}$ BASSOMPI 69 NP B13 189 $\begin{array}{lllll}\text { BISHOP } & 69 & \text { NP } & \text { B } & 9 \\ \text { CRENNELL } & 69 & 403 \\ \text { CRL } 22 & 487 \\ \text { DAVIS } & 69 & \text { PRL } & 22 & 4071\end{array}$

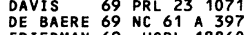

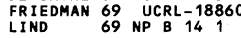
$\begin{array}{llllll}\text { ABRAMS } & 70 & \text { PR } & D & 1 & 2433 \\ \text { AGUILAR } & 70 & \text { PRL } & 25 & 1362\end{array}$ AGUILAR 171 PR $D 42583$ $\begin{array}{llllll}\text { BARNHAM } & 71 & \text { NP } & 8 & 28 & 17 \\ \text { CORDS } & 71 & \text { PR } & 0 & 4 & 1974\end{array}$ BUCHNER 72 NP B 4533

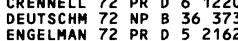
$\begin{array}{llllll}\text { ENGELMAN } & 72 & \text { PR } & 0 & 5 & 2162 \\ \text { FRATI } & 72 & \text { PR } & D & 6 & 236 \\ \text { FRA } & 72 & & \end{array}$

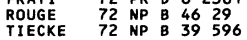
CHARRIER 73 NP B 51317
ALSO 75 PRIVATE COMM.

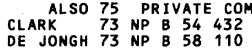
$\begin{array}{llllll}\text { LINGLIN } & 73 & \text { NP } & 8 & 55 & 408 \\ \text { HALUCH } & 73 & \text { PR } & 0 & 8 & 2837\end{array}$ $\begin{array}{lllll}\text { CHUNG } & 74 & \text { PL } & 518 & 413 \\ \text { DEHM } & 74 & \text { NP } & B 75 & 47\end{array}$ $\begin{array}{lllll}\text { ANTIPOV } & 75 & \text { NP } & 886 & 381 \\ \text { LAUSCHER } & 75 & \text { NP } & 886 & 189\end{array}$ $\begin{array}{lllll}\text { LAUSCHER } & 75 & \text { NP } & 886 & 189 \\ \text { MCCUBBIN } & 55 & \text { NP } & 886 & 13 \\ \text { MTTER } & 75 & \text { NP } & \text { B84 } & 333\end{array}$ $\begin{array}{lllll}\text { ETKIN } 76 & \text { PRL } 36 & 1482\end{array}$ HENDRICK 76 NP B 112189

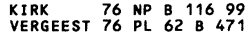
BOWLER 77 NP B $126 \quad 31$ BALDI 78 NP B 134365

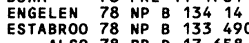

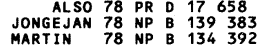
ETKIN 80 PR D 2242 $\begin{array}{llllll}\text { ASTON } & 81 & \text { PL } & 106 & \text { B } & 235 \\ \text { DAUM } & 81 & \text { NP } & \text { B } & 187 & 1\end{array}$ $\begin{array}{llllll}\text { DELFOSSE } & 81 & \text { NP } & B & 183 & 349 \\ \text { TOAFF } & 81 & \text { PR } & \text { D } & 23 & 1500\end{array}$ BAUBILLI 82 NP B 20221

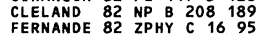
+GOLDBERG, GOZ, BARNES, LEITNER+(BNL+SYRACUSE)
BASSOMPIERRE, GOLDSCHMIDT+ (CERN+BRUX+BIRM) I JP (BNL) HARDY, CHUNG, DAHL, HESS, KIRZ, MILLER (LRL) $\begin{array}{lc}\text { +GOLDSCHMIDT-CLERMONT, HENRI + } & \text { (BRUX+CERN) } \\ \text { +HENDRICKS TICC IONI YAGER } & \text { (LAJOLLA) }\end{array}$ DEUTSCHMANN+ (AACH+BERL+CERN+LOIC+VIENNA) (JHU)
CALLAHAN, CARSON, COX, DENEGRI, +
(GENEVE) L. DUBAL (IOWA)
SCHWEINGRUBER, DERRICK, FIELDS+ (ANL + NWES)
F, L.SCHWEINGRUBER (NORTHWESTERN, EVANSTON) BASSOMPIERE, GOLDSCHMIDT-CLERM.+ (CERN+BRUX) JP +KARSHON, LAL, ONEALL, SCARR (BNL) + GOLDSCHMIAT TE, ALSTON, LYNCH, SOLMITZ (LRL)

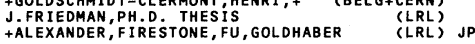
EIISENSTEIN, KIM, MARSHALL, O. HALLORAN, + (ILL)
AGUILAR-BENITEZ, BASSANO, EI SNER, + (BNL+PURD) + EISNER, KINSON + CARMONY, ERWIN, MEIERE, + (PURD+UCD+IUPU) +DEHM, CHARRIERE, CORNET + ${ }^{+}$(MPIM+CERN+BRUX) DEUTSCHMANN, + (ABCLV COLLABORATION) ENGELMANN, MUSGRAVE, FORMAN, + VIDEAU, VOLTE, DE BRION, +GRIJNS, HEINEN, DE GROOT,+ CHARRIERE, DRI JARD, DE BAERE,+ (CERN+BELG) (OXFORD
+ + LYONS, RADOJICIC
+ +CORNET, CHARR IERE, + (BRUX+MONS + CERN + MPIM) D. LINGLIN
+FLATTE, FRIEDMAN +EI SNER, PROTOPOPESCU, SAMIOS, STRAND (BNL) ASCOLI, BUSNELLO, KIENZLE+ (SERP+CERN+ILL) N. AOR COLLABORATION) JP + (AACH+BERL+CERN+LOIC+VIEN+ATHU+ATEN+LIVP) +FOLEY, GOLDMAN, LINDENBAUM, KIM, + (BNL+CUNY)
J.GOLDBERG $+V I G N A U D$, BURLAUD, + (MONS+SACL+LPNP+BELG) +KLEIN, COUNYHAN, + (AACH +BERL+CERN + LOIC+WIIEN)
+ +ENGELEN, JONGEJANS, + (AMST +CERN +NI JM+OXF) JP +DAINTON, DRAKE, WILLIAMS (OXFORD) +BOHR INGER, DORSAZ, HUNGERBUHLER+ (GEVA) + JONGEJANS, HEMINGWAY, + (NIJM+ZEEM+CERN+OXF) 作 JONGEJANS, CERRADA, ( ${ }^{+}$(ZEEM+CERN +NI JM+OXF)
+ +SHIMADA, BALDI, BOHRINGER, DORSAZ+(DURH+GEVA) +FOLEY, LINDENBAUM, KRAMER, + (BNL+CUNY) JP +CARNEGIE, DUNWOODIE, DURKIN+(SLAC+CARL+OTTA) JP GUT + MUSGRAVE, AMMAR, DAVIS, ECKLLUND,+ (ANL+KANS) BAUBILLIER+ (BIRM+CERN+GLAS+MSU+LPNP) +DELFOSSE, DORSAZ, GLOOR (DURH+GEVA+LAUS+PI TT)
FERNANDEZ, AGUILAR+
(MADR+CERN+CDEF+STOH)

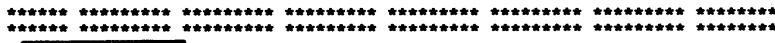
SEEN IN PARTIAL WAVE ANALYSIS OF THE K-PI+PI- SYSTEM COTTER 78). SEE L $L(1770)$ MINIREVIEW.
NEED CONFIRMATION OMITTED FROM TABLE.

39 L(1580) MASS (MEV)

M

(1580.) APPROX. OTTER 79 $10,14,16 \mathrm{~K}-\mathrm{P} \quad 12 / 79$ 
Mesons

$$
\mathrm{L}(1580), \mathrm{K}^{*}(1650), \mathrm{L}(1770)
$$

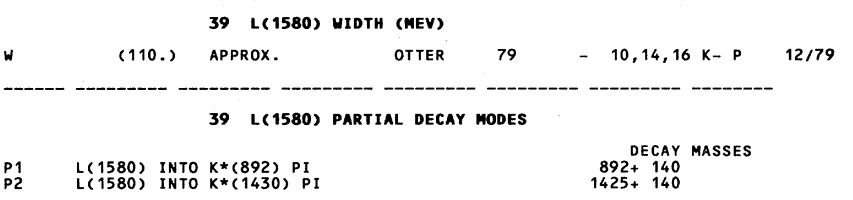

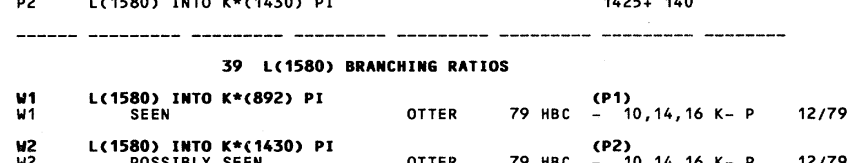

W2 L(1580) INTO
POSSIBLY SEEN

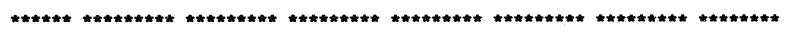

\section{REFERENCES FOR LC(1580)}

OTTER 79 NP B 1471 +RUDOLPH, + (AACH+BERL+CERN+LOIC+WIEN) JP

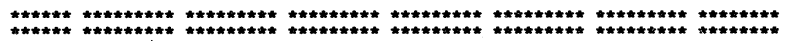

\begin{tabular}{|c|c|}
\hline$K^{*}(1650)$ & $29 K *(1650, J P=1-) \quad I=1 / 2$ \\
\hline
\end{tabular}

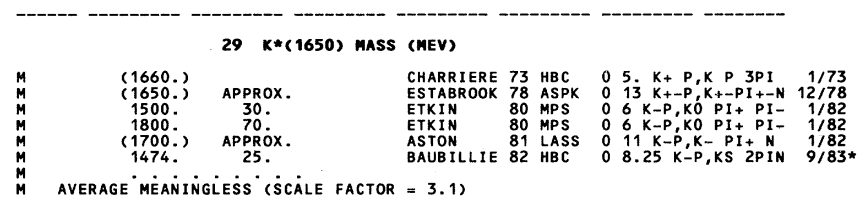

\section{$29 \quad k *(1650)$ UIDTH (MEV)}

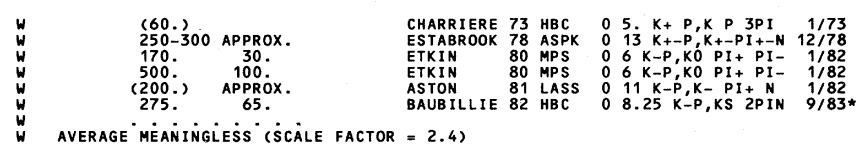

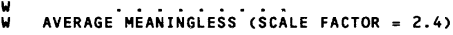

$29 K *(1650)$ PARTIAL DECAY MODES

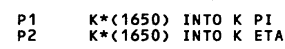

DECAY MASSES

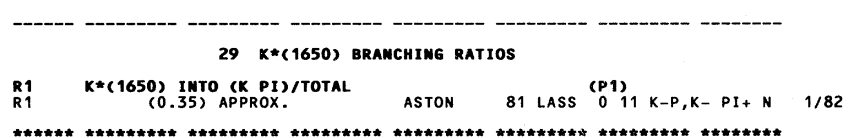

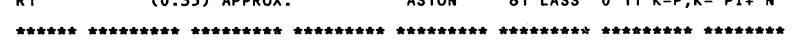

\begin{tabular}{|c|c|c|}
\hline $\begin{array}{l}\text { CHARRIER } \\
\text { ESTABROO }\end{array}$ & & $\begin{array}{l}\text { REFEREMCES FOR } K *(1650) \\
\text { CHARRI ERE, DR I JARD, DE BAERE, }+ \text { (CERN+BELG) } \\
\text { ESTABROOKS, CARNEGIE, (MONT+CARL+DURH+SLAC) }\end{array}$ \\
\hline & 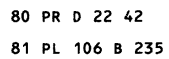 & $\begin{array}{l}\text { +FOLEY, LINDENBAUM, KRAMER,+ (BNL+CUNY) } \\
\text { +CARNEG IE, DUNWOODIE, OURKI + +(SLAC+CARL+OTTA) }\end{array}$ \\
\hline UBILLI & 82 NP в 20221 & (BIRM+CERN+GLAS+MSU+LPNP) \\
\hline
\end{tabular}

BAUBILLI 82 NP B 20221 BAUBILLIER+ (BIRM+CERN+GLAS+MSU+LPNP)

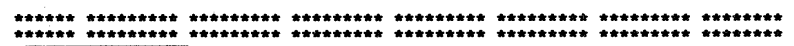

\section{$L(1770) \quad 23 \quad$ L(1770, JP=2-) $1=1 / 2$}

The $\mathrm{L}(1770)$ is seen as a bump at a mass $\sim 1.8 \mathrm{GeV}$ in the diffractive-like processes $\mathrm{KN} \rightarrow(\mathrm{K} \pi \pi) \mathrm{N}$. The effect is largely dominated by the $\mathrm{J}^{\mathrm{P}}=2^{-}$partial waves.

The long-standing questions concerning the resonant nature of the enhancement as well as its possible decay modes have been largely clarified. A detailed partialwave analysis based on 200,000 diffractive $\mathrm{K}^{-} \mathrm{p} \rightarrow$ $\mathrm{K}^{-} \boldsymbol{\pi}^{+} \boldsymbol{\pi}^{-} \mathrm{p}$ events (DAUM 81) establishes resonancelike phase variations and isolates several decay modes. The behavior of the extracted $2^{-}$waves requires the

\section{Data Card Listings}

existence of at least one $\mathrm{L}$ meson, but there are indications suggesting the presence of a second state in this mass region.

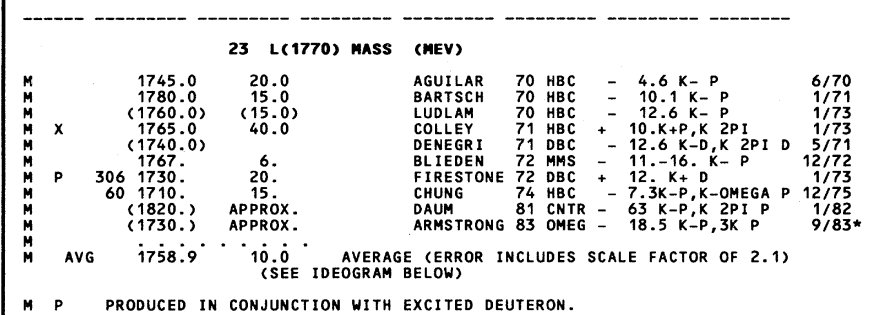

$\begin{array}{lll}M & P & \text { PRODUCED IN CONJUNCIION WITH EXCI IED DEUTERON. } \\ \text { M } & \mathrm{X} \text { SYSTEMATIC ERRORS ADDED CORRESP. TO SPREAD OF DIFERENT FITS. }\end{array}$
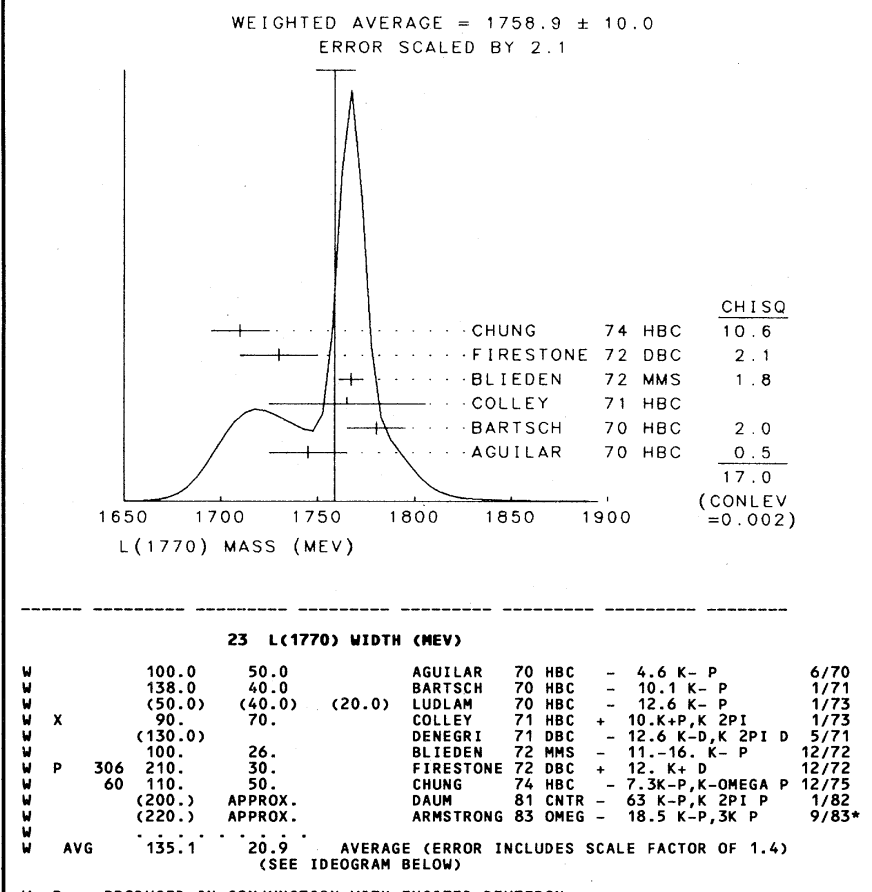

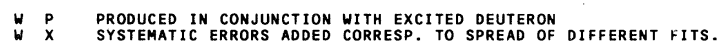

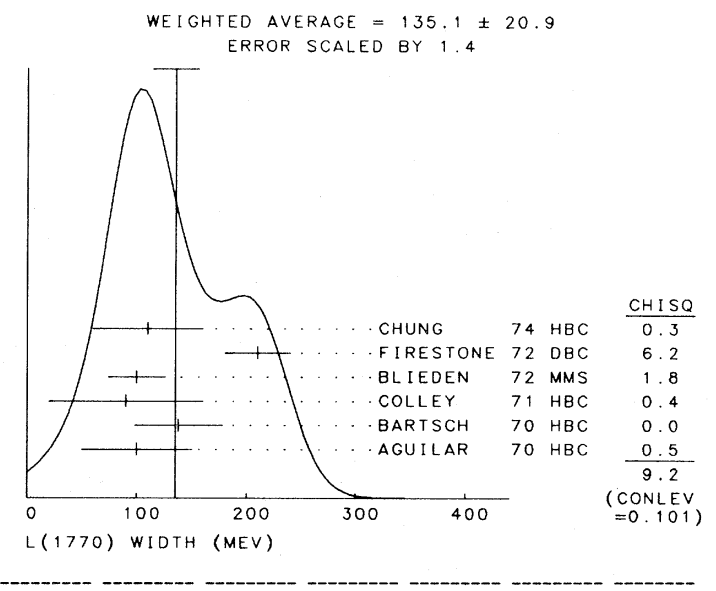




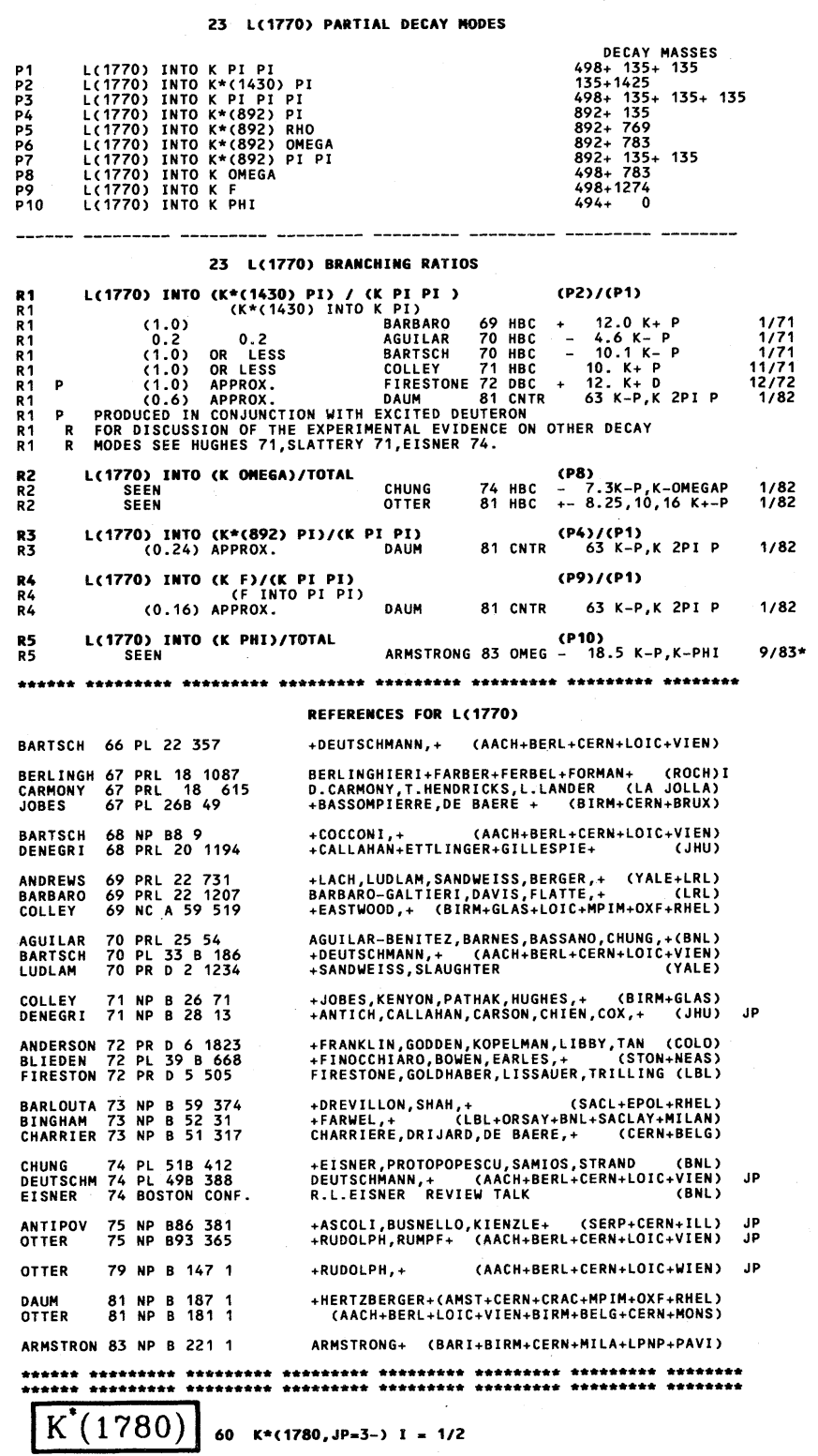

All the recent high-statistics experiments studying the $\mathrm{K} \pi$ system in $\mathrm{KN} \rightarrow \mathrm{K} \pi \mathrm{N}$ interactions have shown clear evidence for the existence of a resonant effect at $\sim 1800 \mathrm{MeV}$ in the $\mathrm{J}^{\mathrm{P}}=3^{-}$partial wave (BALDI 76 , BRANDENBURG 76, CHUNG 78, CLELAND 80, ASTON 81). The intensity of the $3^{-}$partial wave of the $\mathrm{K} \pi \pi$ system produced in the charge-exchange process $\mathrm{K}^{-} \mathrm{p} \rightarrow \mathrm{K}^{0} \pi^{+} \pi^{-} \mathrm{n}$ also shows resonance-like behavior at $\sim 1800 \mathrm{MeV}$ (BEUSCH 78, ETKIN 80, BAUBILLIER 82). Since the mass values quoted for the $K \pi$ and $\mathrm{K} \pi \pi$ modes are not significantly different, it seems natural to consider them as alternative decay modes of a single resonance.

There appears to be some disagreement in the values of the width obtained using the $\mathrm{K} \pi$ channel. The measured values tend to become larger when the number of angular moments included in the fit increases. For the time being the observed discrepancies seem to originate from the explicit parametrization of the experimental distributions rather than from the data themselves.

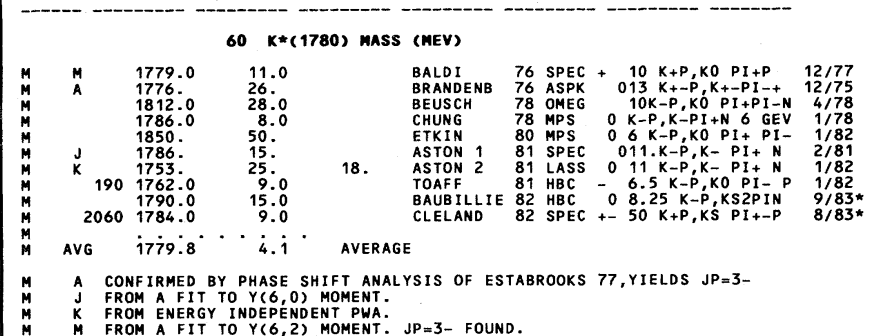
$K$
$M$
$M$
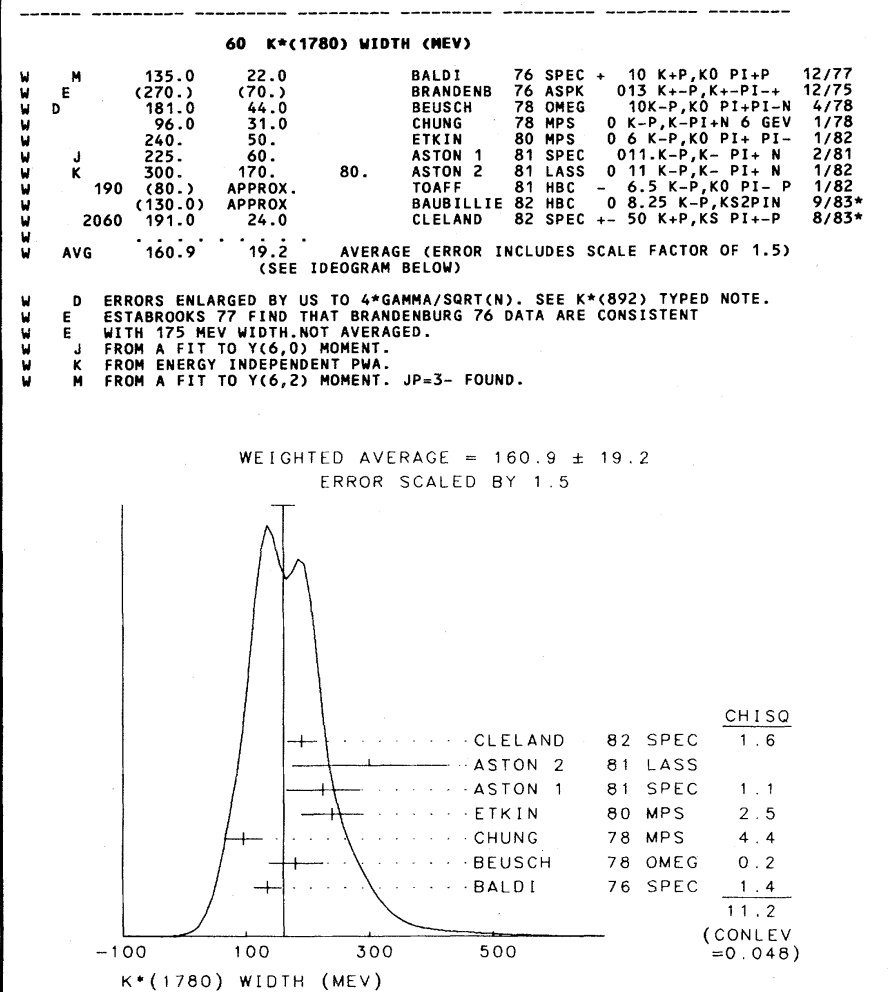

$60 K *(1780)$ PARTIAL DECAY MODES

\begin{tabular}{|c|c|c|c|}
\hline $\begin{array}{l}\text { P1 } \\
\text { P2 } \\
\text { P3 } \\
\text { P4 } \\
\text { P5 } \\
\text { P6 }\end{array}$ & $\begin{array}{l}K *(1780) \\
K *(1780) \\
K *(1780) \\
K *(1780) \\
K *(1780) \\
K *(1780)\end{array}$ & 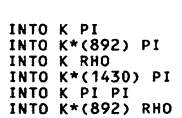 & $\begin{array}{l}\text { DECAY MASSES } \\
494+140 \\
892+140 \\
494+769 \\
1425+140 \\
494+140+140 \\
1275+769\end{array}$ \\
\hline
\end{tabular}


Mesons

$K^{*}(1780), K(1830), K^{*}(2060), K(2250), \quad K(2320)$
$60 \quad K *(1780)$ BRAMCHIMG RATIOS

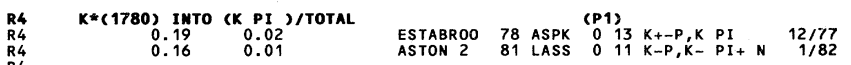
${ }_{R 4}^{R 4}$ AVG $\cdots .166^{\cdots} \cdot 0.012$ aVERAGE (ERROR INCLUDES SCALE FACTOR OF 1.3)

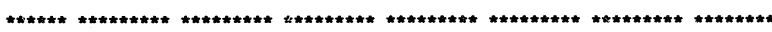

$\begin{array}{llll}\text { CARMONY } & 71 \text { PRL } 27 & 1160 \\ \text { FIRESTON } 71 & \text { PL } 36 \text { B } 513\end{array}$ $\begin{array}{lllll}\text { AGUILAR } & 73 & \text { PRL } & 30 & 672 \\ \text { WALUCH } & 73 & \text { PR } & 0 & 8 \\ \text { W } & 2837\end{array}$ $\begin{array}{llllll}\text { BALDI } & 76 & \text { PL } & 63 & B & 344 \\ \text { BRANDENB } 76 & \text { PL } & 60 & 8 & 478 \\ \text { SPIRO } & 76 & \text { PL } & 60 & \text { B } & 389\end{array}$ BOWLER 77 NP $B$ B 12631
CARMONY 77 PRD 16 1251
GRASSLER 77 NP B 125 189 $\begin{array}{lllll}B \text { BEUSCH } & 78 & \text { PL } 74 & \text { B } 282 \\ \text { CHUNG } & 78 & 282\end{array}$

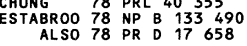
$\begin{array}{lllll}\text { CLELAND } & 80 & \text { PL } & 978 & 465 \\ \text { ENGELEN } & 80 & \text { NP } & B & 167 \\ \text { ETK } & 61\end{array}$

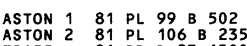
A

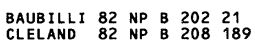

\section{REFEREMCES FOR $K *(1780)$}

+CORDS, CLOPP, ERHIN, MEIERE, (PURD+UCD+IUPU)
FIRESTONE, GOLDHABER, LISSAUER, TRILLING (LBL) +CHUNG, EISNER, PROTOPOPESCU, SAMIOS,+ (BNL)
+FLATTE, FRIEDMAN +BOEHRINGER, DORSAZ, HUNGERBUHLER, (GENEVA) JP

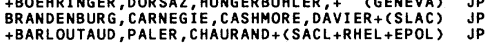
+DAINTON, DRAKE, HILLIAMS (OXFORD) JP +CLOPP, LANDER, $M E$ EERE, YEN,
+KLUGOW, (PURD+UCD+IUPU)
+ (AACHEN+BERLIN+CERN+LOIC+VIENAA)

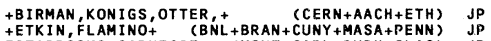
+DORSAZ, MARTIN, NEF, + (PITT+GEVA+LAUS+DURH) JP

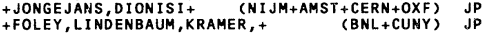
+DUNWOODIE, DURK IN, FIEGUTH+ (SLAC+CARL+OTTA) JP +CARNGG IE, DUNHOOD IE, DURKIN+(SLAC+CARL OTIA) JP
+MUSGRAVE, AMMAR, DAVIS, ECKLUND, + (ANL+KANS)

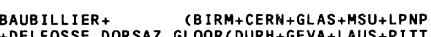
+DELFOSSE, DORSAZ, GLOOR (DURH + GEVA LAUS + PI TT) ESTABROOOS, CARNEGIE, (MONT+CARL DURH SLAC) JP

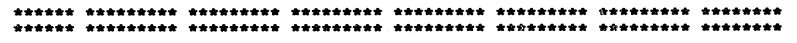

$\mathrm{K}(1830) 88 \mathrm{k}(1830$, sp $=0-) \mathrm{I}=1 / 2$

SEEN IN PARTIAL WAVE ANALYSIS OF K- PHI SYSTEM.
NEEDS CONF IRMATION. OMITTED FROM TABLE.

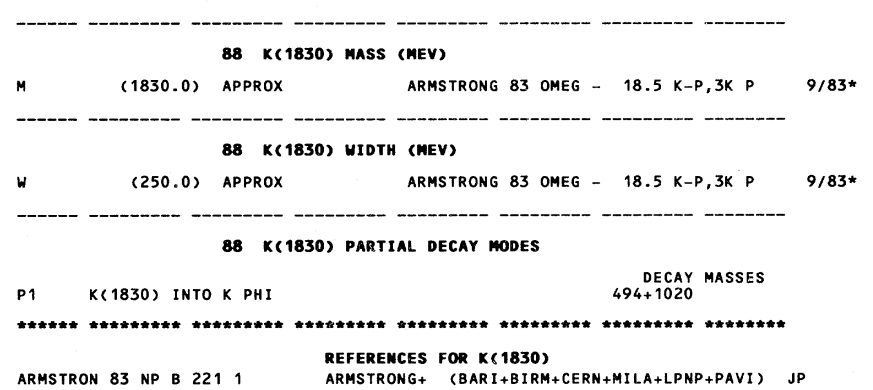

:

$K^{*}(2060) 35 \quad k *(2060, J P=4+) \quad I=1 / 2$

$$
35 K *(2060) \text { MASS (MEV) }
$$

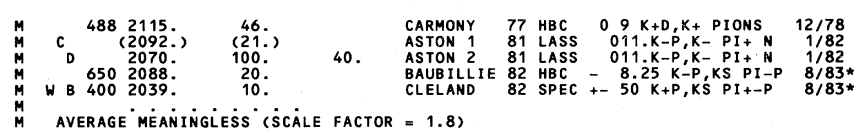

M. B FROM A FIT TO 8 MOMENTS.

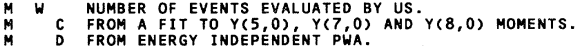

\section{$35 * *(2060)$ HIDTH (MEV)}

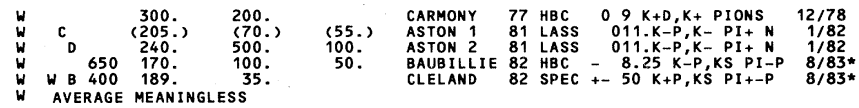

B B FROM A FIT TO 8 MOMENTS.

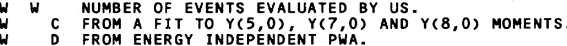

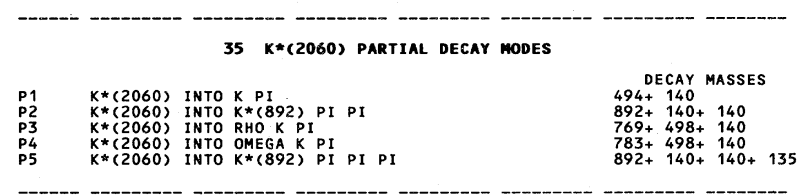

\section{Data Card Listings}

R1
R1 $K *(2060)$
SEEN

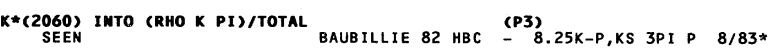
$K *(2060)$
SEN INTO COMEGA K PI)/TOTAL K*(2060) INTO (K* (892) 3 PI)/TOTAL
POSSIBLY SEEN

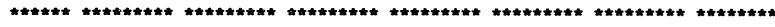

CARMONY 77 PRD 161251 +CLOPP, LANDER, MEIERE, YEN, + (PURD+UCD+IUPU)

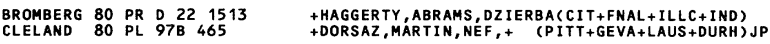

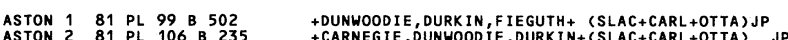

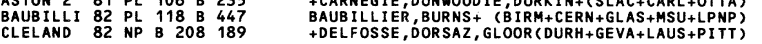

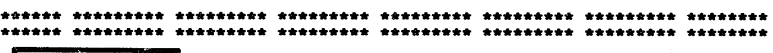

\begin{tabular}{|c|c|}
\hline $\mathrm{K}(2250)$ & 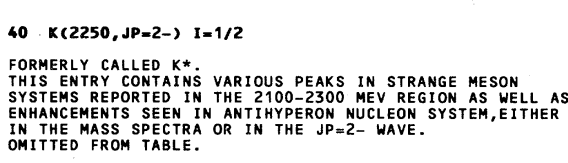 \\
\hline & $40 \mathrm{~K}(2250)$ MASS (MEV) \\
\hline $\begin{array}{ll} & 20(2240 .) \\
& (2200) \\
& 37(2147) \\
2 & 2235 .) \\
\vdots & 2260 . \\
a & 2200.0\end{array}$ & 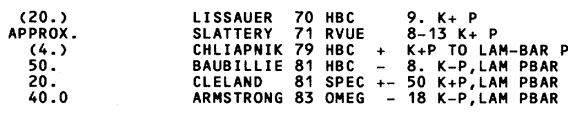 \\
\hline
\end{tabular}

M C COMPTLATION OF (ANTIHYP.-NUCLEON) MASS IN K+P 8.-13. GEV/C

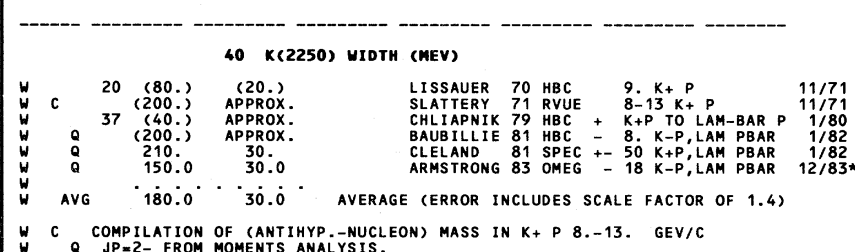

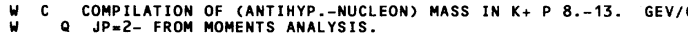

\section{$40 \mathrm{~K}(2250)$ PARTIAL DECAY MODES}

$P 1$
$P 2$
$P 2(2250)$
$P(2250)$ DECAY MASSES
$\begin{aligned} & 498+135+135 \\ & 1116+938\end{aligned}$

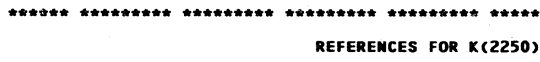

ALEXANDE 68 PRL 20755 ALEXANDER, FI RESTONE, GOLOHABER, SHEN (LRL) LISSAUER 70 NP B 18491 +ALEXANDER, FIRESTONE, GOLDHABER (LBL)

SLATTERY 71 UR-875-332(PREP) P. SLATTERY, A REVIEW OF STRANGE MESONS(ROCH)

CHLIAPNI 79 NP B 158253 CHLIAPNIKOV, GERDYUKOV + (CERN+BELG+MONS)

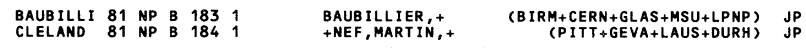

ARMSTRON 83 NP B 227365 ARMSTRONG+ (BARI+BIRM+CERN+MILA+LPNP+PAVI)

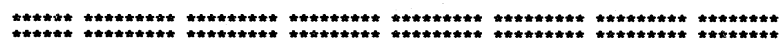

$\mathrm{K}(2320)$ 90 $\mathrm{x}(2320, \mathrm{JP}=3+) \mathrm{I}=1 / 2$

THIS ENTRY CONTAINS ENHANEMENTS SEEN IN THE
JPP $3+$ WAVE OF THE ANTIHPERON NUCLEON SYSTEM

$90 \mathrm{~K}(2320)$ MASS (MEV)

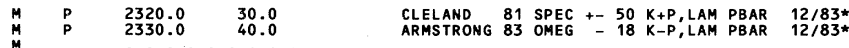
a avg $2333.6^{\circ} 24^{\circ} 0^{\circ}$ average

M P JP $=3+$ FROM MOMENTS ANALYSIS 
$90 \mathrm{~K}(2320)$ WIDTH (MEV)

$\begin{array}{llll}W & P & (250.0) & \text { APPROX. } \\ W & P & 150.0 & 30.0\end{array}$

W $P$ JP $=3+$ FROM MOMENTS ANALYSIS

$90 K(2320)$ PARTIAL DECAY MODES

P1 K(2320) INTO LAMBDA PBAR $1116+938$ DECAY MASSES

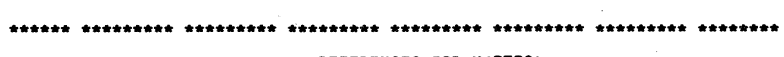

REFEREMCES FOR $K(2320)$

+NEF, MARTIN , + (PITT+GEVA+LAUS + DURH)

ARMSTRON 83 NP B 227365 ARMSTRONG+ (BARI+BIRM+CERN+MILA+LPNP+PAVI)

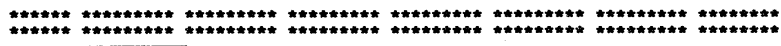

$\mathrm{K}(2500)$ 91 $\mathrm{x}(2500, \mathrm{JP}=4-) \mathrm{I=1/2}$

THIS ENTRY CONTAINS ENHANCEMENTS SEEN IN THE
JP=4- WAVE OF THE ANTIHYPERON NUCLEON SYSTEM
OMITTED FROM TABLE.

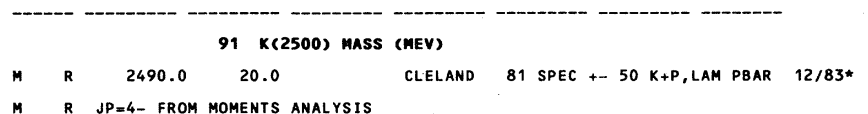

$M \quad R \quad J P=4-$ FROM MOMENTS ANALYSIS

\section{$91 K(2500)$ MIOTH (MEV)}

H $R$ (250.0) APPROX.

CLELAND 81 SPEC +- 50 K+P, LAM PBAR 12/83*

W $R$ JPI4- FROM MOMENTS ANALYSIS

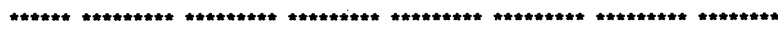

\section{REFERENCES FOR $K(2500)$}

CLELAND 81 NP B 1841 +NEF, MARTIN, + (PITT+GEVA+LAUS+DURH)

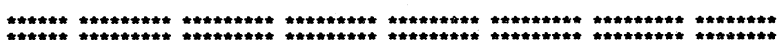

\section{$\mathrm{C}= \pm 1$ MESON STATES}

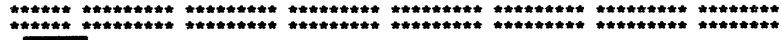

$D^{ \pm} \quad 31$ CHARGED D(1869, JP=0-) I=1/2

SeE StABle PARTICle datA CARD Listings

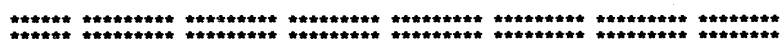

\section{$D^{0} \quad 32$ MEUTRAL $D(1865, J P=0-) \quad I=1 / 2$}

SEe STABLE PARTICLE data CARD LISTINGS

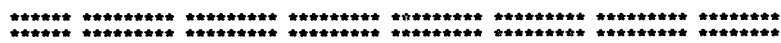

$D^{* \pm}(2010){ }_{62}$ CHARGED $D *(2010, J P=1-) I=1 / 2$

62 CHARGED D*(2010) MASS (MEV)

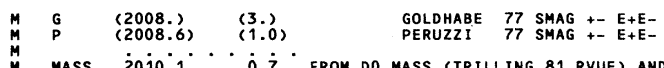

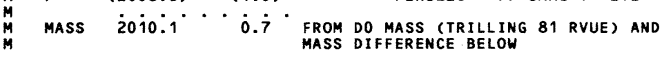

$M \quad G$ FROM SIMULTANEUS FIT TO D**+, D*0, D+, AND DO, NOT INDEPENDENT OF

$\begin{array}{lll}M & G & \text { FELDMAN } 77 \text { MASS DIFFERENCE BELOW. } \\ M & \text { P PERUZZ } 77 \text { MASS NOT INDPENDENT OF FELOMAN } 77 \text { MASS DIFFERENCE } \\ M & \text { P }\end{array}$

$62\left(D_{+}^{*}\right)-(D O)$ MASS DIfFERENCE (MEV)

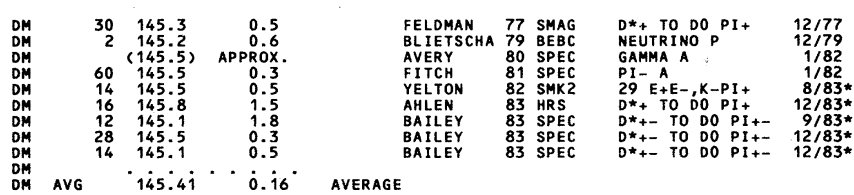

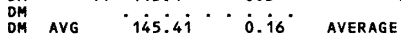

$62(D *+)-(D * 0)$ MASS DifFEREMCE (MEV)

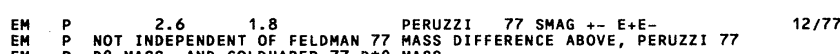
EM P NOT INDEPENDENT OF FELDMAN 77 MASS
EM $P$ DO MASS, AND GOLDHABR 77 D $* 0$ MASS.
EM

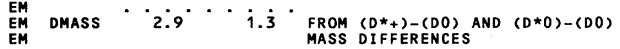

$12 / 777$
62 CHARGED D*(2010) WIDTH (MEV)

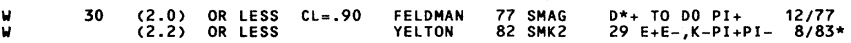

62 Charged D*(2010) PARTIal deECAY modes

P1 D*+(2010) INTO DO PI+ DECAY MASSES

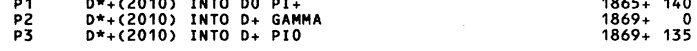

P D*-(2010) MODEs are CHARGe CONJUGates OF above modes

62 CHARGED D*(2010) BRAMCHING RATIOS

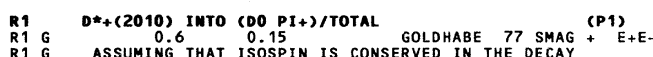

$12 / 77$

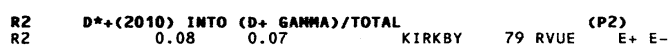

$12 / 79$

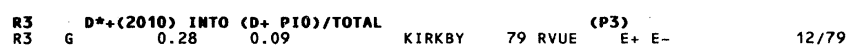

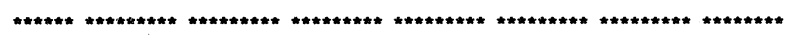

PERUZZI 76 PRL 37569

REFEREMCES FOR CMARGED $D *(2010)$

+PICCOLO, FELDMAN, NGUYEN, WISS, + (SLAC+LBL)

FELDMAN 77 PRL 381313

+ PERUZZI, PICCOLO, ABRAMS, ALAM+ (SLAC+LBL)
+ PICCOLOFELDMAN, PERL, (ALAC,

+ PICCOLO, FELDMAN, PERL, +(SLAC, LBL, NWES + HAWA)
+ +WISS, ABRAMS, ALAM, BOYARSKI, + (LBL+SLAC)

BLIETSCH 79 PL 86 B 108 BLIETSCHAU, + (AACH+BONN+CERN+MPIM+OXF)
(SLAC)

AVERY 80 PRL 441309 +WISS,BINKLEY,ATIYA, + (ILL+FNAL+COLU)
FITCH

FITCH 81 PRL $46761 \quad$ +DEVAUX, CAVAGLIA,MAY, + (PRIN+SACL+TORI+BNL)
(LRILLING 81 PRPL 7557 G.H.TRILLING

$\begin{array}{llll}\text { BEBEK } & 82 & \text { PRL } 49610 & 610 \\ \text { YELTON } & 82 \text { PRL } 49430 & \text { +(HARV+OSU+ROCH+RUTG+SYRA+VAND+CORN+ITHACA) } \\ \text { +FELDMAN,GOLDHABER, } & \text { (SLAC+LBL+UCB+HARV) }\end{array}$

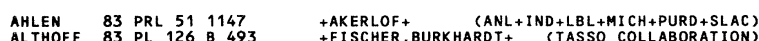

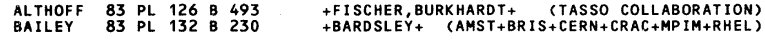

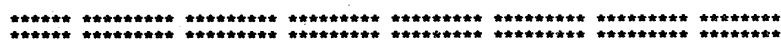

$D^{70}(2010) 61$ meUtral $D *(2010, J P=1-) \quad I=1 / 2$

J CONSistent With 1, Value 0 RUled out (NGUYen 77).

61 MEUTRAL D*(2010) MASS (MEV)

M G $\begin{aligned} & \text { (2006.) } \\ & M\end{aligned}$

MASS $20077^{\circ} \cdots \dot{2} \cdot i^{M}$ FROM DO MASS (TRILLING 81 RVUE) AND

$61(D * 0)-(D 0)$ MASS DIFFEREMCE (MEV)

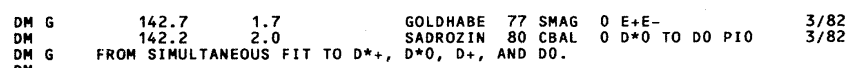

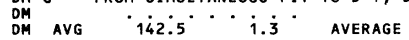

61 MEUTRAL D*(2010) WIDTH (MEV)

\begin{tabular}{|c|c|c|c|c|c|}
\hline$w$ & $(5)$. & OR LESS & GOLDHAB2 & 76 SMAG & $E+E-T O \quad D * D *$ \\
\hline
\end{tabular}

61 MEUTRAL D*(2010) PARTIAL DECAY MODES

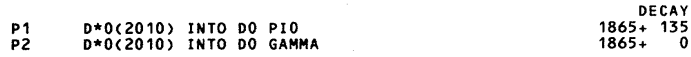

$P$ D*0(2010)Bar modes are charge conjugates of above modes

61 MEUTRAL D*(2010) BRANCHING RATIOS

R1 D*0(2010) INTO (DO GAMMA)/(DO PIO + DO GAMMA)
0.45
0.15

R1 $G$ WE QUOTE THE NORMAL FIT VALUE FROM TABLE 1. THE ISO-SPIN

R1 G CONSTRAINED FIT IS NOW KNOWN TO GIVE A DO GAMMA FRACTION WHICH IS
R1 G TOO LARGE. SEE DETAILS IN FOOTNOTE 21 OF FELDMAN 77 REVIEW.

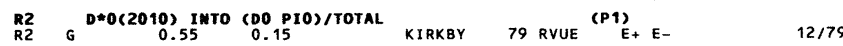

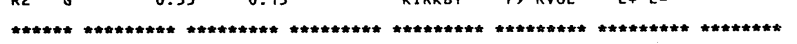
REFEREMCES FOR MEUTRAL $D *(2010)$

$\begin{array}{llll}\text { GOLDHAB1 } 76 & \text { PRL } 37255 & \text { GOLDHABER, PIERRE, ABRAMS, ALAM, } & + \\ \text { GOLDHAB } 76 \text { SLAC CONF. } 379 & \text { G.GOLDHABER (AVAIL. AS LBL-5534) } & \text { (LBL+SLAC) } \\ \text { (LBL+SLAC) }\end{array}$

GOLDHABE $77 \mathrm{PL} 69$ B 503 GOLDHABER, ABRAMS, ALAM+ (LBL+SLAC)

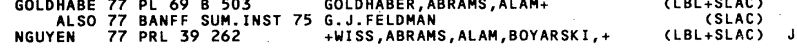

KIRKBY 79 BATAVIA CONF.107 J. KIRKBY (SLAC)

SADROZIN 80 MADISON CONF. 681 SADROZINSKI, + (PRIN+CIT+HARV+SLAC+STAN) 
Mesons

$D^{* 0}(2010), F^{ \pm}, F^{*}(2140), B^{ \pm}, B^{0}$, Exotic Mesons

\section{Data Card Listings}

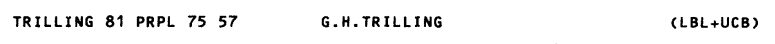

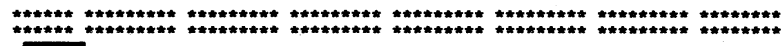

$F^{ \pm} \quad 34 \quad F+-(1970, J P=0-) \quad I=0$

SEE STABLE PARTICLE DATA CARD LISTINGS

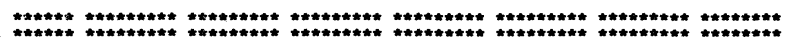

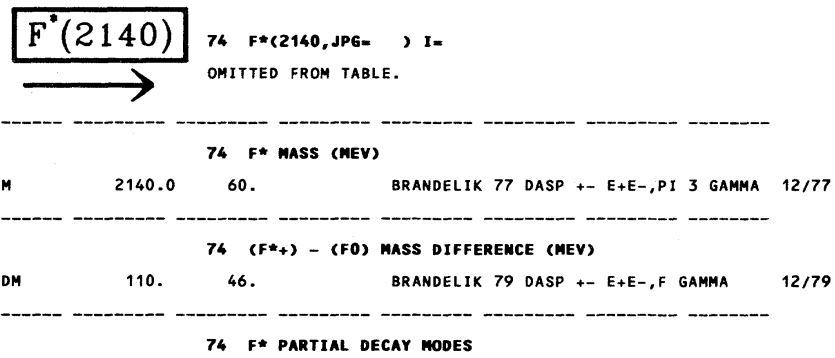

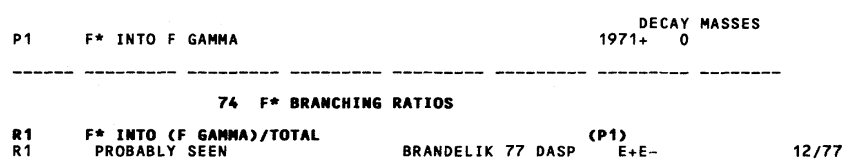

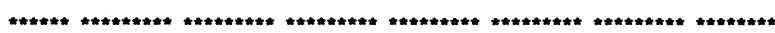

BRANDELI 77 PL 70 \& 132

REFEREMCES FOR $F *(2140)$

BRANDELI 78 PL 76 B 361

BRANDELIK, CORDS, + (AACH+DESY+HAMB+MPIM+TOKY)

BRANDELIK, CORDS, + (AACH+DESY + HAMB+MPIM+TOKY)

BRANDELI 79 PL 80 B 412

BRANDELIK, CORDS, + $(A A C H+D E S Y+H A M B+M P I M+T O K Y)$

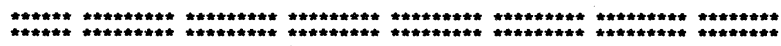

$$
B= \pm 1 \text { MESON STATES }
$$

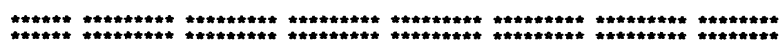

$\mathrm{B}^{ \pm} 41$ CHARGED B(5271, JP= , $\mathrm{I}=$

SEE STABLE PARTICLE DATA CARD LISTINGS

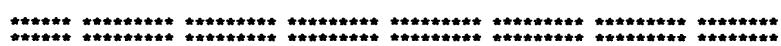

$\mathrm{B}^{0} \quad 42$ meUtral B(5274,JP= , I=

SEe StABle PARTicle data CARD LISTINGS

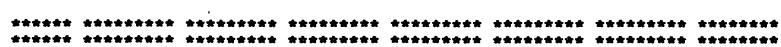

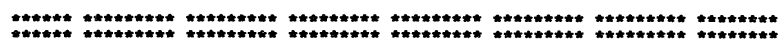
EXOTIC MESON STATES

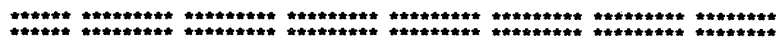

EXOTICS 50 exotrcs

THE PURPOSE OF THIS ENTRY IS TO PROVIDE A LIST OF
REFERENCES FOR EXOTIC MESON SEARCHES (SEE THE SECTION

REFERENCES FOR EXOTIC MESON SEARCHES (SEE THE SECTION

SECTION OF THIS REVIEW), AS WELL AS THEORETI CALLY BASED SUGGESTIONS FOR EXPERIMENTS. NOTE THAT LIPK IN 73
PROPOSES EXPERIMENTS WHICH ARE CONCLUSIVE EVEN IF
NEGATIVE RESULTS ARE OBTAINED.

$* * * * * * * * * * * * * * * * * * * * * * * * * * * * * * * * * * * * * * * * * * * * * * * * * * * * * * * * * * *$
REFERENCES FOR EXOTICS REPORTS ON SEARCHES

ROSENFEL 68 PHILA.CONF.P. 455 A.H.ROSENFELD (LRL)
DODLO

\begin{tabular}{l} 
DODD 69 PR 1771991 +JOLDERSMA, PALMER, SAMIOS (BNL) \\
\hline
\end{tabular}

CHO
CIA PL 32 B 409
GIACOMEL 70 PL 33 B 373 GDERICK, JOHNSON, MUSGRAVE, (ANL+NWES+KANS)
G.GIACOMELLI + (BGNA+SACL+AMST+REHO+EPOL)

GIACOMEL $70 \mathrm{PL} 33.373$ G.GIACOMELLI + (BGNA+SACL+AMST+REHO+EPOL)

LYS
ROSNER 70 PR 022525 .MESON SPECTROSCOPY, JP. C.BALTAY AND A.H.ROSENFELD,P.499

BUHL 72 NP B 37421 +CLINE, TERRELL
(WISCONSIN)

COHEN 73 NP B 531 +FERBEL, SLATTERY, WERNER (ROCHESTER
(LPNT)

ALAM $\quad 74$ PL 538207

\begin{tabular}{ll} 
COHEN & 74 \\
OREN & BOSTON \\
\hline & MP B71 189 \\
\hline
\end{tabular}

+FERBEL, SLATTERY, WERNER
+BAUBILLIER, GEORGE, ARMENISE, + (ROCHESTER)
(LPNP+BARI)

$\begin{array}{lllll}\text { BALTAY } & 75 & \text { PL } & 578 & 293 \\ \text { DAVIS } & 75 & \text { NP } & \text { B96 } & 426\end{array}$

BRUNDIER 76 PL 64 B 107

$\begin{array}{lllllll}\text { BOUCROT } & 77 & \text { NP } & \text { B } & 121 & 251 \\ \text { HOOGLAND } & 77 & \text { NPP } & \text { B } & 126 & 109\end{array}$

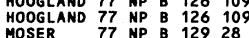

$\begin{array}{lllll}\text { ALAM } & 78 & \text { PRL } & 40 & 1685 \\ \text { ARMSTRON } & 78 & \text { PL } 77 & \text { B } & 447\end{array}$

+BRABSON, GALLOWAY,
D.COHEN REVIEW TALK (IND+PURD+SLAC+VAND)
(COLU) + COOPER, FIELDS, RHINES, WHITMORE, + (ANL+ +OXF) + CAUTIS, COHEN, KALELKAR, PI SELLO + + (COLU+BING)
+ AMMAR, KROPAC, YARGER, + (KANS+CCAC+ANL)

BRUNDIERS, BRUN, FLURI, + (FREIBURG +SACL+ETH)

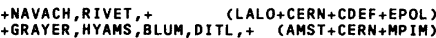
+GRAYER', HYMSS, BLUM, DITL; + (AMST+CERN + MPIM)
F.L.MOSER

+BAGGETT, BAGLIN, BONAMY+(IND+PURD+SLAC+VAND)

LEMOIGNE 79 BATAVIA CONF.524 +ABOLINS, BARATE+ (SACL+LOIC+SHMP+IND)

KOOI JMAN 80 PRL 45316 +ARENTON, AYRES, DIEBOLD, MAY+ (ANL+EFI)

$\begin{array}{llllll}\text { AGUILAR } & 81 & \text { ZPHY C } 6 & 109 & \text { +ALBA JAR, SJOGREN, + (CERN+CDEF+MADR+STOH) } \\ \text { APEL } & 81 \text { NP B } 193 & 269 & \text { (CER + }\end{array}$

$\begin{array}{lll}\text { APEL } & 81 \text { NP B } 193269 & \text { +AUGENSTEIN, BERTOLUCCI, DONSKOV, + (SERP + CERN) } \\ \text { BIONTA } 81 \text { PRL } 46970 & \text { +CARROLL, EDELSTEIN, }\end{array}$

EVANGELIS STA+ (BARI+BONN+CERN+DARE+LIVP+MILA)

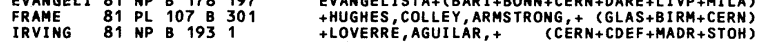

SUGGESTIONS FOR SEARCHES

ROSNER 68 PRL 21950,1468 J.L.ROSNER (TEL-AVIV)

ROSNER 70 EXP.MESON SPECTROSCOPY, ED. C.BALTAY AND A.H.ROSENFELD,P.499

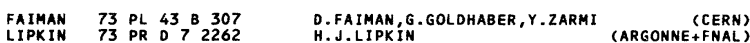

HOLMGREN 78 PL 77 B 304 +PENMINGTON
(STOH+CERN)

ARENTON 82 PR D 252241 +AYRES, DIEBOLD, MAY, SHALLOH+ (ANL+ ILL)

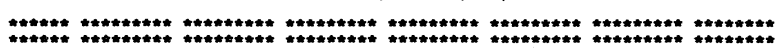


For notation, see key at front of Listings.

Baryons

$N^{\prime} s$ and $\Delta$ 's

\section{NOTE ON N AND $\triangle$ RESONANCES}

\section{Introduction}

The excited states of the nucleon have been studied in a large number of formation and production experiments. Production experiments are not suitable for an accurate determination of resonance parameters, but they are of interest in searching for the many predicted nucleon resonances that decouple from the $\pi \mathrm{N}$ channel. ${ }^{1}$

The masses, widths, and elasticities of the $\mathrm{N}$ and $\Delta$ resonances in the main Baryon Table have been determined almost entirely from partial-wave analyses of $\pi \mathrm{N}$ elastic and charge-exchange scattering data (Sec. II). Similar methods of analysis have been used to get the branching fractions for decay into $\mathrm{N} \eta, \Lambda \mathrm{K}$, and $\Sigma \mathrm{K}$.

The remaining branching fractions are from analyses of $\pi \mathrm{N} \rightarrow \mathrm{N} \pi \pi$ data, which so far have only taken into account the contributions from quasi-2-body intermediate states (Sec. III).

In addition to the usual Breit-Wigner parameters, the Data Card Listings give the locations and the residues of the poles of the resonant partial waves on the second sheet of the complex energy plane as obtained from $\pi \mathrm{N}$ partial-wave analyses and from the isobar model analyses of $\pi N \rightarrow N \pi \pi$. The Listings also give $\gamma \mathrm{N}$ decay amplitudes of the resonances (Sec. IV), and there are brief remarks on electroproduction of nucleon resonances (Sec. V) and on nucleon resonances as seen in production experiments (Sec. VI).

Table 1 lists all the entries in the Listings and gives our evaluation of the status of each, both overall and channel by channel. We have made a number of changes since the 1982 edition. Four $\mathrm{N}$ and three $\Delta$ resonances have been removed from the main Baryon Table: the $N(1990), N(2080), N(2200)$, and $\Delta(1600)$ have been reduced from 3-star to 2-star status, and the $\mathrm{N}(3030), \Delta(2850)$, and $\Delta(3230)$ have been killed altogether [see the notes in the Listings for the $\mathrm{N}(\sim 3000)$ and the $\Delta(\sim 3000)$ for the reasons for this]. A resonance is considered to be well established only if it has been seen in at least two independent analyses and if its partial wave does not behave erratically or have large errors. Good reason for a cautious attitude is the fact that some recent data ${ }^{2}$ differ appreciably from earlier data and from predictions of the analyses. Only the established resonances (overall status 3 or 4 stars) appear in the main Baryon Table.

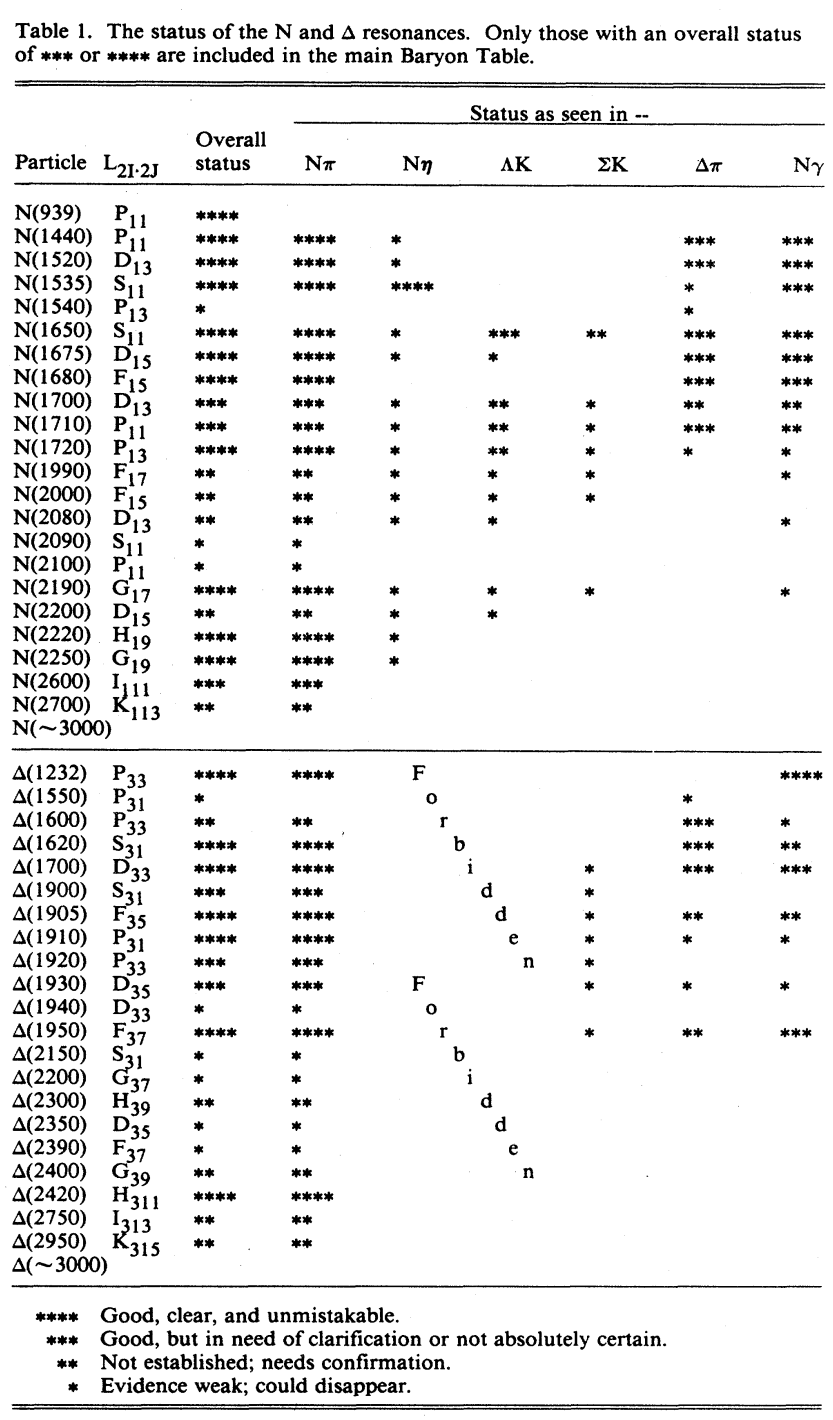

The Data Card Listings in this edition have been much shortened by the omission of many now-obsolete results. Nearly all of the omitted results were published before 1975. There also used to be separate entries for bumps seen in production experiments - bumps with masses in the $1440-\mathrm{MeV}$ region, the $1520-\mathrm{MeV}$ region, etc. - but these have been removed. All the omitted material may be found in our 1982 edition. $^{3}$

There are two recent extensive reviews of nucleon resonances. 4,5

\section{References for section I}

1. R. Koniuk and N. Isgur, Phys. Rev. D21, 1868 (1980). 


\section{Baryons}

$N^{\prime} s$ and $\Delta$ 's

\section{Data Card Listings}

2. $\pi$ N Newsletter No. 1 (1984), eds. G. Höhler and B.M.K. Nefkens.

3. Particle Data Group, Phys. Lett. 111B (1982).

4. G. Höhler, Pion-Nucleon Scattering, LandoltBörnstein Vol. I/9b (1983), ed. H. Schopper, Springer Verlag.

5. A.J.G. Hey and R.L. Kelly, Phys. Reports 96, 71 (1983).

\section{Two-body partial-wave analyses and determination} of resonance parameters

(by G. Höhler, University of Karlsruhe)

$\pi \boldsymbol{N}$ partial-wave analysis: Even if all measurable' $\pi \mathrm{N}$ $\rightarrow \pi \mathrm{N}$ scattering data were measured with infinite accuracy, it would not be possible in the inelastic region to determine a unique set of partial waves from the data alone. It is essential to add theoretical constraints, and unitarity, analyticity, and isospin invariance are chosen in order to avoid using a specific model or parametrization that might bias the solution.

Atkinson et al., ${ }^{1}$ in a continuation of earlier work, have recently investigated how much the amplitudes are restricted by unitarity if the $\mathrm{d} \sigma / \mathrm{d} \Omega$ and $\mathrm{P}$ angular distributions for $\pi^{+} p$ elastic scattering are given at a certain energy with very high precision. They found a variety of solutions, which differ from one another substantially in some of the lower partial waves and strongly in the tail of high partial waves. They concluded that cutting off the partial-wave expansion sharply (which was done in many early and some recent analyses ${ }^{2,3,4}$ ) is not justified.

In QCD, isospin is not exactly conserved in strong interactions because the masses of the up and down quarks are different. At present, the only experimental evidence for a violation is in the $\Delta(1232)$ region, where one expects an effect because of the splitting of the $\Delta^{++}$ and $\Delta^{0}$ masses. Other cases reported in the literature turned out to be caused by errors in the data or the analysis.

The uniqueness problem remains serious even if one includes data for all three reactions and isospin invariance. Therefore it is necessary to add analyticity constraints. Many analyses used as input predictions for the forward amplitudes, which follow from total-crosssection data, the optical theorem, and forward dispersion relations, but this is still not nearly enough.
Constraints based on Mandelstam's 2-variable analyticity have so far been used successfully only in the analyses of the CMU-LBL ${ }^{5}$ and Karlsruhe-Helsinki ${ }^{6}$ groups. In both, long tails of high partial waves were admitted, but only some global effects of these waves should be taken seriously, not the value of a single high partial wave. The resonance masses, widths, and elasticities in the Baryon Table are mainly determined by these two analyses, whose partial-wave amplitudes are shown in Fig. 1.

Results from other recent analyses are suspect due to sharp cutoff of the partial waves and for other reasons (see Sec. 2.1 in Ref. 7). It is necessary to check if Hendry's solution ${ }^{8}$ is compatible with analyticity.

Substantial progress in partial-wave analysis may be expected in 1984/85 when the final results of several experiments ${ }^{9}$ will become available. Furthermore, the analysis will be simplified and improved if predictions for the tail of high partial waves, based on new evaluations of the nearby parts of the Mandelstam double spectral function ${ }^{10}$ and of the left-hand cut singularities of the partial-wave dispersion relation, ${ }^{11}$ are used. A good test of predictions for the highest resonances (masses $>2.2 \mathrm{GeV}$ ) is not yet possible because the data are still too poor. Evidence for resonances in this range has been reported by Koch $^{6}$ and by Hendry. ${ }^{8}$

Determination of resonance parameters: Since a dynamical theory of $\pi \mathrm{N}$ scattering does not yet exist, the "resonance parameters" are not defined in a unique way. One can fit the partial-wave amplitudes to a phenomenological ansatz consisting of a generalized Breit-Wigner form combined with a background term, and most of the earlier analyses, including the first CMU-LBL analysis and the KH 78 analysis, ${ }^{6}$ used a prescription of this type. A more sophisticated multichannel coupled resonance scheme was applied in the recent work of the CMU-LBL group. ${ }^{5}$ The parameters listed in the Baryon Table have been derived by these methods.

This approach has a difficulty which becomes more and more important as the energy increases: some "background terms" such as diffraction and $\rho$-exchange give contributions to the partial waves which resemble highly inelastic resonances (see Sec. 2.4.1.1 in Ref. 7). It is true that the energy dependence is different, but at high energies the speed with which an amplitude 

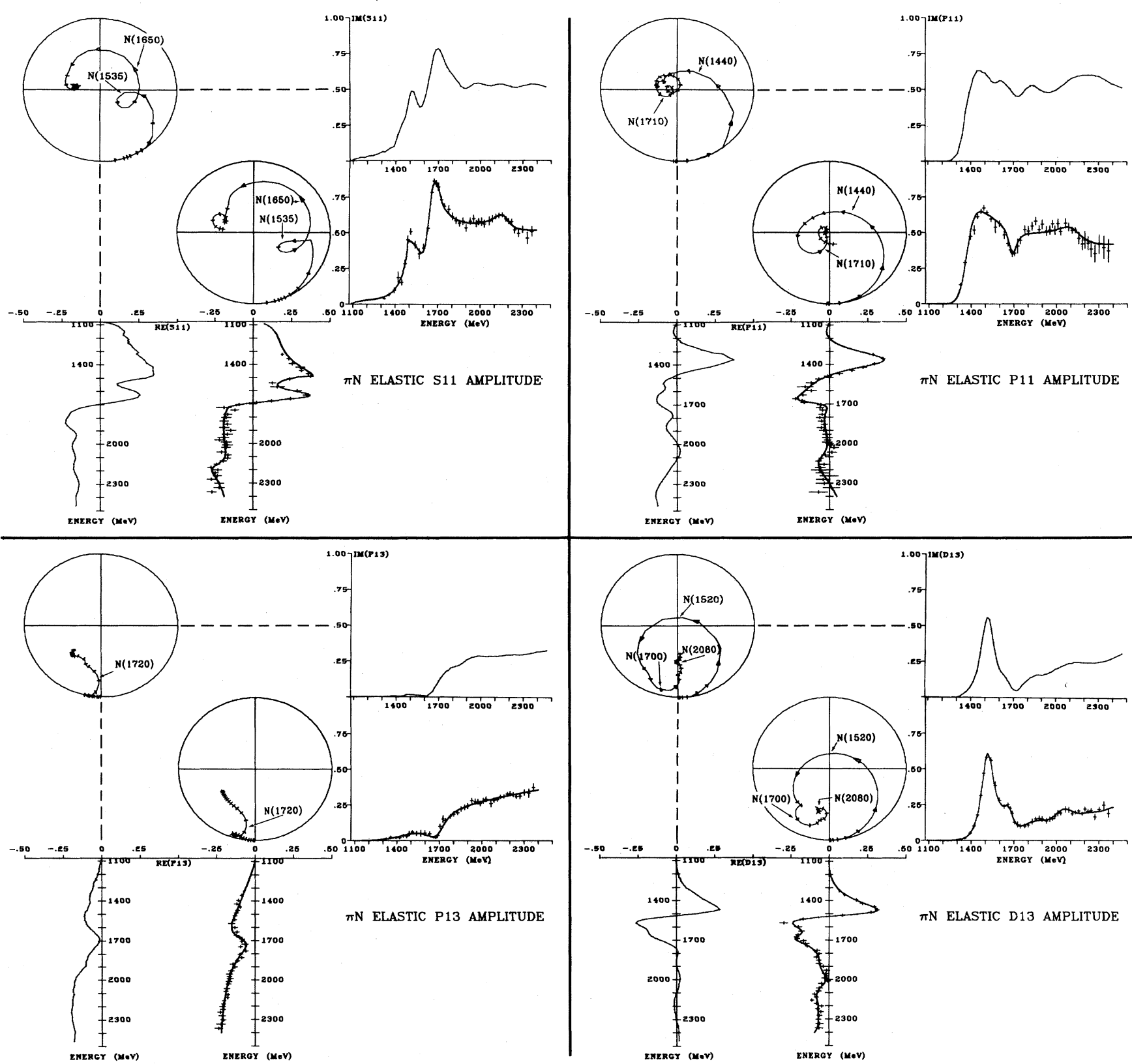

Fig. 1(a). The $L_{2 I \cdot 2 J}=S_{11}, P_{11}, P_{13}$, and $D_{13}$ partial-wave amplitudes for $\pi N$ elastic scattering. The upper plot for each amplitude is from HOEHLER 79 and the lower one is from CUTKOSKY 80. In the Argand plots, the ticks are at integral multiples of $50 \mathrm{MeV}$, and the established resonances are shown at their nominal positions. The real and imaginary parts of the amplitudes as functions of energy are shown projected in alignment with the Argand plots (in the projections of the CUTKOSKY 80 amplitudes, the "data points" are results of energyindependent fits, and the curves are from an energy-dependent fit to join them). 


\section{Baryons}

Data Card Listings

\section{$N^{\prime} s$ and $\Delta^{\prime} s$}

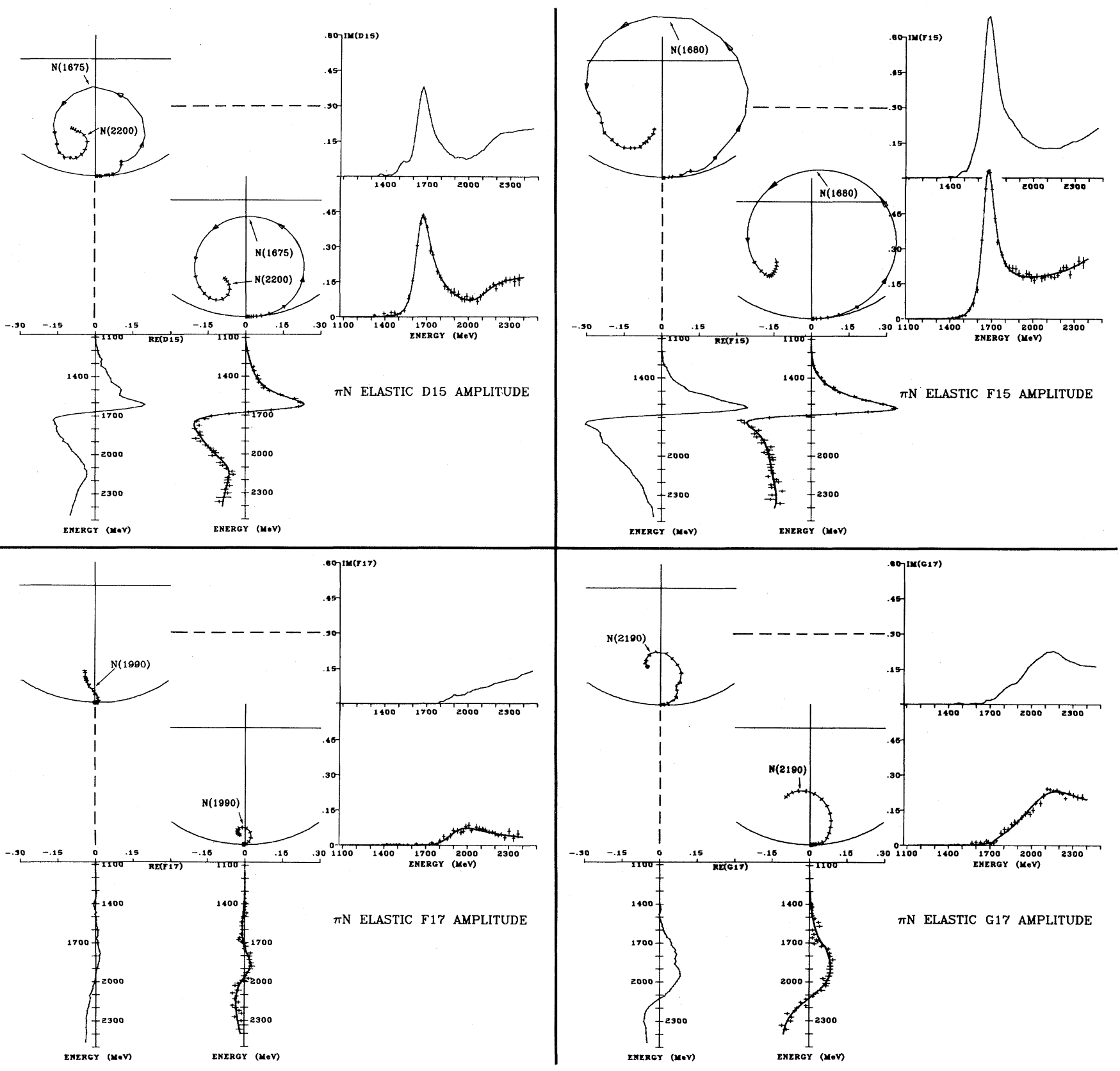

Fig. 1(b). The $\mathrm{L}_{2 \mathrm{I} \cdot 2 \mathrm{~J}}=\mathrm{D}_{15}, \mathrm{~F}_{15}, \mathrm{~F}_{17}$, and $\mathrm{G}_{17}$ partial-wave amplitudes for $\pi \mathrm{N}$ elastic scattering. The upper plot for each amplitude is from HOEHLER 79 and the lower one is from CUTKOSKY 80. In the Argand plots, the ticks are at integral multiples of $50 \mathrm{MeV}$, and the established resonances are shown at their nominal positions. The real and imaginary parts of the amplitudes as functions of energy are shown projected in alignment with the Argand plots (in the projections of the CUTKOSKY 80 amplitudes, the "data points" are results of energyindependent fits, and the curves are from an energy-dependent fit to join them). 

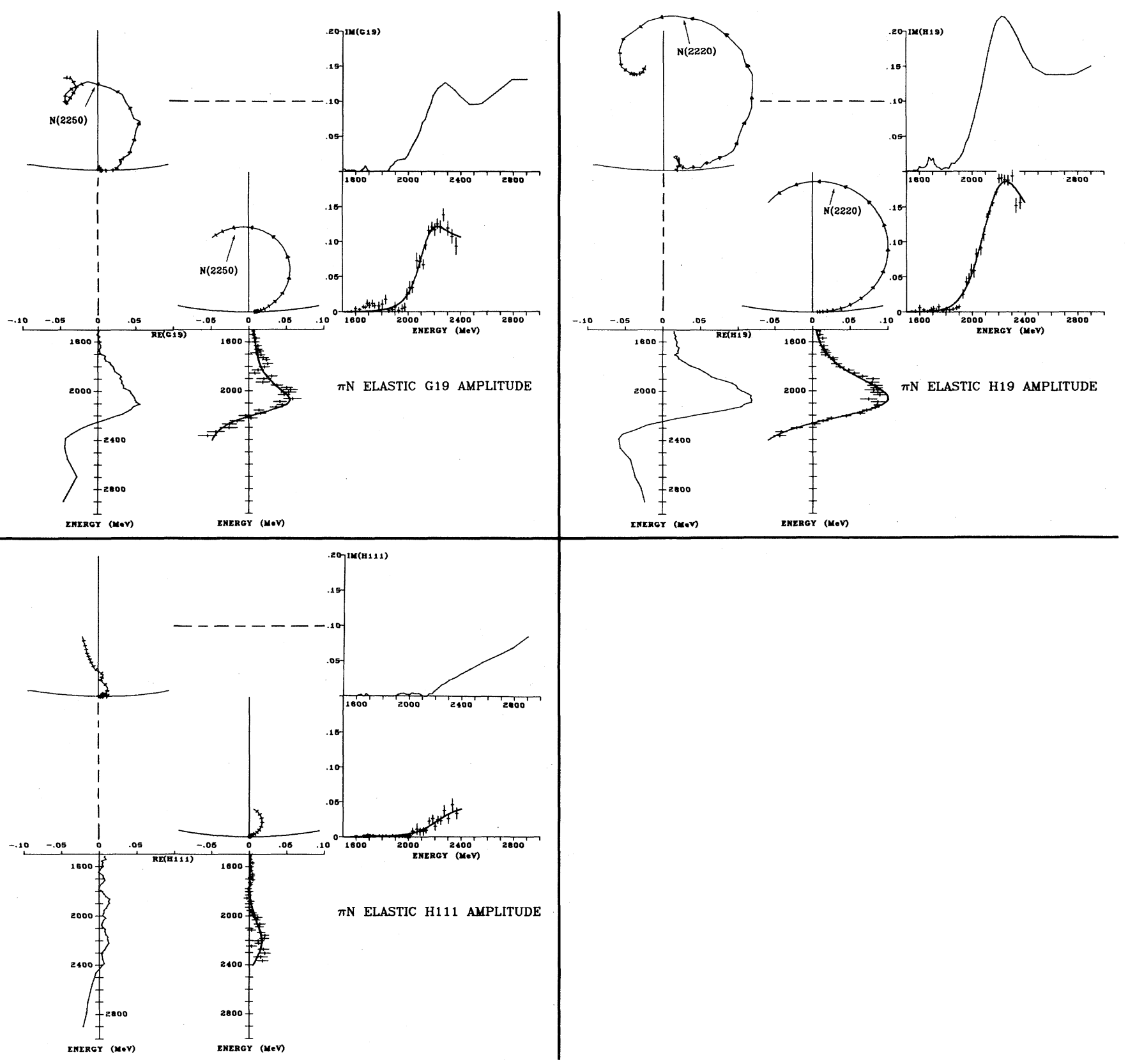

Fig. 1(c). The $\mathrm{L}_{2 \mathrm{I} \cdot 2 \mathrm{~J}}=\mathrm{G}_{19}, \mathrm{H}_{19}$, and $\mathrm{H}_{111}$ partial-wave amplitudes for $\pi \mathrm{N}$ elastic scattering. The upper plot for each amplitude is from HOEHLER 79 and the lower one is from CUTKOSKY 80. In the Argand plots, the ticks are at integral multiples of $50 \mathrm{MeV}$, and the established resonances are shown at their nominal positions. The real and imaginary parts of the amplitudes as functions of energy are shown projected in alignment with the Argand plots (in the projections of the CUTKOSKY 80 amplitudes, the "data points" are results of energyindependent fits, and the curves are from an energy-dependent fit to join them). 


\section{Baryons}

Data Card Listings

N's and $\Delta^{\prime} \mathrm{s}$

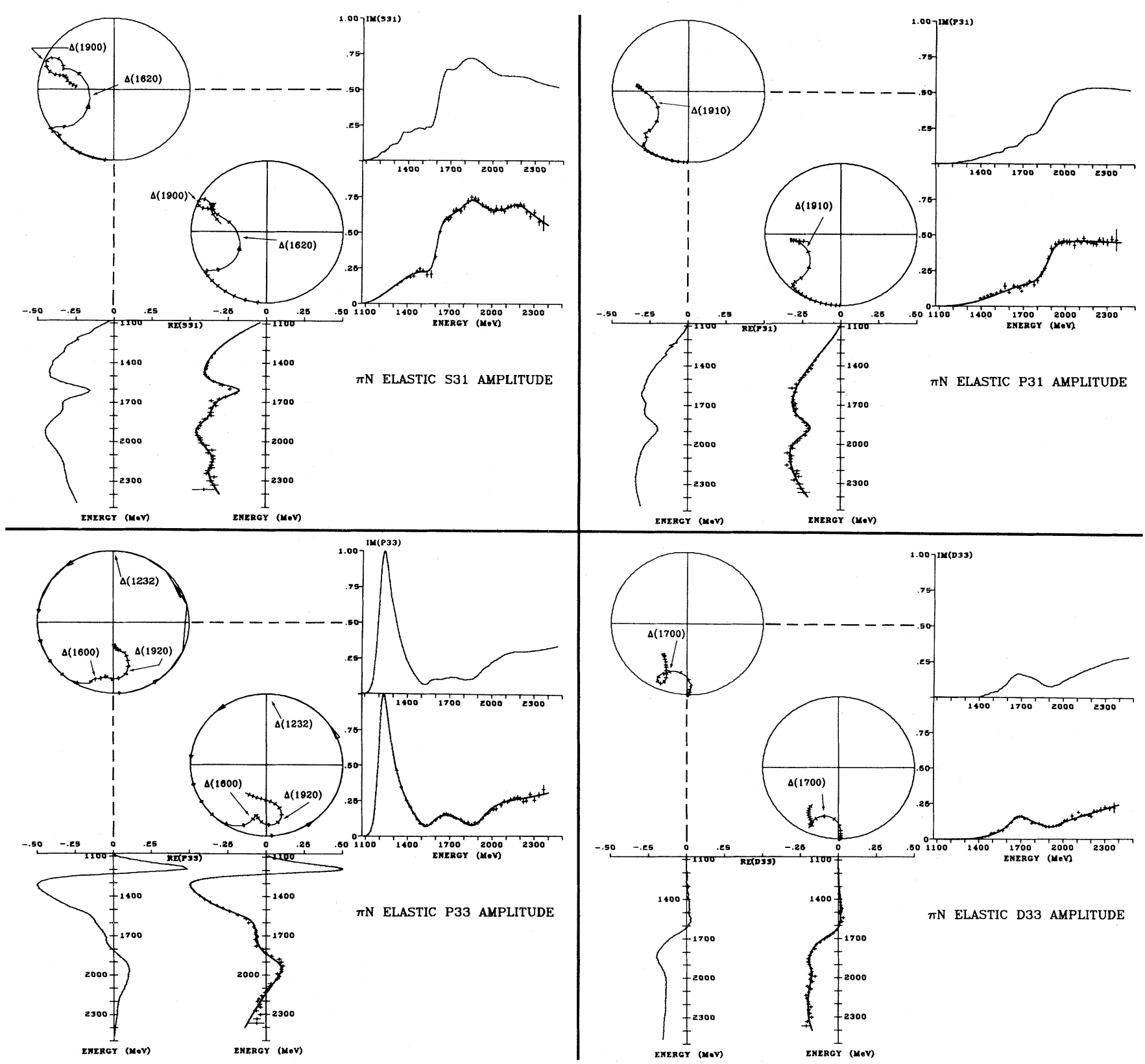

Fig. 1(d). The $L_{2 I \cdot 2 J}=S_{31}, P_{31}, P_{33}$, and $D_{33}$ partial-wave amplitudes for $\pi N$ elastic scattering. The upper plot for each amplitude is from HOEHLER 79 and the lower one is from CUTKOSKY 80. In the Argand plots, the ticks are at integral multiples of $50 \mathrm{MeV}$, and the established resonances are shown at their nominal positions. The real and imaginary parts of the amplitudes as functions of energy are shown projected in alignment with the Argand plots (in the projections of the CUTKOSKY 80 amplitudes, the "data points" are results of energyindependent fits, and the curves are from an energy-dependent fit to join them). 

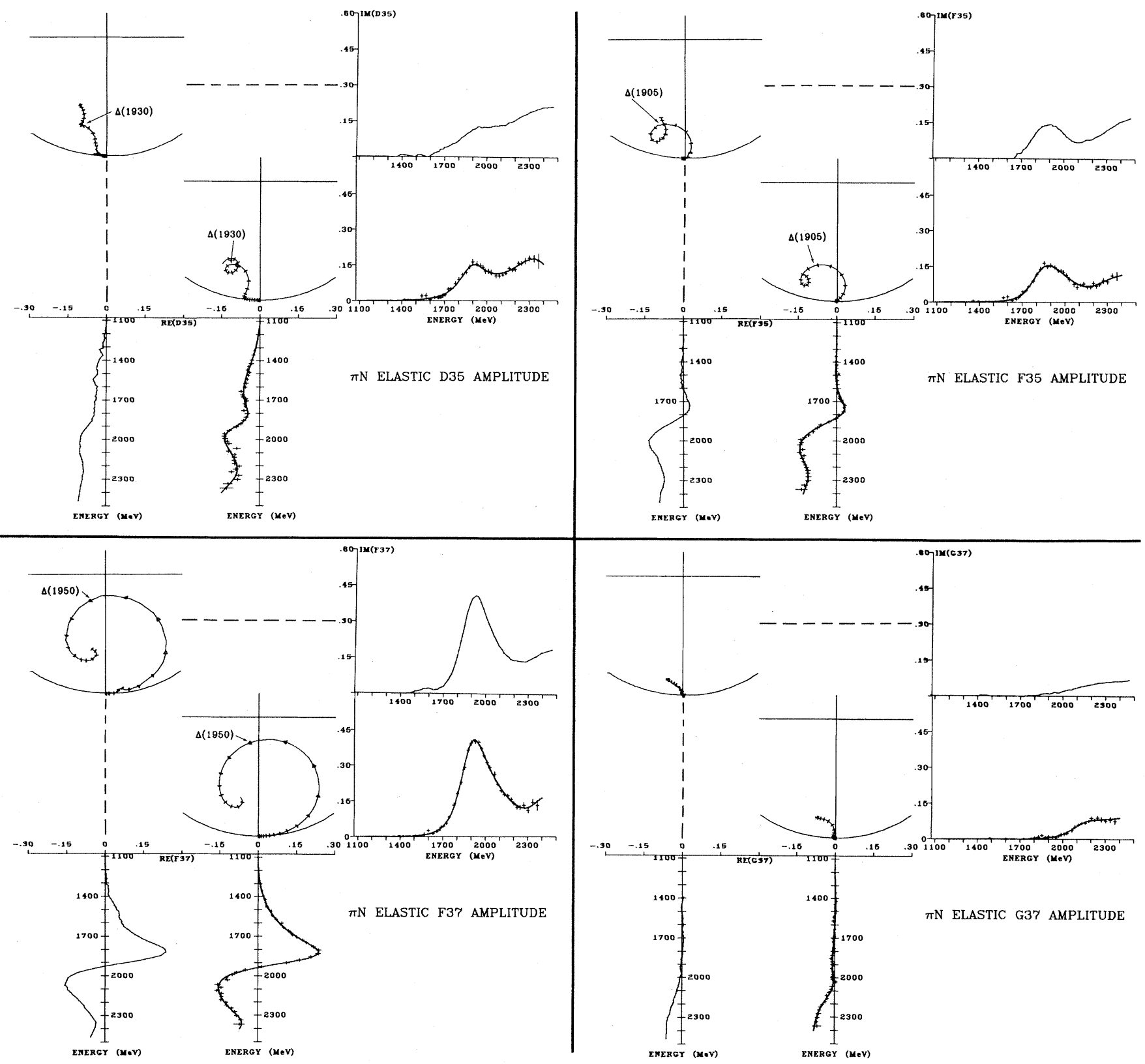

Fig. 1(e). The $\mathrm{L}_{21.2 \mathrm{~J}}=\mathrm{D}_{35}, \mathrm{~F}_{35}, \mathrm{~F}_{37}$, and $\mathrm{G}_{37}$ partial-wave amplitudes for $\pi \mathrm{N}$ elastic scattering. The upper plot for each amplitude is from HOEHLER 79 and the lower one is from CUTKOSKY 80. In the Argand plots, the ticks are at integral multiples of $50 \mathrm{MeV}$, and the established resonances are shown at their nominal positions. The real and imaginary parts of the amplitudes as functions of energy are shown projected in alignment with the Argand plots (in the projections of the CUTKOSKY 80 amplitudes, the "data points" are results of energyindependent fits, and the curves are from an energy-dependent fit to join them). 


\section{Baryons}

Data Card Listings

$\mathrm{N}^{\prime} \mathrm{s}$ and $\Delta^{\prime} \mathrm{s}$
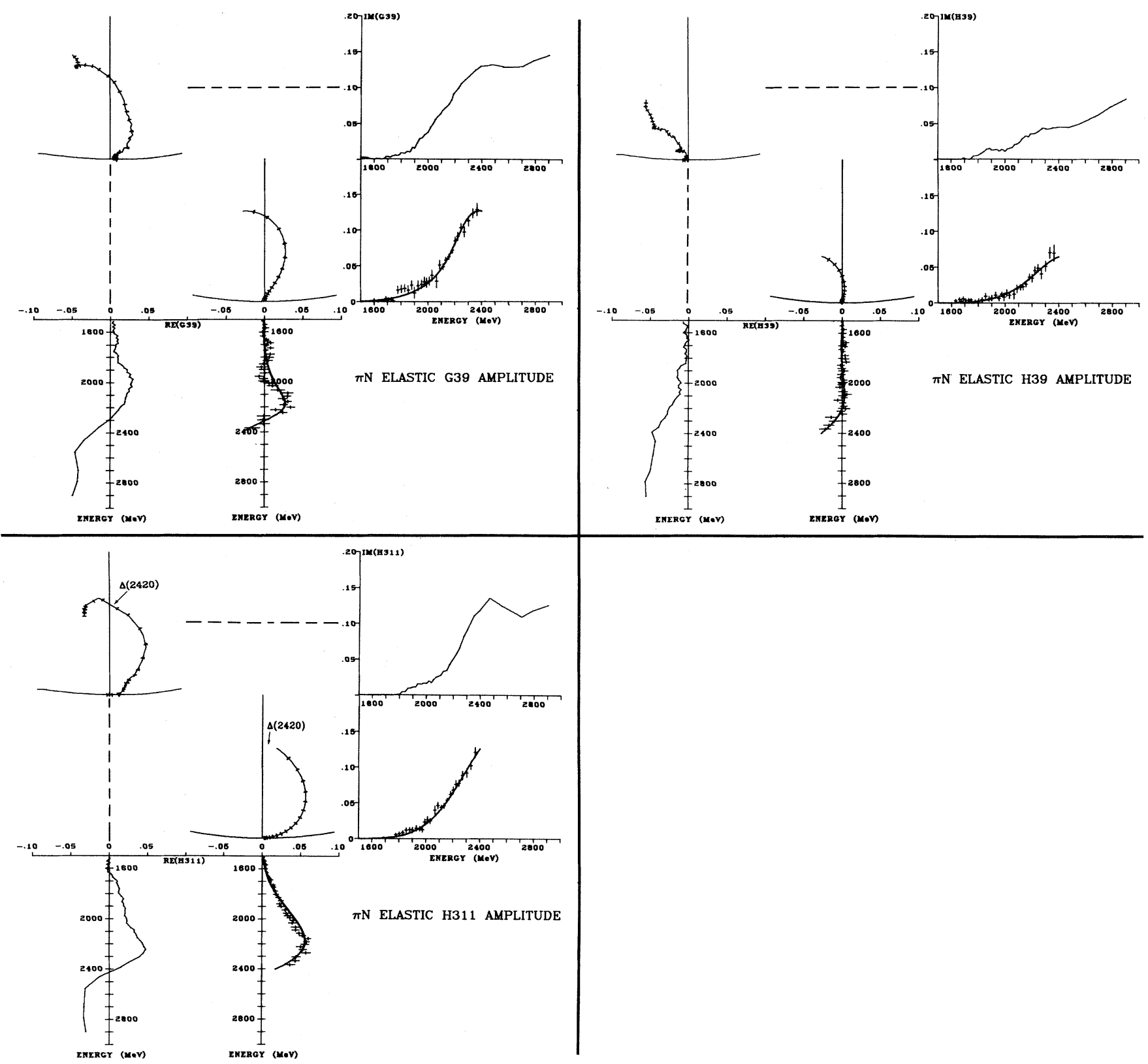

Fig. 1(f). The $L_{2 I \cdot 2 J}=G_{39}, H_{39}$, and $H_{311}$ partial-wave amplitudes for $\pi N$ elastic scattering. The upper plot for each amplitude is from HOEHLER 79 and the lower one is from CUTKOSKY 80. In the Argand plots, the ticks are at integral multiples of $50 \mathrm{MeV}$, and the established resonances are shown at their nominal positions. The real and imaginary parts of the amplitudes as functions of energy are shown projected in alignment with the Argand plots (in the projections of the CUTKOSKY 80 amplitudes, the "data points" are results of energyindependent fits, and the curves are from an energy-dependent fit to join them). 
For notation, see key at front of Listings.

traverses the complex plane cannot be accurately determined due to insufficient data. Furthermore, it is a dynamical question whether this background is part of the resonance mechanism.

If the resonances are ordered according to the shapes of their Argand plots, one finds a continuous transition from textbook-type resonances to tiny wiggles superimposed on a huge background. The Baryon Table lists all objects which have a "resonance-like" shape of the Argand diagram and a maximum of the speed. We have to leave it to the reader to decide which of these objects are "resonances" in the framework of his or her model.

The above discussion shows that a comparison of the resonance masses listed in the Baryon Table with predictions from quark-shell or -bag models or from lattice calculations has appreciable uncertainties, in particular where small mass splittings are concerned, since the models cannot yet treat the scattering process including the background.

The Data Card Listings contain a second set of resonance parameters: the locations and residues of the resonance poles on the second sheet of the s-plane. These numbers can be determined in a (more or less) model-independent way. However, it is a warning that Fonda et al. ${ }^{12}$ were able to fit the resonant $\mathbf{P}_{33}$ amplitude without a pole. One needs a theoretical assumption that excludes parametrizations of this type.

It is remarkable that there exist families of resonances in each of which the splittings of the pole positions are comparable with the errors; i.e., a degeneracy is not excluded. ${ }^{7}$ For example, all six isospin-1/2 partial waves from $S_{11}$ to $F_{15}$ have a well-established resonance with a pole near $\sqrt{\mathrm{s}}=(1665-60 \mathrm{i}) \mathrm{MeV}$, and at least six of the seven possible isospin-3/2 resonances from $S_{31}$ to $F_{37}$ have a pole near (1880-120i) MeV.

Inelastic 2-body reactions: Partial-wave analyses of the inelastic 2-body reactions $\pi \mathrm{N} \rightarrow \mathrm{N} \eta, \Lambda \mathrm{K}$, and $\Sigma \mathrm{K}$ may be carried out in a way similar to the analysis of $\pi \mathrm{N} \rightarrow \pi \mathrm{N}$. However, since the data are less complete and accurate, energy-dependent parametrizations must be used.

The most accurate results, which include information on the resonance masses and widths, follow from the $\pi^{-} \mathrm{p} \rightarrow \Lambda \mathrm{K}^{0}$ data of the Rutherford group. ${ }^{13}$ In an energy-dependent analysis, the nonresonant and high waves were represented by a reggeized $K^{*}$ exchange term. 13,14 Another analysis used a Lagrangian model for the long range forces. ${ }^{15}$ In general, agreement with the $\pi \mathrm{N} \rightarrow \pi \mathrm{N}$ analyses is good, but there are discrepancies for the widths of the $P_{11} N(1710)$ and the $D_{15}$ $N(1675)$ and for the mass of the $D_{15} N(2200)$.

In the analysis of the less accurate $\pi^{-} \mathrm{p} \rightarrow \mathrm{n} \eta$ data, 16 the partial waves were parametrized as Breit-Wigner resonances without background. The resonance spectrum was assumed and the data were used to determine the couplings to the $n \eta$ channel. In some cases of relatively large couplings, the masses and widths were varied in a second step.

The results derived from the bubble chamber data for $\pi^{+} \mathrm{p} \rightarrow \Sigma^{+} \mathrm{K}^{+17}$ have larger uncertainties. Values of the resonance masses were assumed and Breit-Wigner formulas and an empirical ansatz for the background were used for partial waves up to $F$ waves (the $G$ waves are probably not negligible at $1.7 \mathrm{GeV} / \mathrm{c}$ ). The recent addition of precise data from 1820 to $2350 \mathrm{MeV}^{18}$ has allowed an improved analysis. ${ }^{19}$ The solution found is unique. Above $2 \mathrm{GeV}$, all the resonances with two or more stars are seen, but none of the 1-star states is supported.

\section{References for section II}

1. D. Atkinson, M. De Roo, and T. Polman, Groningen preprint (1983).

2. V.S. Zidell, R.A. Arndt, and L.D. Roper, Phys. Rev. D21, 1255 and 1289 (1980).

3. D. Chew, in Proceedings of the IV $V^{\text {th }}$ International Conference on Baryon Resonances (Toronto, 1980), ed. N. Isgur, p. 123.

4. V.V. Abaev, S.P. Kruglov, and Y.A. Malov, Leningrad Report No. 438 (1978).

5. R.E. Cutkosky et al., Phys. Rev. D20, 2804 and 2839 (1979); and in Proceedings of the IV th International Conference on Baryon Resonances (Toronto, 1980), ed. N. Isgur, p. 19.

6. E. Pietarinen, Nucl. Phys. B107, 21 (1976); R. Koch, in Proceedings of the IV th International Conference on Baryon Resonances (Toronto, 1980), ed. N. Isgur, p. 3; G. Höhler et al., Handbook of Pion-Nucleon Scattering, Physics Data 12-1 (1979); and R. Koch and E. Pietarinen, Nucl. Phys. A336, 331 (1980).

7. G. Höhler, Pion-Nucleon Scattering, LandoltBörnstein Vol. I/9b (1983), ed. H. Schopper, Springer Verlag.

8. A.W. Hendry, Ann. of Phys. D21, 1 (1981). 


\section{Baryons}

Data Card Listings

$N^{\prime} s$ and $\Delta^{\prime} s$

9. $\pi \mathrm{N}$ Newsletter No. 1 (1984), eds. G. Höhler and B.M.K. Nefkens.

10. G. Höhler et al., Karlsruhe preprint TKP 83-24 (1983).

11. R. Koch, Karlsruhe preprint TKP 84-1 (1984).

12. L. Fonda, G.C. Ghirardi, and G.L. Shaw, Phys. Rev. D8, 353 (1973).

13. R.D. Baker et al., Nucl. Phys. B141, 29 (1978); D.H. Saxon et al., Nucl. Phys. B162, 522 (1980); and K.W. Bell et al., Nucl. Phys. B222, 389 (1983).

14. R.D. Baker et al., Nucl. Phys. B126, 365 (1977).

15. M. Musette, Nuovo Cim. 57A, 37 (1980).

16. R.D. Baker et al., Nucl. Phys. B156, 93 (1979).

17. Ph. Livanos et al., in Proceedings of the $I V^{\text {th }}$ International Conference on Baryon Resonances (Toronto, 1980), ed. N. Isgur, p. 35.

18. D.J. Candlin et al., Nucl. Phys. B226, 1 (1983).

19. Edinburgh-RAL-Westfield Collaboration, Rutherford preprint RL-83-102 (1983).

\section{The $\pi \mathbf{N} \rightarrow \mathbf{N} \pi \pi$ channel}

(by R.L. Crawford, University of Glasgow)

The $\pi \mathrm{N} \rightarrow \mathrm{N} \pi \pi$ reaction has been analyzed using isobar models, which are prompted by the observation that almost all $\pi \mathrm{N} \rightarrow \mathrm{N} \pi \pi$ events in the resonance region lie in quasi-2-body bands in the Dalitz plot. Thus it is assumed that any purely 3-body interaction is negligible and that the reaction proceeds entirely through quasi-2-body intermediate states.

The resulting parametrization contains the couplings of a number of the $\mathrm{N}$ and $\Delta$ resonances to these quasi2-body states, and it is these which are given in the Listings. A more complete description of the analyses and of the definition of the couplings may be found in our 1982 edition. $^{1}$

The Listings give the results from four analyses, none new to this edition.

LONGACRE 75 (LBL-SLAC) ${ }^{2}$ is based on an analysis of $200,000 \pi^{-} \mathrm{p} \rightarrow \mathrm{p} \pi^{-} \pi^{0}, \pi^{-} \mathrm{p} \rightarrow \mathrm{n} \pi^{-} \pi^{+}$, and $\pi^{+} \mathrm{p} \rightarrow \mathrm{p} \pi^{+} \pi^{0}$ events with the c.m. energy between 1300 and $2000 \mathrm{MeV}$. It includes the intermediate states $\Delta(1232) \pi, N \rho$, and $N \epsilon$ (where $\epsilon$ is the isospin-0 S-wave $\pi \pi$ enhancement). The couplings and the T-matrix poles of 14 resonances are given.

LONGACRE 77 (Saclay) $^{3}$ is a similar analysis that fits $100 \mathrm{~K}$ data points between 1380 and $1740 \mathrm{MeV}$. The couplings and pole positions of 16 resonances are given, including a $P_{13} N(1540)$ and a $P_{31} \Delta(1550)$ suggested for the first time by this analysis.

NOVOSELLER $78(\mathrm{Cal} \mathrm{Tech})^{4}$ is an analysis of $\pi^{-} \mathrm{p}$ $\rightarrow \mathrm{p} \pi^{-} \pi^{0}, \pi^{-} \mathrm{p} \rightarrow \mathrm{n} \pi^{-} \pi^{+}$, and $\pi^{+} \mathrm{p} \rightarrow \mathrm{p} \pi^{+} \pi^{0}$ events from 1650 to $1970 \mathrm{MeV}$ and is based on the earlier LBL-SLAC energy-independent analysis. ${ }^{5}$ Two solutions are given, with the second including the effects of single-pion exchange. They are noted in the Listings as being fits to Longacre 75 and Novoseller 78.

BARNHAM 80 (Imperial College) ${ }^{6}$ is an analysis of $44,000 \pi^{+} \mathrm{p} \rightarrow \mathrm{p} \pi^{+} \pi^{0}$ and $\pi^{+} \mathrm{p} \rightarrow \mathrm{n} \pi^{+} \pi^{+}$events between 1440 and $1700 \mathrm{MeV}$. It thus gives information only about $\Delta$ resonances. Decays into $\Delta(1232) \pi, N \rho$, and $N(1440) \pi$ are considered. It again finds evidence for the $P_{31} \Delta(1550)$, but since it uses data also used by Longacre 77 it is not clear that it confirms this resonance.

It is difficult to assess the systematic uncertainties of the results from these analyses. Again, the reader is referred to our 1982 edition for more details.

\section{References for section III}

1. Particle Data Group, Phys. Lett. 111B (1982).

2. R.S. Longacre et al., Phys. Lett. 55B, 415 (1975); and Phys. Rev. D17, 1795 (1978).

3. R.S. Longacre and J. Dolbeau, Nucl. Phys. B122, 493 (1977).

4. D.E. Novoseller, Nucl. Phys. B137, 509 (1978).

5. D.J. Herndon et al., Phys. Rev. D11, 3183 (1975).

6. K.W.J. Barnham et al., Nucl. Phys. B168, 243 (1980).

\section{Photoproduction and Compton Scattering}

(by R.L. Crawford, University of Glasgow)

Most of the information about the $\gamma \mathrm{N}$ couplings of the $\mathrm{N}$ and $\Delta$ resonances is obtained from partial-wave analyses of single-pion photoproduction. There is now a large amount of data, including many measurements from single and double polarization experiments giving up to six independent experimental observables in some energy ranges. Recently, some couplings have also been obtained from proton Compton scattering. All photoproduction analyses rely heavily on $\pi \mathrm{N} \rightarrow \pi \mathrm{N}$ analyses for knowledge about the existence, masses, and widths of the resonances; there are few photoproduction analyses that treat the masses and widths as free param- 
eters, although the results obtained are of some interest since they give access to the charge +1 states. The results may be found in the appropriate sections of the Data Card Listings. We refer to an earlier edition of this Review ${ }^{1}$ for more about the formalism of singlepion photoproduction.

There are three main methods for the partial-wave analysis of single-pion photoproduction.

(a) The simple isobar model: This is the simplest form of energy-dependent partial-wave analysis (DPWA): the partial waves are parametrized as BreitWigner resonances plus smooth background. The method is sufficiently flexible to give good fits to data, but there are possible problems concerned with the uniqueness of the solutions. This is overcome by the form of the parametrization, but it is not clear how this may introduce bias into the solution.

The Listings contain the couplings from isobar analyses of photoproduction from METCALF 74, TAKEDA 80 , and BRATASHEVSKIJ 80 . ISHII 80 is an isobar analysis of proton Compton scattering in the second resonance region.

(b) Fixed-t dispersion relations (FTDR): In this method, the real parts of the production amplitudes are not parametrized directly but are calculated from the imaginary parts using fixed-t dispersion relations. The latter can be assumed to be resonance dominated and thus can be given a relatively simple parametrization in terms of Breit-Wigner resonances with a little background in the low-angular-momentum partial waves. Alternatively, a K-matrix formalism can be used. Compared to the isobar model, there are fewer parameters and the results may be less sensitive to the details of the parametrization. The method gives fewer problems in obtaining a unique solution than does the isobar model, but it is less flexible and tends to give poorer fits.

The Listings contain the results from the FTDR analyses of AZNAURYAN 77, BARBOUR 78, ARAI 80, CRAWFORD 80, FUJII 81, and AWAJI 81. NOELLE 78 is a hybrid analysis using FTDR in a coupledchannel isobar calculation.

(c) Energy-independent analyses (IPWA): These evaluate the partial waves by fitting at a set of essentially single energies and should be the least biased of all the forms of analysis. At low energies, Watson's theorem ${ }^{2}$ is used to fix the complex phases of many of the partial waves in order to get a single solution. This becomes more difficult as the energy increases due to the onset of inelasticity, and only BERENDS 77 uses this method up to the second resonance region. CRAWFORD 83 is an energy-independent analysis for energies below $1750 \mathrm{MeV}$, based on the CRAWFORD 80 FTDR analysis. It is described below.

New analyses in the Listings: The most recent FTDR analysis, AWAJI 81 (Nagoya), is a revision of the 1979 Tokyo analysis (ARAI 80), and uses new data and more recent resonance parameters from $\pi \mathrm{N}$ elastic partial-wave analyses. It treats the production amplitudes differently depending on the energy. Below 2200 $\mathrm{MeV}$ a 3-channel $\mathrm{K}$-matrix formalism is used, and above $2200 \mathrm{MeV}$ a Regge parametrization is used for the dispersion integrals. Pseudo-resonances are used to describe the imaginary background, The couplings for resonances up to the $F_{17} N(1990)$ are determined for both proton and neutron targets.

CRAWFORD 83 is an energy-independent analysis using only proton data for energies between 1200 and $1920 \mathrm{MeV}$. A unique solution is obtained at each energy by requiring that it not differ radically from the FTDR analysis of CRAWFORD 80. Although the constraints thus applied were the loosest possible that still gave stability of the energy-independent solutions, the two analyses are therefore not totally independent. However, CRAWFORD 83 gives a useful extension of the FTDR solution and achieves a significant improvement in the quality of the fits at all the single energies. The two analyses agree well and again there is weak evidence for the $\mathrm{P}_{31} \Delta(1550)$.

Resonance couplings in the Listings: The Listings in this edition omit a number of analyses that are now obsolete due to later analyses that used improved data sets. The omitted analyses are ROSSI 73, HEMMI1 73, HEMMI2 73, BENEVENTANO 74, KRIVETS 75 (isobar model), MOORHOUSE 73, DEVENISH 73, KNIES 74, MOORHOUSE 74, DEVENISH2 74, CRAWFORD 75, and BARBOUR 76 (FTDR). They may all be found in our 1982 edition. $^{3}$

The errors for the couplings given in the Listings vary very widely for the different analyses since they have been obtained in different ways and are not comparable. METCALF 74, FELLER 76, AZNAURYAN 77, and ARAI 80 quote errors obtained from the sensi- 


\section{Baryons}

Data Card Listings

$N^{\prime} s$ and $\Delta^{\prime} s$

tivity of the "best possible" $\chi^{2}$ to the value of each coupling and thus give only statistical errors based on the data. In BARBOUR 78, CRAWFORD 80, and CRAWFORD 83, it is considered that systematic errors that depend on different forms of parametrization, including that for the background, are more important, and the errors given are an estimate of these. The errors given in AWAJI 81 also include a contribution from the uncertainty in the $\pi \mathrm{N}$ elasticity used to calculate the couplings from the partial waves.

Table 2 gives a compilation of the couplings obtained from BARBOUR 78, ARAI 80, CRAWFORD 80, FUJII 81, AWAJI 81, and CRAWFORD 83. The errors quoted are a combination of the statistical errors from the analyses and of the systematic differences between them. They are compared with the range of predictions from recent quark models. ${ }^{4-7}$ There is qualitative agreement in that (1) any coupling whose sign is the same in all the quark models also has the same sign in the analyses, and (2) those couplings which are predicted to be zero or small do seem to be small.

\section{References for section IV}

1. Particle Data Group, Rev. Mod. Phys. 48, S157 (1976).

2. K.M. Watson, Phys. Rev. 95, 228 (1954).

3. Particle Data Group, Phys. Lett. 111B (1982).

4. T. Kubota and K. Ohta, Phys. Lett. 65B, 374 (1976).

5. I.M. Barbour and D.K. Ponting, Zeit. Physik C4, 119 (1980).

6. R. Koniuk and N. Isgur, Phys. Rev. D21, 1868 (1980).

7. C.P. Forsyth, Carnegie-Mellon University report C00-3066-168 (1981).

\section{Electroproduction}

The excitation of the $N$ and $\Delta$ resonances by virtual photons has been investigated using pion and $\eta$ electroproduction data. For example, a recent measurement of $\pi^{+}$electroproduction ${ }^{1}$ gives additional information about the switching in importance of the helicity $3 / 2$ and $1 / 2$ amplitudes for the $D_{13} N(1520)$ and $F_{15}$ $\mathrm{N}(1680)$ resonances. Such information provides tests of the single-quark transition model. ${ }^{2}$ However, there is not much new information for the present Review of Particle Properties, so we refer to our last edition for a

\begin{tabular}{|c|c|c|c|c|}
\hline \multicolumn{5}{|c|}{ (a) Proton target couplings } \\
\hline \multirow[b]{2}{*}{ Resonance } & \multirow[b]{2}{*}{$\begin{array}{l}\text { Heli- } \\
\text { city }\end{array}$} & \multicolumn{3}{|c|}{ Couplings $\left(\mathrm{GeV}^{-1 / 2} \times 10^{-3}\right)$} \\
\hline & & $\begin{array}{c}\text { Partial-wave } \\
\text { analyses }\end{array}$ & Status & $\begin{array}{c}\text { Quark-model } \\
\text { predictions }\end{array}$ \\
\hline$N(1440) P_{11}$ & $1 / 2$ & $-69 \pm 7$ & good & -50 to -5 \\
\hline$N(1520) D_{13}$ & $\begin{array}{l}1 / 2 \\
3 / 2\end{array}$ & $\begin{array}{r}-22 \pm 10 \\
+167 \pm 10\end{array}$ & $\begin{array}{l}\text { good } \\
\text { good }\end{array}$ & $\begin{array}{l}-41 \text { to }+6 \\
+95 \text { to }+174\end{array}$ \\
\hline$N(1535) S_{11}$ & $1 / 2$ & $+73 \pm 14$ & good & +97 to +147 \\
\hline$N(1650) S_{11}$ & $1 / 2$ & $+48 \pm 16$ & fair & -9 to +95 \\
\hline$N(1675) D_{15}$ & $\begin{array}{l}1 / 2 \\
3 / 2\end{array}$ & $\begin{array}{l}+19 \pm 12 \\
+19 \pm 12\end{array}$ & $\begin{array}{l}\text { good, } \neq 0 \\
\text { good, } \neq 0\end{array}$ & $\begin{array}{l}0 \text { to }+12 \\
0 \text { to }+16\end{array}$ \\
\hline$N(1680) F_{15}$ & $\begin{array}{l}1 / 2 \\
3 / 2\end{array}$ & $\begin{array}{r}-17 \pm 10 \\
+127 \pm 12\end{array}$ & $\begin{array}{c}\text { good, } \neq 0 \\
\text { good }\end{array}$ & $\begin{array}{r}-7 \text { to }+24 \\
+47 \text { to }+154\end{array}$ \\
\hline$N(1700) D_{13}$ & $\begin{array}{l}1 / 2 \\
3 / 2\end{array}$ & $\begin{aligned}-22 & \pm 13 \\
0 & \pm 19\end{aligned}$ & $\begin{array}{l}\text { good, } \approx 0 \\
\text { fair, } \approx 0\end{array}$ & $\begin{array}{r}-7 \text { to }+9 \\
-12 \text { to }+33\end{array}$ \\
\hline$N(1710) P_{11}$ & $1 / 2$ & $+5 \pm 16$ & fair,$\approx 0$ & -47 to -7 \\
\hline$N(1720) P_{13}$ & $\begin{array}{l}1 / 2 \\
3 / 2\end{array}$ & $\begin{array}{l}+52 \pm 39 \\
-35 \pm 24\end{array}$ & $\begin{array}{l}\text { poor } \\
\text { fair }\end{array}$ & $\begin{array}{r}-133 \text { to }+74 \\
-65 \text { to }+46\end{array}$ \\
\hline$N(1990) F_{17}$ & $\begin{array}{l}1 / 2 \\
3 / 2 \\
\end{array}$ & $\begin{array}{r}+24 \pm 30 \\
31 \pm 55 \\
\end{array}$ & $\begin{array}{c}\text { poor } \\
\text { bad }\end{array}$ & $\begin{array}{l}-10 \text { to }-8 \\
-13 \text { to }-10 \\
\end{array}$ \\
\hline$\Delta(1232) \mathrm{P}_{33}$ & $\begin{array}{l}1 / 2 \\
3 / 2\end{array}$ & $\begin{array}{l}-141 \pm 5 \\
-258 \pm 11\end{array}$ & $\begin{array}{l}\text { good } \\
\text { good }\end{array}$ & $\begin{array}{l}-127 \text { to }-94 \\
-220 \text { to }-162\end{array}$ \\
\hline$\Delta(1550) \mathrm{P}_{31}$ & $1 / 2$ & $+16 \pm 16$ & $?$ & $?$ \\
\hline$\Delta(1600) P_{33}$ & $\begin{array}{l}1 / 2 \\
3 / 2\end{array}$ & $\begin{array}{r}-20 \pm 29 \\
+1 \pm 22\end{array}$ & $\begin{array}{l}\text { poor }, \approx 0 \\
\text { fair }, \approx 0\end{array}$ & $\begin{array}{r}-61 \text { to }+2 \\
-107 \text { to }+4\end{array}$ \\
\hline$\Delta(1620) S_{31}$ & $1 / 2$ & $+19 \pm 16$ & fair & +43 to +86 \\
\hline$\Delta(1700) D_{33}$ & $\begin{array}{l}1 / 2 \\
3 / 2\end{array}$ & $\begin{array}{r}+116 \pm 17 \\
+77 \pm 28\end{array}$ & $\begin{array}{l}\text { fair } \\
\text { fair }\end{array}$ & $\begin{array}{l}+78 \text { to }+106 \\
+79 \text { to }+105\end{array}$ \\
\hline$\Delta(1900) S_{31}$ & $1 / 2$ & $+10 \pm ?$ & $?$ & -3 \\
\hline$\Delta(1905) F_{35}$ & $\begin{array}{l}1 / 2 \\
3 / 2\end{array}$ & $\begin{array}{l}+27 \pm 13 \\
-47 \pm 19\end{array}$ & $\begin{array}{l}\text { good } \\
\text { fair }\end{array}$ & $\begin{array}{l}-10 \text { to }+44 \\
-41 \text { to }+15\end{array}$ \\
\hline$\Delta(1910) P_{31}$ & $1 / 2$ & $-12 \pm 30$ & poor & -16 to +15 \\
\hline$\Delta(1920) P_{33}$ & $\begin{array}{l}1 / 2 \\
3 / 2\end{array}$ & $\begin{array}{l}+40 \pm ? \\
+23 \pm ?\end{array}$ & $?$ & $\overline{-}$ \\
\hline$\Delta(1930) D_{35}$ & $\begin{array}{l}1 / 2 \\
3 / 2\end{array}$ & $\begin{array}{l}-30 \pm 40 \\
-10 \pm 35\end{array}$ & $\begin{array}{l}\text { poor } \\
\text { poor }\end{array}$ & $\begin{array}{l}-17 \\
-24\end{array}$ \\
\hline$\Delta(1950) F_{37}$ & $\begin{array}{l}1 / 2 \\
3 / 2\end{array}$ & $\begin{array}{l}-73 \pm 14 \\
-90 \pm 13 \\
\end{array}$ & $\begin{array}{l}\text { good } \\
\text { good }\end{array}$ & $\begin{array}{l}-50 \text { to }-25 \\
-69 \text { to }-32 \\
\end{array}$ \\
\hline
\end{tabular}

(b) Neutron target couplings

\begin{tabular}{llrccc}
\hline & \multicolumn{4}{c}{ Couplings $\left(\mathrm{GeV}^{-1 / 2} \times 10^{-3}\right)$} \\
\cline { 2 - 5 } Resonance & $\begin{array}{l}\text { Heli- } \\
\text { city }\end{array}$ & $\begin{array}{c}\text { Partial-wave } \\
\text { analyses }\end{array}$ & Status & $\begin{array}{l}\text { Quark-model } \\
\text { predictions }\end{array}$ \\
\hline $\mathrm{N}(1440) \mathrm{P}_{11}$ & $1 / 2$ & $+37 \pm 19$ & fair & +4 to +38 \\
$\mathrm{~N}(1520) \mathrm{D}_{13}$ & $1 / 2$ & $-65 \pm 13$ & good & -52 to -23 \\
& $3 / 2$ & $-144 \pm 14$ & good & -144 to -102 \\
$\mathrm{~N}(1535) \mathrm{S}_{11}$ & $1 / 2$ & $-76 \pm 32$ & fair & -119 to -83 \\
$\mathrm{~N}(1650) \mathrm{S}_{11}$ & $1 / 2$ & $-17 \pm 37$ & poor & -45 to +4 \\
$\mathrm{~N}(1675) \mathrm{D}_{15}$ & $1 / 2$ & $-47 \pm 23$ & fair & -55 to -31 \\
& $3 / 2$ & $-69 \pm 19$ & fair & -78 to -44 \\
$\mathrm{~N}(1680) \mathrm{F}_{15}$ & $1 / 2$ & $+31 \pm 13$ & good & -32 to +27 \\
& $3 / 2$ & $-30 \pm 14$ & good & -25 to +2 \\
$\mathrm{~N}(1700) \mathrm{D}_{13}$ & $1 / 2$ & $0 \pm 56$ & bad & -15 to +23 \\
& $3 / 2$ & $-2 \pm 44$ & bad & -76 to -17 \\
$\mathrm{~N}(1710) \mathrm{P}_{11}$ & $1 / 2$ & $-5 \pm 23$ & fair & -21 to +29 \\
$\mathrm{~N}(1720) \mathrm{P}_{13}$ & $1 / 2$ & $-2 \pm 26$ & fair & -23 to +57 \\
& $3 / 2$ & $-43 \pm 94$ & bad & -61 to +12 \\
$\mathrm{~N}(1990) \mathrm{F}_{17}$ & $1 / 2$ & $-49 \pm 45$ & poor & -19 to -18 \\
& $3 / 2$ & $-122 \pm 55$ & poor & -25 to -23 \\
\hline \hline
\end{tabular}


For notation, see key at front of Listings.

Baryons

$\mathrm{N}^{\prime} \mathrm{s}$ and $\Delta^{\prime} s, \mathrm{p}, \mathrm{n}, \mathrm{N}(1440)$

brief review ${ }^{3}$ and to a recent article for an extensive review ${ }^{4}$ of electroproduction.

\section{References for section $V$}

1. H. Breuker et al., Zeit. Physik C13, 113 (1982).

2. F. Foster and G. Hughes, Zeit. Physik C14, 123 (1982).

3. Particle Data Group, Phys. Lett. 111B (1982).

4. F. Foster and G. Hughes, Rep. Prog. Phys. 46, 1445 (1983).

\section{Production experiments}

Partial-wave analyses of course separate partial waves, whereas a peak in a cross section or an invariant mass distribution usually cannot be disentangled from background and analyzed for its quantum numbers; and more than one resonance may be contributing to the peak. We used to have separate entries in the Listings for bumps seen in production experiments in the 1440$\mathrm{MeV}$ region, the $1520-\mathrm{MeV}$ region, etc., but these have been removed from this edition. They may be found in the 1982 edition. $^{1}$

\section{Reference for section VI}

1. Particle Data Group, Phys. Lett. 111B (1982).

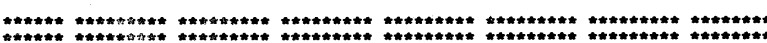
$S=0 \quad l=1 / 2 \quad$ NUCLEON STATES (N)

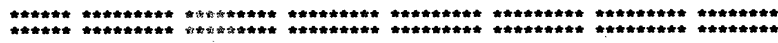

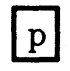

16 PROTOM(938, JP=1/2+) $I=1 / 2$

SEE STABLE PARTICLE DATA CARD LISTINGS

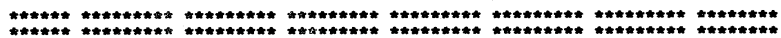

n 17 MEUTROn(939, JP=1/2+) I=1/2

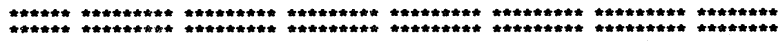

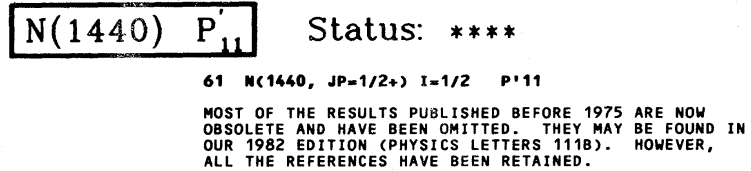

IN ADDITION, RESULTS IN THIS REGION FROM PRODUCTION EXPERIMENTS, WHICH USED TO BE

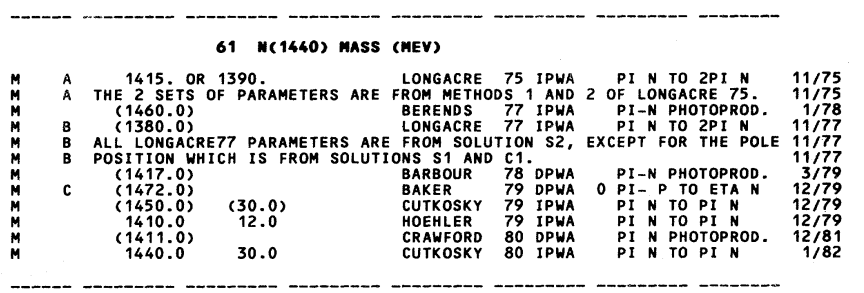

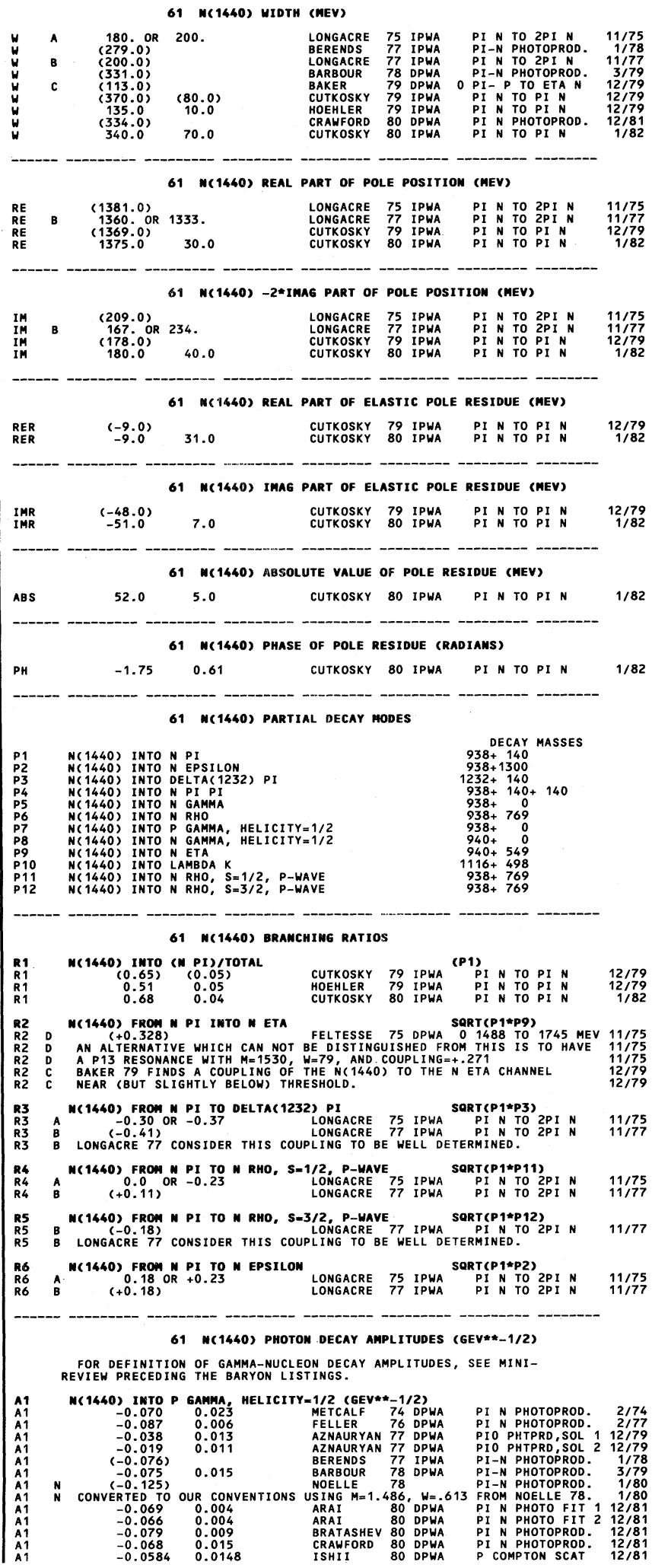


Baryons

Data Card Listings

$\mathrm{N}(1440), \quad \mathrm{N}(1520)$
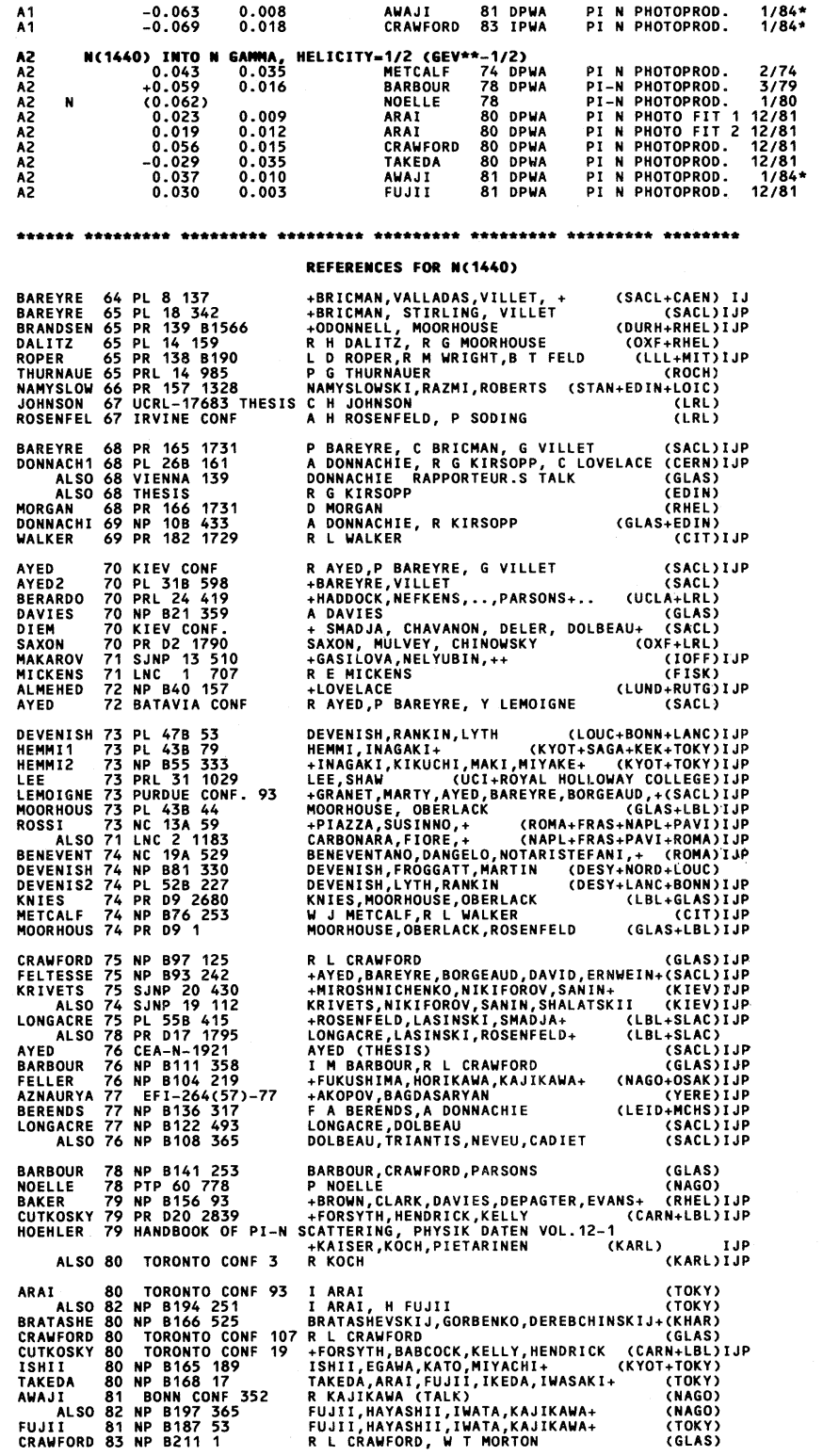

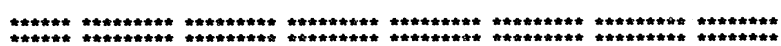

$\mathrm{N}(1520) \quad \mathrm{D}_{13}^{\prime} \quad$ Status: $* * * *$

$62 \mathrm{~N}(1520, J P=3 / 2-) \quad I=1 / 2 \quad D^{\prime} 13$

MOST OF THE RESULTS PUBLISHED BEFORE 1975 ARE NOW

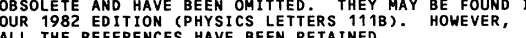

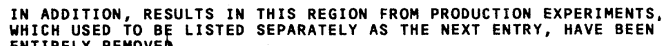

62 M(1520) MASS (MEV)

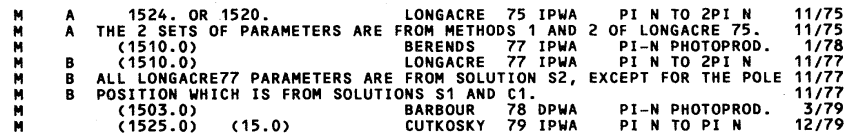

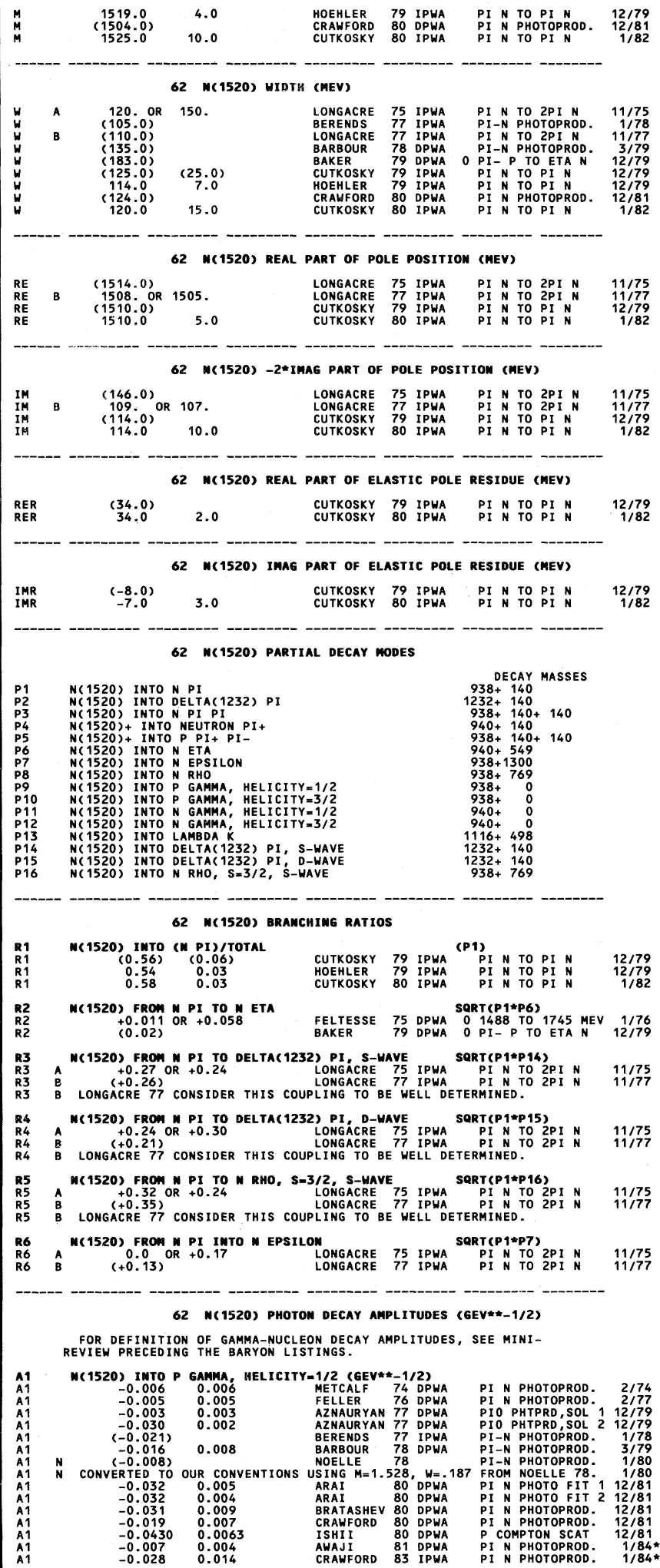



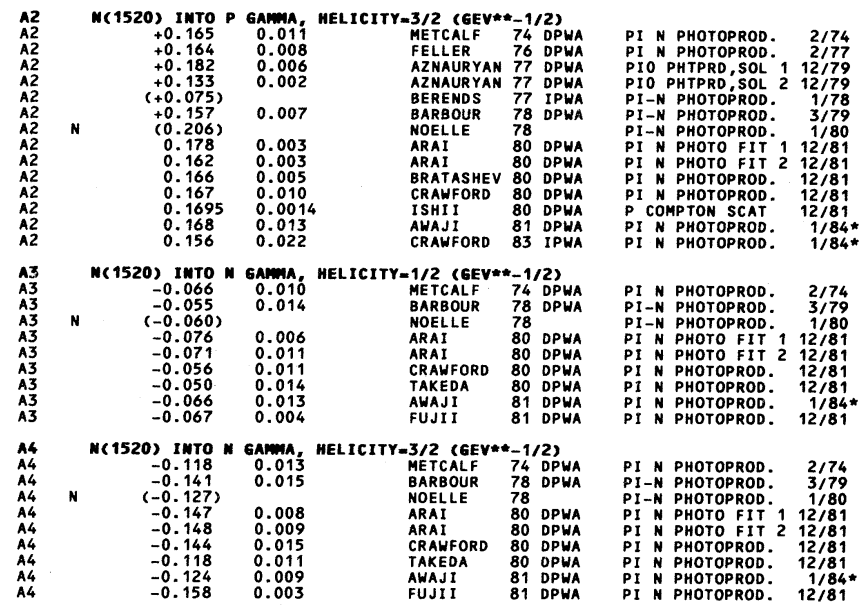

PI N PHOTOPROD.
PI-N PHOTOPROD.
PII/74

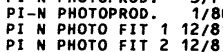

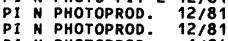
$P 1$
$P 1$

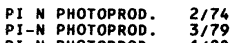

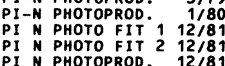
$P I$
$P I$

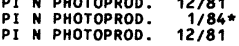

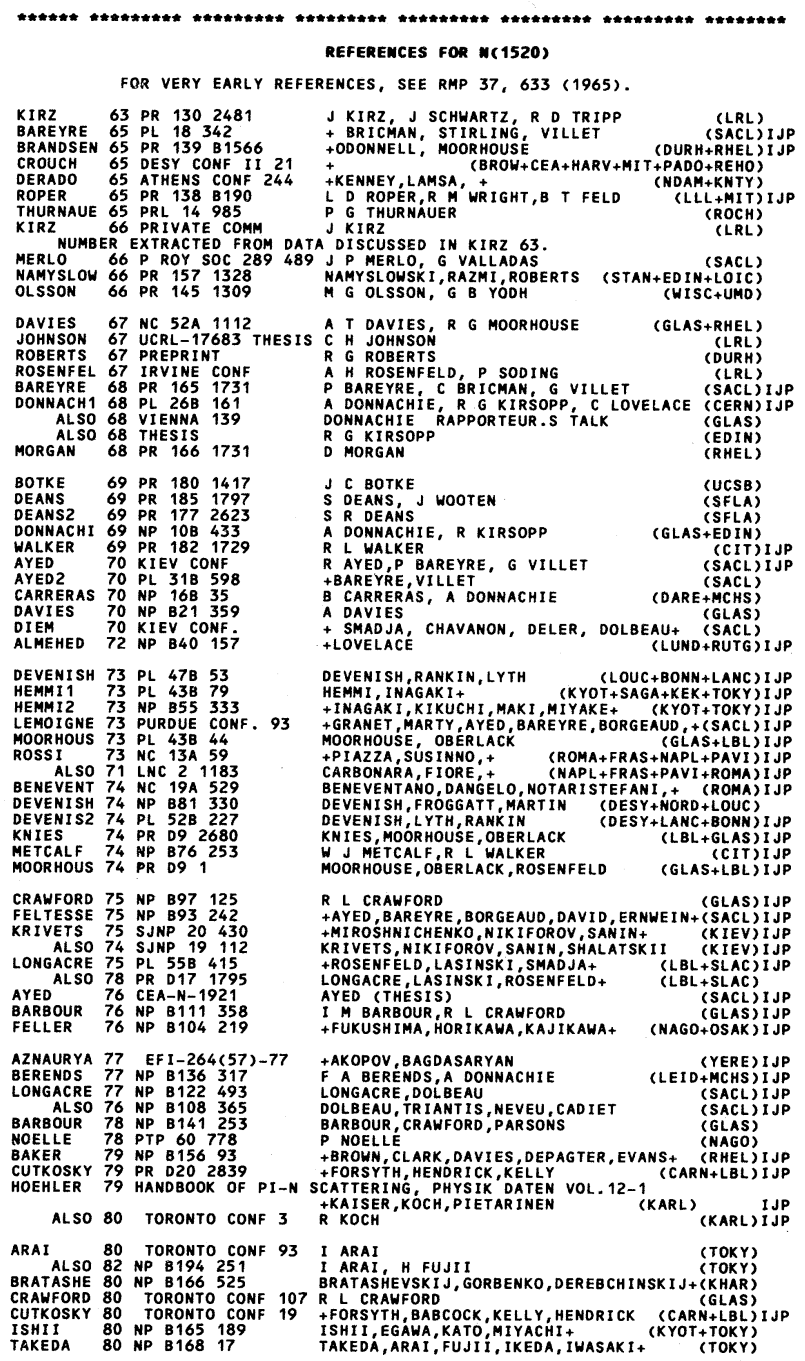

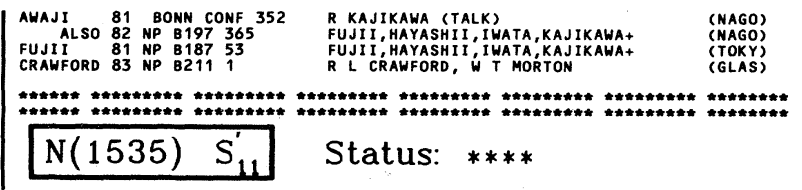

$63 \mathrm{~N}(1535, \mathrm{JP}=1 / 2-) \quad I=1 / 2$ S' 11

MOST OF THE RESULTS PUBLISHED BEFORE 1975 ARE NOW OBSOLETE AND HAVE BEEN OMITTED. THEY MAY BE FOUND IN
OUR 1982 EDITION (PHYSICS LETTERS 1118 ). HOWEVER,

NEARLY ALL THE REFERENCES HAVE BEEN RETAINED.

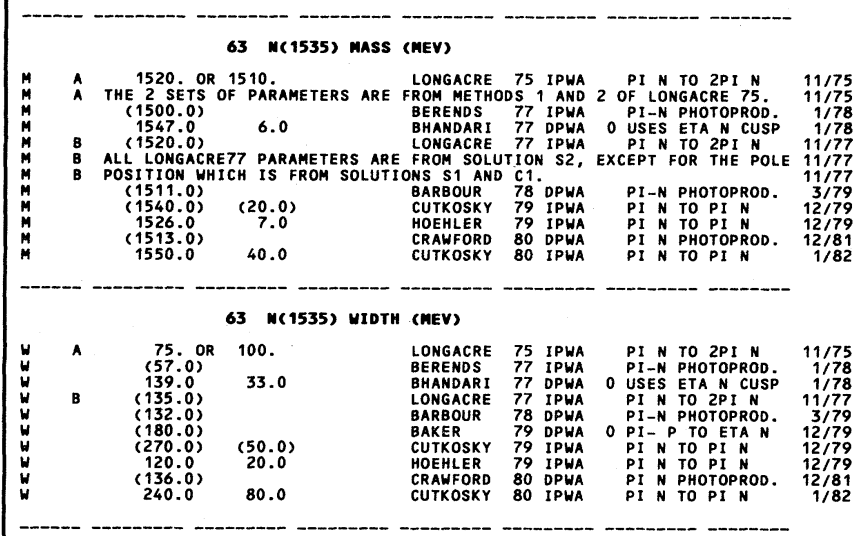

63 N(1535) REAL PART OF POLE POSITION (MEV)

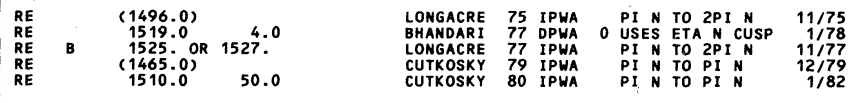

63 M(1535) -2*IMAg PART OF POLE POSITION (MEV)

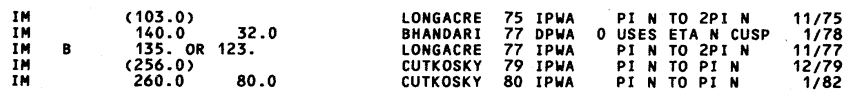

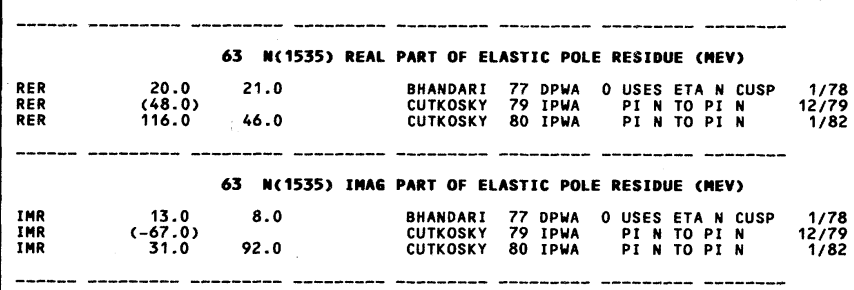

63 M(1535) PARTIAL DECAY MODES

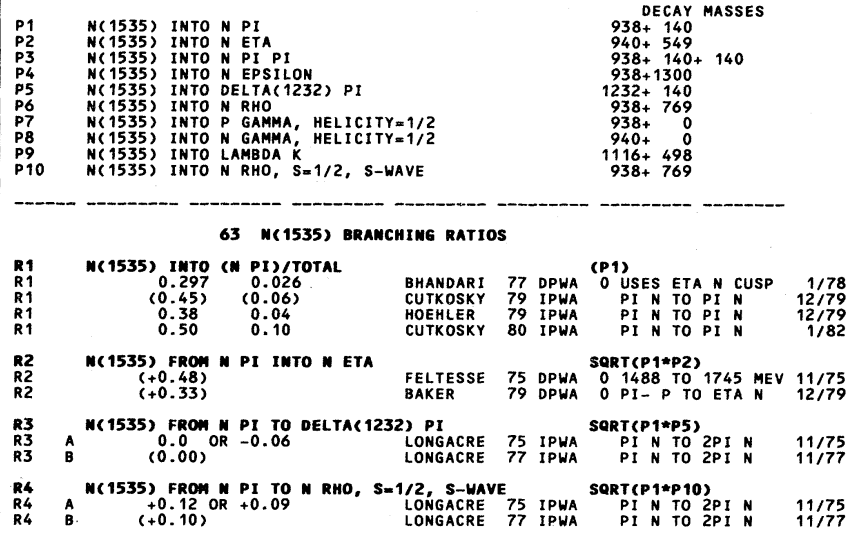


Baryons

$\mathrm{N}(1535), \mathrm{N}(1540), \mathrm{N}(1650)$
Data Card Listings
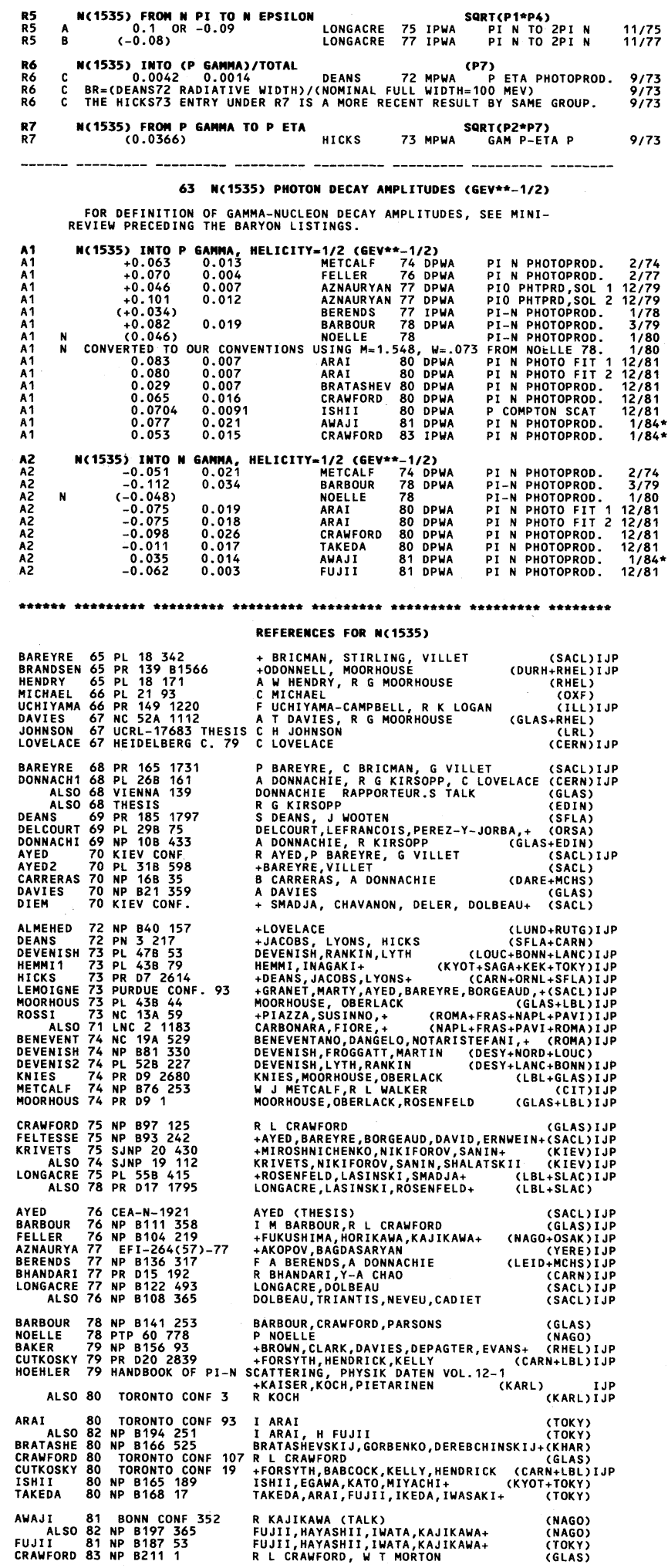

SEE HERE IN THE 1982 EDITION (PHYSICS LETTERS 11 118) FOR ADDITIONAL
EARLY REFERENCES (NONE AFTER 1973) ON THE N ETA THRESHOLD.

\begin{tabular}{|c|c|c|}
\hline$N(1540)$ & $P_{13}^{\prime}$ & Status: * \\
\hline & $\begin{array}{l}109, \\
\text { THIS } \\
\text { ANALY }\end{array}$ & 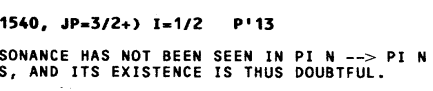 \\
\hline
\end{tabular}

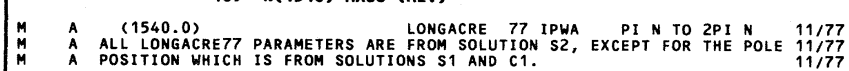

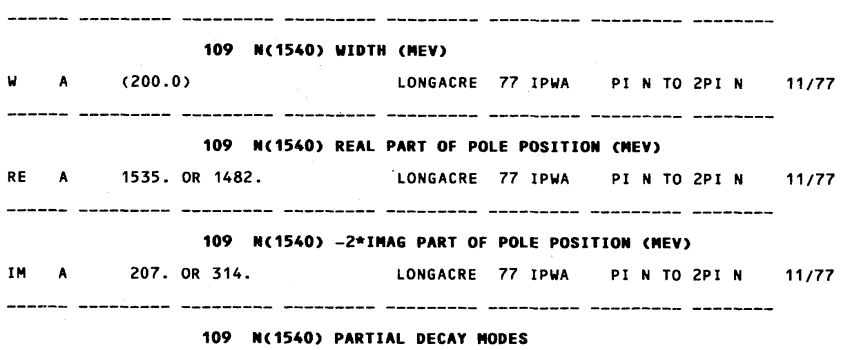

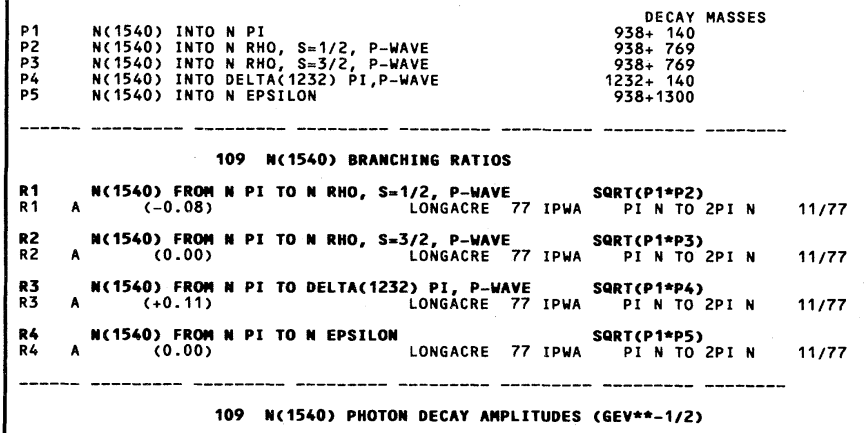

FOR DEFINITION OF GAMMA-NUCLEON DECAY AMPLITUDES, SEE MINI-

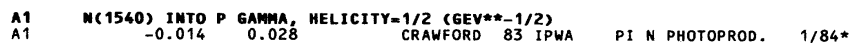

A2 N(1540)
A2 IMTO P GAMmA; MELICITY=3/2 (GEV**-1/2)
0.009
0.027

********************************************************************

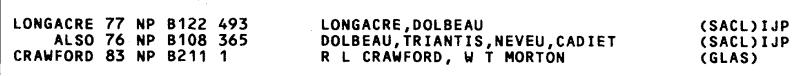

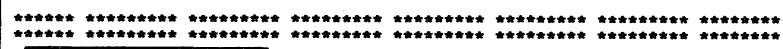

$\mathrm{N}(1650) \mathrm{S}_{11}^{\prime \prime} \quad$ Status: $* * * *$

$66 \mathrm{M}(1650, J P=1 / 2-) \quad I=1 / 2 \quad S \cdot 11$

MOST OF THE RESULTS PUBLISHED BEFORE 1975 ARE NOW
OBSOLETE AND HAVE BEEN OMITED. THEY MAY BE FOUND IN
OUR OUR 1982 EDITIN (PHYSSCS LETTERS 111B). HOWEVER,

66 N(1650) MASS (MEV)

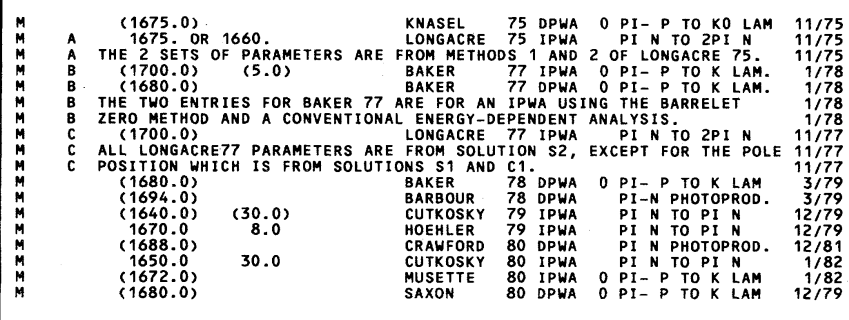




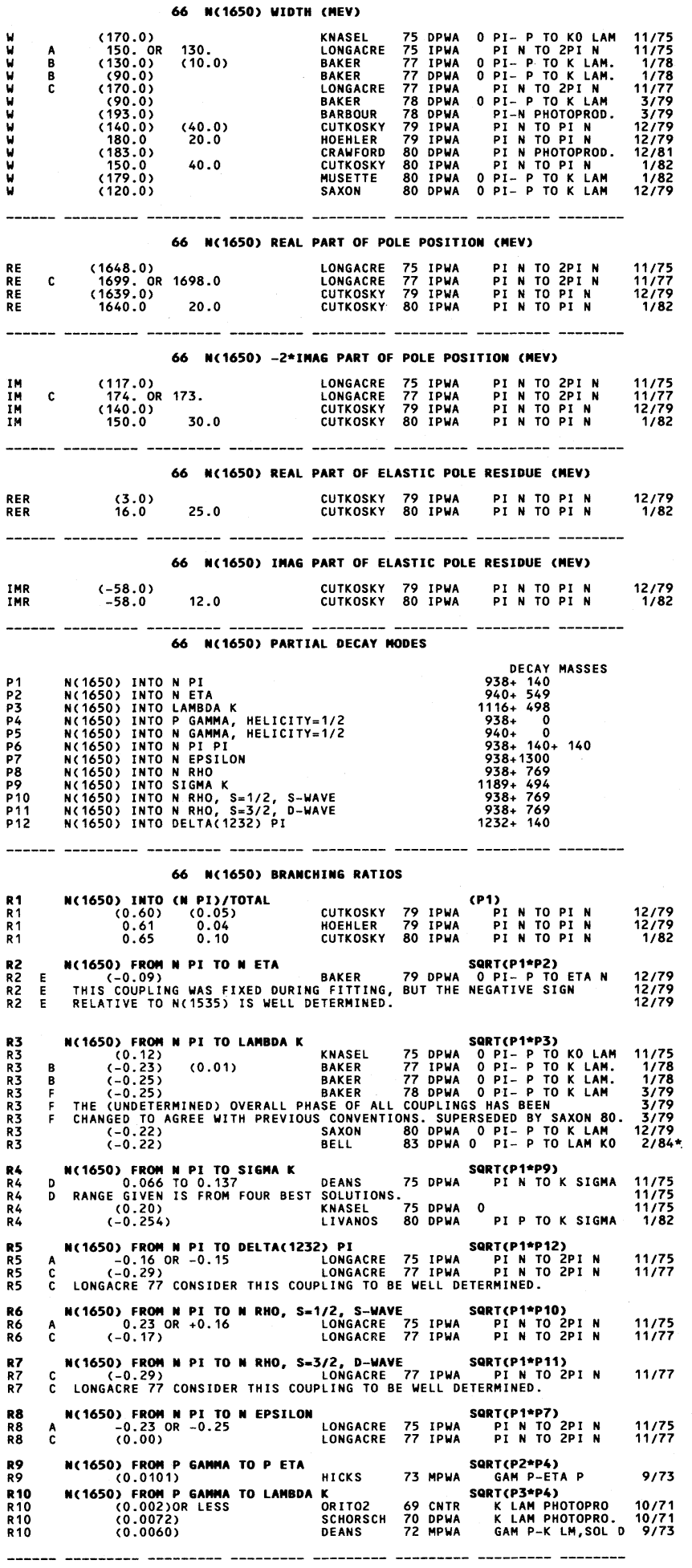

66 M(1650) PHOTON DECAY AMPLITUDES (GEV**-1/2) FOR DEFINITION OF GAMMA-NUCLEON DECAY AMPLITUDES, SEE MINI-
REVIEW PRECEDING THE BARYON LISTINGS.

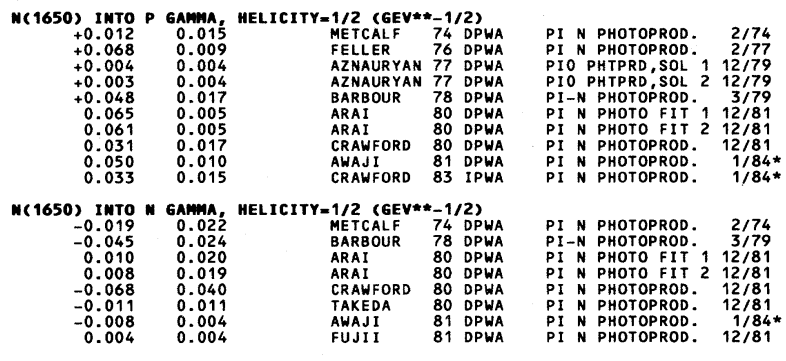

PI N PHOTOPROD.

PI-N PHOTOPROD: $\begin{array}{r}2 / 74 \\ 3 / 79\end{array}$ I PHOTO FIT 2 12/81 PI N PHOTOPRO $\begin{array}{lll}\text { PI } & \text { N PHOTOPROD. } \\ \text { PI N PHOTOPROD. } & 12 / 81 \\ \text { PI } & 1 / 84 *\end{array}$

\section{$\begin{array}{cccc}-0.008 & 0.004 & \text { AWAJI } & 81 \\ 0.004 & 0.004 & \text { FUJII } & 81 \text { DPWA } \\ 0.004 & \end{array}$}

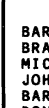

\section{CI $N$ PHOTOPROD.} OHNSON 67 UCRL-17683 THESIS N

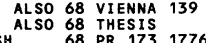
(DURH+RHEL) IJP

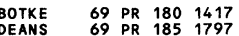
DEANS2 69 PR 1772623

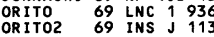

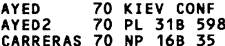

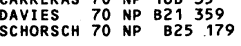

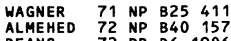
DEVENISH 73 PR 478 P. 53

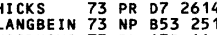

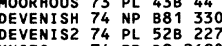

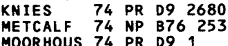
CRAHFORO 75 NP 897125

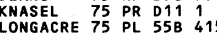
ALSO 78 PR 0171795

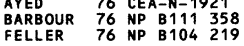
AZNAURYA 77 EF I-264557)-77 ONGACRE 77 NP B122 495

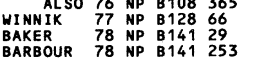

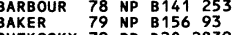
(RLEL) IJP

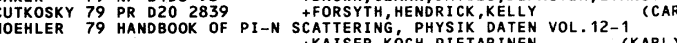
ALSO 80 TORONTO CONF 3 R KOCH, KOCH, PIETARINEN (KARL) (KARL)IJP
+KAISER $\begin{array}{llll}\text { ARAI } & 80 & \text { TORONTO CONF } 93 & \text { I ARAI } \\ \text { ALSO } 82 \text { NP B194 251 } & \text { (TOKY) }\end{array}$

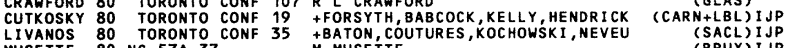

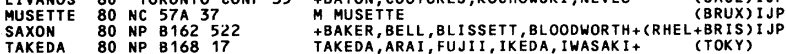
$\begin{array}{llll}\text { AWAJI } 81 \text { BONN CONF } 352 & \text { R KAJIKAWA (TALK) } & \text { (NAGO) } \\ \text { ALSO } 82 \text { NP B197 } 365 & \text { FUJI, HAYASHII, WHATA, KAJIKAWA+ } & \text { (NAGO) }\end{array}$

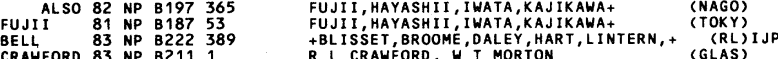

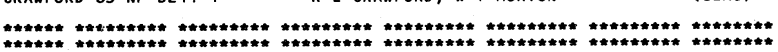
\begin{tabular}{|lll}
\hline $\mathrm{N}(1675)$ & $\mathrm{D}_{15}^{\prime}$ & Status: \\
\hline
\end{tabular}

$64 M(1675, J P=5 / 2-) \quad I=1 / 2 \quad D \cdot 15$

MOST OF THE RESULTS PUBLISHED BEFORE 1975 ARE NOW OBSOLETE AND HAVE BEEN OMITTED. THEY MAY BE FOUND
OUR 1982 EDITION (PHYSICS LETTERS 111 ). HOWEVER,
ALL THE REFERENCES HAVE BEEN RETAINED.

IN ADDITION, RESULTS IN THIS REGION FROM PRODUCTION EXPERIMENTS,
WHICH USED TO BE LISTED SEPARATELY IN AN ENTRY FOLLOWING THE 


\section{Baryons \\ Data Card Listings $\mathrm{N}(1675)$}

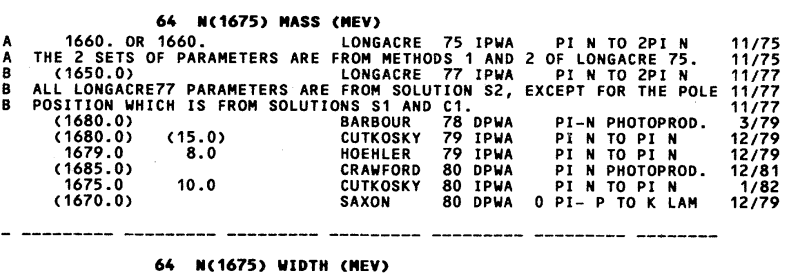

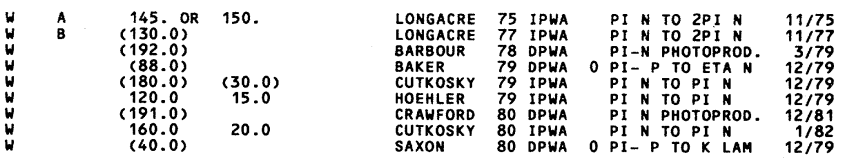

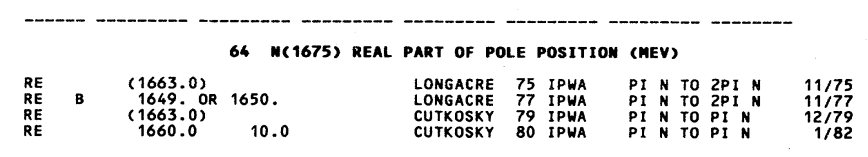

64 W(1675) $-2 *$ IMAG PART OF POLE POSITION (MEV)

\begin{tabular}{|c|c|c|c|c|}
\hline $\lim _{\substack{M \\
\text { IM } \\
\text { IM }}} B$ & 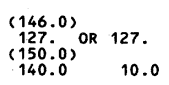 & $\begin{array}{l}\text { LONGACRE } \\
\text { LONGACRE } \\
\text { CUTKOSKY } \\
\text { CUTKOSKY }\end{array}$ & $\begin{array}{l}75 \text { IPWA } \\
77 \text { IPWA } \\
79 \text { IPWA } \\
80 \text { IPWA }\end{array}$ & 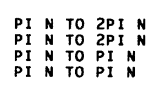 \\
\hline
\end{tabular}

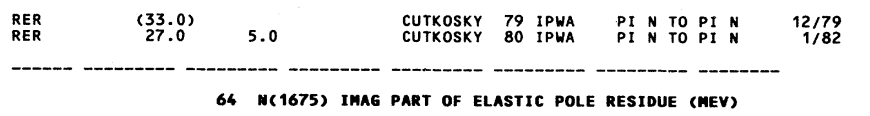

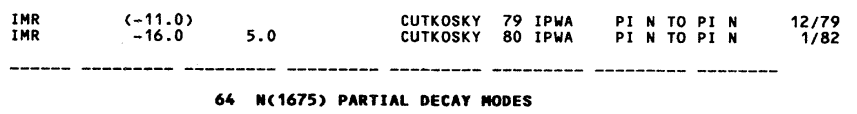

\begin{tabular}{|c|c|}
\hline 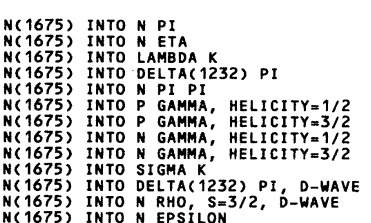 & 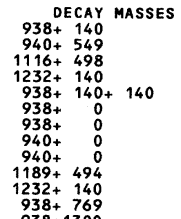 \\
\hline
\end{tabular}

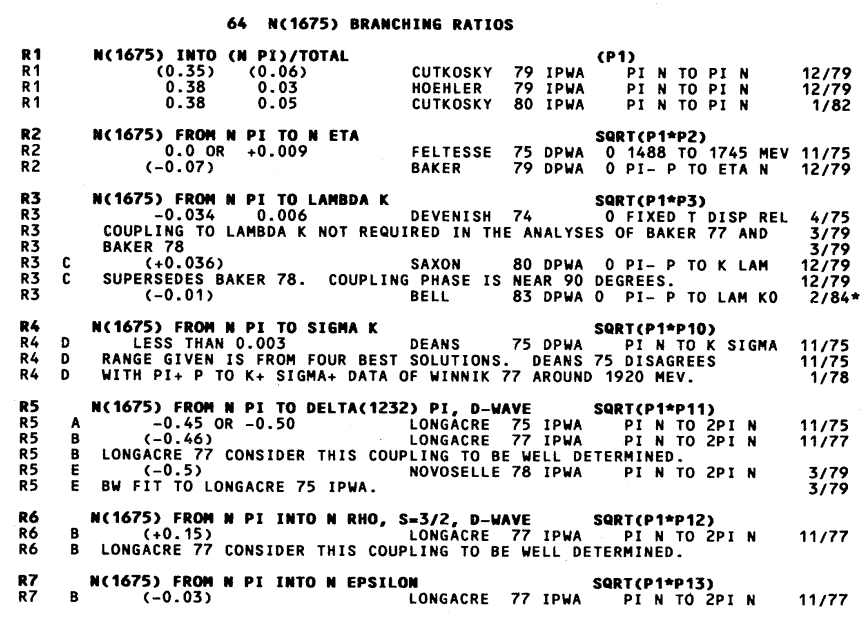

64 M(1675) PHOTON DECAY AMPLITUDES (GEV**-1/2)

FOR DEFINITION OF GAMMA-NUCLEON DECAY AMPLITUDES, SEE MINI-
REVIEW PRECED ING THE BARYON LISTINGS.

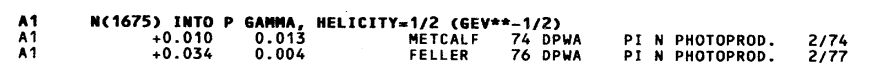

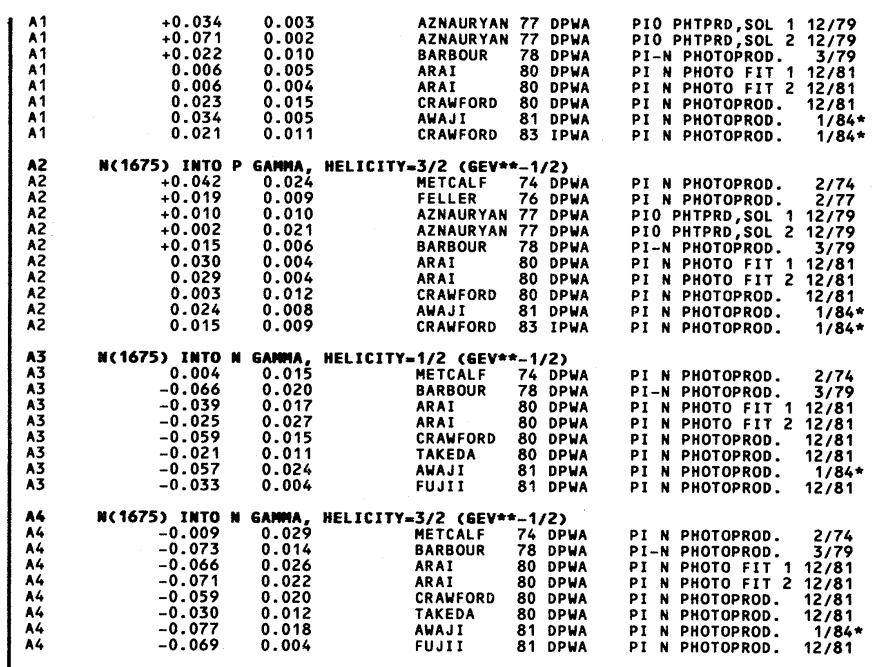

$* * * * * * * * * * * * * * * * * * * * * * * * * * * * * * * * * * * * * * * * * * * * * * * * * 1$
REFEREMCES FOR $N(1675)$

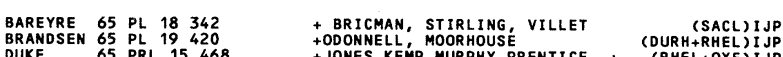

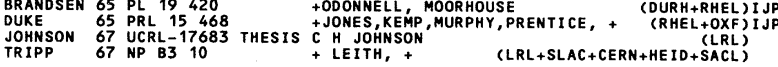

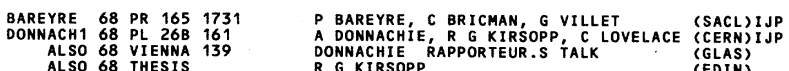

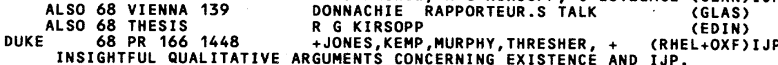

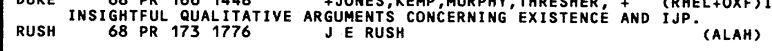

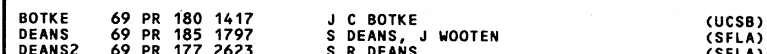

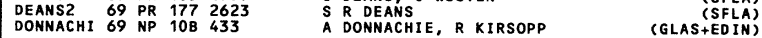

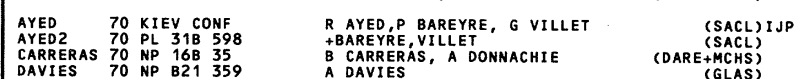

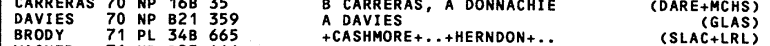

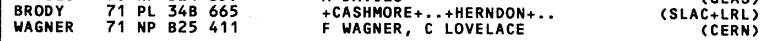
ALMEHED 72
DEVENISH 73 PL 157

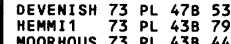

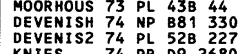

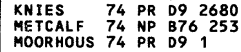
CRAWFORD 75 NP 897125

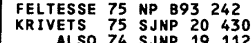

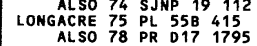

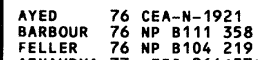

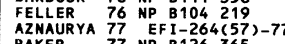

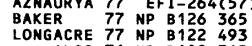

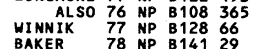

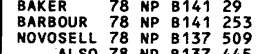
TOVEDCE

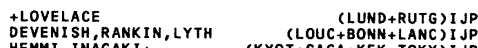
MOORHOUSE, OBERLACK (KTOYSAGA+KEK+ TOKY)IJP DEVENSH, FROGGATT,MART IN (DESY+NORD+LOUC)

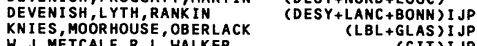

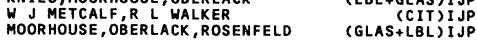
$R$ L CRAWFORD PMITCHELL, MONTGOMERY, + (GLAS) IJP

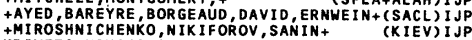

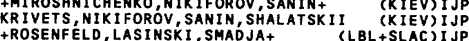
PROSEAFELD, LASINSK, SMADJA+
LONGACRE, LASINSKI, ROSENFELO+ (LBL+SLAC) IJP
(LBL+SLAC)

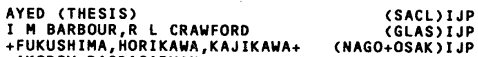
+FUKUSHIMA, HORIKAHA, KAJIKAWA+ (NAGO+OSAR) IJP
+AKOPOV, GAGDASARYAN
(YERE) IP

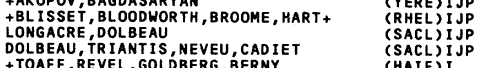
HBLISSET, BLOODHORTH, BROMME+
BARBOUR, CRAHFORD, PARSONS

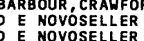

(RL+CAMB) IJP
(GLAS)
(CIT)IJP
(CIT)IJP

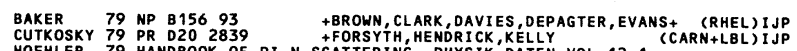

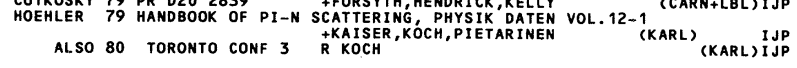

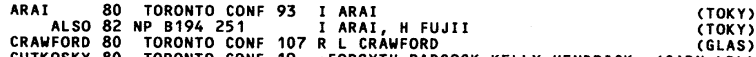

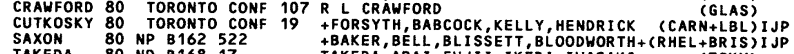

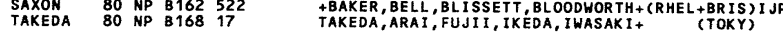

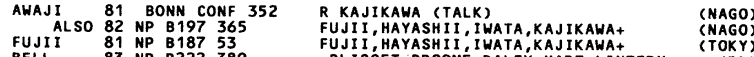

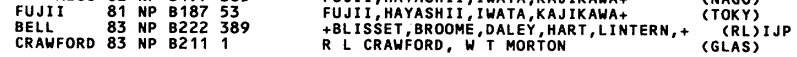

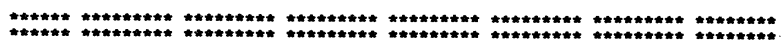




\section{\begin{tabular}{|ll}
$\mathrm{N}(1680)$ & $\mathrm{F}_{15}^{\prime}$ \\
\hline
\end{tabular} \\ $65 \mathrm{M}(1680, J P=5 / 2+) \quad I=1 / 2 \quad F \cdot 15$ \\ MOST OF THE RESULTS PUBLISHED BEFORE 1975 ARE NOW OBSOLETE AND HAVE BEEN OMITTED. THEY MAY BE FOUND IN
OUR 1982 EDITION (PHYSICS LETTERS 1118). HOWEVER,
ALL THE REFERENCES HAVE BEEN RETAINED. IN ADDITION, RESULTS IN THIS REGION FROM PRODUCTION EXPERIMENTS,
WHICH USED TO BE LISTED SEPARATEY IN AN ENTRY FOLLOWING THE}

\section{M(1680) mass (nev)}

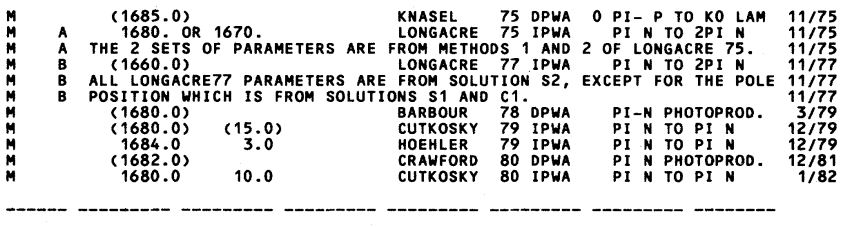

65 M(1680) WIDTH (MEV)

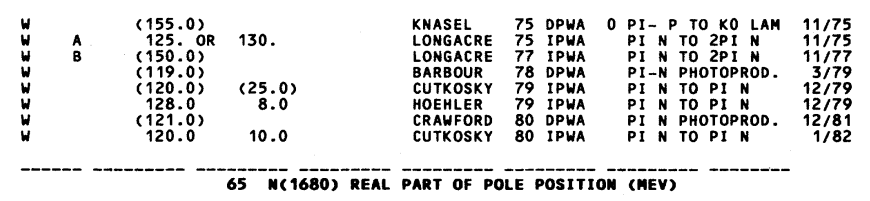

\begin{tabular}{|c|c|c|c|c|c|c|}
\hline $\begin{array}{l}\mathrm{RE} \\
\mathrm{RE} \\
\mathrm{RE} \\
\mathrm{RE}\end{array}$ & B & $\begin{array}{l}(1688.0) \\
1656.0 \mathrm{OR} \\
(1666.0) \\
1667.0\end{array}$ & $\begin{array}{r}1653 . \\
5.0\end{array}$ & $\begin{array}{l}\text { LONGACRE } \\
\text { LONGACRE } \\
\text { CUTKOSKY } \\
\text { CUTKOSKY }\end{array}$ & $\begin{array}{l}75 \text { IPWA } \\
77 \\
79 \\
79 \\
\text { IPWA } \\
80 \text { IPWA }\end{array}$ & 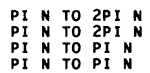 \\
\hline
\end{tabular}

\begin{tabular}{|c|c|c|c|c|c|c|}
\hline B & $\begin{array}{l}(132.0) \\
145.0 \mathrm{OR} \\
(112.0) \\
110.0\end{array}$ & $\begin{array}{l}143 . \\
10.0\end{array}$ & $\begin{array}{l}\text { LONGACRE } \\
\text { LONGACRE } \\
\text { CUTKOSKY } \\
\text { CUTKOSKY }\end{array}$ & $\begin{array}{l}75 \text { IPWA } \\
77 \text { IPWA } \\
79 \\
80 \text { IPWA } \\
80 \text { IPWA }\end{array}$ & 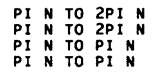 & $\begin{array}{r}11 / 75 \\
11177 \\
12 / 79 \\
1 / 82\end{array}$ \\
\hline
\end{tabular}

\begin{tabular}{|c|c|c|c|c|c|c|c|}
\hline & & 65 & $N(1680)$ & REAL & PART OF E & ELASTIC POLE & RESIDUE (MEV) \\
\hline $\begin{array}{l}\text { RER } \\
\text { RER }\end{array}$ & $\begin{array}{c}(31.0) \\
31.0\end{array}$ & & 2.0 & & $\begin{array}{l}\text { CUTKOSKY } \\
\text { CUTKOSKY }\end{array}$ & $\begin{array}{l}79 \text { IPWA } \\
80 \text { IPHA }\end{array}$ & $\begin{array}{lllll}P 1 & N & \text { TO } & \text { PI } & N \\
\text { PI } & N & \text { TO } & \text { PI } & N\end{array}$ \\
\hline & & 65 & $\omega(1680)$ & IMAG & PART OF E & ELASTIC POLE & RESIDUE (MEV) \\
\hline $\begin{array}{l}\text { IMR } \\
\text { IMR }\end{array}$ & $\begin{array}{l}(-15.0) \\
-14.0\end{array}$ & & 3.0 & & $\begin{array}{l}\text { CUTKOSKY } \\
\text { CUTKOSKY }\end{array}$ & $\begin{array}{ll}79 & \text { IPWA } \\
80 & \text { IPWA }\end{array}$ & $\begin{array}{lllll}\text { PI } & N & \text { TO } & \text { PI } & N \\
\text { PI } & N & \text { TO } & \text { PI } & \text { N }\end{array}$ \\
\hline
\end{tabular}

65 M(1680) PARTIAL decay mODEs

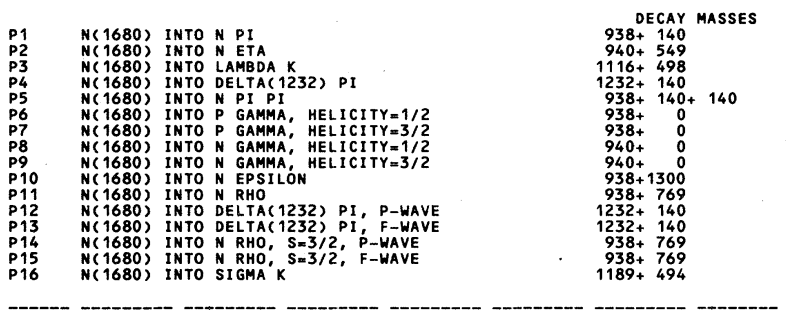

65 M(1680) BRAMCHIMG RATIOS

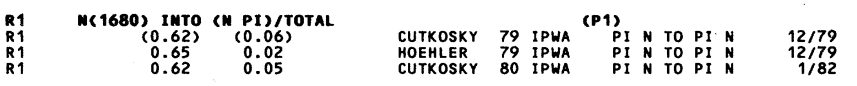

R2 M(1680) FROM N PI TO N ETA
R2 SEEN

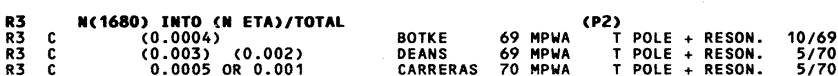

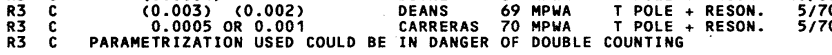

R4 M(1680) INTO (N ETA)/(N PI)
R4 0.027$)$ OR LESS

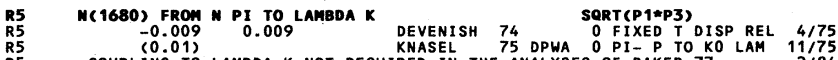

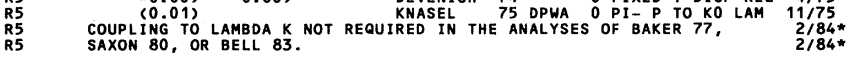

R6 W(1680) FROM M PI TO SIGMA $K$ DEANS 75 DPHA PORT(P1*P16)

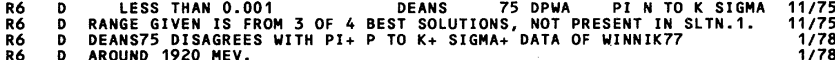

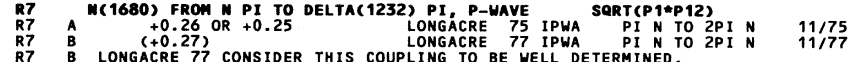

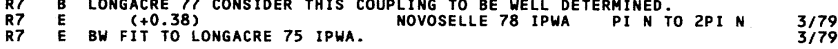

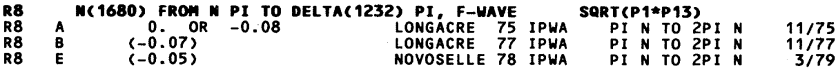

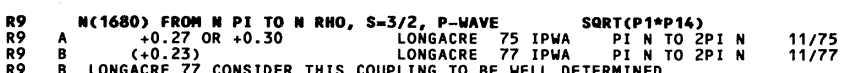

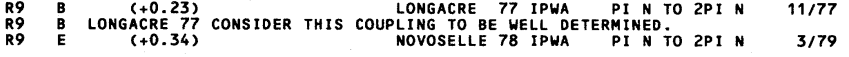

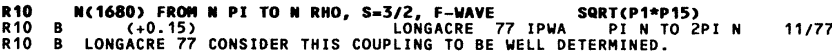

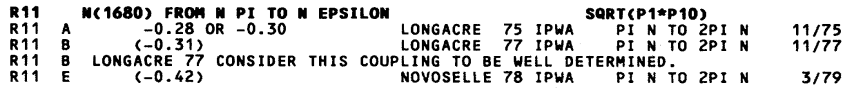

65 M(1680) PHOTON DECAY AMPLITUDES (GEV**-1/2)

FOR DEFINITION OF GAMMA-NUCLEON DECAY AMPLITUDES, SEE MINI-
REVIEW PRECEDING THE BARYN LISTINGS.

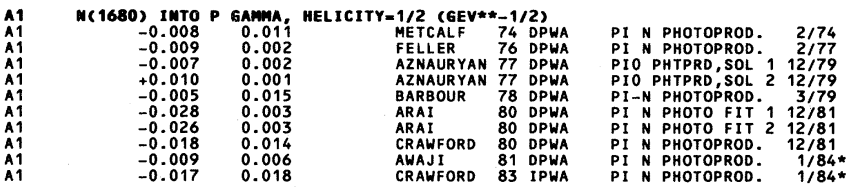

n(1680) IMTO P GAMMA, HELICITY $3 / 2$ (GEV**-1/2)
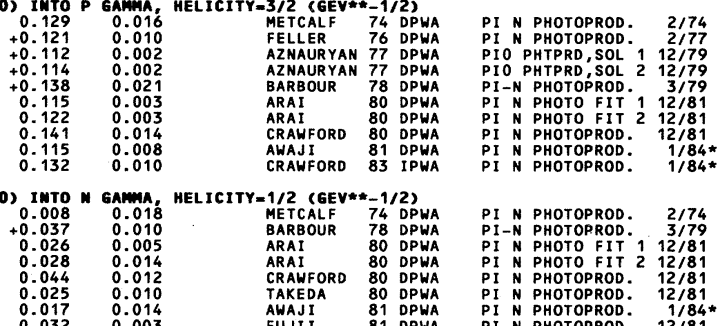

PI N PHOTOPROD. 12/81

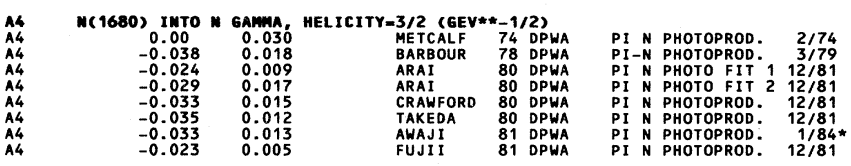

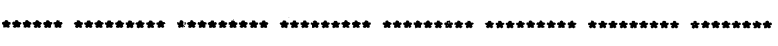

REFEREMCES FOR N(1680)

FOR VERY EARLY REFERENCES, SEE RMP 37, 633 (1965).

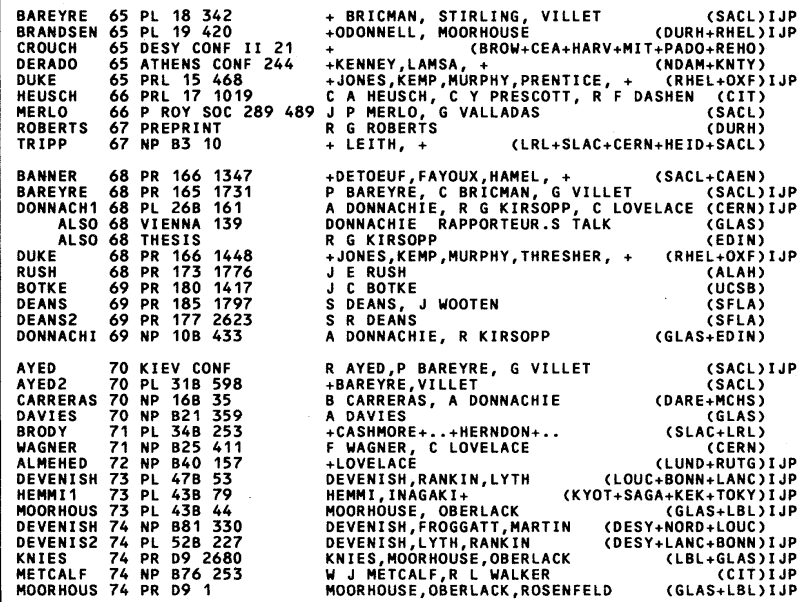




\section{Baryons}

$\mathrm{N}(1680), \mathrm{N}(1700)$

\section{Data Card Listings}

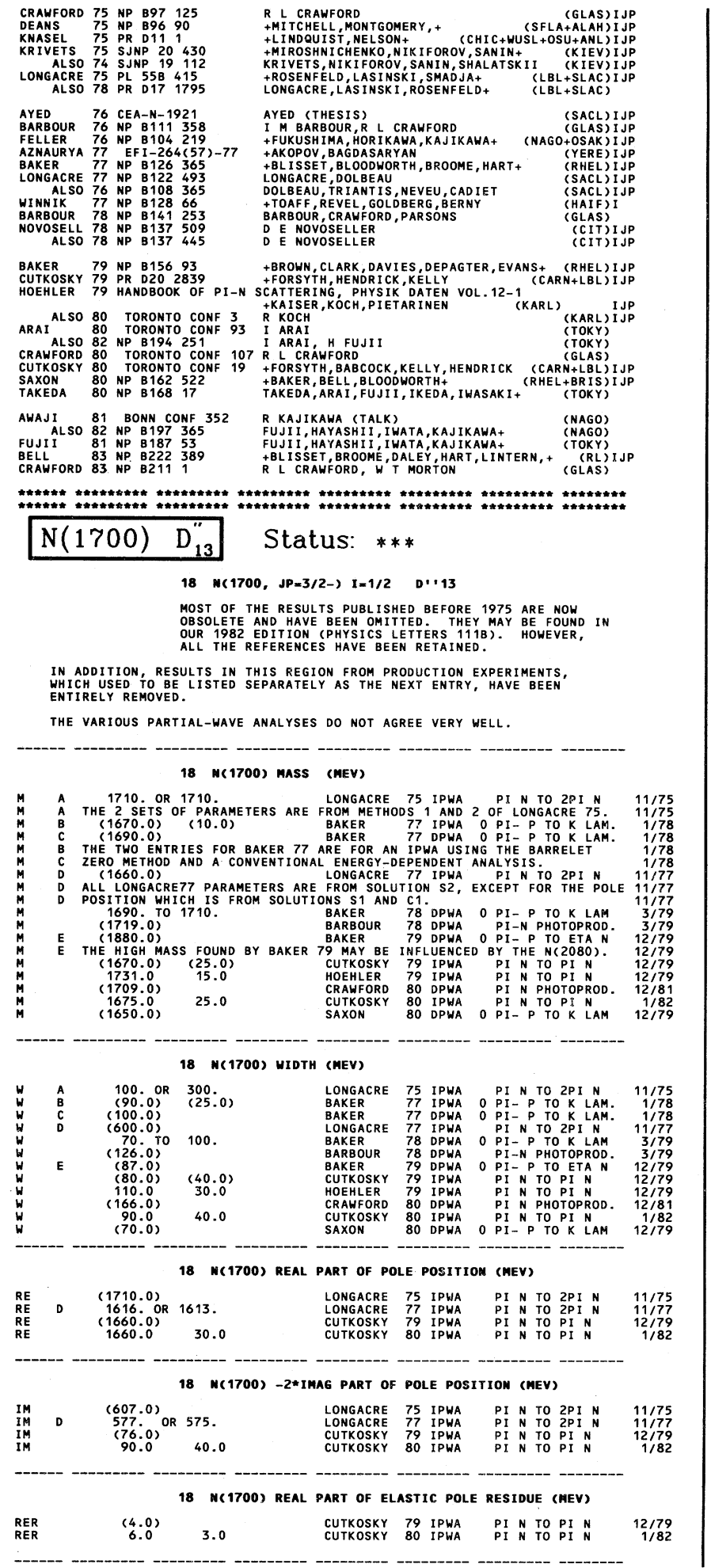

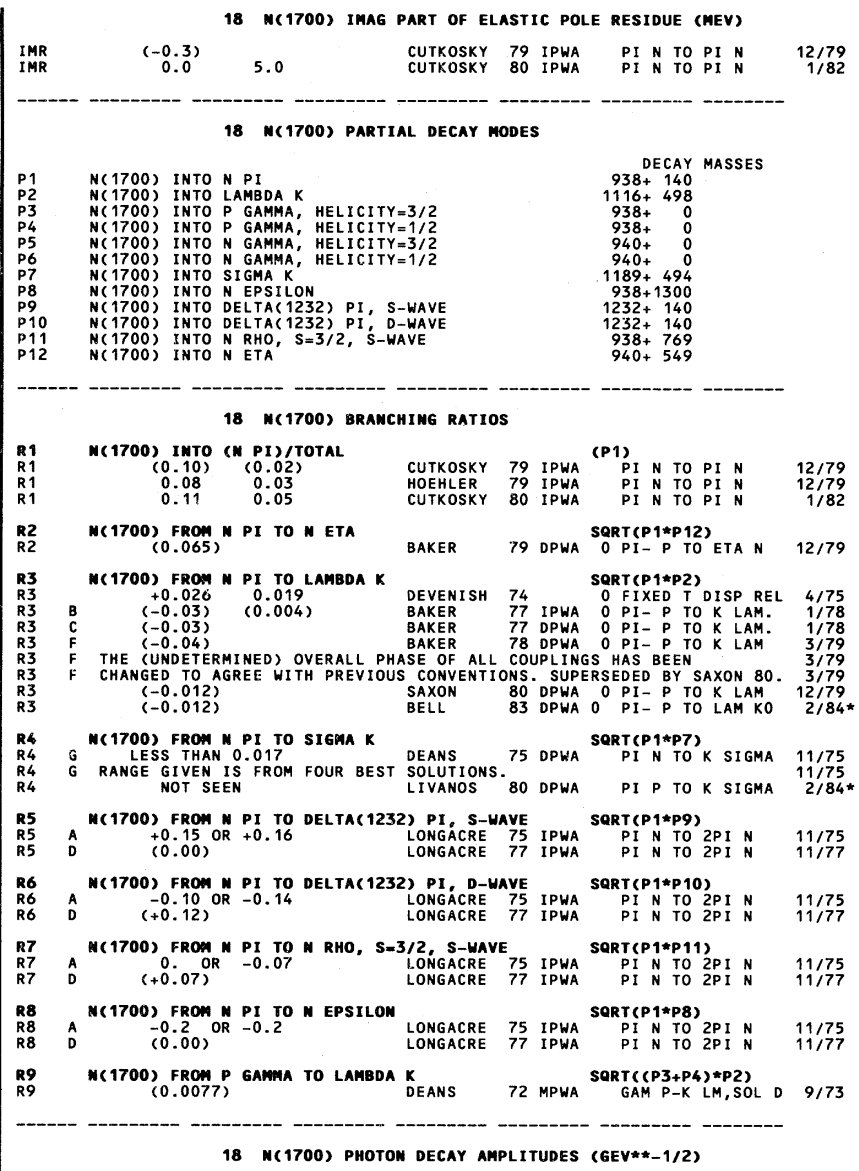
FOR DEFINIIION OF GAMMA-NUCLEON DECAY AMPLITUDES, SEE MINI-

N(1700)

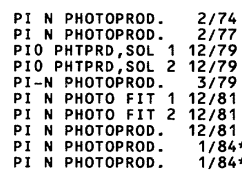

M(1700) INTO P GAMMA, HELICITY $=3 / 2$ (GEV**-1/2)

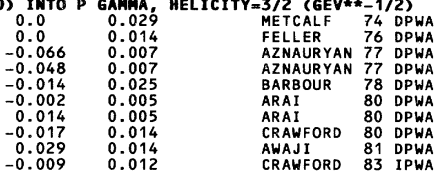

M(1700) INTO N GAMHA, HELICITY=1/2 (GEV**-1/2)

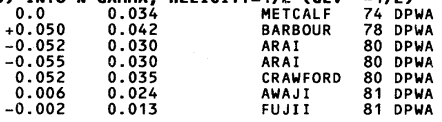

M(1700) IMTO N GAMHA, HELICITY=3/2 (GEV**-1/2)

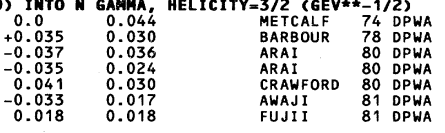

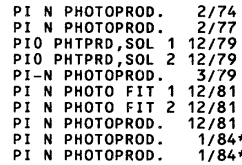

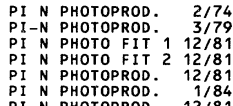

$\begin{array}{ll}\text { PI N PHOTOPROD. } & 2 / 74 \\ \text { PI-N PHOTOPROD: } & 3 / 79\end{array}$ 


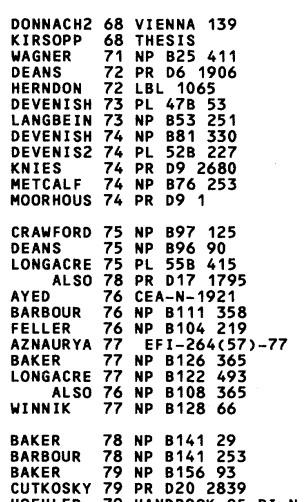

REFERENCES FOR $N(1700)$

DONNACHIE RAPPORTEUR.S TALK

R G KIRSOPP L DETACE

(EDIN)
(EDRN)

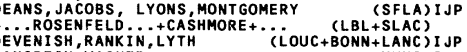

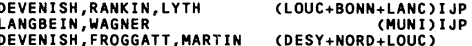

$\begin{array}{ll}\text { DEEVNISH, FROGGATT, MARTIN } & \text { (DESY+NORD+LOUC) } \\ \text { DEVENISH,LYTH, RANIN } & \text { (DESY+LANC+BONN) IJP }\end{array}$

$\begin{array}{ll}\text { KNIES, MOORHOUSE, OBERLACK } & \text { (LBL +GLAS) IJP } \\ \text { WJ METCAL, R L WALKER } & \text { (CIT)IJP } \\ \text { MOORHOUSE, OBERLACK, ROSENFELD } & \text { (GLAS+LBL)IJP }\end{array}$

$R$ L CRAWFORD

$(G L A S)$ IJP
$($ SFLA+ALAH)IJP
$(L B L+S L A C)$ IJP

$\begin{array}{ll}\text { +MITCHELL, MONTGOMERY, } & \text { (SFLA+ALAH) IJP } \\ \text { +ROSENFELD, LASINSKI, SMADJA+ } & \text { (LBL+SLAC)IJP }\end{array}$ AYEGACRE, LASINSKI, ROSENFELD

(GACL)IJP
M BARBOUR, R L CRAWFORD
FUKUSHIMA, HORIKAWA, KAJIKAWA+
(NAGO+OSAK)IJP

+BLISSET, BLOODWORTH, BROOME, HART+

LONGACRE,'DOLBEAU

DOLBEAU, TRIANT IS, NEVEU, CADIET

+BL ISSET, BLOODWORTH, BROOME+
(RL+CAMB) IJP

BARBOUR, CRAAWORD, PARSONS
+BROWN, CARK, DAVIES, DEPAGTER, EVANS+ (GLAS) IJPP
+FORSYTH, HENDRICK, KELLY THEL) (CARN +LBL) IJP

CUTKOSKY 79 PR 0202839

+
A

(KARL) YJP
(KARL)IJP

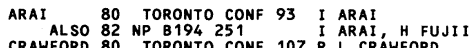

(TOKY)

CUTKOSKY 80 TORONTO CONF 19 +FORSYH, BABCOCK, KELLY, HENDRICK (CARN+LBL) IJP LIVANOS 80 TORONTO CONF 35 + + BATON, COUTURE, KOCHOWSKI NEVEU CARN (SACL) IJP
SAXON 80 NP B162 522

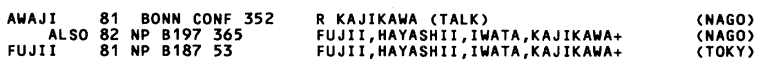

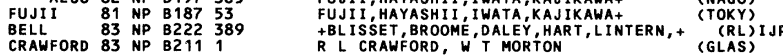

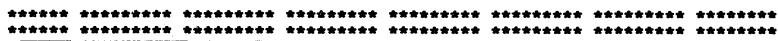

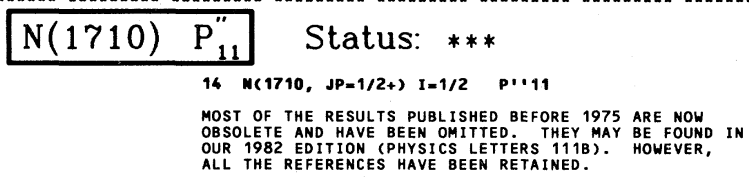

THE VARIOUS PARTIAL-WAVE aNALYSES DO NOT AGREe VERY WELL.

\section{M(1710) MASS (MEV)}

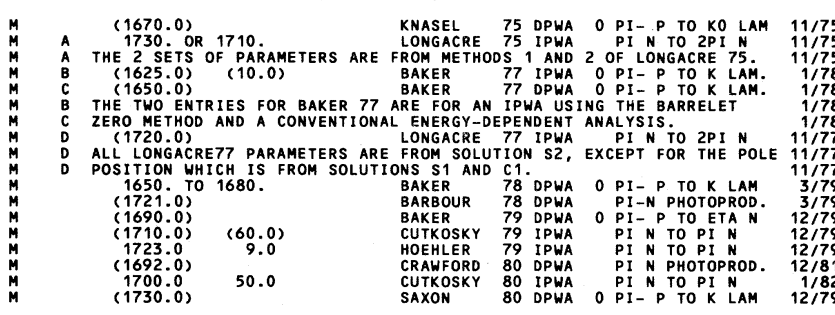

14 H(1710) WIDTH (MEV)

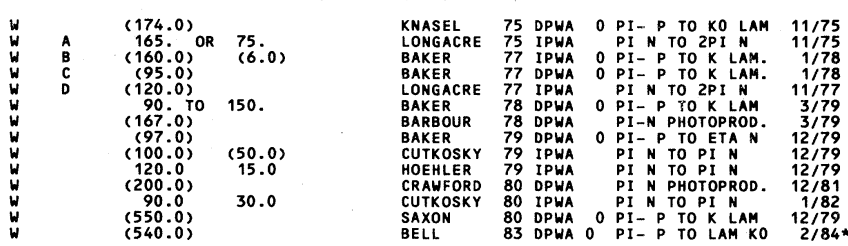

14 M(1710) REAL PART OF POLE POSITION (MEV)

\begin{tabular}{|c|c|c|c|c|c|}
\hline $\begin{array}{l}\text { RE } \\
\text { RE } \\
\text { RE }\end{array}$ & $\begin{array}{l}(1708.0) \\
1720.0 \mathrm{OR} \\
(1692.0) \\
1690.0\end{array}$ & $\begin{array}{r}1711 . \\
20.0\end{array}$ & $\begin{array}{l}\text { LONGACRE } \\
\text { LONGACRE } \\
\text { CUTKOSKY } \\
\text { CUTKOSKY }\end{array}$ & $\begin{array}{l}75 \mathrm{IPW} \\
77 \mathrm{IPW} \\
79 \mathrm{IPW} \\
80 \mathrm{IPW}\end{array}$ & 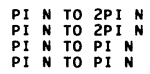 \\
\hline
\end{tabular}

14 M(1710) -2*IMAG PART OF POLE POSITIOM (MEV)

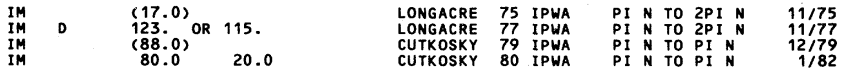

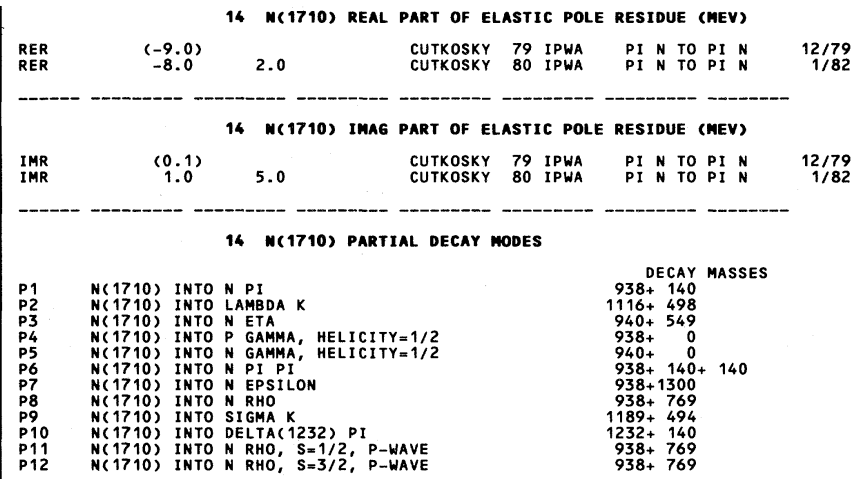

14 M(1710) BRAMCHIMG RATIOS

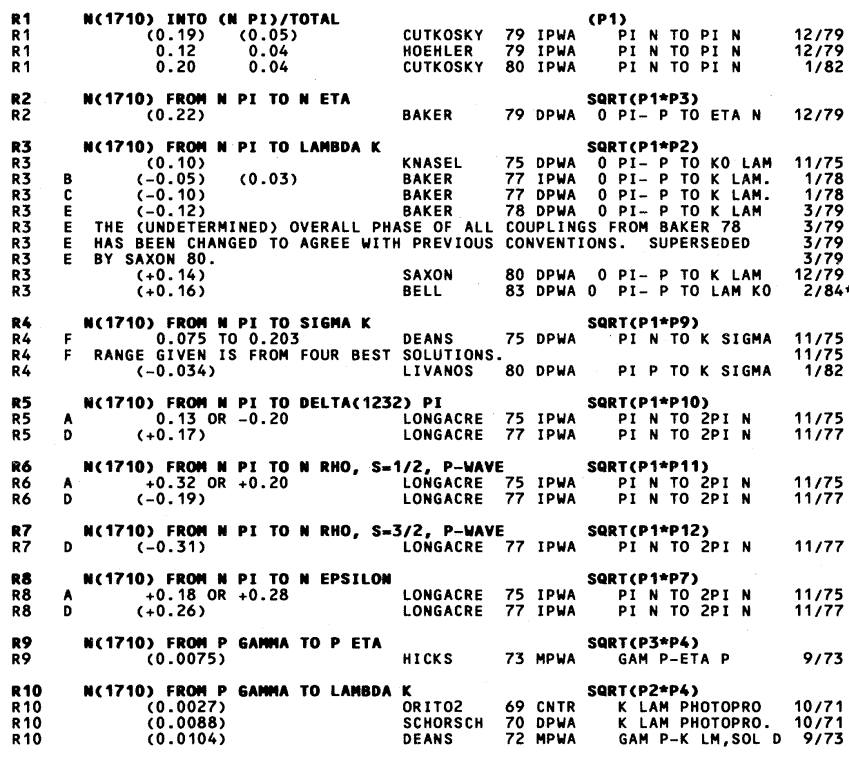

14 M(1710) PHOTON DECAY AMPLITUDES (GEV**-1/2)

FOR DEFINITION OF GAMMA-NUCLEON DECAY AMPLITUDES, SEE MINI-
REVIEW PRECEDING THE BARYN LISTINGS.

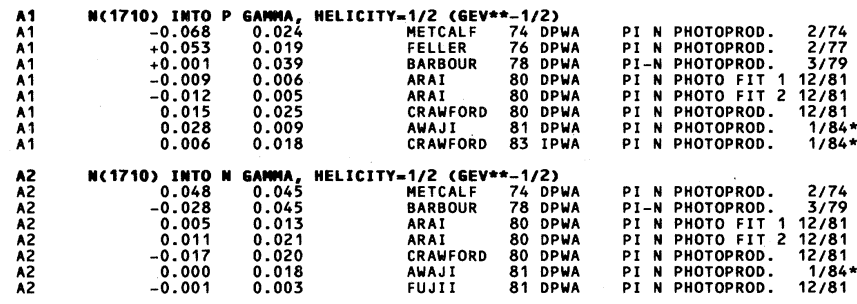

$\begin{array}{lllll}\text { ALSO } & 68 & \text { PL } & 268 & 161 \\ \text { ALIENAA } & 139\end{array}$

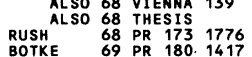

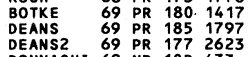

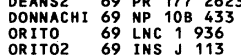

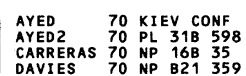


Baryons $\mathrm{N}(1710), \mathrm{N}(1720)$

\section{Data Card Listings}

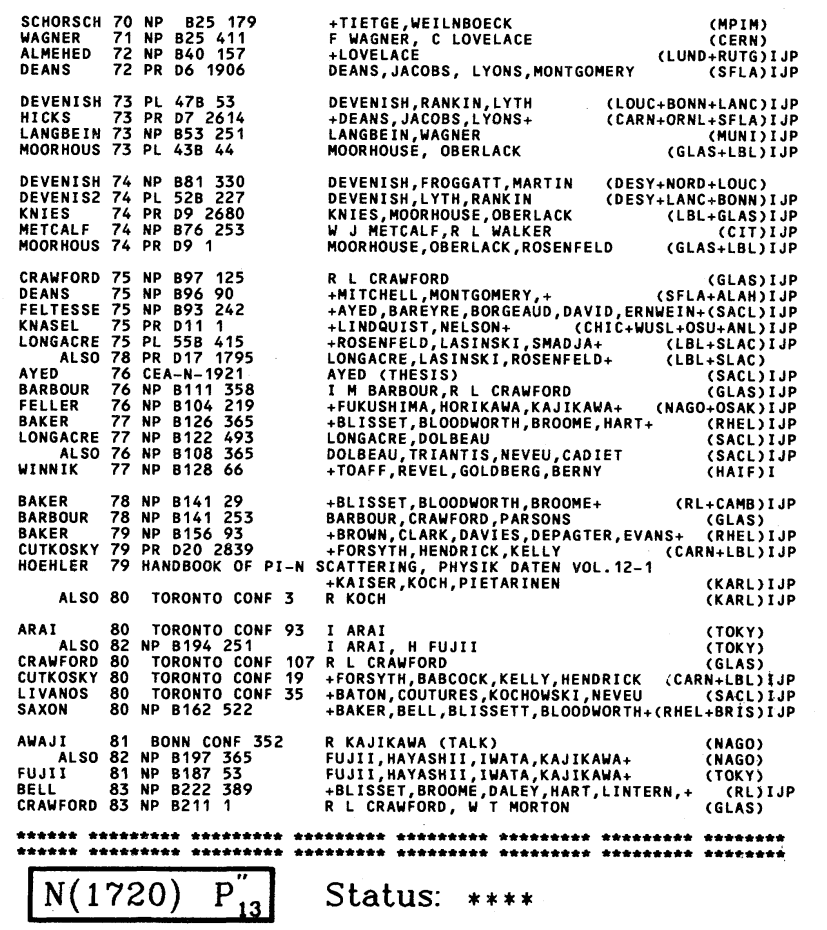

$15 \mathrm{M}(1720, J P=3 / 2+)$ I=1/2 $p \cdots 13$

MOST OF THE RESULTS PUBLISHED BEFORE 1975 ARE NOW
OBSOLETE AND HAVE BEEN OMITTED. THEY MAY BE FOUND IN

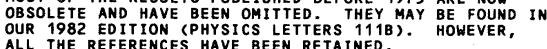

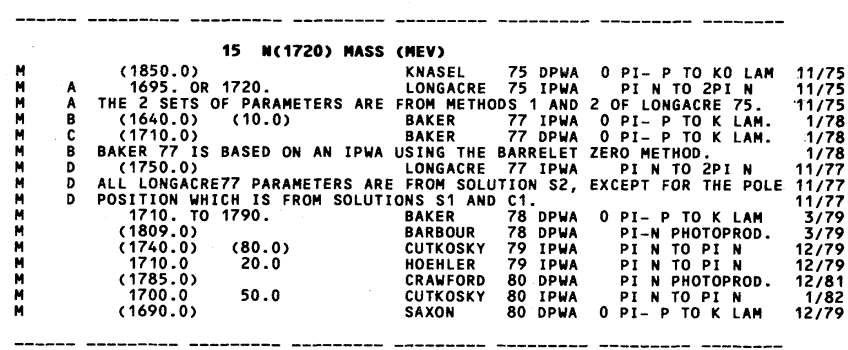

15 N(1720) МIDTH (MEV)

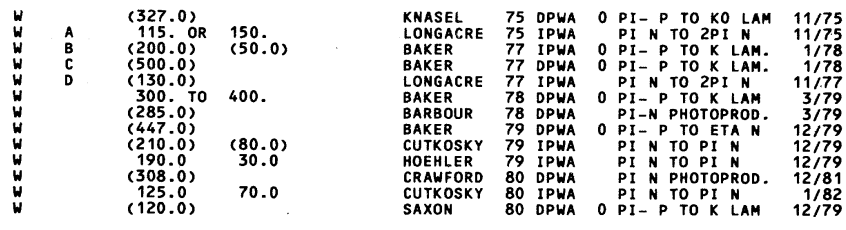

15 M(1720) REAL PART OF POLE POSITION (MEV)

\begin{tabular}{|c|c|c|c|c|}
\hline & 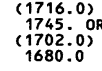 & $\begin{array}{r}1748.0 \\
30.0\end{array}$ & 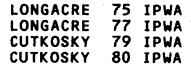 & 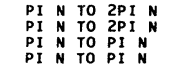 \\
\hline
\end{tabular}

15 M(1720) -2*IMAG PART OF POLE POSITIOW (MEV)

\begin{tabular}{|c|c|c|c|c|c|}
\hline 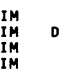 & $\begin{array}{l}(124.0) \\
(155: 0 \mathrm{OR} \\
(158.0)^{2} \\
120.0\end{array}$ & $\begin{array}{l}123 . \\
40.0\end{array}$ & $\begin{array}{l}\text { LONGACRE } \\
\text { LONGACRE } \\
\text { COTKKOSKY } \\
\text { CUTKOSKYY }\end{array}$ & 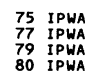 & 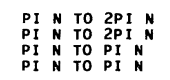 \\
\hline
\end{tabular}

15 N(1720) ReAL PART OF ELASTIC POLE Residue (MEV)

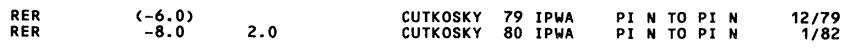

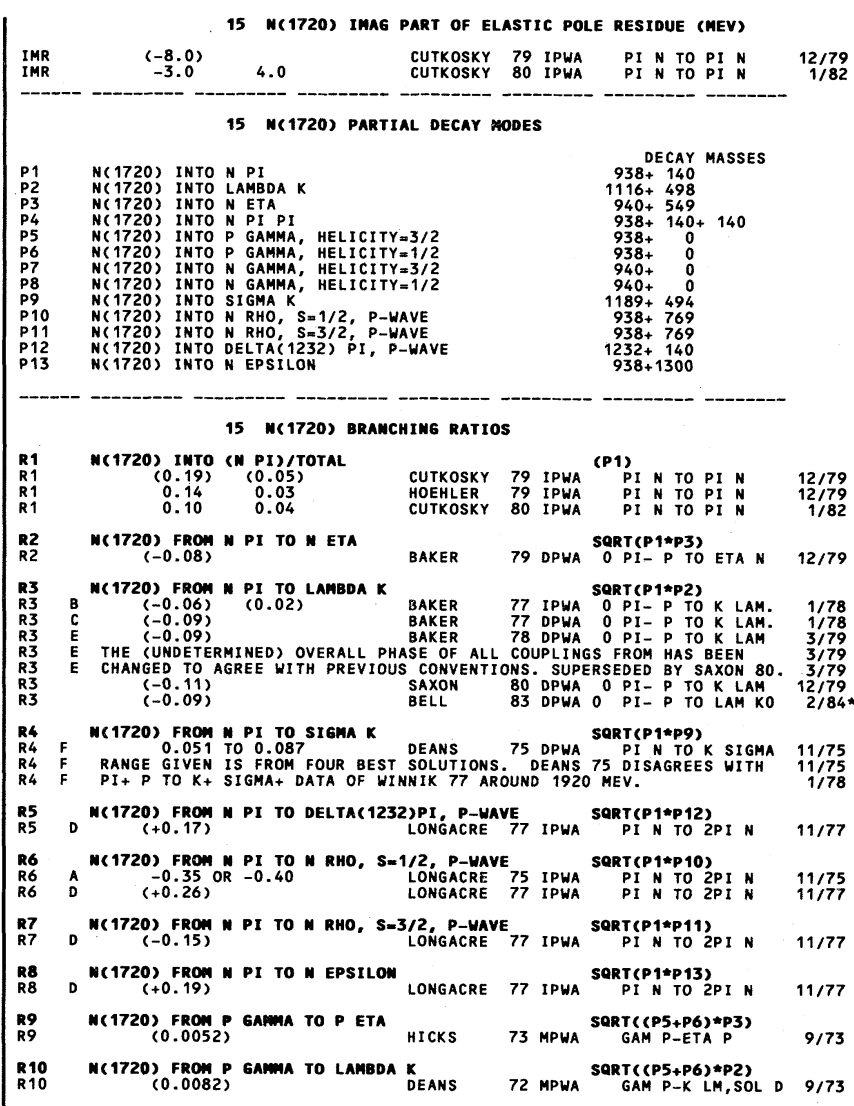

15 N(1720) PHOTON DECAY AMPLITUDES (GEV**-1/2)

FOR DEFINITION OF GAMMA-NUCLEON DECAY AMPLITUDES, SEE MINI-
REVIEW PRECED ING THE BARYON LISTINGS.

(1720) THTO P GAMA MELICITY-1/2

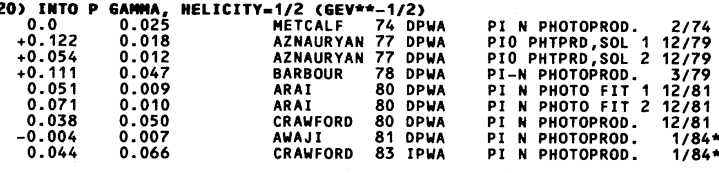

M(1720) IWTO P GAMmA, HELICITY $3 / 2$ (GEE**-1/2)

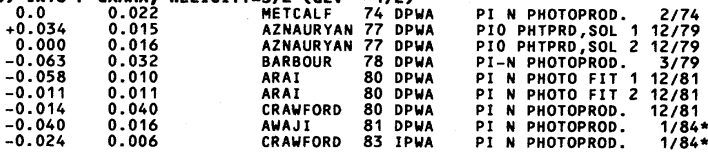

M(1720) INTO N GAMPAA HELICITY=1/2 (GEV**-1/2)

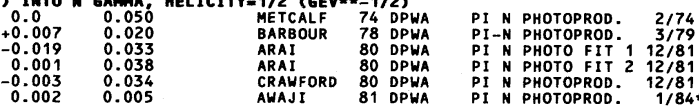

M( 1720) INTO N GAMAM, MEL ICITY-3/2 (GEV**-1/2)

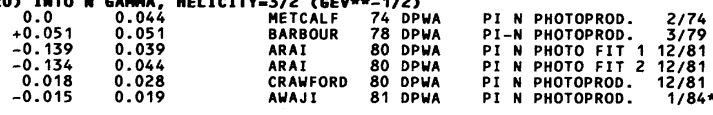

ALSO 68 PL 26 VIENA 16

$\begin{array}{ll}68 \\ 68 \text { PRE } 175 \\ 69 & 1776\end{array}$

$\begin{array}{lllll}\text { BOTKE } & 69 & \text { PR } & 180 & 1417 \\ \text { DEANS } & 69 & P R & 185 & 1797\end{array}$

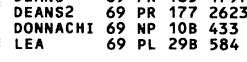

REFEREMCES FOR M(1720)

A OONNACHIE, RPG KIRSOPP, C LOVELACE (CERN) IJP
DONNACHIE RAPPORTEUR.S TALK
(GLAS) $R$
$J$
$J$
E RUSH

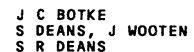

A DONACH
LEA, OADES, HARD R K KOWAOP,
(UCSB)
(SFLA)
(GLAS+EDIN) 


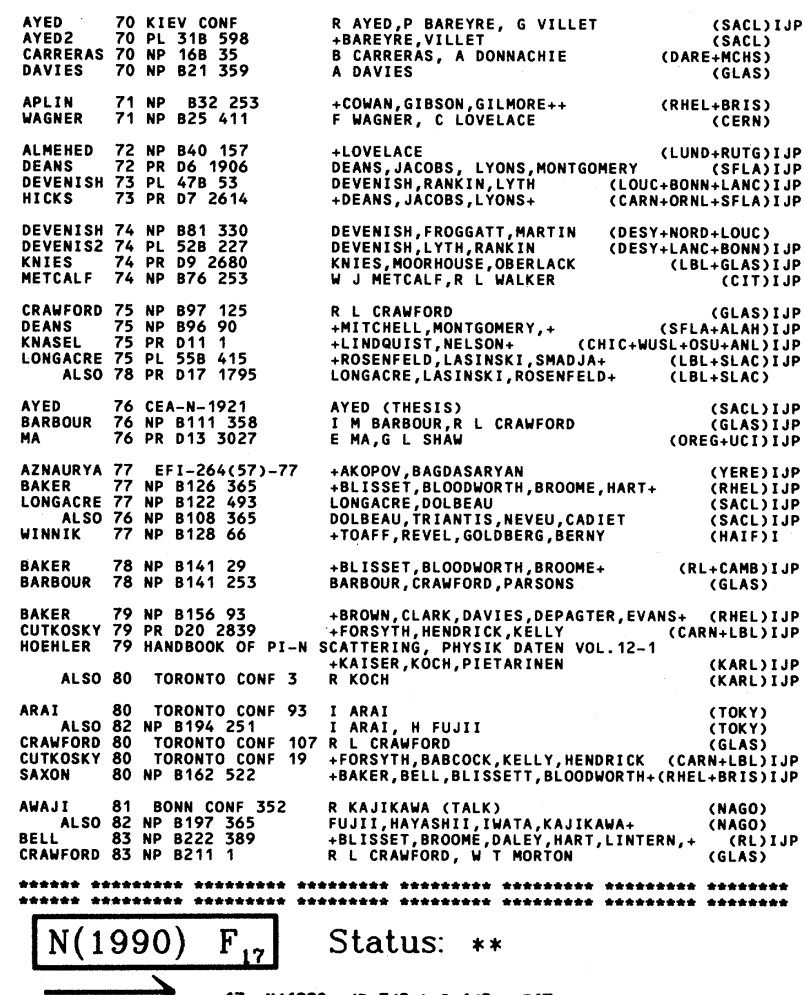

$17 W(1990, J P=7 / 2+) \quad 1=1 / 2 \quad F 17$

MOST OF THE RESULTS PUBL ISHED BEFORE 1975 ARE NOW
OBSOLETE AND HAVE BEEN OMITTED. THEY MAY BE FOUND IN
OUR OUR 1982 EDITION (PHYSICS LETTERS 1118 ). HOWEVER,

THE VARIOUS ANALYSES DO NOT AGREE VERY WELL WITH ONE ANOTHER.

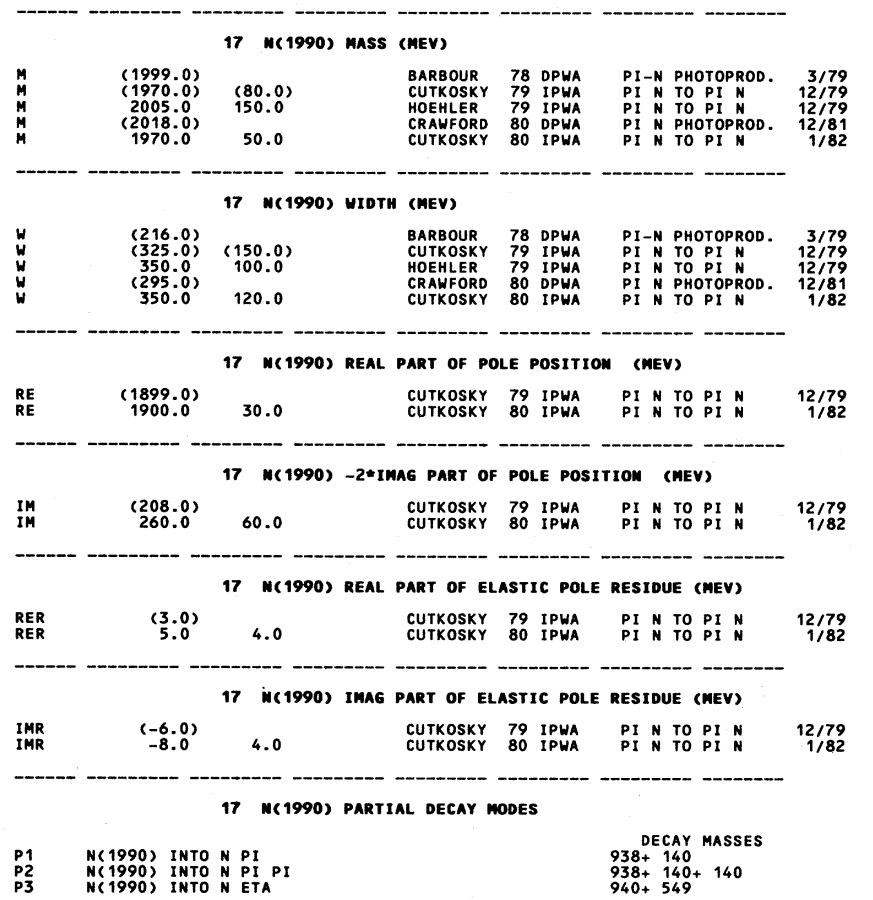

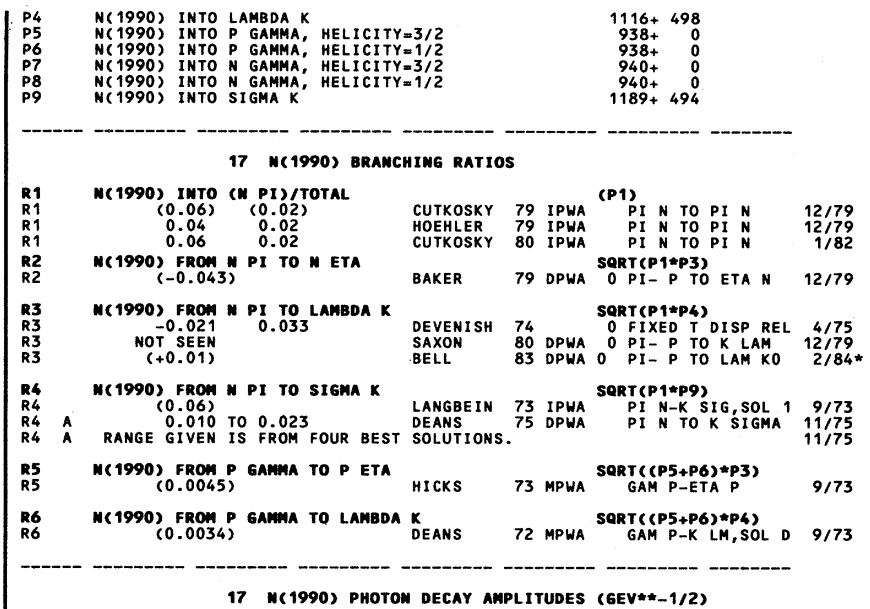

FOR DEFINITION OF GAMMA-NUCLEon deCAY AMPLITUdes, SEe Mini-

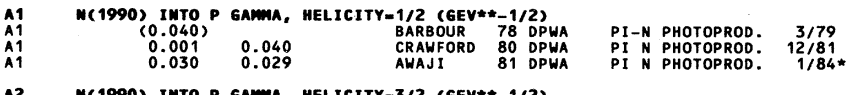

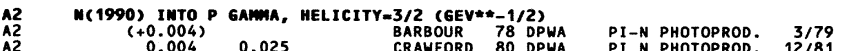

$\begin{array}{lccllll}\text { A2 } & (+0.004) & 0.025 & \text { BARBOUR } & 78 \text { DPWA } & \text { PI-N PHOTOPROD. } & 3 / 79 \\ \text { A2 } & 0.004 & 0.025 & \text { CRAWFOR } & 80 \text { DPWA } & \text { PII N PHOTOPROD: } & 12181 \\ \text { A2 } & 0.086 & 0.060 & \text { AHAJI } & 81 \text { DPWA } & \text { PI N PHOTOPROD. } & 1 / 84 *\end{array}$

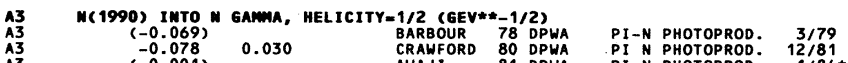

CRAWFORD 81 DPWA

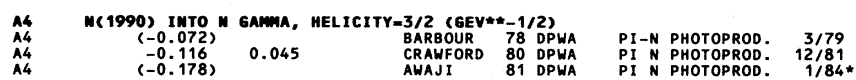

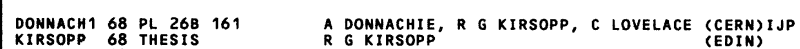

$\begin{array}{lllll}\text { DEANS } & 69 & \text { PR } & 185 & 1797 \\ \text { DEANS2 } & 69 & \text { PR } & 177 & 2623\end{array}$

$\begin{array}{llll}\text { LEA } & 69 & \text { PL } 298584 \\ \text { AYED } & 70 & \text { PL } 318598 \\ \text { APE } & 71 & \end{array}$

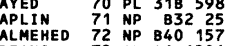

DEANS 72 PR D6 1906

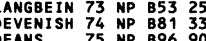

AYED 76 CEA-N-192

WINIIK 77 NP B128 66

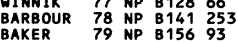

BARBOUR, RRA CFORD, PARSONS
+BROUN, CLARK, DAVIES, DEPAGTER, EVANS+ (GLAS)
(RHEL) IJP

WOEHLER 79 HANDBOOK OF PI-N SCAORSYYH, HENORICK, KELLY

$\begin{array}{lll}\text { ALSO } 80 \text { TORONTO CONF } 3 \text { R KOCH, KOCH, PIETARINEN } & \text { (KARL) IJP } \\ \text { (KARL)IJP }\end{array}$

CRAWFORD 80
COTKOSKY 80 TORONO CONF 107 R L CRAWFORD
TORONTO CONF 19 +FORSYTH, BABCOCK, KELLY, HENDRICK (CARN (GLAS) +BAKER, BELL, BLISSETT, BLOODORTH+(RHEL+BRIS)IJP

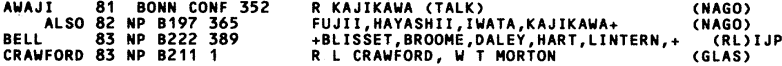

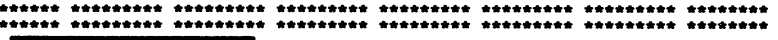 $\begin{array}{ll}\mathrm{N}(2000) & \mathrm{F}_{15}^{\prime \prime}\end{array}$ Status: **} OLDER RESULTS HAVE BEEN RETAINED SIMPLY BECAUSE
THERE IS LITTLE INFORMATION AT ALL ABOUT THIS STATE.

06 M(2000) MASS (MEV)

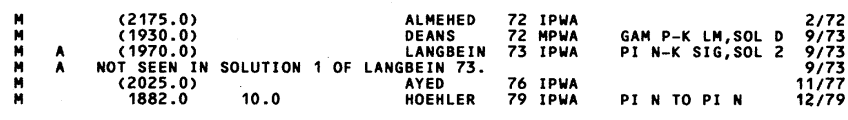


Baryons

$\mathrm{N}(2000), \mathrm{N}(2080)$

\section{Data Card Listings}

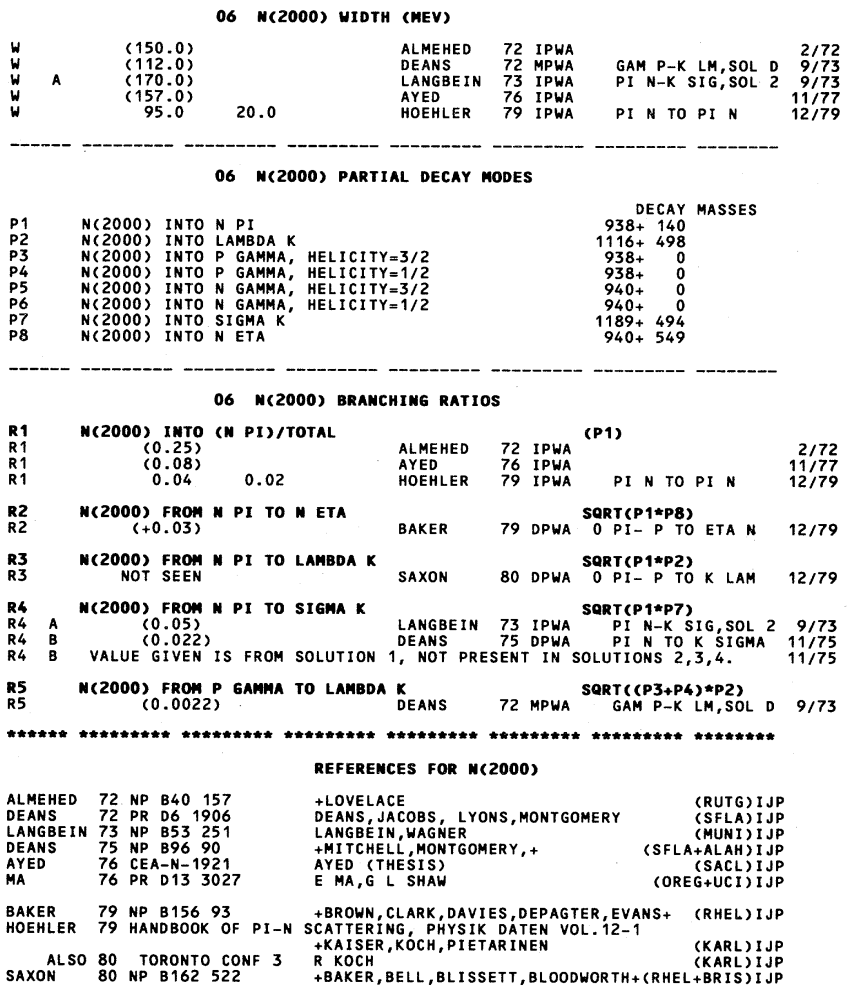

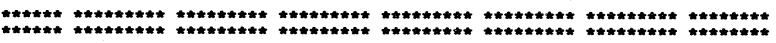

\begin{tabular}{|lll}
$\mathrm{N}(2080)$ & $\mathrm{D}_{13}^{\prime \prime}$
\end{tabular}$\quad$ Status: **

$16 \mathrm{M(2080,JP=3/2-)} I=1 / 2$ D $\cdots 13$

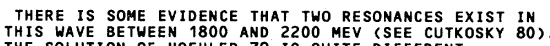

MOST OF THE RESULTS PUBLISHED BEFORE 1975 ARE NOW OBSOLETE AND HAVE
BEEN OMITTED THEY MAY BE FOUNO IN OUR 1982 EDITION CPHYSICS LETTERS

BEEN OMITTED THEY MAY BE FOUND IN OUR 1982 EDITION (PHYSICS LETTERS
111B). HOWEVER, ALL THE REFERENCES HAVE BEEN RETAINED.

\section{M(2080) MASS (MEV)}

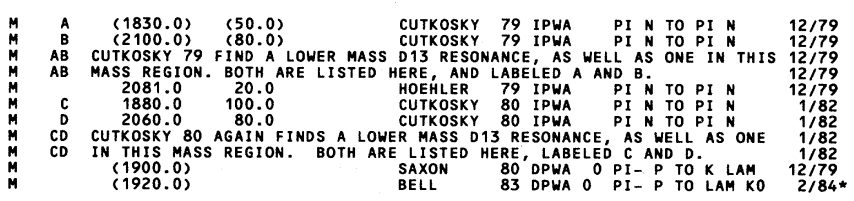

16 M(2080) WIDTH (MEV)

\begin{tabular}{|c|c|c|c|c|c|c|}
\hline W & $\begin{array}{l}(125.0) \\
(300.0) \\
265.0 \\
180.0 \\
300.0 \\
(240.0) \\
(320.0)\end{array}$ & 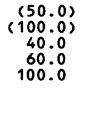 & $\begin{array}{l}\text { CUTKOSKY } \\
\text { CUTKOSKY } \\
\text { HOEHER } \\
\text { CUTKOSKY } \\
\text { CUTKOSKY } \\
\text { CUAXONKY } \\
\text { BAXLL } \\
\text { BELL }\end{array}$ & $\begin{array}{l}79 \text { IPWA } \\
79 \text { IPWA } \\
79 \text { IPWA } \\
80 \text { IPWA } \\
80 \text { IPWA } \\
80 \text { DPWA } \\
83 \text { DPWA } 0\end{array}$ & 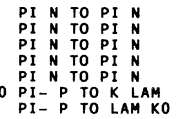 & \\
\hline
\end{tabular}

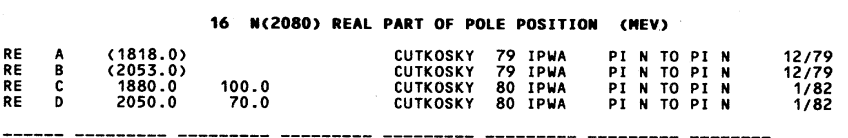

16 M(2080) -2* IMAG PART OF POLE POSITION (MEV)

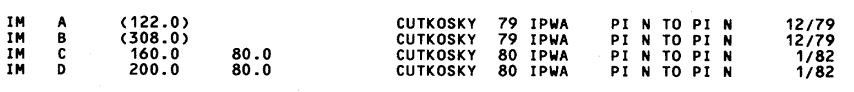

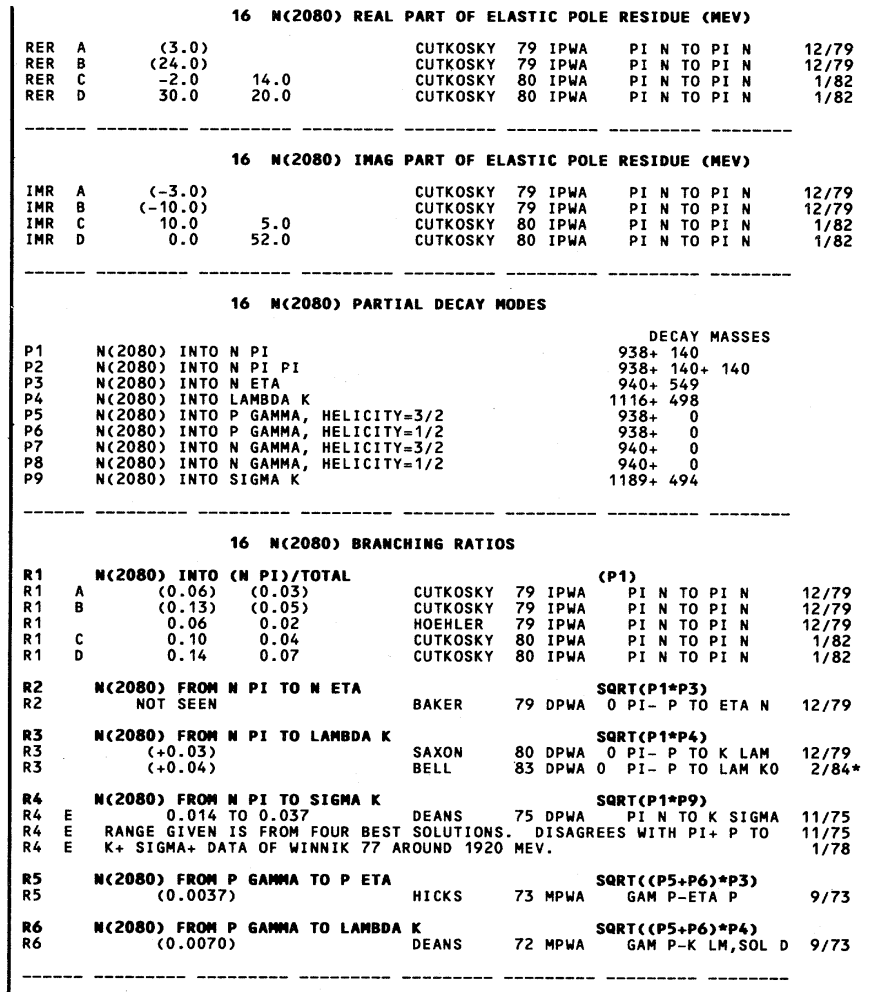

16 M(2080) PHOTON DECAY AMPLITUDES (GEV**-1/2)

EOR DEFINITION OF GAMMA-NUCLEON DECAY AMPLITUDES, SEE MINI-
REVIEU PRECED TNG THE BARYON LISTINGS.

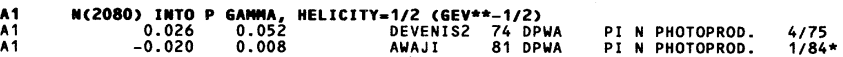

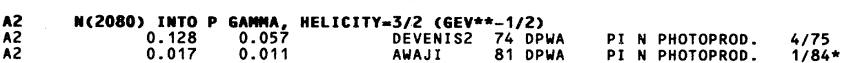

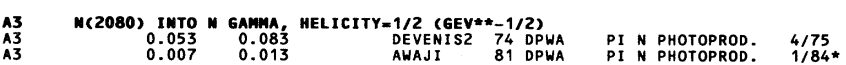

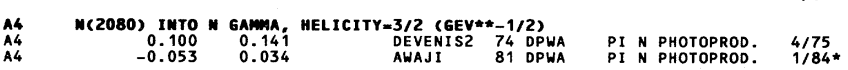

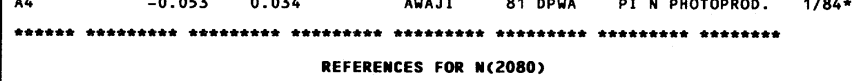

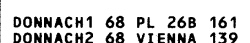

KIRSOPP 68 THESIS

LEA DONACHI 69 PL $29 \mathrm{~B} 584$

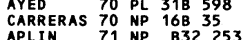

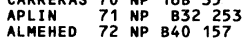

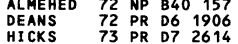

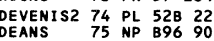

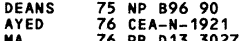

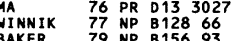

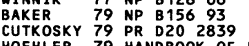

EMCES FOR M(2080)

A DONNACHIE, R G KIRSOPP, C LOVELACE (CERH) IJP
DONAACHIE RAPPORTEUR.S TALK
(GLAS)

$R$ G KIRSOPP

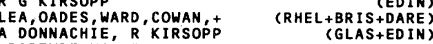

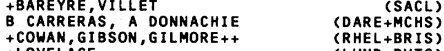

HOVELACE

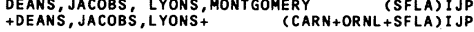

DEVENISH, LYTH, RANKIN
HITCHELL, MONTGOMERY,
MESTH

AYED (THESIS)

TOAF, REVEL, GOLDBERG, BERNY (OREG+UCI) IJ

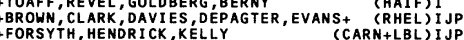

ALSO 80 TORONTO CONF 3 R KOCR, KOCH, PIETAR INEN N
(KARL) IJP
(KARL) IJP

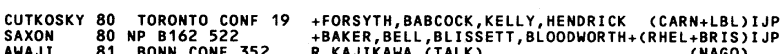

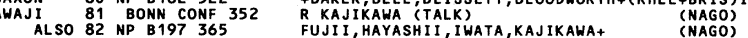

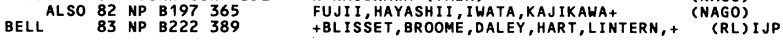

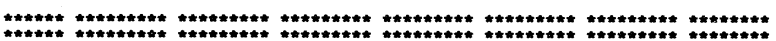




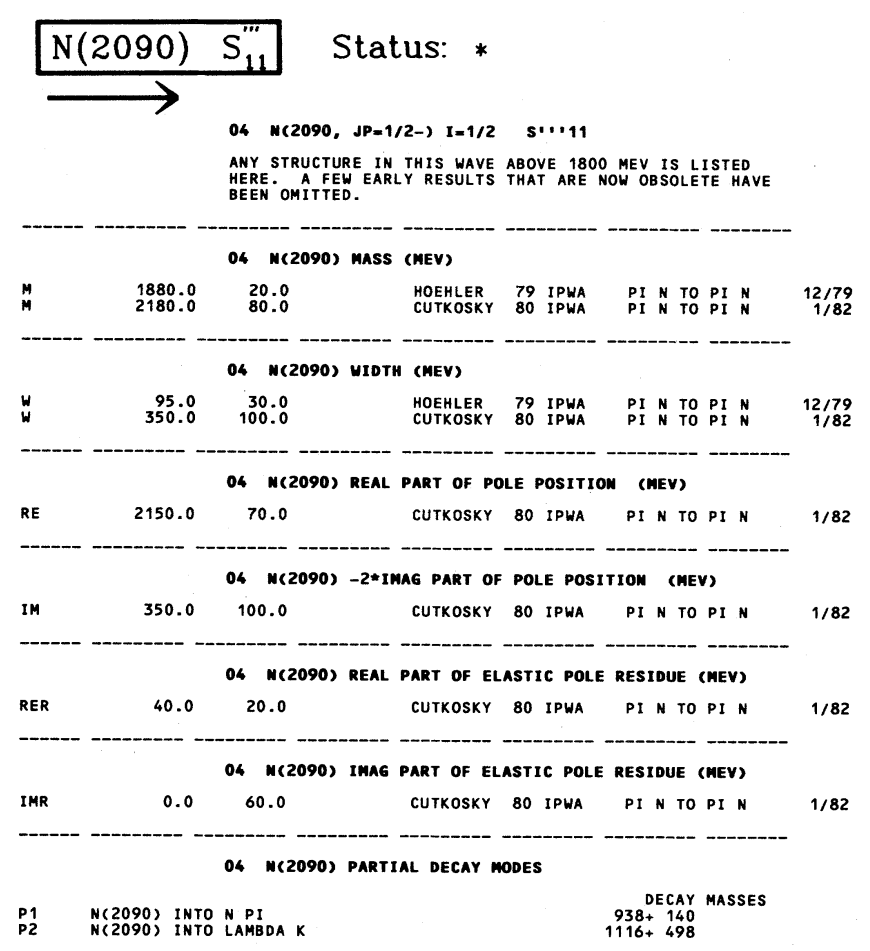

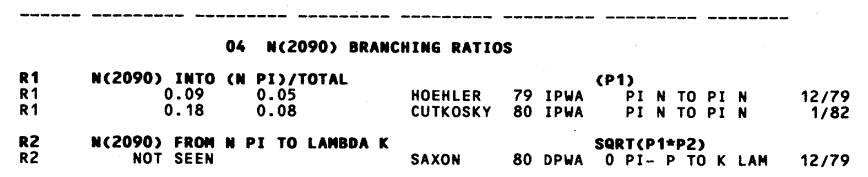

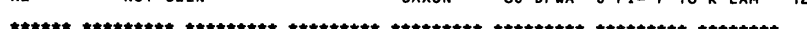

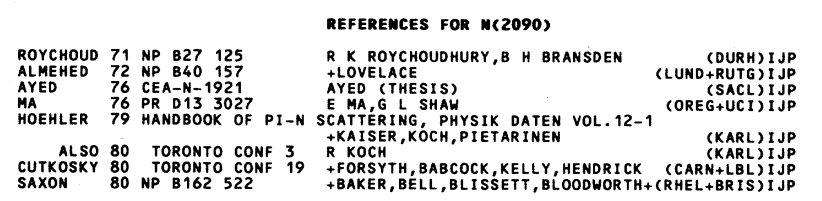

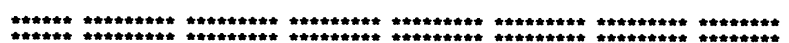

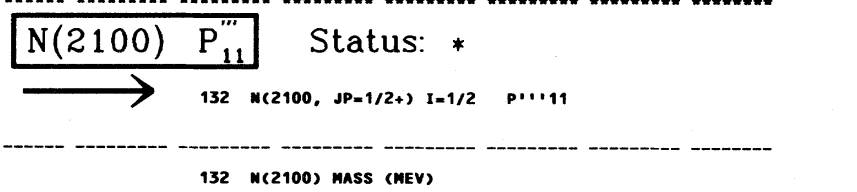

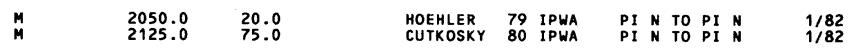

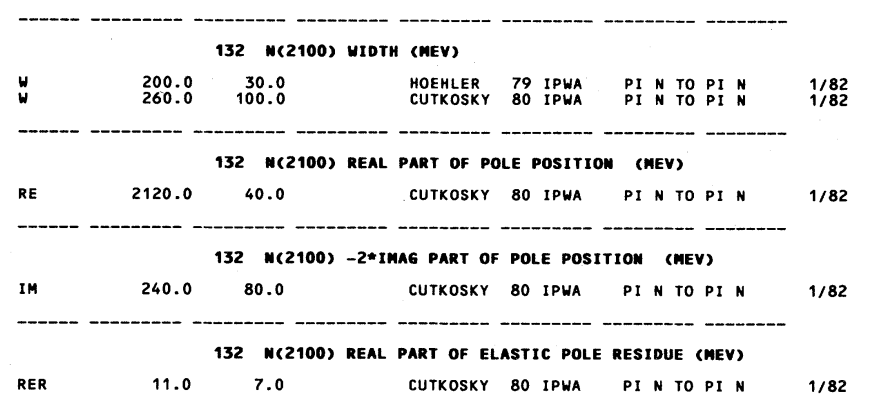

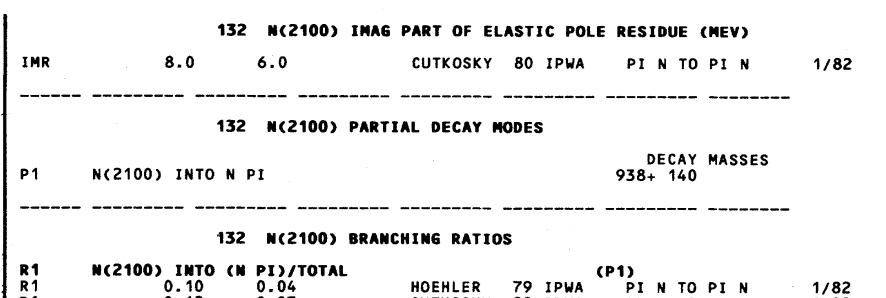

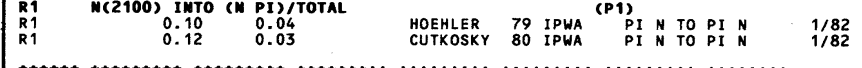
REFEREMCES FOR N(2100)

HOEHLER 79 HANOBOOK OF PI-N SCATTERING, PHYSSK DATEN VOL. 12-1

$\begin{array}{lll}\text { KOCH } 80 \text { TORONTO CONF } 3 \stackrel{\text { KAISER, KOCH, PIETARINEN }}{R} \text { KOCH } & \text { (KARL) IJP } \\ \text { (KARL)IJP }\end{array}$

CORKOSKY BO TORONO CONF 3 G R TORSYTH, BABCOCK, KELLY, HENDRICK (CARN+LBL)IJP

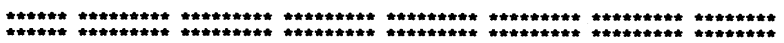

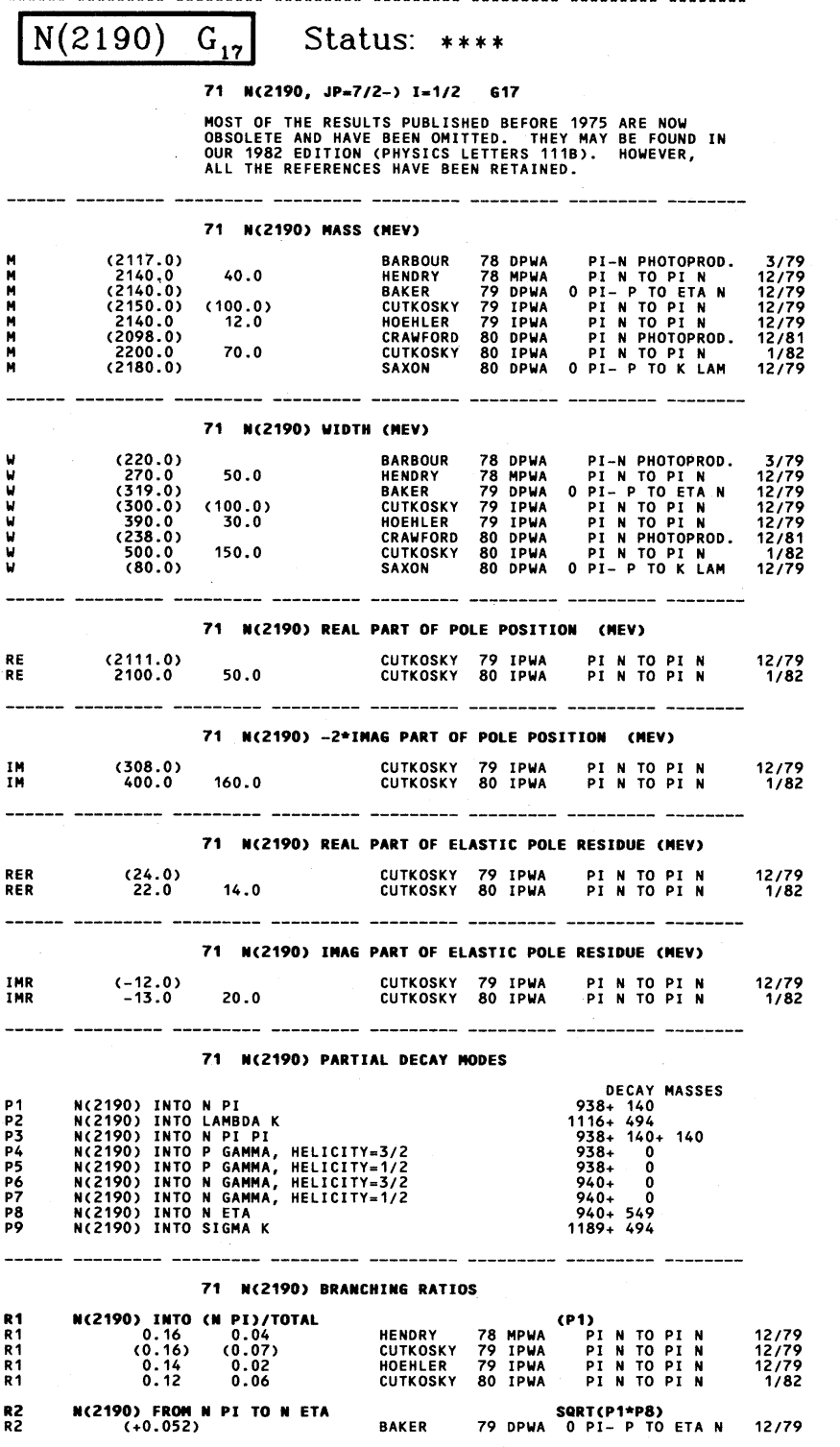


Baryons

Data Card Listings

$\mathrm{N}(2190), \mathrm{N}(2200), \mathrm{N}(2220)$

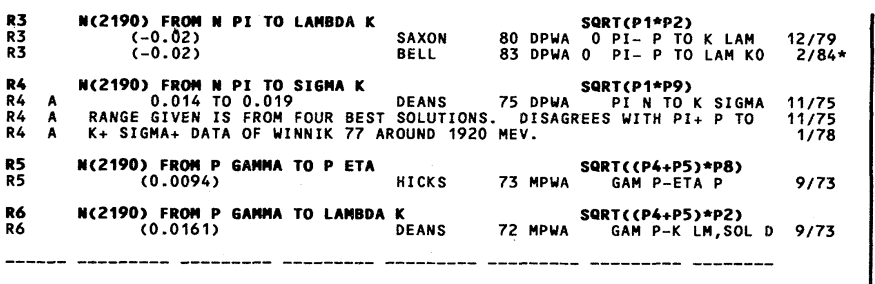

71 N(2190) PHOTON DECAY AMPLITUDES (GEV**-1/2)

FOR DEFINITION OF GAMMA-NUCLEON DECAY AMPLITUDES, SEE MINI-

A1 M(2190) INTO P GAMMA, HELICITY=1/2 (GEV**-1/2)

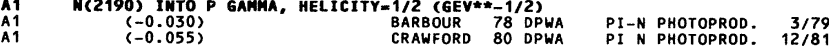

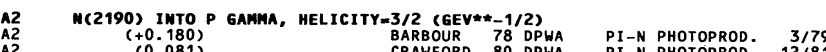

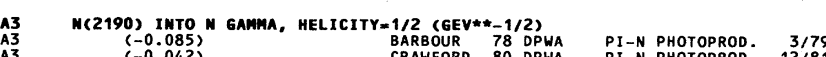

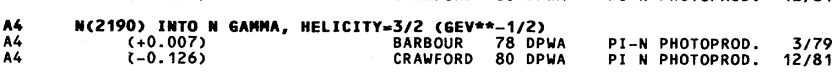

**********************************************************************

REFERENCES FOR M(2190)

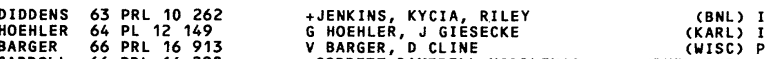

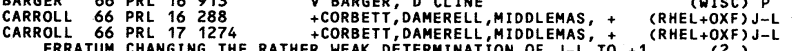

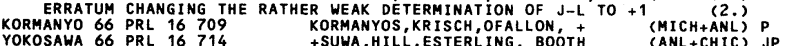

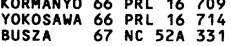

$\begin{array}{rllll}\text { DONNACH1 } & 68 & \text { PL } & 268 & 161 \\ \text { ALSO } & 68 & \text { VIENNA } & 139\end{array}$

\begin{tabular}{lll} 
ALSO 68 THESIS & 69 \\
\hline PL 298 & 58 \\
\hline
\end{tabular}

AYED $70 \mathrm{KIEV}$ CONF

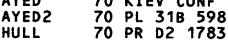

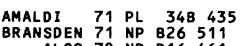

$\begin{array}{llll}\text { ALSO } 70 & \text { NP } & \text { B16 } & 461 \\ \text { ROYCHOUD } 71 & \text { NP } & \text { B27 } & 125 \\ 7 & \text { AMALO }\end{array}$

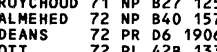

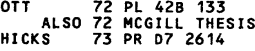

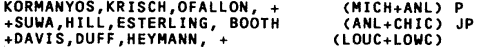

A DONNACHIE, R G KIRSOPP, C LOVELACE (CERN)IJP
DONNACHIE RAPPORTEUR.S TALK

DONNACHIE RAPPORTEUR.S TALK
$R$ KIRSOPP
(ELIAS)

R G KIRSOPP
LEA, OADES, WARD, COWAN, + (RHEL+BRIS+DARE)

+BLEER, BLIEDEN, COLLINS,
R AYED, BAREYRE, G VILLET
+BARYRE, VILLET, (BNL +CARN)
J HULL, R LEACOCK
(SACL) IJP
(SACL)

+BIANCASTELLI, BOS10,+

ROYCHOUDHURY, PERRIN, BRANSDEN

ROYCHOUDHURY, PERRIN, BRANSDEN
$R K$ ROYCHOUDHURY, B H BRANSDEN

DEANS, JACOBS LYONS MONTGOMERY (LUND+RUTG)IJP

TEANS, ACOBS, LYONS, MONTGOMERY (SFLA) IJP
+ TRISCHUK, VAVRA, RICHARDS, + (MCGI+STLO+ IOWA)IJP

J VAVRA , JAVA, LYONS + (CARN+ORNL+SFLA) IJP
+DEANS, JACOBS, LYONS

+ALSPECTOR, BOMBEROWITZ+
+MITHELL, MONTGOMERY, +

AYED (THESIS)

TOAF , REVEL, GOLDBERG, BERNY

BARBOUR, CRAWFORD, PARSONS

(SACL) IJJP
(OREG+UCI) IJJP

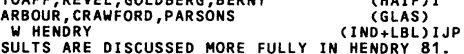

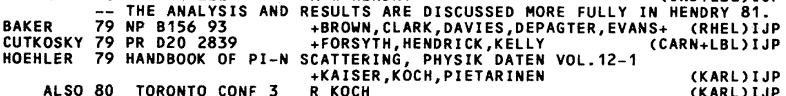

ALSO 80 TORONTO CONF 3 R KOCH

$\begin{array}{llll}\text { CRAWFORD } 80 & \text { TORONTO CONF } 107 \text { R L CRAWFORD } \\ \text { CUTKOSKY } 80 & \text { TORONTO CONF } 19 \text { +FORSYTH, BABCOCK, KELLY, HENDRICK (GLAS) } \\ \text { (CARN+LBL) }\end{array}$

CUTKOSKY 80 TORONTO CONF 19 +FORSYTH, BABCOCK, KELLY, HENDRICK (CARN+LBL) IJP
SAXON 80 NP B B 52 522
+BAKER, BELL,BLISSETT,BLOODORTH+(RHEL+BRIS) IJP

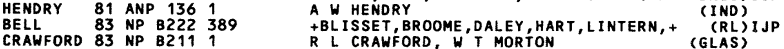

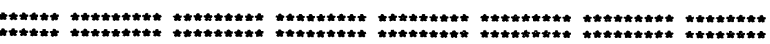

$\begin{array}{|ll|}N(2200) & D_{15}^{\prime \prime} \text { Status: ** } \\ \longrightarrow & 05 \text { N(2200, JP=5/2-) I=1/2 DN15 }\end{array}$

THE MASS IS NOT WELL DETERMINED. A FEW EARLY
RESULTS HAVE BEEN OMITTED.

\begin{tabular}{|c|c|c|c|c|c|c|}
\hline \multirow[b]{2}{*}{$M$} & \multicolumn{2}{|r|}{05 m(2200) MASS } & \multicolumn{3}{|l|}{ (MEV) } & \multirow[b]{2}{*}{$\begin{array}{r}12 / 79 \\
1 / 82 \\
12 / 79 \\
2 / 84\end{array}$} \\
\hline & $\begin{array}{r}2228.0 \\
2180.0 \\
(1920.0) \\
(1900.0)\end{array}$ & $\begin{array}{l}30.0 \\
80.0\end{array}$ & $\begin{array}{l}\text { HOEHLER } \\
\text { CUTKOSKY } \\
\text { SAXON } \\
\text { BELL }\end{array}$ & $\begin{array}{l}79 \text { IPWA } \\
30 \text { IPWA } \\
80 \text { DPWA } \\
83 \text { DPWA } 0\end{array}$ & 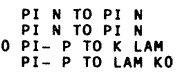 & \\
\hline & & $05 \mathrm{M}(2200)$ MIDTH & H (MEV) & & & \\
\hline$w$ & $\begin{array}{l}310.0 \\
400.0 \\
(2200.0) \\
(130.0)\end{array}$ & $\begin{array}{r}50.0 \\
100.0\end{array}$ & $\begin{array}{l}\text { HOEHLER } \\
\text { CUTKOSKY } \\
\text { SAXON } \\
\text { BELL }\end{array}$ & $\begin{array}{l}79 \text { IPWA } \\
80 \text { IPHA } \\
80 \text { DPHA } \\
83 \text { DPWA } 0\end{array}$ & 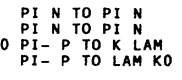 & $\begin{array}{c}12 / 79 \\
1 / 82 \\
12 / 79 \\
2 / 84 *\end{array}$ \\
\hline
\end{tabular}

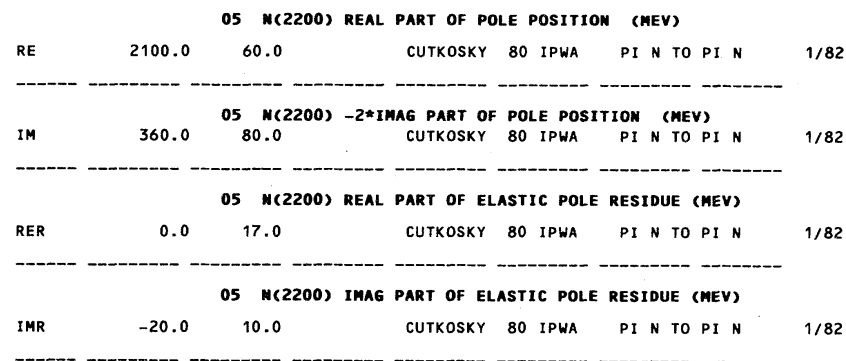

05 N(2200) PARTIAL deCAY MOdES

$\begin{array}{llc} & & \text { DECAY MASSES } \\ \text { P1 } & \text { N(2200) INTO N PI } & 938+140 \\ \text { P2 } & \text { N(2200) INTO N ETA } & 940+549 \\ \text { P3 } & \text { N(2200) INTO LAMBDA K } & 1116+498\end{array}$

O5 M(2200) BRAMCHING RATIOS

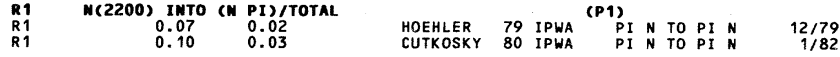

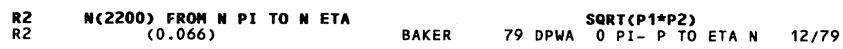

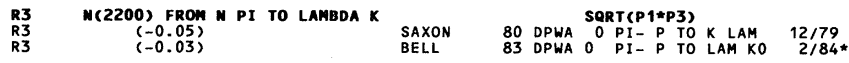

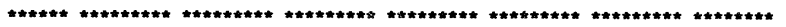

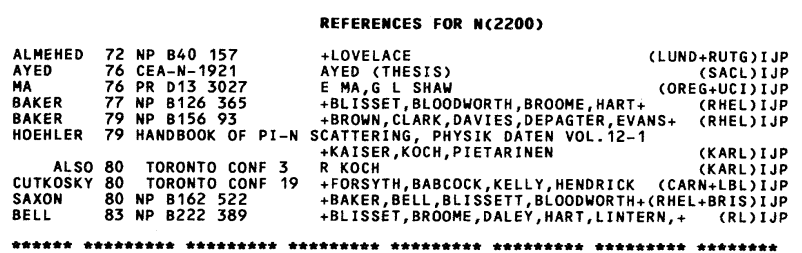

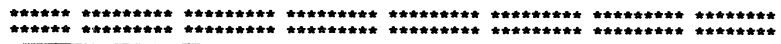

$\mathrm{N}(2220) \quad \mathrm{H}_{19} \quad$ Status: $* * * *$

$90 \mathrm{~N}(2220, \mathrm{JP}=9 / 2+) \quad \mathrm{I}=1 / 2 \quad \mathrm{H} 19$

MOST OF THE RESULTS PUBL ISHED BEFORE 1975 ARE NOW OUR 1982 EDITION (PHYSICS LETTERS 111B) MAY BE FOUND IN ALL THE REFERENCES HAVE BEEN RETAINED.

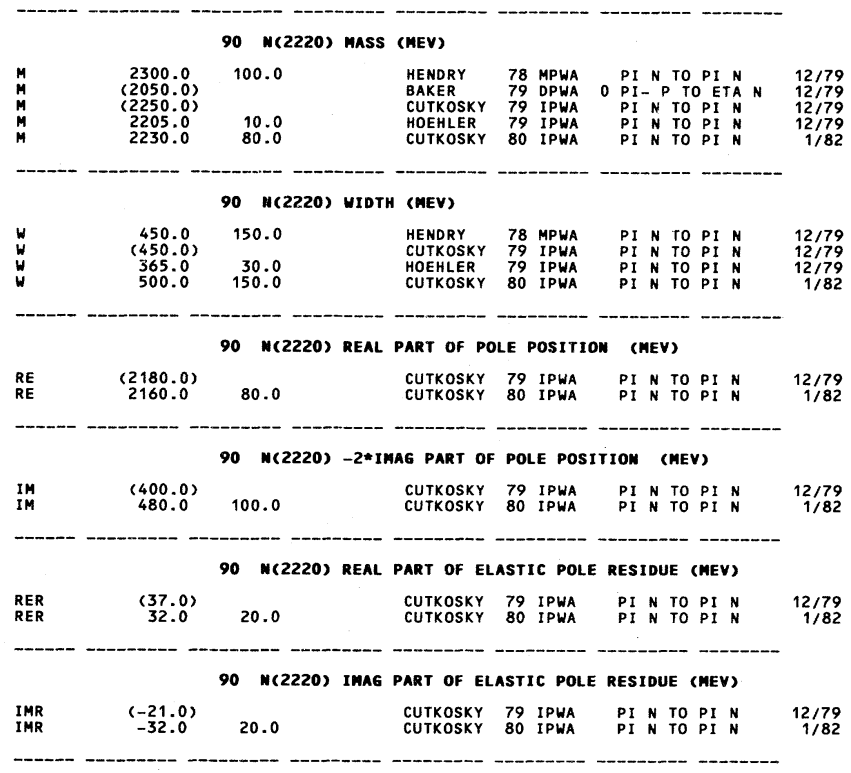


90 n(2220) PARTIAL DECAY MODES

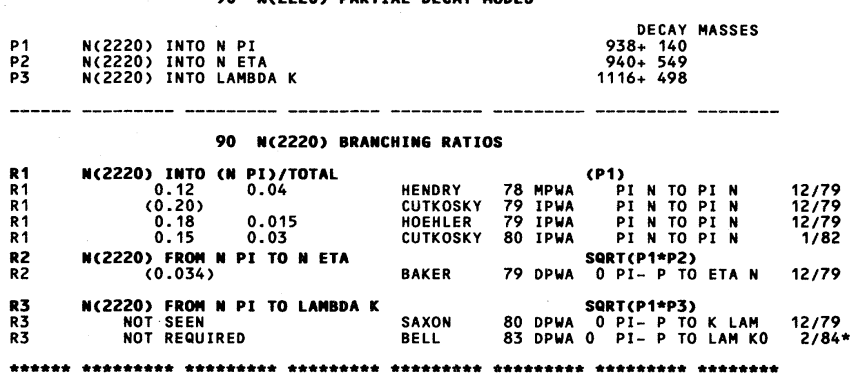

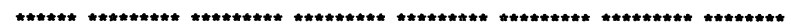

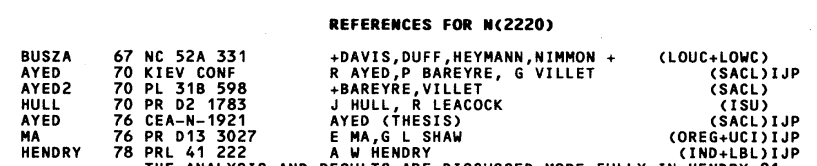

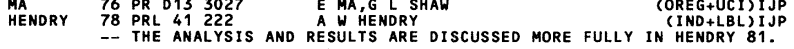

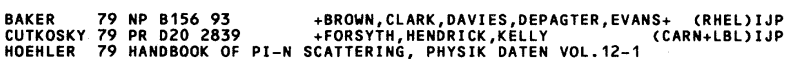

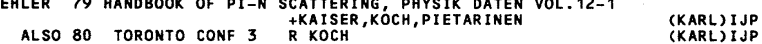

$\begin{array}{llll}\text { CUTKOSKY } 80 & \text { TORONTO CONF } 19 & \text { +FORSYTH, BABCOCK, KELLY, HENDRICK (CARN+LBL) IJP } \\ \text { SAXON } 80 \text { NP B } 162 \text { 522 } & \text { +BAKER, BELL, BLISSETT, BLOODWORTH+(RHEL+BRIS)IJP }\end{array}$

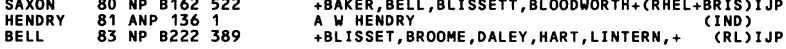

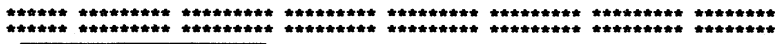

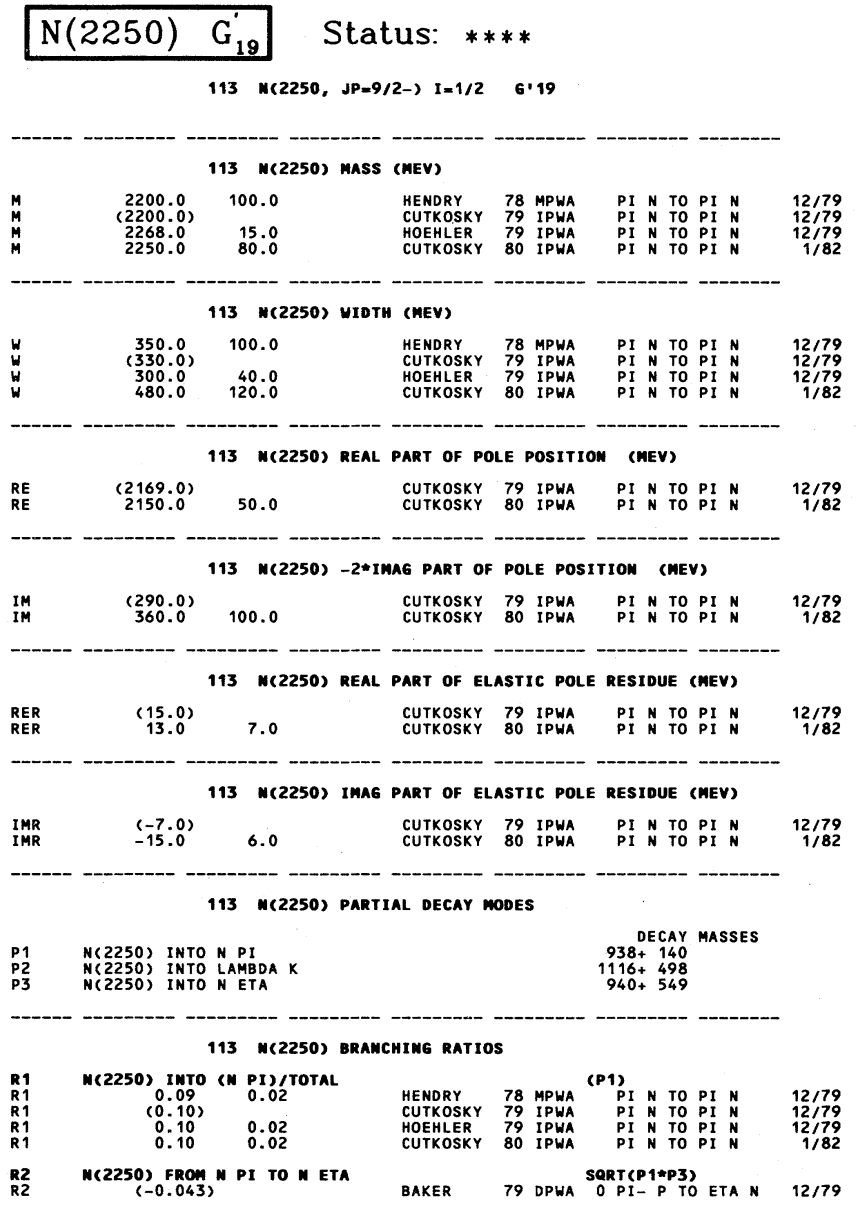

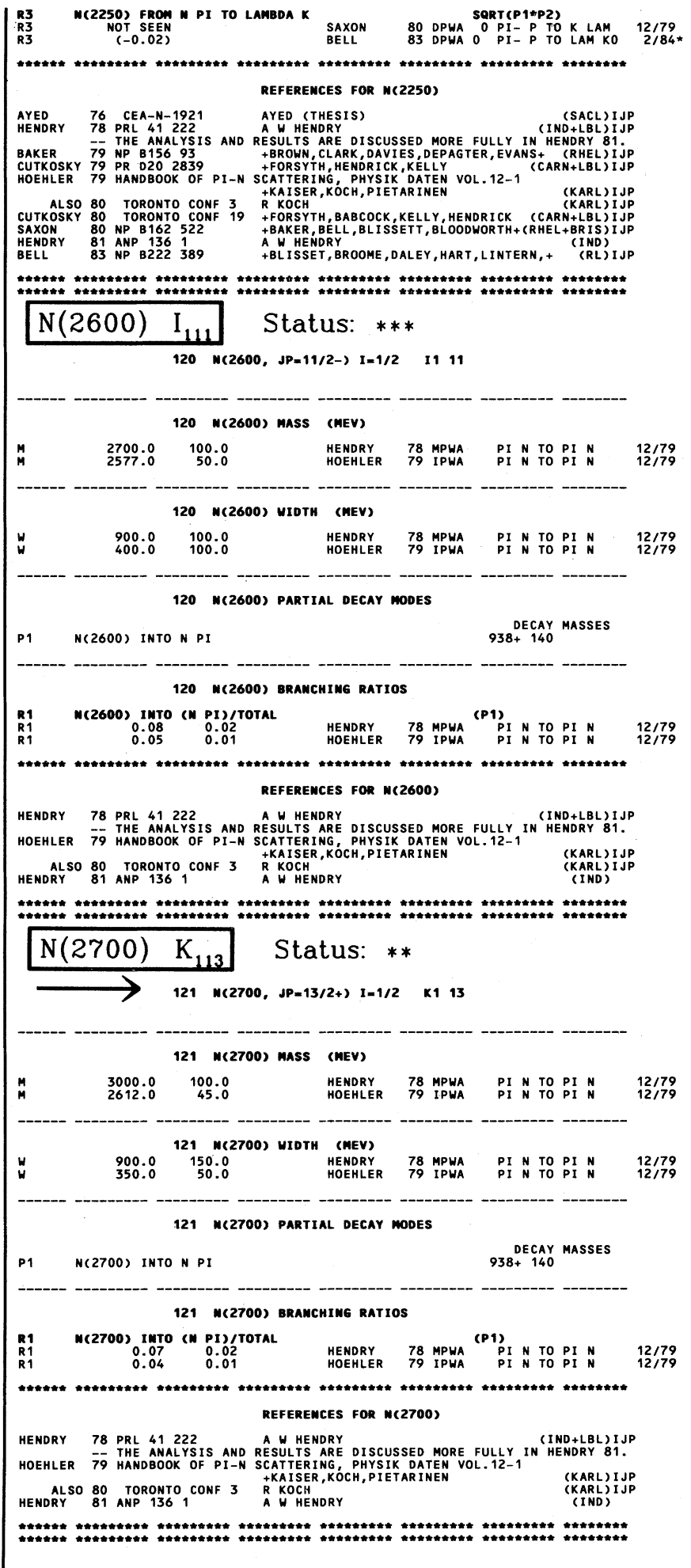




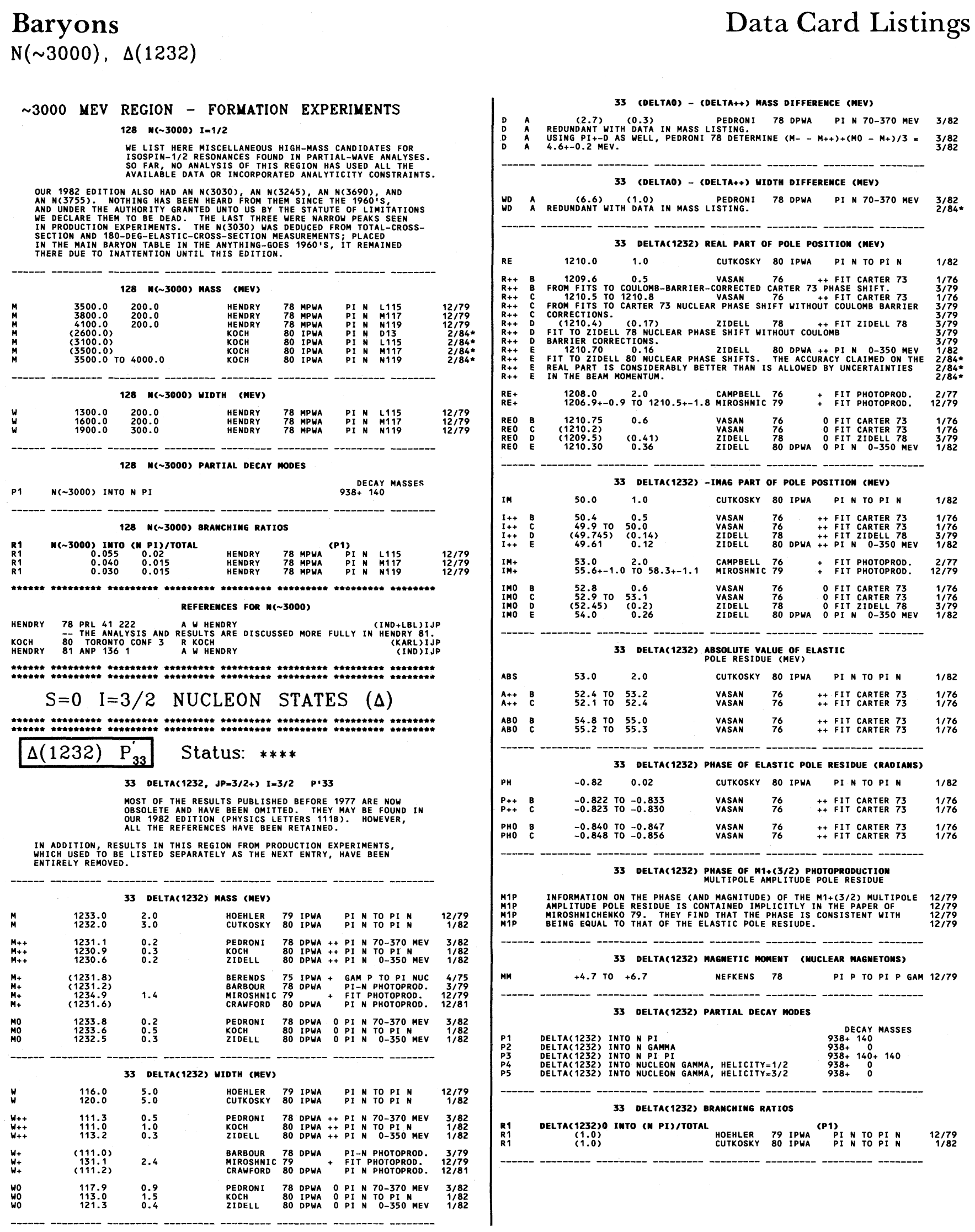



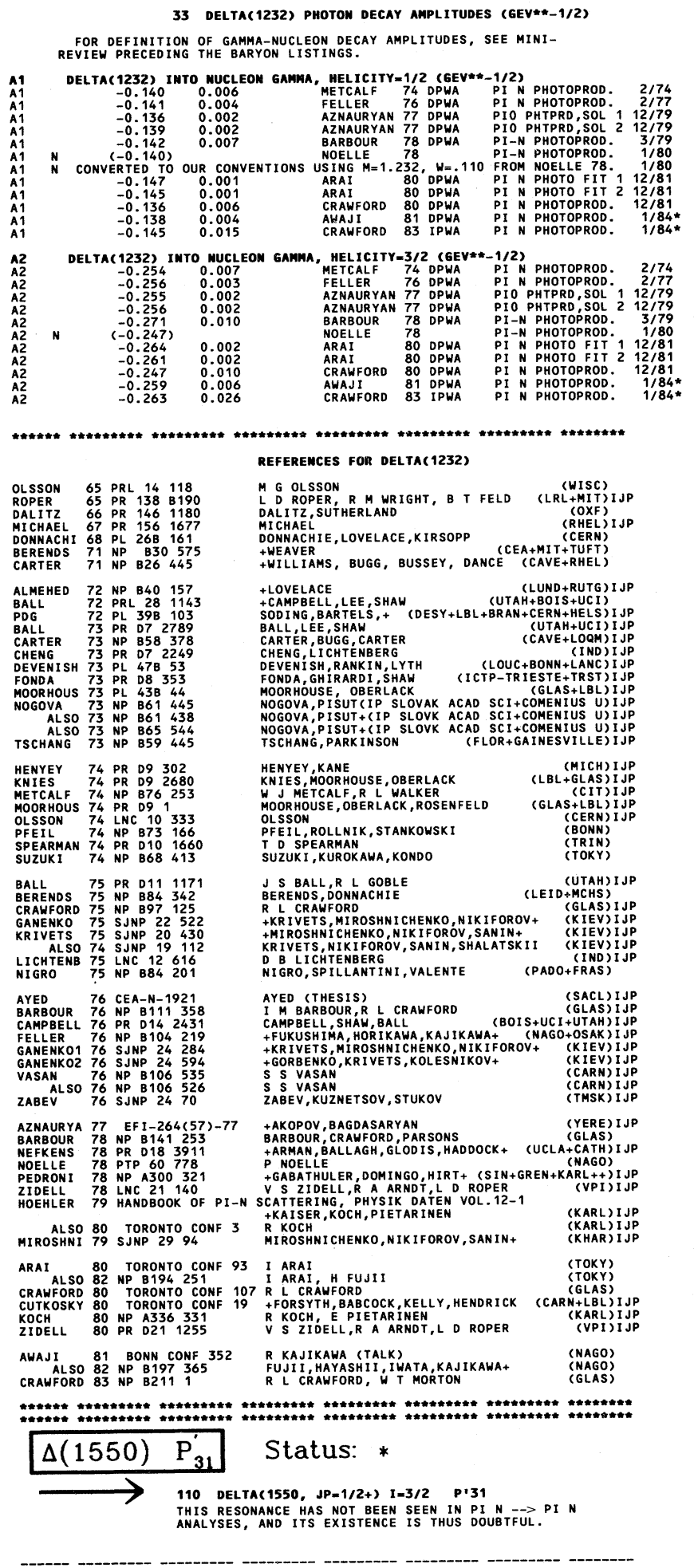

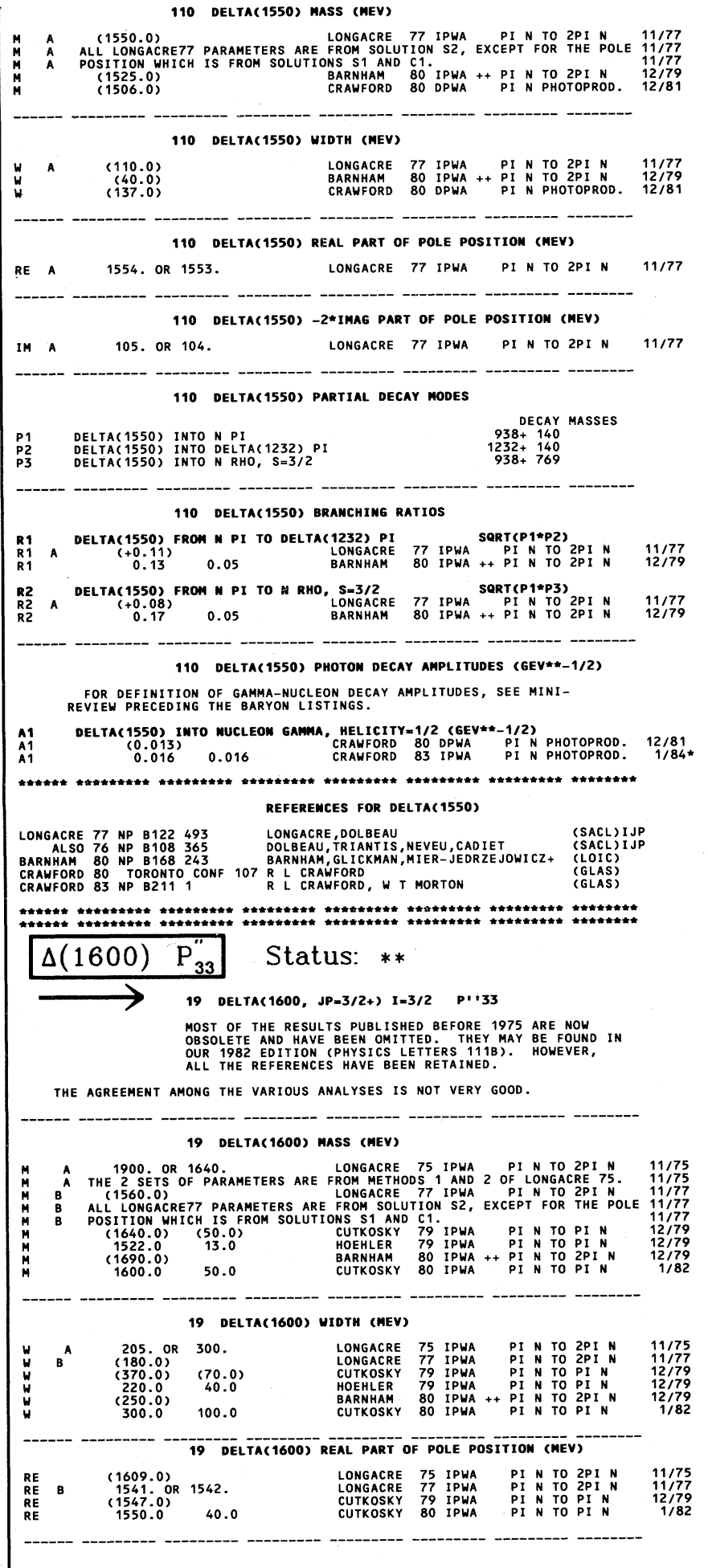


Baryons

$\Delta(1600), \Delta(1620)$

\section{Data Card Listings}

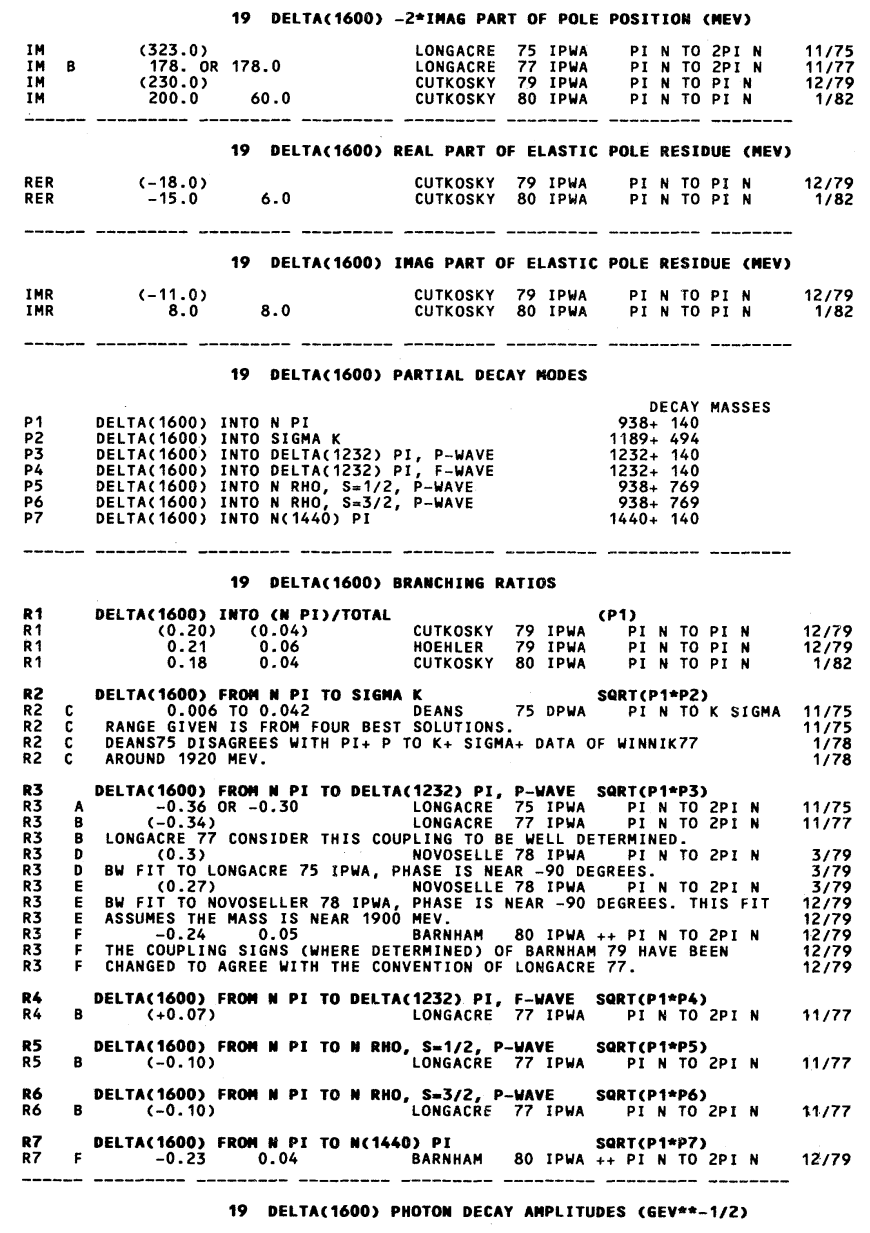

FOR DEFINITION OF GAMMA-NUCLEON DECAY AMPLITUDES, SEE MINI-
REVIEW PRECEDING THE BARYON LISTINGS.

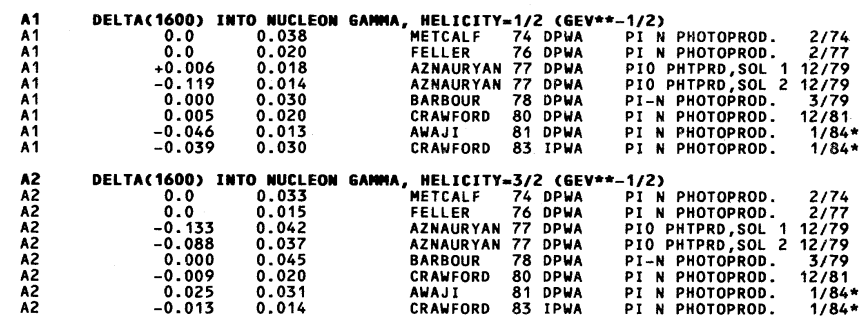

\begin{tabular}{|l} 
CUTKOSKY 79 \\
HOE 0202839 \\
HOEHLR 79 HANDBOOK OF PI-N SCATTERING, HENDRICK, KELLY PHYSIK DATEN VOL. 12-1 (CARN+LBL)IJP
\end{tabular}

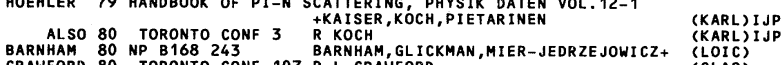

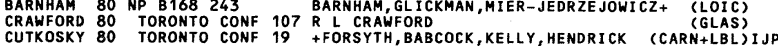
$\begin{array}{llll}\text { AWAJI } 81 & \text { BONN CONF } 352 & \text { R KAJIKAWA (TALK) } & \text { (NAGO) } \\ \text { ALSO 82 NP B197 } 365 & \text { FUIII, HAYASHII, IWATA, KAJIKAWA+ } & \text { (NAGO) } \\ \text { CRAWFORD } 83 \text { NP B211 } 1 & \text { RL CRAWFORD, W T MORTON } & \text { (GLAS) }\end{array}$

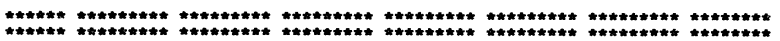
\begin{tabular}{|lll}
$\Delta(1620)$ & $S_{31}$ & Status: $* * * *$
\end{tabular} 82 DELTA(1620, JP=1/2-) I=3/2 S'31 MOST OF THE RESULTS PUBLISHED BEFORE 1975 ARE NOW
OBSOLETE AND HAVE BEEN OMITTED. THEY MAY BE FOUND IN
OUR 1982 EDITION (PHYSICS LETTERS 11 B H HOWEVER OUR 1982 EDITION (PHYSICS LETTERS 111B). HOWEVER,

82 DELTA(1620) MASS (MEV)

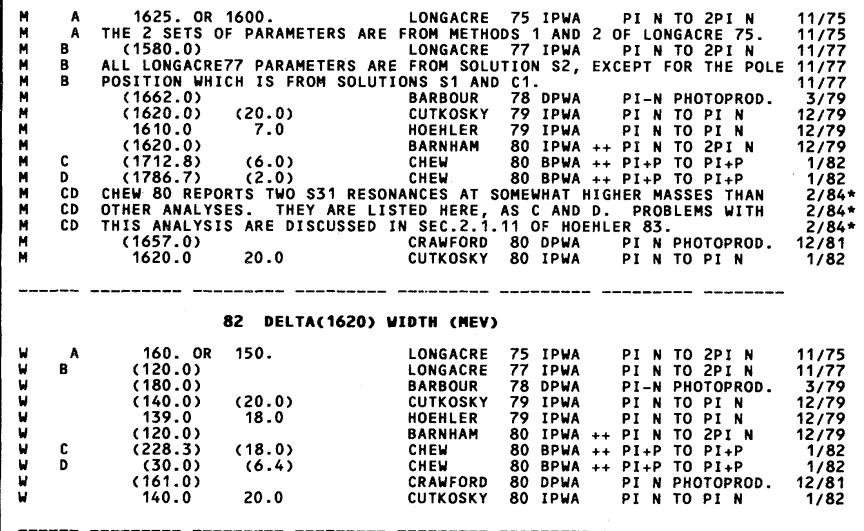

82 DELTA(1620) REAL PART OF POLE POSITION (MEV)

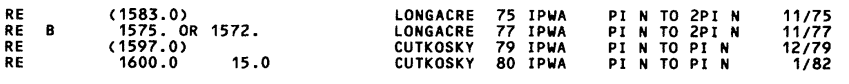
82 DELTA(1620) -2*IMAG PART OF POLE POSITION (MEV)

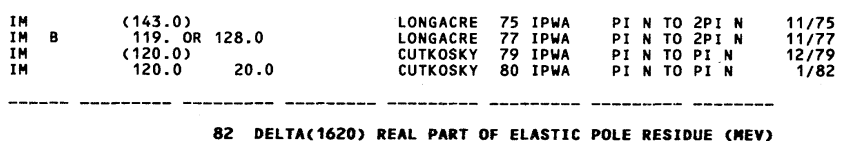

\begin{tabular}{|c|c|c|c|c|c|c|c|}
\hline $\begin{array}{l}\text { RER } \\
\text { RER }\end{array}$ & $\begin{array}{c}(-6.0) \\
-5.0\end{array}$ & 5.0 & $\begin{array}{l}\text { CUTKOSKY } \\
\text { CUTKOSKY }\end{array}$ & & $\begin{array}{l}\text { IPWA } \\
\text { IPWA }\end{array}$ & $\begin{array}{ll}\text { PI } & \text { TO } \\
\text { PI } & \text { TO }\end{array}$ & $\begin{array}{r}12 / 79 \\
1 / 82\end{array}$ \\
\hline
\end{tabular}

\begin{tabular}{|c|c|c|c|c|c|c|}
\hline & & 82 DELTA(1620) & IMAG PART O & F ELASTIC & POLE RESIDUE (MEV) & \\
\hline $\begin{array}{l}\text { IMR } \\
\text { IMR } \\
\text { IMR } \\
\end{array}$ & $\begin{array}{l}(-15.0) \\
-14.0\end{array}$ & 3.0 & $\begin{array}{l}\text { CUTKOSKY } \\
\text { CUTKOSKY }\end{array}$ & $\begin{array}{l}79 \text { IPWA } \\
80 \text { IPWA }\end{array}$ & $\begin{array}{lllll}P I & N & \text { To } & \text { PI } & N \\
\text { PI } & N & \text { TO } & \text { PI } & N\end{array}$ & $\begin{array}{r}12 / 79 \\
1 / 82\end{array}$ \\
\hline
\end{tabular}

82 DELtTa(1620) PARTial deECAY MODES

DELTA(1620) INTO N PI DECAY MASSES

DELTAC 1620) INTO N PI PI GAMMA, HELICITY $=1 / 2$

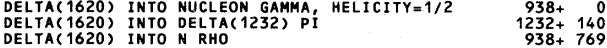
$\begin{array}{ll} & \\ \text { DELTA(1620) INTO N RHO, S=1/2, S-WAVE } & 938+769 \\ \text { DELTA(1620) INTO N RHO; S=3/2, D-WAVE } & 938+769 \\ \text { DELTA } 1620) \text { INTO N }(1440) \text { PI } & 1440+140\end{array}$ DELTA(1620) INTO N(1440) PI $1440+140$

82 Delta(1620) BRAMCHIMG Ratios DELTA(1620) INTO (N PI)/TOTAL
$\begin{gathered}(0.25) \\ 0.35\end{gathered}(0.04)$
$(0.06)$

$(0.60)$
$(0.36)$
0.25 $\quad 0.03$

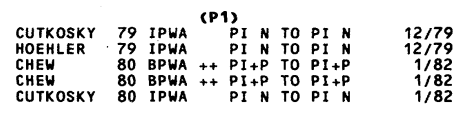
(LBL+SLAC)IJP $(L B L+S L A C)$ ( (SACL) I JP
(NAGO+OSAK) IJP (YERE) IJP
(SACL) IJP
(SACL)IJP .... 


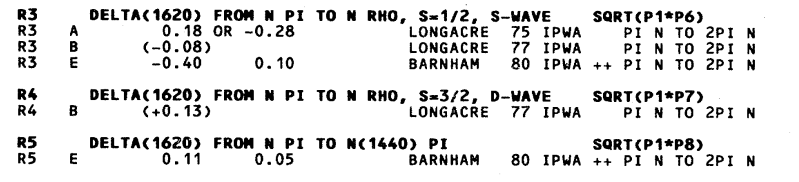

82 DELTA(1620) PHOTON DECAY AMPLITUDES (GEV**-1/2) FOR DEFINITION OF GAMMA-NUCLEON DECAY AMPLITUDES, SEE MINI-
REVIEW PRECEDING THE BARYON LISTINGS.

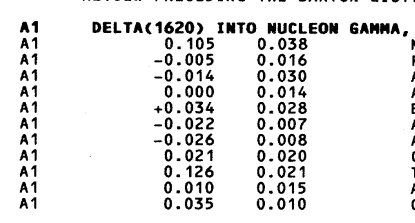

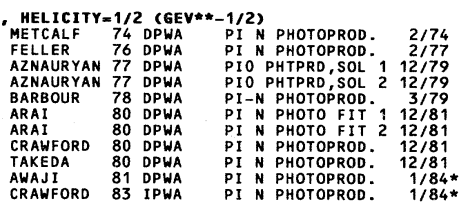

\section{$* * * * * * * * * * * * * * * * * * * * * * * * * * * * * * * * * * * * * * * * * * * * * * * *$
REFERENCES FOR DELTA(1620)}

$\begin{array}{llll}\text { CARRUTHE } 60 & \text { PRL } 4303 & \text { P CARRUTHERS } \\ \text { DEVLIN } 62 \text { PR } 125690 & \text { T J DEVLIN, B J MOYER, V PEREZ-MENDEZ (CORN) I }\end{array}$

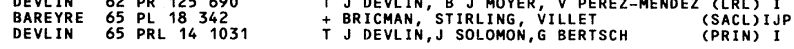

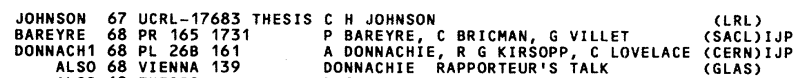
$\begin{array}{llll}\text { ALSO } & 68 & \text { VIENNA } & 13 \\ \text { ALSO } & 68 & \text { THESIS }\end{array}$ DONNACHI 69 NP 108433

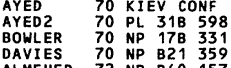
$\begin{array}{lllll}\text { AAVIES } & 70 & \text { NP } & 821 & 359 \\ \text { ALMEHED } & 72 & \text { NP } & 840 & 157 \\ \text { DEVENISH } 73 & \text { PL } & 478 & 53\end{array}$

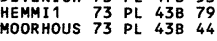
$\begin{array}{lllll}\text { DEVENIS2 } & 74 & \text { PL } & 528 & 227 \\ \text { KNIES } & 74 & \text { PR } & \text { D9 } 2680\end{array}$ $\begin{array}{lllll}\text { MNIES } & 74 & \text { PR } & 59 & 2680 \\ \text { METCAL } 74 & \text { NPP } & 876 & 253\end{array}$

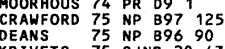

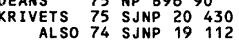

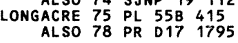
$\begin{array}{llll}\text { AYED } & 76 & \text { CEA-N-1921 } \\ \text { BARBOUR } & 76 & \text { NP B } 111 & 358 \\ \text { FELLER } & 76 & \text { NP } & \text { B } 104 \\ 219\end{array}$

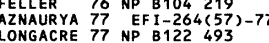
ALSO 76 NP B108 365 CUTKOSKY 79 PR D20 2839
HOEHLER 79 HANDBOOK OF PI-N SCAT ALSO 80 TORONTO CONF 3 R KOCH DONNACHIE RAPPORTEUR'S TALK
RIRSOPP
(EDIN) A DONNACHIE, R KIRSOPP
R AYED, BAREYRE, G VILLET (GLAS+EDIN)
+BAREYRE, VIRLET
(SACL)IJP +CASHMORE
A DAVIES
+ DOVELACE A DAVIES
+LOVELACE
DEVENISH, RANKIN , LYTH (LUND+RUTG) IJP
(LOUC+BONN+LANC)I JP
(KYOT (GLAS+LBL)IJP DEVENISH, LYTH, RANKIN
KNIES, MOORHOUSE, OBERLACK
(DESY+LANC+BONN) IJP
(LBL+GLAS)IJP WJ METCALF, R L WALKER MOORHOUSE, OBERLACK, ROSENFELD (GLAS+LBL) IJP
(GLAS) IJP +MITCHELL, MONTGOMERY,
+ MIROSHNI CHENKO, NIKIFOROV, SANIN + (SFLA+ALAH)IJP
(KIEV) IJP KRIVETS,NIKI FOROV, SANIN, SHALATSK II (KIEV) IJP
ROSENFELD, LASINSKI, SMAD JA+
RONGACLAC)IJP$$
(S A C L) \text { IJP }
$$
AKOPOV, BAGDASARYAN

LONGACRE, DOLBEAU
DOLBEU, TRIANT IS, NEVEU, CADIET ORSYTH, HENDRICK, KELLY
OR

MGO+OSAKIIJP (SACL) IJP
(SACL) IJP

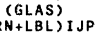
(KARL) IJP
(KARL) IJPP

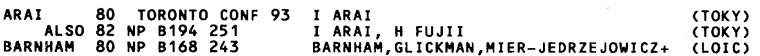
CHEW 80 TORONTO CONF 123 D M CHEW
CRAWFORD 80 TORONTO CONF $107 R$ L CRAWFORD
(GLAL) IJP CUTKOSKY 80 TORONTO CONF 19 +FORSYTH, BABCOCK, KELLY, HENDRICK (CARN+LL) IJP
TAKEDA 80 NP B 16817 $\begin{array}{lll}\text { AWAJI } 81 \text { BONN CONF } 352 & \text { R KAIIKAWA (TAL), IKEDA, WWASAKI+ } & \text { (NAY) } \\ \text { ALSO } 82 \text { NP B197 } 365 & \text { FUJII, HAYASHI, IWATA, KAJIKAWA+ } & \text { (NAGO) }\end{array}$

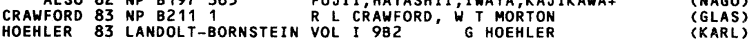

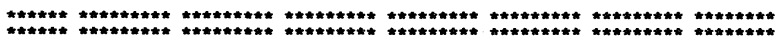

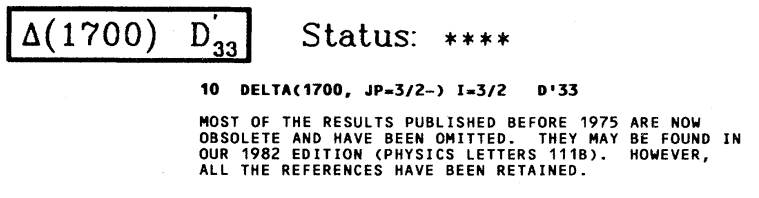

10 DELTA(1700) MASS (MEV)

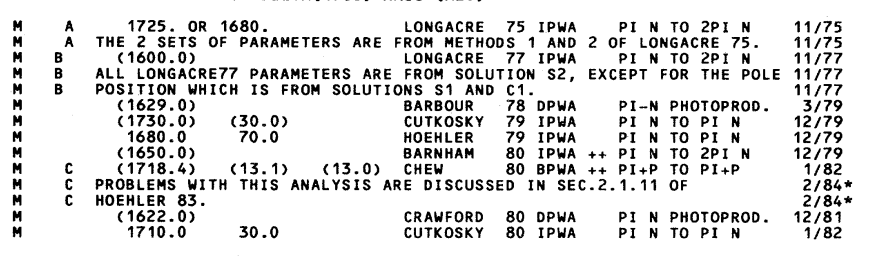

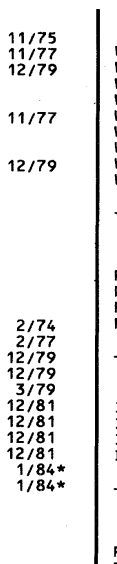
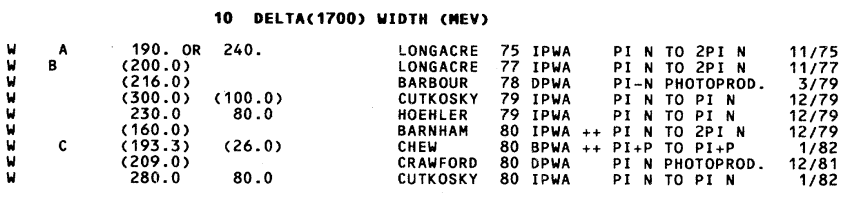

10 DELTA(1700) REAL PART OF POLE POSITION (MEV)

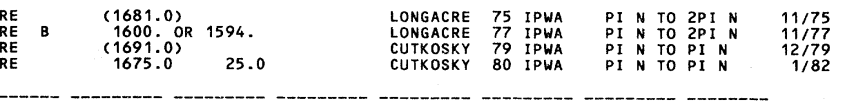

10 DELTA(1700) -2*IMAG PART OF POLE POSITION (MEV

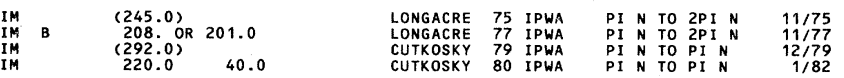

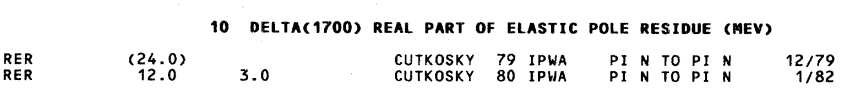

\begin{tabular}{|c|c|c|c|c|c|c|}
\hline & & 10 & DELTA(1700) & IMAG PART & OF ELASTIC & POLE RESIDUE (MEV) \\
\hline $\begin{array}{l}I M R \\
I M R\end{array}$ & $\begin{array}{l}(-2.0) \\
-4.0\end{array}$ & & 5.0 & $\begin{array}{l}\text { CUTKOSKY } \\
\text { CUTKOSKY }\end{array}$ & $\begin{array}{l}79 \text { IPWA } \\
80 \text { IPWA }\end{array}$ & $\begin{array}{llll}P I & N & \text { TO } & P I \\
P I & N \\
P I & \text { TO } & \text { PI } & N\end{array}$ \\
\hline
\end{tabular}

10 delta(1700) Partial decay modes

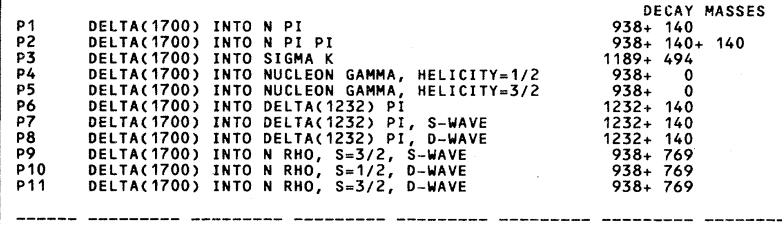

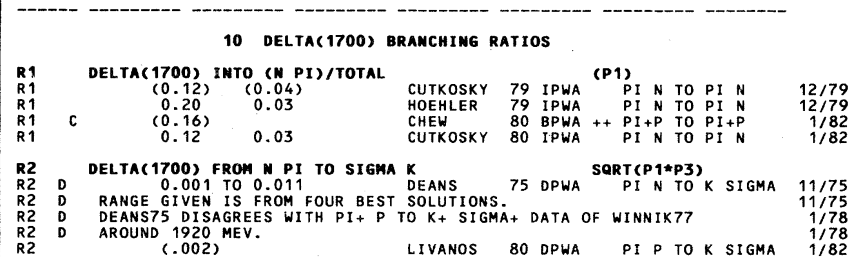

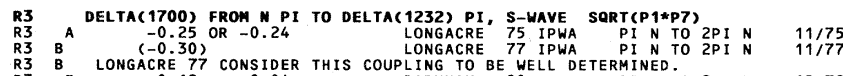

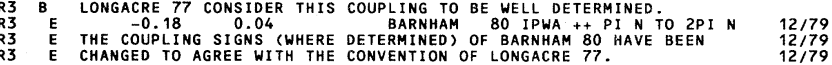

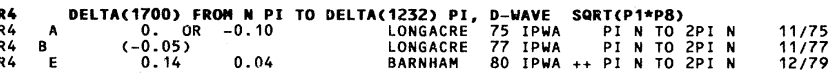

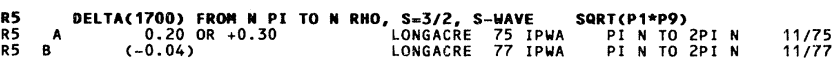

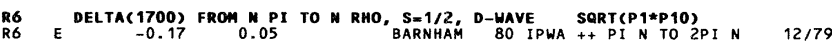

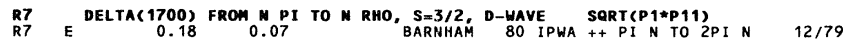

10 DeLta(1700) Photon DeCAY AMPLITUdes (GEV**-1/2)

FOR DEFINITION OF GAMMA-NUCLEON DECAY AMPLITUDES, SEE MINI-
REVIEW PRECEDING THE BARYN LISTINGS.

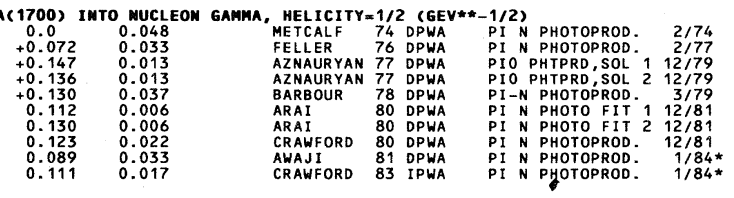

DELTA(1700) INTO NUCLEON GAMMA, MELICITY $=3 / 2$ (GEV**-1/2)

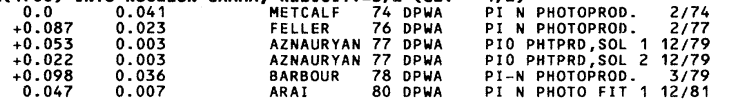


Baryons

$\Delta(1700), \Delta(1900), \Delta(1905)$

Data Card Listings

\begin{tabular}{|c|c|}
\hline $\begin{array}{ll}0.050 & 0.007 \\
0.102 & 0.015 \\
0.000 & 0.015 \\
0.107 & 0.015\end{array}$ & 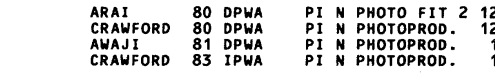 \\
\hline & $\begin{array}{l}* * * * * * * * * * * * * * * * * * * * * * * \\
\text { REFERENCES FOR DELTA(1700) }\end{array}$ \\
\hline 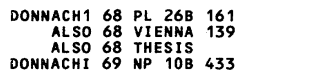 & 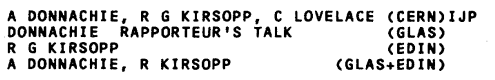 \\
\hline 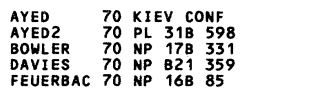 & 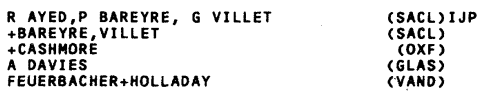 \\
\hline 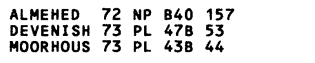 & $\begin{array}{l}\text { LOVELACE } \\
\text { DEVENISH, RANKIN, LYTH } \\
\text { MOORHOUSE, OBERLACK }\end{array}$ \\
\hline 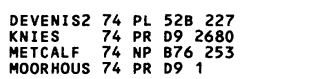 & 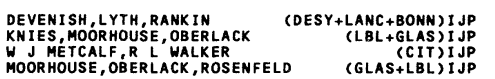 \\
\hline 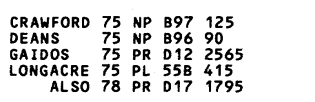 & 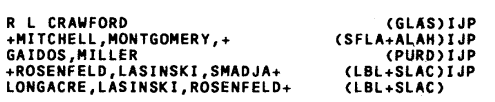 \\
\hline 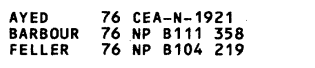 & 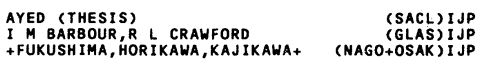 \\
\hline 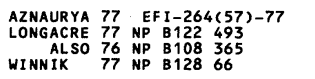 & 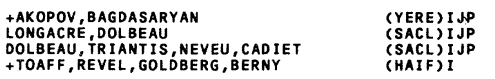 \\
\hline $\begin{array}{l}\text { BARBOUR } 78 \mathrm{NP} 1414253 \\
\text { CUTKOSKY } 79 \text { PPR D20 } 2839 \\
\text { HOEHLER } 79 \text { HANOBOK OF }\end{array}$ & 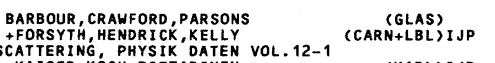 \\
\hline ALSO 80 TORONTO CONF 3 & $\begin{array}{l}\text { KAISER, KOCH, PIETARINEN } \\
\text { R KOCH }\end{array}$ \\
\hline 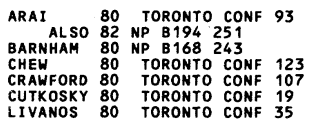 & 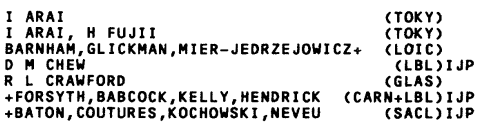 \\
\hline 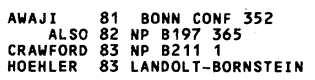 & 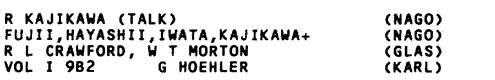 \\
\hline
\end{tabular}

HOEHLR 83 LANDOLT BORNSTEIN VOL 1982 G

$\begin{array}{lll}\Delta(1900) & \mathrm{S}_{31}^{\prime \prime}\end{array}$ Status: ***

30 DELTA(1900, JP=1/2-) $I=3 / 2 \quad S+31$

\begin{tabular}{|c|c|c|c|c|c|c|}
\hline & & 30 DELT & MASS (MEV) & & & \\
\hline & $\begin{array}{l}(1850.0) \\
1908.0 \\
(1918.5) \\
(1803.0) \\
1890.0\end{array}$ & $\begin{array}{l}(35.0) \\
30.0 \\
(23.0) \\
50.0\end{array}$ & $\begin{array}{l}\text { CUTKOSKY } \\
\text { HOEHLER } \\
\text { CHEW } \\
\text { CRAWFORD } \\
\text { CUTKOSKY }\end{array}$ & $\begin{array}{l}79 \text { IPWA } \\
79 \text { IPHA } \\
80 \text { IP } \\
80 \text { BPA } \\
80 \text { PPWA } \\
80 \text { IPWA }\end{array}$ & 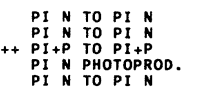 & $\begin{array}{r}12 / 78 \\
12 / 78 \\
11,82 \\
1218 \\
1 / 82\end{array}$ \\
\hline & & 30 DELT & WIDTH (MEV) & & & \\
\hline$\underset{W}{W}$ & $\begin{array}{l}(130.0) \\
140.0 \\
(93.5) \\
(137.0) \\
170.0\end{array}$ & $\begin{array}{c}(40.0) \\
40.0 \\
(54.0) \\
50.0\end{array}$ & $\begin{array}{l}\text { CUTKOSKY } \\
\text { HOEHLR } \\
\text { CHEHER } \\
\text { CRAHFORD } \\
\text { CUTKOSKY }\end{array}$ & 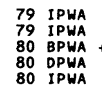 & 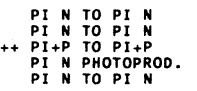 & $\begin{array}{r}12 / 79 \\
1279 \\
12 / 88 \\
128 \\
1 / 82\end{array}$ \\
\hline & & DELT & REAL PART O & OF POLE POS & SITHOM (MEV) & \\
\hline $\begin{array}{l}\mathrm{RE} \\
\mathrm{RE}\end{array}$ & $\begin{array}{c}(1844.0) \\
1870.0\end{array}$ & 40.0 & $\begin{array}{l}\text { CUTKOSKY } \\
\text { cUTKOSKY }\end{array}$ & $\begin{array}{l}79 \text { IPWA } \\
80 \text { IPHA }\end{array}$ & $\begin{array}{llll}P I & N \\
P I & N & P \\
N\end{array}$ & $\begin{array}{r}12 / 78 \\
1 / 82\end{array}$ \\
\hline & & 30 DELT & -2*IMAG PAR & RT OF POLE & POSITIOM (MEV) & \\
\hline $\operatorname{IM}_{\mathrm{IM}}$ & $\begin{array}{l}(142.0) \\
180.0\end{array}$ & & $\begin{array}{l}\text { CUTKOSKY } \\
\text { CUTKOSKY }\end{array}$ & $\begin{array}{l}79 \mathrm{IPHA} \\
80 \mathrm{IPWA}\end{array}$ & $\begin{array}{ll}P I \\
P I\end{array}$ & $\begin{array}{r}12 / 78 \\
1 / 82\end{array}$ \\
\hline & & 30 DELT & REAL PART O & DF ELASTIC & POLE RESIDUE (MEV) & \\
\hline $\begin{array}{l}\substack{R E R \\
R \in R} \\
R \in R\end{array}$ & $\begin{array}{l}(7.0) \\
9.0\end{array}$ & 4.0 & $\begin{array}{l}\text { cuTKoskY } \\
\text { cuTKOSKY }\end{array}$ & $\begin{array}{l}79 \mathrm{IPHA} \\
80 \mathrm{IPHA}\end{array}$ & $\begin{array}{ll}P 1 \\
P I\end{array}$ & $\begin{array}{r}12 / 79 \\
1 / 82\end{array}$ \\
\hline & & 30 DELT & IMAG PART O & PF ELASTIC & POLE RESIDUE (MEV) & \\
\hline $\operatorname{IMR}_{\mathrm{IMR}}$ & $\begin{array}{c}(-1.0) \\
3.0\end{array}$ & & $\begin{array}{l}\text { CUTKOSKY } \\
\text { cUTKOSKY }\end{array}$ & $\begin{array}{l}79 \mathrm{IPWA} \\
80 \mathrm{IPWA}\end{array}$ & $\begin{array}{ll}P_{1} & N \\
P I & \text { TO } \\
\end{array}$ & 12 \\
\hline
\end{tabular}

\author{
30 DELta(1900) PARTIAL DECAY MOdEs

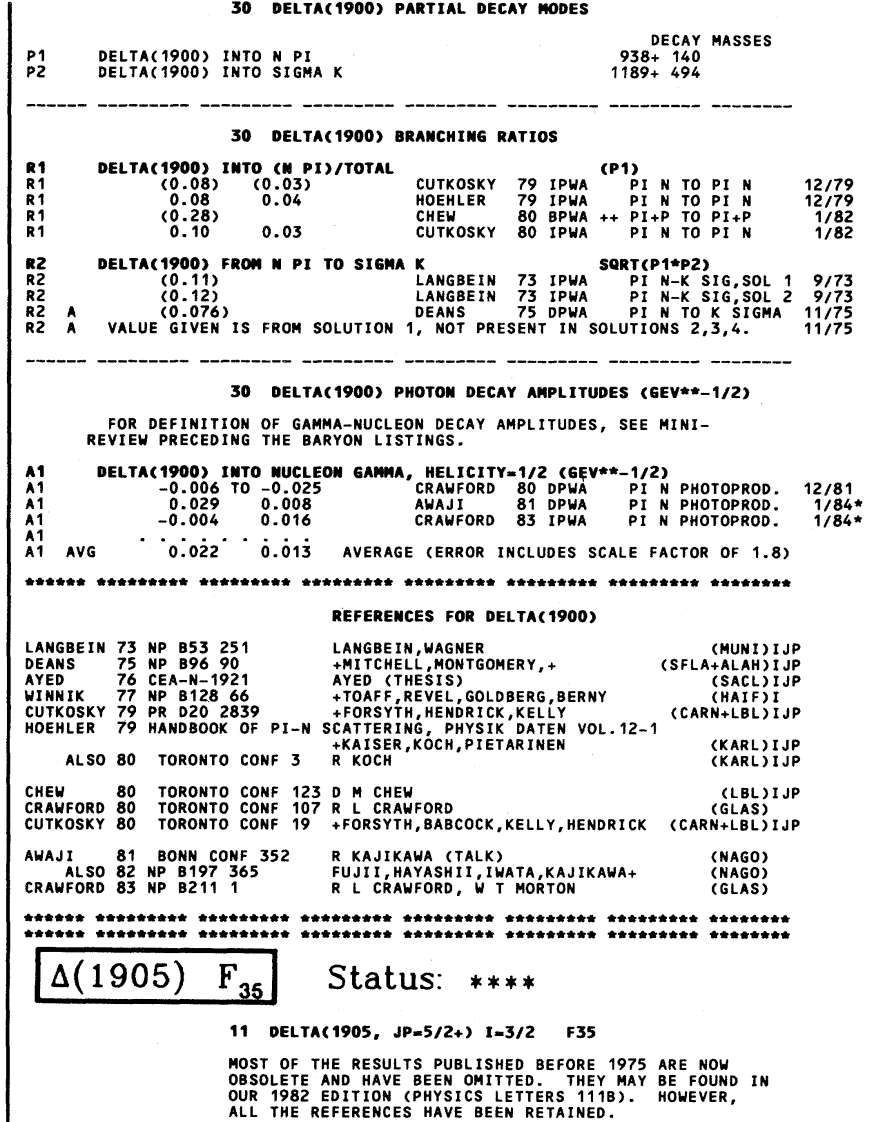

CUTKOSKY 80 ALSO FINDS WEAK EVIDENCE FOR A HIGHER MASS F35 RESONANCE
IN ADDITION TO THIS STATE. BOTH RESONANCES ARE LISTED HERE FOR NOW.

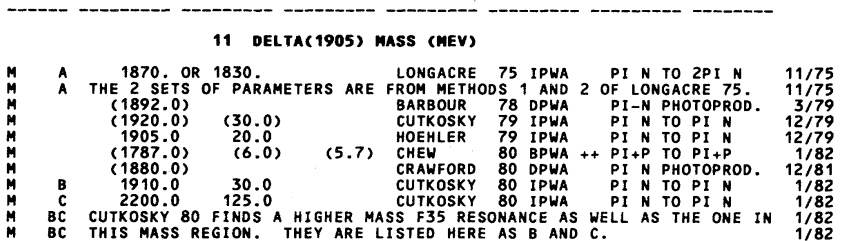

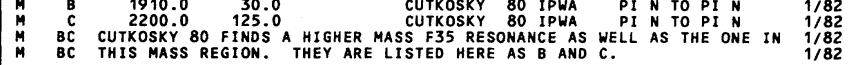

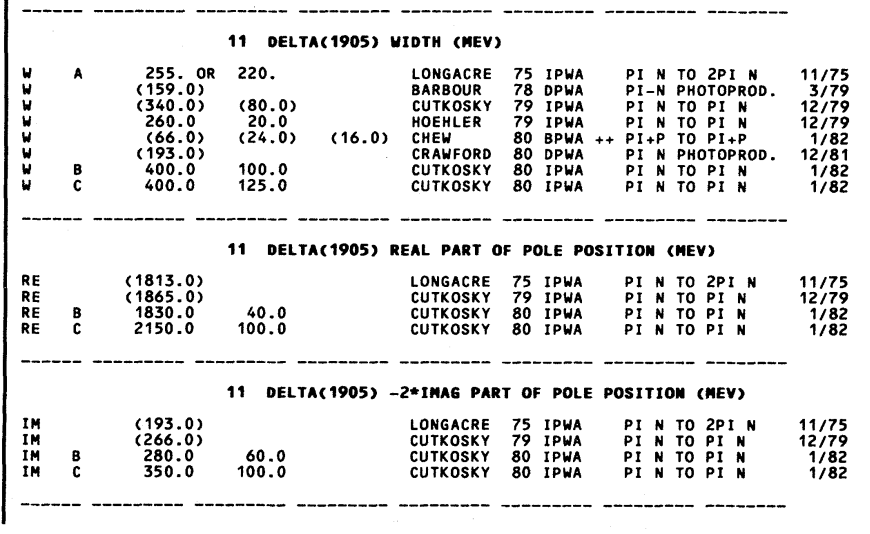




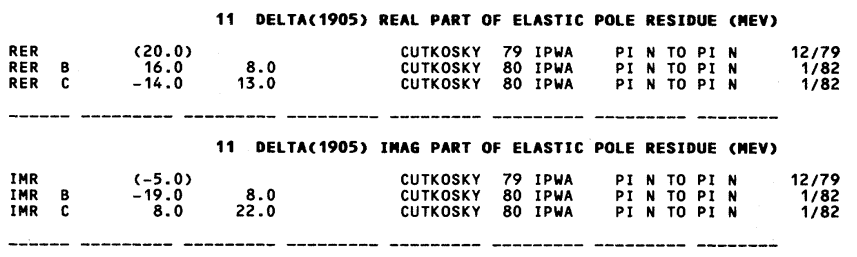

11 delta(1905) partial decay modes

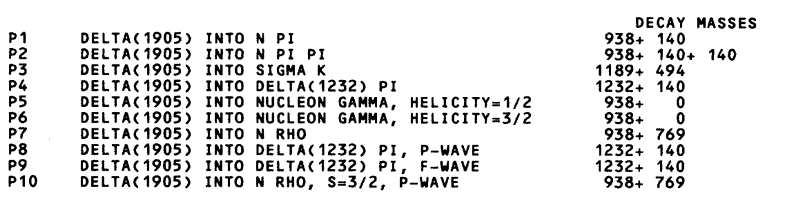

11 Delta( 1905) BRAMCHING Ratios

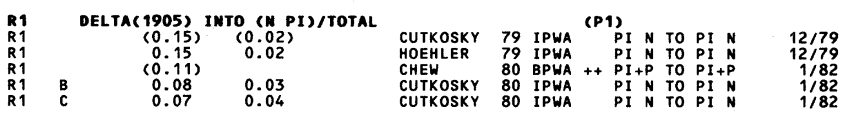

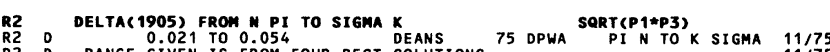

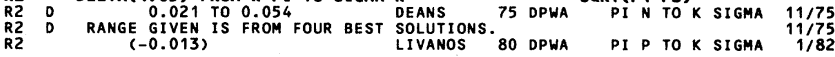

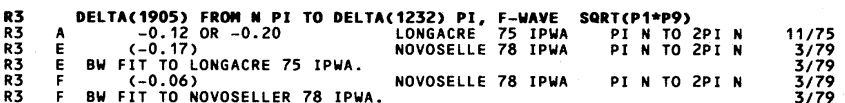

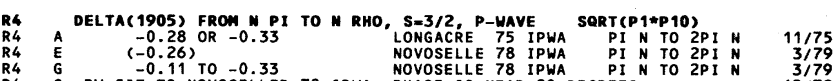

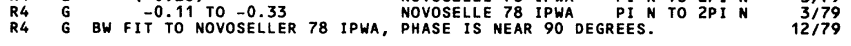

11 DELTA (1905) PHOTON DECAY aMPLITUdES (GEV**-1/2)

FOR DEFINITION OF GAMMA-NUCLEON DECAY AMPLITUDES, SEE MINI-
REVIEW PRECEDING THE BARYN LISTINGS.

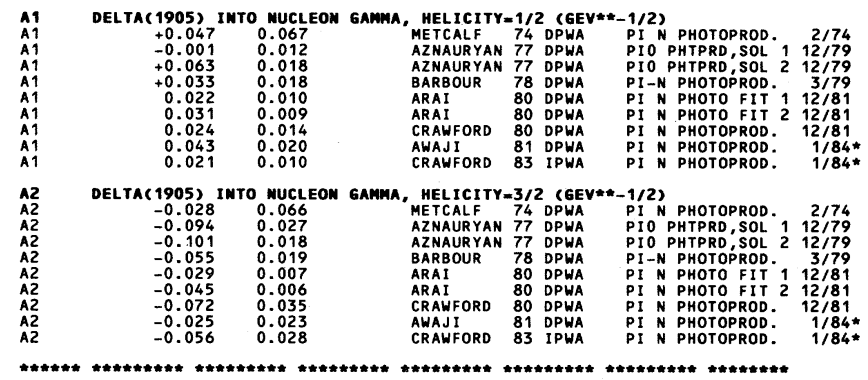

$* * * * * * * * * * * * * * * * * * * * * * * * * * * * * * * * * * * * * * * * * * * * * *$
REFERENCES FOR DELTA(1905)

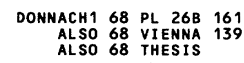

A DONNACHIE, R G KIRSOPP, C LOVELACE (CERN) IJP
DONNACHIE RAPPORTEUR'S TALK (GLAS)

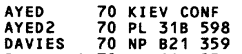

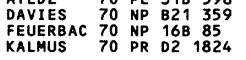

$\begin{array}{llll}\text { ALMEHED } & 72 & \text { NP } B 40 & 157 \\ \text { MEHTANI } & 72 \\ \text { PRL } 29 & 1634\end{array}$

$\begin{array}{llll}\text { MEHTANI } 72 & \text { PRL } 29 & 1634 \\ \text { LANGBEIN } 73 & \text { NPP } 853 & 251 \\ & \end{array}$

$\begin{array}{llll}\text { DEVENIS2 } 74 & \text { PL } & 52 B & 227 \\ \text { KNIES } & 74 & \text { PR D9 } 2680\end{array}$

CRAWFORD 75 NP $B 97125$

$\begin{array}{rllll}\text { DEANS } & 75 & \text { NP } & 896 & 90 \\ \text { LONGACRE } & 75 & \text { PL } & 558 & 415 \\ \text { ALSO } & 78 & \text { PR } & 017 & 1795\end{array}$

AYED 76 CEA-N-1921

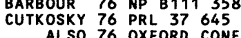

ALSO 76 OXFORD CONF, 49
AZNAURYA 77 EFI-264(57)-77
WINNIK 77 NP 8128 66

BARBOUR 78 NP B141 253

DONNACHIE RAPPORTEUR'S TALK
$R G$ KIRSOPP

(EDIN)

R AYED, P BAREYRE, G VILLLT
+BAREYRE, VILLET
A DAVIES

F DAVIES
FEUERBACHER+ HOLLADAY
G KALMUS, G BORREANI, J LOUIE

(SACL) IJP

( $S A C L) I$
(SACL)
(GLAS)

+ LOVELACE

FUNG, KERNAN, SCHALK, +
LANGBEIN,

DEVENISH, LYTH, RANK IN
KNIES, MOORHOUSE, OBERLACK

(LUND+RUTG) I JP

KNIES, MOORHOUSE, OBERLACK

(UCR + LBL)

$R$ L CRAWFORD

MITCHELL, MONTGOMERY,
+ROSENFELOLASINSKI, SMAD

LONGACRE, LASINSKI, ROSENFELD

(LBL+GLAS) I JP

AYED (THESIS)

CUTKOSKY, HENDR ICK, KELLYY
CUTKOSKY, HENDR ICK, CHAO+

+AKOPOV, BAGDASARYAN
+ TOAFF, REVEL, GOLOBERG, BERNY

BARBOUR, CRAWFORD, PARSONS

D E NOVOSELLER
D E NOVOSELLER
CUTKOSKY 79 PR 0202839
HOEHLER 79 HANDBOOK OF PI-N SCATTERTH, HENDRICK, KELLY
(CARN+LBL) IJP $\begin{array}{ll}\text { ALSO } 80 \text { TORONTO CONF } 3 \text { R KOCH, KOCH, PIETARINEN } & \text { (KARL) IJP } \\ \text { (KARL)IJP }\end{array}$ ARAI 80 TORONTO CONF 93 I ARAI, (TOKY)
ALSO 82 NP B194 251 (TOKY)

$\begin{array}{lll}\text { CHEW } 80 & \text { TORONTO CONF } 123 & \text { D M CHEW } \\ \text { CRAWFORD } 80 & \text { TORONTO CONF } 107 \text { R L CRAWFORD } & \text { (LBL) IJP }\end{array}$ CUTKOSKY 80 TORONTO CONF 19 F FORSYTH, BABCOCK, KELLY, HENDRICK
(CARN+LBL) IJP
LIVANOS 80 TORONTO CONF 35 +BATON, COUTURES, KOCHOWSKI, NEVEU
(SACL)IJP

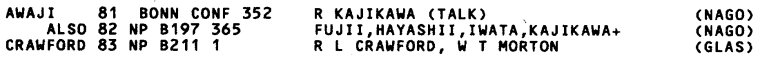

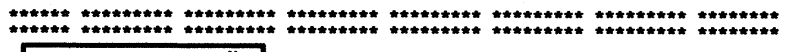
$\begin{array}{lll}\Delta(1910) & \mathrm{P}_{31}^{\prime \prime}\end{array}$ Status: $* * * *$

12 DELTA(1910, JP=1/2+) I=*/2 $P+31$ MOST OF THE RESULTS PUBLISHED BEFORE 1975 ARE NOW OUR 1982 EDITION (PHYSICS LETTERS 111B) HOWEVER,

12 DeLta(1910) mass (MEV)

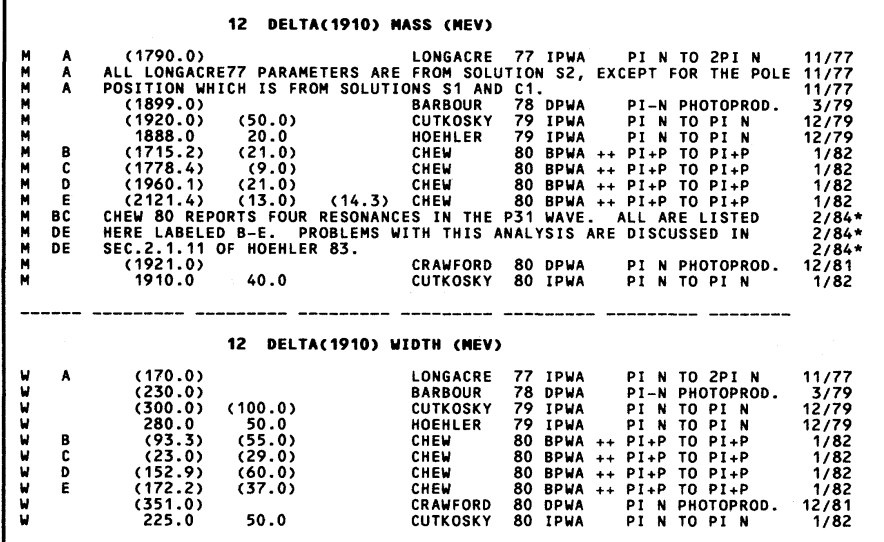

12 DELTA(1910) REAL PART OF POLE POSITION (MEV)

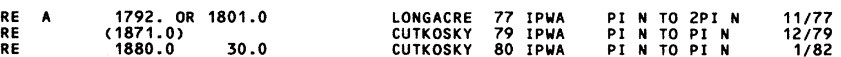

12 DELTA(1910) -2*IMAg PART OF POLE POSITION (MEV)

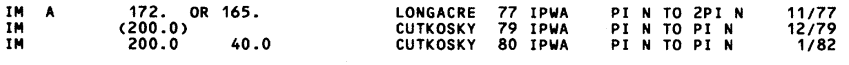

12 deLtA(1910) REAL PART OF ELASTIC POLE RESIDUE (MEV)

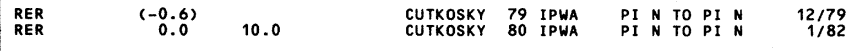

12 deLtA(1910) IMAG PART OF ELASTIC POLE RESIDUE (MEV)

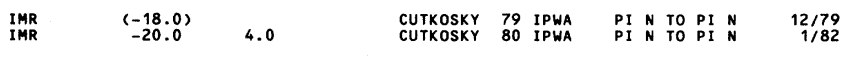

12 delta(1910) Partial decay modes

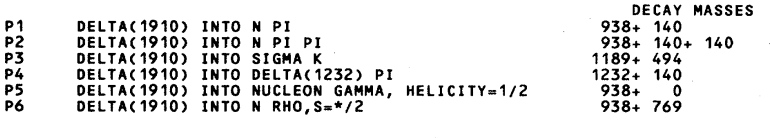

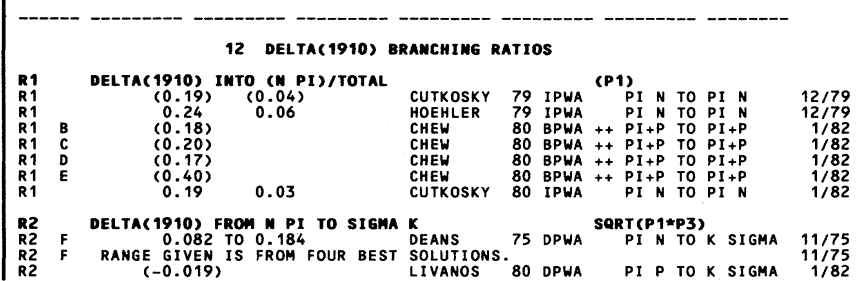


Baryons

$\Delta(1910), \Delta(1920), \Delta(1930)$

\section{Data Card Listings}

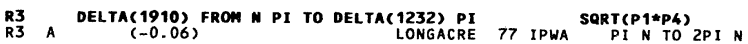

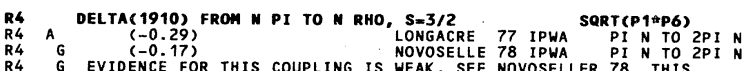

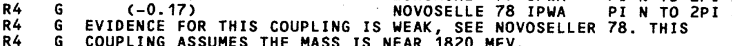
12 DELTA(1910) PHOTON DECAY AMPLITUDES (GEV**-1/2) FOR DEFINITION OF GAMMA-NUCLEON DECAY AMPLITUDES, SEE MINI-
REVIEW PRECED ING THE BARYN LISTINGS.

$\hat{A}$
A
A
A
A.
A.
A.
A.
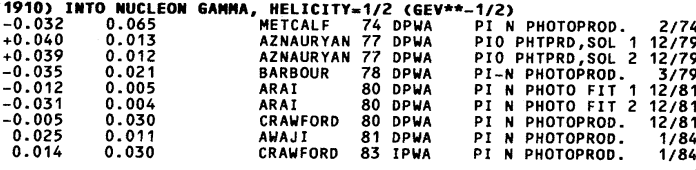

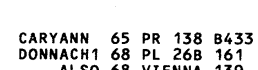

REFEREMCES FOR DELTA(1910)

ALSO 68 VIENAA 13

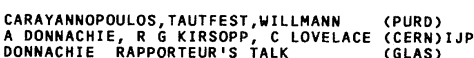

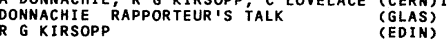
R AYED, PAREYRE, G VILLET ABAREYRE, VILLET

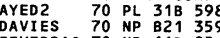

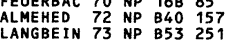

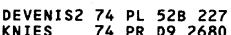
METCALF 74 NP B76 253

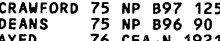
AYED 76 CEA-N-1921 TKOSKY 76 PRL 37645
ALSO 76 OXFORD CONF. 49 AZNAURYA 77 EFI-264(57) -77 AINNISO 76 NP 810836

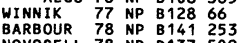
FEUERBACHER+HOLLAD LANGBEIN, WAGNER (SACL)
(GAS)
(YAMON) DEVENISH, LYTH, RANKIN

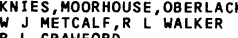

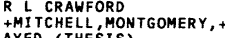
AYED (THESIS)
I $M$ BARBOUR, R L L CRAWFOR CUTKOSKY,HENORICK, KELLY

+akOPOV, BAGOASARYAN LOLGACRE, DOLBEAU

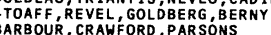

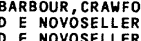
D NOYSELER CUTKKSKY 79 PR D DO 2839
HOEHLER 79 HANDOOK OF ALSO 80 TORONTO CONF 3 K KAIS KOCH

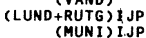
AO TORONTO CONF 93 I ARA CHEW 80 TORONTO CONF 123 O M CHEW ${ }^{2}$ FUJI

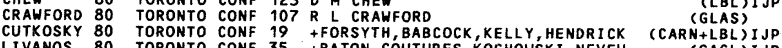
AHAJI 81 BONN CONF 352 R KAJIKAHA (TALK) (NAGO)

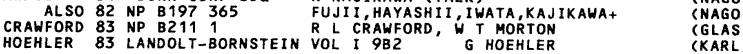

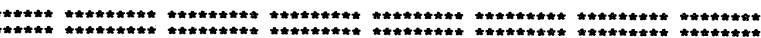

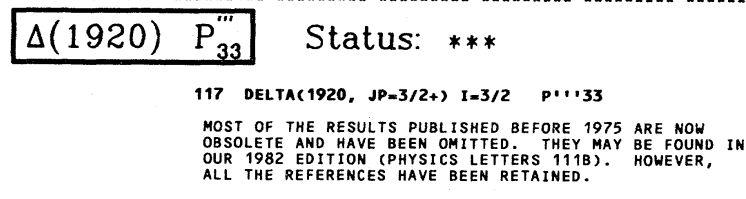
117 DeLta(1920) MASS (MEV)

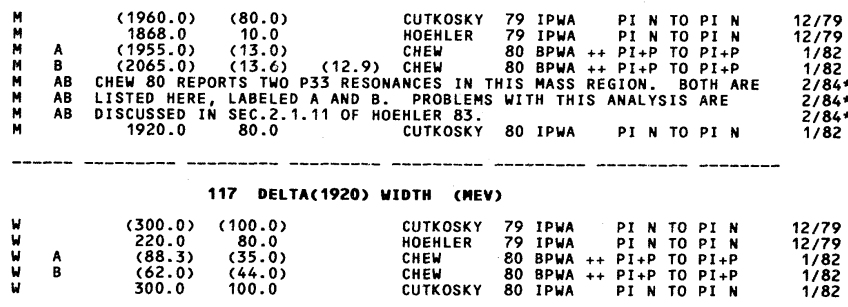

117 DeLtac 1920) ReAl PART Of POLE Position (MEV)

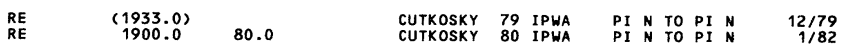

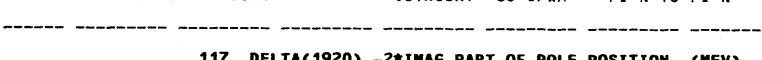

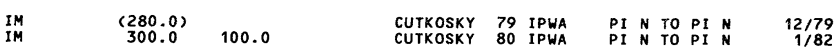

$11 / 77$
$11 / 77$
$32 / 79$
$12 / 79$

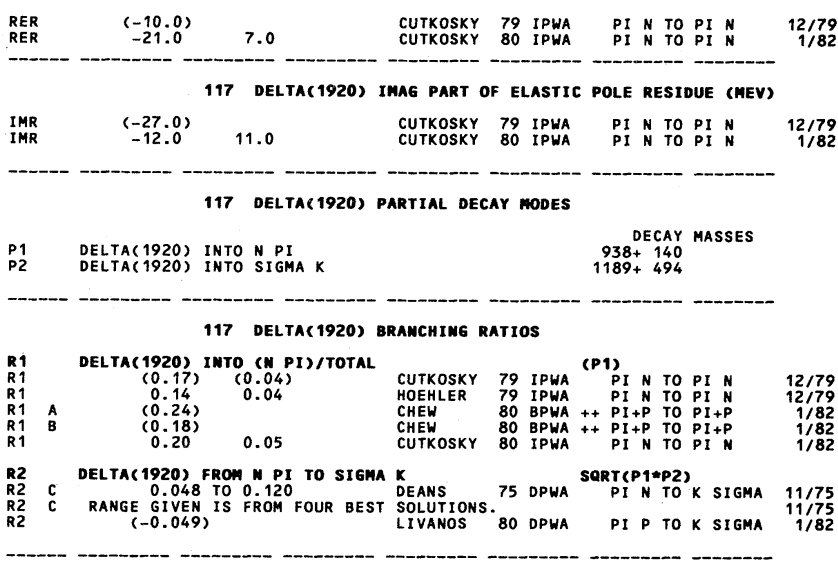

117 DELTAC 1920) PHOTON DECAY AMPLITUDES (GEV**-1/2)

FOR DEFINITION OF GAMMA-NUCLEON DECAY AMPLITUDES, SEE MINI-
REVIEW PRECED ING THE BARYON LISTINGS.

DELTAC 1920) INTO MUCLEOW GAMMA, MELICITY $1 / 2$ (

DELTAC 1920) INTO MUCLEON GAMMA, MELICITY-3/2 (GEV**-1/2)

A2 DETAC

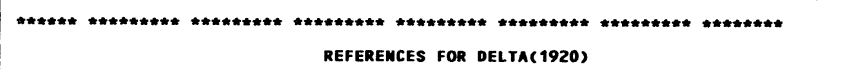

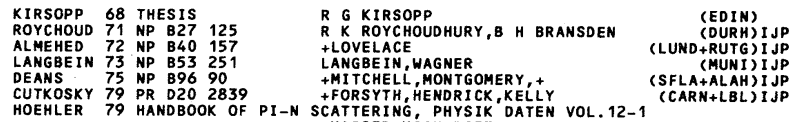
$\begin{array}{ll}\text { ALSO } 80 \text { TORONTO. CONF } 3 \text { R KOCH, KOCH, PIETAR INEN } & \text { (KARL) IJP } \\ \text { (KARL)IJP }\end{array}$

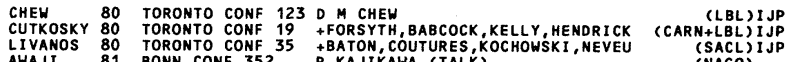

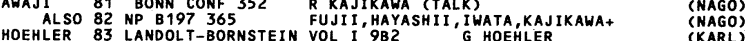

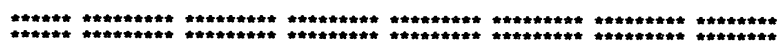

\begin{tabular}{lll}
\hline$(1930)$ & $D_{35}^{\prime}$
\end{tabular} Status: ***

13 DELTA(1930, JP=5/2-) I=3/2 D.35

MOST OF THE RESULTS PUBLISHED BEFORE 1975 ARE NOW

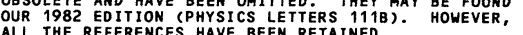

the agreement among the various analyses is not very good.

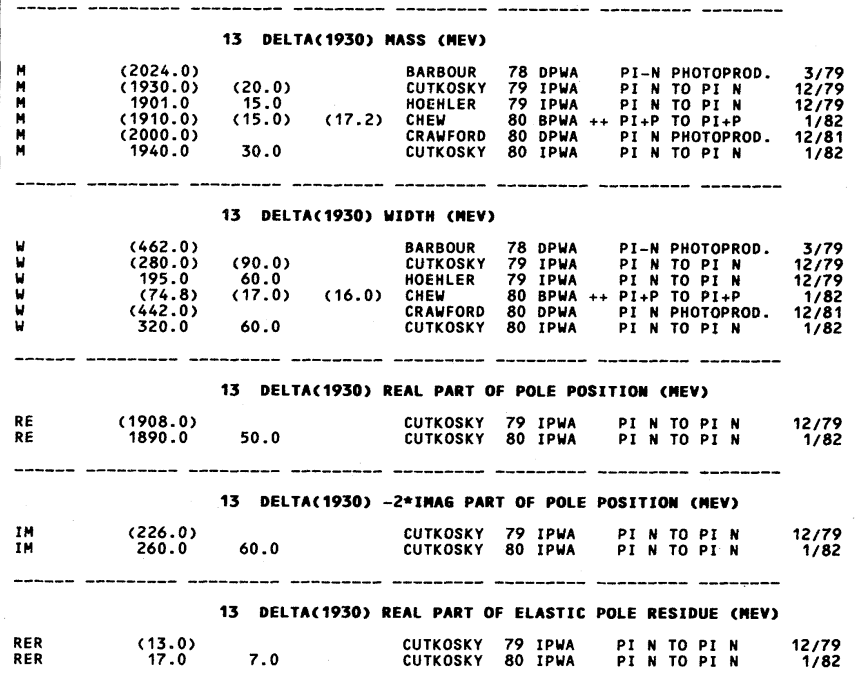




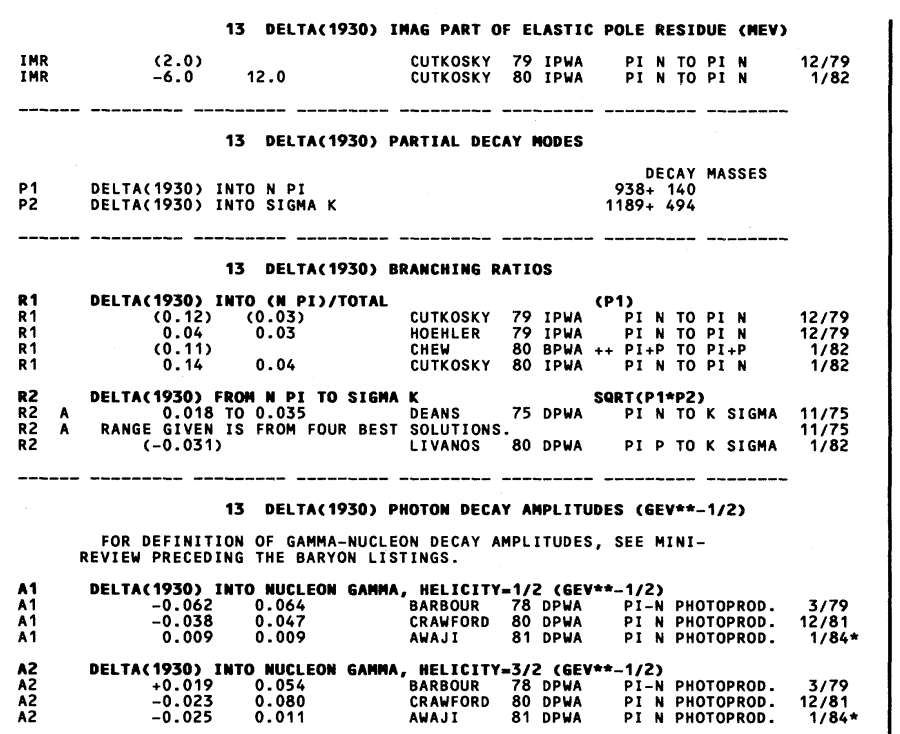

$\begin{array}{llll}\text { OONNACH1 } & 68 & \text { PL } 268 & 161 \\ \text { KIRSOPP } & 88 \text { THESIS }\end{array}$

DONNACHI 69 NP 108433

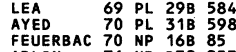

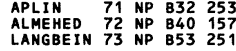

$\begin{array}{ll}\text { CRAWFORD } 75 \mathrm{NP} B 97 & 125 \\ \text { DEANS } & 75 \mathrm{NP} B 9690\end{array}$

AYED 76 CEA-N-1921

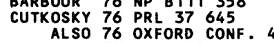

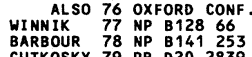

CUTKSKKY 79 PR D20 2839
HOEHLER 79 HANOBOK OF PI-N SCEAT

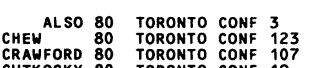

RefEREMCES For deLta( 1930)

$A$ DONNACHIE, R G KIRSOPP, C LOVELACE (CERN) IJP
R
(EDIN K KRSOPP

A DONNACHIE, R KIRSOPP (GLASSEDIN)

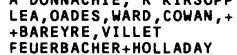

FEUERBACHER+HOLLADAY
+ COWAN, GIBSON,GILMORE++

+LOVELACE
LANGBEIN, WAGNER

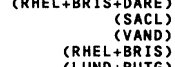

R L CRAWFORD

(RHELLBRIS)
(LUND +RUG) IJP
(MUNI) IJP

CHELL, MONTGOMERY, +

(SFLA+ALAS)IJPP

AYED (THESIS)

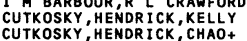

CUTKOSKY,',ENDR ICK, CHAO
TTAFF,
BAEREL, GODDEERG, BERNY

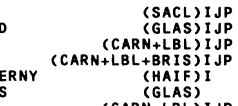

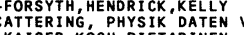

(GLAS)
(CARN+LBL)IJP

(KARL) IJP

KOCH

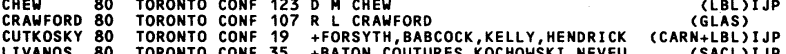

AWAJI 81 BONN CONF 352 R KAJIKAWA (TALK)

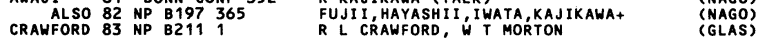

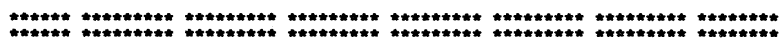

\begin{tabular}{|lll}
\hline$(1940)$ & $D_{33}^{\prime \prime}$ & Status: * \\
$\longrightarrow$ & 136 DELTA(1940, JP=3/2-) I=3/2 $\quad$ Dי33
\end{tabular}

136 DELTA(1940) MASS (MEV)

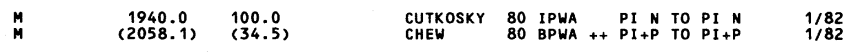

136 deLta(1940) Midth (MEV)

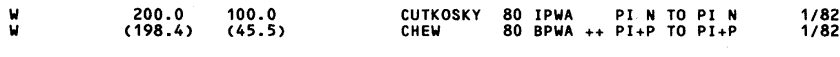

$\begin{array}{llll}136 & \text { DELTA(1940) REAL PART OF POLE POSITION (MEV) } \\ \text { RE CUTKOSKY } 80 \text { IPWA PI N TO PI N } & 1 / 82\end{array}$

$\begin{array}{llllll}136 & \text { DELTA(1940) } & -2 * \text { IMAG PART OF POLE POSITION (MEV) } & \\ \text { IM } & 200.0 & 60.0 & \text { CUTKOSKY } 80 \text { IPWA PI N TO PI N } & 1 / 82\end{array}$

136 DELTA(1940) REAL PART OF ELASTIC POLE RESIDUE (MEV)

$\begin{array}{lllllll}\text { RER } & -6.0 & 5.0 & \text { CUTKOSKY } 80 \text { IPWA PI N TO PI N } & 1 / 82\end{array}$

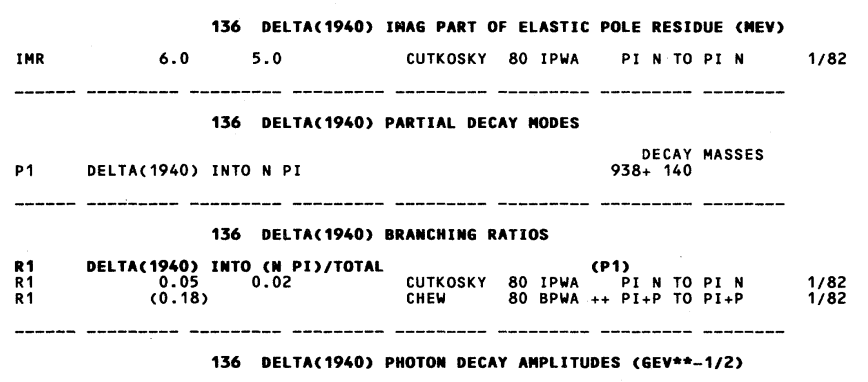

FOR DEFINIIION OF GAMMA-NUCLEON DECAY AMPLITUDES, SEE MINI-
REVIEW PRECEDING THE BARYON LISTINGS.

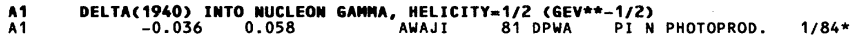

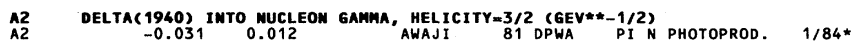

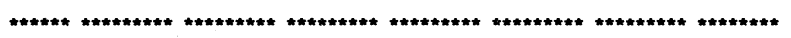
REFERENCES FOR DELTAC 1940)

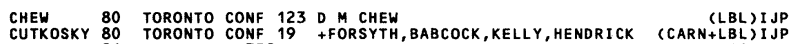

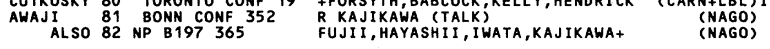

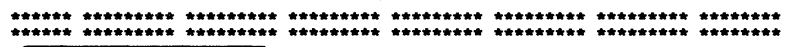
\begin{tabular}{|lll}
\hline$(1950)$ & $\mathrm{F}_{37}^{\prime}$ & Status: $* * * *$
\end{tabular}

83 DELTA(1950, JP=7/2+) I=3/2 F'37

MOST OF THE RESULTS PUBLISHED BEFORE 1975 ARE NOW
OBSOLETE AND HAVE BEEN OMITTED. FTEY MAY BEFFOUND IN

OUR 1982 EDITION (PHYSICS LETTERS 1118 .
ALL THE REFERENCES HAVE BEEN RETAINED.

IN ADDIYION, RESULTS IN THIS REGION FROM PRODUCTION EXPERIMENTS,
WHICH USE TO BE LISTED SEPARATELY AS THE NEXT ENTRY, HAVE BEEN ENT IRELY REMOVED.

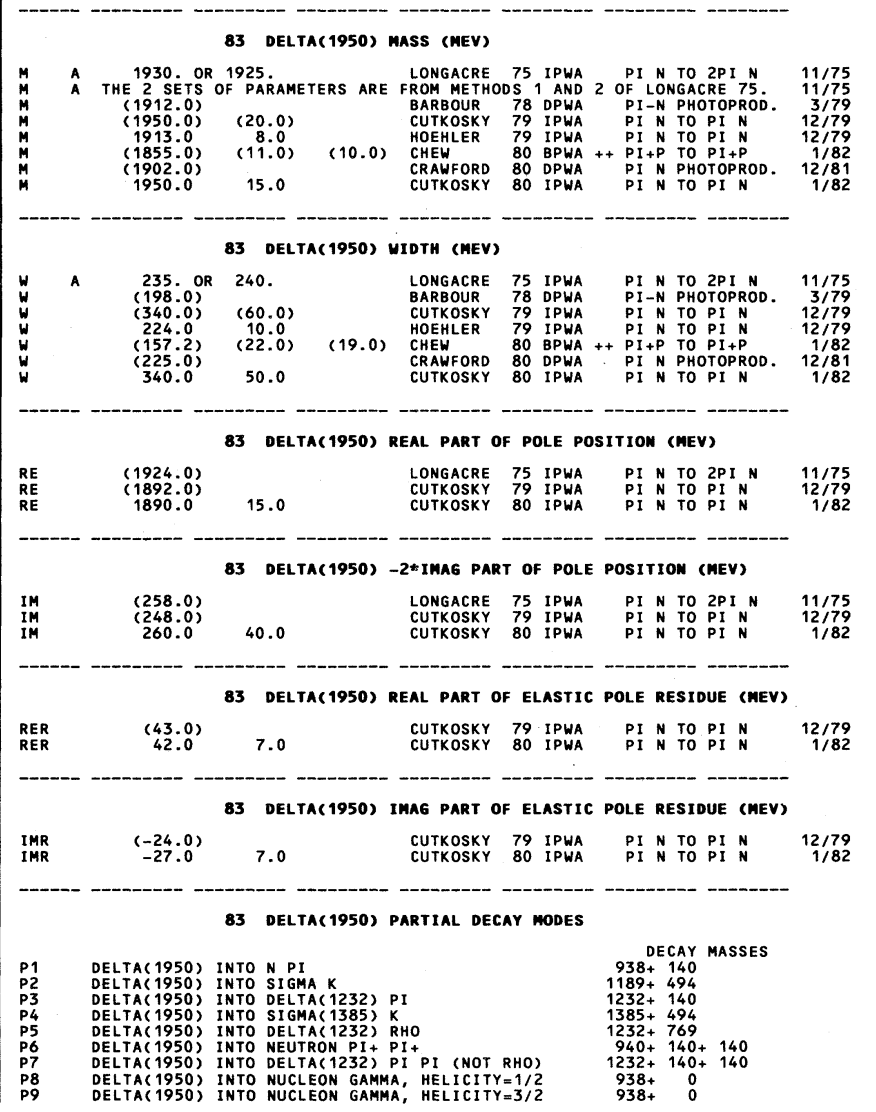


Baryons

$\Delta(1950), \Delta(2150), \Delta(2200)$

Data Card Listings

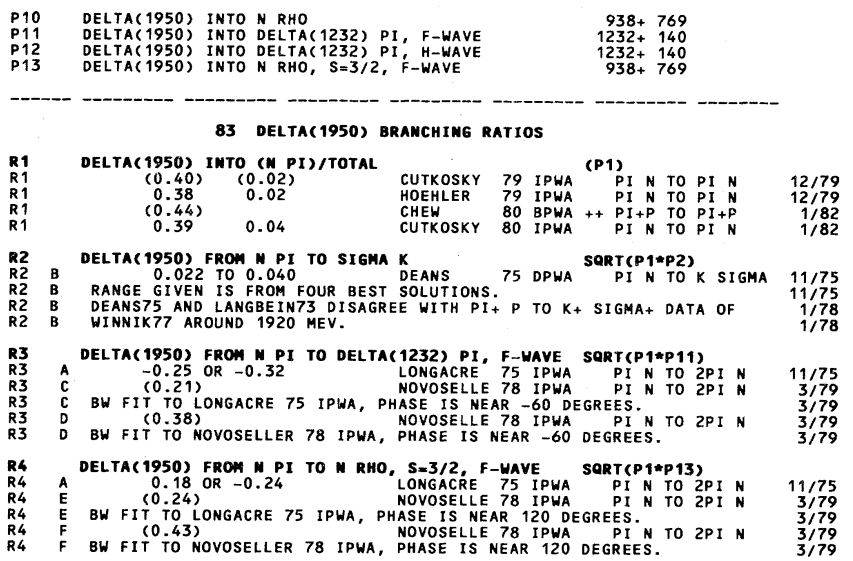

83 DELTA(1950) PHOTON DECAY AMPLITUdES (GEV*\#-1/2) FOR DEFINITION OF GAMMA-NUCLEON DECAY AMPLITUDES, SEE MINI-
REVIEW PRECEDING THE BARYN LISTINGS.

A1
A1
$A 1$
$A 1$
$A 1$
$A 1$
$A 1$
$A 1$
$A 1$
$A 1$
$A 2$
$A 2$
$A 2$
$A 2$
$A 2$
$A 2$
$A 2$
$A 2$
$A 2$
$A 2$ DELTA(1950) INTO MUCLEON GAMMA,
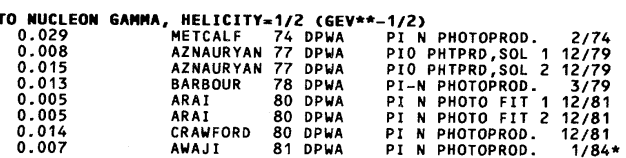

DELTA(1950), INTO WUCLEON GAMMA, HELICITY $=3 / 2$ CGEV**-1
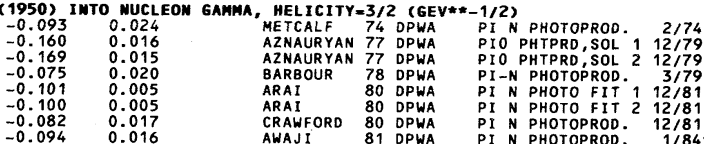

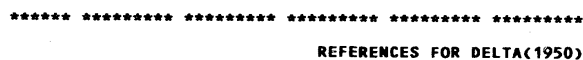

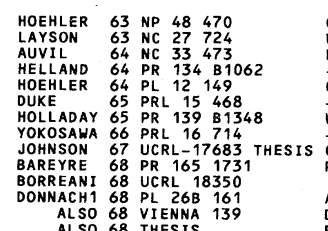

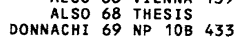

AYED
AY

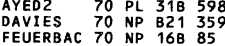

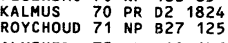

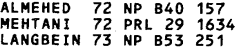

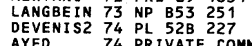

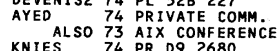

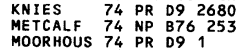

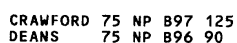

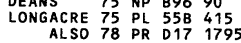

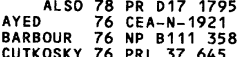

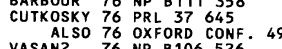

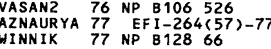

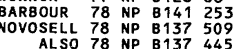

G HOEHLER, 6 EBEL

S LAYSON

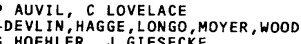

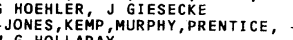

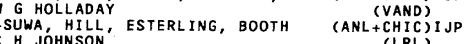

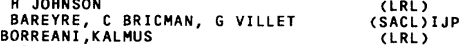

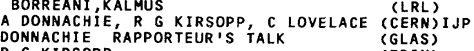

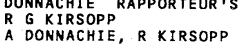

(GLAS+EDTN)

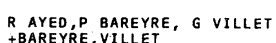

(SACL) IJP
(SACS

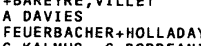

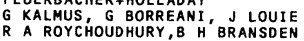

(DURH) IJP

tFUNG, KERNAN, SCHALK, + (LUND+RUTG) IJP

COESY LANC CWUWH) IJP

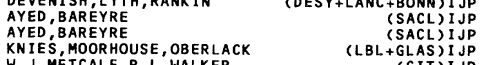

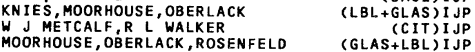

R L CRAWFORD
+ MITCHELL, MONTGOMERY,+
(GLAS) IJPP

ROSENF ELD, LASINSKI, SMADJA+
+RONGACRE LAS (LBL SLAC) IJP

LONGACRE, LASINSKI, ROSENFEL +
AYED (THESIS)

II M BABBOOR, R L CRAHFORD

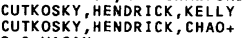

CUTKOSSY,HENDRICK, CMAO
$S \rightarrow$ VASA

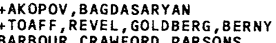

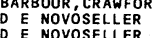

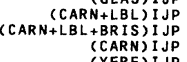

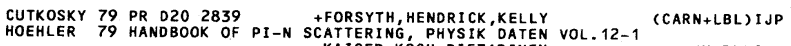
$\begin{array}{lll}\text { ALSO } 80 \text { TORONTO CONF } 3 & \text { KKAISER, KOCCH, PIETARINEN } \\ R & \text { KOCH } & \text { (KARL)IJP } \\ \text { KARLIJP }\end{array}$

CHEH 80 TORONTO CONF 123 D $\mathrm{M}$ CHEI H H FUJI

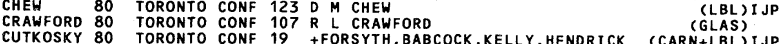

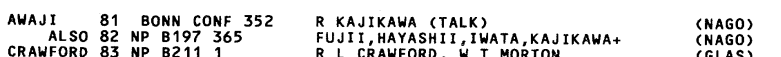

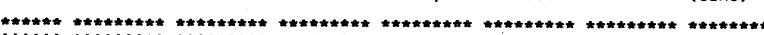

\begin{tabular}{|ll}
\hline$(2150)$ & $\mathrm{S}_{31}^{\prime \prime}$
\end{tabular} Status: *

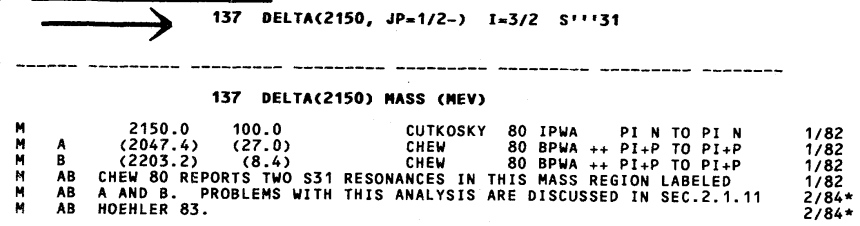

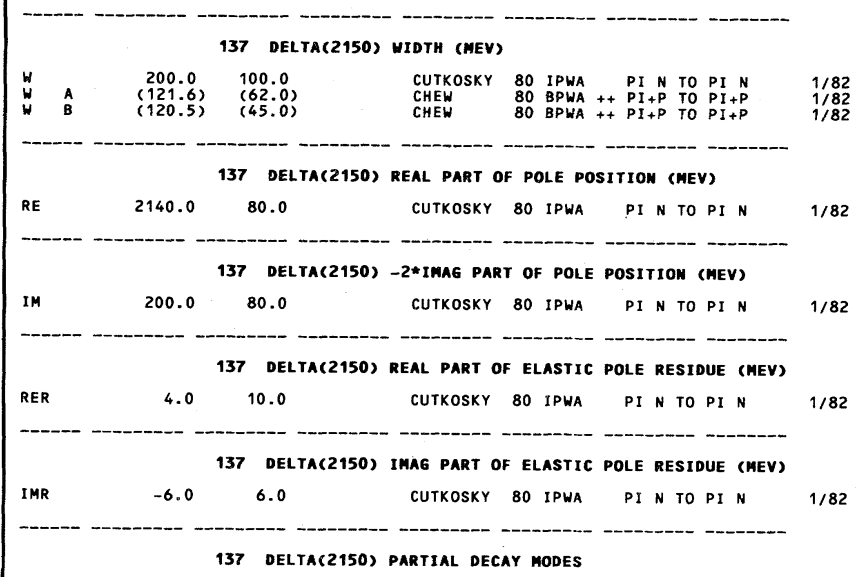

P1 Delta(2150) INTO N PI 938+140 MASSES

137 DELTA(2150) BRAMCHIMG RATIOS

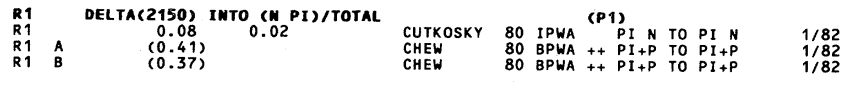

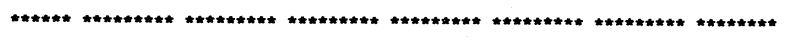

REFEREMCES FOR DELTA(2150)

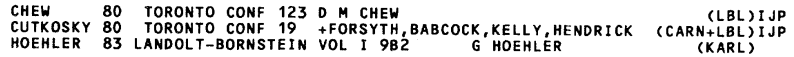

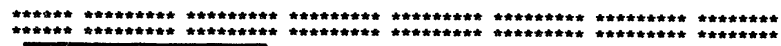

$\Delta(2200) G_{37} \quad$ Status: *

$\longrightarrow 135$ DELTA(2200, JP=7/2-) I=3/2 G37

the agreement among the various analyses is not very good.

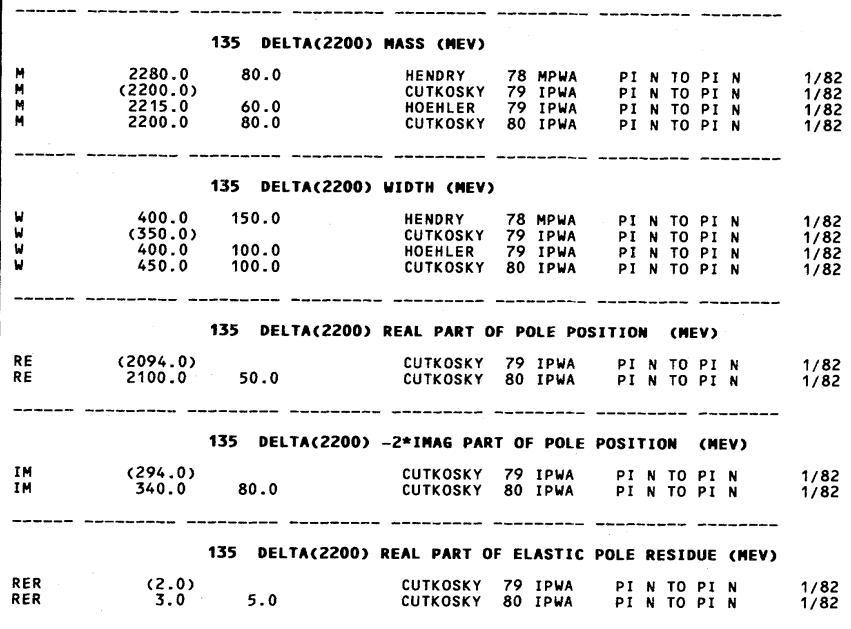




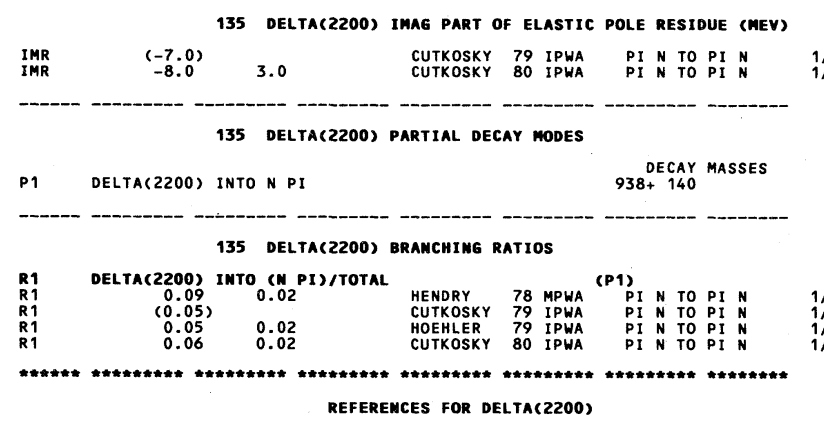

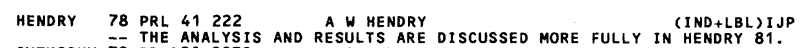

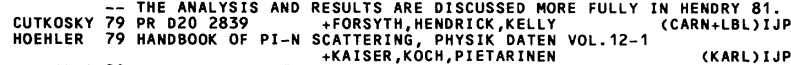
ALSO 80 TORONTO CONF 3 KAISR, KOOCH, PIETARINEN
KKOCH

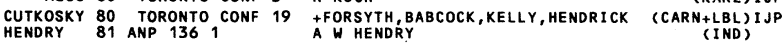

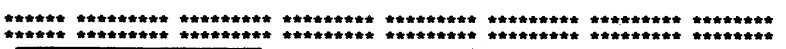
\begin{tabular}{|ll|}
\hline$(2300)$ & $\mathrm{H}_{39}$ \\
$\longrightarrow$ & 123 DELTAC2300, JP-9/2*) I-3/2
\end{tabular}

\begin{tabular}{|c|c|c|c|c|c|c|}
\hline \multirow[b]{2}{*}{$\stackrel{M}{M}$} & \multicolumn{4}{|c|}{123 DELTA(2300) MASS (MEV) } & \multirow[b]{2}{*}{ 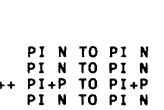 } & \multirow[b]{2}{*}{$\begin{array}{r}12 / 79 \\
12 / 79 \\
1 / 82 \\
1 / 82\end{array}$} \\
\hline & $\begin{array}{r}2450.0 \\
2217.0 \\
(2204.5 \\
2400.0)\end{array}$ & $\begin{array}{c}100.0 \\
80.0 \\
(3.4) \\
125.0\end{array}$ & $\begin{array}{l}\text { HENORY } \\
\text { HEOELLER } \\
\text { CHEWE } \\
\text { CUTKOSKY }\end{array}$ & $\begin{array}{l}78 \text { MPWA } \\
79 \text { IP PAA } \\
80 \text { BPA } \\
80 \text { IPWA }\end{array}$ & & \\
\hline & \multicolumn{4}{|c|}{123 DELTA(2300) MIDTH (MEV) } & & \\
\hline$\underset{W}{W}$ & $\begin{array}{l}500.0 \\
300.0 \\
632.33 \\
425.0\end{array}$ & $\begin{array}{l}200.0 \\
100.0 \\
11.0 \\
150.0\end{array}$ & $\begin{array}{l}\text { HENDRY } \\
\text { HEOHLER } \\
\text { HEEWE } \\
\text { CUTKOSKY }\end{array}$ & 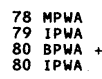 & 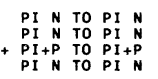 & $\begin{array}{r}12 / 79 \\
12179 \\
1182 \\
1 / 82\end{array}$ \\
\hline
\end{tabular}

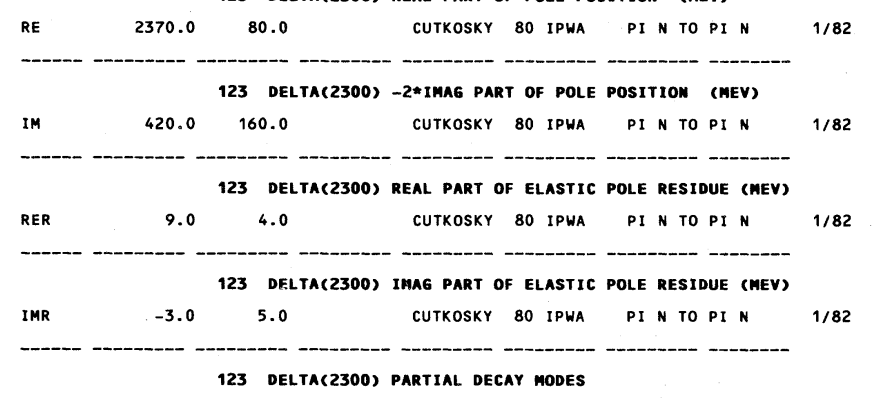

P1 DELTA(2300) INTO N PI DECAY MASSES

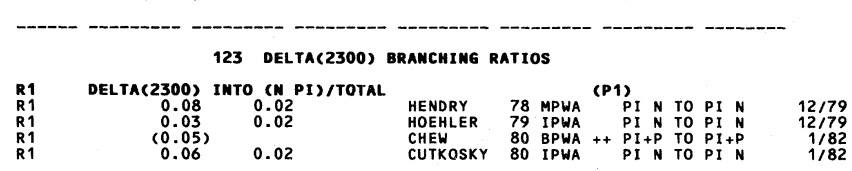
*************************************************************************** ReFEREMCES For DeLta(2300)

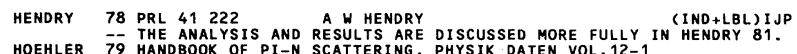

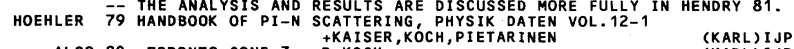

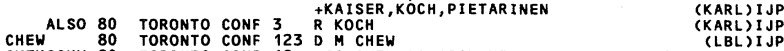

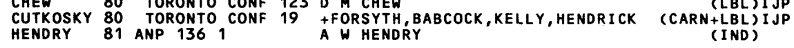

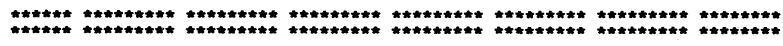

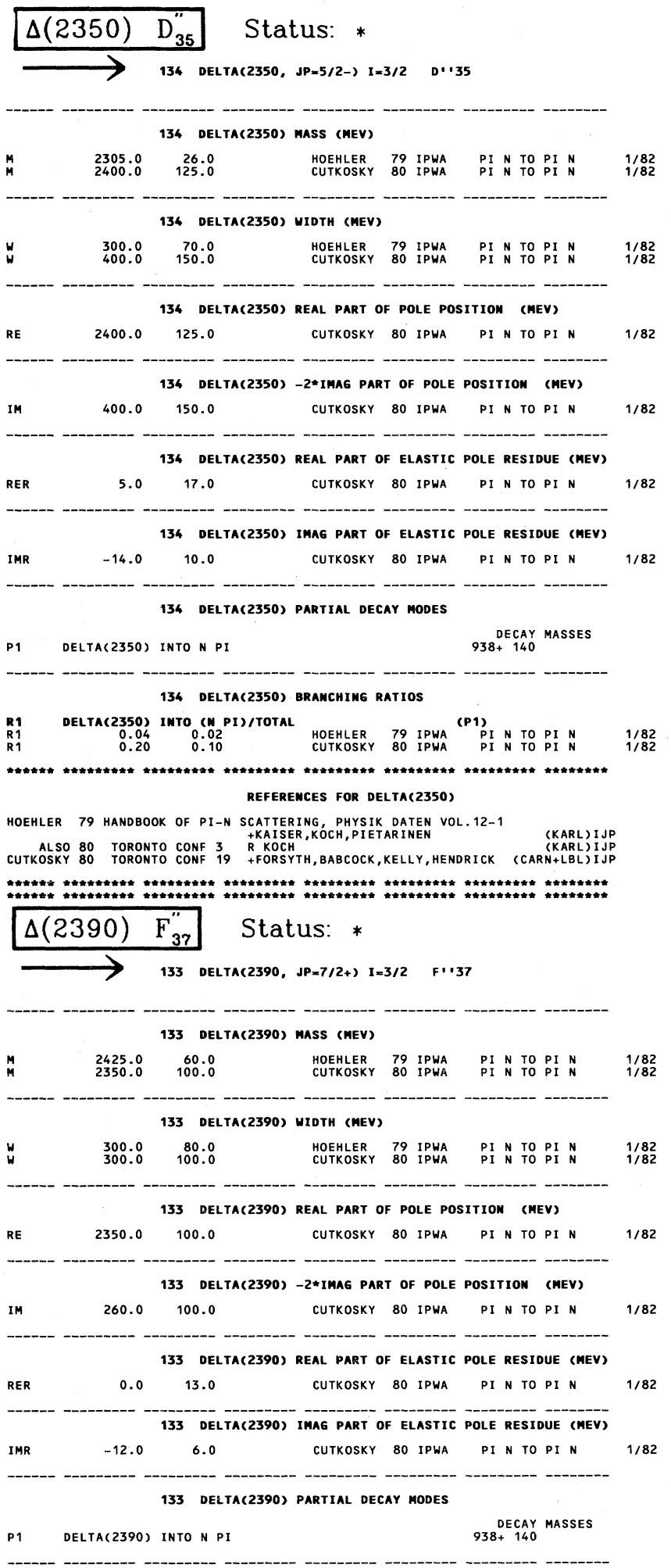


Baryons

$\Delta(2390), \Delta(2400), \Delta(2420), \Delta(2750)$

\section{Data Card Listings}

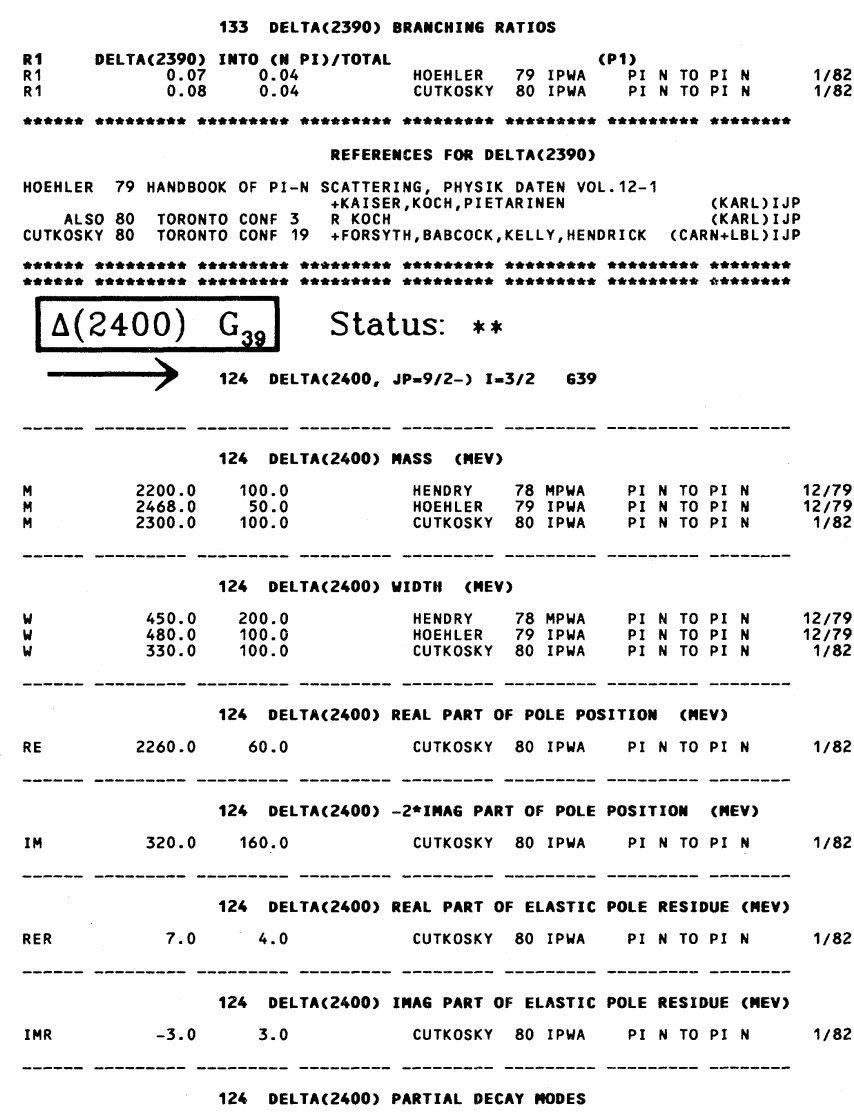

P1 DELTA(2400) INTO N PI $938+140$

124 DELTA(2400) BRaMCHIMG Ratios

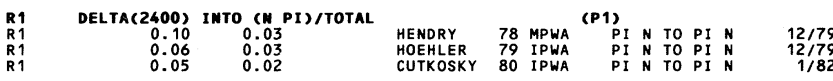

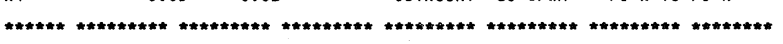
REFERENCES FOR DELTAC2400)

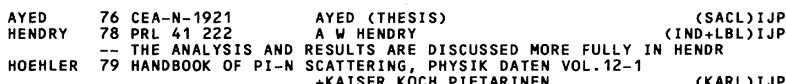

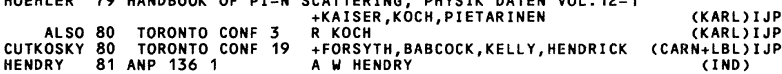

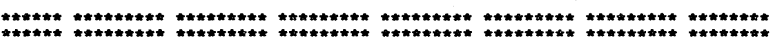

\section{$\Delta(2420) \quad \mathrm{H}_{31} \quad$ Status: ****}

84 DELTA(2420, JP=11/2+) I=3/2 H3 11

MOST OF THE RESULTS PUBLISHED BEFORE 1975 ARE NOW
OBSOLETE AND HAVE BEEN OMITTED. THEY MAY BE FOUND IN OUR 1982 EDITION (PHSSICS LETTERS 111 ). HOWEVER,
ALL THE REFERENES HAVE BEEN RETAINED

IN ADDITION, RESULTS IN THIS REGION FROM PRODUCTION EXPERIMENTS,
WHICH USED TO BE LISTED SEPARATELY AS THE NEXT ENTRY, HAVE BEEN

-

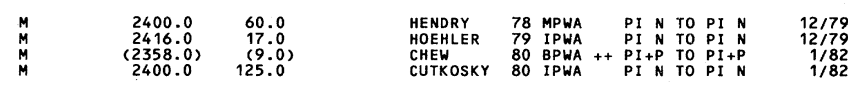

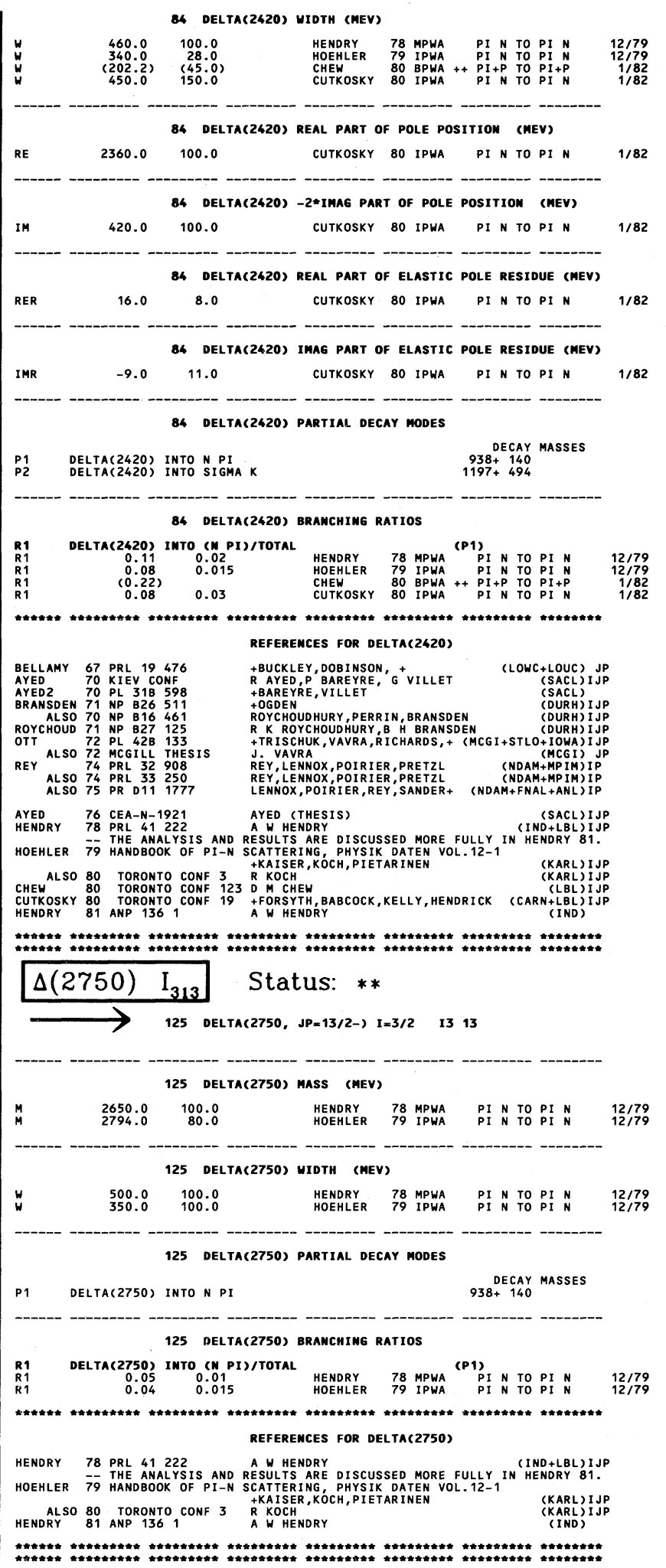




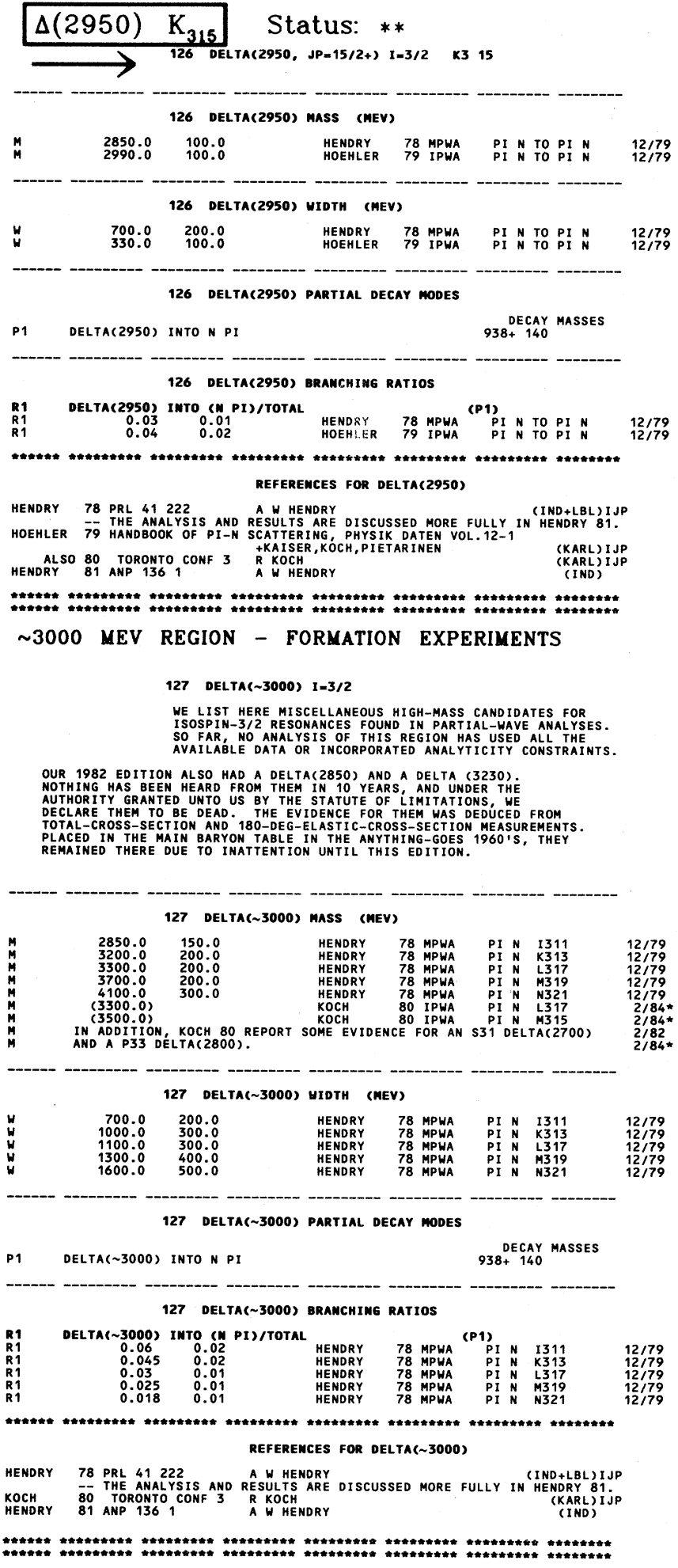

\section{NOTE ON THE $S=$ +1 BARYON SYSTEM}

The evidence for strangeness +1 baryon resonances was thoroughly reviewed in our 1976 edition, ${ }^{1}$ and has been reviewed more recently by Kelly ${ }^{2}$ and by Oades. ${ }^{3}$ One new partial-wave analysis ${ }^{4}$ has been published since our 1982 edition. As usual, the results permit no definite conclusion - the same story heard for 15 years. The general feeling, supported by the prejudice against baryons not make up of three quarks, is that the suggestive counterclockwise movement in the Argand diagram of some of the partial waves is not real evidence for true Breit-Wigner resonances. But until the dynamics of the $\mathrm{KN}$ system is better understood, the possibility that $\mathrm{Z}^{*}$ resonances exist will not be finally laid to rest.

\section{References}

1. Particle Data Group, Rev. Mod. Phys. 48, S188 (1976).

2. R.L. Kelly, in Proceedings of the Meeting on Exotic Resonances (Hiroshima, 1978), ed. I. Endo et al.

3. G.C. Oades, in Low and Intermediate Energy Kaon-Nucleon Physics (1981), ed. E. Ferrari and G. Violini.

4. K. Nakajima et al., Phys. Lett. 112B, 80 (1982).

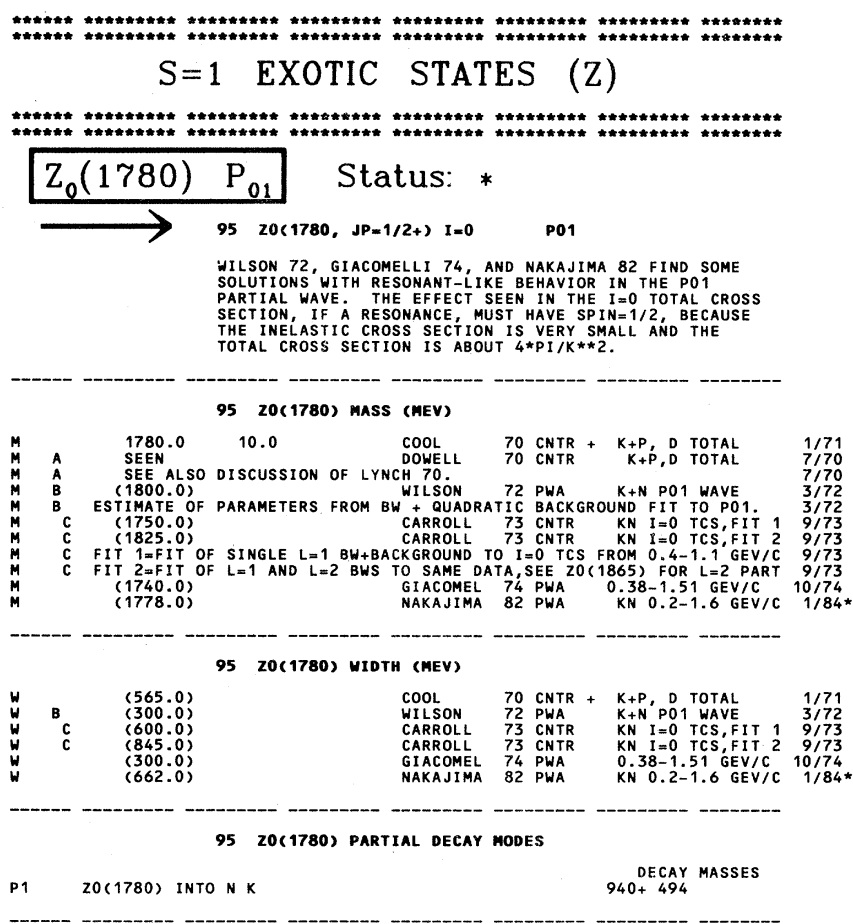




\section{Baryons}

$Z_{0}(1780), Z_{0}(1865), Z_{1}(1900)$

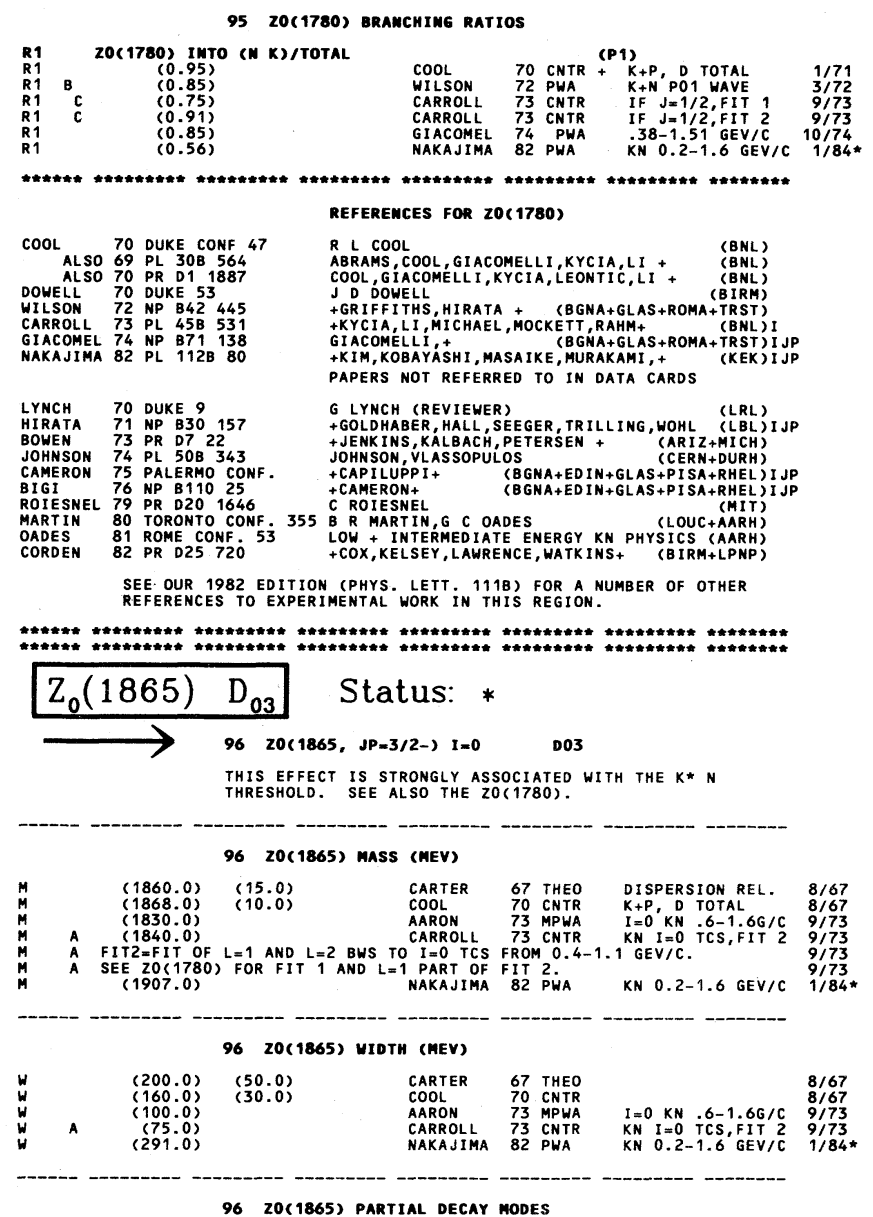

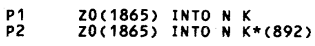

DECAY MASSES

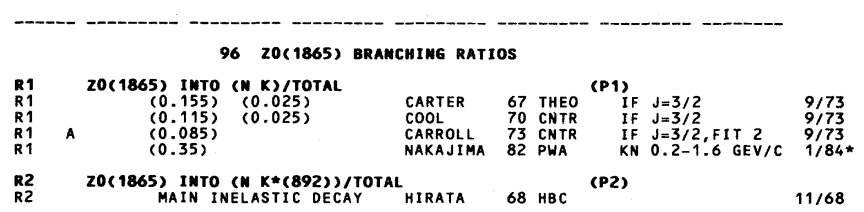

Data Card Listings

P1
P2
Z1(1900) INTO N 1900$)$ INTO N $K$ DLTA(1232) K

DECAY MASSES

$\stackrel{\mathrm{Z}_{1}(1900)}{\longrightarrow} \mathrm{P}_{13}$ Status:

THIS EFFECT IS STRONGLY ASSOCIATED WITH THE K-DELTA

$9721(1900)$ MASS (MEV)

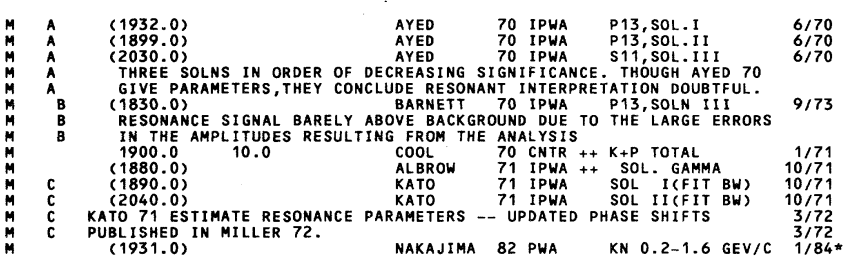

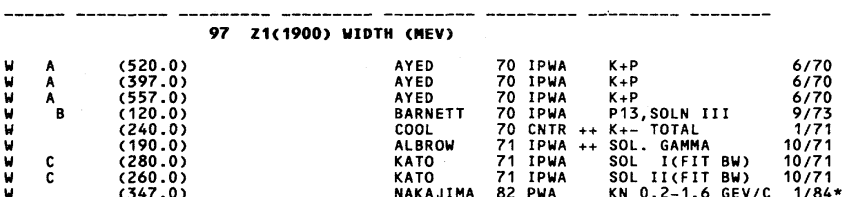

97 21(1900) REAL PART OF POLE POSITION 4 ARNDT 74 DPWA K+ P ELASTIC $4 / 75$

\begin{tabular}{llllll} 
RE & $D$ & $(1787.0)$ \\
RE & SUPERSEED BY ARNDT 78. & ARNDT & 74 DPWA & $K+P$ ELASTIC & $4 / 75$ \\
RE & $(1796.0)$ & ARNDT & 78 DPWA & $K+P$ & 3779 \\
\hline
\end{tabular}

\begin{tabular}{|c|c|c|c|c|c|c|}
\hline & & 97 & $21(1900)$ & MAGIMARY & RT OF POLE & POSIIION \\
\hline $\operatorname{IM}_{I M} D$ & $\begin{array}{l}(100.0) \\
(101.0)\end{array}$ & & & $\begin{array}{l}\text { ARNDT } \\
\text { ARNDT }\end{array}$ & $\begin{array}{l}74 \text { DPWA } \\
78 \text { DPWA }\end{array}$ & $\begin{array}{c}K_{+} P \\
K_{+}\end{array}$ \\
\hline
\end{tabular}

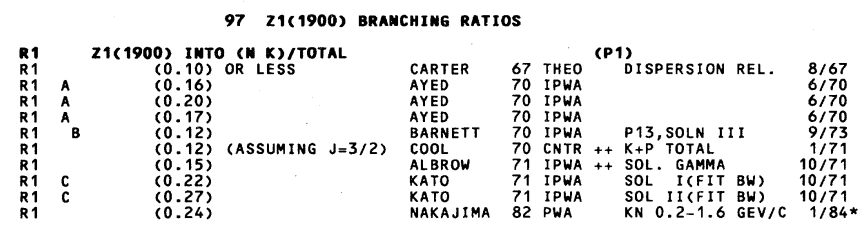

R2 Z1(1900) IMTO (DELTA(1232) K)/TOTAL

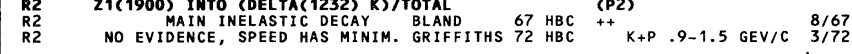

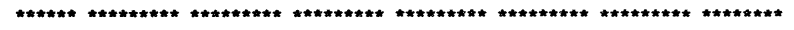

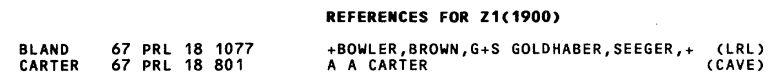

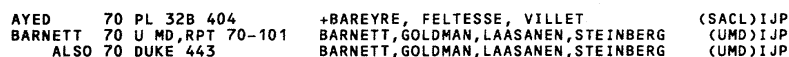

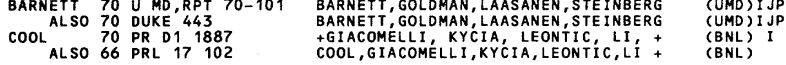

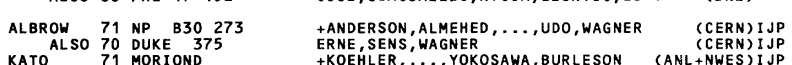

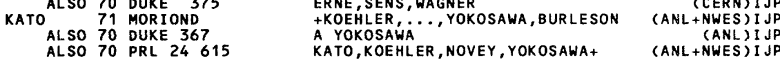

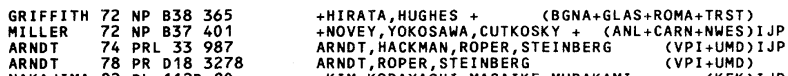

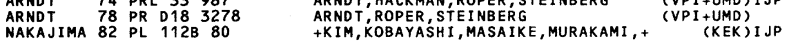

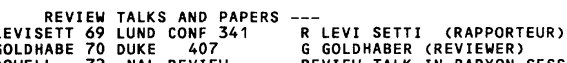

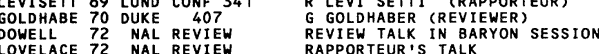

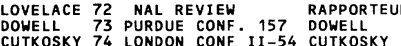


KELLYY 75 ANL-HEP-CP-75-58 REVIEE TALK IN BARYON SESSION
URBAN 75 PL 608 77

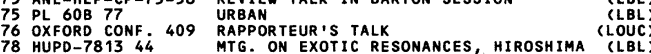

78 HUPD-7813 44 MT MTG. ON EXOTIC RESONANCES, HIROSHIMA (LBL)
81 ROME CONF. 53 LOW INTERMEDATE ENERGY KN PHYSICS (AARH)

SEE ALSO OUR 1982 EDITION (PHYS. LETT. 1118 ) FOR A LARGE NUMBER OF
OTHER REFERENCES TO THEORETICAL AND EXPERIMENTAL WORK IN THIS REGION.

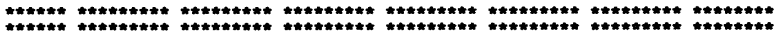

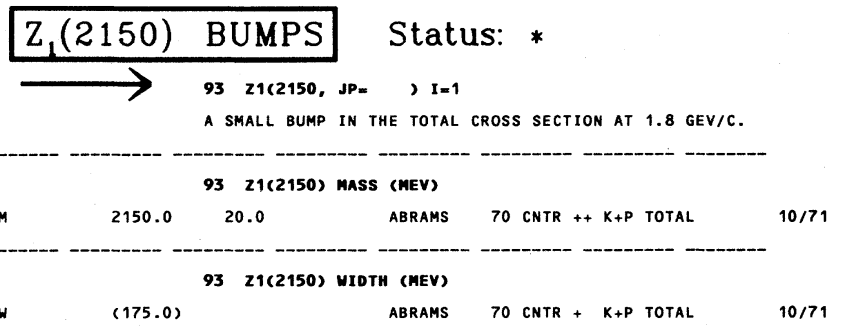

$\begin{array}{lll}\text { (175.0) } & \text { ABRAMS } 70 \mathrm{CNTR}+\mathrm{K}+\mathrm{P} \text { TOTAL } & 10 / 71\end{array}$

93 Z1(2150) PARTIAL DECAY MODES

P1 Z1(2150) INTO $N$ DECAY MASSES

93 21(2150) BRAMCHING RATIOS

R1
R1 1 (2150) THTO (M K)/TOTAL
R1 N NOT KNOWN, THE FOLLOWING IS $(J+1 / 2) * P 1 \quad$ (P1)

70 CNTR + K+P TOTAL $10 / 71$

********************************************************************* REFERENCES FOR $21(2150)$

$\begin{array}{cllll}\text { ABRAMS } & 70 \text { PR } & 01 & 1917 & \text { +COOL, GIACOMELLI, KYCIA, LEONTIC, LI + } \\ \text { ALSO } & 67 & \text { PRL } 19257 & \text { (BNL) }\end{array}$

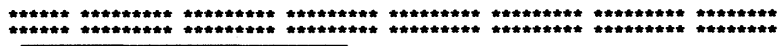

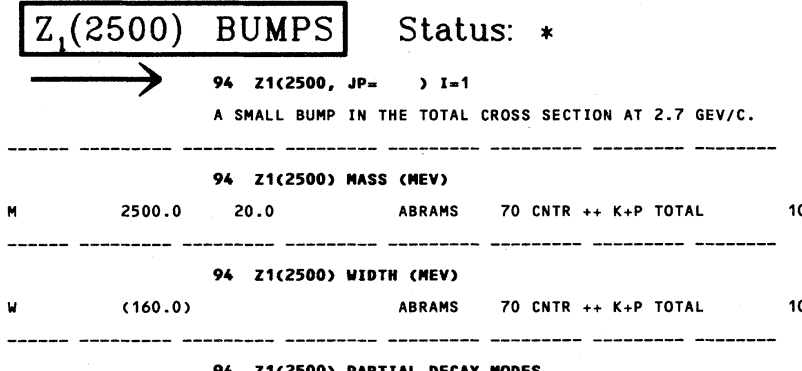

94 Z1(2500) PARTIAL DECAY MODES

P1 Z1(2500) INTO N K PECAY MASSES

94 Z1(2500) BRAMCHING RATIOS

R1 $21(2500)$ INTO (N) K)/TOTAL
R1
R1 IS NOT KNOWN, THE FOLLOWING IS (J+1/2)*P1
( 10.03$)$

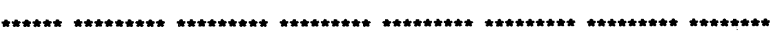

REFERENCES FOR Z1(2500)

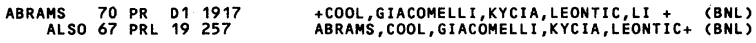

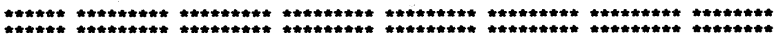

\section{NOTE ON $\triangle$ AND $\Sigma$ RESONANCES}

\section{Introduction}

Progress in $\mathrm{Y}^{*}$ 's has ground to a halt. Whether the field is dead or is merely in suspended animation, to be revived eventually at the lower energy accelerators such as KEK and TRIUMF, remains to be seen. Since the 1982 edition, there has been a paper giving new data on
$\mathrm{K}^{-} \mathrm{n}$ elastic scattering, ${ }^{1}$ and that is it: no new partialwave analyses, no new $Y^{*}$ 's promoted to the Baryon Table (in fact, we have removed three - see below). Nor does there appear to be a single new experiment on $\mathbf{Y}^{*}$ 's, planned or in progress, anywhere in the world.

Table 1 is an attempt to evaluate the status, both overall and channel by channel, of each $\mathrm{Y}^{*}$ in the Listings; the evaluations are of course partly subjective. A blank indicates there is no evidence at all: either the relevant couplings are small or the resonance does not really exist. The main Baryon Table includes only the established resonances (overall status 3 or 4 stars). We have reduced the $\Lambda(2585)$, the $\Sigma(2455)$, and the $\Sigma(2620)$

Table 1. The status of the $\Lambda$ and $\Sigma$ resonances. Only those with an overall status of $* * *$ or $* * * *$ are included in the main Baryon Table.

\begin{tabular}{|c|c|c|c|c|c|c|}
\hline \multirow[b]{2}{*}{ Particle } & \multirow[b]{2}{*}{$\mathrm{L}_{\mathrm{I} \cdot 2 \mathrm{~J}}$} & \multirow[b]{2}{*}{$\begin{array}{l}\begin{array}{l}\text { Overall } \\
\text { status }\end{array} \\
\end{array}$} & \multicolumn{4}{|c|}{ Status as seen in -. } \\
\hline & & & $\mathrm{N} \overline{\mathrm{K}}$ & $\Lambda \pi$ & $\Sigma \pi$ & Other channels \\
\hline$\Lambda(1116)$ & $\mathrm{P}_{01}$ & $* * * *$ & & & & $\mathrm{~N} \pi$ (weakly) \\
\hline$\Lambda(1405)$ & $\mathrm{S}_{01}^{01}$ & $* * * *$ & $* * * *$ & $\mathrm{~F}$ & $* * * *$ & \\
\hline$\Lambda(1520)$ & $\mathrm{D}_{03}$ & $* * * *$ & $* * * *$ & 0 & $* * * *$ & $\Lambda \pi \pi, \Lambda \gamma$ \\
\hline$\Lambda(1600)$ & $P_{01}$ & $* * *$ & $* * *$ & $\mathrm{r}$ & ** & \\
\hline$\Lambda(1670)$ & $\mathrm{S}_{01}$ & $* * * *$ & $* * * *$ & b & $* * * *$ & $\Lambda \eta$ \\
\hline$\Lambda(1690)$ & $\mathrm{D}_{03}$ & $* * * *$ & $* * * *$ & $\mathrm{i}$ & $* * * *$ & $\Lambda \pi \pi, \Sigma \pi \pi$ \\
\hline$\Lambda(1800)$ & $S_{01}$ & $* * *$ & $* * *$ & d & ** & $\mathrm{N} \underline{\bar{K}}^{*}, \Sigma(1385) \pi$ \\
\hline$\Lambda(1800)$ & $\mathrm{P}_{01}$ & $* * *$ & $* * *$ & d & ** & $\mathrm{NK}^{*}$ \\
\hline$\Lambda(1820)$ & $\mathrm{F}_{05}$ & $* * * *$ & $* * * *$ & e & $* * * *$ & $\Sigma(1385) \pi$ \\
\hline$\Lambda(1830)$ & $\mathrm{D}_{05}$ & $* * * *$ & $* * *$ & $\mathrm{n}$ & $* * * *$ & $\Sigma(1385) \pi$ \\
\hline$\Lambda(1890)$ & $P_{03}$ & $* * * *$ & $* * * *$ & $\mathrm{~F}$ & ** & $\mathrm{NK}^{*}, \Sigma(1385) \pi$ \\
\hline$\Lambda(2000)$ & & $*$ & & 0 & $*$ & $\Lambda \omega, \mathrm{NK}^{*}$ \\
\hline$\Lambda(2020)$ & $F_{07}$ & $*$ & * & $\mathrm{r}$ & * & \\
\hline$\Lambda(2100)$ & $\mathrm{G}_{07}$ & $* * * *$ & $* * * *$ & $\mathrm{~b}$ & $* * *$ & $\Lambda \omega, \mathrm{NK}^{*}$ \\
\hline$\Lambda(2110)$ & $F_{05}$ & $* * *$ & ** & $\mathrm{i}$ & $*$ & $\Lambda \omega, \mathrm{N}^{*}$ \\
\hline$\Lambda(2325)$ & $\mathrm{D}_{03}$ & $*$ & * & d & & $\Lambda \omega$ \\
\hline$\Lambda(2350)$ & & $* * *$ & $* * *$ & d & $*$ & \\
\hline$\Lambda(2585)$ & & $* *$ & $* *$ & $\begin{array}{l}\mathrm{e} \\
\mathrm{n}\end{array}$ & & \\
\hline
\end{tabular}

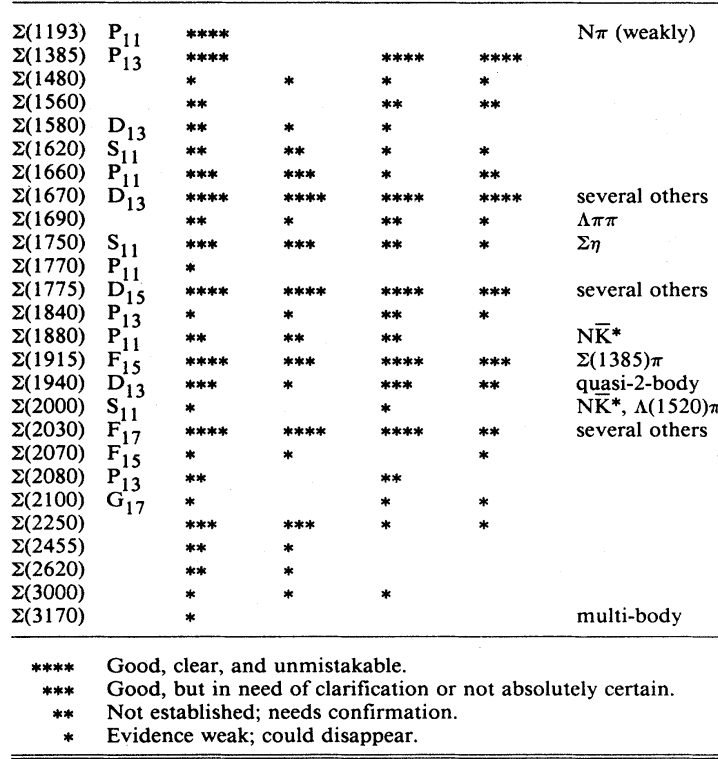




\section{Baryons}

$\Lambda$ 's and $\Sigma$ 's

\section{Data Card Listings}

from 3-star to 2-star status and removed them from the main Table: they are seen only as ripples in isospinunfolded total cross sections, and nothing at all has been learned about them since 1970. Several of the 1- and 2star resonances may eventually disappear, but there are probably many resonances yet to be discovered underlying the established ones.

None of the $\mathrm{Y}^{*}$ 's proposed in the last decade couple strongly to the main 2-body decay channels $\mathrm{N} \overline{\mathrm{K}}, \Lambda \pi$, and $\Sigma \pi$, and thus they seldom appear in cross sections or invariant mass distributions. However, when the reactions $\overline{\mathrm{K}} \mathrm{N} \rightarrow \overline{\mathrm{K}} \mathrm{N}, \overline{\mathrm{K}} \mathrm{N} \rightarrow \Lambda \pi$, and $\overline{\mathrm{K}} \mathrm{N} \rightarrow \Sigma \pi$ are partial-wave analyzed, some of the amplitudes are found to traverse small, more-or-less resonance-like counterclockwise circles. The question in each case is: Is this really a resonance, or is it an idle meander? Is the effect even real, or is it the result of imperfect data and analysis procedures?

What follows is the review of $Y^{*}$ 's, somewhat revised, that appeared in the 1982 edition: it summarizes "recent" progress and problems. (For another brief overview, see Tripp. ${ }^{2}$ ) In the Data Card Listings, some obsolete results, nearly all from before 1975 , have been removed. This has been done only for the established $Y^{*}$ 's, where the addition of much improved data to partial-wave analyses has really made obsolete the older results. Where little new has been learned in the last decade [such as for the $\Lambda(1405)$ ], or where the situation is uncertain, nothing has been removed.

\section{Formation experiments}

\section{(by G.P. Gopal, Rutherford Appleton Laboratory)}

Partial-wave analyses have been made mainly for the $\mathrm{N} \overline{\mathrm{K}}, \Lambda \pi$, and $\Sigma \pi$ channels, but there are also a few results for the $\Xi K, \Lambda \omega$, and some quasi-2-body channels. The early analyses usually covered only the range of a single bubble chamber experiment. Although the amplitudes obtained often did not join smoothly with those from analyses in neighboring mass ranges, they did give fairly reliable information about the strongly coupled resonances. The more recent analyses have used the Breit-Wigner forms of these dominant resonances as input to provide constraints in determining the overall amplitudes and thus to get information about the less strongly coupled resonances. Besides covering wider ranges, some of the more ambitious of the analyses at the lower energies have treated several channels simul- taneously, so that unitarity constraints are automatically satisfied and only a single mass and width is obtained for each resonance.

In the mid and late 1970's, a large amount of new data became available. Results from several large $\mathrm{K}^{-} \mathrm{p}$ bubble chamber experiments were published. ${ }^{3-6}$ Other bubble chamber experiments studied $\mathrm{K}^{-} \mathrm{n}$ reactions ${ }^{7}$ and $K_{L}^{0} p$ reactions. ${ }^{8}$ Counter experiments measured the $\mathbf{K}^{-} \mathbf{p} \rightarrow \overline{\mathbf{K}}^{0} \mathbf{n}$ total and differential cross sections at low energies, ${ }^{9}$ the $\mathrm{K}^{-} \mathrm{p}$ polarizations down to $1630 \mathrm{MeV}$ for the first time, ${ }^{10}$ the $K^{-}$p polarizations from 1700 to $1900 \mathrm{MeV}$ with an order of magnitude increase in statistics, ${ }^{11}$ the $\mathrm{K}^{-} \mathrm{n}$ elastic angular distributions from 1600 to $1800 \mathrm{MeV}^{12}$ and from 1900 to $2300 \mathrm{MeV},{ }^{13}$ and the $180^{\circ} \mathrm{K}^{-} \mathrm{p}$ and $0^{\circ} \mathrm{\Sigma}^{-} \pi^{+}$differential cross sections from 1550 to $1900 \mathrm{MeV} .^{14}$

In the following, we compare the more recent partial-wave analyses with each other and with the data. Some of the data have yet to be incorporated into any analysis.

The $N \bar{K}$ channel: The most recent analysis ${ }^{15}$ is an update of the old Rutherford Lab-Imperial College (RLIC 77) analysis. ${ }^{16}$ As before, it is a conventional energy-dependent analysis with the added constraint that the masses and widths of the resonances had to be consistent with those determined in the inelastic channels analyzed previously $-\Lambda \pi, \Sigma \pi, \Lambda(1520) \pi, \Sigma(1385) \pi$, and $N \overline{\mathrm{K}}^{*}(892)$. The analysis also goes closer to threshold: the range covered is 1470 to $2170 \mathrm{MeV}$. It includes all the $\mathrm{N} \overline{\mathrm{K}}$ data mentioned above except for the high-statistics charge-exchange counter measurements ${ }^{9}$ (which disagree with both the earlier and the latest ${ }^{4}$ high-statistics bubble chamber measurements), the backward elastic data, ${ }^{14}$ and the most recent $K^{-} n$ elastic data. ${ }^{1}$ As before, angular distributions (a total of 5110 data points) were fit directly. The new amplitudes are not very different from the RLIC 77 amplitudes for this channel. However, the $\mathrm{K}^{-} \mathrm{n}$ data removed some of the uncertainties in the $\Sigma$ spectrum.

The LBL-Mt. Holyoke-CERN analysis ${ }^{17}$ covers the narrower range of 1500 to $1940 \mathrm{MeV}$ and also includes most of the new data. It is an energy-dependent analysis using a unitary background parametrized in terms of scattering lengths. The cusp effects at the $\Lambda \eta$ and $\Sigma \eta$ thresholds are included by introducing a squareroot singularity in the energy variation of the widths of the appropriate resonances. This group's own high- 
For notation, see key at front of Listings.

statistics charge-exchange data ${ }^{9}$ (which do not agree with bubble chamber measurements) all but kill the less well-established resonances.

The University College, London (UCL) K-matrix energy-dependent analysis ${ }^{18}$ covers from 1540 to 2000 $\mathrm{MeV}$. The NK amplitudes are consistent with those of the other analyses over most of this range. However, at the low end there are major differences, indicating the absence of constraints from the $\Lambda(1520)$, which lies just outside the range covered. The $\mathrm{K}^{-} \mathrm{n}$ angular distributions and $\mathrm{K}^{-} \mathrm{p}$ polarization measurements are not very well described by this analysis.

The above analyses, all below $2200 \mathrm{MeV}$, are complemented by the College de France-Saclay (CdF-S) energy-dependent analysis ${ }^{6}$ covering from 2070 to 2440 $\mathrm{MeV}$. Besides the conventional polynomial parametrization of the background amplitudes, also tried is a parametrization using constraints imposed by the duality hypothesis (that s-channel backgrounds come exclusively from the $t$-channel Pomeron exchange amplitude). With 30 fewer free parameters, the results are consistent with the conventional approach.

The $\Sigma_{x}$ channel: There is very little agreement, particularly in the lower partial waves, between the two multichannel analyses. ${ }^{16,18}$ The low-energy $\mathrm{K}_{\mathrm{L}}^{0} \mathrm{p} \rightarrow \Sigma^{0} \pi^{+}$data $^{8}$ are better explained by the RLIC 77 amplitudes than by the UCL amplitudes. At the high end, there is good continuity between the RLIC 77 amplitudes and those from the single-channel analysis of the CdF-S collaboration ${ }^{6}$ covering from 2070 to 2440 $\mathrm{MeV}$. The $\Lambda(1520)$ and $\Lambda(2110)$ resonances, which lie outside the range covered by the UCL analysis, clearly provide strong constraints on the amplitudes.

The $\Lambda \pi$ channel: This isospin-1 channel has been the subject of many energy-dependent and -independent analyses (for example, RLIC 77, ${ }^{16} \mathrm{UCL},{ }^{18}$ BaillonLitchfield, ${ }^{19}$ de Bellefon-Berthon, ${ }^{20}$ and Van Horn ${ }^{21}$ ). However, even the widespread use of the method of Barrelet zeroes has not helped to resolve the $\Sigma$ spectrum - probably because most $\Sigma$ resonances simply do not couple strongly to the initial $\mathrm{N} \overline{\mathrm{K}}$ channel.

Quasi-2-body channels: The RLIC group has made energy-dependent analyses of the $\Lambda(1520) \pi, \Sigma(1385) \pi$, and $\mathrm{NK}^{*}(892)$ channels over the widest ranges for which data are available. The data were extracted from the appropriate 3-particle final states by making 4-variable fits to an incoherent superposition of quasi-2-body final states and 3-particle Lorentz-invariant phase space. The quality of the fits suggests a maximum model-dependent systematic uncertainty of $10 \%$. The $\Lambda \omega$ channel has been analyzed from threshold to $2440 \mathrm{MeV}$ by the CdF-S collaboration. 6

Sign conventions for resonance couplings: In terms of the isospin- 0 and -1 elastic scattering amplitudes $A_{0}$ and $A_{1}$, the amplitude for $K^{-} p \rightarrow \bar{K}^{0} n$ scattering is $\pm\left(A_{1}-A_{0}\right) / 2$, where the sign depends on conventions used in conjunction with the Clebsch-Gordan coefficients (such as, is the baryon or the meson the "first" particle). If this reaction is partial-wave analyzed and if the overall phase is chosen so that, say, the $D_{15} \Sigma(1775)$ amplitude at resonance points along the positive imaginary axis (points "up"), then any $\Sigma$ at resonance will point "up" and any $\Lambda$ at resonance will point "down" (along the negative imaginary axis). Thus the phase at resonance determines the isospin. The above ignores background amplitudes in the resonating partial waves.

That is the basic idea. In a similar but somewhat more complicated way, the phases of the $\bar{K} N \rightarrow \Lambda \pi$ and $\overline{\mathbf{K}} \mathrm{N} \rightarrow \Sigma \pi$ amplitudes for a resonating partial wave help determine the SU(3) multiplet to which the resonance belongs. Again, a convention has to be adopted for some overall arbitrary phases: which way is "up"? Our convention is that of Levi-Setti ${ }^{22}$ and is shown in Fig. 1 , which also compares experimental results with theoretical predictions for the signs of several other resonances. In the Listings, a + or - sign in front of a measurement of an inelastic resonance coupling indicates the sign (the absence of a sign means that the sign is not determined, not that it is positive). For more details, see Appendix II of our 1982 edition. ${ }^{23}$

Argand plots: Figure 2 shows some representative Argand plots of partial-wave amplitudes. For the $\mathrm{N} \overline{\mathbf{K}}$ channel we show the amplitudes from RLIC $77^{16}$ and from LBL-Mt. Holyoke-CERN, ${ }^{17}$ and for the $\Lambda \pi$ and $\Sigma \pi$ channels we show those from RLIC $77^{16}$ and from UCL. 18

Errors on masses and widths: The errors quoted on resonance parameters from partial-wave analyses are often only statistical, and the parameters can change by more than these errors when a different parametrization 


\section{Baryons}

Data Card Listings

$\Lambda^{\prime} \mathrm{s}$ and $\Sigma^{\prime} \mathrm{s}$

of the waves is used. Furthermore, the different analyses use more or less the same data, so it is not really appropriate to treat the different determinations of the resonance parameters as independent or to average them together. In any case, the spread of the masses, widths, and branching fractions from the different analyses is certainly a better indication of the uncertainties than are the quoted errors. In the Baryon Table, usually a range reflecting the spread of the values is given rather than a particular value with error.

For three states, the $\Lambda(1520)$, the $\Lambda(1820)$, and the $\Sigma(1775)$, there is enough information to make an overall fit to the various branching fractions. It is then necessary to use the quoted errors, but the errors obtained from the fit should not be taken seriously.

\section{Production experiments}

Partial-wave analyses of course separate partial waves, whereas a peak in a cross section or an invariant mass distribution usually cannot be disentangled from background and analyzed for its quantum numbers; and more than one resonance may be contributing to the peak. Results from partial-wave analyses and from production experiments are generally kept separate in the Listings, and in the Baryon Table results from production experiments are used only for the low mass states: the $\Sigma(1385)$ and $\Lambda(1405)$ of course lie below the $\bar{K} N$ threshold and everything about them comes from pro- duction experiments; and production and formation experiments agree quite well in the case of $\Lambda(1520)$ and results have been combined. There is some disagreement between production and formation experiments in the 1600-1700-MeV region: see the Note on the $\Sigma(1670)$.

\section{References}

1. O. Braun et al., Nucl. Phys. B203, 349 (1982).

2. R.D. Tripp, in Proceedings of the Third LAMPF II Workshop (Los Alamos, 1983), Vol. II, p. 635.

3. T.S. Mast et al., Phys. Rev. D14, 13 (1976), and references cited therein.

4. B. Conforto et al., Nucl. Phys. B105, 189 (1976); and W. Cameron et al., Nucl. Phys. B193, 21 (1981).

5. R.J. Hemingway et al., Nucl. Phys. B91, 12 (1975).

6. A. de Bellefon et al., Nuovo Cim. 42A, 403 (1977); Nuovo Cim. 37A, 175 (1977); Nucl. Phys. B90, 1 (1975); and Nuovo Cim. 41A, 96 (1977).

7. M.J. Corden et al., Nucl. Phys. B125, 61 (1977).

8. A. Engler et al., Phys. Rev D18, 3061 (1978); W. Cameron et al., Nucl. Phys. B132, 189 (1978); and M.J. Corden et al., Nucl. Phys. B155, 13 (1979).

9. M. Alston-Garnjost et al., Phys. Rev. D17, 2216 (1978); and D17, 2226 (1978).

10. R.D. Ehrlich et al., Phys. Lett. 71B, 455 (1977).

11. H.C. Bryant et al., Nucl. Phys. B168, 207 (1980).

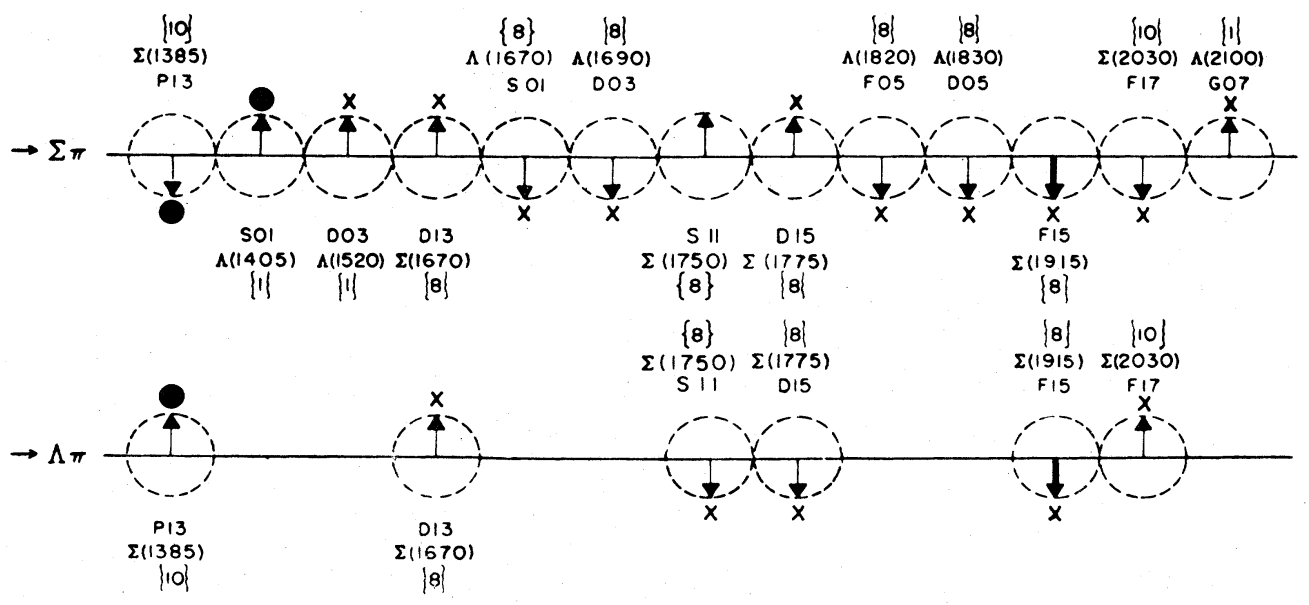

Fig. 1. The signs of the imaginary parts of resonating amplitudes in the $\bar{K} N \rightarrow \Lambda \pi$ and $\Sigma \pi$ channels. The signs of the $\Sigma(1385)$ and $\Lambda(1405)$, marked with a $\bullet$, are set by convention, and then the others are determined relative to them. The signs required by the SU(3) assignments of the resonances are shown with an arrow, and the experimentally determined signs are shown with an $X$. 

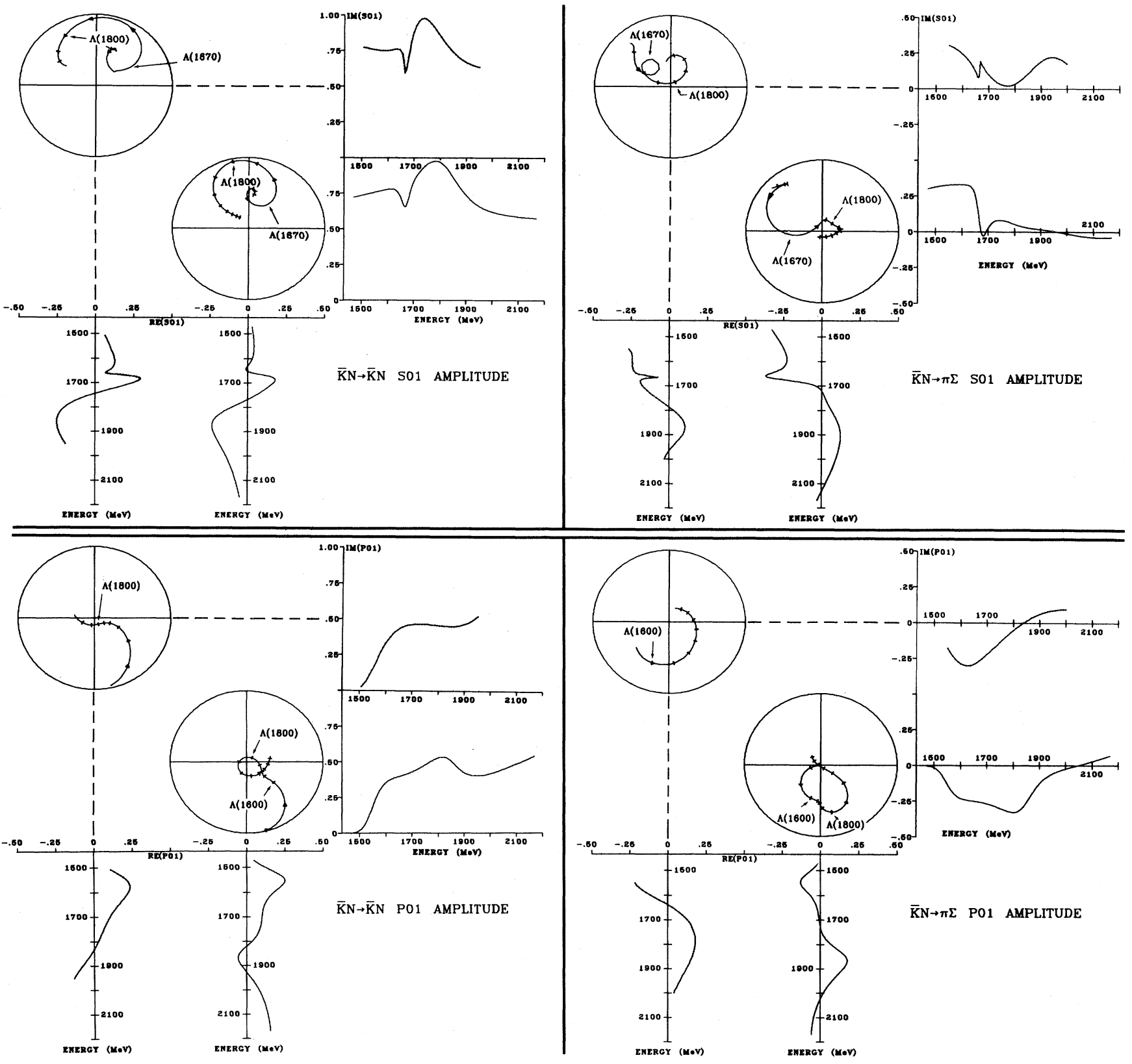

Fig. 2(a). The $\mathrm{L}_{\mathrm{I} \cdot 2 \mathrm{~J}}=\mathrm{S}_{01}$ and $\mathrm{P}_{01}$ partial-wave amplitudes for $\overline{\mathrm{K}} \mathrm{N}$ scattering in the elastic and $\Sigma \pi$ channels. The lower plot for each amplitude is from RLIC 77, the upper plots for the elastic amplitudes are from ALSTON 78, and the upper plots for the $\Sigma \pi$ amplitudes are from MARTIN 77. In the Argand plots, the ticks are at integral multiples of $50 \mathrm{MeV}$, and the established resonances are shown at their nominal positions [the $\mathrm{S}_{01}$ $\Lambda(1405)$ is of course below threshold and is not shown]. The real and imaginary parts of the amplitudes as functions of energy are shown projected in alignment with the Argand plots. 


\section{Baryons}

Data Card Listings

\section{$\Lambda$ 's and $\Sigma^{\prime} \mathrm{s}$}
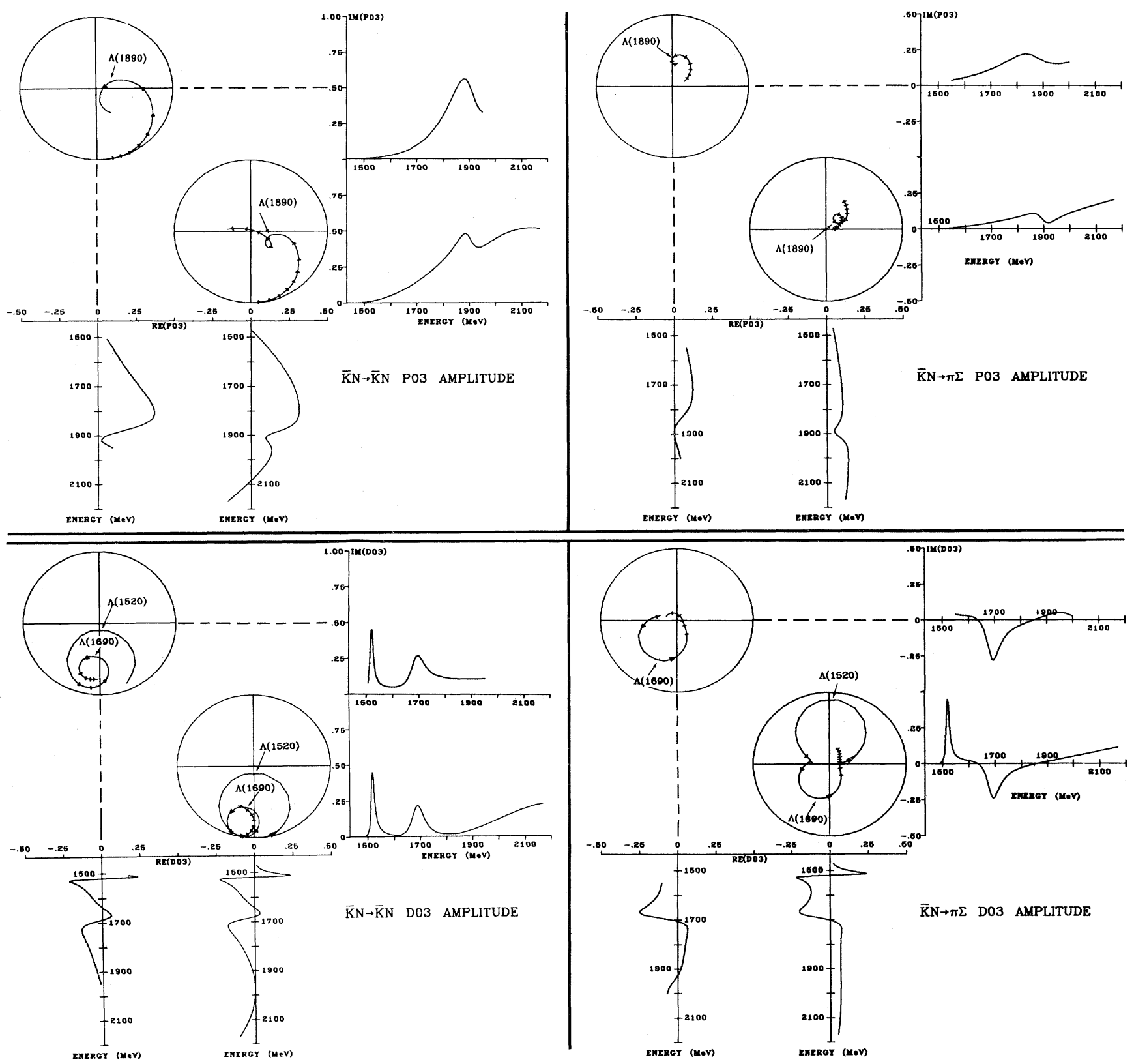

Fig. 2(b). The $\mathrm{L}_{\mathrm{I} \cdot 2 \mathrm{~J}}=\mathrm{P}_{03}$ and $\mathrm{D}_{03}$ partial-wave amplitudes for $\overline{\mathrm{K}} \mathrm{N}$ scattering in the elastic and $\Sigma \pi$ channels. The lower plot for each amplitude is from RLIC 77, the upper plots for the elastic amplitudes are from ALSTON 78 , and the upper plots for the $\Sigma \pi$ amplitudes are from MARTIN 77. In the Argand plots, the ticks are at integral multiples of $50 \mathrm{MeV}$, and the established resonances are shown at their nominal positions. The real and imaginary parts of the amplitudes as functions of energy are shown projected in alignment with the Argand plots. 

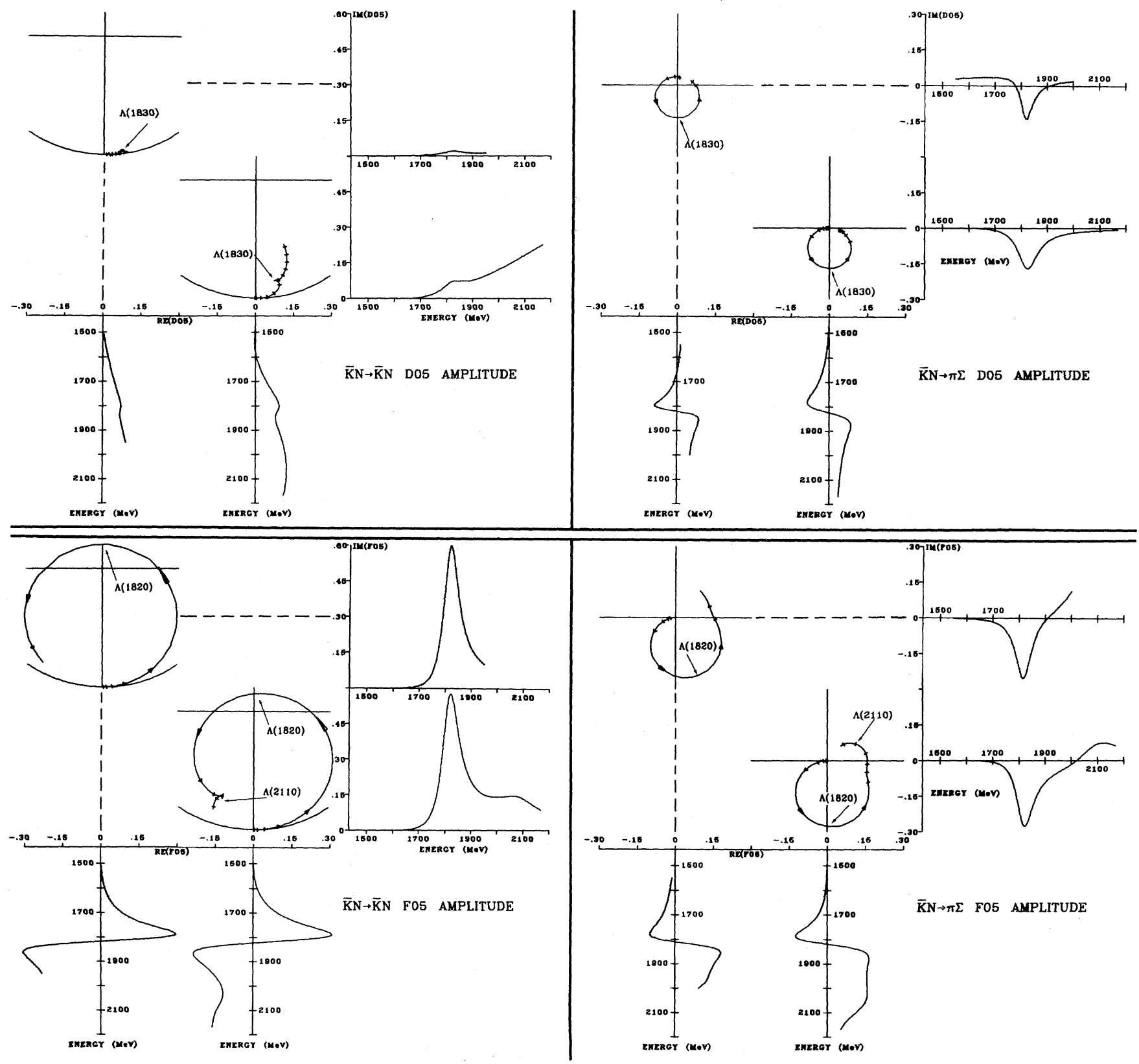

Fig. 2(c). The $\mathrm{L}_{\mathrm{I} \cdot 2 \mathrm{~J}}=\mathrm{D}_{05}$ and $\mathrm{F}_{05}$ partial-wave amplitudes for $\overline{\mathrm{K}} \mathrm{N}$ scattering in the elastic and $\Sigma \pi$ channels. The lower plot for each amplitude is from RLIC 77, the upper plots for the elastic amplitudes are from ALSTON 78, and the upper plots for the $\Sigma \pi$ amplitudes are from MARTIN 77. In the Argand plots, the ticks are at integral multiples of $50 \mathrm{MeV}$, and the established resonances are shown at their nominal positions. The real and imaginary parts of the amplitudes as functions of energy are shown projected in alignment with the Argand plots. 
Baryons

Data Card Listings

$\Lambda$ 's and $\Sigma$ 's
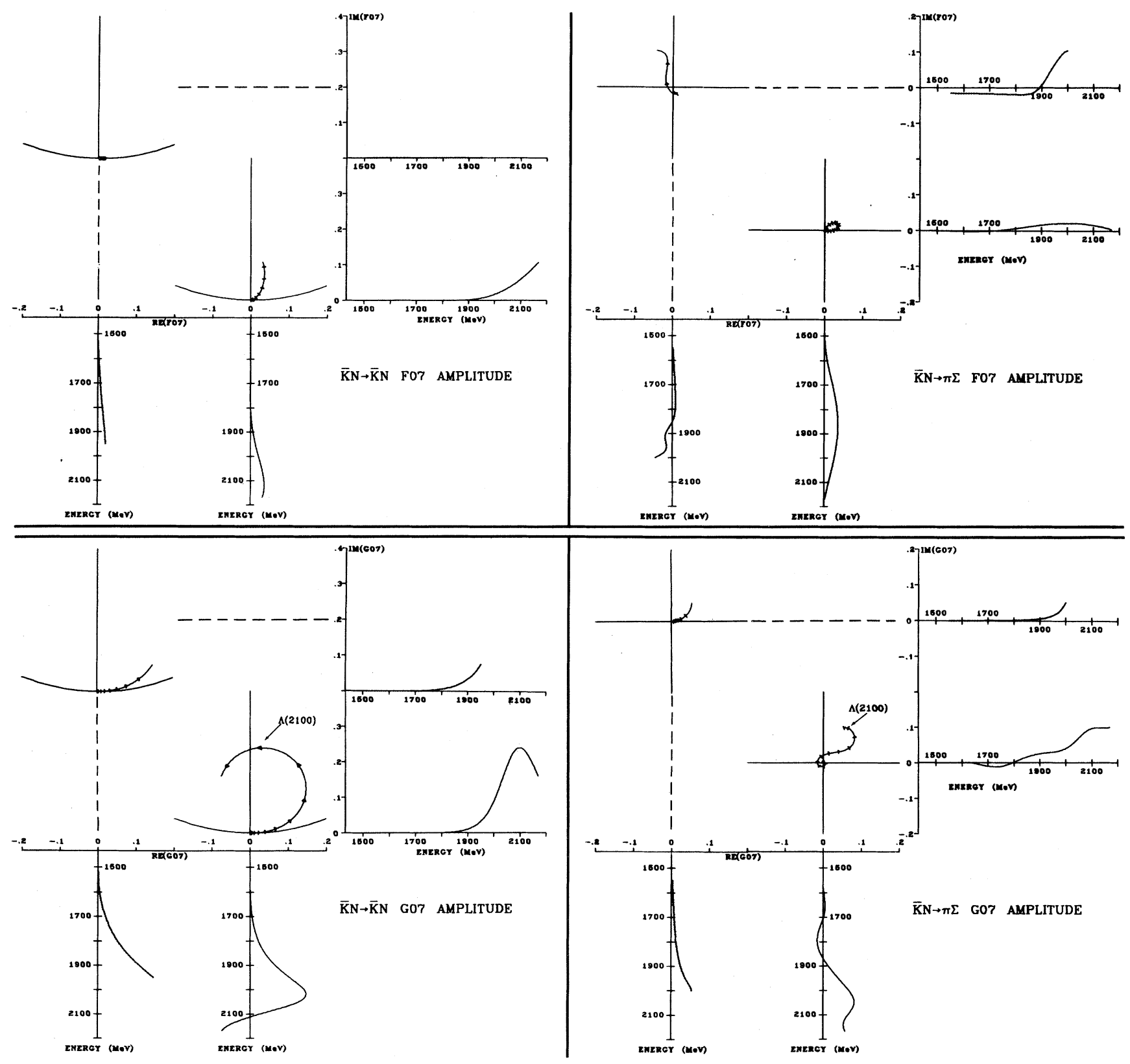

Fig. 2(d). The $\mathrm{L}_{\mathrm{I} \cdot 2 \mathrm{~J}}=\mathrm{F}_{07}$ and $\mathrm{G}_{07}$ partial-wave amplitudes for $\overline{\mathrm{K}} \mathrm{N}$ scattering in the elastic and $\Sigma \pi$ channels. The lower plot for each amplitude is from RLIC 77, the upper plots for the elastic amplitudes are from ALSTON 78 , and the upper plots for the $\Sigma \pi$ amplitudes are from MARTIN 77. In the Argand plots, the ticks are at integral multiples of $50 \mathrm{MeV}$, and the established resonance is shown at its nominal position. The real and imaginary parts of the amplitudes as functions of energy are shown projected in alignment with the Argand plots. 

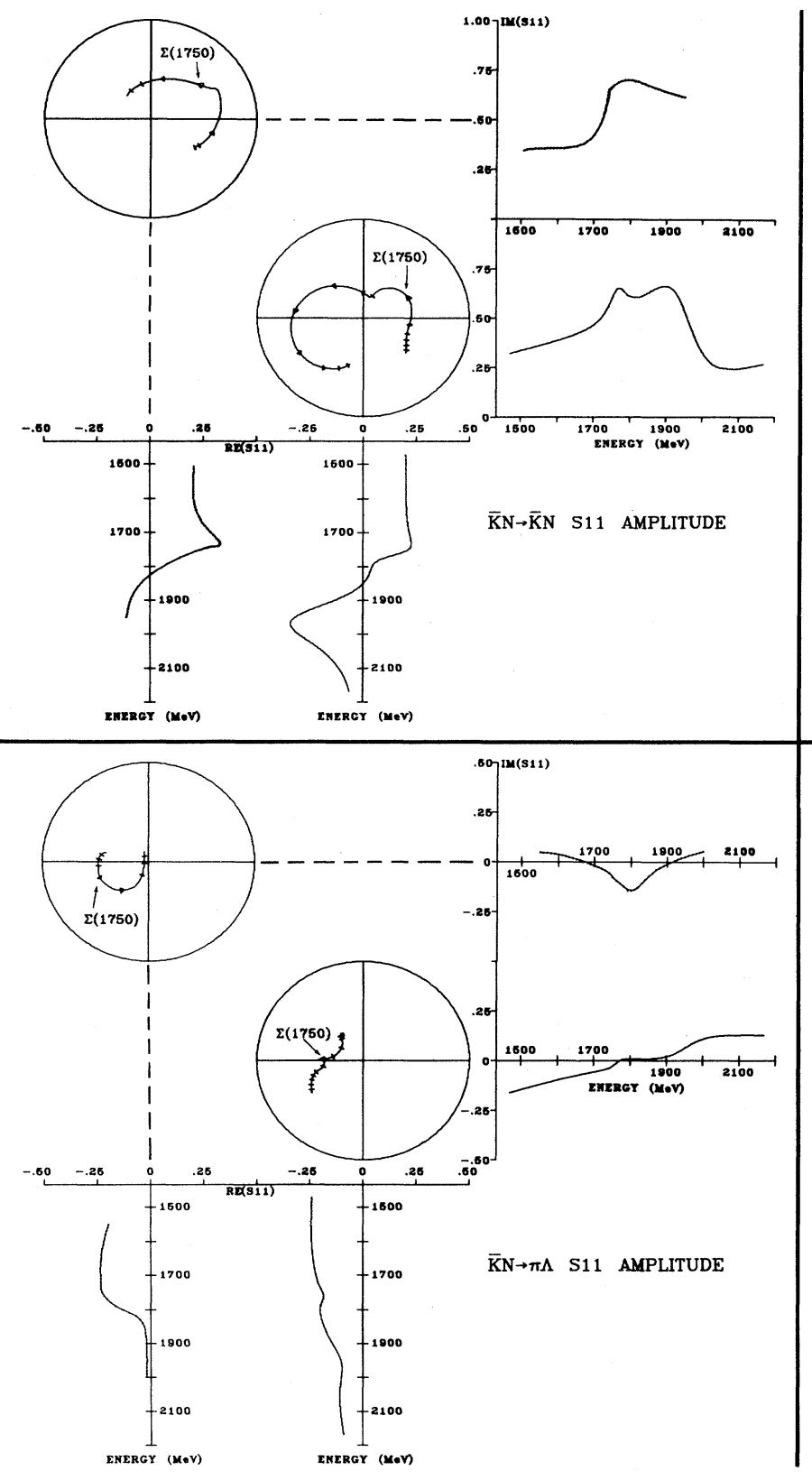

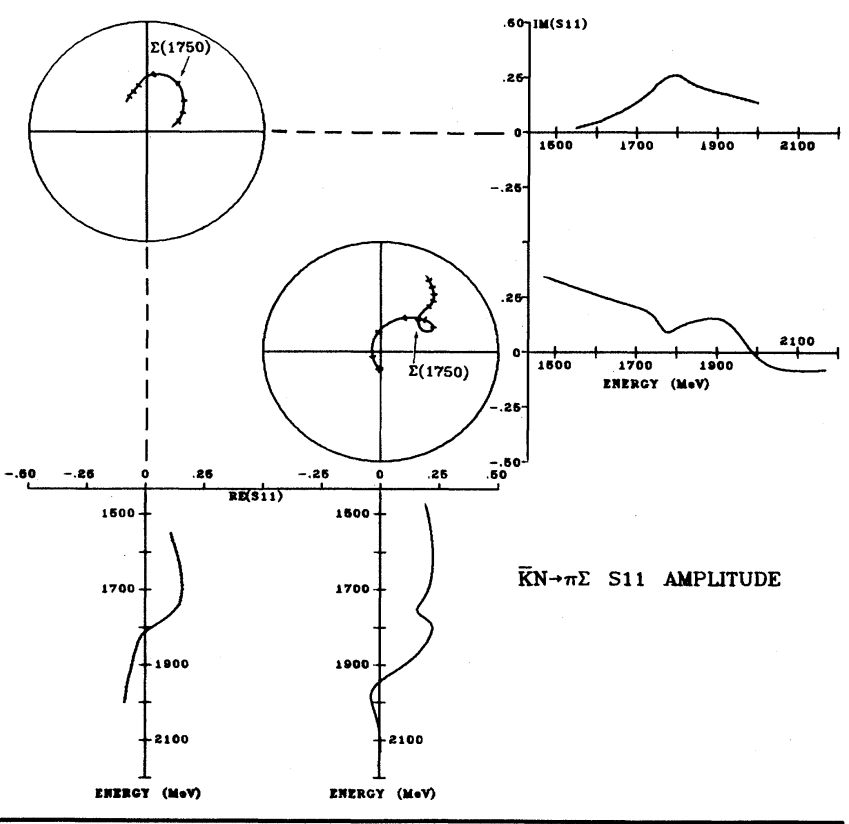

Fig. 2(e). The $L_{1 \cdot 2 J}=S_{11}$ partial-wave amplitudes for $\bar{K} N$ scattering in the elastic, $\Lambda \pi$, and $\Sigma \pi$ channels. The lower plot for each amplitude is from RLIC 77, the upper plot for the elastic amplitude is from ALSTON 78, and the upper plots for the $\Lambda \pi$ and $\Sigma \pi$ channels are from MARTIN 77. In the Argand plots, the ticks are at integral multiples of $50 \mathrm{MeV}$, and the established resonance is shown at its nominal position. The real and imaginary parts of the amplitudes as functions of energy are shown projected in alignment with the Argand plots. 
Baryons

Data Card Listings

$\Lambda^{\prime} \mathrm{s}$ and $\Sigma^{\prime} \mathrm{s}$
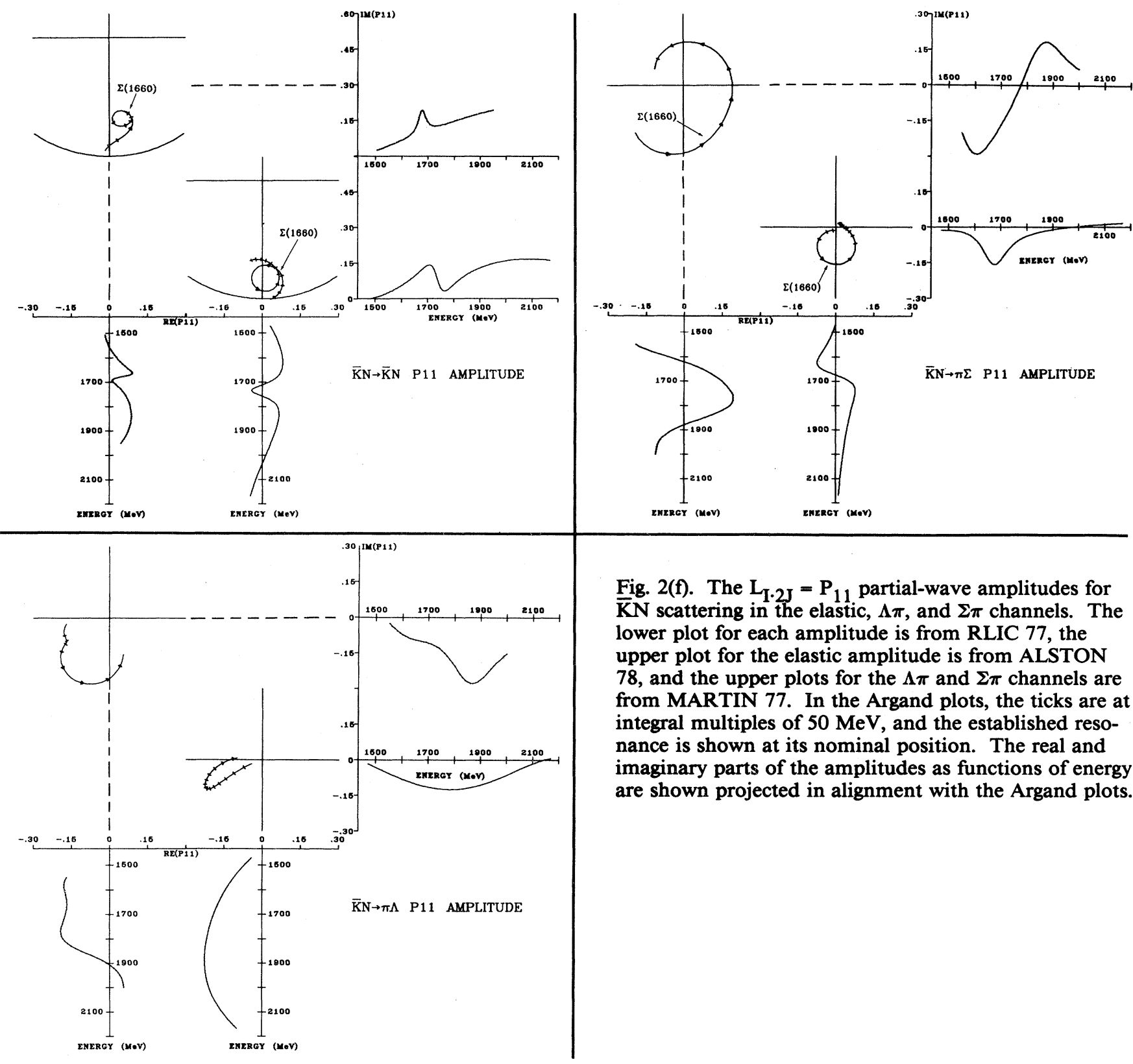

Fig. 2(f). The $\mathrm{L}_{\mathrm{I} \cdot 2 \mathrm{~J}}=\mathrm{P}_{11}$ partial-wave amplitudes for $\overline{\mathrm{K} N}$ scattering in the elastic, $\Lambda \pi$, and $\Sigma \pi$ channels. The lower plot for each amplitude is from RLIC 77, the upper plot for the elastic amplitude is from ALSTON 78 , and the upper plots for the $\Lambda \pi$ and $\Sigma \pi$ channels are from MARTIN 77. In the Argand plots, the ticks are at integral multiples of $50 \mathrm{MeV}$, and the established resonance is shown at its nominal position. The real and imaginary parts of the amplitudes as functions of energy are shown projected in alignment with the Argand plots. 

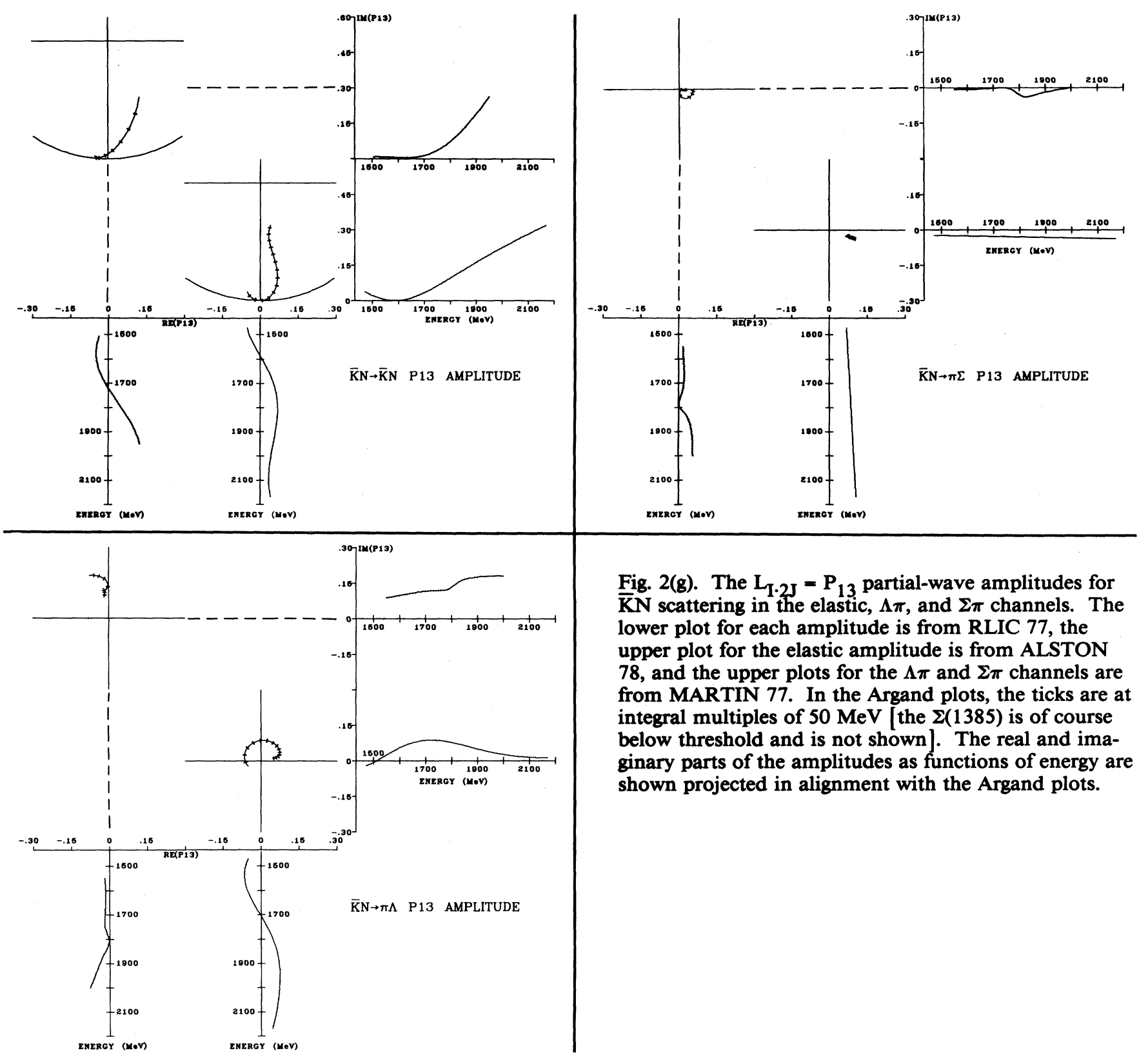

Fig. 2(g). The $L_{1 \cdot 2 J}=P_{13}$ partial-wave amplitudes for $\overline{\mathrm{K}} \mathrm{N}$ scattering in the elastic, $\Lambda \pi$, and $\Sigma \pi$ channels. The lower plot for each amplitude is from RLIC 77, the upper plot for the elastic amplitude is from ALSTON 78, and the upper plots for the $\Lambda \pi$ and $\Sigma \pi$ channels are from MARTIN 77. In the Argand plots, the ticks are at integral multiples of $50 \mathrm{MeV}$ [the $\Sigma(1385)$ is of course below threshold and is not shown]. The real and imaginary parts of the amplitudes as functions of energy are shown projected in alignment with the Argand plots. 


\section{Baryons}

Data Card Listings

$\Lambda$ 's and $\Sigma$ 's
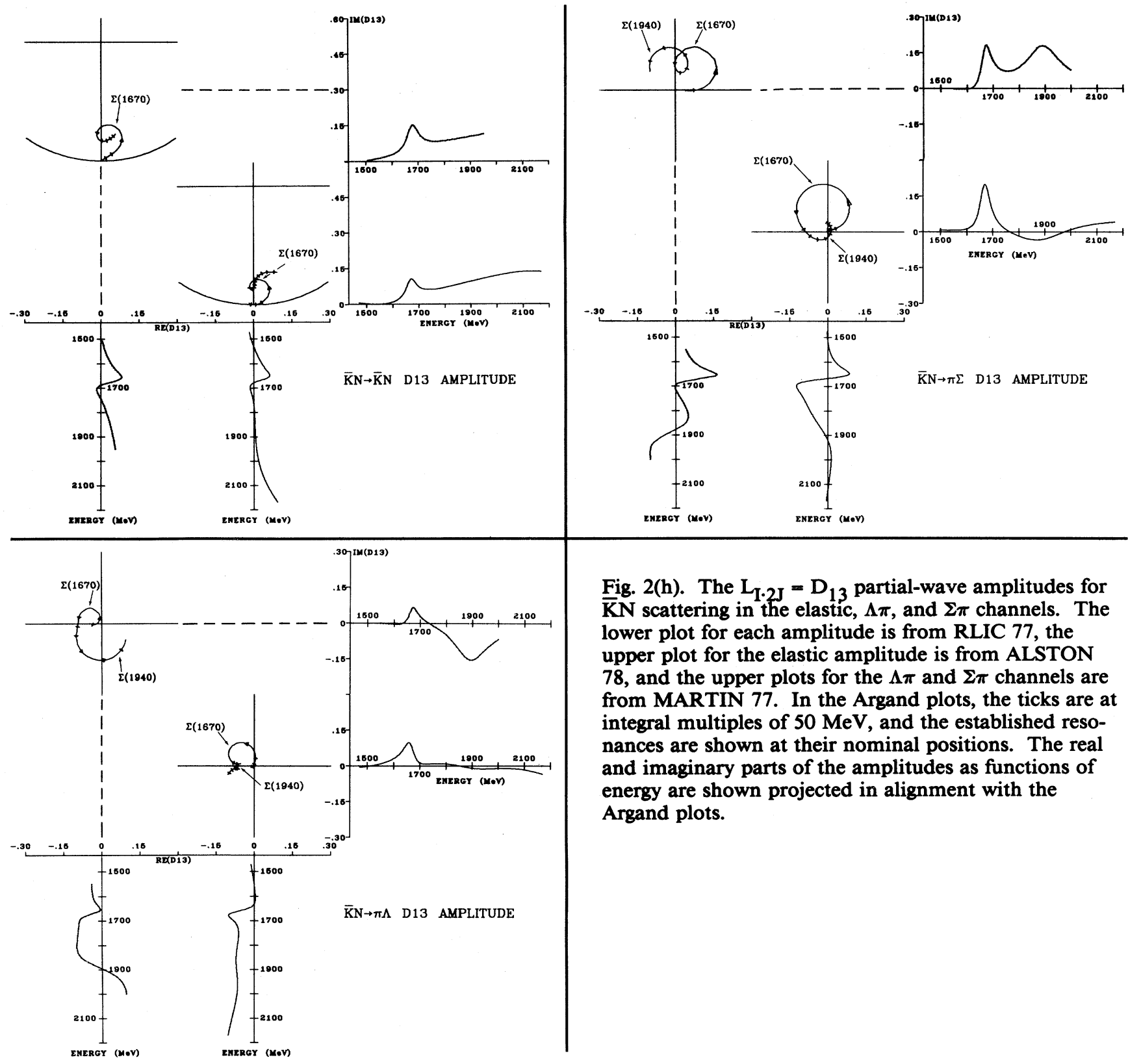

Fig. 2(h). The $\mathrm{L}_{\mathrm{I} \cdot 2 \mathrm{~J}}=\mathrm{D}_{13}$ partial-wave amplitudes for $\overline{\mathbf{K}} \mathbf{N}$ scattering in the elastic, $\Lambda \pi$, and $\Sigma \pi$ channels. The lower plot for each amplitude is from RLIC 77, the upper plot for the elastic amplitude is from ALSTON 78 , and the upper plots for the $\Lambda \pi$ and $\Sigma \pi$ channels are from MARTIN 77. In the Argand plots, the ticks are at integral multiples of $50 \mathrm{MeV}$, and the established resonances are shown at their nominal positions. The real and imaginary parts of the amplitudes as functions of energy are shown projected in alignment with the Argand plots. 

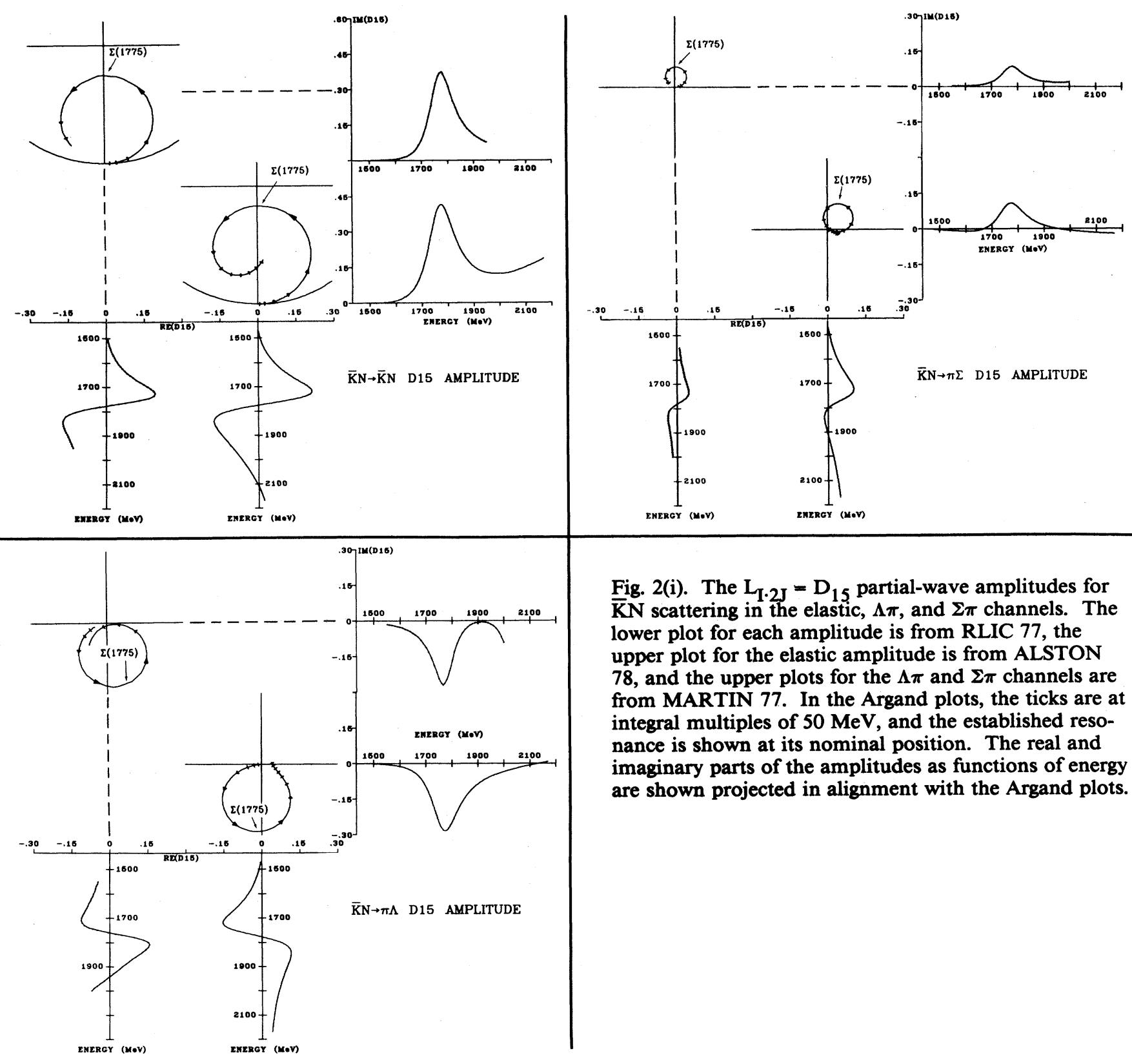

Fig. 2(i). The $L_{I \cdot 2 J}=D_{15}$ partial-wave amplitudes for $\overline{\mathrm{K}} \mathrm{N}$ scattering in the elastic, $\Lambda \pi$, and $\Sigma \pi$ channels. The lower plot for each amplitude is from RLIC 77, the upper plot for the elastic amplitude is from ALSTON 78 , and the upper plots for the $\Lambda \pi$ and $\Sigma \pi$ channels are from MARTIN 77. In the Argand plots, the ticks are at integral multiples of $50 \mathrm{MeV}$, and the established resonance is shown at its nominal position. The real and imaginary parts of the amplitudes as functions of energy are shown projected in alignment with the Argand plots. 
Baryons

Data Card Listings

$\Lambda$ 's and $\Sigma$ 's

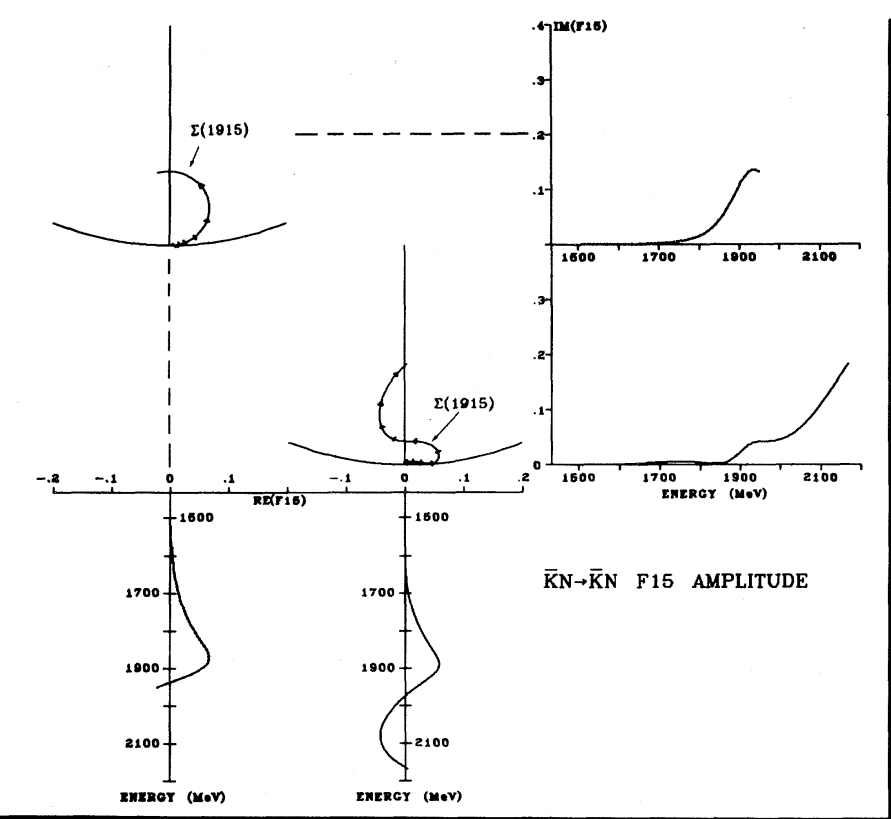

merex (Mon

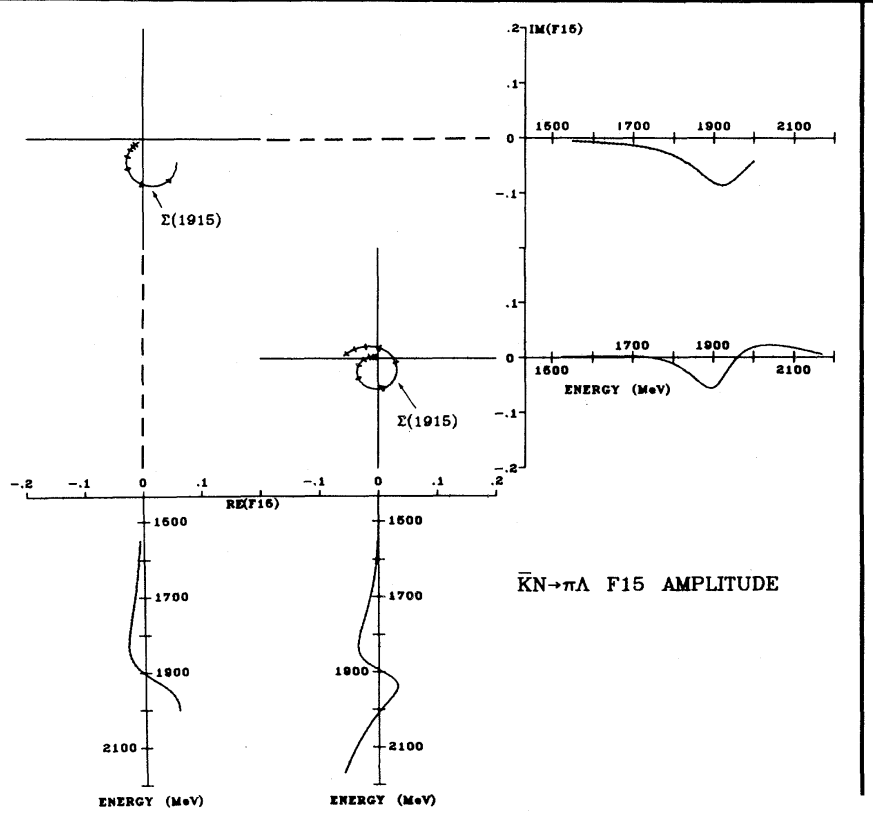

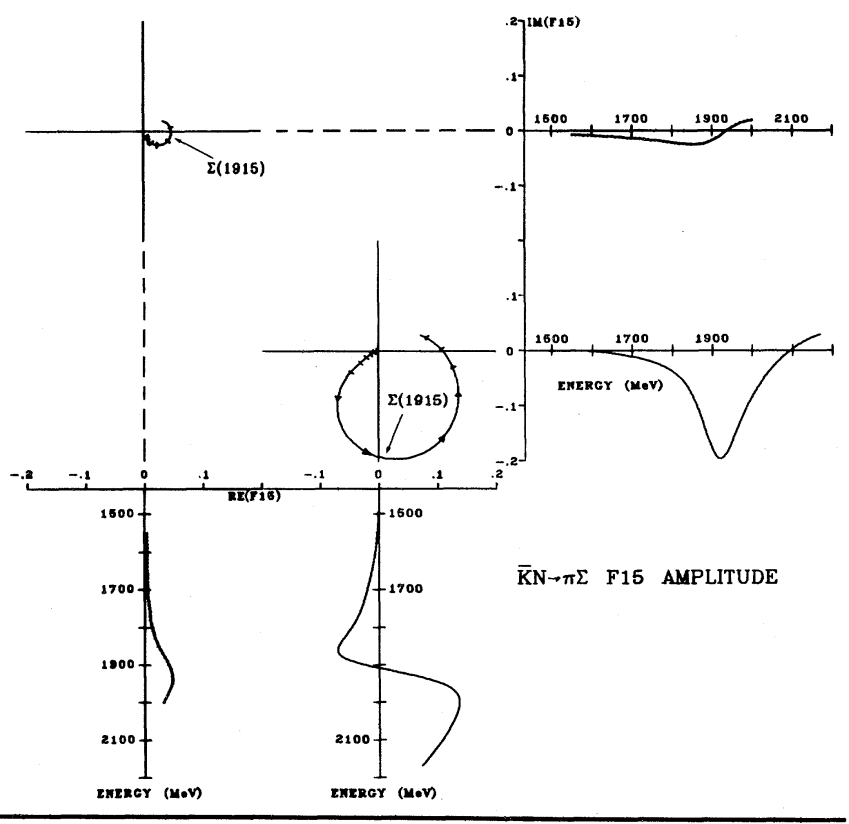

Fig. 2(j). The $L_{1 \cdot 2 J}=F_{15}$ partial-wave amplitudes for $\overline{\mathrm{K}} N$ scattering in the elastic, $\Lambda \pi$, and $\Sigma \pi$ channels. The lower plot for each amplitude is from RLIC 77, the upper plot for the elastic amplitude is from ALSTON 78, and the upper plots for the $\Lambda \pi$ and $\Sigma \pi$ channels are from MARTIN 77. In the Argand plots, the ticks are at integral multiples of $50 \mathrm{MeV}$, and the established resonance is shown at its nominal position. The real and imaginary parts of the amplitudes as functions of energy are shown projected in alignment with the Argand plots. 

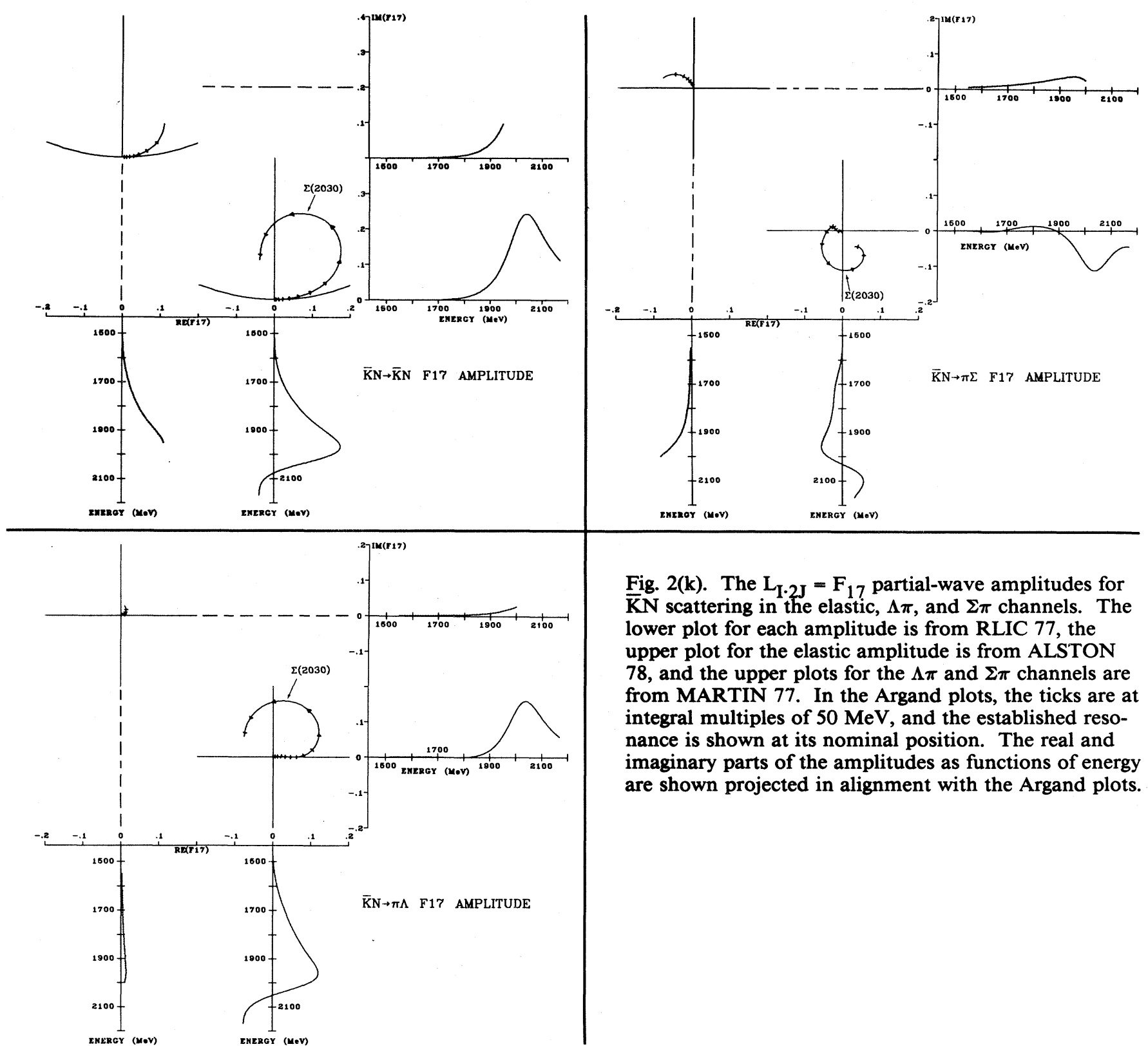

Fig. 2(k). The $\mathrm{L}_{\mathrm{I} \cdot 2 \mathrm{~J}}=\mathrm{F}_{17}$ partial-wave amplitudes for $\bar{K} N$ scattering in the elastic, $\Lambda \pi$, and $\Sigma \pi$ channels. The lower plot for each amplitude is from RLIC 77, the upper plot for the elastic amplitude is from ALSTON 78, and the upper plots for the $\Lambda \pi$ and $\Sigma \pi$ channels are from MARTIN 77. In the Argand plots, the ticks are at integral multiples of $50 \mathrm{MeV}$, and the established resonance is shown at its nominal position. The real and imaginary parts of the amplitudes as functions of energy are shown projected in alignment with the Argand plots. 


\section{Baryons}

Data Card Listings

$\Lambda^{\prime} \mathrm{s}$ and $\Sigma^{\prime} \mathrm{s}, \Lambda, \Lambda(1405), \Lambda(1520)$

12. C.J.S. Damerell et al., Nucl. Phys. B155, 13 (1979).

13. Y. Declais et al., CERN 77-16 (1977).

14. M. Alston-Garnjost et al., Phys. Rev. D21, 1191 (1980).

15. G.P. Gopal, in Proceedings of the IV ${ }^{\text {th }}$ International Conference on Baryon Resonances (Toronto, 1980), edited by N. Isgur, p. 159.

16. G.P. Gopal et al., Nucl. Phys. B119, 362 (1977).

17. M. Alston-Garnjost et al., Phys. Rev. D18, 182 (1978).

18. B.R. Martin et al., Nucl. Phys. B126, 266 (1977); B126, 285 (1977); and B127, 349 (1977).

19. P. Baillon and P.J. Litchfield, Nucl. Phys. B94, 39 (1975).

20. A. de Bellefon and A. Berthon, Nucl. Phys. B109, 129 (1976).

21. A.J. Van Horn, Nucl. Phys. B87, 145 (1975).

22. R. Levi-Setti, in Proceedings of the Lund International Conference on Elementary Particles (Lund, 1969), p. 339.

23. Particle Data Group, Phys. Lett. 111B (1982).

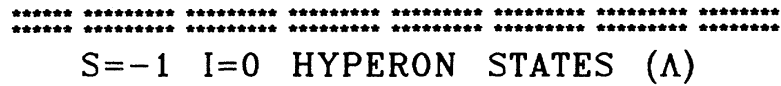

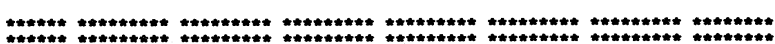

$\Lambda \quad 18$ LAMBDA(1116, JP=1/2+) I=0

SEE STABLE PARTicle datA CARD LISTINGS

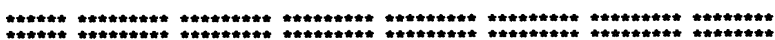

$\Lambda(1405) \quad \mathrm{S}_{01}^{\prime} \quad$ Status: ****

37 LAMBDAC 1405 , JP-1/2-) I I 0
PRODUCTION EXPERIMENTS

THIS RESONANCE IS IDENTIFIED WITH THE VIRTUAL BOUND

STATE IN THE N-KBAR SYSTEM FOUND IN THE ANALYSIS OF
LOW ENERGY K-P INTERACTIONS. WE LIST THOSE RESULTS SEPARATELY IN THE NEXT ENTRY WE USE ONLY PRODUCTION
EXPERIMENTS FOR GETTING THE MASS AND WIDTH FOR THE SEPARATELY TN THE
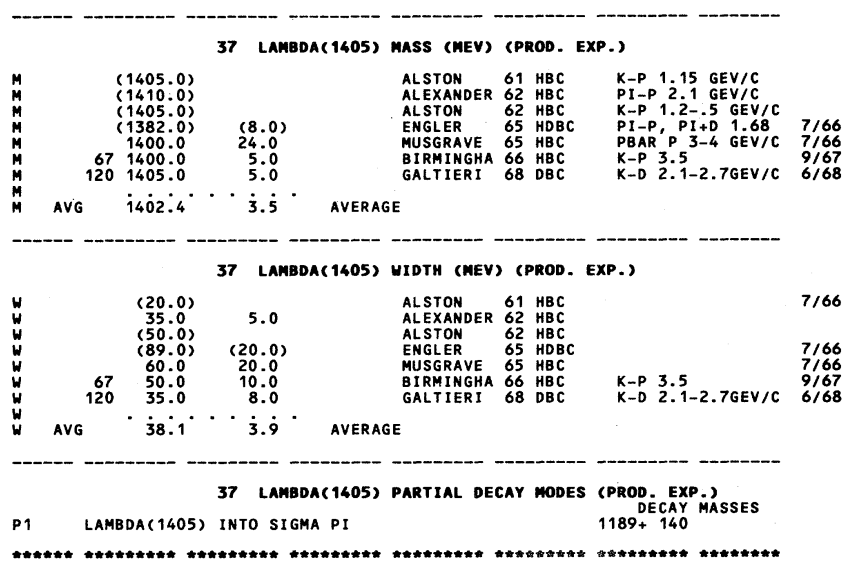

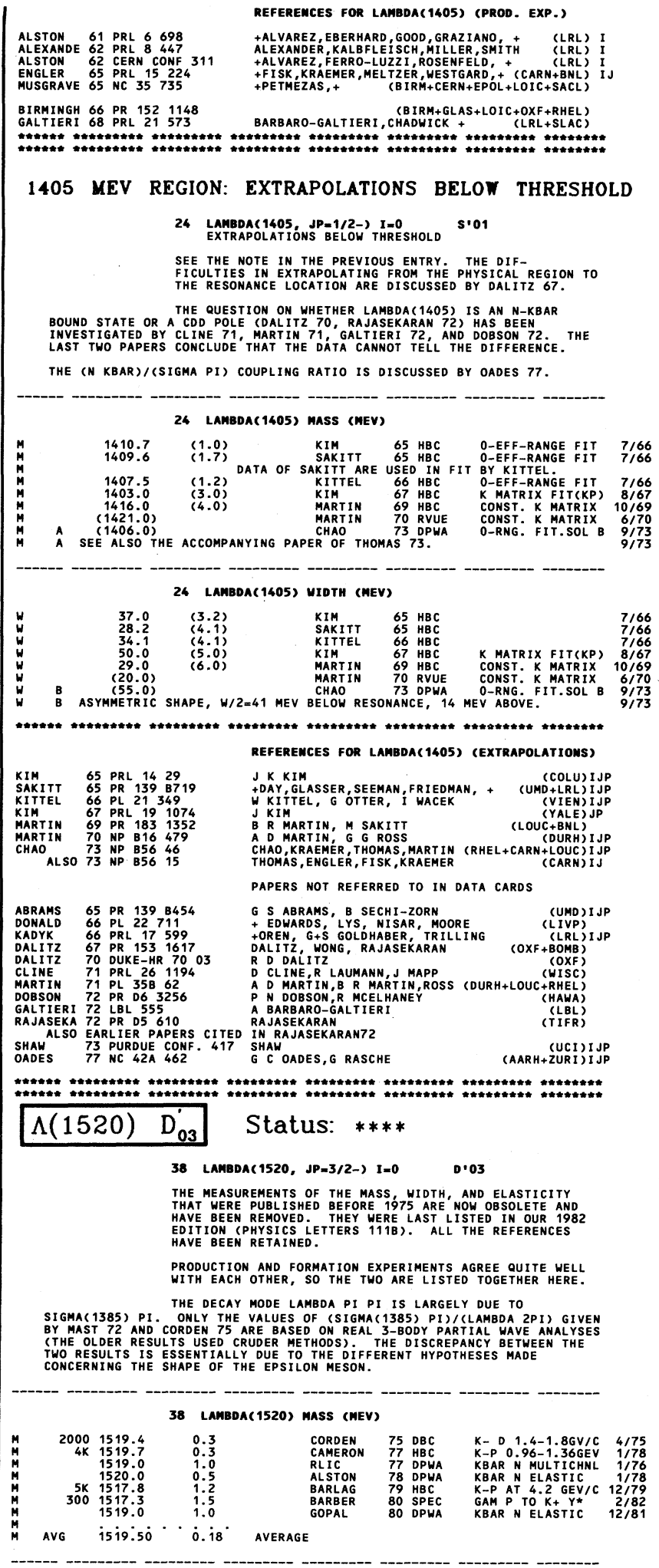




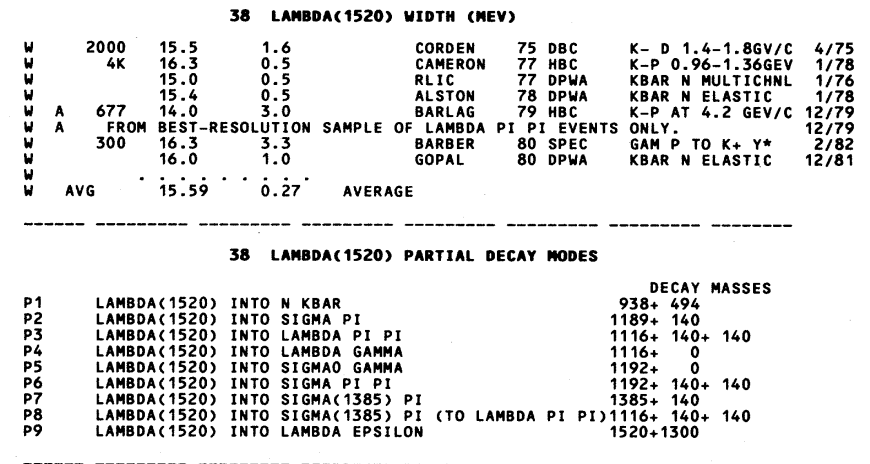

FITTED PARTLAL DECAY MODE BRANCHING FRACTIONS

The matrix below is derived from the error matrix for the fitted partial decay mode branching fractions, $P_{i}$, as follows: The diagonal elements are $P_{i} \neq \delta P_{i}$, where

$\delta P_{i}=\sqrt{\left\langle\delta P_{i} \delta P_{i}\right\rangle}$, while the off-diagonal elements are the normalized correlation coefficients $\left\langle\delta P_{i} \delta P_{j}\right\rangle /\left(\delta P_{i} \cdot \delta P_{j}\right)$. For the definitions of the individual $P_{i}$, see the listings

above; only those $P_{i}$ appearing in the matrix are assumed in the fit to be nonzero and

are thus constrained to add to 1 .

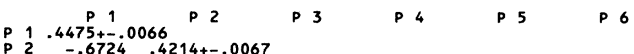

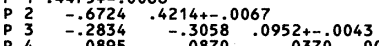

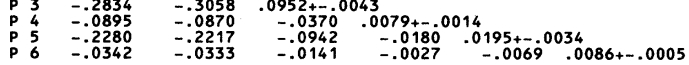

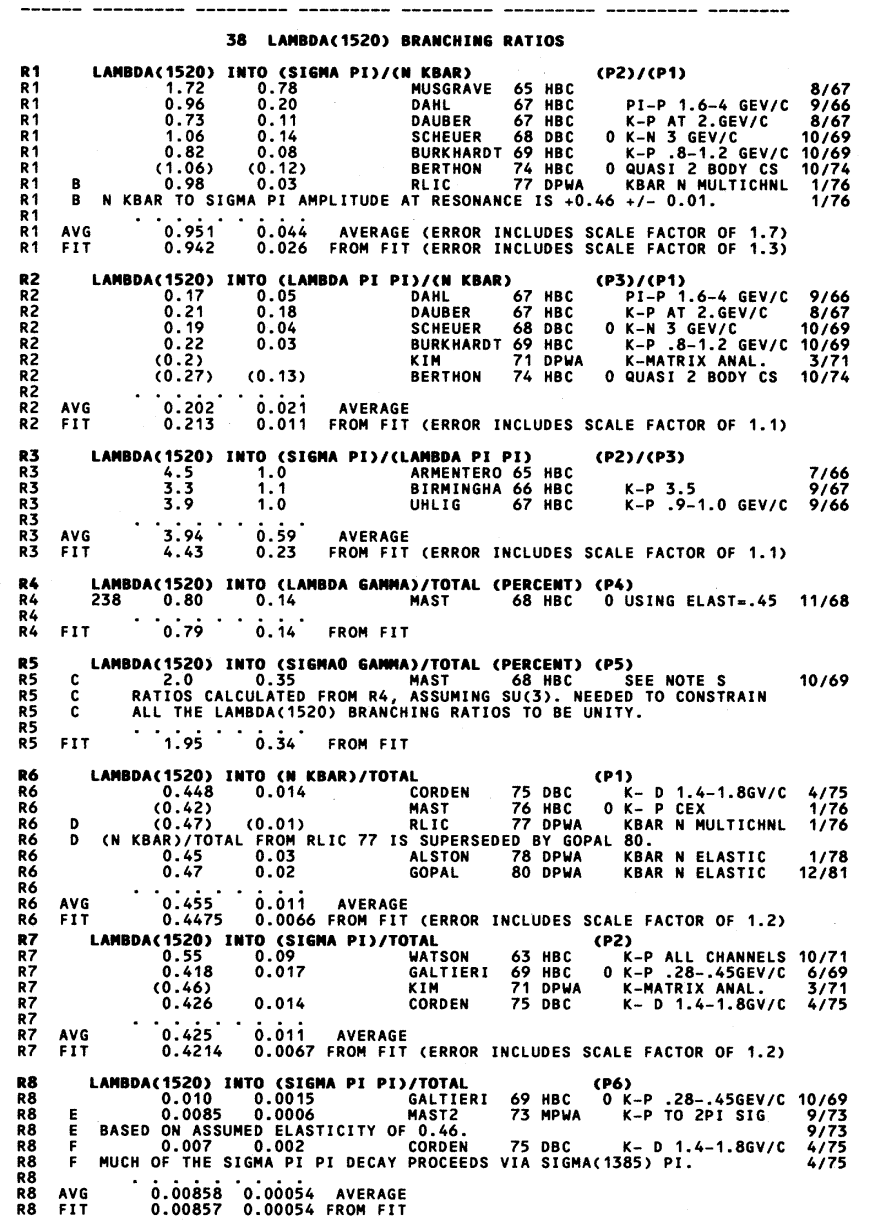

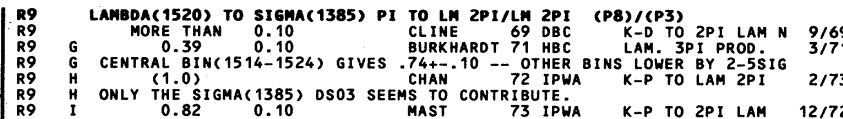
R
R9
I
R9 $\begin{array}{llllll}\text { R10 LAMBDA(1520) INTO (SIGMA(1385) } & \begin{array}{l}\text { PI)/TOTAL } \\ \text { R10 }\end{array} \text { CHAN } & \text { HBC } & \text { (P7) } & \text { K-P TO LAM 2PI } & 3 / 71\end{array}$

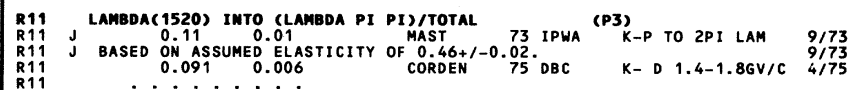

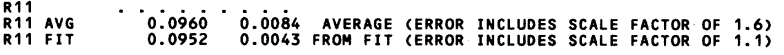

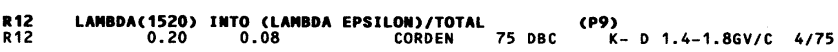

********************************************************************

$\begin{array}{lllll}\text { FERRO-LU } & 62 & \text { PRL } 8 & 28 \\ \text { GALTIERI } & 63 & \text { PL } 6 \text { 8 } 296\end{array}$ $\begin{array}{ll}\text { GALTIERI } & 63 \\ \text { PL } & 6296 \\ \text { WATSON } & 63 \\ \text { PR } & 1312248\end{array}$ ALMEIDA 64 PL 9204
ARMENTER 65 PL 19338
MUSGRAVE 65 NC $35 \quad 735$ $\begin{array}{llllll}\text { BIRMINGH } & 66 & \text { PR } & 152 & 1148 \\ \text { DAHL } & 67 & \text { PR } & 163 & 1377\end{array}$

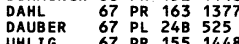
$\begin{array}{lllll}\text { UHLIG } & 67 & \text { PR } & 155 & 1448 \\ \text { MAST } & 68 & \text { PRL } 21 & 1715 \\ \text { SCHEUER } & 68 & \text { NP } & \text { B8 } & 503\end{array}$ $\begin{array}{llll}\text { BURKHARD } & 69 & \text { NP } & B 14 \\ \text { CLINE } & 69 & 106 \\ \text { LNC } 2407\end{array}$ GALTIERI 69 LUND 352 BURKHARDT71 NP B27 64 $\begin{array}{rllll}\text { COLLEY } 71 & \text { NP } & 831 & 61 \\ \text { KIM } & 71 & \text { PRL } & 27 & 356 \\ \text { ALSO } & 70 & \text { DUKE } 161\end{array}$

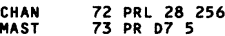
$\begin{array}{lllll}\text { MAST2 } & 73 & \text { PRD } & 3212 \\ \text { BERTHON } & 74 & \text { NC } 214 & 146\end{array}$

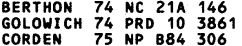

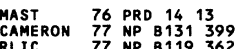

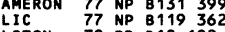

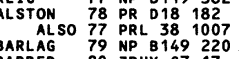

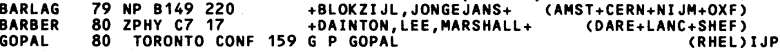

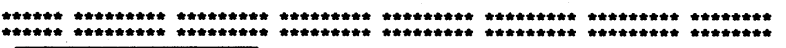
\begin{tabular}{|ll|l}
$\Lambda(1600)$ & $\mathrm{P}_{01}^{\prime}$
\end{tabular} Status: $* * *$

101 LAMBDA(1600, JP=1/2+) I=0 P'01 SEE THE NOTE FOR THE LAMBDA( 1800 , JP=1/2+) PII 01.
SOMEWHERE IN THIS REGION THERE IS PROBABLY ONE, SOMEWHERE IN THIS REGION THERE
AND PERHAPS THO, PO1 STATES.

101 LAMBDA(1600) MASS (MEV)

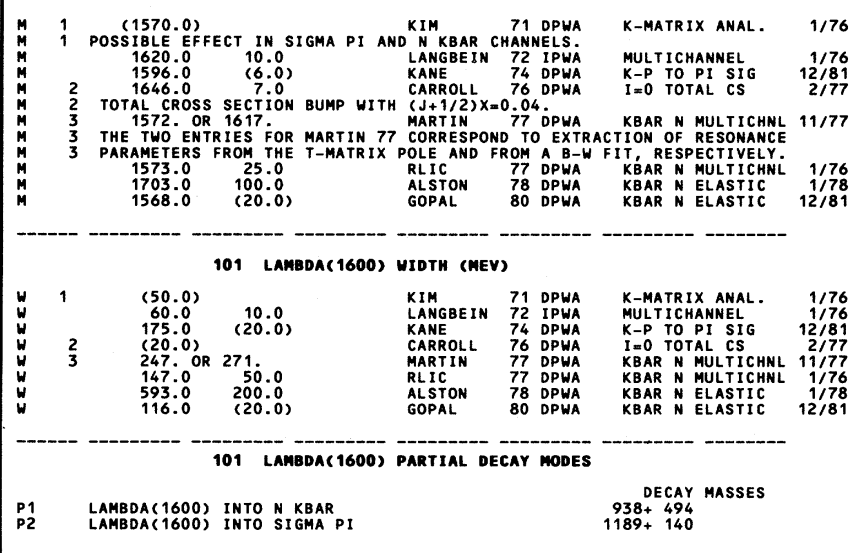


Baryons

$\Lambda(1600), \Lambda(1670), \Lambda(1690)$

\section{Data Card Listings}

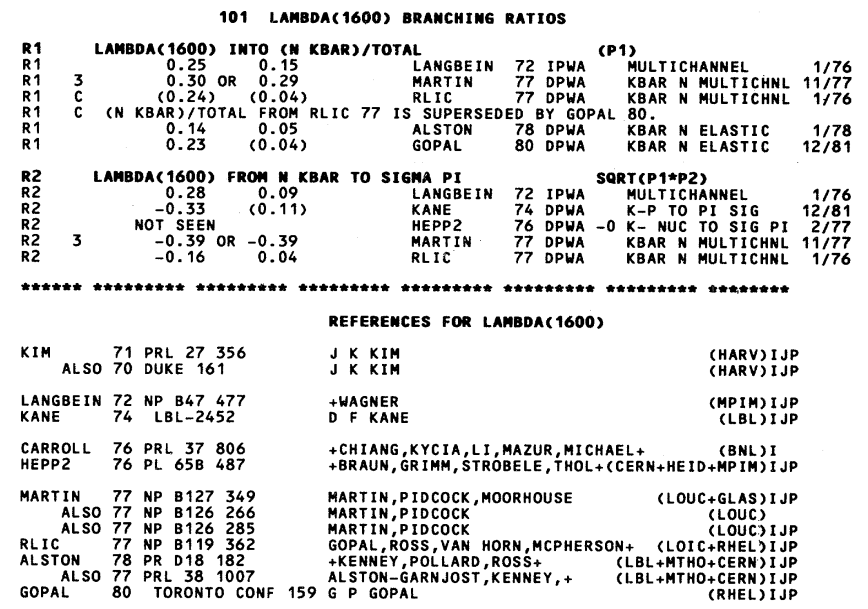

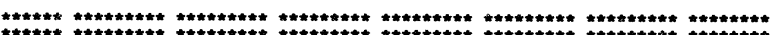

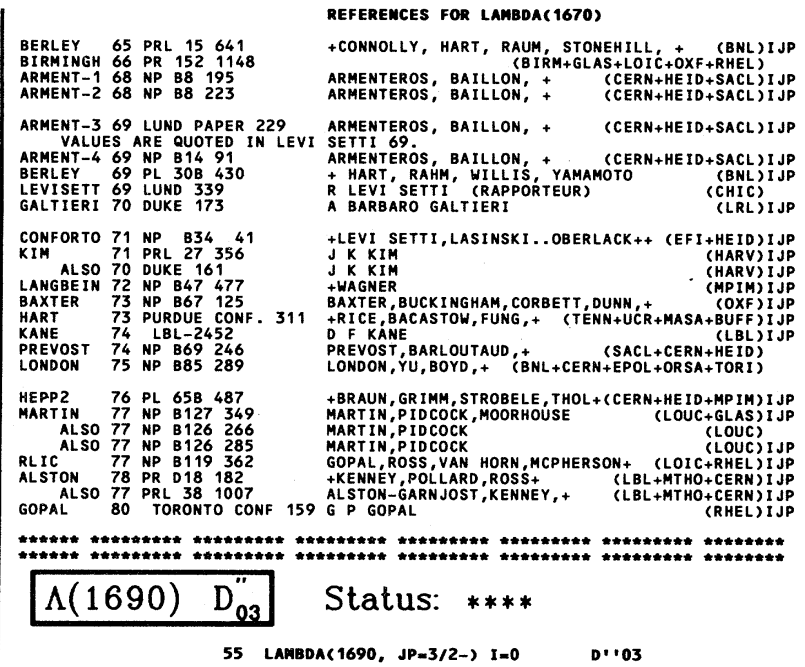

THE MEASUREMENTS OF THE MASS, WIDTH, AND ELASTICITY

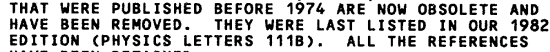

40 LAMBDA(1670, JP=1/2-) I=0 S'י01

THE MEASUREMENS OF THE MASS, HIDTH, AND ELASTICITY
THAT WERE PUBLISHED BEFORE 974 ARE NOW OBSOLETE AND

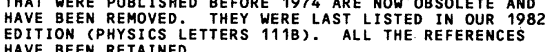

40 LAMBDA(1670) MASS (MEV)

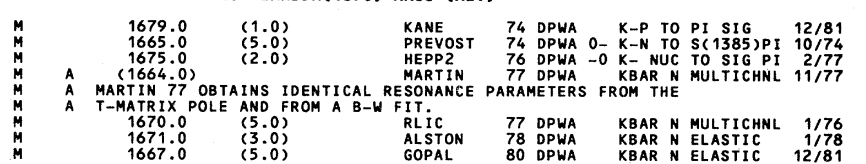
40 LAMBDA(1670) WHDTH (MEV)

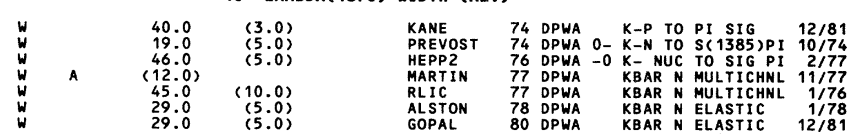

40 Lamboac 1670) PARTIAL DecaY modes

\begin{tabular}{|c|c|c|}
\hline $\begin{array}{l}\text { P1 } \\
\text { P2 }\end{array}$ & 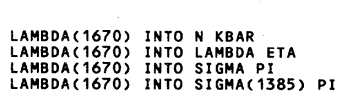 & $\begin{array}{l}\text { DECAY MASSES } \\
938+494 \\
1116+549 \\
1189+140 \\
1385+140\end{array}$ \\
\hline
\end{tabular}

40 LAMBDA( 1670) BRAMCHIMG RATIOS

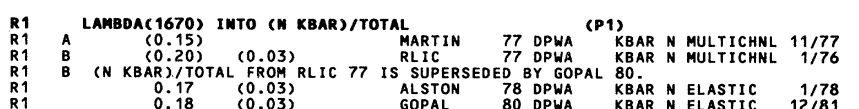

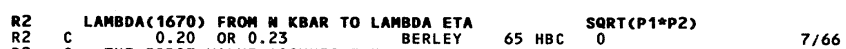

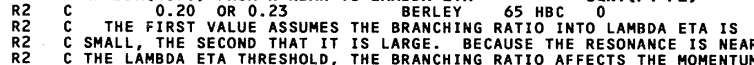

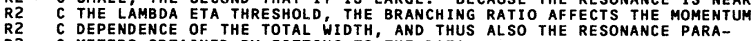

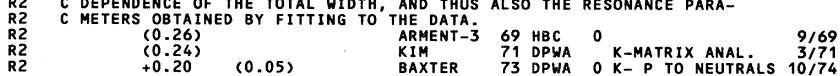

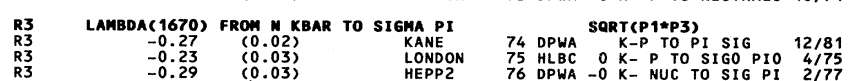

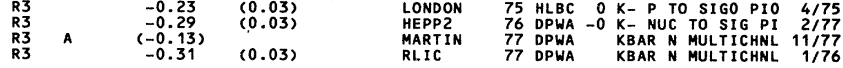

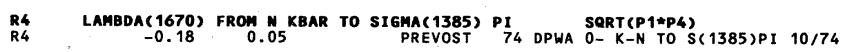

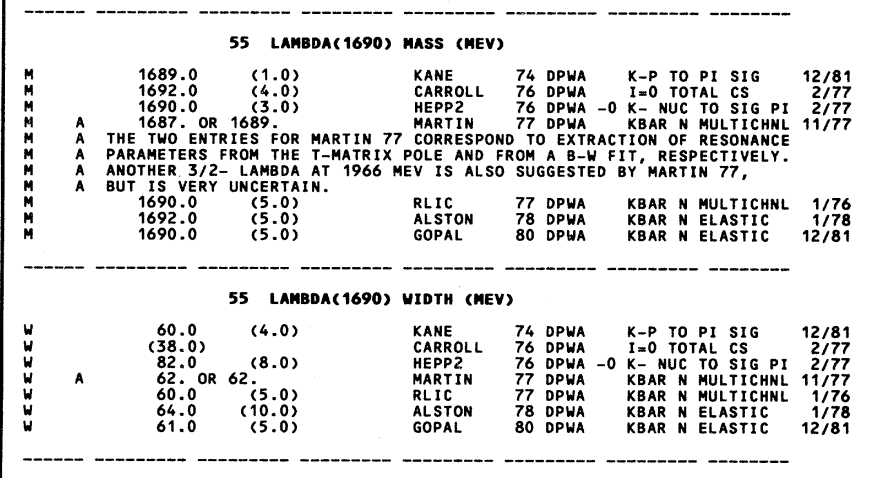

55 LAMBdA( 1690) PARTIAL DECAY MODES

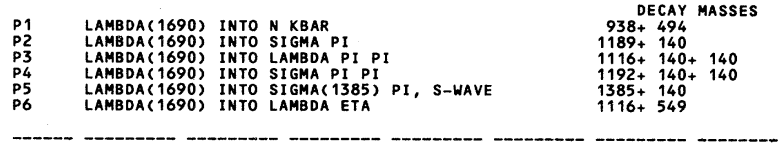

55 LAKBDA( 1690) BRAMCHIMG RATIOS

THE SUM OF ALL THE QUOTED BRANCHING RATIOS IS MORE THAN 1.0 .0$. THE
TWO-BOOY RATIOS ARE FROM PART TAL WAVE ANALYSES AND THUS PROBABLY ARE MORE RELIABLE THAN THE THREE-BODY RATIOS, HHICH ARE DETERMINED EROM
BUMPS IN CROSS SECTIONS. OF THE LATTER, THE SIGMA PI PI BUMP LOOKS

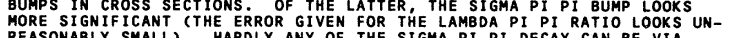

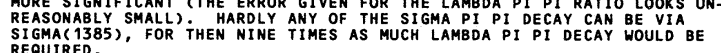

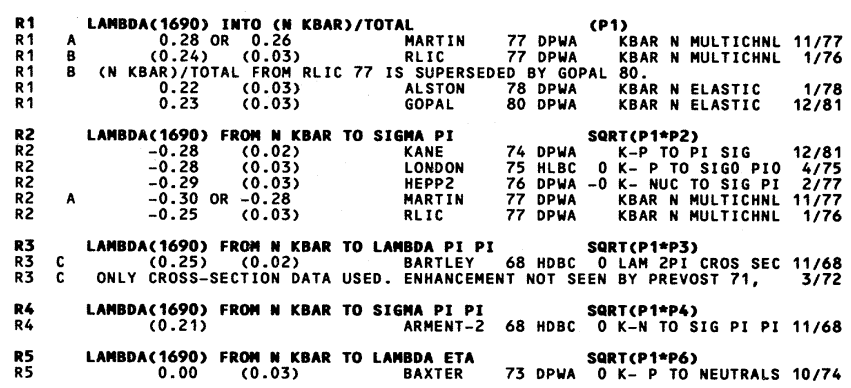




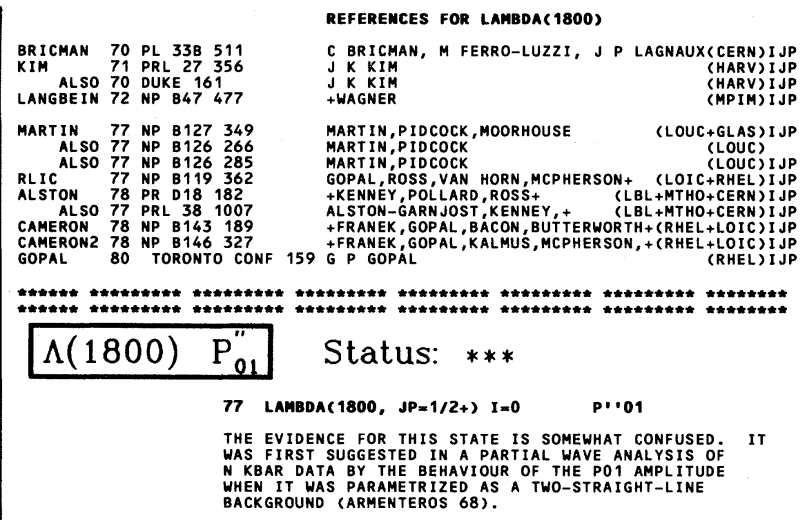

ALMOST ALL THE RECENT ANALYSES CONTAIN A PO1 STATE, AND SOMETIMES TWO, BUT THE MASSE, WHDTHS, AND BRANC.
SEE ALSO THE LAMBOA $(1600)$ POO LISTING.

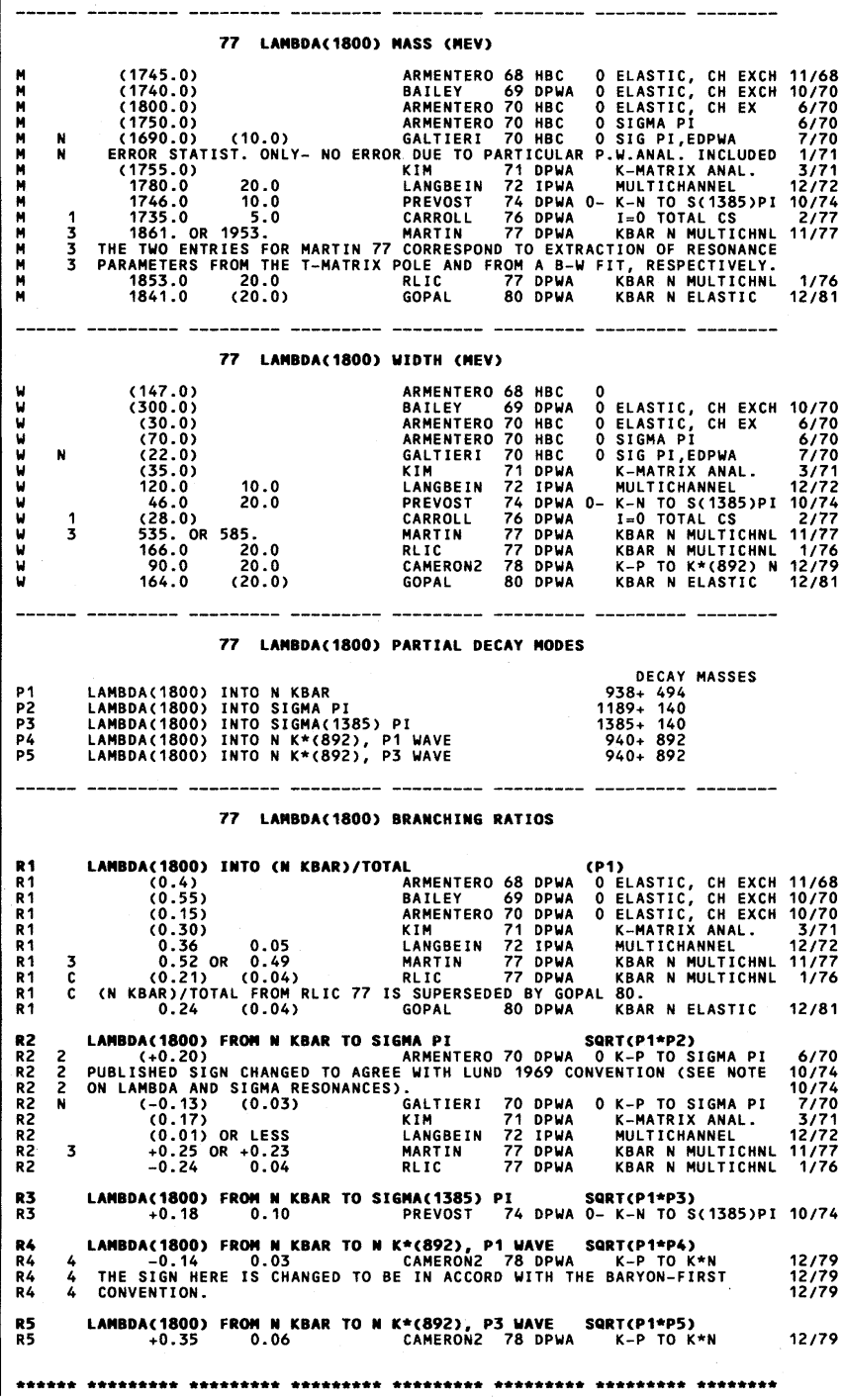




\section{Baryons}

$\Lambda(1800), \Lambda(1820), \Lambda(1830)$
Data Card Listings
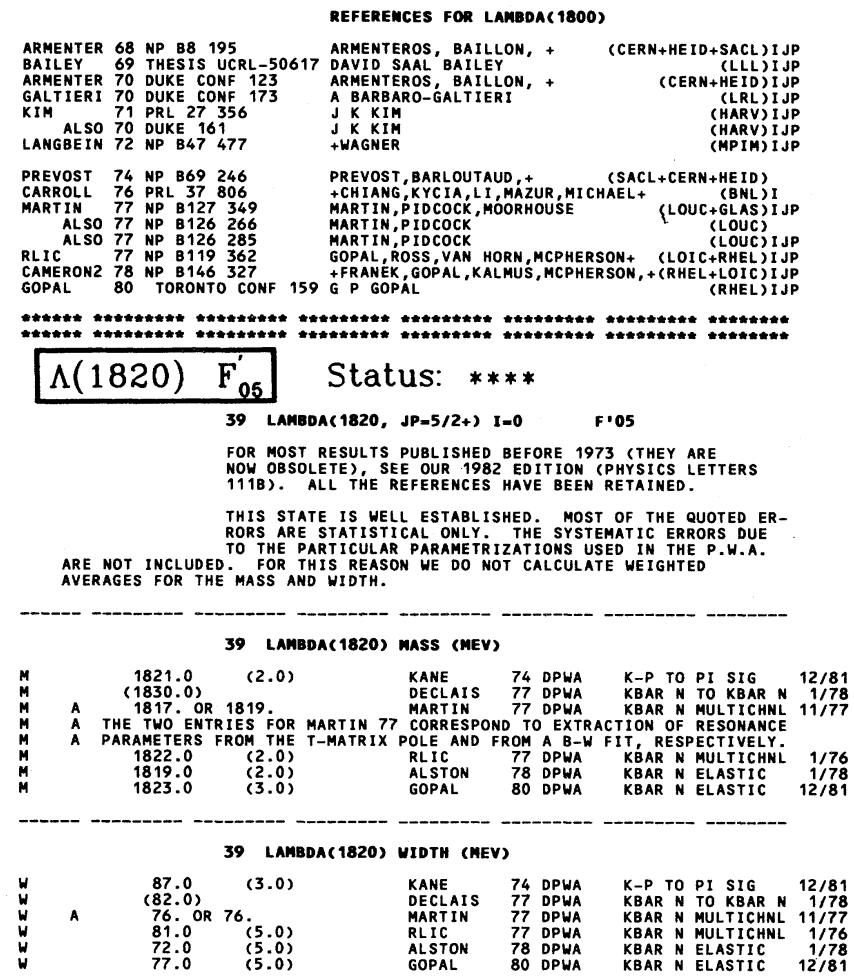

39 LAMBda( 1820) PARTIAL DECAY mOdes

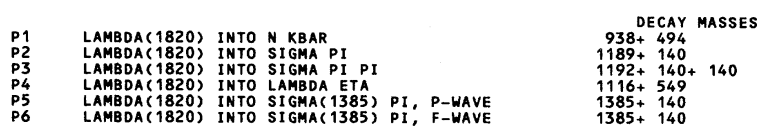

\section{FITTED PARTIAL DECAY MODE BRANCHING FRACTIONS}

The matrix below is derived from the error matrix for the fitted partial decay mode branching fractions, $P_{i}$, as follows: The diagonal elements are $P_{i} \pm \delta P_{i}$, where

$\delta P_{i}=\sqrt{\left.i \delta P_{i} \delta P_{i}\right\rangle}$, while the off-diagonal elements are the normalized correlation coefficients $s\left\langle\mathrm{P}_{i} \delta \mathrm{P}_{j}\right\rangle /\left(\delta \mathrm{P}_{i} \cdot \delta \mathrm{P}_{j}\right)$. For the definitions of the individual $\mathrm{P}_{i,}$ see the listing above; only those $P_{i}$ appearing in the matrix are assumed in the fit to be nonzero and are thus constrained to add to 1 .

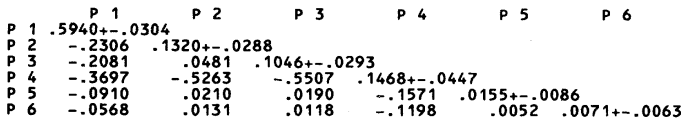

39 LAMBdA(1820) BRAMCHING RATIOS

ERRORS QUOTED BY EXPERIMENTERS DO NOT INCLUDE UNCERTAINTY DUE
TO PARAMETRIZATION USED IN THE P.W.A. THEY SHOULD BE INCREASED.

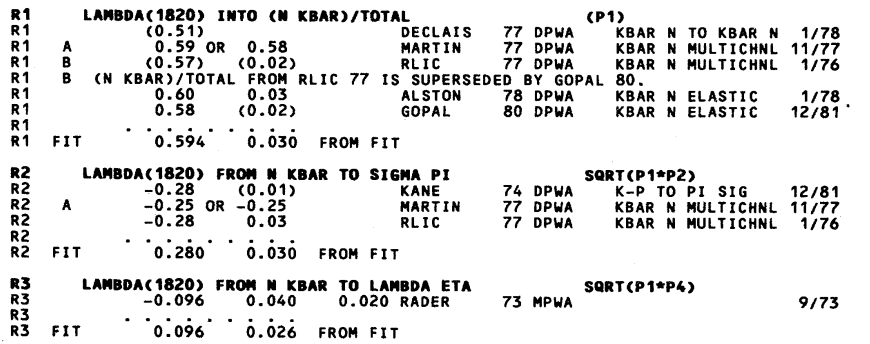

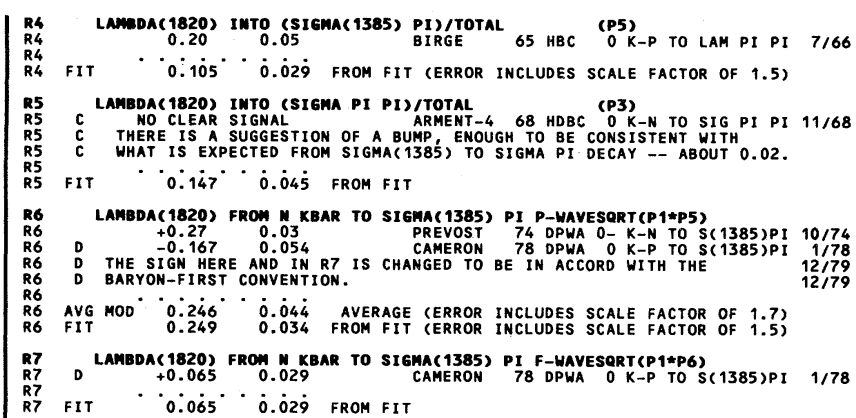

$* * * * * * * * * * * * * * * * * * * * * * * * * * * * * * * * * * * * * * * * * * * * * * *$
REFEREMCES FOR LAMBDA( 1820$)$

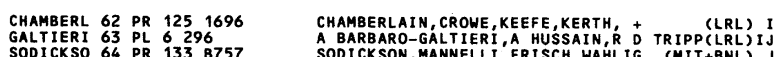

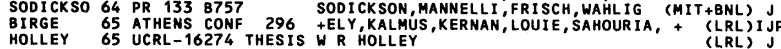
HOLLEY 65 UCRL-16274 THESIS WR HOLLEY (LIRL)
BIRMINGH 66 PR 1521148 (BIRM+GLAS+LOIC+OXF+RHEL)

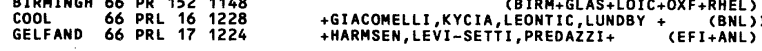

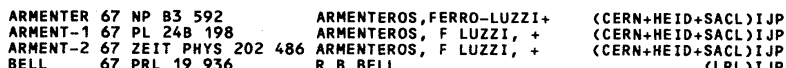

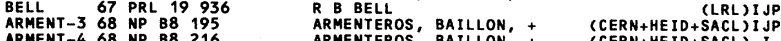

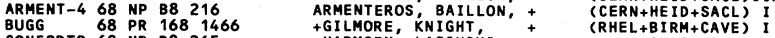

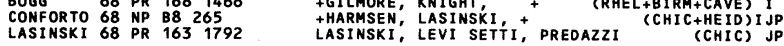
\begin{tabular}{l} 
BRICMAN $70 \mathrm{PL} 31 \mathrm{~B} 152$ \\
BRICMAN1 $70 \mathrm{PL} 33$ 511 \\
\hline
\end{tabular}

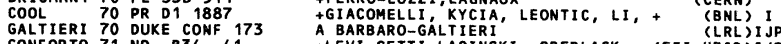

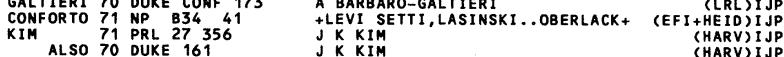
PREVOST 71 AMSTERDAM CONF + CHS COLLABORATION
KANE 72 PR D5 1583 (CERN+HEID+SACL)
(LBL) IJP

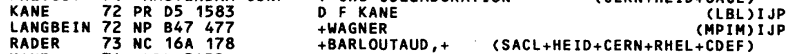

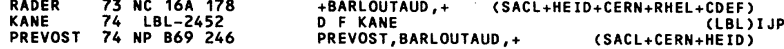
$\begin{array}{lll}\text { DECLAIS } 77 \text { CERN } 77-16 & \text { PDUCHON, LOUVEL, PATRY, SEGUINOT+ } & \text { (CAEN+CERN)IJP } \\ \text { MARTIN } 77 \text { NP B127 } 349 & \text { MARTIN,PIDCOCK,MOORHOUSE } & \text { (LOUC+GLAS)IJP }\end{array}$

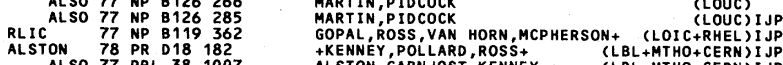

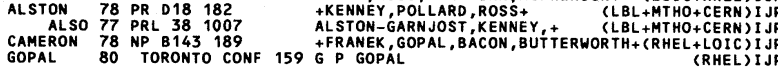

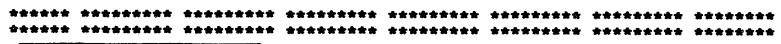

$\Lambda(1830) \quad D_{05}$ Status: ****

56 LAMBDA( 1830, JP=5/2-) I=0 DO5 FOR RESULTS PUBLISHED BEFORE 1973 (THEY ARE
NOW OBSOLETE), SEE OUR 1982 EDITION (PHYSICS LETTERS
$111 B$ ) ALL THE REFRENCES HAVE BEEN RETAINED THE BEST EVIDENCE FOR THIS RESONANCE COMES FROM THE

56 LAMBDAC (1830) MASS (MEV)

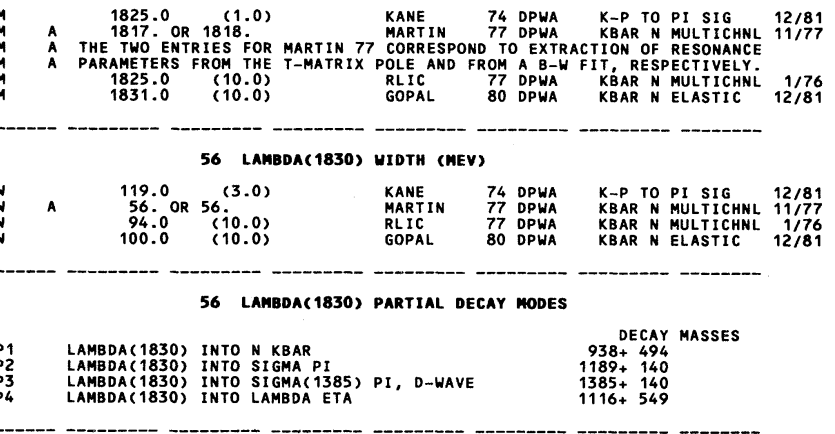


56 LAMBDA( 1830) BRAMCHIMG RATIOS

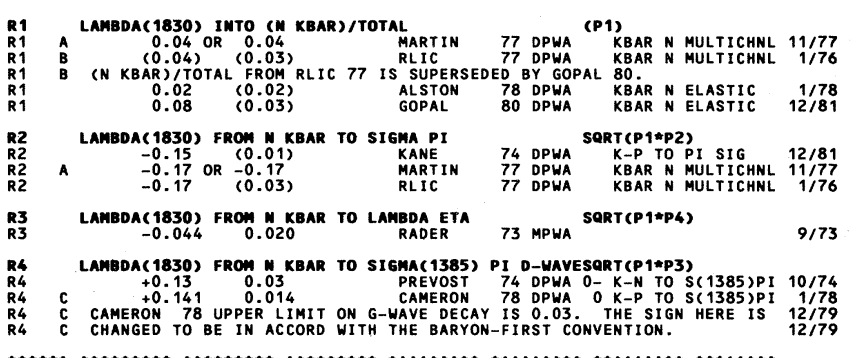

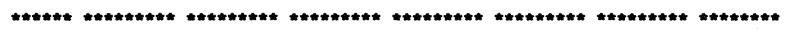

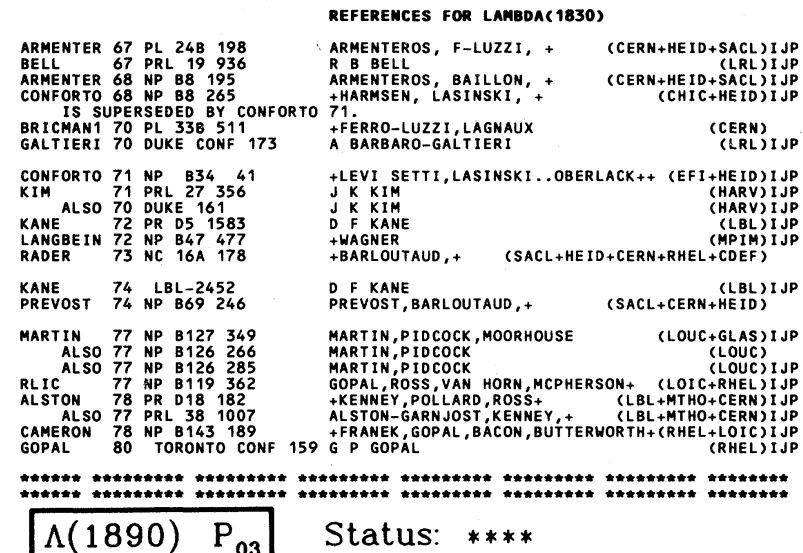

60 LAMBDA( $1890, J P=3 / 2+$ ) I=0 P03 FOR RESULTS PUBLISHED BEFORE 1974 (THEY ARE
NOW OBSOLETE) S SEE OUR 1982 EDITION (PHYSICS LETTERS
$111 B$ ). ALL THE REFERENCES HAVE BEEN RETAINED. THE JP $=3 / 2+$ ASSIGNMENT IS CONSISTENT WITH ALL AVAILABLE DATA (INCLUDING POLARIZATION) AND RECEN
PARTIAL WAVE ANALYSES. THE DOMINANT INELASTIC MODES REMAIN UNKNOWN.

60 LAMBDA (1890) MASS (MEV)
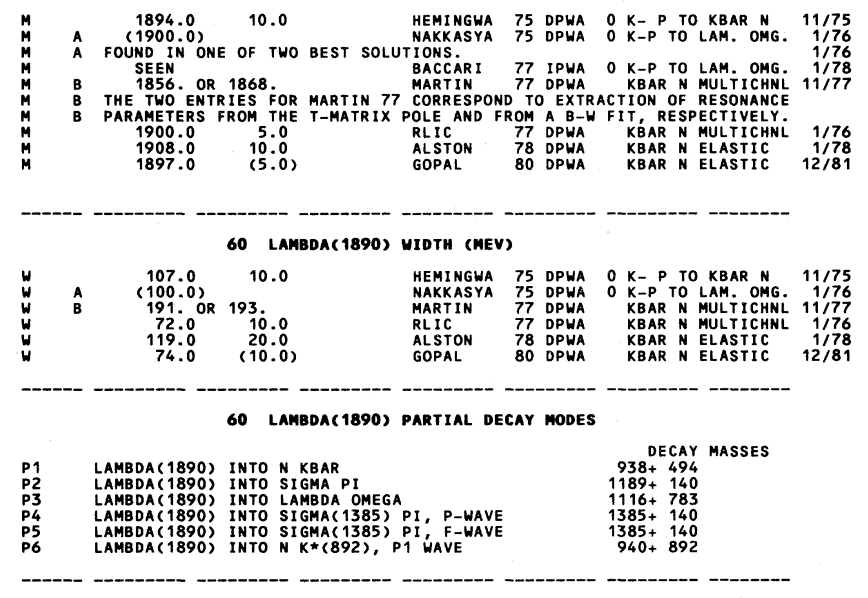

60 LAMBDA( 1890) BRAMCHIMG RATIOS

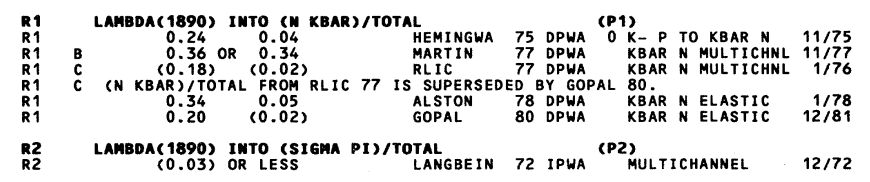

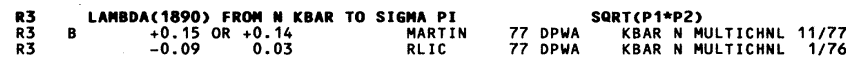

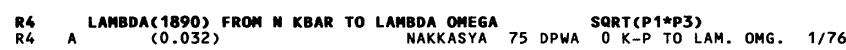

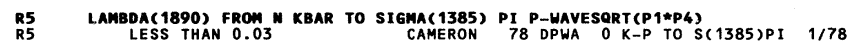

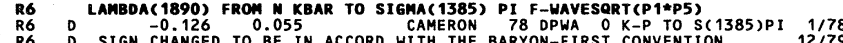

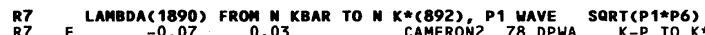

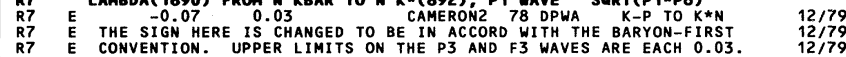

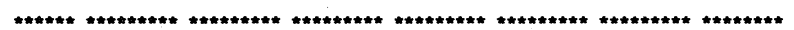

$\begin{array}{llll}\text { ARMENTER } 67 & \text { NP } & 83 & 592 \\ \text { ARMENTERO68 NP } & 88 & 195\end{array}$ ARMENTER 68 PR 1681466
BUGG
CONFRTO 68 NP B8 265 GALTIERI 68 PRL 21573 $\begin{array}{llll}\text { BRICMAN } 70 & \text { PL } 31 B & 152 \\ \text { BRICMAN1 } 70 & \text { PL } 33 B & 511\end{array}$ ALBROW 71 NP $B 29413$
CONFORTO 71 NP 83441
KIM 71 PRL 27356

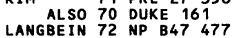
$\begin{array}{lllll}\text { LEA } & 73 & \text { NP } & B 56 & 77 \\ \text { HEMINGWA } & 75 & \text { NP } & 891 & 12 \\ \text { NAKKASYA } & 75 & \text { NP } & 893 & 85\end{array}$

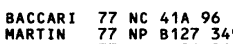

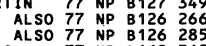

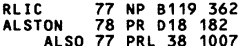
CAMERON 78 NP $B 143189$ CAMERON2 78 NP B B 166327
GOPAL 80 TORONTO CONF 159 G P P GOPAL

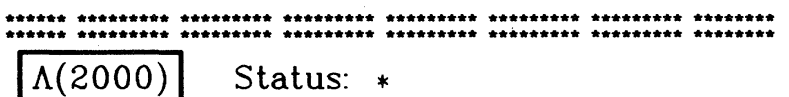

$\Lambda(2000)$ Status: *

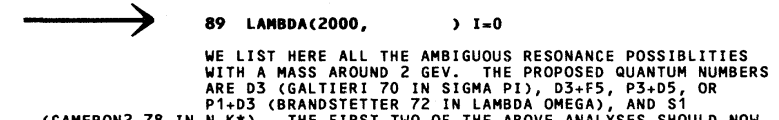
(CAMERON2 78 IN $N$ M $K^{\star}$ ). (BRANDSTETTER 72 IN LAMBDA OMEGA) AND S1 89 LAMBDA(2000) MASS (MEV)

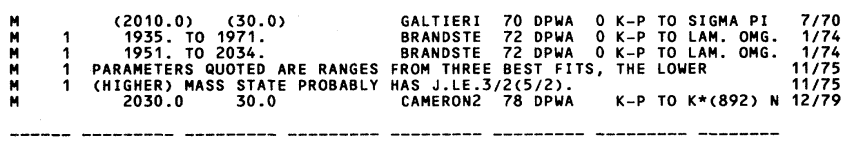

89 LAMEdA (2000) WIDTH (MEV)

W $1(130.0)(50.0)$ GALTIERI 70 DPWA 0 K-P TO SIGMA PI $7 / 70$

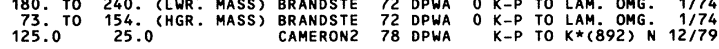
89 LAMBda(2000) PARTIAL deCAY modes

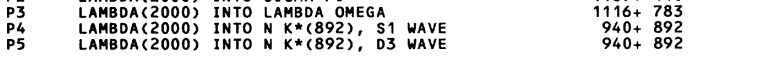

89 LAMBdA(2000) BRAMCHING RATIOS

R1 LAMBDA(2000)
R1 FROM
$(-0.20)$
$(0.04)$

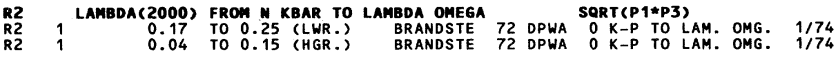

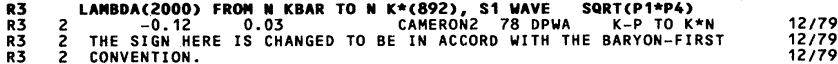

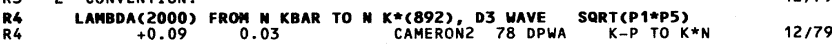

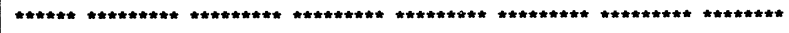
REFEREMCES FOR LAMBda(2000)

GALTIERI 70 DUKE CONF 173 A BARBARO-GALTIERI
(LRL) IJP $\begin{array}{ll}\text { BRANDSTE } 72 \text { NP B39 } 13 & \text { BRANDSTETTER, BUT TERWORTH, + (RHEL+CDEF+SACL) } \\ \text { CAMERON2 } 78 \text { NP B146 } 327 & \text { +FRANEK, GOPAL, KALMUS, MCPHERSON, +(RHEL+LOIC) IJP }\end{array}$ 
Baryons

$\Lambda(2000), \Lambda(2020), \Lambda(2100)$
Data Card Listings

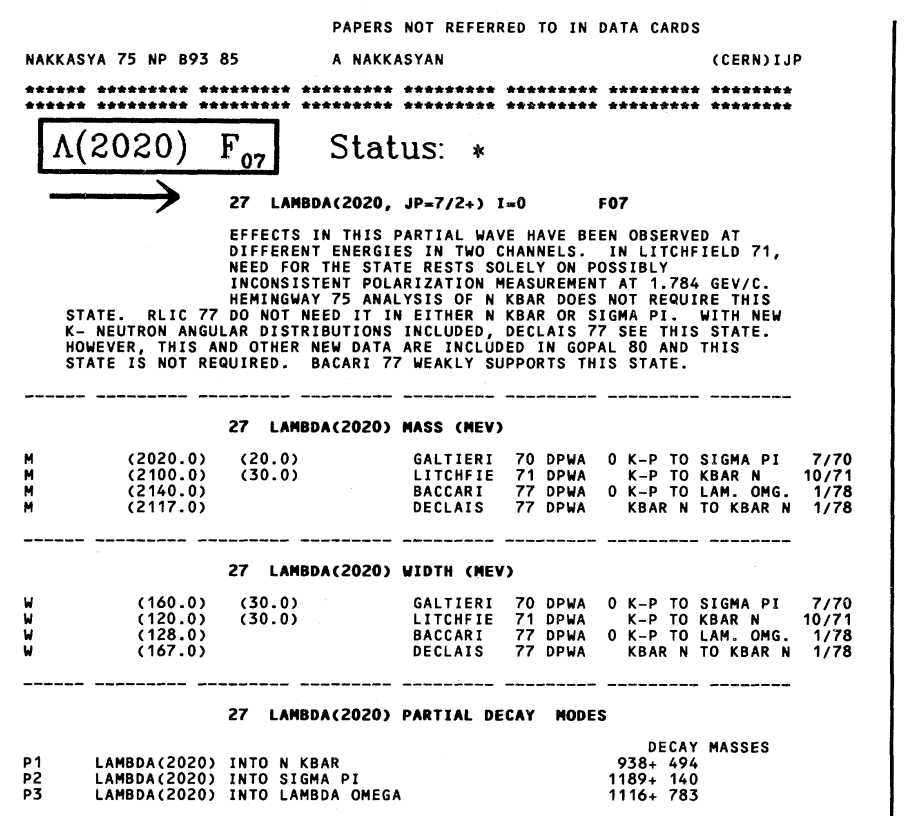

27 LAMBDA(2020) BRAMCHIMG RATIOS

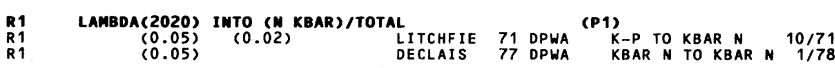

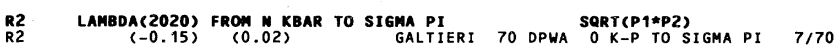

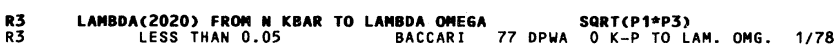

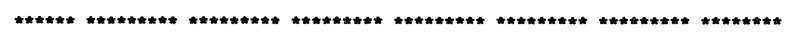
REFEREMCES FOR LAMBDA(2020)

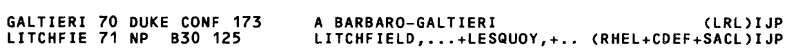

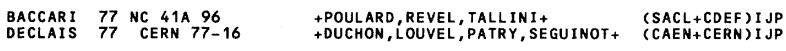
PAPERS NOT REFERRED to IN DATA CARDS HEMINGWA 75 NP 89112 HEMINGWAY, EADES, HARMSEN+ (CERN+HE ID + MP IM) IJP

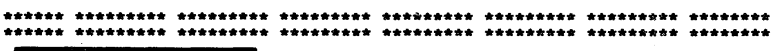

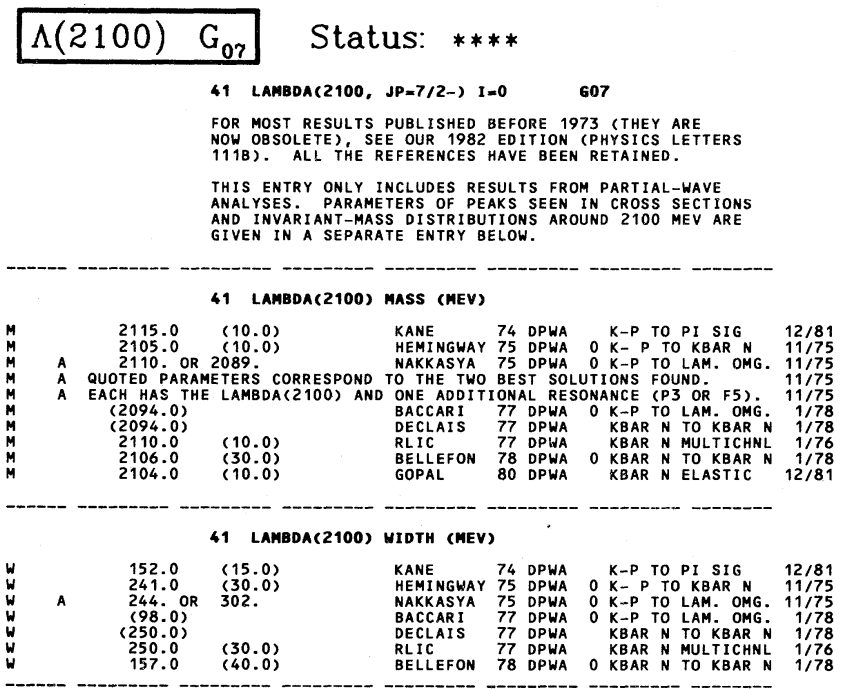

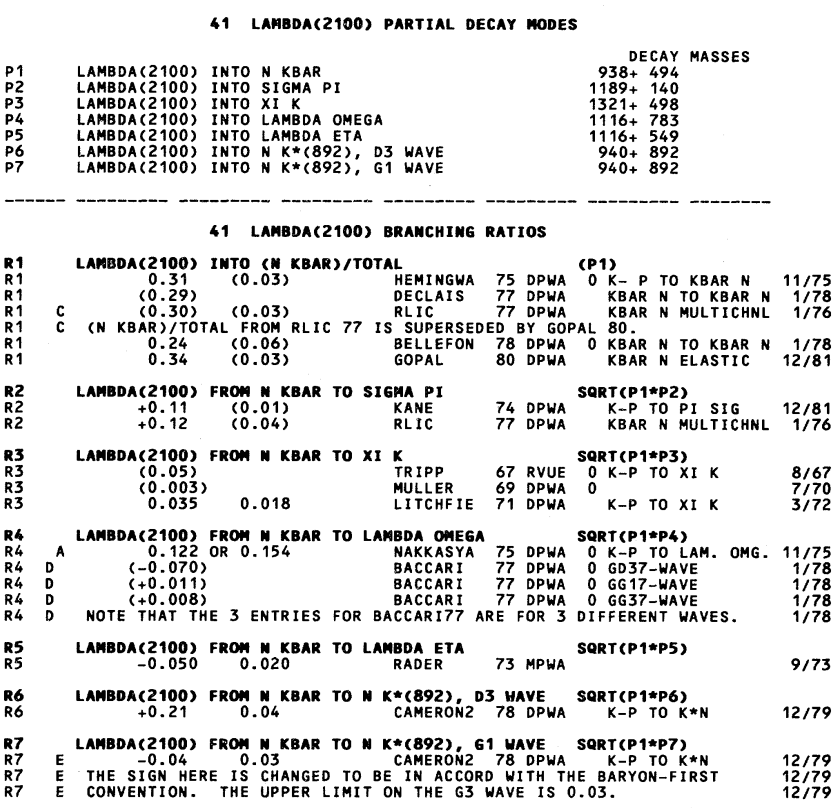

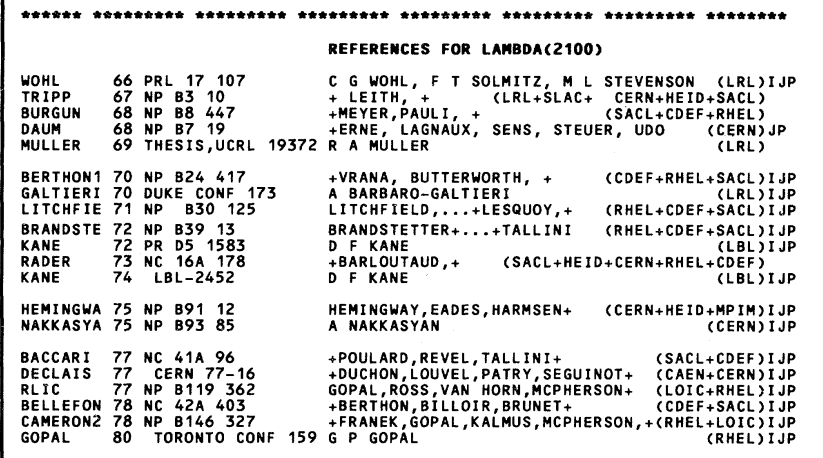

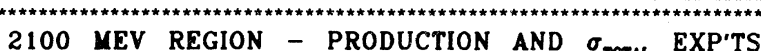

25 LAMBDA(2100, JP $=$, I=0 PRODUCTION EXPERIMENTS SEE THE NOTE TO THE 607 LAMBDA(2100), IN FRONT OF THIS
ENTY. HERE WE LIST ONLY PARAMTERS OF PEAKS IN CROSS

ENTRY HERE WE LIST ONLY PARAMETERS OF PEAKS IN CROSS

SECTION PEAKS ARE AT LEAST DOMINANTLY ASSOCIATED WITH THE LAMBDA(2100),
BUT MAY CONTAIN A SMALL CONTRIBUTION FROM THE SUGGESTED BUT NOT ESTAB-

BUT MAY CONTAIN A SMALL CONTRIBUTION FROM
LISHED OTHER RESONANCES IN THIS REGION.

25 LAMBDA(2100) MASS (MEV) (PROD. EXP.)

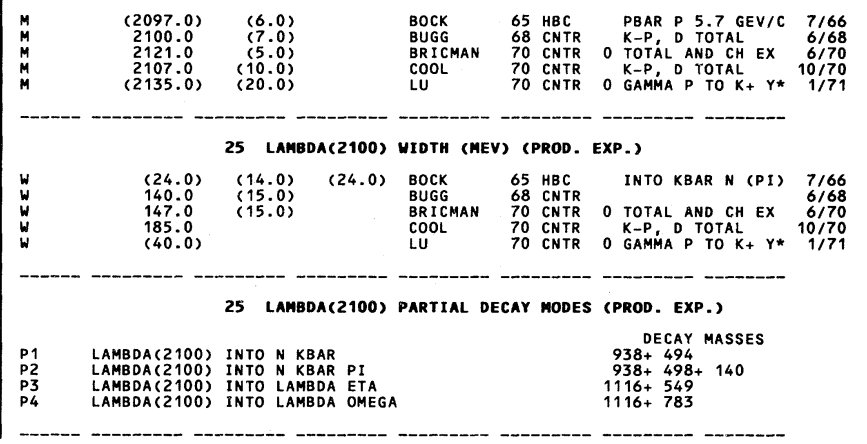




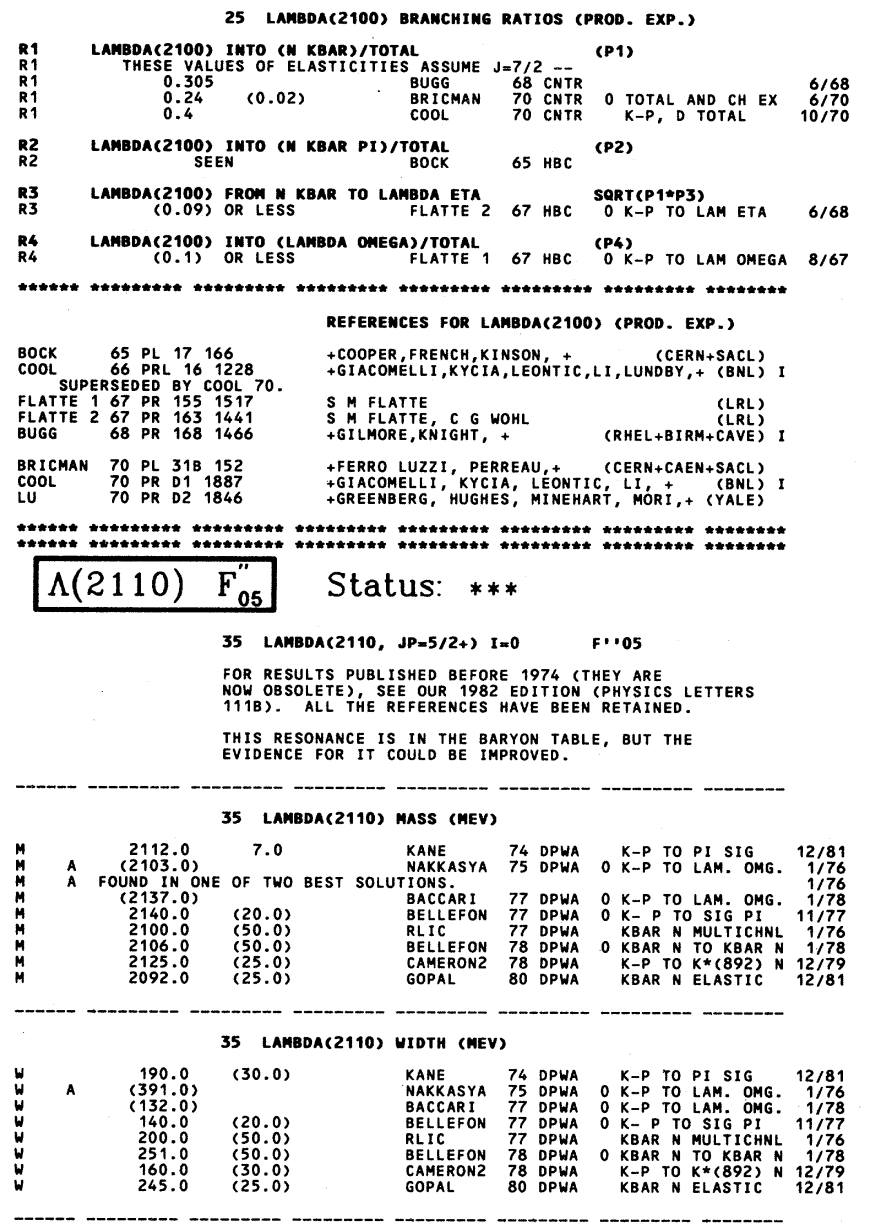

35 LAMBda(2110) PARTIAL decay modes

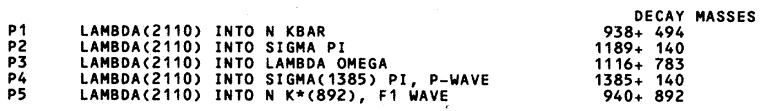

35 LAMBDA(2110) BRAMCHING RATIOS

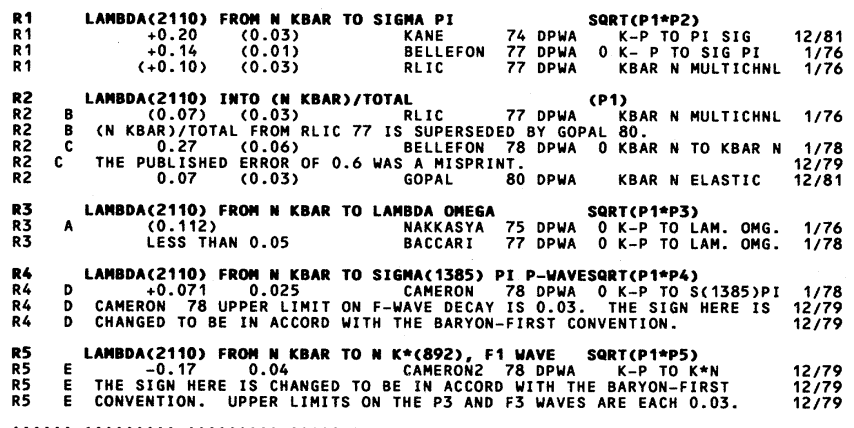

REFEREMCES FOR LAMBDA(2110)

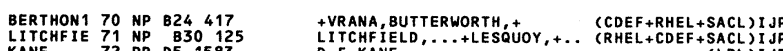

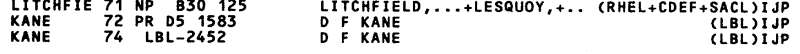

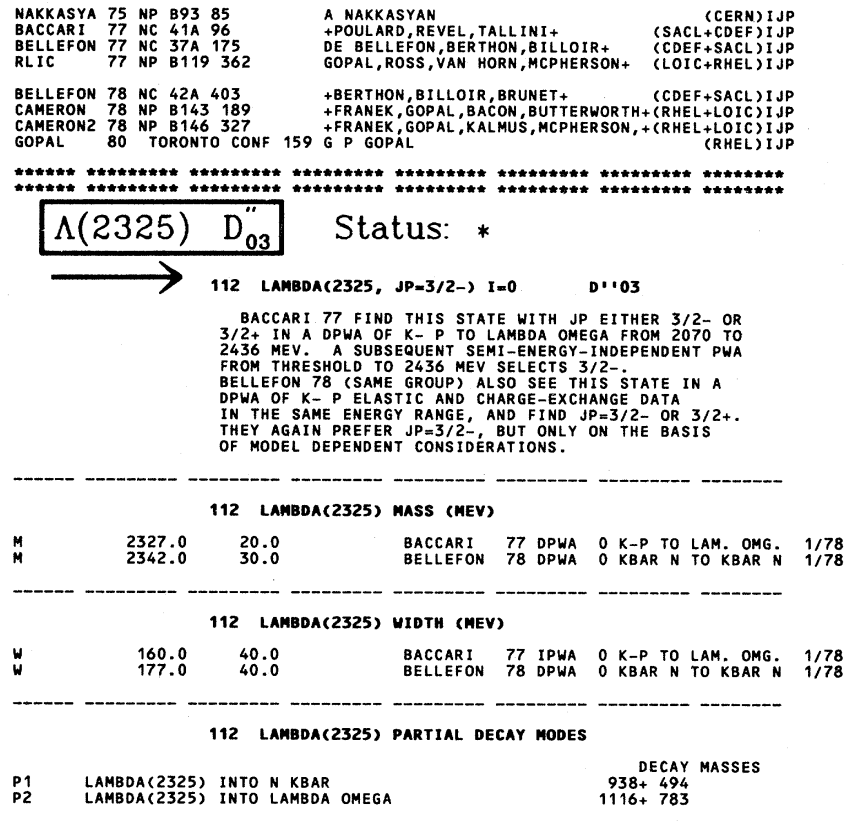

112 LAMBda(2325) BRAMCHIMG RATIOS

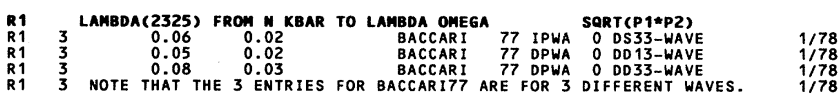

R2 LAMBDA(2325) INTO (N KBAR)/TOTAL
R2
0.19

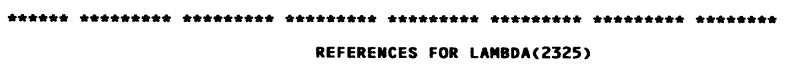

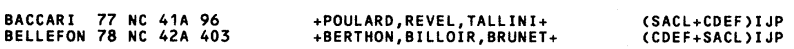

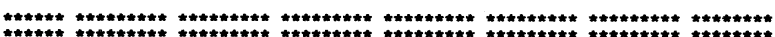

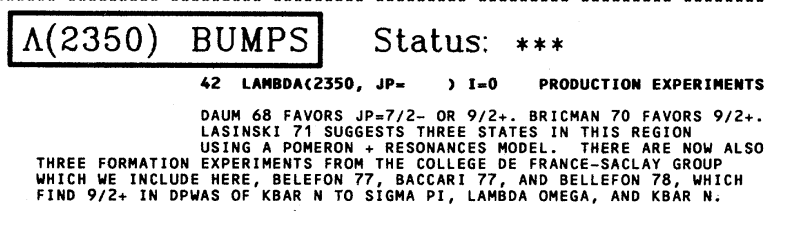

42 LAMBDA(2350) MASS (MEV) (PROD. EXP.)

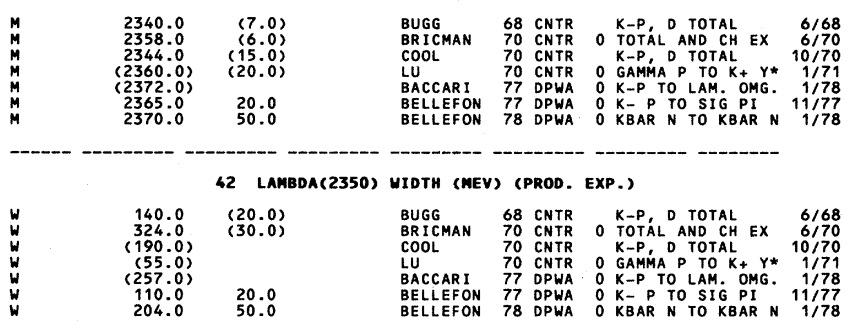

42 Lambda(2350) PARTial decay modes (PROD. EXP.)

$\begin{array}{lll}\text { P1 } & \text { LAMBDA(2350) INTO N KBAR } & \text { DECAY MASSES } \\ \text { P2 } & \text { LAMBDA(2350) INTO SIGMA PI } & 938+494 \\ \text { P3 } & \text { LAHBDA } & 189+140\end{array}$

P3 LAMBDA(2350) INTO LAMBDA OMEGA $1116+783$

42 LAMBDA(2350) BRAMCHING RATIOS (PROD. EXP.)

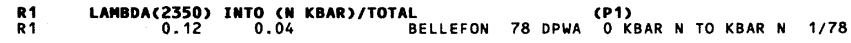




\section{Baryons}

$\Lambda(2350), \Lambda(2585), \Sigma^{+}, \Sigma^{-}, \Sigma^{0}, \Sigma(1385)$

\section{Data Card Listings}

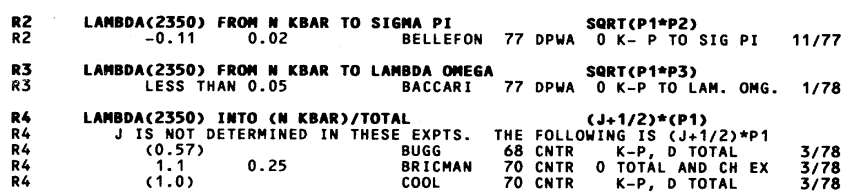

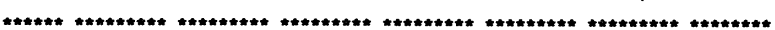
REFEREMCES FOR LAMBDA(2350) (PROD. EXP.)

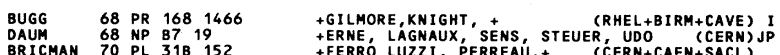

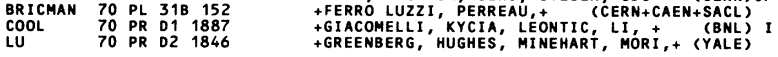

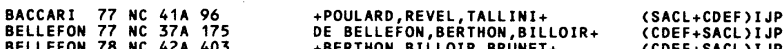
PAPER MOT REFERRED TO IN DATA CARDS
COOL O6 PRL 16 1228
+GIACOMELLI, KYCIA, LEONTIC, LI, LUNDBY, (BNL) I

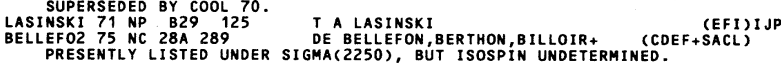

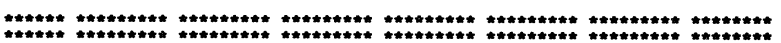
$\Lambda(2585)$ BUMPS Status: **

$\longrightarrow 7$ LAMBdAC2585, JP- , I=0 PRODUCTION EXPERIMENTS

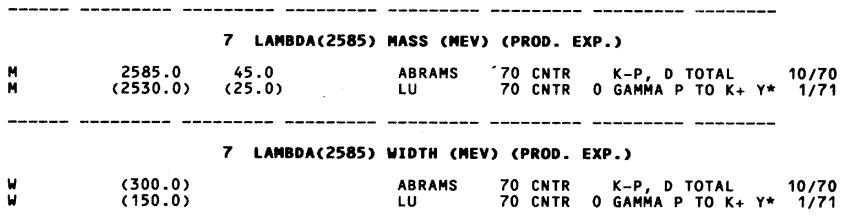

7 LAMBda(2585) PARTIAL DeCAY MODES (PROD. EXP.)

P1 LAMBDA(2585) INTO N KBAR DECAY MASSES
$938+494$

7 LAMBDAC2585) BRAMCHIMG RATIOS (PROD. EXP.)

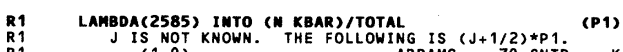

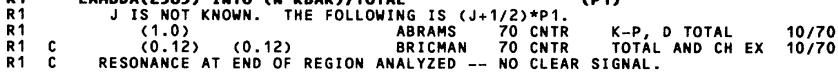

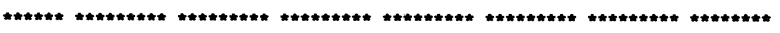
REFEREMCES FOR LAMBDA(2585) (PROD. EXP.)

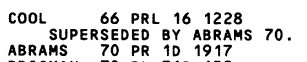

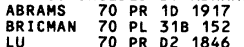
+GIACOMELLI, KYCIA, LEONTIC, LUNDBY + (BNL)I : $S=-1$ I=1 HYPERON STATES $(\Sigma)$ :

$\Sigma^{+} \quad 19$ SIGMA $+(1189, J P=1 / 2+) 1=1$

SEe Stable particle data card Listings

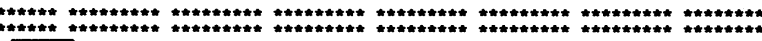
$\Sigma^{-} \quad 20$ SIGMA-(1197, JP=1/2t) I=1 SeE STABle particle data Card LISTINGS

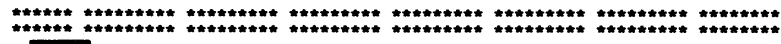
$\Sigma^{0} \quad 21$ SIGMAO(1193, JP=1/2+) I=1 SeE Stable particle data card Listings

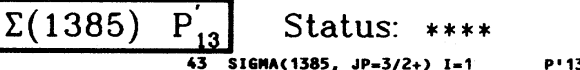

SERIOUS INCOMPATIBILITIES EXIST BETWEEN DIFFERENT INCOMPAT IBILITIES ARE AT LEAST PARTIALLY ACY OUNTED FOR

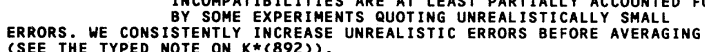

IN THE LISTMGS BELOW WE ATTEMPT TO OBTAIN THE BEST VALUES FOR THE
SEPARAT CHARE STATE MASSES AND WIDTHS. THUS WE DO NOT USE RESULTS WOTED FOR MIXED CHARGES.

WE HO LONGER USE EVERY PUBL ISHED VALUE, BUT AVERAGE ONLY THE MOST

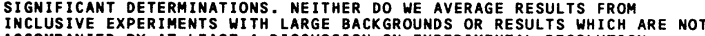

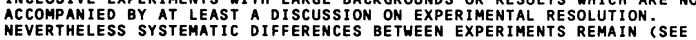
THE IDEOGRAMS INSERTED IN THE DATA CARD LISTINGS BELOWN. THESE
DIFFERENCES COULD ARISE FROM INTERERENE EFECTS THAT CHAMGE PRODUC TION MECHANISM AND/OR BEAM MOMENTUM. THEY CAN ALSO BE ACCOUNTED

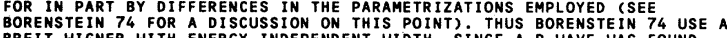
BREIT-MIGNER WIT ENERG INOEPENDENT WIDTH, SINCEA A P-HAVE WAS FOUNO

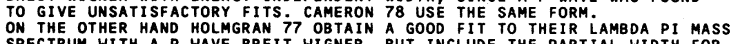
THE SIGMA PI DECAY MODE IN THE PARAMETRIZATION.
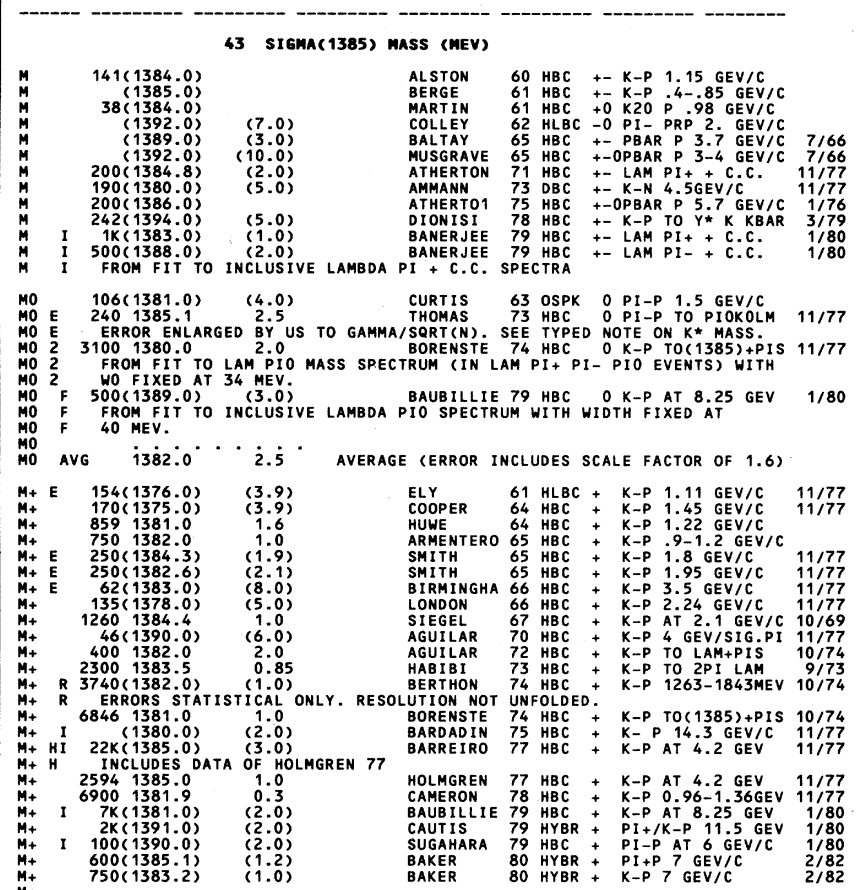

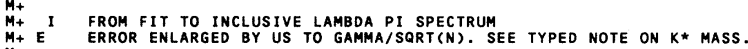

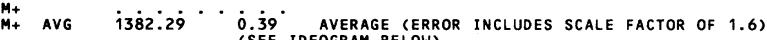
(SEE IDEOGRAM BELOW)

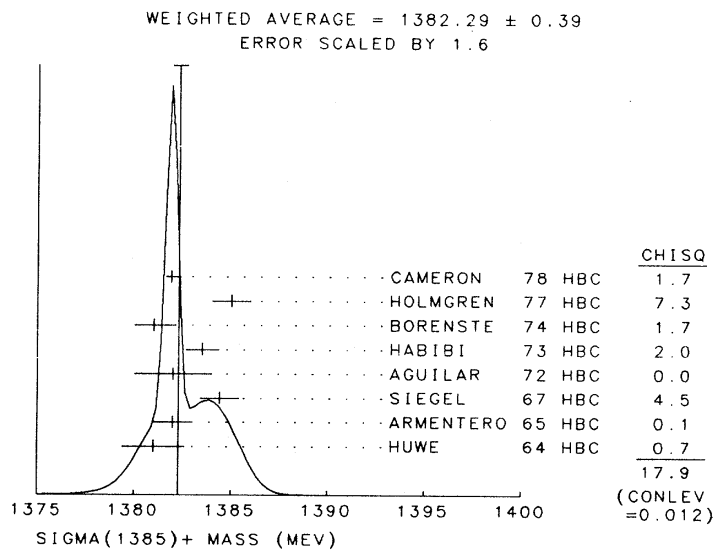




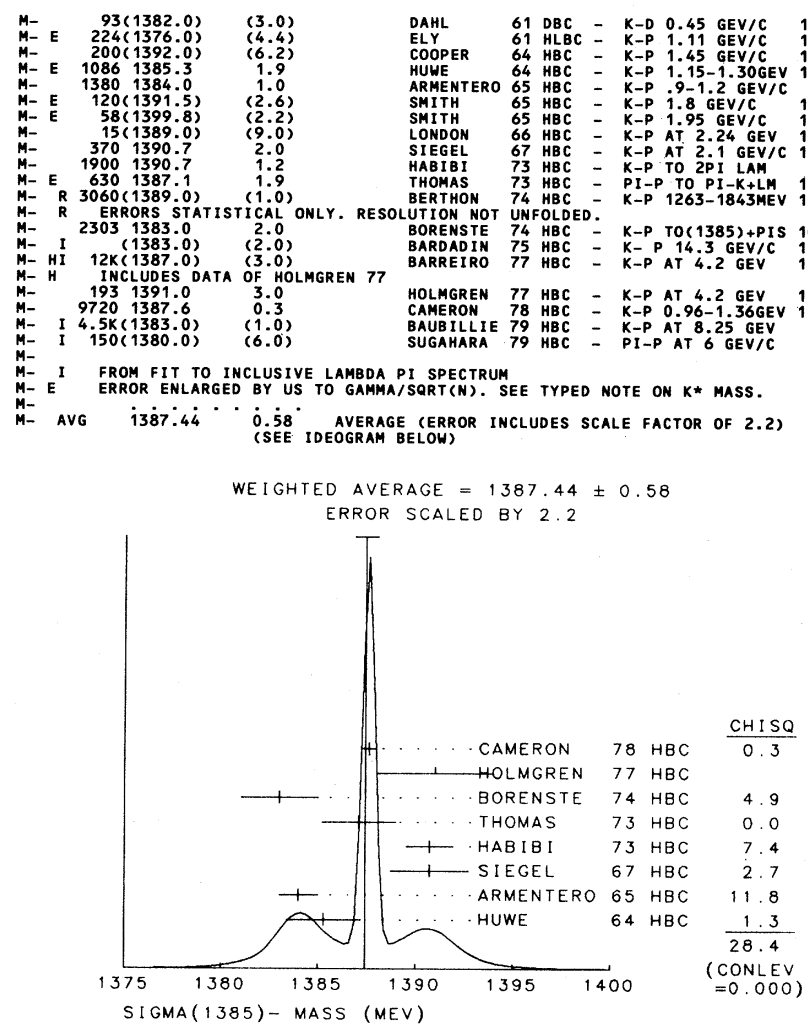

43 (SIGMA(1385)-)-(SIGMA(1385)+) MASS DIFFEREMCE (MEV)

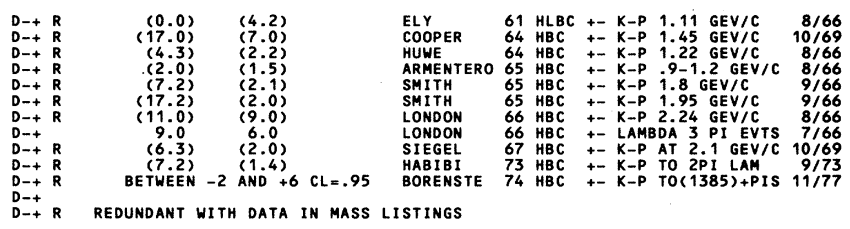

43 (SIGMA(1385)0) - (SIGMA(1385)+) MASS DIFFER. (MEV)

D0+
$D 0+R$ REDUNDANEN -4 AND +4 CL $=95$ BORENSTE 74 HBC +0 K-P TO(1385)+PIS $11 / 77$ -

43 (SIGMA(1385)-) - (SIGMA(1385)0) MASS DIFFER. (MEV)

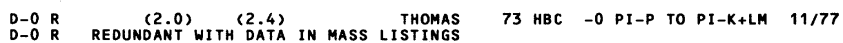

D-O R REDUNDANT WITH DATA IN MASS LISTINGS
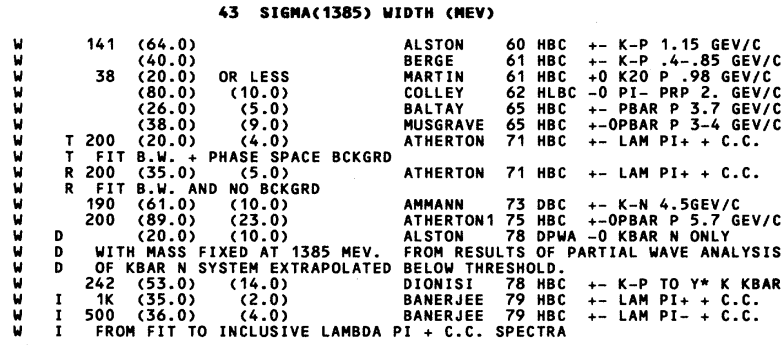

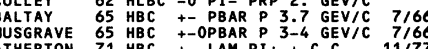

ATHERTON $71 \mathrm{HBC}+-$ LAM PI+ + C.C. $11 / 77$

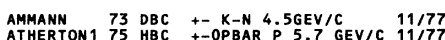

ALSTON 78 DPWA -0 KBAR N ONLY GEV/C $11 / 77$ $\begin{array}{ll}\text { FROM RESULTS OF PARTIAL WAVE ANALYSIS } & 1 / 78 \\ \text { BELOW THRESHOLD. } & 1 / 78 \\ \text { DOONISI } & 78 \text { HBC +- K-P TO Y* K KBAR } 3 / 79\end{array}$ $\begin{array}{llll}\text { DIONISI } & 78 \mathrm{HBC} & +- \text { K-P TO Y* K KBAR } & 3 / 79 \\ \text { BANERJEE } & 79 \mathrm{HBC}+- \text { LAM PI+ + C.C. } & 1 / 80 \\ \text { BANERJEE } & 79 \mathrm{HBC}+\text { - LAM PI + C.C. } & 1 / 80\end{array}$

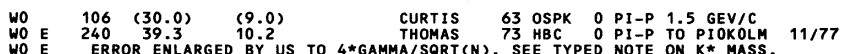

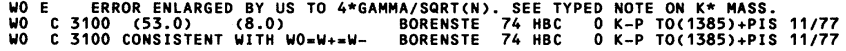

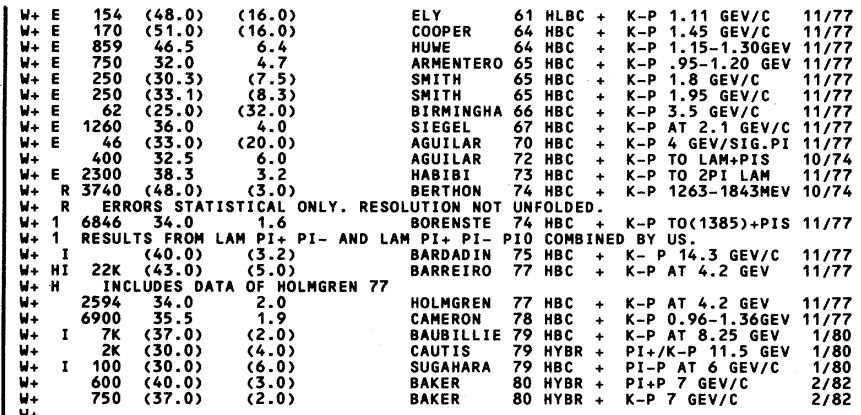

$H_{+}$I FROM FIT TO INCLUSIVE LAMBDA PI SPECTRUM
$W_{+}$ER EROR ENLARGED BY US TO 4 \#GAMMA/SQRT (N). SEE TYPED NOTE ON $K$ * MASS.

${ }^{H_{+}} \mathrm{H}^{+}$aVG $34.96^{\circ} \cdot 0.92^{\circ}$ aVerage

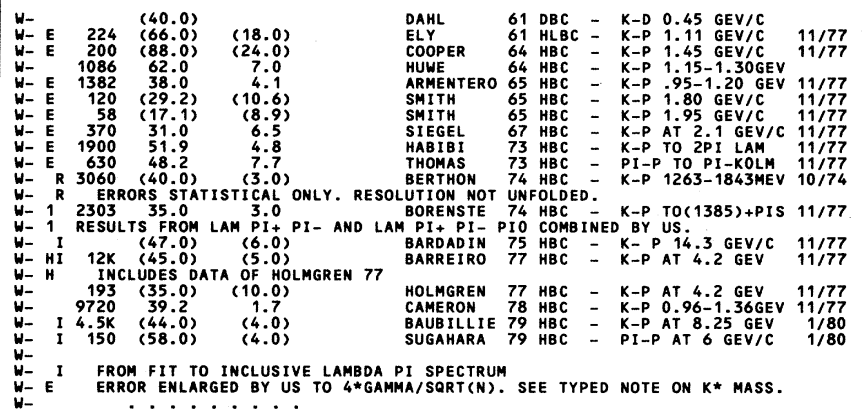

F- I FROM FIT TO INCLUSIVE LAMBDA PI SPECTRUM
W- E ERROR ENLARGED BY US TO 4*GAMMA/SQRT(N). SEE TYPED NOTE ON K* MASS

W- AVG $39^{\circ} .^{\circ} \cdot{ }^{2} .4^{\circ}$ AVERAGE (ERROR INCLUDES SCALE FACTOR OF 1.9 )

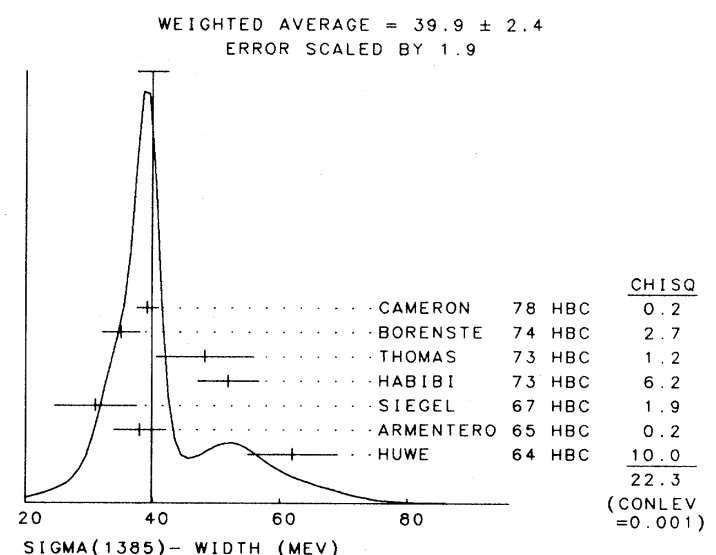

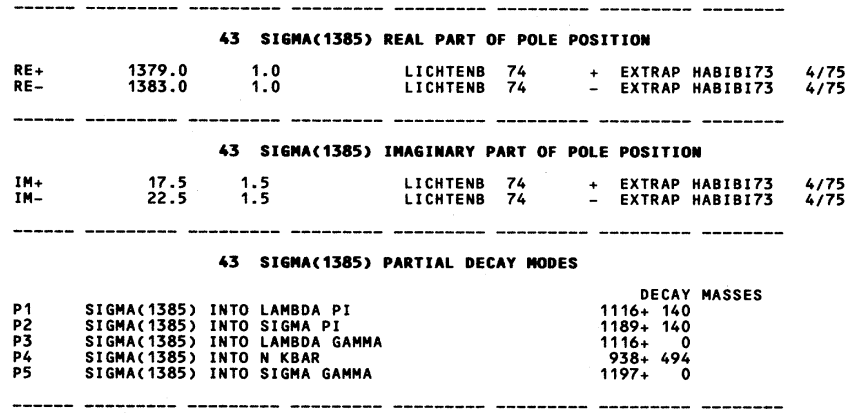




\section{Baryons}

$\Sigma(1385), \Sigma(1480), \Sigma(1560)$

\section{Data Card Listings}

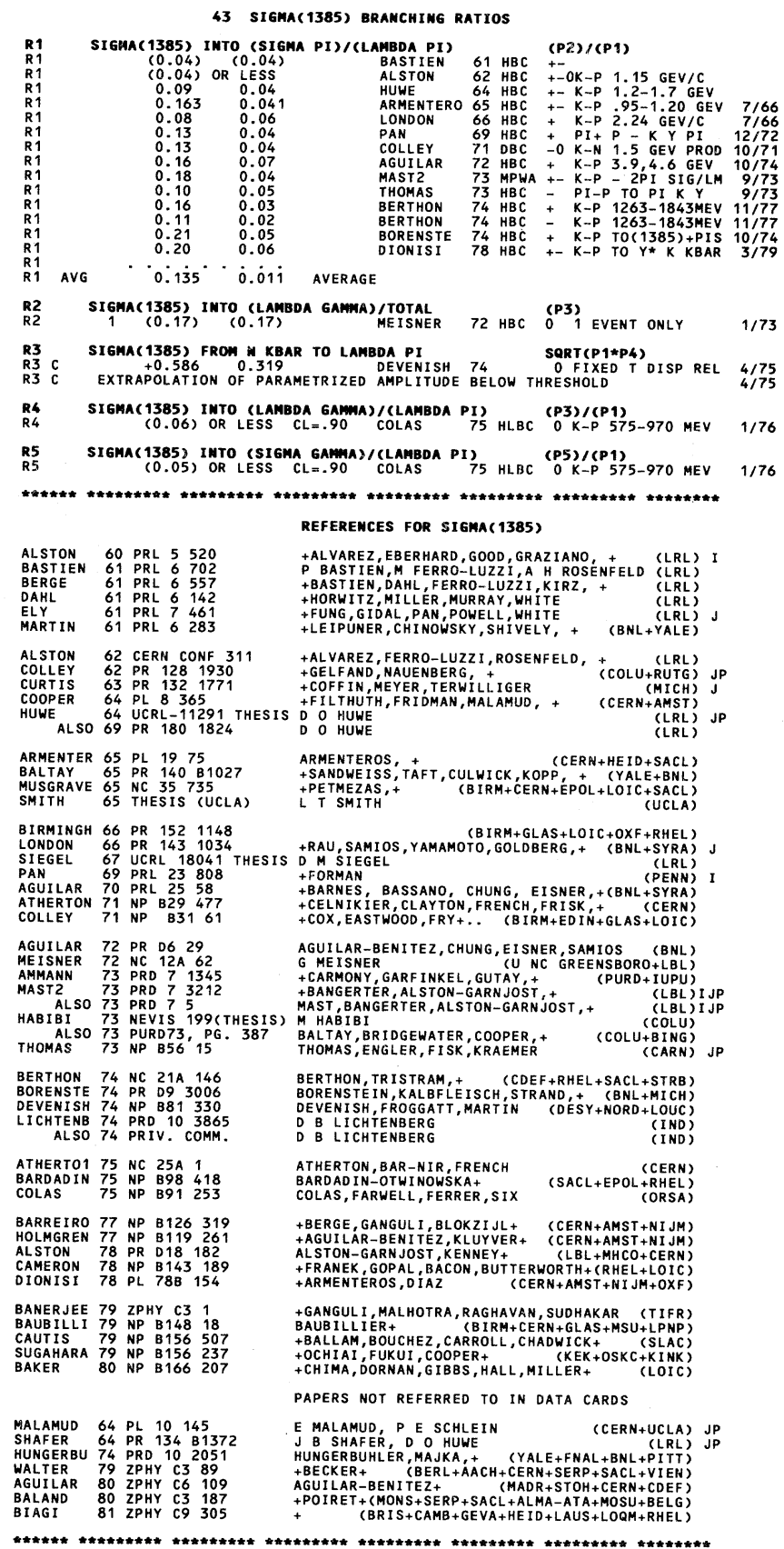

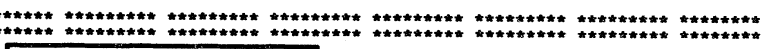

$\Sigma(1480)$ BUMPS Status: *

23 SIGMAC1480, JP= , I=1 PRODUCTION EXPERIMENTS

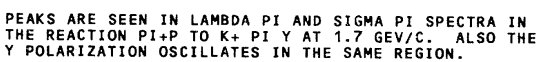

SEE MILLER 70 FOR A DISCUSSION OF THIS STATE. HE SUGGESTS A POSSIBLE
ALTERNATE EXPLANATION IN TERMS OF A REFLECTION OF NG 1675 S DECAY

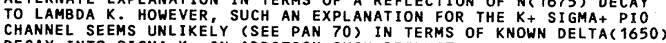
DEAY INTO SIGMA K. IN ADDITION SUCH REFLECIONS WOULD ALSO HAVE
TACAOUNT YOR THE OSCILATION OF THE Y POLARIZATION IN THE 1480
MASS REGION.

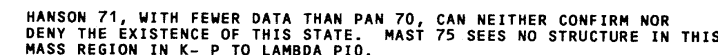

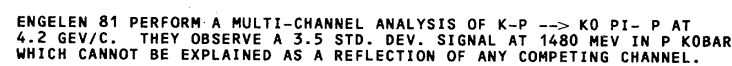

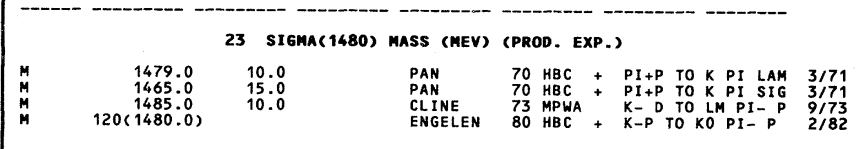
23 SIGMA(1480) UIDTH (MEV) (PROD. EP.)

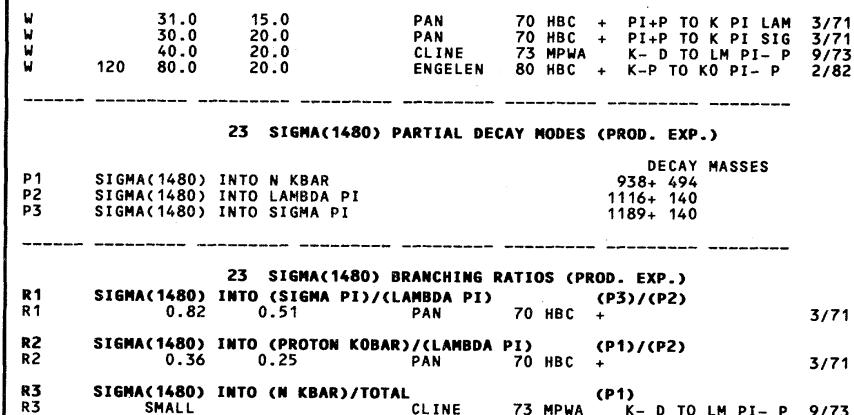
R3 SMALL INTO (N KOAR)/TLTLL CLINE 73 MPWA P K- D TO LM PI- P 9/73

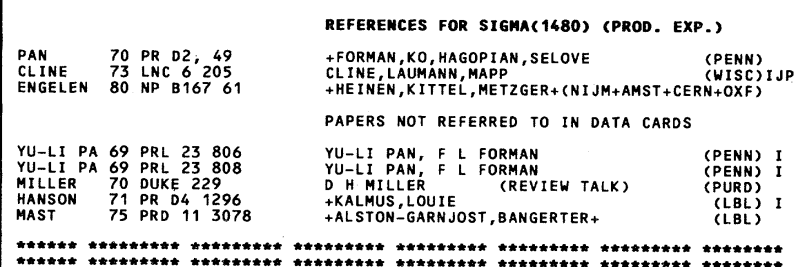

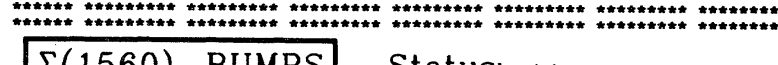
$\Sigma(1560)$ BUMPS Status: ** 80 SIGMAC 1560, JP= , I=1 PRODUCTION EXPERIMENTS THIS ENTRY LISTS PEARS REPORTED IN MASS SPECTRA AROUND
1560 MEVY WITHOUT IMPLYING THAT THEY ARE NEEESSARILY
RELATED. DIONISI 78 OBSERVE A 6 S STD. DEV. ENHANCEMENT AT 1553

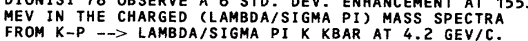

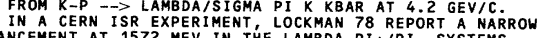

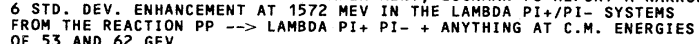

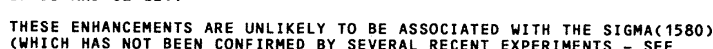

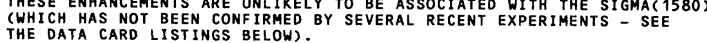
CARROLL 76 OBSERVE A BUMP AT 1550 MEV CAS WELL AS AT 1580 MEV IN THE K-N IET TOTAL CROS SECTION, BUT UNCERAANTIES IN CROSS SECTION
MEAUREMETS OUTSTDE THE MASS RANGE OF THE EXPERIMENT PRECLUDE ING ITS SIGNIFICANCE. SEE ALSO MEADOWS 80 FOR A REview OF THIS STATE.

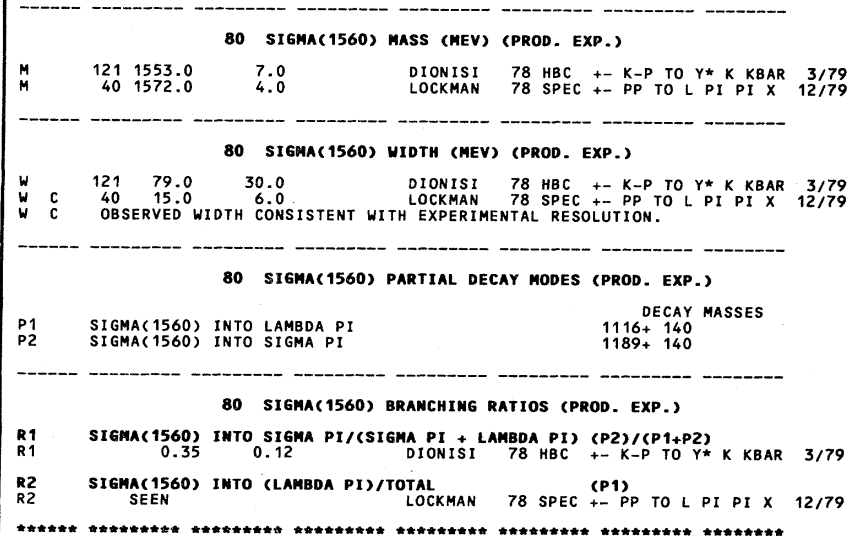




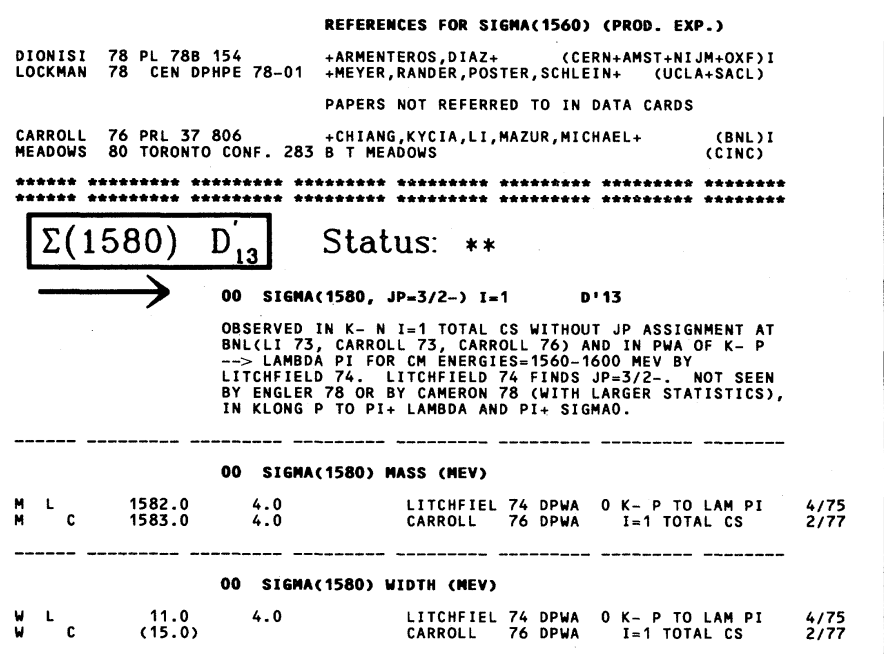

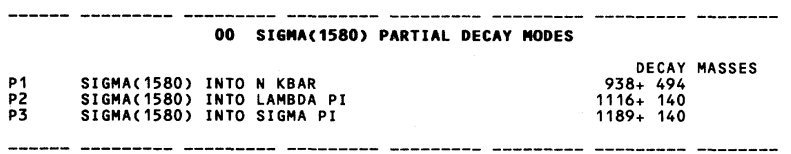

DO SIGMA(1580) BRAMCHIMG RATIOS

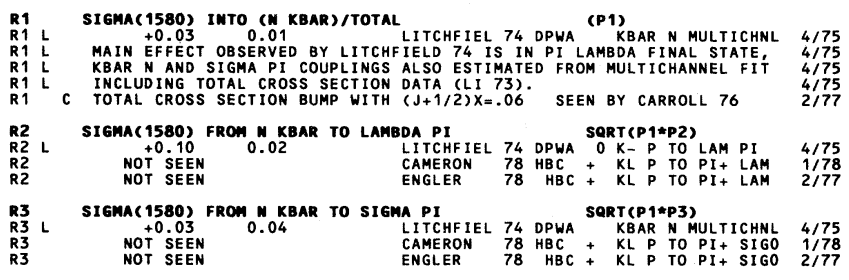

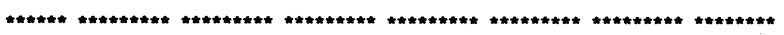

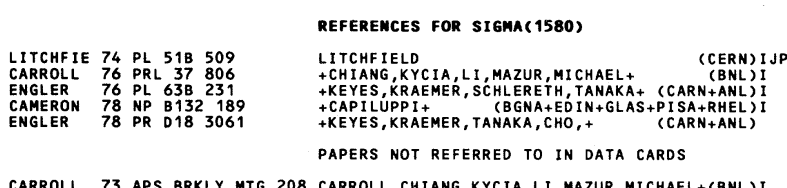

CARROLL 73 APS BRKLY MTG 208 CARROLL, CHIANG, KYCIA, LI, MAZUR, MICHAEL+(BNL) I
LI
(BNL) I

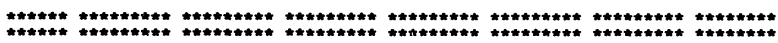

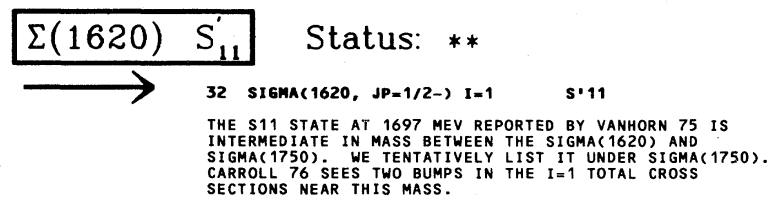

PRODUCTION EXPERIMENTS ARE LISTED SEPARATELY IN THE NEXT ENTRY.

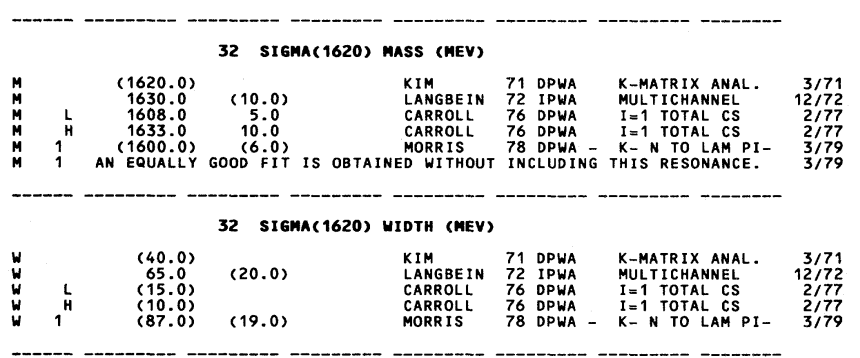

32 Sigma(1620) PARTIAL DECAY MODES

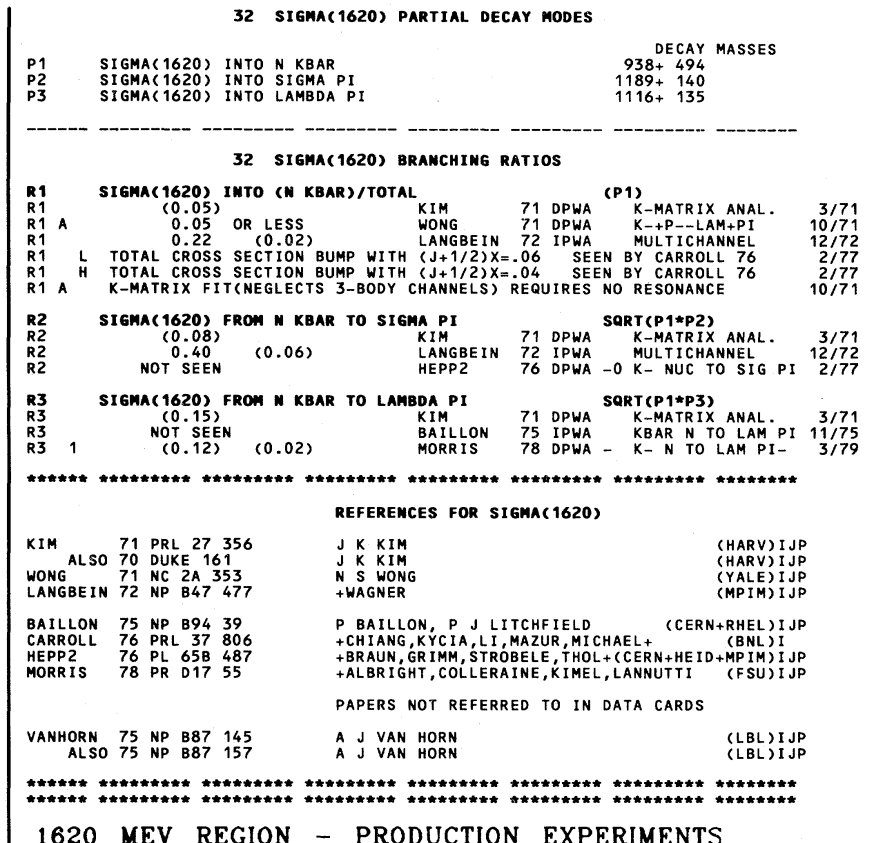

78 SIgmac 1620, JP= , $\mathrm{I}=1$ PRODUCTION EXPERIMENTS

FORMATION EXPERIMENTS ARE LISTED SEPARATELY IN THE

THIS RESONANCE NEEDS CONF IRMATION. THE RESULTS OF
CRENNELL 69 AT 3.9 GEV IC ARE NOT CONFIRMED BY THE SABRE COLLABORATION AT 3.0 GEV /C (SABRE 70). HOWEVER IN AN EXPERIMENT AT 4.5 GEV/C, AMMANN 70 SEE A PEAK AT 1642 MEV WHICH ON THE BASIS OF
BRANCHING RATIOS THEY DO NOT ASSOCIATE WITH THE SIGMA(1670). SEE MILLER 70 FOR A REVIEW OF THESE CONFLICTS.

78 SIGMA (1620) MASS (MEV) (PROD. EXP.)

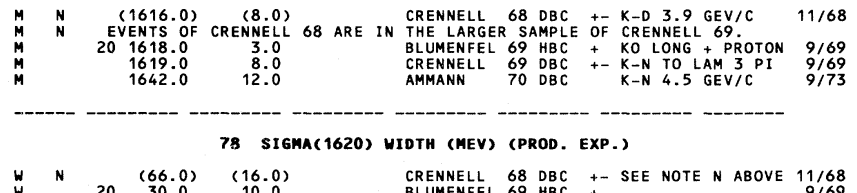

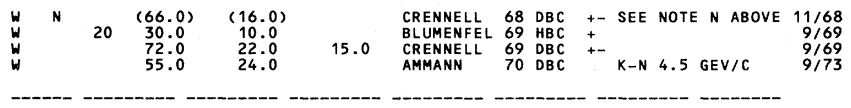

78 SIgma(1620) Partial decay mOdes (PROD. EXP.)

$\begin{array}{lllc} & & \\ \text { P1 } & \text { SIGMA(1620) INTO N KBAR } & \text { DECAY MASSES } \\ \text { P2 } & \text { SIGMA (1620) INTO LAMBDA PI } & 938+494 \\ \text { P3 } & \text { SIGMA(1620) INTO SIGMA(1385) PI } & 1116+140 \\ \text { P4 } & \text { SIGMA(1620) INTO LAMBDA PI PI } & 1385+140 \\ \text { P5 } & \text { SIGMA(1620) INTO SIGMA PI PI } & 1116+140+140 \\ \text { P6 } & \text { SIGMA(1620) INTO LAMBDA(1405) PI } & 1189+140 \\ & & & 1405+140\end{array}$

78 SIGMA(1620) BRANCHING RATIOS (PROD. EXP.)

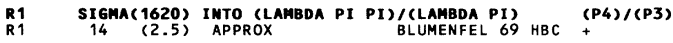

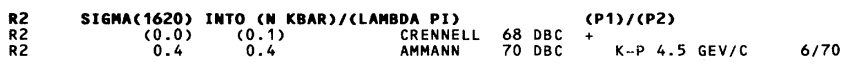

R3 SIGMA( 1620) INTO (LAMBDA PI)/TOTAL
R3 CRENNELL

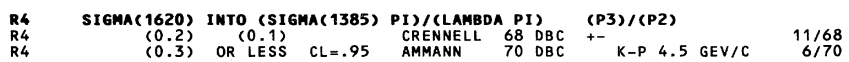

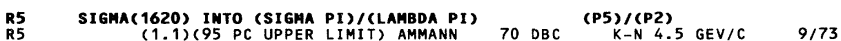

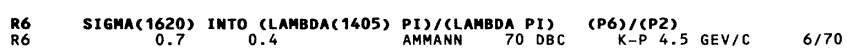




\section{Baryons}

$\Sigma(1620), \Sigma(1660), \quad \Sigma(1670)$

\section{Data Card Listings}

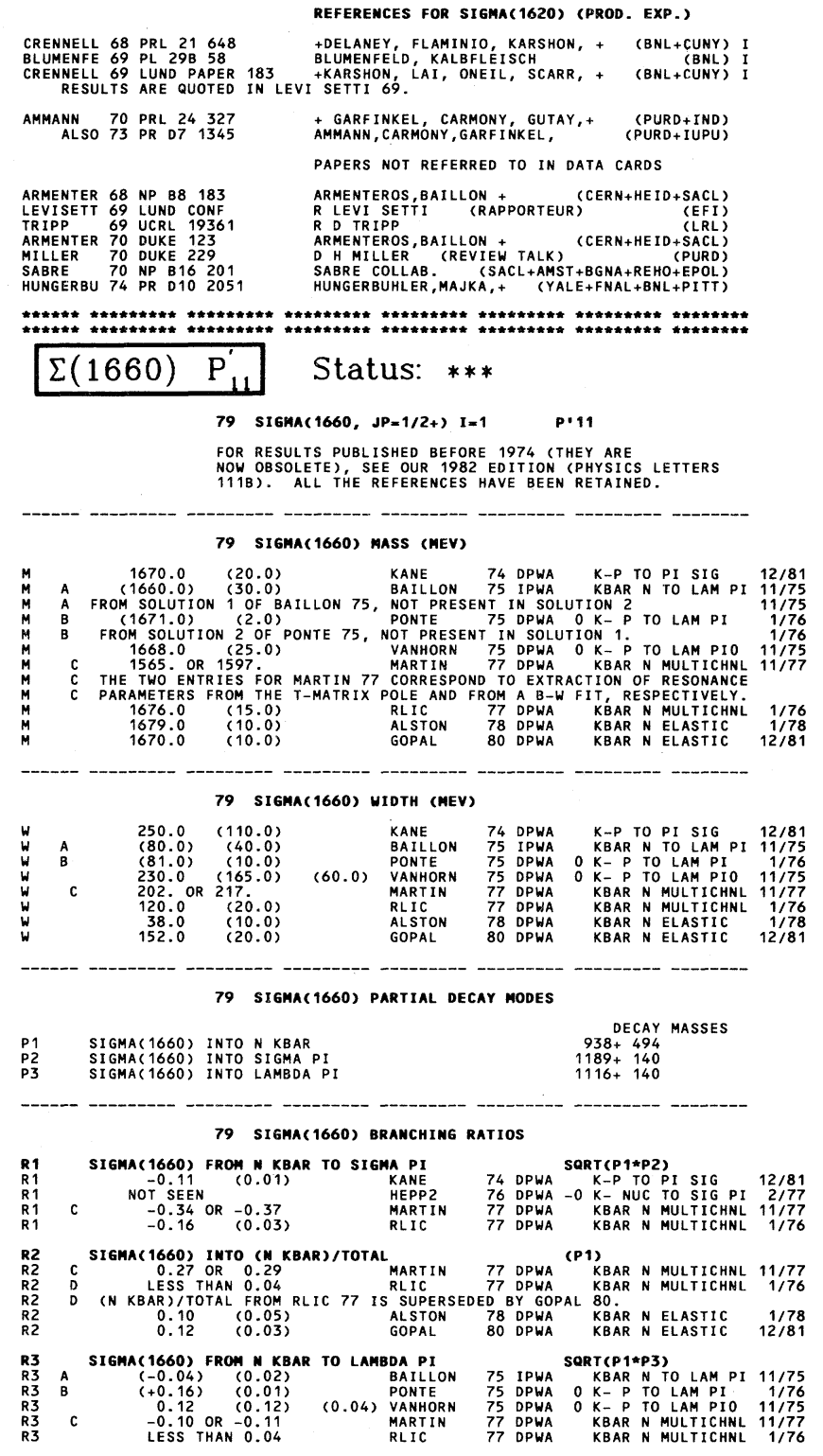

\section{NOTE ON THE $\Sigma(1670)$}

Production experiments: The measured $\Sigma \pi / \Sigma \pi \pi$ branching ratio for produced $\Sigma(1670)$ 's is strongly dependent on momentum transfer. This was first discovered by EBERHARD 69, who suggested that there exist two $\Sigma$ resonances with the same mass and quantum numbers: one with a large $\Sigma \pi \pi$ [mainly $\Lambda(1405) \pi$ ] decay mode produced peripherally, and the other with a large $\Sigma \pi$ decay mode produced at larger angles. These results were confirmed by AGUILAR-BENITEZ 70, ASPELL 74, ESTES 74, and TIMMERMANS 76. The most likely quantum numbers for both the $\Sigma \pi$ and the $\Lambda(1405) \pi$ states are $D_{13}$. There is also possibly a third $\Sigma$, the $\Sigma(1690)$ in the Listings, the main evidence for which is a large $\Lambda \pi / \Sigma \pi$ branching ratio. These topics have been reviewed by EBERHARD 73 and by MILLER 70.

Formation experiments: Two states are also observed near this mass in formation experiments. One of these, the $D_{13} \Sigma(1670)$, has the same quantum numbers as those observed in production and has a large $\Sigma \pi / \Sigma \pi \pi$ branching ratio. It may well be the $\Sigma(1670)$ produced at larger angles (see TIMMERMANS 76). The other state, the $P_{11} \Sigma(1660)$, has different quantum numbers from those seen in production, and its $\Sigma \pi / \Sigma \pi \pi$ branching ratio is unknown. Thus its relation to the produced $\Sigma(1670)$ 's remains obscure.

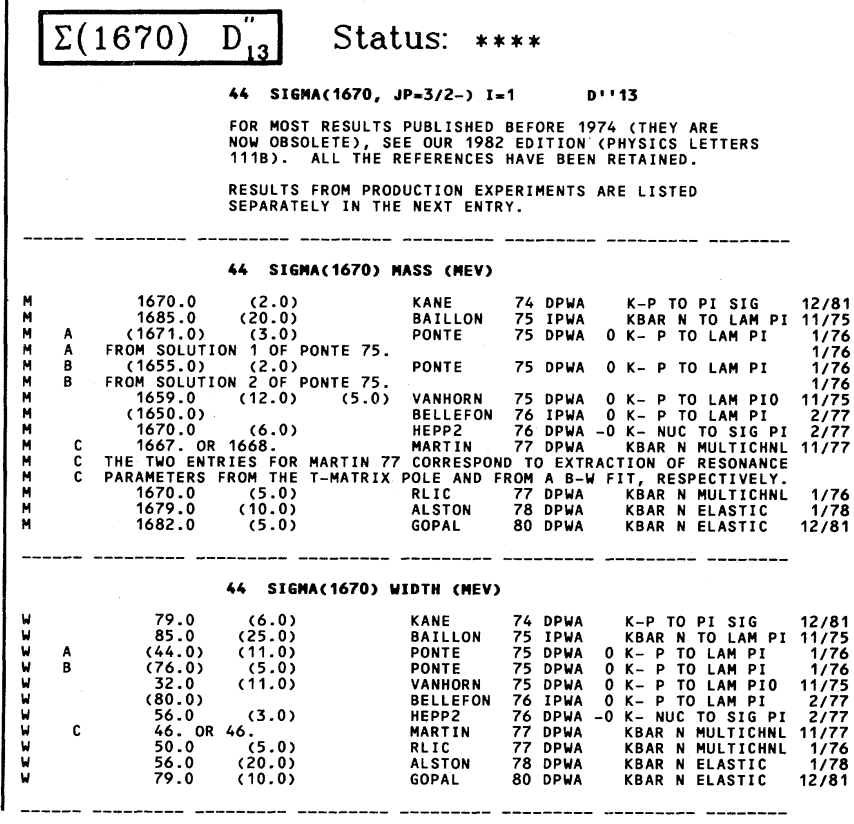




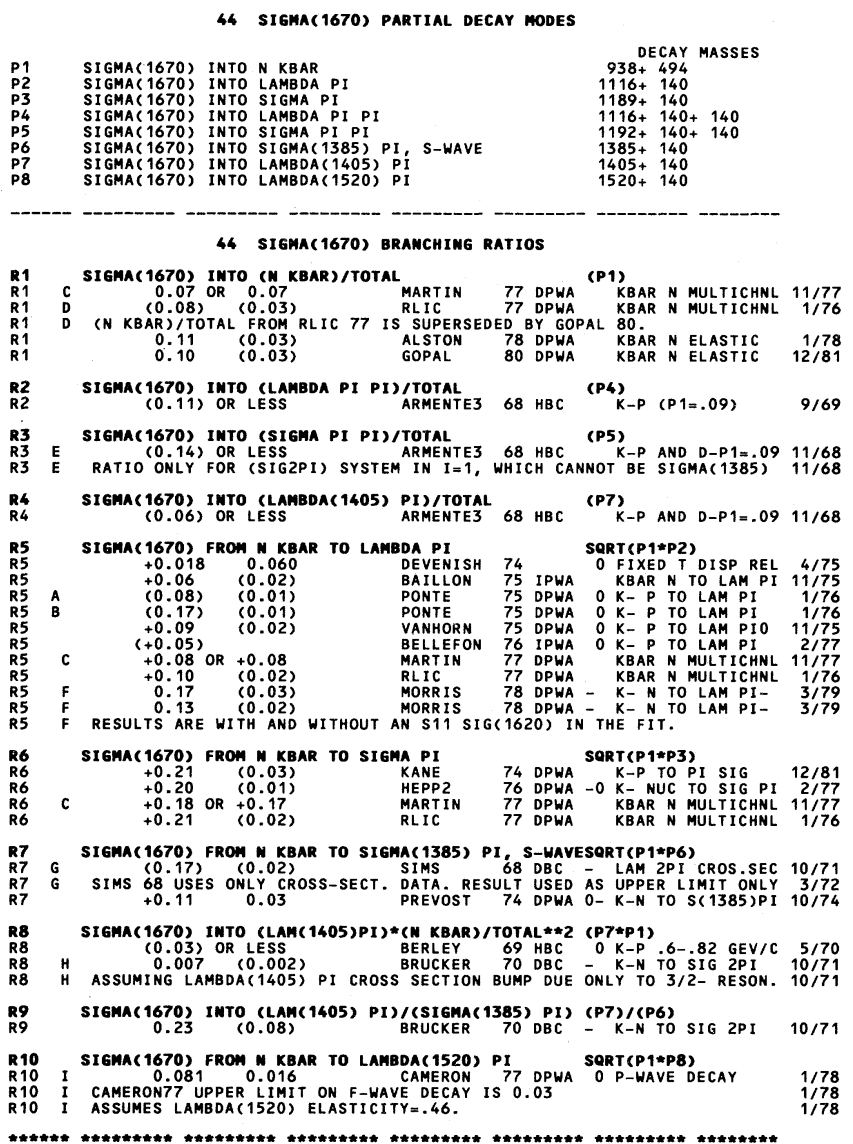

REFEREMCES FOR SIGMA (1670)

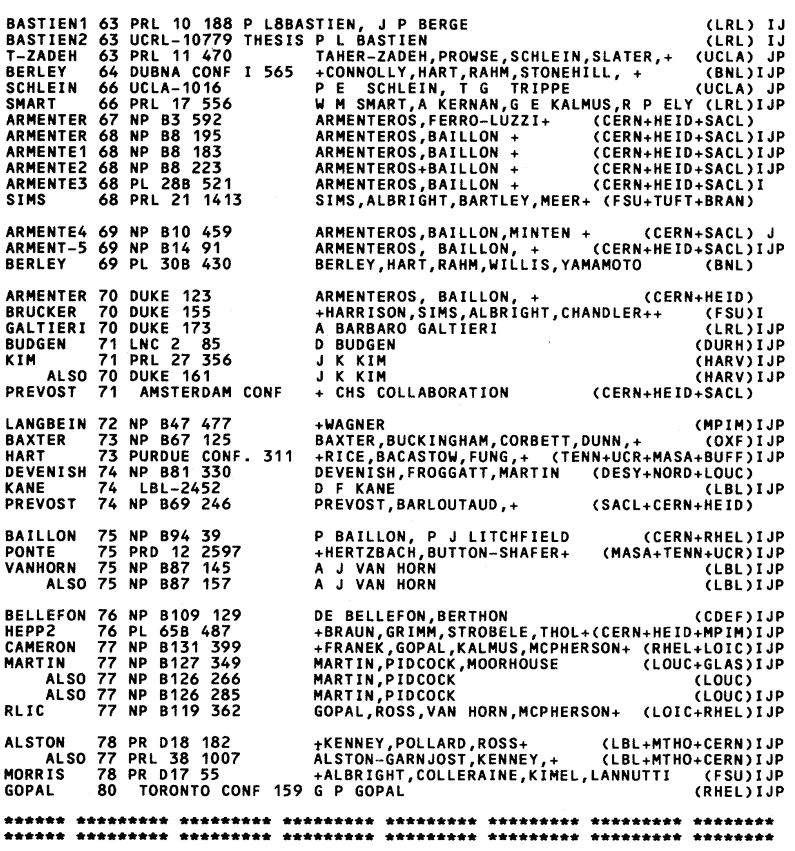

$\Sigma(1670)$ BUMPS

51 SIGMAC1670, JP= , I=1 PROD. AND CROSS SECT. EXPS FORMATION EXPERIMENTS ARE LISTED SEPARATELY IN THE PROBABLY THERE ARE TWO STATES AT SAME MASS WITH SAME QUANTUM NUMBERS, ONE DECAYING INTO SIGMA PI AND LAMBDA PRÉCEDING THE PRECEDING ENTRY.

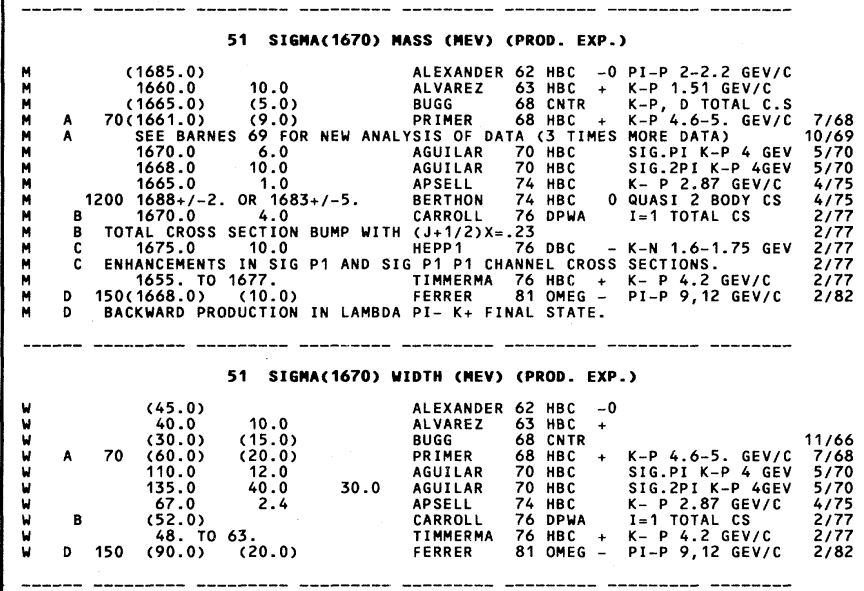

51 Sigma(1670) Partial decaY MOdES (PROD. EXP.)

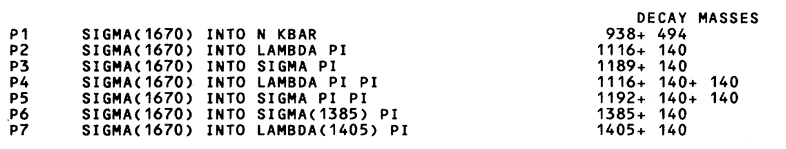

51 SIGMA(1670) BRAMCHING RATIOS (PROD. EXP.)

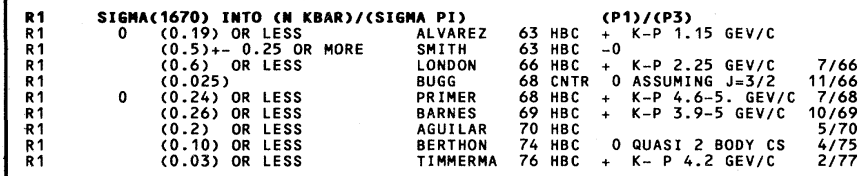

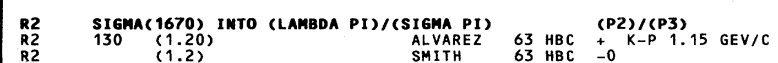

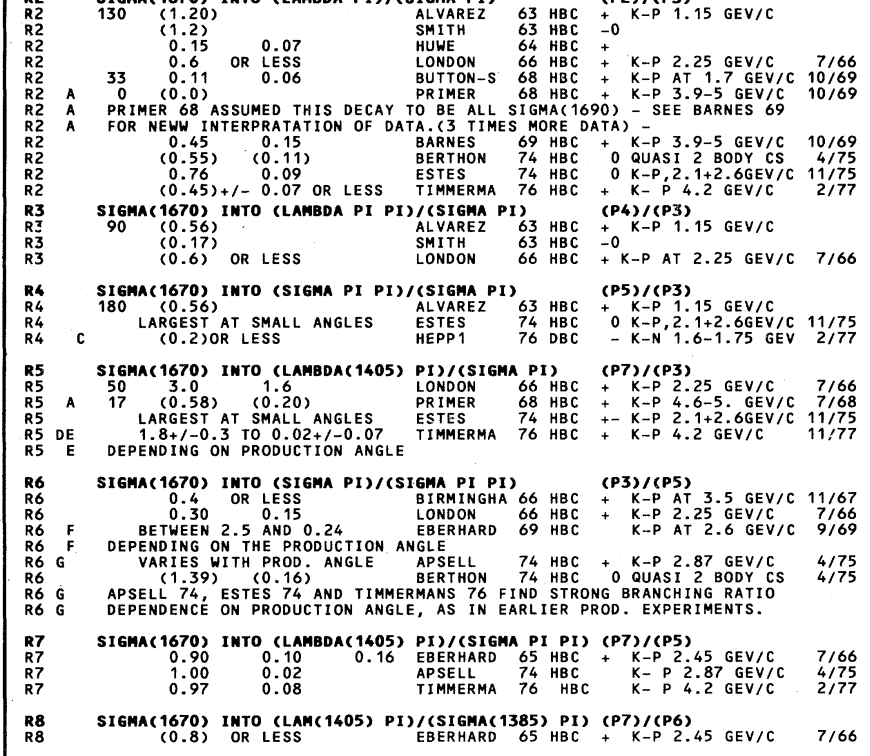


Baryons

$\Sigma(1670), \Sigma(1690), \Sigma(1750)$

\section{Data Card Listings}

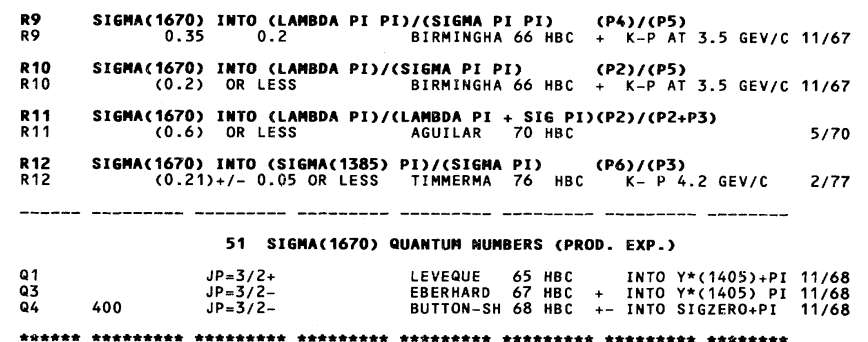
REFERENCES FOR SIGMA(1670) (PROD. EXP.)

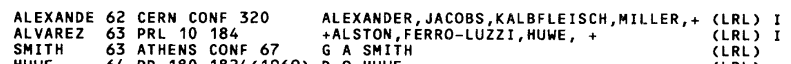

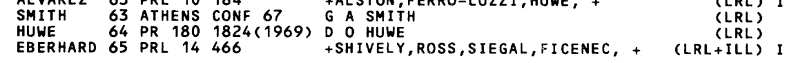

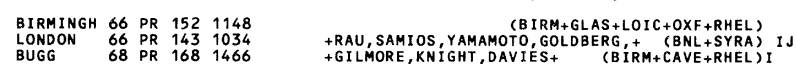

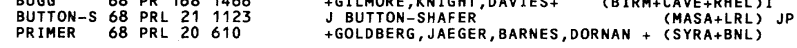

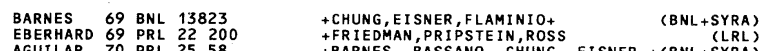
AGUILAR 70 PRL 2558 +BARNES, BASSANO, CHUNG, EISNER, +(BNL+SYRA)

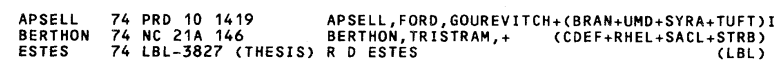

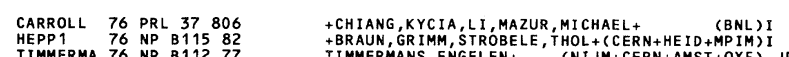

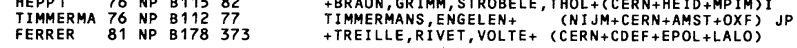
PAPERS NOT REFERRED to IN DATA CARDS

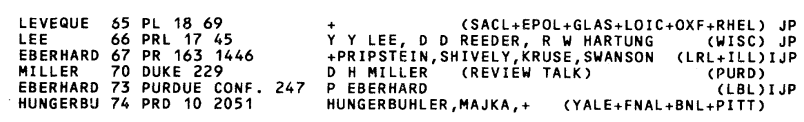

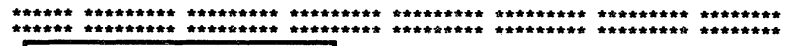
$\Sigma(1690)$ BUMPS Status: **

58 SIGMA $1690, \mathrm{JP}=$, I=1 PRODUCTION EXPERIMENTS SEE THE NOTE PRECEDING THE SIGMA(1670) LISTINGS. SEEN
IN PRODUCTION EXPERIMENTS ONLY, MAINLY IN LAMBDA PI.

58 SIGMA(1690) MASS (MEV) (PROD. EXP.)

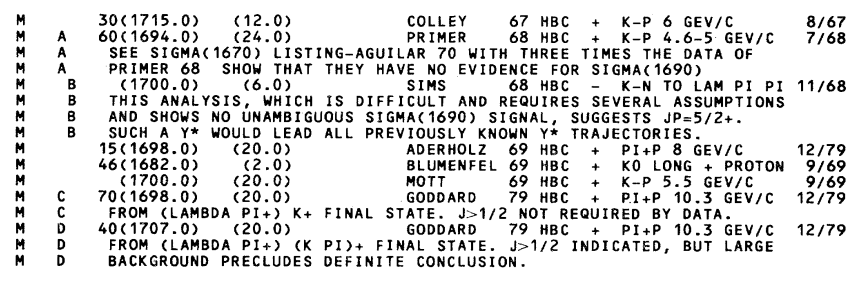

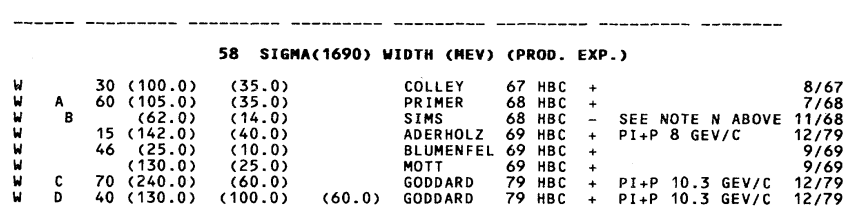

58 SIGMA (1690) PARTIAL deCAY MODES (PROD. EXP.)

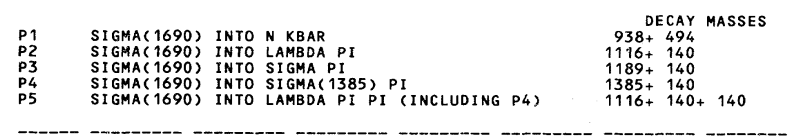

58 SIGMA(1690) BRAMCHIMG RATIOS (PROD. EXP.)

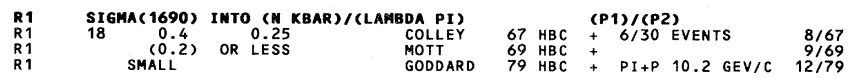

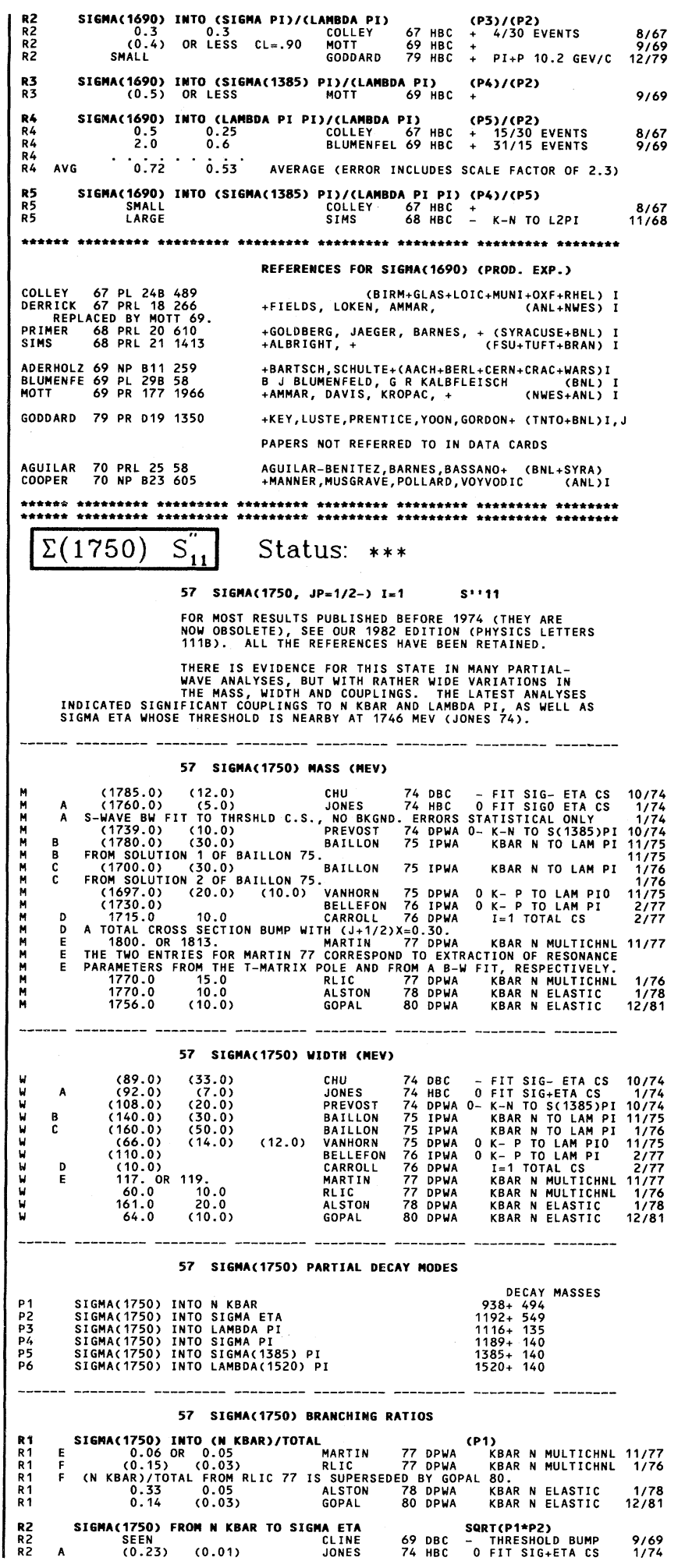




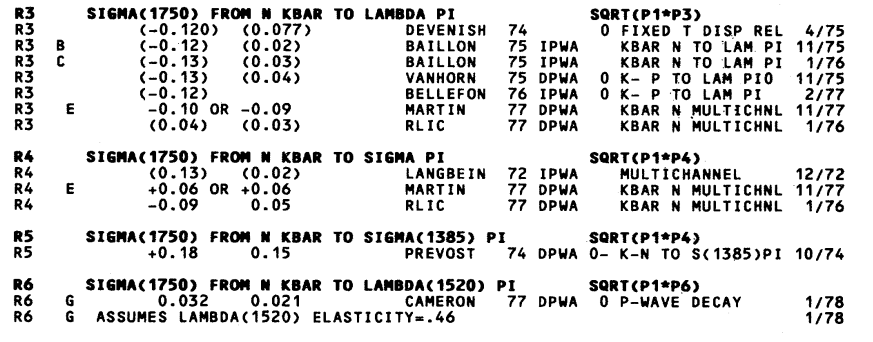

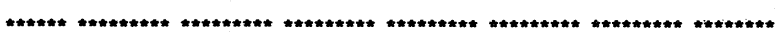
REFERENCES FOR SIGMA(1750)

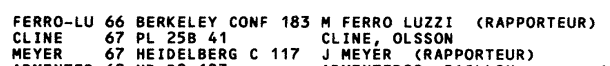

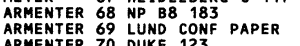

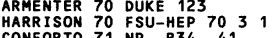

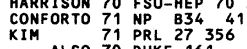

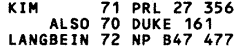

$\begin{array}{llllll}\text { BAXTER } & 73 & \text { NP } & 867 & 125 \\ \text { CHU } & 74 & \text { NC } 20 A & 35 \\ \text { JONES } & 74 & \end{array}$

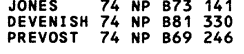

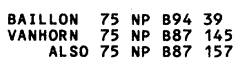

ARMERTEROS, BALLON, +
ARMENTEROS, BAILLON, +

ARMENTERS, BAILLON,

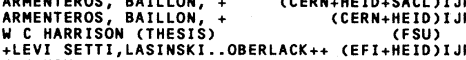

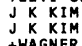

BAXTER, BUCKINGHAM, CORBETT, OUNN, + (OXF) IJP

JONES S $\begin{array}{ll}\text { DEEENISH, FROGGATT, MART IN } & \text { (DESY+NORD CHOUC) } \\ \text { PREVOST, BARLOUTAUD, } & \text { (SACL+CERN+HEID) }\end{array}$

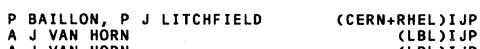

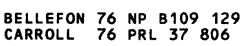

CAMERON 77 NP 8131399

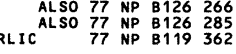

A J VAN HORN (LBL)IJJ

DE BELLEFON, BERTHON
+CHIANG, KYCIA, LI, MAZUR, MICHAEL+
(CDEF) IJP
(BNL)I FFRANEK, GOPAL,KALMUS, MCPHERSON+ (RHEL+LOIC) IJP

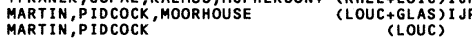

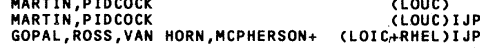

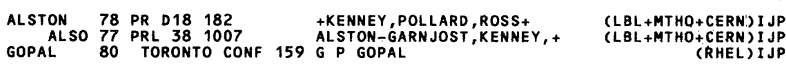

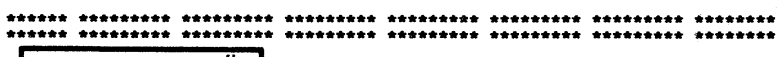
$\begin{array}{lll}\Sigma(1770) & \mathrm{P}_{11}^{\prime \prime}\end{array}$ Status: *

EVIDENCE FOR THIS STATE NOW RESTS SOLELY ON SOLUTION 1

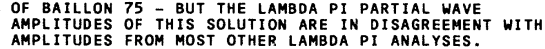

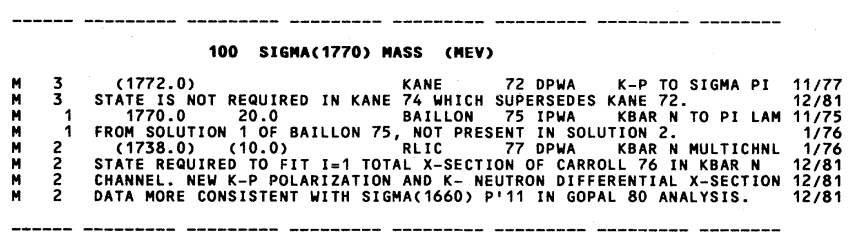

\begin{tabular}{|c|c|c|c|c|c|}
\hline & & si & IIDTH CMEV & & \\
\hline $\begin{array}{ll}W & 3 \\
y & 1\end{array}$ & $\begin{array}{l}(80.0) \\
80.0 \\
(72.0)\end{array}$ & $\begin{array}{r}30.0 \\
(10: 0)\end{array}$ & $\begin{array}{l}\text { KANE } \\
\text { BAILLON } \\
\text { RLIC }\end{array}$ & $\begin{array}{l}72 \text { DPHA } \\
75 \text { IPWA } \\
77 \text { DPWA }\end{array}$ & 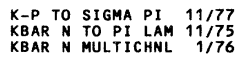 \\
\hline
\end{tabular}

100 Sigma(1770) PARTIAL decaY mODES

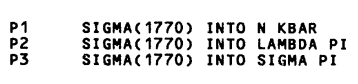

DECAY MASSES $938+494$
$1116+140$
$1189+140$

100 SIGMA(1770) BRAMCHIMG RATIOS

R1 2 SIGMA(1770) INTO (M KBAR)/TOTAL
R1
$(0.14)$
$(0.04)^{2}$

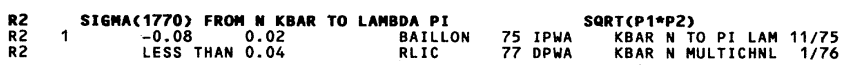

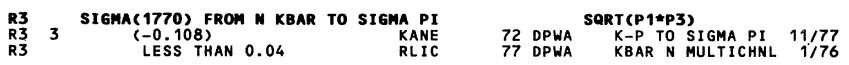

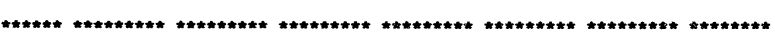

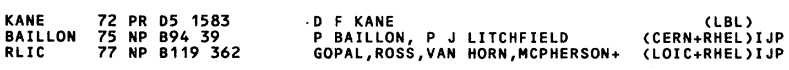

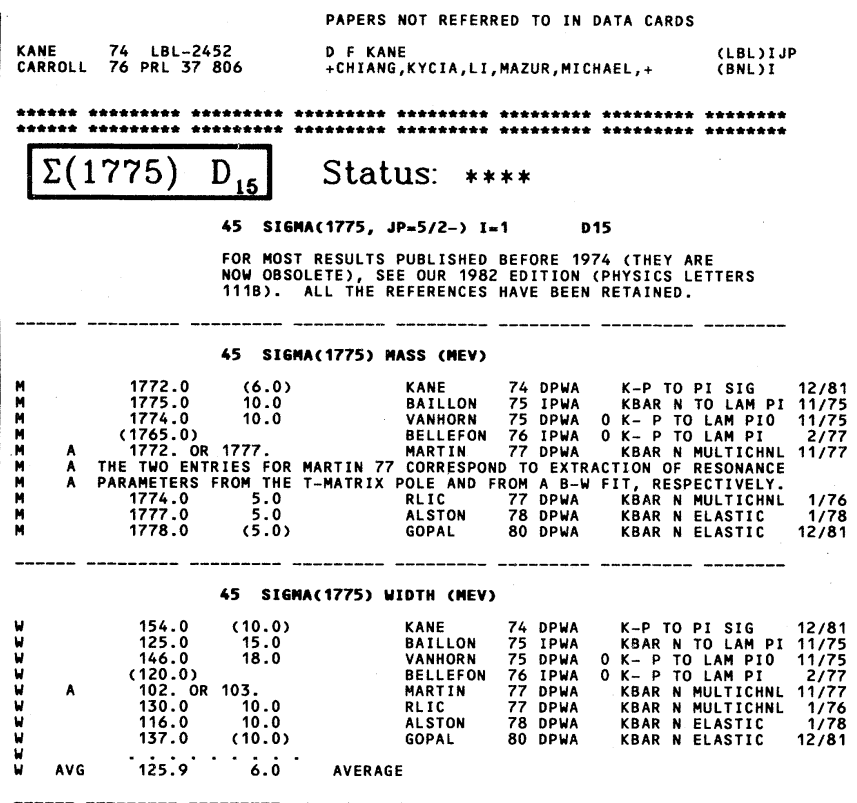

45 SIGMa(1775) PARTIAL DECAY modes

\begin{tabular}{|c|c|c|}
\hline $\begin{array}{l}\text { P1 } \\
\text { P2 } \\
\text { P3 } \\
\text { P4 } \\
\text { P5 } \\
\text { P6 } \\
\text { P7 }\end{array}$ & 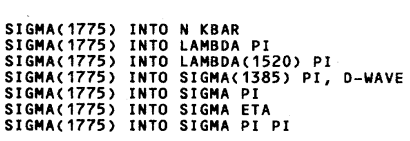 & $\begin{array}{l}\text { DECAY MASSES } \\
938+494 \\
1116+135 \\
1520+140 \\
1385+140 \\
1189+140 \\
1192+549 \\
1192+140+140\end{array}$ \\
\hline
\end{tabular}

\section{FITTED PARTIAL DECAY MODE BRANCHING FRACTIONS}

The matrix below is derived from the error matrix for the fitted partial decay mode branching fractions, $P_{i}$, as follows: The diagonal elements are $P_{i} \pm \delta P_{i}$, where

$\delta \mathrm{P}_{\mathrm{i}}=\sqrt{\left.\delta \mathrm{P}_{\mathrm{i}} \delta \mathrm{P}_{\mathrm{i}}\right\rangle}$, while the off-diagonal elements are the normalized cor relation coefficients $\left\langle\delta \mathrm{P}_{\mathrm{i}} \delta \mathrm{P}_{\mathrm{j}}\right\rangle /\left(\delta \mathrm{P}_{\mathrm{i}} \cdot \delta \mathrm{P}_{\mathrm{j}}\right\rangle$. For the definitions of the individual $\mathrm{P}_{\mathrm{i}}$, see the listings above; only those $P_{i}$ appearing in the matrix are assumed in the fit to be nonzero and are thus constrained to add to 1

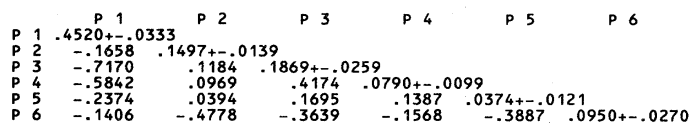

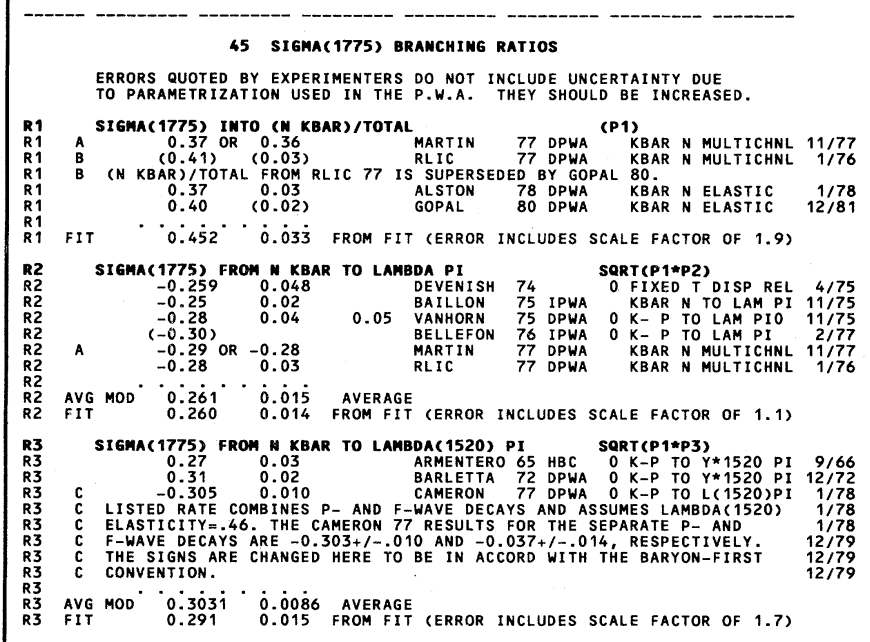


Baryons

$\Sigma(1775), \Sigma(1840), \Sigma(1880)$

\section{Data Card Listings}

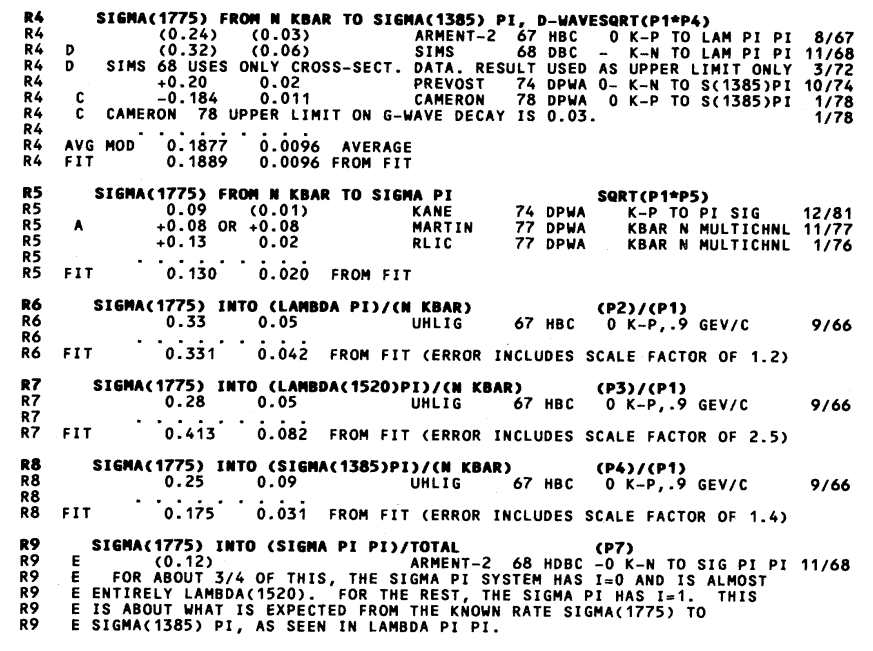

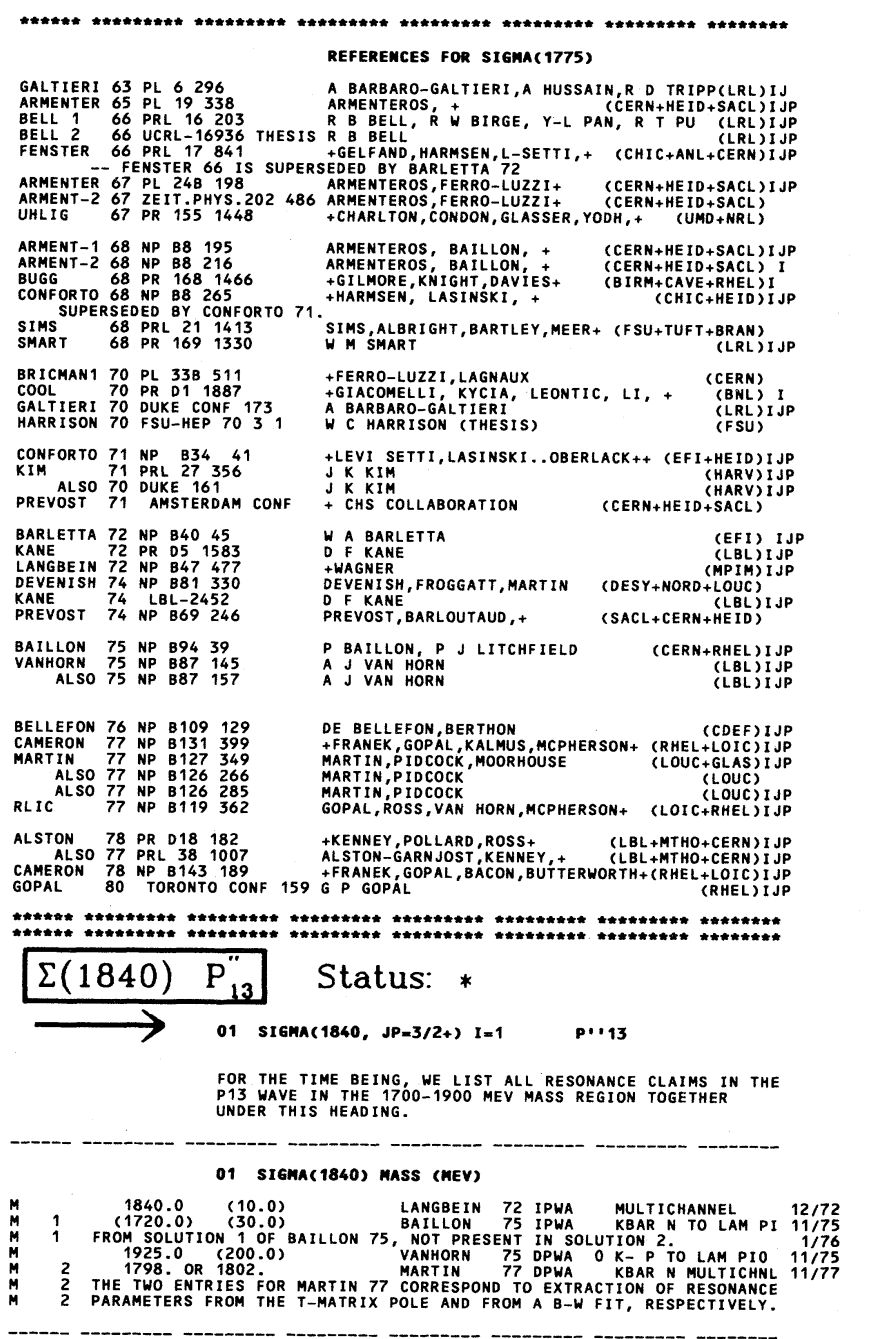

01 SIGMa(1840) MIOTH (MEV)

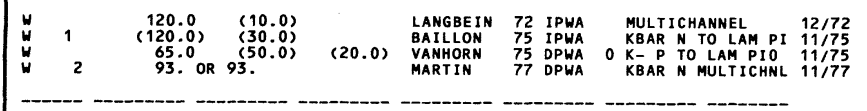

01 Sigma(1840) PARTIAL decay modes

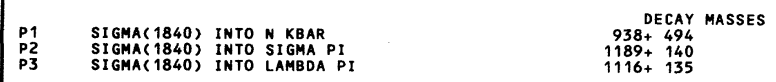

-

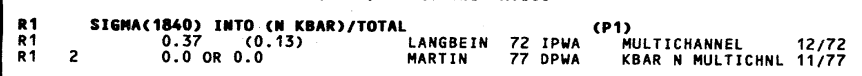

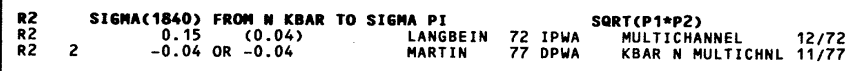

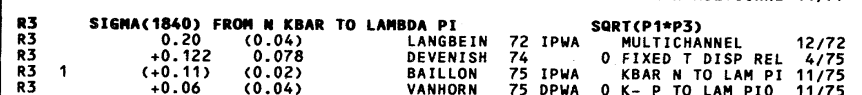

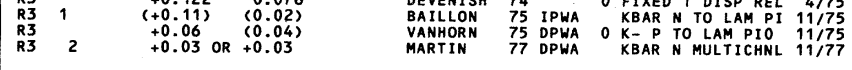

$* * * * * * * * * * * * * * * * * * * * * * * * * * * * * * * * * * * * * * * * * * * * * * * * * * *$
REFEREMCES FOR SIGMAC 1840$)$

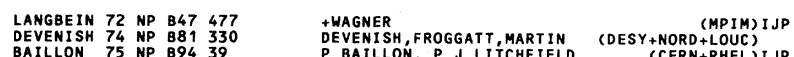

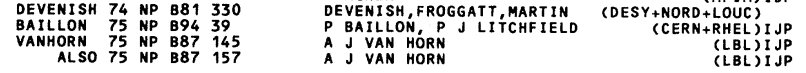

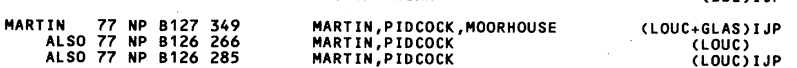

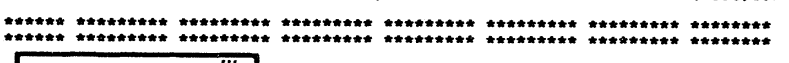

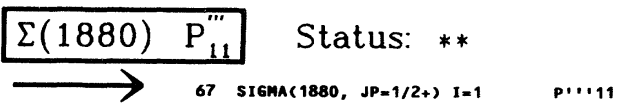

A RESONANCE IS SUGGESTED BY SEVERAL PARTIAL-WAVE
ANAYYSES ACROSS THS REGION, BUT WITH WIDE VARIATIONS IN THE MASS AND OTHER PARAME TERS T WE LIST HERE.
ALL CLAIMS WHICH LIE WELL ABOVE THE SIGMA(1770).

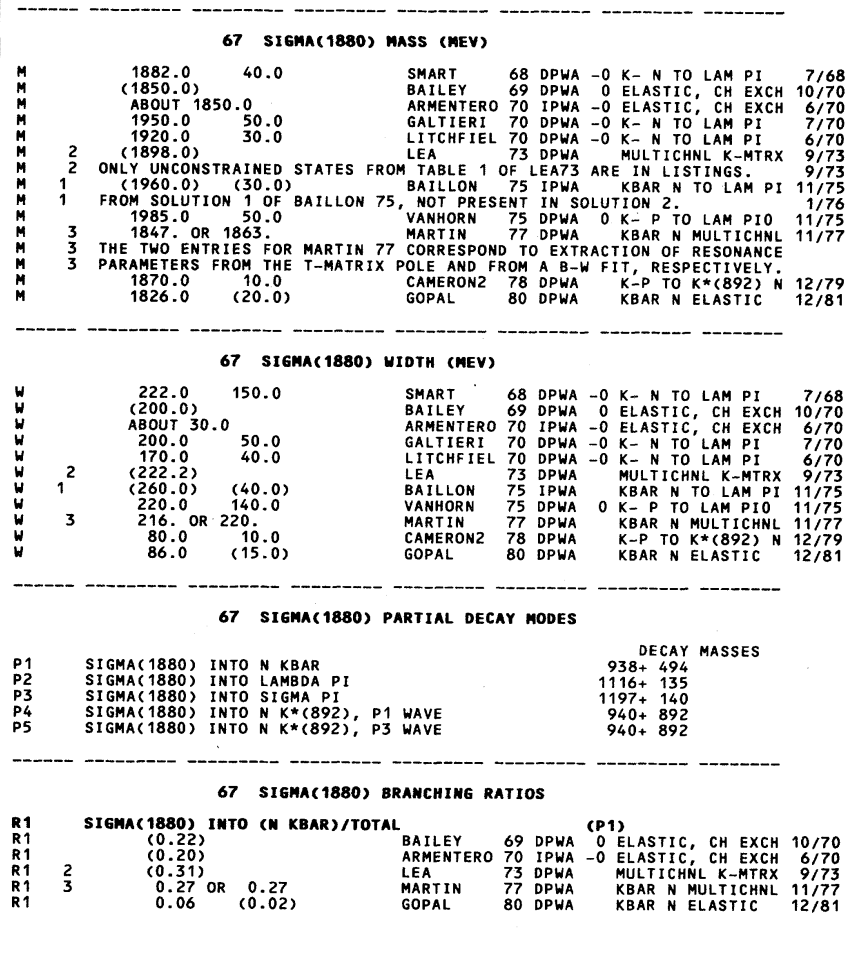



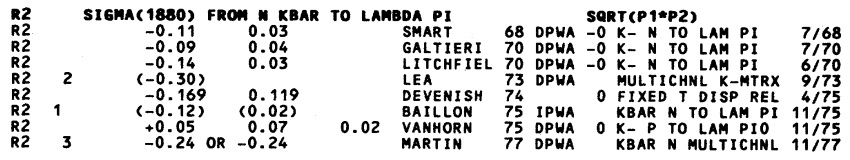

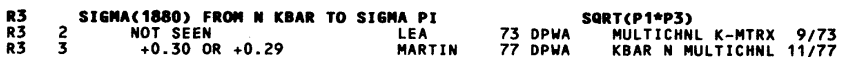

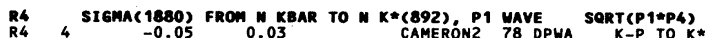

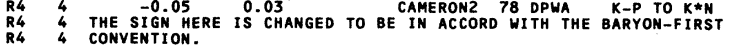

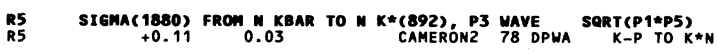

12,79
1279
$12 / 79$ $12 / 79$

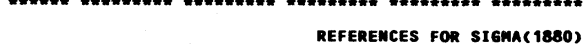

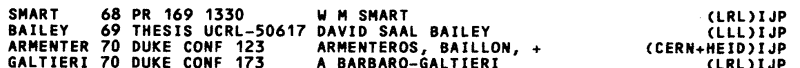

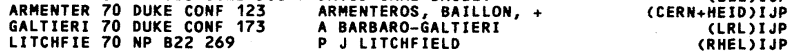

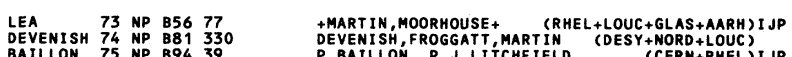

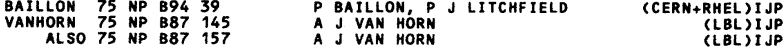

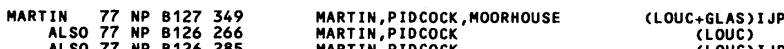

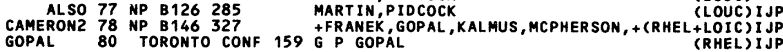

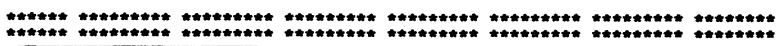

\section{$\Sigma(1915) \quad \mathrm{F}_{15}^{\prime} \quad$ Status: ****}

46 SIGMA(1915, JP=5/2+) I=1 F'15 FOR RESULTS PUBLISHED BEFORE 1974 (THEY ARE
NOH OBSOLETE), SEE OUR 1982 EDITION (PHYSICS LETTERS 1118). ALL the REFERENCES hAVE BEEN RETAINED.

THIS RESONANCE WAS FIRST SEEN IN THE TOTAL-CROSS-SEC-
TION MEASUREMENTS OF COOL 66 . IN THIS ENTRY, HOWEVER,

SEE THE MEXT ENTEY LFR THE PARAMETERS OF PEAKS SEEN AROUND 1900 - 1950

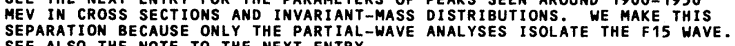

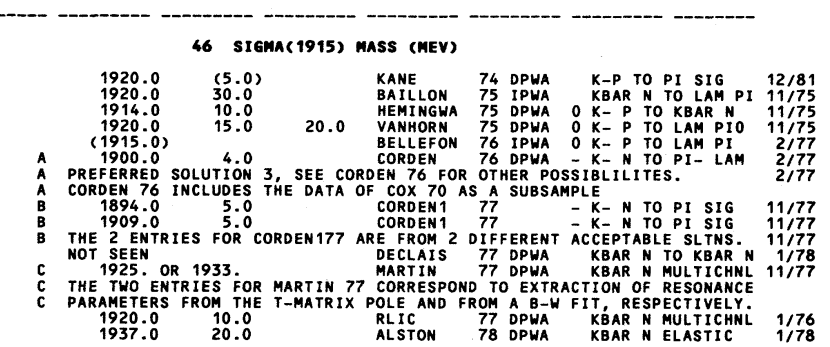

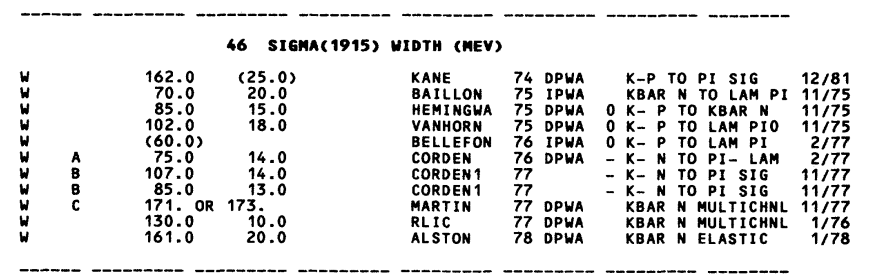

46 SIGMA (1915) PARTIAL DECAY MODES

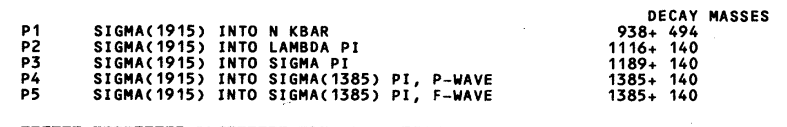

46 SIGMa(1915) BranCHIMG RATHOS

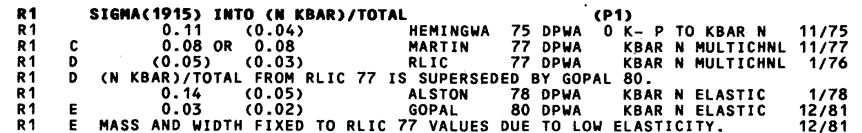

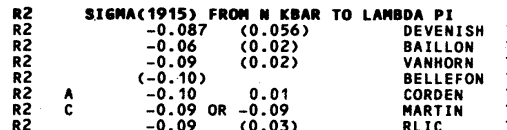

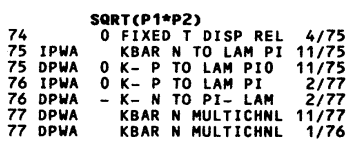

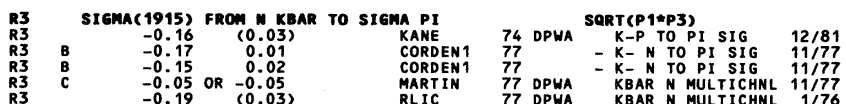

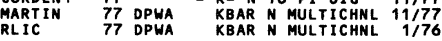

AVG MOD ${ }^{\circ} 0.1660^{\circ} 0.0089$ average

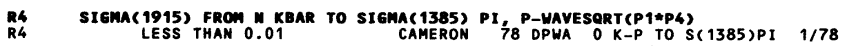

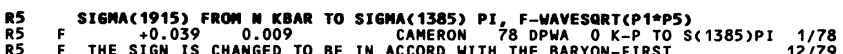

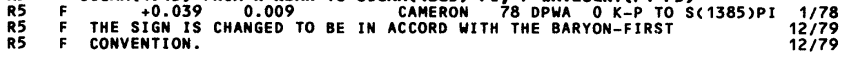

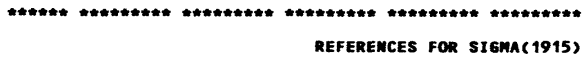

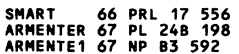

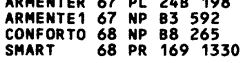

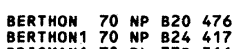

BERTHON1
BRICMAN 70 NP 824.417
COX

CALTIERI 70 DUKE CONF 173
GITCHF IE 70 NP B22 269

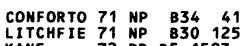

KANE 72 PR 051583
DEVENISH 74 NP 881530
KANE 74 LBL-2452

WM SMART A KERNAN, G E KALMUS, R P ELY (LRL)IJP

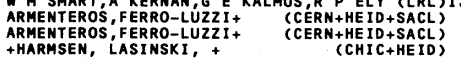

THARMSEN, LASINSKI, +
WM SMART
(CHIC+HED)
(LRL)IJP

$\begin{aligned} & \text { +RANGAN, VRANA, } \\ & \text { +VRANA, BUTTERHORTH, }\end{aligned}+\begin{aligned} & \text { (CDEF+RHELLSACL)IJJP } \\ & \text { (CDEF+RHEL +SACL)IJ }\end{aligned}$

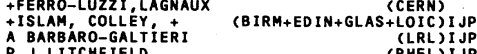

A Jitincto

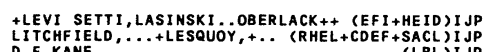

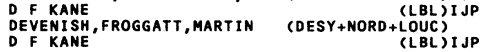

BAILLON 75 NP 89439

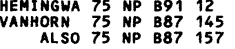

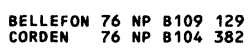

P BAILLON, PJ LITCCHFIELD (CERN+RHEL)IJP

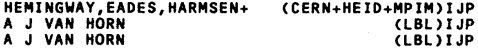
(CDEF) IJP
DE BELLEFON, BERTHON
+ COX, DARTNELL, KENYON, ONEALE, SUMOROK+ (BIRM) IJP

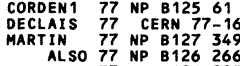
$\begin{array}{lll}\text { ALSO } 77 \text { NP } & 8126 & 266 \\ \text { ALSO } 77 & \text { NP B126 } & 285 \\ \text { B } & 285\end{array}$

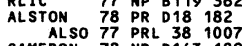

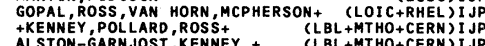

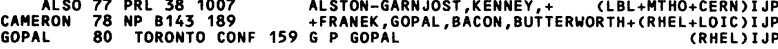

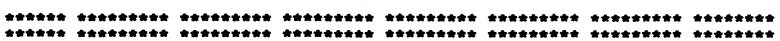

1915 MEV REGION - PRODUCTION AND $\sigma_{\text {TOTAL }}$ EXP'TS

29 SIGMAC1915, JP= , I=1 Production EXPERIMEMTS SEE THE NOTES TO THE SIGMA(1915) AND SIGMA(1940), WHICH AND INVARIANT-MASS DISTRIBUTIONS. THE CROSS-SECTION PEAKS ARE ALMOST

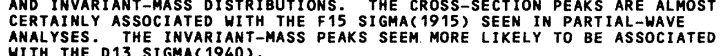

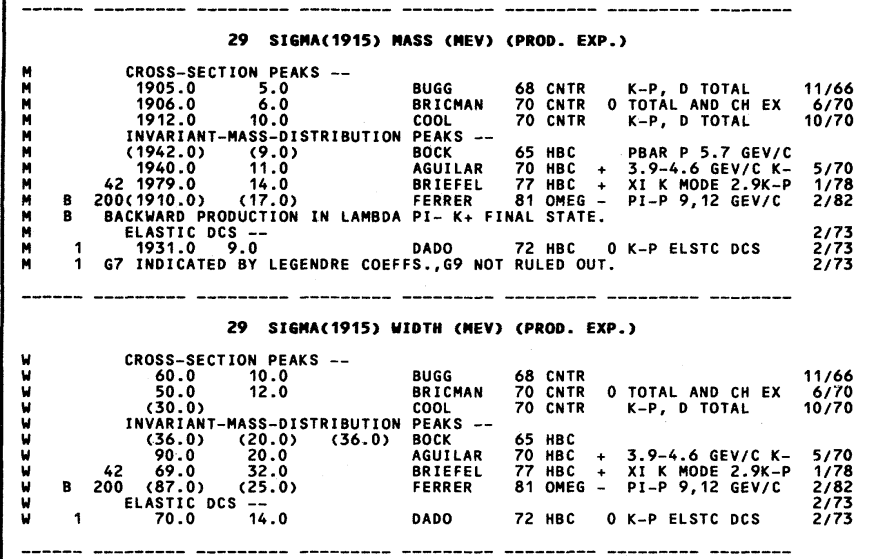




\section{Baryons}

$\Sigma(1915), \Sigma(1940), \Sigma(2000)$

29 SIGMA(1915) PARTIAL DECAY MODES (PROD. EXP.)

$\begin{array}{lllc} & & \text { DECAY MASSES } \\ \text { P1 } & \text { SIGMA(1915) INTO N KBAR } & 938+494 \\ \text { P2 } & \text { SIGMA (1915) INTO LAMBDA PI } & 1116+135 \\ \text { P3 } & \text { SIGMA(1915) INTO SIIGMA PI } & 1189+140 \\ \text { P4 } & \text { SIGMAC 1915) INTO XI K } & 1315+494\end{array}$

29 SIGMAC(1915) BRAMCHIMG RATIOS (PROD. EXP.)

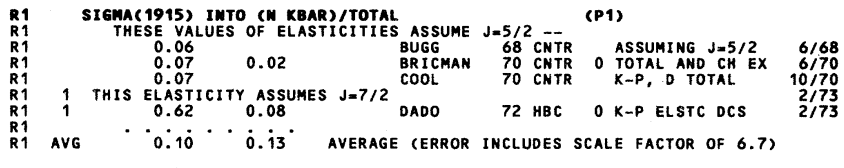

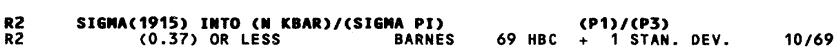

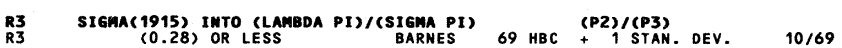

$\begin{array}{llll}\text { R4 SIGHA(1915) IMTO (XI K)/TOTAL BRIEFEL } 77 \mathrm{HBC} & \text { (P4) } \\ \text { R4 K-P } 2.87 \mathrm{GEV} & 1 / 78\end{array}$

***********************************************************************

REFEREMCES FOR SIGHA(1915) (PROD. EXP.)

BOCK 65 PL 17166
COOL 66 PRL 161288 +COOPER, RRENCH, KINSON, +
+GIACOMELLI, KYCIA, LEONTIC, LI, LUNDBY, + (BNL) I

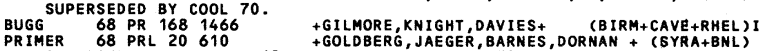

PRIMER
SUPERSEDED BY BARNES 69 AND AGUIAR-BENITEZ 70.
BARES 69 PRL 22479
+FLAMINIO, MONTANET, SAMIOS +
(BNL+SYRA)

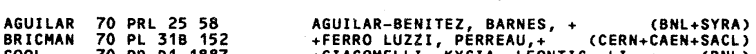

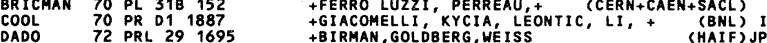

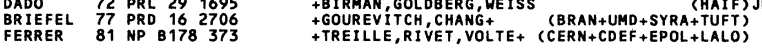

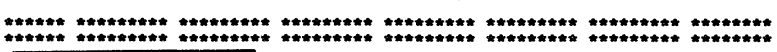
$\Sigma(1940) \quad D_{13}^{\prime \prime \prime} \quad$ Status: $* * *$

98 SIEmA(1940, JPm3/2-) I=1 Di, 13

FOR RESULTS PUBLISHED BEFORE 1974 (THEY ARE
NOH OBSOLETE), SEE OUR 1982 EDITION (PPHSICS LETTERS
11118

SOME, NOT ALL, PARTIAL HAVE ANALYSES SUGgeSt A STATE IN THIS REGION. IT IS PERHAPS ASSOCIATED WITH THE BUMPS SEEN IN PRODUCTION EXPERIMENTS NEAR THIS MASS (SEE THE
PRECEDING ENTRY). THIS STATE IS NOT REQURED IN PRECEDING ENTRY) THIS STATE IS NOT REQIRED IN
K- NEUTRON TO (PI SIGMA) - ANAYSIS OF GOYAL T7. KBAR N
ANALYSIS (GOPAL 80) WITH K- YEUTRON ELASTIC DATA
DOES NOT REQUIRE THIS STATE.

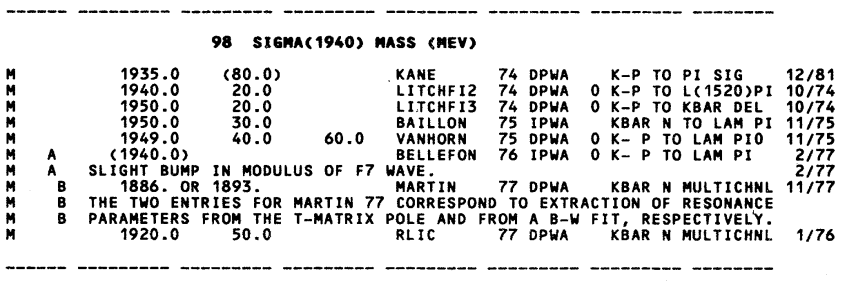

98 SIGMA(1940) WIOTH (MEV)

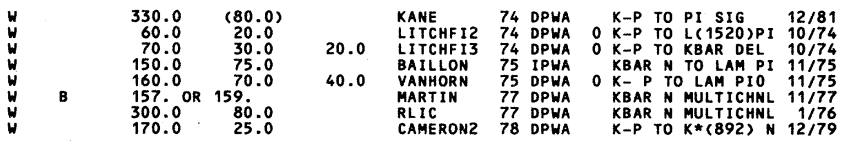

98 SIGMA(1940) PARTIAL DECAY MODES

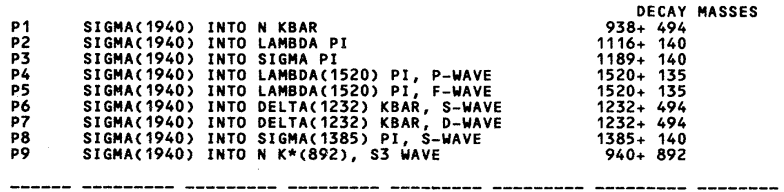

98 SIGMA (1940) BRANCHIMG RATIOS

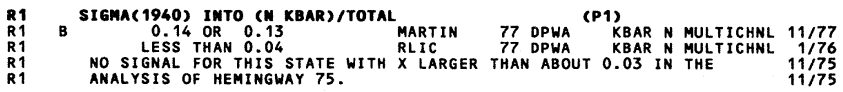

\section{Data Card Listings}

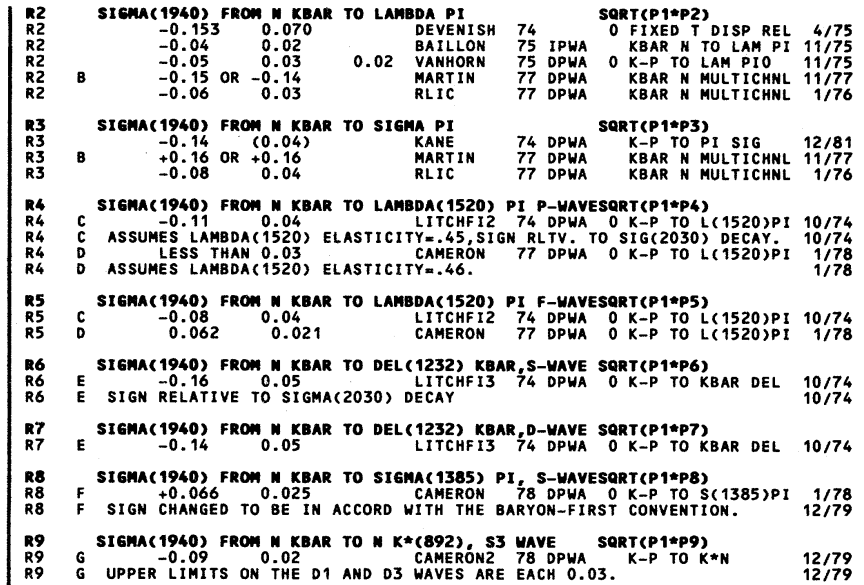

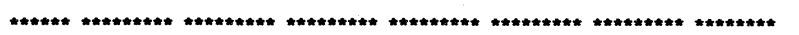
REFEREMCES FOR SIGMA(1940)

$\begin{array}{lll}\text { GALTIERI } 70 \text { DUKE CONF } 173 & \text { A BARBARO-GALTIERI } & \text { (LRL)IJP } \\ \text { LITCHFIE } 700 \text { NP B22 } 269 & \text { P J LITCHFIELD } & \text { (RHEL)IJP }\end{array}$

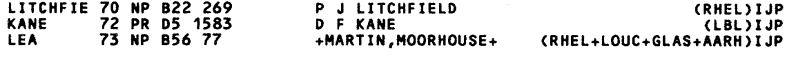
DEVENISH 74 NP 881330 DEVENISH, FROGGATT, MARTIN (DESY+NORD+LOUC)
KANE

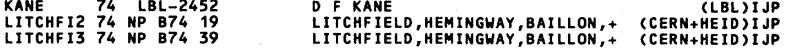

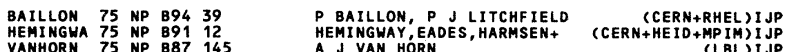
VANHORN 75 NP B87 145
ALSO 75 NP B87 157 BELLEFON 76 NP B109 129
CAMERON 77 NP B131 399
MOE BELLEFON, BERTHON
MARTIN

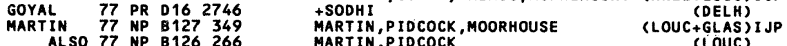

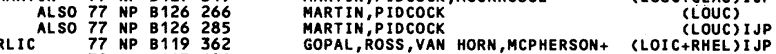

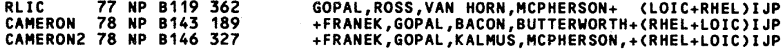

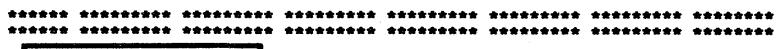
$\Sigma(2000) \mathrm{S}_{\text {U. }}^{\prime \prime}$ Status: *

02 SIEMA(2000, JP=1/2-) $1=1 \quad$ S', 11

WE LIST HERE ALL REPORTED S11 STATES LYING ABOVE

02 SIGMa(2000) MASS (MEV)

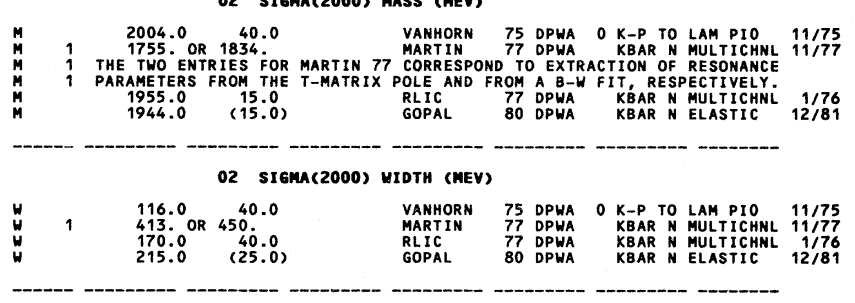

02 Sigma(2000) PARTIAL decaY mOdes

$\begin{array}{llc} & & \\ \text { P1 } & \text { SIGMA(2000) INTO N KBAR } & \text { DECAY MASSES } \\ \text { P2 } & \text { SIGMA(2000) INTO LAMBDA PI } & 938+494 \\ \text { P3 } & \text { SIGMA(2000) INTO SIGMA PI } & 1116+135 \\ \text { P4 } & \text { SIGMA(2000) INTO LAMBA } 1520) \text { PI } & 1197+140 \\ \text { P5 } & \text { SIGMA(2000) INTO N K\$(892), S1 WAVE } & 1520+140 \\ \text { P6 } & \text { SIGMA(2000) INTO N K*(892), D3 WAVE } & 940+892 \\ & & 940+892\end{array}$

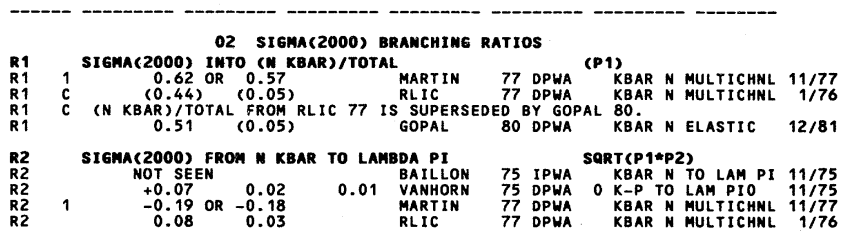




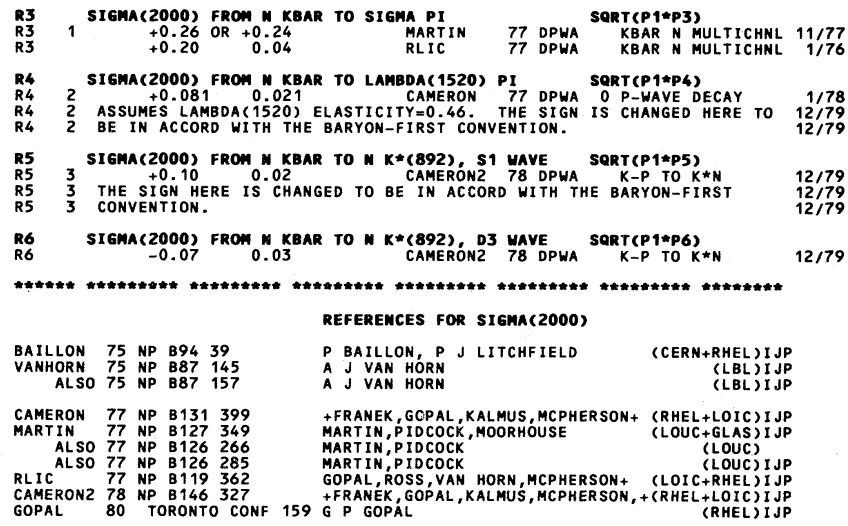

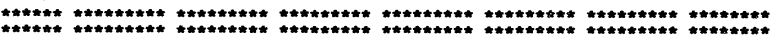

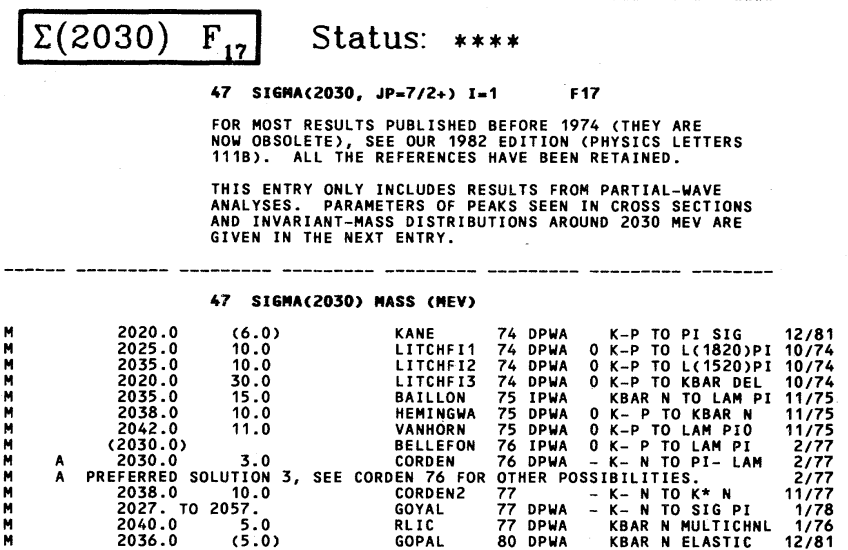

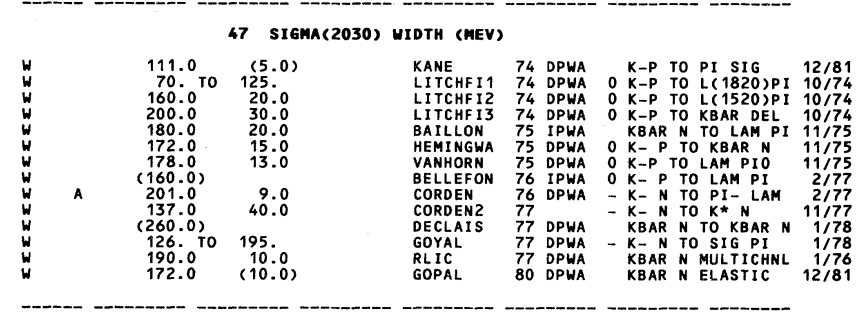

47 SIGMA(2030) PARTIAL DECAY MODES

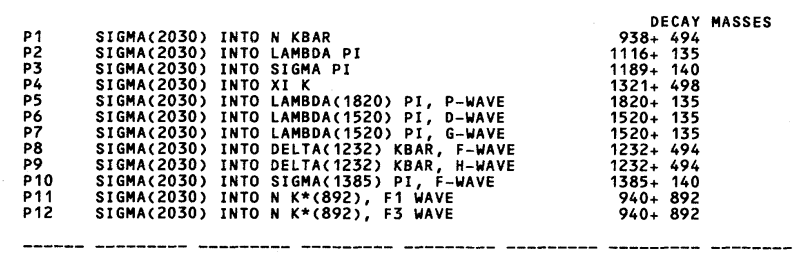

47 SIGMA(2030) BRAMCHIMG RATIOS

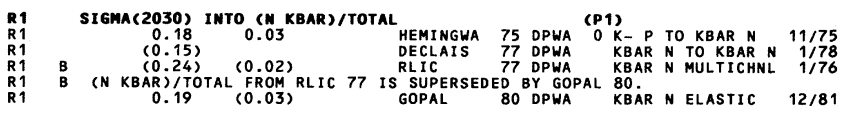

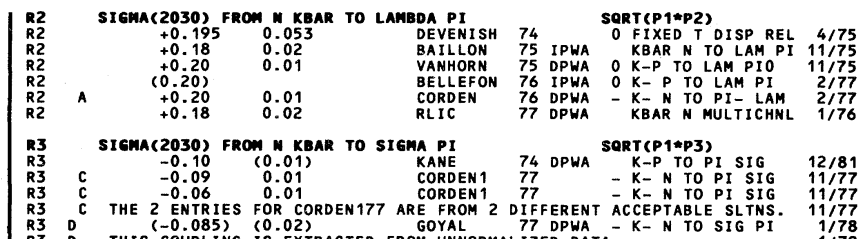

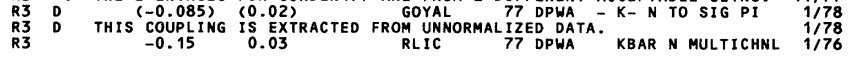

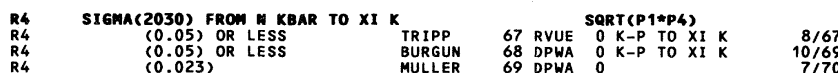

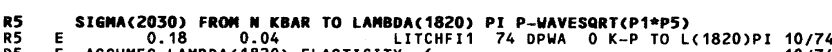

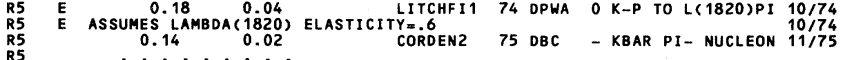
R5 AVG $\cdots 0.148^{\cdots} \cdot 0.018$ aVerage

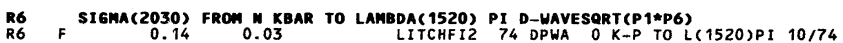

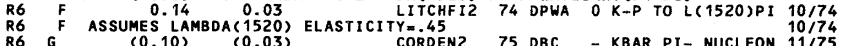

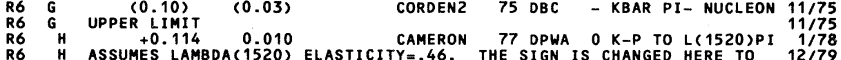

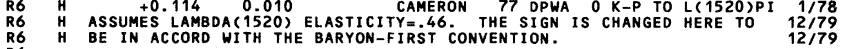
R6 AVG $0.1166^{\circ} 0.0095$ aVerage

R7 SIGMAC2030) FROM N KBAAR TO LAMBDA (1520) PI G-MAVESSRT (P1*P7)

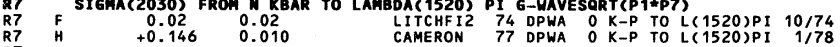
R7 AVG $0.121 \cdots \dot{0} 0.050$ aVERAGE (ERROR INCLUDES SCALE FACTOR OF 5.6) R8 SIGMA(2030) FROM N KBAR TO DEL(1232) KBAR, F-MAVE SQRT(P1*P8)

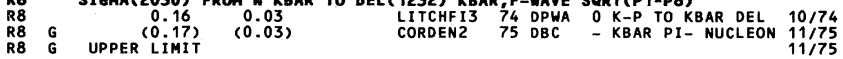

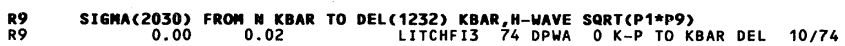

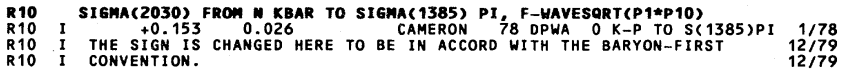

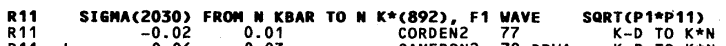

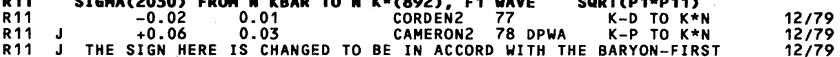
R11 J THE SIGN HERE IS CHANGED TO BE IN ACCORD WITH THE BARYON-FIRST $12 / 79$
R11 JONVENTION.
R11 R11 AVG MOD $0.024^{\circ} \dot{0} \dot{0} 1 \dot{2}$ aVerage (error InCludes SCale factor of 1.3 )

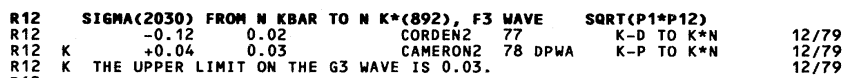
R12 AVG MOD $0.095^{\circ} \cdot \dot{0} .037^{\circ}$ aVERage (erRor InCludes SCale factor of 2.2)

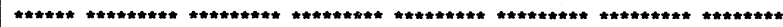

\begin{tabular}{|c|c|c|}
\hline $\begin{array}{l}\text { WOHL } \\
\text { TRIPP } \\
\text { BURGUN } \\
\text { DAUM } \\
\text { SMART } \\
\text { MULLER }\end{array}$ & $\begin{array}{l}66 \text { PRL } 17107 \\
67 \text { NP } B 3107 \\
68 \text { NP } 88447 \\
68 \text { NP } 8719 \\
68 \text { PR } 1691336 \\
69 \text { THESIS, UCRL } 19372\end{array}$ & 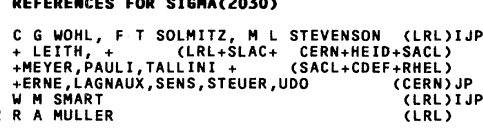 \\
\hline $\begin{array}{l}\text { BERTHON } \\
\text { BERTHON1 } \\
\text { COX } \\
\text { GALTIERI } \\
\text { LITCHFIE }\end{array}$ & 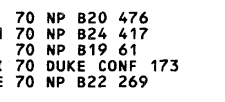 & 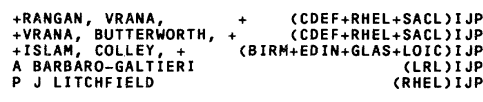 \\
\hline $\begin{array}{l}\text { CAMPBELL } \\
\text { LITCHFIE } \\
\text { KANE }\end{array}$ & $\begin{array}{l}71 \mathrm{NP} 82575 \\
71 \mathrm{NP} B 30125 \\
72 \mathrm{PR} \quad 051583\end{array}$ & 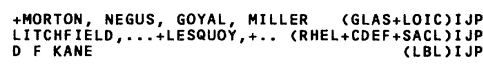 \\
\hline $\begin{array}{l}\text { DEVENISH } \\
\text { KAE SH } \\
\text { LITCHFI1 } \\
\text { LITCHF II } \\
\text { LITCHF I3 }\end{array}$ & 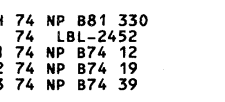 & 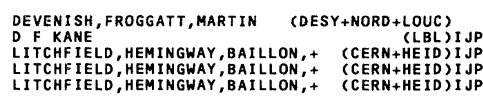 \\
\hline $\begin{array}{l}\text { BAILLON } \\
\text { CORDEN2 } \\
\text { MEMNGMA } \\
\text { VANHORN } \\
\text { BELLLO } \\
\text { BELEF } \\
\text { CORDEN }\end{array}$ & $\begin{array}{llll}75 & \text { NP } & 894 & 39 \\
75 & \text { NP } & 892 & 365 \\
75 & \text { NP } & 892 & 365 \\
75 & \text { NP } & 891 & 12 \\
75 & \text { NP } & 887 & 145 \\
75 & \text { NP } & 887 & 157 \\
76 & \text { N } & 15109 & 129 \\
76 & \text { NP } & 8104 & 382\end{array}$ & 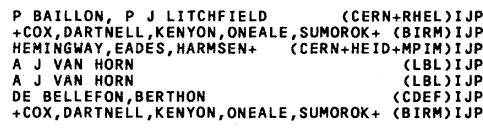 \\
\hline 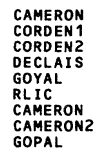 & 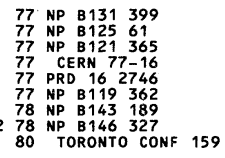 & 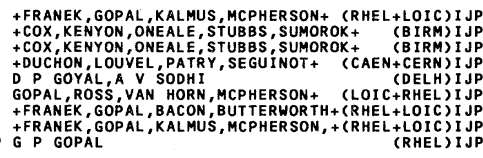 \\
\hline & & \\
\hline
\end{tabular}


Baryons

$\Sigma(2030), \Sigma(2070), \Sigma(2080), \Sigma(2100)$

2030 MEV REGION - PRODUCTION AND $\sigma_{\text {TOTAL }}$ EXP'TS

28 SIGMAC2030, JP= , I=1 PRODUCTIOM EXPERINEMTS

SEE THE NOTE ON THE F17 SIGMAC2030), IN FRONT OF THIS
ENTRY. HERE WE LIST ONLY PARAMETRS OF PEAKS IN CROSS
SECTIONS AND INVARIIANT-MASS DISTRIBUTIONS. THE CROSS-

SECTION PEAKS ARE AT LEAST DDMINANTLY ASSOCIATED WITH THE SI SMA (2030), BUT MAY CONTAIN A SMALL CONTRIBUTION FROH

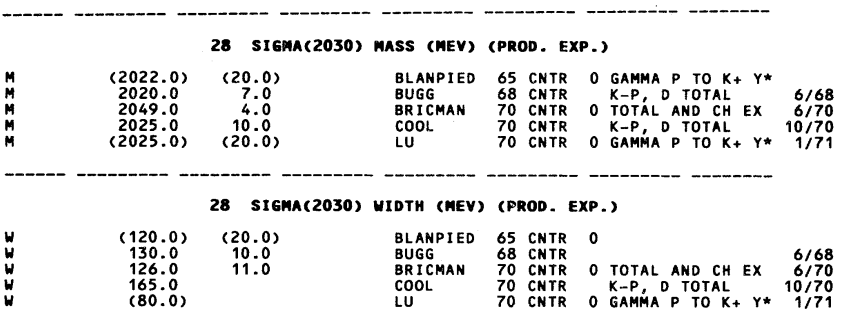

$\begin{array}{llll} & 28 & \text { SIGHA(2030) PARTIAL DECAY MODES (PROD. EXP.) } \\ \text { P1 } & \text { SIGMA(2030) INTO N KBAR } & \text { DECAY MASSES } \\ \text { P2 } & \text { SIGMA(2030) INTO N KBAR PI } & 938+494 \\ & & 938+498+140\end{array}$

28 SIgMa(2030) BRAMCHING RATIOS (PROD. EXP.)

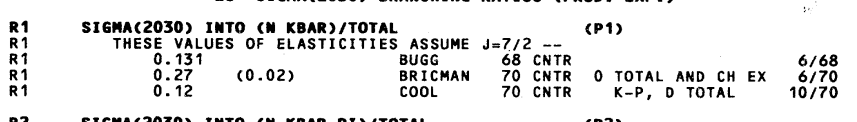
R2 SIGMA(2030) INTO (W KBAR PI)/TOTAL
R2

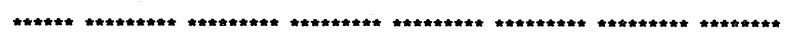

\begin{tabular}{|c|c|c|}
\hline $\begin{array}{l}\text { BLANPI ED } \\
\text { COOL }\end{array}$ & $\begin{array}{llll}65 & \text { PRL } & 14 & 741 \\
66 & P R L & 16 & 1228\end{array}$ & $\begin{array}{l}\text { +GREEBERG, HUGHES, KITCHING, LU, (+ (YALE+CEA) } \\
\text { +GIACOMELLI, KYCIA, LEONTIC,LI, LUNDBY, + (BNL) I }\end{array}$ \\
\hline BUGG & 68 PR 1681466 & +GILMORE, KNIGHT, + \\
\hline $\begin{array}{l}\text { BRICMAN } \\
\text { COOL } \\
\text { LU }\end{array}$ & $\begin{array}{llll}70 & \mathrm{PL} & 31 \mathrm{~B} & 152 \\
70 & \mathrm{PR} & \mathrm{DO} & 1887 \\
70 & \mathrm{PR} & 02 & 1846\end{array}$ & 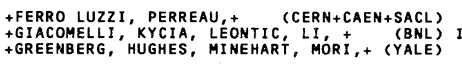 \\
\hline
\end{tabular}

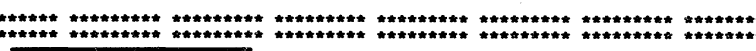

\begin{tabular}{|ll}
\hline$(2070)$ & $F_{15}^{\prime \prime}$
\end{tabular} Status: *

THIS STATE SUGGESTED BY BERTHON 70 NOH FINDS CONFIRMATION IN GOPAL 80 WITH NEW K-P POLARIZATION AND K- NEUTRON ANGULAR DISTRIBUTIONS. THE VERY BROAD STATE
SEN IN KANE 72 IS NOT REQUIRED IN THE LATER (KANE 74) ANALYSIS OF PI SI GMA.

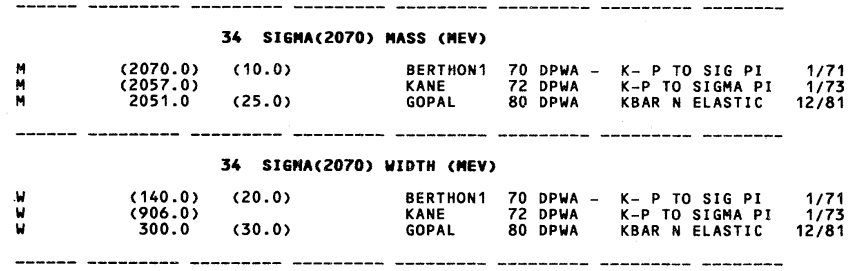

34 Sigma(2070) partial decay modes

$\begin{array}{llr}\text { P1 } & \text { SIGMA(2070) INTO N KBAR } & \text { DECAY MASSES } \\ \text { P2 } & \text { SI GMA(2070) INTO SIGMA PI } & 938+494 \\ & 1189+140\end{array}$

34 SIGMA(2070) BRANCHIMG RATIOS

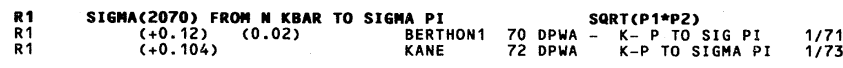

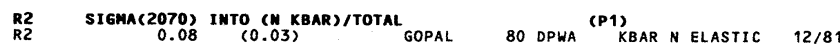
************************************************************************* REFERENCES FOR SIGMA(2070)

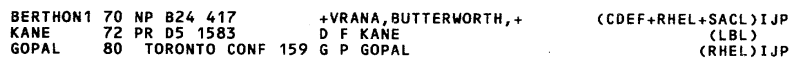

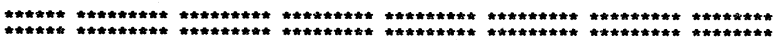

Data Card Listings

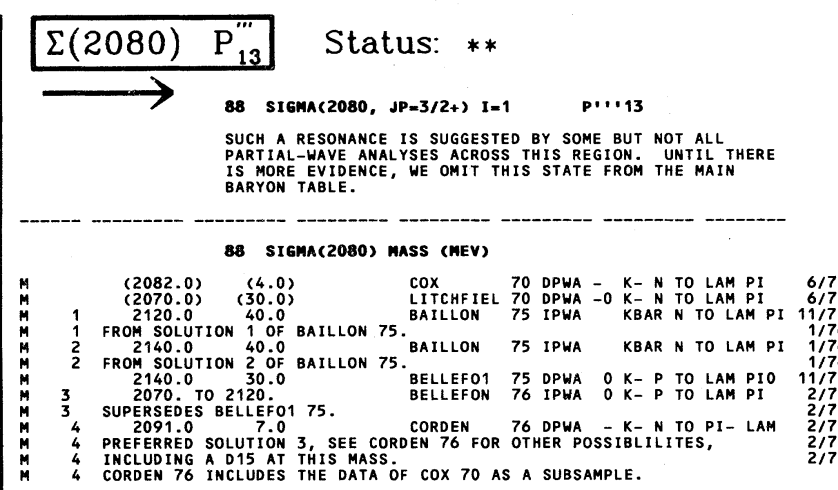

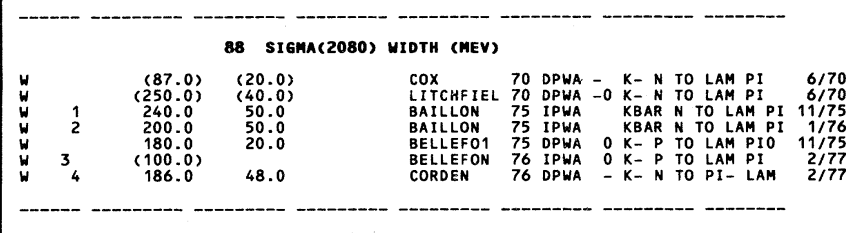

88 SIGMA(2080) PARTIAL DECAY MODES

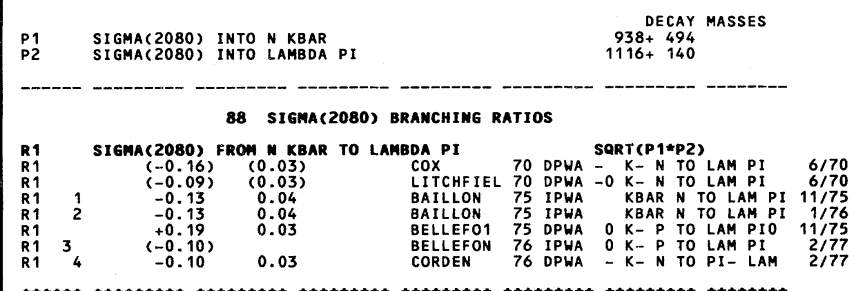

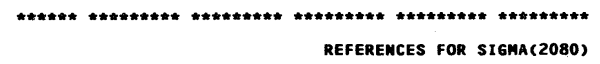

COX 70 NP B19 61
LITCHFIE 70 NP B22 269

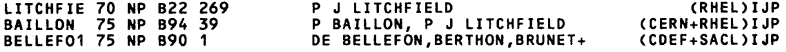
BELLEFON 76 NP $B 109129 \quad$ DE BELLEFON, BERTHON
CORDEN 76 NP B104 382

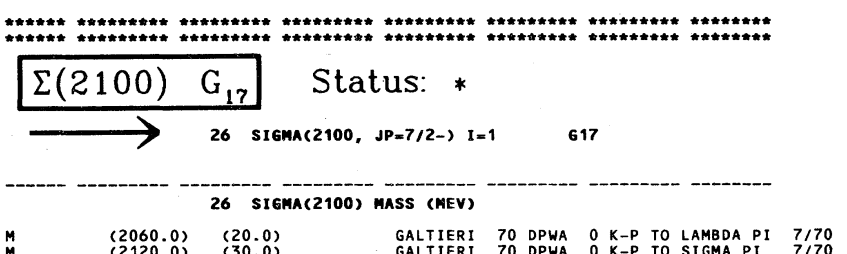

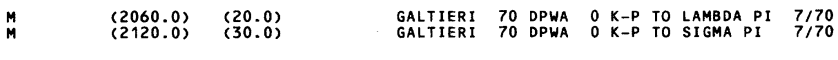

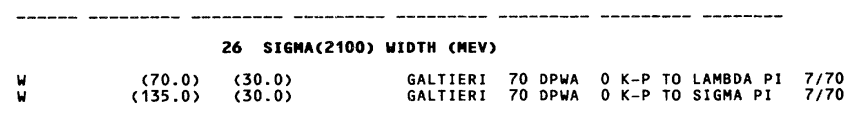

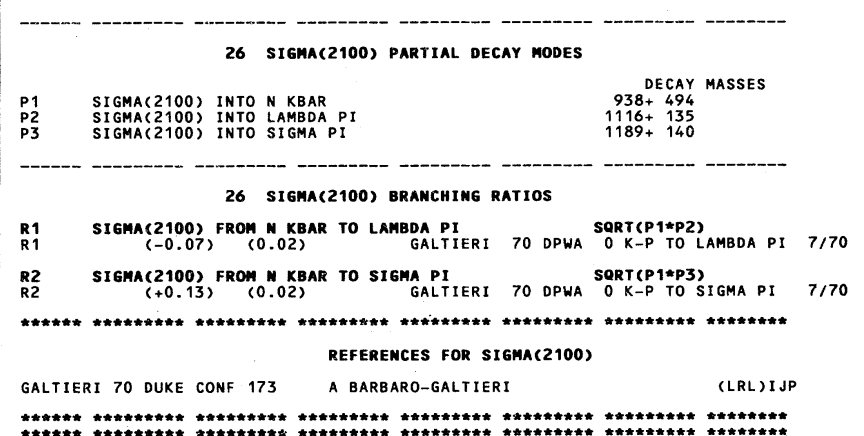


Baryons

$\Sigma(2620), \Sigma(3000), \Sigma(3170), \quad \Xi ' s$
Data Card Listings

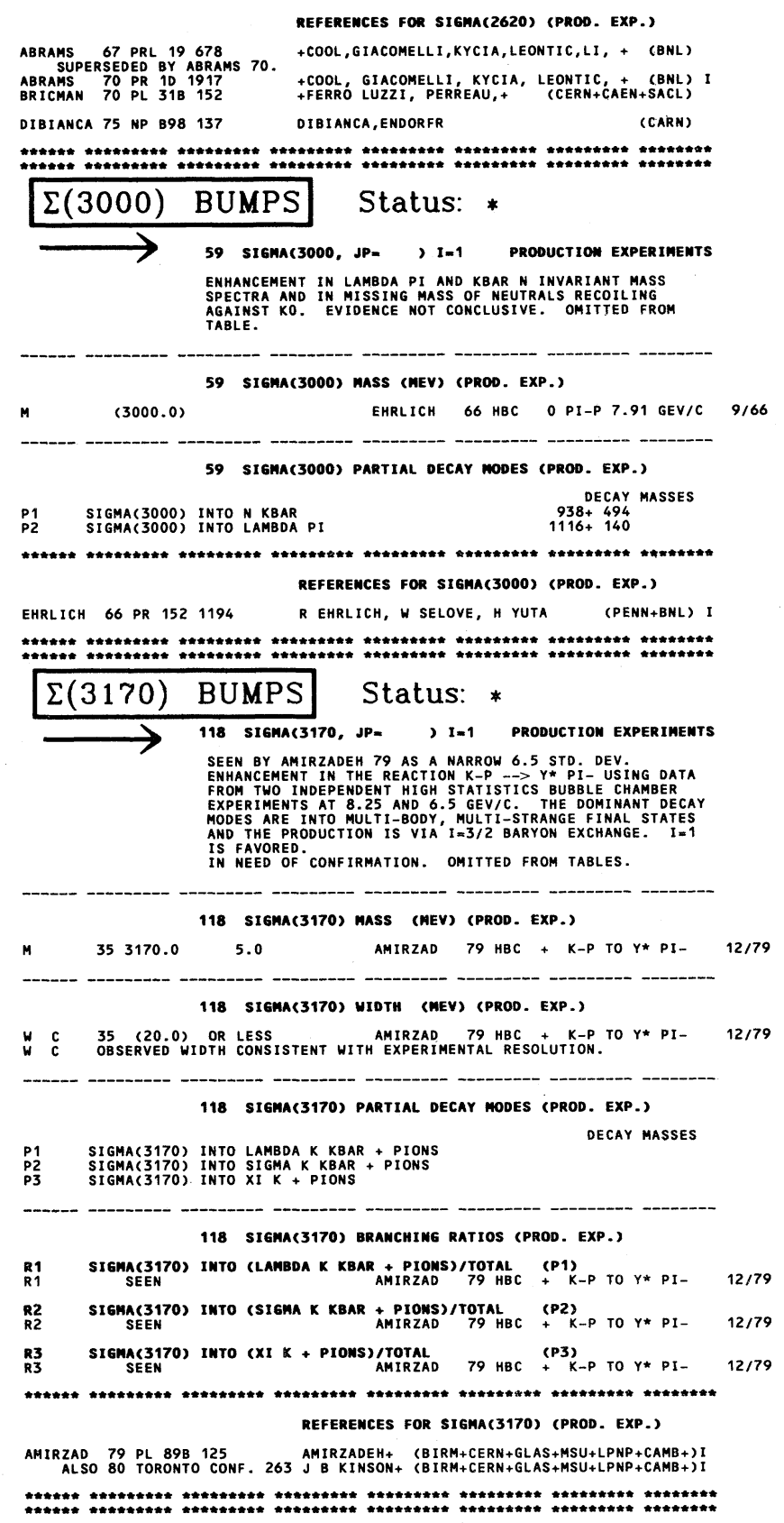

\section{NOTE ON $\Xi$ RESONANCES}

The $\Xi$ resonance situation has always been unhappy. This is because: (1) $\Xi$ resonances can only be produced as a part of a final state, and so the analysis is more complicated than if direct formation were possible; (2) they are produced with small cross sections (typically a few $\mu \mathrm{b}$ ); and (3) the final states are topologically compli- cated and difficult to study with electronic techniques. Thus our early knowledge of $\Xi$ resonances came entirely from bubble chamber experiments, where the numbers of events are small, and our best information about $\Xi$ resonances is still from bubble chamber experiments.

Until fairly recently only the $\Xi(1530)$ was really well established. However, the late 1970's saw a major improvement with the results of GAY 76 and HEMINGWAY 77. The $\Xi(1820)$ and $\Xi(2030)$ were firmly established as narrow states (widths of about $20 \mathrm{MeV}$ ), and the spin of the $\Xi(1820)$ was found to be $3 / 2$ (TEODORO 78).

Since then, however, not much has changed, although there is some improved evidence for the $\Xi(2250)$ and the $\Xi(2370)$. There is probably at least one other $\Xi$ in the $1850-2000-\mathrm{MeV}$ region, and there are indications of several others above $2000 \mathrm{MeV}$. Indeed, there should be many $\Xi$ resonances below $2500 \mathrm{MeV}$, and the broad (and not completely established) $\Xi(1940)$ could well be a mixture of several of them. For now we are forced to group together disparate observations and await new results. The disagreements among experiments are shown in ideograms in the Listings.

Results from experiments using electronic methods have recently become available. BIAGI 81 used the CERN hyperon beam to study inclusive $\Lambda \overline{\mathrm{K}}$ and $\Xi \pi$ mass spectra from 102 and $135 \mathrm{GeV} / \mathrm{c} \Xi^{-}$incident on hydrogen and deuterium. They saw a large $\Xi(1820)$ signal in $\Lambda \overline{\mathrm{K}}$ as well as a peak at about $1700 \mathrm{MeV}$, which might be associated with the threshold enhancement seen by DIONISI 78 . The $\Xi(1940)$ appears as a broad bump in the $\Xi \pi$ mass spectrum, and there is a very clean $\Xi(1530)$ signal. And Brookhaven multiparticle spectrometer measurements of $\mathrm{K}^{-} \mathrm{p} \rightarrow \mathrm{K}^{+}$anything at $5 \mathrm{GeV} / \mathrm{c}$ (JENKINS 83) have seen all the wellestablished $\Xi$ resonances and also the less wellestablished $\Xi(2250), \Xi(2370)$, and $\Xi(2500)$.

The table below gives our evaluation of the present status of the $\Xi$ resonances. For a detailed review, see Meadows. 1

\section{Reference}

1. B.T. Meadows, in Proceedings of the $I V^{\text {th }}$ International Conference on Baryon Resonances (Toronto, 1980), ed. N. Isgur, p. 283. 
Table 1 . The status of the $\Xi$ resonances. Only those with an overall status of $* * *$ or **** are included in the main Baryon Table.

\begin{tabular}{|c|c|c|c|c|c|c|c|}
\hline \multirow[b]{2}{*}{ Particle } & \multirow[b]{2}{*}{$\mathrm{L}_{2 \mathrm{I} \cdot 2 \mathrm{~J}}$} & \multirow[b]{2}{*}{$\begin{array}{l}\text { Overall } \\
\text { status }\end{array}$} & \multicolumn{5}{|c|}{ Status as seen in -- } \\
\hline & & & $\Xi \pi$ & $\Lambda \mathrm{K}$ & $\Sigma \mathrm{K}$ & $\Xi(1530) \pi$ & Other channels \\
\hline$\Xi(1318)$ & $P_{11}$ & $* * * *$ & & & & & Weak to $\Lambda \pi$ \\
\hline$\Xi(1530)$ & $P_{13}^{11}$ & $* * * *$ & $* * * *$ & & & & \\
\hline$\Xi(1630)$ & & $*$ & $*$ & & & & \\
\hline$\Xi(1680)$ & & $* *$ & & * & $* *$ & & \\
\hline$\Xi(1820)$ & 13 & $* * *$ & * & $* * *$ & ** & **** & \\
\hline$\Xi(1940)$ & 13 & $* *$ & $* *$ & & & $* *$ & \\
\hline$\Xi(2030)$ & 1 & $* * *$ & & $* *$ & $* * *$ & & \\
\hline$\Xi(2120)$ & & $*$ & & * & & & \\
\hline$\Xi(2250)$ & & ** & & & & & 3-body decays \\
\hline$\Xi(2370)$ & 1 & ** & & & & & 3-body decays \\
\hline$\Xi(2500)$ & 1 & $*$ & & * & * & & 3-body decays \\
\hline
\end{tabular}

**** Good, clear, and unmistakable.

** Good, but in need of clarification or not absolutely certain

** Not established; needs confirmation.

* Evidence weak; could disappear.

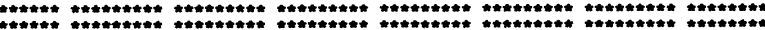

$$
\mathrm{S}=-2 \quad \mathrm{I}=1 / 2 \quad \text { HYPERON STATES }(\Xi)
$$

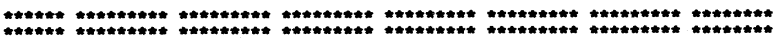

$\Xi^{-} \quad 22 \times I-(1321, J P=1 / 2, I=1 / 2$

SEE STABLE PARTICLE DATA CARD LISTINGS

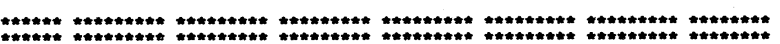

$\Xi^{0} \quad 23 \times x$ IOC(1315, JP =1/2, $\mathrm{I}=1 / 2$

SeE STABLE PARTICLE daTA CARD LISTINGS

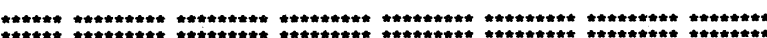

\section{$\Xi(1530) P_{13} \quad$ Status: ****}

$49 X I(1530, J P=3 / 2+) \quad I=1 / 2 \quad P 13$

THIS IS THE ONLY XI RESONANCE HHOSE PROPERTIES ARE
ALL AT LEAST REASONABLY WELL KNOWN. SPIN-PARITY $3 / 2$ -

IS FAVORED BY THE DATA.

WE DO NOT USE DETERMINATIONS OF THE MASS AND THE HIDTH OO THIS
STATE UNLESS THEY ARE ACCOMPANIED BY SOME DISCUSSION OF SYSTEMATICS AND RESOLUTION.

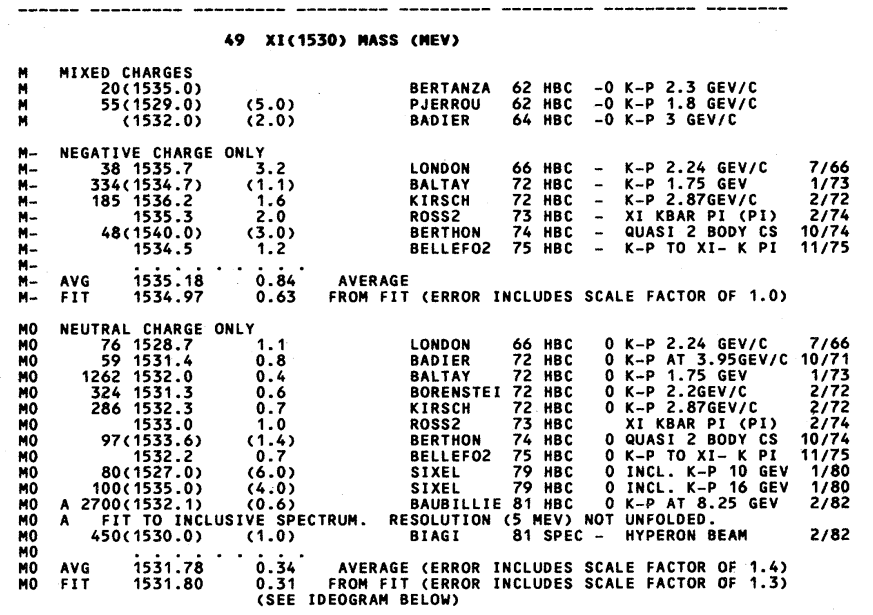

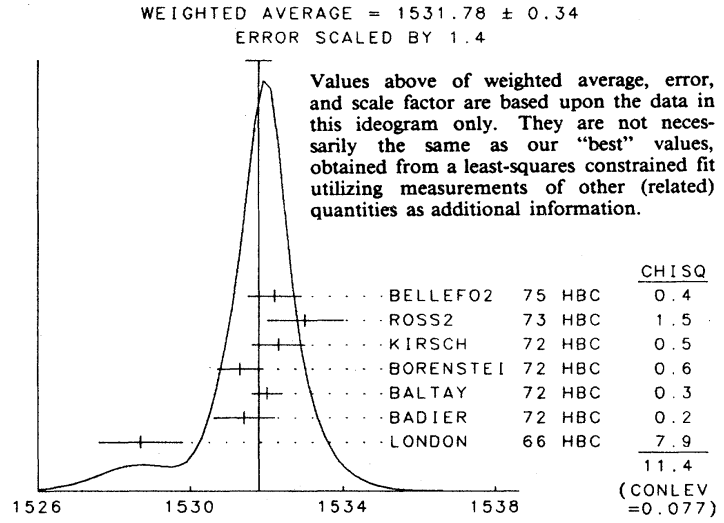

XI (1530)O MASS (MEV)

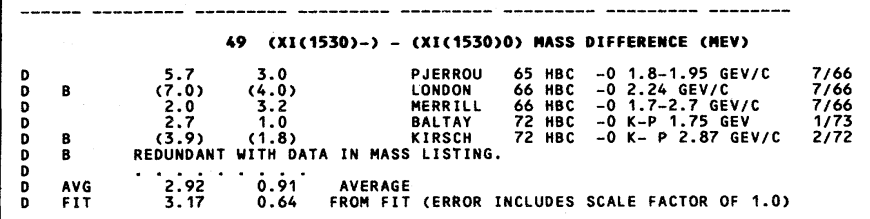

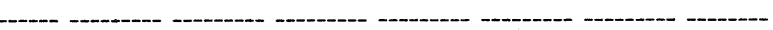

49 XI(1530) MIDTH (MEV)
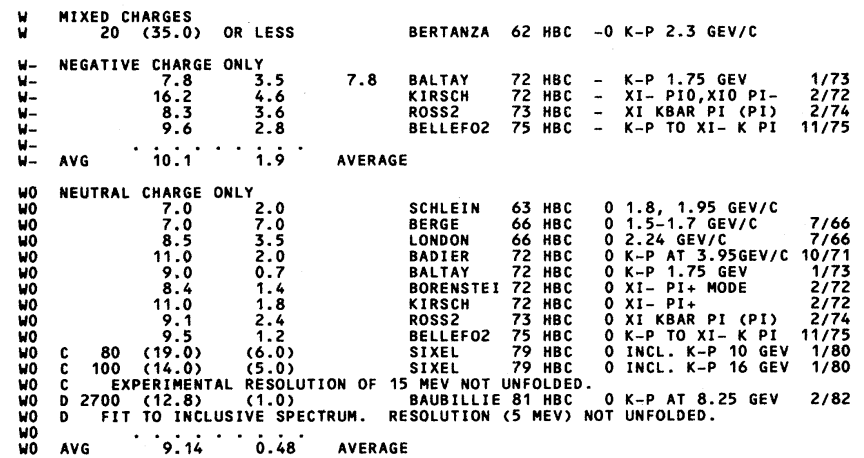

wo avg $9.14^{\cdots} \cdot 0.48^{\circ}$ average

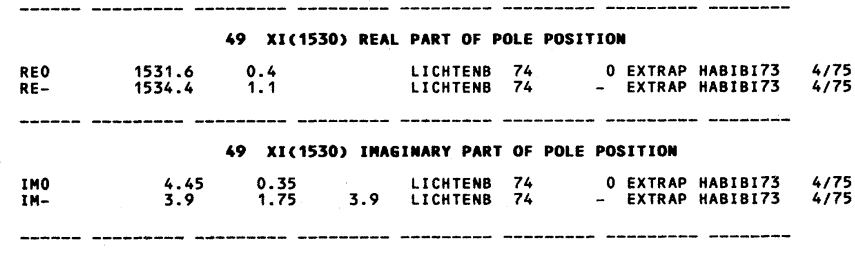

49 XI(1530) Partial decaY modes

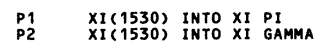

DECAY MASSES

49 XI(1530) BRAMCHIMG RATIOS (MEV)

R1 XI(1530) INTO (XI GAMMA)/TOTAL
R1
$(0.04)$ OR LESS CL=.90 KALBFLEI 75 HBC

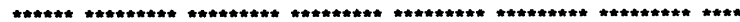

REFEREMCES FOR XI(1530)

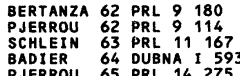

+BRISSON, CONNOLLY, GOLDBERG, GRAY, +(BNL+SYRA) IJ +PROWSE, SCHLEIN, SLATER, STORK, TICHO (UCLA) I
+CARMONY, PJERROU, SLATER, STORK, TICHO (UCLA) IJP +DEMOULIN, GOLDBERG S
+SCHLEIN, SLATER, SMI TH, STORK, TICHO SACL +AMST) I I 


\section{Baryons \\ $\Xi(1530), \quad \Xi(1630), \quad \Xi(1680), \quad \Xi(1820)$

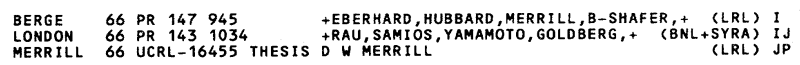

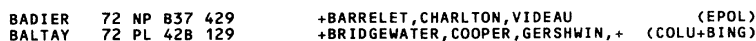

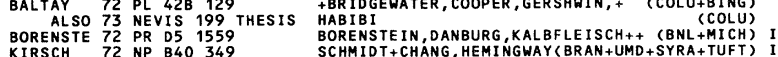

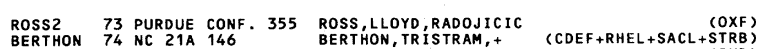

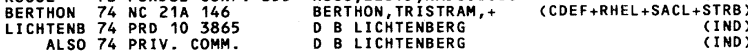

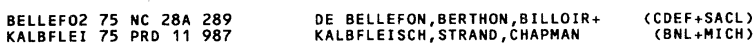

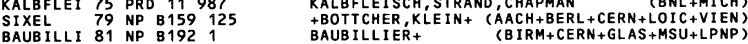

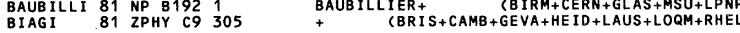

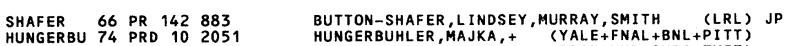

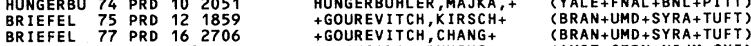 \\ *}

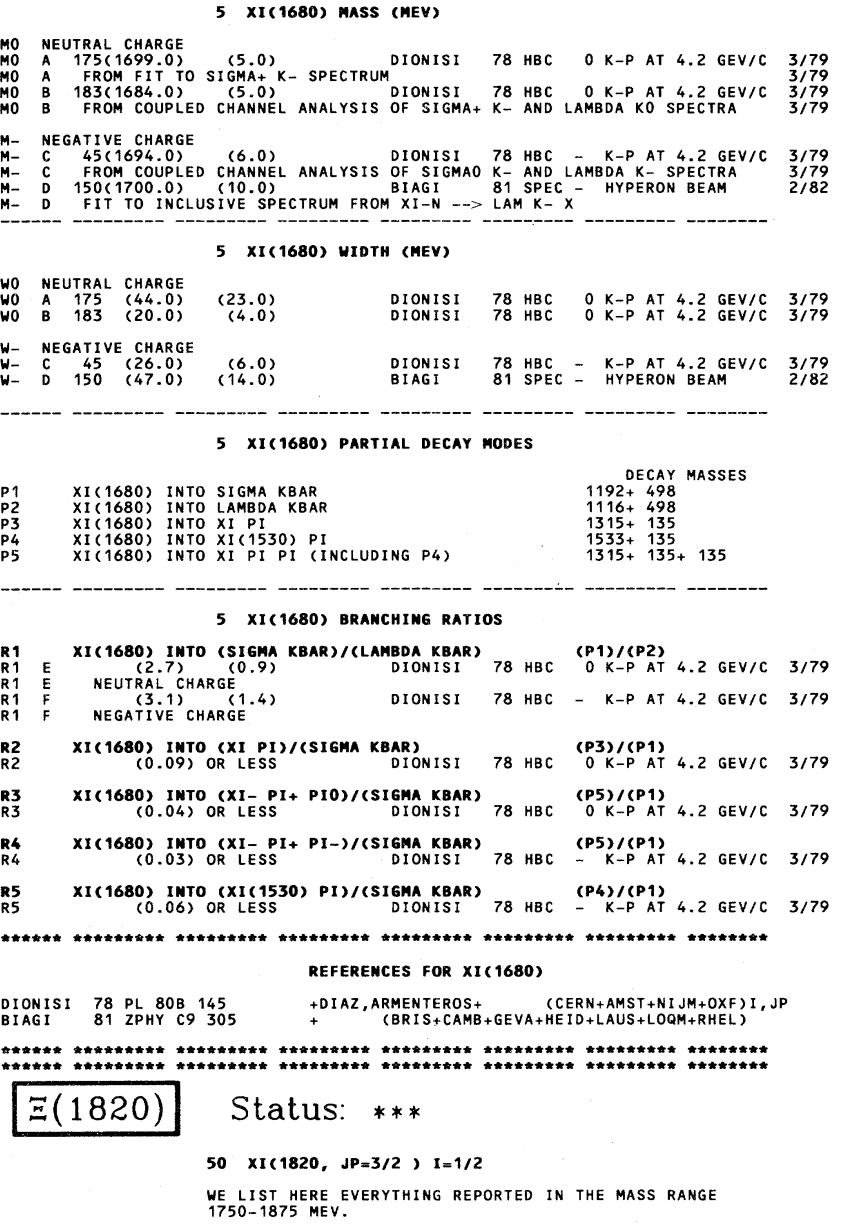

The clearest evidence for this state comes from GAY 76, who saw an 8-standard-deviation peak in $\Lambda \mathrm{K}^{-}$as well as signals in $\Xi(1530) \pi$ and $\Sigma \bar{K}$. The peak is narrow $(\Gamma=21 \pm 7 \mathrm{MeV}$ ), whereas earlier (and much smaller) experiments found widths of up to $100 \mathrm{MeV}$ (see the Listings below). A spin-parity analysis of the GAY 76 data, but with more events (TEODORO 78), favors spin $3 / 2$ but cannot make a parity discrimination.

BIAGI 81 used the CERN hyperon beam to study $\Xi^{-}$interactions in hydrogen and deuterium. The diffractively produced $\Lambda \mathrm{K}^{-}$system has a broad peak $(\Gamma=72 \pm 20 \mathrm{MeV})$ at $1830 \mathrm{MeV}$ on top of a substantial background. There is also a smaller peak in the inclusive $\Lambda \mathrm{K}_{\mathrm{S}}^{0}$ spectrum.

Neither GAY 76 nor BIAGI 81 saw a peak in the $\Xi \pi$ channel. It is possible that $\Xi \pi$ peaks seen in this region by some lower momentum experiments are at least partly due to the $\Xi(1940)$, with a shape distorted by the limited phase space available (SMITH 65). The situa- 
tion is further confused because some of the experiments were forced to add several different channels together to overcome poor statistics (CRENNELL 70, BADIER 71).
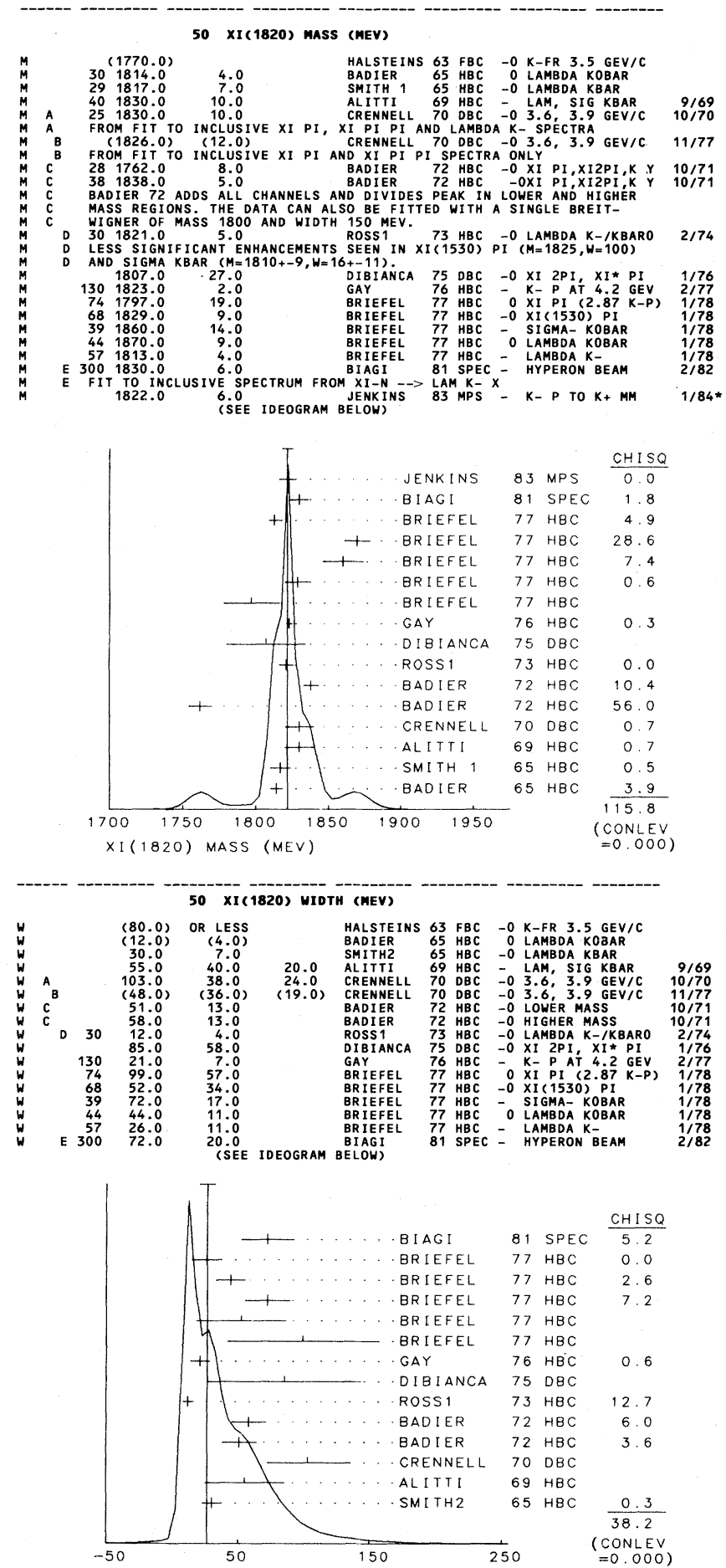

XI (1820) WIDTH (MEV)
$9 / 69$

(1)

50 XI(1820) PARTIAL DECAY MODES

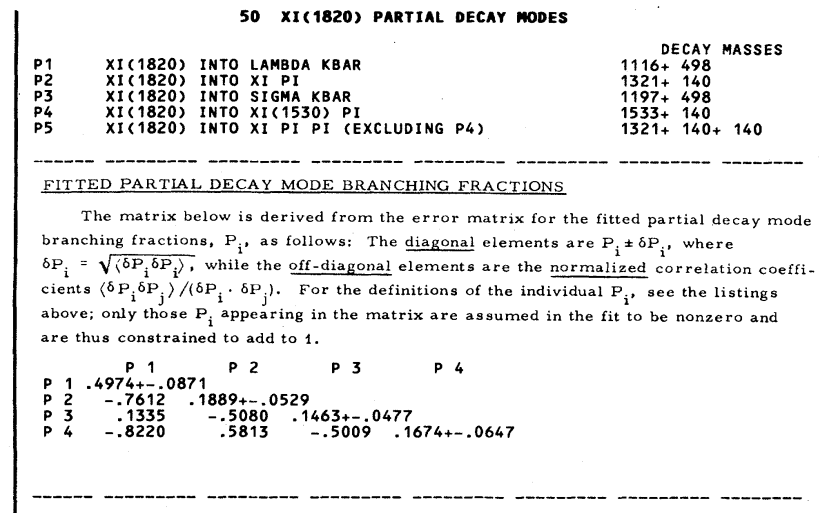

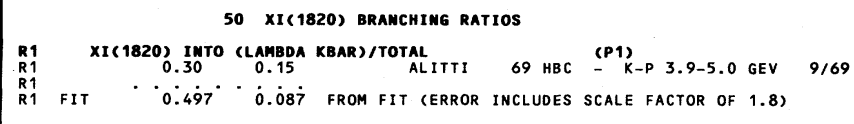
$\begin{array}{llll}R 2 & X I(1820) \\ R 2 & \text { IWTO (XI PI)/TOTAL ALITI } & 69 \mathrm{HBC} & \text { (P2) } \\ \text { R-P } 3.9-5.0 \mathrm{GEV} & 9 / 69\end{array}$ R2 FIT $0.189^{\circ} 0 . \dot{0} 5 \dot{3}$ FROM FIT (ERROR INCLUDES SCALE FACTOR OF 1.2)

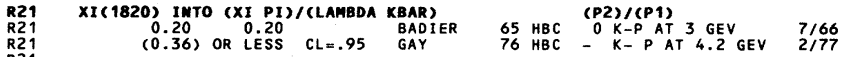
R21 FIT $0.38^{\circ} \cdot \dot{0} .^{\circ} 6^{\circ}$ FROM FIT (ERROR INCLUDES SCALE FACTOR OF 1.5)

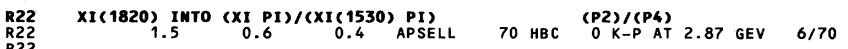
R22 FIT $\cdot \dot{1}_{1.13} \cdots 0.36^{\circ}$ FROM FIT

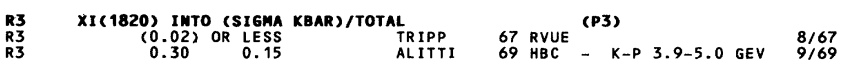

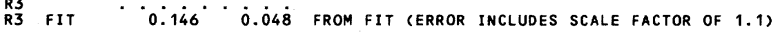
R31 XI(1820) INTO (SIGMA KBAR)/(LAMBDA KBAR)
R31
0.24
0.10

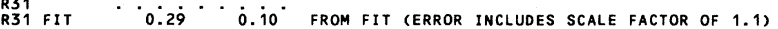

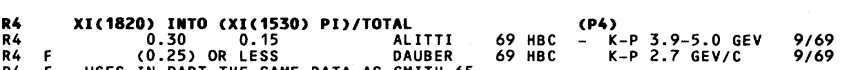

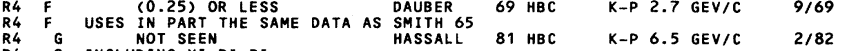
R4 FIT $0.167^{\circ} \cdot 0.065$ FROM FIT (ERROR INCLUDES SCALE FACTOR OF 1.6)

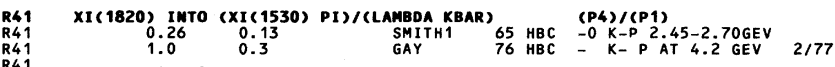

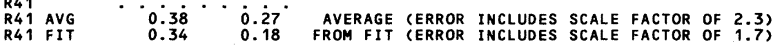

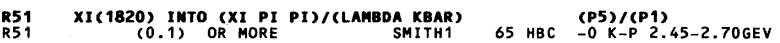
R52 XI(1820) INTO (XI PI PI)/(XI(1530) PI)
R52 H
$(0.5)$

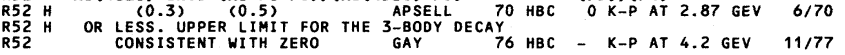
R53 XI(1820) INTO (XI PI PI (IMCL. XI(1530) PI)/(LAMBDA KBAR)

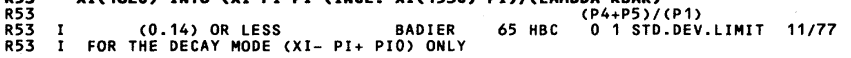

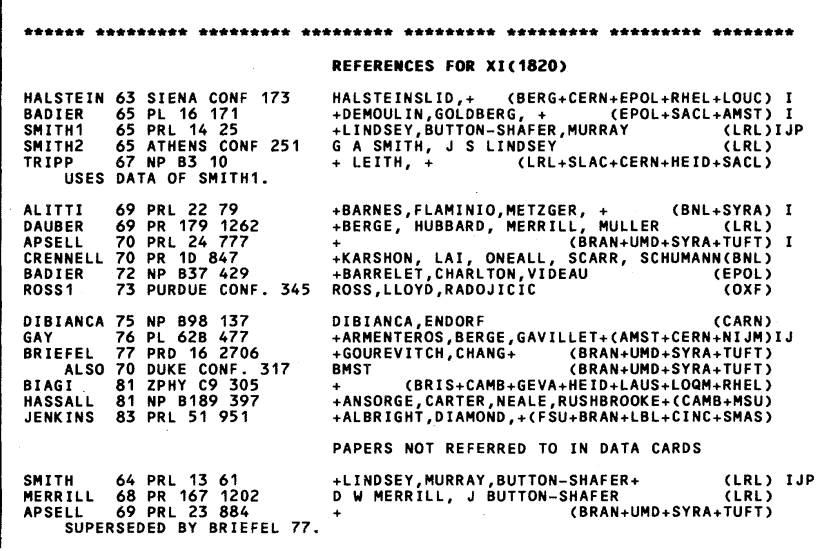


Baryons

$\Xi(1820), \quad \Xi(1940), \quad \Xi(2030)$

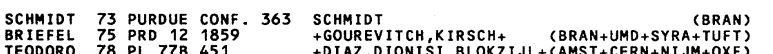

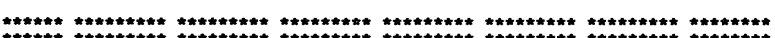

\section{$\Xi(1940) \quad$ Status: ** \\ $52 X I(1940, J P=\quad, I=1 / 2$}

WE LIST UNDER XI(1940) EVERYTHING REPORTED IN THE MASS
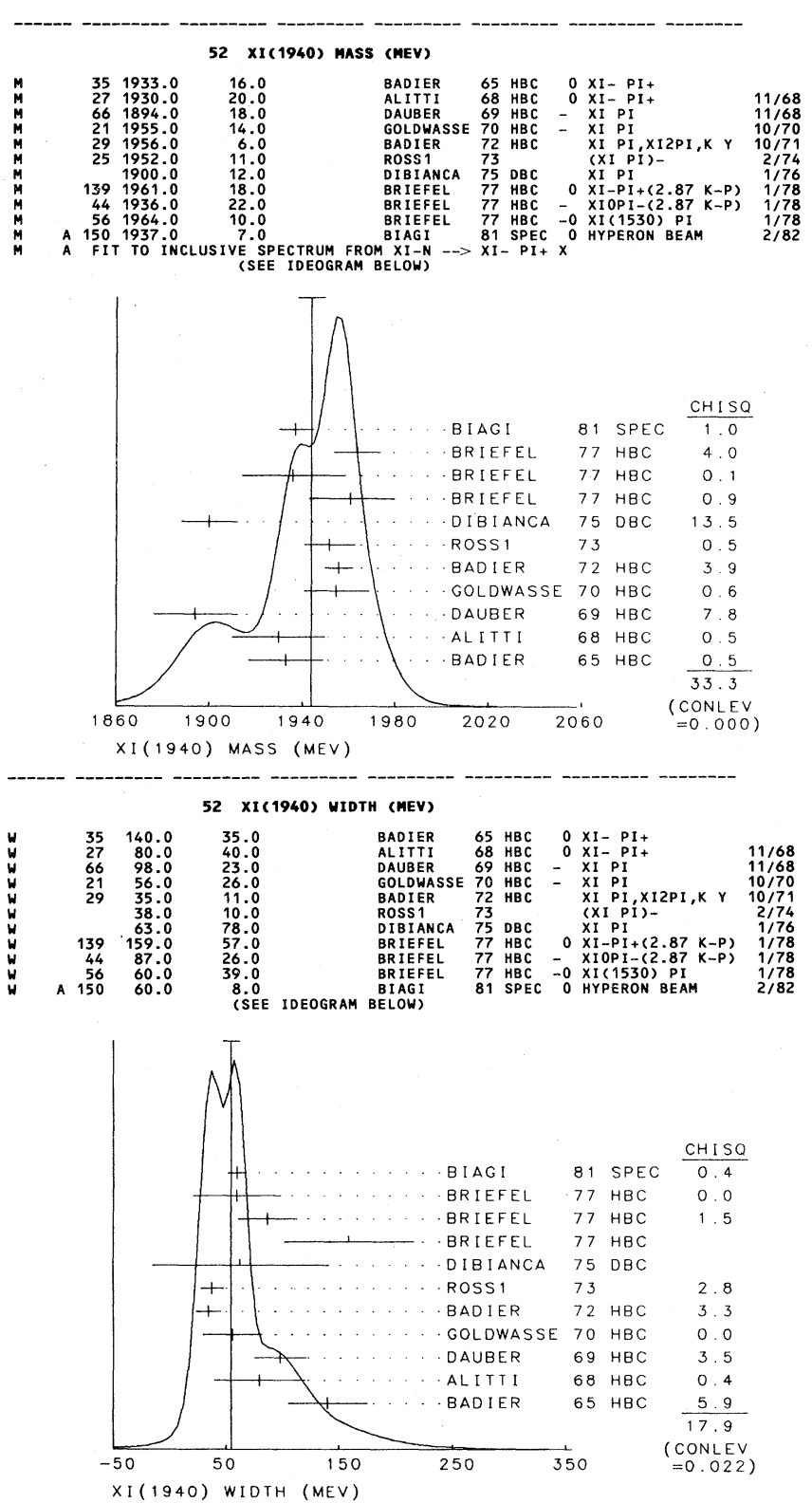

52 XI(1940) PARTIAL DECAY MODES

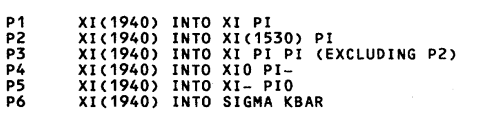

DECAY MASSES

$1321+140$
$1533+140$

1533+ $1400+140$ $1315+140$
$1321+135$
$1197+498$
52 XI(1940) BRAMCHIMG RATIOS THE XI(1940) IS SEEN MAINLY IN XI PI AND SOME IN XI(1530) PI. IT
HAS BEEN LOOKED FOR IN OTHER CHANNELS BUT ONLY OBSERVED BY HASSALL 81
WHO SEE A 3 SIGMA EFFECT IN SIGMA KBAR.

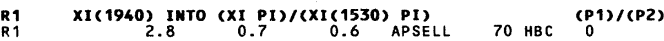

XI(1940) INTO (XI PI PI)/(XI(1530) PI)

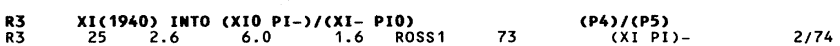

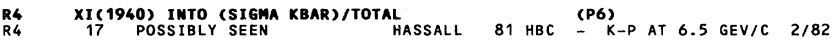

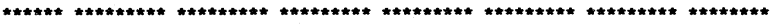

REFEREMCES FOR XI(1940)

BADIER
65

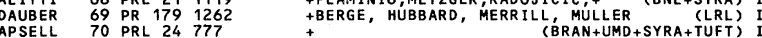

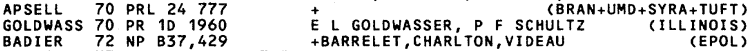

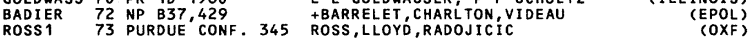

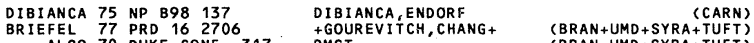

BRIEALO 70 DUKE CONF. 317 BMST

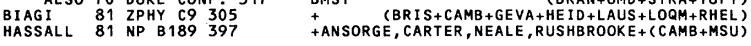

PAPERS NOT REFERRED TO IN DATA CARDS

APSELL 69 PRL $23884+\infty$ (BRAN+UMO+SYRA+TUFT)

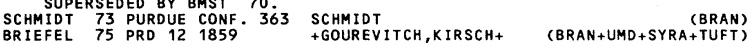

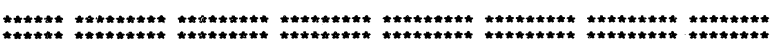

$\Xi(2030) \quad$ Status: $* * *$

$68 \times X(2030, J P=5 / 2$ OR GREATER) $I=1 / 2$

THE EVIDENCE FOR THIS STATE HAS BEEN MUCH IMPROVED

IN SIGMA KAAR AND A WEAKER COUPLING TO LAMBDA KBAR.

XI PI PI COR XI(1530) PI) CHANNEL, IN CONTRAST TO DIBIANCA 75. THE CONF IRMED BY HEMINGWAY 77.

A MOMENTS ANALYSIS OF THE HEMINGWAY 77 DATA INDICATES THAT THE SPIN
IS GREATER THAN OR EQUAL TO $5 / 2$ AT A LEVEL OF 3 STD. DEVIATIONS.

\section{XI(2030) MASS (MEV)}
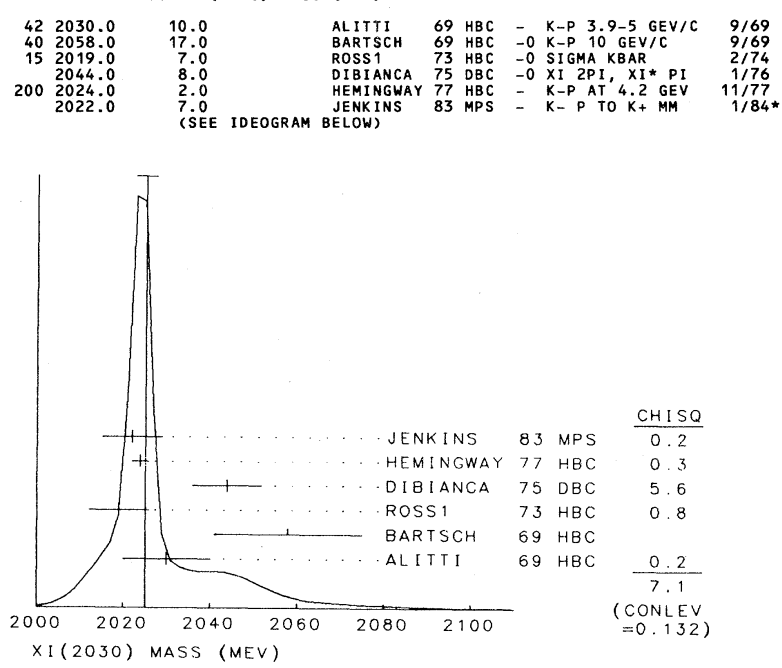

68 XI(2030) WIDTH (MEV)

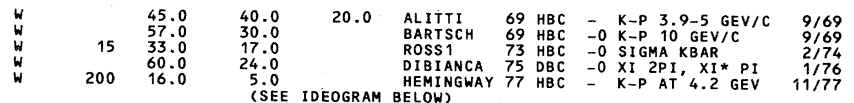




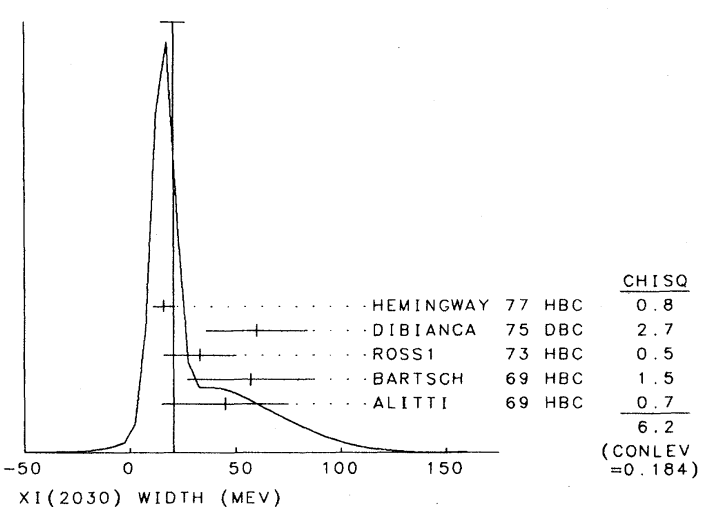

68 XI(2030) Partial decay mOdes

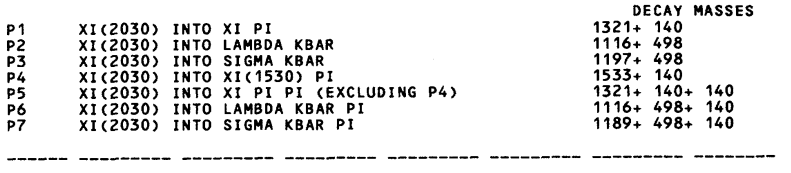

68 XI(2030) BRANCHIMG RATIOS

R1 $X I(2030)$ INTO (XI PI)/(MODES P1 TO P4)
$R 1$
(0.30) OR LESS

R11 $X I(2030)$ INTO (XI PI)/(SIGMA KBAR)
R11
$(0.19)$ OR LESS CL $=.95$

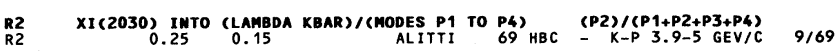

R21 XI(2030) INTO (LAMBDA KBAR)/(SIGMA KBAR)
R21

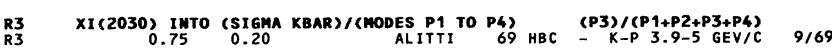

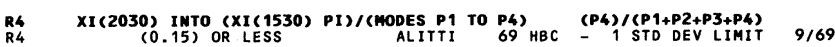

RA1 XI(2030) INTO (XI PI PI INCL. XI(1530) PI)/(SIGMA KBAR)

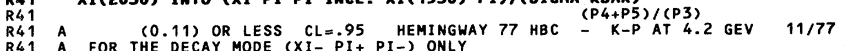

R6
R6

R61 XI(2030) INTO (LAMBDA KBAR PI)/(SIEMA KBAR)
R61
$(0.32)$ OR LESS CL=.95 HEMINGWAY 77 HBC

R7 XI(2030) INTO (SIGMA KBAR PI)/TOTALL
RA

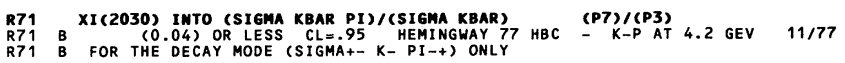

R71 B FOR THE DECAY MODE (SIGMA+- K- PI-+) ONLY

REFERENCES FOR XI(2030)

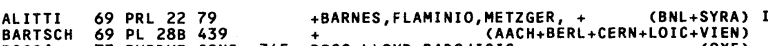

ROSS, LLOYD, RADOJICIC (AACH+BERL+CERN+LOLC+VIEN)
(OXF)

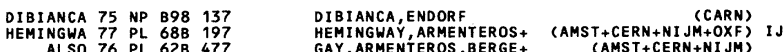
ALSO 76 PL 62B 477 GAY, ARMENTEROS,BERGE+ (AMS (AMST+CERN+NI JM)
JENKINS 83 PRL 51951 +ALBRIGHT, DIAMOND, +(FSU+BRAN+LBL+CINC+SMAS)

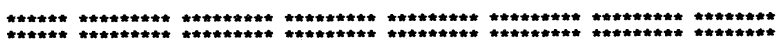

\section{$\Xi(2120) \quad$ Status: *}

$\longrightarrow \quad 103 \times 1(2120, \mathrm{JP}=\quad, \mathrm{I}=1 / 2$

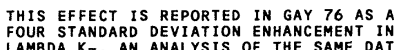

LAMBDA K-. AN ANALYSIS OF THE SAME DATA BY
HEMINGWAY 77, BUT WITH ADDITIONAL STATISTICS

POINTS OUT THAT THE SIGNIFICANCE OF THE ENHANCEMENT IS GREATL'Y REDUCED

POINTS OUT THAT THE SIGNIFICANCE OF THE ENHANCMENT IS GREATL
IF A RESTRICIVE FOUR-MOMENTUM CUT (U-CUT) IS MADE. THS SU
AN ANOMALOUS PRODUCTION MECHANISM IF THE STATE IS GENUINE.

CHLIAPNIKOV 79 REPORT A BUMP OF 18 EVENTS AT 2137 MEV IN AN INCLUSIVE
ANTI-LAMBDA $\mathrm{K}+$ SPECTRUM FROM K+P INTERACTIONS AT 32 GEV/C. THE K+ ARE NOT UNIQUELY IDENTIFIED. BUMPS WI TH LOWER NUMBERS OF EVENTS ARE ALSO

in NEED OF CONFIRMATION, OMITTED fRom tables.

$$
\Xi(2030), \Xi(2120), \Xi(2250), \Xi(2370)
$$

103 XI(2120) MASS (MEV)

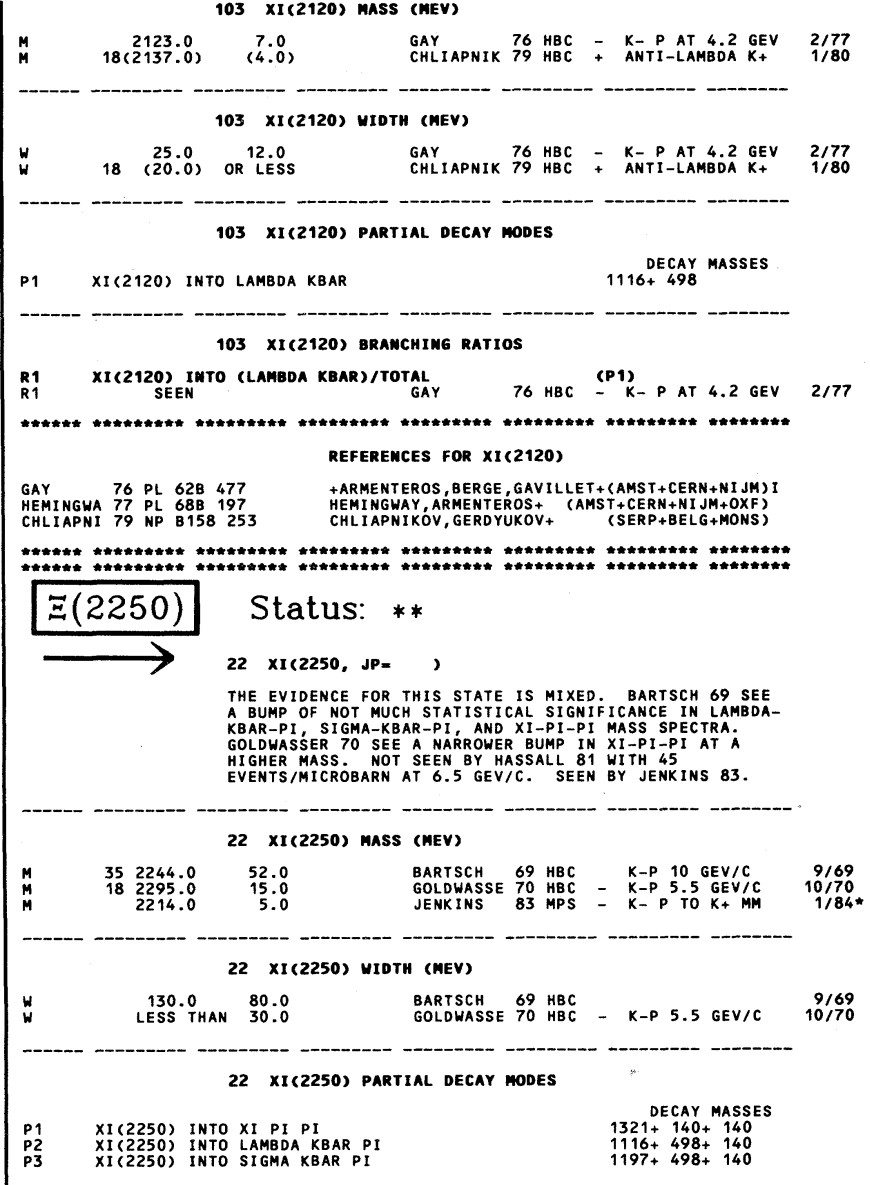

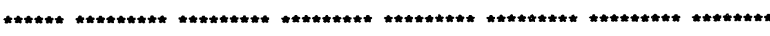

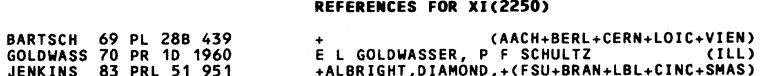
PAPERS NOT REFERRED TO IN DATA EARDS

HASSALL 81 NP B189 397 +ANSORGE, CARTER, NEALE, RUSHBROOKE+ (CAMB +MSU)

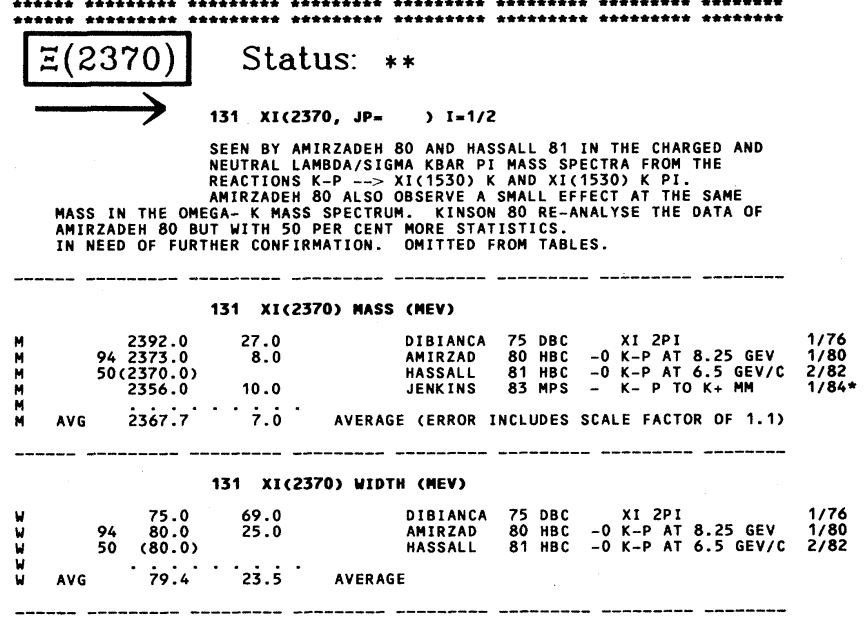


Baryons $\Xi(2370), \Xi(2500), \Omega^{-}, \Lambda_{\mathrm{c}}^{+}, \Sigma_{\mathrm{c}}(2450), \mathrm{A}^{+}, \Lambda_{\mathrm{b}}^{0}$, DIBARYONS
Data Card Listings

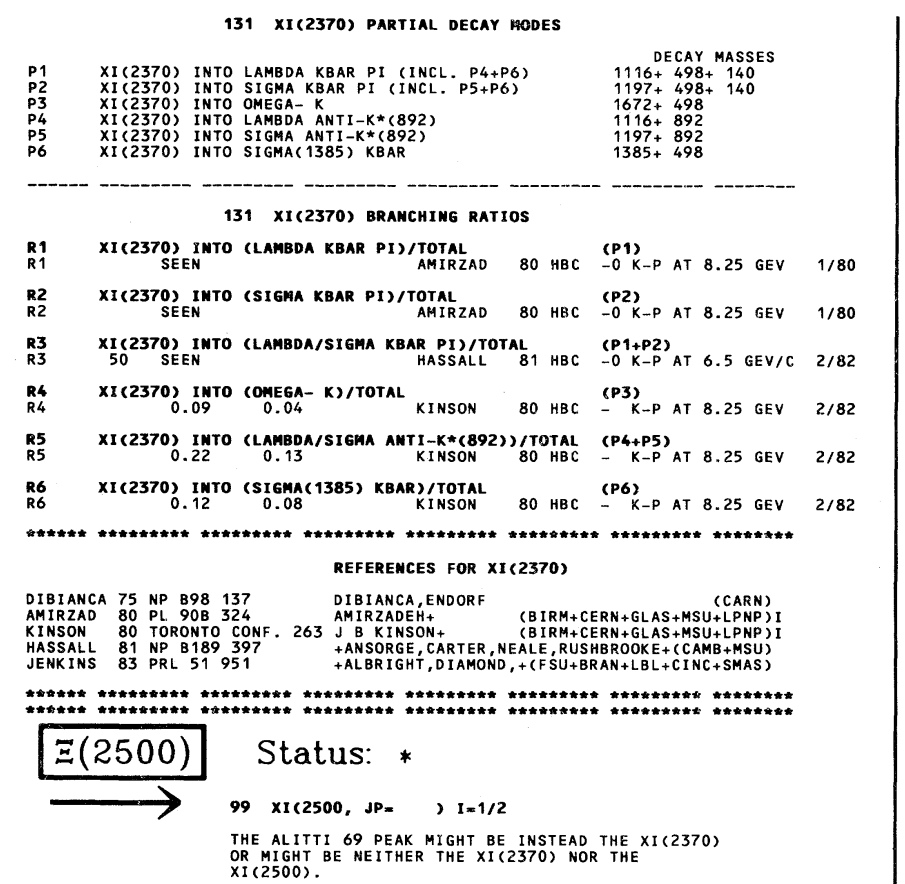

\begin{tabular}{|c|c|c|c|c|c|c|}
\hline & & $99 \times 1(2500)$ MASS & (MEV) & & & \\
\hline M & $\begin{array}{ll}30 & 2430.0 \\
45 & 2500.0 \\
& 2505.0\end{array}$ & $\begin{array}{l}20.0 \\
10.0 \\
10.0\end{array}$ & $\begin{array}{l}\text { ALITTI } \\
\text { BARTSCH } \\
\text { JENKINS }\end{array}$ & $\begin{array}{l}69 \mathrm{HBC} \\
69 \mathrm{HBC} \\
83 \mathrm{MPS}\end{array}$ & $\begin{array}{l}-\quad K-P 4.6-5 \text { GEV } / C \\
=0 \text { K-P } 10 \text { GEV } / \mathrm{C} \\
=K-P \text { TO K+ MM }\end{array}$ & $\begin{array}{l}9 / 69 \\
9169 \\
1 / 84 *\end{array}$ \\
\hline
\end{tabular}

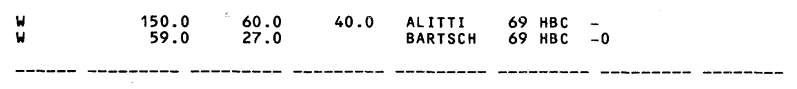

99 XI(2500) PARTIAL DECAY MODES

\begin{tabular}{|c|c|c|}
\hline $\begin{array}{l}\text { P1 } \\
P 2 \\
P 3 \\
P 4 \\
P 5 \\
P 6\end{array}$ & 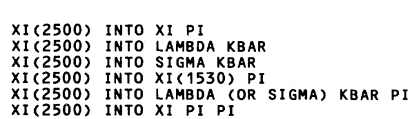 & $\begin{array}{l}\text { DECAY MASSES } \\
1321+140 \\
1116+498 \\
1197+498 \\
1533+140 \\
1116+498+140 \\
1321+140+140\end{array}$ \\
\hline
\end{tabular}

99 XI(2500) BRANCHIMG RATIOS

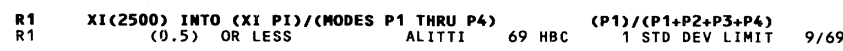

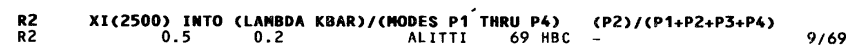

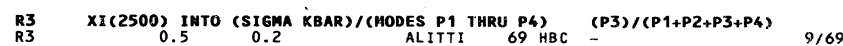

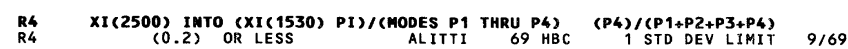

R5
R5 XI (250) INTO (LAMBDA (OR SIGMA) KaAR PII)/TOTAL
SEEN

R6 XI(2500) INTO (XI PI PI)/TOTAL BARTSCH 69 HBC $\stackrel{-0}{\text { RE }}$

***************************************************************************

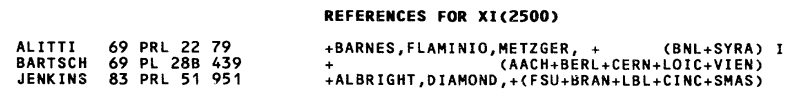

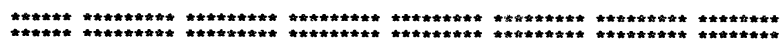

$$
\mathrm{S}=-3 \quad \mathrm{I}=0 \text { HYPERON STATE }(\Omega)
$$

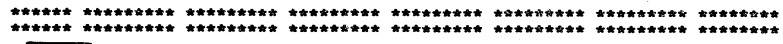

\begin{tabular}{|c|c|}
\hline$\Omega^{-}$ & $\begin{array}{l}24 \text { OMEGA-C } 1672, J P=\quad, I=0 \\
\text { SEE STABLE PARTICLE DATA CARD LISTINGS }\end{array}$ \\
\hline
\end{tabular}

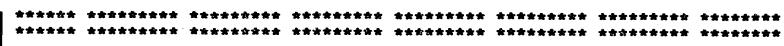
CHARMED BARYONS

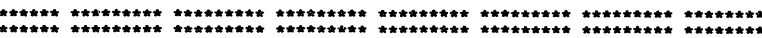

$\Lambda_{c}^{+} \quad 33$ LAMBDA/C+(2282, JP= ,

SeE Stable particle data card Listings

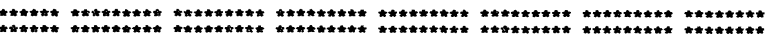

$\sum_{c}(2450) \quad$ Status: **

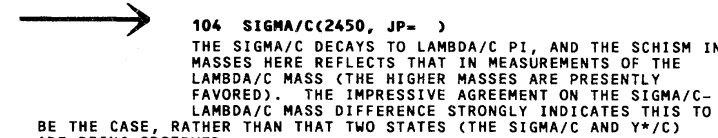

BE THE CASE, RATHED
ARE BEING OBSERYER

THIS PARTICLE IS AT ABOUT THE 2-AND-1/2-StaR LeVEL. A Definitive
EXPERIMENT IS NEEDED.

\begin{tabular}{|c|c|c|c|}
\hline \multicolumn{3}{|c|}{104 SIGMA/C(2450) MASS (MEV) } & \multirow[b]{2}{*}{$\begin{array}{r}31 / 7 \\
11 / 8 \\
3 \\
1217 \\
11 / 8 \\
3 \\
3\end{array}$} \\
\hline $\begin{array}{lc}1 & 2426.0 \\
9 & (2460.0) \\
1 & (2439.0) \\
6 & 2425.0 \\
1 & 2457.0 \\
1 & 2454.0\end{array}$ & $\begin{array}{r}12.0 \\
\text { OR }{ }^{\text {MORE }} \\
10.0 \\
4.0 \\
5.0\end{array}$ & 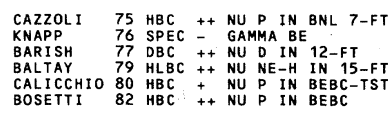 & \\
\hline \multicolumn{4}{|c|}{104 (SIGMA/C)-(LAMBDA/C+) MASS DIFFEREMCE (MEV) } \\
\hline $\begin{array}{ll}1 & 166.0 \\
6 & 168.0 \\
1 & 168.0 \\
1 & 166.0\end{array}$ & $\begin{aligned} & 15.0 \\
& 3=0 \\
& 3.0 \\
& 1.0\end{aligned}$ & 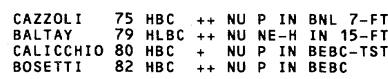 & 18 \\
\hline
\end{tabular}

104 SIGMA/C(2450) PARTIAL DECAY MODES

P1 SIGMA/C(2450) INTO LAMBDA/C+ PI $2282+140$ DECAY MASSES

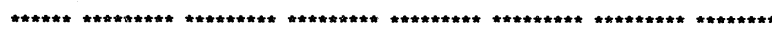

$\begin{array}{lllll}\text { CAZZOLI } & 75 & \text { PRL } & 34 & 1125 \\ \text { KNAPP } & 76 & \text { PRL } & 37 & 882\end{array}$

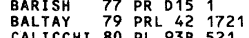

REFEREMCES FOR SIGMA/C(2450)

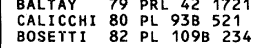

+CNOPS, CONNOLLYY LOUTTIT, MURTAGH
+LEE, (BNL)
+LENG, SMITH

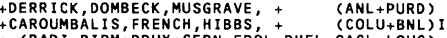

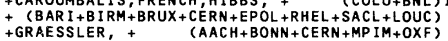

THEORY AND REVIEW

DERUJULA 75 PR $D 12147$ TGEORGI, GLASHOW

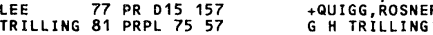

(HARV)
(FNAL)
(LBL)

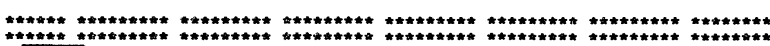

$A^{+} \quad 45 A+(2460, J P=$

$\rightarrow \quad$ SeE stable particle data card listings

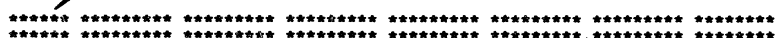

BOTTOM (BEAUTY) BARYON

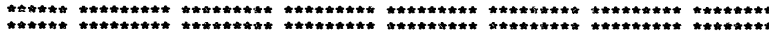

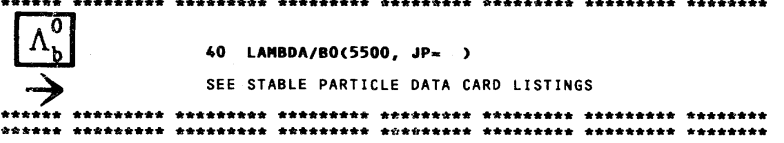

\section{NOTE ON DIBARYON RESONANCES}

(by L.D. Roper, Virginia Polytechnic Institute and State University)

The first modern theoretical discussion of dibaryon resonances was probably by Oakes. ${ }^{1}$ The first experimental hint of them was in a $\Lambda p$ invariant mass distribution by $\mathrm{Dahl},{ }^{2}$ and in a pp partial-wave analysis by 
Arndt ${ }^{3}$ for the ${ }^{1} D_{2}$ state. [The notation is $(2 S+1)_{L_{J}}$, where $S$ is the total spin, $L$ is the orbital angular momentum, and $\mathrm{J}$ is the total angular momentum. The Pauli principle restricts two nucleons to be in one of the following states:

$$
\begin{aligned}
& I=0:\left({ }^{3} S_{1},{ }^{3} D_{1}\right),{ }^{1} P_{1},{ }^{3} D_{2},\left({ }^{3} D_{3},{ }^{3} G_{3}\right),{ }^{1} F_{3},{ }^{3} G_{4}, \cdots \\
& I=1:{ }^{1} S_{0},{ }^{3} P_{0},{ }^{3} P_{1},\left({ }^{3} P_{2},{ }^{3} F_{2}\right),{ }^{1} D_{2},{ }^{3} F_{3}, \cdots \text {. }
\end{aligned}
$$

Here the states that couple together (same $J^{P}$ ) are grouped together in parentheses. Similarly, only certain states are allowed for $\Lambda \Lambda$, etc.]

Interest in dibaryons rose dramatically in 1977 when strong energy dependence was unexpectedly observed in pp polarization experiments at Argonne. ${ }^{4}$ Also, in that year and the next, Hoshizaki claimed the existence of dibaryon resonances in a pp partial-wave analysis. ${ }^{5}$ In the same year Jaffe gave a detailed theoretical treatment of multiquark states. ${ }^{6}$ There is now a vast literature on dibaryon resonances.

However, there is still disagreement about what "dibaryon resonances" are. There is little doubt of the existence of distinct structures in NN partial-wave amplitudes that look very much like ordinary highly inelastic resonances, such as are seen in $\pi \mathrm{N}$ scattering. The question is whether these structures are caused by resonance poles in the complex energy plane or by some other structure of the scattering amplitude.

One aspect of the arguments about dibaryon resonances is whether they are calculable in terms of quark theory or should instead be calculated using some hadron interaction theory without reference to the underlying quarks. Both approaches have had successes and failures, so possibly both will make contributions to unraveling the mysteries of dibaryon resonances.

The idea that dibaryon resonances are "pseudoresonances"7 has taken some new turns. The idea is that box diagrams (e.g., involving $\mathrm{N} \Delta$ in $\mathrm{NN}$ scattering) create resonance-like loops in the Argand diagram without resonance poles actually existing. The problem with believing this is whether poles would be created when one unitarizes the box-diagram calculations in order to calculate physical scattering amplitudes. Kloet and Tjon $^{8}$ have recently shown that a model exists in which, indeed, this is the case. However, resonance hunters should definitely report pole positions rather than looping Argand diagrams in the future. All who suggest that the $\mathrm{NN}^{1} \mathrm{D}_{2}$ or ${ }^{3} \mathrm{~F}_{3}$ resonance-like structure is a resonance or is instead due to some other dynamics must take their case to the world collection of $\mathrm{NN}$ scattering data in the form of a detailed partial-wave analysis, and should report the existence or nonexistence of resonance poles.

Closely related to the work described above is that by Ueda, ${ }^{9}$ in which Faddeev $\pi \mathrm{NN}$ dynamics is fitted to the NN partial-wave amplitudes. Although the fit is not good, the approximately correct structure is present. The interesting point is that poles do occur on the "resonance" sheets in the complex energy plane. Ueda claims this work is important because many claims for resonance poles assume that the poles exist, but the Faddeev approach does not make such prior assumptions.

VerWest ${ }^{10}$ recently reported separable potential model fits to the ${ }^{1} \mathrm{D}_{2}$ and ${ }^{3} \mathrm{~F}_{3} \mathrm{NN}$ amplitudes and claims some solutions had no resonance poles, but Kloet and Tjon ${ }^{11}$ have shown that these potential model fits all do, indeed, have resonance poles.

The dinucleon resonances also communicate with the $\gamma d$ and $\pi d$ channels. There is not much $\gamma d$ data, and the multipole analysis does not yield much certainty about which dibaryons are involved. In the $\pi \mathrm{d}$ case, uncertainties abound, and the partial-wave analysis yields poor fits compared to the NN analyses. In addition to $\mathrm{NN}$ analyses, results from partial-wave analyses of $\pi \mathrm{d} \rightarrow \pi \mathrm{d}, \pi \mathrm{d} \rightarrow \pi \mathrm{pn}, \mathrm{pp} \rightarrow \pi \mathrm{d}$, and $\gamma \mathrm{d} \rightarrow \mathrm{pn}$ scattering are listed below. Most of these strongly indicate the existence of dibaryon resonances in the ${ }^{1} \mathrm{D}_{2}$ and ${ }^{3} \mathrm{~F}_{3} \mathrm{NN}$ states, and some indicate possible resonances in the ${ }^{1} \mathrm{~S}_{0},{ }^{3} \mathrm{~S}_{1},{ }^{3} \mathrm{P}_{1},{ }^{3} \mathrm{P}_{2},{ }^{3} \mathrm{D}_{3},{ }^{1} \mathrm{~F}_{3}$, and ${ }^{1} \mathrm{G}_{4}$ states.

Since our last edition, many papers have been published about dibaryon resonances, but very little new hard information has emerged. There are ten new references for dinucleons and only one new reference for strange dibaryons giving new values for the resonancepole (or Breit-Wigner) parameters. However, most of the new references for dinucleons are merely new fits to NN partial-wave amplitudes.

A notable paper is that of Semianczuk et al., ${ }^{12}$ in which two prominent peaks are seen in the np invariant mass spectrum from dp $\rightarrow$ (np)p deuteron breakup. However, a recent preprint by Katayama et al. ${ }^{13}$ claims to have done a similar experiment and did not see the peaks.

Since our last edition, only one paper has appeared giving data for the strange dibaryon states. It appears 


\section{Baryons DIBARYONS}

that the strangeness -1 dominant resonance is in the ${ }^{3} S_{1}$ state, an SU(3) partner of the deuteron. An excellent review is given by Dalitz. ${ }^{14} \mathrm{He}$ concludes that the $S=-1{ }^{3} S_{1}$ resonance pole probably exists. However, May et al. ${ }^{15}$ report no enhancements in the $\Sigma^{+} n, \Sigma^{0} p$, or $\Lambda p$ invariant mass spectra in the $K^{-} d \rightarrow \pi^{-} X$ reaction, and Arenton et al. ${ }^{16}$ report no enhancement in the $\Lambda \mathrm{p}$ spectrum in the $\mathrm{pp} \rightarrow \Lambda \mathrm{pK}^{+}$reaction.

In the Listings below, we separate the determinations of pole positions and Breit-Wigner parameters. To be a resonance, the pole must occur on the lower half of the second sheet for the elastic channel; it may be a bound state or resonance for inelastic channels.

In summary, this reviewer feels that the evidence, both experimental and theoretical, for the ${ }^{1} \mathrm{D}_{2}$ and ${ }^{3} \mathrm{~F}_{3}$ dinucleon resonances is now very strong. The theoretical calculations almost all now agree that resonance poles occur in the NN amplitudes. The disagreement among production experiments is inherent in the difficulties of that kind of experiment; overlapping highly inelastic resonances are very difficult to see in final states.

For more detailed reviews of dibaryons, with a wide variety of opinions, see Hoshizaki, ${ }^{17} \mathrm{Bugg},{ }^{18} \mathrm{Kamae},{ }^{19}$ Vinh Mau, ${ }^{20}$ Kroll, $^{21}$ and Locher. ${ }^{22}$

\section{References}

1. R.J. Oakes, Phys. Rev. 131, 2239 (1963).

2. O.I. Dahl et al., Phys. Rev. Lett. 6, 142 (1961).

3. R.A. Arndt, Phys. Rev. 165, 1834 (1968).

4. For a list of references and a review, see A. Yokosawa, Phys. Rep. 64, 47 (1980).

5. N. Hoshizaki, Prog. Theor. Phys. 58, 716 (1977), and 60, 1796 (1978).

6. R.L. Jaffe, Phys. Rev. Lett. 38, 195 (1977), and 38, 617 (1977).

7. B.L.G. Bakker, I.M. Narodetsky, and J.A. Simonov, Lett. Nuovo Cim. 19, 265 (1977); Yu.A. Simonov and $M$. van der Velde, Phys. Lett. 76B, 277 (1978); I.M. Narodetsky and Yu.A. Simonov, Sov. J. Nucl. Phys. 28, 698 (1978); Yu.A. Simonov and M. van der Velde, J. Phys. G5, 493 (1979).

8. W.M. Kloet and J.A. Tjon, Phys. Lett. 106B, 24 (1981); and Nucl. Phys. A392, 271 (1983).

9. T. Ueda, Phys. Lett. 119B, 281 (1982).

10. B.J. VerWest, Phys. Rev. C25, 482 (1982).

11. W.M. Kloet and J.A. Tjon, Phys. Rev. C27, 430 (1983).

12. T. Semianczuk et al., Phys. Lett. 128B, 367 (1983).

\section{Data Card Listings}

13. N. Katayama et al., University of Tokyo preprint UT-HE-83/14 (1983).

14. R.H. Dalitz, Nucl. Phys. A354, 101c (1981).

15. M. May et al., Phys. Rev. C25, 1079 (1982).

16. M.W. Arenton et al., Phys. Rev. D28, 657 (1983).

17. N. Hoshizaki, in $V I^{\text {th }}$ International Seminar on High Energy Physics Problems (Dubna, 1981).

18. D. Bugg, Nucl. Phys. A374, 95c (1982).

19. T. Kamae, Nucl. Phys. A374, 25c (1982).

20. R. Vinh Mau, Nucl. Phys. A374, 3c (1982).

21. P. Kroll, in Troisieme Journees d'Etudes SATURNE (Fontevraud, 1983).

22. M.P. Locher, in $10^{\text {th }}$ International Conference on Few Body Problems in Physics (Karlsruhe, 1983).

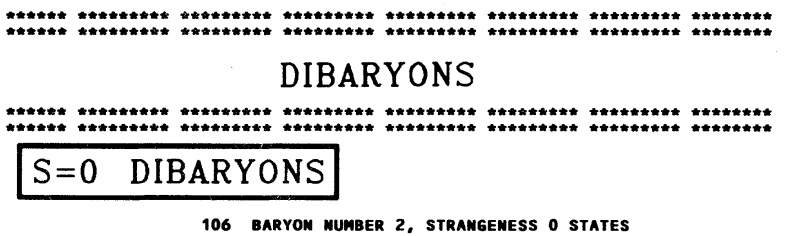

106 Baryon mumber 2, stramgeness O states

IN THIS SECTION WE USE THE FOLLOWING ABBREVIATIONS FOR TYPES
OF ANALYSES--

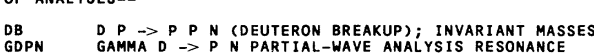

GDPN GAMMA D $\rightarrow$ P N PARTIAL-WAVE ANALYSIS RESONANCE

NN F FIT TO NN ELASTIC PARTIALLWAVE ANALYSIS RESULTS

NNF NP TOTAL CROSS SECTION BREIT-WIGNER FIT

PARAMETRIZATION

PARAMETRIZATION
PIDC
PIDI DIF DIFRENTIAL CROSS SECTION BREIT-WIGNER FIT

PNI
$P$ P P STEIT-HESONANCE

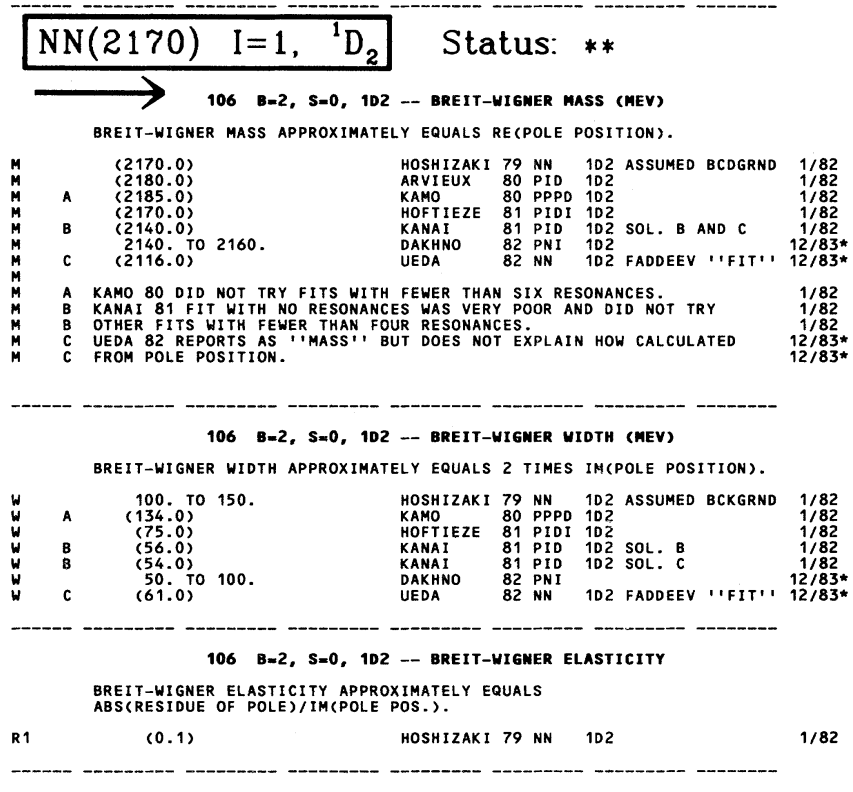




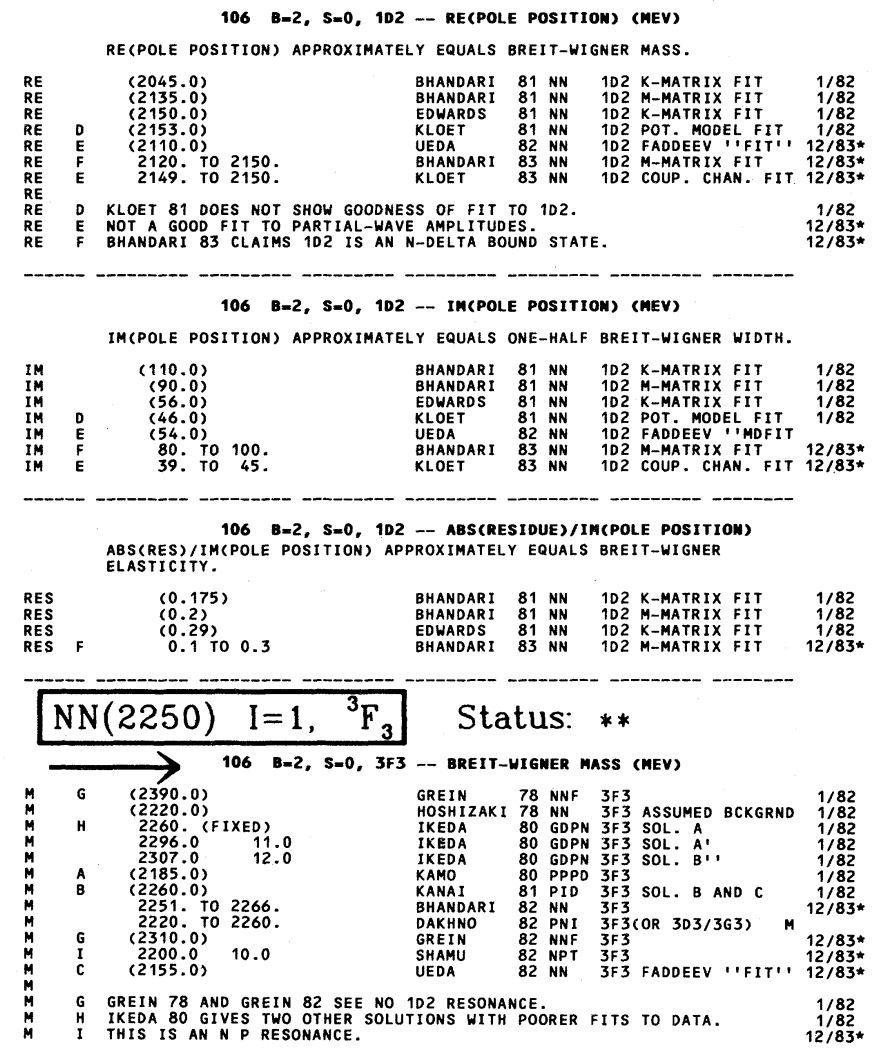

106 B=2, S=0, $3 F 3$-- BREIT-WIGMER WIDTH (MEV)

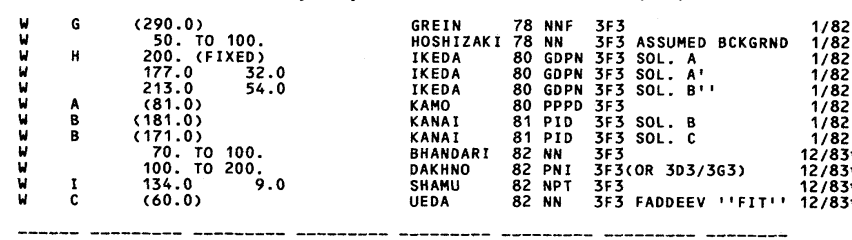

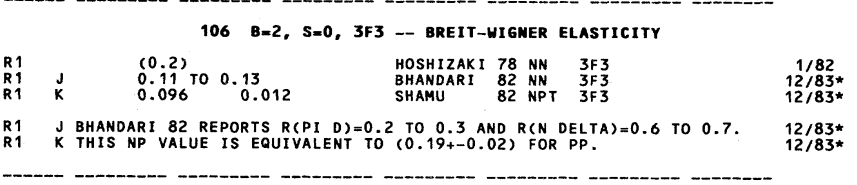
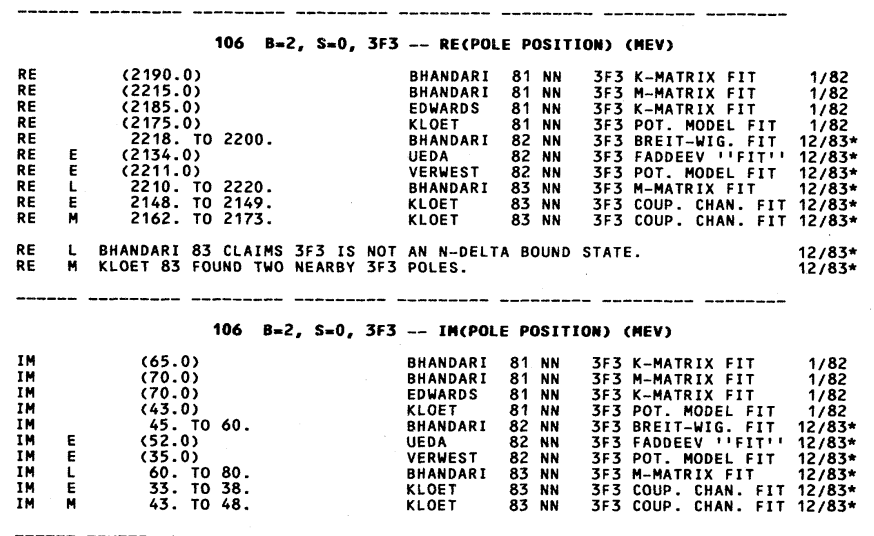

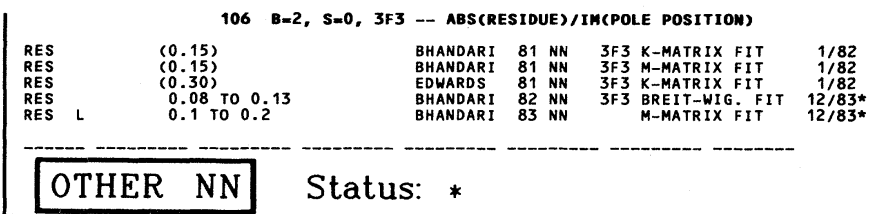

$\longrightarrow 106$ B=2, S=0 MISCELL. -- BREIT-WIGMER MASS (MEV)

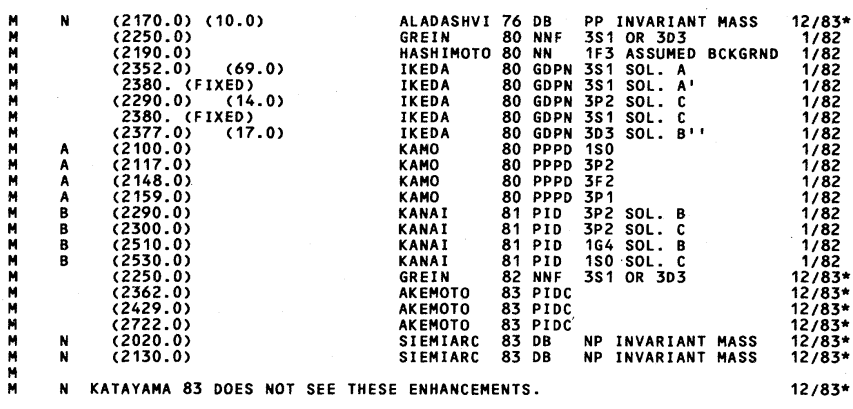

106 B=2, S=0 MISCELL. -- BREIT-HIGMER MIDTH (MEV)

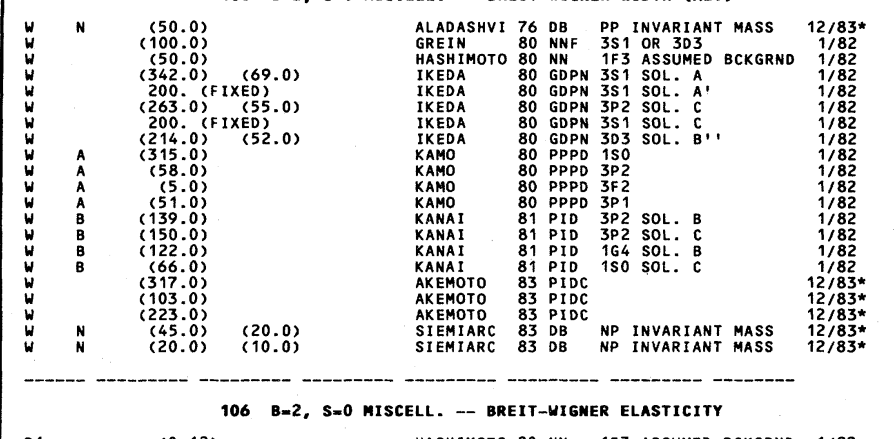

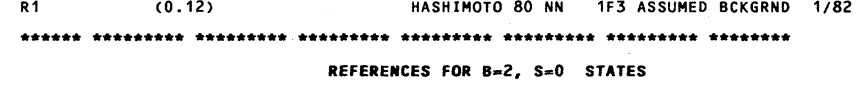

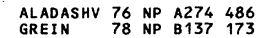

HOSHZAK 78 PTP 60 1796
HOSHIZA 79 PTP 61729
HNH

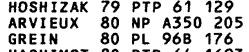

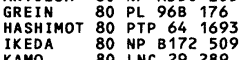

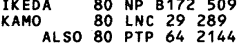

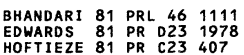

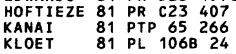

BHANDARI 82 LNC 3465
DAKHNO 82 PL 1148 B 409

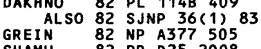

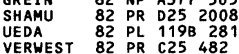

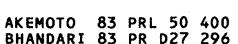

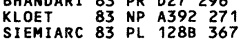

$\begin{array}{llll}\text { HIDAKA } & 77 \text { PL } 708 & 479 \\ \text { FRASCARI } & 80 & \text { PL } 9918 & 345\end{array}$

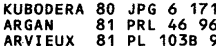

BOLGER
GREIN
HOLT

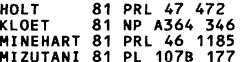

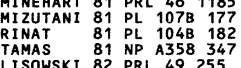

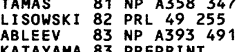

$\begin{array}{ll}\text { ARA } & \text { KAYAMA } 83 \text { PREPRNT } \\ \text { KLOET } 83 \text { PR C27 } 430\end{array}$

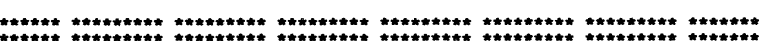




\section{Baryons}

DIBARYONS

\section{$\mathrm{S}=-1 \quad$ DIBARYON}

107 Baryom mumber 2, Stramgemess - 1 states

IN THIS SECTION WE USE THE FOLLOWING ABBREVIATIONS FOR TYPES

BB
BARYON-BARYON SCATTERING COMBINED AMPLITUDE ANALYSIS

LPPIM LAMBDA-P INVARIANT MASS

LPPIMM LAMBDA-P-PI INVARIANT MASS
SPIM

$\Lambda \mathrm{N}(2130) \quad \mathrm{I}=1 / 2,{ }^{3} \mathrm{~S}_{1}$ Status: **
BREIT-WIGNER MASS APPROXIMATELY EQUALS RE(POLE POSITION).

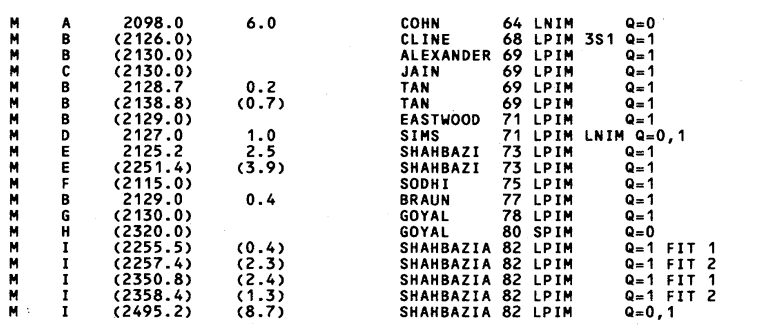

M SIGMA- D TO LAMBDA N X.

$K-D$ TO PI- LAMBDA P.
GOYAL 71 RAISES DOUBTS ABOUT THE EXPERIMENTAL PROCEDURE USED

IN JAIN 69. JAIN STUDIED K- EMULSION TO LAMBDA P.
$K-$ D TO PII- LAMBDA P, PI- LAMBDA PI+ N, AND PI- LAMBDA PIO P.
$N$ P TO LAMBDA $P$ X FOR P IN CARBON 12.

gOYAL 78 SEes anOtheR UNCERTAIN PEAK AT 2195-2210 MEV.

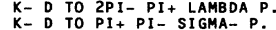

SIMULTANEOUS FIT TO INVARIANT MASS AND LAMBDA-P ELASTIC

107 B=2, S=-1 -- BREIT-HIGMER WIDTH (MEV) BREIT-WIGNER WIDTH APPROXIMATELY EQUALS 2 TIMES IM(POLE POSITION).

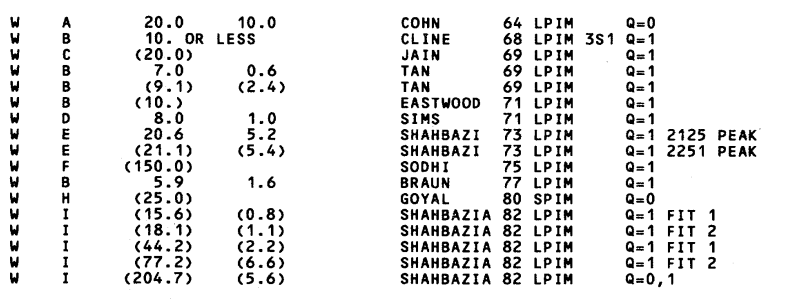

107 B=2, S=-1 -- RE(POLE POSITIOM) (MEV)

RE(POLE POSITION) APPROXIMATELY EQUALS BREIT-WIGNER MASS.

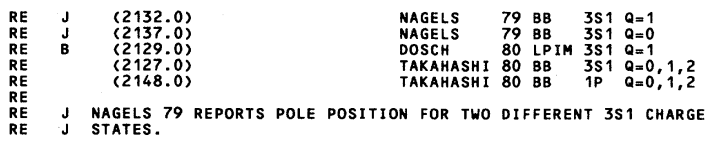

$1 / 82$
$1 / 82$
$1 / 82$
$1 / 82$
$1 / 82$

$1 / 82$

\section{Data Card Listings}

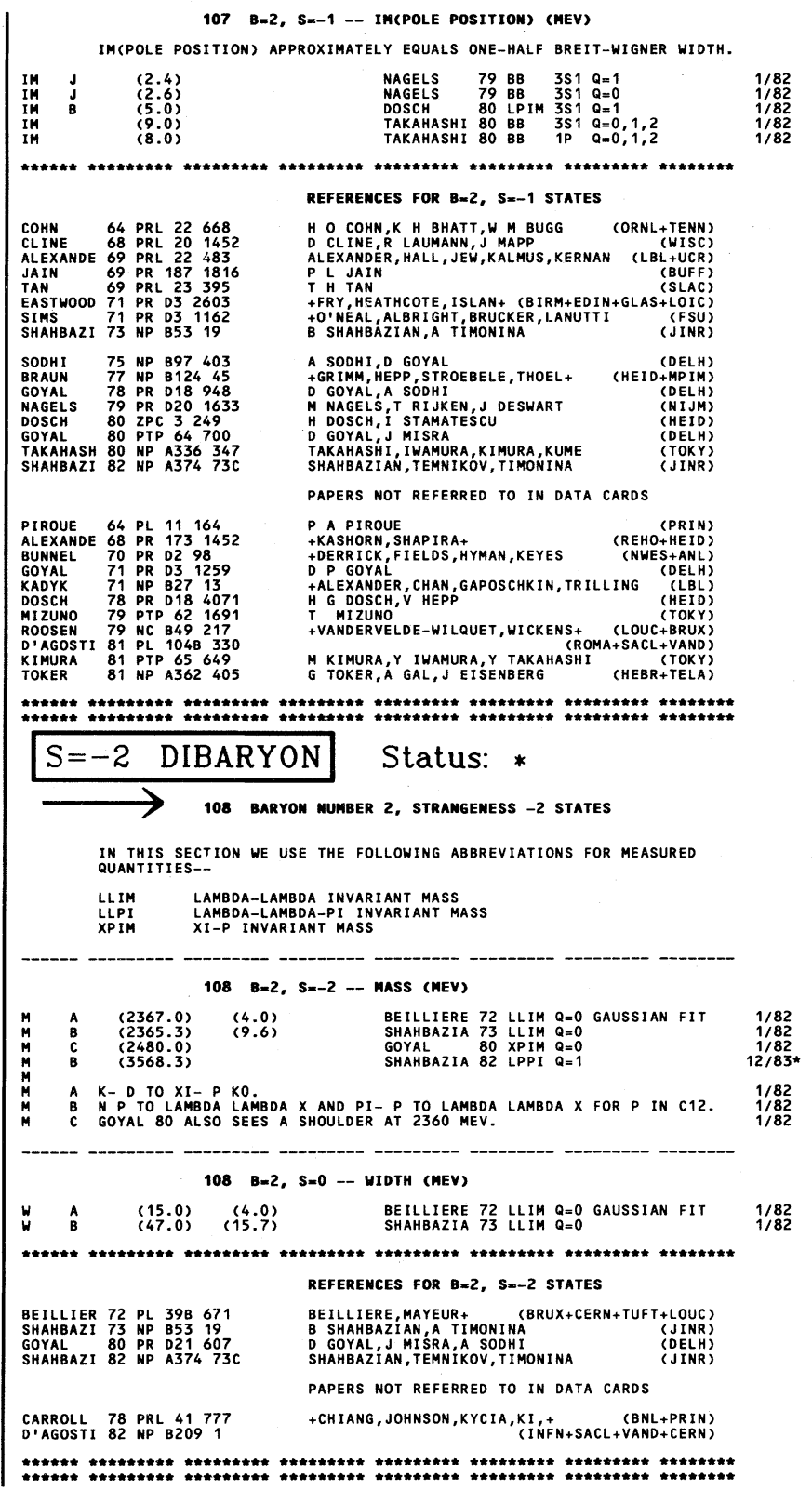




\section{APPENDIX I}

\section{THE STATUS OF THE STANDARD MODEL OF ELECTROWEAK INTERACTIONS}

(by R.N. Cahn, LBL)

The standard SU(2) $\times U(1)$ model of electroweak interactions has (aside from the masses of the fermions and the Higgs boson) three fundamental parameters. ${ }^{1}$ Thus the model is fully specified by measuring three independent constants. Two of these are well established: $G_{F}=1.16637 \times 10^{-5} \mathrm{GeV}^{-2}$ and $\alpha=1 / 137.036$. The measurement of one more parameter suffices to determine all the predictions of the theory, assuming that quark distributions in the hadrons are known and that radiative corrections can be dealt with. For the present we ignore radiative corrections.

Prior to the discoveries of the $\mathrm{W}$ and $\mathrm{Z}$, the third measurement was made in neutral-current experiments and conventionally expressed in terms of $\sin ^{2} \theta_{\mathrm{w}}$. See the section on the Standard Model of Electroweak Interactions. The usual model, which has only doublet Higgs bosons, has the value unity for the parameter $\rho$ $=\mathbf{M}_{\mathbf{W}}^{2} /\left(\mathbf{M}_{\mathbf{Z}}^{2} \cos ^{2} \theta_{\mathbf{W}}\right)$, ignoring radiative corrections. A multitude of neutral-current experiments have produced values for the mixing angle which are in good agreement and thus provide strong evidence for the standard model. Among the experiments are:

1. Neutrino and antineutrino deep inelastic scattering from isosinglet targets.

2. Neutrino and antineutrino deep inelastic scattering by protons.

3. Elastic $\nu_{\mu} \mathrm{p}$ and $\bar{\nu}_{\mu} \mathrm{p}$ scattering.

4. Exclusive and inclusive $\pi$ production in neutral-current events.

5. Neutrino disintegration of the deuteron: $\bar{\nu}_{\mathrm{e}} \mathrm{d} \rightarrow \bar{\nu}_{\mathrm{e}} \mathrm{np}$.

6. Polarized-electron deuteron deep inelastic scattering.

7. Forward-backward asymmetry in $\mathrm{e}^{+} \mathrm{e}^{-} \rightarrow \mu^{+} \mu^{-}$.

8. Elastic ve scattering.

The data for these processes and a comparison with the predictions of the standard model are given in two extensive reviews. ${ }^{2,3}$ The conclusion of these and all similar studies is that all available data are consistent with the standard model. Moreover, if it is treated as a free parameter, the value of $\rho$ is consistent with unity, the value it has in the simplest model. For example, Kim et al. find $\rho=1.002 \pm 0.015 \pm(0.011)$ and $\sin ^{2} \theta_{\mathrm{W}}=0.234 \pm 0.013 \pm$ (0.009), where the parentheses indicate a theoretical uncertainty. If only the data from deep inelastic scattering and from the e-d experiment are used, the results are $\rho=0.992 \pm 0.017 \pm(0.011)$ and $\sin ^{2} \theta_{W}=0.224 \pm 0.015 \pm(0.012)$.

Some of the most precise data are used in the accompanying figure, which shows the region of the $\rho-\sin ^{2} \theta_{W}$ plane allowed by data for deep inelastic scattering from an isoscalar target and for the scattering of polarized electrons by deuterons. It is important to notice that the allowed values of the two variables are correlated. The curves were obtained from the following formulas:

$$
\begin{aligned}
& R_{\nu N}=\frac{\sigma_{\nu N}^{N C}}{\sigma_{\nu N}^{C C}}= \\
& \rho \frac{\frac{1}{2}-\sin ^{2} \theta_{W}+\frac{20}{27} \sin ^{4} \theta_{W}+\epsilon\left(\frac{1}{6}-\frac{1}{3} \sin ^{2} \theta_{W}+\frac{20}{27} \sin ^{4} \theta_{W}\right)}{1+\frac{1}{3} \epsilon}, \\
& R_{\nu N}=\frac{\sigma_{\nu N}^{N C}}{\sigma_{\nu N}^{C C}}= \\
& \rho \frac{\frac{1}{6}-\frac{1}{3} \sin ^{2} \theta_{W}+\frac{20}{27} \sin ^{4} \theta_{W}+\epsilon\left(\frac{1}{2}-\sin ^{2} \theta_{W}+\frac{20}{27} \sin ^{4} \theta_{W}\right)}{\frac{1}{3}+\epsilon} .
\end{aligned}
$$

The parameter $\epsilon$ is the ratio of antiquark momentum to quark momentum in the nucleon. It is related to the ratio of the neutrino charged-current cross section to the antineutrino charged-current cross section:

$$
\frac{\sigma_{\nu \mathrm{N}}^{\mathrm{CC}}}{\sigma_{\nu \mathrm{N}}^{\mathrm{CC}}}=\frac{1+\frac{1}{3} \epsilon}{\frac{1}{3}+\epsilon} .
$$

The curves correspond to the limits $R_{\nu \mathrm{N}}=0.31-0.33, R_{\nu \mathrm{N}}=0.357$ -0.397 , indicative of the results of the CHARM collaboration, ${ }^{4}$ with $\epsilon$ taken to be 0.20 .

The polarized-electron deuteron scattering experiment measured the asymmetry in the cross section for left-handed $\left(\sigma_{L}\right)$ and righthanded $\left(\sigma_{R}\right)$ electrons:

$$
\mathrm{A}=\frac{\sigma_{\mathrm{R}}-\sigma_{\mathrm{L}}}{\sigma_{\mathrm{R}}+\sigma_{\mathrm{L}}}
$$

In the quark parton model, this has the form (with $\mathrm{q}^{2}>0$ for deep inelastic scattering)

$$
\frac{A}{q^{2}}=a_{1}+a_{2}\left[\frac{1-(1-y)^{2}}{1+(1-y)^{2}}\right],
$$

where $y$ is the fraction of the incident lepton's energy lost in the collision. For an isoscalar target like the deuteron, and ignoring the antiquarks, the standard model gives

$$
\begin{aligned}
& a_{1}=-\frac{G_{F}}{2 \sqrt{2} \pi \alpha} \frac{9}{10} \rho\left(1-\frac{20}{9} \sin ^{2} \theta_{W}\right) \\
& a_{2}=-\frac{G_{F}}{2 \sqrt{2} \pi \alpha} \frac{9}{10} \rho\left(1-4 \sin ^{2} \theta_{W}\right) .
\end{aligned}
$$

The best determined linear combination of the parameters $a_{1}$ and $a_{2}$ is

$$
0.24 a_{2}+0.97 a_{1}=(-8.1 \pm 1.1) \times 10^{-5} \mathrm{GeV}^{-2} .
$$

This is inferred from Ref. 5 , and the uncertainty corresponds to $1 \sigma$. The $1 \sigma$ extremes are shown in the figure.

Once the masses of the $W$ and $Z$ are known, either one or their ratio (or any other single combination) can be used as the third

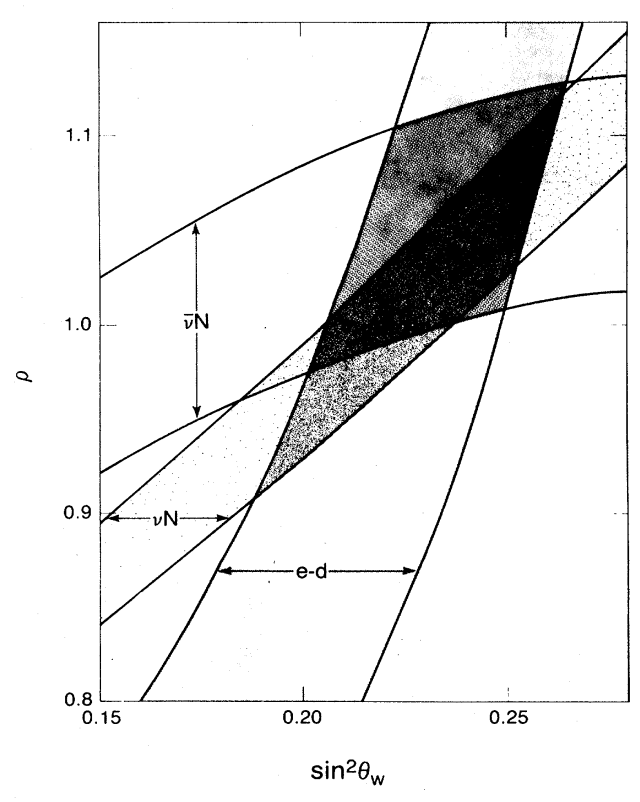


measured parameter, replacing the neutral-current data. For example, we can use the ratio of the masses as the third parameter. Then $\sin ^{2} \theta_{W}$ is simply a parameter derived from the measured quantities $\mathrm{G}_{\mathrm{F}}$ and $\alpha$. It can always be eliminated from any expression in favor of $\mathrm{G}_{\mathrm{F}}, \alpha$, and $\mathrm{M}_{\mathrm{W}} / \mathrm{M}_{\mathrm{Z}}$. It is convenient, however, to retain it by defining:

$$
\cos ^{2} \theta_{\mathbf{W}}=\mathbf{M}_{\mathbf{W}}^{2} / \mathbf{M}_{\mathbf{Z}}^{2} \text {. }
$$

This is now a definition and is not modified by radiative corrections. ${ }^{6}$ However, the lowest order prediction for $\mathrm{M}_{\mathrm{W}}^{2}$ is modified by corrections of order $\alpha$ :

$$
\mathrm{M}_{\mathrm{W}}^{2}=\frac{\pi \alpha}{\sqrt{2} \mathrm{G}_{\mathrm{F}} \sin ^{2} \theta_{\mathrm{W}}(1-\Delta \mathrm{r})} .
$$

The quantity $\Delta r$ is of order $\alpha$ and depends on the masses of the leptons, quarks, and Higgs boson. The dominant terms are due to vacuum polarization by light fermions. If the $t$ quark mass is set to $36 \mathrm{GeV}$ and the Higgs boson mass to the mass of the $Z$, the value of $\Delta r$ is $0.0696 \pm 0.0020$. This value is not very sensitive to these last two assumed values.

As of this writing (December 1983) the most recent results ${ }^{7,8}$ from the UA-1 and and UA-2 experiments at the CERN SPS collider for the $\mathrm{W}$ and $\mathrm{Z}$ masses are (in $\mathrm{GeV}$ )

$$
\begin{array}{ccc} 
& \mathrm{M}_{\mathbf{W}} & \mathrm{M}_{\mathbf{Z}} \\
\cline { 2 - 2 } \text { UA-1 } & 80.9 \pm 1.5(\text { stat }) & 95.6 \pm 1.4(\text { stat }) \\
\text { UA-2 } & 81.0 \pm 2.5 \pm 1.3 & 91.9 \pm 1.3 \pm 1.4
\end{array}
$$

Thus we find from the definition of $\cos ^{2} \theta_{W}$

$$
\begin{array}{ll} 
& \sin ^{2} \theta_{\mathrm{W}} \\
\text { UA-1 } & 0.284 \pm 0.016(\text { stat }) \\
\text { UA-2 } & 0.223 \pm 0.027 \text { (stat) }
\end{array}
$$

The systematic uncertainties may make the total uncertainty about twice as large as the indicated statistical uncertainties.

Given the uncertainties in the data above, a detailed test of the model is not possible. That the neutral-current data are consistent with $\rho=1$ and with a single value of $\sin ^{2} \theta_{W}$ is important evidence for the model. Moreover, the $\mathrm{W}$ and $\mathrm{Z}$ masses are consistent with this value for the weak mixing angle. A detailed test of the model awaits higher precision data on the weak boson masses. Such data will permit a single test involving the four measured parameters $\alpha$, $\mathrm{G}_{\mathrm{F}}, \mathrm{M}_{\mathrm{W}}$, and $\mathrm{M}_{\mathrm{Z}}$, and one additional test comparing the derived quantity $\sin ^{2} \theta_{\mathrm{W}}$ with the value obtained in neutral-current experiments. In making this comparison, it will be necessary to include radiative corrections for the neutral-current experiments. ${ }^{9}$ Their effect is to lower the values of $\sin ^{2} \theta_{W}$ discussed in connection with the neutral-current experiments to a value $0.217 \pm 0.014$.

Should such a high-precision test reveal a discrepancy with the radiatively corrected theory, it could indicate that the model needs fundamental modification. Small deviations from the predictions described above could arise if the $t$ quark mass or Higgs boson mass is far from the value assumed or if there are additional generations of fermions. At present there is no indication of the need for a modification of the theory, but the tests are not yet very stringent.

\section{References}

1. In the Lagrangian they are the $S U(2)$ coupling constant $g$, the $U(1)$ coupling constant $\mathbf{g}^{\prime}$, and the vacuum expectation value of the Higgs field. We do not consider the mass of the Higgs boson here.

2. J.E. Kim et al., Rev. Mod. Phys. 53, 211 (1981).

3. P.Q. Hung and J.J Sakurai, Ann. Rev. Nucl. Part. Sci. 31, 375 (1981).

4. M. Jonker et al., Phys. Lett. 99B, 265 (1981).

5. C. Prescott et al., Phys. Lett. 84B, 524 (1979).

6. Radiative corrections have been discussed by many authors. Here we rely on W.J. Marciano and A. Sirlin, "Testing the Standard Model by Precise Determinations of $\mathrm{W}^{ \pm}$and $\mathrm{Z}$ Masses," Phys. Rev. D29, 945 (1984).

7. UA-1 Collaboration, G. Arnison et al., Phys. Lett. 122B, 103 (1983); ibid. 126B, 398 (1983).

8. UA-2 Collaboration, M. Banner et al., Phys. Lett. 122B, 476 (1983).

9. C.H. Llewellyn-Smith and J. Wheater, Phys. Lett. 105B, 486 (1981).

\section{APPENDIX II \\ THE PERTURBATIVE QCD COUPLING CONSTANT}

\section{(by I. Hinchliffe, LBL)}

This note is concerned with the definition of the running coupling constant and of the scale parameter $\Lambda$ in QCD. It is intended to be pedagogical rather than rigorous; one of the many excellent reviews can be consulted for more details. ${ }^{1}$. Comments will be made on the theoretical uncertainties inherent in attempts to extract $\Lambda$ from the data, but no critique of individual experiments will be given.

In the limit of zero quark masses QCD contains only one fundamental parameter, its coupling constant $\left(\alpha_{\mathrm{s}}\right)$. In a field theory it is necessary to define a coupling constant order by order in perturbation theory. If a process is calculated beyond leading order, divergences can arise in the integrations over momenta flowing in closed loops of Feynman diagrams. These divergences are removed by absorbing them into a renormalized coupling constant $(\alpha)$. In order to do this, $\alpha$ is defined in terms of some quantity calculated to the same order in perturbation theory. This constitutes the renormalization scheme.

In the familiar case of QED, the coupling constant $\left(\alpha_{\mathrm{EM}}\right)$ is defined to be the value of the photon-electron-electron coupling in the limit of zero photon momentum when the electrons are on their mass shells (mass-shell renormalization scheme). This definition is readily related to the scattering rate for low-energy electrons off a static source. Notice that the relevant energy scale characterizing the coupling constant definition is the electron mass $\left(\mathrm{m}_{\mathrm{e}}\right)$. If some other $Q E D$ process with momentum scale $Q$ is calculated (e.g., $\mathrm{e}^{+} \mathrm{e}^{-} \rightarrow \mathrm{e}^{+} \mathrm{e}^{-}$at wide angle and with center-of-mass energy $\sqrt{\mathrm{s}}=\mathrm{Q} \gg \mathrm{m}_{\mathrm{e}}$ ), the cross section can be written as a power series in $\alpha_{\mathrm{EM}}$ and will have the following form

$$
\mathrm{A}\left[1+\frac{\mathrm{B} \alpha_{\mathrm{EM}}}{\pi} \log \left(\mathrm{Q}^{2} / \mathrm{m}_{\mathrm{e}}^{2}\right)+\cdots\right] \text {. }
$$

Here the term $A$ is the rate calculated to lowest nontrivial order in $\alpha_{\mathrm{EM}}$, and $\mathrm{B}$ is constant. In the $\mathrm{e}^{+} \mathrm{e}^{-} \rightarrow \mathrm{e}^{+} \mathrm{e}^{-}$case, $\mathrm{A} \propto \alpha_{\mathrm{EM}}^{2} / \mathrm{s}$. In the asymptotic limit $Q \gg m_{e}$, the term $\frac{B \alpha_{E M}}{\pi} \log \left(Q^{2} / m_{e}^{2}\right)$ could become of order one, and the perturbation series would fail to converge. Fortunately these terms, and all terms of order $\alpha_{\mathrm{EM}^{\mathrm{N}}}^{\mathrm{N}} \log ^{\mathrm{N}}\left(\mathrm{Q}^{2} / \mathrm{m}_{\mathrm{e}}^{2}\right)$, can be summed. This summation leads to the introduction of a coupling constant which depends on $Q$. This running coupling constant $\alpha_{\text {REM }}(\mathrm{Q})$ is such that $\alpha_{\mathrm{REM}}\left(\mathrm{m}_{\mathrm{e}}\right)=\alpha_{\mathrm{EM}}$, and if $\alpha_{\mathrm{REM}}(\mathrm{Q})$ is expanded as a power series in $\alpha_{\mathrm{EM}}$ and inserted 
into the term $\mathrm{A}$, all the terms $\alpha_{\mathrm{EM}}^{\mathrm{N}} \log ^{\mathrm{N}}\left(\mathrm{Q}^{2} / \mathrm{m}_{\mathrm{e}}^{2}\right)$ are reproduced.

In $\mathrm{QED}, \alpha_{\mathrm{REM}}(\mathrm{Q})$ is a rather slow function of $\mathrm{Q}$ increasing with $\mathrm{Q}$ so that $\alpha_{\mathrm{REM}}\left(\mathrm{m}_{\mathrm{w}}\right) \approx 1 / 128 .{ }^{2}$ In contrast, the running coupling constant in QCD decreases relatively rapidly as $Q$ rises due to the self-interactions of the gluons. ${ }^{3}$ The coupling constant is large at $Q$ $\approx \mathrm{m}_{\pi}$, the scale of hadronic binding. A perturbation expansion will not therefore enable one to calculate the hadron spectrum and obtain $\alpha_{\mathrm{s}}$ from the values of the hadron masses.

In the absence of strong coupling techniques we are restricted to using perturbation theory to calculate processes from QCD and to determine $\alpha_{\mathbf{s}}$. We can only use processes which have a large momentum scale and hence a small $\alpha_{\mathbf{s}}$. As an illustration consider the ratio

$$
\begin{aligned}
\mathrm{R} & =\frac{\sigma\left(\mathrm{e}^{+} \mathrm{e}^{-} \rightarrow \text { hadrons }\right)}{\sigma\left(\mathrm{e}^{+} \mathrm{e}^{-} \rightarrow \mu^{+} \mu^{-}\right)} \\
& \equiv 3 \sum_{\mathrm{i}} \mathrm{Q}_{\mathrm{i}}^{2}\left(1+\frac{\alpha_{\mathrm{s}}(\mathrm{s})}{\pi}+\mathrm{N} \frac{\alpha_{\mathrm{s}}^{2}(\mathrm{~s})}{\pi^{2}}+\cdots\right) .
\end{aligned}
$$

Here we are assuming that $\sqrt{\mathrm{s}}$, the center-of-mass energy, is large compared to quark masses; the sum i runs over all quarks of charge $\mathrm{Q}_{\mathrm{i}}$ and $\mathrm{N}$ is a numerical factor. We have used $\alpha_{\mathrm{s}}(\mathrm{s})$ since $\sqrt{\mathrm{s}}$ is the only scale present in the problem. If we assume that $\mathrm{N} \alpha_{\mathbf{s}}(\mathrm{s}) / \pi$ $<1$, then, by comparing with data to leading order in $\alpha_{\mathrm{s}}$ (s) [the $\alpha_{\mathrm{s}}(\mathrm{s}) / \pi$ term], we could determine $\alpha_{\mathrm{s}}(\mathrm{Q})$, confident that the leading terms reproduce the full perturbation series fairly well.

It is usual to introduce the parameter $\Lambda$ to parametrize the $\mathrm{Q}^{2}$ dependence of $\alpha_{\mathrm{s}}\left(\mathrm{Q}^{2}\right):{ }^{3}$

$$
\alpha_{s}\left(Q^{2}\right)=\frac{12 \pi}{(33-2 f) \log \left(Q^{2} / \Lambda^{2}\right)},
$$

where $f$ is the number of quark flavors with mass less than $Q / 2 . \quad \Lambda$ now appears as the fundamental parameter in QCD. Unfortunately if we do not know the coefficient $\mathbf{N}$ in Eq. (1), then $\Lambda$ is arbitrary in the following sense. Rescale $\Lambda=x \Lambda^{\prime}$; then

$$
\alpha_{s}\left(Q^{2}, \Lambda\right)=\alpha_{s}\left(Q^{2}, \Lambda^{\prime}\right)\left[1+\frac{(33-2 f)}{6 \pi} \alpha_{s}\left(Q^{2}, \Lambda^{\prime}\right) \log x+O\left(\alpha_{s}^{2}\right)\right] .
$$

If Eq. (1) is now rewritten as a series in $\alpha_{s}\left(\mathrm{Q}^{2}, \Lambda^{\prime}\right)$, then the coefficient $\mathrm{N}$ will change. If we have neglected this term, then $\Lambda$ and $\Lambda^{\prime}$ are equivalent! A fit to the data to leading order can of course still be used to determine a $\Lambda_{\mathrm{LO}}$ defined by Eq. (2), but we have no guarantee that another process will yield the same $\Lambda_{\mathrm{LO}}$. The situation only clarifies (or becomes more muddled depending on one's point of view) when we know the coefficient $\mathrm{N}$.

We should of course check that $N \alpha_{s}(s) / \pi<<1$, so that the perturbation series is reliable. Unfortunately $N$ is not well defined; it depends on the renormalization scheme. We cannot define $\alpha_{\mathrm{s}}$ in the same way as $\alpha_{\mathrm{EM}}$ was defined in QED, since perturbation theory is unreliable in that region of momenta. One possibility is to define $\alpha_{s}\left(\mathrm{Q}^{2}\right)$ as the value of the three-gluon vertex (or quarkquark-gluon vertex) when the invariant masses of the particles are $-\mathrm{Q}^{2}$. This is the momentum-space scheme, ${ }^{4}$ and $\alpha_{\mathrm{s}}$ is denoted by $\alpha_{\mathrm{MOM}}\left(\mathrm{Q}^{2}\right) . \alpha_{\mathrm{MOM}}\left(\mathrm{Q}^{2}\right)$ is not related directly to any physical process. The scheme is difficult to calculate with and produces a gauge-dependent $\alpha_{\mathrm{s}}\left(\mathrm{Q}^{2}\right)$; much more convenient is the minimalsubtraction scheme (MS). Here loop integrals are evaluated in $n$ dimensions. The divergences appear as singularities of the form $1 /(\mathrm{n}-4)$. These terms are dropped and $\alpha_{\mathrm{MS}}\left(\mathrm{Q}^{2}\right)$ so defined. ${ }^{5}$ These singularities are always accompanied by $\log 4 \pi$ and the Euler constant $\gamma_{E}=0.577 \ldots$; these can be dropped also (the $\overline{\mathrm{MS}}$ scheme) and an $\alpha \overline{\mathrm{MS}}\left(\mathrm{Q}^{2}\right)$ defined. ${ }^{6}$ The coupling constants in all these schemes are related as follows $\left(\alpha_{\mathrm{MOM}}\right.$ is defined in Feynman gauge):

$$
\begin{gathered}
\alpha_{\mathrm{MOM}}\left(\mathrm{Q}^{2}\right)=\alpha_{\mathrm{MS}}\left(\mathrm{Q}^{2}\right)\left[1+\mathrm{x} \alpha_{\mathrm{MS}}\left(\mathrm{Q}^{2}\right) \cdots\right], \\
\mathrm{x}=3.57-0.38 \mathrm{f}
\end{gathered}
$$

$$
\begin{gathered}
\alpha_{\mathrm{MOM}}\left(\mathrm{Q}^{2}\right)=\alpha_{\overline{\mathrm{MS}}}\left(\mathrm{Q}^{2}\right)\left[1+\mathrm{x}^{\prime} \alpha \overline{\mathrm{MS}}\left(\mathrm{Q}^{2}\right) \cdots\right], \\
\mathbf{x}^{\prime}=1.86-0.48 \mathrm{f} .
\end{gathered}
$$

The $\overline{\mathrm{MS}}$ and MOM schemes are most frequently used. Other schemes are also possible; for example we could define $\alpha_{\mathrm{R}}\left(\mathrm{Q}^{2}\right)$ so that

$$
\mathrm{R} \equiv 3 \Sigma \mathrm{Q}_{\mathrm{i}}^{2}\left[1+\frac{\alpha_{\mathrm{R}}\left(\mathrm{Q}^{2}\right)}{\pi}\right]
$$

with no correction at $\mathrm{Q}^{2}=\mathrm{s}_{0}$. Then

$$
\alpha_{\mathbf{R}}\left(\mathrm{s}_{0}\right)=\alpha \overline{\mathrm{MS}}\left(\mathrm{s}_{0}\right)\left[1+\mathrm{N}^{\prime} \frac{\alpha \overline{\mathrm{MS}}\left(\mathrm{s}_{0}\right)}{\pi}\right]
$$

with $\mathrm{N}^{\prime}=2-0.1 \mathrm{f}^{7}$

It is now clear that $\mathbf{N}$ will depend on the scheme so that the size of $\mathrm{N} \alpha_{\mathbf{s}} / \pi$ is a scheme dependent. This simple fact can lead to long discussions about which scheme is best and which processes have reliable perturbation expansions. ${ }^{8}$

Beyond leading order Eq. (2) does not accurately represent the $\mathrm{Q}^{2}$ dependence of $\alpha_{\mathrm{s}}\left(\mathrm{Q}^{2}\right)$. To next order we have

$$
\begin{aligned}
& \alpha_{s}\left(Q^{2}\right)=\frac{12 \pi}{(33-2 f) \log \left(Q^{2} / \Lambda^{2}\right)} \\
& \times\left\{1-\frac{6(153-19 f)}{(33-2 f)^{2}} \frac{\log \left[\log \left(Q^{2} / \Lambda^{2}\right)\right]}{\log \left(Q^{2} / \Lambda^{2}\right)}+\frac{D}{\log \left(Q^{2} / \Lambda^{2}\right)}\right\}
\end{aligned}
$$

where $\mathrm{D}$ is arbitrary to this order. If $\alpha=\alpha \overline{\mathrm{MS}}$, it is customary ${ }^{6}$ to define $\Lambda_{\overline{\mathrm{MS}}}$ from Eq. (3) with $\mathrm{D}=0$; but other definitions are possible. It seems that some confusion could be avoided by abolishing $\Lambda$ and simply quoting $\alpha_{s}\left(Q^{2}\right)$ in some scheme at some $\mathrm{Q}^{2}=\mathrm{Q}_{0}^{2}$.

There are several more theoretical complications in QCD predictions. In the case of $R$ the scale $Q^{2}$ was unambiguous; this is not always the case. For example, the production of three jets in $\mathrm{e}^{+} \mathrm{e}^{-}$ collisions is predicted by $Q C D$ but is $Q^{2}$ equal to $s$ or to the invariant mass of a jet pair? To leading order in QCD, we cannot tell. The coefficient of the next-to-leading term will change if $\mathrm{Q}^{2}$ is changed (c.f., the shift in $N$ as $\Lambda$ was rescaled above), further complicating the issue of whether the perturbation series is reliable.

Many processes are also subject to "higher twist corrections." For example, if we retain quark masses, $\mathrm{R}$ receives corrections depending on $\mathrm{m}_{\mathrm{q}}^{2} / \mathrm{s}$ so that extra parameters enter $\mathrm{QCD}$ predictions. Some higher twist corrections are purely kinematic in origin and can be calculated if the quark masses are known. Unfortunately other higher twist corrections cannot be calculated (e.g., those in deep inelastic scattering); they are all of order $1 / \mathrm{Q}^{2}$ relative to the perturbation theory, but the coefficients are unknown.

In comparing quoted values for $\alpha_{\mathrm{s}}$ or $\Lambda$, the following must be considered:

1. What order in perturbation theory was used?

2. What renormalization scheme was used?

3. What scale $Q^{2}$ was used?

4. If the $\mathrm{Q}$ range of the experiment covers some quark masses, how were thresholds dealt with?

5. How were higher twist terms parametrized?

For example, attempts have been made to extract $\alpha_{\mathrm{s}}$ (or $\Lambda$ ) from data on 3-jet events in $\mathrm{e}^{+} \mathrm{e}^{-}$annihilation. Issues 1 and 5 appear to be pertinent here. The perturbation expansion is not very reliable ${ }^{9}$ so the value of $\Lambda$ depends on the order used. Issue 5 manifests itself in the dependence of $\alpha_{\mathrm{s}}$ upon Monte Carlo program parameters used to parametrize jet fragmentation. ${ }^{10}$ Values of $\alpha_{\mathrm{s}}$ quoted from these data range from 0.12 to $0.21 .^{10}$ In contrast, data on deep inelastic scattering do not suffer from problems concerning the perturbation theory, and values of $\Lambda_{\mathrm{L} 0}$ range from 125 to 275 MeV. ${ }^{11}$ 


\section{References}

1. See, for example, F.J. Ynduráin, "Quantum Chromodynamics", Springer Verlag (1983). G.P. Lepage, from a talk given at Lepton Photon Meeting, Cornell Univ. (1983).

2. W. Marciano and A. Sirlin, Phys. Rev. Lett. 46, 163 (1981).

3. H.D. Politzer, Phys. Rev. Lett. 30, 1346 (1973). D.J. Gross and F. Wilczek, Phys. Rev. Lett. 30, 1323 (1973); Phys. Rev. D8, 3635 (1973); and Phys. Rev. D9, 980 (1974).

4. W. Celmaster and R.J. Gonsalves, Phys. Rev. D20, 1420 (1979).
5. G. t'Hooft, Nucl. Phys. B61, 455 (1973).

6. W.A. Bardeen et al., Phys. Rev. D18, 3998 (1978).

7. M. Dine and J. Saperstein, Phys. Rev. Lett. 43, 668 (1979).

8. I. Hinchliffe, in Proceedings of the APS-DPF Conference, Santa Cruz (1981). See Lepage, Ref. 1.

9. R.K. Ellis, D.A. Ross, and A.T. Terrano, Phys. Rev. Lett. 45, 1226 (1980).

10. J. Dorfan, from a talk given at Lepton Photon Meeting, Cornell (1983).

11. F. Dydak, from a talk given at Lepton Photon Meeting, Cornell (1983).

\section{APPENDIX III}

\section{THE KOBAYASHI-MASKAWA MIXING MATRIX}

\section{(by F.J. Gilman, SLAC)}

In the "standard model" with $\mathrm{SU}(2) \times \mathrm{U}(1)$ as the gauge group of electroweak interactions, both the quarks and leptons are assigned to be left-handed doublets and right-handed singlets. The quark mass eigenstates are not the same as the weak eigenstates, and the matrix connecting them has become known as the KobayashiMaskawa matrix since an explicit parametrization in the six-quark case was first published by them in 1973 .

By convention, the three charge $2 / 3$ quarks ( $u, c$, and $t$ ) are unmixed, and all the mixing is expressed in terms of a $3 \times 3$ unitary matrix $\mathrm{V}$ operating on the charge $-1 / 3$ quarks $(\mathrm{d}, \mathrm{s}, \mathrm{b})$ :

$$
\left(\begin{array}{c}
d^{\prime} \\
s^{\prime} \\
b^{\prime}
\end{array}\right)=\left(\begin{array}{lll}
v_{u d} & v_{u s} & v_{u b} \\
v_{c d} & v_{c s} & v_{c b} \\
v_{t d} & v_{t s} & v_{t b}
\end{array}\right)\left(\begin{array}{l}
d \\
s \\
b
\end{array}\right)
$$

Kobayashi and Maskawa ${ }^{1}$ themselves used a parameterization involving four angles, $\theta_{1}, \theta_{2}, \theta_{3}, \delta$ :

$$
\left(\begin{array}{l}
d^{\prime} \\
s^{\prime} \\
b^{\prime}
\end{array}\right)=\left(\begin{array}{ccc}
c_{1} & -s_{1} c_{3} & -s_{1} s_{3} \\
s_{1} c_{2} & c_{1} c_{2} c_{3}-s_{2} s_{3} e^{i \delta} & c_{1} c_{2} s_{3}+s_{2} c_{3} e^{i \delta} \\
s_{1} s_{2} & c_{1} s_{2} c_{3}+c_{2} s_{3} e^{i \delta} & c_{1} s_{2} s_{3}-c_{2} c_{3} e^{i \delta}
\end{array}\right)\left(\begin{array}{l}
d \\
s \\
b
\end{array}\right),
$$

where $c_{i}=\cos \theta_{i}$ and $s_{i}=\sin \theta_{i}$ for $\mathrm{i}=1,2,3$. In the limit $\theta_{2}=\theta_{3}=$ 0 , this reduces to the usual Cabibbo mixing with $\theta_{1}$ identified with the Cabibbo angle (up to a sign). The angles $\theta_{1}, \theta_{2}, \theta_{3}$ can all be made to lie in the first quadrant (so that all $s_{i}, c_{i}$ are positive) by an appropriate redefinition of quark field phases.

Slightly different forms of the Kobayashi-Maskawa parametrization are found in the literature. The K-M matrix used in the 1982 Review of Particle Properties is obtained by letting $s_{1} \rightarrow-s_{1}$ and $\delta$ $\rightarrow \delta+\pi$ in the matrix given above. An alternative used in another review ${ }^{2}$ is to change Eq. (2) by $s_{1} \rightarrow-s_{1}$ but leave $\delta$ unchanged. With this change in $s_{1}, \theta_{1}$ becomes the usual Cabibbo angle, with the "correct" sign (i.e., $\mathrm{d}^{\prime}=\mathrm{d} \cos \theta_{1}+\mathrm{s} \sin \theta_{1}$ ), in the limit $\theta_{2}=\theta_{3}$ $=0$. The angles $\theta_{1}, \theta_{2}, \theta_{3}$ can, as before, all be taken to lie in the first quadrant by adjusting quark field phases. Since all these parametrizations are referred to as "the" Kobayashi-Maskawa form, some care about which one is being used is needed when the quadrant in which $\delta$ lies is under discussion.

A quite different parametrization is due to Maiani ${ }^{3}$ in terms of angles $\theta, \beta, \gamma$, and $\delta^{\prime}$ :

$$
\mathrm{V}=\left(\begin{array}{ccc}
\mathrm{c}_{\beta} \mathrm{c}_{\theta} & \mathrm{c}_{\beta} \mathrm{s}_{\theta} & \mathrm{s}_{\beta} \\
-\mathrm{s}_{\gamma} \mathrm{c}_{\theta} \mathrm{s}_{\beta} \mathrm{e}^{\mathrm{i} \delta^{\prime}}-\mathrm{s}_{\theta} \mathrm{c}_{\gamma} & \mathrm{c}_{\gamma} \mathrm{c}_{\theta}-\mathrm{s}_{\gamma} \mathrm{s}_{\beta} \mathrm{s}_{\theta} \mathrm{e}^{\mathrm{i} \delta^{\prime}} & \mathrm{s}_{\gamma} \mathrm{c}_{\beta} \mathrm{e}^{\mathrm{i} \delta^{\prime}} \\
-\mathrm{s}_{\beta} \mathrm{c}_{\gamma} \mathrm{c}_{\theta}+\mathrm{s}_{\gamma} \mathrm{s}_{\theta} \mathrm{e}^{-\mathrm{i} \delta^{\prime}} & -\mathrm{c}_{\gamma} \mathrm{s}_{\beta} \mathrm{s}_{\theta}-\mathrm{s}_{\gamma} \mathrm{c}_{\theta} \mathrm{e}^{-\mathrm{i} \delta^{\prime}} & \mathrm{c}_{\gamma} \mathrm{c}_{\beta}
\end{array}\right)
$$

where $c_{\beta}=\cos \beta, s_{\beta}=\sin \beta$, etc. With $\beta=\gamma=0$, the first two generations of quarks decouple from the third, and $\theta$ is directly the
Cabibbo angle. No physics can depend on which of the above parametrizations (or any other) is used as long as it is used consistently and care is taken to be sure that no other choice of phases is in conflict.

The values of individual Kobayashi-Maskawa matrix elements can in principle all be determined from weak decays of the relevant quarks, or, in some cases, from deep inelastic neutrino scattering. Our present knowledge comes from the following sources:

(1) Nuclear beta decay, when compared to muon decay, gives

$$
\left|\mathrm{V}_{\text {ud }}\right|=0.9737 \pm 0.0025
$$

in one evaluation ${ }^{4}$ and

$$
\left|\mathrm{V}_{\mathrm{ud}}\right|=0.9730 \pm 0.0024
$$

in another. ${ }^{5}$

(2) An analysis of hyperon and $K_{\ell 3}$ decays yielded ${ }^{4}$

$$
\left|\mathrm{V}_{\text {us }}\right|=0.219 \pm 0.011 \text {, }
$$

where the quoted uncertainty includes a statistical minimization error of \pm 0.003 and an estimate of $\sim 5 \%$ theoretical uncertainty due to SU(3) symmetry breaking. For the hyperon decays this involved a simultaneous $\chi^{2}$ fit to $V_{\text {us }}$ and the SU(3) parameter $\alpha_{D}=D /(D+F)$. A subsequent analysis using some new data on hyperon decays and similar theoretical inputs for form factors, etc., gave 5

$$
\left|\mathrm{V}_{\text {us }}\right|=0.227 \pm 0.003 \pm 0.013
$$

The first error is statistical and the second represents an estimate of the effect of SU(3) symmetry breaking. A recent CERN hyperon experiment carried out a series of different Cabibbo fits with various experimental and theoretical inputs. ${ }^{6}$ Results are quoted for the effective "vector" and "axial-vector" Cabibbo angles and the overall Cabibbo angles (see Table III of Ref. 6). A representative value is

$$
\left|\mathrm{V}_{\mathrm{us}}\right|=0.231 \pm 0.003
$$

where the quoted error is statistical and does not include an estimate of the uncertainty due to $\mathrm{SU}(3)$ symmetry breaking.

(3) From neutrino and antineutrino production of charm, the CDHS group has deduced ${ }^{7}$

$$
\left|\mathrm{V}_{\mathrm{cd}}\right|=0.24 \pm 0.03 \text {. }
$$

Values of $\left|V_{c s}\right|$ from such experiments are dependent on assumptions about the strange quark density in the parton-sea and are not well enough constrained to give information which is not already in hand from $\left|V_{c d}\right|$ and $\left|V_{c b}\right|$ (see below), plus unitarity.

(4) The ratio $\left|V_{u b} / V_{c b}\right|$ is obtained from the semileptonic decay of $\mathrm{B}$ mesons by fitting to the lepton energy spectrum as a sum of contributions involving $b \rightarrow u$ and $b \rightarrow c$. The relative overall phase space factor between the two processes is calculated from the usual four-fermion interaction with one massive fermion 
(c quark or u quark) in the final state. The value of this factor is between 0.4 and 0.5 , depending on the quark masses used. We use 0.45 , in which case the experimental lack of observation of the hard lepton spectrum characteristic of $b \rightarrow u \overline{\nu_{\ell}}$, which is quoted as a limit $^{8}$

$$
\frac{\Gamma\left(\mathrm{b} \rightarrow \mathrm{u} \overline{\nu_{\ell}}\right)}{\Gamma\left(\mathrm{b} \rightarrow \overline{c \bar{\nu}_{\ell}}\right)}<0.05,
$$

translates to

$$
\frac{\left|\mathrm{v}_{\mathrm{ub}}\right|}{\left|\mathrm{v}_{\mathrm{uc}}\right|}<0.15
$$

There are some theoretical uncertainties in this analysis stemming from the fact that the physical decays involve actual hadrons, primarily pseudoscalar B mesons, and not free quarks as is assumed in the calculations of the lepton spectra for $b \rightarrow u \overline{\nu_{\ell}}$ and $b \rightarrow$ $\overline{c \nu}$.

(5) The magnitude of $V_{c b}$ itself can be determined if the meas ured semileptonic bottom hadron partial width is assumed to be that of $a b$ quark decaying through the usual V-A interaction:

$$
\Gamma\left(\mathrm{b} \rightarrow \overline{c \nu_{\ell}}\right)=\frac{B R\left(b \rightarrow c \overline{\ell \nu}_{\ell}\right)}{\tau_{b}}=\frac{G_{\mathrm{F}}^{2} \mathrm{~m}_{\mathrm{b}}^{5}}{192 \pi^{3}} \mathrm{~F}\left(\mathrm{~m}_{\mathrm{b}}, \mathrm{m}_{\mathrm{c}}\right)\left|\mathrm{V}_{\mathrm{cb}}\right|^{2},
$$

where $\tau_{b}$ is the $b$ lifetime and $F\left(m_{b}, m_{c}\right)$ is the phase space factor chosen above as 0.45 . Following $\mathrm{S}$. Stone ${ }^{8}$ and using an average semileptonic branching ratio of $0.116 \pm 0.006$ (which from Eq. (10) is $\operatorname{BR}\left(\mathrm{b} \rightarrow c \overline{\ell \nu}_{\rho}\right)$ to withing $\left.5 \%\right)$, a bottom hadron lifetime between 0.6 and $1.4 \times 10^{-12} \mathrm{sec}$, and $\mathrm{m}_{\mathrm{b}}$ between 4.8 and $5.2 \mathrm{GeV} / \mathrm{c}^{2}$ :

$$
0.036<\left|V_{c b}\right|<0.070 \text {. }
$$

Using Eqs. (4), (6), (11), and (13) together with unitarity and the assumption that there are only three generations, the $90 \%$ confidence limits on the magnitude of matrix elements of the complete matrix are: 8

$$
\left(\begin{array}{lllllr}
0.9705 & \text { to } 0.9770 & 0.21 & \text { to } 0.24 & 0 . & \text { to } 0.014 \\
0.21 & \text { to } 0.24 & 0.971 & \text { to } 0.973 & 0.036 & \text { to } 0.070 \\
0 . & \text { to } 0.024 & 0.036 \text { to } 0.069 & 0.997 \text { to } 0.999
\end{array}\right) \text {. }
$$

Similar values for these matrix elements, are found in several other analyses. 9,10 The ranges shown are for the individual matrix elements. The constraints of unitarity connect different elements, so choosing a specific value for one element restricts the range of the others. The data do not preclude there being more than three generations. However, the entries deduced from unitarity might be altered when the K-M matrix is expanded to accommodate more generations.
Further information on the angles requires theoretical assumptions. In particular, as CP-violating amplitudes involve $\sin \delta$, assuming that observed $\mathrm{CP}$ violation is solely related to a nonzero value of $\delta$ allows additional constraints to be brought to bear. While hadronic matrix elements whose values are imprecisely known now enter, the constraints from $\mathrm{CP}$ violation in the neutral kaon system are tight enough that there may be no solution at all for certain quark masses, values of $\delta$, etc. See Refs. 11, 12, and 13. Additional correlated bounds on quark masses and mixing angles can be obtained from $\mathrm{K}_{\mathrm{L}}^{0} \rightarrow \mu^{+} \mu^{-}$, which involves a hadronic matrix element known from $\mathrm{K}_{\ell 3}$ decay. ${ }^{14}$

\section{References}

1. M. Kobayashi and K. Maskawa, Prog. Theor. Phys. 49, 652 (1983).

2. L.L Chau, Phys. Rep. 95, 1 (1983).

3. L. Maiani, in Proceedings of the 1977 International Symposium on Lepton and Photon Interactions at High Energies (DESY, Hamburg, 1977), p. 867.

4. R.E. Shrock and L.L Wang, Phys. Rev. Lett. 41, 1692 (1978).

5. E.A. Paschos and U. Turke, Phys. Lett. 116B, 360 (1982).

6. M. Bourquin et al., Z. Phys. C21, 27 (1983).

7. H. Abramowicz et al., Z. Phys. C15, 19 (1982).

8. The $\mathbf{b}$ decay data are reviewed by $\mathbf{S}$. Stone in Proceedings of the International Symposium on Lepton and Photon Interactions at High Energies (Cornell University, Ithaca, 1984), p. 203. See also C. Klopfenstein et al., Phys. Lett. 130B, 444 (1983), who give 0.055 for the ratio in Eq. (10).

9. K. Kleinknecht and B. Renk, Phys. Lett. 130B, 459 (1983).

10. E.A. Paschos and U. Turke, Institute of Theoretical Physics preprint NSF-ITP-83-168, 1983 (unpublished).

11. P. Ginsparg, S. Glashow, and M. Wise, Phys. Rev. Lett. 50, 1415 (1983), consider constraints on the t quark mass.

12. F.J. Gilman and J.S. Hagelin, Phys. Lett. 133B, 433 (1983), consider constraints on $\epsilon^{\prime} / \epsilon$.

13. Paschos and Turke (Ref. 10), and S. Pakvasa, Phys. Rev. D28, 2915 (1983), consider constraints on $B^{0}-\bar{B}^{0}$ mixing.

14. R.E. Shrock and M.B. Voloshin, Phys. Lett. 87B, 375 (1979); and T. Inami and C.S. Lim, Prog. Theor. Phys. 65, 297 (1981). Dispersive contributions are further discussed by $\mathrm{V}$. Barger et al., Phys. Rev. D25, 1860 (1981); it has recently been argued by F.J. Gilman and J.S. Hagelin, Phys. Lett. 126B, 111 (1983), that these are small, in agreement with the original analysis above. 


\section{RECENT PARTICLE PHYSICS COMPILATIONS}

\section{Compilation of Coupling Constants and Low-Energy Parameters}

O. Dumbrajs, R. Koch, H. Pilkuhn, G.C. Oades, H. Behrens, J.J. de Swart, P. Kroll Nuclear Physics B216, 277 (1983)

Pion Nucleon Scattering: 1) Tables of Data, 2) Methods and Results of Phenomenological Analyses G. Höhler (ed. H. Schopper) Landolt-Börnstein New Series Volumes I/9 b1 (1982) and I/9 b2 (1983)

Compilation of Cross Sections I: $\pi^{+}$and $\pi^{-}$Induced Reactions V. Flaminio, W.G. Moorhead, D.R.O. Morrison, N. Rivoire CERN-HERA Report 83-01 (1983)

Compilation of Cross Sections II: $\mathrm{K}^{+}$and $\mathrm{K}^{-}$Induced Reactions V. Flaminio, W.G. Moorhead, D.R.O. Morrison, N. Rivoire CERN-HERA Report 83-02 (1983)

Compilation of Cross Sections III: $p$ and $\bar{p}$ Induced Reactions

V. Flaminio, W.G. Moorhead, D.R.O. Morrison, N. Rivoire CERN-HERA Report 84-01 (1984)

Scattering of Elementary Particles: NN and KN

J. Bystricky, P. Carlson, C. Lechanoine, F. Lehar, F. Mönnig, K.R. Schubert (ed. H. Schopper) Landolt-Börnstein New Series Volume I/9 a (1980)

Current Experiments in Elementary Particle Physics

C.G. Wohl, F.E. Armstrong, A. Rittenberg, T.G. Trippe, G.P. Yost, L. Addis, P. Joos, Y. Oyanagi,

D.C. Dodder, S.N. Grudtsin, Yu.G. Ryabov, R. Frosch, A. Olin Lawrence Berkeley Laboratory Report LBL-91, revised (1983)

Major Detectors in Elementary Particle Physics

G. Gidal, B. Armstrong, A. Rittenberg Lawrence Berkeley Laboratory Report LBL-91, supplement (1983)

Table of Isotopes, $7^{\text {th }}$ Edition

C.M. Lederer, V.S. Shirley, E. Browne, J.M. Dairiki, R.E. Doebler, A.A. Shihab-Eldin, L.J. Jardine, J.K. Tuli, A.B. Buyrn John Wiley \& Sons, New York (1978) 


\section{ACCESSING AND USING PARTICLE PHYSICS DATABASES}

A number of publicly accessible computer databases containing particle physics information now exist at various institutions. Some of these databases are for literature searching, allowing the user to locate papers of interest, while others contain actual numerical data. The following discussion gives some idea of what is available and how to get started using these databases. The two locations covered are SLAC and Rutherford Appleton Laboratory (RAL).

\section{The SLAC Particle Physics Databases}

The databases of interest at SLAC are: (1) HEP, a literature-searching guide for all particle physics journal articles, preprints, reports, theses, etc., indexed by the standard bibliographic quantities and, starting sometime in mid1984, also indexed by accelerator, detector, beam momentum, reactions and particles studied, etc.; (2) RPP, containing the Data Card Listings from the Review of Particle Properties, indexed by particle property; and (3) EXPERIMENTS, a guide to current and past particle physics experiments, indexed similarly to the HEP database. In addition, it is hoped that the Durham-RAL databases discussed below will also be available at SLAC in the near future.

All these databases are managed by the SPIRES database management system, which runs interactively under VM/CMS on SLAC's mainframe IBM computers. To enter SPIRES, once you are logged onto the computer, key in CALL SPIRES. You can then obtain information about the database you are interested in by typing in, say, EXPLAIN RPP. To actually access the database, enter, for example, SELECT RPP. You may then find out what terms are available for searching on by keying in SHOW INDEXES. To see the form of the contents of a particular index, say the PP (particle property) index of the RPP database, key in BROWSE PP; this will give you an idea of what kinds of expressions appear in this index, and thus will suggest what form you should use in your search. Then to do an actual search for information, say for the RPP Data Card Listings on the $\eta$ meson mass, you would key in a command like FIND PP ETA MASS, followed by the command TYPE; this would print out the Listings for the $\eta$ mass. At any time, you may get help by typing in such commands as EXPLAIN EXPLAIN, EXPLAIN SHOW INDEXES, EXPLAIN BROWSE, EXPLAIN FIND, EXPLAIN TYPE, etc. When you are finished searching, key in EXIT, which gets you out of SPIRES.

Anyone who has an account on the SLAC computing system can access these databases online. If you do not have an account and cannot find anyone who does (at main laboratories, ask at the library), please contact SLAC directly. An extensive wall poster, "A Guide to VM Spires," is available from the SLAC library. For more information, contact Alan Rittenberg at LBL (CMS-id AXRVX, tel. 415-486-4723, or 451-4723 on FTS), or Louise Addis at SLAC (CMS-id ADDIS, tel. 415-854-3300, ext. 2411).

\section{The Durham-RAL Particle Physics Databases}

These databases contain compilations of current and past experimental particle physics data (e.g., reaction cross sections), and are available for interactive searching under CMS on Rutherford Appleton Laboratory's mainframe IBM computer. The topics included are: (1) two-body (and quasi-two-body) reactions; (2) hadron and photon one- and two-particle inclusive distributions; (3) lepton-produced inclusive data (i.e., deep inelastic scattering, structure functions, etc.); and (4) data from $\mathrm{e}^{+} \mathrm{e}^{-}$annihilations. The databases also contain complete bibliographic information on these and other related topics, plus status information of current particle physics experiments. To insure that the databases are up to date, experimentalists are urged to send their data to the compilers immediately on completion.

The databases can be easily used by anyone having network access to the RAL computers; a guest identifier PDG (password PDG) is available for those who do not have their own CMS account. An EXEC file, HEPDATA, on the UDISK gives direct access to the databases, and contains an extensive built-in HELP facility to assist the unfamiliar user. Data is retrieved using a simple keywordbased search, and can be displayed in either tabular or graphical form.

For more information, or a guide to the service, please contact Michael Whalley at Durham University, England (CMS-id MRW; tel. 0385-64971, ext. 591), or Richard Roberts at RAL (CMS-id RGR; tel. 0235-21900, ext. 5259). 


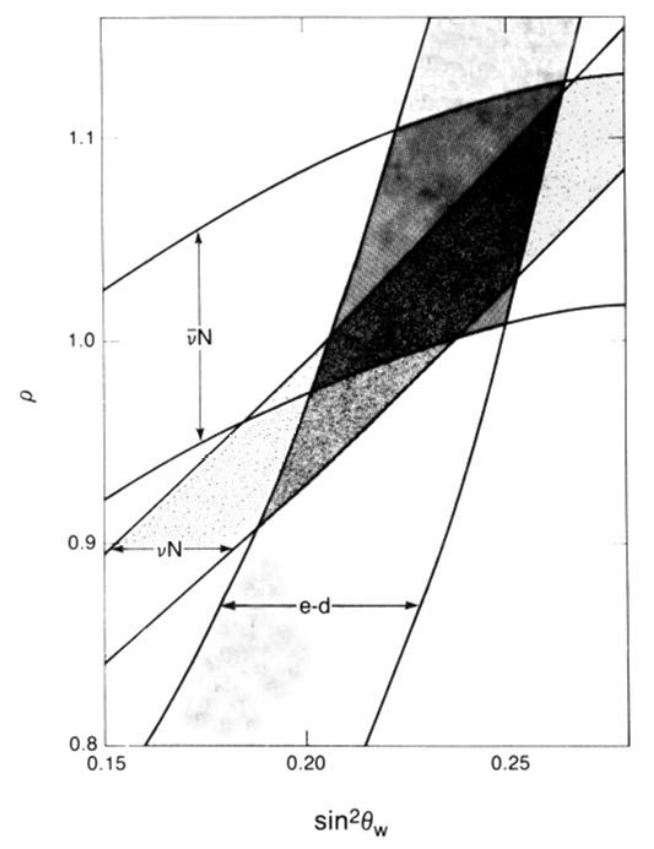

